\title{
ONTOGENY AND \\ PHYLOGENY OF BRAIN BARRIER MECHANISMS
}

EDITED BY: Norman R. Saunders, Helen B. Stolp and Shane A. Liddelow PUBLISHED IN : Frontiers in Neuroscience

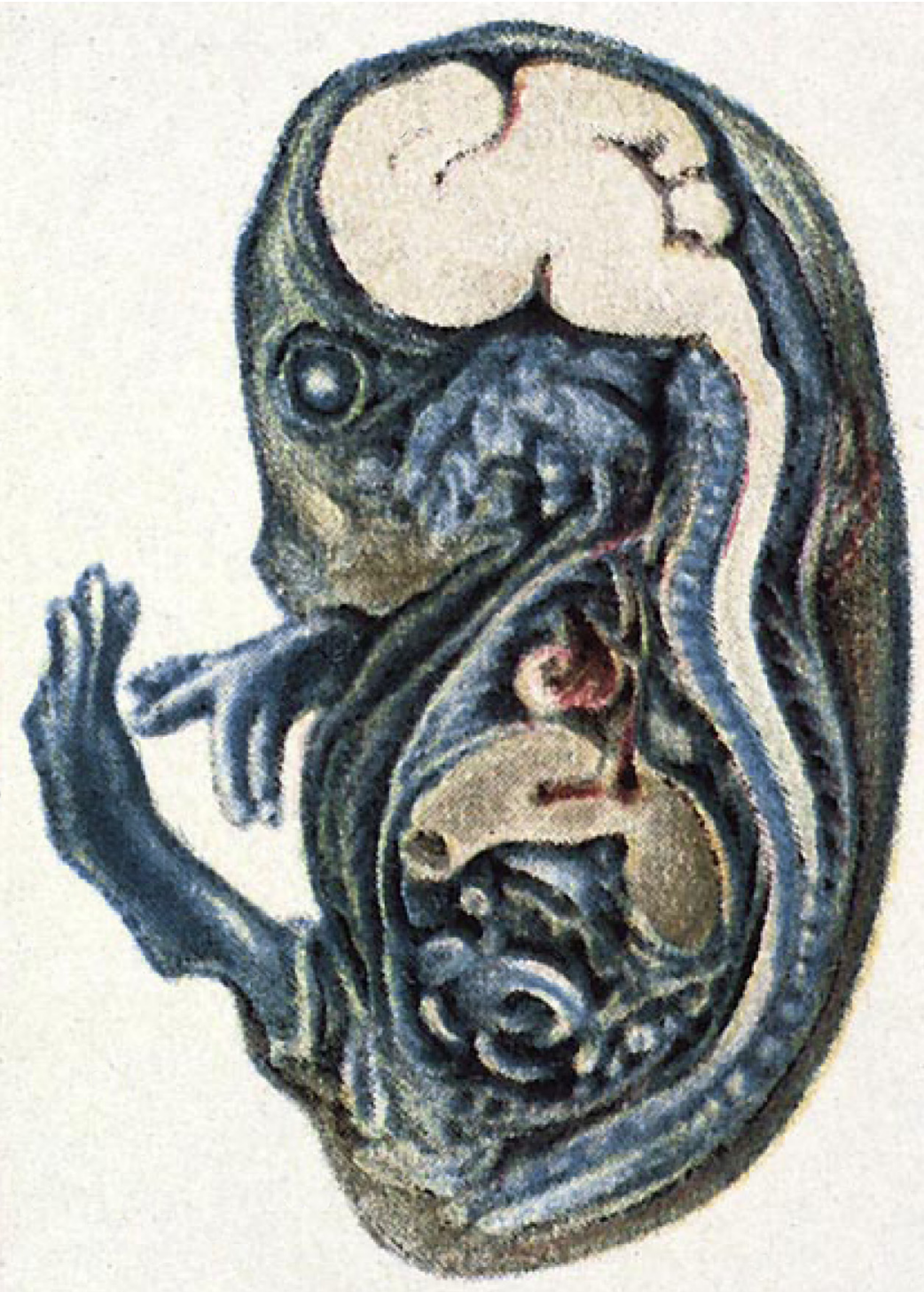




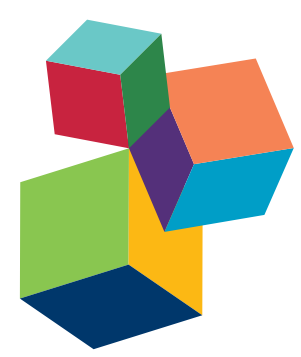

Frontiers Copyright Statement

(c) Copyright 2007-2016 Frontiers Media SA. All rights reserved.

All content included on this site, such as text, graphics, logos, button icons, images, video/audio clips, downloads, data compilations and software, is the property of or is licensed to Frontiers Media SA

("Frontiers") or its licensees and/or subcontractors. The copyright in the text of individual articles is the property of their respective authors, subject to a license granted to Frontiers.

The compilation of articles constituting this e-book, wherever published, as well as the compilation of all other content on this site, is the exclusive property of Frontiers. For the conditions for downloading and copying of e-books from Frontiers' website, please see the Terms for Website Use. If purchasing Frontiers e-books from other websites or sources, the conditions of the website concerned apply.

Images and graphics not forming part of user-contributed materials may not be downloaded or copied without permission.

Individual articles may be downloaded and reproduced in accordance with the principles of the CC-BY licence subject to any copyright or other notices. They may not be re-sold as an e-book.

As author or other contributor you grant a CC-BY licence to others to reproduce your articles, including any graphics and third-party materials supplied by you, in accordance with the Conditions for Website Use and subject to any copyright notices which you include in connection with your articles and materials.

All copyright, and all rights therein, are protected by national and international copyright laws.

The above represents a summary only. For the full conditions see the Conditions for Authors and the Conditions for Website Use.

ISSN 1664-8714 ISBN 978-2-88919-810-8

DOI 10.3389/978-2-88919-810-8

\section{About Frontiers}

Frontiers is more than just an open-access publisher of scholarly articles: it is a pioneering approach to the world of academia, radically improving the way scholarly research is managed. The grand vision of Frontiers is a world where all people have an equal opportunity to seek, share and generate knowledge. Frontiers provides immediate and permanent online open access to all its publications, but this alone is not enough to realize our grand goals.

\section{Frontiers Journal Series}

The Frontiers Journal Series is a multi-tier and interdisciplinary set of open-access, online journals, promising a paradigm shift from the current review, selection and dissemination processes in academic publishing. All Frontiers journals are driven by researchers for researchers; therefore, they constitute a service to the scholarly community. At the same time, the Frontiers Journal Series operates on a revolutionary invention, the tiered publishing system, initially addressing specific communities of scholars, and gradually climbing up to broader public understanding, thus serving the interests of the lay society, too.

\section{Dedication to Quality}

Each Frontiers article is a landmark of the highest quality, thanks to genuinely collaborative interactions between authors and review editors, who include some of the world's best academicians. Research must be certified by peers before entering a stream of knowledge that may eventually reach the public - and shape society; therefore, Frontiers only applies the most rigorous and unbiased reviews.

Frontiers revolutionizes research publishing by freely delivering the most outstanding research, evaluated with no bias from both the academic and social point of view. By applying the most advanced information technologies, Frontiers is catapulting scholarly publishing into a new generation.

\section{What are Frontiers Research Topics?}

Frontiers Research Topics are very popular trademarks of the Frontiers Journals Series: they are collections of at least ten articles, all centered on a particular subject. With their unique mix of varied contributions from Original Research to Review Articles, Frontiers Research Topics unify the most influential researchers, the latest key findings and historical advances in a hot research area! Find out more on how to host your own Frontiers Research Topic or contribute to one as an author by contacting the Frontiers Editorial Office: researchtopics@frontiersin.org 


\section{ONTOGENY AND PHYLOGENY OF BRAIN BARRIER MECHANISMS}

Topic Editors:

Norman R. Saunders, The University of Melbourne, Australia

Helen B. Stolp, King's College London, UK

Shane A. Liddelow, Stanford University, Stanford, USA

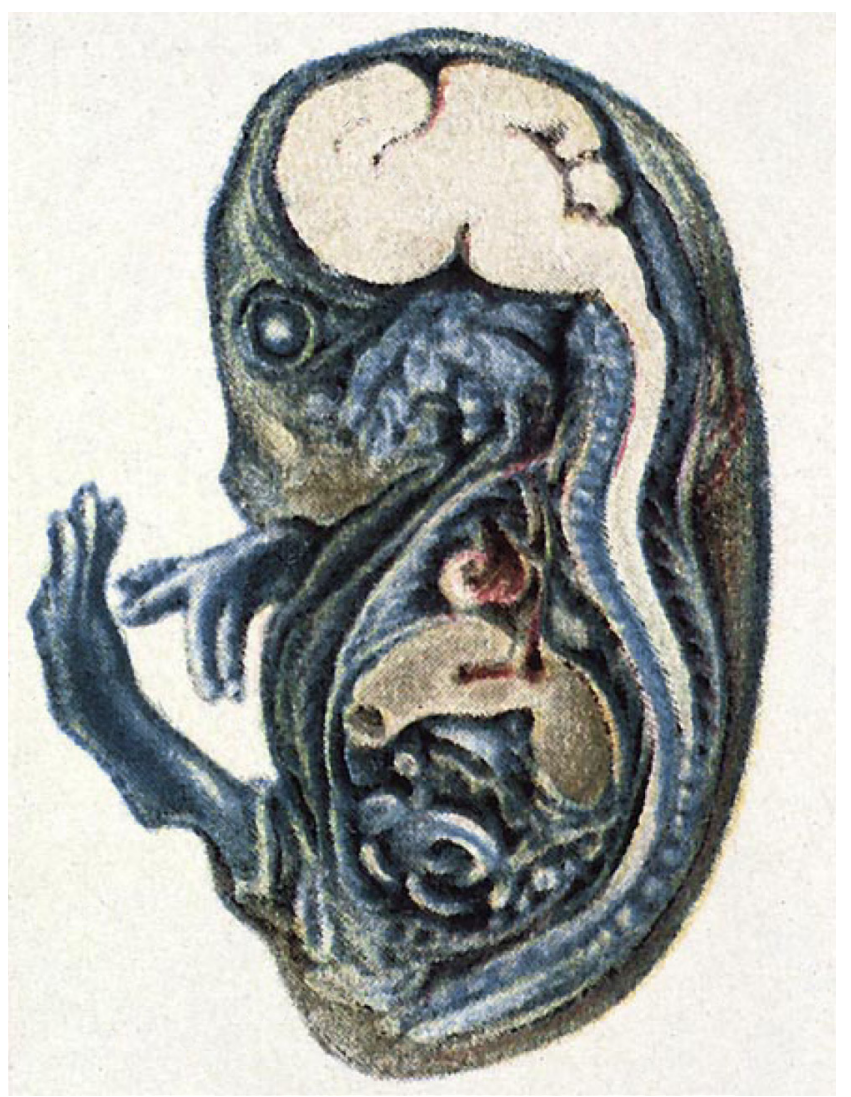

Early demonstration of blood-brain barrier phenomenon in developing brain. This is probably the first documented evidence, which makes this image of particular historical importance. Mid-gestation guinea pig embryo injected with trypan blue (Wislocki,1920). Note lack of staining of brain and spinal cord.

Image taken from Saunders NR, Dreifuss J-J, Dziegielewska KM, Johansson PA, Habgood MD,

Møllgård K and Bauer H-C (2014) The rights and wrongs of blood-brain barrier permeability studies: a walk through 100 years of history. Front. Neurosci. 8:404. doi: 10.3389/fnins.2014.00404 
The brain functions within an internal environment that is determined and controlled by morphological structures and cellular mechanisms present at interfaces between the brain and the rest of the body. In vertebrates these interfaces are across cerebral blood vessels (blood-brain barrier) choroid plexuses (blood-cerebrospinal fluid barrier) and pia-arachnoid. There is a CSF-brain barrier in the neuroepithelium lining the ventricular system that is only present in embryos. There is now substantial evidence that many brain barrier mechanisms develop early and that in some cases they are functionally more active and even more specialized compared to adult barriers. Therefore barriers in developing brain should be viewed as adapted appropriately for the growing brain and not, as is still widely believed, immature.

Considerable advances in our understanding of these barrier mechanisms have come from studies of the developing brain and invertebrates. A striking aspect, to be highlighted in this special edition, is that many of the molecular mechanisms in these very diverse species are similar despite differences in the cellular composition of the interfaces.

This Frontiers Topic comprises articles in three sections: Original studies, Reviews and Myths \& Misconceptions. Original articles provide new information on molecular and cellular barrier mechanisms in developing brains of primates, including human embryos (Brøchner et al., Ek et al., Errede et al.), rodents (Bauer et al., Liddelow, Strazielle \& Ghersi-Egea, Saunders et al., Whish et al.), chick (Bueno et al.) and zebrafish (Henson et al.) as well as studies in drosophila (Hindle \& Bainton, De Salvo et al., Limmer et al.). The Reviews section includes evolutionary perspectives of the blood-brain and blood-CSF barriers (Bueno et al., Bill \& Korzh). There are also detailed reviews of the current state of understanding of different interfaces and their functional mechanisms in developing brain (Bauer et al., Strazielle \& Gjersi-Egea, Liddelow, Richardson et al., Errede et al., Henson et al., Brøchner et al.) and in invertebrates (Hindle \& Bainton, De Salvo et al., Limmer et al.). Different aspects of the relationship between properties of the internal environment of the brain and its development are discussed. (Stolp \& Molnar, Johansson, Prasongchean et al.). A neglected area, namely barriers over the surface of the brain during development is also covered (Brøchner et al.). Clinically related perspectives on barrier disruption in neonatal stroke are provided by Kratzer et al. and other aspects of dysfunction by Morretti et al. and by Palmeta et al. on the continuing problem of bilirubin toxicity.

Progress in this field is hampered by many prevailing myths about barrier function, combined with methodologies that are not always appropriately selected or interpreted. These are covered in the Misconceptions, Myths and Methods section, including historical aspects and discussion of the paracellular pathway, a central dogma of epithelial and endothelial biology (Saunders et al.) and a review of markers used to define brain barrier integrity in development and in pathological conditions (Saunders et al.). Use of inappropriate markers has caused considerable confusion and unreliable interpretation in many published studies. Torbett et al. deal with the complexities of the new field of applying proteomics to understanding blood-brain barrier properties as do Huntley at al with respect to applying modern high throughput gene expression methods (Huntley et al.). The Editorial summarizes the contributions from all authors. This includes mention of some the main unanswered but answerable questions in the field and what the impediments to progress may be.

Citation: Saunders, N. R., Stolp H. B., Liddelow, S. A., eds. (2016). Ontogeny and Phylogeny of Brain Barrier Mechanisms. Lausanne: Frontiers Media. doi: 10.3389/978-2-88919-810-8 


\section{Table of Contents}

07 Editorial: Ontogeny and Phylogeny of Brain Barrier Mechanisms

Helen B. Stolp, Shane A. Liddelow and Norman R. Saunders

\section{Development of brain barrier mechanisms}

Reviews

10 "You Shall Not Pass"-tight junctions of the blood brain barrier

Hans-Christian Bauer, István A. Krizbai, Hannelore Bauer and Andreas Traweger

31 Choroid plexus in developmental and evolutionary perspective

Brent Roy Bill and Vladimir Korzh

42 Embryonic blood-cerebrospinal fluid barrier formation and function

David Bueno, Maryam Parvas, Ismaill Hermelo and Jordi Garcia-Fernàndez

54 Development of the choroid plexus and blood-CSF barrier

Shane A. Liddelow

\section{Original Research papers}

67 Outer brain barriers in rat and human development

Christian B. Brøchner, Camilla B. Holst and Kjeld Møllgård

83 Transcriptomal changes and functional annotation of the developing nonhuman primate choroid plexus

C. Joakim Ek, Peter Nathanielsz, Cun Li and Carina Mallard

96 The contribution of CXCL12-expressing radial glia cells to neuro-vascular patterning during human cerebral cortex development

Mariella Errede,Francesco Girolamo,Marco Rizzi, Mirella Bertossi, Luisa Roncali, and Daniela Virgintino

107 The inner CSF-brain barrier: developmentally controlled access to the brain via intercellular junctions

Sophie Whish, Katarzyna M. Dziegielewska, Kjeld Møllgård, Natassya M. Noor, Shane A. Liddelow, Mark D. Habgood, Samantha J. Richardson

and Norman R. Saunders

\section{Transport}

\section{Reviews}

122 Transport of thyroid hormones via the choroid plexus into the brain: the roles of transthyretin and thyroid hormone transmembrane transporters Samantha J. Richardson, Roshen C. Wijayagunaratne, Damian G. D'Souza, Veerle M. Darras and Stijn L. J. Van Herck 


\section{Original Research papers}

141 Influx mechanisms in the embryonic and adult rat choroid plexus: a transcriptome study

Norman R. Saunders, Katarzyna M. Dziegielewska, Kjeld Møllgård,

Mark D. Habgood, Matthew J. Wakefield, Helen Lindsay, Nathalie Stratzielle, Jean-Francois Ghersi-Egea and Shane A. Liddelow

\section{Invertebrate models}

\section{Reviews}

162 Barrier mechanisms in the Drosophila blood-brain barrier

Samantha J. Hindle and Roland J. Bainton

174 The Drosophila blood-brain barrier: development and function of a glial endothelium

Stefanie Limmer, Astrid Weiler, Anne Volkenhoff, Felix Babatz and Christian Klämbt

\section{Original Research papers}

193 The Drosophila surface glia transcriptome: evolutionary conserved blood-brain barrier processes

Michael K. DeSalvo, Samantha J. Hindle, Zeid M. Rusan, Souvinh Orng, Mark Eddison, Kyle Halliwill and Roland J. Bainton

215 Functional and genetic analysis of choroid plexus development in zebrafish Hannah E. Henson, Chaithanyarani Parupalli, Bensheng Ju and Michael R. Taylor

Methods, Myths and Misconceptions

Reviews

234 Dissecting gene expression at the blood-brain barrier

Melanie A. Huntley, Nga Bien-Ly, Richard Daneman and Ryan J. Watts

248 Markers for blood-brain barrier integrity: how appropriate is Evans blue in the twenty-first century and what are the alternatives?

Norman R. Saunders, Katarzyna M. Dziegielewska, Kjeld Møllgård and

Mark D. Habgood

264 The rights and wrongs of blood-brain barrier permeability studies: a walk through 100 years of history

Norman R. Saunders, Jean-Jacques Dreifuss, Katarzyna M. Dziegielewska,

Pia A. Johansson, Mark D. Habgood, Kjeld Møllgård and Hans-Christian Bauer

290 Understanding the rules of the road: proteomic approaches to interrogate the blood brain barrier

Bruce E. Torbett, Andrew Baird and Brian P. Eliceiri

Barrier mechanisms in normal brain development and in response to pathological insults

Reviews

296 The choroid plexuses and their impact on developmental neurogenesis

Pia A. Johansson 
305 Barrier mechanisms in neonatal stroke

Ingrid Kratzer, Sophorn Chip and Zinaida S. Vexler

313 Blood-brain barrier dysfunction in disorders of the developing brain

Raffaella Moretti, Julien Pansiot, Donatella Bettati, Nathalie Strazielle, Jean-François

Ghersi-Egea, Giuseppe Damante, Bobbi Fleiss, Luigi Titomanlio and Pierre Gressens

328 Neurogenic niches in the brain: help and hindrance of the barrier systems

Helen B. Stolp and Zoltán Molnár

\section{Original Research papers}

335 Hydrophilic bile acids protect human blood-brain barrier endothelial cells from disruption by unconjugated bilirubin: an in vitro study

Inês Palmela, Leonor Correia, Rui F. M. Silva, Hiroyuki Sasaki, Kwang S. Kim, Dora Brites and Maria A. Brito

346 The neural milieu of the developing choroid plexus: neural stem cells, neurons and innervation

Weerapong Prasongchean, Bertrand Vernay, Zeinab Asgarian, Nahin Jannatul and

Patrizia Ferretti 


\title{
Editorial: Ontogeny and Phylogeny of Brain Barrier Mechanisms
}

\author{
Helen B. Stolp ${ }^{1}$, Shane A. Liddelow ${ }^{2,3}$ and Norman R. Saunders ${ }^{2 *}$ \\ ${ }^{1}$ Division of Biomedical Engineering and Health Sciences, Department of Perinatal Imaging and Health, King's College \\ London, London, UK, ${ }^{2}$ Pharmacology and Therapeutics, Developmental Neurobiology and Neurotrauma, University of \\ Melbourne, Parkville, VIC, Australia, ${ }^{3}$ Neurobiology, Stanford University, West Stanford, CA, USA
}

Keywords: blood-brain barrier, choroid plexus, tight junctions, influx mechanisms, efflux mechanisms, development, Drosophila, zebra fish

\section{The Editorial on the Research Topic}

\section{Ontogeny and Phylogeny of Brain Barrier Mechanisms}

The protective barriers of the central nervous system, positioned at interfaces between the brain, spinal cord and the periphery, are often considered as gatekeepers-restricting entry of unwanted molecules and ensuring an effective maintenance of the delicate microenvironment of the central nervous system. While there is truth in this depiction, it does not fully capture the highly dynamic nature of these brain barrier systems; the specific regulation of transporters, the signaling between endothelial cells and other components of the neurovascular unit; or the precise regulation of cerebrospinal fluid composition via the choroid plexus, that changes appropriately both during development and throughout aging.

In this Frontiers Topic on Ontogeny and phylogeny of brain barrier mechanisms, we celebrate the advances made in understanding the normal structure and function of these mechanisms in a diverse range of organisms. The study of development and non-mammalian species shows the similarities of many brain barrier mechanisms as well some diversity.

Substantial information is now available about the protein composition of tight junctions,

Edited and reviewed by: Robert W. Williams, University of Tennessee Health Science Center, USA

*Correspondence:

Norman R. Saunders n.saunders@unimelb.edu.au

Specialty section: This article was submitted to Neurogenomics, a section of the journal Frontiers in Neuroscience

Received: 20 January 2016 Accepted: 01 February 2016 Published: 16 February 2016

Citation:

Stolp HB, Liddelow SA and Saunders NR (2016) Editorial: Ontogeny and Phylogeny of Brain

Barrier Mechanisms.

Front. Neurosci. 10:41. doi: 10.3389/fnins.2016.00041 an integral component of barrier structure. As well as recent investigations on interaction of protein components, we are gaining insights into the polarity changes in endothelial and epithelial cells required for correct establishment of tight junctions (Bauer et al.). The polarization of cells at brain barriers is also key for regulation of transport, such has been clearly indicated for influx (Saunders et al.) and efflux transporters (Strazielle and Ghersi-Egea). The important transport role of the barrier systems in relation to brain development has been highlighted for thyroid hormone in the review by Richardson et al. Genomic studies highlight molecular diversity of the barriers, which show substantial changes in gene regulation with age, species and at different barrier sites (Bill and Korzh; Bueno et al. DeSalvo et al.; Johansson; Limmer et al.; Ek et al.; Saunders et al.; Strazielle and Ghersi-Egea). The use of non-mammalian animal models has advanced the capacity to interrogate the molecular diversity of the barrier systems (DeSalvo et al.; Henson et al.; Hindle and Bainton; Limmer et al.). Zebrafish in particular, because of their transparency, provide an unparalleled opportunity to observe directly at high resolution the development of particular aspects of barrier function (Bill and Korzh; Henson et al.). This plethora of species represents fantastic opportunities for further investigation of how regulation of gene or protein production affects barrier function.

The new genomic studies included in this Topic also report enrichment of genes not just for cell adhesion and solute transport, but also metabolism and cell signaling (DeSalvo et al.; Limmer et al.) indicate a more integrated, deterministic role of the barrier systems in brain function. This integration is particularly evident in neurogenic niches where proliferation and differentiation of cells are in part regulated by contact or release of signaling molecules from cells that comprise these 
barriers (Stolp and Molnár). To further emphasize this point, Errede et al. provide an elegant study showing cross-talk between blood vessels and radial glia within the developing human cerebral cortex.

For research into this complex biological system to advance further, it is necessary for a number of important concepts to be addressed. First, there needs to be consistent and accurate definitions of brain barrier systems. Second, recognition is required that barrier systems in development are not just present and functional, but also integral for facilitating normal brain growth. And third, there is an urgent need for identification and community-wide implementation of welldescribed, reproducible and meaningful assessments of barrier function.

Significant progress has been made regarding the first two of these concepts. In this special Topic Whish et al. and Brøchner et al. explore two under-studied barriers in the developing brain. These authors elegantly demonstrate multiple components of the barrier system at the inner and outer CSF-brain interfaces, and how these differ from blood-brain and blood-CSF barriers. Brøchner et al. extend on the barrier definitions outlined by Saunders et al. (2008). In particular they identify 3 distinct morphological components (arachnoid barrier cell layer, pial microvessels, and glial end feet/pial surface layer) that contribute to the outer CSF-brain barrier interface that has previously been treated as a single entity. Bueno et al. review experiments on very early stages of brain development shortly after neural tube closure in the chick embryo. This is a stage that has otherwise received very little attention. They describe a subset of endothelial cells in the ventral mesencephalon and anterior ventral prosencephalon with transient transport properties that they suggest parallel the functions of the choroid plexuses before these structures differentiate.

The misconception that brain barrier systems are immature in the developing brain is still frequently touted in the literature, despite evidence to the contrary dating back to experiments conducted in the early twentieth century (see Saunders et al.). In this special Topic, further evidence is presented on ageand region-specific regulation of influx and efflux transporters (Saunders et al.; Strazielle and Ghersi-Egea) - as described above, the developing brain actually has more individual barrier systems than in the adult (Bueno et al.; Brøchner et al.; Whish et al.). The choroid plexus in particular is a complex set of barrier mechanisms that are present from an extremely early stage of brain development (Liddelow). The evidence presented on progenitor cells specifically sitting in CSF and vascular niches points to a tightly controlled environment with specific signaling mechanisms appropriate for different stages of the development of the brain is particularly striking. This contradicts the long held immature brain barrier hypothesis (Bueno et al.; Johansson; Stolp and Molnár). There is also evidence for an additional neurogenic niche in the choroid plexus stroma (Prasongchean et al.), which these authors suggest contributes specifically to prenatal innervation of the choroid plexus and regulation of CSF secretion.

While there are huge advances in our understanding of structural (e.g., tight junction) proteins and transporter systems at brain barriers during development and aging in a wide variety of species, we are still limited in our understanding of how these elements of the barrier systems are altered following injury or during disease. The field is currently most interested in barrier dysfunction in the adult and aging brain, aspects that are outside the scope of this Topic. However, barrier dysfunction during development has implications not only for the fetus or newborn acutely at the time of the disorder but also chronically with the possibility of long-term consequences. Here Moretti et al. provide an overview of disorders that involve brain barrier mechanisms including their long-term sequelae. Kratzer et al. deal with the specific developmental problem of neonatal stroke; while Palmela et al. provide some in vitro data on the problem of neonatal kernicterus. The role of the blood-brain barrier in neonatal bilirubin encephalopathy is much more complex than the longstanding simple view that it occurs because of barrier absence or immaturity (see Saunders et al.).

Both in the developmental and adult brain barrier fields we are missing a consensus on the appropriate methodologies that should be used to understand barrier function. For example, quantification of tight junction proteins is a common assessment in injury models to determine whether structural elements of barriers are functioning effectively. However, there is no clear indication of (a) what effect reduction in tight junction proteins produces, or (b) how changes in expression of tight junction protein genes or cellular content of these proteins relate to structural integrity of junctions. To make claims about loss of barrier integrity requires ultrastructural studies with suitable markers that are visible at high resolution, something that is rarely done. If brain barrier studies are to integrate with a proper understanding of neurological injury we need substantial improvement in our assessments of barrier function. This focus on structural proteins of brain barriers also means that active transport systems, important for understanding of energetics of the barriers, are rarely studied. This is despite evidence of high metabolic function of cerebral endothelial cells compared to those in the periphery (Oldendorf et al., 1977). While tracer studies still represent a functional measure, irrespective of mechanism, there are substantial problems with how these have been administered and interpreted (see Saunders et al.).

Leukocyte infiltration into the brain during injury is often used as an indication of barrier dysfunction, but is a finding that is not interpreted so easily. Leukocytes cross into the brain under control conditions as part of the normal immune surveillance of the brain (Ransohoff and Engelhardt, 2012), but it is unclear whether the massive influx of leukocytes into the brain (e.g., in Multiple Sclerosis active lesions) is a dysfunction of the barrier, or a normal pathological response resulting from abnormal signaling in the brain. There is evidence from perinatal brain injury of leukocyte populations entering the brain and contributing to injury at a time when the adaptive immune system had previously been considered immature and unresponsive (Wang and Mallard, 2016). It is unclear whether some of these cells are also contributing to resolution of the lesion, or whether they only produce negative effects on the brain. Whatever the reason for peripheral immune cell infiltration, there is increasing evidence of beneficial effects of controlled 
neuroinflammation in pathological conditions (Mardiguian et al., 2013; Evans et al., 2014; Amantea and Bagetta, 2016).

Clear guidelines for conducting genomic or proteomic studies of barrier systems are now provided (Huntley et al.; Torbett et al.). What remains is for these studies to extend from an analysis of normal barrier interfaces, to studies of heterogeneity of barrier systems and of cerebral endothelial cells (Macdonald et al., 2010) as well as responses to injury and other pathological conditions. Similarly, there is need for a battery of standardized tests for barrier function, including tracers and other measures of barrier integrity as well as transporter presence and function. For a full understanding of normal and abnormal barrier function these should be performed together, in whole systems studies at macro- and microscopic levels. The wide range of molecular and physiological tools available, as well as the multiple in vitro and in vivo models, means that these aims are well within reach.

Two problems that have been a major preoccupation in the blood-brain barrier field over recent decades have been how to screen drugs with potential therapeutic value for treating neurological disorders, and how to devise methods for effective delivery of such drugs to the brain. In spite of a considerable expenditure of time and resources outcomes have been disappointing, perhaps in part because of an undue focus on in vitro methods. Although not a specific focus of

\section{REFERENCES}

Amantea, D., and Bagetta, G. (2016). Drug repurposing for immune modulation in acute ischemic stroke. Curr. Opin. Pharmacol. 26, 124-130. doi: 10.1016/j.coph.2015.11.006

Evans, M. C., Gaillard, P. J., de Boer, M., Appeldoorn, C., Dorland, N., et al. (2014). CNS-targeted glucocorticoid reduces pathology in mouse model of amyotrophic lateral sclerosis. Acta Neuropathol. Commun. 2:66. doi: 10.1186/2051-5960-2-66

Macdonald, J. A., Murugesan, N., and Pachter, J. S. (2010). Endothelial cell heterogeneity of blood-brain barrier gene expression along the cerebral microvasculature. J. Neurosci. Res. 88, 1457-1474. doi: 10.1002/jnr.22316

Mardiguian, S., Serres, S., Ladds, E., Campbell, S. J., Wilainam, P., McFadyen, C., et al. (2013). Anti-IL-17A treatment reduces clinical score and VCAM-1 expression detected by in vivo magnetic resonance imaging in chronic relapsing EAE ABH mice. Am. J. Pathol. 182, 2071-2081. doi: 10.1016/j.ajpath.2013.02.029

Oldendorf, W. H., Cornford, M. E., and Brown, W. J. (1977). The large apparent work capability of the blood-brain barrier: a study of the mitochondrial content of capillary endothelial cells in brain and other tissues of the rat. Ann. Neurol. 1, 409-417. this Topic, several of the papers point to contributions that are, or could be made by approaches described here. Thus, Hindle and Bainton describe promising live imaging approaches in Drosophila, supported by the report of DeSalvo et al. that the transcriptomes of fly and mouse show many similarities particularly with respect to the $\mathrm{ABC}$ efflux transporters that prevent the entry of so many drugs into the central nervous system. The advances in understanding of many different aspects of barrier function described in the papers in this Topic hold promise for developing better drug delivery systems both in vivo and in improved in vitro systems.

The twenty-five reviews and original research articles in this Topic, provide a comprehensive update on the state of these research fields, their interrelations and implications for the blood-brain barrier field as a whole. We should like to thank all the authors and additional editors for their splendid and patient contributions to the Topic. We hope that the Topic articles will provide both a benchmark and reference point for future studies.

\section{AUTHOR CONTRIBUTIONS}

All authors listed, have made substantial, direct and intellectual contribution to the work, and approved it for publication.

Ransohoff, R. M., and Engelhardt, B. (2012). The anatomical and cellular basis of immune surveillance in the central nervous system. Nat. Rev. Immunol. 12, 623-635. doi: 10.1038/nri3265

Saunders, N. R., Ek, C. J., Habgood, M. D., and Dziegielewska, K. M. (2008). Barriers in the brain: a renaissance? Trends Neurosci. 31, 279-286. doi: 10.1016/j.tins.2008.03.003

Wang, X., and Mallard, C. (2016). Editorial: white blood cells matter in neonatal white-matter injury. J. Leukoc. Biol. 99, 4-6. doi: 10.1189/jlb.3CE06 $15-242 \mathrm{R}$

Conflict of Interest Statement: The authors declare that the research was conducted in the absence of any commercial or financial relationships that could be construed as a potential conflict of interest.

Copyright (c) 2016 Stolp, Liddelow and Saunders. This is an open-access article distributed under the terms of the Creative Commons Attribution License (CC BY). The use, distribution or reproduction in other forums is permitted, provided the original author(s) or licensor are credited and that the original publication in this journal is cited, in accordance with accepted academic practice. No use, distribution or reproduction is permitted which does not comply with these terms. 


\title{
"You Shall Not Pass"-tight junctions of the blood brain barrier
}

\author{
Hans-Christian Bauer 1,2,3, István A. Krizbai ${ }^{4,5}$, Hannelore Bauer ${ }^{6}$ and Andreas Traweger ${ }^{1,3 *}$ \\ 1 Institute of Tendon and Bone Regeneration, Paracelsus Medical University - Spinal Cord Injury and Tissue Regeneration Center Salzburg, Salzburg, Austria \\ 2 Department of Traumatology and Sports Injuries, Paracelsus Medical University, Salzburg, Austria \\ ${ }^{3}$ Austrian Cluster for Tissue Regeneration, Vienna, Austria \\ ${ }^{4}$ Biological Research Centre, Institute of Biophysics, Hungarian Academy of Sciences, Szeged, Hungary \\ ${ }^{5}$ Institute of Life Sciences, Vasile Goldis Western University of Arad, Arad, Romania \\ ${ }^{6}$ Department of Organismic Biology, University of Salzburg, Salzburg, Austria
}

\section{Edited by:}

Helen B. Stolp, King's College

London, UK

\section{Reviewed by:}

Thomas Paul Davis, University of Arizona College Of Medicine, USA

Stefan Liebner, Goethe University Clinic, Germany

Richard Daneman, University of California, San Diego, USA

\section{*Correspondence:}

Andreas Traweger, Institute of Tendon and Bone Regeneration, Paracelsus Medical University Spinal Cord Injury and Tissue Regeneration Center Salzburg,

Strubergasse 22,

A-5020 Salzburg, Austria

e-mail: andreas.traweger@pmu.ac.at
The structure and function of the barrier layers restricting the free diffusion of substances between the central nervous system (brain and spinal cord) and the systemic circulation is of great medical interest as various pathological conditions often lead to their impairment. Excessive leakage of blood-borne molecules into the parenchyma and the concomitant fluctuations in the microenvironment following a transient breakdown of the blood-brain barrier (BBB) during ischemic/hypoxic conditions or because of an autoimmune disease are detrimental to the physiological functioning of nervous tissue. On the other hand, the treatment of neurological disorders is often hampered as only minimal amounts of therapeutic agents are able to penetrate a fully functional BBB or blood cerebrospinal fluid barrier. An in-depth understanding of the molecular machinery governing the establishment and maintenance of these barriers is necessary to develop rational strategies allowing a controlled delivery of appropriate drugs to the CNS. At the basis of such tissue barriers are intimate cell-cell contacts (zonulae occludentes, tight junctions) which are present in all polarized epithelia and endothelia. By creating a paracellular diffusion constraint TJs enable the vectorial transport across cell monolayers. More recent findings indicate that functional barriers are already established during development, protecting the fetal brain. As an understanding of the biogenesis of TJs might reveal the underlying mechanisms of barrier formation during ontogenic development numerous in vitro systems have been developed to study the assembly and disassembly of TJs. In addition, monitoring the stage-specific expression of TJ-associated proteins during development has brought much insight into the "developmental tightening" of tissue barriers. Over the last two decades a detailed molecular map of transmembrane and cytoplasmic TJ-proteins has been identified. These proteins not only form a cell-cell adhesion structure, but integrate various signaling pathways, thereby directly or indirectly impacting upon processes such as cell-cell adhesion, cytoskeletal rearrangement, and transcriptional control. This review will provide a brief overview on the establishment of the BBB during embryonic development in mammals and a detailed description of the ultrastructure, biogenesis, and molecular composition of epithelial and endothelial TJs will be given.

\section{Keywords: blood-brain barrier, tight junctions, PDZ scaffolds, MAGUK proteins, cell polarity, brain capillary} endothelial cells, vascular permeability
Abbreviations: CD31/34, cluster of differentiation 31/34; Cdc42, cell division control protein 42 ; CD2AP, CD2-associated protein; $\mathrm{CDK} 4$, cyclin-dependent kinase 4/cell division protein kinase 4; C/EBP, CCAAT/enhancer-binding-protein; cECs, capillary endothelial cells; CNS, central nervous system; ECM, extracellular matrix; ES cells, embryonic stem cells; GAP, GTPase activating protein; GDNF, glialderived neurotrophic factor; GEF-H1, guanine nucleotide exchange factor- $\mathrm{H} 1$ Glut-1, glucose transporter-1; GUK, guanylate kinase; hCMEC/D3, human cerebral microvascular endothelial cell line/D3; hnRNP, heterogeneous nuclear ribonucleoprotein; MAGI, membrane-associated guanylate kinase inverted; MAGUK, membrane-associated guanylate kinase; MARVEL, MAL (myelin and lymphocyte) and related proteins for vesicle trafficking and membrane link; NVU, neurovascular unit; PDZ, PSD-95, discs large, ZO-1; SMAD, SMA (small)/ MAD: Mothers against decapentaplegic; TAMPs, TJ-associated MARVEL proteins; TEER,

\section{ESTABLISHMENT OF THE BBB DURING MAMMALIAN EMBRYOGENESIS}

During evolution organisms with a complex nervous system have developed mechanisms to selectively restrict the bloodto-brain traffic of compounds allowing a tight regulation of the neuroparenchymal microenvironment. Such cerebral

transepithelial electrical resistance; Unc-5, uncoordinated locomotion-5; VEGF, vascular endothelial growth factor; WD40, tryptophan-aspartic acid (WD) repeat; Wnt, wingless/Int-1. 
homeostasis is fundamental for proper function of synapses and neural networks. However, despite the burgeoning literature on when the BBB is established in the course of mammalian development, the topic is still heavily debated. This is largely the result of inconsistent and partially contradictive results stemming from different animal models used and due to misinterpretation of experiments (for review see Saunders et al. in this volume). In addition, not a single barrier, but the sum of several cellular and molecular barriers ensures the stable environment needed for the proper function of the central nervous system, further complicating the analysis. Of the three main CNS barriers the brain endothelium constituting the blood-brain barrier (BBB) represents the major interface between the blood and the brain interstitial fluid with an estimated total surface area of $20 \mathrm{~m}^{2}$ (Begley and Brightman, 2003). The endothelial cells are fitted with specialized structures, such as cell-cell adhesion complexes obliterating the paracellular space (tight junctions) and an array of transport and shuttle proteins controlling the flux of ions and solutes. The term BBB has been coined nearly 100 years ago by Stern et al. (Stern and Rothlin, 1918; Stern and Gauthier, 1921), implying a structure completely preventing an exchange between blood and nervous tissue. However, since then it has become increasingly clear that the BBB is a complex and dynamic structure allowing a controlled passage of blood-borne substances. In addition, the functional $\mathrm{BBB}$, at least in the adult, is not only composed of the endothelial cells (ECs) lining the microvasculature but also requires the contact and/or input of other cell types, including astrocytes, neurons, and pericytes separated only by a basement membrane. All these contributing building blocks of the BBB are commonly being referred to as "neurovascular unit," a term which has been introduced for the first time by members of the Stroke Progress Review Group in 2002 at the NIH, USA. During embryonic development however, the composition of the NVU and the nature of the cerebral capillary endothelial cells (cECs) most likely is very different from those seen in the adult. At an even more fundamental level, at which developmental stage can we speak of a structure exerting barrier functions and does $\mathrm{BBB}$ formation in $\mathrm{cEC}$ coincide with the early vascularization of the growing CNS?

In mice angiogenesis in the brain begins at day E9 of development, when pial vessels encircle the telencephalon (Vasudevan et al., 2008). By E10 pial vessels sprout and start forming a primitive vascular plexus within the telencephalon. The newly formed vessels have a premature appearance (see Figures 1A,B) but allow already erythrocyte traffic (Bauer et al., 1993, 1995). In parallel, periventricular vessels migrate into the dorsal telencephalon resulting in a ventrolateral to dorsomedial angiogenesis gradient (Vasudevan et al., 2008). It is proposed that the pial vessels develop into venous sinuses and the periventricular vessels into arteries. The assumption that the plexus of the CNS likely arises from two different endothelial cell populations is further supported by the demonstration of different expression patterns of homeobox transcription factors, namely Dlx1/2, Nkx2.1 in periventricular ventral vessels and Pax6 in pial vessels. Intriguingly, these transcription factors also regulate neuroepithelial cell proliferation and neuronal migration (Sussel et al., 1999; Nery et al., 2003). Therefore, it is tempting to speculate that angiogenesis in due course influences and regulates neuronal proliferation and differentiation.

Several proteins, morphogenetic molecules and their receptors are involved in both, angiogenesis of the brain and in the formation of the BBB: VEGF has been identified as one of the first of these effectors. This morphogenetic molecule is secreted by neuronal precursor cells and promotes via binding to endothelial tyrosine kinase receptors (FLT-1/VEGFR-1; FLK1/VEGFR-2) vessel sprouting during the early phases of CNS development (Breier et al., 1992; Raab et al., 2004). VEGF is also secreted by brain endothelial cells and exerts its influence on the cortical cytoarchitecture during later stages of embryonic development allowing the establishment of a functional neuronal architecture in the CNS (Li et al., 2013). Other effector molecules include members of the Wnt family (Wnt7a/b, Wnt3a) together with the action of $B$-catenin, Tie-2 (an endothelial cellspecific receptor tyrosine kinase) and its ligands angiopoietin-1 and angiopoietin-2 (Ang-1, Ang-2), as well as sonic hedgehog and Norrin (Engelhardt and Liebner, 2014). The important role of Ang-1 during BBB formation is additionally strengthened by the identification of SSeCKS, a soluble protein which induces the expression of Ang-1 in cEC of the BBB (Lee et al., 2003). Next to these effectors which exert their action throughout the entire CNS, others are restricted to specific areas. For example GPR124 (orphan G protein-coupled receptor 124) is specifically expressed in endothelial cells regulating sprouting in the forebrain and in spinal cord but not in other regions of the CNS (Kuhnert et al., 2010). GPR124 also modulates TGF- $B$ signaling, thereby influencing pericyte and astrocyte differentiation and further impacts upon glucose transport by regulating the expression of Glut-1 in cECs. Taken together, the factors described above are intimately related to the induction of a functional BBB as they promote differentiation of the cECs, ultimately resulting in the formation of occluding cell adhesion structures and the expression of specific transporter receptors and shuttling molecules such as the multidrug resistance molecule.

The early transplantation experiments by Stewart and Wiley and Ikeda et al. demonstrated the induction of BBB features of invading endothelial cells by ectopic transplantation of embryonic brain tissue, indicating that the acquisition of BBB function is not merely an intrinsic property of cerebral endothelial cells (Stewart and Wiley, 1981; Ikeda et al., 1996) but requires the direct and indirect communication with neighboring neuronal cells. In the early brain these are the neuroepithelial cells and neuroblasts and later in embryonic development and postnatally, neurons, pericytes and glial cells (Bauer and Bauer, 2000). However, the underlying molecular machinery and ramifications of this interaction are not fully understood mainly due to its complex nature and its constant modulation during embryonic and postnatal development. Lindahl et al. showed that platelet derived growth factor-B (PDGF-B) secreted from migrating cECs led to the recruitment of pericytes and their attachment to the newly formed vessels (Lindahl et al., 1997). In the following years in vivo and in vitro studies demonstrated that pericytes are imperative for BBB formation (Deli et al., 2005; Armulik et al., 2010; Daneman et al., 2010b). Recently, another effector was described by Ben-Zvi et al. (2014) named Mfsd2a, a transmembrane protein 


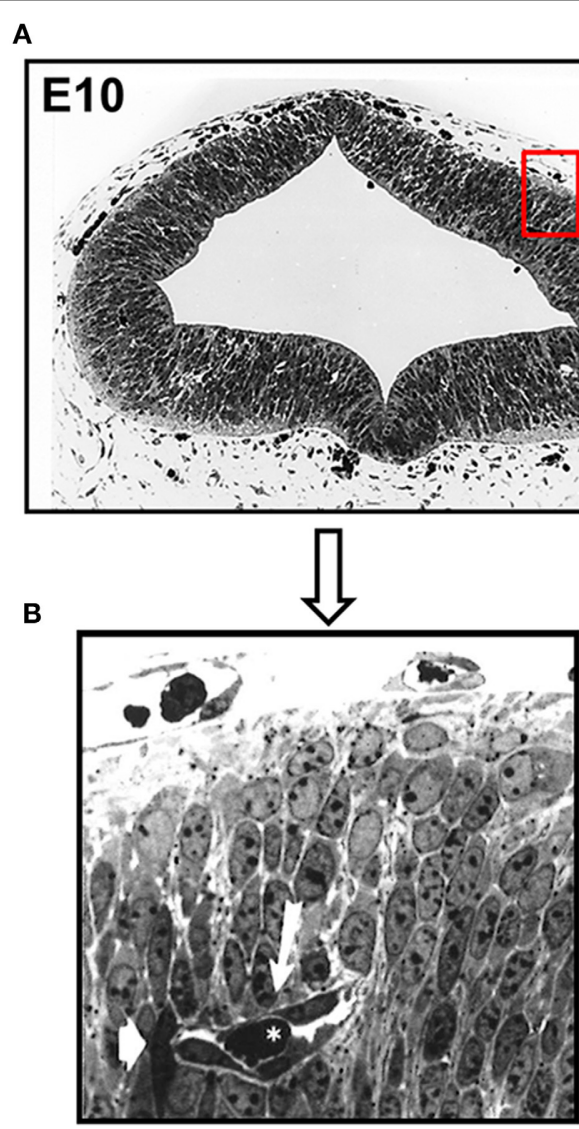

FIGURE 1 | Establishment of the intraneural vascular plexus and a functional BBB during mouse embryogenesis. (A) Semithin section of embryonic mouse neocortex at E10 showing the intraneural and parts of the surrounding perineural domain (B) neuroepithelial cells surround a primitive
C
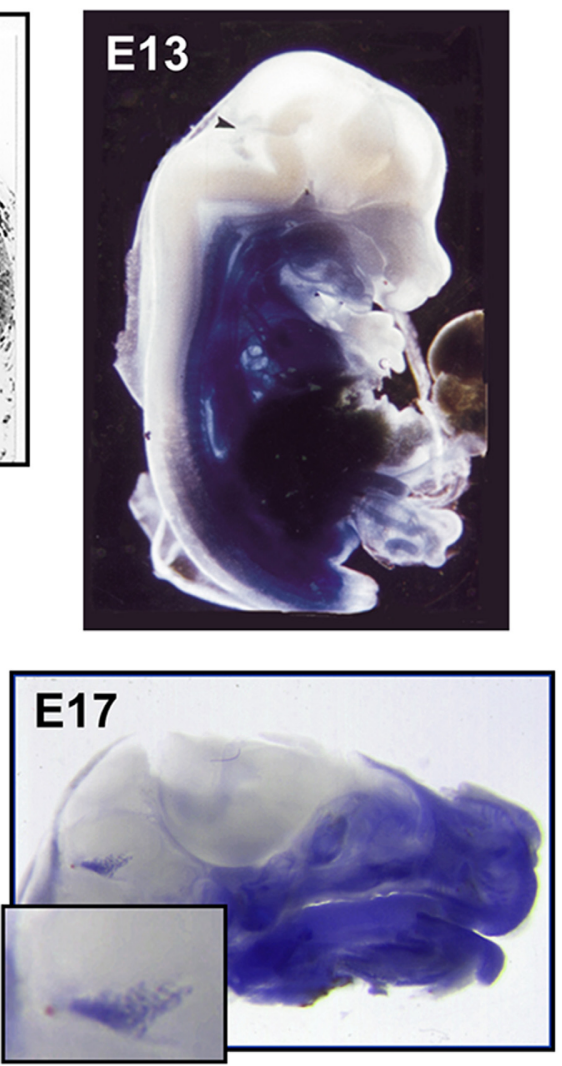

capillary (white arrow) containing a red blood cell (asterisk). Murine E13 (C) and E17 (D) embryos microperfused with 0.5\% Trypan blue solution. After fixation specimens were cut medially and photographed. Arrowhead indicates and enlarged area depicts stained choroid plexus (Bauer et al., 1993, 1995). exclusively expressed in cerebral endothelial cells. Mfsd2a suppresses transcytosis in endothelial cells and thus the transport of plasma proteins. Interestingly, the protein is expressed in mice at embryonic day 13.5 and its expression depends on the presence of pericytes. In Mfsd2a-knockout mice the BBB is impaired from embryonic day 15.5 to adulthood what led the authors to the conclusion that vascularization and the establishment of a functional $\mathrm{BBB}$ are not coinciding. In earlier findings however it has been shown that the BBB becomes functional even before E15 (Bauer et al., 1993, 1995). Microperfusion of E12-E13 mouse embryos with Trypan Blue revealed that the CNS remained void of staining except for the choroid plexus anlage, indicating that at least certain plasma proteins have been excluded (see Figures 1C,D). Further, the number of pinocytotic vesicles in endothelial cells of intraneural capillaries decreased from E12 onwards, whereas the number of vesicles in endothelial cells of the perineural domain in the CNS increased (see Table 1).

In summary, the formation of a functional $\mathrm{BBB}$, or more precisely, of a neurovascular unit with specific barrier structures and transport functions is a complex, multifactorial process requiring the concerted action and interaction of various cell types including cECs, astrocytes, neuronal and glial cells, as well as pericytes. Finally, although the developmental stage at which a functional $\mathrm{BBB}$ is present is still under debate, there is good evidence that a barrier toward large molecules exists very early in embryonic brain development, which then fully matures postnatally.

\section{TIGHT JUNCTION MORPHOLOGY AND FUNCTION}

Next to an extensive transport machinery, tight junctions sealing the microvascular endothelium represent the core structure of the $\mathrm{BBB}$. The tight junction (TJ) is the most apical cell-cell junction complex in polarized epithelia and endothelia and can be visualized by ultrathin-section electron microscopy as focal points or "kissing points" where membranes of adjacent cells come into close apposition, completely obliterating the intercellular cleft (Farquhar and Palade, 1963). The application of freeze-fracture electron microscopy provided a more detailed description of TJs. TJs of epithelial cells appear as a complex network of anastomosing and continuous cylindrical strands on the protoplasmic leaflet (P-face) of the plasma membrane, with complementary grooves on the exoplasmic leaflet (E-face), completely encircling the apical aspect of the cell (Chalcroft and Bullivant, 1970; Staehelin, 1973, 1974; Van Deurs and Koehler, 1979). In contrast, depending 
Table 1 | Frequency of endothelial fenestrations (f), junctional complexes (j.c.) and pinocytotic vesicles (p.v.) in intraneural and perineural capillaries during murine embryonic development.

\begin{tabular}{|c|c|c|c|c|c|c|}
\hline Day of embryonic development & \multicolumn{3}{|c|}{ Intraneural capillaries } & \multicolumn{3}{|c|}{ Perineural capillaries } \\
\hline E9 & \multicolumn{3}{|c|}{ No capillaries present } & 0.9 & 3.2 & 2.0 \\
\hline E10 & nd & 1.7 & 2.9 & 0.13 & 2.9 & 1.9 \\
\hline E12 & nd & 2.58 & 2.33 & nd & 3.25 & 3.0 \\
\hline E14 & nd & 2.29 & 1.57 & nd & 2.43 & 3.29 \\
\hline
\end{tabular}

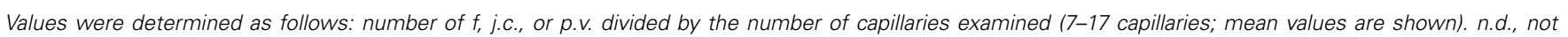
detected (Bauer et al., 1993).

on the vascular bed investigated, TJ strands of endothelial cells generally display a significantly lower level of P-face association. Whereas endothelial cells of the peripheral vasculature show predominantly E-face associated strands (Simionescu et al., 1976; Muhleisen et al., 1989), freeze fracture replicas of TJs located at the $\mathrm{BBB}$ reveal a high degree of $\mathrm{P}$-face association and are among the most complex found in the entire vasculature (Nagy et al., 1984; Liebner et al., 2000b). It seems that not only the complexity of the strand network but also the association of the TJ-strands with the P- or E-face reflects the functional quality (i.e., permeability and electrical resistance) of the barrier (Lippoldt et al., 2000). The varying P-face/E-face ratios possibly are a consequence of altered attachment of the TJ strands to the epithelial or endothelial cytoskeleton, but much detail is still missing.

Next to the establishment and maintenance of a size and charge selective paracellular barrier (Gumbiner, 1987, 1993; Cereijido et al., 1993), TJs also serve to create an intramembrane diffusion barrier ("fence") restricting the intermixing of apical and basolateral components within the exoplasmic leaflet of plasma membranes (Van Meer et al., 1986). In addition, they guide a selective distribution and clustering of membrane components to distinct cell-surfaces (Fanning and Anderson, 1999) and assemble large protein complexes around scaffolding proteins at their cytoplasmic aspect. The modular nature of these adaptor proteins allows the spatial and temporal assembly of multi-protein complexes not only ensuring the structural integrity of the TJ, but also integrating various regulatory pathways which are pivotal for TJ physiology, i.e., establishment of apico-basal polarity, gene expression, and cell proliferation (Gonzalez-Mariscal et al., 2008; Guillemot et al., 2008; Balda and Matter, 2009; McCrea et al., 2009). Finally, the junctional cytoplasmic plaque provides a direct and indirect link to the cytoskeleton.

Generally, much of our understanding of TJ biology stems from studying polarized epithelial cells. However, several aspects of the core structure of endothelial and epithelial TJs are similar and many of the reported findings apply most likely for both cell types. The identification of an ever increasing number of TJ-associated proteins (Gonzalez-Mariscal and Nava, 2005; Wang and Margolis, 2007; Ebnet, 2008; Gonzalez-Mariscal et al., 2008; Giepmans and Van Ijzendoorn, 2009; Steed et al., 2010; Bauer et al., 2011) has led to a wide acceptance of the "protein model", proposing that linear arrays of polymerized transmembrane adhesion molecules form TJ-strands and adhere with strands from apposing plasma membranes (Staehelin, 1973; Tsukita et al., 2001; Furuse, 2010). However, it remains to be unequivocally proven that the high transcellular electrical resistance of epithelia and endothelia can solely be attributed to the molecular interaction of TJ-strands. The involvement of lipid components ("lipid model") cannot be fully ruled out and a "lipid-protein hybrid model" might explain the complexity of TJ physiology (Kan, 1993; Yamagata et al., 2003; Lee et al., 2008; Chen-Quay et al., 2009). But information on the functional and structural relationship of proteins and lipids in the junctional complex remain scarce.

\section{THE BIOGENESIS OF TIGHT JUNCTIONS}

Functional TJs not only seal the paracellular space between epithelial and endothelial cells, they are also imperative for the establishment of a polarized, transporting epithelial and endothelial phenotype. The cellular constituents of tissue barriers are polarized, displaying apico-basal polarity through the selective distribution of specific lipid- and protein complexes to distinct sites at the cell surface. Generally, the cells are oriented with their apical aspect of the plasma membrane toward the lumen ("free surface"), whereas the lateral domains are in close contact with neighboring cells and basally adhere to other solid tissues (or parenchyme), only separated by a basement membrane. Epithelial and endothelial cells grown in suspension, or loss of matrix anchorage results in programmed cell death (anoikis), whereas grown on a surface, even single cells polarize. Therefore, the contact to the extracellular matrix, mainly conferred by the family of integrin transmembrane proteins, is crucial to ensure robust cell polarization and morphogenesis in vivo (Lee and Streuli, 2014). As soon as an appropriate vectorial transport machinery is in place, epithelia and endothelia are considered fully differentiated, separating tissue and organ compartments and allowing the establishment and maintenance of distinct internal and external milieus.

However, in spite of considerable structural similarities the functional properties of epithelial and endothelial barriers in various tissues and organs can significantly vary. This is largely due to the expression of different transmembrane (i.e., claudins; see further below) and accessory TJ proteins. However, also parameters 
other than mere structural features need to be taken into account when comparing barriers of various origin and organs. For example, environmental factors (i.e., soluble factors or contacting cells) can influence the barrier properties in developing and mature organs. A beautiful example is the intimate association of cerebral endothelial cells with neuroectoderm-derived accessory cells (astroglia, neurons, and neural precursor cells), which is a unique feature of the $\mathrm{BBB}$ and imperative for its physiological functioning. These cells not only contribute physically to the barrier but also induce barrier properties in differentiating cerebral endothelial cells (Bauer and Bauer, 2000; Abbott, 2002; Haseloff et al., 2005; Abbott et al., 2006; Weidenfeller et al., 2007).

Most of our knowledge about tissue barrier formation stems from studies on epithelial and endothelial cells in vitro (GonzalezMariscal et al., 1985; Stevenson et al., 1988; Bauer et al., 2011; Watson et al., 2013; Czupalla et al., 2014; Wilhelm and Krizbai, 2014). In this respect, particularly the re-establishment of TJs following calcium depletion and the concomitant breakdown of cell-cell contacts has been investigated (Gonzalez-Mariscal et al., 1990; Contreras et al., 1992; Stuart et al., 1994). Much of our understanding of TJ formation in primary (and secondary) epithelia also comes from studying embryogenesis of D. melanogaster (cellularization) and C. elegans (Knust and Bossinger, 2002; St Johnston and Ahringer, 2010). Mammalian TJ biogenesis has mainly been described in cultured preimplantation mouse embryos, which serve as a valuable model to determine the expression of junctional proteins during the earliest steps of cell lineage differentiation. Cultured preimplantation mouse embryos can easily be manipulated to precisely determine the role of junction-related proteins at early developmental stages such as compaction and cavitation (formation of the blastocoel) (Dard et al., 2008; Eckert and Fleming, 2008). Early signs of junction establishment appear during formation of tubular structures or cavities, a process which is fundamental to organ development in both vertebrates and invertebrates. From a range of investigations describing the epithelial and endothelial tubular biogenesis, three major morphologic mechanisms are being proposed, commonly referred to as cell hollowing, cord hollowing, and cavitation. In all of them, tubes or cavities arise from lumenless, unpolarized cell aggregates or single unpolarized cells. While cell and cord hollowing are common in vascular structures, cavitation is rather attributed to epithelial tubulogenesis and is only scarcely observed in endothelial vessel formation (Lubarsky and Krasnow, 2003). Cavitation has extensively been described in the preimplantation mouse embryo and coincides with the first occurrence of a differentiated epithelium in the mammalian embryo (Eckert and Fleming, 2008). The undifferentiated mammalian blastomeres are subjected to three rounds of cleavage, resulting in the formation of the eight-cell embryo, also referred to as the "early morula" stage. Subsequently, compaction of the embryo is initiated, representing the moment of establishment of an epithelial phenotype and the generation of an apical-basal axis. This asymmetric cellular organization is then coupled with a specific orientation of the mitotic spindle during cell division, resulting in asymmetric division of the blastomeres giving rise to two different cell populations. The inner, non-polarized blastomeres form the inner cell mass (ICM) of the blastocyst, and the outer polarized blastomeres giving rise to the first polarized epithelium, the trophectoderm (TE) which develops into the chorioallantoic placenta later during development. The ICM then develops into the epiblast, the origin of all tissues of the embryo proper, and the primitive endoderm which gives rise to the extra-embryonic membranes. A more detailed description on embryonic axis establishment and blastocyst lineage formation is beyond the scope of this review and the reader is referred to a series of excellent reviews (Larue et al., 1994; Arnold and Robertson, 2009; Rossant and Tam, 2009; Morris and Zernicka-Goetz, 2012; Takaoka and Hamada, 2012).

Overall, premature intercellular contacts serve as an initial spatial cue for the establishment of polarity not only in blastomeres but also in cells in general. Early studies then identified E-cadherin as the prime epithelial signature marker. During compaction of the early mouse embryo, diffusely localized E-cadherin becomes redistributed to long filopodia protruding from the blastomeres which facilitate the attachment of two neighboring cells via homophilic binding of opposing E-cadherin clusters (FierroGonzalez et al., 2013). Interestingly, mouse embryos deficient in E-cadherin fail to form a proper trophectoderm and die around the time of implantation, compaction not being affected. This late phenotype is explained by the presence of a residual maternal E-cadherin pool, which is sufficient to drive compaction (Larue et al., 1994). Next to E-cadherin, the homophilic binding of nectin family members at developing adhesive structures takes place very early during the establishment of the polarized epithelial phenotype (Sato et al., 2006; Takai et al., 2008b). Nectins constitute a family of single-pass, calcium-independent transmembrane adhesive molecules which, together with clusters of cadherins form mature adherens junctions (Indra et al., 2013). During the early phases of polarization it is believed that nectin controls the initiation of cadherin clustering by preventing the endocytotic removal of cadherin (Takai et al., 2008a). The majority of nectin function is conferred via its association with the cytoplasmic protein afadin, which allows cross-talk with other junctional complexes. Afadin not only functionally couples the E-cadherin and nectin complex (Tachibana et al., 2000), but also directly interacts with the TJ-associated protein JAMA, thereby influencing tight junction assembly during further progression of the polarity program (Fukuhara et al., 2002). JAMA assembles at the cell membranes of the early 8-cell mouse embryo prior to compaction and unlike any other TJ constituent its recruitment to the cell-cell contact initiation sites is independent of cadherin-mediated adhesion (Thomas et al., 2004). Therefore, JAM-A recruitment occurs substantially ahead of other TJ transmembrane proteins, with occludin and claudin assembly taking place from the late morula stage onwards (Thomas et al., 2004; Eckert and Fleming, 2008). Importantly, only compact embryos treated with an E-cadherin neutralizing antibody disassemble rapidly, whereas inactivation of JAM-A does not perturb compaction and only results in a delayed cavitation of the embryo (Thomas et al., 2004). This result underscores the importance of cadherin-based cell-cell adhesion during compaction and indicates that JAM-A cell surface expression does not contribute to the initiation of cell adhesion per se within the embryo. 


\section{CELL POLARIZATION-A KEY STEP TOWARD BARRIER FORMATION}

The establishment of mutually exclusive cortical domains in epithelia and endothelia in part relies on the asymmetric distribution of a set of evolutionary conserved biochemical factors controlling cell polarity. Genetic studies in C. elegans and D. melanogaster have identified three polarity groups, the Crb, Par, and Scrib groups, with the members of each group having interdependent and partly antagonistic functions. Although the majority of our understanding of the complex polarity signaling pathways in mammals is based on studies carried out in $2 \mathrm{D}$ and $3 \mathrm{D}$ cultured epithelial cells more recent studies indicate that the same set of proteins is critical for endothelial polarity (Lizama and Zovein, 2013). The Par complex is located sub-apically in epithelial cells and assembles around Par-6 isoforms (Macara, 2004). Par6 binds atypical protein kinase $\mathrm{C}$ (aPKC) $\lambda / \iota$ or $\xi$ and selectively engages GTP-bound Cdc42 (Joberty et al., 2000; Johansson et al., 2000; Lin et al., 2000), which stimulates the activity of Par6-associated aPKC several-fold, potentially through a conformational rearrangement of the complex. The Par6/aPKC complex appears to be a general determinant of polarity, which undergoes cell-specific interactions with other polarity proteins. Members of the Par-complex act in synergy with the Crumbs/Pals/Patj group of proteins to define the apical cell surface (Hurd et al., 2003; Ebnet et al., 2008). As Jam-A (and all other isoforms; see further below) directly interacts with Par3, it most likely serves as a spatial landmark to tether the Par3/Par6/aPKC polarity complex to the primordial junction. The expression of the Par6/aPKC complex has been confirmed in brain endothelial cells (Daneman et al., 2010a) and it has also been shown to be important for the establishment of endothelial polarity and lumen formation in general (Zovein et al., 2010). Genetic deletion of $\beta 1$-integrin results in decreased Par3 expression, leading to a loss of endothelial polarity (Osada et al., 2011). More recently, it has been shown that the non-canonical Wnt/planar cell polarity (PCP) pathway contributes to TJ integrity of hCMEC/D3 cell in vitro, suggesting that this pathway might act as a key regulator of the $\mathrm{BBB}$ in vivo (Artus et al., 2014). However, similarly to what has been reported for epithelial cells (Chen and Macara, 2005; Qin et al., 2005) the knock-down of Par3 only delays but does not fully inhibit TJ formation in brain endothelial cells.

The Crumbs group (Crb) localizes apically or at apical junctions and consists of the transmembrane Crb proteins and the large scaffolding proteins Pals1 and Patj through which the complex physically is connected to the Par group via Par6 (Bilder et al., 2003; Hurd et al., 2003; Assemat et al., 2008; Tepass, 2012). In ECs tight junction integrity is also maintained by angiomotin and angiomotin-like proteins (Amot, AmotL1), in part through the action of the associated RhoGEF Syx and loss of any of these proteins results in increased vascular permeability and/or reduced sprouting angiogenesis (Garnaas et al., 2008; Zheng et al., 2009; Ngok et al., 2012).

The basolateral region of epithelial cells is specified by a group of tumor suppressor genes, the Scrib complex. These include Scribble, which encodes a LAP protein (with leucine-rich repeats and four PDZ domains), Dlg, a MAGUK protein, and Lgl, comprised of WD40 repeats (Yamanaka and Ohno, 2008). Scrib,
Dlg and Lgl are membrane-associated adaptors implicated in cytoskeletal organization and protein trafficking and potentially in coupling tissue architecture to withdrawal from the cell cycle. Although little is known about Scribble in endothelial cells, a recent study provides evidence that loss of Scrib does exhibit vascular hemorrhaging suggestive of defective permeability barriers and a likely role in endothelial cell tube formation and angiogenesis (Michaelis et al., 2013). In breast cancer epithelia Scribble has been demonstrated to interact with TAZ, a member of the Hippo signaling pathway (Cordenonsi et al., 2011). Interestingly, knock-down of angiomotin-like 2 (amotL2), another member of the hippo cascade with ties to the Crb-polarity complex, results in decreased tubulogenesis in vitro (Wang et al., 2011). Therefore, it is tempting to speculate that the opposing functions of Crumbs/Amot and Scribble polarity complexes also antagonistically impact upon the Hippo pathway to regulate endothelial polarity and lumen formation.

One of the essential polarity regulators from yeast to man is the RhoGTPase Cdc42. In epithelial cells PTEN-mediated segregation of phosphoinositides has been shown to initiate polarization by recruiting Cdc42 to apical domains (Martin-Belmonte et al., 2007). Further, the small GTPase Rac1 has been shown to play an important role downstream of the Par complex (Iden and Collard, 2008). Independent of their polarity actions the best studied function of Rho GTPases is their role als molecular organizers of the actin cytoskeleton. These two processes are interconnected as cell polarization and the establishment of a functional TJ also entails a complex rearrangement of the actin cytoskeleton, microtubule organizing centers, and the establishment of a vesicle trafficking machinery, all together facilitating vectorial transport functions (Rodriguez-Boulan and Macara, 2014).

After establishment of cell polarity additional junctional proteins are being sequentially delivered to the site of the adhesion structure resulting in the maturation of the adherens junction (AJ) and TJ (Eckert and Fleming, 2008). Initially, during compaction an immature junction, containing AJ and TJ constituents, is formed serving as an initial landmark which is required for proper cell polarization. Next to cadherin-catenin components, the TJ-related proteins ZO-1 (the shorter ZO- $1 \alpha$ isoform) and JAM-A localize to the immature junction. In addition, the rab GTPase rab13 relocates early to the apico-lateral contact site (Sheth et al., 2000). During the following 16-cell stage the TJ proteins ZO-2 (Sheth et al., 2008) and cingulin (Javed et al., 1993) enter the stage of junctional biogenesis and subsequently the transmembrane proteins occludin and claudin-1 and -3 are targeted to the junction. Concomitantly the segregation into an apical tight junction and an adherens junction located more basally takes place (Rajasekaran et al., 1996; Sheth et al., 2000). Interestingly, although the formation of tight junctions and cell polarity are intimately related, in vitro the Par protein- and Scribble-complex have minor effects on TJ establishment in epithelial cells. The knock-down of either protein only delays but does not inhibit TJ formation (Chen and Macara, 2005; Qin et al., 2005). In contrast, perturbation of the Crbs/Pals1/Patj complex results in persistent TJ defects (Fogg et al., 2005; Shin et al., 2005; Wang et al., 2007). As the junction further matures, together with a complex rearrangement of the cytoskeleton and 
the establishment of a vectorial transport system, a permeability seal is formed which is the prerequisite for the formation of the nascent blastocoel cavity.

Similar to epithelial differentiation, blood vessel formation relies on cell-cell contact and apical-basal polarization of endothelial cells prior to lumen formation and junctional maturation. As already mentioned above, various models have been proposed to explain vascular lumenization, including the intracellular vacuole coalescence model (cell hollowing), or extracellular vacuole exocytosis and lumenal repulsion model (cord hollowing) (Iruela-Arispe and Davis, 2009; Zeeb et al., 2010; Axnick and Lammert, 2012). While cell hollowing involves the generation and fusion of intracellular vacuoles of individual cells, cord hollowing is based on lumen formation between two or more ECs (or an EC cord) (Lubarsky and Krasnow, 2003; Blum et al., 2008; Chung and Andrew, 2008; Lee and Bautch, 2011). Both types of lumen formation make use of a similar set of proteins and pathways and distinguishing them from each other in vivo is a difficult task. Generally, lumina of small capillaries (maximum diameter of a single cell) are formed by cell hollowing while lumenization of larger vessels follows the process of cord hollowing. However, using a computational model of lumen formation it was recently suggested that cell hollowing and cord hollowing may operate in parallel (Boas and Merks, 2014).

The initial phase of cord hollowing is characterized by cellcell adhesion, exocytosis and apical membrane repulsion (see Figure 2). Initial intercellular contacts are established by homotypic interaction of VE-cadherin and Pecam-1/CD31 which are distributed along the entire contacting plasma membrane. Additionally, the uniform distribution of the polarity proteins Par3 in contacting ECs is indicative of a yet unpolarized state of the cells. Besides VE-cadherin also $\mathrm{N}$-cadherin was found at EC contact sites, which is believed to increase the amount of VE-cadherin at cell-cell contacts (Luo and Radice, 2005). Subsequently, de-adhesive glycoproteins (CD34-sialomucins) are delivered from cytoplasmic vesicles to the contacting plasma membrane delineating the future apical surface. The specific targeting of CD34-sialomucins to EC contact sites depends on VEcadherin and involves PTEN signaling together with PIP2/PIP3 conversion (Zeeb et al., 2010). It is believed that the negatively

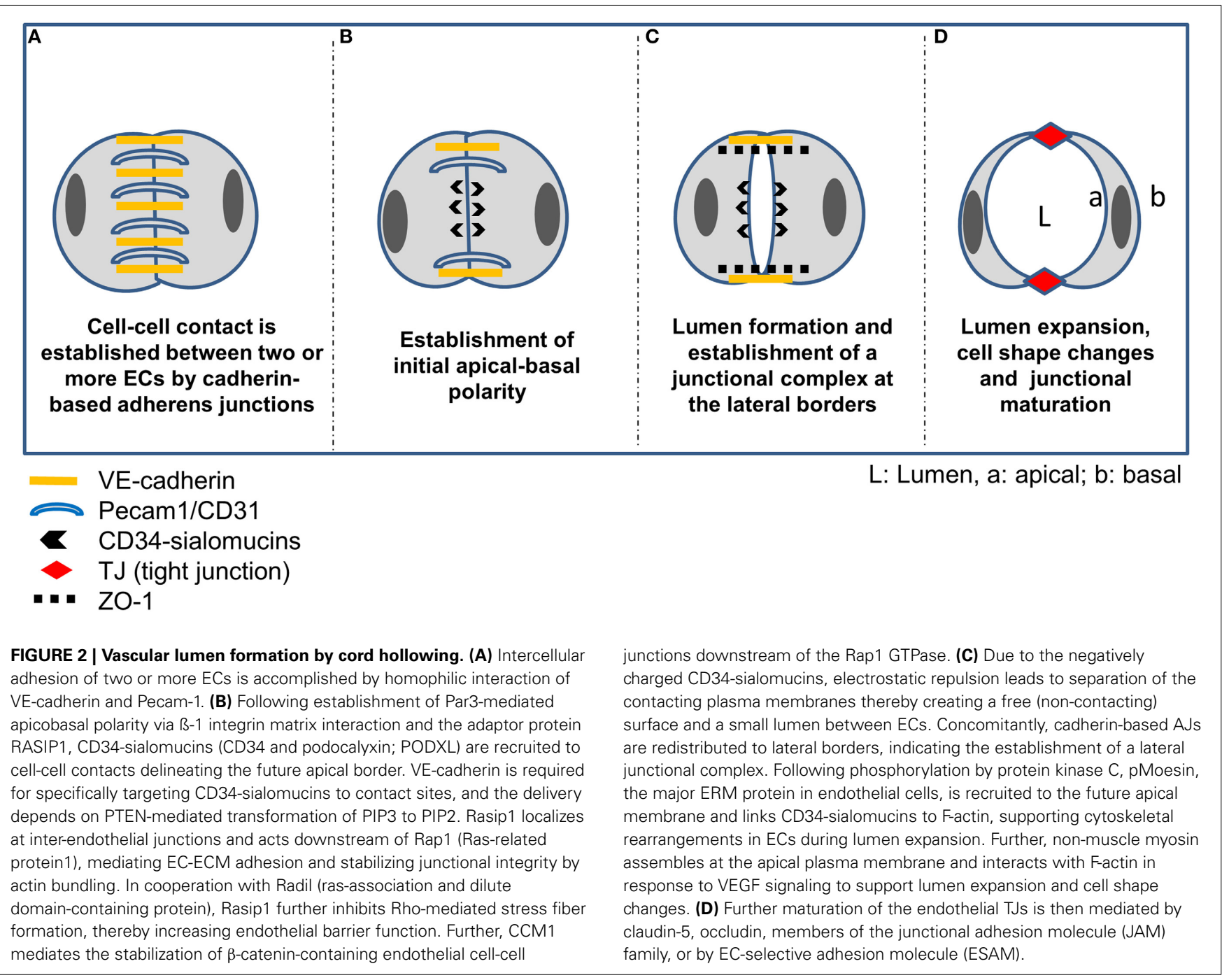


charged extracellular domains of CD34 sialomucins initiate the repulsion of opposing leaflets creating a narrow space between the cells, commonly referred to as "slit formation" (Robbins and Beitel, 2010; Strilic et al., 2010). One of the CD34-related sialomucins is podocalyxin (PODXL). Originally, PODXL has been described as the major sialoprotein in the glycocalyx of glomerular podocytes (Kerjaschki et al., 1986). However, it has been recognized early that PODXL is a widespread component of vascular endothelial cells (Born and Palinski, 1985; Horvat et al., 1986). It localizes to the luminal domain of endothelial plasma membranes in a patchy distribution and is absent from the abluminal aspect (Horvat et al., 1986). Although the function of endothelial sialoproteins has long remained elusive, a role in regulating the thrombogenic properties of the endothelial surface was proposed (Gorog et al., 1982; Born and Palinski, 1985). More recently it has been shown that PODXL (syn. gp135) is a key regulator of apical domain structure establishment in epithelial cells (Meder et al., 2005) and it recruits filamentous actin and ezrin to the plasma membrane, inducing microvillus formation (Nielsen et al., 2007). Interestingly, embryoid bodies lacking ZO-1 or ZO-1/ZO-2 (but not ZO-2 alone) exhibit reduced expression of PODXL at the apical plasma membrane and lack TJs in the extraembryonic endoderm (Phua et al., 2014). Further, quantitative PCR profiling of RNA samples from laser capture microdissected microvessels revealed that PODXL is preferably expressed in brain microvessels (Agarwal et al., 2010) suggesting a role in BBB establishment and/or function.

CD34-sialomucins are further linked to the actin cytoskeleton via protein kinase $\mathrm{C}$ - (PKC) phosphorylated moesin, thereby contributing to cell shape changes during lumenization (Strilic et al., 2009). Lumen extension then depends on cytoskeletal rearrangements involving non-muscle myosin and recruitment thereof to the apical F-actin is accomplished by activation of the Rho-associated protein kinase (ROCK) and VEGF-A signaling (reviewed in Lee and Bautch, 2011). Finally, the assembly of CD34-sialomucins at the future apical membrane triggers the redistribution of junctional proteins to the lateral border, constituting the initial step in the formation of a lateral junctional complex.

Disturbance of endothelial polarization is observed in cerebral cavernous malformations (CCM), the most common vascular dysplasia in the brain, which is characterized by hemorrhagic stroke, focal neurological deficits and seizures (reviewed in Draheim et al., 2014). CCM1 binds to and is regulated by the small GTPase Rap1, enhancing the adhesive properties of VEcadherin (Glading et al., 2007). The CCM1 FERM domain is unmasked by the activated Rap1, targeting CCM1 to the junction and promoting endothelial polarization in a Par-dependent manner (Lampugnani et al., 2010) (see Figure 3). In the absence of CCM1, endothelial lumenization is severely disturbed, as evidenced by grossly dilated capillaries and multicavernous structures (Clatterbuck et al., 2001). Endothelial-specific disruption of the CCM1 gene in mice induces endothelial to mesenchymal transition due to increased BMP6-SMAD signaling, a process which is characterized by the loss of cell polarity, increased cell proliferation and migratory capacity (Maddaluno et al., 2013). Although CCM genes are inactivated in all types of endothelial cells under pathological conditions, CCM lesions are mostly apparent in the brain vasculature. Therefore, the loss of endothelial polarity and the concomitant impaired astroglia-endothelial interaction, which is imperative for BBB function, seems to be the underlying cause of frequent vascular lesions in the brain (Maddaluno et al., 2013). This and other studies elegantly demonstrate that cell-cell adhesion and apical-basal polarization appears to be a universal event preceding epithelial and endothelial tubulogenesis, a mechanism imperative for organ development and vascularization.

Results from numerous studies on various developmental models converge on a crucial role for apical membrane specialization prior to junctional maturation. The establishment of a cadherin-based primordial junctional complex prior to segregation of a more apically located TJ appears to be a conserved step in epithelial and endothelial differentiation. Besides the indispensability of cadherin-based cell-cell contacts, the early and bi-phasic role of ZO-1 isoforms appears to be crucial for endothelial and epithelial barrier formation. Following de novo establishment of tight junctions, a dizzying number of proteins localize to the cytoplasmic aspect of TJ not only ensuring the integrity of the barrier but also allowing a remodeling and change in permeability properties in response to physiological and pathological stimuli. Of the known TJ constituents, the zonula occludens ( $\mathrm{ZO}$ ) proteins lie at the heart of the TJ cytoplasmic plaque. Below, the major tight junction proteins are being discussed, focusing on proteins that have been shown to localize to endothelial TJs.

\section{TIGHT JUNCTION PROTEINS MEMBRANE-SPANNING TJ CONSTITUENTS}

Overall, 3 distinct TJ-associated transmembrane protein groups can be distinguished (see Figure 4B): (1) claudins, a family of proteins comprising at least 27 different members in mammals (Mineta et al., 2011); (2) TAMPs (TJ-associated MARVEL proteins) - a group of proteins containing the structural tetraspanning MARVEL motif, including occludin (Furuse et al., 1993), tricellulin (Ikenouchi et al., 2005), and MarvelD3 (Steed et al., 2009); (3) Immunoglobulin superfamily membrane proteins JAM-A/-B/-C (Martin-Padura et al., 1998; AurrandLions et al., 2000; Palmeri et al., 2000; Ebnet et al., 2003), coxsackie adenovirus receptor (CAR) (Carson et al., 1999; Cohen et al., 2001), and endothelial cell-selective adhesion molecule (ESAM) (Nasdala et al., 2002).

Occludin was the first integral membrane protein shown to localize to TJs (Furuse et al., 1993) and is characterized by four transmembrane domains, a short intracellular turn, two extracellular loops, and a long carboxy- and a short amino-terminal cytoplasmic domain. Cis-oligomerization of occludin seems to be mediated by the MARVEL motif (Blasig et al., 2006; Yaffe et al., 2012), whereas the extracellular loops are believed to contribute to the gate-keeping function of TJs. The last 150 aa of the carboxyl terminus of occludin interact directly with F-actin (Wittchen et al., 1999) and the scaffold proteins ZO-1 (Furuse et al., 1994), ZO-2 (Itoh et al., 1999b), and ZO-3 (Haskins et al., 1998). Information regarding the physiological relevance of the $\mathrm{N}$ terminal domain remains scarce. Using a yeast-based two-hybrid screen the E3 ubiquitin protein ligase Itch was found to directly 


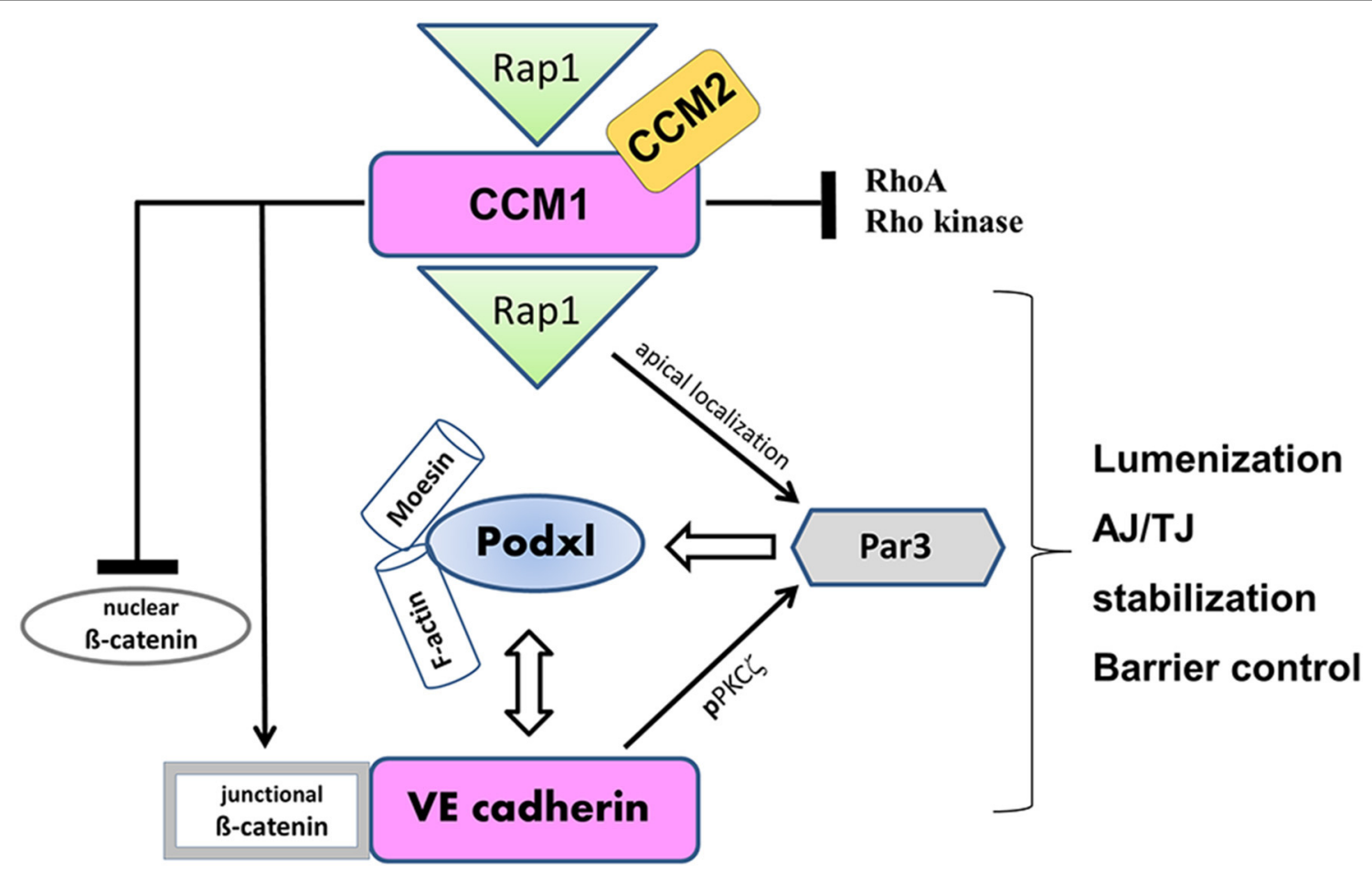

FIGURE 3 | VE-cadherin and CCM1 act in concert to establish endothelial polarization and lumen formation. Rap1 regulates the junctional localization of CCM1 and mediates CCM1 activity in endothelial polarization and lumen formation. CCM1 stabilizes VE-cadherin at AJs, by associating with $ß$-catenin and inhibiting dissociation of $ß$-catenin from AJs. Thereby, nuclear accumulation of ß-catenin and ß-catenin transcriptional signaling is impaired. VE-cadherin promotes the apical localization and activation of the Par3 polarity complex. While VE-cadherin is needed for phosphorylation of $\mathrm{PKC} \xi, \mathrm{CCM} 1 / \mathrm{Rap} 1$ is responsible for the junctional recruitment of the polarity complex. The accumulation of podocalyxin (PODXL) at the presumptive apical membrane is controlled by VE-cadherin and $B 1$ integrin. PODXL in turn recruits moesin and filamentous actin (F-actin) to the apical domain initiating endothelial lumen formation. interact with the outermost $\mathrm{NH}_{2}$-terminal domain of occludin (Traweger et al., 2002). Indeed, following VEGF-induced phosphorylation occludin is targeted for proteasomal degradation via Itch, resulting in increased endothelial permeability (Murakami et al., 2009). Occludin is targeted by several kinases and is phosphorylated on serine, threonine (Sakakibara et al., 1997; Wong, 1997), and tyrosine residues (Tsukamoto and Nigam, 1999; Chen et al., 2002) and reversible phosphorylation is an important regulatory mechanism to control TJ assembly and maintenance. For example, endothelial cells subjected to shear stress showed a significantly reduced, but tyrosine-phosphorylated pool of occludin (DeMaio et al., 2001). However, controversial results on the role of occludin phosphorylation in epithelial and endothelial cells exist.

Early investigations led to the assumption that occludin is the core transmembrane protein of TJs (Furuse et al., 1996; McCarthy et al., 1996). However, the TJ scientific community was stunned to learn that occludin is dispensable for the formation of tight junction strands. Occludin-deficient ES cells were found to be morphologically indistinguishable from their wild type counterparts (Saitou et al., 1998) and occludin-deficient mouse embryos were viable and did not show any gross morphological alterations of TJs (Saitou et al., 2000). However, phenotypically ocln-/animals displayed multiple histological abnormalities, including chronic inflammation and hyperplasia of the gastric epithelium, calcification of the brain, cortical bone thinning, and testicular atrophy. Subsequent analysis of the knockout animals suggested occludin may play a role in epithelial differentiation and proliferation (Schulzke et al., 2005). In man, mutations of the occludin gene cause band-like calcification with simplified gyration and polymicrogyria (BLC-PMG), a rare neurological disorder resulting in malformations of cortical development, implicating a role of occludin for TJs at the BBB (O'Driscoll et al., 2010). Most likely, other proteins of the TAMP family (i.e., tricellulin or MarvelD3) act redundantly partially masking the function of occludin. In summary, although occludin clearly is not of fundamental importance for the core TJ structure the findings outlined above suggest that it has a regulatory and accessory function in $\mathrm{TJ}$ formation and physiology.

After having realized that occludin is not the major TJstrand protein, the search for a new protein began. Furuse and Tsukita identified small proteins that co-fractionated with occludin in a junction-enriched chicken liver fraction (Furuse et al., 1998). Since the initial discovery of these small proteins, termed claudin- 1 and claudin-2, a total of 27 different claudin family members have been identified in man and mouse (Mineta et al., 2011). Although the predicted protein folding topology of claudins $(21-28 \mathrm{kD})$ is very similar to that of occludin, they do 


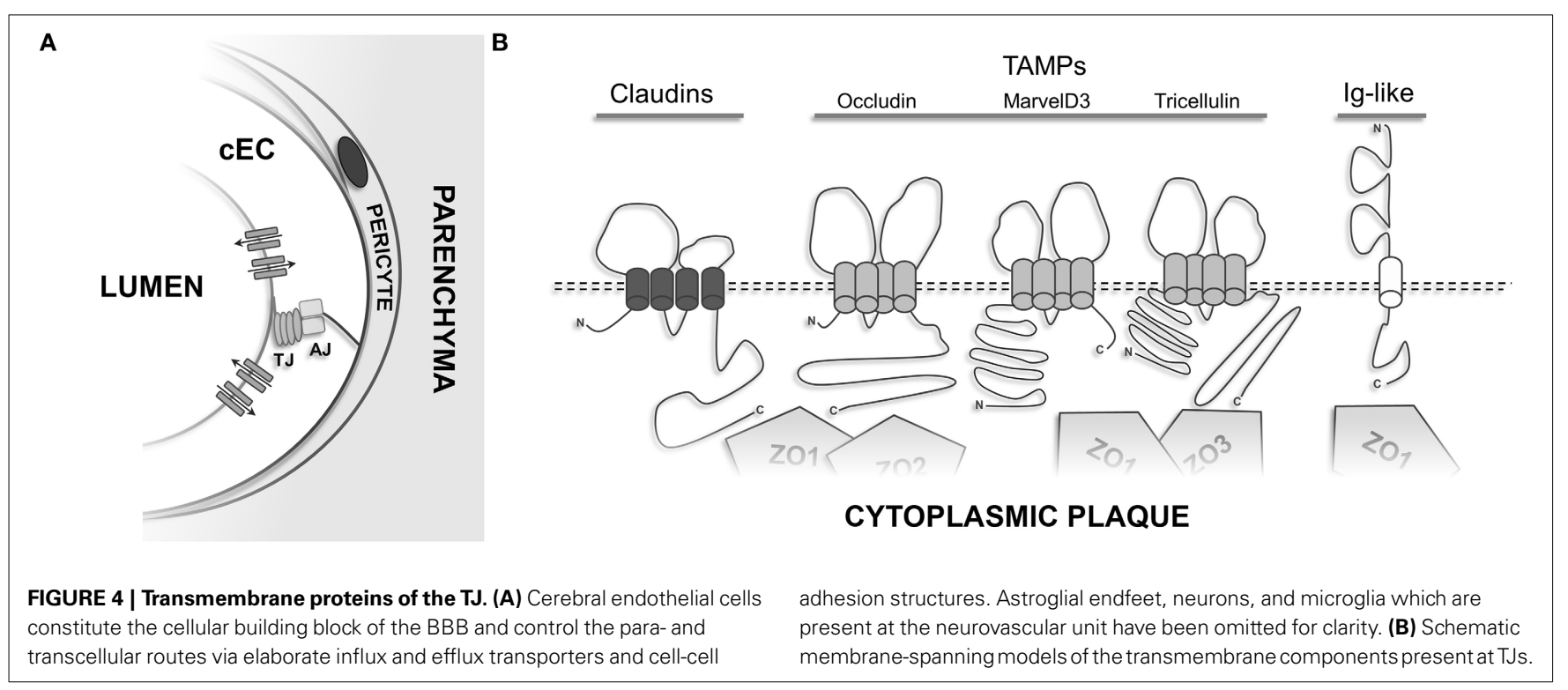

not share any sequence homology (Furuse et al., 1998). Several gain-of-function and loss-of-function studies have led to the current model that claudins are the prime transmembrane proteins responsible for the formation of higher-order tight junction strands. For example, claudin-1 deficient mice die shortly after birth due to excessive transepidermal water loss (Furuse et al., 2002) and claudin-11 knockout animals show a complete loss of TJs in Sertoli cells, oligodendrocytes, and the marginal cells of the inner ear (Gow et al., 1999, 2004; Mazaud-Guittot et al., 2010).

It is believed that claudins and TAMPs oligomerize in a homoand heterophilic fashion and TJ-strands are formed by mosaics of different claudin-family members and the combination and stoichiometry of various isoforms determines the charge- and size-selective properties of the barrier (Sonoda et al., 1999; Anderson and Van Itallie, 2009). Further, claudins show a tissuespecific expression pattern, contributing to tissue specific barrier properties. Therefore, for each tissue a unique combination of claudins which oligomerize with other TAMPS most likely determines the paracellular tightness of the junction toward different solutes.

Overall, claudins- $1,-3,-5,-11,-14,-19$ appear to be sealing components of tight junctions and for several of them human mutations have been characterized. Mutations in claudin-1 cause the neonatal ichtyosis and sclerosing cholangitis (NISCH) syndrome. Claudin-14 mutations are the underlying cause of autosomal recessive deafness 29 and 49 and mutations in claudin-19 cause renal hypomagnesemia with ocular involvement. Next to acting as "sealants" claudins have been proposed to form paracellular ion-selective channels (Amasheh et al., 2002) and so far claudin-2, $-10 \mathrm{a},-10 \mathrm{~b},-15$, and -17 have been shown to have channel-forming properties. Whereas claudins-2, 10b, and 15 display cation selectivity, $-10 \mathrm{a}$ and 17 have been described as anion-selective channels (reviewed in Gunzel and Fromm, 2012).

At the BBB, mainly claudin-3 (Wolburg et al., 2003) and claudin-5 (Morita et al., 1999) are being expressed, claudin-12 likely also being part of the BBB-TJ (Ohtsuki et al., 2007; Schrade et al., 2012). Early studies also have demonstrated the presence of claudin-1 at brain capillary endothelial cells (Liebner et al., 2000a), however this was a false report due to antibody crossreactivity with claudin-3. Subsequent studies using claudin-1 specific antibodies failed to detect any claudin-1 in CNS parenchymal microvessels and/or in primary mouse or human brain endothelial cells (Wolburg et al., 2003; Hamm et al., 2004; Coisne et al., 2005; Weksler et al., 2005).

Of those having been described so far, claudin- 5 is the most abundant isoform at the BBB as also shown by a SAGE analysis of rat brain microvasculature (Enerson and Drewes, 2006). It is crucial for the "tightness" of TJs at the mammalian BBB as its deletion resulted in a size-selective opening of the $\mathrm{BBB}$ in vivo, allowing the diffusion of molecules smaller than $800 \mathrm{Da}$ (Nitta et al., 2003). Interestingly, the TJs of the BBB were morphologically normal, nevertheless the knockout of claudin-5 resulted in early neonatal death. This study also shows that the increase in paracellular flux is tolerated during embryogenesis, potentially due to the presence of the placental barrier. This indicates that the BBB in the mouse embryo is "immature," even though it already restricts the flux of blood-borne products into the neuroparenchyma (see Figures 1C,D).

Additional data establishes the key role of claudin-5 and claudin-3 in TJ integrity at the BBB. For example, overexpression of claudin-5 increases the paracellular tightness in cultured brain microvascular endothelial cells (Ohtsuki et al., 2007) and the selective loss of claudin-3 in experimental autoimmune encephalomyelitis or human glioblastoma multiforme is associated with BBB breakdown, suggesting that it also contributes to BBB integrity and function (Wolburg et al., 2003). Interestingly, a specific role of claudin-3 at the BBB is also underscored by the observation that its expression correlates with the maturation of the $\mathrm{BBB}$ in response to $\mathrm{Wnt} / \beta$-catenin signaling during development (Liebner et al., 2008). However, the exact role of claudin-3 at the $\mathrm{BBB}$ remains to be seen.

The greatest sequence diversity of claudins is located in the C-terminal domain, suggesting that their diverse functions are in part conferred by their association with cytoplasmic proteins. 
Generally, the last C-terminal amino acids constitute a PDZ binding motif resulting in the recruitment of the TJ-plaque proteins ZO-1, ZO-2, ZO-3 (Itoh et al., 1999a), which seem to instruct claudins to form functional TJ strands (Umeda et al., 2006). Similarly to occludin, claudins are also phosphorylated on serine or threonine residues, regulating their function and intracellular localization (Ishizaki et al., 2003; D’Souza et al., 2005; Ikari et al., 2006; Aono and Hirai, 2008).

Next to bicellular contacts, the formation of specialized tricellular contacts is required to maintain the integrity of cellular sheets. At these contacts a specialized TJ structure (tTJs) is formed where three sealing elements are in close apposition and form a narrow tube in the intercellular cleft (Friend and Gilula, 1972; Staehelin, 1973; Ikenouchi et al., 2005). Specialized transmembrane proteins, tricellulin and proteins of the angulin family are located at these contacts and recently have been identified in endothelial cells of the brain and retina (Mariano et al., 2013; Iwamoto et al., 2014). Tricellulin shares some sequence similarity with occludin and depending on the cell type can also be found at bicellular contacts where it possibly acts as a substitute for occludin (Ikenouchi et al., 2005; Krug et al., 2009). Taken together, TJ-associated MARVEL proteins are able to partially exert redundant functions, but also show tissue-specific expression and are involved in distinct aspects of tight junction assembly, maintenance, and regulation (Raleigh et al., 2010).

So far, the last class of transmembrane proteins found to localize to the TJ are members of the immunoglobulin superfamily. Junction adhesion molecules or more precisely the first isoform JAM-A was identified by using monoclonal antibodies raised against endothelial antigens (Martin-Padura et al., 1998). They form homotypic cell-cell contacts between endothelial and epithelial cells and are highly enriched at TJs (Bazzoni et al., 2000a; Liu et al., 2000). JAMs are generally glycosilated and encompass two extracellular variable type Ig domains and a single transmembrane domain. Through a classical type II PDZ binding motif located in its outermost C-terminus JAM-A interacts with several cytoplasmic proteins, aiding in the establishment of the cytoplasmic plaque of TJs (Bazzoni et al., 2000b; Ebnet et al., 2000; Martinez-Estrada et al., 2001; Hamazaki et al., 2002). Interestingly, JAM-/- mice show little alterations of the overall epithelial architecture in the intestinal tract, but display increased colonic inflammation and paracellular permeability (Laukoetter et al., 2007). Next to JAM-A, the closely related proteins JAM-B (JAM2) and JAM-C (JAM3) have been cloned and characterized (Aurrand-Lions et al., 2000; Cunningham et al., 2000; Palmeri et al., 2000; Arrate et al., 2001; Aurrand-Lions et al., 2001). In contrast to JAM-A, JAM-B/-C seem restricted to endothelial cells and have been demonstrated to form heterodimers in cell-cell contacts, thereby counteracting the interaction with the leukocyte receptor alpha(M)beta2 integrin (Cunningham et al., 2002; Lamagna et al., 2005). In humans, homozygous mutations of JAM-C result in intracerebral hemorrhages, subependymal calcification, and congenital cataracts (Mochida et al., 2010). However, overall little is known about their exact role at the BBB.

Strikingly, among all TJ-associated transmembrane proteins only JAM-A/-B/-C interact directly with the cell polarity protein Par3 (Ebnet et al., 2001, 2003; Itoh et al., 2001). JAMs localize to sites of early cell-cell contact formation, so called puncta or primordial spot-like junctions, where they most likely "flag" this region for the recruitment of the Par3/Par6/aPKC polarity complex during early junctional biogenesis in epithelial and endothelial cells (see also further above). Next to their involvement in cell-cell adhesion JAMs also mediate the transendothelial migration of leukocytes which is of particular importance for BBB function (Fraemohs et al., 2004; Aurrand-Lions et al., 2005).

Four additional Ig-superfamily protein members have been found to localize to TJs: CAR - coxsackie- and adenovirus receptor (Cohen et al., 2001; Raschperger et al., 2006), CLMPcoxsackie- and adenovirus receptor-like membrane protein (Raschperger et al., 2004), JAM 4 (Hirabayashi et al., 2003), and ESAM-endothelial cell-selective adhesion molecule (Hirata et al., 2001; Nasdala et al., 2002). Of these, ESAM seems to be the only endothelial-specific transmembrane protein. ESAM-/- mice did not show any major vascular defects, however endothelial tube formation was impaired suggesting a role of ESAM in cellcell contact formation (Ishida et al., 2003). More recently, ESAM was also suggested to support the extravasation of neutrophils during the early phase of an inflammatory response (Wegmann et al., 2006).

Taken together, transmembrane proteins of the TJ not only facilitate cell-cell adhesion and impede paracellular flux, they also target cytoplasmic protein assemblies to distinct cell membrane domains, endowing endothelial and epithelial cells with their critical biological properties.

\section{TJ CYTOPLASMIC PLAQUE PROTEINS}

The most prominent subgroup of scaffolding proteins localizing to tight junctions is represented by the MAGUK (membraneassociated guanylate kinase) proteins (Dimitratos et al., 1999; Gonzalez-Mariscal et al., 2000; Funke et al., 2005). The modular nature of MAGUK proteins was early recognized as they all share a common structural core consisting of one or several PDZ domains and an SH3 domain followed by a catalytically inactive guanylate kinase (GUK) domain (see Figure 5A). Originally, MAGUKs only referred to a group of proteins including the mammalian synaptic scaffold protein Psd-95, the Drosophila tumor suppressor Dlg, and the tight junction protein ZO-1. Since then a large number of scaffold proteins belonging to this family have been identified. Generally, at junctions they serve as molecular hubs coordinating large protein assemblies which transduce the signals impinging on and emanating from the apical plasma membrane, thereby influencing diverse cellular processes, including the establishment and maintenance of cell polarity and cell-cell adhesion complexes, synaptic plasticity, and cell survival (Funke et al., 2005; Te Velthuis et al., 2007; De Mendoza et al., 2010).

Stevenson and Goodenough identified the first TJ-associated MAGUK ZO-1 (zonula occludens-1) by generating monoclonal antibodies against a TJ-enriched plasma membrane fraction from rodent liver (Stevenson et al., 1986). Subsequently, ZO-2 (Gumbiner et al., 1991) and ZO-3 (Balda et al., 1993; Haskins et al., 1998) were discovered by co-immunoprecipitation studies. The $\mathrm{ZO}$ proteins are at the core of large protein networks which are anchored to the TJ through their association with the 


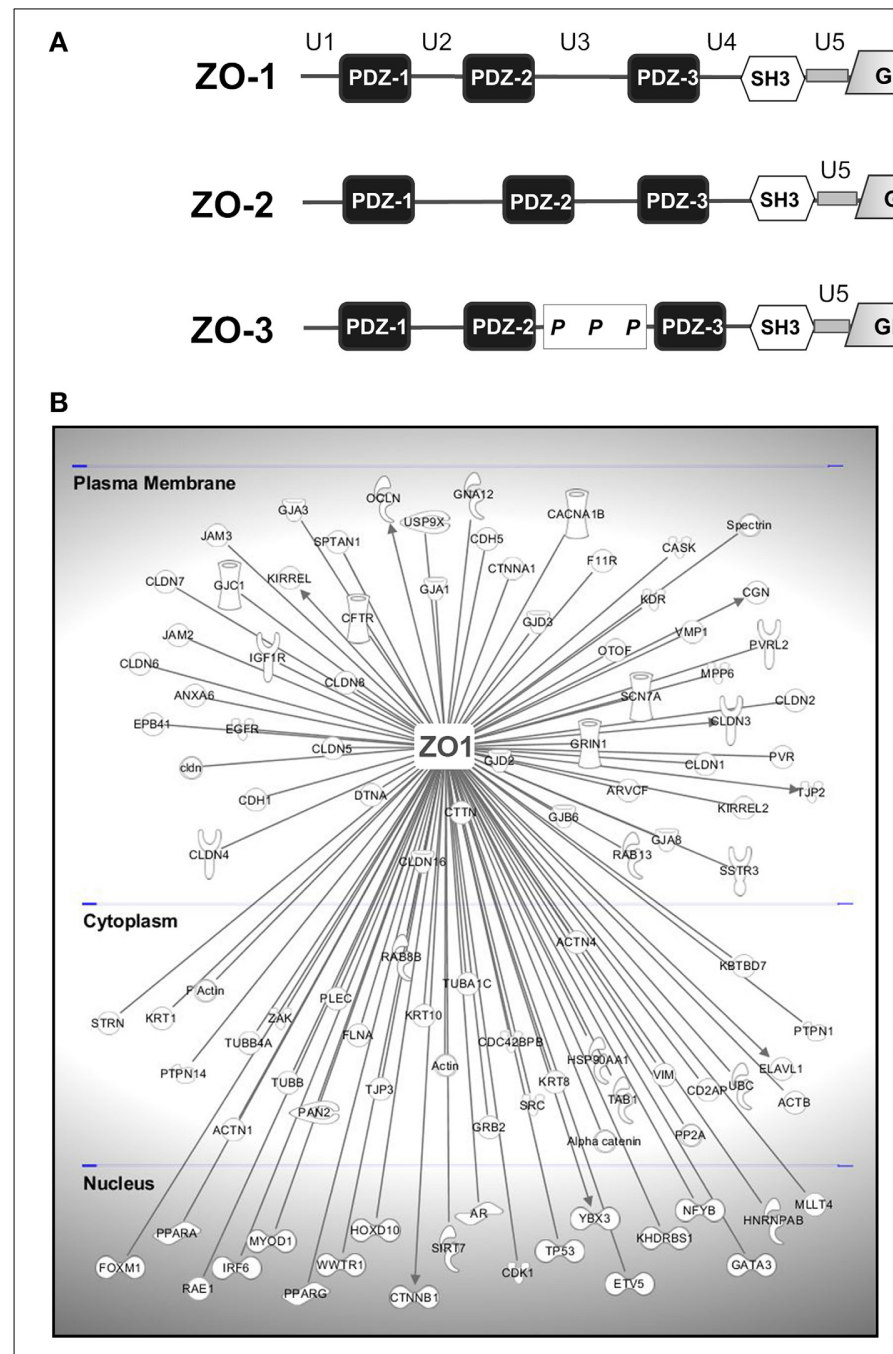

FIGURE 5 | TJ-MAGUKS are membrane-associated adaptor proteins assembling the tight junction plaque. (A) ZO-1, ZO-2, and ZO-3 are multi-domain proteins composed of three PDZ domains, one $\mathrm{SH} 3$ and a catalytically inactive GUK domain. Next to these MAGUK-related domains, ZO proteins also contain various ZO-specific unique $(U)$ and proline-rich $(P)$

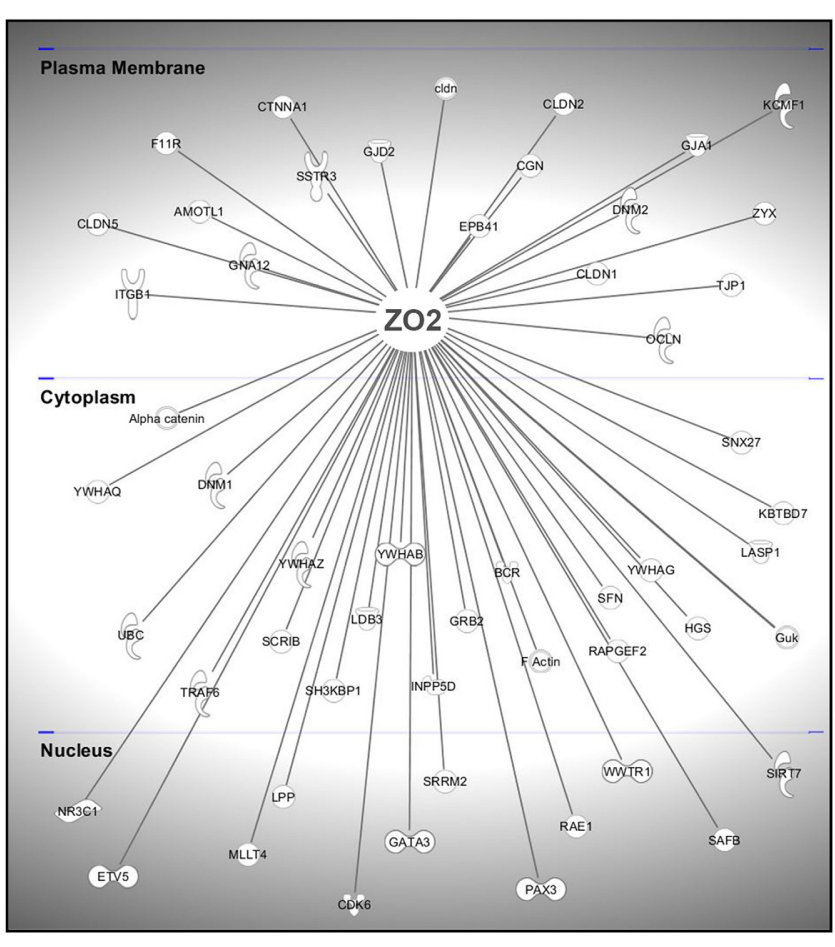

regions. ZU5 is a region found in ZO-1 and Unc5-like netrin receptors. (B) ZO-1 and ZO-2 are at the basis of large protein assemblies containing structural and signaling molecules. Shown are proteins that have been demonstrated to interact with ZO-1 or ZO-2 in epithelial and endothelial cells. Gene symbols according to the H.G.N.C. nomenclature are indicated.
C-terminal domains of JAM-A (Bazzoni et al., 2000b; Ebnet et al., 2000; Itoh et al., 2001), occludin (Furuse et al., 1994; Fanning et al., 1998), and most claudins (Itoh et al., 1999a). They further interact physically or functionally with a multitude of cytoplasmic proteins forming the TJ-plaque and they provide a direct link to the actin cytoskeleton via a direct interaction with actin filaments (Itoh et al., 1997; Fanning et al., 1998; Wittchen et al., 1999; Fanning et al., 2002) or the actin binding proteins fodrin/spectrin, cortactin, and protein 4.1R (Katsube et al., 1998; Mattagajasingh et al., 2000). This association to intact cytoskeletal arrays is critical for the physiology of cell-cell junctions.

ZO-1 and ZO-2 are expressed in both epithelial and endothelial cells and have been shown to be indispensable for TJ- strand formation (Umeda et al., 2006; Katsuno et al., 2008). ZO-3 seems to be confined to epithelial cells (Inoko et al., 2003) and is less critical for TJ function (Adachi et al., 2006; Xu et al., 2008).
Structurally, ZO proteins share a high degree of sequence similarity across their protein-binding modules, however display significant variability within their C-terminus suggesting that these regions are functionally distinct. Next to the core MAGUK domains of the $\mathrm{ZO}$ proteins, several unique $(\mathrm{U})$ regions have been identified (see Figure 5A). In the case of ZO-1 these regions influence its intracellular localization and its scaffolding properties (Fanning et al., 2007; Fanning and Anderson, 2009), but little is known about their relevance for ZO-2 and ZO-3. Although the high sequence similarity also suggests a fair degree of functional redundancy, specific roles have been described for the different ZO proteins. Deficiency of ZO-1 results in mislocalization of endothelial junctional adhesion complexes and defects in angiogenesis were evident, resulting in early embryonic lethality (Katsuno et al., 2008). Deletion of ZO-2 in mice is embryonic lethal shortly after implantation due to an arrest in early 
gastrulation, embryos showing a decreased proliferation rate and an increase in apoptotic cells (Xu et al., 2008).

In brain cECs a decrease in the expression of ZO-1 and occludin after cerebral embolism has been reported (Kago et al., 2006) and in vitro under hypoxic conditions the localization of ZO-1 and ZO-2 was altered with a concurrent decrease in TEER (Mark and Davis, 2002; Fischer et al., 2004). Further, disruption of the TJs at the BBB occurs in many neurological disorders (Rosenberg, 2012). However, in most cases the changes in TJ structure and function are most likely the result of indirect downstream signaling events triggered secondary to an inflammatory or traumatic event and are not directly exerted by the junctional proteins themselves. Generally, the reduced expression of ZO-1 correlates with increased proliferation of cells and/or transformation. For example, in highly proliferative brain microvascular endothelial cells from human brain tumors ZO-1 levels are low. Apart from reduced expression, $\mathrm{ZO}$ proteins may also regulate proliferation by nuclear translocation. A large body of evidence demonstrates that ZO-1 and ZO-2 are not only present at cellcell junctions but also target the cell nucleus. Even though the majority of these findings relate to epithelial cells, it is likely that many of these processes also take place in endothelial cells. All $\mathrm{ZO}$ proteins harbor conserved nuclear localization and nuclear export sequences (Lopez-Bayghen et al., 2006) and in sparse cultures, ZO-1 and ZO-2 are present in cell nuclei but become re-distributed to the plasma membrane as soon as cells reach confluence (Gottardi et al., 1996; Islas et al., 2002; Traweger et al., 2003; Jaramillo et al., 2004; Gonzalez-Mariscal et al., 2006). ZO-1 further perturbs gene expression by sequestering the Y-box transcription factor ZONAB to the cytoplasm. ZONAB interacts with the cell cycle regulator CDK4 and controls expression of cell cycle regulators such as cyclin D1 and PCNA (for review see Balda and Matter, 2009). ZO-2 has been shown to inhibit the transcription of G1 cyclin after translocating to the nucleus (Gonzalez-Mariscal et al., 2009). In addition, several proteins interact with ZO-2 during nuclear shuttling or within the nucleus itself. For example, the interaction of the armadillo-repeat protein ARVCF (armadillo repeat gene deleted in velocardiofacial syndrome) depends on an N-terminal PDZ domain of ZO-2 (Kausalya et al., 2004), a region which is also targeted by the non-receptor tyrosine kinase JAK1. Also, the transcription factors Jun, Fos, and C/EBP were shown to associate with ZO-2 within the nucleus and at TJs in epithelial cells (Betanzos et al., 2004). Next to directly associating with and/or influencing transcription factors, ZO-2 possibly impacts upon the transcription machinery via its association with the hnRNP SAF-B/HET (Traweger et al., 2003), a molecular platform which assembles a transcription complex in the vicinity of actively transcribed genes (Renz and Fackelmayer, 1996). Taken together, ZO proteins potentially act as direct or indirect regulators of cell proliferation through nuclear translocation and/or their ability to sequester transcription factors and signaling molecules to the cytoplasm. However, our understanding of the signaling pathways and their biochemical properties through which $\mathrm{ZO}$ proteins exert their effects beyond cell-cell adhesion remains fragmentary.

Next to the TJ-MAGUKs members of the so called MAGI protein family (membrane-associated guanylate kinase with inverted orientation) localize to TJs. They are large adaptor proteins containing 6 PDZ domains and a module of a GuK domain and two WW domains C-terminal to the first PDZ domain. Of the identified MAGI proteins, only MAGI-1 has been shown to localize to TJs of brain endothelial cells where it interacts with ESAM and promotes maturation of TJs in a Rho-dependent manner (Wegmann et al., 2004; Kimura et al., 2010). In vascular endothelial cells MAGI- 1 physically interacts with $\beta$-catenin and localizes to VE-cadherin-based AJs (Sakurai et al., 2006), however much detail about the role of MAGI-1 at AJs and TJs is still missing.

Several other regulatory cytoplasmic plaque proteins have been demonstrated to localize to the TJ-plaque, including heterotrimeric G-proteins (Denker et al., 1996; Fabian et al., 1998; Saha et al., 1998). In brain capillary endothelial cells $G_{\alpha \mathrm{i} 2}$ is found in a complex together with claudin-5 and its depletion results in increased paracellular permeability and delayed TJformation after a hyperosmotic shock of cultured cECs (Luissint et al., 2012). Another class of proteins influencing TJ physiology are Rho family GTPases. In rat brain endothelial cells C3 transferase, a toxin which specifically inactivates RhoA-C, abrogated lymphocyte transmigration, suggesting that Rho is required for junction intergrity (Adamson et al., 1999). In addition, expression of mutant Rho GTPases in epithelial and endothelial cells affected barrier properties (Nusrat et al., 1995; Jou et al., 1998; WojciakStothard et al., 2001; Bruewer et al., 2004) and the paracellular flux of sucrose was increased in cerebral endothelial cells treated with lysophoshatidic acid (Schulze et al., 1997). So far, 3 TJassociated Rho-specific regulators have been identified. (1) The Rho-activator GEF-H1 has been shown to regulate paracellular permeability in epithelial and endothelial cells (Benais-Pont et al., 2003; Birukova et al., 2006). (2) Tuba, an activator for Cdc42, which is recruited to epithelial TJs in a ZO-1-dependent manner (Otani et al., 2006). (3) The GAP protein SH3BP1 which restricts the activity of Cdc42 and Rac when complexed with the scaffold protein CD2AP, parcingulin, and the actin filament capping protein CapZ (Elbediwy et al., 2012).

Cingulin and the closely related protein paracingulin (JACOP) have also been shown to concentrate at TJs of epithelial and endothelial cells, providing a direct link to the cytoskeleton and to regulate the activity of Rho family GTPases at junctions by recruiting guanidine exchange factors of RhoA and Rac1 (Paschoud et al., 2011). Overall, little is known about how the activities of RhoGTPases are regulated and how they feed into the signaling networks that control tight junction formation and integrity. Most likely a Rho-dependent regulation of the actinomyosin cytoskeleton perturbs TJ integrity.

Taken together, the modular structure of a set of adaptor proteins allows the spatio-temporal assembly of multi-protein complexes (see Figure 5B) at discrete regions of epithelial and endothelial cells through their interaction with clustered transmembrane TJ proteins, not only ensuring the structural integrity of the TJ, but also integrating various regulatory pathways at the cytoplasmic plaque which are pivotal for TJ physiology.

\section{THE NVU—COUPLING BBB INTEGRITY AND NEURONAL ACTIVITY}

The endothelial cells forming the lining of the microvasculature of the brain are set up to actively provide the brain with oxygen and 
essential nutrients, allowing not only a timely response to local demands but also buffering, which is crucial to physiological neuronal function (Abbott et al., 2006; Abbott, 2013). Brain cECs are wrapped by pericytes, both sharing a common basement membrane onto which astrocytes project their endfeet, ensheathing over $95 \%$ of the abluminal microvascular surface (see Figure 4A). In addition, cECs receive input from neurons.

Early in vivo grafting studies and experiments using astrocyteconditioned media highlighted the role of astrocyte-derived secreted factors to influence the BBB properties of brain cECs by inducing junction formation (Arthur et al., 1987; Janzer and Raff, 1987; Tao-Cheng et al., 1987; Haseloff et al., 2005). Glialderived neurotrophic factor (GDNF) has been demonstrated to enhance the barrier properties of brain cECs in vitro (Igarashi et al., 1999) and FGF was found to decrease BBB permeability (El Hafny et al., 1996), which is in line with the BBB integrity loss seen in FGF-2-/-/FGF-5 $5^{-/-}$double mutant mice (Reuss et al., 2003). Recently, sonic hedgehog (Shh) has been shown to be secreted by perivascular astrocytes and endothelial-specific deletion of Smoothened (Smo), a downstream target of Shh, resulted in incomplete $\mathrm{BBB}$ maturation early during mouse embryonic development (Alvarez et al., 2011). Further, Shh knockout mice displayed a decrease in TJ proteins in brain cECs.

Next to astrocytes, pericytes have been shown to directly influence the BBB (Hori et al., 2004; Daneman et al., 2010b). The canonical $\mathrm{Wnt} / \beta$-catenin has also been shown to influence $\mathrm{TJ}$ integrity and $\mathrm{BBB}$ formation, increasing the expression of several genes, including the TJ protein claudin-3 (Liebner et al., 2008; Daneman et al., 2009). In vitro, activation of Wnt pathway controls the expression several TJ proteins (Paolinelli et al., 2013). Further, genetic ablation of $\mathrm{Wnt7a} / \mathrm{b}$ results in the breakdown of the BBB and a severe CNS-specific hemorrhaging phenotype early in embryonic development (Stenman et al., 2008).

Next to astrocytes and pericytes, noradrenergic, serotonergic, cholinergic, and GABA-ergic neurons have been found to directly contact brain cECs (Cohen et al., 1996, 1997; Tong and Hamel, 1999; Vaucher et al., 2000). It is believed that neurons innervating the brain cECs and astrocytes of the NVU regulate BBB permeability; however the mechanism of action remains unknown.

Taken together, the $\mathrm{BBB}$ is a dynamic structure receiving continuous input from the CNS. It is this intimate association of cerebral endothelial cells with neuroectoderm-derived accessory cells that influences barrier function (i.e., tightness) in a spatio-temporal manner in the developing and mature brain.

\section{CONCLUDING REMARKS}

The basic structure of the BBB has been described more than 100 years ago. Much progress has been made on elucidating the molecular programs that govern tight junction biogenesis and many of the core molecular components of the endothelial TJ complex which constitutes the main physical paracellular barrier at the BBB have been identified. TJs are now appreciated as dynamic structures where multiple signaling pathways converge, fitting the $\mathrm{BBB}$ of the cerebral vascular bed with a high degree of plasticity in response to physiological and pathological stimuli. Next to cell-cell adhesion, processes such as cellular polarization, cytoskeletal rearrangements, integrin-mediated attachment to the ECM, and polarized trafficking are pivotal to the physiological functioning of the BBB. These mechanisms have so far mainly been described in epithelial cells and although most of the key players are present in endothelial cells, their actions may vary markedly in different physiological contexts. Therefore, it will be central to gain a better understanding of the spatio-temporal establishment of functional TJs at the BBB during early vascularization of the embryonic brain. Clearly, further insights have largely been hampered by the lack of suitable in vitro culture systems appropriately replicating the vascular properties of the brain microcapillaries. As for many neurological diseases it remains problematic to deliver appropriate drugs to the CNS, a transient modulation of the TJs or transcytosis at the BBB may constitute an alternative approach. Undoubtedly, a more detailed understanding of the complex structure and physiology of endothelial TJs will aid in developing practical solutions for the future.

\section{ACKNOWLEDGMENTS}

We apologize to those authors whose work we were unable to cite because of space limitations. Hans-Christian Bauer, Hannelore Bauer, and Andreas Traweger gratefully acknowledge funding by the Paracelsus Medical University Research Fund (PMU-FFF), the European Community's 7th framework program NEUROBID, and the Lorenz Böhler Fund. The authors have no potential conflicts of interest.

\section{REFERENCES}

Abbott, N. J. (2002). Astrocyte-endothelial interactions and blood-brain barrier permeability. J. Anat. 200, 629-638. doi: 10.1046/j.1469-7580.2002.00064.x

Abbott, N. J. (2013). Blood-brain barrier structure and function and the challenges for CNS drug delivery. J. Inherit. Metab. Dis. 36, 437-449. doi: 10.1007/s10545013-9608-0

Abbott, N. J., Ronnback, L., and Hansson, E. (2006). Astrocyte-endothelial interactions at the blood-brain barrier. Nat. Rev. Neurosci. 7, 41-53. doi: $10.1038 / \mathrm{nrn} 1824$

Adachi, M., Inoko, A., Hata, M., Furuse, K., Umeda, K., Itoh, M., et al. (2006) Normal establishment of epithelial tight junctions in mice and cultured cells lacking expression of ZO-3, a tight-junction MAGUK protein. Mol. Cell. Biol. 26, 9003-9015. doi: 10.1128/MCB.01811-05

Adamson, P., Etienne, S., Couraud, P. O., Calder, V., and Greenwood, J. (1999). Lymphocyte migration through brain endothelial cell monolayers involves signaling through endothelial ICAM-1 via a rho-dependent pathway. J. Immunol. 162, 2964-2973.

Agarwal, N., Lippmann, E. S., and Shusta, E. V. (2010). Identification and expression profiling of blood-brain barrier membrane proteins. J. Neurochem. 112, 625-635. doi: 10.1111/j.1471-4159.2009.06481.x

Alvarez, J. I., Dodelet-Devillers, A., Kebir, H., Ifergan, I., Fabre, P. J., Terouz, S., et al. (2011). The Hedgehog pathway promotes blood-brain barrier integrity and CNS immune quiescence. Science 334, 1727-1731. doi: 10.1126/science. 1206936

Amasheh, S., Meiri, N., Gitter, A. H., Schoneberg, T., Mankertz, J., Schulzke, J. D., et al. (2002). Claudin-2 expression induces cation-selective channels in tight junctions of epithelial cells. J. Cell Sci. 115, 4969-4976. doi: 10.1242/jcs.00165

Anderson, J. M., and Van Itallie, C. M. (2009). Physiology and function of the tight junction. Cold Spring Harb. Perspect. Biol. 1:a002584. doi: 10.1101/ cshperspect.a002584

Aono, S., and Hirai, Y. (2008). Phosphorylation of claudin-4 is required for tight junction formation in a human keratinocyte cell line. Exp. Cell Res. 314, 3326-3339. doi: 10.1016/j.yexcr.2008.08.012

Armulik, A., Genove, G., Mae, M., Nisancioglu, M. H., Wallgard, E., Niaudet, C. et al. (2010). Pericytes regulate the blood-brain barrier. Nature 468, 557-561. doi: $10.1038 /$ nature 09522 
Arnold, S. J., and Robertson, E. J. (2009). Making a commitment: cell lineage allocation and axis patterning in the early mouse embryo. Nat. Rev. Mol. Cell Biol. 10, 91-103. doi: $10.1038 /$ nrm 2618

Arrate, M. P., Rodriguez, J. M., Tran, T. M., Brock, T. A., and Cunningham, S. A. (2001). Cloning of human junctional adhesion molecule 3 (JAM3) and its identification as the JAM2 counter-receptor. J. Biol. Chem. 276, 45826-45832. doi: 10.1074/jbc.M105972200

Arthur, F. E., Shivers, R. R., and Bowman, P. D. (1987). Astrocyte-mediated induction of tight junctions in brain capillary endothelium: an efficient in vitro model. Brain Res. 433, 155-159. doi: 10.1016/0165-3806(87)90075-7

Artus, C., Glacial, F., Ganeshamoorthy, K., Ziegler, N., Godet, M., Guilbert, T., et al. (2014). The Wnt/planar cell polarity signaling pathway contributes to the integrity of tight junctions in brain endothelial cells. J. Cereb. Blood Flow Metab. 34, 433-440. doi: 10.1038/jcbfm.2013.213

Assemat, E., Bazellieres, E., Pallesi-Pocachard, E., Le Bivic, A., and MasseyHarroche, D. (2008). Polarity complex proteins. Biochim. Biophys. Acta 1778, 614-630. doi: 10.1016/j.bbamem.2007.08.029

Aurrand-Lions, M., Duncan, L., Ballestrem, C., and Imhof, B. A. (2001). JAM-2, a novel immunoglobulin superfamily molecule, expressed by endothelial and lymphatic cells. J. Biol. Chem. 276, 2733-2741. doi: 10.1074/jbc.M005458200

Aurrand-Lions, M., Lamagna, C., Dangerfield, J. P., Wang, S., Herrera, P., Nourshargh, S., et al. (2005). Junctional adhesion molecule-C regulates the early influx of leukocytes into tissues during inflammation. J. Immunol. 174, 6406-6415. doi: 10.4049/jimmunol.174.10.6406

Aurrand-Lions, M. A., Duncan, L., Du Pasquier, L., and Imhof, B. A. (2000). Cloning of JAM-2 and JAM-3: an emerging junctional adhesion molecular family? Curr. Top. Microbiol. Immunol. 251, 91-98. doi: 10.1007/978-3-64257276-0_12

Axnick, J., and Lammert, E. (2012). Vascular lumen formation. Curr. Opin. Hematol. 19, 192-198. doi: 10.1097/MOH.0b013e3283523ebc

Balda, M. S., Gonzalez-Mariscal, L., Matter, K., Cereijido, M., and Anderson, J. M. (1993). Assembly of the tight junction: the role of diacylglycerol. J. Cell Biol. 123, 293-302. doi: 10.1083/jcb.123.2.293

Balda, M. S., and Matter, K. (2009). Tight junctions and the regulation of gene expression. Biochim. Biophys. Acta 1788, 761-767. doi: 10.1016/j.bbamem.2008.11.024

Bauer, H., Sonnleitner, U., Lametschwandtner, A., Steiner, M., Adam, H., and Bauer, H. C. (1995). Ontogenic expression of the erythroid-type glucose transporter (Glut 1) in the telencephalon of the mouse: correlation to the tightening of the blood-brain barrier. Brain Res. Dev. Brain Res. 86, 317-325. doi: 10.1016/0165-3806(95)00044-E

Bauer, H. C., and Bauer, H. (2000). Neural induction of the blood-brain barrier: still an enigma. Cell. Mol. Neurobiol. 20, 13-28. doi: 10.1023/A:1006939 825857

Bauer, H. C., Bauer, H., Lametschwandtner, A., Amberger, A., Ruiz, P., and Steiner, M. (1993). Neovascularization and the appearance of morphological characteristics of the blood-brain barrier in the embryonic mouse central nervous system. Brain Res. Dev. Brain Res. 75, 269-278. doi: 10.1016/0165-3806(93)90031-5

Bauer, H. C., Traweger, A., Zweimueller-Mayer, J., Lehner, C., Tempfer, H., Krizbai, I., et al. (2011). New aspects of the molecular constituents of tissue barriers. J. Neural Transm. 118, 7-21. doi: 10.1007/s00702-010-0484-6

Bazzoni, G., Martinez-Estrada, O. M., Mueller, F., Nelboeck, P., Schmid, G., Bartfai, T., et al. (2000a). Homophilic interaction of junctional adhesion molecule. J. Biol. Chem. 275, 30970-30976. doi: 10.1074/jbc.M003946200

Bazzoni, G., Martinez-Estrada, O. M., Orsenigo, F., Cordenonsi, M., Citi, S., and Dejana, E. (2000b). Interaction of junctional adhesion molecule with the tight junction components ZO-1, cingulin, and occludin. J. Biol. Chem. 275, 20520-20526. doi: 10.1074/jbc.M905251199

Begley, D. J., and Brightman, M. W. (2003). Structural and functional aspects of the blood-brain barrier. Prog. Drug Res. 61, 39-78.

Benais-Pont, G., Punn, A., Flores-Maldonado, C., Eckert, J., Raposo, G., Fleming, T. P., et al. (2003). Identification of a tight junction-associated guanine nucleotide exchange factor that activates Rho and regulates paracellular permeability. J. Cell Biol. 160, 729-740. doi: 10.1083/jcb.200211047

Ben-Zvi, A., Lacoste, B., Kur, E., Andreone, B. J., Mayshar, Y., Yan, H., et al. (2014). Mfsd2a is critical for the formation and function of the blood-brain barrier. Nature 509, 507-511. doi: 10.1038/nature13324

Betanzos, A., Huerta, M., Lopez-Bayghen, E., Azuara, E., Amerena, J., and Gonzalez-Mariscal, L. (2004). The tight junction protein ZO-2 associates with
Jun, Fos and C/EBP transcription factors in epithelial cells. Exp. Cell Res. 292, 51-66. doi: 10.1016/j.yexcr.2003.08.007

Bilder, D., Schober, M., and Perrimon, N. (2003). Integrated activity of PDZ protein complexes regulates epithelial polarity. Nat. Cell Biol. 5, 53-58. doi: $10.1038 /$ ncb897

Birukova, A. A., Adyshev, D., Gorshkov, B., Bokoch, G. M., Birukov, K. G., and Verin, A. D. (2006). GEF-H1 is involved in agonist-induced human pulmonary endothelial barrier dysfunction. Am. J. Physiol. Lung Cell. Mol. Physiol. 290, L540-L548. doi: 10.1152/ajplung.00259.2005

Blasig, I. E., Winkler, L., Lassowski, B., Mueller, S. L., Zuleger, N., Krause, E., et al. (2006). On the self-association potential of transmembrane tight junction proteins. Cell. Mol. Life Sci. 63, 505-514. doi: 10.1007/s00018-005-5472-x

Blum, Y., Belting, H. G., Ellertsdottir, E., Herwig, L., Luders, F., and Affolter M. (2008). Complex cell rearrangements during intersegmental vessel sprouting and vessel fusion in the zebrafish embryo. Dev. Biol. 316, 312-322. doi: 10.1016/j.ydbio.2008.01.038

Boas, S. E., and Merks, R. M. (2014). Synergy of cell-cell repulsion and vacuolation in a computational model of lumen formation. J. R. Soc. Interface 11:20131049. doi: 10.1098/rsif.2013.1049

Born, G. V., and Palinski, W. (1985). Unusually high concentrations of sialic acids on the surface of vascular endothelia. Br. J. Exp. Pathol. 66, 543-549.

Breier, G., Albrecht, U., Sterrer, S., and Risau, W. (1992). Expression of vascular endothelial growth factor during embryonic angiogenesis and endothelial cell differentiation. Development 114, 521-532.

Bruewer, M., Hopkins, A. M., Hobert, M. E., Nusrat, A., and Madara, J. L. (2004) RhoA, Rac1, and Cdc42 exert distinct effects on epithelial barrier via selective structural and biochemical modulation of junctional proteins and F-actin. Am. J. Physiol. Cell Physiol. 287, C327-C335. doi: 10.1152/ajpcell.00087.2004

Carson, S. D., Hobbs, J. T., Tracy, S. M., and Chapman, N. M. (1999). Expression of the coxsackievirus and adenovirus receptor in cultured human umbilical vein endothelial cells: regulation in response to cell density. J. Virol. 73, 7077-7079.

Cereijido, M., Gonzalez-Mariscal, L., Contreras, R. G., Gallardo, J. M., GarciaVillegas, R., and Valdes, J. (1993). The making of a tight junction. J. Cell Sci. Suppl. 17, 127-132. doi: 10.1242/jcs.1993.Supplement_17.18

Chalcroft, J. P., and Bullivant, S. (1970). An interpretation of liver cell membrane and junction structure based on observation of freeze-fracture replicas of both sides of the fracture. J. Cell Biol. 47, 49-60.

Chen, X., and Macara, I. G. (2005). Par-3 controls tight junction assembly through the Rac exchange factor Tiam1. Nat. Cell Biol. 7, 262-269. doi: 10.1038/ncb1226

Chen, Y. H., Lu, Q., Goodenough, D. A., and Jeansonne, B. (2002). Nonreceptor tyrosine kinase c-Yes interacts with occludin during tight junction formation in canine kidney epithelial cells. Mol. Biol. Cell 13, 1227-1237. doi: 10.1091/mbc.01-08-0423

Chen-Quay, S. C., Eiting, K. T., Li, A. W., Lamharzi, N., and Quay, S. C. (2009). Identification of tight junction modulating lipids. J. Pharm. Sci. 98, 606-619. doi: $10.1002 /$ jps. 21462

Chung, S., and Andrew, D. J. (2008). The formation of epithelial tubes. J. Cell Sci. 121, 3501-3504. doi: 10.1242/jcs.037887

Clatterbuck, R. E., Eberhart, C. G., Crain, B. J., and Rigamonti, D. (2001). Ultrastructural and immunocytochemical evidence that an incompetent bloodbrain barrier is related to the pathophysiology of cavernous malformations. J. Neurol. Neurosurg. Psychiatr. 71, 188-192. doi: 10.1136/jnnp.71.2.188

Cohen, C. J., Shieh, J. T., Pickles, R. J., Okegawa, T., Hsieh, J. T., and Bergelson, J. M. (2001). The coxsackievirus and adenovirus receptor is a transmembrane component of the tight junction. Proc. Natl. Acad. Sci. U.S.A. 98, 15191-15196. doi: 10.1073/pnas.261452898

Cohen, Z., Bonvento, G., Lacombe, P., and Hamel, E. (1996). Serotonin in the regulation of brain microcirculation. Prog. Neurobiol. 50, 335-362. doi: 10.1016/S0301-0082(96)00033-0

Cohen, Z., Molinatti, G., and Hamel, E. (1997). Astroglial and vascular interactions of noradrenaline terminals in the rat cerebral cortex. J. Cereb. Blood Flow Metab. 17, 894-904. doi: 10.1097/00004647-199708000-00008

Coisne, C., Dehouck, L., Faveeuw, C., Delplace, Y., Miller, F., Landry, C., et al. (2005). Mouse syngenic in vitro blood-brain barrier model: a new tool to examine inflammatory events in cerebral endothelium. Lab. Invest. 85, 734-746. doi: 10.1038/labinvest.3700281

Contreras, R. G., Miller, J. H., Zamora, M., Gonzalez-Mariscal, L., and Cereijido, M. (1992). Interaction of calcium with plasma membrane of epithelial (MDCK) cells during junction formation. Am. J. Physiol. 263, C313-C318. 
Cordenonsi, M., Zanconato, F., Azzolin, L., Forcato, M., Rosato, A., Frasson, C., et al. (2011). The Hippo transducer TAZ confers cancer stem cellrelated traits on breast cancer cells. Cell 147, 759-772. doi: 10.1016/j.cell. 2011.09.048

Cunningham, S. A., Arrate, M. P., Rodriguez, J. M., Bjercke, R. J., Vanderslice, P., Morris, A. P., et al. (2000). A novel protein with homology to the junctional adhesion molecule. Characterization of leukocyte interactions. J. Biol. Chem. 275, 34750-34756. doi: 10.1074/jbc.M002718200

Cunningham, S. A., Rodriguez, J. M., Arrate, M. P., Tran, T. M., and Brock, T. A. (2002). JAM2 interacts with alpha4beta1. Facilitation by JAM3. J. Biol. Chem. 277, 27589-27592. doi: 10.1074/jbc.C200331200

Czupalla, C. J., Liebner, S., and Devraj, K. (2014). In vitro models of the blood-brain barrier. Methods Mol. Biol. 1135, 415-437. doi: 10.1007/978-1-4939-0320-7_34

Daneman, R., Agalliu, D., Zhou, L., Kuhnert, F., Kuo, C. J., and Barres, B. A. (2009). Wnt/beta-catenin signaling is required for CNS, but not non-CNS, angiogenesis. Proc. Natl. Acad. Sci. U.S.A. 106, 641-646. doi: 10.1073/pnas. 0805165106

Daneman, R., Zhou, L., Agalliu, D., Cahoy, J. D., Kaushal, A., and Barres, B. A. (2010a). The mouse blood-brain barrier transcriptome: a new resource for understanding the development and function of brain endothelial cells. PLoS ONE 5:e13741. doi: 10.1371/journal.pone.0013741

Daneman, R., Zhou, L., Kebede, A. A., and Barres, B. A. (2010b). Pericytes are required for blood-brain barrier integrity during embryogenesis. Nature 468, 562-566. doi: 10.1038/nature09513

Dard, N., Breuer, M., Maro, B., and Louvet-Vallee, S. (2008). Morphogenesis of the mammalian blastocyst. Mol. Cell. Endocrinol. 282, 70-77. doi: 10.1016/j.mce.2007.11.004

De Mendoza, A., Suga, H., and Ruiz-Trillo, I. (2010). Evolution of the MAGUK protein gene family in premetazoan lineages. BMC Evol. Biol. 10:93. doi: 10.1186/1471-2148-10-93

Deli, M. A., Abraham, C. S., Kataoka, Y., and Niwa, M. (2005). Permeability studies on in vitro blood-brain barrier models: physiology, pathology, and pharmacology. Cell. Mol. Neurobiol. 25, 59-127. doi: 10.1007/s10571-004-1377-8

DeMaio, L., Chang, Y. S., Gardner, T. W., Tarbell, J. M., and Antonetti, D. A. (2001). Shear stress regulates occludin content and phosphorylation. Am. J. Physiol. Heart Circ. Physiol. 281, H105-H113.

Denker, B. M., Saha, C., Khawaja, S., and Nigam, S. K. (1996). Involvement of a heterotrimeric $\mathrm{G}$ protein alpha subunit in tight junction biogenesis. J. Biol. Chem. 271, 25750-25753. doi: 10.1074/jbc.271.42.25750

Dimitratos, S. D., Woods, D. F., Stathakis, D. G., and Bryant, P. J. (1999). Signaling pathways are focused at specialized regions of the plasma membrane by scaffolding proteins of the MAGUK family. Bioessays 21, 912-921.

Draheim, K. M., Fisher, O. S., Boggon, T. J., and Calderwood, D. A. (2014). Cerebral cavernous malformation proteins at a glance. J. Cell Sci. 127, 701-707. doi: $10.1242 /$ jcs. 138388

D’Souza, T., Agarwal, R., and Morin, P. J. (2005). Phosphorylation of claudin-3 at threonine 192 by cAMP-dependent protein kinase regulates tight junction barrier function in ovarian cancer cells. J. Biol. Chem. 280, 26233-26240. doi: 10.1074/jbc.M502003200

Ebnet, K. (2008). Organization of multiprotein complexes at cell-cell junctions. Histochem. Cell Biol. 130, 1-20. doi: 10.1007/s00418-008-0418-7

Ebnet, K., Aurrand-Lions, M., Kuhn, A., Kiefer, F., Butz, S., Zander, K., et al. (2003). The junctional adhesion molecule (JAM) family members JAM-2 and JAM3 associate with the cell polarity protein PAR-3: a possible role for JAMs in endothelial cell polarity. J. Cell Sci. 116, 3879-3891. doi: 10.1242/jcs.00704

Ebnet, K., Iden, S., Gerke, V., and Suzuki, A. (2008). Regulation of epithelial and endothelial junctions by PAR proteins. Front. Biosci. 13, 6520-6536. doi: $10.2741 / 3172$

Ebnet, K., Schulz, C. U., Meyer Zu Brickwedde, M. K., Pendl, G. G., and Vestweber, D. (2000). Junctional adhesion molecule interacts with the PDZ domaincontaining proteins AF-6 and ZO-1. J. Biol. Chem. 275, 27979-27988. doi: 10.1074/jbc.M002363200

Ebnet, K., Suzuki, A., Horikoshi, Y., Hirose, T., Meyer Zu Brickwedde, M. K., Ohno, S., et al. (2001). The cell polarity protein ASIP/PAR-3 directly associates with junctional adhesion molecule (JAM). EMBO J. 20, 3738-3748. doi: 10.1093/emboj/20.14.3738

Eckert, J. J., and Fleming, T. P. (2008). Tight junction biogenesis during early development. Biochim. Biophys. Acta 1778, 717-728. doi: 10.1016/j.bbamem.2007.09.031
Elbediwy, A., Zihni, C., Terry, S. J., Clark, P., Matter, K., and Balda, M. S. (2012). Epithelial junction formation requires confinement of Cdc42 activity by a novel SH3BP1 complex. J. Cell Biol. 198, 677-693. doi: 10.1083/jcb.201202094

El Hafny, B., Bourre, J. M., and Roux, F. (1996). Synergistic stimulation of gammaglutamyl transpeptidase and alkaline phosphatase activities by retinoic acid and astroglial factors in immortalized rat brain microvessel endothelial cells. J. Cell. Physiol. 167, 451-460.

Enerson, B. E., and Drewes, L. R. (2006). The rat blood-brain barrier transcriptome. J. Cereb. Blood Flow Metab. 26, 959-973. doi: 10.1038/sj.jcbfm.9600249

Engelhardt, B., and Liebner, S. (2014). Novel insights into the development and maintenance of the blood-brain barrier. Cell Tissue Res. 355, 687-699. doi: 10.1007/s00441-014-1811-2

Fabian, G., Szabo, C. A., Bozo, B., Greenwood, J., Adamson, P., Deli, M. A., et al. (1998). Expression of G-protein subtypes in cultured cerebral endothelial cells. Neurochem. Int. 33, 179-185. doi: 10.1016/S0197-0186(98)00008-4

Fanning, A. S., and Anderson, J. M. (1999). Protein modules as organizers of membrane structure. Curr. Opin. Cell Biol. 11, 432-439. doi: 10.1016/S09550674(99)80062-3

Fanning, A. S., and Anderson, J. M. (2009). Zonula occludens-1 and -2 are cytosolic scaffolds that regulate the assembly of cellular junctions. Ann. N.Y. Acad. Sci. 1165, 113-120. doi: 10.1111/j.1749-6632.2009.04440.x

Fanning, A. S., Jameson, B. J., Jesaitis, L. A., and Anderson, J. M. (1998). The tight junction protein $\mathrm{ZO}-1$ establishes a link between the transmembrane protein occludin and the actin cytoskeleton. J. Biol. Chem. 273, 29745-29753. doi: 10.1074/jbc.273.45.29745

Fanning, A. S., Little, B. P., Rahner, C., Utepbergenov, D., Walther, Z., and Anderson, J. M. (2007). The unique-5 and -6 motifs of ZO-1 regulate tight junction strand localization and scaffolding properties. Mol. Biol. Cell 18, 721-731. doi: 10.1091/mbc.E06-08-0764

Fanning, A. S., Ma, T. Y., and Anderson, J. M. (2002). Isolation and functional characterization of the actin binding region in the tight junction protein $\mathrm{ZO}-1$. FASEB J. 16, 1835-1837. doi: 10.1096/fj.02-0121fje

Farquhar, M. G., and Palade, G. E. (1963). Junctional complexes in various epithelia. J. Cell Biol. 17, 375-412. doi: 10.1083/jcb.17.2.375

Fierro-Gonzalez, J. C., White, M. D., Silva, J. C., and Plachta, N. (2013). Cadherindependent filopodia control preimplantation embryo compaction. Nat. Cell Biol. 15, 1424-1433. doi: 10.1038/ncb2875

Fischer, S., Wiesnet, M., Marti, H. H., Renz, D., and Schaper, W. (2004). Simultaneous activation of several second messengers in hypoxia-induced hyperpermeability of brain derived endothelial cells. J. Cell. Physiol. 198, 359-369. doi: 10.1002/jcp.10417

Fogg, V. C., Liu, C. J., and Margolis, B. (2005). Multiple regions of Crumbs3 are required for tight junction formation in MCF10A cells. J. Cell Sci. 118, 2859-2869. doi: 10.1242/jcs.02412

Fraemohs, L., Koenen, R. R., Ostermann, G., Heinemann, B., and Weber, C. (2004). The functional interaction of the beta 2 integrin lymphocyte functionassociated antigen-1 with junctional adhesion molecule-A is mediated by the I domain. J. Immunol. 173, 6259-6264. doi: 10.4049/jimmunol.173.10.6259

Friend, D. S., and Gilula, N. B. (1972). Variations in tight and gap junctions in mammalian tissues. J. Cell Biol. 53, 758-776. doi: 10.1083/jcb.53.3.758

Fukuhara, A., Irie, K., Nakanishi, H., Takekuni, K., Kawakatsu, T., Ikeda, W., et al. (2002). Involvement of nectin in the localization of junctional adhesion molecule at tight junctions. Oncogene 21, 7642-7655. doi: 10.1038/sj.onc. 1205875

Funke, L., Dakoji, S., and Bredt, D. S. (2005). Membrane-associated guanylate kinases regulate adhesion and plasticity at cell junctions. Annu. Rev. Biochem. 74, 219-245. doi: 10.1146/annurev.biochem.74.082803.133339

Furuse, M. (2010). Molecular basis of the core structure of tight junctions. Cold Spring Harb. Perspect. Biol. 2:a002907. doi: 10.1101/cshperspect.a002907

Furuse, M., Fujimoto, K., Sato, N., Hirase, T., and Tsukita, S. (1996). Overexpression of occludin, a tight junction-associated integral membrane protein, induces the formation of intracellular multilamellar bodies bearing tight junction-like structures. J. Cell Sci. 109(Pt 2), 429-435.

Furuse, M., Fujita, K., Hiragi, T., Fujimoto, K., and Tsukita, S. (1998). Claudin1 and -2 : novel integral membrane proteins localizing at tight junctions with no sequence similarity to occludin. J. Cell Biol. 141, 1539-1550. doi: 10.1083/jcb.141.7.1539

Furuse, M., Hata, M., Furuse, K., Yoshida, Y., Haratake, A., Sugitani, Y., et al. (2002). Claudin-based tight junctions are crucial for the mammalian epidermal 
barrier: a lesson from claudin-1-deficient mice. J. Cell Biol. 156, 1099-1111. doi: 10.1083/jcb.200110122

Furuse, M., Hirase, T., Itoh, M., Nagafuchi, A., Yonemura, S., and Tsukita, S. (1993). Occludin: a novel integral membrane protein localizing at tight junctions [see comments]. J. Cell Biol. 123, 1777-1788. doi: 10.1083/jcb.123. 6.1777

Furuse, M., Itoh, M., Hirase, T., Nagafuchi, A., Yonemura, S., Tsukita, S., et al. (1994). Direct association of occludin with ZO-1 and its possible involvement in the localization of occludin at tight junctions. J. Cell Biol. 127, 1617-1626. doi: $10.1083 /$ jcb.127.6.1617

Garnaas, M. K., Moodie, K. L., Liu, M. L., Samant, G. V., Li, K., Marx, R., et al. (2008). Syx, a RhoA guanine exchange factor, is essential for angiogenesis in vivo. Circ. Res. 103, 710-716. doi: 10.1161/CIRCRESAHA.108.181388

Giepmans, B. N., and Van Ijzendoorn, S. C. (2009). Epithelial cell-cell junctions and plasma membrane domains. Biochim. Biophys. Acta 1788, 820-831. doi: 10.1016/j.bbamem.2008.07.015

Glading, A., Han, J., Stockton, R. A., and Ginsberg, M. H. (2007). KRIT-1/CCM1 is a Rap1 effector that regulates endothelial cell cell junctions. J. Cell Biol. 179, 247-254. doi: 10.1083/jcb.200705175

Gonzalez-Mariscal, L., Betanzos, A., and Avila-Flores, A. (2000). MAGUK proteins: structure and role in the tight junction. Semin. Cell Dev. Biol. 11, 315-324. doi: $10.1006 / \mathrm{scdb} .2000 .0178$

Gonzalez-Mariscal, L., Chavez De Ramirez, B., and Cereijido, M. (1985). Tight junction formation in cultured epithelial cells (MDCK). J. Membr. Biol. 86, 113-125. doi: 10.1007/BF01870778

Gonzalez-Mariscal, L., Contreras, R. G., Bolivar, J. J., Ponce, A., Chavez De Ramirez, B., and Cereijido, M. (1990). Role of calcium in tight junction formation between epithelial cells. Am. J. Physiol. 259, C978-C986.

Gonzalez-Mariscal, L., and Nava, P. (2005). Tight junctions, from tight intercellular seals to sophisticated protein complexes involved in drug delivery, pathogens interaction and cell proliferation. Adv. Drug Deliv. Rev. 57, 811-814. doi: 10.1016/j.addr.2005.01.004

Gonzalez-Mariscal, L., Ponce, A., Alarcon, L., and Jaramillo, B. E. (2006). The tight junction protein ZO-2 has several functional nuclear export signals. Exp. Cell Res. 312, 3323-3335. doi: 10.1016/j.yexcr.2006.07.006

Gonzalez-Mariscal, L., Tapia, R., and Chamorro, D. (2008). Crosstalk of tight junction components with signaling pathways. Biochim. Biophys. Acta 1778, 729-756. doi: 10.1016/j.bbamem.2007.08.018

Gonzalez-Mariscal, L., Tapia, R., Huerta, M., and Lopez-Bayghen, E. (2009). The tight junction protein ZO-2 blocks cell cycle progression and inhibits cyclin D1 expression. Ann. N.Y. Acad. Sci. 1165, 121-125. doi: 10.1111/j.17496632.2009.04024.x

Gorog, P., Schraufstatter, I., and Born, G. V. (1982). Effect of removing sialic acids from endothelium on the adherence of circulating platelets in arteries in vivo. Proc. R. Soc. Lond. B Biol. Sci. 214, 471-480. doi: 10.1098/rspb.1982.0022

Gottardi, C. J., Arpin, M., Fanning, A. S., and Louvard, D. (1996). The junctionassociated protein, zonula occludens-1, localizes to the nucleus before the maturation and during the remodeling of cell-cell contacts. Proc. Natl. Acad. Sci. U.S.A. 93, 10779-10784. doi: 10.1073/pnas.93.20.10779

Gow, A., Davies, C., Southwood, C. M., Frolenkov, G., Chrustowski, M., Ng, L., et al. (2004). Deafness in Claudin 11-null mice reveals the critical contribution of basal cell tight junctions to stria vascularis function. J. Neurosci. 24, 7051-7062. doi: 10.1523/JNEUROSCI.1640-04.2004

Gow, A., Southwood, C. M., Li, J. S., Pariali, M., Riordan, G. P., Brodie, S. E., et al. (1999). CNS myelin and sertoli cell tight junction strands are absent in Osp/claudin-11 null mice. Cell 99, 649-659. doi: 10.1016/S00928674(00)81553-6

Guillemot, L., Paschoud, S., Pulimeno, P., Foglia, A., and Citi, S. (2008). The cytoplasmic plaque of tight junctions: a scaffolding and signalling center. Biochim. Biophys. Acta 1778, 601-613. doi: 10.1016/j.bbamem.2007.09.032

Gumbiner, B. (1987). Structure, biochemistry, and assembly of epithelial tight junctions. Am. J. Physiol. 253, C749-C758.

Gumbiner, B., Lowenkopf, T., and Apatira, D. (1991). Identification of a 160-kDa polypeptide that binds to the tight junction protein ZO-1. Proc. Natl. Acad. Sci. U.S.A. 88, 3460-3464. doi: 10.1073/pnas.88.8.3460

Gumbiner, B. M. (1993). Breaking through the tight junction barrier [comment]. J. Cell Biol. 123, 1631-1633. doi: 10.1083/jcb.123.6.1631

Gunzel, D., and Fromm, M. (2012). Claudins and other tight junction proteins. Compr. Physiol. 2, 1819-1852. doi: 10.1002/cphy.c110045
Hamazaki, Y., Itoh, M., Sasaki, H., Furuse, M., and Tsukita, S. (2002). MultiPDZ domain protein 1 (MUPP1) is concentrated at tight junctions through its possible interaction with claudin-1 and junctional adhesion molecule. J. Biol. Chem. 277, 455-461. doi: 10.1074/jbc.M109005200

Hamm, S., Dehouck, B., Kraus, J., Wolburg-Buchholz, K., Wolburg, H., Risau, W., et al. (2004). Astrocyte mediated modulation of blood-brain barrier permeability does not correlate with a loss of tight junction proteins from the cellular contacts. Cell Tissue Res. 315, 157-166. doi: 10.1007/s00441-003-0825-y

Haseloff, R. F., Blasig, I. E., Bauer, H. C., and Bauer, H. (2005). In search of the astrocytic factor(s) modulating blood-brain barrier functions in brain capillary endothelial cells in vitro. Cell. Mol. Neurobiol. 25, 25-39. doi: 10.1007/s10571004-1375-x

Haskins, J., Gu, L., Wittchen, E. S., Hibbard, J., and Stevenson, B. R. (1998). ZO-3, a novel member of the MAGUK protein family found at the tight junction, interacts with ZO-1 and occludin. J. Cell Biol. 141, 199-208. doi: 10.1083/jcb.141.1.199

Hirabayashi, S., Tajima, M., Yao, I., Nishimura, W., Mori, H., and Hata, Y. (2003). JAM4, a junctional cell adhesion molecule interacting with a tight junction protein, MAGI-1. Mol. Cell. Biol. 23, 4267-4282. doi: 10.1128/MCB.23.12.42674282.2003

Hirata, K., Ishida, T., Penta, K., Rezaee, M., Yang, E., Wohlgemuth, J., et al. (2001). Cloning of an immunoglobulin family adhesion molecule selectively expressed by endothelial cells. J. Biol. Chem. 276, 16223-16231. doi: 10.1074/jbc.M100630200

Hori, S., Ohtsuki, S., Hosoya, K., Nakashima, E., and Terasaki, T. (2004). A pericyte-derived angiopoietin-1 multimeric complex induces occludin gene expression in brain capillary endothelial cells through Tie-2 activation in vitro. J. Neurochem. 89, 503-513. doi: 10.1111/j.1471-4159.2004.02343.x

Horvat, R., Hovorka, A., Dekan, G., Poczewski, H., and Kerjaschki, D. (1986). Endothelial cell membranes contain podocalyxin-the major sialoprotein of visceral glomerular epithelial cells. J. Cell Biol. 102, 484-491. doi: $10.1083 /$ jcb.102.2.484

Hurd, T. W., Gao, L., Roh, M. H., Macara, I. G., and Margolis, B. (2003). Direct interaction of two polarity complexes implicated in epithelial tight junction assembly. Nat. Cell Biol. 5, 137-142. doi: 10.1038/ncb923

Iden, S., and Collard, J. G. (2008). Crosstalk between small GTPases and polarity proteins in cell polarization. Nat. Rev. Mol. Cell Biol. 9, 846-859. doi: $10.1038 / \mathrm{nrm} 2521$

Igarashi, Y., Utsumi, H., Chiba, H., Yamada-Sasamori, Y., Tobioka, H., Kamimura, Y., et al. (1999). Glial cell line-derived neurotrophic factor induces barrier function of endothelial cells forming the blood-brain barrier. Biochem. Biophys. Res. Commun. 261, 108-112. doi: 10.1006/bbrc.1999.0992

Ikari, A., Matsumoto, S., Harada, H., Takagi, K., Hayashi, H., Suzuki, Y., et al. (2006). Phosphorylation of paracellin-1 at Ser217 by protein kinase A is essential for localization in tight junctions. J. Cell Sci. 119, 1781-1789. doi: $10.1242 /$ jcs. 02901

Ikeda, E., Flamme, I., and Risau, W. (1996). Developing brain cells produce factors capable of inducing the HT7 antigen, a blood-brain barrier-specific molecule, in chick endothelial cells. Neurosci. Lett. 209, 149-152. doi: 10.1016/03043940(96) 12625-2

Ikenouchi, J., Furuse, M., Furuse, K., Sasaki, H., and Tsukita, S. (2005). Tricellulin constitutes a novel barrier at tricellular contacts of epithelial cells. J. Cell Biol. 171, 939-945. doi: 10.1083/jcb.200510043

Indra, I., Hong, S., Troyanovsky, R., Kormos, B., and Troyanovsky, S. (2013). The adherens junction: a mosaic of cadherin and nectin clusters bundled by actin filaments. J. Invest. Dermatol. 133, 2546-2554. doi: 10.1038/jid.2013.200

Inoko, A., Itoh, M., Tamura, A., Matsuda, M., Furuse, M., and Tsukita, S. (2003). Expression and distribution of ZO-3, a tight junction MAGUK protein, in mouse tissues. Genes cells 8, 837-845. doi: 10.1046/j.1365-2443.2003.00681.x

Iruela-Arispe, M. L., and Davis, G. E. (2009). Cellular and molecular mechanisms of vascular lumen formation. Dev. Cell 16, 222-231. doi: 10.1016/j.devcel.2009.01.013

Ishida, T., Kundu, R. K., Yang, E., Hirata, K., Ho, Y. D., and Quertermous, T. (2003). Targeted disruption of endothelial cell-selective adhesion molecule inhibits angiogenic processes in vitro and in vivo. J. Biol. Chem. 278, 34598-34604. doi: 10.1074/jbc.M304890200

Ishizaki, T., Chiba, H., Kojima, T., Fujibe, M., Soma, T., Miyajima, H., et al. (2003). Cyclic AMP induces phosphorylation of claudin-5 immunoprecipitates and expression of claudin-5 gene in blood-brain-barrier endothelial cells via protein 
kinase A-dependent and -independent pathways. Exp. Cell Res. 290, 275-288. doi: 10.1016/S0014-4827(03)00354-9

Islas, S., Vega, J., Ponce, L., and Gonzalez-Mariscal, L. (2002). Nuclear localization of the tight junction protein ZO-2 in epithelial cells. Exp. Cell Res. 274, 138-148. doi: 10.1006/excr.2001.5457

Itoh, M., Furuse, M., Morita, K., Kubota, K., Saitou, M., and Tsukita, S. (1999a). Direct binding of three tight junction-associated MAGUKs, ZO-1, ZO-2, and ZO-3, with the COOH termini of claudins. J. Cell Biol. 147, 1351-1363. doi: 10.1083/jcb.147.6.1351

Itoh, M., Morita, K., and Tsukita, S. (1999b). Characterization of ZO-2 as a MAGUK family member associated with tight as well as adherens junctions with a binding affinity to occludin and alpha catenin. J. Biol. Chem. 274, 5981-5986. doi: 10.1074/jbc.274.9.5981

Itoh, M., Nagafuchi, A., Moroi, S., and Tsukita, S. (1997). Involvement of ZO-1 in cadherin-based cell adhesion through its direct binding to alpha catenin and actin filaments. J. Cell Biol. 138, 181-192. doi: 10.1083/jcb.138.1.181

Itoh, M., Sasaki, H., Furuse, M., Ozaki, H., Kita, T., and Tsukita, S. (2001). Junctional adhesion molecule (JAM) binds to PAR-3: a possible mechanism for the recruitment of PAR-3 to tight junctions. J. Cell Biol. 154, 491-497. doi: 10.1083/jcb.200103047

Iwamoto, N., Higashi, T., and Furuse, M. (2014). Localization of Angulin-1/LSR and Tricellulin at Tricellular Contacts of Brain and Retinal Endothelial Cells in vivo. Cell Struct. Funct. 39, 1-8. doi: 10.1247/csf.13015

Janzer, R. C., and Raff, M. C. (1987). Astrocytes induce blood-brain barrier properties in endothelial cells. Nature 325, 253-257. doi: 10.1038/325253a0

Jaramillo, B. E., Ponce, A., Moreno, J., Betanzos, A., Huerta, M., Lopez-Bayghen, E., et al. (2004). Characterization of the tight junction protein ZO-2 localized at the nucleus of epithelial cells. Exp. Cell Res. 297, 247-258. doi: 10.1016/j.yexcr.2004.03.021

Javed, Q., Fleming, T. P., Hay, M., and Citi, S. (1993). Tight junction protein cingulin is expressed by maternal and embryonic genomes during early mouse development. Development 117, 1145-1151.

Joberty, G., Petersen, C., Gao, L., and Macara, I. G. (2000). The cell-polarity protein Par6 links Par3 and atypical protein kinase C to Cdc42. Nat. Cell Biol. 2, 531-539. doi: 10.1038/35019573

Johansson, A., Driessens, M., and Aspenstrom, P. (2000). The mammalian homologue of the Caenorhabditis elegans polarity protein PAR-6 is a binding partner for the Rho GTPases Cdc42 and Racl. J. Cell Sci. 113(Pt 18), 3267-3275.

Jou, T. S., Schneeberger, E. E., and Nelson, W. J. (1998). Structural and functional regulation of tight junctions by RhoA and Racl small GTPases. J. Cell Biol. 142, 101-115. doi: 10.1083/jcb.142.1.101

Kago, T., Takagi, N., Date, I., Takenaga, Y., Takagi, K., and Takeo, S. (2006). Cerebral ischemia enhances tyrosine phosphorylation of occludin in brain capillaries. Biochem. Biophys. Res. Commun. 339, 1197-1203. doi: 10.1016/j.bbrc.2005.11.133

Kan, F. W. (1993). Cytochemical evidence for the presence of phospholipids in epithelial tight junction strands. J. Histochem. Cytochem. 41, 649-656. doi: 10.1177/41.5.8468446

Katsube, T., Takahisa, M., Ueda, R., Hashimoto, N., Kobayashi, M., and Togashi, S. (1998). Cortactin associates with the cell-cell junction protein ZO-1 in both Drosophila and mouse. J. Biol. Chem. 273, 29672-29677. doi: $10.1074 /$ jbc. 273.45 .29672

Katsuno, T., Umeda, K., Matsui, T., Hata, M., Tamura, A., Itoh, M., et al. (2008). Deficiency of zonula occludens- 1 causes embryonic lethal phenotype associated with defected yolk sac angiogenesis and apoptosis of embryonic cells. Mol. Biol. Cell 19, 2465-2475. doi: 10.1091/mbc.E07-12-1215

Kausalya, P. J., Phua, D. C., and Hunziker, W. (2004). Association of ARVCF with zonula occludens (ZO)-1 and ZO-2: binding to PDZ-domain proteins and cellcell adhesion regulate plasma membrane and nuclear localization of ARVCF. Mol. Biol. Cell 15, 5503-5515. doi: 10.1091/mbc.E04-04-0350

Kerjaschki, D., Poczewski, H., Dekan, G., Horvat, R., Balzar, E., Kraft, N., et al. (1986). Identification of a major sialoprotein in the glycocalyx of human visceral glomerular epithelial cells. J. Clin. Invest. 78, 1142-1149. doi: 10.1172/JCI112694

Kimura, R., Ishida, T., Kuriyama, M., Hirata, K., and Hayashi, Y. (2010). Interaction of endothelial cell-selective adhesion molecule and MAGI-1 promotes mature cell-cell adhesion via activation of RhoA. Genes Cells 15, 385-396. doi: 10.1111/j.1365-2443.2010.01387.x
Knust, E., and Bossinger, O. (2002). Composition and formation of intercellular junctions in epithelial cells. Science 298, 1955-1959. doi: 10.1126/science. 1072161

Krug, S. M., Amasheh, S., Richter, J. F., Milatz, S., Gunzel, D., Westphal, J. K., et al. (2009). Tricellulin forms a barrier to macromolecules in tricellular tight junctions without affecting ion permeability. Mol. Biol. Cell 20, 3713-3724. doi: 10.1091/mbc.E09-01-0080

Kuhnert, F., Mancuso, M. R., Shamloo, A., Wang, H. T., Choksi, V., Florek, M., et al. (2010). Essential regulation of CNS angiogenesis by the orphan G proteincoupled receptor GPR124. Science 330, 985-989. doi: 10.1126/science.1196554

Lamagna, C., Meda, P., Mandicourt, G., Brown, J., Gilbert, R. J., Jones, E. Y., et al. (2005). Dual interaction of JAM-C with JAM-B and alpha(M)beta2 integrin: function in junctional complexes and leukocyte adhesion. Mol. Biol. Cell 16, 4992-5003. doi: 10.1091/mbc.E05-04-0310

Lampugnani, M. G., Orsenigo, F., Rudini, N., Maddaluno, L., Boulday, G., Chapon, F., et al. (2010). CCM1 regulates vascular-lumen organization by inducing endothelial polarity. J. Cell Sci. 123, 1073-1080. doi: 10.1242/jcs.059329

Larue, L., Ohsugi, M., Hirchenhain, J., and Kemler, R. (1994). E-cadherin null mutant embryos fail to form a trophectoderm epithelium. Proc. Natl. Acad. Sci. U.S.A. 91, 8263-8267. doi: 10.1073/pnas.91.17.8263

Laukoetter, M. G., Nava, P., Lee, W. Y., Severson, E. A., Capaldo, C. T., Babbin, B. A., et al. (2007). JAM-A regulates permeability and inflammation in the intestine in vivo. J. Exp. Med. 204, 3067-3076. doi: 10.1084/jem.20071416

Lee, C. Y., and Bautch, V. L. (2011). Ups and downs of guided vessel sprouting: the role of polarity. Physiology (Bethesda). 26, 326-333. doi: 10.1152/physiol.00018.2011

Lee, D. B., Jamgotchian, N., Allen, S. G., Abeles, M. B., and Ward, H. J. (2008). A lipid-protein hybrid model for tight junction. Am. J. Physiolo. Renal Physiol. 295, F1601-F1612. doi: 10.1152/ajprenal.00097.2008

Lee, J. L., and Streuli, C. H. (2014). Integrins and epithelial cell polarity. J. Cell Sci. 127, 3217-3225. doi: 10.1242/jcs.146142

Lee, S. W., Kim, W. J., Choi, Y. K., Song, H. S., Son, M. J., Gelman, I. H., et al. (2003). SSeCKS regulates angiogenesis and tight junction formation in bloodbrain barrier. Nat. Med. 9, 900-906. doi: 10.1038/nm889

Li, S., Haigh, K., Haigh, J. J., and Vasudevan, A. (2013). Endothelial VEGF sculpts cortical cytoarchitecture. J. Neurosci. 33, 14809-14815. doi: 10.1523/JNEUROSCI.1368-13.2013

Liebner, S., Corada, M., Bangsow, T., Babbage, J., Taddei, A., Czupalla, C. J., et al. (2008). Wnt/beta-catenin signaling controls development of the blood-brain barrier. J. Cell Biol. 183, 409-417. doi: 10.1083/jcb.200806024

Liebner, S., Fischmann, A., Rascher, G., Duffner, F., Grote, E. H., Kalbacher, H., et al. (2000a). Claudin-1 and claudin-5 expression and tight junction morphology are altered in blood vessels of human glioblastoma multiforme. Acta Neuropathol. (Berl.) 100, 323-331. doi: 10.1007/s004010000180

Liebner, S., Kniesel, U., Kalbacher, H., and Wolburg, H. (2000b). Correlation of tight junction morphology with the expression of tight junction proteins in blood-brain barrier endothelial cells. Eur. J. Cell Biol. 79, 707-717. doi: 10.1078/0171-9335-00101

Lin, D., Edwards, A. S., Fawcett, J. P., Mbamalu, G., Scott, J. D., and Pawson, T. (2000). A mammalian PAR-3-PAR-6 complex implicated in Cdc42/Racl and aPKC signalling and cell polarity. Nat. Cell Biol. 2, 540-547. doi: $10.1038 / 35019592$

Lindahl, P., Johansson, B. R., Leveen, P., and Betsholtz, C. (1997). Pericyte loss and microaneurysm formation in PDGF-B-deficient mice. Science 277, 242-245. doi: 10.1126/science.277.5323.242

Lippoldt, A., Kniesel, U., Liebner, S., Kalbacher, H., Kirsch, T., Wolburg, H., et al. (2000). Structural alterations of tight junctions are associated with loss of polarity in stroke-prone spontaneously hypertensive rat blood-brain barrier endothelial cells. Brain Res. 885, 251-261. doi: 10.1016/S0006-8993(00)02954-1

Liu, Y., Nusrat, A., Schnell, F. J., Reaves, T. A., Walsh, S., Pochet, M., et al. (2000). Human junction adhesion molecule regulates tight junction resealing in epithelia [In Process Citation]. J. Cell Sci. 113, 2363-2374.

Lizama, C. O., and Zovein, A. C. (2013). Polarizing pathways: balancing endothelial polarity, permeability, and lumen formation. Exp. Cell Res. 319, 1247-1254. doi: 10.1016/j.yexcr.2013.03.028

Lopez-Bayghen, E., Jaramillo, B., Huerta, M., Betanzos, A., and Gonzalez-Mariscal, L. (2006). "TJ proteins that make round trips to the nucleus," in Tight Junctions, ed L. Gonzalez-Mariscal (Georgetown, TX: Landes Bioscience, Springer), 76-100. 
Lubarsky, B., and Krasnow, M. A. (2003). Tube morphogenesis: making and shaping biological tubes. Cell 112, 19-28. doi: 10.1016/S0092-8674(02)01283-7

Luissint, A. C., Federici, C., Guillonneau, F., Chretien, F., Camoin, L., Glacial, F., et al. (2012). Guanine nucleotide-binding protein Galphai2: a new partner of claudin-5 that regulates tight junction integrity in human brain endothelial cells. J. Cereb. Blood Flow Metab. 32, 860-873. doi: 10.1038/jcbfm.2011.202

Luo, Y., and Radice, G. L. (2005). N-cadherin acts upstream of VE-cadherin in controlling vascular morphogenesis. J. Cell Biol. 169, 29-34. doi: 10.1083/jcb. 200411127

Macara, I. G. (2004). Parsing the polarity code. Nat. Rev. Mol. Cell Biol. 5, 220-231. doi: $10.1038 / \mathrm{nrm} 1332$

Maddaluno, L., Rudini, N., Cuttano, R., Bravi, L., Giampietro, C., Corada, M., et al. (2013). EndMT contributes to the onset and progression of cerebral cavernous malformations. Nature 498, 492-496. doi: 10.1038/nature12207

Mariano, C., Palmela, I., Pereira, P., Fernandes, A., Falcao, A. S., Cardoso, F. L., et al. (2013). Tricellulin expression in brain endothelial and neural cells. Cell Tissue Res. 351, 397-407. doi: 10.1007/s00441-012-1529-y

Mark, K. S., and Davis, T. P. (2002). Cerebral microvascular changes in permeability and tight junctions induced by hypoxia-reoxygenation. Am. J. Physiol. Heart Circ. Physiol. 282, H1485-H1494. doi: 10.1152/ajpheart.00645.2001

Martin-Belmonte, F., Gassama, A., Datta, A., Yu, W., Rescher, U., Gerke, V., et al. (2007). PTEN-mediated apical segregation of phosphoinositides controls epithelial morphogenesis through Cdc42. Cell 128, 383-397. doi: 10.1016/j.cell.2006.11.051

Martin-Padura, I., Lostaglio, S., Schneemann, M., Williams, L., Romano, M., Fruscella, P., et al. (1998). Junctional adhesion molecule, a novel member of the immunoglobulin superfamily that distributes at intercellular junctions and modulates monocyte transmigration. J. Cell Biol. 142, 117-127. doi: 10.1083/jcb.142.1.117

Martinez-Estrada, O. M., Villa, A., Breviario, F., Orsenigo, F., Dejana, E., and Bazzoni, G. (2001). Association of junctional adhesion molecule with calcium/calmodulin-dependent serine protein kinase (CASK/LIN-2) in human epithelial caco-2 cells. J. Biol. Chem. 276, 9291-9296. doi: 10.1074/jbc. M006991200

Mattagajasingh, S. N., Huang, S. C., Hartenstein, J. S., and Benz, E. J. Jr. (2000). Characterization of the interaction between protein 4.1R and ZO-2: a possible link between the tight junction and the actin cytoskeleton. J. Biol. Chem. 275, 30573-30585. doi: 10.1074/jbc.M004578200

Mazaud-Guittot, S., Meugnier, E., Pesenti, S., Wu, X., Vidal, H., Gow, A., et al. (2010). Claudin 11 deficiency in mice results in loss of the Sertoli cell epithelial phenotype in the testis. Biol. Reprod. 82, 202-213. doi: 10.1095/biolreprod.109.078907

McCarthy, K. M., Skare, I. B., Stankewich, M. C., Furuse, M., Tsukita, S., Rogers, R. A., et al. (1996). Occludin is a functional component of the tight junction. J. Cell Sci. 109, 2287-2298.

McCrea, P. D., Gu, D., and Balda, M. S. (2009). Junctional music that the nucleus hears: cell-cell contact signaling and the modulation of gene activity. Cold Spring Harb. Perspect. Biol. 1:a002923. doi: 10.1101/cshperspect.a002923

Meder, D., Shevchenko, A., Simons, K., and Fullekrug, J. (2005). Gp135/podocalyxin and NHERF-2 participate in the formation of a preapical domain during polarization of MDCK cells. J. Cell Biol. 168, 303-313. doi: $10.1083 /$ jcb. 200407072

Michaelis, U. R., Chavakis, E., Kruse, C., Jungblut, B., Kaluza, D., Wandzioch, K., et al. (2013). The polarity protein Scrib is essential for directed endothelial cell migration. Circ. Res. 112, 924-934. doi: 10.1161/CIRCRESAHA.112.300592

Mineta, K., Yamamoto, Y., Yamazaki, Y., Tanaka, H., Tada, Y., Saito, K., et al. (2011). Predicted expansion of the claudin multigene family. FEBS Lett. 585, 606-612. doi: 10.1016/j.febslet.2011.01.028

Mochida, G. H., Ganesh, V. S., Felie, J. M., Gleason, D., Hill, R. S., Clapham, K. R., et al. (2010). A homozygous mutation in the tight-junction protein JAM3 causes hemorrhagic destruction of the brain, subependymal calcification, and congenital cataracts. Am. J. Hum. Genet. 87, 882-889. doi: 10.1016/j.ajhg.2010. 10.026

Morita, K., Sasaki, H., Furuse, M., and Tsukita, S. (1999). Endothelial claudin: claudin-5/TMVCF constitutes tight junction strands in endothelial cells. J. Cell Biol. 147, 185-194. doi: 10.1083/jcb.147.1.185

Morris, S. A., and Zernicka-Goetz, M. (2012). Formation of distinct cell types in the mouse blastocyst. Results Probl. Cell Differ. 55, 203-217. doi: 10.1007/978-3642-30406-4_11
Muhleisen, H., Wolburg, H., and Betz, E. (1989). Freeze-fracture analysis of endothelial cell membranes in rabbit carotid arteries subjected to short-term atherogenic stimuli. Virchows Arch. B Cell Pathol. Incl. Mol. Pathol. 56, 413-417. doi: 10.1007/BF02890045

Murakami, T., Felinski, E. A., and Antonetti, D. A. (2009). Occludin phosphorylation and ubiquitination regulate tight junction trafficking and vascular endothelial growth factor-induced permeability. J. Biol. Chem. 284, 21036-21046. doi: 10.1074/jbc.M109.016766

Nagy, Z., Peters, H., and Huttner, I. (1984). Fracture faces of cell junctions in cerebral endothelium during normal and hyperosmotic conditions. Lab. Invest. 50, 313-322.

Nasdala, I., Wolburg-Buchholz, K., Wolburg, H., Kuhn, A., Ebnet, K., Brachtendorf, G., et al. (2002). A transmembrane tight junction protein selectively expressed on endothelial cells and platelets. J. Biol. Chem. 277, 16294-16303. doi: 10.1074/jbc.M111999200

Nery, S., Corbin, J. G., and Fishell, G. (2003). Dlx2 progenitor migration in wild type and Nkx2.1 mutant telencephalon. Cereb. Cortex 13, 895-903. doi: 10.1093/cercor/13.9.895

Ngok, S. P., Geyer, R., Liu, M., Kourtidis, A., Agrawal, S., Wu, C., et al. (2012). VEGF and Angiopoietin-1 exert opposing effects on cell junctions by regulating the Rho GEF Syx. J. Cell Biol. 199, 1103-1115. doi: 10.1083/jcb.201207009

Nielsen, J. S., Graves, M. L., Chelliah, S., Vogl, A. W., Roskelley, C. D., and McNagny, K. M. (2007). The CD34-related molecule podocalyxin is a potent inducer of microvillus formation. PLoS ONE 2:e237. doi: 10.1371/journal. pone.0000237

Nitta, T., Hata, M., Gotoh, S., Seo, Y., Sasaki, H., Hashimoto, N., et al. (2003). Sizeselective loosening of the blood-brain barrier in claudin-5-deficient mice. J. Cell Biol. 161, 653-660. doi: 10.1083/jcb.200302070

Nusrat, A., Giry, M., Turner, J. R., Colgan, S. P., Parkos, C. A., Carnes, D., et al. (1995). Rho protein regulates tight junctions and perijunctional actin organization in polarized epithelia. Proc. Natl. Acad. Sci. U.S.A. 92, 10629-10633. doi: 10.1073/pnas.92.23.10629

O’Driscoll, M. C., Daly, S. B., Urquhart, J. E., Black, G. C., Pilz, D. T., Brockmann, K., et al. (2010). Recessive mutations in the gene encoding the tight junction protein occludin cause band-like calcification with simplified gyration and polymicrogyria. Am. J. Hum. Genet. 87, 354-364. doi: 10.1016/j.ajhg.2010.07.012

Ohtsuki, S., Sato, S., Yamaguchi, H., Kamoi, M., Asashima, T., and Terasaki, T. (2007). Exogenous expression of claudin-5 induces barrier properties in cultured rat brain capillary endothelial cells. J. Cell. Physiol. 210, 81-86. doi: $10.1002 /$ jcp. 20823

Osada, T., Gu, Y. H., Kanazawa, M., Tsubota, Y., Hawkins, B. T., Spatz, M., et al. (2011). Interendothelial claudin-5 expression depends on cerebral endothelial cell-matrix adhesion by beta(1)-integrins. J. Cerebral Blood Flow Metab. 31, 1972-1985. doi: 10.1038/jcbfm.2011.99

Otani, T., Ichii, T., Aono, S., and Takeichi, M. (2006). Cdc42 GEF Tuba regulates the junctional configuration of simple epithelial cells. J. Cell Biol. 175, 135-146. doi: $10.1083 /$ jcb. 200605012

Palmeri, D., Van Zante, A., Huang, C. C., Hemmerich, S., and Rosen, S. D. (2000). Vascular endothelial junction-associated molecule, a novel member of the immunoglobulin superfamily, is localized to intercellular boundaries of endothelial cells. J. Biol. Chem. 275, 19139-19145. doi: 10.1074/jbc.M003189200

Paolinelli, R., Corada, M., Ferrarini, L., Devraj, K., Artus, C., Czupalla, C. J., et al. (2013). Wnt activation of immortalized brain endothelial cells as a tool for generating a standardized model of the blood brain barrier in vitro. PLoS ONE 8:e70233. doi: 10.1371/journal.pone.0070233

Paschoud, S., Yu, D., Pulimeno, P., Jond, L., Turner, J. R., and Citi, S. (2011). Cingulin and paracingulin show similar dynamic behaviour, but are recruited independently to junctions. Mol. Membr. Biol. 28, 123-135. doi: 10.3109/09687688.2010.538937

Phua, D. C., Xu, J., Ali, S. M., Boey, A., Gounko, N. V., and Hunziker, W. (2014). ZO-1 and ZO-2 are required for extra-embryonic endoderm integrity, primitive ectoderm survival and normal cavitation in embryoid bodies derived from mouse embryonic stem cells. PLoS ONE 9:e99532. doi: 10.1371/journal.pone.0099532

Qin, Y., Capaldo, C., Gumbiner, B. M., and Macara, I. G. (2005). The mammalian Scribble polarity protein regulates epithelial cell adhesion and migration through E-cadherin. J. Cell Biol. 171, 1061-1071. doi: 10.1083/jcb.200506094 
Raab, S., Beck, H., Gaumann, A., Yuce, A., Gerber, H. P., Plate, K., et al. (2004). Impaired brain angiogenesis and neuronal apoptosis induced by conditional homozygous inactivation of vascular endothelial growth factor. Thromb. Haemost. 91, 595-605. doi: 10.1160/TH03-09-0582

Rajasekaran, A. K., Hojo, M., Huima, T., and Rodriguez-Boulan, E. (1996). Catenins and zonula occludens-1 form a complex during early stages in the assembly of tight junctions. J. Cell Biol. 132, 451-463. doi: 10.1083/jcb.132.3.451

Raleigh, D. R., Marchiando, A. M., Zhang, Y., Shen, L., Sasaki, H., Wang, Y., et al. (2010). Tight junction-associated MARVEL proteins marveld3, tricellulin, and occludin have distinct but overlapping functions. Mol. Biol. Cell 21, 1200-1213. doi: 10.1091/mbc.E09-08-0734

Raschperger, E., Engstrom, U., Pettersson, R. F., and Fuxe, J. (2004). CLMP, a novel member of the CTX family and a new component of epithelial tight junctions. J. Biol. Chem. 279, 796-804. doi: 10.1074/jbc.M308249200

Raschperger, E., Thyberg, J., Pettersson, S., Philipson, L., Fuxe, J., and Pettersson, R. F. (2006). The coxsackie- and adenovirus receptor (CAR) is an in vivo marker for epithelial tight junctions, with a potential role in regulating permeability and tissue homeostasis. Exp. Cell Res. 312, 1566-1580. doi: 10.1016/j.yexcr.2006.01.025

Renz, A., and Fackelmayer, F. O. (1996). Purification and molecular cloning of the scaffold attachment factor B (SAF-B), a novel human nuclear protein that specifically binds to S/MAR-DNA. Nucleic Acids Res. 24, 843-849. doi: 10.1093/nar/24.5.843

Reuss, B., Dono, R., and Unsicker, K. (2003). Functions of fibroblast growth factor (FGF)-2 and FGF-5 in astroglial differentiation and blood-brain barrier permeability: evidence from mouse mutants. J. Neurosci. 23, 6404-6412.

Robbins, R. M., and Beitel, G. J. (2010). Vascular lumen formation: negativity will tear us apart. Curr. Biol. 20, R973-R975. doi: 10.1016/j.cub.2010.10.032

Rodriguez-Boulan, E., and Macara, I. G. (2014). Organization and execution of the epithelial polarity programme. Nat. Rev. Mol. Cell Biol. 15, 225-242. doi: $10.1038 / \mathrm{nrm} 3775$

Rosenberg, G. A. (2012). Neurological diseases in relation to the blood-brain barrier. J. Cereb. Blood Flow Metab. 32, 1139-1151. doi: 10.1038/jcbfm.2011.197

Rossant, J., and Tam, P. P. (2009). Blastocyst lineage formation, early embryonic asymmetries and axis patterning in the mouse. Development 136, 701-713. doi: 10.1242/dev.017178

Saha, C., Nigam, S. K., and Denker, B. M. (1998). Involvement of Galphai2 in the maintenance and biogenesis of epithelial cell tight junctions. J. Biol. Chem. 273, 21629-21633. doi: 10.1074/jbc.273.34.21629

Saitou, M., Fujimoto, K., Doi, Y., Itoh, M., Fujimoto, T., Furuse, M., et al. (1998). Occludin-deficient embryonic stem cells can differentiate into polarized epithelial cells bearing tight junctions. J. Cell Biol. 141, 397-408. doi: 10.1083/jcb.141.2.397

Saitou, M., Furuse, M., Sasaki, H., Schulzke, J. D., Fromm, M., Takano, H., et al. (2000). Complex phenotype of mice lacking occludin, a component of tight junction strands. Mol. Biol. Cell 11, 4131-4142. doi: 10.1091/mbc.11.12.4131

Sakakibara, A., Furuse, M., Saitou, M., Ando-Akatsuka, Y., and Tsukita, S. (1997). Possible involvement of phosphorylation of occludin in tight junction formation. J. Cell Biol. 137, 1393-1401. doi: 10.1083/jcb.137.6.1393

Sakurai, A., Fukuhara, S., Yamagishi, A., Sako, K., Kamioka, Y., Masuda, M., et al. (2006). MAGI-1 is required for Rap1 activation upon cell-cell contact and for enhancement of vascular endothelial cadherin-mediated cell adhesion. Mol. Biol. Cell 17, 966-976. doi: 10.1091/mbc.E05-07-0647

Sato, T., Fujita, N., Yamada, A., Ooshio, T., Okamoto, R., Irie, K., et al. (2006). Regulation of the assembly and adhesion activity of E-cadherin by nectin and afadin for the formation of adherens junctions in Madin-Darby canine kidney cells. J. Biol. Chem. 281, 5288-5299. doi: 10.1074/jbc.M510070200

Schrade, A., Sade, H., Couraud, P. O., Romero, I. A., Weksler, B. B., and Niewoehner, J. (2012). Expression and localization of claudins-3 and -12 in transformed human brain endothelium. Fluids Barriers CNS 9:6. doi: 10.1186/2045-8118-9-6

Schulze, C., Smales, C., Rubin, L. L., and Staddon, J. M. (1997). Lysophosphatidic acid increases tight junction permeability in cultured brain endothelial cells. J. Neurochem. 68, 991-1000. doi: 10.1046/j.1471-4159.1997. 68030991.x

Schulzke, J. D., Gitter, A. H., Mankertz, J., Spiegel, S., Seidler, U., Amasheh, S., et al. (2005). Epithelial transport and barrier function in occludin-deficient mice. Biochim. Biophys. Acta 1669, 34-42. doi: 10.1016/j.bbamem.2005.01.008

Sheth, B., Fontaine, J. J., Ponza, E., McCallum, A., Page, A., Citi, S., et al. (2000). Differentiation of the epithelial apical junctional complex during mouse preimplantation development: a role for rab13 in the early maturation of the tight junction. Mech. Dev. 97, 93-104. doi: 10.1016/S0925-4773(00)00416-0

Sheth, B., Nowak, R. L., Anderson, R., Kwong, W. Y., Papenbrock, T., and Fleming, T. P. (2008). Tight junction protein ZO-2 expression and relative function of ZO-1 and ZO-2 during mouse blastocyst formation. Exp. Cell Res. 314, 3356-3368. doi: 10.1016/j.yexcr.2008.08.021

Shin, K., Straight, S., and Margolis, B. (2005). PATJ regulates tight junction formation and polarity in mammalian epithelial cells. J. Cell Biol. 168, 705-711. doi: 10.1083/jcb.200408064

Simionescu, M., Simionescu, N., and Palade, G. E. (1976). Segmental differentiations of cell junctions in the vascular endothelium. Arteries and veins. J. Cell Biol. 68, 705-723. doi: 10.1083/jcb.68.3.705

Sonoda, N., Furuse, M., Sasaki, H., Yonemura, S., Katahira, J., Horiguchi, Y., et al. (1999). Clostridium perfringens enterotoxin fragment removes specific claudins from tight junction strands: evidence for direct involvement of claudins in tight junction barrier. J. Cell Biol. 147, 195-204. doi: 10.1083/jcb.147.1.195

St Johnston, D., and Ahringer, J. (2010). Cell polarity in eggs and epithelia: parallels and diversity. Cell 141, 757-774. doi: 10.1016/j.cell.2010.05.011

Staehelin, L. A. (1973). Further observations on the fine structure of freeze-cleaved tight junctions. J. Cell Sci. 13, 763-786.

Staehelin, L. A. (1974). Structure and function of intercellular junctions. Int. Rev. Cytol. 39, 191-283. doi: 10.1016/S0074-7696(08)60940-7

Steed, E., Balda, M. S., and Matter, K. (2010). Dynamics and functions of tight junctions. Trends Cell Biol. 20, 142-149. doi: 10.1016/j.tcb.2009.12.002

Steed, E., Rodrigues, N. T., Balda, M. S., and Matter, K. (2009). Identification of MarvelD3 as a tight junction-associated transmembrane protein of the occludin family. BMC Cell Biol. 10:95. doi: 10.1186/1471-2121-10-95

Stenman, J. M., Rajagopal, J., Carroll, T. J., Ishibashi, M., McMahon, J., and McMahon, A. P. (2008). Canonical Wnt signaling regulates organ-specific assembly and differentiation of CNS vasculature. Science 322, 1247-1250. doi: 10.1126/science. 1164594

Stern, L., and Gauthier, R. (1921). Recherches sur le liquid cephalo-rachidien. 1. Les rapports entre le liquide cephalo-rachidien et la circulatioon sanguine. Arch. Int. Physiol. 17, 138-192. doi: 10.3109/13813452109146211

Stern, L., and Rothlin, E. (1918). Effets de l'action directe du curare sur les différentes parties du cervelet. Schweiz Arch. Neurol. Psychiatry 3, 234-254.

Stevenson, B. R., Anderson, J. M., and Bullivant, S. (1988). The epithelial tight junction: structure, function and preliminary biochemical characterization. Mol. Cell. Biochem. 83, 129-145. doi: 10.1007/BF00226141

Stevenson, B. R., Siliciano, J. D., Mooseker, M. S., and Goodenough, D. A. (1986). Identification of ZO-1: a high molecular weight polypeptide associated with the tight junction (zonula occludens) in a variety of epithelia. J. Cell Biol. 103, 755-766. doi: 10.1083/jcb.103.3.755

Stewart, P. A., and Wiley, M. J. (1981). Developing nervous tissue induces formation of blood-brain barrier characteristics in invading endothelial cells: a study using quail-chick transplantation chimeras. Dev. Biol. 84, 183-192. doi: 10.1016/0012-1606(81)90382-1

Strilic, B., Eglinger, J., Krieg, M., Zeeb, M., Axnick, J., Babal, P., et al. (2010). Electrostatic cell-surface repulsion initiates lumen formation in developing blood vessels. Curr. Biol. 20, 2003-2009. doi: 10.1016/j.cub.2010.09.061

Strilic, B., Kucera, T., Eglinger, J., Hughes, M. R., McNagny, K. M., Tsukita, S., et al. (2009). The molecular basis of vascular lumen formation in the developing mouse aorta. Dev. Cell 17, 505-515. doi: 10.1016/j.devcel.2009.08.011

Stuart, R. O., Sun, A., Panichas, M., Hebert, S. C., Brenner, B. M., and Nigam, S. K. (1994). Critical role for intracellular calcium in tight junction biogenesis. J. Cell. Physiol. 159, 423-433. doi: 10.1002/jcp.1041590306

Sussel, L., Marin, O., Kimura, S., and Rubenstein, J. L. (1999). Loss of Nkx2.1 homeobox gene function results in a ventral to dorsal molecular respecification within the basal telencephalon: evidence for a transformation of the pallidum into the striatum. Development 126, 3359-3370.

Tachibana, K., Nakanishi, H., Mandai, K., Ozaki, K., Ikeda, W., Yamamoto, Y., et al. (2000). Two cell adhesion molecules, nectin and cadherin, interact through their cytoplasmic domain-associated proteins. J. Cell Biol. 150, 1161-1176. doi: 10.1083/jcb.150.5.1161

Takai, Y., Ikeda, W., Ogita, H., and Rikitake, Y. (2008a). The immunoglobulin-like cell adhesion molecule nectin and its associated protein afadin. Annu. Rev. Cell Dev. Biol. 24, 309-342. doi: 10.1146/annurev.cellbio.24.110707.175339

Takai, Y., Miyoshi, J., Ikeda, W., and Ogita, H. (2008b). Nectins and nectin-like molecules: roles in contact inhibition of cell movement and proliferation. Nat. Rev. Mol. Cell Biol. 9, 603-615. doi: 10.1038/nrm2457 
Takaoka, K., and Hamada, H. (2012). Cell fate decisions and axis determination in the early mouse embryo. Development 139, 3-14. doi: 10.1242/dev.060095

Tao-Cheng, J. H., Nagy, Z., and Brightman, M. W. (1987). Tight junctions of brain endothelium in vitro are enhanced by astroglia. J. Neurosci. 7, 3293-3299.

Te Velthuis, A. J., Admiraal, J. F., and Bagowski, C. P. (2007). Molecular evolution of the MAGUK family in metazoan genomes. BMC Evol. Biol. 7:129. doi: $10.1186 / 1471-2148-7-129$

Tepass, U. (2012). The apical polarity protein network in Drosophila epithelial cells: regulation of polarity, junctions, morphogenesis, cell growth, and survival. Annu. Rev. Cell Dev. Biol. 28, 655-685. doi: 10.1146/annurev-cellbio-092910154033

Thomas, F. C., Sheth, B., Eckert, J. J., Bazzoni, G., Dejana, E., and Fleming, T. P. (2004). Contribution of JAM-1 to epithelial differentiation and tight-junction biogenesis in the mouse preimplantation embryo. J. Cell Sci. 117, 5599-5608. doi: $10.1242 /$ jcs. 01424

Tong, X. K., and Hamel, E. (1999). Regional cholinergic denervation of cortical microvessels and nitric oxide synthase-containing neurons in Alzheimer's disease. Neuroscience 92, 163-175. doi: 10.1016/S0306-4522(98)00750-7

Traweger, A., Fang, D., Liu, Y. C., Stelzhammer, W., Krizbai, I. A., Fresser, F., et al. (2002). The Tight Junction-specific Protein Occludin Is a Functional Target of the E3 Ubiquitin-protein Ligase Itch. J. Biol. Chem. 277, 10201-10208. doi: 10.1074/jbc.M111384200

Traweger, A., Fuchs, R., Krizbai, I. A., Weiger, T. M., Bauer, H. C., and Bauer, H. (2003). The tight junction protein ZO-2 localizes to the nucleus and interacts with the heterogeneous nuclear ribonucleoprotein scaffold attachment factor-B. J. Biol. Chem. 278, 2692-2700. doi: 10.1074/jbc.M206821200

Tsukamoto, T., and Nigam, S. K. (1999). Role of tyrosine phosphorylation in the reassembly of occludin and other tight junction proteins. Am. J. Physiol. 276, F737-F750.

Tsukita, S., Furuse, M., and Itoh, M. (2001). Multifunctional strands in tight junctions. Nat. Rev. Mol. Cell Biol. 2, 285-293. doi: 10.1038/35067088

Umeda, K., Ikenouchi, J., Katahira-Tayama, S., Furuse, K., Sasaki, H., Nakayama, M., et al. (2006). ZO-1 and ZO-2 independently determine where claudins are polymerized in tight-junction strand formation. Cell 126, 741-754. doi: 10.1016/j.cell.2006.06.043

Van Deurs, B., and Koehler, J. K. (1979). Tight junctions in the choroid plexus epithelium. A freeze-fracture study including complementary replicas. J. Cell Biol. 80, 662-673. doi: 10.1083/jcb.80.3.662

Van Meer, G., Gumbiner, B., and Simons, K. (1986). The tight junction does not allow lipid molecules to diffuse from one epithelial cell to the next. Nature 322, 639-641. doi: 10.1038/322639a0

Vasudevan, A., Long, J. E., Crandall, J. E., Rubenstein, J. L., and Bhide, P. G. (2008). Compartment-specific transcription factors orchestrate angiogenesis gradients in the embryonic brain. Nat. Neurosci. 11, 429-439. doi: 10.1038/nn2074

Vaucher, E., Tong, X. K., Cholet, N., Lantin, S., and Hamel, E. (2000). GABA neurons provide a rich input to microvessels but not nitric oxide neurons in the rat cerebral cortex: a means for direct regulation of local cerebral blood flow. J. Comp. Neurol. 421, 161-171. doi: 10.1002/(SICI)1096-9861(20000529)421:2 $<161:: A I D-C N E 3>3.0 . C O ; 2-\mathrm{F}$

Wang, Q., Chen, X. W., and Margolis, B. (2007). PALS1 regulates E-cadherin trafficking in mammalian epithelial cells. Mol. Biol. Cell 18, 874-885. doi: 10.1091/mbc.E06-07-0651

Wang, Q., and Margolis, B. (2007). Apical junctional complexes and cell polarity. Kidney Int. 72, 1448-1458. doi: 10.1038/sj.ki.5002579

Wang, Y., Li, Z., Xu, P., Huang, L., Tong, J., Huang, H., et al. (2011). Angiomotin-like2 gene (amotl2) is required for migration and proliferation of endothelial cells during angiogenesis. J. Biol. Chem. 286, 41095-41104. doi: 10.1074/jbc.M111.296806

Watson, P. M., Paterson, J. C., Thom, G., Ginman, U., Lundquist, S., and Webster, C. I. (2013). Modelling the endothelial blood-CNS barriers: a method for the production of robust in vitro models of the rat blood-brain barrier and bloodspinal cord barrier. BMC Neurosci. 14:59. doi: 10.1186/1471-2202-14-59

Wegmann, F., Ebnet, K., Du Pasquier, L., Vestweber, D., and Butz, S. (2004). Endothelial adhesion molecule ESAM binds directly to the multidomain adaptor MAGI-1 and recruits it to cell contacts. Exp. Cell Res. 300, 121-133. doi: 10.1016/j.yexcr.2004.07.010

Wegmann, F., Petri, B., Khandoga, A. G., Moser, C., Khandoga, A., Volkery, S., et al. (2006). ESAM supports neutrophil extravasation, activation of Rho, and VEGF-induced vascular permeability. J. Exp. Med. 203, 1671-1677. doi: 10.1084/jem.20060565
Weidenfeller, C., Svendsen, C. N., and Shusta, E. V. (2007). Differentiating embryonic neural progenitor cells induce blood-brain barrier properties. J. Neurochem. 101, 555-565. doi: 10.1111/j.1471-4159.2006.04394.x

Weksler, B. B., Subileau, E. A., Perriere, N., Charneau, P., Holloway, K., Leveque, M., et al. (2005). Blood-brain barrier-specific properties of a human adult brain endothelial cell line. FASEB J. 19, 1872-1874. doi: 10.1096/fj.043458 fje

Wilhelm, I., and Krizbai, I. A. (2014). In vitro models of the blood-brain barrier for the study of drug delivery to the brain. Mol. Pharm. 11, 1949-1963. doi: $10.1021 / \mathrm{mp} 500046 \mathrm{f}$

Wittchen, E. S., Haskins, J., and Stevenson, B. R. (1999). Protein interactions at the tight junction. Actin has multiple binding partners, and ZO-1 forms independent complexes with ZO-2 and ZO-3. J. Biol. Chem. 274, 35179-35185. doi: $10.1074 /$ jbc.274.49.35179

Wojciak-Stothard, B., Potempa, S., Eichholtz, T., and Ridley, A. J. (2001). Rho and Rac but not Cdc42 regulate endothelial cell permeability. J. Cell Sci. 114, 1343-1355.

Wolburg, H., Wolburg-Buchholz, K., Kraus, J., Rascher-Eggstein, G., Liebner, S., Hamm, S., et al. (2003). Localization of claudin-3 in tight junctions of the blood-brain barrier is selectively lost during experimental autoimmune encephalomyelitis and human glioblastoma multiforme. Acta Neuropathol. 105, 586-592. doi: 10.1007/s00401-003-0688-z

Wong, V. (1997). Phosphorylation of occludin correlates with occludin localization and function at the tight junction. Am. J. Physiol. 273, C1859-C1867.

Xu, J., Kausalya, P. J., Phua, D. C., Ali, S. M., Hossain, Z., and Hunziker, W. (2008). Early embryonic lethality of mice lacking ZO-2, but Not ZO-3, reveals critical and nonredundant roles for individual zonula occludens proteins in mammalian development. Mol. Cell. Biol. 28, 1669-1678. doi: 10.1128/MCB. 00891-07

Yaffe, Y., Shepshelovitch, J., Nevo-Yassaf, I., Yeheskel, A., Shmerling, H., Kwiatek, J. M., et al. (2012). The MARVEL transmembrane motif of occludin mediates oligomerization and targeting to the basolateral surface in epithelia. J. Cell Sci. 125, 3545-3556. doi: $10.1242 /$ jcs. 100289

Yamagata, K., Tagami, M., Takenaga, F., Yamori, Y., Nara, Y., and Itoh, S. (2003). Polyunsaturated fatty acids induce tight junctions to form in brain capillary endothelial cells. Neuroscience 116, 649-656. doi: 10.1016/S03064522(02)00715-7

Yamanaka, T., and Ohno, S. (2008). Role of $\mathrm{Lgl} / \mathrm{Dlg} / \mathrm{Scribble}$ in the regulation of epithelial junction, polarity and growth. Front. Biosci. 13, 6693-6707. doi: $10.2741 / 3182$

Zeeb, M., Strilic, B., and Lammert, E. (2010). Resolving cell-cell junctions: lumen formation in blood vessels. Curr. Opin. Cell Biol. 22, 626-632. doi: 10.1016/j.ceb.2010.07.003

Zheng, Y., Vertuani, S., Nystrom, S., Audebert, S., Meijer, I., Tegnebratt, T., et al. (2009). Angiomotin-like protein 1 controls endothelial polarity and junction stability during sprouting angiogenesis. Circ. Res. 105, 260-270. doi: 10.1161/CIRCRESAHA.109.195156

Zovein, A. C., Luque, A., Turlo, K. A., Hofmann, J. J., Yee, K. M., Becker, M. S., et al. (2010). Betal integrin establishes endothelial cell polarity and arteriolar lumen formation via a Par3-dependent mechanism. Dev. Cell 18, 39-51. doi: 10.1016/j.devcel.2009.12.006

Conflict of Interest Statement: The authors declare that the research was conducted in the absence of any commercial or financial relationships that could be construed as a potential conflict of interest.

Received: 11 September 2014; accepted: 14 November 2014; published online: 03 December 2014

Citation: Bauer H-C, Krizbai IA, Bauer H and Traweger A (2014) "You Shall Not Pass"-tight junctions of the blood brain barrier. Front. Neurosci. 8:392. doi: 10.3389/ frins.2014.00392

This article was submitted to Neurogenomics, a section of the journal Frontiers in Neuroscience.

Copyright () 2014 Bauer, Krizbai, Bauer and Traweger. This is an open-access article distributed under the terms of the Creative Commons Attribution License (CC BY). The use, distribution or reproduction in other forums is permitted, provided the original author(s) or licensor are credited and that the original publication in this journal is cited, in accordance with accepted academic practice. No use, distribution or reproduction is permitted which does not comply with these terms. 


\title{
Choroid plexus in developmental and evolutionary perspective
}

\author{
Brent Roy Bill ${ }^{1+}$ and Vladimir Korzh ${ }^{2,3 *}$ \\ 1 Semel Institute for Neuroscience and Human Behavior, University of California Los Angeles, Los Angeles, CA, USA \\ ${ }^{2}$ Agency for Science, Technology and Research of Singapore, Institute of Molecular and Cell Biology, Singapore, Singapore \\ ${ }^{3}$ National University of Singapore, Department of Biological Sciences, Singapore, Singapore
}

\section{Edited by:}

Norman Ruthven Saunders, University of Melbourne, Australia

Reviewed by:

Mengqing Xiang, Rutgers

University-Robert Wood Johnson

Medical School, USA

Michael Robert Taylor, University of

Wisconsin-Madison, USA

*Correspondence:

Vladimir Korzh, Institute of

Molecular and Cell Biology, A-STAR

(Agency for Science, Technology and

Research), 61 Biopolis Dr., Proteos,

Singapore 138673, Singapore

e-mail:vlad@imcb.a-star.edu.sg

${ }^{\dagger}$ Present address:

Brent Roy Bill, Department of

Biology, The University of Texas at

Tyler, Tyler, TX, USA
The blood-cerebrospinal fluid boundary is present at the level of epithelial cells of the choroid plexus. As one of the sources of the cerebrospinal fluid (CSF), the choroid plexus (CP) plays an important role during brain development and function. Its formation has been studied largely in mammalian species. Lately, progress in other model animals, in particular the zebrafish, has brought a deeper understanding of CP formation, due in part to the ability to observe CP development in vivo. At the same time, advances in comparative genomics began providing information, which opens a possibility to understand further the molecular mechanisms involved in evolution of the CP and the blood-cerebrospinal fluid boundary formation. Hence this review focuses on analysis of the CP from developmental and evolutionary perspectives.

\section{Keywords: brain-CSF, transgenics, epithelium, vasculature, stroma, glia}

\section{WHAT IS THE CHOROID PLEXUS?}

The choroid plexus (CP) is a set of ependymal-derived structures that regulate the composition of cerebrospinal fluid (CSF). Historically, the term "CP" (from Latin: chorion-delicate, plexus-knot) was defined by the vasculature; however, it now refers to multiple layers and cell types that form this organ (Strong, 1956; Netsky et al., 1975; Johanson, 2003; Redzic et al., 2005; Bill et al., 2008; Garcia-Lecea et al., 2008). The CP structure is conserved in vertebrate species, and consists of: a fenestrated vasculature core, an interstitial stromal layer, and a single layer of polarized cuboidal epithelia. Endothelial-derived and epithelialderived basement membranes flank the stroma providing for a unique method of semipermeable filtration (Johanson et al., 2011). In most vertebrates there are four CP's: CPIII, CPIV, and two lateral CPs, inset within each ventricle. Due to differences in cortical development, teleosts have only two CPs, the diencephalic and myelencephalic $\mathrm{CP}(\mathrm{dCP}$ and $\mathrm{mCP}$ ) homologous with the midline CPs (CPIII and IV, respectively). For simplicity, we will primarily focus on comparisons between $\mathrm{mCP}$ and CPIV development.

Despite vital roles in development, physiology, and protection of the brain, the $\mathrm{CP}$ has remained outside of a sphere of interest for many mainstream neuroscientists. There are numerous reasons why this is the case including: reductionist approaches that refine work to a targeted neural tissue, a misconception that the CSF is only for metabolic support and waste removal, and a limited view of the pathologies that affect the $\mathrm{CP}$. A renaissance in $\mathrm{CP}$ research has occurred over the past few years placing the $\mathrm{CP}$ as a critical modulator of the central nervous system elucidating roles during sleep, appetite, neural transmitter availability, and development of the brain (Zappaterra and Lehtinen, 2012). It has been implicated in over 30 multi-systemic disorders (Emerich et al., 2005; Lavezzi et al., 2013). Furthermore, it has presented itself as a potential target for pharmaceutical and cell-based treatments for Amblyopia, Stroke, Parkinson's disease, Huntington's disease, and Alzheimer's disease (Borlongan et al., 2004a,b; Emerich et al., 2006; Luo et al., 2013; Spatazza et al., 2013; Bolos et al., 2014). This clearly illustrates the need for a better understanding of the development and function of this vital organ.

A strong case can be made that the CP should be considered one of the circumventricular organs (Weindl and Joynt, 1973; Tsuneki, 1986; Ganong, 2000; Joly et al., 2007), as they have many similarities; for example, location at a periphery of the brain in close proximity to the ventricles, high levels of vascularization, functional roles in endocrine signaling, the production of proteins for secretion into the CSF, and the presence of fenestrated vessels (Molnar and Stolp; Richardson, this volume). However, the barrier function performed by the epithelia tight junctions of the $\mathrm{CP}$ has generated discussion as to its inclusion amongst circumventricular organs (Jeong et al., 2008; Umans and Taylor, 2012). Hence attempts to analyze the evolutionary and developmental origins of $\mathrm{CP}$ and some circumventricular organs such as subcommissural organ might shed light on this topic. 
Comparative genomics early on demonstrated its importance for analysis of expression of imprinted genes in the developing CP (Overall et al., 1997), and genes critical for its development (Johansson et al., 2013). Recently, the detailed characterization of CP development in teleosts (Bill et al., 2008; Garcia-Lecea et al., 2008) and the significant progress in genome sequencing of model animals, including several fish (see, for example, Howe et al., 2013; Venkatesh et al., 2014) opened new horizons for advancement of comparative genomics of the $\mathrm{CP}$ on a much broader scale.

\section{DEVELOPMENT OF CP IN MAMMALS}

In all vertebrates, the CPs develop in a posterior to anterior order with the $\mathrm{CP}$ of the IVth ventricle developing prior to more anterior CPs (Netsky et al., 1975; Knott et al., 1997; Garcia-Lecea et al., 2008). This is unlike many other developmental processes that progress from anterior to posterior, including, but not limited to, somitogenesis and neurodifferentiation (Korzh et al., 1993; Kimmel et al., 1995). It is tempting to link this sequence of events with other preceding neurodevelopmental events such as, closure of the neural tube in amphibians and mammals, which is a bidirectional process progressing both anteriorly and posteriorly from the hindbrain (Copp et al., 2003; Korzh, 2014).

The hindbrain roof plate epithelium has been considered to serve two major roles: the dorsal organizing center (Liem et al.,
1997; Lee and Jessell, 1999; Lee et al., 2000; Millonig et al., 2000; Broom et al., 2012), and the source of the intermediate epithelium that transforms into cerebrospinal fluid-producing epithelium of the CPIV (Wilting and Christ, 1989; Thomas and Dziadek, 1993). As a dorsal organizing center, the hindbrain roof plate epithelium influences dorsal neuroectoderm, by secreting signaling molecules, such as BMPs and WNTs, to direct transcription in domains along the dorsal-ventral axis, specifically within the rhombic lip (Chizhikov and Millen, 2004; Currle et al., 2005). Reciprocal Notch signaling maintains the boundary between the hindbrain roof plate epithelium and the rhombic lip (Broom et al., 2012). Given that the cells of the CP epithelium and vascular are drawn to this location, it is tempting to hypothesize that the hindbrain roof plate epithelium secretes factors that recruit these cell types.

It is thought that in mammals the hindbrain roof plate epithelium is primarily derived from the dorsal most neural ectoderm of the lateral rhombic lips, and these cells differ at the molecular level along the anterior-posterior and dorsal-ventral axes (Awatramani et al., 2003; Chizhikov et al., 2006; Hunter and Dymecki, 2007). The hindbrain roof plate epithelium consists of three distinct fields (Figure 1). The two primary fields are the medial and lateral fields, and they are distinct in developmental timing and mitotic activity. The lateral field expresses several molecular markers that further separate it into the lateral-anterior

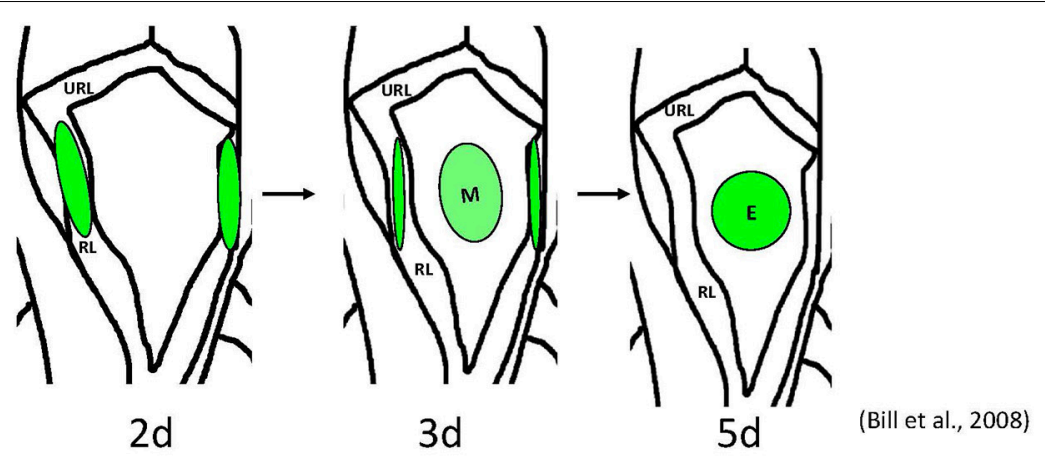

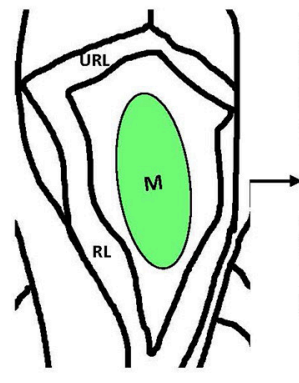

$36 \mathrm{~h}$

$3 d$

$5 d$

(Bill et al., 2008)

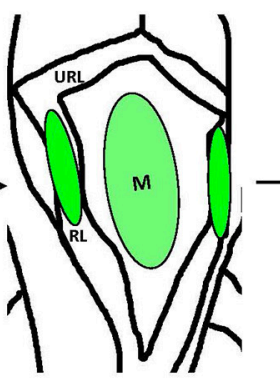

$2 d$

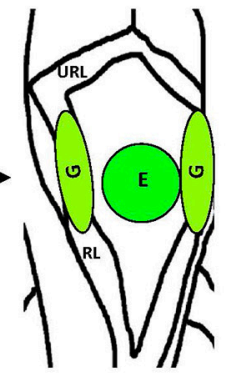

$3 d$

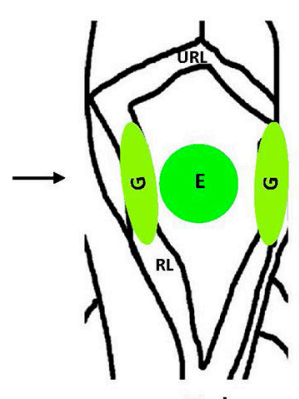

$5 d$
FIGURE 1 | Schemata of formation of the choroid plexus of IV ventricle in zebrafish as revealed by analysis of different transgenics. Above- mn16Et, below - Gateways. The cytosolic GFP, first detected the lateral clusters, could be detected in the prospective epithelial cells of mn16Et at $3 \mathrm{dpf}$, i.e., after these coalesced toward the midline. This mimics events of the $\mathrm{CP}$ morphogenesis as hypothesized in mammals. In contrast, in the Gateway transgenics, the GFP is rather prominent in the midline cluster prior to its coalescence, i.e. during a stage of tela choroidea. Abbreviations: E, epidermal cells of the prospective choroid plexus of IV ventricle; G, glial cells of the prospective choroid plexus of IV ventricle; $M$, midline cluster; RL, rhombic lips; URL, upper rhombic lips. 
and lateral-posterior fields (Hunter and Dymecki, 2007). It appears that the lateral fields are the primary contributor of cells to CPIV, which initially develops as an invagination of the hindbrain roof plate epithelium (Awatramani et al., 2003). CPIV will subsequently receive migratory cells directly from the rhombic lip (Hunter and Dymecki, 2007). The fate-mapping studies of these fields lead to a number of questions. What is the fate of the midline field of hindbrain roof plate epithelium, and what are its contributions to CPIV development? Are the midline and lateral fields the sole source of cells present in the CPIV differentiated epithelium? Does the hindbrain roof plate epithelium also contribute to the CPIV vasculature or stroma? Is there a roof plate at the hindbrain (myelencephalon) level adjacent to the CPIV $(\mathrm{mCP})$ ? What genes are involved in specification of the different cell lineages in the CPIV? Are there cell adhesion molecules present on the cellular surface of developing CPIV cells that restrict cell type mixing, or do they segregate by developmental turnover as previously determined for the lateral CP (Liddelow et al., 2010)?

\section{CAN FISH PROVIDE INFORMATION RELEVANT TO CP DEVELOPMENT IN MAMMALS?}

Given that all vertebrates, including fish have brain ventricles (Lowery and Sive, 2005), it is reasonable to expect that the molecular mechanisms underlying their formation were conserved during evolution, at least to some extent. For example, the Engrailed family of evolutionarily conserved transcriptional repressors plays an important role during formation of the midbrain-hindbrain boundary and adjacent tissues, the posterior midbrain and cerebellum. Engrailed loss-of-function mutations result in a reduction and/or loss of these structures, whereas gain-of-function mutations in Engrailed family members expressed at the dorsal midline cause misspecification of roof plate cells thus perturbing axonal navigation and interfering with the development of structures deriving from the dorsal neuroepithelium, such as the CP, epiphysis and subcommissural organ in fish, birds and rodents (Araki and Nakamura, 1999; Ristoratore et al., 1999; Louvi and Wassef, 2000). This suggests that the developmental program of the $\mathrm{CP}$ and some circumventricular organs is evolutionarily conserved opening a possibility to study these processes in the abundantly available and transparent embryos of fish (Figure 2). Indeed, the first in vivo studies of several enhancer-trap transgenic lines of zebrafish expressing cytosolic GFP (Table 1) brought about significant progress in understanding the development of CP.

\section{THE FATE OF THE MIDLINE FIELD AND SPECIFICATION OF CP LINEAGES}

As mentioned above, cell fate studies of CPIV in mice demonstrated a role of cells of the rhombic lips (lateral fields) in this process; however, the role of the hindbrain roof plate epithelium, in the absence of suitable markers, is not well-understood (Awatramani et al., 2003; Landsberg et al., 2005; Hunter and Dymecki, 2007). Interestingly, in zebrafish, a very similar developmental scenario can be proposed based upon observations of formation of the $\mathrm{mCP}$. In the zebrafish transgenic line, mn16Et GFP expression is initially confined to the rhombic lips (lateral

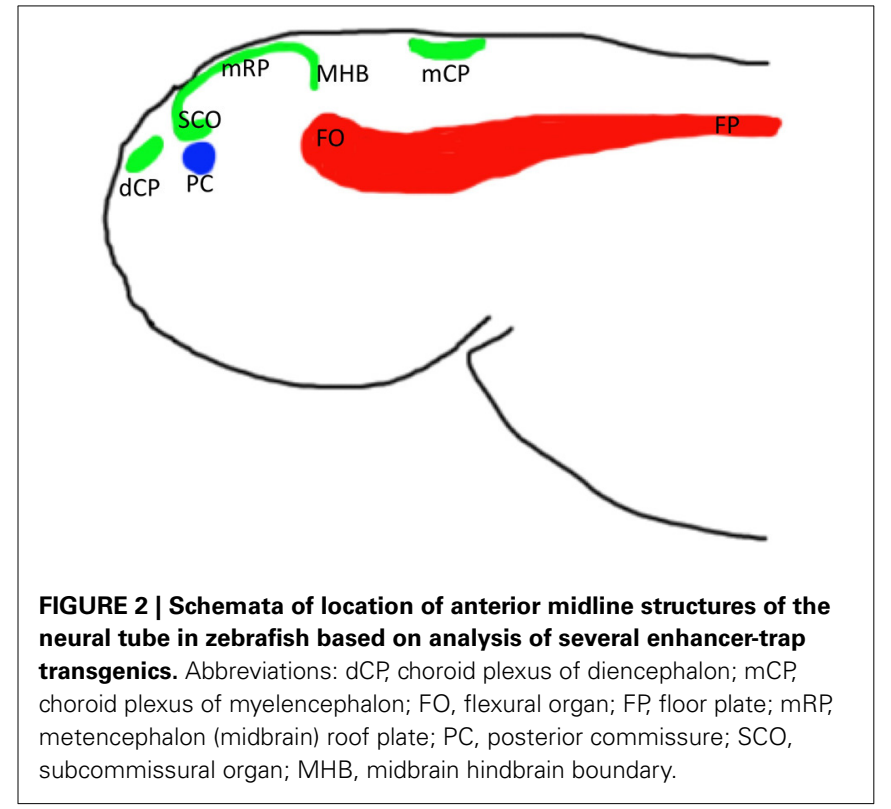

clusters), then spreads toward the midline. In time, only the midline cluster remains fluorescent (Bill et al., 2008). This could be interpreted in a manner similar to that in mice, i.e., that the lateral clusters of the rhombic lips are the only structures giving rise to the tela choroidea, the primordium of the CP.

Fortunately, zebrafish are amenable to large-scale genetic manipulation, thus additional in vivo markers for tela choroidea and CP were generated within our large-scale screen for enhancer-trap lines in zebrafish (Parinov et al., 2004; Kondrychyn et al., 2009). This provided the opportunity to study CP formation in real time. In particular, the transgenic line Gateways (sqet33e20Et) revealed formation of the $\mathrm{dCP}$ and $\mathrm{mCP}$, each from several distinct cell groups, which is similar to that during formation of CPs in mice (Currle et al., 2005; Garcia-Lecea et al., 2008). The following phases of mCP formation were defined: (i) the tela choroidea forms from the neuroepithelial cells of the IVth ventricle roof, simultaneously the vasculature grows toward the final mCP location; (ii) some cells of the rhombic lips join the tela choroidea by migrating from the upper and lateral rhombic lips, reminiscent of the migration observed in mice and mn16Et zebrafish; (iii) the tela choroidea coalesces forming the button-like structure (medial cluster or mCP epithelial cells) in the roof of the IVth ventricle; (iv) the capillary encircles the structure and invades its interior; (v) as the tela choroidea coalesces, the remaining cells of the lateral clusters develop an astroglial phenotype, and (vi) these cells later converge with the medial cluster due to a reduction of the ventricle and ventricular roof. Therefore, this analysis revealed contribution into the $\mathrm{MCP}$ from at least four different cell lineages - the primary medial cluster derived from a roof of IVth ventricle, the secondary cells of medial cluster derived from lateral rhombic lips, the endothelial cells of the invading vasculature, and astroglial cells of the rhombic lips. Based on morphological criteria, the former two lineages contribute to the epithelial and stromal components of the CP, and the latter two correspond to endothelial and astroglial components. To support 
Table 1 | Zebrafish transgenic lines that label the choroid plexus.

\begin{tabular}{|c|c|c|c|}
\hline Transgenic line & Tissue & Genes linked & References \\
\hline sqet33Et & $\mathrm{CP}$, dorsal interneurons, NCC, meninx & zic3-zic6 & Kondrychyn et al., 2011; Winata et al., 2013 \\
\hline sqet3310Et & $\begin{array}{l}\mathrm{CP} \text { (epithelium), roof and floor plates, pigment } \\
\text { and olfactory }\end{array}$ & & Kondrychyn et al., 2011 \\
\hline sqet33e20Et (Gateways) & $\begin{array}{l}\text { CP (medial and lateral clusters, tela choroidea, } \\
\text { epithelium and glia), roof plate, NCC, CVOs, } \\
\text { lens, pronephros, olfactory }\end{array}$ & csrnp1b, sulf1, slc05A1 & Jiao et al., 2011; Kondrychyn et al., 2011 \\
\hline sqKR19Et & $\begin{array}{l}\text { Expression pattern identical to that in } \\
\text { SqET33-E20-CP (medial and lateral clusters, } \\
\text { tela choroidea, epithelium and glia), roof plate, } \\
\text { NCC, CVOs, lens, pronephros, olfactory }\end{array}$ & sema3gb & Teh et al., 2010 \\
\hline sqet33-mi84Et & $\mathrm{CP}$ (vasculature), general vasculature & pard3 & Kondrychyn et al., 2011 \\
\hline sqgw18aEt & $\mathrm{CP}$, roof plate, ear, eyes & Not mapped & Kondrychyn et al., 2011 \\
\hline sqgw19cEt & $\mathrm{CP}$, roof plate, ventral neurons of spinal cord & Not mapped & Kondrychyn et al., 2011 \\
\hline sqgw42aEt & CP (epithelium), roof plate, ventral neurons & csrnp1b, sulf1, slc05A1 & Garcia-Lecea et al., 2008 \\
\hline sqgw84bEt & $\mathrm{CP}$, roof plate, pronephros, neurons & slc22A13 & Kondrychyn et al., 2011 \\
\hline mn16Et & $\begin{array}{l}\text { Lateral and medial clusters, tela choroidea, } \\
\text { epithelium. }\end{array}$ & Not mapped & Bill et al., 2008 \\
\hline
\end{tabular}

and develop this idea further more experimental evidence will be required. Nevertheless, based on unpublished results of other zebrafish transgenics, it is clear that these highlight events somewhat different from those seen in mn16Et and Gateways. It is critical that these transgenic and other developmental markers of CP be characterized, as they will further delineate unique contributions of different cell populations into the CP.

\section{ANTERIOR ROOF PLATE: DISAPPEARING ACT OR MODIFYING MORPHOGENESIS?}

The study of formation of the midline signaling structures in zebrafish transgenics revealed similar organization of the roof and floor plates along the spinal cord. During late neurulation, when the primitive lumen is reduced into a central canal, the roof plate is extended significantly along the dorsal-ventral axis, whereas floor plate is extended a little (Kondrychyn et al., 2013; Korzh, 2014). This organization reverses anteriorly, where starting from the myelencephalon level the floor plate progressively extends along the dorsal-ventral axis and the roof plate either is converted into other dorsal midline structures such as CP, or is significantly reduced as it happens at the level of mesencephalon (Korzh, 2007, 2014). Several observations support this interpretation. First, none of several roof plate transgenics analyzed so far express a fluorescent marker in the roof plate only; other expression domains represent the neural crest derivatives, interneurons, CP (see Table 1), i.e., structures derived from common progenitors (Papan and Campos-Ortega, 1999; Garcia-Lecea et al., 2008).

This raises an intriguing question: does the roof plate disappear partially and/or convert into other structures (CP or circumventricular organs)? Is this unique to bony fish? As mentioned above, there are several studies documenting the roof plate at the myelencephalon level in terrestrial vertebrates (Liem et al., 1997; Lee and Jessell, 1999; Lee et al., 2000; Millonig et al., 2000). Hence, the dorsal neural tube of bony fish may be evolutionary ancient and reminiscent of a common ancestor of vertebrates. And yet a presence of the most of circumventricular organs in zebrafish and terrestrial vertebrates (except those at the telencephalon level) makes this doubtful. One should not forget that current theories of formation of CP in bony fish and mammals still differ somewhat (Awatramani et al., 2003; Landsberg et al., 2005; Hunter and Dymecki, 2007; Korzh, 2007, 2014; Garcia-Lecea et al., 2008). Perhaps, the roof plate development in terrestrial vertebrates could be studied in vivo, at some point, or with help from comparative genomics, and results of such studies will contribute toward solving this interesting evolutionary puzzle.

Since the CP occupies a position at the dorsal midline, it is reasonable to predict that it forms by early neural progenitors found in this place. The roof plate occupies the dorsal midline of spinal cord. This poses a question, does the roof plate occupy the dorsal midline of the fourth (myelencephalic) ventricle, or do these cells somewhat differ? Dorsal neuroepithelium of the spinal cord prior to conversion into the roof plate undergoes topological transformation without cell migration. As a result the cells polarized initially along the medial-lateral axis become polarized along dorsal-ventral axis and elongate (Kondrychyn et al., 2013). In contrast, cells of midline dorsal neuroepithelium at the myelencephalic level fail to undergo such transformation. Instead, they coalesce into a cluster representing the epithelial lineage of CP. In parallel, the lateral clusters develop as glial cells morphologically reminiscent of the roof plate, but their presence is limited by an extent of 2-3 rhombomeres proximal to the medial cluster. These will fuse later on with the medial cluster as the glial lineage of the same CP (Garcia-Lecea et al., 2008). Each of these clusters represents approximately one half of the myelencephalic roof plate progenitors. Hence one may speculate that a ventricle separates the roof plate progenitors into two lateral clusters, and that the ventricle formation drives morphogenetic transformation of the dorsal midline progenitors into CP.

Hence at least for now there is no reason to believe that at the level of brain ventricles the roof plate is maintained. This consideration is supported by analysis of the roof plate formation 
in other brain regions, for example, the mesencephalon. At this level one more cavity reminiscent of a ventricle forms early on in parallel with the myelencephalon (hindbrain, IVth) and diencephalon (IIIrd) bona fide ventricles. This transient cavity known as the opthocoele develops an equivalent of the Sylvius aqueduct connecting two ventricles. At this level the roof plate cells are not as elongated as those at the spinal cord level, but they do have morphological similarity to the spinal cord roof plate. So, it seems we may formulate a simple hypothesis: "The roof plate is present at the dorsal midline of the neural tube only in absence of ventricles. In presence of ventricles it is converted into other derivatives, such as CP, etc."

Does the roof plate contribute into any other midline derivatives? One of these is the subcommissural organ. It has been suggested that the subcommissural organ is derived from the neuroepithelial cells that line the lumen of the dorsal-caudal aspect of the diencephalon (Huh et al., 2009). In view of the fact that the subcommissural organ expresses several transgenes as the roof plate and/or CP, we suggest that this organ, located posterior and ventral to the epiphysis and, immediately below the posterior commissure, develops as the modified extension of the mesencephalic roof plate (Korzh, 2014).

\section{GENES OF THE DORSAL MIDLINE}

At the molecular level, there are at least two differences that have been identified in development of the CP in mammals and teleosts. First, Otx2 null mice fail to develop all four CP; however, in teleosts, otx2 expression appears limited to the diencephalon (Li et al., 1994; Johansson et al., 2013) thus potentially restricting its effects on the dCP. Despite several morpholinos designed for knockdown of Otx 2 and available zebrafish mutants, the CPs in Otx2-deficient animals have not been investigated further. Second, transthyretinin (TTR), a common marker of the CP in amniotes, is not expressed in the $\mathrm{CP}$ of amphibians and teleost fish (Power et al., 2000). Given that in these species high levels of $t t r$ are produced by the liver, future studies will be required to determine whether liver-derived $t t r$ can functionally substitute for CP-derived $t$ tr.

There are many more similarities in expression of molecular markers in the CP. Clusterin is the cytoprotective secreted chaperone protein linked to protection of cells from oxidative stress, and its deficiency has been implicated in Alzheimer's, metabolic and cardiovascular diseases (Trougakos, 2013; Park et al., 2014). Despite being vertebrate-specific, when expressed in Drosophila, Clusterin increases resistance to heat-shock, wet starvation, oxidative stress and increases the lifespan of flies (Lee et al., 2012). During mouse development its expression is enriched in the CP and ependyma (Charnay et al., 2008), and in zebrafish, it is expressed in developing mCP (Jiao et al., 2011). Clusterin may represent the earliest CSF protein secreted by the CP that suppresses proteotoxic stress. Clusterin expression is regulated by Notch signaling (Jiao et al., 2011). The developmental roles of both Notch and Shh signaling are conserved between fish and mammals, and Notch is known to regulate CP development (Hunter and Dymecki, 2007; Bill et al., 2008; Garcia-Lecea et al., 2008; Nielsen and Dymecki, 2010).
There are also several areas that will require more research to determine the similarities and differences in developmental activity of evolutionary conserved genes. The CPIII fails to develop in the Gli3-deficient mouse embryos (Grove et al., 1998). In zebrafish Gli3 is broadly expressed in the dorsal neural tube (Tyurina et al., 2005); however functional studies of CP in the Gli3-deficient zebrafish have not been performed. Amongst genes expressed in the $\mathrm{CP}$ of both mice and zebrafish, there are several Msx genes (Nishikawa et al., 1994; Landsberg et al., 2005; Thisse and Thisse, 2005); however, systematic comparisons will need to be performed and their specific roles in CP development established. Lastly, Gdf7 marks the primordium of the CP epithelium in mice, and has been utilized by many studies to drive expression within the CPIV (Currle et al., 2005); however, gdf7 expression and function have not been analyzed in zebrafish in sufficient detail. These studies will be critical to determine the level of evolutionary conservation of developmental mechanisms of the $\mathrm{CP}$ and fill in a gap in this knowledge using molecular tools already available.

Translation of teleost findings to the mammalian systems could also be highly informative. The transposon-mediated enhancer-trap study identified several cell populations that contribute to the formation of the $\mathrm{CP}$, and the insertion sites mapped identified the mCP-associated genes. In particular, the Gateway (sqet33e20Et) transgene has been mapped into a region containing csrnp1a (axud1), sulf1, and slc5a1 (Garcia-Lecea et al., 2008). Transposon insertions of two other transgenic lines with expression in the CP (sqgw42aEt and sqgw45cEt) were mapped into the same genomic area supporting a role for one of these genes in the CP development or function. csrnp1, whose expression is controlled by Shh and PDGF, was proposed to be involved in regulating cell migration. It is essential for the development of neural-crest-derived cephalic structures (Schmahl et al., 2007; Feijoo et al., 2009). slc5a1 (sglt1) has been studied for its role in glucose re-absorption in kidney and intestine (Gorboulev et al., 2012). Given that the functionally related glucose transporter 1 (GLUT1) is expressed in the CP of mammals, as well as that of bony and cartilaginous fish, this would make slc5a1, which encodes the sodium/glucose cotransporter rather interesting to investigate further despite the fact that its expression pattern might preclude a localized function (Farrell and Pardridge, 1991; Farrell et al., 1992; Planas et al., 2000; Jensen et al., 2010; Balmaceda-Aguilera et al., 2012). sulf1 is expressed in the developing CP of mice (Ohto et al., 2002; Kalus et al., 2009; Ratzka et al., 2010), where its deficiency causes only minor defects or no defects in development of the CP probably due to functional redundancy with other Sulf genes (Holst et al., 2007; Kalus et al., 2009). Its orthologue in zebrafish is also expressed in the CP along with two other related genes-sulf 2 and sulf $2 a$ (Gorsi et al., 2010). Since sulf1 has been linked to formation of the BBB, it is probably the best candidate gene, which expression is mimicked in the Gateways. Coincidentantly, the posttranslational modification of components of the extracellular matrix by sulfation plays an important role in regulation of cellular signaling (FGF, $\mathrm{Hh}$, and Wnt) and mutations affecting components of sulfation machinery and its substrates are known to cause a variety of 
developmental defects linked to deficient cell signaling (Luders et al., 2003).

Lastly, several of the genes, specifically those that mediate the tight junctions between the CP epithelial cells are conserved across multiple phyla. These are not specific to the CP alone, but are a consistent feature between blood-CSF barrier, which is mediated at the level of epithelial cells of the CP (Dziegielewska et al., 2001); and BBB, which in all vertebrates is mediated at the level of endothelial cells of brain microvessels except sharks and rays, where it is at the level of astrocytes (Abbott et al., 2006; Bundgaard and Abbott, 2008). In the blood-CSF barrier the tight junction (or zonula occludens, $\mathrm{ZO}$ ) is located in apical regions between adjacent epithelial cells, and filters the bloodto-CSF passage of hydrophilic molecules and ions (Johanson, 2003). Tight junctions are dynamic multimolecular complexes of membrane-associated proteins active in intracellular signaling, which include $\mathrm{ZO}$ proteins and claudins and a number of other proteins (Luissint et al., 2012). In the zebrafish there are three maternally-expressed genes encoding $\mathrm{ZO}$ proteins, so the $\mathrm{ZO}$ proteins are available from the very beginning of development (Kiener et al., 2007). Claudin 5a is also expressed in the brain during a period prior to and during CP formation (Zhang et al., 2010). Although a systematic analysis of genes expressed in the CP epithelia in evolutionary perspective is still in its infancy, there is a progress in this area. Hence, we will know to which extent the $\mathrm{CP}$ epithelium is conserved in evolution rather soon.

\section{DEVELOPMENT OF THE CP VASCULATURE}

The vasculature of the human CPIV is supplied by branches that originate primarily from the posterior inferior cerebellar artery, and to a lesser extent the anterior inferior cerebellar artery (mostly in the anterior CP portion closest to the foramen of Lushka). In rare cases, branches of the superior cerebellar, the basilar artery, or other arteries extend branches to supply the CP (Sharifi et al., 2005). Once inside the CP the vessels become highly branched and interconnected providing a vast network. The vessels are unique from other brain regions in that they lack the endothelial barrier protein and astrocytic processes in the basement membrane (Bouchaud et al., 1989; Rosenstein et al., 1992), and contain 60-80 nm transcellular pores or fenestrations, allowing for the exit of substrates into the stroma (Strazielle and Ghersi-Egea, 2000; Stan et al., 2012). The fenestrations are covered by diaphragms that have tufts of heparan sulfate proteoglycans extending into the lumen suggesting a role in selective permeability (Stan et al., 2012). The dilation of these vessels is mediated by pericytes, innervating nerves, and interepithelial pressure sensors (Mortazavi et al., 2014), and has a direct effect on the composition of the CSF; therefore, they are highly regulated in response to CSF conditions independent of the physiological blood pressure (Scala et al., 2011). A specialized basement membrane, critical for serum filtering, is secreted by the vasculature very similar to that secreted by the fenestrated vasculature of the kidney (Johanson et al., 2011).

\section{DEVELOPMENT OF THE MAMMALIAN CP VASCULATURE}

In human, the posterior inferior cerebellar artery and anterior inferior cerebellar artery begin sprouting at 44 and 40 days, respectively, and the posterior inferior cerebellar artery terminates in the $\mathrm{CP}$ at 52 days. Its arrival is concurrent with that of the first epithelial cells suggesting that signaling from the roof plate is critical for angiogenesis (Macchi et al., 2005). Development of the posterior inferior cerebellar artery is highly variable, with a variety of origins and paths consistent with a model in which hypoxia induces angiogenic factors, for example VEGF, and consequently angiogenic sprouting induced (Macchi et al., 2005; Yang et al., 2010). Angiogenic sprouting has been described in detail for the $\mathrm{CP}$ vasculature in other organisms as well. Strong described the process in 14-day rabbit embryos observing a reorganization of vessels occurring, with the pontobulbar artery running into the area that develops as the tela choroidea (Strong, 1956). Over the next few days the vasculature continues to develop, and anastomose being drawn into each progressive epithelial invagination (Strong, 1956). A second more rapid angiogenic process, called splitting angiogenesis or intussusceptive angiogenesis, is involved in the enumeration of vessels within the invaginations. This process was inferred from the identification of pillar structures bisecting larger vessels of the $\mathrm{CP}$ by electron microscopy (Scala et al., 2011). This developmental process has been primarily described in other tissues; however, it could be extrapolated to the $\mathrm{CP}$ vasculature. Intussusceptive angiogenesis describes three separate processes for vascular enumeration: microvascular growth, arborization, and remodeling (Makanya et al., 2009).

The zebrafish is one of the premiere animal models for studying vascular development due to its ability to develop without a functioning circulatory system or blood (Stainier, 2001), and large number of transgenics that label the vasculature (Lawson and Weinstein, 2002; Jin et al., 2005, 2007; Rehn et al., 2011; Trinh le et al., 2011; Zhou et al., 2011; Sacilotto et al., 2013). Unfortunately, the vasculature structure of the adult $\mathrm{mCP}$ has not been characterized as it has been in mammals. This is primarily due to an emphasis within the zebrafish community on early developmental processes, thus $\mathrm{mCP}$ characterization has been limited to the first 6 days post fertilization (dpf). Angiogenic sprouting occurs from the middle cerebral vessel at $48 \mathrm{hpf}$ creating the dorsal longitudinal vein. The dorsal longitudinal vein extends along the dorsal midline turning at $\sim 50 \mathrm{hpf}$ to fuse with either left or right posterior cerebral vein (Bill et al., 2008; Lenard et al., 2013). The tip cells of the dorsal longitudinal vein and posterior cerebral vein initiate this junction, subsequently rearranging, and splitting to produce a multicellular lumen (Lenard et al., 2013). Consistent with events in the mammalian CPIV, the angiogenic sprout arrival occurs simultaneous to the migration of the epithelial field suggesting that the roof plate organizer is required to attract both sets of cells to their final localization. A secondary branch forms off of the main dorsal longitudinal vein branch and then fuses with the opposing posterior cerebral vein, and a third branch forms to connect the left and right posterior cerebral veins. We named this connecting vessel the trans-choroid plexus branch as it crosses near the posterior margin of mCP (Bill et al., 2008). Further branching between the dorsal longitudinal vein, posterior cerebral vein, and trans-choroid plexus branch are observed by $5 \mathrm{dpf}$ internal to the mCP region defined by transgenic lines that label the $\mathrm{mCP}$ epithelia. The resulting structure is 
highly fragile and prone to hemorrhage (Bill, 2008) much like the mammalian CP (Scott and Bergevin, 2005). Given this fragility, it is not surprising, that the $\mathrm{CP}$ vasculature is constantly being repaired utilizing bone marrow-derived endothelial progenitors (Zhang et al., 2002). Unfortunately, processes such as invagination of the $\mathrm{mCP}$, intussusceptive angiogenesis, and the integration of bone marrow-derived endothelial progenitors have not been studied in zebrafish. One striking difference is that the zebrafish appears to have a relatively invariant growth profile, while the vascular growth in humans is much more variable. There are two potential reasons for its invariant nature in the zebrafish. The first is that the corresponding period of zebrafish developmental program is much more rapid than that in human ( $2 \mathrm{~h}$ vs. 8 days). The increased developmental window could allow for competing attractive and repulsive angiogenic factors time to alter the trajectory of the main vessel. A second hypothesis could be related to the levels of oxygen that are driving vascular development. We know that the zebrafish are able to survive for up to $7 \mathrm{dpf}$ without a functioning circulatory system, due to the permeability of the embryo to oxygen; therefore, hypoxic conditions within the developing brain would be much more common during human development. This would lead to involvement of competing angiogenic factors as other brain regions would attempt to obtain oxygen, and thus development would take a much more tortuous path.

At the molecular level sprouting angiogenesis, intussusceptive angiogenesis, and development of fenestrations are controlled by interactions between the VEGF, NOTCH, and SHH signaling pathways (Kamba et al., 2006; Nielsen and Dymecki, 2010; Yang et al., 2010; Dimova et al., 2013). The CP epithelial cells continuously secrete VEGF at high levels even in adults suggesting multiple roles for VEGF in vascular development (Esser et al., 1998), while the endothelial cells express the VEGF receptors. While these processes have been investigated extensively in the tail of the zebrafish, work on the cranial vasculature has lagged behind, and very little is known about the genes required for the dorsal longitudinal vein attraction to the $\mathrm{mCP}$, branching, and production of the fenestrated vessels. Part of the problem is a general role of some of these pathways (Notch and Shh) in morphogenesis of the CP (Garcia-Lecea et al., 2008). In the future, extension of the developmental and molecular studies of the $\mathrm{mCP}$ vasculature will be required to determine the extent of conservation.

\section{DEVELOPMENT OF THE CP STROMA}

The stroma lies between the vascular basement membrane and the epithelial basement membrane. It contains a wide variety of cells including: fibroblasts, telocytes, stem cells, dendritic cells, leukocytes, macrophages, kolmer cells (a specialized monocyte, also called epiplexus cells), and in some cases T-cells (Strazielle and Ghersi-Egea, 2000; Nataf et al., 2006; Popescu et al., 2012). The stroma is hypothesized to function as a stem cell niche with the nestin-positive fibroblasts and telocytes supporting myeloid progenitors and neural stem cells, respectively (Nataf et al., 2006; Popescu et al., 2012).

The stroma develops from invagination of the mesenchyme that lies dorsal to the hindbrain roof plate epithelium, and progressively transitions to being composed of high levels of connective tissues with low density of mesenchymal cells (Shuangshoti and Netsky, 1966). Little is known about the development of this region; however, there are indications that the stromal composition may be highly dynamic and change during development, and in response to immunologic insult. For example, it is hypothesized that the fibroblast niche provides different developmental signals during development than during adulthood based on the progeny of the myeloid progenitors; embryonic-derived progenitors produce dendritic cells, while adult-derived progenitors produce macrophages (Nataf et al., 2006). Another difference is that kolmer cells are only present in the adult CP (Strazielle and Ghersi-Egea, 2000). Given the immunologic complexity of the stroma, much of the cell diversity is driven by immunologic insult, or CNS injury (Mitchell et al., 2009; Wojcik et al., 2011; Hasegawa-Ishii et al., 2013; Shechter et al., 2013; Szmydynger-Chodobska et al., 2013). Previous analysis in zebrafish failed to demonstrate presence of unlabelled cells in the $\mathrm{mCP}$ of transgenics suggesting that all cell lineages except vasculature derive from GFP-positive cells (Garcia-Lecea et al., 2008). This raises a criticism regarding contribution of mesenchymal cells into the mCP of this species; however, as mentioned all analyses on zebrafish have focused on early development opening the possibility that the mesenchymal-derived cells enter at later developmental time points. New in vitro systems and transgenics in combination with high-resolution bioimaging may lead to a much better definition of this portion of the $\mathrm{mCP}$.

\section{CONCLUSIONS}

The CP has been a popular organ system to investigate in a variety of vertebrates including fish, lizards, amphibians, birds, and mammals; however, the number of systematic studies that look at a single feature of the $\mathrm{CP}$ across the phyla have been very few, and primarily restricted to the CP epithelia. Given the CP's importance in a variety of physiologic brain functions and pathologies, we suggest a need for these types of studies, as they provide valuable insights into understanding CP development and function. In this review, we have tried to highlight how several studies in the zebrafish have expanded our knowledge of CP development in general, and shed light on several aspects that remain not fully understood, including developmental fate of the medial group of hindbrain roof cells. Based on comparisons of fish and mice, we observe that the CP derives from cells that share their origin with the roof plate and neural crest, and its development is driven by the evolutionary conserved molecular determinants. Indeed, this is a renaissance in $\mathrm{CP}$ research, and there are many questions that are yet to be answered. For example, what are the tropic factors that are secreted from the dorsal organizing center to attract the vasculature and epithelia cells to their final location? What are the molecular differences that exist within the $\mathrm{CP}$, as cells can be derived from either the roof plate or neural crest? What is the molecular identity of the stem cell niche? And lastly, to what extent are features of the CP conserved? Given an abundance of modern developmental tools (transgenics and cell transplantation), and genomic technologies (single cell transcriptome and genome engineering), we are poised to answer these questions, and feel that they can be answered soon. 


\section{ACKNOWLEDGMENTS}

Authors are indebted to the collective input of colleagues from the Steve Ekker, Lisa Schimenti and Vladimir Korzh laboratories, where most of experimental data in support of our ideas have been generated and personnel of the Fish Facilities of the University of Minnesota and the Institute of Molecular and Cell Biology, Singapore for care and maintenance of fish.

\section{REFERENCES}

Abbott, N. J., Ronnback, L., and Hansson, E. (2006). Astrocyte-endothelial interactions at the blood-brain barrier. Nat. Rev. Neurosci. 7, 41-53. doi: $10.1038 / \mathrm{nrn} 1824$

Araki, I., and Nakamura, H. (1999). Engrailed defines the position of dorsal dimesencephalic boundary by repressing diencephalic fate. Development 126, 5127-5135.

Awatramani, R., Soriano, P., Rodriguez, C., Mai, J. J., and Dymecki, S. M. (2003). Cryptic boundaries in roof plate and choroid plexus identified by intersectional gene activation. Nat. Genet. 35, 70-75. doi: 10.1038/ng1228

Balmaceda-Aguilera, C., Cortes-Campos, C., Cifuentes, M., Peruzzo, B., Mack, L., Tapia, J. C., et al. (2012). Glucose transporter 1 and monocarboxylate transporters 1,2, and 4 localization within the glial cells of shark blood-brainbarriers. PLoS ONE 7:e32409. doi: 10.1371/journal.pone.0032409

Bill, B. R. (2008). Utilization of Forward and Reverse Genetic Approaches to Inform Ocular and Choroid Plexus Development. Molecular, Cellular, Developmental Biology, and Genetics. Minneapolis, MN: University of Minnesota.

Bill, B. R., Balciunas, D., McCarra, J. A., Young, E. D., Xiong, T., Spahn, A. M., et al. (2008). Development and Notch signaling requirements of the zebrafish choroid plexus. PLoS ONE 3:e3114. doi: 10.1371/journal.pone.0003114

Bolos, M., Antequera, D., Aldudo, J., Kristen, H., Bullido, M. J., and Carro, E. (2014). Choroid plexus implants rescue Alzheimer's disease-like pathologies by modulating amyloid-beta degradation. Cell. Mol. Life Sci. 71, 2947-2955. doi: 10.1007/s00018-013-1529-4

Borlongan, C. V., Skinner, S. J., Geaney, M., Vasconcellos, A. V., Elliott, R. B., and Emerich, D. F. (2004a). Intracerebral transplantation of porcine choroid plexus provides structural and functional neuroprotection in a rodent model of stroke. Stroke 35, 2206-2210. doi: 10.1161/01.STR.0000138954.25825.0b

Borlongan, C. V., Skinner, S. J., Geaney, M., Vasconcellos, A. V., Elliott, R. B., and Emerich, D. F. (2004b). Neuroprotection by encapsulated choroid plexus in a rodent model of Huntington's disease. Neuroreport 15, 2521-2525. doi: 10.1097/00001756-200411150-00018

Bouchaud, C., Le Bert, M., and Dupouey, P. (1989). Are close contacts between astrocytes and endothelial cells a prerequisite condition of a blood-brain barrier? The rat subfornical organ as an example. Biol. Cell 67, 159-165.

Broom, E. R., Gilthorpe, J. D., Butts, T., Campo-Paysaa, F., and Wingate, R. J. (2012). The roof plate boundary is a bi-directional organiser of dorsal neural tube and choroid plexus development. Development 139, 4261-4270. doi: 10.1242/dev.082255

Bundgaard, M., and Abbott, N. J. (2008). All vertebrates started out with a glial blood-brain barrier 4-500 million years ago. Glia 56, 699-708. doi: 10.1002/glia.20642

Charnay, Y., Imhof, A., Vallet, P. G., Hakkoum, D., Lathuiliere, A., Poku, N., et al. (2008). Giannakopoulos, Clusterin expression during fetal and postnatal CNS development in mouse. Neuroscience 155, 714-724. doi: 10.1016/j.neuroscience.2008.06.022

Chizhikov, V. V., and Millen, K. J. (2004). Mechanisms of roof plate formation in the vertebrate CNS. Nat. Rev. Neurosci. 5, 808-812. doi: 10.1038/ nrn 1520

Chizhikov, V. V., Lindgren, A. G., Currle, D. S., Rose, M. F., Monuki, E. S., and Millen, K. J. (2006). The roof plate regulates cerebellar cell-type specification and proliferation. Development 133, 2793-2804. doi: 10.1242/dev.02441

Copp, A. J., Greene, N. D., and Murdoch, J. N. (2003). The genetic basis of mammalian neurulation. Nat. Rev. Genet. 4, 784-793. doi: 10.1038/nrg1181

Currle, D. S., Cheng, X., Hsu, C. M., and Monuki, E. S. (2005). Direct and indirect roles of CNS dorsal midline cells in choroid plexus epithelia formation. Development 132, 3549-3559. doi: 10.1242/dev.01915

Dimova, I., Hlushchuk, R., Makanya, A., Styp-Rekowska, B., Ceausu, A., Flueckiger, S., et al. (2013). Inhibition of Notch signaling induces extensive intussusceptive neo-angiogenesis by recruitment of mononuclear cells. Angiogenesis 16, 921-937. doi: 10.1007/s10456-013-9366-5

Dziegielewska, K. M., Ek, J., Habgood, M. D., and Saunders, N. R. (2001). Development of the choroid plexus. Microsc Res. Tech. 52, 5-20. doi: 10.1002/1097-0029(20010101)52:1<5::AID-JEMT3 > 3.0.CO;2-J

Emerich, D. F., Skinner, S. J., Borlongan, C. V., Vasconcellos, A. V., and Thanos, C. G. (2005). The choroid plexus in the rise, fall and repair of the brain. Bioessays 27, 262-274. doi: 10.1002/bies.20193

Emerich, D. F., Thanos, C. G., Goddard, M., Skinner, S. J., Geany, M. S., Bell, W. J., et al. (2006). Extensive neuroprotection by choroid plexus transplants in excitotoxin lesioned monkeys. Neurobiol. Dis. 23, 471-480. doi: 10.1016/j.nbd.2006.04.014

Esser, S., Wolburg, K., Wolburg, H., Breier, G., Kurzchalia, T., and Risau, W. (1998). Vascular endothelial growth factor induces endothelial fenestrations in vitro. J. Cell Biol. 140, 947-959. doi: 10.1083/jcb.140.4.947

Farrell, C. L., and Pardridge, W. M. (1991). Blood-brain barrier glucose transporter is asymmetrically distributed on brain capillary endothelial lumenal and ablumenal membranes: an electron microscopic immunogold study. Proc. Natl. Acad. Sci. U.S.A. 88, 5779-5783. doi: 10.1073/pnas.88.13.5779

Farrell, C. L., Yang, J., and Pardridge, W. M. (1992). GLUT-1 glucose transporter is present within apical and basolateral membranes of brain epithelial interfaces and in microvascular endothelia with and without tight junctions. J. Histochem. Cytochem. 40, 193-199. doi: 10.1177/40.2.1552163

Feijoo, C. G., Sarrazin, A. F., Allende, M. L., and Glavic, A. (2009). Cysteinserine-rich nuclear protein 1, Axud1/Csrnp1, is essential for cephalic neural progenitor proliferation and survival in zebrafish. Dev. Dyn. 238, 2034-2043. doi: $10.1002 /$ dvdy. 22006

Ganong, W. F. (2000). Circumventricular organs: definition and role in the regulation of endocrine and autonomic function. Clin. Exp. Pharmacol. Physiol. 27, 422-427. doi: 10.1046/j.1440-1681.2000.03259.x

Garcia-Lecea, M., Kondrychyn, I., Fong, S. H., Ye, Z. R., and Korzh, V. (2008). In vivo analysis of choroid plexus morphogenesis in zebrafish. PLoS ONE 3:e3090. doi: 10.1371/journal.pone.0003090

Gorboulev, V., Schurmann, A., Vallon, V., Kipp, H., Jaschke, A., Klessen, D., et al. (2012). $\mathrm{Na}(+)$-D-glucose cotransporter SGLT1 is pivotal for intestinal glucose absorption and glucose-dependent incretin secretion. Diabetes 61, 187-196. doi: $10.2337 / \mathrm{db} 11-1029$

Gorsi, B., Whelan, S., and Stringer, S. E. (2010). Dynamic expression patterns of 6$\mathrm{O}$ endosulfatases during zebrafish development suggest a subfunctionalisation event for sulf2. Dev. Dyn. 239, 3312-3323. doi: 10.1002/dvdy.22456

Grove, E. A., Tole, S., Limon, J., Yip, L., and Ragsdale, C. W. (1998). The hem of the embryonic cerebral cortex is defined by the expression of multiple Wnt genes and is compromised in Gli3-deficient mice. Development 125, 2315-2325.

Hasegawa-Ishii, S., Shimada, A., Inaba, M., Li, M., Shi, M., Kawamura, N., et al. (2013). Selective localization of bone marrow-derived ramified cells in the brain adjacent to the attachments of choroid plexus. Brain Behav. Immun. 29, 82-97. doi: 10.1016/j.bbi.2012.12.010

Holst, C. R., Bou-Reslan, H., Gore, B. B., Wong, K., Grant, D., Chalasani, S., et al. (2007). Secreted sulfatases Sulf1 and Sulf2 have overlapping yet essential roles in mouse neonatal survival. PLoS ONE 2:e575. doi: 10.1371/journal.pone.0000575

Howe, K., Clark, M. D., Torroja, C. F., Torrance, J., Berthelot, C., Muffato, M. et al. (2013). The zebrafish reference genome sequence and its relationship to the human genome. Nature 496, 498-503. doi: 10.1038/nature12111

Huh, M. S., Todd, M. A., and Picketts, D. J. (2009). SCO-ping out the mechanisms underlying the etiology of hydrocephalus. Physiology 24, 117-126. doi: 10.1152/physiol.00039.2008

Hunter, N. L., and Dymecki, S. M. (2007). Molecularly and temporally separable lineages form the hindbrain roof plate and contribute differentially to the choroid plexus. Development 134, 3449-3460. doi: 10.1242/dev.003095

Jensen, P. J., Gunter, L. B., and Carayannopoulos, M. O. (2010). Akt2 modulates glucose availability and downstream apoptotic pathways during development. J. Biol. Chem. 285, 17673-17680. doi: 10.1074/jbc.M109.079343

Jeong, J. Y., Kwon, H. B., Ahn, J. C., Kang, D., Kwon, S. H., Park, J. A., et al. (2008) Functional and developmental analysis of the blood-brain barrier in zebrafish. Brain Res. Bull. 75, 619-628. doi: 10.1016/j.brainresbull.2007.10.043

Jiao, S., Dai, W., Lu, L., Liu, Y., Zhou, J., Li, Y., et al. (2011). The conserved clusterin gene is expressed in the developing choroid plexus under the regulation of notch but not IGF signaling in zebrafish. Endocrinology 152, 1860-1871. doi: 10.1210/en.2010-1183 
Jin, S. W., Beis, D., Mitchell, T., Chen, J. N., and Stainier, D. Y. (2005). Cellular and molecular analyses of vascular tube and lumen formation in zebrafish. Development 132, 5199-5209. doi: 10.1242/dev.02087

Jin, S. W., Herzog, W., Santoro, M. M., Mitchell, T. S., Frantsve, J., Jungblut, B., et al. (2007). A transgene-assisted genetic screen identifies essential regulators of vascular development in vertebrate embryos. Dev. Biol. 307, 29-42. doi: 10.1016/j.ydbio.2007.03.526

Johanson, C. (2003). "The choroid plexus-CSF nexus," in Neuroscience in Medicine, ed P. M. Conn (Totowa, NJ: Humana Press), 165-195.

Johanson, C. E., Stopa, E. G., and McMillan, P. N. (2011). The blood-cerebrospinal fluid barrier: structure and functional significance. Methods Mol. Biol. 686, 101-131. doi: 10.1007/978-1-60761-938-3_4

Johansson, P. A., Irmler, M., Acampora, D., Beckers, J., Simeone, A., and Gotz, M. (2013). The transcription factor Otx2 regulates choroid plexus development and function. Development 140, 1055-1066. doi: 10.1242/dev. 090860

Joly, J. S., Osorio, J., Alunni, A., Auger, H., Kano, S., and Retaux, S. (2007). Windows of the brain: towards a developmental biology of circumventricular and other neurohemal organs. Semin. Cell Dev. Biol. 18, 512-524. doi: 10.1016/j.semcdb.2007.06.001

Kalus, I., Salmen, B., Viebahn, C., von Figura, K., Schmitz, D., D’Hooge, R., et al. (2009). Differential involvement of the extracellular 6-O-endosulfatases Sulf1 and Sulf2 in brain development and neuronal and behavioural plasticity. J. Cell. Mol. Med. 13, 4505-4521. doi: 10.1111/j.1582-4934.2008. 00558.x

Kamba, T., Tam, B. Y., Hashizume, H., Haskell, A., Sennino, B., Mancuso, M. R., et al. (2006). VEGF-dependent plasticity of fenestrated capillaries in the normal adult microvasculature. Am. J. Physiol. Heart Circ. Physiol. 290, H560-H576. doi: 10.1152/ajpheart.00133.2005

Kiener, T. K., Sleptsova-Friedrich, I., and Hunziker, W. (2007). Identification, tissue distribution and developmental expression of tjp1/zo-1, tjp2/zo-2 and tjp3/zo-3 in the zebrafish, Danio rerio. Gene Expr. Patterns 7, 767-776. doi: 10.1016/j.modgep.2007.05.006

Kimmel, C. B., Ballard, W. W., Kimmel, S. R., Ullmann, B., and Schilling, T. F. (1995). Stages of embryonic development of the zebrafish. Dev. Dyn. 203, 253-310. doi: 10.1002/aja.1002030302

Knott, G. W., Dziegielewska, K. M., Habgood, M. D., Li, Z. S., and Saunders, N. R. (1997). Albumin transfer across the choroid plexus of South American opossum (Monodelphis domestica). J. Physiol. 499(Pt 1), 179-194.

Kondrychyn, I., Garcia-Lecea, M., Emelyanov, A., Parinov, S., and Korzh, V. (2009). Genome-wide analysis of Tol2 transposon reintegration in zebrafish. BMC Genomics 10:418. doi: 10.1186/1471-2164-10-418

Kondrychyn, I., Teh, C., Garcia-Lecea, M., Guan, Y., Kang, A., and Korzh, V. (2011). Zebrafish Enhancer TRAP transgenic line database ZETRAP 2.0. Zebrafish 8, 181-182. doi: 10.1089/zeb.2011.0718

Kondrychyn, I., Teh, C., Sin, M., and Korzh, V. (2013). Stretching morphogenesis of the roof plate and formation of the central canal. PLOS ONE 8:e56219. doi: 10.1371/journal.pone.0056219

Korzh, V. (2007). Transposons as tools for enhancer trap screens in vertebrates. Genome Biol. 8(Suppl. 1):S8. doi: 10.1186/gb-2007-8-s1-s8

Korzh, V. (2014). Stretching cell morphogenesis during late neurulation and mild neural tube defects. Dev. Growth Differ. 56, 425-433. doi: 10.1111/dgd.12143

Korzh, V., Edlund, T., and Thor, S. (1993). Zebrafish primary neurons initiate expression of the LIM homeodomain protein Isl-1 at the end of gastrulation. Development 118, 417-425.

Landsberg, R. L., Awatramani, R. B., Hunter, N. L., Farago, A. F., DiPietrantonio, H. J., Rodriguez, C. I., et al. (2005). Hindbrain rhombic lip is comprised of discrete progenitor cell populations allocated by Pax6. Neuron 48, 933-947. doi: 10.1016/j.neuron.2005.11.031

Lavezzi, A. M., Matturri, L., Del Corno, G., and Johanson, C. E. (2013). Vulnerability of fourth ventricle choroid plexus in sudden unexplained fetal and infant death syndromes related to smoking mothers. Int. J. Dev. Neurosci. 31, 319-327. doi: 10.1016/j.ijdevneu.2013.04.006

Lawson, N. D., and Weinstein, B. M. (2002). In vivo imaging of embryonic vascular development using transgenic zebrafish. Dev. Biol. 248, 307-318. doi: 10.1006/dbio.2002.0711

Lee, K. J., and Jessell, T. M. (1999). The specification of dorsal cell fates in the vertebrate central nervous system. Annu. Rev. Neurosci. 22, 261-294. doi: 10.1146/annurev.neuro.22.1.261
Lee, K. J., Dietrich, P., and Jessell, T. M. (2000). Genetic ablation reveals that the roof plate is essential for dorsal interneuron specification. Nature 403, 734-740. doi: 10.1038/35001507

Lee, Y. N., Shim, Y. J., Kang, B. H., Park, J. J., and Min, B. H. (2012). Overexpression of human clusterin increases stress resistance and extends lifespan in Drosophila melanogaster. Biochem. Biophys. Res. Commun. 420, 851-856. doi: 10.1016/j.bbrc.2012.03.087

Lenard, A., Ellertsdottir, E., Herwig, L., Krudewig, A., Sauteur, L., Belting, H. G., et al. (2013). In vivo analysis reveals a highly stereotypic morphogenetic pathway of vascular anastomosis. Dev. Cell 25, 492-506. doi: 10.1016/j.devcel.2013.05.010

Li, Y., Allende, M. L., Finkelstein, R., and Weinberg, E. S. (1994). Expression of two zebrafish orthodenticle-related genes in the embryonic brain. Mech. Dev. 48, 229-244. doi: 10.1016/0925-4773(94)90062-0

Liddelow, S. A., Dziegielewska, K. M., Vandeberg, J. L., and Saunders, N. R. (2010). Development of the lateral ventricular choroid plexus in a marsupial, Monodelphis domestica. Cerebrospinal Fluid Res. 7:16. doi: 10.1186/1743-84547-16

Liem, K. F. Jr., Tremml, G., and Jessell, T. M. (1997). A role for the roof plate and its resident TGFbeta-related proteins in neuronal patterning in the dorsal spinal cord. Cell 91, 127-138. doi: 10.1016/S0092-8674(01)80015-5

Louvi, A., and Wassef, M. (2000). Ectopic engrailed 1 expression in the dorsal midline causes cell death, abnormal differentiation of circumventricular organs and errors in axonal pathfinding. Development 127, 4061-4071.

Lowery, L. A., and Sive, H. (2005). Initial formation of zebrafish brain ventricles occurs independently of circulation and requires the nagie oko and snakehead/atplala.1 gene products. Development 132, 2057-2067. doi: 10.1242/dev.01791

Luders, F., Segawa, H., Stein, D., Selva, E. M., Perrimon, N., Turco, S. J., et al. (2003). Slalom encodes an adenosine $3^{\prime}$-phosphate $5^{\prime}$-phosphosulfate transporter essential for development in Drosophila. EMBO J. 22, 3635-3644. doi: 10.1093/emboj/cdg345

Luissint, A. C., Artus, C., Glacial, F., Ganeshamoorthy, K., and Couraud, P. O. (2012). Tight junctions at the blood brain barrier: physiological architecture and disease-associated dysregulation. Fluids Barriers CNS 9:23. doi: 10.1186/2045-8118-9-23

Luo, X. M., Lin, H., Wang, W., Geaney, M. S., Law, L., Wynyard, S., et al. (2013). Recovery of neurological functions in non-human primate model of Parkinson's disease by transplantation of encapsulated neonatal porcine choroid plexus cells. J. Parkinsons Dis. 3, 275-291. doi: 10.3233/JPD-130214

Macchi, V., Porzionato, A., Guidolin, D., Parenti, A., and De Caro, R. (2005). Morphogenesis of the posterior inferior cerebellar artery with threedimensional reconstruction of the late embryonic vertebrobasilar system. Surg. Radiol. Anat. 27, 56-60. doi: 10.1007/s00276-004-0303-6

Makanya, A. N., Hlushchuk, R., and Djonov, V. G. (2009). Intussusceptive angiogenesis and its role in vascular morphogenesis, patterning, and remodeling. Angiogenesis 12, 113-123. doi: 10.1007/s10456-009-9129-5

Millonig, J. H., Millen, K. J., and Hatten, M. E. (2000). The mouse Dreher gene Lmxla controls formation of the roof plate in the vertebrate CNS. Nature 403 764-769. doi: 10.1038/35001573

Mitchell, K., Yang, H. Y., Berk, J. D., Tran, J. H., and Iadarola, M. J. (2009). Monocyte chemoattractant protein-1 in the choroid plexus: a potential link between vascular pro-inflammatory mediators and the CNS during peripheral tissue inflammation. Neuroscience 158, 885-895. doi: 10.1016/j.neuroscience.2008.10.047

Mortazavi, M. M., Griessenauer, C. J., Adeeb, N., Deep, A., Bavarsad Shahripour, R., Loukas, M., et al. (2014). The choroid plexus: a comprehensive review of its history, anatomy, function, histology, embryology, and surgical considerations. Childs Nerv. Syst. 30, 205-214. doi: 10.1007/s00381-013-2326-y

Nataf, S., Strazielle, N., Hatterer, E., Mouchiroud, G., Belin, M. F., and Ghersi-Egea, J. F. (2006). Rat choroid plexuses contain myeloid progenitors capable of differentiation toward macrophage or dendritic cell phenotypes. Glia 54, 160-171. doi: 10.1002/glia.20373

Netsky, M. G., Shuangshoti, S., Tennyson, V. M., Brightman, M. W., Becker, N. H., Sutton, C. H., et al. (1975). The Choroid Plexus in Health and Disease. Charlottesville, VA: University Press of Virginia.

Nielsen, C. M., and Dymecki, S. M. (2010). Sonic hedgehog is required for vascular outgrowth in the hindbrain choroid plexus. Dev. Biol. 340, 430-437. doi: 10.1016/j.ydbio.2010.01.032 
Nishikawa, K., Nakanishi, T., Aoki, C., Hattori, T., Takahashi, K., and Taniguchi, S. (1994). Differential expression of homeobox-containing genes Msx-1 and Msx2 and homeoprotein Msx-2 expression during chick craniofacial development. Biochem. Mol. Biol. Int. 32, 763-771.

Ohto, T., Uchida, H., Yamazaki, H., Keino-Masu, K., Matsui, A., and Masu, M. (2002). Identification of a novel nonlysosomal sulphatase expressed in the floor plate, choroid plexus and cartilage. Genes Cells 7, 173-185. doi: 10.1046/j.13569597.2001.00502.x

Overall, M., Bakker, M., Spencer, J., Parker, N., Smith, P., and Dziadek, M. (1997). Genomic imprinting in the rat: linkage of Igf2 and H19 genes and opposite parental allele-specific expression during embryogenesis. Genomics 45, 416-420. doi: 10.1006/geno.1997.4933

Papan, C., and Campos-Ortega, J. A. (1999). Region-specific cell clones in the developing spinal cord of the zebrafish. Dev. Genes Evol. 209, 135-144. doi: $10.1007 / \mathrm{s} 004270050237$

Parinov, S., Kondrichin, I., Korzh, V., and Emelyanov, A. (2004). Tol2 transposonmediated enhancer trap to identify developmentally regulated zebrafish genes in vivo. Dev. Dyn. 231, 449-459. doi: 10.1002/dvdy.20157

Park, S., Mathis, K. W., and Lee, I. K. (2014). The physiological roles of apolipoprotein J/clusterin in metabolic and cardiovascular diseases. Rev. Endocr. Metab. Disord. 15, 45-53. doi: 10.1007/s11154-013-9275-3

Planas, J. V., Capilla, E., and Gutierrez, J. (2000). Molecular identification of a glucose transporter from fish muscle. FEBS Lett. 481, 266-270. doi: 10.1016/S00145793(00)02020-2

Popescu, B. O., Gherghiceanu, M., Kostin, S., Ceafalan, L., and Popescu, L. M. (2012). Telocytes in meninges and choroid plexus. Neurosci. Lett. 516, 265-269. doi: 10.1016/j.neulet.2012.04.006

Power, D. M., Elias, N. P., Richardson, S. J., Mendes, J., Soares, C. M., and Santos, C. R. (2000). Evolution of the thyroid hormone-binding protein, transthyretin. Gen. Comp. Endocrinol. 119, 241-255. doi: 10.1006/gcen.2000.7520

Ratzka, A., Mundlos, S., and Vortkamp, A. (2010). Expression patterns of sulfatase genes in the developing mouse embryo. Dev. Dyn. 239, 1779-1788. doi: $10.1002 /$ dvdy.22294

Redzic, Z. B., Preston, J. E., Duncan, J. A., Chodobski, A., and SzmydyngerChodobska, J. (2005). The choroid plexus-cerebrospinal fluid system: from development to aging. Curr. Top. Dev. Biol. 71, 1-52. doi: 10.1016/S00702153(05)71001-2

Rehn, K., Wong, K. S., Balciunas, D., and Sumanas, S. (2011). Zebrafish enhancer trap line recapitulates embryonic aquaporin la expression pattern in vascular endothelial cells. Int. J. Dev. Biol. 55, 613-618. doi: 10.1387/ijdb.103249kp

Ristoratore, F., Carl, M., Deschet, K., Richard-Parpaillon, L., Boujard, D., Wittbrodt, J., et al. (1999). The midbrain-hindbrain boundary genetic cascade is activated ectopically in the diencephalon in response to the widespread expression of one of its components, the medaka gene Ol-eng2. Development 126, 3769-3779.

Rosenstein, J. M., Krum, J. M., Sternberger, L. A., Pulley, M. T., and Sternberger, N. H. (1992). Immunocytochemical expression of the endothelial barrier antigen (EBA) during brain angiogenesis. Brain Res. Dev. Brain Res. 66, 47-54. doi: 10.1016/0165-3806(92)90138-M

Sacilotto, N., Monteiro, R., Fritzsche, M., Becker, P. W., Sanchez-Del-Campo, L., Liu, K., et al. (2013). Analysis of Dll4 regulation reveals a combinatorial role for Sox and Notch in arterial development. Proc. Natl. Acad. Sci. U.S. A. 110, 11893-11898. doi: 10.1073/pnas.1300805110

Scala, G., Corona, M., Langella, E., and Maruccio, L. (2011). Microvasculature of the buffalo (Bubalus bubalis) choroid plexuses: structural, histochemical, and immunocytochemical study. Microsc. Res. Tech. 74, 67-75. doi: 10.1002/jemt.20875

Schmahl, J., Raymond, C. S., and Soriano, P. (2007). PDGF signaling specificity is mediated through multiple immediate early genes. Nat. Genet. 39, 52-60. doi: $10.1038 / \mathrm{ng} 1922$

Scott, D. E., and Bergevin, M. (2005). Fine structural correlates of the choroid plexus of the lateral cerebral ventricle of the human fetal brain. Anat. Rec. 282, 8-12. doi: 10.1002/ar.a.20104

Sharifi, M., Ciolkowski, M., Krajewski, P., and Ciszek, B. (2005). The choroid plexus of the fourth ventricle and its arteries. Folia Morphol. 64, 194-198.

Shechter, R., Miller, O., Yovel, G., Rosenzweig, N., London, A., Ruckh, J., et al. (2013). Recruitment of beneficial M2 macrophages to injured spinal cord is orchestrated by remote brain choroid plexus. Immunity 38, 555-569. doi: 10.1016/j.immuni.2013.02.012
Shuangshoti, S., and Netsky, M. G. (1966). Histogenesis of choroid plexus in man. Am. J. Anat. 118, 283-316. doi: 10.1002/aja.1001180114

Spatazza, J., Lee, H. H., Di Nardo, A. A., Tibaldi, L., Joliot, A., Hensch, T. K., et al. (2013). Choroid-plexus-derived Otx2 homeoprotein constrains adult cortical plasticity. Cell Rep. 3, 1815-1823. doi: 10.1016/j.celrep.2013.05.014

Stainier, D. Y. (2001). Zebrafish genetics and vertebrate heart formation. Nat. Rev. Genet. 2, 39-48. doi: 10.1038/35047564

Stan, R. V., Tse, D., Deharvengt, S. J., Smits, N. C., Xu, Y., Luciano, M. R., et al. (2012). The diaphragms of fenestrated endothelia: gatekeepers of vascular permeability and blood composition. Dev. Cell 23, 1203-1218. doi: 10.1016/j.devcel.2012.11.003

Strazielle, N., and Ghersi-Egea, J. F. (2000). Choroid plexus in the central nervous system: biology and physiopathology. J. Neuropathol. Exp. Neurol. 59, 561-574.

Strong, L. H. (1956). Early development of the ependyma and vascular pattern of the fourth ventricular choroid plexus in the rabbit. Am. J. Anat. 99 249-290. doi: 10.1002/aja.1000990204

Szmydynger-Chodobska, J., Gandy, J. R., Varone, A., Shan, R., and Chodobski, A. (2013). Synergistic interactions between cytokines and AVP at the bloodCSF barrier result in increased chemokine production and augmented influx of leukocytes after brain injury. PLoS ONE 8:e79328. doi: 10.1371/journal.pone.0079328

Teh, C., Chudakov, D. M., Poon, K. L., Mamedov, I. Z., Sek, J. Y., Shidlovsky, K., et al. (2010). Optogenetic in vivo cell manipulation in KillerRed-expressing zebrafish transgenics. BMC Dev. Biol. 10:110. doi: 10.1186/1471-213X10-110

Thisse, B., and Thisse, C. (2005). Functions and regulations of fibroblast growth factor signaling during embryonic development. Dev. Biol. 287, 390-402. doi: 10.1016/j.ydbio.2005.09.011

Thomas, T., and Dziadek, M. (1993). Capacity to form choroid plexus-like cells in vitro is restricted to specific regions of the mouse neural ectoderm. Development 117, 253-262.

Trinh le, A., Hochgreb, T., Graham, M., Wu, D., Ruf-Zamojski, F., Jayasena, C. S., et al. (2011). A versatile gene trap to visualize and interrogate the function of the vertebrate proteome. Genes Dev. 25, 2306-2320. doi: 10.1101/gad. 174037.111

Trougakos, I. P. (2013). The molecular chaperone apolipoprotein J/clusterin as a sensor of oxidative stress: implications in therapeutic approaches - a minireview. Gerontology 59, 514-523. doi: 10.1159/000351207

Tsuneki, K. (1986). A survey of occurrence of about seventeen circumventricular organs in brains of various vertebrates with special reference to lower groups. J. Hirnforsch. 27, 441-470.

Tyurina, O. V., Guner, B., Popova, E., Feng, J., Schier, A. F., Kohtz, J. D., et al. (2005) Zebrafish Gli3 functions as both an activator and a repressor in Hedgehog signaling. Dev. Biol. 277, 537-556. doi: 10.1016/j.ydbio.2004.10.003

Umans, R. A., and Taylor, M. R. (2012). Zebrafish as a model to study drug transporters at the blood-brain barrier. Clin. Pharmacol. Ther. 92, 567-570. doi: 10.1038/clpt.2012.168

Venkatesh, B., Lee, A. P., Ravi, V., Maurya, A. K., Lian, M. M., Swann, J. B., et al. (2014). Elephant shark genome provides unique insights into gnathostome evolution. Nature 505, 174-179. doi: 10.1038/nature12826

Weindl, A., and Joynt, R. J. (1973). Barrier properties of the subcommissural organ. Arch. Neurol. 29, 16-22. doi: 10.1001/archneur.1973.004902500 34004

Wilting, J., and Christ, B. (1989). An experimental and ultrastructural study on the development of the avian choroid plexus. Cell Tissue Res. 255, 487-494. doi: 10.1007/BF00218783

Winata, C. L., Kondrychyn, I., Kumar, V., Srinivasan, K. G., Orlov, Y., Ravishankar, A., et al. (2013). Genome wide analysis reveals Zic3 interaction with distal regulatory elements of stage specific developmental genes in zebrafish. PLoS Genet. 9:e1003852. doi: 10.1371/journal.pgen.1003852

Wojcik, E., Carrithers, L. M., and Carrithers, M. D. (2011). Characterization of epithelial V-like antigen in human choroid plexus epithelial cells: potential role in CNS immune surveillance. Neurosci. Lett. 495, 115-120. doi: 10.1016/j.neulet.2011.03.051

Yang, J., Dombrowski, S. M., Deshpande, A., Krajcir, N., and Luciano, M. G. (2010). VEGF/VEGFR-2 changes in frontal cortex, choroid plexus, and CSF after chronic obstructive hydrocephalus. J. Neurol. Sci. 296, 39-46. doi: 10.1016/j.jns.2010.06.012 
Zappaterra, M. W., and Lehtinen, M. K. (2012). The cerebrospinal fluid: regulator of neurogenesis, behavior, and beyond. Cell. Mol. Life Sci. 69, 2863-2878. doi: 10.1007/s00018-012-0957-x

Zhang, J., Piontek, J., Wolburg, H., Piehl, C., Liss, M., Otten, C., et al. (2010). Establishment of a neuroepithelial barrier by Claudin5a is essential for zebrafish brain ventricular lumen expansion. Proc. Natl. Acad. Sci. U.S.A. 107, 1425-1430. doi: 10.1073/pnas.0911996107

Zhang, Z. G., Zhang, L., Jiang, Q., and Chopp, M. (2002). Bone marrowderived endothelial progenitor cells participate in cerebral neovascularization after focal cerebral ischemia in the adult mouse. Circ. Res. 90, 284-288. doi: 10.1161/hh0302.104460

Zhou, T., Wang, L., Zhu, K. Y., Dong, M., Xu, P. F., Chen, Y., et al. (2011). Dominant-negative C/ebpalpha and polycomb group protein Bmil extend short-lived hematopoietic stem/progenitor cell life span and induce lethal dyserythropoiesis. Blood 118, 3842-3852. doi: 10.1182/blood-2010-12327908
Conflict of Interest Statement: The authors declare that the research was conducted in the absence of any commercial or financial relationships that could be construed as a potential conflict of interest.

Received: 29 August 2014; accepted: 22 October 2014; published online: 14 November 2014.

Citation: Bill BR and Korzh V (2014) Choroid plexus in developmental and evolutionary perspective. Front. Neurosci. 8:363. doi: 10.3389/fnins.2014.00363

This article was submitted to Neurogenomics, a section of the journal Frontiers in Neuroscience.

Copyright (C) 2014 Bill and Korzh. This is an open-access article distributed under the terms of the Creative Commons Attribution License (CC BY). The use, distribution or reproduction in other forums is permitted, provided the original author(s) or licensor are credited and that the original publication in this journal is cited, in accordance with accepted academic practice. No use, distribution or reproduction is permitted which does not comply with these terms. 


\section{Embryonic blood-cerebrospinal fluid barrier formation and function}

\section{David Bueno*, Maryam Parvas, Ismail Hermelo and Jordi Garcia-Fernàndez}

Department of Genetics, Faculty of Biological Sciences, University of Barcelona, Barcelona, Spain

\section{Edited by:}

Norman Ruthven Saunders,

University of Melbourne, Australia

Reviewed by:

Patrizia Ferretti, University College London, UK

Jaleel A. Miyan, The University of

Manchester, UK

\section{${ }^{*}$ Correspondence:}

David Bueno, Departament de

Genètica, Facultat de Biologia,

Universitat de Barcelona, Av.

Diagonal 643, 08028 Barcelona,

Spain

e-mail:dbueno@ub.edu
During embryonic development and adult life, brain cavities and ventricles are filled with cerebrospinal fluid (CSF). CSF has attracted interest as an active signaling medium that regulates brain development, homeostasis and disease. CSF is a complex protein-rich fluid containing growth factors and signaling molecules that regulate multiple cell functions in the central nervous system (CNS). The composition and substance concentrations of CSF are tightly controlled. In recent years, it has been demonstrated that embryonic CSF (eCSF) has a key function as a fluid pathway for delivering diffusible signals to the developing brain, thus contributing to the proliferation, differentiation and survival of neural progenitor cells, and to the expansion and patterning of the brain. From fetal stages through to adult life, CSF is primarily produced by the choroid plexus. The development and functional activities of the choroid plexus and other blood-brain barrier (BBB) systems in adults and fetuses have been extensively analyzed. However, eCSF production and control of its homeostasis in embryos, from the closure of the anterior neuropore when the brain cavities become physiologically sealed, to the formation of the functional fetal choroid plexus, has not been studied in as much depth and remains open to debate. This review brings together the existing literature, some of which is based on experiments conducted by our research group, concerning the formation and function of a temporary embryonic blood-CSF barrier in the context of the crucial roles played by the molecules in eCSF.

Keywords: embryonic cerebrospinal fluid, blood-eCSF barrier, primary neurogenesis, neural progenitor cells, brain development, cephalic vesicles

\section{INTRODUCTION}

Many civilizations have developed along riverbanks and seashores, in order to use the fluid medium to promote cohesion and transport, and to favor the survival of the people living at its edge. Surprisingly, the brain is also organized, from its embryonic beginnings and throughout adult life, around an extraordinarily dynamic and complex fluid, called cerebrospinal fluid (CSF), which the cells lining the brain and spinal cord bathe in. CSF is a subject that attracts growing interest in brain development research. In the last two decades, this has led to an understanding of normal adult brain function and disease, including migration of neuroblasts created in the subventricular zone of the adult brain from the lateral ventricles to the olfactory bulb (Sawamoto et al., 2006). CSF composition during embryogenesis and the fetal period is highly complex, as demonstrated by several proteomic analyses (Dziegielewska et al., 1980a, 1981; Fielitz et al., 1984; Gato et al., 2004; Parada et al., 2005a, 2006; Zappaterra et al., 2007). In addition, CSF composition and its biological

\footnotetext{
Abbreviations: BBB, blood-brain barrier; CG, Carnegie Stage (for human embryo development); CNS, central nervous system; CSF, cerebrospinal fluid; E, embryonic developmental day; eCSF, embryonic cerebrospinal fluid; EGF, epidermal growth factor ESI-MS/MS: electrospray ionization coupled with tandem mass spectrometry; fCSF, fetal cerebrospinal fluid; FGF2, fibroblast growth factor no. 2; HRP, horse radish peroxidase; IsO, mesencephalic-rhombencephalic isthmus organizing center; RBP, retinol binding protein; SDS-PAGE, sodium dodecyl sulfate polyacrylamide gel electrophoresis.
}

properties change throughout development in a tightly regulated manner in parallel with its function, as demonstrated for some of the molecules contained within the embryonic CSF (eCSF), e.g., retinol binding protein (RBP), and fibroblast growth factor no. 2 (FGF2), with regard to the initiation of primary brain neurogenesis (Parvas et al., 2008a).

The development of the brain involves three main distinct phases: formation of the tube from the neural plate; polarization of the tube into a posterior spinal cord, and an anterior expanded brain, which is initially a hollow eCSF-filled vesicle surrounded by a pseudo-monostratified neuroepithelium; and histiogenesis of the neuroepithelium (Figure 1; for a general description of central nervous system (CNS) development, see Smith and Schoenwolf, 1997; Copp et al., 2003; Copp, 2005). During most of the last century, developmental neuroscience focused primarily on neuroepithelial events at the molecular, cellular, and tissue level. For example, it has been demonstrated that diffusible molecules such as growth factors and morphogens secreted locally by organizing centers regulate these processes by controlling neighboring cells in an autocrine/paracrine way (Bally-Cuif and Boncinelli, 1997), and that they regulate the expression of a number of transcription factors through which complex molecular, and genetic networks are established.

Only in the last two decades has the eCSF in the cavities of the brain primordium started to attract attention and be considered 
an essential part of the developing brain which exerts a direct influence on basic neuroepithelial cell behavior, i.e., promoting cellular survival, replication, and neurogenic differentiation, and also contributing to the regulation of neuroepithelial gene expression, thus playing a key role in brain development (Gato et al., 2005; Martin et al., 2006, 2009; Parada et al., 2008a; Alonso et al., 2011; Feliciano et al., 2014). In fetuses and adults, these cavities become the brain's ventricular system, since eCSF is the precursor

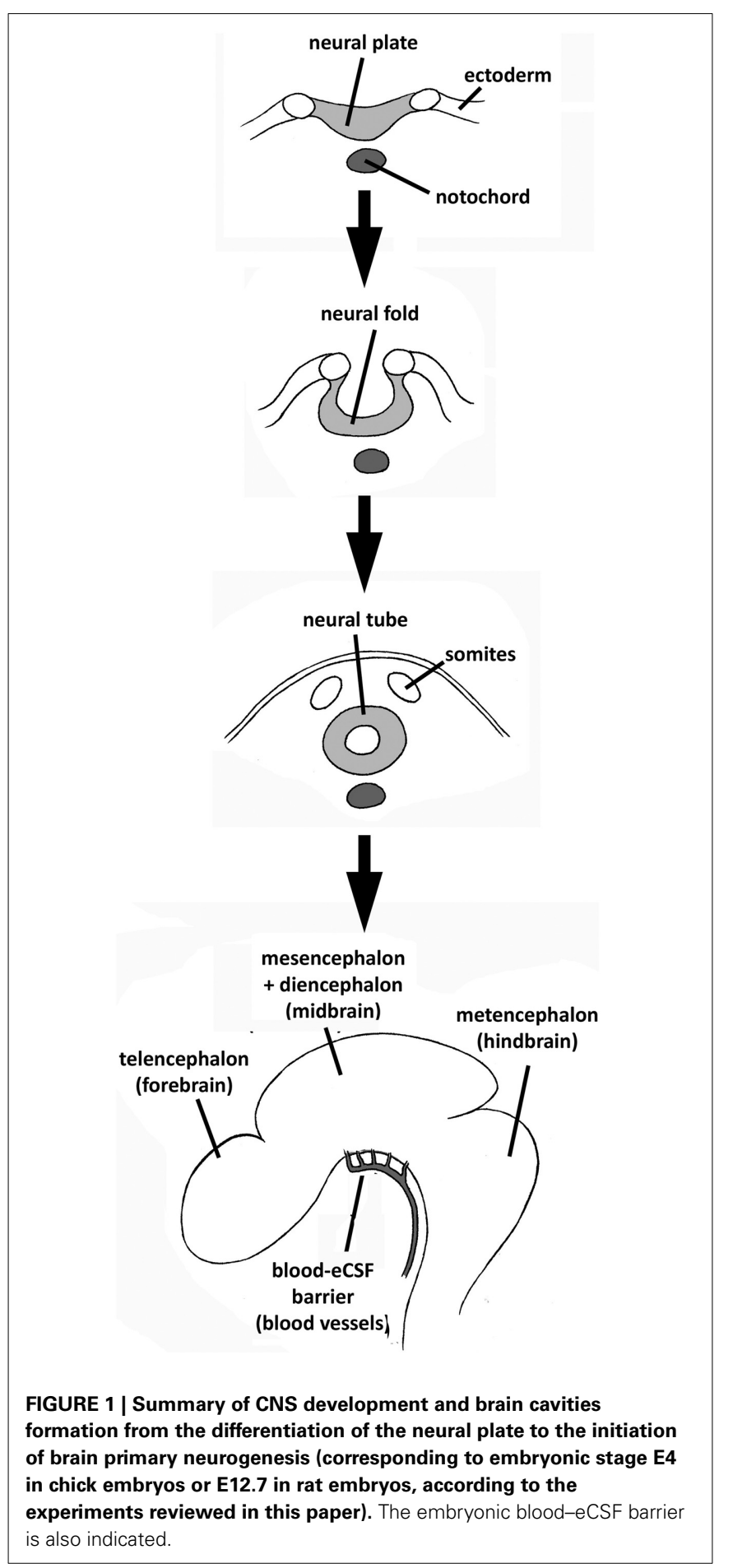

of fetal and adult CSF (reviewed by Miyan et al., 2003). It has also been demonstrated that fetal CSF (fCSF) contributes to cortical development (Miyan et al., 2006).

CSF composition and homeostasis in fetuses and the adult brain are tightly regulated by the choroid plexus, whose epithelial cells establish a blood-CSF barrier (Figure 2; Møllgård et al., 1979; Tauc et al., 1984). The choroid plexus is a vascular structure in the brain ventricles that manufactures CSF by promoting the transport of certain molecules from blood plasma and producing others that are delivered directly to the ventricles. Acting in parallel, CNS homeostasis in adult vertebrates is also controlled by the blood-brain barrier (BBB) vessels in

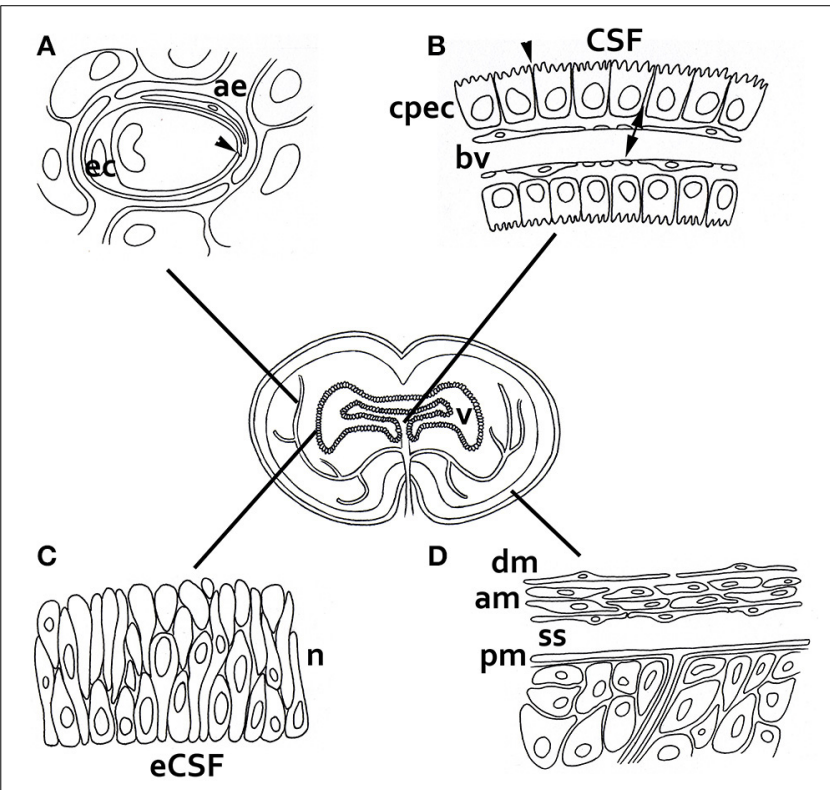

FIGURE 2 | Barriers of the developing and adult brain. (A) The blood-brain barrier between the lumen of cerebral blood vessels and the brain parenchyma. The endothelial cells have luminal tight junctions (arrowheads) that form a physical barrier, preventing the movement of molecules from the blood plasma, except those that are allowed to enter the brain via active and specific transport. In embryos, some blood vessels and the neuroectodermal cells adjacent to them also perform this function. (B) The blood-CSF barrier between choroid plexus blood vessels and the adult and fetal CSF. These blood vessels are fenestrated and form a non-restrictive barrier (arrows), but the choroid plexus epithelial cells are joined by tight junctions to stop the movement of molecules (arrowheads). (C) The inner CSF-brain barrier between the eCSF and the brain parenchyma, which is present only during early development. The neuroependymal cells lining the brain cavities do not allow the exchange of large molecules such as proteins between the eCSF and brain, but they do allow exchange of smaller molecules such as sucrose. This implies that the influence of eCSF on basic neuroepithelial cell behavior-e.g., promoting cellular survival, replication and neurogenic differentiation-has to be accounted for through specific receptors located at the apical pole of neural progenitor cells, which are in contact with the lumen of the cavities. There is no crossing restriction in the adult brain. (D) The outer CSF-brain barrier between the subarachnoid space and overlaying structures. The blood vessels are fenestrated, but the outer cells of the arachnoid membrane are connected by tight junctions. Abbreviations: ae, astrocitic endfoot; am, arachnoid membrane; bv, blood vessel; cpec, choroid plexus endothelial cell; dm, dura mater; ec, endothelial cell; n, neuroepithelium; pm, pia mater; ss, subarachnoid space; $v$, ventricle. 
the brain; this significantly impedes entry from the blood to the brain of virtually all molecules, except those that are small and lipophilic. However, BBB vessels also allow sets of small and large hydrophilic molecules, e.g., gene products, to enter the brain via active transport (reviewed by Rubin and Staddon, 1999; see Figure 2 for a more extensive summary of brain barriers).

In parallel with eCSF studies, control of eCSF production and homeostasis in vertebrate embryos from the closure of the anterior neuropore to the formation of the choroid plexus during fetal stages have not been analyzed until recently. However, this developmental stage is particularly interesting for brain formation, since it is mainly characterized by rapid brain anlagen growth and initiation of primary neurogenesis in the neural progenitor cells lining the cavities. This review brings together the existing literature on the formation and function of embryonic blood-CSF barriers, from the closure of the anterior neuropore to the formation of choroid plexuses in vertebrates, using avians (chicks), and mammals (mainly mice and rats) as model systems. We highlight the crucial role played by eCSF in neural development. As stated by Gato (2013) in a short but revealing commentary, "eCSF-and by extension the barriers and mechanisms controlling its formation, composition and homeostasis_constitute a rising subject in brain development."

\section{PHYLOGENETIC ORIGIN OF THE CSF SYSTEM}

It is thought that the CSF system evolved in the deuterostome lineage from the ancestor of echinoderms and chordates to maintain the chemical environment necessary for the functioning of the cells of the CNS, including the neuroendocrine pathways (Brocklehurst, 1979). For example, it is known that in starfish, the neurons of the radial nerves and "brain" are always in contact with seawater; this allows them to detect the composition of the seawater, as well as other physical parameters such as water temperature, and modulate the activity of the nervous system accordingly. However, its open nature means that this system does not respond to neuroendocrine or other signaling molecule pathways (Figure 3).

During the larval stages of morphologically and evolutionarily more complex lancelets, such as the chordate amphioxus Branchiostoma lanceolatum, seawater enters the ventricular lumen of the "brain" through the anterior neuropore, which remains open until adulthood. Seawater represents the first "internal" fluid environment of lancelet brain tissue and depends directly on the environment. However, when lancelets reach adulthood, the anterior neuropore closes and the ventricular lumen of the "brain" becomes a closed system (Figure 3). Thus, the composition of this fluid no longer depends directly on the environment, but on the activity and metabolism of the organism. Finally, the composition of eCSF in craniata, such as lampreys, and vertebrates depends exclusively on the surrounding tissue and the rest of the embryo from early embryonic stages, since the anterior neuropore closes very early in neural tube development, before brain primary neurogenesis starts. This suggests that this condition was first developed by the ancestor of these two phylogenetic groups (Figure 3; reviewed by Vígh et al., 2004).

\section{eCSF PROTEIN CONTENT}

In vertebrates, brain development depends on regional character specification, which in turn depends on a progressive series of precisely coordinated morphogenetic movements in combination with cell proliferation and differentiation. These processes are controlled by the sequential action of patterning molecules, which give neural progenitor cells specific characteristics, including transcription factors and diffusible molecules such as growth factors and morphogens (Martinez, 2001; Stern, 2001, 2002). During early stages of brain development, when the architecture of the brain anlagen is formed by a pseudo-monostratified neuroepithelium that develops around the cephalic cavities with its lining surface in close contact with the eCSF, these diffusible molecules may come from both the surrounding tissues, such as the mesencephalic-rhombencephalic isthmus ( $\mathrm{IsO}$ ) organizing center (Nakamura et al., 2005), and eCSF, which may be used as a fluid pathway for their delivery.

Classic studies performed on chick embryos at developmental stages E2.5 to E6.5 ( $\mathrm{E}=$ embryonic developmental day) showed that eCSF has a higher concentration of total proteins than adult CSF (Birge et al., 1974; Gato et al., 2004). A similar situation has been observed in sheep at E35 (youngest fetus) as compared with E60 (sheep gestation, 145 days), and in rat from E12 to E22 (birth), and neonatal. Some phylogenetic differences in CSF maturation have been identified, however, when comparing these mammalian species, regarding, for example, the developmental stages at which the reduction in total CSF protein occurs (Dziegielewska et al., 1980a,b, 1981; Checiu et al., 1984; Fielitz et al., 1984).

The first published studies of the specific protein content of eCSF were based on crossed immunoelectrophoresis to identify individual proteins, and radial immunodiffusion to quantitate them. It was suggested that the protein fractions identified in sheep eCSF were albumin, fetuin, alpha-fetoprotein, transferrin, lipoproteins, alpha1-antitypsin, and prealbumin (Dziegielewska et al., 1980a, 1981). Rat eCSF exhibits a similar electrophoretic pattern, with the possible presence of IgGs (Dziegielewska et al., 1981). As regards chick eCSF, Gato et al. (2004) identified 21 protein fractions by SDS-PAGE protein separation and molecular mass inference, which coincide with previously reported proteins such as transferrin, alpha-fetoprotein, immunoglobulins, and transthyretin.

Proteomic analyses of avian and mammalian eCSF that confirm and significantly expand on most of the abovementioned findings have also been published. The first reports to use conventional proteomic techniques (2D-electrophoresis, in-gel digestion, and ESI-MS/MS mass spectrometry analysis) on chick and rat eCSF at E4 and E12.5, respectively, which correspond to the developmental stages in which brain development is characterized by maximum neuroepithelial progenitor cell proliferation and the beginning of neural differentiation, showed the presence of dozens of different specific proteins within this fluid, most of which may be involved in brain development (Parada et al., 2005a, 2006). These proteins were classified into several different groups according to their functional characteristics, as previously reported in systems other than eCSF (Table 1). The most remarkable dissimilarity between chick and rat eCSF proteomes is the 


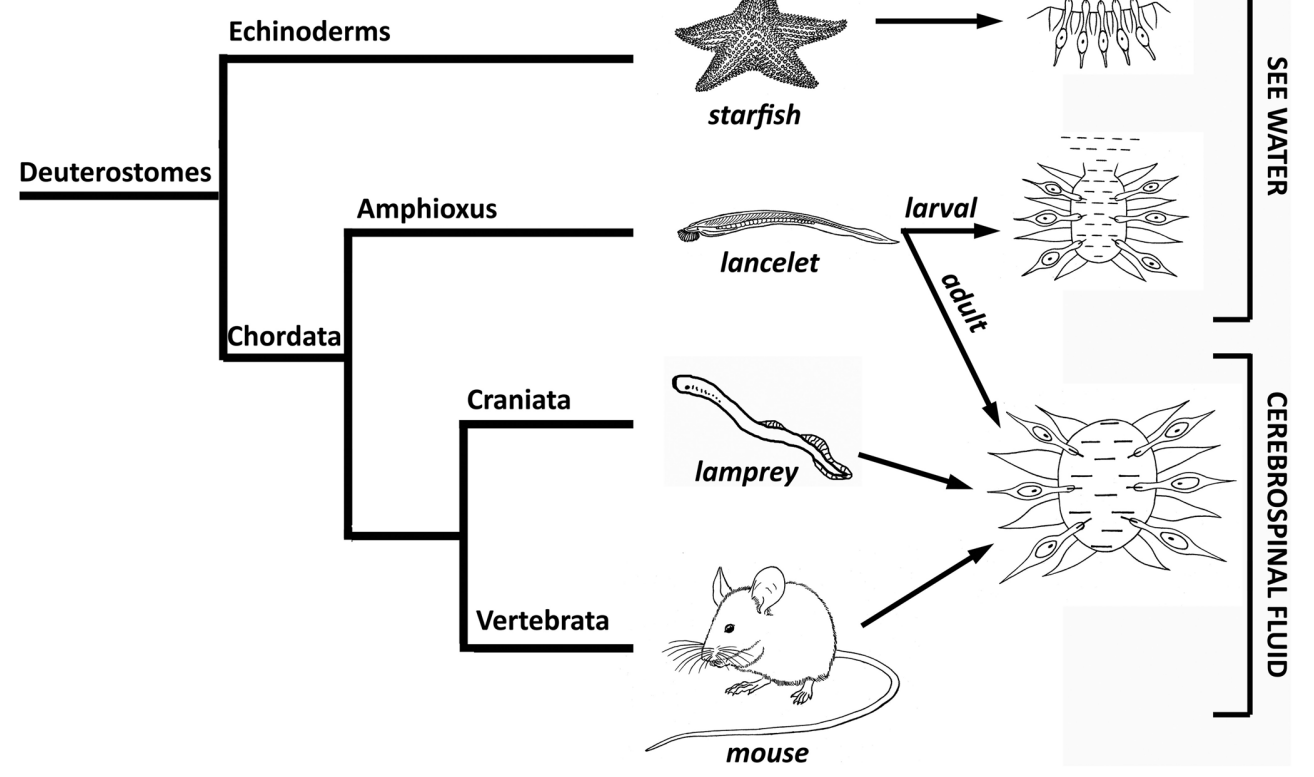

FIGURE 3 | Scheme of the evolution of the phylogenetic increase in the control that the chemical environment has over the cerebrospinal fluid.

presence of enzymes and enzyme regulators in rat eCSF, as well as an increased number of members of the apolipoprotein family, from two different apolipoproteins identified in chicks, AI and AIV, to at least five in rats, including AI and AIV, as well as $B, E$, and $M$. It has been suggested that this relates to the higher complexity of the CNS in mammals, although apolipoprotein B was later identified in chick eCSF by Western-blot analysis using specific antibodies (Parada et al., 2008a). These dissimilarities may also be due to differences in choroid plexus formation. E4.5 chick embryos correspond to a developmental stage 2.5 days before the first appearance of the choroid plexus; while in E12.5 rat embryos, transthyretin-positive choroid plexus primordium starts to be detected. However, rat choroid plexus primordium at this developmental stage is not fully functional and, as discussed below (see Sections Protein Traffic Occurs in Very Specific Blood Vessels and Includes Both Endothelial and Neuroepithelial Cells, and Traffic of Water, ions, And Glucose Occurs at the Same Transport Site as Proteins), these rat embryos still maintain a specific and temporary embryonic blood-eCSF barrier.

Zappaterra et al. (2007) also reported an extensive and thorough proteome analysis of rat eCSF from three different stages: E12.5, E14.5 from both the lateral and fourth ventricles, and E17.5 during cortical development. They identified 423, 318, 249, and 382 proteins, respectively, which were also classified into several groups according to their function (Table 1). The human eCSF proteome has been analyzed at Carnegie Stage (CG) 20 (Zappaterra et al., 2007), revealing the presence of 188 proteins; 130 of which are shared with rats. The categorization of these proteins based on their molecular function and the biological processes they are involved in are almost identical, which would suggest that they represent essential eCSF functions.
More recently, proteomic analysis performed on both eCSF from chick and rat embryos at E4 and E13 respectively, but using a more sensitive technique (a high-performance liquid chromatography ion-trap column coupled to an ESI MS/MS mass spectrometer), showed the presence of 121 (chick), and 100 (rat) different proteins within the fluid, although all of them may be classified in the same general groups (Bueno et al., unpublished results). All of these studies, whose overall results are relatively similar, have proved to be complementary and reinforce the putative capacity of eCSF to influence the behavior of neuroepithelial cells through the molecules it contains, as has been demonstrated in several studies (Gato et al., 2005; Martin et al., 2006, 2009; Parada et al., 2008a,b).

However, one of the limitations of this proteomic analysis is the almost complete lack of growth factors and cytokines within eCSF proteomes, since these molecules, which are crucial for controlling developmental processes, exert their actions at very low concentrations. They may therefore be masked by the more abundant proteins or may be below the detection threshold of the techniques used. Nevertheless, Western-blot analysis of both avian and mammalian eCSF using specific antibodies revealed the presence of some growth factors and cytokines within this fluid. These include fibroblast growth factor 2 (FGF2) (Martin et al., 2006), epidermal growth factor (EGF) (Birecree et al., 1991), and leukemia inhibitory factor (Hatta et al., 2006), which are known to be involved in the regulation of a number of developmental processes.

Although interpreting these different results is complex because of the different classifications used (see Table 1), and the fact that some gene products may correspond to different functional groups in different studies, it is evident that eCSF acts as 
Table 1 | Summary of the classifications of proteins identified in chick, rat and human eCSF proteomes.

\begin{tabular}{ll}
\hline Chick eCSF & Rat eCSF \\
\hline Parada et al., 2006 & Parada et al., 2005a \\
Bueno et al., unpublished results & 1. Extracellular matrix \\
1. Extracellular matrix & 2. Osmotic pressure and ion carriers \\
2. Osmotic pressure and ion & 3. Regulators of lipid metabolism \\
carriers & 4. Retinol and corticosteroid carriers \\
3. Cell death and quiescence & 5. Enzymes and enzyme regulators \\
4. Apolipoproteins & 6. Antioxidant and antimicrobial \\
5. Retinol and vitamin D carriers & 7. Unknown function during \\
6. Antioxidant and antimicrobial & embryonic development
\end{tabular}

7. Intracellular

8. Unknown

Zappaterra et al., 2007
Bueno et al., unpublished results

According to subcellular location

1. Secreted

2. Secreted, extracellular matrix

3. Cell membrane

4. Cytoplasm

5. Nucleus

6. Intracellular

7. Golgi

8. Lysosome

9. ER

According to molecular function

1. Cell adhesion

2. Chaperone

3. Cytoskeletal

4. Defense/immunity

5. Extracellular matrix

6. Hydrolase

7. Kinase

8. Ligase

9. Membrane traffic

10. Miscellanea

11. Unclassified

12. Nucleic acid binding

13. Oxidoreductase

14. Phosphatase

15. Protease

16. Receptor

17. Calcium binding

18. Regulatory molecule

19. Signaling molecule

20. Synthase and synthetase

21. Transcription factor

22. Transfer

23. Transferase

24. Transporter

25. Isomerase

According to biological process

1. Neuronal activities

2. Signal transduction

3. Developmental processes

4. Cell proliferation and

differentiation

5. Coenzyme and prosthetic group metabolism

\section{Human eCSF}

Zappaterra et al., 2007

According to subcellular location

1. Secreted

2. Secreted, extracellular matrix

3. Cell membrane

4. Cytoplasm

5. Nucleus

6. Intracellular

7. Golgi

8. Lysosome

According to molecular function

1. Cell adhesion

2. Chaperone

3. Cytoskeletal

4. Defense/immunity

5. Extracellular matrix

6. Hydrolase

7. Kinase

8. Ligase

9. Membrane traffic

10. Miscellanea

11. Unclassified

12. Nucleic acid binding

13. Oxidoreductase

14. Phosphatase

15. Protease

16. Receptor

17. Calcium binding

18. Regulatory molecule

19. Signaling molecule

20. Synthase and synthetase

21. Transcription factor

22. Transfer

23. Transferase

24. Transporter

25. Cell junction protein

According to biological process

1. Neuronal activities

2. Signal transduction

3. Developmental processes

4. Cell proliferation and differentiation

5. Coenzyme and prosthetic group metabolism

6. Cell structure and motility

7. Immunity and defense

8. Apoptosis

9. Oncogenesis

10. Muscle contraction

11. Transport

12. Blood circulation and gas exchange

13. Carbohydrate metabolism

14. Nucleoside, nucleotide and nucleic acid metabolism

15. Homeostasis

16. Protein metabolism and modification

17. Cell cycle 
Table 1 | Continued

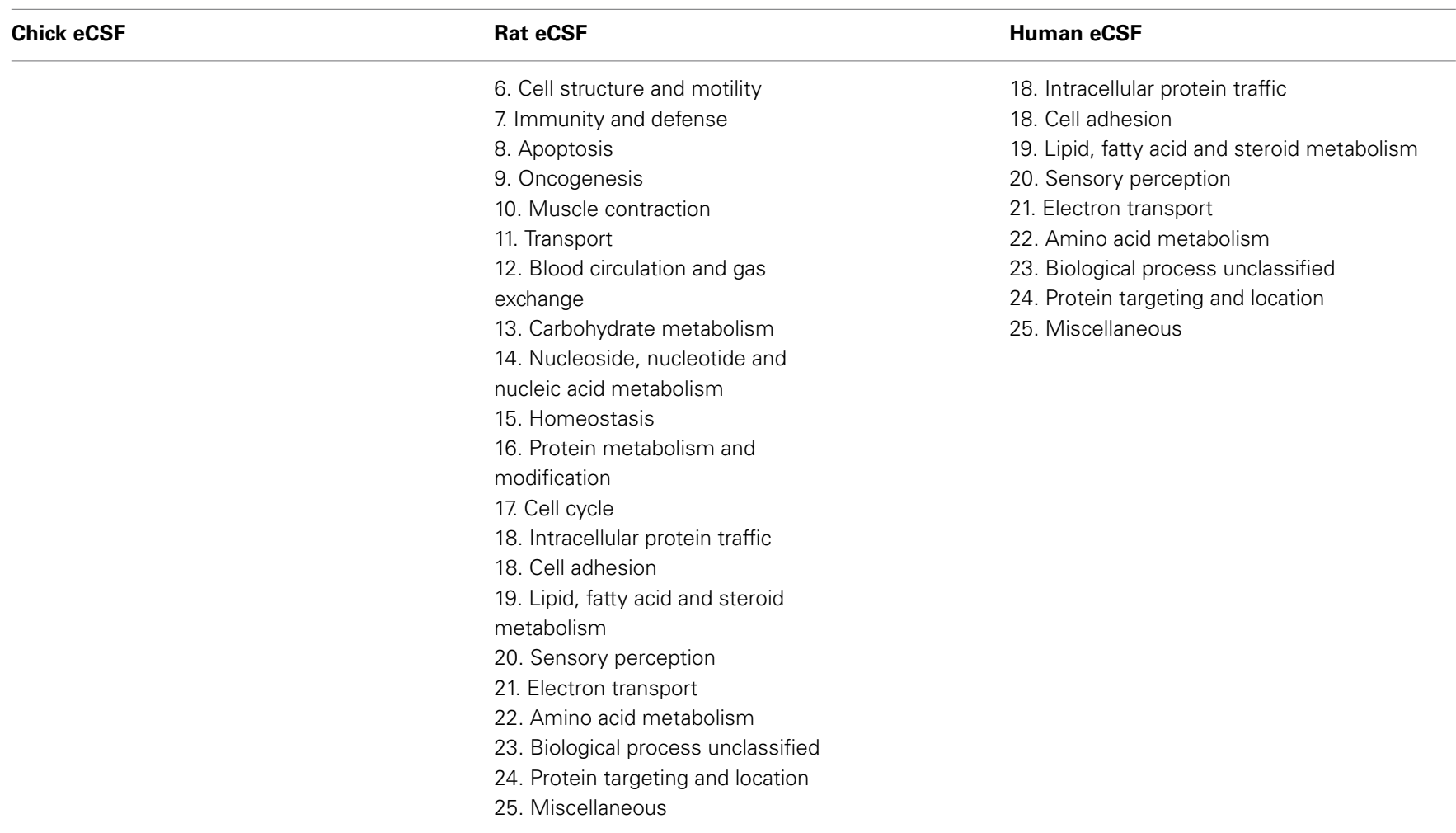

References are shown in the table.

a fluid pathway to transfer proteins to and from the neuroepithelial cells. It thus influences neuroepithelial cell behavior and, hence, brain development (Gato et al., 2005; Parada et al., 2005a, 2008a,b; Martin et al., 2006, 2009; Alonso et al., 2011; Castells et al., 2012; see Gato and Desmond, 2009 for a review).

\section{eCSF FUNCTION}

Through the specific molecules it contains and certain of its mechanical properties, eCSF plays several crucial roles during the early stages of brain development: just after the closure of the anterior neuropore when the brain ventricles become physiologically sealed cavities, during initial brain anlagen growth, and during the initiation of the primary neurogenesis of the neural progenitor cells. Using several experimental approaches in chick and mouse embryos, it has been reported that the progressive increase in eCSF volume exerts positive pressure against the neuroepithelial walls and generates an expansive force, thus contributing to brain expansion. An experimentally inflicted decrease in eCSF pressure leads to severe dysmorphogenesis and brain collapse (Desmond and Jacobson, 1977; Desmond and Levitan, 2002). The rapid expansion, which accounts for $70 \%$ of brain enlargement in the early stages, occurs in parallel with occlusion of the spinal neurocoele (Desmond and Jacobson, 1977). This renders the cephalic cavities a physiologically sealed system, which would indicate the existence of mechanisms that control the influx of water and ions into the brain cavities. Moreover, as proteoglycans are the major components of the extracellular matrix in embryo brain cavities (Alonso et al., 1998, 1999), it has been suggested that the special osmotic properties of chondroitin sulfate proteoglycan and other proteoglycans in eCSF may cause water retention in the cavities. This would generate and regulate the inner cephalic hydrostatic pressure, although other proteins, e.g., major eCSF proteins, may also contribute to this function.

In addition to the mechanical properties of eCSF, it has also been shown to contribute to the regulation of some basic cellular functions, e.g., the survival, proliferation, and differentiation of neural progenitor cells. Through the use of in vitro cultures of mesencephalic neuroectodermal explants from both chick and rat embryos at E4 and E12.5, respectively, the diffusible molecules in eCSF have been shown to actively contribute to the regulation of the survival, proliferation, and neurogenesis of neuroepithelial progenitor cells, directly from this embryonic fluid (Gato et al., 2005; Martin et al., 2009). Mesencephalic explants cultured with only a chemically defined medium show a reduction in the number of proliferating cells, an increased rate of apoptotic cells, and a severe decrease in the number of cells engaged in the process of neural differentiation (i.e., primary neurogenesis) compared to control embryos. However, when the explants are cultured with a chemically defined medium supplemented with eCSF, the cellular parameters remain close to those of the control embryos.

Most of the proteins identified in eCSF in the abovementioned proteomic analysis have known physiological functions during embryonic development that are consistent with the overall roles reported for eCSF during CNS development. Functional in vivo and in vitro analysis of the particular role of some of these gene products at the beginning of primary neurogenesis, before 
the formation of functional choroid plexuses, has revealed their specific roles in the overall function of eCSF in neuroepithelial progenitor cell behavior. For example, it has been reported that the deglycosylation of eCSF proteoglycans with beta-d-xyloside alters brain enlargement in chick embryos (Alonso et al., 1998), and that immunoblocking of the FGF2 in eCSF severely disrupts neuroepithelial stem cell proliferation and differentiation (Martin et al., 2006). Similarly, it has been reported that the LDL lipid fraction, transported by apolipoprotein B in eCSF, is also involved in regulating neuroepithelial progenitor cell proliferation and differentiation (Parada et al., 2008a). Other reports have shown that retinol-binding protein $(\mathrm{RBP})$ is responsible for transporting and transferring all-trans-retinol from the embryonic plasma to the eCSF, from where it reaches the neuroepithelium and is transformed into retinoic acid, the actual morphogen, by retinoic acid-synthesizing enzymes expressed in the neuroepithelium (Parada et al., 2008b; Alonso et al., 2011). Also that large glycoprotein SCO-spondin synthesized by the by the diencephalic roof plate contributes to the control of neuroepithelial cell proliferation via eCSF (Vera et al., 2013). As well as that eCSF nanovesicles carry evolutionarily conserved molecules that promote neural stem cell amplification (Feliciano et al., 2014); among other findings.

In addition to the effects of eCSF on neural progenitor cell behavior through some of the specific molecules it contains, its involvement in regulating the expression of genes known to be involved in brain patterning has also been established (Parada et al., 2005b). When dorsal mesencephalic neuroepithelial explants lacking IsO (a known organizing center for mesencephalon and brain development) or dorsal mesencephalic neuroepithelial explants including IsO are cultured in a chemically defined medium but in the absence of eCSF, the typical expression of dorsal mesencephalic, and IsO genes, i.e., $f g f 8$ and $o t x 2$, is disrupted. Conversely, when dorsal mesencephalic explants including IsO are cultured in an eCSF-supplemented medium, they do exhibit this expression. Interestingly, however, when dorsal mesencephalic explants lacking IsO are cultured with an eCSF-supplemented medium, they also show ectopic expression domains of Shh in the mesencephalic neuroectoderm; Shh is a gene that is typically expressed in the ventral but not dorsal neuroectoderm. Only the concurrence of eCSF within the culture medium and IsO in the dorsal mesencephalic explant makes this tissue mimic its typical pattern of gene expression, which would suggest that $\mathrm{IsO}$ and some molecules in eCSF exert a synergistic action.

\section{MECHANISMS CONTROLLING ECSF FORMATION AND HOMEOSTASIS}

The crucial roles played by eCSF during initial brain development together with the complexity of its composition (it includes proteins that may account for the overall function of this fluid in neuroepithelial development) raise some interesting questions about the origin of the gene products and other molecules within it. Further questions concern how the homeostasis of this fluid is controlled at the beginning of primary neurogenesis, just after the closure of the anterior neuropore when the cephalic cavities become physiologically sealed, since the development of a functional choroid plexus is known to occur a bit later in development.

\section{THE NEED FOR A BLOOD-eCSF BARRIER FUNCTION: A HISTORICAL PERSPECTIVE}

Developing choroid plexuses are first detected at E7 in chicks, at E12.5-E13 in rats and mice, and during the seventh week of gestation in humans (Bellairs and Osmond, 2005; Emerich et al., 2005). Despite the large amount of accurate information available on brain barriers encompassing fetal stages through to adulthood (Ferguson and Woodbury, 1969; Parada et al., 2007; Johanson et al., 2008, 2011; Gato and Desmond, 2009; Liddelow, 2011; Ek et al., 2012; Zappaterra and Lehtinen, 2012), the existence of an embryonic blood-eCSF barrier that controls eCSF composition and homeostasis during early embryonic development remains in doubt. This is partially due to a common belief that barriers in the developing brain are immature, although this implies neither the absence of mechanisms that control eCSF composition and homeostasis, nor that the embryonic brain does not require an environment of great chemical stability.

A further complicating factor is the way in which bloodCSF barrier permeability has traditionally been interpreted. Conventionally, the fact that the ratio of the concentration of particular molecules in CSF with respect to that in blood plasma is much higher in the developing brain than in the adult brain (Ferguson and Woodbury, 1969; Dziegielewska et al., 1979; Habgood et al., 1993; Ek et al., 2001) has been interpreted as evidence of greater barrier permeability (Spector and Johanson, 1989; Kniesel et al., 1996; Engelhardt, 2003; Lee et al., 2003). However, the morphological basis of these barriers formed by means of tight junctions is present from the earliest stages of development between endothelial blood vessel cells in BBB areas, as well as in the epithelial cells of the choroid plexuses in the blood-CSF barrier (Møllgård et al., 1979; Saunders and Møllgård, 1984; Tauc et al., 1984). To reconcile these two sets of evidence, it has been suggested that different transcellular mechanisms operate for protein and small molecule transfer across the embryonic blood-CSF interface (Johansson et al., 2006).

In the past 8 years, a number of studies have provided increasing evidence of the existence of a transient blood-eCSF barrier mechanism in both avian and mammal embryos between the closure of the anterior neuropore and the start of choroid plexus functionality (see below). It has also been demonstrated that at this developmental stage in avians, major eCSF protein fractions are not synthesized by the neuroectodermal cells, but are synthesized in other embryonic tissues or derived from the egg, and subsequently transported from the blood plasma to the cephalic cavities, most probably in a selective manner (Parvas et al., 2008 b). These data suggest the need for a physiological bloodeCSF barrier to control the initial composition and homeostasis of the eCSF before the formation of a functional fetal choroid plexus.

\section{THE CEPHALIC NEUROECTODERM BECOMES IMPERMEABLE JUST AFTER CLOSURE OF THE ANTERIOR NEUROPORE}

The impermeability of the cephalic neuroectoderm is one of the key factors in the formation of a physiologically sealed system 
of brain cavities. Early experiments to test the integrity of the embryonic neuroectoderm relied on injected dyes such as trypan, or Evans blue, or enzymes such as horseradish peroxidase (HRP). They produced conflicting results, probably because of the fragility of embryos (Habgood et al., 1992). For example, Wakai and Hirokawa (1978) showed that blood-CSF interface permeability to HRP may follow a free diffusion pattern beginning to decrease at E12-E14 in chick embryos; although their results are probably an artifact due to the very high concentrations of HRP used in the work. However, much more recent experiments in which small concentrations of FGF2 conjugated to FITC or alternatively other proteins of a similar size not normally present in the eCSF are injected into the outflow of the embryo heart, show the existence of selective transport from the blood plasma to the eCSF from E4 onwards (Martin et al., 2006; Parvas et al., 2008a).

In a similar way, the microinjection of a small-sized tracer, biotin dextran amine of $3 \mathrm{kDa}$ (BDA3000), into the outflow of the heart or the cephalic cavity of embryos at E3 and E4, showed that from E4 onwards its transport is restricted to a small subset of endothelial cells and cells adjacent to neuroepithelial cells, located in the ventral mesencephalon and the most anterior part of the ventral prosencephalon, lateral to the floor plate, via transcellular routes (Parvas et al., 2008a) (Figure 4). The existence of these specialist transport cells is a key factor in blood-eCSF barrier activity and in some respects may parallel the function of the fetal choroid plexus (Møllgård and Saunders, 1977; Balslev et al., 1997; Ek et al., 2001; Johansson et al., 2006).

\section{PROTEIN TRAFFIC IS SPECIFIC AND HOMEOSTASIS TIGHTLY REGULATED}

Initially, eCSF is derived from trapped amniotic fluid; a situation formally equivalent to that of lancelets with respect to seawater during the shift from larval to adult life (see Section Phylogenetic Origin of the CSF System Above; Vígh et al., 2004). After the closure of the anterior neuropore, however, the brain cavities

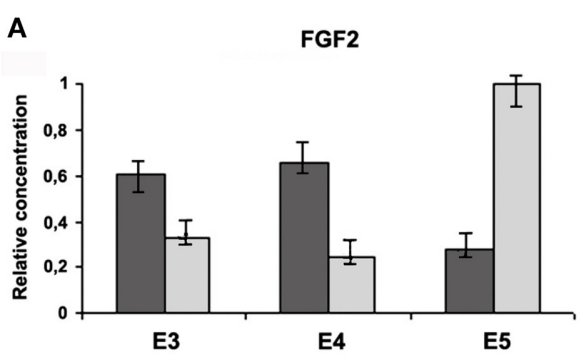

B

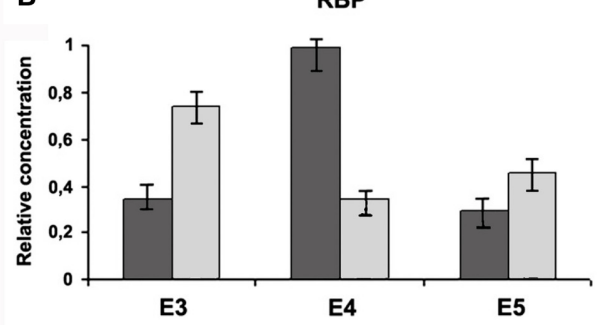

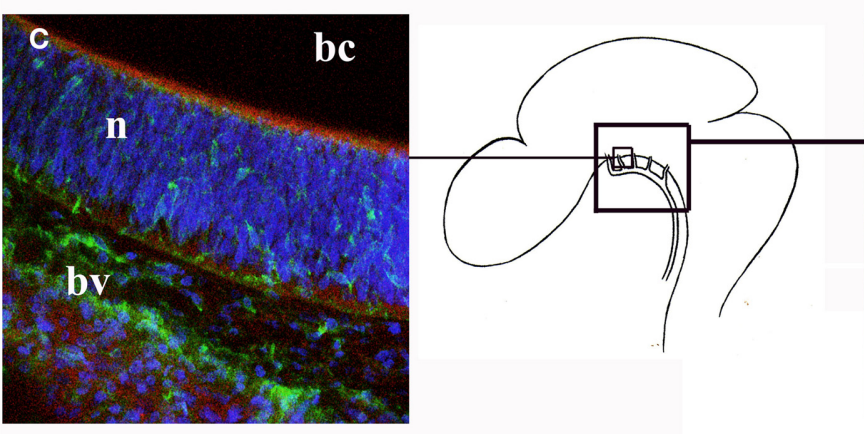
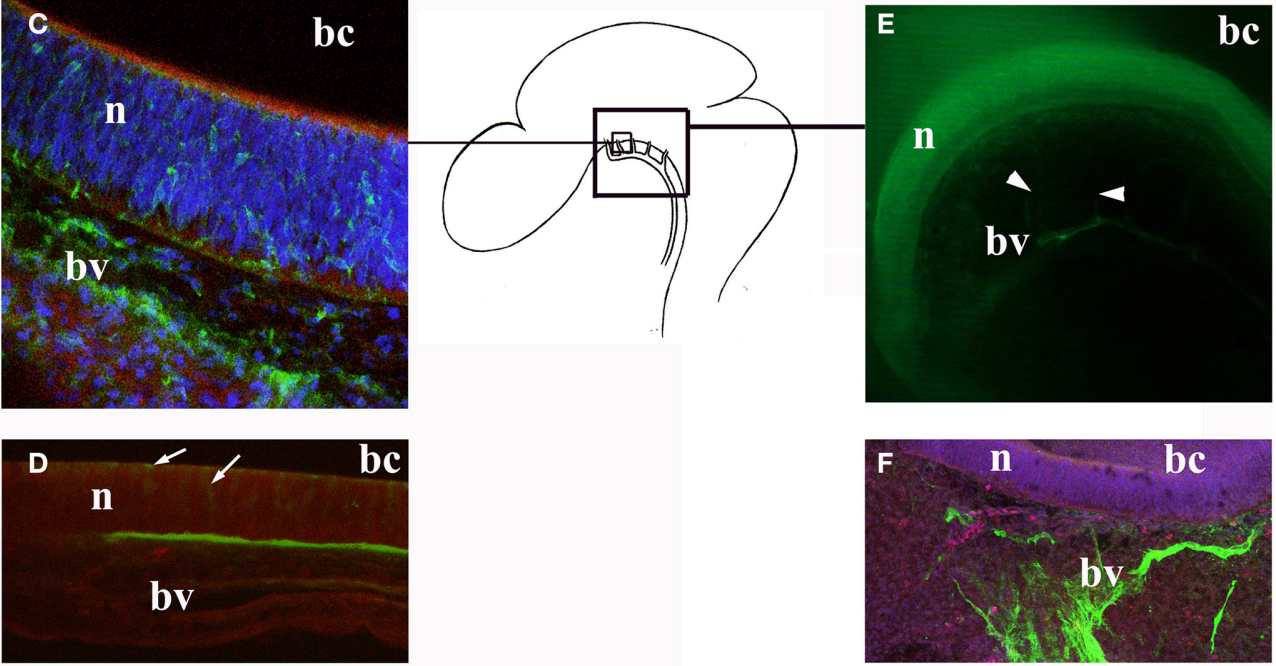

FIGURE 4 | Transport of molecules across the blood-eCSF barrier in chick embryos. (A-B) Relative concentrations of some endogenous proteins in the embryonic serum (light gray), and in the eCSF (dark gray), at E3, E4, and E5 developmental stages (Parvas et al., 2008a). (C) Vibratome section showing transport of BDA3000 injected into the cephalic cavities at the ventral mesencephalon, lateral to the floor plate, at E4 (Parvas et al., 2008a). Note the presence of fluorescent dextran in both blood vessels (bv) and across the neuroectoderm (n). (D) Vibratome section showing transport of BSA microinjected into the cephalic cavities from the eCSF to the embryonic serum, at E4 (Parvas et al., 2008a). Note the accumulation of BSA in the mesenchyme, at the interface between the neuroectoderm ( $n$ ) and the blood vessels (bv). Also note the presence of BSA within neuroectodermal cells (arrows). (E) AQP1 immunostaining of half head, at E5. Note the immunostained blood vessels and capillary sprouts (arrowheads) (Parvas and Bueno, 2010b). (F) Magnification of a blood vessel immunostained with an anti-AQP1 antibody, at E6. Abbreviations: bc, brain cavity; bv, brain vesicles; $n$, neuroectoderm. 
become a physiologically sealed system, hence its composition becomes tightly regulated. Interestingly, most of the molecules identified in chick eCSF are not produced by the neuroectoderm itself. They are stored in the yolk or white of the egg and taken up by the chorioallantoic membrane, or produced by other embryonic structures. This would suggest that they are transported from the production or storage site to the eCSF, most probably via the blood plasma (Martin et al., 2006; Parvas et al., 2008a,b).

In this way, the quantification of the relative concentration of endogenous chick proteins normally present in both blood plasma and the eCSF, e.g., ovalbumin, pRBP, and FGF2, at different developmental stages, demonstrated that their physiological ratio is developmentally regulated. They vary from E3 to E5 in a way which is consistent with their known activities in brain development (Parvas et al., 2008a; Figure 4). For example, the eCSF/blood plasma ratio for FGF2 is higher at E5 than at E3 or E4, the developmental period at which this growth factor acts from the eCSF to influence neuroepithelial progenitor cell proliferation (Martin et al., 2006). While for pRBP, the eCSF/blood plasma ratio is much higher at E4 than at E3 or E5, coinciding exactly with its reported period of activity in retinol transport from blood plasma to the eCSF (Parada et al., 2008b).

It has also been demonstrated that the transfer of microinjected proteins across the blood-eCSF interface is protein-specific (Parvas et al., 2008a). Thus, proteins from non-chick sources are never transported from the blood plasma to the eCSF when experimentally microinjected into the outflow of the heart; with the notable exception of HRP, as discussed above. In the same way, neither were proteins which are not normally present in eCSF but are in the blood plasma transported from the blood plasma to this fluid, even when their concentration was experimentally increased 10- to 100-fold by microinjection (Parvas et al., 2008a).

Conversely, when chick proteins such as ovalbumin, FGF2 and pRBP, which are transported between these two embryonic compartments under normal physiological conditions, were bound to FITC to distinguish them from their endogenously produced counterparts and experimentally microinjected into the outflow of the heart, they were actively transported across the blood-eCSF interface (Parvas et al., 2008a; Figure 4). However, the transfer ratio of these proteins was maintained in its normal range, indicating that eCSF homeostasis is strongly controlled by the barrier system. For example, when ovalbumin, FGF2 or pRBP bound to FITC were microinjected into the outflow of the heart of chick embryos at E4, they were effectively transferred from the blood plasma to the eCSF, but the total concentration of each of these molecules did not increase in eCSF: it remained stable despite their 10- to 100-fold increase in the blood plasma (Parvas et al., 2008a). In other words, the rate of traffic does not depend on the relative concentrations between the blood plasma and the eCSF, but on some kind of "sensor" that controls eCSF homeostasis, paralleling those identified in the choroid plexus for several sets of molecules.

Moreover, the experimental microinjection of these chick endogenous proteins into the cephalic cavities to directly raise their concentration in the eCSF was rapidly compensated by the eCSF-blood plasma transport system (Parvas et al., 2008a). Similarly, when the microinjected proteins came from non-chick sources, they were rapidly eliminated from the eCSF (Figure 4). All of these data strongly suggest blood-eCSF barrier activity in both directions (from blood plasma to eCSF and vice versa) to control eCSF composition before the formation of the functional fetal choroid plexus, thereby ensuring that the eCSF activity is maximally efficient during neural development (Parvas and Bueno, 2011; Castells et al., 2012).

The importance of the blood-eCSF barrier in the control of eCSF composition and homeostasis, and thus, indirectly, in early brain development, has been tested by disrupting it with 6-aminonicotinamide gliotoxin (6-AN); a known antimetabolite of nicotinamide that blocks protein transport across the BBB by halting transcellular caveolae transport (Bertossi et al., 2003; Parvas and Bueno, 2011). Chick embryos treated with 6-AN showed caveolae transport disruption and a lack of protein transport across the blood-eCSF barrier; and this had a clear effect on neuroepithelial cell survival, proliferation and neurogenesis. This blockage also disrupted water influx to the eCSF, leading to an alteration in brain anlagen growth.

\section{PROTEIN TRAFFIC OCCURS IN VERY SPECIFIC BLOOD VESSELS AND INCLUDES BOTH ENDOTHELIAL AND NEUROEPITHELIAL CELLS}

Histological analysis was carried out on embryos when transporting endogenous proteins linked to FITC microinjected into the outflow of the heart from the blood plasma to the eCSF, or when microinjected into the cephalic cavities from the eCSF to the blood plasma, as well as proteins from non-chick sources in the same direction. The results demonstrated that, within the developmental period analyzed, transfer from blood plasma to the eCSF, and vice versa occurs only in a specific area of the embryo: in the brain stem lateral to the floor plate, in the ventral mesencephalon and the most anterior part of the ventral prosencephalon (Figure 4). Interestingly, this location does not correspond to the area in which fetal choroid plexus development starts, i.e., from an invagination of the dorsal roof plate along the midline of the neural tube. This would seem to indicate that barrier properties are controlled by different independent systems at different stages of development. Moreover, the histological analysis showed that this blood-eCSF barrier function includes endothelial cells of specific perineural blood vessels and some vascular sprouts located within the neuroectoderm cells and adjacent neuroepithelial cells, as well as their adjacent neuroectodermal cells (Parvas et al., 2008a).

Similarly, immunohistological analysis revealed the presence of caveolin 1 in the endothelial cells of blood vessels involved in the blood-eCSF barrier function and in the adjacent neuroectodermal cells, which would indicate that proteins are transported via transcellular routes (Parvas and Bueno, 2010a). Caveolin 1 is the main structural component of caveolae, which are 50 to $100 \mathrm{~nm}$ vesicular invaginations of the plasma membrane that are involved in transcellular molecular transport, cell adhesion and signal transduction. Endothelial cells usually show the highest expression of caveolin 1, which is also found in the human BBB vessels (Dermeitzel and Krause, 1991; Laterra and Goldstein, 1993). 
Interestingly, caveolin 1 has also been detected in the same embryonic area in rat embryos, including endothelial cells of blood vessels and the adjacent neuroectodermal cells, at an equivalent developmental stage (E12.7) (Parvas and Bueno, 2010a). Although few data are available on protein transport from blood plasma to eCSF in mammals at this developmental stage, the presence of caveolin 1 in the same region that involves both endothelial and neuroepithelial cells has led to the suggestion that there is a barrier function in mammals that acts in the same way as the function that has been demonstrated in chick embryos (Parvas and Bueno, 2010a).

\section{TRAFFIC OF WATER, IONS AND GLUCOSE OCCURS AT THE SAME TRANSPORT SITE AS PROTEINS}

During the initial stages of brain development, the control of water flux and regulation of solute transport is critical, since the increase in brain cavity volume is accompanied by a parallel increase in eCSF volume (reviewed by Gato and Desmond, 2009). Aquaporins (AQPs), a family of transmembrane proteins that form molecular water channels that mediate rapid membrane water transport, have been identified in epithelial and endothelial cells at numerous locations in higher vertebrates (Nielsen and Agre, 1995; Verkman et al., 1996; Agre et al., 1998, 2002). Of the 12 AQPs described to date (AQP0 to AQP11), AQP1 and AQP4 appear to play a role in water transport across brain barriers. AQP1 is expressed at very early stages of choroid plexus development in a range of mammalian species (Johansson et al., 2005), and AQP4 is present in adult brain endothelial cells (AmiryMoghaddam et al., 2004). Findings from several studies suggest that choroid plexus apical expression of AQP1 is closely related to the rate of CSF formation. For example, AQP1-null mice have lowered CSF formation and pressure (Oshio et al., 2005).

Similarly, ion transfer appears to be critical for eCSF production and brain anlagen growth. Ions can move through cell membranes, both actively via ion pumps and passively via ion channels. In the adult, AQP4 co-localizes with the inwardly rectifying $\mathrm{K}^{+}$channel Kir4.1 (Nagelhus et al., 2004), and it has been suggested that together, these two proteins play a key role in $\mathrm{K}^{+}$ and water balance. A study using PCR analysis and immunohistochemical procedures revealed that Kir4.1, AQP1, and AQP4 are present in both chick and rat embryos in the same blood vessels where specific protein transport has also been detected (Parvas and Bueno, 2010b; Figure 4). In chick embryos, AQP1 is present from E4, and AQP4, and Kir4.1 from E5; and in rat embryos AQP1 is detected from E12.7 and AQP4, and Kir4.1 from E13.7, i.e., at the equivalent developmental stages. These results may explain the control of the rapid expansion of brain anlagen at this developmental stage by means of water and ion influx, as previously suggested by Desmond and Jacobson (1977).

In addition to water and ion influx, the transfer of energy molecules such as glucose is crucial for the developing neuroepithelium. Glucose is an important energy source for the brain, and although few data are available on embryos, it has been recently shown that several glucose transporters are expressed at a much higher level in the rat embryonic choroid plexus than in the adult (Liddelow et al., 2014). Glucose is transported across cell membranes mainly by the GLUT family of solute carriers.
In adults, GLUT1 is located in the brain barriers and also in the choroid plexus epithelial cells (Saunders et al., 2013). Similarly, a study using PCR analysis and immunohistochemical procedures demonstrated that in chick and rat embryos, GLUT1 is also present in the same blood vessels where protein transport and the presence of AQP1, AQP4, and Kir4.1 have been detected (Parvas and Bueno, 2010b).

\section{CONCLUSION}

Taken together, all of these findings seem to confirm that a blood-eCSF barrier function controls eCSF composition and homeostasis from early stages of brain development in chick embryos. That control includes protein, glucose, water, and ion influx, and thus regulates eCSF osmolarity. It has been proposed that a similar blood-eCSF barrier is also present in mammals at the equivalent brain development stages (Parvas and Bueno, $2010 \mathrm{a}, \mathrm{b})$. The roles ascribed to this transient barrier confirm the crucial importance of the eCSF milieu for brain development at the beginning of primary neurogenesis; after the closure of the anterior neuropore and before the fetal choroid plexus is fully functional, which occurs shortly afterwards. Thus, the bloodeCSF barrier function should be considered as an actual barrier system that operates in early embryos in a transcellular way, and which includes specific transporters for particular molecules and sensors to monitor eCSF homeostasis. Just as most civilizations develop along riverbanks and seashores, using the fluid medium that is immediately available to them to promote cohesion and transport, and to enhance the chances of survival of the people who live at the edge of the liquid medium, so the brain is also organized, from its embryonic beginnings and throughout adult life, around an extraordinarily dynamic, and complex fluid: the CSF. Continuing this simile, as civilizations build harbors from which to ship goods and control transport that becomes ever more complex as they develop, so the brain has evolved barrier mechanisms, which start to form very early in brain development and change their morphology and physiology in accordance with the changing developmental stages.

\section{ACKNOWLEDGMENTS}

Financial support from the Spanish Ministerio de Educación y Ciencia, via grant BFU2011-23921 is gratefully acknowledged. We also thank the reviewers and editors for comments and suggestions that have made the paper more comprehensive and sounder.

\section{REFERENCES}

Agre, P., Bonhivers, M., and Borgnia, M. J. (1998). The aquaporins, blueprints for cellular plumbing systems. J. Biol. Chem. 273, 14659-14662.

Agre, P., King, L. S., Yasui, M., Guggino, W. B., Ottersen, O. P., Fujiyoshi, Y., et al. (2002). Aquaporin water channels-from atomic structure to clinical medicine. J. Physiol. 542, 3-16. doi: 10.1113/jphysiol.2002.020818

Alonso, M. I., Gato, A., Moro, J. A., and Barbosa, E. (1998). Disruption of proteoglycans in neural tube fluid by beta-D-xyloside alters brain enlargement in chick embryos. Anat. Rec. 252, 499-508.

Alonso, M. I., Gato, A., Moro, J. A., Martin, P., and Barbosa, E. (1999). Involvement of sulfated proteoglycans in embryonic brain expansion at earliest stages of development in rat embryos. Cells Tissues Organs 165, 1-9. doi: $10.1159 / 000016667$ 
Alonso, M. I., Martín, C., Carnicero, E., Bueno, D., and Gato, A. (2011). Cerebrospinal fluid control of neurogenesis induced by retinoic acid during early brain development. Dev. Dyn. 240, 1650-1659. doi: 10.1002/dvdy.22657

Amiry-Moghaddam, M., Frydenlund, D. S., and Ottersen, O. P. (2004). Anchoring of aquaporin-4 in brain: molecular mechanisms and implications for the physiology and pathophysiology of water transport. Neuroscience 129, 999-1010. doi: 10.1016/j.neuroscience.2004.08.049

Bally-Cuif, L., and Boncinelli, E. (1997). Transcription factors and head formation in vertebrates. Bioessays 19, 127-135. doi: 10.1002/bies.950190207

Balslev, Y., Dziegielewska, K. M., Møllgard, K., and Saunders, N. R. (1997). Intercellular barriers to and transcellular transfer of albumin in the fetal sheep brain. Anat. Embryol. (Berl.) 195, 229-236. doi: 10.1007/s004290050042

Bellairs, R., and Osmond, M. (2005). Atlas of Chick Development. London: Elsevier Academy Press.

Bertossi, M., Girolamo, F., Errede, M., Virgintino, D., and Roncali, L. (2003). Effects of 6-aminonicotinamide gliotoxin on blood-brain barrier differentiation in the chick embryo cerebellum. Anat. Embryol. (Berl.) 207, 209-219. doi: 10.1007/s00429-003-0335-4

Birecree, E., King, L. E. J., and Nanney, L. B. (1991). Epidermal growth factor and its receptor in the developing human nervous system. Brain Res. Dev. Brain Res. 60, 145-154. doi: 10.1016/0165-3806(91)90043-I

Birge, W. J., Rose, A. D., Haywood, J. R., and Doolin, P. F. (1974). Development of the blood-cerebrospinal fluid barrier to proteins and differentiation of cerebrospinal fluid in the chick embryo. Dev. Biol. 41, 245-254.

Brocklehurst, G. (1979). The significance of the evolution of the cerebrospinal fluid system. Ann. Rev. Coll. Surg. Engl. 61, 349-356.

Castells, A., Parvas, M., and Bueno, D. (2012). Homeostasis of cerebrospinal fluid has a role in early brain development. Neuroreport 23, 917-921. doi: 10.1097/WNR.0b013e3283582067

Checiu, I., Prelipceanu, O., and Popescu, O. (1984). The role of cerebrospinal fluid during embryonic development. A biochemical study. Morphol. Embryol. 30, 243-250.

Copp, A. J. (2005). Neurulation in the cranial region-normal and abnormal. J. Anat. 207, 623-635. doi: 10.1111/j.1469-7580.2005.00476.x

Copp, A. J., Greene, N. D., and Murdoch, J. N. (2003). The genetic basis of mammalian neurulation. Nat. Rev. Genet. 4, 784-793. doi: 10.1038/nrg1181

Dermeitzel, R., and Krause, D. (1991). "Molecular anatomy of the blood brain barrier as defined by immunocyto-chemistry," in International Review of Cytology, eds K. W. Jeon and M. Friedlander (New York, NY: Academic Press), 57-109.

Desmond, M. E., and Jacobson, A. G. (1977). Embryonic brain enlargement requires cerebrospinal fluid pressure. Dev. Biol. 57, 188-198. doi: 10.1016/0012 1606(77)90364-5

Desmond, M. E., and Levitan, M. L. (2002). Brain expansion in the chick embryo initiated by experimentally produced occlusion of the spinal neurocoel. Anat. Rec. 268, 147-159. doi: 10.1002/ar.10146

Dziegielewska, K. M., Evans, C. A., and Fossan, G. (1980a). Proteins in cerebrospinal fluid and plasma of fetal sheep during development. J. Physiol. 300, 441-455.

Dziegielewska, K. M., Evans, C. A., and Lai, P. C. (1981). Proteins in cerebrospinal fluid and plasma of fetal rats during development. Dev. Biol. 83, 193-200. doi: 10.1016/S0012-1606(81)80024-3

Dziegielewska, K. M., Evans, C. A., Malinowska, D. H., Møllgård, K., Reynolds, J. M., Reynolds, M. L., et al. (1979). Studies of the development of brain barrier systems to lipid insoluble molecules in fetal sheep. J. Physiol. 292, 207-231.

Dziegielewska, K. M., Evans, C. A., Malinowska, D. H., Møllgård, K., Reynolds, M. L., and Saunders, N. R. (1980b). Blood-cerebrospinal fluid transfer of plasma proteins during fetal development in the sheep. J. Physiol. 300, 457-465.

Ek, C. J., Dziegielewska, K. M., Habgood, M. D., and Saunders, N. R. (2012). Barriers in the developing brain and Neurotoxicology. Neurotoxicology 33, 586-604. doi: 10.1016/j.neuro.2011.12.009

Ek, C. J., Habgood, M. D., Dziegielewska, K. M., Potter, A., and Saunders, N. R. (2001). Permeability and route of entry for lipid-insoluble molecules across brain barriers in developing Monodelphis domestica. J. Physiol. 536, 841-853. doi: 10.1111/j.1469-7793.2001.00841

Emerich, D. F., Skinner, S. J. M., Borlongan, C. V., Vasconcellos, A. V., and Thanos, C. G. (2005). The choroid plexus in the rise, fall and repair of the brain. Bioessays 27, 262-274. doi: 10.1002/bies.20193

Engelhardt, B. (2003). Development of the blood-brain barrier. Cell Tissue Res. 314, 119-129. doi: 10.1387/ijdb.103224sl
Feliciano, D. M., Zhang, S., Nasrallah, C. M., Lisgo, S. N., and Bordey, A. (2014). Embryonic cerebrospinal fluid nanovesicles carry evolutionarily conserved molecules that promote neural stem cell amplification. PLOS ONE 9:e88810. doi: 10.1371/journal.pone.0088810

Ferguson, R. K., and Woodbury, D. M. (1969). Penetration of 14C-inulin and 14Csucrose into brain, cerebrospinal fluid, and skeletal muscle of developing rats. Exp. Brain Res. 7, 181-194.

Fielitz, W., Esteves, A., and Moro, R. (1984). Protein composition of cerebrospinal fluid in the developing chick embryo. Brain Res. 315, 111-115. doi: 10.1016/0165-3806(84)90082-8

Gato, A. (2013). Cerebrospinal fluid: a rising subject in brain development. Front. Mol. Neurosci. 6, 1. doi: 10.3389/fnmol.2013.00030

Gato, A., and Desmond, M. E. (2009). Why the embryo still matters: CSF and the neuroepithelium as interdependent regulators of embryonic brain growth, morphogenesis and histiogenesis. Dev. Biol. 327, 263-272. doi: 10.1016/j.ydbio.2008.12.029

Gato, A., Martín, P., Alonso, M. I., Martín, C., Pulgar, M. A., and Moro, J. A. (2004). Analysis of cerebro-spinal fluid protein composition in early developmental stages in chick embryos. J. Exp. Zool. A Comp. Exp. Biol. 301, 280-289. doi: 10.1002/jez.a.20035

Gato, A., Moro, J. A., Alonso, M. I., Bueno, D., De La Mano, A., and Martín, C. (2005). Embryonic cerebrospinal fluid regulates neuroepithelial survival, proliferation, and neurogenesis in chick embryos. Anat. Rec. A Discov. Mol. Cell Evol. Biol. 284, 475-484. doi: 10.1002/ar.a.20185

Habgood, M. D., Knott, G. W., Dziegielewska, K. M., and Saunders, N. R. (1993). The nature of the decrease in blood-cerebrospinal fluid barrier exchange during postnatal brain development in the rat. J. Physiol. 468, 73-83.

Habgood, M. D., Sedgwick, J. E., Dziegielewska, K. M., and Saunders, N. R. (1992). A developmentally regulated blood-cerebrospinal fluid transfer mechanism for albumin in immature rats. J. Physiol. 456, 181-192.

Hatta, T., Matsumoto, A., Ono, A., Udagawa, J., Nimura, M., Hashimoto, R., et al. (2006). Quantitative analyses of leukemia inhibitory factor in the cerebrospinal fluid in mouse embryos. Neuroreport 17, 1863-1866. doi: 10.1097/WNR.0b013e3280113962

Johanson, C. E., Duncan, J. A., Klinge, P. M., Brinker, T., Stopa, E. G., and Silverberg, G. D. (2008). Multiplicity of cerebrospinal fluid functions: new challenges in health and disease. Cerebrospinal Fluid Res. 5, 10. doi: 10.1186/17438454-5-10

Johanson, C. E., Stopa, E. G., and McMillan, P. N. (2011). The blood-cerebrospinal fluid barrier: structure and functional significance. Methods Mol. Biol. 686, 101-131. doi: 10.1007/978-1-60761-938-3_4

Johansson, P. A., Dziegielewska, K. M., Ek, C. J., Habgood, M. D., Liddelow, S. A., Potter, A. M., et al. (2006). Blood-CSF barrier function in the rat embryo. Eur. J. Neurosci. 24, 65-76. doi: 10.1111/j.1460-9568.2006.04904.x

Johansson, P. A., Dziegielewska, K. M., Ek, C. J., Habgood, M. D., Møllgård, K., Potter, A., et al. (2005). Aquaporin-1 in the choroid plexuses of developing mammalian brain. Cell Tissue Res. 322, 353-364. doi: 10.1007/s00441-0051120-x

Kniesel, U., Risau, W., and Wolburg, H. (1996). Development of blood-brain barrier tight junctions in the rat cortex. Brain Res. Dev. Brain Res. 96, 229-240.

Laterra, J., and Goldstein, G. W. (1993). "Brain microves-sels and microvascular cells in vitro," in The Blood-Brain Barrier Pardridge, ed W. M. Pardridge (New York, NY: Raven Press), 1-24.

Lee, S. W., Kim, W. J., Choi, Y. K., Song, H. S., Son, M. J., Gelman, I. H., et al. (2003). SSeCKS regulates angiogenesis and tight junction formation in bloodbrain barrier. Nat. Med. 9, 900-906. doi: 10.1038/nm889

Liddelow, S. A. (2011). Fluids and barriers of the CNS: a historical viewpoint. Fluids Barriers CNS 8:2. doi: 10.1186/2045-8118-8-2

Liddelow, S. H., Dziegielewska, K. M., Møllgård, K., Habgood, M. D., Wakefield, M. J., Lyndsay, H., et al. (2014). Influx mechanisms in the embryonic and adult rat choroid plexus: a transcriptome study. Front. Neurosci.

Martin, C., Alonso, M. I., Santiago, C., Moro, J. A., De la Mano, A., Carretero, R., et al. (2009). Early embryonic brain development in rats requires the trophic influence of cerebrospinal fluid. Int. J. Dev. Neurosci. 27, 733-740. doi: 10.1016/j.ijdevneu.2009.06.002

Martin, C., Bueno, D., Alonso, M. I., Moro, J. A., Callejo, S., Parada, C., et al. (2006). FGF2 plays a key role in embryonic cerebrospinal fluid trophic properties over chick embryo neuroepithelial stem cells. Dev. Biol. 297, 402-416 doi: 10.1016/j.ydbio.2006.05.010 
Martinez, S. (2001). The isthmic organizer and brain regionalization. Int. J. Dev. Biol. 44, 367-371. Available online at: http://www.ijdb.ehu.es/web/paper.php? doi $=11291867$

Miyan, J. A., Nabiyouni, M., and Zendah, M. (2003). Development of the brain: a vital role for cerebrospinal fluid. Can. J. Physiol. Pharmacol. 81, 317-328. doi: 10.1139/y03-027

Miyan, J. A., Zendah, M., Mashayekhi, F., and Owen-Lynch, P. J. (2006). Cerebrospinal fluid supports viability and proliferation of cortical cells in vitro, mirroring in vivo development. Cerebrospinal Fluid Res. 3:2. doi: 10.1186/17438454-3-2

Møllgård, K., Lauritzen, B., and Saunders, N. R. (1979). Double replica technique applied to choroid plexus from early foetal sheep: completeness and complexity of tight junctions. J. Neurocytol. 8, 139-149. doi: 10.1007/BF01175557

Møllgård, K., and Saunders, N. R. (1977). A possible transepithelial pathway via endoplasmic reticulum in foetal sheep choroid plexus. Proc. R. Soc. Lond. B Biol. Sci. 199, 321-326. doi: 10.1098/rspb.1977.0142

Nagelhus, E. A., Mathiisen, T. M., and Ottersen, O. P. (2004). Aquaporin-4 in the central nervous system: cellular and subcellular distribution and coexpression with KIR4.1. Neuroscience 129, 905-913. doi: 10.1016/j.neuroscience.2004.08.053

Nakamura, H., Katahira, T., Matsunaga, E., and Sato, T. (2005). Isthmus organizer for midbrain and hindbrain development. Brain Res. Brain Res. Rev. 49, 120-126. doi: 10.1016/j.brainresrev.2004.10.005

Nielsen, S., and Agre, P. (1995). The aquaporin family of water channels in kidney. Kidney Int. 48, 1057-1068.

Oshio, K., Watanabe, H., Song, Y., Verkman, A. S., and Manley, G. T. (2005). Reduced cerebrospinal fluid production and intracranial pressure in mice lacking choroid plexus water channel Aquaporin-1. FASEB J. 19, 76-78. doi: 10.1096/fj.04-1711fje

Parada, C., Gato, A., Aparicio, M., and Bueno, D. (2006). Proteome analysis of chick embryonic cerebrospinal fluid. Proteomics 6, 312-320. doi: 10.1002/pmic.200500085

Parada, C., Escolà-Gil, J. C., and Bueno, D. (2008a). Low-density lipoproteins from embryonic cerebrospinal fluid are required for neural differentiation. J. Neurosci. Res. 86, 2674-2684. doi: 10.1002/jnr.21724

Parada, C., Gato, A., and Bueno, D. (2005a). Mammalian embryonic cerebrospinal fluid proteome has greater apolipoprotein and enzyme pattern complexity than the avian proteome. J. Proteome Res. 4, 2420-2428. doi: 10.1021/pr050213t

Parada, C., Gato, A., and Bueno, D. (2008b). All-trans retinol and retinol-binding protein from embryonic cerebrospinal fluid exhibit dynamic behavior during early central nervous system development. Neuroreport 19, 945-950. doi: 10.1097/WNR.0b013e3283021c94

Parada, C., Martín, C., Alonso, M. I., Moro, J. A., Bueno, D., and Gato, A. (2005b). Embryonic cerebrospinal fluid collaborates with the isthmic organizer to regulate mesencephalic gene expression. J. Neurosci. Res. 82, 333-345. doi: 10.1002/jnr.20618

Parada, C., Parvas, M., and Bueno, D. (2007). Cerebrospinal fluid proteomes: from neural development to neurodegenerative diseases. Curr. Proteomics 4, 89-106. doi: 10.2174/157016407782194611

Parvas, M., and Bueno, D. (2010a). The embryonic blood-CSF barrier has molecular elements for specific glucose transport and for the general transport of molecules via transcellular routes. Adv. Biosci. Biotechnol. 1, 315-321. doi: 10.4236/abb.2010.14041

Parvas, M., and Bueno, D. (2010b). The embryonic blood-CSF barrier has molecular elements to control E-CSF osmolarity during early CNS development. J. Neurosci. Res. 88, 1205-1212. doi: 10.1002/jnr.22293

Parvas, M., and Bueno, D. (2011). Disruption of embryonic blood-CSF barrier in chick embryos reveals the actual importance of this barrier to control E-CSF composition and homeostasis in early brain development. Open J. Neurosci. 1, 1-11. Available online at: http://rossscience.org/ojns/articles/ 2075-9088-1-3.pdf

Parvas, M., Parada, C., and Bueno, D. (2008a). A blood-CSF barrier function controls embryonic CSF protein composition and homeostasis during early CNS development. Dev. Biol. 321, 51-63. doi: 10.1016/j.ydbio.2008.05.552
Parvas, M., Rius, M., and Bueno, D. (2008b). Most of the abundant protein fractions of embryonic cerebrospinal fluid are produced out of the brain anlagen. Open Proteomics J. 1, 1-4. doi: 10.2174/187503970080 1010001

Rubin, L. L., and Staddon, J. M. (1999). The cell biology of the blood-brain barrier. Annu. Rev. Neurosci. 22, 11-28. doi: 10.1146/annurev.neuro.22.1.11

Saunders, N. R., Daneman, R., Dziegielewska, K. M., and Liddelow, S. A. (2013). Transporters of the blood-brain and blood-CSF interfaces in development and in the adult. Mol. Aspects Med. 34, 742-752. doi: 10.1016/j.mam.2012.11.006

Saunders, N. R., and Møllgård, K. (1984). Development of the blood-brain barrier. J. Dev. Physiol. 6, 45-57.

Sawamoto, K., Wichterle, H., Gonzalez-Perez, O., Cholfin, J. A., Yamada, M., Spassky, N., et al. (2006). New neurons follow the flow of cerebrospinal fluid in the adult brain. Science 311, 629-632 doi: 10.1126/science.1119133

Smith, J. L., and Schoenwolf, G. C. (1997). Neurulation: coming to closure. Trends Neurosci. 20, 510-517.

Spector, R., and Johanson, C. E. (1989). The mammalian choroid plexus. Sci. Am. 261, 68-74.

Stern, C. D. (2001). Initial patterning of the central nervous system: how many organizers? Nat. Rev. Neurosci. 2, 92-98. doi: 10.1038/35053563

Stern, C. D. (2002). Induction and initial patterning of the nervous systemthe chick embryo enters the scene. Curr. Opin. Genet. Dev. 12, 447-451. doi: 10.1016/S0959-437X(02)00324-6

Tauc, M., Vignon, X., and Bouchaud, C. (1984). Evidence for the effectiveness of the blood-CSF barrier in the fetal rat choroid plexus. A freeze-fracture and peroxidase diffusion study. Tissue Cell 16, 65-74.

Vera, A., Stanic, K., Montecinos, H., Torrejón, M., Marcellini, S., and Caprile, T. (2013). SCO-spondin from embryonic cerebrospinal fluid is required for neurogenesis during early brain development. Front. Cell Neurosci. 7:80. doi: 10.3389/fncel.2013.00080

Verkman, A. S., van Hoek, A. N., Ma, T., Frigeri, A., Skach, W. R., Mitra, A., et al. (1996). Water transport across mammalian cell membranes. Am. J. Physiol. 270, C12-30.

Vígh, B., Manzano e Silva, M. J., Frank, C. L., Vincze, C., Czirok, S. J., Szabo, A., et al. (2004). The system of cerebrospinal fluid-contacting neurons. Its supposed role in the nonsynaptic signal transmission of the brain. Histol. Histopathol. 19, 607-628. Available online at: http://www.hh.um.es/pdf/Vol_19/19_2/Vigh-19-607-628-2004.pdf

Wakai, S., and Hirokawa, N. (1978). Development of the blood-brain barrier to horseradish peroxidase in the chick embryo. Cell Tissue Res. 195, 195-203. doi: 10.1007/BF00236719

Zappaterra, M. D., and Lehtinen, M. K. (2012). The cerebrospinal fluid: regulator of neurogenesis, behavior, and beyond. Cell Mol. Life Sci. 69, 2863-2878. doi: 10.1007/s00018-012-0957-x

Zappaterra, M. D., Lisgo, S. N., Lindsay, S., Gygi, S. P., Walsh, C. A., and Ballif, B. A. (2007). A comparative proteomic analysis of human and rat embryonic cerebrospinal fluid. J. Proteome Res. 6, 3537-3548. doi: 10.1021/pr070247w

Conflict of Interest Statement: The authors declare that the research was conducted in the absence of any commercial or financial relationships that could be construed as a potential conflict of interest.

Received: 25 June 2014; accepted: 08 October 2014; published online: 28 October 2014. Citation: Bueno D, Parvas M, Hermelo I and Garcia-Fernàndez J (2014) Embryonic blood-cerebrospinal fluid barrier formation and function. Front. Neurosci. 8:343. doi: 10.3389/fnins.2014.00343

This article was submitted to Neurogenomics, a section of the journal Frontiers in Neuroscience.

Copyright (C) 2014 Bueno, Parvas, Hermelo and Garcia-Fernàndez. This is an openaccess article distributed under the terms of the Creative Commons Attribution License (CC BY). The use, distribution or reproduction in other forums is permitted, provided the original author(s) or licensor are credited and that the original publication in this journal is cited, in accordance with accepted academic practice. No use, distribution or reproduction is permitted which does not comply with these terms. 


\title{
Development of the choroid plexus and blood-CSF barrier
}

\author{
Shane A. Liddelow ${ }^{1,2 *}$ \\ ${ }^{1}$ Department of Neurobiology, Stanford University, CA, USA \\ ${ }^{2}$ Department of Pharmacology and Therapeutics, The University of Melbourne, Parkville, VIC, Australia
}

\section{Edited by:}

Joana A. Palha, University of Minho, Portugal

Reviewed by:

Eva Carro, Instituto de Investigacion Hospital 12 de Octubre, Spain Helle Hasager Damkier, University of Copenhagen, Denmark David John Begley, Kings College London, UK

*Correspondence:

Shane A. Liddelow, Department of Neurobiology, Stanford University, Level 2 Fairchild Building, 299

Campus Drive West Stanford, CA 94305, USA

e-mail: liddelow@stanford.edu
Well-known as one of the main sources of cerebrospinal fluid (CSF), the choroid plexuses have been, and still remain, a relatively understudied tissue in neuroscience. The choroid plexus and CSF (along with the blood-brain barrier proper) are recognized to provide a robust protective effort for the brain: a physical barrier to impede entrance of toxic metabolites to the brain; a "biochemical" barrier that facilitates removal of moieties that circumvent this physical barrier; and buoyant physical protection by CSF itself. In addition, the choroid plexus-CSF system has been shown to be integral for normal brain development, central nervous system (CNS) homeostasis, and repair after disease and trauma. It has been suggested to provide a stem-cell like repository for neuronal and astrocyte glial cell progenitors. By far, the most widely recognized choroid plexus role is as the site of the blood-CSF barrier, controller of the internal CNS microenvironment. Mechanisms involved combine structural diffusion restraint from tight junctions between plexus epithelial cells (physical barrier) and specific exchange mechanisms across the interface (enzymatic barrier). The current hypothesis states that early in development this interface is functional and more specific than in the adult, with differences historically termed as "immaturity" actually correctly reflecting developmental specialization. The advanced knowledge of the choroid plexus-CSF system proves itself imperative to understand a range of neurological diseases, from those caused by plexus or CSF drainage dysfunction (e.g., hydrocephalus) to more complicated late-stage diseases (e.g., Alzheimer's) and failure of CNS regeneration. This review will focus on choroid plexus development, outlining how early specializations may be exploited clinically.

Keywords: choroid plexus, development, blood-CSF barrier, cerebrospinal fluid, brain patterning, epithelia, neuroependyma

\section{INTRODUCTION}

The first description of the lateral ventricular choroid plexuses is credited to Herophilos (c. 335-280 B.C.) by Galen of Pergamon (see Liddelow, 2011)—he named the structure the "chorioid mennix," "chorioid" being taken from the outer vascular plexus of the fetus. Two centuries years later, Rufus of Ephesus (c. 100 A.D.) suggested the term "chorioid tunic" be used to describe both the ependyma and choroid plexus. A century and a half of silence followed which was broken when Vesalius reported the gross anatomy of the choroid plexus of the lateral ventricles in his De humani corporis fabrica libri septem (On the fabric of the human body. Book 7: The brain - Vesalius, 1543) before Willis (1664) described the choroid plexus of the fourth ventricle and hypothesized the choroid plexus contained gland-like structures which produced the fluid found in the ventricles. The entire extent of the choroid plexuses was known when Ridley (1695) described the choroid plexus of the third ventricle. After these ancient and distant times, there was a resurgence of descriptions of choroid plexus pathologies in the 1910-1930s (Dandy, 1918; Somerford, 1933; Cohen and Davies, 1939 among others)

Abbreviations: CNS, central nervous system; CSF, cerebrospinal fluid; SLC, solute carrier and more traditional investigative descriptions from the 1960s onwards, first on micrographs about the structure and morphological changes in the shape and distribution of plexus epithelial cells and vasculature (Tennyson and Pappas, 1964; Cancilla et al., 1966; Netsky and Shuangshoti, 1970; Stastný and Rychter, 1976; Sturrock, 1979; Keep and Jones, 1990; Dziegielewska et al., 2001; Liddelow et al., 2010) and later on alterations in the perceived function of the plexus by investigations of cerebrospinal fluid (CSF) (Evans et al., 1974; Johanson et al., 1976; Habgood et al., 1992; Liddelow et al., 2014). Though a wealth of information has been gathered about early developmental origins and growth of the choroid plexus through these means, only recently has research moved to the application of modern technologies to study cues involved in choroid plexus development and maturation. Recent studies on plexus epithelial cell differentiation and genetic lineage analyses along with high throughput genetic expression datasets have provided a wide assortment of data for further investigation. Beyond the historic descriptions of its emergence from neuroectodermal progenitor pools, these recent studies have put emphasis on the importance of the choroid plexuses for normal and regulated development of the remainder of the brain, as well as the role this seemingly small structure plays in normal aging and disease. 
In the mouse embryo, the choroid plexus accounts for $27 \%$ of the total ventricular wall area (Knudsen, 1964) and in human embryos this value is even larger (approximately 63\% of the ventricular surface; Voetmann, 1949), the importance of the choroid plexus in normal brain development and function is therefore immense. This review will spend some time discussing the molecular expression of transcription factors and emergence of the choroid plexuses during early embryogenesis, before embarking on a discussion on disruption of normal plexus mechanisms of action during aging and disease. Due to the excess of information available on the telecephalic (lateral ventricular) choroid plexus, the majority of discussions will be focussed on this plexus, however, when other structures are considered they will be listed as such.

\section{NORMAL DEVELOPMENT}

The choroid plexuses, found in the lateral, third and fourth ventricles of the brain (adjacent to the embryonic dorsal midline in the hindbrain, diencephalon, and telencephalon, respectively) are epithelial tissue masses highly vascularized with fenestrated blood vessels (Figure 1). These structures constitute a transfer interface between blood and CSF in the ventricles of the brain. Approximately two thirds of this CSF is produced and secreted by the choroid plexus, the remainder produced by other areas such as the ependymal cells of the ventricular surface and those cells lining the subarachnoid space (see Davson and Segal, 1996; Zheng and Chodobski, 2005 for review). This fluid circulates in the ventricular system, subarachnoid spaces and spinal canal (for review see Davson and Segal, 1996; Saunders et al., 2000; Zheng and Chodobski, 2005) and the plexuses are able to control the homeostasis of its composition by regulation of movement of essential ions and molecules into, and metabolites out of the CSF (Kusuhara and Sugiyama, 2004; Saunders et al., 2013). The choroid plexuses are the main structures that comprise the bloodCSF barrier, the other contributors being the arachnoid and arachnoid villi on the outer surface of the brain (Wright, 1978; and for review see Davson and Segal, 1996). Together these interfaces provide a protective restriction barrier formed by bands of tight junctions between adjacent cells that impede the movement of molecules into the central nervous system (CNS). In addition to this mechanical barrier, the barrier interfaces comprise a biochemical barrier - an additional protective mechanism that effluxes toxic molecules and drugs taken up by cells in the bloodCSF barrier (see Ek et al., 2010; Kratzer et al., 2013). This review will focus on the choroid plexus over the arachnoid membrane, however many properties are retained in both interfaces.

\section{GROSS MORPHOLOGICAL DEVELOPMENT}

As the choroid plexuses themselves do not have distinct proliferative zones; there is much evidence showing (and is widely accepted) the choroid plexus stroma has mesenchymal origins, like meningeal cells, while the actual plexus epithelial cells are derived from neuroepithelium. Genetic lineage analyses indicate the plexus epithelium originates from the roof plate (Awatramani et al., 2003; Currle et al., 2005; Hunter and Dymecki, 2007) a well-known signaling center in early CNS development. From this area the migration of pre-plexus cells can be traced. The post differentiated development of the choroid plexus cells themselves has proved a more difficult beast to investigate. Much study using cell division markers have shown no examples of choroid plexus epithelial cells undergoing division to produce additional plexus epithelium, while in contrast, many mitotic figures have been described in the nearby neuroependyma (Zand, 1930; Kappers et al., 1958). An aside should be noted however that there are examples of a proliferative choroid plexus giving rise to neuronal and glial lineage cells (Itokazu et al., 2006; Bolos et al., 2013). The neuroependyma, originally formed by invagination of the anterior end of the neural tube (Imayoshi et al., 2008), proceeds to divide into three regions:

(1) the most lateral region gives rise to cortical neuroepithelium, which further develop to neurons and glia of the cortex;

(2) the medial or dorsal telencephalic midline regions is split in two, with the cortical hem being a major source of neocortical Cajal-Retzius cells; and

(3) the most medial region of the dorsal telencephalic midline gives rise to choroid plexus epithelium.

Systematic studies on histogenesis of plexus epithelial cells report it arises from an infolding of the multilayered roof plate of the neural tube, between the paraphyseal arch and the medial wall (Bailey, 1916; Tennyson and Pappas, 1964; Sturrock, 1979; Thomas and Dziadek, 1993; Awatramani et al., 2003; Currle et al., 2005; Hunter and Dymecki, 2007). In addition, it has been shown with chick-quail chimeras (Wilting and Christ, 1989) that specific cells destined to develop into choroid plexus are detectable up to $72 \mathrm{~h}$ before the structure even emerges from the neuroependymal wall of the ventricles. This "pre plexus" empendyma has been shown to envelope connective tissue and develop blood vessels, more representative of a mature plexus, upon direct contact with tissue derived from the mesodermal germ layer (Sarnat, 1998), suggesting cues for plexus differentiation are indeed released by nearby cell types in the developing CNS.

Once entering the plexus, newly differentiated cells undergo maturation through four distinct stages; described in many different species with the distinct difference in marsupials that glycogen is absent (Dziegielewska et al., 2001; Ek et al., 2003). The four stages as described by Tennyson and Pappas (1964) and Sturrock (1979) are as follows: Stage I (see Figure 1C): the early epithelial cells are pseudostratified with nuclei that are centrally located. Stage I plexus epithelia are widely accepted to not contain extensive apical villi (common in the adult plexus). Stage II: low columnar to cuboidal epithelial cell shape, with an emerging basal connective tissue. Apical villi are still absent, though some villi-like extensions are seen in late-stage cells. Nuclei move more apically in the cell. Stage III: now with cuboidal epithelial cells and basally-located nuclei it is in this stage cilia on the apical cell surface appear, along with a great increase in complexity of the capillary network, with an accompanied increase in the number of villi. Stage IV (see Figure 1C): in the final stage of plexus epithelial cell development transition to cuboidal cells is complete and the cells become slightly smaller (approximately $10 \mu \mathrm{m}^{2}$ in profile), with most nuclei situated centrally-to-basally within the cytoplasm. While additional cells are continuously added to the choroid plexus throughout early development and cell maturation, these newly added cells will 


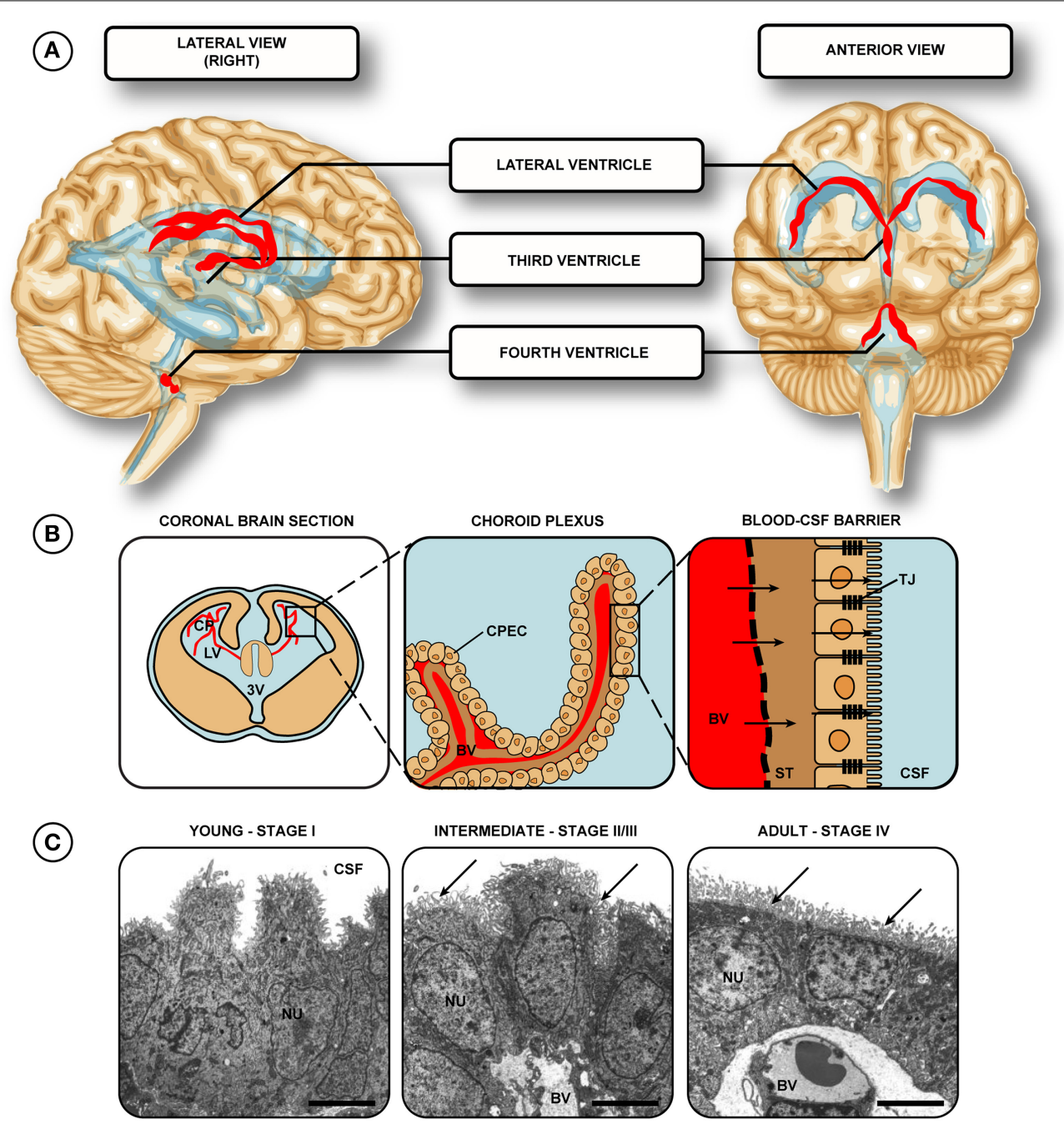

FIGURE 1 | Location of choroid plexuses in the human brain. (A) The choroid plexuses are present in the two lateral, third and fourth ventricles (red ribbons). (B) Each of the plexuses is comprised of fenestrated vessels, with a single layer of intimately opposed choroid epithelial cells, joined by tight junctions-forming the bloodcerebrospinal fluid barrier. (C) Transmission electron micrographs of lateral ventricular choroid plexus. Stage I-the epithelial cells are pseudostratified with centrally located nuclei. Stage $\|/\| I I-$ low columnar to cuboidal, basal-to-centrally located nuclei, with apical villi present (arrows, a characteristic from stage III onwards). Stage IV-cuboidal epithelial cells with central-to-apical nuclei and many villi (arrows). (C) reproduced from Ek et al. (2003) Copyright (C2003 Wiley All rights reserved. Abbreviations: $\mathrm{BV}$, blood vessel; $\mathrm{CP}$, choroid plexus; $\mathrm{CPEC}$, choroid plexus epithelial cell; CSF, cerebrospinal fluid; LV, lateral ventricle; NU, nucleus; ST, stroma/basement membrane; TJ, tight junction; 3V, third ventricle. transition through the four stages of development outlined. A continued proliferative effort by plexus epithelial cells has been shown to occur even after the original spreading from the neuroependymal wall, though it should be noted that this division usually gives rise to cells that are not reminiscent of plexus epithelium. The choroid plexus epithelial cells from rats from newborns to 8 weeks of age have been shown to function as neural progenitor cells (which can give rise to astrocytes) however this ability decreases with age, with cells from P1 animals twice as likely to undergo this change as those from adult (8 week) animals (Itokazu et al., 2006). It is important to note however this proliferation only occurred after explanting to an ectopic location - continued proliferation of the neuroepedyma which adds additional choroid plexus epithelial cells occurs in adult rat only at an extremely low rate (less than $0.1 \%$ of total plexus cells, see Altman and Das, 1965; Doetsch et al., 1999; Liddelow et al., 2010; Johansson et al., 2013). It is interesting to note that in APP/PS1 mice that overexpress amyloid precursor protein and express mutated presilin 1 (important in generation of beta amyloid) there is an almost 5-fold increase in the number of newly divided cells in the choroid plexus at 1 year of age (Bolos et al., 2013). The authors however comment that these newly dividing cells represent proliferation of immature neurons in the choroid plexus and not new plexus epithelial cells per-sesuggesting the choroid plexus as an important adult stem cell niche. 


\section{TRANSCRIPTION FACTORS INVOLVED IN PLEXUS PATTERNING AND DEVELOPMENT}

Like all forms of early brain development, growth and maturation of choroid plexus is regulated by coordinated actions of multiple signaling centers at key boundaries between nearby anatomical compartments (refer to Table 1 for a non-complete list). Three neighboring telencephalic midline structures are situated to perform such roles in forebrain patterning: the cortical hem, the septum, and the thalamic eminence at the diencephalictelencephalic boundary. These structures all express unique complements of signaling molecules. The medial-lateral patterning of dorsal regions of the telencephalon is regulated by a large number of transcription and secreted signaling factors. Some secreted factors, including members of the bone morphogenic protein (BMP) family of proteins, regulate specification of the choroid plexus epithelium by inducing MSX1 and repressing

Table 1 | Factors involved in the development of the choroid plexus epithelial cells.

\begin{tabular}{lll}
\hline Gene/Factor & Function & Species \\
\hline Bmps & Misexpression causes expansion of choroid plexus at the expense of cortical & Mouse \\
neuroepithelium. Deficits cause loss of specification of plexus epithelial cells. & Panchision et al., \\
& & $2001 ;$ Hébert et al., \\
& & $2002 ;$ Fernandes rise to choroid plexus epithelial cells from E10.5 in mouse \\
Imayoshi et al., 2008
\end{tabular}

\begin{tabular}{ll}
\hline E(spl) Homologs of genes present in mammalian species Kageyama et al., $\quad$ Mouse, rat \\
\hline
\end{tabular}

E2F5, fox J1, p73 Lack of functional transcription factor causes hydrocephalus due to
dysfunction of choroid plexus, mostly dysfunctional cilia on CSF surface of
cells and AQP3 channels

Mouse, human (cells) Chen et al., 1998; Kume et al., 1998; Lindeman et al., 1998; Zheng and Zhao, 2002; Swetloff and Ferretti, 2005; Swetloff et al., 2006

\begin{tabular}{|c|c|c|c|}
\hline Hairy, E(spl) & $\begin{array}{l}\text { bHLH repressor genes regulate non-neuronal versus neuronal fate } \\
\text { specification in the ectoderm. Up-regulation of } E(s p l) \text { represses neuronal } \\
\text { specification, promoting non-neuronal cell fate }\end{array}$ & Drosophila & $\begin{array}{l}\text { Campos-Ortega and } \\
\text { Jan, } 1991\end{array}$ \\
\hline Hes1, Hes3, Hes5 & $\begin{array}{l}\text { Gives rise to choroid plexus epithelial. Knock-out mice have no lateral } \\
\text { ventricular choroid plexus }\end{array}$ & Mouse & Imayoshi et al., 2008 \\
\hline $\operatorname{Lm} x 1 a$ & Gives rise to choroid plexus epithelial cells, from E10.5 onwards in mouse & Mouse & Imayoshi et al., 2008 \\
\hline$M s \times 1 / 2$ & Development of dorsal midline region & Mouse & $\begin{array}{l}\text { Hébert et al., 2002; } \\
\text { Bach et al., } 2003\end{array}$ \\
\hline$M s \times 1 / 2$ & $\begin{array}{l}\text { Induce Fox and Msx genes, causing regulation of specification of choroid } \\
\text { plexus epithelium }\end{array}$ & Mouse & $\begin{array}{l}\text { Xuan et al., 1995; } \\
\text { Furuta et al., 1997; } \\
\text { Porter et al., 1997; } \\
\text { Monuki et al., } 2001\end{array}$ \\
\hline Ngn2 & $\begin{array}{l}\text { Gives rise to choroid plexus epithelial cells and Cajal-Retzius cells, } \\
\text { upregulation causes a loss of choroid plexus epithelial cells }\end{array}$ & Mouse & Imayoshi et al., 2008 \\
\hline Notch1 & $\begin{array}{l}\text { Expression of activated Notch } 1 \text { results in overproduction of hindbrain choroid } \\
\text { plexus epithelium (but not of other rhombic lip lineages) }\end{array}$ & Mouse & $\begin{array}{l}\text { Hunter and Dymecki, } \\
2007\end{array}$ \\
\hline Otx2 & $\begin{array}{l}\text { Master regulator of choroid plexus development. Knock-out causes failure of } \\
\text { choroid plexus to develop }\end{array}$ & Mouse & $\begin{array}{l}\text { Johansson et al., } \\
2013\end{array}$ \\
\hline Wnt2b & Gives rise to choroid plexus epithelial cells, from E10.5 onwards in mouse & Mouse & Imayoshi et al., 2008 \\
\hline
\end{tabular}

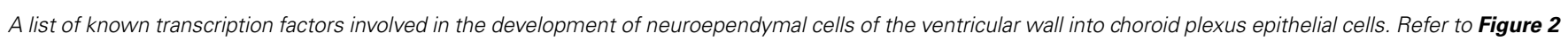

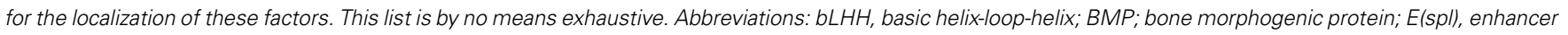

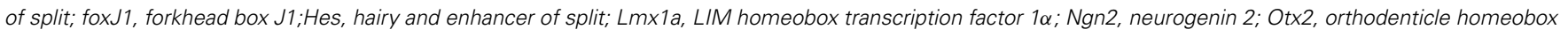
2; p73, tumor protein 73; Wnt2b, wingless-type MMTV integration site family member $2 B$. 
LHX2/FOXG1 levels (Xuan et al., 1995; Furuta et al., 1997; Porter et al., 1997; Monuki et al., 2001; Panchision et al., 2001; Hébert et al., 2002; Bach et al., 2003; Fernandes et al., 2007; Johansson et al., 2013). The cortical hem is a WNT and BMP rich signaling center connected to the choroid plexus on one side and the cortical neuroepithelium on the other (Grove et al., 1998). A lack of the constitutively active form of the receptors for BMPs results in a massive expansion of the choroid plexus epithelium at the expense of the cortical neuroepithelium (Panchision et al., 2001). In contrast, inactivation of BMP receptors results in retarded growth of the choroid plexus, specifically of the epithelial cells (Hébert et al., 2002; Fernandes et al., 2007). One could argue a reduction of BMP receptors on cells in the ventral surface of the neuroepithelium close to the root of the plexus and a large number of these receptors on the dorsal surface may aid in the one-sided growth pattern outlined by Liddelow et al. (2010).

The fruit fly Drosophila has recently become an invaluable tool in the rapid expansion of the molecular knowledge on the fate specification of cells in the ectoderm. There are several Drosophila basic helix-loop-helix repressor genes, such as Hairy/Enhancer of Split $[E(s p l)]$, able to regulate the non-neuronal versus neuronal specification of cells (Campos-Ortega and Jan, 1991). When cells adopt the neuronal fate they express Delta, which in turn activates Notch signaling in neighboring cells, in turn up-regulating $E(s p l)$ expression, promoting non-neuronal cell fate (CamposOrtega and Jan, 1991). This raises the possibility these repressor genes are involved in the formation of non-neuronal tissues in the brain. Mammalian homologs of these genes, the Hes gene family, have been shown to be involved in formation of non-neuronal tissue in developing mouse brain (Imayoshi et al., 2008). In these experiments, expression of Bmp4, Hes1, and Hes5 is seen in the neuroepithelium directly adjacent to the choroid plexus on the upper (dorsal) surface. This localizes the factors in the correct position to aid in the switch to non-neuronal cell fate required for choroid plexus development. In addition Hes 1 and Hes5 expression is absent on the lower (ventral) surface of the choroid plexus root, while Bmp4 expression is still present- possibly causing impedance of mitosis or non-neuronal fate in this area. In addition, inactivation of the repressor genes Hes 1, Hes3, and Hes5 causes up-regulation of the proneural gene neurogenin-2 (Ngn2), prematurely depleting $B m p$ expressing progenitor cells causing loss of choroid plexus epithelial cells (due to the enhanced formation of Cajal-Retzius cells; Imayoshi et al., 2008). A similar effect is seen when bypassing the Hes genes and simply up-regulating Ngn2 expression.

Another set of transcription factors present in choroid plexus epithelial cells immediately after their differentiation from the neuroependyma, E2f5, FoxJ1, and P73, when expressed in aberrant levels cause non-obstructive hydrocephalus in mouse (Swetloff and Ferretti, 2005). The levels of E2F5 protein in the brain are highest in embryonic development and lower in the adult in mouse (Dagnino et al., 1997; Persengiev et al., 1999; Swetloff and Ferretti, 2005) which correlates well with plexus growth patterns - which is most rapid during early development, but reaches a plateau shortly after birth (Liddelow et al., 2010). The amount of E2F5 protein is also increased in nuclei of choroid plexus epithelium of both mouse and human early in development, suggesting it may be more important for maturation of plexus epithelial cells rather than for the original transition from their neuroependymal cell beginnings (Swetloff and Ferretti, 2005). A summary of the position of these transcription factors is outlined in Table 1 and Figure 2. The proliferative nature of choroid plexus epithelial cells has also been shown to occur even after the original dissemination from the neuroependymal wall and a possible neuronal fate. After a graft of plexus epithelium into the spinal cord of the adult rodent, plexus epithelium is able to differentiate into astrocytes (Ide et al., 2001; Kitada et al., 2001). Though the authors do not comment on what may have caused the switch from epithelial cell to astrocyte, it appears likely some local factor in the environment of the spinal cord has caused the transformation. In addition, choroid plexus epithelial cells from rats at a range of developmental ages from postnatal day 1 to 8 weeks have been shown to have an ability to function as neural progenitor cells, however this ability decreases with age, with the plexus epithelium from P1 animals twice as likely to undergo the change than those from adult (8 week) animals (Itokazu et al., 2006). The considerable potential for growth by the adult choroid plexus is demonstrated by growth of the choroid plexus (almost

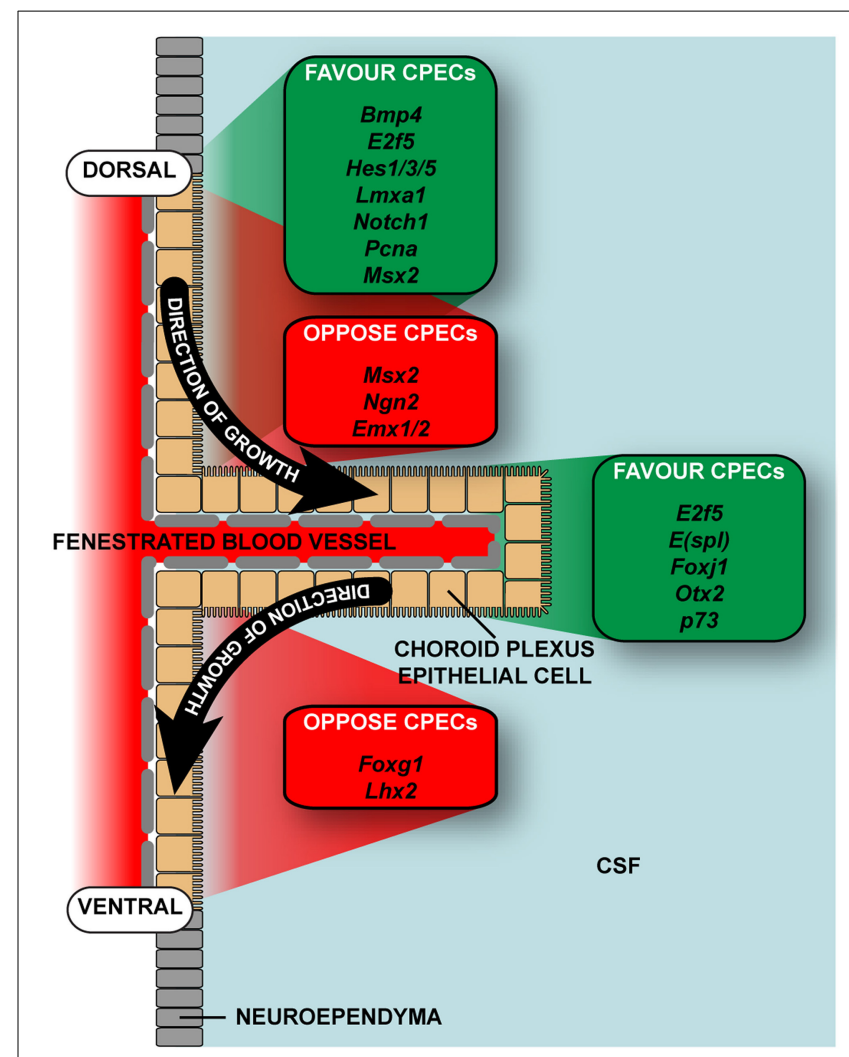

FIGURE 2 | Location of expression of transcription factors known to be important in plexus development and growth. The choroid plexus epithelial cells develop from modified neuroepithelium and are only added to the structure from the dorsal surface. No addition of cells is described from the ventral surface, and no transcription factors that promote differentiation into choroid plexus epithelium have been reported as expressed in this region. For full list of transcripts and references refer to Table 1. CSF, cerebrospinal fluid. 
40-fold in the adult) by intrathecal infusion of fibroblast growth factor 2 or epidermal growth factor (Itokazu et al., 2006).

More recently, Otx2 (orthodenticle homeobox 2) has been implicated as the master regulator of choroid plexus differentiation (Johansson et al., 2013). Like Emx2, Otx2 is expressed in the dorsal roof plate and both play important roles in specification of neuroepithelial versus choroid plexus regions (von Frowein et al., 2006). Emx1 and Emx2 are regulators able to supress plexus cell fate differentiation, while $O t x 2$ pushes cells into a plexus epithelial lineage, the two regulators working in tandem to ensure correct pattering early in development. Indeed, deletion of Otx2 sufficiently early in development causes failure of the choroid plexus to occur, while deletion once the plexus has developed causes epithelial cells to enter an apoptotic pathway, though no apoptotic cells were visualized by the authors (Johansson et al., 2013). These data hypothesize Otx2 is absolutely required for normal choroid plexus development; however its importance in the general maintenance in the mature/adult choroid plexus is yet to be ascertained. It is known however in humans the obliteration of the lateral ventricular choroid plexus as a treatment for hydrocephalus results in no regeneration of the structure (Hallaert et al., 2012; Ogiwara et al., 2014). This suggests early developmental growth factor patterning cues are paramount for normal plexus development, and the structure cannot regenerate from already-present plexus epithelium.

\section{FUNCTION OF CHOROID PLEXUS EPITHELIAL CELLS}

The epithelial cells of the choroid plexus have many functions important for normal embryo development. They are principally recognized as producing a large proportion of the CSF, and acting as site of the blood-CSF barrier, a protective mechanism that ensures the stability of the CSF milieu (Davson and Segal, 1996; Zheng and Chodobski, 2005; Saunders et al., 2008; also see Introduction). Like other brain barriers, the choroid plexus blood-CSF barrier is formed by presence of specialized junctions between adjacent epithelial cells (Wolburg et al., 2001; Saunders et al., 2008; Liebner et al., 2011). The molecular make-up of tight junctions of the blood-CSF barrier is less well-known than those that comprise the blood-brain barrier (Wolburg et al., 2001). A recent study of tight junction protein expression in mouse embryos (E15) and adult choroid plexus has shown several key junctional genes are expressed at a higher level in embryos than in the adults (e.g., Pcdh, Cldn5), whereas for several other genes the reverse is the case (e.g., Igsf5, Cldn2, see Liddelow et al., 2012). This is consistent with previous findings that the fundamental functional basis of this barrier, namely occlusion of the paracellular diffusion pathway, is well-established from the earliest stages of differentiation of the choroid plexuses (Bauer et al., 1993; Ek et al., 2003, 2006). The critical function of these junctions is to join the cells together to create a physical barrier to paracellular diffusion, allowing cells to polarize with distinct luminal and abluminal components. The presence of these junctions between cells of the interface between the periphery and the CNS allows cellular transporters to be effective in controlling the distribution of solutes on either side, and thus set up concentration gradients. These gradients are not only important for mature brain function, but are also likely to be significant for essential features of early brain development (cell division, migration, differentiation and synaptogenesis). For instance, the most active transporter in the choroid plexus appears to be the $\mathrm{Na}^{+} / \mathrm{K}^{+}$ATPase pump-integral for maintenance of the ion gradient that draws water into the ventricles via aquaporin-1 (AQP1) channels in the plexus epithelium (see below). Plexus barrier junctions are tight to molecules as small as lanthanum ion (139 Da; Brightman and Reese, 1969) and tracer molecules such as BED (286 Da; see Ek et al., 2003) and larger dextrans from $30 \mathrm{kDa}$ (Ek et al., 2003, 2006) up to $70 \mathrm{kDa}$ (Liddelow et al., 2009)—more in line with the size of plasma proteins. The presence of this functional barrier is an essential prerequisite for the establishment and maintenance of concentration gradient for ions and proteins between the blood (basal side of the cells) and the CSF on the apical side (see Speake and Brown, 2004). Indeed earlier permeability experiments have already shown that when administered peripherally acidic dyes (Ehrlich, 1885; Goldmann, 1909) or more recently biotinylated and fluorescently-labeled dextran molecules (Ek et al., 2003) only enter the plexus structure and do not enter the CNS milieu. These junctions of the plexus are present from the first emergence of the structure from the wall of the ventricles, however as transcriptome profiling of the possible molecular make-up of this structure has only recently been completed (Marques et al., 2009; Liddelow et al., 2012; Kratzer et al., 2013) there are no plexusspecific knock-out animal models available to further investigate the role of these individual molecules in dysfunctions of plexus development and function.

At all stages of development, the plexus epithelial cells are able to produce and secrete CSF and regulate transfer of molecules across the blood/CSF interface, as tight junctions are present between adjacent cells immediately following their entry from the neuroepithelium. For lipid insoluble substances, such as proteins, this transfer has been shown to be across choroid plexus epithelial cells both in development and in the adult (Møllgård et al., 1979; Ek et al., 2003; Johansson et al., 2006; Liddelow et al., 2009). However, not all choroid plexus cells seem to be involved in this process, the proportion ranges between less than $5 \%$ in the adult to about $15 \%$ during early stages of brain development in opossum (Liddelow et al., 2009) and rat (Johansson et al., 2006), to over 40\% in sheep (Balslev et al., 1997) and humans (Møllgård and Saunders, 1977). These processes of early plexus epithelia will be covered in more detail below.

\section{SOLUTE CARRIERS}

Transport across the blood-brain barrier and blood-CSF barrier is directional, with different classes of transporters involved in movement into (e.g., most SLC transporters) and out of (e.g., ABC efflux pumps, see Figure 3). Additionally, there are both active and passive transporters: active mechanisms move solutes up their concentration gradients and require energy (ATP, e.g., $\mathrm{Na}^{+} / \mathrm{K}^{+}$ATPase), while passive mechanisms move solutes down their concentration gradients and are energy-independent processes. Together, these combined transporters can have a multitude of effects from removal of solutes from the CSF, preventing their entry into the brain (efflux mechanisms), initiation of ion gradients or delivery of specific nutrients, ions and other required molecules to the brain cells (influx mechanisms). 


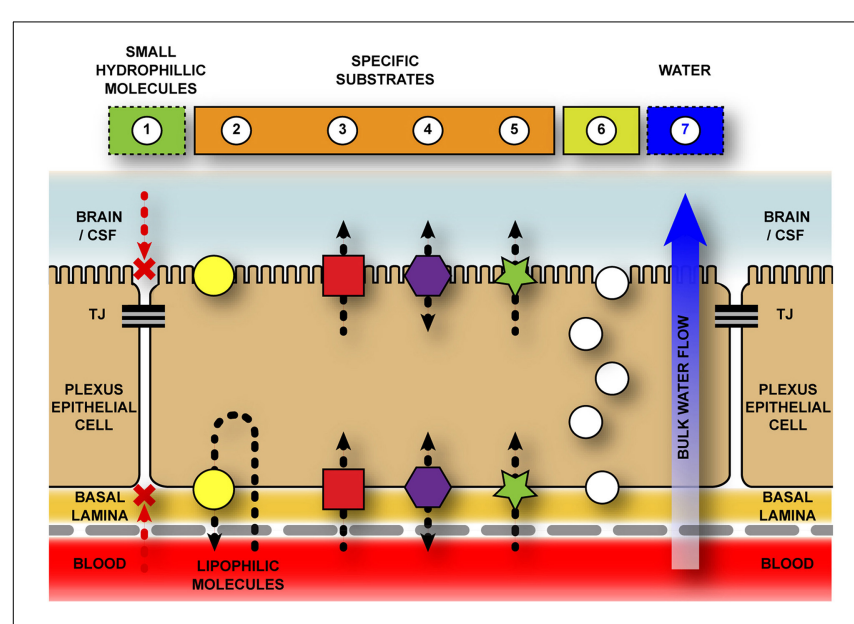

FIGURE 3 | Schematic representation of transport pathways across the blood-cerebrospinal fluid barrier. The blood-cerebrospinal fluid (CSF) barrier is formed by tight junctions between neighboring choroid plexus epithelial cells-halting the paracellular movement of molecules both into, and out of, the brain. Additional chemical barriers exist to impede movement of molecules into the central nervous system. 1-Diffusion for hydrophilic molecules through a paracellular pathway is halted by tight junctions (TJ) between adjacent choroid plexus epithelial cells. 2-Efflux transporters (e.g., ABC family) actively remove specific (mostly) lipophilic solutes from cell cytoplasm and extracellular space. Though the majority of evidence suggests a basolateral removal of molecules to the blood space, there is some evidence that ABCB1 (PGP) and ABCG2 (BCRP) localize to the apical membrane of the choroid plexus (Rao et al., 1999; Gao and Meier, 2001; Gazzin et al., 2008; Niehof and Borlak, 2009; Ek et al., 2010; Reichel et al., 2011), however their function in this position is unknown. 3-Inward transporters (e.g., SLC family) actively transport ions, amino acids, and other small molecules across both basolateral and apical surfaces of plexus epithelial cells into the CSF. Without this active transport these molecules would be unable to cross the blood-CSF barriers as they are too hydrophilic and/or highly polarized. 4-Bi-directional transporters (e.g., OAT family). 5-Protein transporters (e.g., SPARC for albumin) specifically target individual proteins (or classes of protein) and transport them across the cells. 6-Vesicular transport/endocytosis due to presence of specific receptors (e.g., transthyretin receptor, TTR; insulin receptor, INSR) or non-specific mediators (e.g., vesicle-associated membrane proteins, VAMPs). 7-Bulk water flow from blood to CSF via aquaporin transporters, specifically AQP1 (Johansson et al., 2005).

From early in development and throughout adulthood, the occlusion of pathways between plexus epithelial cells produce an environment that allows for gradients to exist across the interface. These ion gradients are due to the presence of transporters on both sides of the choroid plexus epithelium and can transport multiple ions such as $\mathrm{Cl}^{-} / \mathrm{HCO}^{3-}$ on the basolateral surface (Lindsey et al., 1990) or $\mathrm{Na}^{+} / \mathrm{K}^{+} / \mathrm{Cl}^{2-}$ on the apical surface (Pershing and Johanson, 1982; Plotkin et al., 1997). In addition water channels, namely aquaporin-1, important for water transfer across choroid plexus both in the adult and during development, are found on the epithelial cell surfaces Nielsen et al., 1993; Johansson et al., 2005). Plexus epithelial cells are also able to aid in removal of compounds toxic to the nervous system. In one bizarre case, a person with a bullet wound to the head was reported to have a lateral ventricular choroid plexus engorged with copper and wrapped around the bullet lodged in the ventricle (Buwembo and DeVilliers, 1995)—with $\mathrm{Cu}^{2+}$ ion likely being transported out of the ventricles through SLC31A1/SLC31A2 copper transporters on the choroid plexus epithelium (see Saunders et al., 2013).

It has been known for many years that solute carrier (SLC) transporters (for review see Hediger et al., 2004; www.genenames. org/genefamilies/SLC) are both expressed and functionally active in the cerebral endothelial cells that comprise the blood-brain barrier (Kusuhara and Sugiyama, 2004; Simpson et al., 2007; Dahlin et al., 2009; Ho et al., 2012). It is now known that over $65 \%$ of the nearly 400 SLC transporters are also expressed by epithelial cells of the choroid plexus (Saunders et al., 2013). The main transporters defined from physiological studies are those for glucose (GLUT-1), amino acids (acidic, basic, neutral) monocarboxylic acids and ions, including metabolically important ions such as $\mathrm{Fe}^{2+}, \mathrm{Cu}^{2+}$ and $\mathrm{Mg}^{+}$(Bito and Myers, 1970; Bradbury et al., 1972; Amtorp and Sørensen, 1974; Oldendorf, 1977; Cornford et al., 1982; Morgan and Moos, 2002; amongst others). As there is such a large number of SLC transcripts, it can prove difficult to assign these examples of more specific molecules for which there is already a wealth of physiological evidence of transport available. It was assumed in many early studies focussed on entry of molecules into brain that entry was via the blood-brain barrier interface (e.g., Oldendorf, 1977; Cornford et al., 1982; Lefauconnier and Trouvé, 1983) and the extent of transport of a second entry route possible via the choroid plexuses was not fully investigated. In addition, it was not clear from some of these reports if the CSF and choroid plexuses had been removed prior to analysis of brain samples - meaning any choroid plexus tissue or CSF included in brain samples may have led to an overestimate of the contribution of blood-brain barrier transport of the amino acids into the brain. This contamination though small is possible to alter the outcome of experiments as at least some amino acids are reported to accumulate in the choroid plexuses (al-Sarraf et al., 1997a) in addition to entering the CSF directly. Separate assessment of CSF in the developing brain, mainly reflecting entry across choroid plexuses, has been examined by Segal and colleagues (al-Sarraf et al., 1997b). More recently, expression of a large number of SLC transporters has been reported in the embryonic and adult choroid plexus (Marques et al., 2009; Liddelow et al., 2010, 2012; Ho et al., 2012; Saunders et al., 2013), however little physiological investigation has been completed on their function or activity.

Aquaporin-1, the main water channel in the choroid plexus, is present in plexus epithelial cells as soon as they begin differentiation (Johansson et al., 2005), making water transport possible from the earliest moments in choroid plexus development. The gradient that drives the water transport however does not appear to be regulated in development in the same manner as in the adult (Johansson et al., 2008a; Liddelow et al., 2013). The two components that have proved crucial for driving an influx of water and thus production of CSF: $\mathrm{Na}+/ \mathrm{K}+$ ATPase and carbonic anhydrase (for review see Davson and Segal, 1996; Catala, 1998; Zheng and Chodobski, 2005), are not present in the early developing choroid plexus (Johansson et al., 2008a). An early function 
water influx may be due to the high protein concentration in the CSF of these young animals - causing an osmotic pressure gradient across the choroid plexus (see below and Johansson et al., 2008b).

The ever complex mammalian brain contains diverse cell types and distinct microstructures bound and protected by a number of both physical and enzymatic/physiological brain barriers. The large numbers of transporters present at the blood-CSF barrier likely play crucial roles in energy metabolism, nutrient supply, as well as CSF production and neurotransmitter regulation in the brain, particularly during development. The now debunked dogma that the brain develops without functional brain barriers has been replaced with the realization that dysfunction of specific transporter mechanisms, either genetic or acquired through some pathological process, likely play more important roles in subsequent mal-development of the brain or neuropsychiatric disorder later in life than. Thus these transporters are also potential pharmaceutical targets for treatment of such diseases, as they are possible targets for drug entry and toxin removal in both the developing and adult brain.

\section{PROTEIN}

CSF in the developing brain contains characteristically high concentrations of plasma-derived protein when compared to the adult. Levels for embryonic mice and rats are in the range of several $100 \mathrm{mg} / 100 \mathrm{ml}$, whereas in the adult these levels fall to around $10 \mathrm{mg} / 100 \mathrm{ml}$ (Davson and Segal, 1996), while over the same time the level of plasma proteins circulating in the blood increases from several $100 \mathrm{mg} / 100 \mathrm{ml}$ to several thousand. The same trends are consistent for rodents, marsupials and humans. The main route of entry for this protein from plasma into CSF and brain is via specific protein-transferring cells of the choroid plexus (Møllgård and Saunders, 1977; Dziegielewska et al., 1980; Johansson et al., 2006; Liddelow et al., 2009). Although the route of this transfer has been identified as intracellular, the actual mechanism remains unknown (Knott et al., 1997; Liddelow et al., 2009). Once proteins have transferred across the choroid plexus into CSF, some are taken up into cells in the brain, while others continue through the ventricular system to be reabsorbed by the arachnoid granulations on the outer surface of the brain. For example some neuroependymal cells lining the cerebral ventricles take up proteins such as albumin and the fetal protein fetuin (Dziegielewska et al., 2000). The initial cells that form the first layers of the neocortex in the embryo take up fetuin via apical dendrites in contact with the dorsal surface of the cortex (Dziegielewska et al., 2000). Other plasma proteins such as albumin and alfa-fetoprotein are also transported via the same route from early during development, however, this uptake has been little studied and it is not clear whether the proteins themselves are functionally important or bound ligands such as hormones and growth factors are the precious cargo. A recent publication indicated the number of plasma protein positive cells in the ventricular zone of a fetal mouse can be increased following an inflammatory response of the dam-indicating protein uptake by the brain can be physiologically responsive to alterations in uterine environment (Stolp et al., 2011). It is also known that this choroid plexus protein transport system is dynamic - able to adapt to acute alterations in levels of individual proteins circulating in the plasma (Liddelow et al., 2014). What is not known is whether or not the choroid plexus is able to maintain the protein composition of the CSF after chronic alterations in circulating proteins.

The first cells that differentiate to become choroid plexus epithelial cells appear able to transport protein immediately, with no apparent preference provided to cells of different stages of development. Although there appears to be a higher degree of specificity for individual proteins earlier in the development (Liddelow et al., 2010), adult cells are equally able to bulk transfer a range of proteins into the CSF without there being any trauma or damage to the blood-CSF barrier interface. The requirement for protein in the CSF and CNS is likely two-fold: initially the high protein concentration reported in the CSF of early developing animals sets up an osmotic pressure gradient causing the influx of water and consequently improving ventricular expansion and normal brain growth and development (Johansson et al., 2005); and secondly, these plasma proteins are constantly attached with large number of growth factors and other required molecules for normal CNS maintenance-thus active transport of protein into the CSF is a way to control import of other factors not produced locally (Liddelow et al., 2012).

\section{AGING AND DISEASE}

The choroid plexus is unique in the CNS in that the cells, once born and fully matured, do not undergo replacement or degeneration under normal conditions. In the adult, the proliferation of choroid plexus epithelium has been shown to occur at a low rate-less than $0.1 \%$ of total plexus cells per day (Altman and Das, 1965; Doetsch et al., 1999; Liddelow et al., 2010), but no data are available on the senescent plexus. There are few reports of disease of the choroid plexus epithelium itself many cases of plexus disability result from endothelioma of the blood vessels, invading metastatic cancerous growth (accounting for less than $0.14 \%$ of all cerebral metastases-Gopal et al., 2008), or inflammatory lesions, however papilloma of the epithelium itself seems to be the most common, though still only accounting for less than $1 \%$ of all brain tumors (see Rickert and Paulus, 2000 for review). It is important to note however the high expression of Vimentin (Vim) by nearly all choroid plexus tumors suggests a vascular cell abnormality, while the presence of the astrocytic marker glial fibrilliary acidic protein $(G f a p)$ in up to $50 \%$ of plexus neoplasms suggests some conversion of epithelial cells to glial lineages (Rickert and Paulus, 2000), as has been reported for plexus implantation in spinal cord (Ide et al., 2001; Kitada et al., 2001). Other reports in multiple sclerosis describe HLA-DR (a MHC class II cell surface receptor encoded by the human leukocyte antigen) composites on choroid plexus epithelial cells (Vercellino et al., 2008), however closer inspection of the micrographs suggest these deposits are actually present in epiplexus cells as they are never central to the epithelial cell and always contain a separate nucleus. It seems that regardless of case-study age, race, or location, these diseases of the choroid plexus are rare, and to date no study descriptions or animal models are able to accurately describe insufficiencies in normal plexus function (although anencephalic expansion due to excess CSF production by the choroid plexuses is widely reported). 
Rarer still are descriptions of changes in the choroid plexus during normal aging. The choroid plexus performs continuous and vital function, producing up to $75-90 \%$ (approximately 450 $1000 \mathrm{ml} /$ day) of the CSF (see Davson and Segal, 1996). This fluid adequately nourishes the brain prior to full vascularization (see above) and provides buoyant suspension and protection to the brain and spinal cord. During normal aging there have been reports of filamentous, ring-like or arc-like structures in the epithelium of the choroid plexus, termed Biondi bodies (Biondi, 1933; Oksche and Kirschstein, 1972; Kiktenko, 1986; Wen et al., 1999; see also Figure 4). Structurally these filamentous rings are associated with lipid droplets, and appear to develop within the epithelial cells themselves (and thus may be agents of cellular destruction) rather than in the extracellular matrix, however the study by Kiktenko (1986) makes special mention of the fact they were unable to find convincing signs of damage in plexus epithelial cells with large-sized Biondi bodies. Inspection of the electron micrographs however clearly shows examples of these tangled rings bursting forth from ruptured plexus epithelial cells, while nearby ring-free cells are noticeably undamaged (see Figure 4B). These Biondi bodies, or Biondi ring tangles, are not seen between adjacent cells - only in the cytoplasm of plexus epithelia. This intracellular location of the Biondi bodies and their state of preservation compared to other cytoplasmic elements suggest a destructive effect on epithelial cells of choroid plexuses. The more common occurrence in Alzheimer's disease (Miklossy et al., 1998) suggests dementia disease states may cause premature aging of the choroid plexus, as epithelial atrophy is significantly accentuated (as reported by a decrease in cell height is observed compared to age-matched controls (Serot et al., 2000). Choroid epithelial cells also acquire numerous other lipofuscin vacuoles (along with Biondi bodies) in Alzheimer's patients (Miklossy et al., 1998; Wen et al., 1999; Alvira-Botero and Carro, 2010). These Biondi inclusions have thus far proved difficult to image extensively, as the presence only in higher-order primates and humans presents issues for both tissue availability and preservation. The light and transmission electron micrographs provided by Wen et al. (1999; see Figures 4A,C) come from material obtained from tissue banks and as such no information is provided on tissue preparation protocols. Older material prepared for scanning electron microscopy by Kiktenko (1986; see Figure 4B) was prepared from a wide range of aged and otherwise healthy human autopsies that were fixed within $1.5-4.5 \mathrm{~h}$ after death. In these images it is possible to see choroid plexus epithelial cells bursting, possibly due to Biondi ring inclusions and not an artifact of fixation as all burst cells contain these ring structures. Whatever their effect on the plexus, it is true to say that Biondi bodies are characteristic of choroidal epithelia of aged humans. Their absence in young-to-middle aged non-human primates, as well as their absence in various senescent mammals (rodents, dogs, and cats) and birds, has led to suggestions they may relate to differences in brain senescence between humans and other animals. However, Biondi-like inclusions have been identified in an aged (43 year old) male chimpanzee (Oksche et al., 1984).

The choroid plexus therefore demonstrates a robust accumulation of pathological changes, in the form of Biondi bodies, with normal aging, however the changes to normal function are
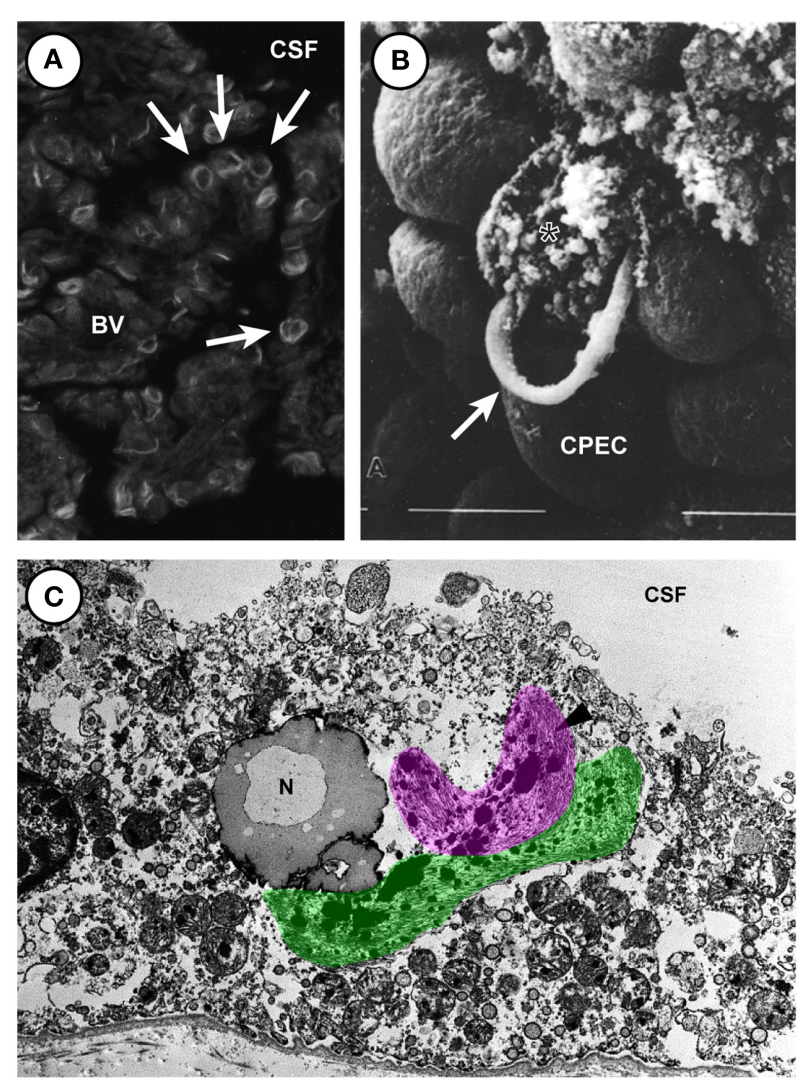

FIGURE 4 | Biondi ring tangles in aged human choroid plexus of the lateral and fourth choroid plexus. (A) Fluorescent light micrograph of thioflavin S-stained Biondi ring tangles (arrows) in the choroid plexus of the brain of a 78-year-old human female with Alzheimer's disease showing Biondi ring tangles appear as ring, tangle, serpentine, and curled profiles. (Magnification: $530 \times$ ). (B) Scanning electron micrograph (Magnification $2500 \times$ ) showing destruction of plexus epithelial cell containing ring-like Biondi inclusions. Arrow marks a ring bursting from an individual plexus epithelial cell. Material from 78 year old woman. (C) Electron micrograph of a choroid plexus from a 70-year-old male with Alzheimer's disease showing the fibrous Biondi ring tangles (one highlighted in pink, the other in green) associated with lipofuscin granules, mitochondria, and other cellular components. (Magnification: 10,300x). (A,C) Reproduced from Wen et al., 1999 Copyright @1999 Elsevier Science B.V. All rights reserved. (B) Reproduced from (Kiktenko, 1986) Copyright (C1986 Springer All rights reserved. Abbreviations: BV, blood vessel; CPEC, choroid plexus epithelial cell; CSF, cerebrospinal fluid; $N$, nucleus.

difficult to ascertain, as no rodent examples are reported thus study of their effects cannot be completed. It is likely however these modifications could alter choroid plexus function, including synthesis, secretion, and transport of proteins and other molecules. A possible reason for this amazing cellular longevity is the high expression of the aging repressor Klotho ( $K l$, see Liddelow et al., 2012). KLOTHO protein is a serum circulating factor that declines with age (Kuro-o et al., 1997; Xiao et al., 2004). Mice deficient for KLOTHO protein manifest a syndrome similar to accelerated human aging and display rapid and extensive arteriosclerosis. Although the vast majority of research has been based on lack of KLOTHO, it is demonstrated an overexpression 
of $K l$ transcript in mice extends their average life span between 19 and 31\% compared to normal mice (Kurosu et al., 2005). The high expression of $K l$ throughout plexus development and into the adult suggests some protective effect on plexus epithelial cells themselves, and also on other CNS cells - especially considering evidence that KLOTHO protein levels in the CSF are decreased in Alzheimer's disease humans (Semba et al., 2014).

To date, the only study of the choroid plexus in systemic disease has been reported by Dohrmann and Herdson (1969). They used NZB/NZW hybrid mice, a strain which develops a disease resembling human systemic lupus erythematosus. Fine structural examination of the choroid plexus revealed an irregular, homogeneous thickening of the capillary basement membranes. Other miscellaneous reports on the pathology of the choroid plexus include Hoff (1930), Rand and Courville (1931) and Leviton et al. (1977). Hoff (1930) reported on increased permeability of the choroid plexus in experimental head injury. Another survey of 62 cases of fatal cerebral trauma noted oedema of the stroma and vacuolation of the epithelial cells of the choroid plexus (Rand and Courville, 1931). The presence of amyloid in the choroid plexus of elderly brains was described by Divry (in Blackwood et al., 1963). Amyloid was present within the choroidal epithelium, confined to the free margins of the cells.

\section{CONCLUDING REMARKS}

On February 7th 1922, Sir John Bland-Sutton gave what was by all accounts an energetic emeritus lecture at Middlesex Hospital, Enland, entitled "The choroid plexus and psammomas." As an introduction to the choroid plexus he commented "The rufflelike structures, called choroid plexuses, which float in the ventricular fluid of man's brain, have aroused the curiosity of anatomists for centuries, yet they took little more interest in them than gardeners take in weeds." Though we have moved on from humble gardeners, until recently the neuroscience community has lagged in the uptake of important studies focussed on the choroid plexus. As a structure that produces and secretes CSF, controls and protects the internal environment of the adult CNS, and is present even before vascularization of other cortical structures, surely we have sufficient evidence to justify serious investigations into this small epithelial tissue mass in the ventricles of all vertebrates. This review has touched on the early development of the choroid plexuses, and its emergence as a possible location of serious complications in aging and disease. Indeed the close homeostatic control of the internal milieu of the CNS: the CSF, glia, and neurons, plays a vital role in normal and abnormal brain function. Dysfunctions of all of the brain barriers contribute heavily to the pathology of neurological conditions, however the added detriment of a dysfunctional choroid plexus during development is additional reason for concern. A proper understanding of the choroid plexus will likely prove important for the production of drug delivery and therapies to help ameliorate a wide range of neurological diseases.

\section{REFERENCES}

Altman, J., and Das, G. D. (1965). Autoradiographic and histological evidence of postnatal hippocampal neurogenesis in rats. J. Comp. Neurol. 124, 319-335. doi: $10.1002 /$ cne. 901240303
al-Sarraf, H., Preston, J. E., and Segal, M. B. (1997a). Acidic amino acid accumulation by rat choroid plexus during development. Brain Res. Dev. Brain Res. 102, 47-52. doi: 10.1016/S0165-3806(97)00075-8

al-Sarraf, H., Preston, J. E., and Segal, M. B. (1997b). Changes in the kinetics of the acidic amino acid brain and CSF uptake during development in the rat. Brain Res. Dev. Brain Res. 102, 27-134.

Alvira-Botero, X., and Carro, E. M. (2010). Clearance of amyloid- $\beta$ peptide across the choroid plexus in Alzheimer's disease. Curr. Aging Sci. 3, 19-229. doi: $10.2174 / 1874609811003030219$

Amtorp, O., and Sørensen, S. C. (1974). The ontogenetic development of concentration differences for protein and ions between plasma and cerebrospinal fluid in rabbits and rats. J. Physiol. 243, 387-400. doi: 10.1113/jphysiol.1974.sp010759

Awatramani, R., Soriano, P., Rodriguez, C., Mai, J. J., and Dymecki, S. M. (2003). Cryptic boundaries in roof plate and choroid plexus identified by intersectional gene activation. Nat. Genet. 35, 70-75. doi: 10.1038/ng1228

Bach, A., Lallemand, Y., Nicola, M. A., Ramos, C., Mathis, L., Maufras, M., et al. (2003). Msx1 is required for dorsal diencephalon patterning. Development 130, 4025-4036. doi: 10.1242/dev.00609

Bailey, P. (1916). Morphology of the roof plate of the fore-brain and the lateral choroid plexuses in the human embryo. J. Comp. Neurol. 26, 79-120. doi: 10.1002/cne.900260104

Balslev, Y., Dziegielewska, K. M., Møllgård, K., and Saunders, N. R. (1997). Intercellular barriers to and transcellular transfer of albumin in the fetal sheep brain. Anat. Embryol. (Berl.) 195, 229-236. doi: 10.1007/s0042900 50042

Bauer, H. C., Bauer, H., Lametschwandtner, A., Amberger, A., Ruiz, P., and Steiner, M. (1993). Neovascularization and the appearance of morphological characteristics of the blood-brain barrier in the embryonic mouse central nervous system. Brain Res. Dev. Brain Res. 75, 269-278. doi: 10.1016/0165-3806(93) 90031-5

Biondi, G. (1933). Ein neuer histologischer befund am epithel des plexus chorioideus. Z. Ges. Neurol. Psychiatry 144, 161-165. doi: 10.1007/BF02870278

Bito, L. Z., and Myers, R. E. (1970). The ontogenesis of haematoencephalic cation transport processes in the rhesus monkey. J. Physiol. 208, 153-170. doi: 10.1113/jphysiol.1970.sp009111

Blackwood, W., McMenemey, W. H., Meyer, A., Norman, R. M., and Russell, D. S. (1963). Greenfield's Neuropathology. Baltimore, MD: Williams and Wilkins.

Bolos, M., Spuch, C., Ordoñez-Gutierrez, L., Wandosell, F., Ferrer, I., and Carro, E. (2013). Neurogenic effects of $\beta$-amyloid in the choroid plexus epithelial cells in Alzheimer's disease. Cell. Mol. Life Sci. 70, 2787-2797. doi: 10.1007/s00018-0131300-x

Bradbury, M. W., Crowder, J., Desai, S., Reynolds, J. M., Reynolds, M., and Saunders, N. R. (1972). Electrolytes and water in the brain and cerebrospinal fluid of the foetal sheep and guinea-pig. J. Physiol. 227, 591-610. doi: 10.1113/jphysiol.1972.sp010049

Brightman, M. W., and Reese, T. S. (1969). Junctions between intimately apposed cell membranes in the vertebrate brain. J. Cell Biol. 40, 648-677. doi: 10.1083/jcb.40.3.648

Buwembo, J. D., and DeVilliers, J. C. (1995). Migrating retained intracranial missiles. African J. Neurol. Sci. 14:189.

Campos-Ortega, J. A., and Jan, Y. N. (1991). Genetic and molecular bases of neurogenesis in drosophila melanogaster. Annu. Rev. Neurosci. 14, 399-420. doi: 10.1146/annurev.ne.14.030191.002151

Cancilla, P. A., Zimmerman, H. M., and Becker, N. H. (1966). A histochemical and fine structure study of the developing rat choroid plexus. Acta Neuropathol. 6, 188-200. doi: 10.1007/BF00686764

Catala, M. (1998). Embryonic and fetal development of structures associated with the cerebro-spinal fluid in man and other species. Part I: The ventricular system, meninges and choroid plexuses. Arch. Anat. Cytol. Pathol. 46, 153-169.

Chen, J., Knowles, H. J., Hebert, J. L., and Hackett, B. P. (1998). Mutation of the mouse hepatocyte nuclear factor/forkhead homologue 4 gene results in an absence of cilia and random left-right asymmetry. J. Clin. Invest. 102, 1077-1082. doi: 10.1172/JCI4786

Cohen, H., and Davies, S. (1939). A specific factor in extracts of the choroid plexus influencing the permeability of the myelencephalic roof. J. Anat. 73, 525-539.

Cornford, E. M., Braun, L. D., and Oldendorf, W. H. (1982). Developmental modulations of blood-brain barrier permeability as an indicator of changing nutritional requirements in the brain. Pediatr. Res. 16(4 Pt 1), 324-328. doi: 10.1203/00006450-198204000-00017 
Currle, D. S., Cheng, X., Hsu, C. M., and Monuki, E. S. (2005). Direct and indirect roles of CNS dorsal midline cells in choroid plexus epithelia formation. Development 132, 3549-3559. doi: 10.1242/dev.01915

Dagnino, L., Fry, C. J., Bartley, S. M., Farnham, P., Gallie, B. L., and Phillips, R. A. (1997). Expression patterns of the E2F family of transcription factors during mouse nervous system development. Mech. Dev. 66, 13-25. doi: 10.1016/S09254773(97)00083-X

Dahlin, A., Royall, J., Hohmann, J. G., and Wang, J. (2009). Expression profiling of the solute carrier gene family in the mouse brain. J. Pharmacol. Exp. Ther. 329, 558-570. doi: 10.1124/jpet.108.149831

Dandy, W. E. (1918). Extirpation of the choroid plexus of the lateral ventricles in communicating hydrocephalus. Ann. Surg. 68, 569-579. doi: 10.1097/00000658191812000-00001

Davson, H., and Segal, M. B. (1996). Physiology of The CSF and Blood-Brain Barriers. Boca Raton, FL: CRC Press.

Doetsch, F., Caille, I., Lim, D. A., Garcia-Verdugo, J. M., and Alvarez-Buylla, A. (1999). Subventricular zone astrocytes are neural stem cells in the adult mammalian brain. Cell 97, 703-716. doi: 10.1016/S0092-8674(00)80783-7

Dohrmann, G. J., and Herdson, P. B. (1969). Fine structural studies of capillaries in NZB/NZW mice. Exp. Mol. Path. 11, 163-171. doi: 10.1016/00144800(69)90005-7

Dziegielewska, K. M., Daikuhara, Y., Ohnishi, T., Waite, M. P., Ek, J., Habgood, M. D., et al. (2000). Fetuin in the developing neocortex of the rat: distribution and origin. J. Comp. Neurol. 423, 373-388. doi: 10.1002/1096-9861(20000731) 423:3<373::AID-CNE2>3.0.CO;2-D

Dziegielewska, K. M., Ek, C. J., Habgood, M. D., and Saunders, N. R. (2001). Development of the choroid plexus. Miscrosc. Res. Tech. 52, 5-20. doi: 10.1002/ 1097-0029(20010101)52:1<5::AID-JEMT3>3.3.CO;2-A

Dziegielewska, K. M., Evans, C. A., Malinowska, D. H., Møllgård, K., Reynolds, M. L., and Saunders, N. R. (1980). Blood-cerebrospinal fluid transfer of plasma proteins during fetal development in the sheep. J. Physiol. 300, 457-465. doi: 10.1113/jphysiol.1980.sp013172

Ek, C. J., Dziegielewska, K. M., Stolp, H., and Saunders, N. R. (2006). Functional effectiveness of the blood-brain barrier to small water-soluble molecules in developing and adult opossum (Monodelphis domestica). J. Comp. Neurol. 496, 13-26. doi: 10.1002/cne.20885

Ek, C. J., Habgood, M. D., Dziegielewska, K. M., and Saunders, N. R. (2003). Structural characteristics and barrier properties of the choroid plexuses in developing brain of the opossum (Monodelphis domestica). J. Comp. Neurol. 460, 451-464. doi: 10.1002/cne.10661

Ek, C. J., Wong, A., Liddelow, S. A., Johansson, P. A., Dzeigielewska, K. M., and Saunders, N. R. (2010). Efflux mechanisms at the developing brain barriers: ABC-transporters in the fetal and postnatal rat. Toxicol. Lett. 197, 51-59. doi: 10.1016/j.toxlet.2010.04.025

Ehrlich, P. (1885). Sauerstoff-Bedürfniss des Organismus Eine Farbenanalytische Studie. Berlin: Hirschwald.

Evans, C. A., Reynolds, J. M., Reynolds, M., Saunders, N. R., and Segal, M. B. (1974). The development of the blood-brain barrier and choroid plexus function in immature foetal sheep. J. Physiol. 224, 15P-16P. doi: 10.1113/jphysiol.1974.sp010596

Fernandes, M., Gutin, G., Alcorn, H., McConnell, S. K., and Hébert, J. M. (2007). Mutations in the BMP pathway in mice support the existence of two molecular classes of holoprosencephaly. Development 134, 3789-3794. doi: 10.1242/dev.004325

Furuta, Y., Piston, D. W., and Hogan, B. L. (1997). Bone morphogenic proteins (BMPs) as regulators of dorsal forebrain development. Development 124, 2203-2212.

Gao, N., and Meier, P. J. (2001). Organic anion transport across the choroid plexus. Microsc. Res. Tech. 52, 60-64. doi: 10.1002/1096-9861(20000731)423:3<373:: AID-CNE2>3.0.CO;2-D

Gazzin, S., Strazielle, N., Schmitt, C., Fevre-Montange, M., Ostrow, J. D., Tiribelli, C., et al. (2008). Differential expression of the multidrug resistance-related proteins $\mathrm{ABCb} 1$ and $\mathrm{ABCcl}$ between blood-brain interfaces. J. Comp. Neurol. 520, 497-507. doi: 10.1002/cne.21808

Goldmann, E. E. (1909). Die äussere und Sekretion des gesunden und kranken organismus im lichte der "vitalen färbung." Beitr. Klin. Chirurz. 64, 192-265.

Gopal, P., Park, J. R., Debski, R., and Parker, J. C. (2008). Choroid plexus carcinoma. Arch. Pathol. Lab. Med. 132, 1350-1354. doi: 10.1043/15432165(2008)132[1350:CPC]2.0.CO;2
Grove, E. A., Tole, S., Limon, J., Yip, L., and Ragsdale, C. W. (1998). The hem of the embryonic cerebral cortex is defined by the expression of multiple Wnt genes and is compromised in Gli3-deficient mice. Development 125, 2315-2325.

Habgood, M. D., Sedgwick, J. E., Dziegielewska, K. M., and Saunders, N. R. (1992). A developmentally regulated blood-cerebrospinal fluid transfer mechanism for albumin in immature rats. J. Physiol. 456, 181-192. doi: 10.1113/jphysiol.1992.sp019332

Hallaert, G. G., Vanhauwaert, D. J., Loggle, K., Van den Broecke, C., Baert, E., Van Roost, D., et al. (2012). Endoscopic coagulation of choroid plexus hyperplasia. J. Neurosurg. Pediatr. 9, 169-177. doi: 10.3171/2011.11.PEDS11154

Hébert, J. M., Mishina, Y., and McConnel, S. K. (2002). BMP signalling is required locally to pattern the dorsal telencephalic midline. Neuron 35, 1029-1041. doi: 10.1016/S0896-6273(02)00900-5

Hediger, M. A., Romero, M. F., Peng, J. B., Rolfs, A., Takanaga, H., and Bruford, E. A. (2004). The ABCs of solute carriers: physiological, pathological and therapeutic implications of human membrane transport proteins. Pflugers Arch. 447, 465-468. doi: 10.1007/s00424-003-1192-y

Ho, H. T., Dahlin, A., and Wang, J. (2012). Expression profiling of solute carrier gene families at the blood-CSF barrier. Front. Pharmacol. 3:154. doi: 10.3389/fphar.2012.00154

Hoff, H. (1930). Experimentelle Studien zur Frage des postkommotionellen Hirnödems. Z. Ges. Neurol. Psychiat. 129, 583-590. doi: 10.1007/BF02865077

Hunter, N. L., and Dymecki, S. M. (2007). Molecularly and temporally separable lineages form the hindbrain roof plate and contribute differentially to the choroid plexus. Development 134, 3449-3460. doi: 10.1242/dev.003095

Ide, C., Kitada, M., Chakrabortty, S., Taketomi, M., Matsumoto, N., Kikukawa, S., et al. (2001). Grafting of choroid plexus ependymal cells promotes the growth of regenerating axons in the dorsal funiculus of rat spinal cord: a preliminary report. Exp. Neurol. 167, 242-251. doi: 10.1006/exnr.2000.7566

Imayoshi, I., Shimogori, T., Ohtsuka, T., and Kageyama, R. (2008). Hes genes and neurogenin regulate non-neural versus neural fate specification in the dorsal telencephalic midline. Development 135, 2531-2541. doi: 10.1242/dev.021535

Itokazu, Y., Kitada, M., Dezawa, M., Mizoguchi, A., Matsumoto, N., Shimizu, A., et al. (2006). Choroid plexus ependymal cells host neural progenitor cells in the rat. Glia 53, 32-42. doi: 10.1002/glia.20255

Johanson, C. E., Reed, D. J., and Woodbury, D. M. (1976). Developmental studies of the compartmentalization of water and electrolytes in the choroid plexus of the neonatal rat brain. Brain Res. 116, 35-48. doi: 10.1016/0006-8993(76)90247-X

Johansson, P. A., Dziegielewska, K. M., Ek, C. J., Habgood, M. D., Liddelow, S. A., Potter, A. M., et al. (2006). Blood-CSF barrier function in the rat embryo. Eur. J. Neurosci. 24, 65-76. doi: 10.1111/j.1460-9568.2006.04904.x

Johansson, P. A., Dziegielewska, K. M., Ek, C. J., Habgood, M. D., Møllgård, K., Potter, A., et al. (2005). Aquaporin-1 in the choroid plexuses of developing mammalian brain. Cell Tissue. Res. 322, 353-364. doi: 10.1007/s00441-0051120-x

Johansson, P. A., Dziegielewska, K. M., Liddelow, S. A., and Saunders, N. R. (2008b). The blood-CSF barrier explained: when development is not immaturity. Bioessays 30, 237-248. doi: 10.1002/bies.20718

Johansson, P. A., Dziegielewska, K. M., and Saunders, N. (2008a). Low levels of $\mathrm{Na}, \mathrm{K}$-ATPase and carbonic anhydrase II during choroid plexus development suggest limited involvement in early CSF secretion. Neurosci. Lett. 442, 77-80. doi: 10.1016/j.neulet.2008.06.068

Johansson, P. A., Irmler, M., Acampora, D., Beckers, J., Simeone, A., and Götz, M. (2013). The transcription factor Otx2 regulates choroid plexus development and function. Development 140, 1055-1066. doi: 10.1242/dev.090860

Kageyama, R., Ohtsuka, T., and Kobayashi, T. (2007). The Hes gene family: repressors and oscillators that orchestrate embryogenesis. Development 134, 1243-1351. doi: 10.1242/dev.000786

Kappers, J. A., Ten Kate, I., and De Bruyn, H. J. (1958). On mast cells in the choroid plexus of the axolotl (Ambystoma mex.). Z. Zellforsch. Mikrosk. Anat. 48, 617-634. doi: 10.1007/BF00398650

Keep, R. F., and Jones, H. C. (1990). A morphometric study on the development of the lateral ventricle choroid plexus, choroid plexus capillaries and ventricular ependymal in the rat. Brain Res. Dev. Brain Res. 56, 47-53. doi: 10.1016/01653806(90)90163-S

Kiktenko, A. I. (1986). Biondi bodies in the choroid plexus epithelium of the human brain. A scanning electron-microscopic study. Cell Tissue Res. 244, 239-240. doi: 10.1007/BF00218405 
Kitada, M., Chakrabortty, S., Matsumoto, N., Taketomi, M., and Ide, C. (2001). Differentiation of choroid plexus ependymal cells into astrocytes after grafting into the pre-lesioned spinal cord in mice. Glia 36, 364-374. doi: 10.1002/glia.1123

Knott, G. W., Dziegielewska, K. M., Habgood, M. D., Li, Z. S., and Saunders, N. R. (1997). Albumin transfer across the choroid plexus of South American opossum (Monodelphis domestica). J. Physiol. 499, 179-194. doi: 10.1113/jphysiol.1997.sp021919

Knudsen, P. A. (1964). The surface area of the choroid plexus in normal mouse embryos. Acta. Anat. 58, 355-367. doi: 10.1159/000142595

Kratzer, I., Liddelow, S. A., Saunders, N. R., Dziegielewska, K. M., Strazielle, N., and Ghersi-Egea, J. F. (2013). Developmental changes in the transcriptome of the rat choroid plexus in relation to neuroprotection. Fluids Barriers CNS 10:25. doi: 10.1186/2045-8118-10-25

Kume, H., Maruyama, K., Shinozaki, K., Kuzume, H., and Obata, K. (1998). Phosphorylation and spatiotemporal distribution of KW8 (NDRF/NeuroD2) a NeuroD family basic helix-loop-helix protein. Brain Res. Mol. Brain Res. 60 107-114. doi: 10.1016/S0169-328X(98)00176-4

Kuro-o, M., Matsumura, Y., Aizawa, H., Kawaguchi, H., Suga, T., Utsugi, T., et al. (1997). Mutation of the mouse klotho gene leads to a syndrome resembling ageing. Nature 390, 45-51. doi: 10.1038/36285

Kurosu, H., Yamamoto, M., Clark, J. D., Pastor, J. V., Nandi, A., Gurnani, P., et al. (2005). Suppression of aging in mice by the hormone Klotho. Science 309, 1829-1833. doi: 10.1126/science.1112766

Kusuhara, H., and Sugiyama, Y. (2004). Efflux transport systems for organic anions and cations at the blood-CSF barrier. Adv. Drug Deliv. Rev. 56, 1741-1763. doi: 10.1016/j.addr.2004.07.007

Lefauconnier, J. M., and Trouvé, R. (1983). Developmental changes in the pattern of amino acid transport at the blood-brain barrier in rats. Brain Res. 282, 175-182. doi: 10.1016/0165-3806(83)90095-0

Leviton, A., Gilles, F., and Strassfeld, R. (1977). The influence of route of delivery and hyaline membranes on the risk of neonatal intracranial hemorrhages. Ann. Neurol. 2, 451-454. doi: 10.1002/ana.410020602

Liddelow, S. A. (2011). Fluids and barriers of the CNS: a historical viewpoint. Fluids Barriers CNS 8:2. doi: 10.1186/2045-8118-8-2

Liddelow, S. A., Dziegielewska, K. M., Ek, C. J., Habgood, M. D., Bauer, H., Bauer, H. C., et al. (2013). Mechanisms that determine the internal environment of the developing brain: a transcriptomic, functional and ultrastructural approach. PLoS ONE 8:e65629. doi: 10.1371/journal.pone.006 5629

Liddelow, S. A., Dziegielewska, K. M., Ek, C. J., Johansson, P. A., Potter, A. M., and Saunders, N. R. (2009). Cellular transfer of macromolecules across the developing choroid plexus of Monodelphis domestica. Eur. J. Neurosci. 29, 253-266. doi: 10.1111/j.1460-9568.2008.06571.x

Liddelow, S. A., Dziegielewska, K. M., Møllgard, K. M., Noor, N. M., Wheaton, B. J., Gehwolf, R., et al. (2014). Cellular specificity of the blood-CSF barrier for albumin transfer across the choroid plexus epithelium. PLOS ONE 9:e106592. doi: 10.1371/journal.pone.0106592

Liddelow, S. A., Dziegielewska, K. M., Vandeberg, J. L., and Saunders, N. R. (2010). Development of the lateral ventricular choroid plexus in a marsupial, Monodelphis domestica. Cerebrospinal Fluid Res. 7:16. doi: 10.1186/1743-84547-16

Liddelow, S. A., Temple, S., Møllgård, K., Gehwolf, R., Wagner, A., Bauer, H., et al. (2012). Molecular characterisation of transport mechanisms at the developing mouse blood-CSF interface: a transcriptome approach. PLoS ONE 7:e33554. doi: 10.1371/journal.pone.0033554

Lindeman, G. J., Dagnino, L., Gaubatz, S., Xu, Y., Bronson, R. T., Warren, H. B., et al. (1998). A specific, nonproliferative role for E2F-5 in choroid plexus function revealed by gene targeting. Genes Dev. 12, 1092-1098. doi: 10.1101/gad.12.8.1092

Liebner, S., Czupalla, C. J., and Wolburg, H. (2011). Current concepts of blood-brain barrier development. Int. J. Dev. Biol. 55, 467-476. doi: 10.1387/ijdb.103224sl

Lindsey, A. E., Schneider, K., Simmons, D. M., Baron, R., Lee, B. S., and Kopito, R. R. (1990). Functional expression and subcellular localization of an anion exchanger cloned from choroid plexus. Proc. Natl. Acad. Sci. U.S.A. 87, 5278-5282. doi: 10.1073/pnas.87.14.5278

Marques, F., Sousa, J. C., Coppola, G., Falcao, A. M., Rodrigues, A. J., Geschwind, D. H., et al. (2009). Kinetic profile of the transcriptome changes induced in the choroid plexus by peripheral inflammation. J. Cereb. Blood Flow Metab. 29, 921-932. doi: 10.1038/jcbfm.2009.15

Miklossy, J., Kraftsik, R., Pillevuit, O., Lepori, D., Genton, C., and Bosman, F. T. (1998). Curly fiber and tangle-like inclusions in the ependyma and choroid plexus-a pathogenetic relationship with the cortical Alzheimer-type changes? J. Neuropathol. Exp. Neurol. 57, 1202-1212. doi: 10.1097/00005072-19981200000012

Møllgård, K., Jacobsen, M., Jacobsen, G. K., Clausen, P. P., and Saunders, N. R. (1979). Immunohistochemical evidence for an intracellular localization of plasma proteins in human foetal choroid plexus and brain. Neurosci. Lett. 14, 85-90. doi: 10.1016/0304-3940(79)95349-7

Møllgård, K., and Saunders, N. R. (1977). A possible transepithelial pathway via endoplasmic reticulum in foetal sheep choroid plexus. Proc. R. Soc. Lond. B. Biol. Sci. 199, 321-326. doi: 10.1098/rspb.1977.0142

Monuki, E. S., Porter, F. D., and Walsh, C. A. (2001). Patterning of the dorsal telencephalon and cerebral cortex by a roof plate-Lhx2 pathway. Neuron. 32 , 591-604. doi: 10.1016/S0896-6273(01)00504-9

Morgan, E. H., and Moos, T. (2002). Mechanism and developmental changes in iron transport across the blood-brain barrier. Dev. Neurosci. 24, 106-113. doi: $10.1159 / 000065699$

Netsky, M. G., and Shuangshoti, S. (1970). Studies on the choroid plexus. Neurosci. Res. 3, 131-173. doi: 10.1016/B978-0-12-512503-1.50009-8

Niehof, M., and Borlak, J. (2009). Expression of HNF4alpha in the human and rat choroid plexus: implications for drug transport across the blood-cerebrospinalfluid (CSF) barrier. BMC Mol. Biol. 10:68. doi: 10.1186/1471-2199-10-68

Nielsen, S., Smith, B. L., Christensen, E. I., and Agre, P. (1993). Distribution of the aquaporin CHIP in secretory and resorptive epithelia and capillary endothelia. Proc. Natl. Acad. Sci. U.S.A. 90, 7275-7259. doi: 10.1073/pnas.90.15.7275

Ogiwara, H., Uematsu, K., and Morota, N. (2014). Obliteration of the choroid plexus after endoscopic coagulation. J. Neurosurg. Pediatr. 4, 1-4. doi: 10.3171/2014.10.JNS132643

Oksche, A., and Kirschstein, H. (1972). Formation and ultrastructure of Biondi bodies in the human choroid plexus (biopsy material). Z. Zellforsch. Mikrosk. Anat. 124, 320-341. doi: 10.1007/BF00355034

Oksche, A., Liesner, R., Tigges, J., and Tigges, M. (1984). Intraepithelial inclusions resembling human biondi bodies in the choroid plexus of an aged chimpanzee. Cell Tissue Res. 235, 467-469. doi: 10.1007/BF00217876

Oldendorf, W. H. (1977). The blood-brain barrier. Exp. Eye Res. 25, 177-190. doi: 10.1016/S0014-4835(77)80016-X

Panchision, D. M., Pickel, J. M., Studer, L., Lee, S. H., Turner, P. A., Hazel, T. G., et al. (2001). Sequential actions of BMP receptors control neural precursor cell production and fate. Genes Dev. 15, 2094-2110. doi: 10.1101/gad.894701

Persengiev, S. P., Kondova, I. I., and Kilpatrick, D. L. (1999). E2F4 actively promotes the initiation and maintenance of nerve growth factor-induced cell differentiation. Mol. Cell. Biol. 19, 6048-6056.

Pershing, L. K., and Johanson, C. E. (1982). Acidosis-induced enhanced activity of the Na-K exchange pump in the in vivo choroid plexus: an ontogenetic analysis of possible role in cerebrospinal fluid $\mathrm{pH}$ homeostasis. J. Neurochem. 38, 322-332. doi: 10.1111/j.1471-4159.1982.tb08632.x

Plotkin, M. D., Snyder, E. Y., Hebert, S. C., and Delpire, E. (1997). Expression of the $\mathrm{Na}-\mathrm{K}-2 \mathrm{Cl}$ cotransporter is developmentally regulated in postnatal rat brains: a possible mechanism underlying GABA's excitatory role in immature brain. J. Neurobiol. 33, 781-795.

Porter, F. D., Drago, J., Xu, Y., Cheema, S. S., Wassif, C., Huang, S. P., et al. (1997) Lhx2, a LIM homeobox gene, is required for eye, forebrain, and definitive erythrocyte development. Development 124, 2935-2944.

Rand, C. W., and Courville, C. B. (1931). Histologic studies in cases of fatal injury to the head. II. Changes in the choroid plexus and ependymal. Arch. Surg. 23 357-425. doi: 10.1001/archsurg.1931.01160090002001

Rao, V. V., Dahlheimer, J. L., Bardgett, M. E., Snyder, A. Z., Finch, R. A., Sartorelli, A. C., et al. (1999). Choroid plexus epithelial expression of MDR1 P glycoprotein and multidrug resistance-associated protein contribute to the bloodcerebrospinal-fluid drug-permeability barrier. Proc. Natl. Acad. Sci. U.S.A. 96, 3900-3905. doi: 10.1073/pnas.96.7.3900

Reichel, V., Burghard, S., John, I., and Huber, O. (2011). P-glycoprotein and breast cancer resistance protein expression and function at the blood-brain barrier and blood-cerebrospinal fluid barrier (choroid plexus) in streptozotocininduced diabetes in rats. Brain Res. 1370, 238-245. doi: 10.1016/j.brainres.2010. 11.012 
Ridley, H. (1695). The Anatomy of the Brain. London: S Smith \& B Walford.

Rickert, C. H., and Paulus, W. (2000). Tumors of the choroid plexus. Microsc. Res. Tech. 52, 104-111. doi: 10.1002/1097-0029(20010101)52:1<104::AIDJEMT12>3.0.CO;2-3

Serot, J. M., Béné, M. C., Foliguet, B., and Faure, G. C. (2000). Morphological alterations of the choroid plexus in late-onset Alzheimer's disease. Acta Neuropathol. 99, 105-108. doi: 10.1007/PL00007412

Sarnat, H. B. (1998). Histochemistry and immunocytochemistry of the developing ependyma and choroid plexus. Microsc. Res. Tech. 41, 14-28.

Saunders, N. R., Daneman, R., Dziegielewska, K. M., and Liddelow, S. A. (2013). Barriers of the blood-brain and blood-CSF interfaces in development and in the adult. Mol. Aspects Med. 34, 742-752. doi: 10.1016/j.mam.2012. 11.006

Saunders, N. R., Ek, C. J., Habgood, M. D., and Dziegielewska, K. M. (2008). Barriers in the brain: a renaissance? Trends Neurosci. 31, 279-286. doi: 10.1016/j.tins.2008.03.003

Saunders, N. R., Knott, G. W., and Dziegielewska, K. M. (2000). Barriers in the immature brain. Cell. Mol. Neurobiol. 20, 29-40. doi: 10.1023/A:10069918 09927

Semba, R. D., Moghekar, A. R., Hu, J., Sun, K., Turner, R., Ferrucci, L., et al. (2014) Klotho in the cerebrospinal fluid of adults with and without Alzheimer's disease. Neurosci. Lett. 558, 37-40. doi: 10.1016/j.neulet.2013.10.058

Simpson, I. A., Carruthers, A., and Vannucci, S. J. (2007). Supply and demand in cerebral energy metabolism: the role of nutrient transporters. J. Cereb. Blood Flow Metab. 27, 1766-1791. doi: 10.1038/sj.jcbfm.9600521

Somerford, A. E. (1933). A case of papilloma of the choroid plexus. Arch. Dis. Child 8, 53-56. doi: 10.1136/adc.8.43.53

Speake, T., and Brown, P. D. (2004). Ion channels in epithelial cells of the choroid plexus isolated from the lateral ventricle of rat brain. Brain Res. 1005, 60-66. doi: 10.1016/j.brainres.2004.01.034

Stastný, F., and Rychter, Z. (1976). Quantitative development of choroid plexuses in chick embryo cerebral ventricles. Acta Neurol. Scand. 53, 251-259. doi: 10.1111/j.1600-0404.1976.tb04346.x

Stolp, H. B., Johansson, P. A., Habgood, M. D., Dziegielewska, K. M., Saunders, N. R., and Ek, C. J. (2011). Effects of neonatal systemic inflammation on bloodbrain barrier permeability and behaviour in juvenile and adult rats. Cardiovasc. Psychiatry Neurol. 2011:469046. doi: 10.1155/2011/469046

Sturrock, R. R. (1979). A morphological study of the development of the mouse choroid plexus. J. Anat. 129, 777-796.

Swetloff, A., and Ferretti, P. (2005). Changes in E2F5 intracellular localization in mouse and human choroid plexus epithelium with development. Int. J. Dev. Biol. 49, 859-865. doi: 10.1387/ijdb.051996as

Swetloff, A., Greenwood, S., Wade, A. M., and Ferretti, P. (2006). Growth of choroid plexus epithelium vesicles in vitro depends on secretory activity. J. Cell. Physiol. 208, 549-555. doi: 10.1002/jcp.20687

Tennyson, V. M., and Pappas, G. D. (1964). Fine structure of the developing telencephalic and myencephalic choroid plexus in the rabbit. J. Comp. Neurol. 123 379-411. doi: 10.1002/cne.901230307

Thomas, T., and Dziadek, M. (1993). Capacity to form choroid plexus-like cells in vitro is restricted to specific regions of the mouse neural ectoderm. Development $117,253-262$.

Vercellino, M., Votta, B., Condello, C., Piacentino, C., Romagnolo, A., Merola, A., et al. (2008). Involvement of the choroid plexus in multiple sclerosis autoimmune inflammation: a neuropathological study. J. Neuroimmunol. 199 133-141. doi: 10.1016/j.jneuroim.2008.04.035

Voetmann, E. (1949). On the structure and surface area of the human choroid plexuses. Ugeskr. Laeger. 111, 1051.

von Frowein, J., Wizenmann, A., and Götz, M. (2006). The transcription factors Emx1 and Emx2 suppress choroid plexus development and promote neuroepithelial cell fate. Dev. Biol. 296, 239-252. doi: 10.1016/j.ydbio.2006. 04.461

Vesalius, A. (1543). De humani Corporis Fabrica Libri Septem. Padua: School of Medicine. Available online at: www.archive.org

Wen, G. Y., Wisniewski, H. M., and Kacsak, R. J. (1999). Biondi ring tangles in the choroid plexus of Alzheimer's disease and normal aging brains: a quantitative study. Brain Res. 832, 40-46. doi: 10.1016/S0006-8993(99)01466-3

Willis, T. (1664). Cerebri Anatome. London: Martyn and Allestree.

Wilting, J., and Christ, B. (1989). An experimental and ultrastructural study on the development of the avian choroid plexus. Cell Tissue Res. 255, 487-494. doi: 10.1007/BF00218783

Wolburg, H., Wolburg-Buchholz, K., Liebner, S., and Engelhardt, B. (2001) Claudin-1, claudin-2 and claudin-11 are present in tight junctions of choroid plexus epithelium of the mouse. Neurosci. Lett. 307, 77-80. doi: 10.1016/S03043940(01)01927-9

Wright, E. M. (1978). Transport processes in the formation of the cerebrospinal fluid. Rev. Physiol. Biochem. Pharmacol. 83, 3-34.

Xiao, N. M., Zhang, Y. M., Zheng, Q., and Gu, J. (2004). Klotho is a serum factor related to human aging. Chin. Med. J. 117, 742-747.

Xuan, S., Baptista, C. A., Balas, G., Talo, W., Soares, V. C., and Lai, E. (1995). Winged helix transcription factor BF-1 is essential for the development of the cerebral hemispheres. Neuron 14, 1141-1152. doi: 10.1016/08966273(95)90262-7

Zand, N. (1930). Les Plexus Choroïdes, Anatomie, Physiologie, Pathologie. Paris: Masson \& Cie.

Zheng, W., and Chodobski, A. (2005). The Blood-Cerebrospinal Fluid Barrier. Boca Raton, FL: CRC Press.

Zheng, W., and Zhao, Q. (2002). Establishment and characterization of an immortalized Z310 choroidal epithelial cell line from murine choroid plexus. Brain Res. 958, 371-380. doi: 10.1016/S0006-8993(02)03683-1

Conflict of Interest Statement: The author declares that the research was conducted in the absence of any commercial or financial relationships that could be construed as a potential conflict of interest.

Received: 21 October 2014; accepted: 22 January 2015; published online: 03 March 2015

Citation: Liddelow SA (2015) Development of the choroid plexus and blood-CSF barrier. Front. Neurosci. 9:32. doi: 10.3389/fnins.2015.00032

This article was submitted to Neurogenomics, a section of the journal Frontiers in Neuroscience.

Copyright (C) 2015 Liddelow. This is an open-access article distributed under the terms of the Creative Commons Attribution License (CC BY). The use, distribution or reproduction in other forums is permitted, provided the original author(s) or licensor are credited and that the original publication in this journal is cited, in accordance with accepted academic practice. No use, distribution or reproduction is permitted which does not comply with these terms. 


\title{
Outer brain barriers in rat and human development
}

\author{
Christian B. Brøchner ${ }^{1}$, Camilla B. Holst ${ }^{2}$ and Kjeld Møllgård ${ }^{1 *}$ \\ ${ }^{1}$ Department of Cellular and Molecular Medicine, Faculty of Health and Medical Sciences, University of Copenhagen, \\ Copenhagen, Denmark, ${ }^{2}$ Department of Oncology, Copenhagen University Hospital, Copenhagen, Denmark
}

\section{OPEN ACCESS}

Edited by:

Helen B. Stolp,

King's College London, UK

Reviewed by:

Gavin John Clowry,

Newcastle University, UK

Daniela Virgintino,

Bari University School of Medicine,

Udo Schuma

University of Hamburg, Germany

*Correspondence:

Kjeld Møllgård,

Department of Cellular and

Molecular Medicine,

Faculty of Health and Medical Sciences,

University of Copenhagen Blegdamsvej 3 ,

DK-2200 Copenhagen, Denmark kjm@sund.ku.dk

Specialty section:

This article was submitted to Neurogenomics, a section of the journal Frontiers in Neuroscience

Received: 24 November 2014

Accepted: 20 February 2015

Published: 16 March 2015

Citation:

Brøchner CB, Holst CB and Møllgård $K$ (2015) Outer brain barriers in rat and

human development.

Front. Neurosci. 9:75.

doi: 10.3389/fnins.2015.00075
Complex barriers at the brain's surface, particularly in development, are poorly defined. In the adult, arachnoid blood-cerebrospinal fluid (CSF) barrier separates the fenestrated dural vessels from the CSF by means of a cell layer joined by tight junctions. Outer CSF-brain barrier provides diffusion restriction between brain and subarachnoid CSF through an initial radial glial end feet layer covered with a pial surface layer. To further characterize these interfaces we examined embryonic rat brains from E10 to P0 and forebrains from human embryos and fetuses (6-21st weeks post-conception) and adults using immunohistochemistry and confocal microscopy. Antibodies against claudin-11, BLBP, collagen 1, SSEA-4, MAP2, YKL-40, and its receptor IL-13R $\alpha 2$ and EAAT1 were used to describe morphological characteristics and functional aspects of the outer brain barriers. Claudin-11 was a reliable marker of the arachnoid blood-CSF barrier. Collagen 1 delineated the subarachnoid space and stained pial surface layer. BLBP defined radial glial end feet layer and SSEA-4 and YKL-40 were present in both leptomeningeal cells and end feet layer, which transformed into glial limitans. IL-13R $\alpha 2$ and EAAT1 were present in the end feet layer illustrating transporter/receptor presence in the outer CSF-brain barrier. MAP2 immunostaining in adult brain outlined the lower border of glia limitans; remnants of end feet were $\mathrm{YKL}-40$ positive in some areas. We propose that outer brain barriers are composed of at least 3 interfaces: blood-CSF barrier across arachnoid barrier cell layer, blood-CSF barrier across pial microvessels, and outer CSF-brain barrier comprising glial end feet layer/pial surface layer.

Keywords: arachnoid blood-CSF barrier, development, outer CSF-brain barrier, pial microvessel blood-CSF barrier, SSEA-4, subarachnoid space, YKL-40

\section{Introduction}

For decades the meninges have been considered to be no more than a protective shield, but increasing evidence suggests that the complex outer barriers of the brain also function as morphogenic signaling centers (Siegenthaler and Pleasure, 2011; Decimo et al., 2012), dynamic transport systems (Yasuda et al., 2013), a stem cell niche (Decimo et al., 2012) and regulators of immune cell entry to the CNS (Stolp et al., 2013). Outer brain barriers have so far been treated as a single entity although it is well-established that they are composed of several independent cellular components. In this study we aimed to combine a short review of what is known in the field with new data describing

Abbreviations: BBB, blood-brain barrier; BLBP, brain specific lipid-binding protein; CSF, cerebrospinal fluid; EFL, end feet layer; EM, electron microscopy; GFAP, glial fibrillary acidic protein; PSL, pial surface layer; SAS, subarachnoid space; SSEA-4, stage-specific embryonic antigen-4. 
arachnoid, pial, and brain surface development in rat and human.

\section{The Meninges}

In addition to the blood-brain and the classical blood-CSF (across the choroid plexus) barriers, the arachnoid barrier is a third barrier forming an important interface between blood and CSF in the brain. Dyes injected into the blood tend to stain dura but not the arachnoid suggesting that the arachnoid rather than the dura prevents entry of blood-borne substances into the subarachnoid space (Broman, 1949). Later, ultrastructural electron microscopical (EM) and freeze fracture studies described the arachnoid as a multi-layered epithelium with tight junctions between cells of the outer continuous 1-3 cellular layers-the arachnoid barrier cell layer-that forms an effective seal covering the inner dural surface (Nabeshima et al., 1975; Rascher and Wolburg, 1997). The pia and the inner layer of arachnoid comprise one cell typethe leptomeningeal cell-covering the outer-most layer of nervous tissue of the brain, the glia limitans, which is composed of a dense multilayered meshwork of astrocytic processes covered by an outer basement membrane. The layers of developing human meninges were investigated and defined by O'Rahilly and Muller (1986) starting with the primary meninx comprising neural crest-derived cells (Decimo et al., 2012) that initially surround the neural tube and at around 6th week post-conception (wpc) are present around most parts of the brain. Later 6 layers develop consisting of subcutaneous tissue, the skeletogenous layer (the dense mesenchyme between the subcutaneous layer and at first the primary meninx), dura mater, the dural limiting layer (probably contributing to both dura and arachnoid), leptomeninx (arachnoid and pia), and cerebral wall (O'Rahilly and Muller, 1986).

Differentiation of the meninges also involves formation of the subarachnoid space. Thus, the spaces that appear in the inner reticulated layer of the primary meninx become confluent and develop into the subarachnoid space and cisterns during late embryonic and early fetal period. Leptomeningeal cells condense at the inner surface of the dura and become arachnoid barrier cells and at the brain surface they form the pial surface layer. In newborn rat and in early fetal human brain the subarachnoid space extends from the arachnoid barrier cell layer to the basement membrane of the pial surface layer and contains CSF and blood vessels bridged by leptomeningeal cells that also form traversing arachnoid trabeculae (see Discussion).

Recent investigations have changed the established view of the meninges as merely protective membranes suggesting that they contribute to neural tissue homeostasis by secreting several trophic factors including FGF2, insulin-like growth factor-II and CXCL12/SDF-1 (Decimo et al., 2012) and they are highly responsive to principal mitogens and necessary for survival and growth of the developing brain (Decimo et al., 2012). In the fetal brain the meninges act as a morphogenetic center via secretion of retinoic acid which regulates neural migration and positioning, and organize the pial basement membrane, a critical anchor point for the radially oriented fibers of neuroepithelial stem cells and radial glial cells, through a variety of other secreted diffusible factors (Siegenthaler and Pleasure, 2011; Decimo et al., 2012).

\section{The End Feet Layer}

The neuroepithelial cell layer of the prosencephalon and early telencephalon covered with basement membrane faces the outside world prior to a forth-coming subarachnoid space. Along with the differentiation of neuroepithelial cells to radial glia the radial glial end feet layer covered with a basement membrane and flattened leptomeningeal cells forms a continuous interface between the telencephalic wall and the early subarachnoid space. Glial transformation from radial glial cells to astrocytes begins in the second trimester in humans and between 25 and $28 \mathrm{wpc}$ the subpial radial glial end feet layer seems to start transforming into a subpial glia limitans (Kadhim et al., 1988) consisting of interdigitating astrocytic processes and a few fibrous astrocytes. Kadhim et al. (1988) found that late subpial end feet were almost free of autophagic and lysosomal hyperactivity indicating that the end feet layer and subsequent glia limitans are created from radial glial cells that do not undergo lysosomal autolysis.

The morphology of the barrier interface over the surface of the brain is most complex during its early development. Thus, in addition to the adult barrier of tight junctions linking subdural arachnoid cells and endothelial cells of blood vessels in the subarachnoid space (Nabeshima et al., 1975), there is a whole array of specialized intercellular junctions over the pial surface of the brain. This has been described in the rat embryo where a progressive appearance of distinct junctional structures between the glial end feet was observed from E14; analysis of albumin distribution at the EM level suggested that the junctions may contribute to restriction of diffusion between the subarachnoid space and the brain extracellular fluid (Balslev et al., 1997). Nabeshima et al. (1975) noted gap junctions between end feet in the glia limitans in different mammals, which has been confirmed by others (e.g., Balslev et al., 1997; Feig and Haberly, 2011). The glia limitans has also been found to harbor specialized transmembrane protein complexes, and may contribute to neural regulation of blood flow through pial arteries, homeostatic regulation of $\mathrm{K}+$ and water and modulation of neural activity (Feig and Haberly, 2011). It must therefore be perceived as an important interface in the brain barrier system, albeit studies regarding the barrier properties of this outer CSF-brain barrier are scarce.

\section{Brain Barriers and Terminology}

Many names have been applied to the outer brain barriers, which complicate interpretation of results. The outer CSF-arachnoid barrier layer is identical with the blood-arachnoid barrier and the arachnoid barrier cell layer. The subarachnoid space with its contents extends from the arachnoid barrier cell layer to the outer CSF-brain barrier. The outer CSF-brain barrier changes during development from a radial glial end feet layer covered by the inner-most part of the pial surface layer which initially consists of a pial basement membrane and a condensed layer of single leptomeningeal cells to a glia limitans formed by astrocytic end feet and surface associated astrocytes covered by a pial basement membrane and scattered associated leptomeningeal cells. This outer CSF-brain barrier and its individual components have also been referred to as glial limiting membrane, external pial limiting membrane, glial end feet barrier, pia-arachnoid end feet barrier and other names, which sometimes interchange and therefore 
further obscure results. The arachnoid barrier cell layer and outer CSF-brain interface have also been classified as one barrier and labeled the pia-arachnoid-brain barrier and in some cases the outer CSF-brain barrier is completely ignored.

The prevailing classification of brain barriers in the developing brain has until now included an arachnoid barrier, a blood-brain barrier, a blood-CSF barrier (across the choroid plexus) and a CSF-brain barrier (across the ventricular zone) (Saunders et al., 2008, 2013). In this study we define brain interfaces that provide diffusion restriction as barriers, and taking into account the confusion in terminology, we define the terms used: The bloodCSF barrier, often only defined as the barrier across the choroid plexus, also encompass the barrier across the arachnoid barrier cell layer and the pial microvessels, since these interfaces provide a barrier between blood and the CSF. We name them the arachnoid blood-CSF barrier across the arachnoid barrier cell layer and the pial microvessel blood-CSF barrier across pial microvessels, with the proviso that the subarachnoid space in the very early embryonic brain does not exist yet nor does it contain CSF, since the choroid plexuses have not started their production yet. The barrier in the pial microvessels has been used as a surrogate for the blood-brain barrier (Allt and Lawrenson, 1997). This is incorrect since the neurovascular unit, which contributes to the fully differentiated blood-brain barrier (Engelhardt and Coisne, 2011) is not present in the subarachnoid space, and given the fact that pial microvessels are not part of the blood-brain interface, only the barrier across brain parenchymal blood vessels should be referred to as a blood-brain barrier proper (BBB). The CSF-brain barrier consists of both an outer CSF-brain barrier (described here) across the radial glial end feet layer covered by a pial surface layer and later in development glia limitans with accompanying pial basement membrane, and an inner CSF-brain barrier (generally referred to as CSF-brain barrier) across the ventricular zone cell layer.

\section{Outer Brain Barriers in Rat and Human Development}

The interfaces at the surface of the developing brain comprise the arachnoid blood-CSF barrier and outer CSF-brain barrier separated by a subarachnoid space containing pial microvessels, which form the pial microvessel blood-CSF barrier. As stated above, little is known about the changes in outer barriers of the brain during development. In addition the outer CSF-brain barrier has been largely ignored partly because of known lack of obvious tight junctions (Balslev et al., 1997). It is now evident that brain barriers are more than just the presence of tight junctions, and it is therefore necessary to characterize, divide and define these complex barriers at the surface of the developing brain. To this end, we examined embryonic rat brains from E10 to P0 and the outer brain barrier site in human forebrain samples from human embryos and fetuses (6-21st weeks post-conception) and adult brain samples using immunohistochemistry and confocal microscopy. An array of various specific antibodies was used against tight junctions (claudins), radial glial cells, glial cells, and neurons (BLBP, EAAT1, GFAP, MAP2).

In a recent study we found YKL-40 mRNA expression in meninges, telencephalon and choroid plexus and YKL-40 immunostaining of sites at all interfaces of the barriers in the developing human brain (Bjørnbak et al., 2014). Our results suggested that YKL-40 secretion from choroid plexus epithelium, leptomeningeal cells, and pericytes may play a main role associated with transport across the entire mesenchy$\mathrm{mal} /$ neuroectodermal interface (Bjørnbak et al., 2014). Furthermore, the overall distribution of YKL-40 in the developing human forebrain was noted to be very similar to that of SSEA4 in forebrains of embryos and early fetuses (Barraud et al., 2007) from this same collection. Therefore, we included antibodies against SSEA-4, YKL-40 and IL-13R $\alpha 2$, an YKL-40 receptor.

\section{Materials and Methods}

\section{Tissue Samples}

Forebrains from two human embryos [ 7 and $31 \mathrm{~mm}$ crown-rump length (CRL)] and 11 fetuses (38-200 mm CRL) corresponding to 6-21st weeks post-conception (wpc) and two adult brain specimens were examined. The embryos and the fetuses were obtained from legal abortions, as previously described (Bjørnbak et al., 2014). The adult brain samples were donated for research following neurosurgery. Informed consent was obtained from all contributing women following oral and written information, in accordance with the Helsinki declaration II, and approved by the Research Ethics Committee of the Capital Region (KFV.100.1735/90). Post-operational treatment consisted of immediate dissection of the samples into blocks, that were fixed for 12 $24 \mathrm{~h}$ at $4^{\circ} \mathrm{C}$ in either $10 \%$ neutral buffered formalin, $4 \%$ FormolCalcium, Lillie's or Bouin's fixatives. Rat embryos, 4 for each age at E12, 14, 16, 18, and P0 (the day of birth) fixed by immersion in either $10 \%$ neutral buffered formalin or Bouin's fixatives were available from a previous investigation (Balslev et al., 1997). Prior to paraffin embedding, the specimens were dehydrated with graded alcohols, and cleared in xylene. For single and double immunohistochemical experiments, 3-10 $\mu \mathrm{m}$ thick serial sections were cut in transverse, sagittal or horizontal planes, and placed on silanized glass slides.

\section{Immunohistochemistry}

Sections were deparaffinized in xylene and rehydrated using graded alcohols following standard protocols. Endogenous peroxidase was quenched using a $0.5 \%$ solution of hydrogen peroxide in methanol for $15 \mathrm{~min}$. Following rinses with TRIS buffered saline (TBS, $5 \mathrm{mM}$ Tris- $\mathrm{HCl}, 146 \mathrm{mM} \mathrm{NaCl}, \mathrm{pH}$ 7.6), non-specific binding was inhibited by incubation for $30 \mathrm{~min}$ with blocking buffer (Dako REAL ${ }^{\mathrm{TM}}$, S2022, Dako, Glostrup, Denmark) or $0.2 \%$ casein (C-7078, Sigma-Aldrich, St. Louis, MO, USA) at room temperature. Prior to overnight incubation at $4^{\circ} \mathrm{C}$ with primary antibodies diluted in blocking buffer, some antibodies required heat induced epitope retrieval with either citrate buffer (pH6) or TEG buffer (pH9) (Table 1). Before developing the signal, sections were washed with TBS. For bright field light microscopy analysis, the REAL ${ }^{\mathrm{TM}}$ EnVision $^{\mathrm{TM}}$ Detection System, Peroxidase/DAB+ rabbit/mouse, (K5007, Dako, Glostrup, Denmark) was used for detecting mouse and rabbit primary antibodies. The sections were washed with TBS, followed by incubation for $10 \mathrm{~min}$ with $3,3^{\prime}$-diamino-benzidine chromogen 
TABLE 1 | List of primary antibodies.

\begin{tabular}{|c|c|c|c|c|c|}
\hline Primary antibodies & Host IgG & Dilution & HIER & Producer & Code number \\
\hline BLBP & Rabbit IgG & 1:2000 & - & Millipore & ABN14 \\
\hline Claudin-11/OSP & Rabbit IgG & $1: 1500$ & - & Abcam & ab53041 \\
\hline Collagen 1 & Rabbit lgG & $1: 500$ & citrate, $\mathrm{pH} 6$ & Abcam & ab292 \\
\hline EAAT1 & Rabbit IgG & $1: 400$ & TEG, pH9 & Abcam & ab416 \\
\hline GFAP & Rabbit IgG & $1: 10000$ & TEG, pH9 & Dako & Z0334 \\
\hline IL-13R $\alpha 2$ & Rabbit IgG & $1: 100$ & - & Proteintech & 11059-1-AP \\
\hline MAP2 & Clon HM-2 & $1: 10000$ & TEG, pH9 & Sigma Aldrich & M4403 \\
\hline SSEA-4 & Mouse lgG3 & $1: 50 / 1: 100$ & - & Millipore & MAB4304 \\
\hline YKL-40 & Rabbit IgG & $1: 10$ & - & Proteintech & 12036-1-AP \\
\hline YKL-40 & Mouse, IgG2kb & 1:50/1:100 & - & * & 201.F9 \\
\hline
\end{tabular}

HIER, Heat Induced Epitope Retrival. TEG, TRIS + ethylene glycol tetraacetic acid buffer. Producers, Abcam, Cambridge, United Kingdom; Millipore, Merck Life Science, Hellerup, Denmark. Dako, Glostrup, Denmark. Sigma-Aldrich, St. Louis, MO, USA. Proteintech, Manchester, United Kingdom. *Kind gift from Prof. Paul A. Price, UCSD, USA.

solution. Positive staining was recognized as a brown color. The sections were counterstained with Mayers hematoxylin and dehydrated in graded alcohols followed by xylene and coverslipped with DPX mounting media. For double labeling immunofluorescence, sections were incubated for $30 \mathrm{~min}$ at room temperature with labeled polymer-HRP anti-mouse (EnVision ${ }^{\mathrm{TM}}+$ System/HRP, K4007, Dako, Glostrup, Denmark). In order to amplify the immunoreaction, this was followed by incubation with a tyramide signal amplification kit with Alexa Fluor 488labeled tyramide (1:200, T20912, Life Technologies, Carlsbad, CA, USA) for $7 \mathrm{~min}$ at room temperature. Subsequently, the sections were incubated for $30 \mathrm{~min}$ at room temperature with biotin-SP-conjugated $\mathrm{F}\left(\mathrm{ab}^{\prime}\right)_{2}$ fragment donkey anti-rabbit antibodies (1:200, 711-066-152, Jackson ImmunoResearch Europe, Suffolk, United Kingdom) followed by streptavidin-conjugated DyLight 594 (1:200, SA5594, Vector Laboratories Europe, Peterborough, United Kingdom). Finally, a nuclear counterstain with DAPI (1:1000, D1306, Life Technologies, Carlsbad, CA, USA) was added for $3 \mathrm{~min}$, before sections were coverslipped. Double labeling was performed with the combination of antibodies against BLBP and SSEA-4, YKL-40 and SSEA-4, YKL-40 and IL13-R $\alpha 2$, and YKL-40 and EAAT1. Details of the primary antibodies including dilutions and suppliers are listed in Table 1. Staining specificity of the monoclonal YKL-40 antibody was thoroughly tested on the same material in a recent study (see Figure 1 in Bjørnbak et al., 2014), and in addition the polyclonal anti-YKL-40 used here resulted in an immunostaining identical to that of monoclonal YKL-40. Control sections were incubated with mouse IgG1, IgG2a or irrelevant rabbit antibodies, as well as subjected to omission of primary or secondary antibodies. These were always blank. The staining patterns were similar irrespective of different methods of fixation used as also emphasized in many studies based on the same collection (e.g., Møllgård and Jacobsen, 1984; Johansen et al., 2007).

For laser scanning confocal microscopy a Carl Zeiss LSM 780 was used. During image acquisition, a sequential scan procedure through the $\mathrm{z}$-axis of the double-labeled sections was applied when applicable, covering in total $9-11 \mu \mathrm{m}$ in depth. Confocal images were taken and analyzed throughout the $\mathrm{z}$-axis of the section, and individual optical sections were stored as TIFF files using Zeiss ZEN Vision v10. Representative images were chosen for figure editing using Adobe Photoshop CS6.

\section{Results}

The meninges and outer brain barriers form during embryogenesis and early fetal life. Many factors are involved in this dynamic process and are necessary for adequate brain development. O'Rahilly and Muller (1986) described development of the human meninges according to Carnegie stages, but have recently revised this staging model (O'Rahilly and Muller, 2010). In Table 2 a summary of important events in the development of the human meninges and outer CSF-brain barrier approximated to weeks post-conception (wpc) is provided. Results in Carnegie stages have been translated according to the revision by O'Rahilly and Muller (2010) and from mammals to human according to the work of Clancy et al. (2001).

We aimed to characterize the interfaces at the surface of the developing brain revealing a claudin-11 positive arachnoid blood-CSF barrier, collagen 1 positive subarachnoid space and pial surface layer, SSEA-4/YKL-40 positive leptomeningeal cells and SSEA-4/YKL-40/IL-13R $\alpha 2 / \mathrm{BLBP}$ positive radial glial end feet. Each interface is described and discussed in the context of possible functional significance for the developing brain.

\section{The Arachnoid Blood-CSF Barrier Becomes Increasingly Claudin-11 Positive During Rat and Human Brain Development}

Previously we have described the developing rat glial end feet barrier prior to the appearance of the arachnoid membrane and thus before the establishment of a well-defined subarachnoid space (Balslev et al., 1997). In a recent study of the embryonic mouse brain immunostained for claudin-11, a distinct reactivity corresponding to the developing arachnoid blood-CSF barrier was found (Whish et al., 2015). Therefore, we decided to test claudin11 as a possible marker for the arachnoid blood-CSF barrier development in rat and human in order to compare this barrier with the developing outer CSF-brain barrier described previously in the rat (Balslev et al., 1997). 
TABLE 2 | Features of outer brain barrier and meningeal development.

\begin{tabular}{|c|c|c|c|}
\hline WPC & Feature & Species & References \\
\hline \multirow[t]{2}{*}{ 6th } & Appearance of a primary meninx around most parts of the brain & Human & O’Rahilly and Muller, 1986 \\
\hline & Amoeboid microglial cells penetrate the brain by crossing the pial basement membrane & Human & Verney et al., 2010 \\
\hline \multirow[t]{2}{*}{ 7th } & The skeletogenous layer becomes visible & Human & O’Rahilly and Muller, 1986 \\
\hline & $\begin{array}{l}\text { Differentiation of a leptomeningeal meshwork that is presumed to contain liquid and represent } \\
\text { the future SAS }\end{array}$ & Human & O'Rahilly and Muller, 1986 \\
\hline \multirow[t]{3}{*}{ 8th } & $\begin{array}{l}\text { The dural limiting layer is almost complete in hindbrain and midbrain but only present in the } \\
\text { area adjacent to the lateral fossa in the forebrain }\end{array}$ & Human & O'Rahilly and Muller, 1986 \\
\hline & The fenestrated sinusoids of the pia-arachnoid become non-fenestrated (E14) & Rat (E14) & Balslev et al., 1997 \\
\hline & Most of the cisternae of the adult is already present & Human & O'Rahilly and Muller, 1986 \\
\hline 7-10th & Initial communication between the ventricular and subarachnoid compartments & Human and rat (E17) & $\begin{array}{l}\text { Brocklehurst, 1969; } \\
\text { Johansson et al., } 2008\end{array}$ \\
\hline 11th & $\begin{array}{l}\text { Completion of the subpial end feet layer (E16) and claudin-11 positive arachnoid blood-CSF } \\
\text { barrier (E18) and thereby appearance of a clearly defined subarachnoid space }\end{array}$ & Rat (E16 and E18) & Balslev et al., 1997 \\
\hline 12-13th & Second wave of microglial cells penetrate the brain via the BBB and inner CSF-brain barrier & Human & Verney et al., 2010 \\
\hline 12 (13-15th) & RGCs begin to transform into astrocytes & $\begin{array}{l}\text { Rhesus monkey (E64) } \\
\text { and newborn ferret }\end{array}$ & $\begin{array}{l}\text { Schmechel and Rakic, } \\
\text { 1979; Voigt, } 1989\end{array}$ \\
\hline 25-28th & Transition from subpial end feet layer to glia limitans & Human & Kadhim et al., 1988 \\
\hline
\end{tabular}

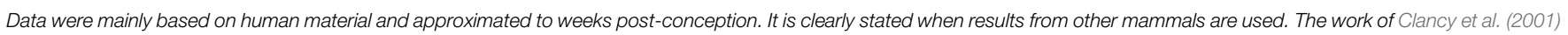
was used for conversion to estimated human age.

Early developing rat brain from E10 to E14 showed a complete lack of claudin-11 immunoreactivity. At E15 a faint immunostaining for claudin-11 was present corresponding to loosely packed arachnoid barrier cells at the base of the brain and to the developing barrier layer of the future tentorium cerebelli (not illustrated). At E18 meninges, the subarachnoid space and cisterns were fully developed and immunostaining for claudin-11 demonstrated a strong distinct reactivity of the entire arachnoid blood-CSF barrier (Figures 1A,B). The barrier cell layer was particularly prominent corresponding to tentorium cerebelli and the base of the brain and interrupted only where cranial nerves penetrated the combined dura-arachnoid (Figure 1A). The arachnoid barrier cell layer of the forebrain consisted of a single cell layer shown in Figure 1B. In late embryonic human brain claudin-11 reactivity was first observed as a loose faintly stained meshwork of leptomeningeal cells surrounding the lower brain stem. At 8th wpc arachnoid barrier cells at tentorium cerebelli and at the base of the brain were positive. In the second trimester meninges, the subarachnoid space and cisterns were fully developed. At mid-gestation the arachnoid blood-CSF barrier of the forebrain consisted of a single claudin-11 positive cell layer (Figure 1C).

\section{The Subarachnoid Space is Defined by Collagen 1 Immunoreactivity}

The second trimester human fetal subarachnoid space between the arachnoid blood-CSF barrier and the outer CSF-brain barrier contained numerous bundles of collagen, many blood vessels and a meshwork of leptomeningeal cells forming trabeculae. Immunostaining for collagen 1 clearly delineated the subarachnoid space as shown in a section from a mid-gestational human fetus in Figure 2A. The arachnoid barrier cell layer was unstained in contrast to the strongly immunostained pial surface layer which faced an unstained glial end feet layer. The basement membranes of the pial blood vessels lost their collagen 1 immunoreactivity upon entering the end feet layer, revealed as a continuous layer of glial end feet only interrupted by penetrating blood vessels after immunostaining for brain specific lipidbinding protein (BLBP) (Figure 2B). The leptomeningeal cells in the subarachnoid space including the arachnoid barrier cell layer were positively stained for both YKL-40 and SSEA-4 (Figure 3) but not for BLBP (Figure 2B). Where blood vessels penetrated the end feet layer the subarachnoid space extended down into a subpial compartment in the marginal zone. Double-labeling with antibodies against BLBP and SSEA-4, showed SSEA-4 immunoreactivity of the pericytes and BLBP defined the outer invaginating surface of the end feet layer, corresponding to the perivascular Virchow-Robin space between vascular basement membrane and surface of end feet (Figures 2B,C). The Virchow-Robin space is obliterated when the glial and endothelial basement membranes join (Brinker et al., 2014) consistent with depletion of BLBP and SSEA-4 immunoreactivity.

\section{From Radial Glial End Feet Layer, Characterized by BLBP-, SSEA-4 and YKL-40 Immunoreactivity, to Glia Limitans \\ Radial Glial End Feet Layer in Fetal Human Brain}

In late embryos and early fetuses immunostained for BLBP, SSEA-4, and YKL-40 the entire parenchymal lining of the 

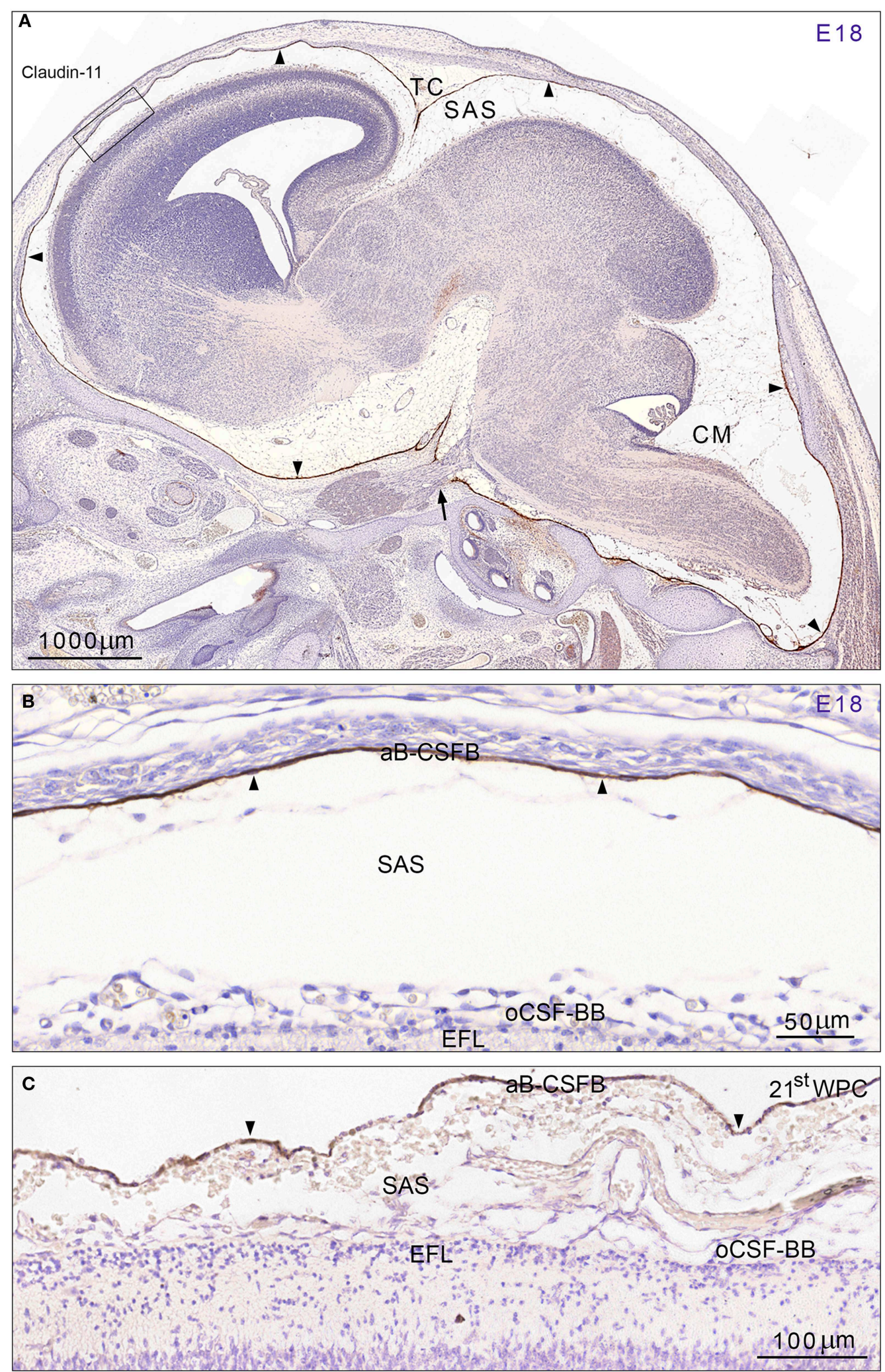

FIGURE 1 | Distribution of claudin-11 immunoreactivity in sagittal sections of E18 rat $(A, B)$ and 21st wpc human (C) brain. (A)

Immunostaining for claudin-11 in a developing rat brain at E18 demonstrates a strong reactivity of the entire arachnoid barrier cell layer (= arachnoid blood-CSF barrier (aB-CSFB)) (arrowheads) in marked contrast to unstained (Continued) 


\section{FIGURE 1 | Continued}

leptomeningeal cells in the subarachnoid space (SAS) and in the entire brain. Cisterns at the base of the brain and cisterna magna (CM) are well-developed. Note the very strongly stained arachnoid covering of the tentorium cerebelli (TC) and the interrupted barrier cell layer where the trigeminal nerve perforates the dura-arachnoid (arrow). The area outlined with a rectangle is shown at higher magnification in $\mathbf{( B )}$ and demonstrates that the barrier cell layer (aB-CSFB) covering the forebrain consists of a strongly stained single cell layer. (C) At mid-gestation the human fetal arachnoid barrier cell layer (aB-CSFB) is also formed by a single claudin-11 positive cell layer. In $\mathbf{( B , C )}$ note that neither the E18 rat radial glial end feet layer (EFL) and the pial surface layer forming the outer CSF brain barrier (oCSF-BB) facing the subarachnoid space (SAS) nor the human fetal radial glial end feet layer (EFL) and pial surface layer show claudin-11 immunoreactivity. Scale bars: (A) $1000 \mu \mathrm{m}$, (B) $50 \mu \mathrm{m}$, (C) $100 \mu \mathrm{m}$. outer CSF-brain barrier, corresponding to radial glial end feet, exhibited strong membranous immunoreactivity of the conically or deltoid shaped end feet with simple straight membranes. The parenchymal lining in the second trimester showed multiple curly closely apposed delicate end feet membranes best appreciated in a stacked series of confocal images of YKL40 stained marginal zone (Figure 3A). At mid-gestation the individual end feet appeared as narrow densely packed parallel tubules outlined by rather straight apical and apical-lateral cell membranes (not shown). Double immunolabeling with antibodies against BLBP and SSEA-4 (Figure 2C) and with antibodies against YKL-40 and SSEA-4 (Figures 3A-C) demonstrated that all end feet possessed a considerable overlapping membrane-associated immunoreactivity for BLBP, SSEA-4 and YKL-40.

\section{Receptors and Transporters Associated with the End Feet Layer}

IL-13R $\alpha 2$, which was recently shown to function as an YKL40/CHI3L1 receptor (He et al., 2013) and EAAT1/GLAST, a well-known glutamate-aspartate influx transporter (also known as Slc1a3), were used as representative examples to illustrate dynamic functions of the end feet layer. Double-immunolabeling of brains from human fetuses in the second trimester with antibodies against IL-13R $\alpha 2$ and YKL-40 (Figure 4A) showed that the entire continuously YKL-40 immunoreactive end feet layer contained evenly distributed IL-13R $\alpha 2$ positive membrane domains in infoldings of the end feet surface membrane. Immunostaining with antibodies against the excitatory amino acid influx transporter EAAT1 resulted in immunopositive punctate or reticular structures along the membranes of the end feet layer and surrounding blood vessels in the outer neocortex at mid-gestation (Figure 4B). Double-immunolabeling with antibodies against EAAT1 and YKL-40 (Figure 4C) showed a similar distribution of influx transporter and YKL-40 within the end feet layer and around blood vessels.

\section{Glia Limitans in Adult Human Brain}

Glia limitans, which forms the outermost part of the molecular layer in adult human cerebral cortex, consisted of a dense, 20-40 $\mu \mathrm{m}$ thick network of intermingled GFAP-positive astrocytic processes and some small fibrous astrocytes, which were not surface-associated (Figure 5A). These astrocytes and the outer surface of the glia limitans were unstained following immunohistochemistry with an antibody against the neuronal marker MAP2. The positive immunoreaction clearly defined the border zone toward the rest of the molecular layer (Figure 5B).
In some regions the outermost layer of the glia limitans consisted of GFAP-negative empty-looking processes reminiscent of second trimester end feet, referred to as remnants of end feet (Figures 5A-C) that protruded into the subarachnoid space. Many of these protrusions showed distinct apical membrane reactivity for YKL-40 (Figure 5C). YKL-40 staining also revealed many YKL-40 positive spheroid bodies corresponding to corpora amylacea particularly within the glia limitans but also in the border zone toward the MAP2 positive part of the molecular layer (Figure 5C) and in large perivascular spaces (not shown).

\section{Discussion}

\section{The Arachnoid Barrier Cell Layer}

Until recently the brain barriers in the developing brain were considered absent or leaky by many investigators (see Discussion in Saunders et al., 2014). Evidence is now emerging that these barriers are functioning and adapted to fetal environment already in the earliest stages of brain development (Saunders et al., 2012, 2013).

When the subarachnoid space is formed (see Table 2), the arachnoid barrier cells facing the inner meningeal dural border cells have closely apposed cell membranes and are joined to each other by numerous tight junctions. It has been shown in many publications that this barrier cell layer is the structural basis for the arachnoid blood-CSF barrier (see e.g., Nabeshima et al., 1975). However, so far it is not known which tight junctional proteins contribute to the paracellular diffusion restriction. We tested the arachnoid blood-CSF barrier for the presence of a number of different claudins using immunostaining and found that claudin-11 may serve as a reliable marker for the arachnoid barrier cells, as suggested from parallel finding in developing mouse brain (Whish et al., 2015). Claudin-11, initially introduced as oligodendrocyte-specific protein (OSP), is involved in formation of tight junctions and is best known for its implication in interlamellar tight junction strands in oligodendrocytes (myelin sheath tight junctions) as well as tight junction strands of the Sertoli cells of the testes (the blood-testis barrier, Morita et al., 1999; Morrow et al., 2010). Our results suggest that the arachnoid blood-CSF barrier is present and characterized by claudin-11 in early fetal life.

Since the arachnoid blood-CSF barrier is avascular and provides a relatively small surface area, it is believed that it does not contribute significantly to blood-brain exchange (Abbott et al., 2010). However, in light of recent findings of drug transporters and drug-metabolizing enzymes in the arachnoid blood-CSF barrier (Yasuda et al., 2013) and taken into account that the 


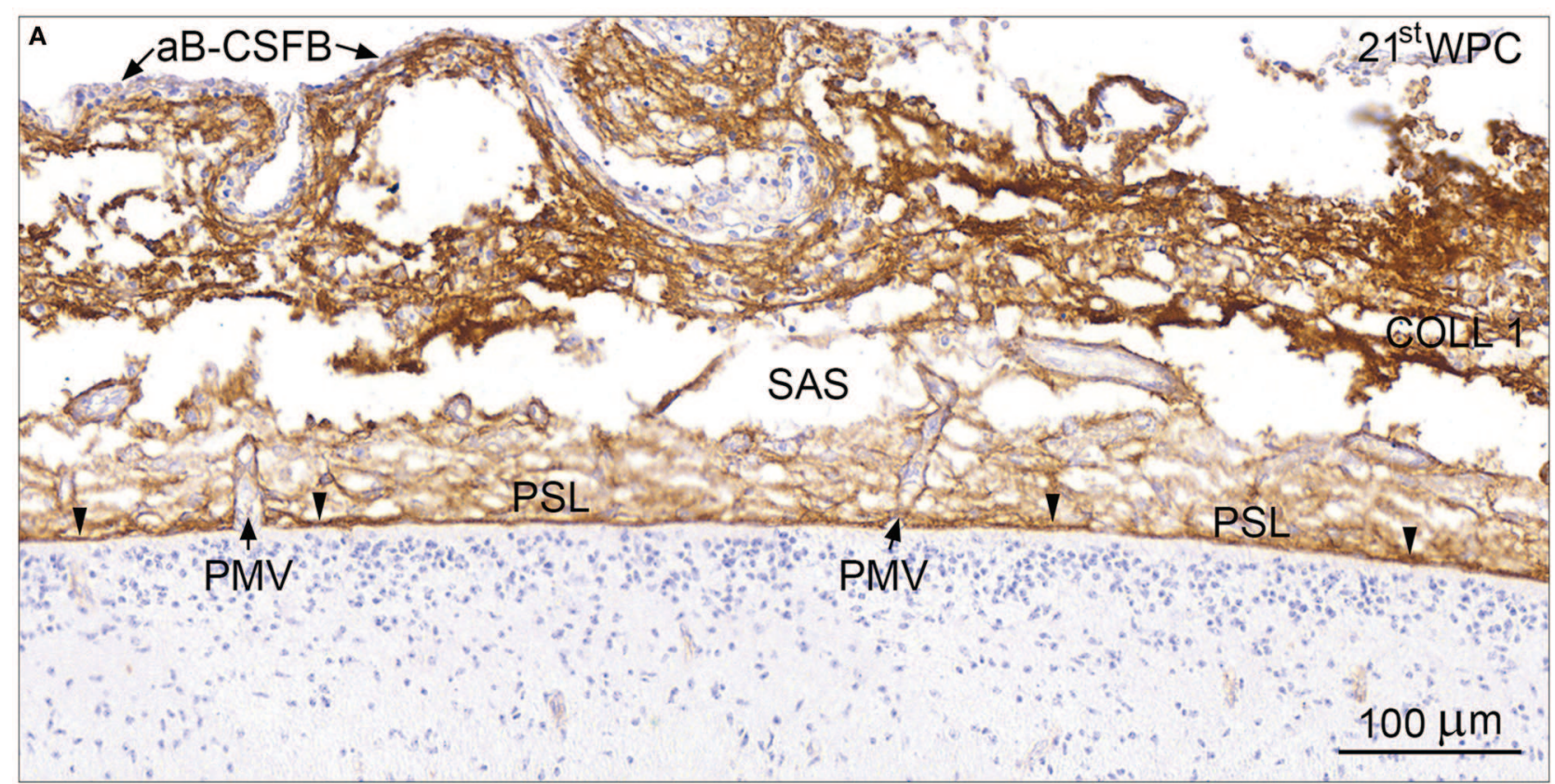

B

\section{SAS}

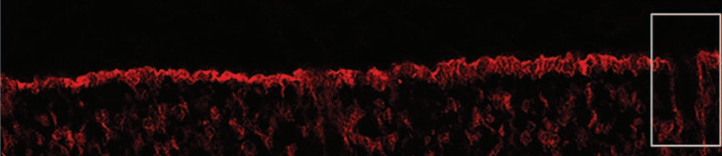

BV

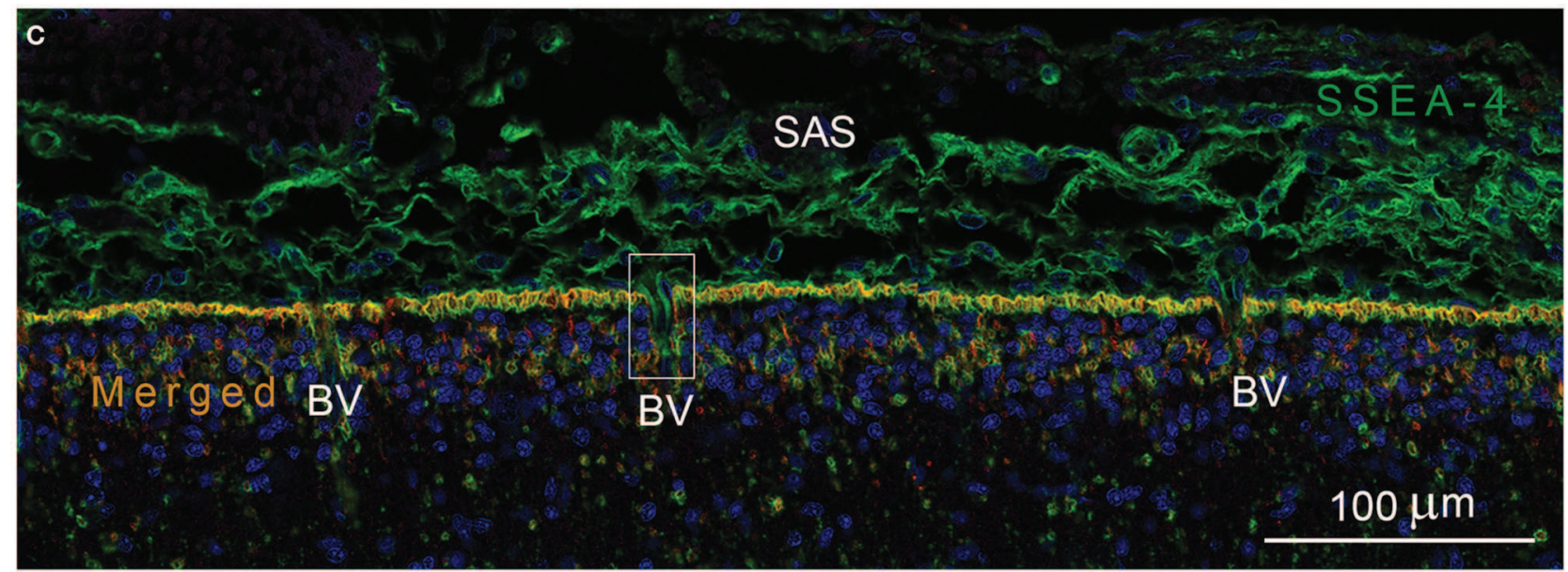

FIGURE 2 | Arachnoid blood-CSF barrier, subarachnoid space, pial surface, and glial end feet layers immunostained for collagen 1 (A) and double immunolabeled with antibodies against BLBP and SSEA-4 (B,C) from occipital cortex of a 21st wpc human fetus. (A) Immunostaining for collagen 1 depicts the limitation of the subarachnoid space (SAS) of a 21st wpc human fetus. The arachnoid barrier cell layer
(aB-CSFB) is unstained (arrows) and the strongly immunostained pial surface layer (PSL) faces an unstained glial end feet layer. The basement membranes of the pial microvessels (BV) loose their stainability abruptly when the vessels enter the end feet layer and fuse with the inner-most part of the PSL (arrowheads). (B) In a section adjacent to that shown in (A), the entire end

(Continued) 


\section{FIGURE 2 | Continued}

feet layer is immunolabeled with anti-BLBP corresponding to the radial glial end feet. Note the spaces in the end feet layer where blood vessels

penetrate into the parenchyma. (C) Double-labeling with antibodies against

BLBP and SSEA-4, nuclear stained with DAPI, reveals an evenly distributed co-localization in the end feet layer. Where pial microvessels penetrate into the marginal zone, the perivascular canals are lined with BLBP, revealing Virchow-Robins space (illustrated with rectangles in B,C). The subarachnoid space contains SSEA-4 positive leptomeningeal cells but shows no BLBP reactivity. (B,C) same magnification. Scale bars: $100 \mu \mathrm{m}$. arachnoid barrier cell layer constitute a much larger fraction of the brain surface area during early development, it is clear that the arachnoid blood-CSF barrier is an important and changing interface, that should not be ignored. It clearly warrants further investigation in the developing and adult brain.

\section{YKL-40/SSEA-4 in Outer CSF-Brain Barrier}

After the penetration of the first vessels into the telencephalic wall and the appearance of the choroid plexus the following mesenchymal/neuroectodermal interfaces comprise the initial brain barrier system: (i) The blood-brain barrier proper across the cerebral endothelial cells where YKL-40/SSEA-4 are present in pericytes (Bjørnbak et al., 2014), (ii) the blood-CSF barrier at the choroid plexus epithelium that is strongly YKL-40/SSEA-4 positive (Barraud et al., 2007; Bjørnbak et al., 2014), (iii) the claudin-11 positive arachnoid blood-CSF barrier delineating the subarachnoidal space which contains strongly YKL-40/SSEA-4 positive leptomeningeal cells and vessels, (iv) YKL-40/SSEA-4 positive pial microvessel blood-CSF barrier, and (v) an outer CSF-brain barrier with a collagen 1 positive pial surface layer covering a glial end feet layer comprising BLBP/SSEA-4/YKL$40 / \mathrm{IL}-13 \mathrm{R} \alpha 2$ positive radial glial end feet. In addition, there is (vi) an inner CSF-brain barrier interface between the ventricular CSF and the brain interstitial fluid at the apical neuroepithelial cell membranes linked by strap junctions (Møllgård et al., 1987) and lined by dense YKL-40 immunoreactivity in the early developing human.

In parallel to developmental processes at the inner CSF-brain barrier, where strap junctions disappear when radial glial cells differentiate to ependymal cells (see Whish et al., 2015), the outer CSF-brain barrier seems to change character when the most superficial subpial glial end feet layer transform into a subpial glia limitans between 25 and 28 wpc in the human brain (Table 2).

An essential morphological feature of the blood-brain barrier proper (BBB) lies in the presence of tight junctions between the cerebral endothelial cells of the vasculature of the brain parenchyma. Solute transport mechanisms and restrictions have been studied thoroughly in developing and adult blood-brain barrier and choroid plexuses blood-CSF barrier, discovering distinct, comprehensive and changing transport systems, which indicate that the choroid plexuses blood-CSF and blood-brain barriers are functionally mature with respect to small molecules in early development (Saunders et al., 2013). In contrast, little is known about the developing outer CSF-brain barrier that separates the outer CSF from the brain's internal milieu. In the rat brain Balslev et al. (1997) described an incomplete layer of radial glial end feet as a part of the brain surface and large fenestrated sinusoid vessels in the subarachnoid space at E12, but there was no albumin reactivity inside the marginal zone suggesting that there must be some diffusional restriction to entry of proteins even in early development. From E14 the pia-arachnoid vessels became non-fenestrated and the developing end feet layer was connected by junctional structures that changed over time and offered restriction to protein penetration, most likely supplemented by the basement membrane (Balslev et al., 1997). In the present study we found immunostaining of the glial end feet layer with both EAAT1 (glutamate transporter), IL-13R $\alpha 2$ (YKL-40 receptor) and BLBP, which transports fatty acids (Kipp et al., 2011) indicating that this outer CSF-brain barrier may well be involved initially in controlling the transfer and diffusion of solutes including morphogens into the brain and cellular transmigration (see below).

Furthermore we observed that the closely packed layer of leptomeningeal cells in the initial pial surface layer covering the radial glial end feet became less coherent in parallel with the formation of the glia limitans, indicating that the pial surface layer contributes to diffusion restriction in early development, a finding consistent with the results of Balslev et al. (1997).

YKL-40 mRNA expression was strong in the pia-arachnoid tissue and prominent YKL-40 staining was evident already from the emergence of the meningeal tissues (Bjørnbak et al., 2014). Our results suggest that YKL-40 is produced by the leptomeninges and secreted into the subarachnoid space. Since the junctional structures between glial end feet restrict diffusion and based on the presence of the YKL-40 receptor IL-13R $\alpha 2$, YKL-40 itself, as shown by the pronounced immunoreactivity of the entire end feet layer, is likely to be due to receptor-mediated uptake from the subarachnoid space.

\section{Involvement of Arachnoid Blood-CSF and Outer CSF-Brain Barriers in Brain Inflammation}

Until recently the central nervous system has been considered an immune-privileged site, but it is now evident that monocytederived macrophages also play an important role in its inflammation and maintenance of the functional plasticity of the healthy brain (Schwartz et al., 2013). The brain is under constant immune surveillance by both blood-born immune cells in leptomeningeal and perivascular spaces and resident microglia (Engelhardt and Coisne, 2011; Stolp et al., 2013). The brain barriers provide relative specialized immune privilege controlling leukocyte trafficking, which is increased considerably during inflammation and disease (Muldoon et al., 2013). Muldoon and colleagues describe four routes for leukocyte CNS entry: (1) blood-tosubarachnoid space via leptomeningeal vessels (pia microvessel blood-CSF barrier); (2) blood-to-parenchymal perivascular space through the BBB; (3) blood-to-cerebrospinal fluid via the choroid plexus (choroid plexuses blood-CSF barrier); (4) blood-to-CSF via meningeal spaces (arachnoid blood-CSF barrier) and through the ependymal lining ventricles (inner CSF-brain barrier) (Ransohoff et al., 2003; Engelhardt and Coisne, 2011; Muldoon et al., 

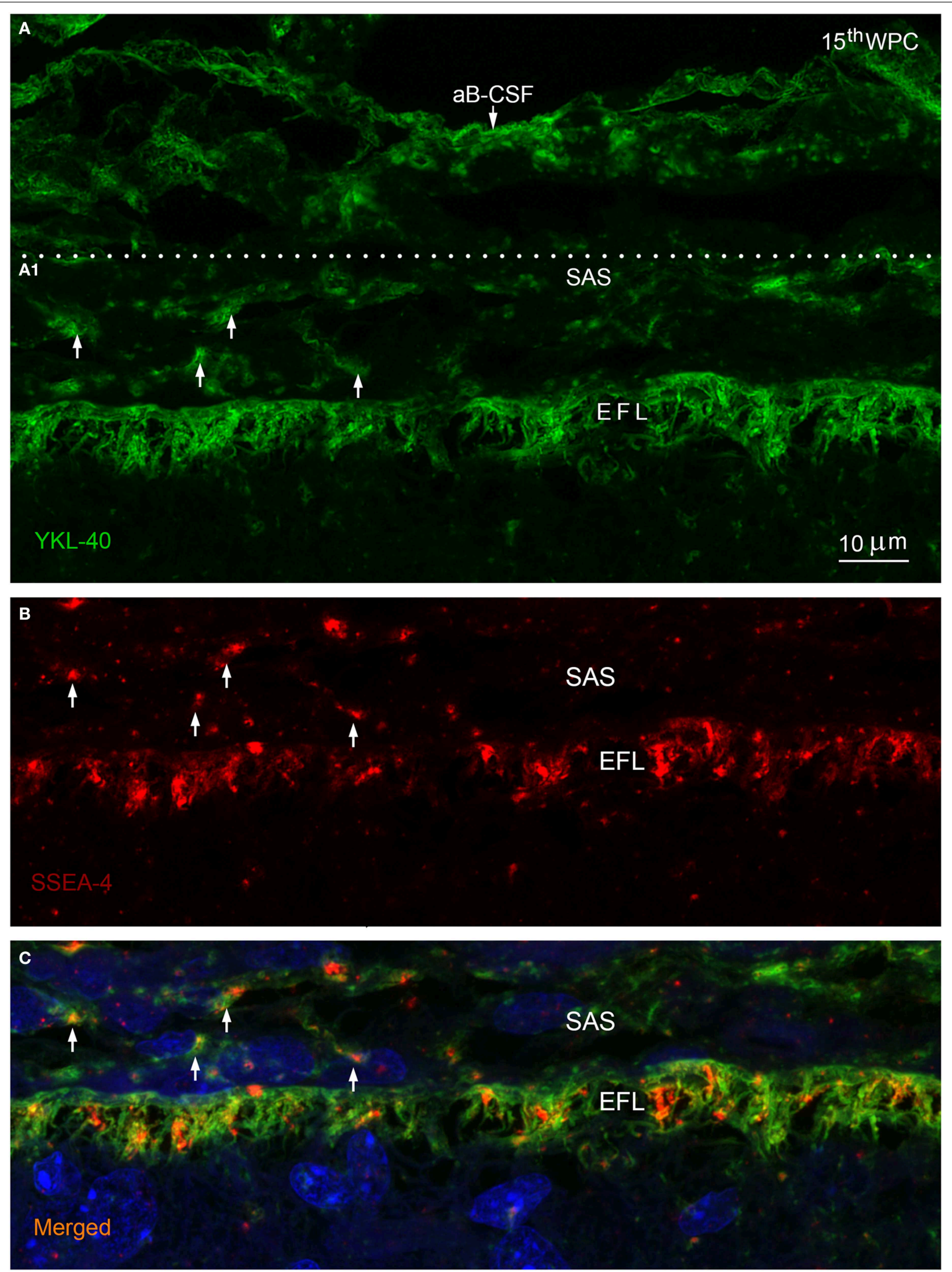

FIGURE 3 | Arachnoid blood-CSF barrier, subarachnoid space, pial surface, and glial end feet layers double immunolabeled with antibodies against YKL-40 and SSEA-4 (A-C) in parietal cortex of a 15th wpc human fetus. Parietal cortex of a 15th wpc fetus, immunolabeled with anti-YKL-40 (A) and anti-SSEA-4 (B), counterstained with DAPI and

(Continued) 


\section{FIGURE 3 | Continued}

merged (A1,B) in (C). The images are representations of a stacked series of 10 steps, with $1 \mu \mathrm{m}$ intervals, processed as maximum projection intensity. The staining of the end feet layer (EFL) shows multiple delicate and curled end feet membranes. YKL-40 immunoreactivity is also present in the arachnoid blood-CSF barrier
(aB-CSFB in (A) and in leptomeningeal cells in the subarachnoid space (arrows in A1). In (B) the pattern of SSEA-4 distribution is seemingly equal to that of YKL-40, and the merged image of the stacked images confirms the co-localization of YKL-40 and SSEA-4 in leptomeningeal cells within the subarachnoid space (SAS) and in the end feet layer of the radial glial cells (C). Scale bar: $10 \mu \mathrm{m}$.
2013; Schwartz et al., 2013). In the subarachnoid space specific antigen triggering enables the immune cell to penetrate the glia limitans (outer CSF-brain barrier) (Bartholomaus et al., 2009; Engelhardt and Coisne, 2011), which requires the activity of matrix metalloproteinases MMP-2 and MMP-9 (Agrawal et al., 2006). Thus, leucocyte trafficking involves all interfaces of the brain barrier system.

It is generally believed that inflammation produces a breakdown of blood-brain barrier at some stages of brain development (Stolp et al., 2005). Ichikawa and Itoh (2011) found that experimental meningitis in rats at first caused increased permeability of the arachnoid blood-CSF barrier rather than the BBB or choroid plexuses blood-CSF barrier indicating, that breakdown of the $\mathrm{BBB}$ and choroid plexuses blood-CSF barrier is secondary. However, further studies are necessary to clarify the role of arachnoid blood-CSF and outer CSF-brain barriers in response to inflammation in the developing brain. Nevertheless, the presence of an early claudin-11 positive arachnoid blood-CSF barrier, diffusion restraint through the outer CSF-brain barrier and distinct transport systems and markers found in critical barrier sites in the present study may be important for securing homeostasis and normal immune function.

\section{Brain Barriers, Inflammation and YKL-40}

In a recent study of YKL-40 in the developing human forebrain, we showed a marked YKL-40 immunoreactivity in neuroepithelial cells, radial glial end feet, leptomeninges, and choroid plexus epithelial cells in early stages. Later developmental features included an increasing number of YKL-40 immunopositive pericytes particularly in the intermediate and subventricular zones and a population of small rounded YKL- 40 positive possible astroglial progenitors migrating along radial glial fibers in the subventricular zone. The choroid plexus, glial end feet and the astrocyte-resembling cells observed in the developing hippocampus were strongly YKL-40 immunopositive (Bjørnbak et al., 2014). In the present study we confirmed the YKL-40 reactivity and found overlapping immunostaining of SSEA-4 and YKL-40 in leptomeningeal cells and the end feet layer.

Current knowledge suggests that neuroinflammation is of great importance in almost all neurological disorders, and that interaction between the brain barriers and the immune system contribute to this process (Stolp et al., 2013). YKL-40 immunoreactivity is specifically detected at sites of brain barriers and entry points for microglia in the developing human brain and is known to be involved in angiogenesis, inflammation and is overexpressed in glioblastoma (brain cancer, GBM) (Lal et al., 1999; Nigro et al., 2005; Colin et al., 2006) and brain tissue and/or CSF in several neurological disorders that commence in the developing brain or/and involve dysfunction of brain barriers
(Chung et al., 2003; Colton et al., 2006; Arion et al., 2007; Garbett et al., 2008; Bonneh-Barkay et al., 2010). YKL-40 has also been found to regulate MMP-2 (Ku et al., 2011) and MMP-9 in some cases (Michelsen et al., 2010). The general spatio-temporal distribution of YKL-40 immunoreactivity suggests that YKL-40 secretion from choroid plexus epithelium, leptomeningeal cells and pericytes is involved in controlling local angiogenesis, access of peripheral cells (microglia and perhaps macrophages) to the developing brain and its specialized immunity. The correlation between YKL-40 and MMPs, presence of YKL-40 in brain barriers and necessity of MMP-2 and MMP-9 in immune cell transmigration through the glia limitans further support the hypothesis, that YKL-40 has a role in immune cell transmigration across brain barriers. Whether YKL-40 expression in the brain barriers is increased in response to neuroinflammation warrants further investigation.

\section{Stem Cells, Brain Barriers and SSEA-4}

In a previous study of human embryonic central nervous system, the stage-specific embryonic antigen-4 (SSEA-4) was found in neural progenitor cells in forebrains of human embryos and early fetuses (Barraud et al., 2007), and was shown in a population of differentiating cells in an intermediate stage between pluripotent human embryonic stem cells (hESCs) and neural progenitor cells (Noisa et al., 2012). SSEA-4 belongs to the group of globoseries glycosphingolipids, and is widely used in characterization of hESC lines (Adewumi et al., 2007). Furthermore, it is highly expressed in GBM compared to normal tissue and an SSEA-4 specific antibody suppresses GBM tumor growth in mice (Lou et al., 2014). In other cancers SSEA-4 has been established as a cancer stem cell marker (Malecki et al., 2012; Noto et al., 2013). The glycoprotein YKL-40 has been associated with hESC and neural stem cell differentiation (Brøchner et al., 2012), and a marked similarity of the distribution patterns in developing human forebrain was noted (Barraud et al., 2007; Bjørnbak et al., 2014). In this study the localization of YKL-40 and SSEA-4 was almost identical corresponding to earlier findings. The role of SSEA-4 in brain barriers remains to be elucidated but its known presence in hESC, GBM and cancer stem cells and co-localization with YKL-40 in the brain barriers suggest that the outer brain barriers participate in organization and development of the brain through several different mediators including SSEA-4.

\section{BLBP and the Morphogenetic Role of Meninges}

Brain specific lipid-binding protein (BLBP) is a member of the lipid-binding protein family that carries small hydrophobic signaling molecules between cellular compartments but also a marker of radial glial cells (Feng et al., 1994; Howard et al., 2006), astrocytes (Kipp et al., 2011) and adult neural stem cells (mice) 


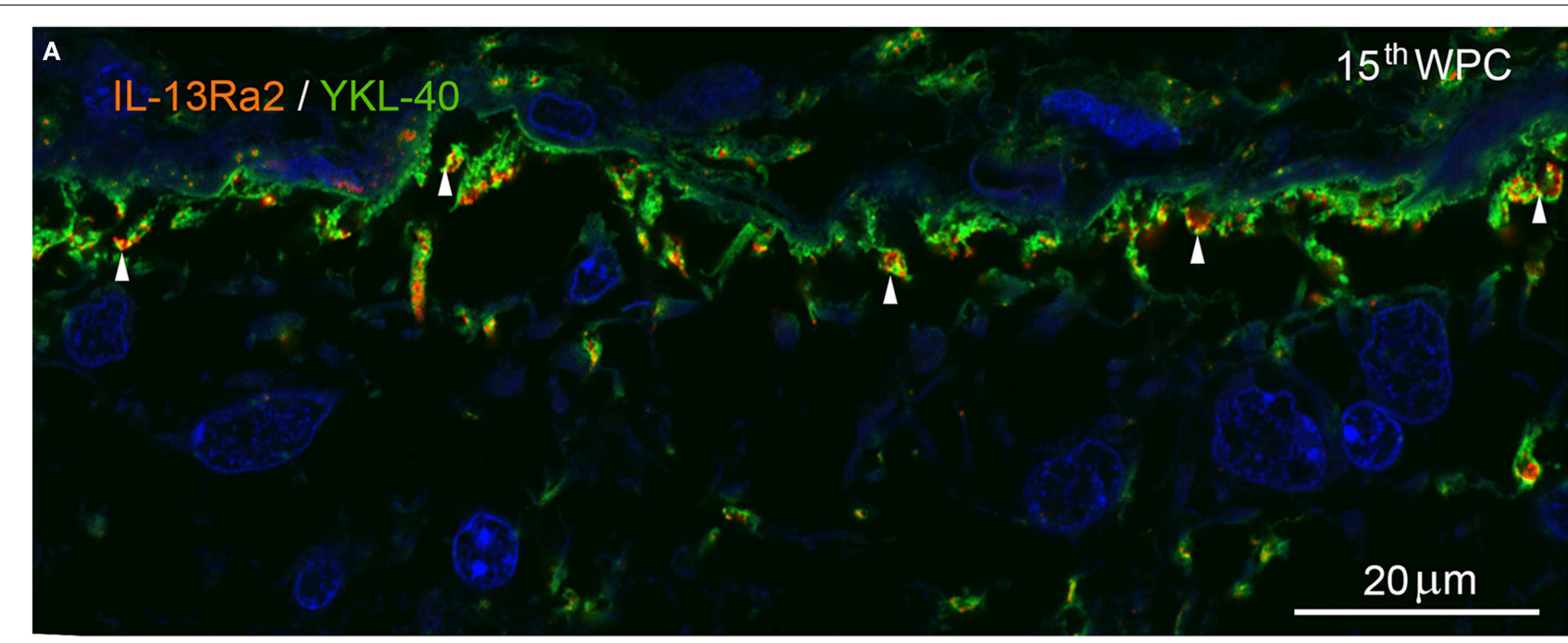

B

\section{EAAT1}

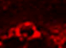

SAS

\section{EFL}

\section{BV}

\section{BV}

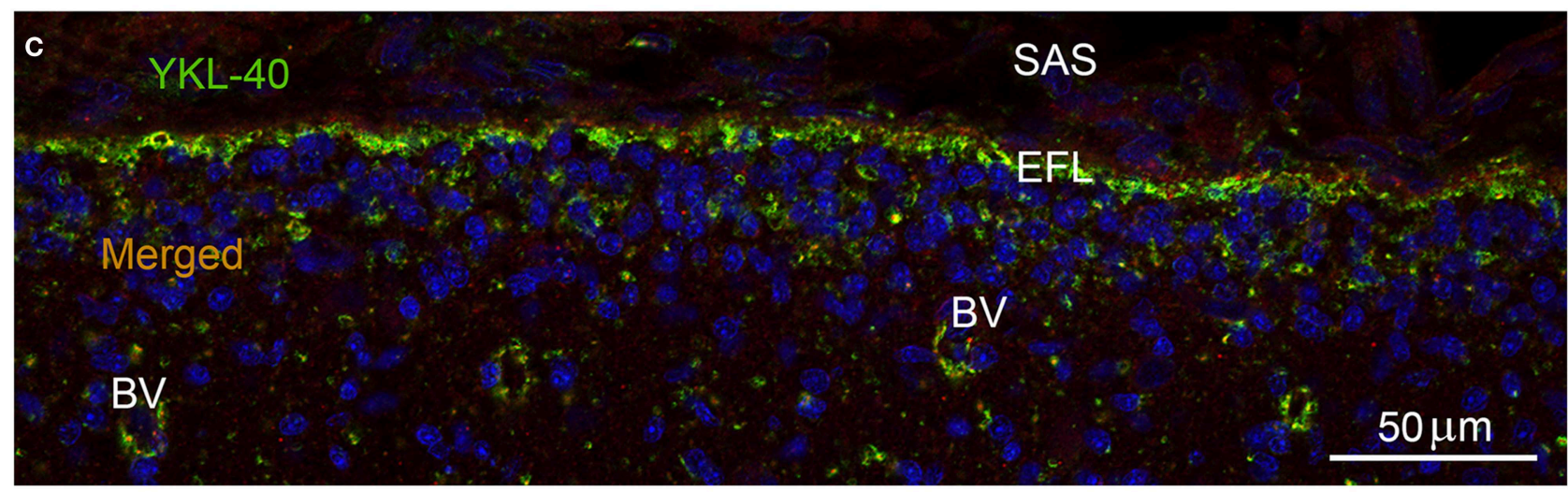

FIGURE 4 | Marginal zone in parietal cortex of a 15th wpc human fetus double immunolabeled with antibodies against YKL-40 and IL-13R $\alpha 2$ (A) and in occipital cortex of a 21st wpc human fetus (B,C) labeled with antibodies against EAAT1 (B) and against EAAT1 and YKL-40 (C). Parietal cortex of a 15th wpc fetus, immunolabeled with antibodies against IL-13R $\alpha 2$ and $\mathrm{YKL}-40$, merged and nuclear counterstained with DAPI (A). The YKL-40 immunoreactive end feet layer contains evenly distributed interleukin-13R $\alpha 2$ receptors (white arrowheads). (B) The excitatory amino acid transporter (EAAT1) is visualized in occipital cortex of a 21st wpc fetus, below the subarachnoid space (SAS) within the end feet layer (EFL) and in surrounding parenchymal blood vessels (BV). The merged image with YKL-40 (C) shows a similar distribution within the end feet layer and around the blood vessels. (A) Scale bar: $20 \mu \mathrm{m}$. (B,C) same magnification. Scale bar: $50 \mu \mathrm{m}$.
(Giachino et al., 2014). BLBP expression is increased in brain tumor tissue and associated with progression and/or decreased survival in glioblastoma (GBM). Depending on the ratio of fatty acid ligands BLBP can increase or decrease migration in GBM
(Kipp et al., 2011). A variety of secreted diffusible factors from fetal meninges regulate neural migration and positioning and organize the pial basement membrane, which is a critical anchor point for the radially oriented fibers of neuroepithelial stem cells 

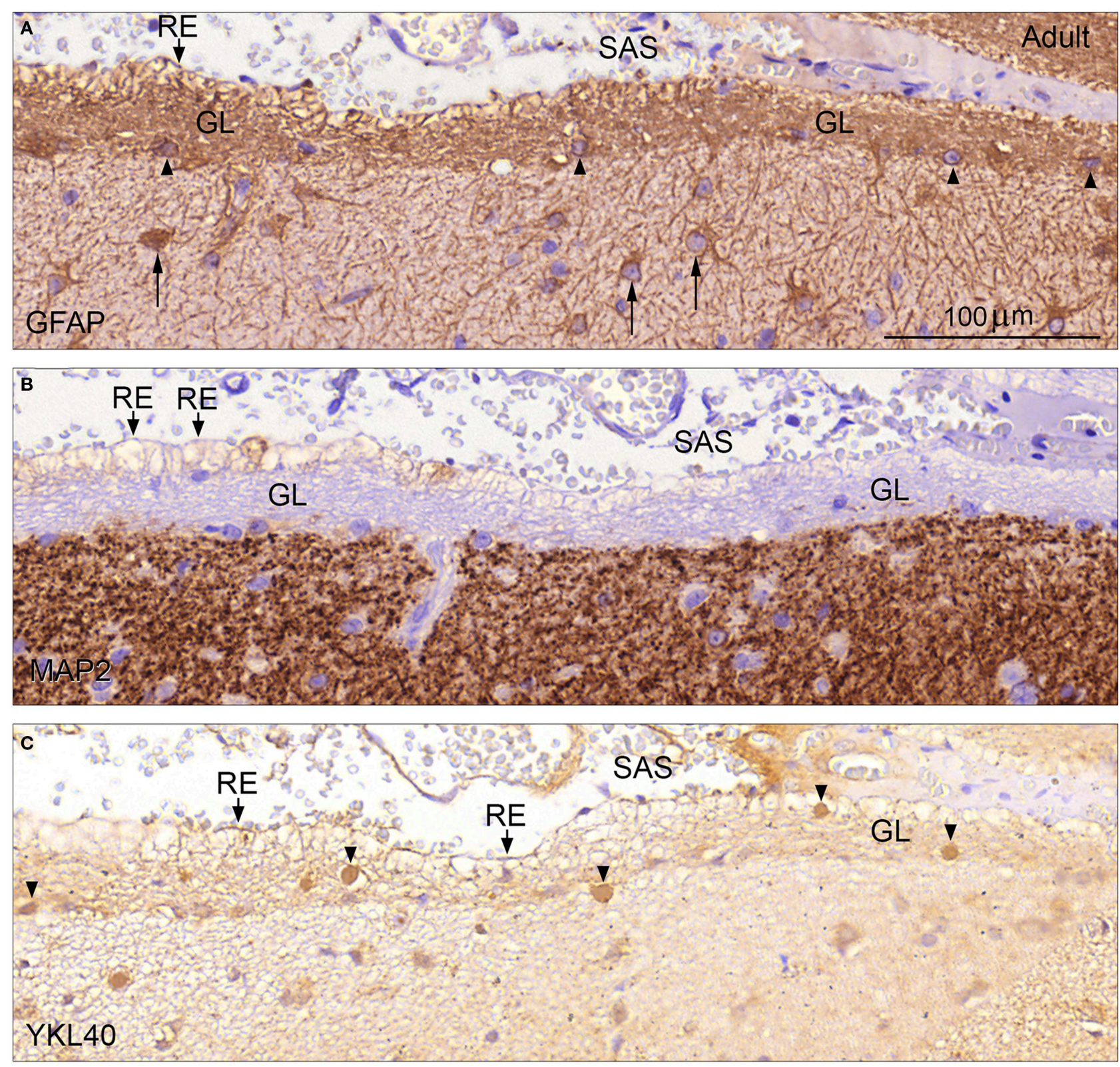

\section{FIGURE 5 | Glia limitans in adult human brain stained for GFAP,}

MAP2, and YKL-40 (A-C). Adult human cerebral cortex immunostained for GFAP in (A) depicts the glia limitans (GL) as a dense multilayered network of GFAP positive astrocytic processes and few small fibrous astrocytes (arrowheads). Note the larger protoplasmic astrocytes (arrows) deeper in the molecular layer. Protrusions of astrocytic processes, which look like remnants of end feet (RE) are seen as patches facing the subarachnoid space (SAS). Immunostaining for MAP2 (B) defines the inner border of glia limitans (GL) toward the rest of the molecular layer and the unstained outer border with the empty-looking protrusions/remnants of end feet (RE) toward the subarachnoid space (SAS) is clearly depicted. Immunoreactivity for YKL-40 of glia limitans (GL) is seen in (C). Many protrusions exhibit distinct apical membrane reactivity for $\mathrm{YKL}-40$, and $\mathrm{YKL}-40$ positive spheroid bodies corresponding to corpora amylacea are seen within the glia limitans but also in the border zone toward the MAP2 positive part of the molecular layer (arrowheads). (A-C) same magnification. (A) Scale bar: (A) $20 \mu \mathrm{m}$.
(Siegenthaler and Pleasure, 2011). Signaling molecules identified so far include the chemokine SDF-1, BMP7, and the morphogen all-trans retinoic acid (atRA) which seems to be involved in the decision of neuroepithelial cells to generate neurons and intermediate progenitor cells (IPCs) destined for the subventricular zone via communications with adjacent radial glial end feet (Siegenthaler et al., 2009). Feng et al. (1994) suggested that BLBP mediates aspects of neuron-glia interaction and is involved in neural differentiation and maintenance of the glial fiber scaffold. We found BLBP in the end feet layer in the developing outer CSF-brain barrier, establishing BLBP as a reliable marker for the radial glial end feet layer and thereby the outer CSF-brain barrier. Furthermore, BLBP has a preference for binding long chain polyunsaturated fatty acids with a possible ligand (Xu et al., 1996) 
being docosahexaenoic acid, also involved in CNS development (Xu et al., 1996), which in light of our findings support that BLBP in the outer CSF-brain barrier participate in neural development and positioning possibly also orchestrated from the meninges.

\section{Concluding Remarks}

Research regarding the brain barrier system has evolved considerably over the last decades and now the focus is on both physical, transport, and metabolic barrier properties. In this study we described the changing barriers at the surface of the developing brain, but to characterize and understand these complex structures and their interaction, we found it necessary to revise the barrier concept based merely on anatomical features. Furthermore, the terminology in this area is at best confusing and inconsistent. Based on these considerations we argued that the outer brain barriers consist of (i) an arachnoid bloodCSF barrier across the arachnoid barrier cell layer, (ii) a pial microvessel blood-CSF barrier across the pial microvessels and (iii) an outer CSF-brain barrier across the radial glial end feet layer covered by a pial surface layer and later in development glia limitans with accompanying pial basement membrane. Our findings of claudin-11 in the arachnoid blood-CSF barrier and EAAT1 and IL-13R $\alpha 2$ in the outer CSF-brain barrier confirm their barrier properties and the spatiotemporal distribution of YKL-40/BLBP/SSEA-4 further establish the necessity of regarding these interfaces as separate entities, since they may play

\section{References}

Abbott, N. J., Patabendige, A. A., Dolman, D. E., Yusof, S. R., and Begley, D. J. (2010). Structure and function of the blood-brain barrier. Neurobiol. Dis. 37, 13-25. doi: 10.1016/j.nbd.2009.07.030

Adewumi, O., Aflatoonian, B., Ahrlund-Richter, L., Amit, M., Andrews, P. W., Beighton, G., et al. (2007). Characterization of human embryonic stem cell lines by the International Stem Cell Initiative. Nat. Biotechnol. 25, 803-816. doi: $10.1038 / \mathrm{nbt} 1318$

Agrawal, S., Anderson, P., Durbeej, M., van Rooijen, N., Ivars, F., Opdenakker, G., et al. (2006). Dystroglycan is selectively cleaved at the parenchymal basement membrane at sites of leukocyte extravasation in experimental autoimmune encephalomyelitis. J. Exp. Med. 203, 1007-1019. doi: 10.1084/jem. 20051342

Allt, G., and Lawrenson, J. G. (1997). Is the pial microvessel a good model for blood-brain barrier studies? Brain Res. Brain Res. Rev. 24, 67-76. doi: 10.1016/S0165-0173(97)00011-8

Arion, D., Unger, T., Lewis, D. A., Levitt, P., and Mirnics, K. (2007). Molecular evidence for increased expression of genes related to immune and chaperone function in the prefrontal cortex in schizophrenia. Biol. Psychiatry 62, 711-721. doi: 10.1016/j.biopsych.2006.12.021

Balslev, Y., Saunders, N. R., and Møllgård, K. (1997). Ontogenetic development of diffusional restriction to protein at the pial surface of the rat brain: an electron microscopical study. J. Neurocytol. 26, 133-148. doi: 10.1023/A:10185279 28760

Barraud, P., Stott, S., Møllgård, K., Parmar, M., and Björklund, A. (2007). In vitro characterization of a human neural progenitor cell coexpressing SSEA4 and CD133. J. Neurosci. Res. 85, 250-259. doi: 10.1002/jnr.21116

Bartholomaus, I., Kawakami, N., Odoardi, F., Schlager, C., Miljkovic, D., Ellwart, J. W., et al. (2009). Effector T cell interactions with meningeal vascular structures in nascent autoimmune CNS lesions. Nature 462, 94-98. doi: 10.1038 /nature 08478 different roles in normal brain development and possibly in several neurological disorders. We therefore propose an elaboration of the model for developing brain barriers as defined by Saunders et al. (2008) and divide the brain interfaces into six barriers at three different sites: (1) The blood-brain barrier proper (i), (2) the blood-CSF barrier constituting (ii) the choroid plexuses blood-CSF barrier across the choroid plexus, (iii) the arachnoid blood-CSF barrier across the arachnoid barrier cell layer and (iv) the pial microvessel blood-CSF barrier across the pial microvessels; (3) The CSF-brain barrier comprising (v) the outer CSF-brain barrier (described earlier in this paragraph) and (vi) inner CSF-brain barrier across the ventricular zone cell layer.

\section{Acknowledgments}

We thank H. Hadberg, P. S. Froh, and K. Ottosen, Department of Cellular and Molecular Medicine, Faculty of Health and Medical Sciences, University of Copenhagen, Copenhagen, Denmark for excellent technical assistance. We thank Professor Paul A. Price, Department of Biology, University of California, San Diego, USA, for providing the monoclonal YKL-40 antibody, and Professor Julia S. Johansen, M. D. for thorough discussions of the manuscript. A Graduate Scholarship from The Faculty of Health and Medical Sciences, University of Copenhagen, Denmark (CBB), and a grant from Vera and Carl Johan Michaelsen Foundation (KM, grant number 34077$)$ supported this work.
Bonneh-Barkay, D., Wang, G., Starkey, A., Hamilton, R. L., and Wiley, C. A. (2010). In vivo CHI3L1 (YKL-40) expression in astrocytes in acute and chronic neurological diseases. J. Neuroinflammation 7:34. doi: 10.1186/1742-2094-7-34

Brinker, T., Stopa, E., Morrison, J., and Klinge, P. (2014). A new look at cerebrospinal fluid circulation. Fluids Barriers CNS 11:10. doi: 10.1186/2045-811811-10

Brøchner, C. B., Johansen, J. S., Larsen, L. A., Bak, M., Mikkelsen, H. B., Byskov, A. G., et al. (2012). YKL-40 is differentially expressed in human embryonic stem cells and in cell progeny of the three germ layers. J. Histochem. Cytochem. 60, 188-204. doi: 10.1369/0022155411433331

Brocklehurst, G. (1969). The development of the human cerebrospinal fluid pathway with particular reference to the roof of the fourth ventricle. J. Anat. 105, 467-475.

Broman, T. (1949). The Permeability of Cerebrospinal Vessels in Normal and Pathological Conditions. Copenhagen: Ejnar Munksgaard.

Bjørnbak, C., Brøchner, C. B., Larsen, L. A., Johansen, J. S., and Møllgård, K. (2014). Brain barriers and a subpopulation of astroglial progenitors of developing human forebrain are immunostained for the glycoprotein YKL-40. J. Histochem. Cytochem. 62, 369-388. doi: 10.1369/002215541 4528514

Chung, C., Tallerico, T., and Seeman, P. (2003). Schizophrenia hippocampus has elevated expression of chondrex glycoprotein gene. Synapse 50, 29-34. doi: $10.1002 /$ syn. 10228

Clancy, B., Darlington, R. B., and Finlay, B. L. (2001). Translating developmental time across mammalian species. Neuroscience 105, 7-17. doi: 10.1016/S03064522(01)00171-3

Colin, C., Baeza, N., Bartoli, C., Fina, F., Eudes, N., Nanni, I., et al. (2006). Identification of genes differentially expressed in glioblastoma versus pilocytic astrocytoma using Suppression Subtractive Hybridization. Oncogene 25, 2818-2826. doi: 10.1038/sj.onc.1209305

Colton, C. A., Mott, R. T., Sharpe, H., Xu, Q., Van Nostrand, W. E., and Vitek, M. P. (2006). Expression profiles for macrophage alternative activation genes in AD 
and in mouse models of AD. J. Neuroinflammation 3:27. doi: 10.1186/17422094-3-27

Decimo, I., Fumagalli, G., Berton, V., Krampera, M., and Bifari, F. (2012). Meninges: from protective membrane to stem cell niche. Am. J. Stem Cells 1, 92-105.

Engelhardt, B., and Coisne, C. (2011). Fluids and barriers of the CNS establish immune privilege by confining immune surveillance to a two-walled castle moat surrounding the CNS castle. Fluids Barriers CNS 8:4. doi: 10.1186/20458118-8-4

Feig, S. L., and Haberly, L. B. (2011). Surface-associated astrocytes, not endfeet, form the glia limitans in posterior piriform cortex and have a spatially distributed, not a domain, organization. J. Comp. Neurol. 519, 1952-1969. doi: $10.1002 /$ cne. 22615

Feng, L., Hatten, M. E., and Heintz, N. (1994). Brain lipid-binding protein (BLBP): a novel signaling system in the developing mammalian CNS. Neuron 12, 895-908. doi: 10.1016/0896-6273(94)90341-7

Garbett, K., Ebert, P. J., Mitchell, A., Lintas, C., Manzi, B., Mirnics, K., et al. (2008). Immune transcriptome alterations in the temporal cortex of subjects with autism. Neurobiol. Dis. 30, 303-311. doi: 10.1016/j.nbd.2008.01.012

Giachino, C., Basak, O., Lugert, S., Knuckles, P., Obernier, K., Fiorelli, R., et al. (2014). Molecular diversity subdivides the adult forebrain neural stem cell population. Stem Cells 32, 70-84. doi: 10.1002/stem.1520

He, C. H., Lee, C. G., Dela Cruz, C. S., Lee, C. M., Zhou, Y., Ahangari, F., et al. (2013). Chitinase 3-like 1 regulates cellular and tissue responses via IL-13 receptor alpha2. Cell Rep. 4, 830-841. doi: 10.1016/j.celrep.2013.07.032

Howard, B., Chen, Y., and Zecevic, N. (2006). Cortical progenitor cells in the developing human telencephalon. Glia 53, 57-66. doi: 10.1002/glia. 20259

Ichikawa, H., and Itoh, K. (2011). Blood-arachnoid barrier disruption in experimental rat meningitis detected using gadolinium-enhancement ratio imaging. Brain Res. 1390, 142-149. doi: 10.1016/j.brainres.2011.03.035

Johansson, P. A., Dziegielewska, K. M., Liddelow, S. A., and Saunders, N. R. (2008). The blood-CSF barrier explained: when development is not immaturity. Bioessays 30, 237-248. doi: 10.1002/bies.20718

Johansen, J. S., Høyer, P. E., Larsen, L. A., Price, P. A., and Møllgård, K. (2007). YKL-40 protein expression in the early developing human musculoskeletal system. J. Histochem. Cytochem. 55, 1213-1228. doi: 10.1369/jhc.7A7245.2007

Kadhim, H. J., Gadisseux, J. F., and Evrard, P. (1988). Topographical and cytological evolution of the glial phase during prenatal development of the human brain: histochemical and electron microscopic study. J. Neuropathol. Exp. Neurol. 47, 166-188. doi: 10.1097/00005072-198803000-00009

Kipp, M., Gingele, S., Pott, F., Clarner, T., van der Valk, P., Denecke, B., et al. (2011). BLBP-expression in astrocytes during experimental demyelination and in human multiple sclerosis lesions. Brain Behav. Immun. 25, 1554-1568. doi: 10.1016/j.bbi.2011.05.003

Ku, B. M., Lee, Y. K., Ryu, J., Jeong, J. Y., Choi, J., Eun, K. M., et al. (2011). CHI3L1 (YKL-40) is expressed in human gliomas and regulates the invasion, growth and survival of glioma cells. Int. J. Cancer 128, 1316-1326. doi: 10.1002/ijc.25466

Lal, A., Lash, A. E., Altschul, S. F., Velculescu, V., Zhang, L., McLendon, R. E., et al. (1999). A public database for gene expression in human cancers. Cancer Res. 59, 5403-5407.

Lou, Y. W., Wang, P. Y., Yeh, S. C., Chuang, P. K., Li, S. T., Wu, C. Y., et al. (2014). Stage-specific embryonic antigen-4 as a potential therapeutic target in glioblastoma multiforme and other cancers. Proc. Natl. Acad. Sci. U.S.A. 111, 2482-2487. doi: 10.1073/pnas.1400283111

Malecki, M., Anderson, M., Beauchaine, M., Seo, S., Tombokan, X., and Malecki, R. (2012). TRA-1-60, SSEA-4, Oct4A, nanog clones of pluripotent stem cells in the embryonal carcinomas of the ovaries. J. Stem Cell Res. Ther. 2, 130.

Michelsen, A. E., Rathcke, C. N., Skjelland, M., Holm, S., Ranheim, T., Krohg-Sorensen, K., et al. (2010). Increased YKL-40 expression in patients with carotid atherosclerosis. Atherosclerosis 211, 589-595. doi: 10.1016/j.atherosclerosis.2010.02.035

Møllgård, K., Balslev, Y., Lauritzen, B., and Saunders, N. R. (1987). Cell junctions and membrane specializations in the ventricular zone (germinal matrix) of the developing sheep brain: a CSF-brain barrier. J. Neurocytol. 16, 433-444. doi: 10.1007/BF01668498

Møllgård, K., and Jacobsen, M. (1984). Immunohistochemical identification of some plasma proteins in human embryonic and fetal forebrain with particular reference to the development of the neocortex. Brain Res. 315, 49-63. doi: 10.1016/0165-3806(84)90076-2

Morita, K., Sasaki, H., Fujimoto, K., Furuse, M., and Tsukita, S. (1999). Claudin11/OSP-based tight junctions of myelin sheaths in brain and Sertoli cells in testis. J. Cell Biol. 145, 579-588. doi: 10.1083/jcb.145.3.579

Morrow, C. M., Mruk, D., Cheng, C. Y., and Hess, R. A. (2010). Claudin and occludin expression and function in the seminiferous epithelium. Philos. Trans. R. Soc. Lond B Biol. Sci. 365, 1679-1696. doi: 10.1098/rstb.2010.0025

Muldoon, L. L., Alvarez, J. I., Begley, D. J., Boado, R. J., Del Zoppo, G. J., Doolittle, N. D., et al. (2013). Immunologic privilege in the central nervous system and the blood-brain barrier. J. Cereb. Blood Flow Metab 33, 13-21. doi: 10.1038/jcbfm.2012.153

Nabeshima, S., Reese, T. S., Landis, D. M., and Brightman, M. W. (1975). Junctions in the meninges and marginal glia. J. Comp. Neurol. 164, 127-169. doi: 10.1002/cne.901640202

Nigro, J. M., Misra, A., Zhang, L., Smirnov, I., Colman, H., Griffin, C., et al. (2005). Integrated array-comparative genomic hybridization and expression array profiles identify clinically relevant molecular subtypes of glioblastoma. Cancer Res. 65, 1678-1686. doi: 10.1158/0008-5472.CAN-04-2921

Noisa, P., Ramasamy, T. S., Lamont, F. R., Yu, J. S., Sheldon, M. J., Russell, A., et al. (2012). Identification and characterisation of the early differentiating cells in neural differentiation of human embryonic stem cells. PLoS ONE 7:e37129. doi: 10.1371/journal.pone.0037129

Noto, Z., Yoshida, T., Okabe, M., Koike, C., Fathy, M., Tsuno, H., et al. (2013). CD44 and SSEA-4 positive cells in an oral cancer cell line HSC4 possess cancer stem-like cell characteristics. Oral Oncol. 49, 787-795. doi: 10.1016/j.oraloncology.2013.04.012

O'Rahilly, R., and Muller, F. (1986). The meninges in human development. J. Neuropathol. Exp. Neurol. 45, 588-608. doi: 10.1097/00005072-19860900000008

O'Rahilly, R., and Muller, F. (2010). Developmental stages in human embryos: revised and new measurements. Cells Tissues Organs 192, 73-84. doi: $10.1159 / 000289817$

Ransohoff, R. M., Kivisakk, P., and Kidd, G. (2003). Three or more routes for leukocyte migration into the central nervous system. Nat. Rev. Immunol. 3, 569-581. doi: $10.1038 /$ nri1 130

Rascher, G., and Wolburg, H. (1997). The tight junctions of the leptomeningeal blood-cerebrospinal fluid barrier during development. J. Hirnforsch. 38, 525-540.

Saunders, N. R., Daneman, R., Dziegielewska, K. M., and Liddelow, S. A. (2013). Transporters of the blood-brain and blood-CSF interfaces in development and in the adult. Mol. Aspects Med. 34, 742-752. doi: 10.1016/j.mam.2012.11.006

Saunders, N. R., Dreifuss, J.-J., Dziegielewska, K. M., Johansson, P. A., Habgood, M. D., Møllgård., K., et al. (2014). The rights and wrongs of blood-brain barrier permeability studies: a walk through 100 years of history. Front. Neurosci. 8:404. doi: 10.3389/fnins.2014.00404

Saunders, N. R., Ek, C. J., Habgood, M. D., and Dziegielewska, K. M. (2008). Barriers in the brain: a renaissance? Trends Neurosci. 31, 279-286. doi: 10.1016/j.tins.2008.03.003

Saunders, N. R., Liddelow, S. A., and Dziegielewska, K. M. (2012). Barrier mechanisms in the developing brain. Front. Pharmacol. 3:46. doi: 10.3389/fphar.2012.00046

Schmechel, D. E., and Rakic, P. (1979). A Golgi study of radial glial cells in developing monkey telencephalon: morphogenesis and transformation into astrocytes. Anat. Embryol. (Berl.) 156, 115-152.

Schwartz, M., Kipnis, J., Rivest, S., and Prat, A. (2013). How do immune cells support and shape the brain in health, disease, and aging? J. Neurosci. 33, 17587-17596. doi: 10.1523/JNEUROSCI.3241-13.2013

Siegenthaler, J. A., Ashque, A. M., Zarbalis, K., Patterson, K. P., Hecth, J. H., Kane, M. A., et al. (2009). Retinoic acid from the meninges regulates cortical neuron generation. Cell 139, 597-609. doi: 10.1016/j.cell.2009.10.004

Siegenthaler, J. A., and Pleasure, S. J. (2011). We have got you 'covered': how the meninges control brain development. Curr. Opin. Genet. Dev. 21, 249-255. doi: 10.1016/j.gde.2010.12.005

Stolp, H. B., Dziegielewska, K. M., Ek, C. J., Habgood, M. D., Lane, M. A., Potter, A. M., et al. (2005). Breakdown of the blood-brain barrier to proteins in white matter of the developing brain following systemic inflammation. Cell Tissue Res. 320, 369-378. doi: 10.1007/s00441-005-1088-6 
Stolp, H. B., Liddelow, S. A., Sa-Pereira, I., Dziegielewska, K. M., and Saunders, N. R. (2013). Immune responses at brain barriers and implications for brain development and neurological function in later life. Front. Integr. Neurosci. 7:61. doi: 10.3389/fnint.2013.00061

Verney, C., Monier, A., Fallet-Bianco, C., and Gressens, P. (2010). Early microglial colonization of the human forebrain and possible involvement in periventricular white-matter injury of preterm infants. J. Anat. 217, 436-448. doi: 10.1111/j.1469-7580.2010.01245.x

Voigt, T. (1989). Development of glial cells in the cerebral wall of ferrets: direct tracing of their transformation from radial glia into astrocytes. J. Comp. Neurol. 289, 74-88. doi: $10.1002 /$ cne. 902890106

Whish, S., Dziegielewska, K. M., Møllgård, K., Noor, N. M., Liddelow, S. A., Habgood, M. D., et al. (2015). The inner CSF-brain barrier: developmentally controlled access to the brain. Front. Neurosci. 9:16. doi: 10.3389/fnins.2015.00016

Xu, L. Z., Sánchez, R., Sali, A., and Heintz, N. (1996). Ligand specificity of brain lipid-binding protein. J. Biol. Chem. 271, 24711-24719. doi: 10.1074/jbc.271.40. 24711
Yasuda, K., Cline, C., Vogel, P., Onciu, M., Fatima, S., Sorrentino, B. P., et al. (2013). Drug transporters on arachnoid barrier cells contribute to the blood-cerebrospinal fluid barrier. Drug Metab Dispos. 41, 923-931. doi: $10.1124 / \mathrm{dmd} .112 .050344$

Conflict of Interest Statement: The Review Editor Gavin Clowry declares that, despite having collaborated with author Kjeld Mollgard, the review process was handled objectively. The authors declare that the research was conducted in the absence of any commercial or financial relationships that could be construed as a potential conflict of interest.

Copyright $\odot 2015$ Brøchner, Holst and Møllgård. This is an open-access article distributed under the terms of the Creative Commons Attribution License (CC BY). The use, distribution or reproduction in other forums is permitted, provided the original author(s) or licensor are credited and that the original publication in this journal is cited, in accordance with accepted academic practice. No use, distribution or reproduction is permitted which does not comply with these terms. 


\title{
Transcriptomal changes and functional annotation of the developing non-human primate choroid plexus
}

\author{
C. Joakim Ek ${ }^{1 *}$, Peter Nathanielsz ${ }^{2}$, Cun $\mathrm{Li}^{2+}$ and Carina Mallard ${ }^{1+}$ \\ ${ }^{1}$ Department of Physiology, Institute for Neuroscience and Physiology, University of Gothenburg, Sahlgrenska Academy, \\ Gothenburg, Sweden, ${ }^{2}$ Department of Obstetrics, Center for Pregnancy and Newborn Research, The University of Texas \\ Health Science Center, San Antonio, TX, USA
}

\section{OPEN ACCESS}

Edited by:

Lester R. Drewes,

University of Minnesota Medical

School Duluth, USA

Reviewed by:

Fernanda Marques,

University of Minho, Portugal

Eva Katrin Wirth,

Charité-Universitätsmedizin Berlin,

Germany

${ }^{*}$ Correspondence:

C. Joakim Ek,

Department of Physiology, Institute for

Neuroscience and Physiology,

University of Gothenburg, Sahlgrenska

Academy, Medicinaregatan 11,

Box 432, 40530 Göteborg, Sweden

joakim.ek@neuro.gu.se

${ }^{\dagger}$ Shared senior authorship.

Specialty section:

This article was submitted to

Neurogenomics, a section of the

journal Frontiers in Neuroscience

Received: 29 September 2014

Paper pending published:

15 November 2014

Accepted: 25 February 2015

Published: 12 March 2015

Citation:

Ek CJ, Nathanielsz P, Li C and Mallard

C (2015) Transcriptomal changes and

functional annotation of the

developing non-human primate

choroid plexus. Front. Neurosci. 9:82.

doi: 10.3389/fnins.2015.00082
The choroid plexuses are small organs that protrude into each brain ventricle producing cerebrospinal fluid that constantly bathes the brain. These organs differentiate early in development just after neural closure at a stage when the brain is little vascularized. In recent years the plexus has been shown to have a much more active role in brain development than previously appreciated thereby it can influence both neurogenesis and neural migration by secreting factors into the CSF. However, much of choroid plexus developmental function is still unclear. Most previous studies on this organ have been undertaken in rodents but translation into humans is not straightforward since they have a different timing of brain maturation processes. We have collected choroid plexus from three fetal gestational ages of a non-human primate, the baboon, which has much closer brain development to humans. The transcriptome of the plexuses was determined by next generation sequencing and Ingenuity Pathway Analysis software was used to annotate functions and enrichment of pathways of changes in the transcriptome. The number of unique transcripts decreased with development and the majority of differentially expressed transcripts were down-regulated through development suggesting a more complex and active plexus earlier in fetal development. The functional annotation indicated changes across widespread biological functions in plexus development. In particular we find age-dependent regulation of genes associated with annotation categories: Gene Expression, Development of Cardiovascular System, Nervous System Development and Molecular Transport. Our observations support the idea that the choroid plexus has roles in shaping brain development.

Keywords: choroid plexus, development, RNAseq, fetus, primate, non-human, baboon, transcriptome

\section{Introduction}

The choroid plexus is a small organ situated in the roof of each brain ventricle. Its main interface is the epithelium that overlays the vascularized core. Its role is often stated as production of cerebrospinal fluid (CSF), which acts to flush out waste products of the central nervous system (CNS), a role that is often referred to as the sink effect. However, there is an increasing awareness of the importance of this organ and the CSF in relation to brain development and 
normal brain function throughout the life-course. The CSF influences both neurogenesis and neuronal migration having a role during development, in the adult, in aging and after brain trauma (Miyan et al., 2003; Redzic et al., 2005; Sawamoto et al., 2006; Falcao et al., 2012; Zappaterra and Lehtinen, 2012; Lehtinen et al., 2013). In addition, the choroid plexus is today thought to play a role as an immune sensing organ for the brain as well as serving as a point of entry for immune cells into the CNS (Ransohoff and Engelhardt, 2012). CSF secretion by the plexuses is impressive. It turns over the total CSF volume (about $150 \mathrm{~mL}$ in a human adult) 3-4 times each day. In addition, the plexuses secrete a wide range of signaling molecules (Lehtinen et al., 2013).

The role of the choroid plexus in development may be of particular importance since it differentiates very early during fetal life, just after neural tube closure, at a time of early cortical vascularization. In the mouse, the rombencephalic choroid plexuses start to differentiate at around E12 and in humans it occurs at around 7 weeks gestation with the telencephalic choroid plexus (tCP) and diencephalic soon after (Jacobsen et al., 1982; Dziegielewska et al., 2001). The size of the plexuses is very much larger in the embryo/fetus in relation to brain size than in the adult (Johanson, 1995). Soon after the plexuses are formed they occupy almost the whole ventricular space at a stage when the cortex is just a thin layer of cells. One important anatomical relationship is the close opposition of the lateral choroid plexus to the ventricular surface of the developing brain where most neurogenesis occurs, suggesting that factors secreted by this plexus are in a position to influence brain development (Falcao et al., 2012). CSF in developing animals has a much higher protein concentration than in adults (Dziegielewska et al., 2000), a difference that was previously thought to be a consequence of an open blood-CSF barrier in early life. However, there is now significant evidence that this barrier closes early in fetal life (Ek et al., 2003; Liddelow et al., 2013). However, the exact contribution of the choroid plexus to the protein composition of fetal CSF is still not clear, as it is able to contribute to both secretion and absorption of a variety of molecules thereby regulating the CSF environment.

With the availability of powerful new molecular techniques, some of the early signaling systems, such as the bone morphogenetic proteins and the sonic hedgehog signaling, that control plexus differentiation have now been proposed (Lehtinen et al., 2013). These authors highlight that the choroid plexus is one of the most under-studied organs in the brain suggesting that new roles for the plexus remain to be discovered. Nearly all the studies of development and function of the choroid plexus have been undertaken in altricial rodent models (Kratzer et al., 2013; Liddelow et al., 2013; Saunder et al., 2013), which have a shorter prenatal development than more precocial species such as humans and non-human primates. There are key differences in the developmental trajectory and regulation between precocial and altricial species (Rabadan-Diehl and Nathanielsz, 2013). Thus, studies in precocial species are needed to remove this barrier to our understanding. To obtain information that will help address this need, we present here information on gene expression in the lateral choroid plexus tissue at three developmental stages in the baboon fetus which shows much closer development to humans. Baboons present one of the best opportunities for comparative studies of maturation of neural structures in fetal life with human fetuses. For example, they show features such as cerebral gyrification index in development similar to humans (Rogers et al., 2010). Our study of choroid plexus development was conducted during the second half of gestation when most of the cortical expansion is occurring in primates (Kroenke et al., 2007). We analyzed the full transcriptome identified using Next Generation Sequencing and used the Ingenuity knowledge base to annotate functions, enrichment of pathways and to predict regulatory networks of changes in the transcriptome.

\section{Materials and Methods}

\section{Care of Animals}

Fourteen female baboons (Papio hamadryas) from the Southwest National Primate Research Center (San Antonio, TX, USA), were recruited for this study and maintained in group housing. All procedures were approved by the Texas Biomedical Research Institute Institutional Animal Care and Use Committee and conducted in AAALAC-approved facilities. The caging system allows control and monitoring of food intake while still maintaining female baboons in group housing, thereby permitting normal social and physical activity (Schlabritz-Loutsevitch et al., 2004). Feeding and management have previously been described in detail (Li et al., 2013). Cesarean sections were performed between 0800 and $1000 \mathrm{~h}$ at 90,120 , and 165 days of gestation (term 184 days) under general anesthesia using techniques previously reported in detail (Schlabritz-Loutsevitch et al., 2007). Food was withdrawn for $16 \mathrm{~h}$ before surgery. Post-operative analgesia was provided with buprenorphine hydrochloride $0.015 \mathrm{mg} / \mathrm{kg}$ per day (Hospira, Inc., Lake Forest, IL, USA) for three post-operative days. Fetal tCP was collected and flash frozen in liquid nitrogen at 90 days gestation ( 3 males/ 1 female), 120 ( 2 males/ 2 females) and 165 day ( 3 males/ 3 females) of gestation, corresponding to $0.5,0.7$, and 0.9 gestation.

\section{RNA Preparation and Illumina Next Generation Sequencing}

Frozen tissues were homogenized with a mechanical homogenizer in $400 \mu \mathrm{L}$ RNase free PBS. RNA was extracted with an RNAeasy mini-kit (Qiagen) according to manufacturer's recommendations along with DNA digestion. RNA quality was analyzed by Biorad Experion electrophoresis. RNA sequencing was performed at the Genomics Core Facility at University of Gothenburg. $1 \mu \mathrm{g}$ of high quality total RNA (RIN $>8$ ) was used for library preparation. Libraries were created using the TruSeq $^{\mathrm{TM}}$ RNA Sample Preparation v2 kit, according to the manufacturer's protocol (TruSeq ${ }^{\mathrm{TM}}$ RNA Sample Preparation v2 Guide). The library was subjected to $50 \mathrm{bp}$ single end read cycles of sequencing on an Illumina HiScan SQ as per manufacturer protocol.

\section{Analysis of Sequencing Data}

Quality assessment of the sequence reads was performed by generating QC statistics with FastQC (http://www. bioinformatics.bbsrc.ac.uk/projects/fastqc). RNA-seq reads were mapped to the current baboon genome assembly, 
Pham_1.01 (https://www.hgsc.bcm.edu/content/baboon-geno me-project) using Tophat (Trapnell et al., 2009). Cufflinks (Trapnell et al., 2012) was used for transcript assembly of individual samples. All assemblies were merged to create a reference transcript, which was used to calculate gene counts with HTSeq (http://www-huber.embl.de/users/anders/ HTSeq/doc/overview.html). One of the replicates from GD90 was removed from the analysis since it was found to be extremely different from the other replicates. DESeq (Anders and Huber, 2010) was then used to find differentially expressed genes. Annotation of genes was done through blasting (Altschul et al., 1990) each transcript against the Uniprot database (UniProt Consortium, 2012). Transcripts with a $p$-value of less than 0.05 after false discovery rate correction were considered to be significantly differentially expressed. Proteins with human homologs were used as input to for analysis in Ingenuity Pathway Analysis software (IPA), a program that serves for the interpretation of large scale transcriptome data across species, with a fold change cut-off of 2.0 and $p<0.05$ across all analysis. This program creates these analyses using the Ingenuity knowledge base, a huge database containing millions of individual interactions between genes and proteins. Analysis using IPA was conducted between June-September 2014 (Ingenuity version 18841524; www.ingenuity.com). A core analysis was created in IPA using standard settings with duplicates resolved with average fold changes.

\section{Results}

\section{Whole Genome Expression Profiling}

We used next generation sequencing to analyze fetal developmental changes in the tCP transcriptome. In total 75,760 transcripts were detected ( $\geq 1$ transcript) across all tCP samples with 70,821 transcripts at GD90, 71,625 at GD120 and 72,651 at GD165.

In Figure 1A the number of transcripts in common and unique in the tCP at GD90, GD120 and GD165, is depicted by a Venn-diagram. For this illustration, a transcript was considered present when the majority of animals had $>0$ transcripts and the median number of transcripts (read counts) was $\geq 10$ at each gestational age. This shows that the majority of transcripts $(78.4 \%)$ were common across all gestational ages. The number of transcripts unique (i.e., only present within a single group) at GD90 was 2790 transcripts (5.8\% of total within this group), at GD120 was 1362 (2.8\%) and at GD165 was 1147 (2.4\%). The number of transcripts unique to the GD90/GD120 groups was 3276 (6.7\% of total), in the GD120/165 groups it was $1242(2.6 \%)$ and in the GD90/165 groups it was $598(1.2 \%)$. Pairwise analysis of differentially expressed transcripts between gestational ages was carried out for the input into IPA. As seen in Figure 1B, the total number of differentially expressed transcripts was greater between GD120 and GD165 (3052 or 4.1\% of total) than between GD90 and GD120 (856 or 1.1\%) and overall between GD90 and GD165 more than 10,000 transcripts (13.6\%) were differentially expressed. There was also a greater number of significantly down-regulated over up-regulated transcripts both from GD90 to GD120 (45\% up, 55\% down) and from GD120 to GD165 (28\% up, $72 \%$ down). The number of differentially expressed transcripts in common between the pairwise comparisons is presented in Figure 1C. About 29\% of transcripts that were significantly up-regulated between GD90 and GD120 were also significantly up-regulated between GD120 and GD165 and about $49 \%$ of transcripts significantly down-regulated between GD90 and GD120 were also down-regulated between GD120 and GD165. Only 20 transcripts in total were significantly regulated in opposite directions (up/down or down/up) between the two pairwise comparisons.

\section{Functional Annotation: GD90/GD120 and GD120/GD165}

Ingenuity pathway analysis software was used for the functional annotation of differentially expressed genes. This uses the Ingenuity knowledge base to functionally annotate genes and to predict the biological functions of these changes (downstream effect analysis). The tendency (direction) of a biological function can in this way be predicted (activation $\mathrm{z}$-score; $>2.0$ or $<-2$ is significantly predictive). These functions are classified under three main groups: Physiological System Development and Function, Molecular and Cellular Mechanisms and Diseases \& Disorders. The functional annotation showed that the top five functional categories were similar between the pairwise comparisons of GD90/GD120 and GD120/GD165. For Physiological Systems the annotations were all related to tissue development or survival with Embryonic development, Organ development and Organismal development common in the top five for the two pairwise comparisons. Similarly, for Molecular and Cellular Functions 3/5 groupings were in common (Cellular movement, Cellular Assembly and Organization, and Cell Morphology). It should also be mentioned that 4/5 top Diseases and Disorders in the functional annotation were identical in the two pairwise analysis (Cancer, Organismal Injury and Abnormalities, Reproductive System Disease and Gastrointestinal Disease). Furthermore, a more comprehensive comparison analysis of the pairwise functional annotation was also made between the GD90/GD120 and GD120/GD165. In Table 1 are the top 25 activation $\mathrm{z}$-scores presented for all biological functions. Only biological functions where an activation $\mathrm{z}$-score could be calculated for both pairwise comparisons have been included. Twenty-two out of top 25 Disease/Biofunctions showed the same directionality and the three opposing activation scores were all found in the lower half of the Table with two of these being closely related (Proliferation of cells and Quantity of cells). The most notable difference between age comparisons was that Migration of Cells had a positive activation score for the GD90/GD120 comparison ( +1.15 ; non-significant), whereas a negative activation score was found for the GD120/GD165 comparison (-4.06; significant). Although many of these predicted effects such as disease related changes may not be directly relevant for choroid plexus developmental function, it indicates that the functional annotations of the transcriptional changes throughout fetal tCP development are quite uniform. For these reasons further analysis was focused on differential changes between GD90 and GD165 and annotations to diseases and disorders were not further considered. 


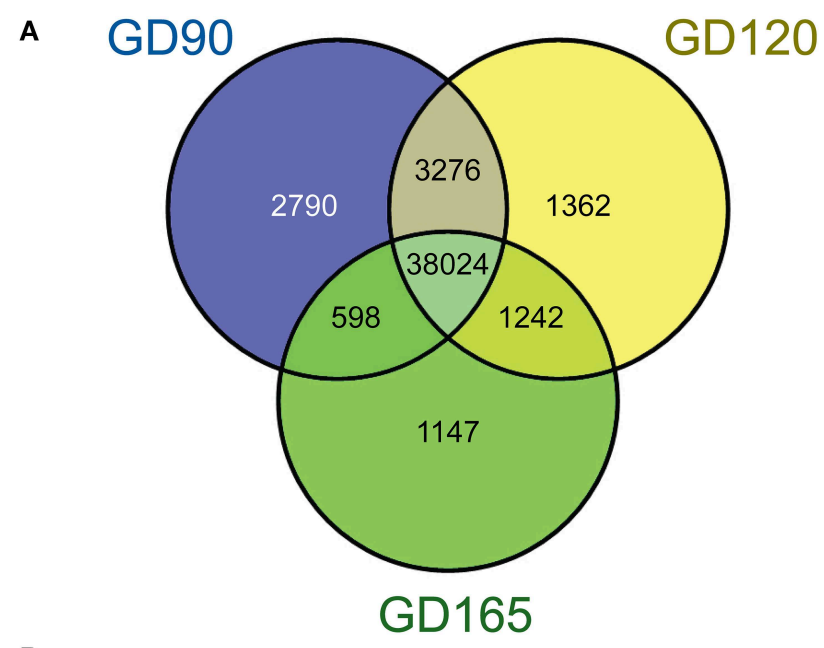

B

\begin{tabular}{rrcccc}
\hline & TOTAL & UP & DOWN & SIGN UP & SIGN DOWN \\
\hline GD90 to GD120 & 72930 & 36030 & 36900 & 386 & 470 \\
GD120 to GD165 & 73798 & 38755 & 35043 & 856 & 2196 \\
GD90 to GD165 & 73751 & 39328 & 34423 & 3739 & 6317 \\
\hline
\end{tabular}

C

all transcripts

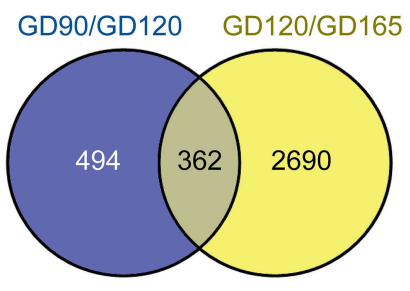

up-regulated

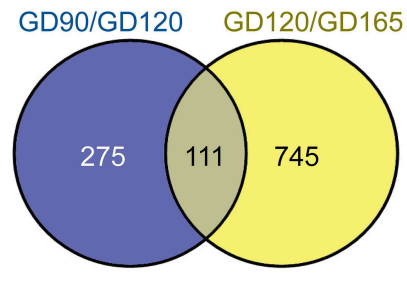

down-regulated

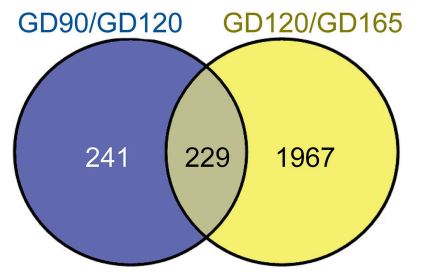

FIGURE 1 | (A) Venn-diagram showing the number of transcripts present at gestational day (GD) 90 (blue), 120 (yellow), and 165 (green) in the fetal baboon choroid plexus. A transcript was considered present when the majority of animal at each age had $>1$ transcript and the median number of transcripts was $>10$. The majority of transcripts were present at all ages (78\%). The number of unique transcripts decreased with developmental age with 2790 at GD90, 1362 at GD120 and 1147 at GD165. (B) Pairwise comparisons of the number of up/down regulated transcripts (non-significant) as well as significantly regulated transcripts between the three fetal ages. The total number of significantly regulated transcripts was less between GD90 and GD120 (856) than between GD120 and GD165 (3052) and greatest between GD90 and GD165 (10056). (C) Number of significantly regulated transcripts that were in common between the two pairwise comparisons GD90/GD120 and GD120/GD165 (left) as well as up-(middle) and down-regulated (right) transcripts.

\section{Analysis of Transcriptional Changes between GD90 and GD165}

\section{Functional Annotation}

All significant biological functions of highly regulated categories are presented in Tables 2A,B sorted after $p$-values with 19 categories for Molecular and Cellular Mechanisms and with 20 categories for Physiological Systems and Development. A complete list with all subcategories is presented in Supplementary Table 1 for all annotated functions with a significant activation z-score. Based on the potential significance specifically for choroid plexus function and development we selected four highly regulated categories (Gene Expression; Development of Cardiovascular
System; Nervous System Development and Molecular Transport) for further detailed analysis. These functions were all significantly enriched in the functional annotation (Tables 2A,B).

\section{Gene expression}

The majority of significantly regulated transcripts were downregulated in our dataset between GD90 and GD165 (see Figure 1). Table 3A lists all sub-categorized functions for gene expression functions. All genes annotated to Transcription, Transcription of RNA/DNA, Expression of RNA/DNA and Transactivation showed overall significant negative activation scores $(-2.84$ to -5.22$)$ predicting inhibition in all these functions. 
TABLE 1 | Comparison analy1sis of functional annotation for the pairwise GD90/GD120 and GD120/GD165 comparisons.

\begin{tabular}{l|r|r}
\hline Diseases and bio functions & GD90/GD120 & GD120/GD165 \\
\hline Organismal death & 3.05 & 12.80 \\
\hline Congenital anomaly of musculoskeletal system & 3.19 & 5.51 \\
\hline Growth failure & 0.93 & 7.09 \\
\hline Congenital anomaly of skeletal bone & 2.76 & 5.03 \\
Craniofacial abnormality & 2.76 & 4.94 \\
\hline Midline defect & 2.59 & 4.66 \\
\hline Size of body & -1.02 & -6.22 \\
\hline Polydactyly & 2.42 & 3.43 \\
Edema & 1.63 & 3.99 \\
\hline Dysplasia & 2.22 & 3.36 \\
\hline Congenital anomaly of limb & 2.27 & 3.26 \\
\hline Morphology of head & 2.40 & 3.10 \\
\hline Migration of cells & 1.15 & -4.09 \\
Morphology of nervous system & 1.91 & 3.26 \\
Abnormal morphology of head & 2.21 & 2.96 \\
\hline Abnormal morphology of nervous system & 2.43 & 2.61 \\
\hline Proliferation of cells & 0.21 & -4.78 \\
\hline Multiple congenital anomalies & 1.47 & 3.50 \\
\hline Formation of cellular protrusions & -0.01 & -4.60 \\
\hline Morphology of central nervous system & 1.98 & 2.61 \\
\hline Dyspnea & 1.20 & 3.33 \\
\hline Abnormal morphology of brain & 1.98 & 2.43 \\
Abnormal morphology of central nervous system & 1.98 & 2.43 \\
\hline Quantity of cells & 0.73 & -3.67 \\
\hline Valus action & & \\
\hline
\end{tabular}

Values are activation z-scores of Diseases and Biofunctions in order of highest scoring with values color indexed red for positive scores and blue for negative scores. All but three functions show the same directionality in z-score (most notably exception being Migration of cells). Many of these functions can be expected to have little relevance for choroid plexus function but still suggests that the transcriptomal changes in plexus are relatively consistent. Note that the comparison GD120/GD165 consistently show higher/lower activation scores than the GD90/GD120

It should be noted that there is a very high level of overlap between the genes associated with the first five of these functions $(>90 \%)$ but also Transactivation show substantial overlap of genes with Transcription (about 75\%). We have presented the 20 most regulated genes (based on fold change) predicting decrease in Transactivation (elements working in trans to regulate transcription), the category which showed the most negative activation score $(-5.22)$, see Table 3B. In total 98 gene changes predicted a decrease in Transactivation and 38 predicted an increase out of a total of 149 genes annotated to this function. The number of findings of each predicted direction of function (increase or decrease) for each gene is also presented in this Table as a measure of robustness for the prediction. In Table 3B , and in other similar Tables showing top regulated genes, we have only included genes for which there are at least 2 findings in the Ingenuity knowledge base predicting consistent change in function (checked for each gene in all lists).

\section{Cardiovascular system development and function}

Since the choroid plexus is a highly vascularized tissue we were particularity interested in genes related to cardiovascular
TABLE 2A | Functional annotation under Physiological System Development and Function of genes significantly changed between GD90 and GD165.

Category p-values

Organismal survival

1.50E-14-1.23E-03

Cardiovascular system development and function

1.50E-14-1.25E-03

Embryonic development

3.49E-14-1.19E-03

Organismal development

2.87E-11-8.44E-04

Nervous system development and function

2.87E-11-1.23E-03

Tissue development

Organ development

4.04E-10-1.46E-05

Organ morphology

5.25E-10-1.23E-03

4.23E-09-1.23E-03

Respiratory system development and function

$6.01 \mathrm{E}-08-1.11 \mathrm{E}-03$

Tissue morphology

$6.01 \mathrm{E}-08-1.23 \mathrm{E}-03$

Visual system development and function

$6.01 \mathrm{E}-08-1.09 \mathrm{E}-03$

Connective tissue development and function

3.03E-07-1.19E-03

Skeletal and muscular system development and function $\quad 3.05 \mathrm{E}-07-3.05 \mathrm{E}-07$

Digestive system development and function

Behavior

Hair and skin development and function

9.14E-07-1.25E-03

3.76E-06-6.16E-04

1.00E-04-9.29E-04

$1.92 \mathrm{E}-04-1.2 \mathrm{E}-03$

4.02E-04-4.02E-04

Reproductive system development and function

Lymphoid tissue structure and development

4.02E-04-1.11E-03

Hematological system development and function

4.38E-04-9.29E-04

The $p$-value is the probability that the gene changes are related to a particular function just by chance. These are given as ranges since there are several subcategories under each of these top categories.

function. In the functional annotation for System Biology this category scored the second most significant $p$-value (Table 2A). Functions categorized to Cardiovascular Development and Function are presented in Table 4A with predicted inhibition in Development of blood vessels, Angiogenesis, Morphogenesis, Vasculogenesis, and Formation of blood vessels whereas the Morphology of Cardiovascular System was predicted to increase, Table 4A. There is a considerable overlap of genes annotated to these functions so we have only presented genes associated with Vasculogenesis which had the most significant $\mathrm{z}$-score. From a total of 245 genes of genes annotated to Vasculogenesis, 107 were predictive of decrease in function whereas 67 predicted increase. In Table 4B we have presented the 20 most regulated genes predicting decrease in Vasculogenesis.

\section{Nervous system development and function}

The tCP is situated in close proximity to the subventricular zone, an area important for neurogenesis. In the downstream effect analysis of System Biology the category Nervous System Development scored the 5th most significant $p$-value (Table 2A). Subcategory functions are presented in Table 5A along with activation $\mathrm{z}$-scores. All functions scored negative activation scores (range -2.01 to -3.23 ) except those related to Morphology of Central Nervous System, which had positive activation scores $(+3.13$ to +4.01$)$. We have further presented genes for Formation of neurites and Morphology of CNS as these two had the most negative and positive activation $\mathrm{z}$-scores (Tables 5B,C). 
TABLE 2B | Functional annotation under Molecular and Cellular Functions of genes significantly changed between GD90 and GD165.

\begin{tabular}{|c|c|c|c|}
\hline \multicolumn{2}{|l|}{ Category } & \multicolumn{2}{|c|}{$p$-values } \\
\hline \multicolumn{2}{|c|}{ Cellular assembly and organization } & \multicolumn{2}{|c|}{ 2.64E-18-9.48E-04 } \\
\hline \multicolumn{2}{|c|}{ Cellular function and maintenance } & \multicolumn{2}{|c|}{$2.64 \mathrm{E}-18-9.48 \mathrm{E}-04$} \\
\hline \multicolumn{2}{|c|}{ Cellular growth and proliferation } & \multicolumn{2}{|c|}{$7.27 \mathrm{E}-14-1.22 \mathrm{E}-03$} \\
\hline \multicolumn{2}{|l|}{ Cell morphology } & \multicolumn{2}{|c|}{ 1.33E-12-1.16E-03 } \\
\hline \multicolumn{2}{|l|}{ Cellular movement } & \multicolumn{2}{|c|}{$2.87 \mathrm{E}-11-1.2 \mathrm{E}-03$} \\
\hline \multicolumn{2}{|l|}{ Cellular development } & \multicolumn{2}{|c|}{ 2.83E-10-5.57E-04 } \\
\hline \multicolumn{2}{|l|}{ Cell death and survival } & \multicolumn{2}{|c|}{ 4.06E-07-1.11E-03 } \\
\hline \multicolumn{2}{|c|}{ Post-translational modification } & \multicolumn{2}{|c|}{$6.93 \mathrm{E}-07-1.07 \mathrm{E}-03$} \\
\hline \multicolumn{2}{|l|}{ Cell cycle } & \multicolumn{2}{|c|}{$6.95 \mathrm{E}-06-1.11 \mathrm{E}-03$} \\
\hline \multicolumn{2}{|c|}{ Cell-to-cell signaling and interaction } & \multicolumn{2}{|c|}{ 2.57E-05-6.44E-04 } \\
\hline \multicolumn{2}{|l|}{ Gene expression } & \multicolumn{2}{|c|}{ 9.51E-05-2.8E-04 } \\
\hline \multicolumn{2}{|l|}{ Molecular transport } & \multicolumn{2}{|c|}{$9.51 \mathrm{E}-05-1.07 \mathrm{E}-03$} \\
\hline \multicolumn{2}{|c|}{ DNA replication, recombination, and repair } & \multicolumn{2}{|c|}{ 1.9E-04-1.07E-03 } \\
\hline \multicolumn{2}{|l|}{ Amino acid metabolism } & \multicolumn{2}{|c|}{ 2.05E-04-2.05E-04 } \\
\hline \multicolumn{2}{|c|}{ Small molecule biochemistry } & \multicolumn{2}{|c|}{ 2.52E-04-4.29E-04 } \\
\hline \multicolumn{2}{|l|}{ Cell signaling } & $4.53 \mathrm{E}$ & $3 \mathrm{E}-04$ \\
\hline Carbohydrate metabolis & & 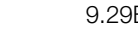 & 9E-04 \\
\hline Cellular compromise & & 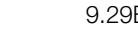 & $9 \mathrm{E}-04$ \\
\hline Protein synthesis & & 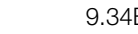 & 1E-03 \\
\hline $\begin{array}{l}\text { The } p \text {-value is the probab } \\
\text { just by chance. These are } \\
\text { each of these top categor }\end{array}$ & $\begin{array}{l}\text { e gene cha } \\
\text { ranges sinc }\end{array}$ & $\begin{array}{l}\text { related to a pe } \\
\text { e several subc }\end{array}$ & $\begin{array}{l}\text { nction } \\
\text { under }\end{array}$ \\
\hline $\begin{array}{l}\text { TABLE 3A | Subcateg } \\
\text { expression along with }\end{array}$ & $\begin{array}{l}\text { ions for ge } \\
\text { ons for ac }\end{array}$ & $\begin{array}{l}\text { lotated to ge } \\
\text { state. }\end{array}$ & \\
\hline Functions annotation & z-score & Prediction & \# \\
\hline Transcription & -4.00 & Decreased & 428 \\
\hline Transcription of DNA & -2.84 & Decreased & 319 \\
\hline Transcription of RNA & -3.80 & Decreased & 419 \\
\hline Expression of DNA & -3.36 & Decreased & 337 \\
\hline Expression of RNA & -4.15 & Decreased & 464 \\
\hline Transactivation & -5.22 & Decreased & 149 \\
\hline
\end{tabular}

$\#$, Number of annotated genes to function.

\section{Transport of molecule}

Since the choroid plexuses is one of the interfaces between blood and the CNS, is the main source of CSF and is known to be rich in transporter proteins we have further explored genes associated to Transport of Molecule. This subcategory scored an activation $\mathrm{z}$-score of -1.81 (non-significant). The top 25 regulated up/down genes between GD90 and GD165 are presented in Table 6.

\section{Generation of heatmaps}

Differentially expressed transcripts/genes, annotated to a certain function, were visualized as heatmaps. Cluster analysis and heatmaps were generated in the statistical software $\mathrm{R}$ and presented in Figure 2.

\section{Regulatory Networks}

Regulatory networks were built from biological functions using transcript profiles from the dataset and required direct
TABLE 3B | The 20 most genes regulated genes predicting a decrease in Transactivation between GD90 and GD165.

\begin{tabular}{lclc}
\hline Gene ID & Fold change & Findings & $\#$ \\
\hline CREBBP & -5.30 & Increases & $(30)$ \\
NCOA2 & -4.53 & Increases & $(32)$ \\
EP300 & -4.32 & Increases & $(38)$ \\
MYBL2 & -3.99 & Increases & $(2)$ \\
SMARCC1 & -3.96 & Increases & $(2)$ \\
TRRAP & -3.89 & Increases & $(4)$ \\
NFE2 & -3.74 & Increases & $(2)$ \\
CCNT1 & -3.72 & Increases & $(4)$ \\
BMPR1B & -3.46 & Increases & $(2)$ \\
MITF & -3.43 & Increases & $(2)$ \\
GLI2 & -3.35 & Increases & $(2)$ \\
TWIST1 & -3.25 & Increases & $(2)$ \\
NCOA1 & -3.23 & Increases & $(35)$ \\
MED1 & -3.22 & Increases & $(4)$ \\
UBR5 & -2.97 & Increases & $(2)$ \\
NOTCH1 & -2.95 & Increases & $(7)$ \\
E2F1 & -2.86 & Increases & $(6)$ \\
PRKG1 & -2.83 & Increases & $(2)$ \\
NR3C1 & -2.75 & Increases & $(38)$ \\
IRF7 & +2.74 & Decreases & $(2)$ \\
& & &
\end{tabular}

In total 98 gene changes predicted a decrease in transactivation and 38 predicted an increase out of total 149 genes in data set. Number in brackets is number of findings that the prediction is based upon.

TABLE 4A | Subcategory functions for genes annotated to Cardiovascular System along with predictions for activation state.

\begin{tabular}{lccc}
\hline Functions annotation & z-score & Prediction & $\#$ \\
\hline Development of cardiovascular system & -2.58 & Decreased & 328 \\
Angiogenesis & -2.39 & Decreased & 234 \\
Morphogenesis of cardiovascular system & -3.01 & Decreased & 72 \\
Development of blood vessel & -2.46 & Decreased & 268 \\
Vasculogenesis & -3.15 & Decreased & 245 \\
Formation of blood vessel & -2.15 & Decreased & 33 \\
Morphology of cardiovascular system & 3.06 & Increased & 224
\end{tabular}

\#, Number of annotated genes to function.

connections between molecules based on experimental evidence in the IPA Knowledge Base. The Regulatory networks connect biological functions of regulated genes in the dataset with upstream (top) regulators. We present the most consistent networks relating to the biological functions within Gene Expression, Cardiovascular System Development and Function, Nervous System Development and Function. No extensive and consistent networks could be built from genes categorized to Transport of molecule and none is illustrated. Networks are presented in Figure 3. The top consistent network related to gene expression was associated with transcription of RNA and transactivation (consistency score +6.75 ) predicting 
TABLE 4B | The 20 most regulated genes predicting a decrease in Vasculogenesis between GD90 and GD165.

\begin{tabular}{|c|c|c|c|c|}
\hline Gene ID & Fold change & \multicolumn{2}{|c|}{ Findings } & $\#$ \\
\hline $\mathrm{CDH} 13$ & -52.11 & \multicolumn{2}{|c|}{ Increases } & (5) \\
\hline NPPC & 43.88 & \multicolumn{2}{|c|}{ Decreases } & (2) \\
\hline SLC8A1 & -11.58 & \multicolumn{2}{|c|}{ Increases } & (2) \\
\hline EDIL3 & -8.34 & \multicolumn{2}{|c|}{ Increases } & (2) \\
\hline SPP1 & -8.13 & \multicolumn{2}{|c|}{ Increases } & (3) \\
\hline MEOX2 & -6.87 & \multicolumn{2}{|c|}{ Increases } & (2) \\
\hline VASH2 & -6.87 & \multicolumn{2}{|c|}{ Increases } & (2) \\
\hline SEMA5A & -6.01 & \multicolumn{2}{|c|}{ Increases } & (2) \\
\hline SLIT2 & -5.99 & \multicolumn{2}{|c|}{ Increases } & (2) \\
\hline COL4A3 & 5.50 & \multicolumn{2}{|c|}{ Decreases } & (3) \\
\hline FGF13 & -5.19 & \multicolumn{2}{|c|}{ Increases } & (6) \\
\hline ANPEP & -4.36 & \multicolumn{2}{|c|}{ Increases } & (2) \\
\hline ETS1 & -4.23 & \multicolumn{2}{|c|}{ Increases } & (3) \\
\hline MDK & -4.15 & \multicolumn{2}{|c|}{ Increases } & (3) \\
\hline ANGPT1 & -4.11 & \multicolumn{2}{|c|}{ Increases } & $(20)$ \\
\hline EFNB2 & -4.04 & \multicolumn{2}{|c|}{ Increases } & $(24)$ \\
\hline PIKЗС2A & -4.04 & \multicolumn{2}{|c|}{ Increases } & (2) \\
\hline $\mathrm{CDH} 5$ & -4.02 & \multicolumn{2}{|c|}{ Increases } & $(22)$ \\
\hline MTOR & -3.95 & \multicolumn{2}{|c|}{ Increases } & (3) \\
\hline THBS2 & 3.75 & \multicolumn{2}{|c|}{ Decreases } & (3) \\
\hline \multicolumn{5}{|c|}{$\begin{array}{l}\text { From a total of } 245 \text { annotated genes, } 107 \text { are predictive of inhibition of function whereas } \\
67 \text { predict activation. Number in brackets is number of findings that the prediction is based } \\
\text { upon. }\end{array}$} \\
\hline \multicolumn{5}{|c|}{$\begin{array}{l}\text { TABLE 5A | Functions annotation for Nervous System Development along } \\
\text { with predictions for activation state (z-score). }\end{array}$} \\
\hline \multicolumn{2}{|c|}{ Functions annotation } & \multicolumn{2}{|l|}{ z-score } & $\#$ \\
\hline Developm & & -2.01 & Decreased & 157 \\
\hline Migration & & -2.66 & Decreased & 89 \\
\hline Proliferatio & al cells & -2.75 & Decreased & 174 \\
\hline Extension & & -2.20 & Decreased & 58 \\
\hline Guidance & & -2.59 & Decreased & 55 \\
\hline Formation & & -3.23 & Decreased & 59 \\
\hline Long-term & of brain & -2.04 & Decreased & 43 \\
\hline Long-term & , hippocampus & -2.36 & Decreased & 38 \\
\hline Long-term & , cerebral cortex & -2.38 & Decreased & 39 \\
\hline Plasticity c & & -2.72 & Decreased & 36 \\
\hline Morpholos & & 4.06 & Increased & 162 \\
\hline Morpholos & & 4.06 & Increased & 148 \\
\hline Morpholog & & 3.13 & Increased & 75 \\
\hline Morpholog & phalon & 3.13 & Increased & 60 \\
\hline
\end{tabular}

\#, Number of annotated genes to function.

regulating of these functions by an activation of miRNA-243-p, miRNA-34a-5p, while inhibition of MSGN and IHH. The most consistent network within Cardiovascular functions was related to Blood vessel development, Angiogenesis, and Migration/Movement of endothelial cells (consistency score +11.35 ). Top regulators of these functions are predicted to be an activation
TABLE 5B | Genes predicting an inhibition of Formation of neurites between GD90 and GD165.

\begin{tabular}{lrlr}
\hline Gene ID & Fold change & Finding & \# \\
\hline MAP1B & -7.15 & Increases & (6) \\
ROBO1 & -4.52 & Increases & $(2)$ \\
CDH2 & -3.46 & Increases & $(2)$ \\
EFNB1 & -3.18 & Increases & $(3)$ \\
SLC9A1 & -2.48 & Increases & $(4)$ \\
SKIL & -2.42 & Increases & $(2)$ \\
DIXDC1 & -2.40 & Increases & $(2)$ \\
SNCA & -2.32 & Increases & (3) \\
SEMA4D & -2.20 & Increases & (3)
\end{tabular}

In total 20 genes predicted an increase in morphology of CNS with 5 predicting a decrease from total of 59 genes. Number in brackets is the number of findings in in ingenuity knowledge base predicting the functional outcome.

TABLE 5C | All genes predicting an increase in morphology of CNS between GD90 and GD165.

\begin{tabular}{lcll}
\hline Gene ID & Fold change & Finding & $\#$ \\
\hline FEZF1 & -16.15 & Decreases & $(2)$ \\
MAP1B & -7.15 & Decreases & $(3)$ \\
DCC & -5.54 & Decreases & $(2)$ \\
ZNF423 & -4.90 & Decreases & $(2)$ \\
GLI3 & -4.07 & Decreases & $(2)$ \\
NFIB & -3.61 & Decreases & $(2)$ \\
NDST1 & -3.38 & Decreases & $(2)$ \\
EPHA4 & -3.00 & Decreases & $(3)$ \\
NR1H3 & -2.49 & Decreases & $(2)$ \\
NFIA & -2.42 & Decreases & $(3)$ \\
LHX2 & -2.42 & Decreases & $(3)$ \\
CCND2 & -2.27 & Decreases & $(2)$
\end{tabular}

In total 17 genes predicted an increase in morphology of CNS with none predicting an increase from total of 162 genes. Number in brackets is number of findings that the prediction is based upon.

of RUNX4, HAND1, SAV1, TFAP2C while inhibition of NOTCH4, TERT, F2R, MYB, and GNA12. The most consistent network for Nervous System functions was related to Formation of neurites and Guidance of axons (consistency score +3.67) with top regulators of this network being NFIA (inhibited) and NEUROG3 (inhibited).

\section{Canonical Pathways}

Canonical Pathways are well defined pathways involved in intra- and inter-cellular functions. The number of significantly enriched canonical pathways $(p<0.01)$ between GD90 and GD165 was in total 18. For these there is an overrepresentation of genes in our data set for what can be expected from chance. These pathways are presented in Table 7. It should be noted that some of these pathways do have a large overlap of molecules, for instance Axon guidance signaling and Ephrin signaling shares 40 molecules. In addition, we used the Molecular activity predictor in IPA to 
TABLE 6 | Top 25 changed genes annotated to Transport of Molecule based on fold change (both up/down-regulated genes).

\begin{tabular}{|c|c|c|c|c|}
\hline Gene ID & Prediction & FC & Findings & \# \\
\hline KCNJ5 & Increased & -51.21 & Decreases & (2) \\
\hline KCNJ1 & Decreased & -39.25 & Increases & (3) \\
\hline SLCO1C1 & Increased & +27.45 & Increases & (10) \\
\hline CFTR & Decreased & -14.00 & Increases & (30) \\
\hline$C P$ & Increased & +10.78 & Increases & (7) \\
\hline IL6 & Increased & +8.97 & Increases & (10) \\
\hline SLC1A7 & Decreased & -7.12 & Increases & (5) \\
\hline SGK1 & Increased & +6.94 & Increases & (7) \\
\hline$A B C A 1$ & Decreased & -6.22 & Increases & (27) \\
\hline PLA2G1B & Increased & +6.08 & Increases & (4) \\
\hline SLC6A4 & Decreased & -5.77 & Increases & (37) \\
\hline SLC15A2 & Decreased & -5.16 & Increases & (5) \\
\hline SCN5A & Decreased & -5.11 & Increases & (3) \\
\hline LRP1 & Decreased & -5.04 & Increases & (3) \\
\hline SLC17A8 & Decreased & -5.01 & Increases & (5) \\
\hline SLC5A3 & Decreased & -4.88 & Increases & (3) \\
\hline ATP7A & Decreased & -4.86 & Increases & (9) \\
\hline SLC39A8 & Decreased & -4.81 & Increases & (5) \\
\hline XPOT & Decreased & -4.62 & Increases & (2) \\
\hline SLC4A1 & Decreased & -4.53 & Increases & (13) \\
\hline SLCO1A2 & Increased & +4.17 & Increases & (19) \\
\hline HTT & Increased & -3.96 & Decreases & (25) \\
\hline ERBB4 & Decreased & -3.78 & Increases & (5) \\
\hline SLC7A3 & Decreased & -3.52 & Increases & (5) \\
\hline SLC7A2 & Decreased & -3.37 & Increases & (6) \\
\hline
\end{tabular}

FC, Fold change; \#, Number in brackets is number of findings that the prediction is based upon.

predict the outcome of changes in these pathways on biological functions.

\section{Discussion}

The choroid plexus is the site of the blood-CSF barrier. This organ acts like a barrier early in development (Ek et al., 2003; Kratzer et al., 2012; Liddelow et al., 2013). A well-formed barrier is the basis for selective bi-directional transfer of molecules across the plexus. The CSF flow through the ventricular system is often simply thought of as the lymph system of the brain serving as a system for the brain to rid itself of waste products. However, there is now a growing body of evidence that indicating the plexus and its regulation of CSF play an active role in brain development (Falcao et al., 2012; Zappaterra and Lehtinen, 2012). Understanding choroid plexus function during fetal development is central to comprehending normal and abnormal brain development. In order to enhance our understanding of this tissue's normal development we obtained tCP from a primate, the hamadryas baboon, during three different fetal ages, from midterm to late gestation, and sequenced the RNA transcriptome. The strength of this study is that the species studied represents the closest experimental model available to human development and can be obtained in a physiological state at several developmental stages. Post-mortem human tissues are liable to be obtained with questionable quality. Although adult studies have suggested high similarity between the mouse and human choroid plexus transcriptome (Janssen et al., 2013), other studies have revealed mixed overlap of genes across studies of solely the adult mouse choroid plexuses (Marques et al., 2011). These differences may be due to the design of these comparisons. In addition, translation in development from rodents to humans is not straightforward since these two groups of mammals show a different developmental timing of many brain maturation processes. One limitation of the present analysis is that the full baboon genome is not known and we could therefore not fully annotate all transcripts for this primate.

Our analysis reveals that the developmental profile of the plexus transcriptome is age distinct. For every set of transcripts analyzed the three different gestational ages cluster within their age group and with the GD90 and GD120 age groups showing the closest signature of their transcriptome (Figure 2). There are some marked overall developmental changes in the transcriptome. The overall number of transcripts that are age unique in the tCP decreases progressively during fetal development with the greatest number at GD90 and the least at GD165. The exact number is influenced by what is set as "detected" but even when we used different settings for detection level a similar progressive decrease in number of unique transcripts with age was observed, indicating robustness of the analysis. The differential analysis of transcripts also highlights this change in transcriptome in that the majority of significantly changed transcripts were downregulated both between GD90 and GD120 and between GD120 and GD165; overall from GD90 to GD165 about 2/3 of all significantly changed transcripts were down-regulated. Not only does this observation point to the existence of significant changes in the plexus during development it also suggest that the choroid plexus is more active in younger fetuses than at late gestation with many transcripts only turned on earlier in development. In order to further analyze these changes we used the Ingenuity knowledge base to annotate functions, enrichment of pathways and to predict regulatory networks. The functional annotation suggested very widespread functions across many biological processes during plexus development predicting a decrease in most functions also suggestive of a more active plexus in the younger fetus (see Supplementary Table 1 ).

In accordance with the decrease in transcripts during development, the functional annotation predicted significant decrease in all biological functions related to gene expression during tCP development with Transactivation showing the most negative activation score (See Table 3A). Table 3B presents the most regulated genes associated with decreased transactivation. Five of these genes have a very strong supporting base for their predicted function and activation state ( $>30$ findings). These are CREPBP gene (encoding for cAMP-response element binding protein), NCA1 and NCA2 gene (encoding for nuclear receptor coactivator $1 / 2$ proteins), EP300 (encoding for E1A binding protein p300) and NR3C1 (encoding for the glucocorticoid receptor). The first four of these have histone acytyltransferase activity, which acylates histones making DNA more accessible for transcription and also helps numerous nuclear receptors in transcription. $C R E B B P$ and its paralog EP300 can work as epigenetic regulators 


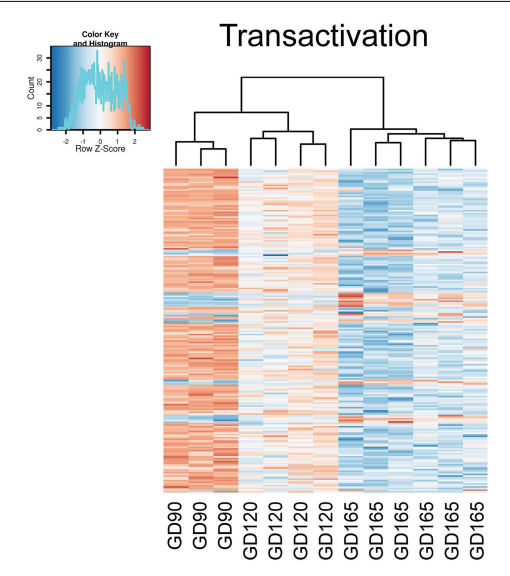

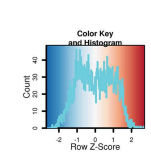

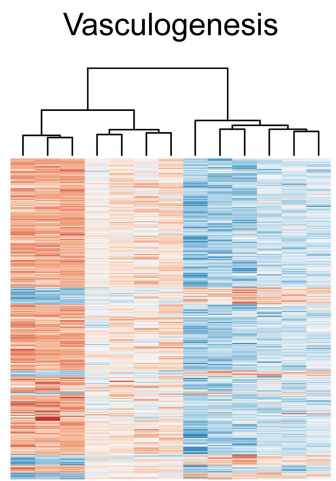

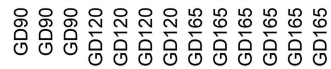

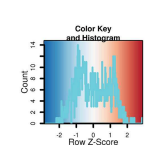

Formation Neurites

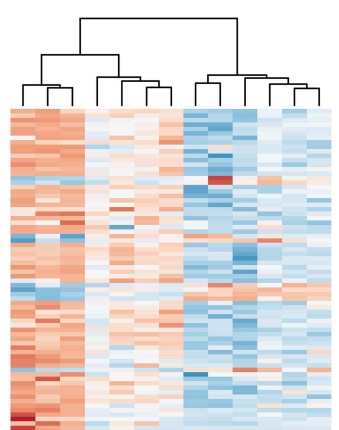

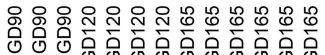
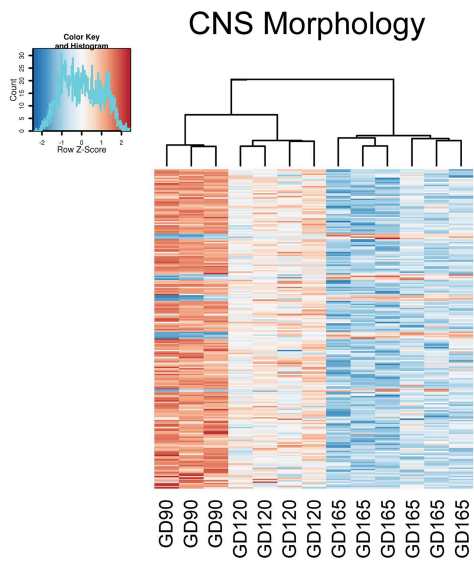

FIGURE 2 | Heatmaps generated from the read counts for each transcript related to certain gene sets for choroid plexuses at gestation day (GD) 90, GD120 and GD165. The gene sets were genes

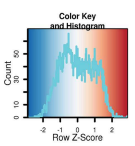

\section{Transport Molecule}

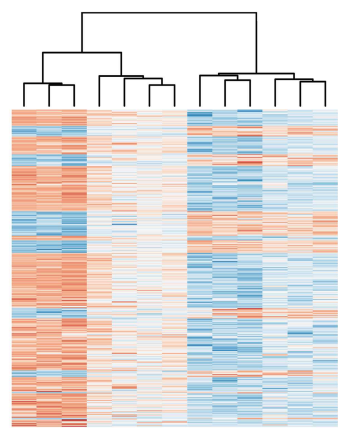

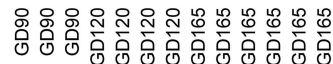

annotated to Transactivation, Vasculogenesis, Formation of neurites, Morphology of CNS and Transport of molecule. Note that the cluster analysis shows that animals group into their respective gestational age.

acetylating key transcription factors such as p53 (Wang et al., 2013). The cytosolic glucocorticoid receptor has many glucocorticoids as ligands and binds to response elements on DNA to turn on genes and has been implicated in many developmental processes.

We found changes similar to those reported in the adult mouse choroid plexus transcriptome in many genes associated to cardiovascular and nervous system development (Marques et al., 2011). In addition, functional annotation showed that Cardiovascular System Development and Function had the second most significant $p$-values (Table 2B). We further explored changes in genes related to the vascular system and in particular development of blood vessels. In general, transcriptional changes predicted a decrease in development of the vascular system during fetal development (Table 4A). In relation to vasculogenesis, it is notable that five of these genes have $>20$ findings in the knowledge base predicting decrease activation state: ANGPT1, encoding for angiopoietin-1 well known to play a role in angiogenesis and stability of blood vessels. EFNB2 gene, encoding for the ephrin-B2 protein, as well as being involved in angiogenesis it is also known to be involved in the development of the nervous system (Kullander and Klein, 2002). The third is CDH5, encoding an endothelial specific cadherin (also known as vascular endothelial cadherin) closely associated to angiogenesis (Daniel and Abrahamson, 2000). The most regulated of the genes associate with inhibition of vasculogenesis was $C D H 13$, encoding for cadherin-13 protein (also known as t-cadherin), although studies suggest that this is important for revascularization of tissues (Parker-Duffen et al., 2013), little is known about its role during normal development.

Previous studies of adult choroid plexus transcriptome have shown expression of many genes associated to Nervous System function (Marques et al., 2011; Janssen et al., 2013; Liddelow et al., 2013). In our analysis, the functional annotation showed that Nervous System Development and Function scored one of the highest significant $p$-values for system biology categories (Table 2B). The functional annotation suggests a great variety of effects on the nervous system including neuronal migration, axonal guidance and formation of neurites involving a large number of effector molecules, which cannot all be detailed. However, each individual predicted function only involves quite a small number of proteins (compared to other functions outlined 

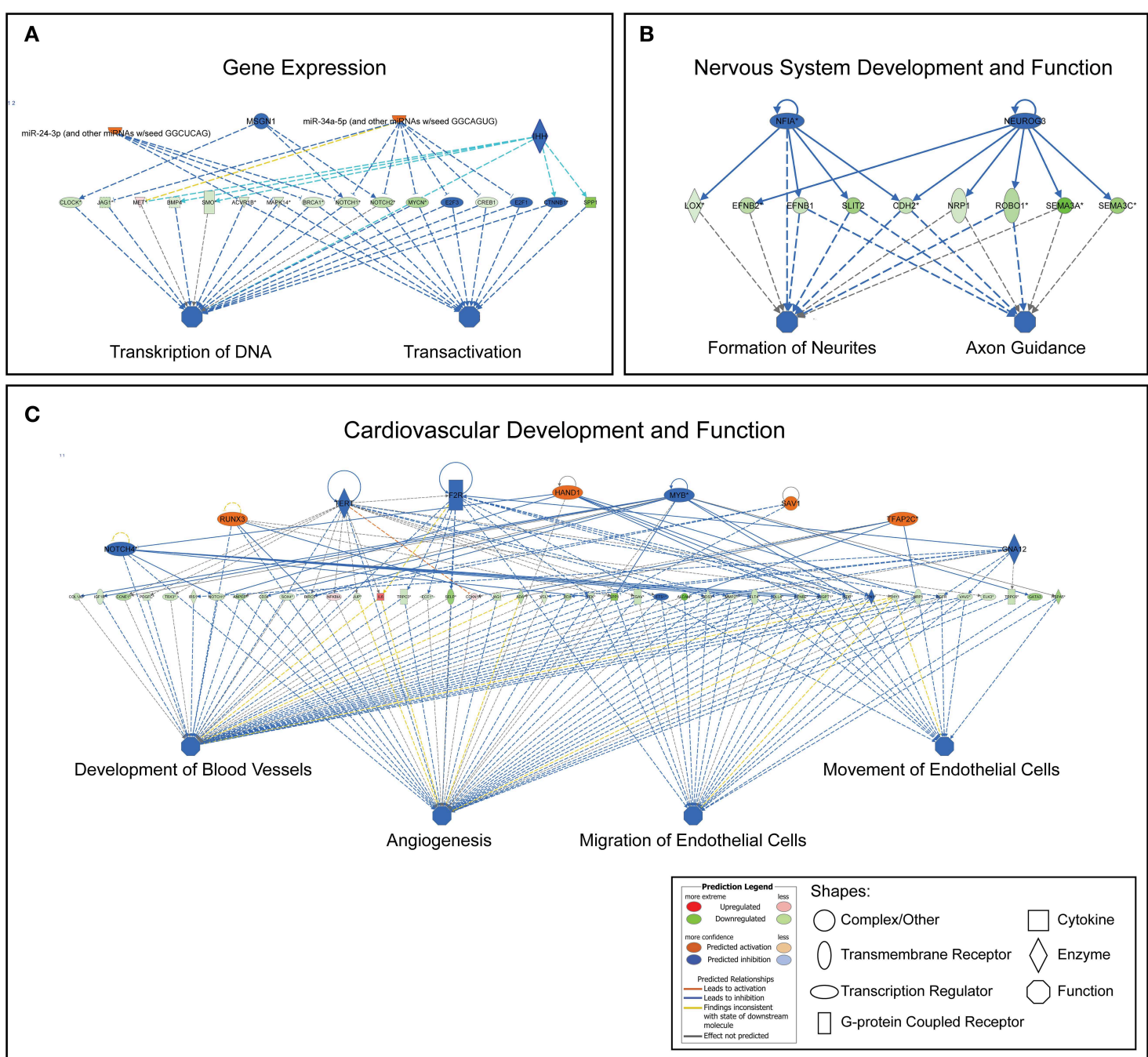

FIGURE 3 | Most consistent regulatory networks generated for the functions of gene expression (A), nervous system and development (B), cardiovascular system development and function (C). This predicts the upstream regulators for the functions tied into the changes in the gene sets. Upstream regulators mainly include transcription factors, enzymes and miRNAs. here). Table 5B, listing all changes predicting a decrease in neurite formation, for instance only has nine molecules in total. These changes could be circumstantial, however, interestingly the most enriched pathways in our data set also suggests that there are many gene changes in relation to functions in the nervous system such as axonal guidance signaling, ephrin signaling, CDK5 signaling and dopamine-DARPP32 feedback in cAMP signaling (Table 7). Ephrin and CDK5 signaling pathways overlaid with changes in genes during development are depicted in Supplementary Figures 1, 2. These results do support the notion that signaling systems within the plexus may be regulating some processes during brain development potentially affecting several maturation processes of the brain. There is much evidence that secretory signals from the choroid plexus do affect brain processes both during development and in adult. In the adult it has been shown that neuroblast migration follows the flow of CSF
(Sawamoto et al., 2006) and that the choroid plexus can even play a role in plasticity of the visual cortex (Spatazza et al., 2013). Furthermore, fibroblast growth factors, bone morphogenetic proteins, Wnts, and platelet derived growth factors produced by the choroid plexus have been shown to affect brain processes (Falcao et al., 2012). The insulin growth factors in CSF have been shown to be important for brain subventricular zone processes. IGF-1 appears to promote proliferation in the subventricular zone and IGF-2, which is much higher in developing brain CSF, can increase neuronal cell division (Mairet-Coello et al., 2009; Lehtinen et al., 2011). Since the ventricular zone expresses receptors for ephrins it is intriguing that ephrin receptor signaling is one of the most enriched pathways in the development of the primate tCP. It is, however, also possible that these genes and signaling pathways have a specific, as yet unknown role within the plexus itself. There is evidence that axonal guidance molecules 
TABLE 7 | Significantly Enriched canonical pathways $(p<0.01)$ between GD90 and GD165 in the choroid plexus.

\begin{tabular}{ll}
\hline Canonical pathways & $\boldsymbol{p}$-value \\
\hline Axonal guidance signaling & $2.34 \mathrm{E}-06$ \\
Molecular mechanisms of cancer & $7.24 \mathrm{E}-04$ \\
Ephrin receptor signaling & $7.59 \mathrm{E}-04$ \\
Epithelial adherens junction signaling & $9.33 \mathrm{E}-04$ \\
CDK5 signaling & $1.17 \mathrm{E}-03$ \\
PTEN signaling & $2.09 \mathrm{E}-03$ \\
Protein Kinase A signaling & $2.29 \mathrm{E}-03$ \\
Actin cytoskeleton signaling & $2.69 \mathrm{E}-03$ \\
Hepatic fibrosis/Hepatic stellate cell activation & $3.98 \mathrm{E}-03$ \\
Wnt/ $\beta$-catenin signaling & $4.17 \mathrm{E}-03$ \\
Ephrin A signaling & $4.17 \mathrm{E}-03$ \\
Insulin receptor signaling & $4.47 \mathrm{E}-03$ \\
Dopamine-DARPP32 feedback in cAMP signaling & $7.94 \mathrm{E}-03$ \\
RhoGDI signaling & $8.32 \mathrm{E}-03$ \\
Role of NFAT in cardiac hypertrophy & $8.51 \mathrm{E}-03$ \\
Sertoli cell-sertoli cell junction signaling & $8.91 \mathrm{E}-03$ \\
Gap junction signaling & $9.77 \mathrm{E}-03$ \\
GNRH signaling & $9.77 \mathrm{E}-03$ \\
\hline
\end{tabular}

are involved in processes in both epithelial as well as endothelial cells (Hinck, 2004).

The choroid plexus contains many transporting systems to exclude or transport molecules into the CNS. There have previously been several studies examining transporters in the plexus in the rodent (Kratzer et al., 2013; Liddelow et al., 2013; Saunder et al., 2013). These studies have shown that many mRNAs for transporters related to protection from xenobiotics are already present in the embryonic rat. In addition, barrier associated genes show little developmental regulation indicating a mature blood-CSF barrier already in the rodent embryo. Although we have not made such a detailed exploration specifically of these genes in the baboon in the present study, we did not obtain any significant $\mathrm{z}$-scores for any transport function of different molecules. In Table 6 we have listed the most regulated genes annotated to Transport of molecules. These are mainly associated to transport of ions, amino acids and neurotransmitters. Notable is that we found a pronounced increase during development in SLCO1C1 encoding for organic anion transporting polypeptide $1 \mathrm{Cl}$ which mediates high affinity transport of thyroxin and reverse-T3 (Pizzagalli et al., 2002). A similar marked increase of Slcolc1 has been shown to occur between the embryonic to the adult choroid plexus in rats (Kratzer et al., 2013). Since it is known that thyroxin is important for brain development the authors suggested that there must be other transporters mediating sufficient thyroxin to the embryonic brain. Likewise $C P$, encoding for ceruloplasmin, a main carrier of copper/iron ions in the blood, showed a strong increase in development in the baboon. Ceruloplasmin content in choroid plexus has been shown to be very high in the adult and localizes specifically to choroid plexus (Rouault et al., 2009). On the other hand there was a decrease in ATP7A and SLC39A8 transporters transcripts which have affinity for copper/zinc. There was also a decrease in genes encoding for cationic amino acid transporters in the plexus (SLC7A2, SLC7A3) as well as proteins associated to neurotransmitter transport (SLC1A7, SLC6A4, SLC17A8). This is consistent with previous studies which have showed both higher gene expression of the amino acid solute carriers in the antenatal choroid plexus as well as higher amino acid transport into the fetal brain as compared to the adult (Saunder et al., 2013).

Functional annotation to Cellular Organization and Assembly function had the most significant $p$-value of all functions and Organization of cytoskeleton one of the most negative activation scores (-7.02; Supplementary Table 1). In addition, several of the enriched canonical pathways are related to cellular assembly and particular actin cytoskeletal processes such as the actin cytoskeletal signaling, RhoGI signaling, gap junction signaling, and GNRH signaling pathway. We further explored the gene changes within these pathways using molecular activity predictions in IPA, which showed that these pathways predicted an increase in actin stabilization while a decrease in actin reorganization with development (see Supplementary Figure 3 for example of molecular predictions). Taken together this suggests that many of the gene changes in the tCP during development are linked to cytoskeletal processes and strengthening the cytoskeleton appears to be one such cellular process as the epithelium matures.

In conclusion, to our knowledge this is the first analysis of development of the fetal non-human primate choroid plexus transcriptome. Our data point to a more active choroid plexus earlier in fetal development with regulation of a more unique set of genes. We report progressive distinct transcriptome changes with widespread changes to genes across a great range of biological functions. Many of these functional changes are those that could be predicted on the basis of known changes during plexus development. The extent of the changes and nature of the genes involved indicate that the developing plexus is undertaking specific active functions with precise regulation and not just acting as a developmental barrier. Importantly, our observations support the notion that the choroid plexus has a direct, active role in influencing brain development. Future studies should further examine the role of these pathways in the choroid plexus.

\section{Acknowledgments}

This work was supported by NICHD 21350 (PN, CL), Swedish Research Council (VR2012-2992, CM), Government grant in Public Health Service at the Sahlgrenska University Hospital (ALFGBG-142881, CM), European Union grant FP7 (Neurobid, HEALTHF2-2009-241778, CM and CJE), the Leducq foundation (DSRR_P34404, CM), Åhlén Foundation (CM), Olle Enqvist Foundation (CM) and the Swedish Brain Foundation (FO2013095, CM), Åke Wiberg Foundation (CJE) and Magnus Bergvall Foundation (CJE).

\section{Supplementary Material}

The Supplementary Material for this article can be found online at: http://www.frontiersin.org/journal/10.3389/fnins. 2015.00082/abstract 
Supplementary Figure 1 Diagrams of canonical ephrin signaling pathway showing up (red) and down (green) regulated genes between GD90 and GD165 along with predictions on biological functions.

Supplementary Figure 2 | Diagrams of canonical CDK5 signaling pathway showing up (red) and down (green) regulated genes between GD90 and GD165 along with predictions on biological functions.

\section{References}

Altschul, S. F., Gish, W., Miller, W., Myers, E. W., and Lipman, D. J. (1990). Basic local alignment search tool. J. Mol. Biol. 215, 403-410. doi: 10.1016/S00222836(05)80360-2

Anders, S., and Huber, W. (2010). Differential expression analysis for sequence count data. Genome Biol. 11:R106. doi: 10.1186/gb-2010-11-10-r106

Daniel, T. O., and Abrahamson, D. (2000). Endothelial signal integration in vascular assembly. Annu. Rev. Physiol. 62, 649-671. doi: 10.1146/annurev.physiol.62.1.649

Dziegielewska, K. M., Ek, J., Habgood, M. D., and Saunders, N. R. (2001). Development of the choroid plexus. Microsc. Res. Tech. 52, 5-20. doi: 10.1002/10970029(20010101)52:1<5::AID-JEMT3>3.0.CO;2-J

Dziegielewska, K. M., Knott, G. W., and Saunders, N. R. (2000). The nature and composition of the internal environment of the developing brain. Cell. Mol. Neurobiol. 20, 41-56. doi: 10.1023/A:1006943926765

Ek, C. J., Habgood, M. D., Dziegielewska, K. M., and Saunders, N. R. (2003). Structural characteristics and barrier properties of the choroid plexuses in developing brain of the opossum (Monodelphis Domestica). J. Comp. Neurol. 460, 451-464. doi: 10.1002/cne.10661

Falcao, A. M., Marques, F., Novais, A., Sousa, N., Palha, J. A., and Sousa, J. C. (2012). The path from the choroid plexus to the subventricular zone: go with the flow! Front. Cell. Neurosci. 6:34. doi: 10.3389/fncel.2012.00034

Hinck, L. (2004). The versatile roles of "axon guidance" cues in tissue morphogenesis. Dev. Cell 7, 783-793. doi: 10.1016/j.devcel.2004.11.002

Jacobsen, M., Clausen, P. P., Jacobsen, G. K., Saunders, N. R., and Mollgard, K. (1982). Intracellular plasma proteins in human fetal choroid plexus during development. I. Developmental stages in relation to the number of epithelial cells which contain albumin in telencephalic, diencephalic and myelencephalic choroid plexus. Brain Res. 255, 239-250. doi: 10.1016/0165-3806(82) 90024-4

Janssen, S. F., Van Der Spek, S. J., Ten Brink, J. B., Essing, A. H., Gorgels, T. G., Van Der Spek, P. J., et al. (2013). Gene expression and functional annotation of the human and mouse choroid plexus epithelium. PLoS ONE 8:e83345. doi: 10.1371/journal.pone. 0083345

Johanson, C. E. (1995). "Ventricles and cerebrospinal fluid," in Neuroscience in Medicine, ed P. M. Conn (Philadelphia: J. B Lippincott Company), 171-196.

Kratzer, I., Liddelow, S. A., Saunders, N. R., Dziegielewska, K. M., Strazielle, N., and Ghersi-Egea, J. F. (2013). Developmental changes in the transcriptome of the rat choroid plexus in relation to neuroprotection. Fluids Barriers CNS 10:25. doi: $10.1186 / 2045-8118-10-25$

Kratzer, I., Vasiljevic, A., Rey, C., Fevre-Montange, M., Saunders, N., Strazielle, N., et al. (2012). Complexity and developmental changes in the expression pattern of claudins at the blood-CSF barrier. Histochem. Cell Biol. 138, 861-879. doi: 10.1007/s00418-012-1001-9

Kroenke, C. D., Van Essen, D. C., Inder, T. E., Rees, S., Bretthorst, G. L., and Neil, J. J. (2007). Microstructural changes of the baboon cerebral cortex during gestational development reflected in magnetic resonance imaging diffusion anisotropy. J. Neurosci. 27, 12506-12515. doi: 10.1523/JNEUROSCI.306307.2007

Kullander, K., and Klein, R. (2002). Mechanisms and functions of Eph and ephrin signalling. Nat. Rev. Mol. Cell Biol. 3, 475-486. doi: 10.1038/nrm856

Lehtinen, M. K., Bjornsson, C. S., Dymecki, S. M., Gilbertson, R. J., Holtzman, D. M., and Monuki, E. S. (2013). The choroid plexus and cerebrospinal
Supplementary Figure 3 | Example of molecular activity prediction using Ingenuity Pathway Analysis software for canonical Actin signaling pathway from differentially expressed genes between GD90 and GD165.

This connects the gene changes in the actin signaling pathway to biological functions. The changes to this pathway predict an increase in actin polymerization and actin stabilization whereas actin reorganization is decreased.

Supplementary Table 1 | Function annotated genes to all subcategory functions with a significant activation $z$-score $(>+2$ or $<-2)$. \#, Number of annotated genes to function.

fluid: emerging roles in development, disease, and therapy. J. Neurosci. 33, 17553-17559. doi: 10.1523/JNEUROSCI.3258-13.2013

Lehtinen, M. K., Zappaterra, M. W., Chen, X., Yang, Y. J., Hill, A. D., Lun, M., et al. (2011). The cerebrospinal fluid provides a proliferative niche for neural progenitor cells. Neuron 69, 893-905. doi: 10.1016/j.neuron.2011.01.023

Li, C., McDonald, T. J., Wu, G., Nijland, M. J., and Nathanielsz, P. W. (2013). Intrauterine growth restriction alters term fetal baboon hypothalamic appetitive peptide balance. J. Endocrinol. 217, 275-282. doi: 10.1530/JOE-13-0012

Liddelow, S. A., Dziegielewska, K. M., Ek, C. J., Habgood, M. D., Bauer, H., Bauer H. C., et al. (2013). Mechanisms that determine the internal environment of the developing brain: a transcriptomic, functional and ultrastructural approach. PLoS ONE 8:e65629. doi: 10.1371/journal.pone.0065629

Mairet-Coello, G., Tury, A., and Dicicco-Bloom, E. (2009). Insulin-like growth factor-1 promotes $\mathrm{G}(1) / \mathrm{S}$ cell cycle progression through bidirectional regulation of cyclins and cyclin-dependent kinase inhibitors via the phosphatidylinositol 3-kinase/Akt pathway in developing rat cerebral cortex. J. Neurosci. 29, 775-788. doi: 10.1523/JNEUROSCI.1700-08.2009

Marques, F., Sousa, J. C., Coppola, G., Gao, F., Puga, R., Brentani, H., et al. (2011). Transcriptome signature of the adult mouse choroid plexus. Fluids Barriers CNS 8:10. doi: 10.1186/2045-8118-8-10

Miyan, J. A., Nabiyouni, M., and Zendah, M. (2003). Development of the brain: a vital role for cerebrospinal fluid. Can. J. Physiol. Pharmacol. 81, 317-328. doi: 10.1139/y03-027

Parker-Duffen, J. L., Nakamura, K., Silver, M., Kikuchi, R., Tigges, U., Yoshida, S., et al. (2013). T-cadherin is essential for adiponectin-mediated revascularization. J. Biol. Chem. 288, 24886-24897. doi: 10.1074/jbc.M113.454835

Pizzagalli, F., Hagenbuch, B., Stieger, B., Klenk, U., Folkers, G., and Meier, P. J. (2002). Identification of a novel human organic anion transporting polypeptide as a high affinity thyroxine transporter. Mol. Endocrinol. 16, 2283-2296. doi: 10.1210/me.2001-0309

Rabadan-Diehl, C., and Nathanielsz, P. (2013). From Mice to Men: research models of developmental programming. J. Dev. Orig. Health Dis. 4, 3-9. doi: 10.1017/S2040174412000487

Ransohoff, R. M., and Engelhardt, B. (2012). The anatomical and cellular basis of immune surveillance in the central nervous system. Nat. Rev. Immunol. 12, 623-635. doi: 10.1038/nri3265

Redzic, Z. B., Preston, J. E., Duncan, J. A., Chodobski, A., and SzmydyngerChodobska, J. (2005). The choroid plexus-cerebrospinal fluid system: from development to aging. Curr. Top. Dev. Biol. 71, 1-52. doi: 10.1016/S00702153(05)71001-2

Rogers, J., Kochunov, P., Zilles, K., Shelledy, W., Lancaster, J., Thompson, P., et al. (2010). On the genetic architecture of cortical folding and brain volume in primates. Neuroimage 53, 1103-1108. doi: 10.1016/j.neuroimage.2010.02.020

Rouault, T. A., Zhang, D. L., and Jeong, S. Y. (2009). Brain iron homeostasis, the choroid plexus, and localization of iron transport proteins. Metab. Brain Dis. 24, 673-684. doi: 10.1007/s11011-009-9169-y

Saunder, N. R., Daneman, R., Dziegielewska, K. M., and Liddelow, S. M. (2013). Transporters of the blood-brain and blood-CSF interfaces in development and in the adult. Mol. Aspects Med. 34, 742-752. doi: 10.1016/j.mam.2012.11.006

Sawamoto, K., Wichterle, H., Gonzalez-Perez, O., Cholfin, J. A., Yamada, M., Spassky, N., et al. (2006). New neurons follow the flow of cerebrospinal fluid in the adult brain. Science 311, 629-632. doi: 10.1126/science.1119133

Schlabritz-Loutsevitch, N., Ballesteros, B., Dudley, C., Jenkins, S., Hubbard, G., Burton, G. J., et al. (2007). Moderate maternal nutrient restriction, but not 
glucocorticoid administration, leads to placental morphological changes in the baboon (Papio sp.). Placenta 28, 783-793. doi: 10.1016/j.placenta.2006.11.012

Schlabritz-Loutsevitch, N. E., Hubbard, G. B., Frost, P. A., Cummins, L. B., Dick, E. J. Jr., Nathanielsz, P. W., et al. (2004). Abdominal pregnancy in a baboon: a first case report. J. Med. Primatol. 33, 55-59. doi: 10.1046/j.1600-0684.2003.00044.x

Spatazza, J., Lee, H. H., Di Nardo, A. A., Tibaldi, L., Joliot, A., Hensch, T. K., et al. (2013). Choroid-plexus-derived Otx2 homeoprotein constrains adult cortical plasticity. Cell Rep. 3, 1815-1823. doi: 10.1016/j.celrep.2013.05.014

Trapnell, C., Pachter, L., and Salzberg, S. L. (2009). TopHat: discovering splice junctions with RNA-Seq. Bioinformatics 25, 1105-1111. doi: 10.1093/bioinformatics/btp120

Trapnell, C., Roberts, A., Goff, L., Pertea, G., Kim, D., Kelley, D. R., et al. (2012). Differential gene and transcript expression analysis of RNA-seq experiments with TopHat and Cufflinks. Nat. Protoc. 7, 562-578. doi: $10.1038 /$ nprot.2012.016

UniProt Consortium. (2012). Reorganizing the protein space at the Universal Protein Resource (UniProt). Nucleic Acids Res. 40, D71-D75. doi: 10.1093/nar/gkr981
Wang, F., Marshall, C. B., and Ikura, M. (2013). Transcriptional/epigenetic regulator $\mathrm{CBP} / \mathrm{p} 300$ in tumorigenesis: structural and functional versatility in target recognition. Cell. Mol. Life Sci. 70, 3989-4008. doi: 10.1007/s00018-012$1254-4$

Zappaterra, M. W., and Lehtinen, M. K. (2012). The cerebrospinal fluid: regulator of neurogenesis, behavior, and beyond. Cell. Mol. Life Sci. 69, 2863-2878. doi: $10.1007 /$ s00018-012-0957-x

Conflict of Interest Statement: The authors declare that the research was conducted in the absence of any commercial or financial relationships that could be construed as a potential conflict of interest.

Copyright $\odot 2015 \mathrm{Ek}$, Nathanielsz, Li and Mallard. This is an open-access article distributed under the terms of the Creative Commons Attribution License (CC BY). The use, distribution or reproduction in other forums is permitted, provided the original author(s) or licensor are credited and that the original publication in this journal is cited, in accordance with accepted academic practice. No use, distribution or reproduction is permitted which does not comply with these terms. 


\title{
The contribution of CXCL12-expressing radial glia cells to neuro-vascular patterning during human cerebral cortex development
}

\author{
Mariella Errede ${ }^{\dagger}$, Francesco Girolamo ${ }^{\dagger}$, Marco Rizzi, Mirella Bertossi, Luisa Roncali and \\ Daniela Virgintino *
}

Department of Basic Medical Sciences, Neurosciences and Sensory Organs, University of Bari School of Medicine, Bari, Italy

\section{Edited by:}

Norman Ruthven Saunders,

University of Melbourne, Australia

\section{Reviewed by:}

Kjeld Møllgård, University of

Copenhagen, Denmark

Udo Schumacher, University of

Hamburg, Germany

\section{*Correspondence:}

Daniela Virgintino, Human Anatomy and Histology Unit, Department of

Basic Medical Sciences,

Neurosciences, and Sensory

Organs, Bari University Medical

School, Piazza Giulio Cesare,

Policlinico-70124 Bari, Italy

e-mail: daniela.virgintino@uniba.it

tThese authors have contributed equally as first authors.
This study was conducted on human developing brain by laser confocal and transmission electron microscopy (TEM) to make a detailed analysis of important features of blood-brain barrier (BBB) microvessels and possible control mechanisms of vessel growth and differentiation during cerebral cortex vascularization. The BBB status of cortex microvessels was examined at a defined stage of cortex development, at the end of neuroblast waves of migration, and before cortex lamination, with BBB-endothelial cell markers, namely tight junction (TJ) proteins (occludin and claudin-5) and influx and efflux transporters (Glut-1 and P-glycoprotein), the latter supporting evidence for functional effectiveness of the fetal BBB. According to the well-known roles of astroglia cells on microvessel growth and differentiation, the early composition of astroglia/endothelial cell relationships was analyzed by detecting the appropriate astroglia, endothelial, and pericyte markers. GFAP, chemokine CXCL12, and connexin 43 (Cx43) were utilized as markers of radial glia cells, CD105 (endoglin) as a marker of angiogenically activated endothelial cells (ECs), and proteoglycan NG2 as a marker of immature pericytes. Immunolabeling for CXCL12 showed the highest level of the ligand in radial glial (RG) fibers in contact with the growing cortex microvessels. These specialized contacts, recognizable on both perforating radial vessels and growing collaterals, appeared as CXCL12-reactive en passant, symmetrical and asymmetrical, vessel-specific RG fiber swellings. At the highest confocal resolution, these RG varicosities showed a CXCL12-reactive dot-like content whose microvesicular nature was confirmed by ultrastructural observations. A further analysis of $R G$ varicosities reveals colocalization of CXCL12 with $\mathrm{Cx} 43$, which is possibly implicated in vessel-specific chemokine signaling.

Keywords: neuroangiogenesis, blood-brain barrier, tight junctions, transporters, radial glia, chemokine CXCL12, connexin 43 , human fetus

\section{INTRODUCTION}

Radial glial (RG) cells were first described in the developing CNS by Magini and Ramón y Cajal in the late 19th century (Bentivoglio and Mazzarello, 1999). Their function as scaffolding for neuroblast migration during cortical histogenesis was postulated and demonstrated by Rakic in the early 1970s (Rakic, 1971, 1972) and then, at the beginning of the new millennium, their role as neuronal and glial precursors in the developing CNS was definitively demonstrated (Malatesta et al., 2000; Malatesta and Götz, 2013). The evidence that RG cells fulfill many functions has attracted new attention to these cells and new discoveries about their diverse functions in the developing brain are rapidly accumulating.

\footnotetext{
Abbreviations: $\mathrm{BBB}$, blood-brain barrier; CD105, endoglin; CNS, Central Nervous System; Coll IV, collagen type IV; Cx43, connexin 43; CXCL12, C-X-C motif chemokine 12; EC, endothelial cells; GFAP, glial fibrillary acidic protein; Glut-1, glucose transporter isoform 1; MVs, microvesicles; NG2, nerve/glial antigen 2; P-gp, P-glycoprotein; RG, radial glia; TJ, tight junction.
}

RG cells differ taxonomically in primates and other mammalian species due to differences in their involvement in neurogenesis, and the timing and region of their appearance, as regards the expression of typical RG immunomarkers (Howard et al., 2008; Xu et al., 2014). For example, in humans, the intermediate filament proteins, glial fibrillary acidic protein (GFAP), and vimentin, are expressed concomitantly in RG from the start of neurogenesis (Choi and Lapham, 1978; Virgintino et al., 1998; Howard et al., 2006). In contrast, in rodents, RG cells undergo a more protracted maturation and become GFAP-positive only at late fetal stages, after neurogenesis is complete, and vimentin is no longer detectable (Rickmann and Wolff, 1985). Moreover, in human telencephalon, due to the larger size of the cerebral cortex compared with the rodent cortex, a "mature" GFAP-expressing RG precociously develops long shafts that may reach a length of $3000-7000 \mu \mathrm{m}$ and show early, special vascular relations (Rakic, 1972; Virgintino et al., 1998).

Pioneering studies have demonstrated that angiogenically formed microvessels invade the developing brain by perforating 
Table 1 | Synopsis of CXCL12 and CXCR4/CXCR7 immunoreactivity in developing brain.

\begin{tabular}{|c|c|c|c|}
\hline Cell type & CXCL12 & CXCR4 & CXCR7 \\
\hline Radial glia cells & cytoplasm ${ }^{\text {high }}$ & - & - \\
\hline Perivascular astrocytes & cytoplasm ${ }^{\text {high }}$ & - & - \\
\hline Neuroblasts & - & nucleus high & nucleus high \\
\hline Endothelial cells & cytoplasm low & - & - \\
\hline Pericytes & - & cytoplasm high & cytoplasm ${ }^{\text {high }}$ \\
\hline
\end{tabular}

Modified from Virgintino et al. (2013).

the basement membrane and penetrating through the external glial limiting membrane to supply the developing nervous tissue, under the control of a concentration gradient of soluble growth factors (Risau et al., 1988; Risau and Wolburg, 1990; Engelhardt and Risau, 1995). In humans the growing microvessels arrange themselves according to geometrically precise paths that parallel the $\mathrm{RG}$ arrangement, taking on a common radial pattern and forming mutual, multiple contacts (Bär, 1980; MarinPadilla, 1985; Virgintino et al., 1998; Rakic, 2007; Marín-Padilla, 2012). In recent years it has become increasingly evident that the role of RG cells during brain vascularization may be more specific than was previously thought. In fact, besides a simple activity providing tracks for growing vessels, RG cells have been suggested to be the source of factors such as vascular endothelial growth factor (VEGF) and Wnt, that intertwine with each other and other angiogenic pathways to facilitate the guidance of penetrating microvessels, stimulate proliferation of endothelial cells (ECs) and sustain vessel stabilization (Liebner and Plate, 2010; Bussmann et al., 2011; Quaegebeur et al., 2011).

During human cerebral cortex vascularization, subsets of RG cells and perivascular astrocytes have been demonstrated, showing high levels of chemokine CXCL12 expression (Virgintino et al., 2013). This chemokine, also known as stromal-derived factor-1 (SDF-1), plays multiple roles in CNS development, its expression in the brain being involved in neural progenitor differentiation, and neuronal cell migration (Tiveron et al., 2006; Peng et al., 2007; Stumm and Höllt, 2007; Li et al., 2008; Tiveron and Cremer, 2008). Moreover, cells of neural crest origin, located in the developing, innermost layer of the meninges, have been demonstrated to be involved in neurogenesis, secreting molecular cues including chemokine CXCL12, that seem to regulate cortical interneuron settlement, and Cajal-Retzius cell tangential migration (Stumm et al., 2003; Borrell and Marín, 2006; Paredes et al., 2006; Li et al., 2008; Siegenthaler et al., 2009).

In addition, CXCL12 and its receptor CXCR4 (C-X-C chemokine receptor) belong to a restricted group of pro-angiogenic molecules that, albeit within a limited developmental window, display a neuro-specific angiogenic activity (Bussmann et al., 2011; Quaegebeur et al., 2011). Overall, these data are consistent with previous observations of human developing cerebral cortex, which have described CXCR4/CXCR7-reactive neuroblasts and subsets of CXCL12 RG cells and astrocytes in close association with CXCR4/CXCR7-reactive microvascular ECs and pericytes (Virgintino et al., 2013; see also Table 1). Accordingly, a model
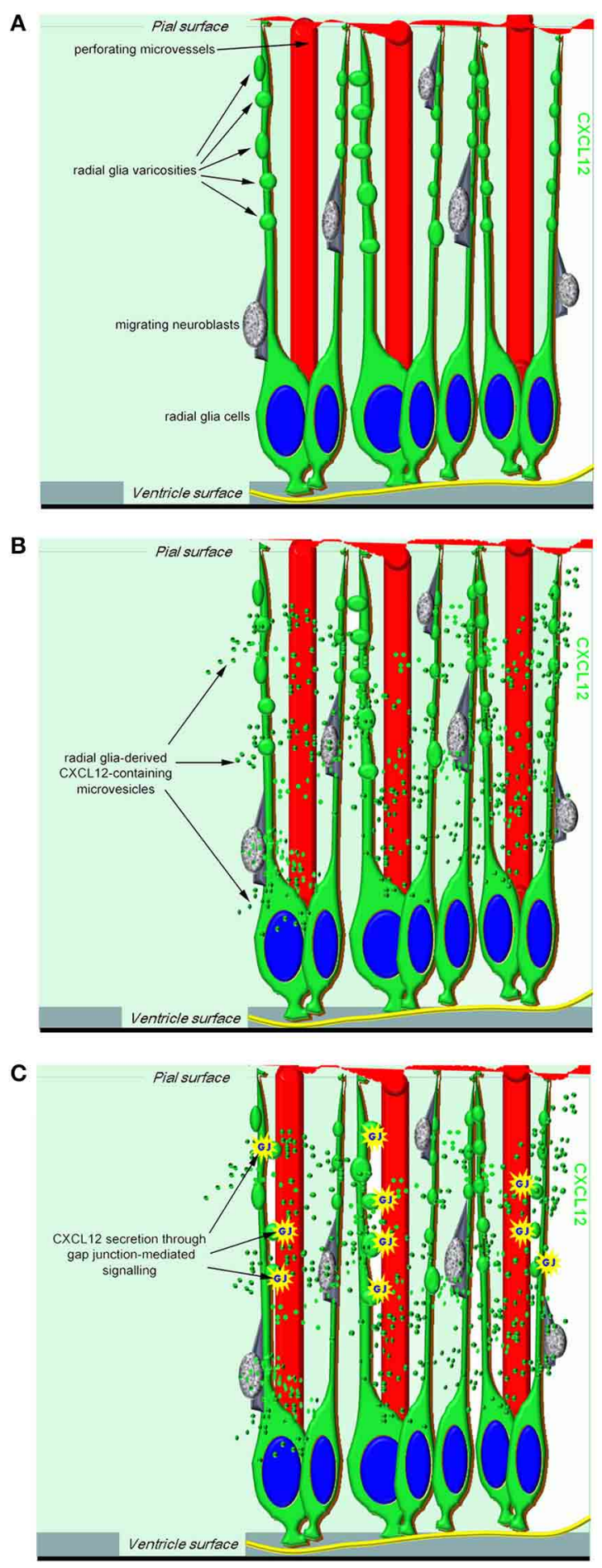

FIGURE 1 | A proposed model of RG-derived CXCL12 regulation of cerebral cortex development and vascularization. (A) In the working model, CXCL12 radial glia cells have a central role not only in neurogenesis and neuroblast migration but also in neuroangiogenesis, being ideally positioned to coordinate neuro-vascular cerebral cortex patterning. (B) Radial glia-derived CXCL12-containing microvesicles may convey their signal to the ECs of growing microvessels. (C) One potential mechanism by which CXCL12 secretion can be modulated in radial glia cells is through gap junction-mediated signaling. 
of synchronized neuro-vascular patterning centered on CXCL12 RG cells has been proposed (Figure 1) and appears to be supported by the present results that have identified, by parallel laser confocal and electron microscopy analyses, RG-microvessel subcellular units shaped by RG varicosities containing CXCL12 microvesicles (MVs) and connexin $43(\mathrm{Cx} 43)$ as a regulatory molecule.

\section{MATERIALS AND METHODS HISTOLOGY}

Autopsy specimens of telencephalon were collected from 4 human fetuses spontaneously aborted due to preterm rupture of the placental membranes (22 weeks of gestation). The sampling and handling of human specimens conformed to the ethical rules of the Department of Pathology, Medical School, University of Bari, Italy, and approval was gained from the local Ethics Committee of the National Health System in compliance with the principles stated in the Declaration of Helsinki. The fetal age was estimated on the basis of the crown-rump length and/or pregnancy records (counting from the last menstrual period). At autopsy, the selected fetuses did not reveal macroscopic structural abnormalities and/or malformations of the central nervous system. The dorso-lateral wall of the telencephalic vesicles (future neocortex) was dissected and fixed for $2-3 \mathrm{~h}$ at $4^{\circ} \mathrm{C}$ by immersion in $2 \%$ paraformaldehyde plus $0.2 \%$ glutaraldehyde solution. Specimens were then washed in phosphate buffered saline (PBS, pH 7.6) and serially sectioned by a vibrating microtome. $20 \mu \mathrm{m}$ sections were collected at regular intervals and processed for conventional histological analysis with toluidine blue staining to ascertain the absence of microscopic malformations; all the other sections were stored in PBS plus $0.02 \%$ paraformaldehyde for fluorescence immunolabeling.

\section{IMMUNOFLUORESCENCE AND LASER CONFOCAL MICROSCOPY}

Multiple immunostainings were carried out with the following polyclonal antisera (pAbs) and monoclonal antibodies (mAbs), all diluted in blocking buffer ( $1 \%$ bovine serum albumin and $2 \%$ fetal calf serum in PBS): rabbit pAb anti-Glut 1 , mouse mAb anti-P-gp, mouse $\mathrm{mAb}$ anti-Claudin-5, rabbit $\mathrm{pAb}$ anti-Occludin, mouse $\mathrm{mAb}$ anti-CXCL12/SDF-1, rabbit $\mathrm{pAb}$ anti-CD105, rabbit pAb anti-NG2 (generous gift from W. B. Stallcup, The Burnham Institute for Medical Research, La Jolla, CA, USA), mouse $\mathrm{mAb}$ anti-GFAP, rat $\mathrm{mAb}$ anti-GFAP, rabbit $\mathrm{pAb}$ anti-collagen type IV (Coll IV), rabbit pAb anti-Cx43 (Table 2). After adhesion on polylysine slides (Menzel-Glaser, GmbH, Braunschweig, Germany) by drying for $10 \mathrm{~min}$ at room temperature, the sections were submitted to the following protocols: rehydration with PBS for $5 \mathrm{~min}$ at room temperature or microwave pretreatment in $0.01 \mathrm{M}$ citrate buffer $(\mathrm{pH} 6.0$ ) for $15 \mathrm{~min}$ at $750 \mathrm{~W}$ when required (Table 2 ); incubation with $0.5 \%$ Triton X-100 in PBS for $30 \mathrm{~min}$ at room temperature and with blocking buffer $30 \mathrm{~min}$ at room temperature, with single or combined primary antibodies overnight at $4^{\circ} \mathrm{C}$, then with appropriate fluorophoreconjugated secondary antibodies (Table 2 ). Finally, the sections were washed for $10 \mathrm{~min} \times 3$ in PBS between each step, counterstained with either TO-PRO- $3^{\mathrm{TM}}$ (1:10K dilution; Invitrogen Corporation, Carlsbad, CA, USA) or Sytox ${ }^{\circledR}$ Green (1:5K dilution; Invitrogen), mounted in Vectashield (Vector Laboratories, Inc. Burlingame, CA, USA), and finally sealed with nail varnish. Negative controls were prepared by using primary antibodies to

Table 2 | List of primary and secondary antibodies combined in multiple immunolabelings.

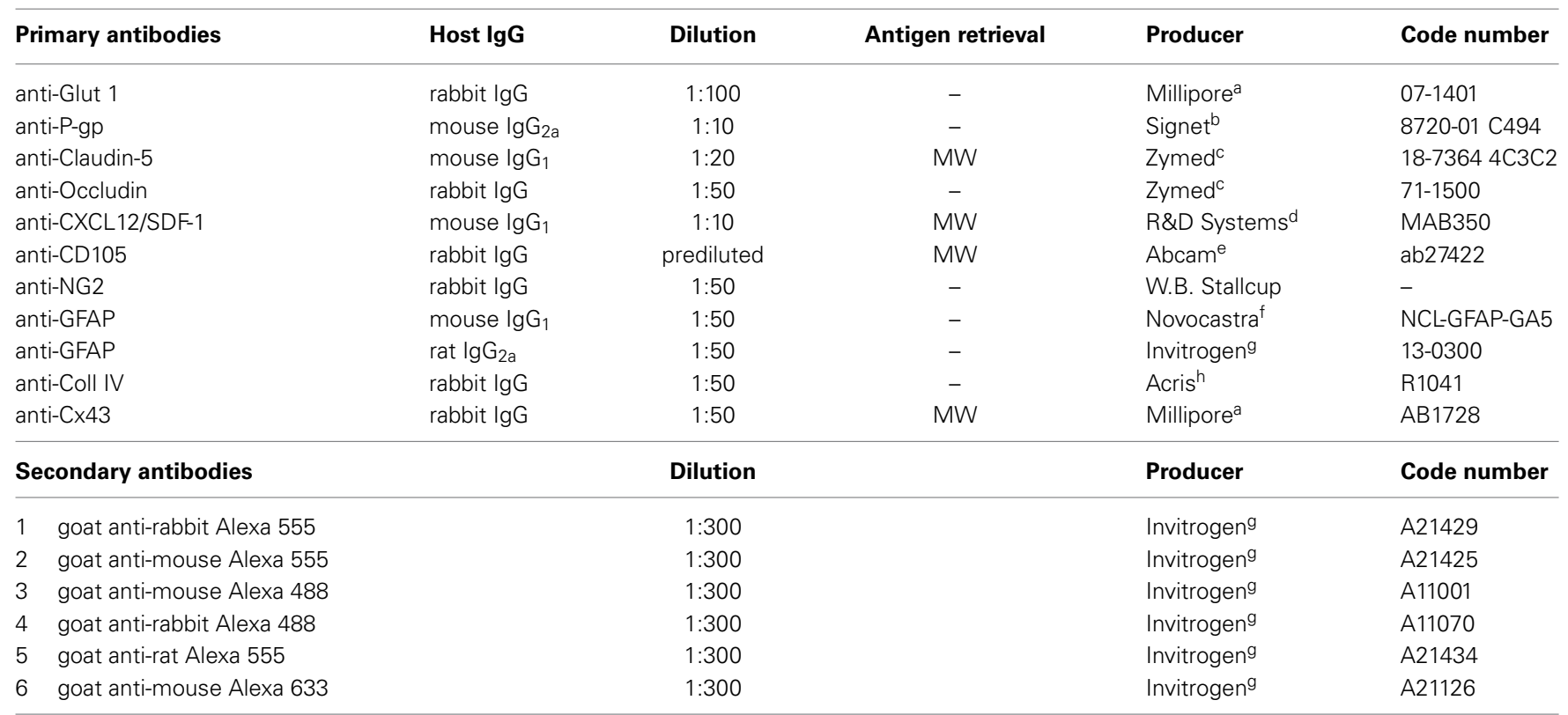

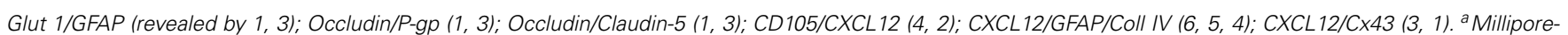
Chemicon; Billerica, MA, USA. 'b Signet Laboratories, Dedham, MA, USA. 'Cymed Laboratories, Invitrogen Corporation, Carlsbad, CA, USA. ${ }^{d}$ R\&D Systems, Minneapolis, MN, USA. ${ }^{e}$ Abcam, Cambridge, UK. ${ }^{f}$ Vision Biosystem Novocastra, Newcastle upon Tyne, UK. ${ }^{9}$ Invitrogen, Eugene, OR, USA. hicris Antibodies GmbH; Herford, Germany. MW, high-temperature microwave pre-treatment of tissue sections. 
stain inappropriate tissues, omitting the primary antibodies, preadsorbing the primary antibodies with an excess of antigen when available, or mismatching the secondary antibodies on appropriate preparations. When not ascertained in previous experiments, the specificity of primary antibodies was tested on tissue sections known to contain the antigen, as positive controls, and applying the same immunostaining protocols described above. Human adult brain sections were used to test the BBB-specific markers (Glut 1, P-gp, Claudin-5, Occludin), Cx43 antibody was tested on human and mouse brain sections and on mouse heart sections, while sections from human tonsils were useful for assessing CXCL12 and CD105, immunoreactivity (control sections were kindly provided by E. Maiorano, Department of Emergency and
Organ Transplantation, Pathological Anatomy Unit, Faculty of Medicine, University of Bari, Bari, Italy). The sections were examined under the Leica TCS SP5 confocal laser-scanning microscope (Leica Microsystems, Mannheim, Germany) using a sequential scan procedure. Confocal images were taken at $250 \mathrm{~nm}$ intervals through the $\mathrm{z}$-axis of the sections. Confocal images were taken with 40X and 63X oil lenses. Z-stacks of serial optical planes (projection images) and single optical planes were analyzed by Leica confocal software (Multicolor Package; Leica Microsystems). The size of RG varicosities and MVs was evaluated with LAS-AF SP5 software (Leica Microsystems) on 63X magnification fields zoomed 3 times. MVs diameter $(\mathrm{nm})$ was measured on single optical planes from CXCL12-labeled sections $(n=18)$ for a total
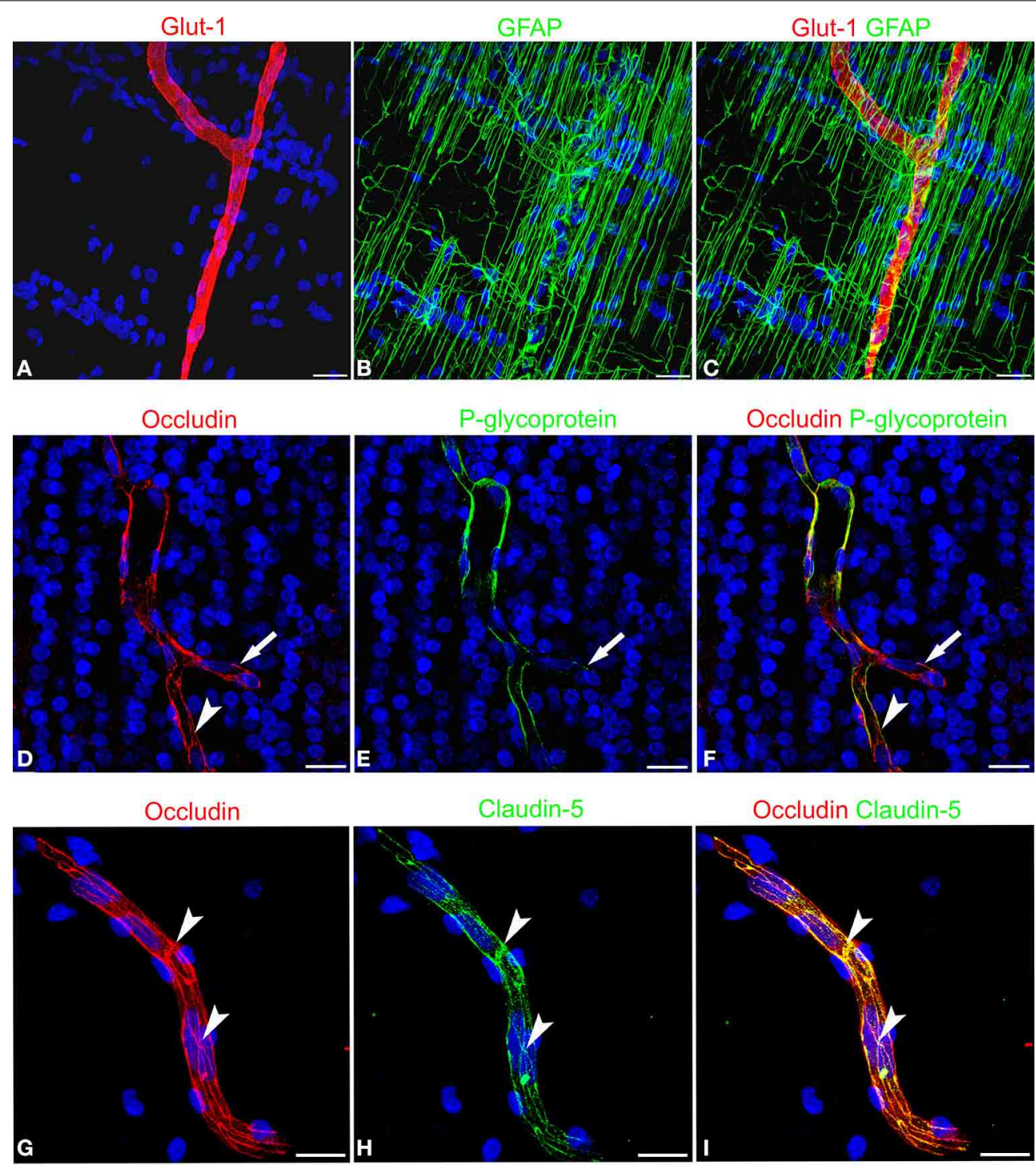

FIGURE 2 | Confocal images of human cerebral cortex at midgestation double labeled with Glut-1/GFAP, occludin/P-glycoprotein, and occludin/claudin-5. (A-C) A radial microvessel surrounded by GFAP-reactive RG fibers is revealed by the Glut-1 reactive endothelial cells. (D-F) On a stem vessel and its collateral (arrow) endothelial P-glycoprotein colocalize with occludin reactivity; note in (E) the lower levels of P-glycoprotein expression on the newly formed vessel branch (arrow) and in (D) and (F) the junctional linear pattern of occludin (arrowhead). (G-I) Occludin appears arranged according to a typical junctional pattern and colocalizes at points with claudin-5 (arrowheads). Bars: A-C and D-F $25 \mu \mathrm{m} ; \mathbf{G}-\mathbf{I} 30 \mu \mathrm{m}$. 


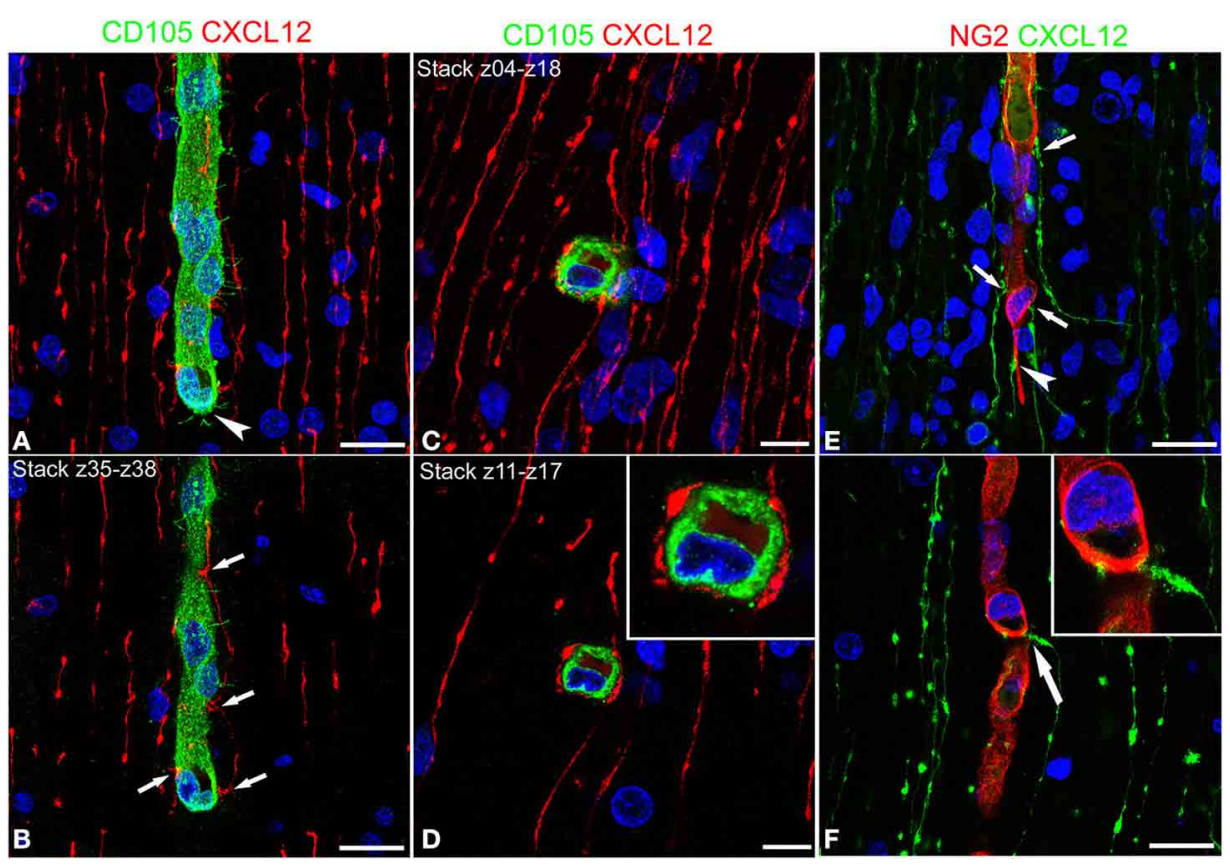

FIGURE 3 | Confocal images of human cerebral cortex at midgestation double labeled with CD105/CXCL12 (A-D) and NG2/CXCL12 (E,F).

CXCL12-reactive RG fibers show evenly spaced varicosities (A) that make contacts at regular intervals (better recognizable on the selection of optical planes z35-z38; B, arrows) with a CD105 labeled, perforating cortex

microvessel; note in (A) the tip endothelial cell on the growing vessel front (arrowhead). (C,D) Transverse view of a vascular branch completely surrounded by CXCL12 RG fibers, whose intimate relationships are better recognizable on a shorter stack of optical planes (z11-z17, D; inset). (E) Multiple CXCL12-reactive varicosities touching the NG2 pericyte cover (arrows) and a pericyte leading process (arrowhead); (F) One CXCL12-labeled varicosity bents to contact the vessel wall (arrow in F; inset). Bars: A,B $20 \mu \mathrm{m} ; \mathbf{C}, \mathbf{D} 10 \mu \mathrm{m} ; \mathbf{E}, \mathbf{F} 20 \mu \mathrm{m}$.

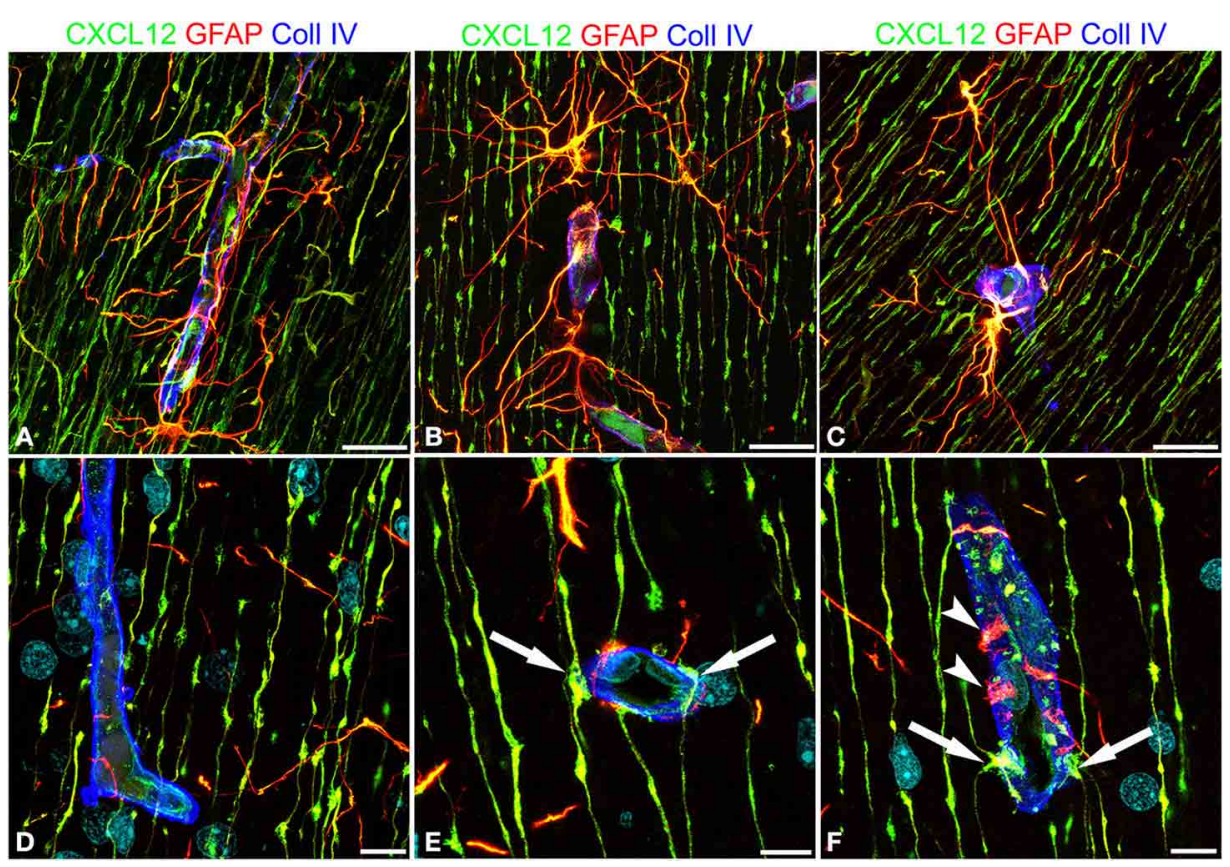

FIGURE 4 | Confocal images of human cerebral cortex at midgestation after multiple labeling with CXCL12, GFAP, and Coll IV. (A-C) GFAP-CXCL12-reactive perivascular astrocytes and CXCL12-reactive RG cells contributing perivascular endings to the microvessel wall revealed by collagen IV (b/ue). (D-F) On selected single optical planes the RG vascular contacts are distinguishable, two en passant, symmetrical varicosities on the vessel wall (E, arrows) and two omega-shaped asymmetrical varicosities on either sides of the vessel profile ( $\mathbf{F}$, arrows); note in (F) classical GFAP-labeled astrocyte end-feet (arrowheads). Bars: A-C $20 \mu \mathrm{m}$; D-F $10 \mu \mathrm{m}$ 


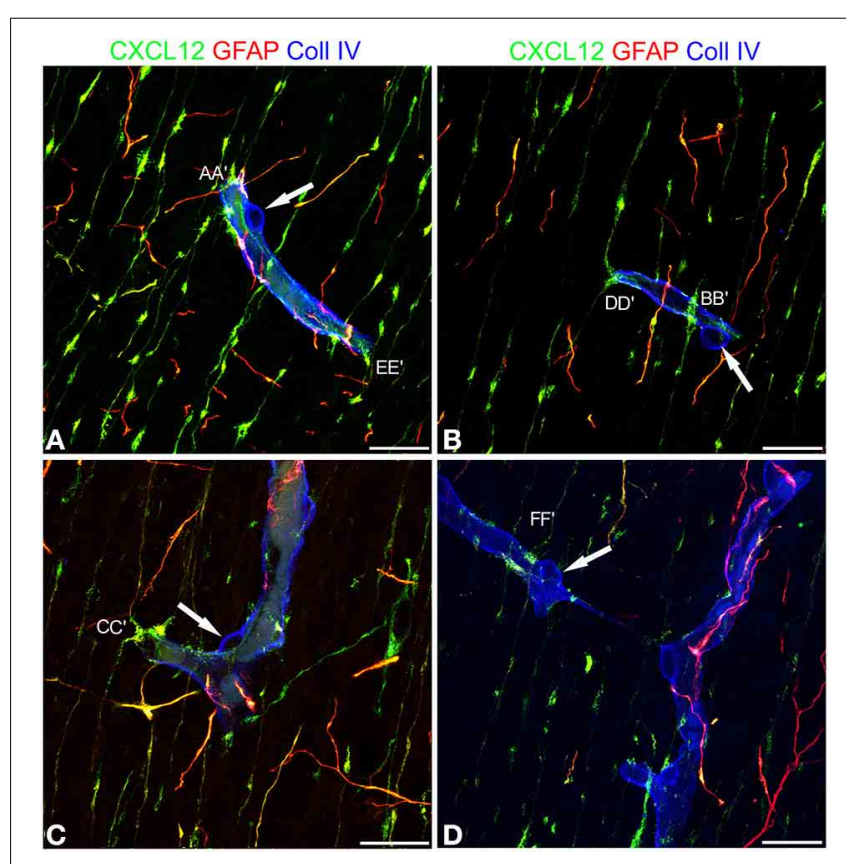

FIGURE 5 | Confocal images of human cerebral cortex at midgestation after multiple labeling with CXCL12, GFAP, and Coll IV. (A-D) A further collection of CXCL12-reactive RG varicosities in contact with the vessel wall, whose Coll IV staining also shows the bulging nuclei of pericytes (arrows in A-D). Single RG-vascular contacts are recognizable in these pictures ( $\mathbf{A A}^{\prime}, \mathbf{B B} \mathbf{B}^{\prime}, \mathbf{C} \mathbf{C}^{\prime}, \mathbf{D D ^ { \prime }}, \mathbf{E} \mathbf{E}^{\prime}$, and $\left.\mathbf{F F}^{\prime}\right)$ and are shown as enlarged details in Figure 6. Bars: A-D $20 \mu \mathrm{m}$.

of 50 fields. The results were expressed as a "range" of size from a minimum to a maximum value.

\section{TRANSMISSION ELECTRON MICROSCOPY}

Small samples collected from the previously described telencephalon specimens were submitted to electron microscopy procedures. Briefly, samples were fixed in $0.1 \mathrm{M}$ phosphate-buffered $3 \%$ glutaraldehyde, post-fixed in phosphate-buffered $1 \%$ OsO4, dehydrated in serial alcohols, and embedded in Epon 812. Ultrathin sections were cut with an LKB V ultramicrotome (LKB Bromma, Sollentuna, Sweden), stained with uranyl acetate and lead citrate, and observed under a CM 10 Philips electron microscope (Philips, Eindhoven, The Netherlands).

\section{RESULTS \\ CEREBRAL CORTEX MICROVESSELS SHOW A BBB-SPECIFIC PHENOTYPE}

The expression of blood-brain barrier (BBB)-specific markers was analyzed in microvessels that, at the end of neuronal migration and beginning of cortex lamination, in the fetal age of around midgestation, vascularize the cerebral cortex, radially invading the neural wall from the pial surface to the deeper subcortical layers. Their endothelial lining is characterized by a strong reactivity to fundamental metabolic and efflux barrierspecific transporters, such as the glucose transporter isoform 1, Glut-1, and to a member of the ABC (ATP-binding cassette) superfamily, the multidrug transporter P-glycoprotein (P-gp or
$\mathrm{ABCB} 1$ ). On double staining with Glut-1 and the astroglia marker GFAP, radial vessels appear surrounded by a curtain of GFAPreactive RG fibers and their ECs express high levels of Glut-1 (Figures 2A-C). Cortex stem microvessels appear aligned with rows of neuroblasts and are seen to form their first collaterals, stem vessels and branches being revealed by the endothelial expression of P-gp (Figures 2E,F). The presence of two BBB transporters indirectly implies that tight junction (TJ) formation is in progress in ECs and that the barrier is partially functional. In fact, on double staining with P-gp and the TJ protein occludin, the latter appears highly expressed on both stem vessels and collaterals, and shows a linear, junctional staining pattern (Figures 2D-F). Also on double staining with the TJ protein claudin-5, occludin displays a clearly junctional distribution, whereas a punctate cytoplasmic pattern still prevails for claudin- 5 (Figures 2G-I). Overall, these data demonstrate an ongoing process of BBB differentiation in human cerebral cortex microvessels at midgestation.

\section{VESSEL-SPECIFIC CONTACTS OCCUR BETWEEN CXCL12-REACTIVE RG FIBERS AND CORTEX MICROVESSELS}

Cerebral cortex microvessels are also revealed by the endothelial marker CD105, which also allows recognition of the growing front of the invading vessels, that is characterized by CD105-reactive endothelial tip cells (Figures 3A,B). On double immunostaining for CXCL12, CD105 labeled microvessels appear surrounded by palisades of CXCL12-reactive RG fibers and in direct contact with sequences of swellings characterizing the RG fibers (Figures 3A,B) that are also seen in close contact with radial vessel collaterals (Figures 3C,D). Double staining with proteoglycan NG2 antibody as a pericyte marker also demonstrates several points of apposition between regular sequences of RG vascular varicosities and the microvessel wall (Figures 3E,F).

These first observations were extended by triple immunostaining with GFAP and CXCL12, as a classical and a novel marker of RG cells, respectively, and with collagen type IV (Coll IV) as a marker of the vascular basal lamina (Figures $4 \mathrm{~A}-\mathrm{F}$ ). By this staining, in the subcortical layers, where the earliest astrocytes become recognizable, the microvessels appear surrounded by a perivascular subset of astrocytes that express high levels of both GFAP and CXCL12 (see also Virgintino et al., 2013) (Figures 4A,B). On the same sections, RG fibers reveal a lower GFAP immunostaining and a higher staining of CXCL12 (Figures 4A-C). The two markers colocalize on RG vessel-specific varicosities that make en passant contacts maintaining a symmetrical profile, denoted as symmetrical varicosities, or assume an omega-like shape and are therefore indicated as asymmetrical varicosities (Figures 4D-F). In favorable conditions of tissue and antigenicity preservation, and with the opportune setting of laser potency and confocal parameters, the described RG symmetrical and asymmetrical varicosities appear filled by CXCL12-labeled dot-like structures that sometimes display a microvesicular nature (Figures 5A-D, 6A-F, $\mathbf{A}^{\prime}, \mathbf{F}^{\prime}$ ). The CXCL12 MVs are densely packed within varicosities measuring about $1-1.5 \mu \mathrm{m}$ and show a diameter of 100-200 nm (Figures 5A-D, $\left.\mathbf{6 A}-\mathbf{F}, \mathbf{A}^{\prime}-\mathbf{F}^{\prime}\right)$. At sites of vascular contact, CXCL12 densities 

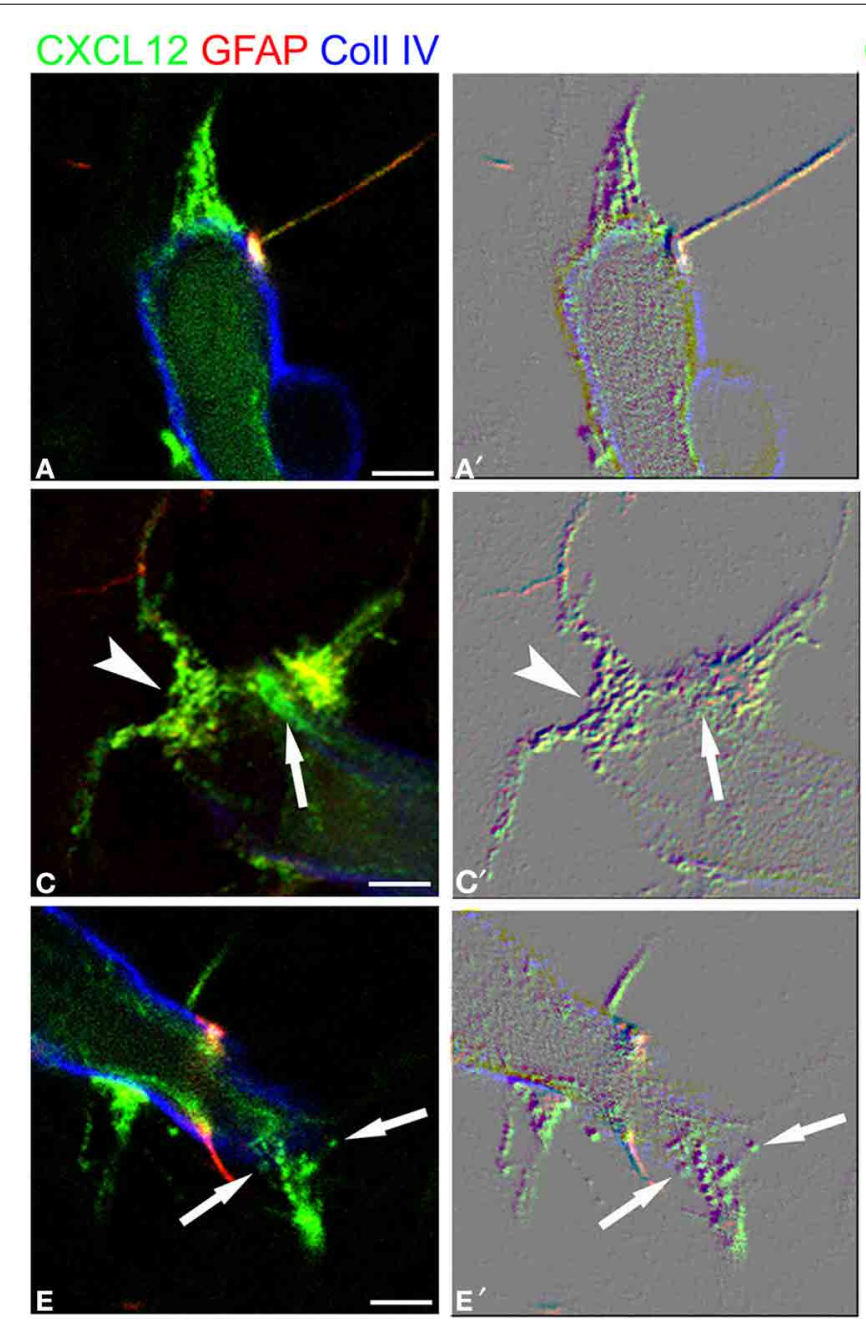

FIGURE 6 | RG-vascular contacts shown in Figure 5, (A-F) and the same pictures rendered with an embossed image filter $\left(\mathbf{A}^{\prime}-\mathbf{F}^{\prime}\right)$. The profile in relief of $\mathrm{MV}$-like subcellular structures is amplified and well-recognizable on the flat surface; note in $\left(\mathbf{A}, \mathbf{A}^{\prime}\right)$ gathered $M V_{s}$, in $\left(\mathbf{B}, \mathbf{B}^{\prime}\right)$ an en passant, symmetrical

\section{CXCL12 GFAP Coll IV}
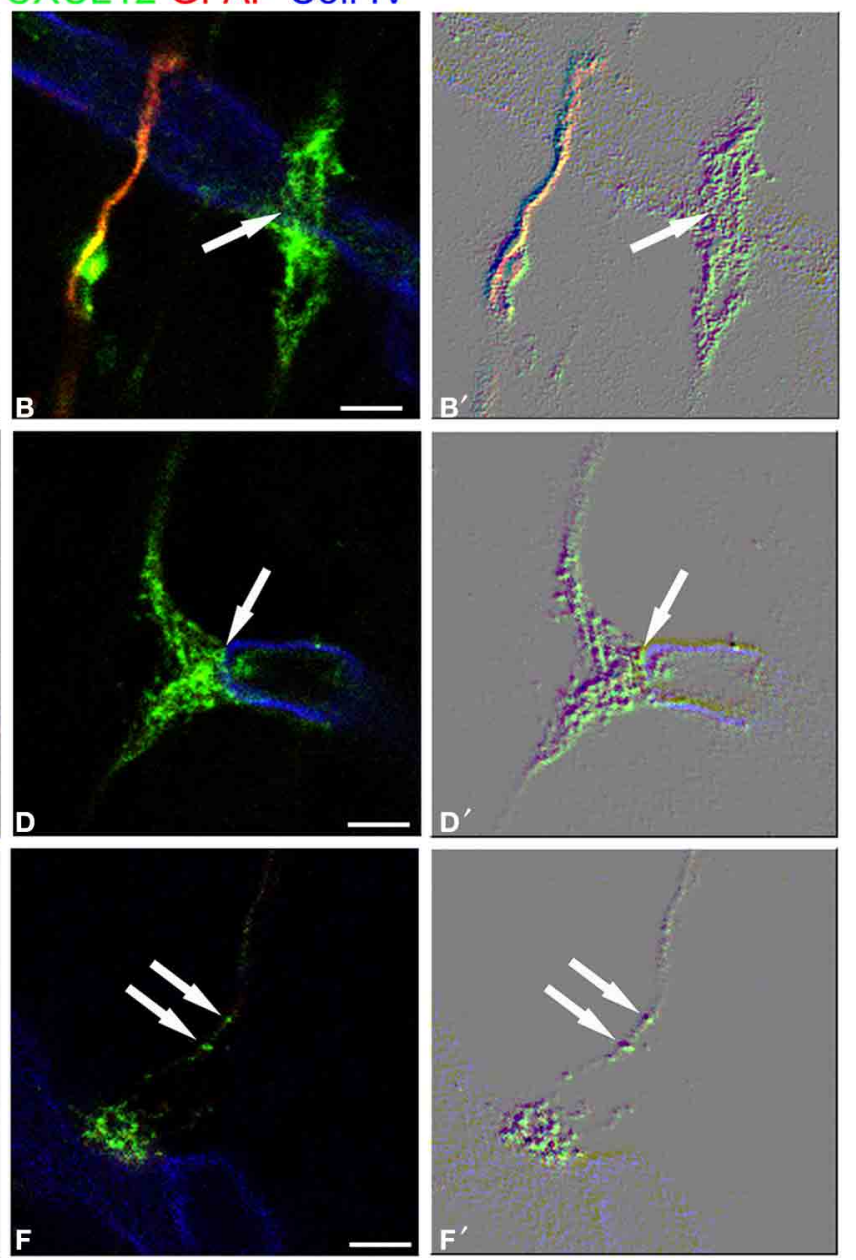

varicosity (arrow), in (C, $\mathbf{C}^{\prime}$ ) an asymmetrical varicosity (arrowhead) and an endothelial CXCL12-reactive density faced by a RG contact (arrow), in (D, $\mathbf{D}^{\prime}$ ) a clearly recognizable MV (arrow), in (E, $\mathbf{E}^{\prime}$ ) released MVs (arrows), and in (F,F') MVs within the RG fiber (arrows). Bars: A-F $2.5 \mu \mathrm{m}$. are also recognizable beneath the Coll IV basal membrane (Figure 6C).

\section{RG VARICOSITY AND MVs ARE CLEARLY REVEALED BY TRANSMISSION ELECTRON MICROSCOPY}

When comparing confocal images of radial microvessels, after triple immunostaining for CXCL12, GFAP, and Coll IV (Figures 7A,B), with similar fields studied by transmission electron microscopy (TEM), some details, appreciated in the former, become clearly defined by ultrastructural observations (Figures 7C,D). The vesicular nature of the subcellular structures observed by confocal microscopy after CXCL12 immunolabeling is confirmed (Figures 7A-D), while RG varicosities and the vascular basal membrane are seen in close contact at several points of the vessel wall (Figures 7C,D). MVs appear homogeneous in size and aligned against the varicosity membrane facing the vessel contact site (Figures 7C,D).

\section{GAP JUNCTION PROTEIN CX43 COLOCALIZES WITH CHEMOKINE CXCL12 IN RG VARICOSITIES}

The presence of $\mathrm{Cx} 43$ was revealed in double immunostaining for CXCL12 and Cx43, the rationale for this approach being sustained by data on Cx43 involvement in RG-guided neuronal migration (Elias et al., 2007; Matsuuchi and Naus, 2013) and by studies on Cx43 in RG cells (Virgintino et al., 2001; Errede et al., 2002). Cx43 plaques are revealed along the RG fibers and concentrate in vessel-contacting RG varicosities, where $\mathrm{Cx} 43$ extensively colocalizes with CXCL12 (Figures 8A-D).

\section{DISCUSSION}

\section{RADIAL GLIAL CELL VARICOSITIES AND CXCL12 MVs INVOLVED IN} CEREBRAL CORTEX VASCULARIZATION

In 1887, when Giuseppe Magini utilized for the first time the Golgi impregnation technique on mammalian developing cerebral cortex, he described "neuroglial cells with very long 


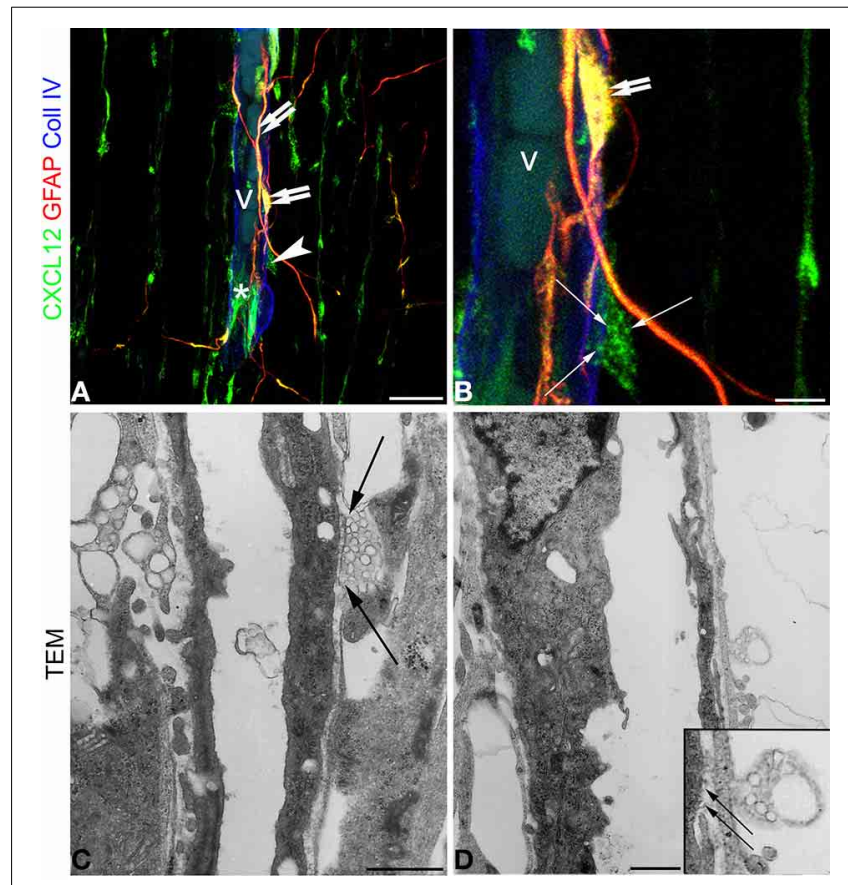

FIGURE 7 | Confocal images (A,B) and transmission electron microscopy (C,D) of human cerebral cortex at midgestation. (A,B) Triple labeling with CXCL12, GFAP, and Coll IV shows the profile of a radial vessel (v) contacted by GFAP/CXCL12-reactive perivascular astrocyte processes (double arrows) and CXCL12-reactive RG fibers (asterisk); a RG varicosity (arrowhead) in contact with the vessel wall is enlarged in (B) and appears filled by CXCL12 labeled MVs (arrows). (C,D) At ultrastructural level, perivascular RG varicosities show their content of membrane-bound MVs regularly aligned with the vessel contact side (C and inset in $\mathbf{D}$; arrows). Bars: A $20 \mu \mathrm{m}$; B $5 \mu \mathrm{m}$; C,D $5 \mu \mathrm{m}$.

and thin filaments bearing numerous varicosities or swellings" (Bentivoglio and Mazzarello, 1999). Subsequently, in 1904, Cajal confirmed the existence of radiating fibers and extended Magini's work, definitely describing "swelling intercalated along the radial glia fibers protoplasmic accumulation” (Ramón y Cajal, 1904). In recent studies on neurogenic RG in rat embryos, DiI-labeled RG cells have shown a "Golgi-like" morphology, including the small varicosities, that have been described as points of cytoplasm accumulation in the flux and reflux of fluid through the cell during mitosis (Weissman et al., 2003; Kriegstein and Alvarez-Buylla, 2009). Surprisingly, CXCL12, a chemokine that accumulates in RG fibers, can show these cellular structures in detail, going beyond the mere profile of cytoplasmic swelling, and instead suggesting a role as specialized cellular sites. RG cells, like cerebellar Bergmann glia and retinal Müller cells, both examples of RG-like glial cells in adult CNS, may display a differential distribution of organelles and membrane domains along their length (Fedoroff and Vernadakis, 1986). The described RG varicosities can be regarded as special compartments of the cell devoted to closely adjoining the vessel wall to form specific vascular contacts.

CXCL12-bearing MVs, as cell-derived MVs (diameter ranging from 0.1 to $1 \mu \mathrm{m}$ ) involved in cell-to-cell communication,

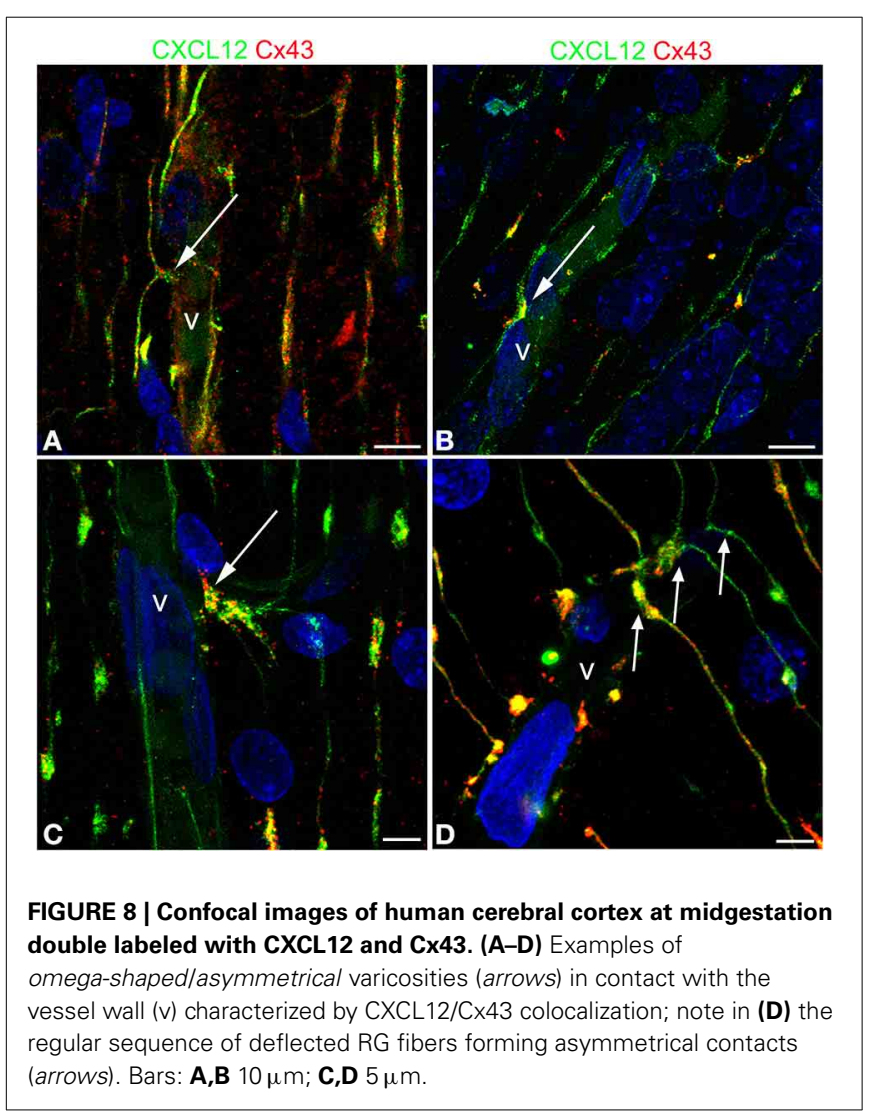

may directly bud from the plasma membrane to convey their membrane-associated bioactive molecule to the target cells (György et al., 2011; Frühbeis et al., 2012; Kalra et al., 2012). It has been demonstrated that cultured astrocytes shed MVs from cell domains interpreted as astrocyte end-feet, which contain angiogenic factors, FGF-2, and VEGF, and membrane associated B1-integrin (Proia et al., 2008), MVs can, therefore, participate in angiogenic events, transferring pro-angiogenic factors to ECs (Boulanger and Tedgui, 2005; Muralidharan-Chari et al., 2010; Martinez and Andriantsitohaina, 2011). Moreover, it has also been shown that tumor-derived MVs released by tumor ECs can be taken up by normal ECs through endocytosis, and promote motility and tube formation, conferring proangiogenic properties to quiescent ECs (Kawamoto et al., 2012). It has been demonstrated that in zebrafish brain and in rat developing retina, chemokine CXCL12 signaling specifically controls the correct pathfinding of newly formed microvessels (Strasser et al., 2010; Unoki et al., 2010; Bussmann et al., 2011; Fujita et al., 2011). Our findings support these roles, suggesting that the chemokine can become available, through the shedding of MVs from RG varicosities, for ligand/receptor interactions on ECs or, alternatively, after MVs endocytosis, for direct EC activation.

\section{CXCL12 VESSEL-SPECIFIC ACTIVITY REGULATED BY CX43 NON-CHANNEL ROLES}

The preliminary results that show a CXCL12/Cx43 colocalization in RG glio-vascular contacts suggest that the release of 
CXCL12 can be regulated by the activation of Cx43 hemichannels, non-junctional structures that can be involved in the release of signaling molecules, and that are a common attribute of cells during development, forming functional hexameric rings before proper gap junctions appear (Nagy et al., 2004; Nielsen et al., 2012; Stehberg et al., 2012; Giaume et al., 2013; Zhou and Jiang, 2014). In fact, during cerebral cortex development, Cx43 displays channel-independent roles also when regulating neuroblast migration (Matsuuchi and Naus, 2013). Moreover, as demonstrated for human bone marrow stromal cells, the secretion of functional CXCL12 by RG cells could be mediated by $\mathrm{Cx} 43$ (Schajnovitz et al., 2011), and $\mathrm{Cx} 43$ has been found to regulate CXCL12-mediated Rap1 EC spreading “in vitro" (Machtaler et al., 2011).

\section{ACTIVATION AND COOPERATION OF MULTIPLE PRO-ANGIO-MORPHOGENETIC PATHWAYS}

The emergence of the chemokine system as a key signal during CNS development and vascularization raises the issue of a possible functional interaction with other pro-angiogenic pathways such as the canonical $\mathrm{Wnt} / \beta$-catenin pathway, whose involvement in regulating vascular morphogenesis has already been described, together with a CNS-specific angiogenic, and barriergenesis function in concert with other signaling pathways (Daneman et al., 2009; Dejana, 2010; Reis and Liebner, 2013). As suggested by studies on the effect of RG cells ablation, a cooperative effect of CXCL12/CXCR4-Wnt/ $\beta$-catenin exists during brain vascularization. In fact, in absence of RG cells, a significant reduction has been observed of cortical thickness, and the regression of nascent brain vessels, via the inhibition of EC Wnt signaling in a contact and stage-dependent manner (Ma et al., 2013). Moreover, the interaction between $\mathrm{Wnt} / \beta$-catenin signaling and the chemokine receptors CXCR4 and CXCR7 leads to a coordinated cell migration via differential receptor regulation (Aman and Piotrowski, 2008); this same cooperation may generate localized molecular cues, inducing differentiation of BBB properties and cortex microvessel maturation (Engelhardt and Liebner, 2014).

In conclusion, this study, carried out during human cerebral cortex development, supports the concept of an involvement of RG cells in the synchronized regulation of neuro-vascular patterning and early BBB differentiation. According to our model (Figures 1A-C), this may take place thanks to the unique, spatial distribution of RG cells, the varicosity/MV/CXCL12/Cx43 machinery, and the help of an overlapping repertoire of signaling molecules (Carmeliet and Tessier-Lavigne, 2005; Vasudevan and Bhide, 2008; Tam and Watts, 2010).

\section{AUTHOR CONTRIBUTIONS}

Mariella Errede Substantial contribution to the design of the work, acquisition, electron microscopy analysis, interpretation of data. Francesco Girolamo Substantial contribution to the design of the work; acquisition, confocal microscopy analysis, interpretation of data. Marco Rizzi Substantial contribution to acquisition and confocal microscopy analysis, interpretation of data. Mirella Bertossi Substantial contribution to interpretation of electron microscopy data and revising the work critically for important intellectual content. Luisa Roncali Substantial contribution in revising the work critically for important intellectual content and final approval of the version to be published. Daniela Virgintino Substantial contributions to the conception of the work, drafting the work and revising it critically; final approval of the version to be published.

\section{ACKNOWLEDGMENTS}

We are indebted to W. B. Stallcup (The Sanford-Burnham Institute for Molecular Medicine, La Jolla, CA, USA) for polyclonal anti-NG2/CSPG4 D2 antiserum and to E. Maiorano (Department of Emergency and Organ Transplantation, Pathological Anatomy Unit, Faculty of Medicine, University of Bari, Bari, Italy) for histological, control sections. Mary Victoria Pragnell is gratefully acknowledged for language help. This work has been funded by grants from Fondazione Cassa di Risparmio di Puglia to Daniela Virgintino and from the Italian Ministry of the University and Scientific Research (MIUR), Progetto di Ricerca d'Interesse Nazionale to Luisa Roncali.

\section{REFERENCES}

Aman, A., and Piotrowski, T. (2008). Wnt/beta-catenin and Fgf signaling control collective cell migration by restricting chemokine receptor expression. Dev. Cell 15, 749-761. doi: 10.1016/j.devcel.2008.10.002

Bär, T. (1980). The vascular system of the cerebral cortex. Adv. Anat. Embryol. Cell Biol. 59, 1-62. doi: 10.1007/978-3-642-67432-7_1

Bentivoglio, M., and Mazzarello, P. (1999). The history of radial glia. Brain Res. Bull. 49, 305-315. doi: 10.1016/S0361-9230(99)00065-9

Borrell, V., and Marín, O. (2006). Meninges control tangential migration of hemderived Cajal-Retzius cells via CXCL12/CXCR4 signaling. Nat. Neurosci. 9, 1284-1293. doi: 10.1038/nn1764

Boulanger, C. M., and Tedgui, A. (2005). Dying for attention: microparticles and angiogenesis. Cardiovasc. Res. 67, 1-3. doi: 10.1016/j.cardiores.2005.05.001

Bussmann, J., Wolfe, S. A., and Siekmannm, A. F. (2011). Arterial-venous network formation during brain vascularization involves hemodynamic regulation of chemokine signaling. Development 138, 1717-1726. doi: 10.1242/dev.059881

Carmeliet, P., and Tessier-Lavigne, M. (2005). Common mechanisms of nerve and blood vessel wiring. Nature 436, 193-200. doi: 10.1038/nature03875

Choi, S. H., and Lapham, L. W. (1978). Radial glia In the human letal cerebrum a combined Golgi, immunofluorescent and electron microscopic study. Brain Res. 148, 295-311. doi: 10.1016/0006-8993(78)90721-7

Daneman, R., Agallin, D., Zhou, L., Kubnert, F., Kuo, J. C., and Barres, B. A. (2009). $\mathrm{Wnt} / \beta$-catenin signaling is required for CNS, but not non-CNS angiogenesis. Proc. Natl. Acad. Sci. U.S.A. 106, 641-646. doi: 10.1073/pnas.0805165106

Dejana, E. (2010). The role of wnt signaling in physiological and pathological angiogenesis. Circ. Res. 107, 943-952. doi: 10.1161/CIRCRESAHA.110.223750

Elias, L. A., Wang, D. D., and Kriegstein, A. R. (2007). Gap junction adhesion is necessary for radial migration in the neocortex. Nature 448, 901-907. doi: 10.1038 /nature06063

Engelhardt, B., and Liebner, S. (2014). Novel insights into the development and maintenance of the blood-brain barrier. Cell Tiss. Res. 355, 687-699. doi: 10.1007/s00441-014-1811-2

Engelhardt, B., and Risau, W. (1995). “The development of the blood-brain barrier” in The Development of the Blood-Brain Barrier, eds J. Greenwood, D. Begley, M. Segal (London: Plenum), 10-100.

Errede, M., Benagiano, V., Girolamo, F., Flace, P., Bertossi, M., Roncali, L., et al. (2002). Differential expression of connexin43 in foetal, adult and tumourassociated human brain endothelial cells. Histochem. J. 34, 265-271. doi: 10.1023/A:1023344106815

Fedoroff, S., and Vernadakis, A. (1986). Astrocytes. Development, Morphology and Regional Specialization of Astrocytes. London: Academic Press.

Frühbeis, C., Fröhlich, D., and Krämer-Albers, E. M. (2012). Emerging roles of exosomes in neuron-glia communication. Front. Physiol. 3:119. doi: 10.3389/fphys.2012.00119 
Fujita, M., Cha, Y. R., Pham, V. N., Sakurai, A., Roman, B. L., Gutkind, J. S., et al. (2011). Assembly and patterning of the vascular network of the vertebrate hindbrain. Development 138, 1705-1715. doi: 10.1242/dev.058776

Giaume, C., Leybaert, L., Naus, C. C., and Sáez, J. C. (2013). Connexin and pannexin hemichannels in brain glial cells: properties, pharmacology, and roles. Front. Pharmacol. 4:88. doi: 10.3389/fphar.2013.00088

György, B., Szabó, T. G., Pásztói, M., Pál, Z., Misják, P., Aradi, B., et al. (2011). Membrane vesicles, current state-of-the-art: emerging role of extracellular vesicles. Cell. Mol. Life Sci. 68, 2667-2688. doi: 10.1007/s00018-0110689-3

Howard, B., Chen, Y., and Zecevic, N. (2006). Cortical progenitor cells in the developing human telencephalon. Glia 53, 57-66. doi: 10.1002/glia.20259

Howard, B. M., Mo, Z., Filipovic, R., Moore, A. R., Antic, S. D., and Zecevic, D. (2008). Radial glia cells in the developing human brain. Neuroscientist 14, 459-473. doi: 10.1177/1073858407313512

Kalra, H., Simpson, R. J., Ji, H., Aikawa, E., Altevogt, P., Askenase, P., et al. (2012). Vesiclepedia: a compendium for extracellular vesicles with continuous community annotation. PLoS Biol. 10:e1001450. doi: 10.1371/journal.pbio.1001450

Kawamoto, T., Ohga, N., Akiyama, K., Hirata, N., Kitahara, S., Maishi, N., et al. (2012). Tumor-derived microvesicles induce proangiogenic phenotype in endothelial cells via endocytosis. PLoS ONE 7:e34045. doi: 10.1371/journal.pone.0034045

Kriegstein, A., and Alvarez-Buylla, A. (2009). The glial nature of embryonic and adult neural stem cells. Annu. Rev. Neurosci. 32, 149-184. doi: 10.1146/annurev.neuro.051508.135600

Li, G., Adesnik, H., Li, J., Long, J., Nicoll, R. A., Rubenstein, J. L., et al. (2008). Regional distribution of cortical interneurons and development of inhibitory tone are regulated by Cxcl12/Cxcr4 signaling. J. Neurosci. 28, 1085-1098. doi: 10.1523/JNEUROSCI.4602-07.2008

Liebner, S., and Plate, K. H. (2010). Differentiation of the brain vasculature: the answer came blowing by the Wnt. J. Angiogenesis Res. 2, 1-10. doi: 10.1186/2040-2384-2-1

Ma, S., Kwon, H. J., Johng, H., Zang, K., and Huang, Z. (2013). Radial glial neural progenitors regulate nascent brain vascular network stabilization via inhibition of Wnt signaling. PLoS Biol. 11:e1001469. doi: 10.1371/journal.pbio. 1001469

Machtaler, S., Dang-Lawson, M., Choi, K., Jang, C., Naus, C. C., and Matsuuchi, L. (2011). The gap junction protein Cx43 regulates B-lymphocyte spreading and adhesion. J. Cell Sci. 124, 2611-2621. doi: 10.1242/jcs.089532

Malatesta, P., and Götz, M. (2013). Radial glia - from boring cables to stem cell stars. Development 140, 483-486. doi: 10.1242/dev.085852

Malatesta, P., Hartfuss, E., and Götz, M. (2000). Isolation of radial glial cells by fluorescent-activated cell sorting reveals a neuronal lineage. Development 127 5253-5263.

Marin-Padilla, M. (1985). Early vascularization of the embryonic cerebral cortex: Golgi and electron microscopic studies. J. Comp. Neurol. 241, 237-249. doi: $10.1002 /$ cne. 902410210

Marín-Padilla, M. (2012). The human brain intracerebral microvascular system: development and structure. Front. Neuroanat. 6:38. doi: 10.3389/fnana.2012.00038

Martinez, M. C., and Andriantsitohaina, R. (2011). Microparticles in angiogenesis: therapeutic potential. Circ. Res. 109, 110-119. doi: 10.1161/CIRCRESAHA.110.233049

Matsuuchi, L., and Naus, C. C. (2013). Gap junction proteins on the move: connexins, the cytoskeleton and migration. Biochim. Biophys. Acta 1828, 94-108. doi: 10.1016/j.bbamem.2012.05.014

Muralidharan-Chari, V., Clancy, J. W., Sedgwick, A., and D'Souza-Schorey, C. (2010). Microvesicles: mediators of extracellular communication during cancer progression. J. Cell Sci. 123, 1603-1611. doi: 10.1242/jcs.064386

Nagy, J. I., Dudek, F. E., and Rash, J. E. (2004). Update on connexins and gap junctions in neurons and glia in the mammalian nervous system. Brain Res. Brain Res. Rev. 47, 191-215. doi: 10.1016/j.brainresrev.2004.05.005

Nielsen, M. S., Nygaard Axelsen, L., Sorgen, P. L., Verma, V., Delmar, M., and Holstein-Rathlou, N. H. (2012). Gap junctions. Compr. Physiol. 2, 1981-2035. doi: 10.1002/cphy.c110051

Paredes, M. F., Li, G. N., Berger, O., Baraban, S. C., and Pleasure, S. J. (2006). Stromal-derived factor-1 (CXCL12) regulates laminar position of CajalRetzius cells in normal and dysplastic brains. J. Neurosci. 26, 9404-9412. doi: 10.1523/JNEUROSCI.2575-06.2006
Peng, H., Kolb, R., and Kennedy, J. E. (2007). Differential expression of CXCL12 and CXCR4 during human fetal neural progenitor cell differentiation. J. Neuroimmun. Pharmacol. 2, 251-258. doi: 10.1007/s11481-007-9081-3

Proia, P., Schiera, G., Mineo, M., Ingrassia, A. M., Santoro, G., Savettieri, G. et al. (2008). Astrocytes shed extracellular vesicles that contain fibroblast growth factor-2 and vascular endothelial growth factor. Int. J. Mol. Med. 21, 63-67. doi: 10.3892/ijmm.21.1.63

Quaegebeur, A., Lange, C., and Carmeliet, P. (2011). The neurovascular link in health and disease: molecular mechanisms and therapeutic implications. Neuron 71, 406-424. doi: 10.1016/j.neuron.2011.07.013

Rakic, P. (1971). Neuron-glia relationship during granule cell migration in developing cerebellar cortex. A Golgi and electronmicroscopic study in Macacus Rhesus. J. Comp. Neurol. 141, 283-312. doi: 10.1002/cne.901410303

Rakic, P. (1972). Mode of cell migration to the superficial layers of fetal monkey neocortex. J. Comp. Neurol. 145, 61-83. doi: 10.1002/cne.901450105

Rakic, P. (2007). The radial edifice of cortical architecture. Brain Res. Rev. 55, 204-219. doi: 10.1016/j.brainresrev.2007.02.010

Ramón y Cajal, S. (1904). Textura del Sistema Nervioso del Hombre y de los Vertebrados. Madrid: Moya.

Reis, M., and Liebner, S. (2013). Wnt signaling in the vasculature. Exp. Cell Res. 319, 1317-1323. doi: 10.1016/j.yexcr.2012.12.023

Rickmann, M., and Wolff, J. R. (1985). Prenatal gliogenesis in the neopallium of the rat. Adv. Anat. Embryol. Cell Biol. 93, 37-66.

Risau, W., Gautschi-Sova, P., and Bohlen, P. (1988). Endothelial cell growth factors in embryonic and adult chick brain are related to human acidic fibroblast growth factor. EMBO J. 7, 959-962.

Risau, W., and Wolburg, H. (1990). Development of the blood-brain barrier. Trends Neurosci. 13, 174-178. doi: 10.1016/0166-2236(90)90043-A

Schajnovitz, A., Itkin, T., D’Uva, G., Kalinkovich, A., Golan, K., Ludin, A., et al. (2011). CXCL12 secretion by bone marrow stromal cells is dependent on cell contact and mediated by connexin-43 and connexin-45 gap junctions. Nat. Immunol. 12, 391-398. doi: 10.1038/ni.2017

Siegenthaler, J. A., Ashique, A. M., Zarbalis, K., Patterson, K. P., Hecht, J. H., Kane, M. A., et al. (2009). Retinoic acid from the meninges regulates cortical neuron generation. Cell 139, 597-609. doi: 10.1016/j.cell.2009.10.004

Stehberg, J., Moraga-Amaro, R., Salazar, C., Becerra, A., Echeverría, C., Orellana, J. A., et al. (2012). Release of gliotransmitters through astroglial connexin 43 hemichannels is necessary for fear memory consolidation in the basolateral amygdala. FASEB J. 26, 3649-3657. doi: 10.1096/fj.11-198416

Strasser, G. A., Kaminker, J. S., and Tessier-Lavigne, M. (2010). Microarray analysis of retinal endothelial tip cells identifies CXCR4 as a mediator of tip cell morphology and branching. Blood 115, 5102-5110. doi: 10.1182/blood-200907-230284

Stumm, R., and Höllt, V. (2007). CXC chemokine receptor 4 regulates neuronal migration and axonal pathfinding in the developing nervous system: implications for neuronal regeneration in the adult brain. J. Mol. Endocrinol. 38, 377-382. doi: 10.1677/JME-06-0032

Stumm, R. K., Zhou, C., Ara, T., Lazarini, F., Dubois-Dalcq, M., Nagasawa, T., et al. (2003). CXCR4 regulates interneuron migration in the developing neocortex. J. Neurosci. 23, 5123-5130.

Tam, S. J., and Watts, R. J. (2010). Connecting vascular and nervous system development: angiogenesis and the blood-brain barrier. Annu. Rev. Neurosci. 33, 379-408. doi: 10.1146/annurev-neuro-060909-152829

Tiveron, M. C., and Cremer, H. (2008). CXCL12/CXCR4 signalling in neuronal cell migration. Curr. Opin. Neurobiol. 18, 237-244. doi: 10.1016/j.conb.2008. 06.004

Tiveron, M. C., Rossel, M., Moepps, B., Zhang, Y. L., Seidenfaden, R., and Favor, J., et al (2006). Molecular interaction between projection neuron precursors and invading interneurons via stromal-derived factor 1 (CXCL12)/CXCR4 signaling in the cortical subventricular zone/intermediate zone. J. Neurosci. 26, 13273-13278. doi: 10.1523/JNEUROSCI.4162-06.2006

Unoki, N., Murakami, T., Nishijima, K., Ogino, K., van Rooijen, N., and Yoshimura, N. (2010). SDF-1/CXCR4 contributes to the activation of tip cells and microglia in retinal angiogenesis. Invest. Ophthalmol. Vis. Sci. 51, 3362-3371. doi: 10.1167/iovs.09-4978

Vasudevan, A., and Bhide, P. G. (2008). Angiogenesis in the embryonic CNS: a new twist on an old tale. Cell Adh. Migr. 2, 167-169. doi: 10.4161/cam.2.3.6485

Virgintino, D., Errede, M., Rizzi, M., Girolamo, F., Strippoli, M., Wälchli, T., et al. (2013). The CXCL12/CXCR4/CXCR7 ligand-receptor system regulates 
neuro-glio-vascular interactions and vessel growth during human brain development. J. Inherit. Metab. Dis. 36, 455-466. doi: 10.1007/s10545-0129574-y

Virgintino, D., Maiorano, E., Errede, M., Vimercati, A., Greco, P., Selvaggi, L., et al. (1998). Astroglia-microvessel relationship in the developing human telencephalon. Int. J. Dev. Biol. 42, 1165-1168.

Virgintino, D., Robertson, D., Errede, M., Benagiano, V., Bertossi, M., Ambrosi, G., et al. (2001). Expression of the gap junction protein connexin43 in human telencephalon microvessels. Microvasc. Res. 62, 435-439. doi: 10.1006/mvre.2001.2345

Weissman, T., Noctor, S. C., Clinton, B. K., Honig, L. S., and Kriegstein, A. R. (2003). Neurogenic radial glial cells in reptile, rodent and human: from mitosis to migration. Cereb. Cortex 13, 550-559. doi: 10.1093/cercor/13.6.550

Xu, L., Tang, X., Wang, Y., Xu, H., and Fan, X. (2014). Radial glia, the keystone of the development of the hippocampal dentate gyrus. Mol. Neurobiol. doi: 10.1007/ s12035-014-8692-y. [Epub ahead of print].

Zhou, J. Z., and Jiang, J. X. (2014). Gap junction and hemichannel-independent actions of connexins on cell and tissue functions - An update. FEBS Lett. 588, 1186-1192. doi: 10.1016/j.febslet.2014.01.001
Conflict of Interest Statement: The authors declare that the research was conducted in the absence of any commercial or financial relationships that could be construed as a potential conflict of interest.

Received: 15 July 2014; paper pending published: 11 August 2014; accepted: 25 September 2014; published online: 15 October 2014.

Citation: Errede M, Girolamo F, Rizzi M, Bertossi M, Roncali L and Virgintino D (2014) The contribution of CXCL12-expressing radial glia cells to neuro-vascular patterning during human cerebral cortex development. Front. Neurosci. 8:324. doi: 10.3389/fnins.2014.00324

This article was submitted to Neurogenomics, a section of the journal Frontiers in Neuroscience.

Copyright (C) 2014 Errede, Girolamo, Rizzi, Bertossi, Roncali and Virgintino. This is an open-access article distributed under the terms of the Creative Commons Attribution License (CC BY). The use, distribution or reproduction in other forums is permitted, provided the original author(s) or licensor are credited and that the original publication in this journal is cited, in accordance with accepted academic practice. No use, distribution or reproduction is permitted which does not comply with these terms. 


\title{
The inner CSF-brain barrier: developmentally controlled access to the brain via intercellular junctions
}

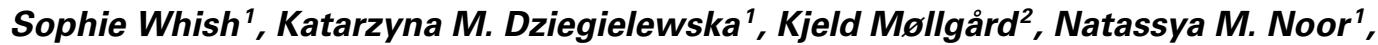 \\ Shane A. Liddelow ${ }^{1,3}$, Mark D. Habgood ${ }^{1}$, Samantha J. Richardson ${ }^{4}$ and Norman R. Saunders ${ }^{1 *}$ \\ Department of Pharmacology and Therapeutics, University of Melbourne, Parkville, VIC, Australia \\ ${ }^{2}$ Department of Cellular and Molecular Medicine, Institute of Cellular and Molecular Medicine, University of Copenhagen, Copenhagen, Denmark \\ ${ }^{3}$ Department of Neurobiology, Stanford University, Palo Alto, CA, USA \\ ${ }^{4}$ School of Medical Sciences, RMIT University, Bundoora, VIC, Australia
}

Edited by:

Joana Pahla, University of Minho,

Portugal

\section{Reviewed by:}

Luc Leybaert, Ghent University, Belgium

Miguel Castanho, University of Lisbon, Portugal

*Correspondence:

Norman R. Saunders, Department of Pharmacology and Therapeutics, University of Melbourne, Grattan Street, Parkville, VIC 3010, Australia e-mail: n.saunders@unimelb.edu.au
In the adult the interface between the cerebrospinal fluid and the brain is lined by the ependymal cells, which are joined by gap junctions. These intercellular connections do not provide a diffusional restrain between the two compartments. However, during development this interface, initially consisting of neuroepithelial cells and later radial glial cells, is characterized by "strap" junctions, which limit the exchange of different sized molecules between cerebrospinal fluid and the brain parenchyma. Here we provide a systematic study of permeability properties of this inner cerebrospinal fluid-brain barrier during mouse development from embryonic day, E17 until adult. Results show that at fetal stages exchange across this barrier is restricted to the smallest molecules (286Da) and the diffusional restraint is progressively removed as the brain develops. By postnatal day P20, molecules the size of plasma proteins $(70 \mathrm{kDa})$ diffuse freely. Transcriptomic analysis of junctional proteins present in the cerebrospinal fluid-brain interface showed expression of adherens junctional proteins, actins, cadherins and catenins changing in a development manner consistent with the observed changes in the permeability studies. Gap junction proteins were only identified in the adult as was claudin-11. Immunohistochemistry was used to localize at the cellular level some of the adherens junctional proteins of genes identified from transcriptomic analysis. $\mathrm{N}$-cadherin, $\beta$ - and $\alpha$-catenin immunoreactivity was detected outlining the inner CSF-brain interface from E16; most of these markers were not present in the adult ependyma. Claudin- 5 was present in the apical-most part of radial glial cells and in endothelial cells in embryos, but only in endothelial cells including plexus endothelial cells in adults. Claudin-11 was only immunopositive in the adult, consistent with results obtained from transcriptomic analysis. These results provide information about physiological, molecular and morphological-related permeability changes occurring at the inner cerebrospinal fluid-brain barrier during brain development.

\section{Keywords: cerebrospinal fluid, brain development, barriers, permeability, ventricular zone}

\section{INTRODUCTION}

The exchange of molecules between the brain and the periphery is restricted by specific cellular and biochemical mechanisms present at several interfaces between these two compartments: the blood-brain barrier proper, the blood-cerebrospinal fluid (CSF) barrier, the blood-arachnoid barrier and the (inner) CSF-brain barrier only present during brain development (Møllgård et al., 1987; Saunders et al., 2012). The morphological basis of bloodbrain and blood-CSF barriers is the presence of tight junctions between cerebral vascular endothelial cells and choroid plexus epithelial cells respectively. These junctions restrict free diffusion between the blood, the brain parenchyma and the CSF (for

Abbreviations: CSF, cerebrospinal fluid; RNA-Seq, RNA sequencing; BDA, biotinylated dextran amine; BED; biotin ethylenediamine; $\mathrm{kDa}$, kilodalton. reviews see Saunders et al., 1999, 2000, 2012; Dziegielewska and Saunders, 2002; Ek et al., 2012; Engelhardt and Liebner, 2014). Much less is known about the development of CSF-brain interface. Junctions between cells lining the ventricular system of the developing brain were first described on the basis of conventional electron microscopy as modified tight or intermediate junctions, or as desmosomes (Tennyson and Pappas, 1962; Duckett, 1968; Levitt et al., 1981). Subsequent studies characterized these junctions in fetal sheep using thin-section and freeze-fracture electron microscopy and established that they had a very different structural arrangement compared with tight junctions. Unlike tight junctions, which appear to join cells in a "belt-like fashion" at their luminal lateral cell membranes, these novel junctions, named "strap junctions," appeared to encircle cells from their ventricular surface (Møllgård et al., 1987) and were shown to 
hinder the free diffusion of solutes from the CSF into the fetal brain (Fossan et al., 1985). In contrast to tight junctions of bloodbrain and blood-CSF barriers, which are present from the earliest stages of brain development and remain throughout adulthood (Møllgård and Saunders, 1986; Mishra and Teale, 2012; Saunders et al., 2012), strap junctions are only present during development, with minimal gap junctions between some neuroepithelial cells; in the mature brain only gap junctions are present (Brightman and Reese, 1969; Møllgård and Saunders, 1986; Møllgård et al., 1987; Iliff et al., 2012). Additional evidence for the presence of a developmentally regulated CSF-brain barrier came from a study examining the cellular distribution of native and foreign albumins injected into fetal and neonatal lateral ventricles of rats (Cavanagh and Warren, 1985). This study showed that both types of albumin were distributed in some (but not all) cells lining the ventricles suggesting specialized uptake rather than paracellular unrestricted diffusion (Cavanagh and Warren, 1985).

The fetal-specific interface that separates CSF from the brain's internal milieu is thought to control the transfer and diffusion of solutes into the brain and play an important role in restricting access of protein, present in high concentration in fetal CSF (Dziegielewska et al., 1980, 1981, 1986, 1989; Adinolfi, 1985; Dziegielewska and Saunders, 1988), across the ventricular zone, which initially consists of neuroepithelial cells that subsequently differentiate to radial glial cells prior to neurogenesis. This, combined with an increase in colloidal osmotic pressure due to the high protein concentration responsible for the expansion of the ventricles and outward growth of the brain (Desmond and Jacobson, 1977), are fundamental mechanisms controlling normal brain development (Saunders, 1992; Johansson et al., 2008; Saunders et al., 2012). The presence of this diffusion restraint during brain development has been suggested to be important for normal cortical development (Stolp et al., 2011b) and its disturbance is thought to contribute to several neurodevelopmental pathologies such as autism (Stolp, 2013; Stolp et al., 2013).

The present study is the first comprehensive investigation into the permeability properties of the inner CSF-brain interface during development, using lipid insoluble molecular markers with a range of sizes from 286 to $70,000 \mathrm{Da}$. We report that permeability of this barrier in mouse brain gradually changes from highly restrictive at fetal ages to an unrestricted diffusion in the adult. Molecular characterization, using RNA sequencing performed at a fetal and adult age, identified several known junctional proteins that are expressed at the CSF-brain barrier in a developmentally regulated manner. In addition to the transcriptomic analysis we also demonstrated that the change in the permeability characteristics coincided with changes in the cellular distribution of several junctional proteins such as cadherins, catenins and claudin-5. We propose that these junctional proteins are involved in the formation of strap junctions and in regulation of the changing diffusional properties of this barrier.

\section{MATERIALS AND METHODS ANIMAL EXPERIMENTATION \\ Ethics statement}

All experimental protocols were approved by the Animal Ethics Committee, University of Melbourne (Application ID 1112115.2).

\section{Animal experiments}

Time-mated pregnant Swiss Pro mice were obtained from the Animal Resource Centre Pty. Ltd. (ARC, Perth, Australia). Animals were housed in the Biomedical Sciences Animal Facility (University of Melbourne) in sterile micro-isolator cages at $20^{\circ} \mathrm{C}$ on a $12 \mathrm{~h}$ light/dark cycle with food and water ad libitum.

Ages of mice used for permeability studies and for estimates of total protein concentration in plasma and CSF were: embryonic day (E) 17, E19 and postnatal day (P) 0, P10, P20 and adult (20-25 g). For transcriptome analysis, RNA-Seq, ventricular/ependymal layer was dissected out from fetal brains at E17/18, and adult (see below). For morphological studies paraffin embedded sections from fetal stages, E15/16, E17-19 and postnatal stages, P15, P20 and adult mice brains were used. These were obtained from previous studies (Liddelow et al., 2012).

\section{Anesthesia}

In experiments involving fetal animals, pregnant mice (E17-E19) were anesthetized by an intraperitoneal (i.p.) injection of urethane $(30 \%$ in distilled water, Veterinary Companies of Australia (VCA), Australia). Two doses of $0.2 \mathrm{ml}$ were given $20 \mathrm{~min}$ apart. Animals were monitored during this process and pain reflexes were observed. When the pain reflexes were no longer evident, mice were placed supine on a heated pad $\left(33^{\circ} \mathrm{C}\right)$ and the uterine horns were exposed. Each fetus was removed consecutively from the uterine horn. After all fetuses were removed, the mother was killed by transection of the heart.

Postnatal pups of both sexes (P0, P10, and P20) and adult female mice were individually anesthetized using inhaled Isoflurane (VCA). Once deep anesthesia was obtained the animals were placed under the dissecting microscope, and several experimental procedures were conducted as described below.

\section{Total protein concentration}

Blood and CSF were collected from 3 to 4 pups at each age and from 3 to 4 adults. Blood was collected from the heart of terminally anesthetized animals using either a heparinized $1 \mathrm{ml}$ syringe with a 26-gauge needle (adults) or a heparinized pulled glass micro-capillary (fetuses and postnatal pups) and centrifuged to separate plasma. CSF was collected from the cisterna magna via gentle suction using a fine glass micro-capillary and PVC tubing to obtain paired samples. CSF samples were inspected under a microscope for blood contamination (Habgood et al., 1993). For fetal animals CSF samples were pooled from several littermates to obtain a large enough volume. Plasma and CSF samples were stored at $-20^{\circ} \mathrm{C}$ until use.

Total protein concentrations in plasma and CSF samples were estimated using the Bradford method (Bradford, 1976) and Protein Standard (Binding Sites, UK). All dilutions of the standards in the range of $1-10 \mathrm{ug} / 100 \mathrm{ul}$ (in triplicate) and samples (in duplicate) were made in sterile saline. Plasma samples were diluted 1000x while CSF ranged from 100x to 10x depending on the age (Dziegielewska et al., 1979). The volume of both the standards and the samples was always $100 \mu \mathrm{l}$ as described in the original method (Bradford, 1976). The concentration of the total protein in each sample was measured from constructed concentration curves and expressed in $\mathrm{mg} 100 \mathrm{ml}^{-1}$ a standard unit in the field (Dziegielewska et al., 1979, 1989). 


\section{CSF-brain barrier permeability experiments}

Rhodamine-conjugated biotinylated dextran amines, BDAs, of molecular masses $3000 \mathrm{Da}(3 \mathrm{kDa}), 10000 \mathrm{Da}(10 \mathrm{kDa})$ and $70000 \mathrm{Da}(70 \mathrm{kDa})$ and a $286 \mathrm{Da}$ biotin ethylenediamine hydrobromide (BED) obtained from Molecular Probes (USA) were used in these experiments. Three to four individual mice from at least two separate litters were obtained for each experiment. All probes were diluted in sterile saline $\left(25 \mathrm{mgml}^{-1}\right)$ and were injected into the right ventricle of anesthetized animals via a glass microcapillary and gentle pressure (see Figure 1). Injected volumes are listed in Table 1. Following injection the markers were allowed to distribute for a further 2-3 min (fetal), $5 \mathrm{~min}$ (postnatal ages to P20) or $10 \mathrm{~min}$ (adults) so that each dextran would flow into the contralateral ventricle. Brains of animals injected with fluorescence BDAs were dissected out from the skull and fixed by immersion in $4 \%$ paraformaldehyde (PFA) for $24 \mathrm{~h}$ at $4^{\circ} \mathrm{C}$. Brains from pups injected with BED were fixed in Bouin's fixative for $24 \mathrm{~h}$ and processed for paraffin embedding (see below).

\section{Fluorescence microscopy}

At the end of permeability experiments and following fixation, brains were embedded in high gel strength 4\% Agar (Sigma Aldrich) and sectioned in the coronal plane at $80 \mu \mathrm{m}$ using a vibrating microtome (Leica). All sections containing the lateral

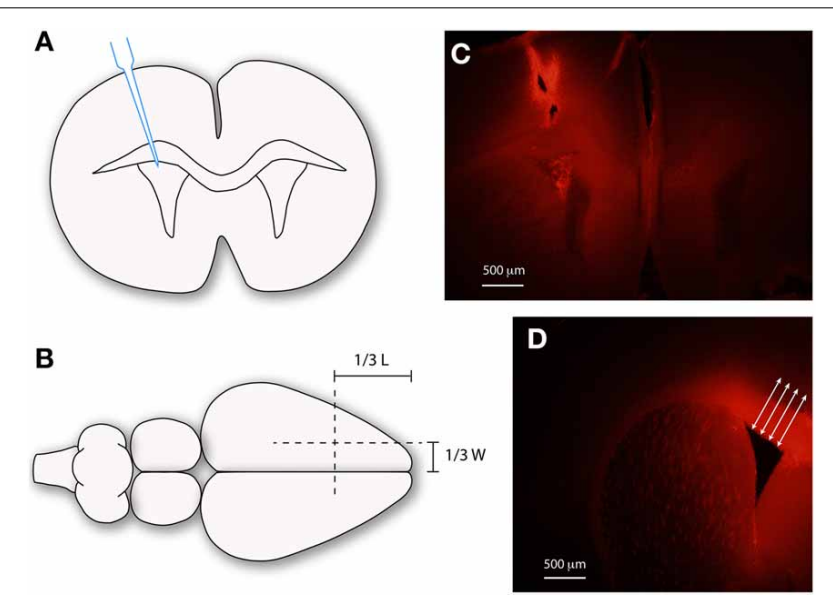

FIGURE 1 | Injection of biotin dextran amines into the ventricular system of mice. Schematic diagrams (A,B) and images (C,D) illustrating the methods used to inject dextrans into the lateral ventricles of mouse brain. (A) Micropipette injection of dextran into lateral ventricle. (B) Illustrates surface landmarks for injection site. (C) Distribution of dextran following injection. (D) Method used to measure diffusion distance of dextrans (red) using ImageJ64. Mean of 10 measurements, spanning the ventricular zone at right angles to ventricular surface (arrows). Scale bars $500 \mu \mathrm{m}$ in (C,D). L, length; W, width.

Table 1 | Volumes and times of injected probes at different ages.

\begin{tabular}{lcc}
\hline Age & Volume & Time \\
\hline E17, P0 & $1 \mu \mathrm{l}$ & $2,3 \mathrm{~min}$ \\
P5, P10 & $5 \mu \mathrm{l}$ & $5 \mathrm{~min}$ \\
P20, Adult & $10 \mu \mathrm{l}$ & $5,10 \mathrm{~min}$
\end{tabular}

ventricles were collected for analysis. Each section was mounted on a glass slide using fluorescence mounting medium (DAKO). Slides were kept at $4^{\circ} \mathrm{C}$ and covered with foil to restrict light exposure. All sections were viewed with an Olympus BX50 fluorescent light microscope with a filter specific for rhodamine. Digitized photographs were taken using an Olympus DP70 camera that was attached to the microscope and processed in Adobe Photoshop $\mathrm{CC}^{\circledR}$ (Adobe ${ }^{\circledR}$ Systems Incorporated, USA). The brightness and color balance functions were used to obtain images with a background close to black to enhance contrast and to standardize obtained images.

\section{Immunohistochemistry}

Paraffin-embedded coronal sections from mice from fetal ages E15-16, E17-19 and postnatal ages P15, P20 and adult were obtained from previous studies (Liddelow et al., 2012). Prior to immunohistochemical processing sections were deparaffinized and rehydrated in xylene followed by a series of graded alcohols, treated with a $0.5 \%$ solution of hydrogen peroxide in methanol for $15 \mathrm{~min}$ to quench endogenous peroxidase, and then rinsed in TRIS buffered saline (TBS, $5 \mathrm{mM}$ Tris- $\mathrm{HCl}, 146 \mathrm{mM}$ $\mathrm{NaCl}, \mathrm{pH}$ 7.6). Non-specific binding was removed by incubation for 30 min with blocking buffer (ChemMate antibody diluent S2022, DakoCytomation, Glostrup, Denmark) at room temperature. The sections were incubated overnight at $4^{\circ} \mathrm{C}$ with rabbit polyclonal or mouse monoclonal antibodies against claudin5 , claudin-11, $\alpha$-catenin, $\beta$-catenin and $\mathrm{N}$-cadherin (details in Table 2). Following primary antibody incubation the sections were washed with TBS and incubated for $30 \mathrm{~min}$ with the REAL EnVision $^{\mathrm{TM}}$ Detection System, Peroxidase/DAB+, Rabbit/Mouse, (K5007) from DakoCytomation. The sections were counterstained with Mayer's hematoxylin and dehydrated in graded alcohols followed by xylene and coverslipped with DPX mounting medium. Control sections were incubated with mouse IgG1, IgG2a or non-specific unrelated rabbit antibodies, as well as subjected to omission of primary or secondary antibodies. These were always blank.

Sections were viewed with an Olympus bright field microscope and photographs taken with only brightness manipulated to obtain comparable images. Three to four sections from a minimum of three brains at each age were developed with each antibody.

\section{Detection of BED}

Brains from BED injected fetal mice were paraffin-embedded as described previously (Liddelow et al., 2011) and $5 \mu \mathrm{m}$ serial coronal sections cut through the cortex encompassing

Table 2 | Antibodies used for immunohistochemistry.

\begin{tabular}{lllll}
\hline Antigen & Manufacturer & Code no. & Species & Dilution \\
\hline Cadherin N & Abcam & ab12221 & Rabbit & $1: 500$ \\
Catenin a E & Santa cruz & sc-7894 & Rabbit & $1: 100$ \\
Catenin ß & Zymed & $13-8400$ & Mouse & $1: 100$ \\
Claudin 5 & Abcam & ab15108 & Rabbit & $1: 150$ \\
Claudin 11 & Abcam & ab53041 & Rabbit & $1: 1500$
\end{tabular}


the lateral ventricles. Five sections spanning the whole lateral ventricular system were processed for histological staining using the $\mathrm{ABC}$ detection method (Vector laboratories) and 3, 3'-diaminobenzidine (DAB) chromagen substrate developer (DAKO) following manufactures' instructions. De-waxing, rehydration, dehydration and mounting of sections with DPX were performed as described above. Sections from brains not injected with BED served as controls and these were always blank.

\section{RNA sequencing analysis}

The lateral aspect of the ventricular zone of E17/18 and adult mouse brains was carefully dissected (Figure 1). Tissue from five brains, collected from three different litters, was pooled randomly to obtain three equal samples at each age and was processed for RNA sequencing.

\section{RNA extraction}

Total RNA was extracted from pools of E17/18 lateral ventricular zone and adult lateral ependymal zone $(n=3$ for both ages) using the RNeasy Mini Kit, Qiashredder columns and gDNA removal columns (Qiagen, Valencia, CA, USA) according to standard supplier protocol. Total RNA samples were quantified using a NanoDrop ND-1000 UV-VIS spectrophotometer (Thermo Scientific, Wilmington, DE, USA).

\section{Illumina next generation RNA sequencing}

As previously described for an RNA-Seq study of developing choroid plexus (Liddelow et al., 2013) sequencing was performed at the Australian Genome Research Facility (Melbourne, VIC, Australia). A cDNA library was prepared from $10 \mu \mathrm{g}$ of total RNA using the mRNA-Seq Sample Preparation Kit (Illumina, San Diego, CA, USA) according to standard manufacturer protocol. Quality of the library was verified using a DNA 1000 chip using the Agilent 2100 Bioanalyzer (Agilent) and quantified by fluorimetry. Only samples with an RNA Integrity Number close to 10.0 were kept for further sequencing experiments. The library was subjected to $100 \mathrm{bp}$ single end read cycles of sequencing on an Illumina HiSeq 2000 sequencer as per manufacturer protocol. Cluster generation was performed on a c-Bot (Illumina) with a single read cluster generation kit.

\section{RNA sequencing data analysis}

Short reads were trimmed to remove ambiguous bases from the start and segments with low quality scores from the end, as indicated by the ascii character " $\mathrm{B}$ " in Illumina 1.5 phred score encoding. Trimmed reads were mapped with Bowtie version 0.12.7 (Langmead et al., 2009) to the Ensembl (Hubbard et al., 2009) rat genome, release 61. Reads that did not map uniquely were discarded. The number of reads mapped to nuclear genes was determined with HTSeq (Anders et al., 2015) version 0.4.7p4, using the default "union" counting option. Differential expression between the adult and embryonic samples was detected using an exact test in the Bioconductor (Gentleman et al., 2004) edgeR package, version 2.4.0 (Robinson et al., 2010), with common dispersion used to estimate variance between samples. Genes considered significantly differentially expressed were those with a $p$-value of less than 0.05 after Benjamini-Hochberg false discovery rate correction. A combination of gene ontology annotation and manual curation was used to select genes encoding proteins that form part of a cell junction. Gene ontology descriptions for rat were downloaded from Biomart (Hubbard et al., 2009), and genes with "junction" mentioned in their gene ontology description were selected. Junction genes of interest were then extracted from this list. Similar searches were carried for other functional categories as described in the Results/Discussion below. For initial analysis genes with $>100$ sequence reads and age-related fold changes $(\mathrm{FC})>2.0\left(\log _{2} \mathrm{FC}>1.0\right)$ were collated and are summarized in Tables 1-4. For more specific analysis of some particular function categories a lower cut-off of 10 sequence reads was used. Complete sequencing data have been deposited in the Gene

\section{Table 3 | RNASeq expression of junction-associated genes in embryonic day 17 (E17) and adult ependymal cells.}

\begin{tabular}{|c|c|c|c|}
\hline \multicolumn{2}{|c|}{ (A) E17 expression } & \multicolumn{2}{|c|}{ (B) Adult expression } \\
\hline Gene ID & FPKM (AVE) & Gene ID & FPKM (AVE) \\
\hline Actb & 805.8 & Cldn11 & 304.7 \\
\hline Ctnnb1 & 114.6 & Actb & 115.8 \\
\hline Actr1a & 39.1 & Gja1 & 35.8 \\
\hline Cdh2 & 23.4 & Pkp4 & 31.2 \\
\hline Actr2 & 14.2 & Ctnnb1 & 29.0 \\
\hline Actl6b & 7.7 & Actr1a & 10.3 \\
\hline Ctnna2 & 4.6 & Actr1b & 6.8 \\
\hline Actr3 & 4.3 & Actr2 & 6.0 \\
\hline Pcdhgc3 & 3.8 & Gjc3 & 5.5 \\
\hline Ctnnd2 & 3.8 & Jam3 & 3.5 \\
\hline Actr10 & 2.7 & Ctnna1 & 2.6 \\
\hline Actr1b & 2.1 & Actn4 & 2.2 \\
\hline $\operatorname{Actg} 1$ & 2.0 & Ctnnd2 & 2.0 \\
\hline Ctnnbip1 & 1.6 & Cdh2 & 1.9 \\
\hline Ctnna 1 & 1.4 & Gjb1 & 1.9 \\
\hline Actn4 & 1.3 & Ctnna2 & 1.3 \\
\hline Ctnnb/1 & 1.3 & Actr10 & 1.2 \\
\hline Cdh13 & 1.2 & Actr3b & 1.1 \\
\hline Cldn5 & 1.0 & Pcdhgc3 & 1.1 \\
\hline Cdh11 & $\underline{0.8}$ & Actr3b & 1.1 \\
\hline Cdh4 & $\underline{0.7}$ & $\underline{A c t 16 b}$ & $\underline{0.9}$ \\
\hline Actr3b & $\underline{0.7}$ & $\underline{\text { Pcdh10 }}$ & $\underline{0.8}$ \\
\hline Pkp4 & $\underline{0.6}$ & $\underline{T j p 1}$ & $\underline{0.7}$ \\
\hline Pcdh10 & $\underline{0.5}$ & Cdh13 & $\underline{0.5}$ \\
\hline Gja1 & $\underline{0.3}$ & $\underline{J a m 2}$ & $\underline{0.4}$ \\
\hline Pcdh17 & $\underline{0.3}$ & $\underline{\text { Cldn5 }}$ & $\underline{0.3}$ \\
\hline Tip1 & $\underline{0.2}$ & Ctnnbip1 & $\underline{0.3}$ \\
\hline Jam2 & $\underline{0.2}$ & $\underline{C d h 11}$ & $\underline{0.2}$ \\
\hline Jam3 & $\underline{0.2}$ & Ctnnb/1 & $\underline{0.2}$ \\
\hline Cldn 11 & $\underline{0.0}$ & $\underline{P c d h 17}$ & $\underline{0.2}$ \\
\hline Gjb1 & $\underline{0.0}$ & $\underline{\operatorname{Actg} 1}$ & $\underline{0.2}$ \\
\hline Gjc3 & $\underline{0.0}$ & $\underline{C d h 4}$ & $\underline{0.0}$ \\
\hline
\end{tabular}

Data are average FPKM (fragments per kilobase of exon per million fragments mapped) from three independent pooled samples of ventricular zone from embryonic day 17 (E17) and adult rats. Genes with FPKM expression $<1.0$ were considered not expressed (underlined in Table). 
Table 4 | Gene expression of junction related genes in embryonic ventricular zone and adult ventricular ependyma.

\begin{tabular}{|c|c|c|}
\hline Gene ID & Fold change(E17/Adult) & Fold change(Adult/E17) \\
\hline \multicolumn{3}{|l|}{ ACTINS } \\
\hline Actb & 6.96 & 0.14 \\
\hline $\operatorname{Actg} 1$ & \multicolumn{2}{|c|}{ Present in embryo only } \\
\hline$A c t / 6 b$ & \multicolumn{2}{|c|}{ Present in embryo only } \\
\hline Actn4 & \multicolumn{2}{|c|}{ No difference between ages } \\
\hline Actr10 & 2.32 & 0.43 \\
\hline Actr1a & 3.79 & 0.26 \\
\hline Actr1b & 0.32 & 3.17 \\
\hline Actr2 & 2.36 & 0.42 \\
\hline Actr3 & 3.94 & 0.25 \\
\hline Actr3b & \multicolumn{2}{|c|}{ Present in adult only } \\
\hline \multicolumn{3}{|c|}{ CADHERINS } \\
\hline Cdh13 & \multicolumn{2}{|c|}{ Present in embryo only } \\
\hline Cdh2 & 12.53 & 0.08 \\
\hline \multicolumn{3}{|c|}{ CLAUDINS } \\
\hline Cldn11 & \multicolumn{2}{|c|}{ Present in adult only } \\
\hline Cldn5 & \multicolumn{2}{|c|}{ Present in embryo only } \\
\hline \multicolumn{3}{|c|}{ CATENINS } \\
\hline Ctnna1 & \multicolumn{2}{|c|}{ No difference between ages } \\
\hline Ctnna2 & 3.54 & 0.28 \\
\hline Ctnnb1 & 3.95 & 0.25 \\
\hline Ctnnbip1 & \multicolumn{2}{|c|}{ Present in embryo only } \\
\hline Ctnnb/1 & \multicolumn{2}{|c|}{ Present in embryo only } \\
\hline Ctnnd2 & \multicolumn{2}{|c|}{ No difference between ages } \\
\hline \multicolumn{3}{|c|}{ GAP JUNCTION PROTEINS } \\
\hline Gja1 & \multicolumn{2}{|c|}{ Present in adult only } \\
\hline Gjb1 & \multicolumn{2}{|c|}{ Present in adult only } \\
\hline Gjc3 & \multicolumn{2}{|c|}{ Present in adult only } \\
\hline \multicolumn{3}{|c|}{ JUNCTIONAL ADHESION MOLECULES } \\
\hline Jam2 & \multicolumn{2}{|c|}{ Present in adult only } \\
\hline Jam3 & \multicolumn{2}{|c|}{ Present in adult only } \\
\hline \multicolumn{3}{|c|}{ PROTOCADHERIN } \\
\hline Pcdhgc3 & 3.49 & 0.29 \\
\hline \multicolumn{3}{|c|}{ PLAKOPHILIN } \\
\hline Pkp4 & Present & ult only \\
\hline
\end{tabular}

Average fold change in FPKM (fragments per kilobase of exon per million frag ments mapped) from three independent pooled samples of ventricular zone from embryonic day 17 (E17) and ependyma from adult rats.

Expression Omnibus (http://www.ncbi.nlm.nih.gov/geo/) under accession code GSE44072.

\section{Statistical analysis}

Where applicable, data are expressed as mean \pm SEM, or as a single mean value (RNA sequencing data). Where no SEM is provided the data are either a single value or a mean of two.

\section{RESULTS}

This study aimed to characterize the permeability properties of the CSF-brain interface during mouse development and to correlate it with changes in the expression of identified junctional proteins and cellular distribution of their protein products.

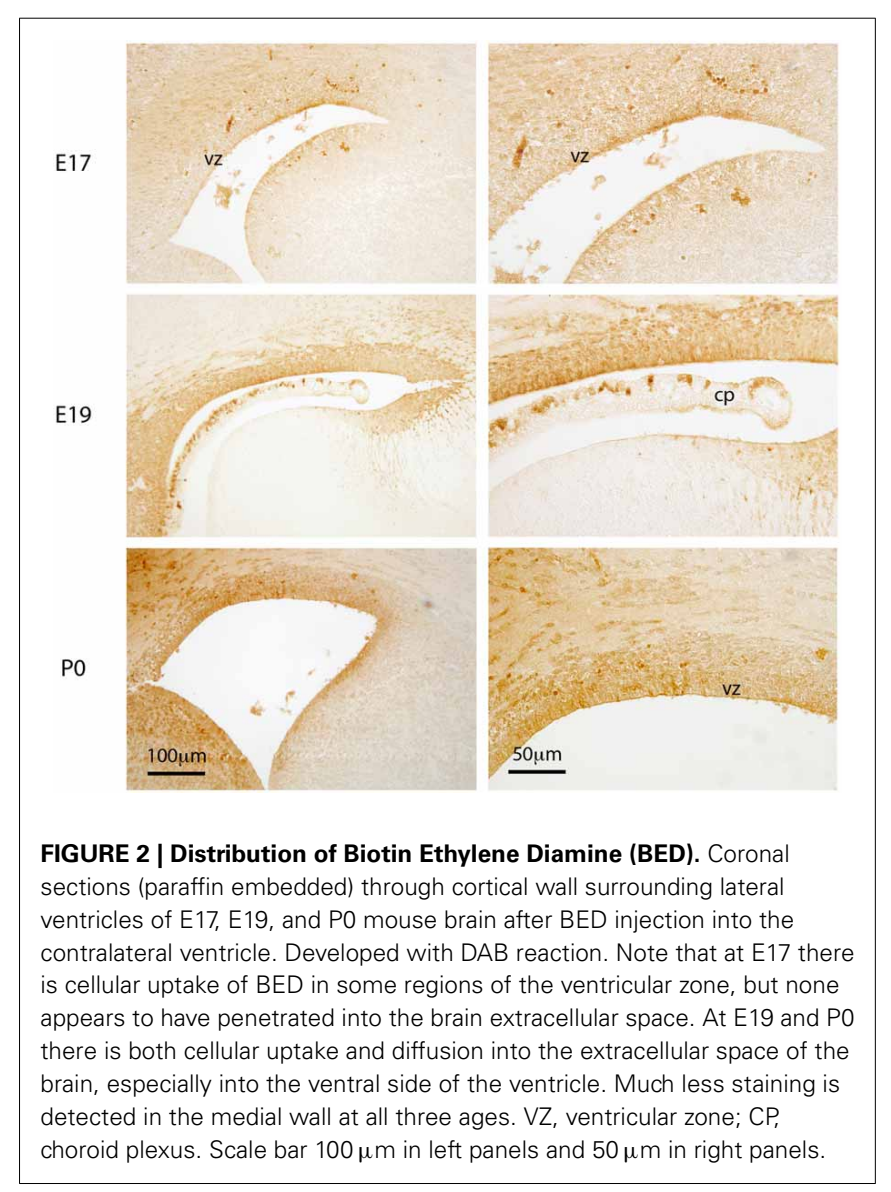

\section{PERMEABILITY PROPERTIES OF THE CSF-BRAIN BARRIER IN DEVELOPMENT}

The permeability characteristics of the CSF-brain barrier were investigated using three different sized biotinylated dextran amines conjugated with rhodamine: 3,10 , and $70 \mathrm{kDa}$. In addition a small marker of $268 \mathrm{Da}$ (BED) was used in fetal animals at E17, E19, and in P0. All animals, from E17 to adult, received an injection of $25 \mathrm{mg} \mathrm{ml}^{-1}$ of marker in sterile saline into one lateral ventricle as described in the Materials and Methods. Vibratome and paraffin-embedded microtome-cut sections from the ventricle contralateral to the injected side were analyzed. Results are illustrated in Figures 2, 3 and summarized in Figure 4.

\section{DA BIOTIN ETHYLENEDIAMINE (BED)}

Following injections into the lateral ventricle of E17 mice, BED was detected in many cells lining the ventricular system but not in at all aspects of the ventricular lining. BED reaction product was also faintly visible in the extracellular space of the ventricular zone between the cells (Figure 2). By E19 and P0, cellular uptake of BED was visible at all aspects of the ventricular lining and the extracellular staining in the brain parenchyma was clearly positive (illustrated in Figure 3). The results from fetal animals indicated that at these ages there is limited diffusion of molecules as small as 286 Da BED, between radial glial cells lining the CSF-brain interface. Uptake by individual ventricular cells was also present (Figure 2). 


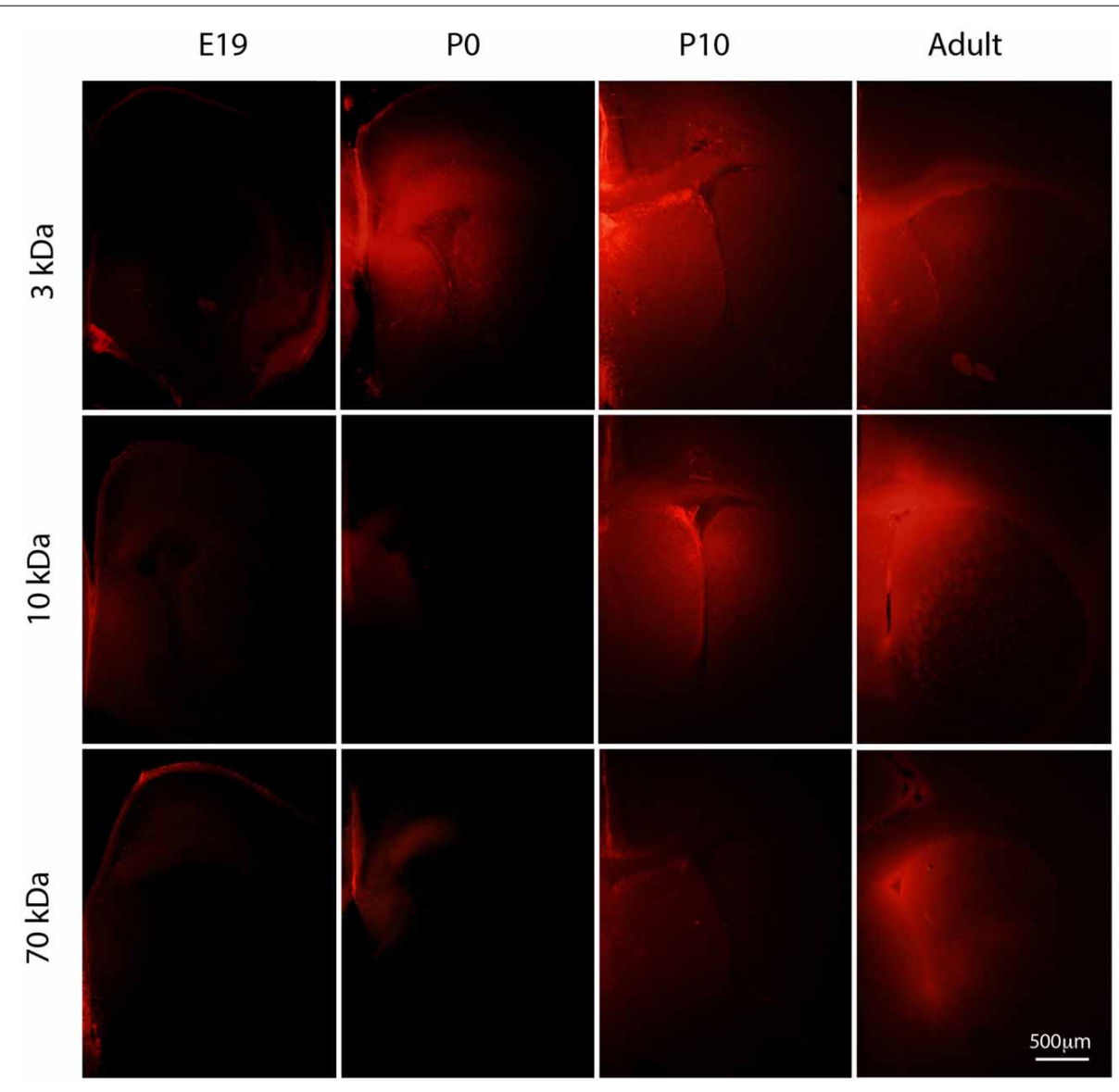

FIGURE 3 | Diffusion of different sized rhodamine labeled biotinylated dextrans (BDA) into postnatal mouse brain. Vibratome coronal sections ( $80 \mu \mathrm{m}$ thick) through cortex of mice injected with different sized BDAs $(3,10$, and $70 \mathrm{kDa}$ ). Markers were injected into the contralateral ventricle and left to diffuse for 2-3 min at E19 and P0, 5 min at P10 and $10 \mathrm{~min}$ in the adult. Note that none of the dextrans entered the brain at E19. In older brains there was a progressive increase in penetration depending on the size of the dextran. By adulthood all three dextrans were entering the brain to a similar extent. Apparent diffusion distances shown in Figure 4. Distribution of BED (286 Da) is illustrated in Figure 2 for comparison. E, embryonic day; P, postnatal day. Scale bar $500 \mu \mathrm{m}$.

\section{KDA RHODAMINE BDA (BDA3 KDA)}

Following injections of BDA3 $\mathrm{kDa}$ into fetal (E17 and E19) lateral ventricles BDA3 $\mathrm{kDa}$ was detected in some cells lining the ventricular zone (Figure 3) but was not detected in the extracellular space of the brain, indicating that at these ages the diffusion of molecules of at least $3 \mathrm{kDa}$ across the CSF-brain barrier was limited. It is possible that the specialized cellular uptake of small molecules may be the primary mechanism allowing their access to the early developing brain.

The earliest age at which diffuse labeling in the extracellular space of the brain was detected was in newborn pups (P0). At that age more $\mathrm{BDA} 3 \mathrm{kDa}$ appeared to diffuse into the medial aspect of the ventricle (as shown by a greater intensity of fluorescent marker, Figure 3) and indicates that the permeability of the inner CSF-brain barrier allows unhindered diffusion of a $3 \mathrm{kDa}$ molecule from about the time of birth in mice (Figures 3, 4).

\section{0 kDa RHODAMINE BDA (BDA10 kDa)}

Diffusion of BDA10 kDa into the brain around the whole ventricular zone interface was prevented in E17 and E19 mice, as no fluorescent labeling was visible at either age in the brain (see
Figure 3). At P0 results for $\mathrm{BDA} 10 \mathrm{kDa}$ were variable-a small amount of the marker (not measurable) was detected diffusing into the extracellular space of the ventricular zone in one pup from one litter, while in 2 other pups no diffusion was observed. This may indicate that at this stage of development there is a change in the permeability properties of the CSF-brain interface and a small difference (hours) in the age of the pups could explain the observed results. At older ages (P10 to adult) BDA10 kDa was found in brains from all animals including some intercellular presence in the ependymal layer. Thus, the permeability of the CSF-brain barrier changes between P0 and P10 to allow the diffusion of molecules up to $10 \mathrm{kDa}$ (Figures 3, 4).

\section{0 kDa RHODAMINE BDA (BDA70 kDa)}

The earliest age at which BDA70 kDa was observed to diffuse into the brain was in P10 animals (Figure 3); however, this was only detected in the medial and lateral aspects of the ventricle. At P20 results were variable. In one litter, where two pups received an injection of $70 \mathrm{kDa}$, diffusion into the ventricular zone was apparent in one brain but not in the other. In pups from a second litter $(n=2)$, diffusion was not observed in the brain of either 
A

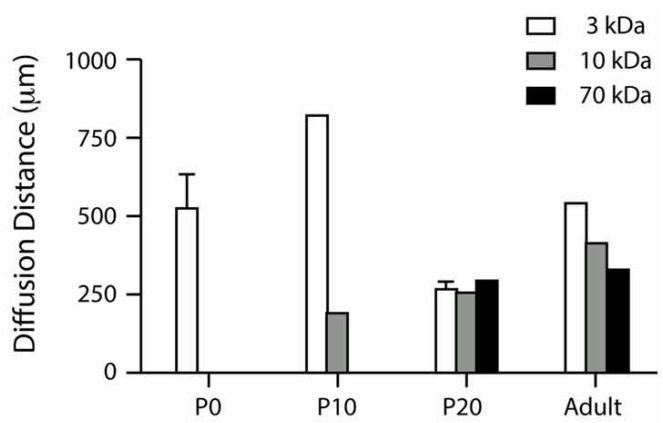

B

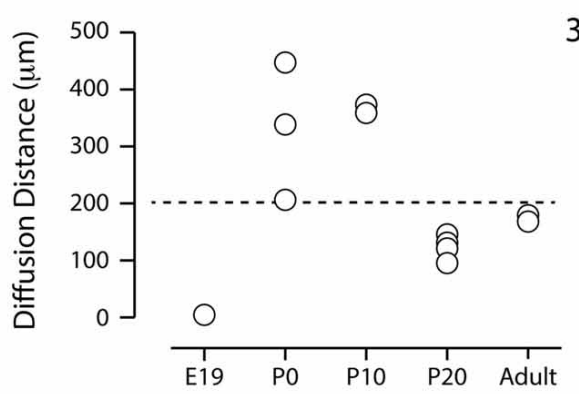

C

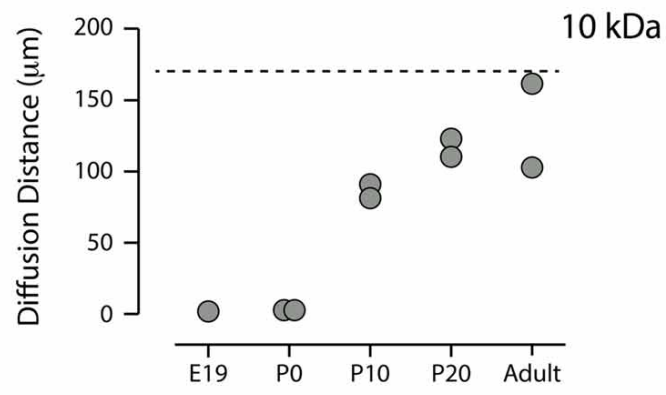

D

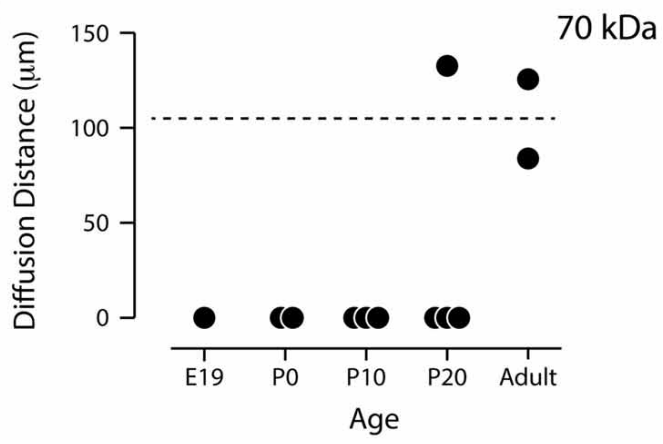

FIGURE 4 | (A) Apparent diffusion distance for three different sized biotinylated dextrans. The values were obtained by measuring the distance to which each marker penetrated into the dorso-lateral wall of the lateral ventricle after injections were made in the contralateral side. Mean \pm SEM (only if $n>2$ ), values from individual experiments are illustrated as circles in (B-D). P, postnatal day. Note that none of the dextrans penetrated at E19 (see B-D and Figure 3). (B,C) Standardized apparent diffusion distances compared to theoretical values. Apparent diffusion distances were standardized by estimating the apparent diffusion distance at 1 min after intraventricular injection of each dextran at each age using Fick's second law of diffusion as different sized dextrans were left to diffuse for different periods of time (see Materials and Methods). The broken lines represent the calculated theoretical diffusion distance at $1 \mathrm{~min}$. Each circle represents

(Continued)

\section{FIGURE 4 | Continued}

a value obtained from individual pups. (B) BDA3 $\mathrm{kDa}$ appeared to diffuse without restraint from P0. There was no diffusion at E17 or E19 (only E19 illustrated). (C) BDA10 kDa did not enter the ventricular zone at P0 and appeared to diffuse less than the theoretical distance at P10 and P20. (D) BDA70 kDa only penetrated the ependyma at P20 in one pup out or four and in the adult. E, embryonic; P, postnatal.

animal. In the adult brain $(n=2)$ diffusion of the probe was observed from the ventricle into the brain in both animals. These results indicate that after the age of weaning $(>$ P20) in the mouse there is no longer any size related diffusion restraint present at the CSF-brain interface for molecules up to $70 \mathrm{kDa}$ (Figures 3, 4).

\section{TOTAL PROTEIN CONCENTRATION IN CSF AND PLASMA OF MICE FROM E15 UNTIL ADULT}

In order to establish if there is a correlation between changes in the permeability properties of the inner CSF-brain barrier and protein concentration in the CSF during brain development, total protein concentration in plasma and CSF of mice from E17 to adult was measured using the Bradford method (Bradford, 1976 see Materials and Methods). Results are shown in Figure 5. For completeness values obtained from a previous study (Liddelow et al., 2012) from E15 mice are also included.

As can be seen from Figure 5A the concentration of total protein in the CSF was higher in younger than in adult animals. There was no statistically significant difference between late fetal (E17 and E19) and P0 protein concentrations in CSF with values ranging from $227 \pm 1.7 \mathrm{mg} 100 \mathrm{ml}^{-1}$ at E17 to $259 \pm$ $23.7 \mathrm{mg} 100 \mathrm{ml}^{-1}$ at P0. However, there was a statistically significant difference between protein concentrations in the CSF at P0

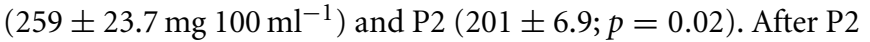
there was a decline until adult levels were reached $(26 \pm 1.5 \mathrm{mg}$ $100 \mathrm{ml}^{-1}$ ). In contrast, total protein concentration in plasma was lowest at E15 $\left(465 \pm 32.4 \mathrm{mg} 100 \mathrm{ml}^{-1}\right)$ and increased to $6188 \pm 51.5 \mathrm{mg} 100 \mathrm{ml}^{-1}$ in adult plasma (Figure 5B). When these results are compared with the results obtained from permeability studies, it appears that the CSF-brain interface becomes less size-restricted for molecular diffusion at the time when the CSF protein levels begin to decline (i.e., after P0).

\section{RNA SEOUENCING ANALYSIS OF EMBRYONIC VENTRICULAR ZONE AND ADULT EPENDYMA}

In order to understand the molecular structure of strap junctions present between adjacent neuroepithelial ventricular zone cells in mouse fetal brain we employed RNA Sequencing analysis of lateral ventricular zone tissue from E17/18 embryos and ependyma from adult mice. Only the dorsolateral part of the lateral ventricular wall was sampled (see Materials and Methods). In this paper we focus only on genes that are thought to play a role in intercellular junctional structures. However, a more comprehensive analysis of the full RNA-Seq dataset dealing with the general development of the ventricular zone will be published elsewhere.

Table 3 lists the junction-related genes that were identified in embryonic ventricular zone (A) and adult ependyma (B). The Table also lists numerous genes known to be associated with tight junctions that were below the detection threshold of the method. 
In the embryonic ventricular zone only 19 junction-related genes were present with FPKM (fragments per kilobase of exon per million fragments mapped) values above the cut off of 1.0. In the adult ependyma there were 23 detectable junction-related genes. In the embryonic ventricular zone 9 genes belonging to the actins were represented, 6 belonged to catenin gene family, 3 were cadherins and one was a claudin $(C l d n 5)$. No detectable levels of genes coding for gap junction proteins were observed. In the adult ependyma three genes for gap junction proteins were detected, eight genes were from the actin family, four were catenins and there was only one cadherin. Claudin-11 (Cldn11) was the gene expressed at the highest level in the adult ependyma (Table 3 ). Genes from this first pass analysis were subsequently grouped by their molecular categories and represented as differentially regulated between the two ages (i.e., the fold differences between E17 and adult). These are summarized in Table 4. In addition, genes that did not show age-related expression differences are also listed. Some genes were only present in the embryo in detectable copy numbers whereas others were present only in the adult. The largest single category was actin and actin-related genestwo (Actg1, Actl6b) were present only in the embryo; one (Actr3b)

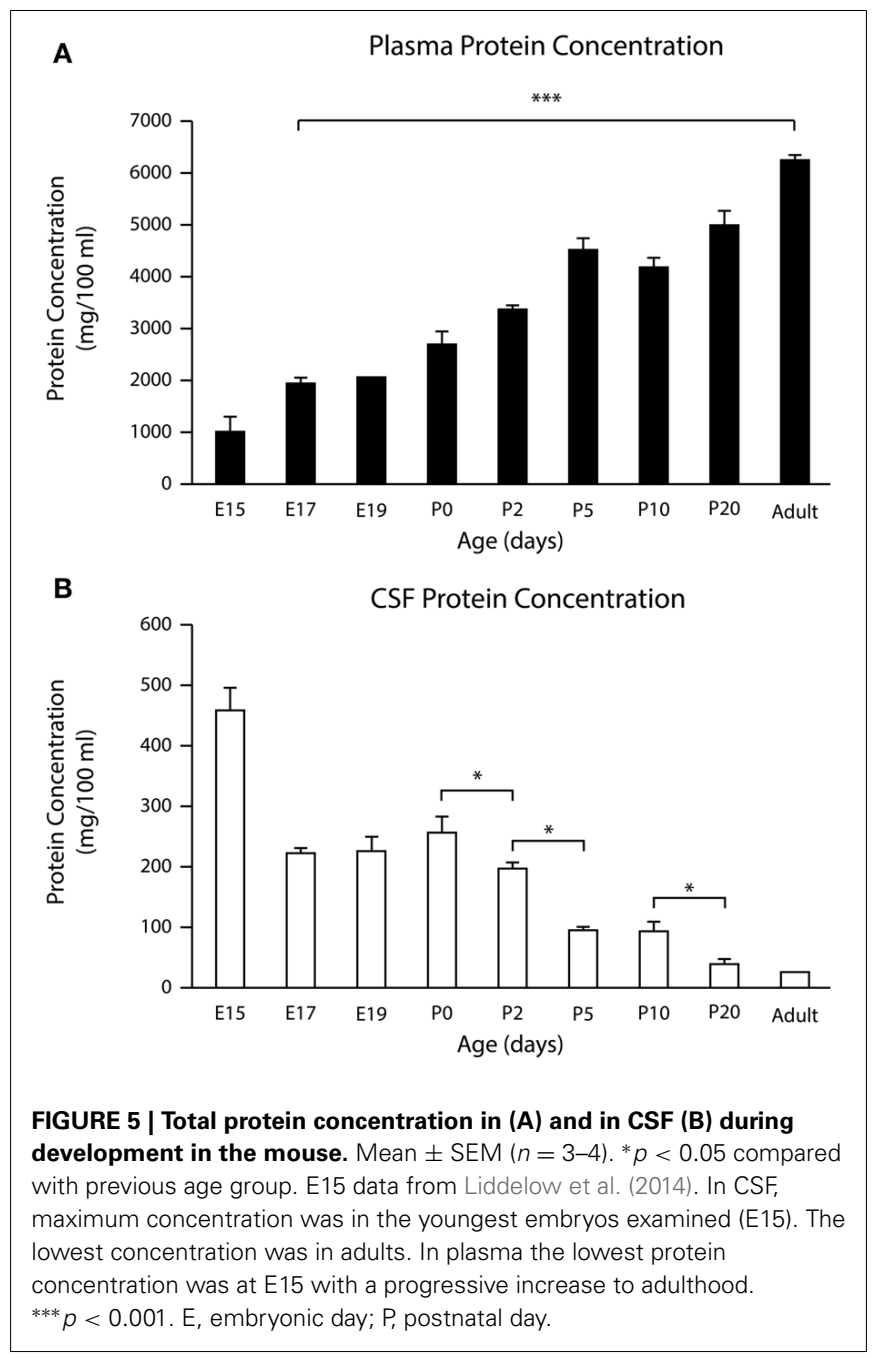

was present only in the adult. Another five were expressed at a higher level in the embryo than in the adult (Actb, Actr3, Actrla, Actr2, Actr10) in the range 2.3-7.0-fold enrichment. In adult ependyma only one actin-related gene $(A c t r 1 b)$ was expressed at a higher level than in the embryo. The cadherin, Cdh13 was only present in the embryo, while $C d h 2$ was expressed at both ages but 13-fold higher in embryos than in the adult. Two claudins were detected, Cldn 5 only in the embryo and Cldn11 only in the adult. Four catenins showed age-related differences in expression, all at a higher level in embryos. Two were present only in the embryo (Ctnnbip1, Ctnnbl1) and 2 were expressed at a higher level in the embryo (Ctnna2, 3.5-fold and Ctnnb1, 3.9-fold); the one protocadherin detected $(P c d h g c 3)$ was also expressed only in the embryo. The gap junction ( Gja1, Gjb1, Gjc3), junctional adhesion molecules (Jam3, Jam3) and one plakophilin (Pkp4) were all only expressed in the adult ependyma.

A summary of genes that were only present in embryonic ventricular zone or adult ependyma is listed in Table 5, together with genes that were expressed at similar levels at both ages.

\section{DISTRIBUTION OF JUNCTIONAL PROTEINS AT THE INNER CSF-BRAIN INTERFACE}

The overall distribution and cellular localization of the adherens junctional proteins $\mathrm{N}$-cadherin (product of gene $C d h 2$ ), $\alpha$ - and $\beta$-catenin (Ctnnal and Ctnnb1) and of the tight junctional proteins claudin-5 and $-11,(C l d n 5$ and $C l d n 11)$, were investigated

Table 5 | Junction-associated genes present only at E17 or adult or not different at these ages in mouse ventricular zone/ependyma.

\begin{tabular}{lcc}
\hline Gene ID & E17(AVE FPKM) & Adult(AVE FPKM) \\
\hline PRESENT AT E17 ONLY & & \\
Actl6b & 7.7 & 0.9 \\
Actg1 & 2.0 & 0.2 \\
Ctnnbip1 & 1.6 & 0.3 \\
Ctnnb/1 & 1.3 & 0.2 \\
Cdh13 & 1.2 & 0.5 \\
Cldn5 & 1.0 & 0.3 \\
PRESENT IN ADULT ONLY & & \\
Cldn11 & 0.0 & 304.7 \\
Gja1 & 0.3 & 35.9 \\
Pkp4 & 0.6 & 31.2 \\
Gjc3 & 0.0 & 5.5 \\
Jam3 & 0.2 & 3.5 \\
Gjb1 & 0.0 & 1.9 \\
Actr3b & 0.7 & 1.1 \\
NO CHANGE IN EXPRESSION & \\
Ctnnd & 3.8 & 2.0 \\
Actn4 & 1.3 & 2.2 \\
Ctnna1 & 1.4 & 2.6 \\
& &
\end{tabular}

Data are FPKM (fragments per kilobase of exon per million fragments mapped) from three independent pooled samples of ventricular zone/ependyma from embryonic day 17 (E17) and adult mice. Full gene lists were truncated to remove transcripts with FPKM $<1.00$. A fold change was considered significant if it was greater than 2.00 . 
using immunohistochemistry in paraffin embedded sections of mouse brains from E15/16 to adult. These protein candidates were chosen based on the transcriptomic analysis of the dataset as described above (see Tables 3-5). N-cadherin (Cdh2) and both catenin genes (Ctnnal and Ctnnb1) were expressed at levels that were higher in the fetal brains. Cldn 5 was only detected in the fetal ventricular zone while Cldn11 was only detected in the adult. Although transcriptomic analysis was performed on samples dissected out from only the lateral wall of the lateral ventricles and most of the permeability data were also calculated from this area, results from immunohistochemical staining are described for all areas lining the ventricles and are illustrated in Figures 6-8.

\section{Overall distribution}

Immunostaining at E15/16 for junctional proteins identified in the transciptomic analysis as those expressed differentially between fetal and adult ages showed a weak surface staining for claudin-5 (between the two arrows in Figure 6A) in contrast to a much stronger reactivity for $\alpha$-catenin, $\mathrm{N}$-cadherin and $\beta-$ catenin, which lined the entire lateral ventricular zone surface including the ganglionic eminence (Figures 6B,C). N-cadherin immunoreactivity was similar to that of $\alpha$-catenin and $\beta$-catenin although it appeared stronger and also clearly included the surface of the ganglionic eminence (Figure 6C). All three adherens junctional proteins showed increased staining of the borders of the ganglionic eminence, at the bottom of the septal fork of the lateral ventricle and the pallio-subpallial boundary (arrows in Figure 6C).

\section{Cellular distribution}

A comparison of immunostaining for junctional proteins of E15/16 and P20/adult lateral telencephalic wall at high magnification (Figure 7 from boxed areas in Figures 6A-D) showed a prominent claudin-5 reactivity in endothelial cells of the sinusoid blood vessels in the subventricular zone but also a distinct staining corresponding to the apical-most radial glial cell membranes (Figure 7A). In contrast the ventricular-facing surface of the ependymal cell lining in lateral ventricle of adult brain was devoid of claudin-5 reactivity (Figure 7A1), whereas the endothelial cells of fenestrated blood vessels of the choroid plexus were positive. Immunostaining for $\alpha$-catenin, $\mathrm{N}$-cadherin and $\beta$-catenin outlined the apical and apico-lateral most part of the ventricular zone cells in the lateral ventricular zone (Figures 7B-D). Ncadherin and $\beta$-catenin immunoreactivity included the cytoplasm of the radial glial cells (Figures 7C,D arrowheads). In the adult immunoreactivity for $\alpha$-catenin was not observed in telencephalic wall, ependymal cells nor in choroid plexus (Figure 7B1). The apical ependymal surface was strongly stained for $\mathrm{N}$-cadherin (Figure 7C1) but virtually no reactivity was found for $\beta$-catenin in the P20/adult forebrain in either the ependyma or in the subependymal zone (Figure 7D1).

Since Cldn11 was only detected in the adult mouse telencephalic wall we compared immunostaining for claudin-11/OSP of E16 and P15 mouse forebrain (Figures 8A,B). The early developing forebrain showed a lack of reactivity in the entire telencephalic wall. Thus, the apical surface of ventricular
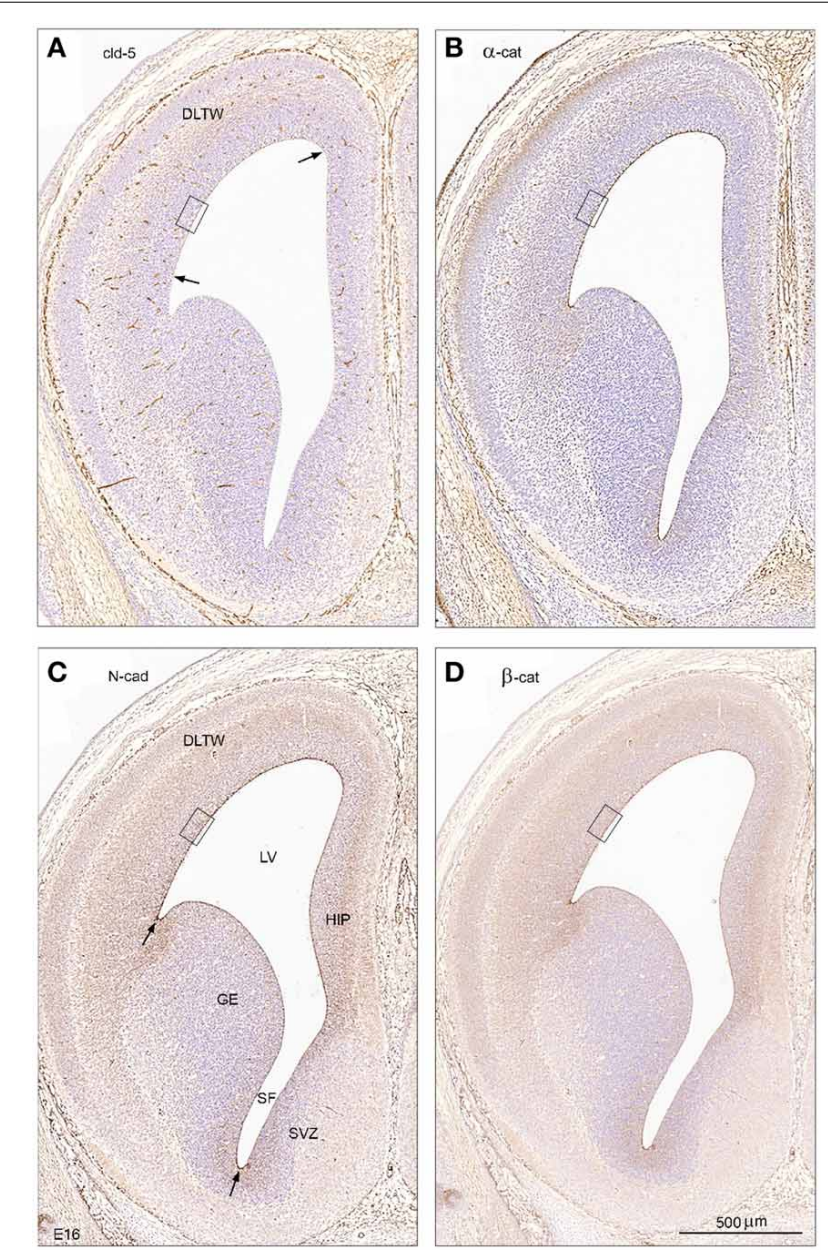

FIGURE 6 | Tight and adherens junctional proteins in embryonic mouse brain. An overall view of tight and adherens junctional proteins in the ventricular zone of early mouse CSF-brain interface at low magnification in coronal sections of E16 brain showing immunostaining for claudin-5 (A), $\alpha$-catenin (B), N-cadherin (C), and $\beta$-catenin (D). Note the differences in distribution of immunopositive staining between different regions of the surface of the ventricular zone with strongest staining of the dorsolateral telencephalic wall (DLTW) and a weak or lacking immunoreactivity of the ganglionic eminence (GE). Arrows in A point to the border of faint claudin-5 reactivity of the ventricular surface of the dorsolateral wall, and in (C) to the increased staining of the dorsolateral and ventral borders of the ganglionic eminence. The boxed areas in (A-D) are shown in higher magnification in Figure 7. HIP, hippocampus; LV, lateral ventricle; SF, septal fork of the lateral ventricle; SVZ, septal ventricular zone. (A-D) Same magnification, scale bar $500 \mu \mathrm{m}$

zone (VZ) facing the lateral ventricle (LV) was completely devoid of claudin-11 immunoreactivity. The developing arachnoid barrier layer (arrowheads) was clearly claudin11-positive. Leptomeningeal cells in the subarachnoidal space and on the outer surface of the telencephalic wall were not stained (Figure 8A). At P15 the ependymal and the subependymal zone of the lateral ventricle (LV) was unstained in marked contrast to the strongly stained myelinated early subependymal oligodendrocytes (Figure 8B, arrows). 


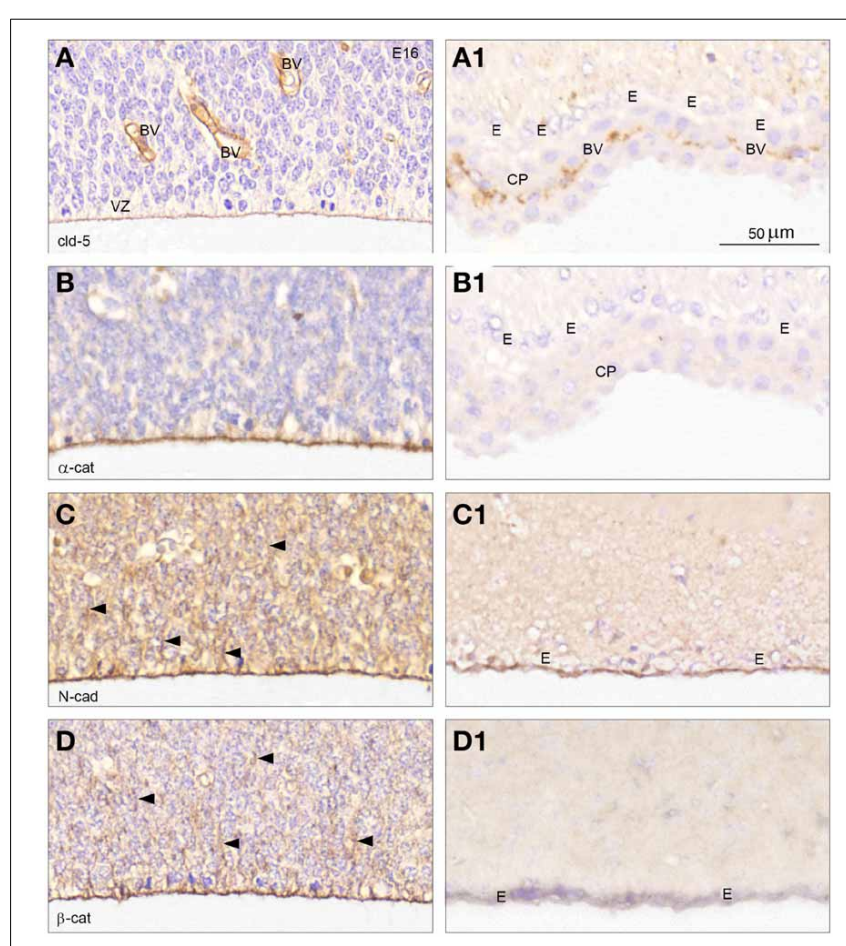

FIGURE 7 | Cellular distribution of adherens and tight junctional proteins at the lateral CSF-brain interface. High magnification coronal sections of E16 (A-D) from boxed areas in Figure $\mathbf{6}$ and of adult (A1-C1) and P20 (D1) forebrain immunostained for claudin-5 (A,A1), $\alpha$-catenin (B,B1), N-cadherin (C,C1), and for $\beta$-catenin (D,D1). (A) At E16 claudin-5 reactivity is prominent in endothelial cells of blood vessels (BV), but a distinct staining is also present corresponding to the apical-most part of the ventricular zone cells (VZ). (A1) In the adult forebrain ependymal cells (E) show no claudin- 5 immunoreactivity in marked contrast to the positively stained endothelial cells of fenestrated blood vessels of the choroid plexus (CP). (B-D) At E16 immunostaining for $\alpha$-catenin (B), N-cadherin (C) and $\beta$-catenin (D) outlines the apical and apico-lateral most part of the ventricular zone cells,-compare (A) (apical) and (D) (apical and apico-lateral staining). $\mathrm{N}$-cadherin and $\beta$-catenin immunostaining extends into the cytoplasm of the ventricular zone cells (arrowheads in C,D). (B1) Immunoreactivity for $\alpha$-catenin is not present in adult forebrain, neither in ependymal cells (E) nor in the choroid plexus (CHP). (C1,D1) The surface of the ependymal cells $(E)$ is strongly stained for $\mathrm{N}$-cadherin but only little reactivity is observed after staining for $\beta$-catenin in the adult forebrain and virtually no reactivity is observed in the subependymal zone. Same magnification in (A-D) and (A1-D1). Scale bar $50 \mu \mathrm{m}$.

\section{DISCUSSION}

The brain develops within a well-defined environment that is controlled by a set of mechanisms present at the blood-brain barriers, which determine the exchange of molecules between the brain and the periphery (Saunders et al., 2012). The molecular exchange between the brain interstitial fluid, peripheral circulation and the CSF occurs across the interfaces between the brain, the blood and the CSF (Saunders et al., 2012). Properties of two of these barriers, the blood-brain barrier proper (between the brain and the blood) and the blood-CSF barrier (between the blood and the CSF) have been extensively studied, including during development (for review see Saunders et al., 2012) but the inner CSF-brain barrier, the last frontier together with the outer

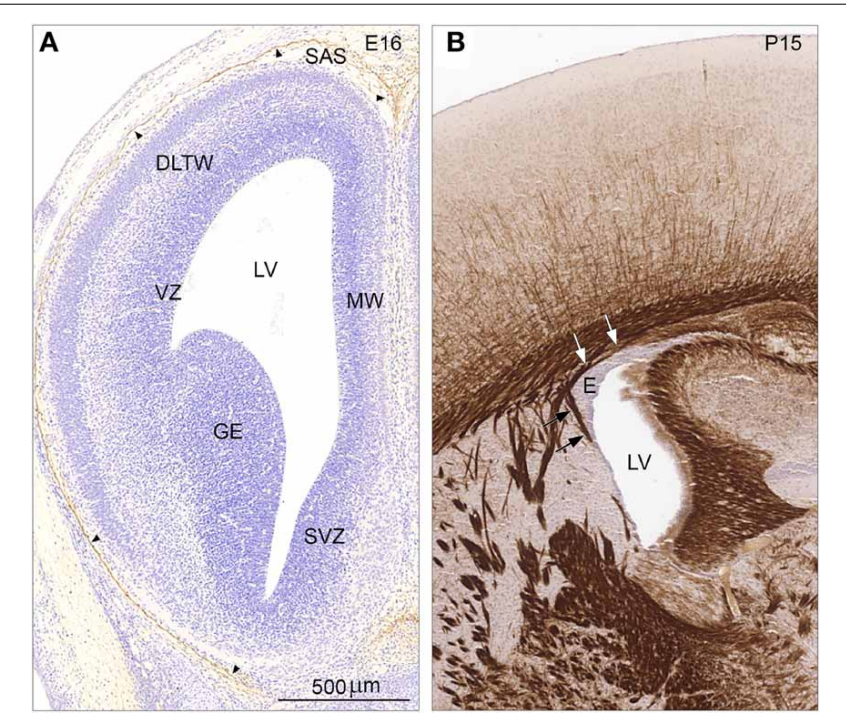

FIGURE 8 | Distribution of claudin-11/OSP immunoreactivity in coronal sections of E16 (A) and P15 (B) mouse forebrain. (A) Immunostaining for claudin-11/OSP of the early developing forebrain at E16 demonstrates a lack of reactivity in the entire telencephalic wall. Thus, the apical surface of ventricular zone (VZ) facing the lateral ventricle (LV) is devoid of immunostaining in all subregions- DLTW, dorsolateral telencephalic wall; MW, medial wall; SVZ, septal ventricular zone and GE, ganglionic eminence. The developing arachnoid barrier layer (arrowheads) is however, claudin-11/OSP positive. Leptomeningeal cells in the subarachnoidal space (SAS) and on the outer surface of the telencephalic wall are not stained. (B). At P15 the ependymal zone (E) of lateral ventricle (LV) is unstained in marked contrast to the strongly stained tight junctions of myelin sheaths in early subependymal oligodendrocytes (arrows). Same magnification in (A,B), scale bar $200 \mu \mathrm{m}$.

CSF-brain barrier as it were (see Brøchner et al., in preparation), is not well understood.

\section{PERMEABILITY OF THE INNER CSF-BRAIN BARRIER}

Here we have presented a study to investigate systematically the permeability properties of this inner barrier during development. Only one study has previously attempted to do so and also found that there was a size restriction at earlier ages compared to free diffusion at later stages of brain maturation (Fossan et al., 1985). However, in the study of Fossan et al. (1985) only one sized marker (HRP, $40 \mathrm{kDa}$ ) was used and only two developmental stages in fetal sheep were compared. The only other studies of ventricular zone in embryos appear to be those of Chang and Sive $(2012 a, b)$ in zebrafish. As in the present report, these authors used a series of different molecular sized dextrans $(10-400 \mathrm{kDa}$ FITC-conjugated) injected into the cerebral ventricles of zebrafish embryos as early as $22 \mathrm{~h}$ post fertilization. In $24 \mathrm{~h}$ post fertilization embryos the smallest $10 \mathrm{kDa}$ dextran "leaked almost immediately into the neuroepithelium, whereas the $70 \mathrm{kDa}$ dextrans moved only slowly into that tissue and $2000 \mathrm{kDa}$ not at all." This contrasts with our results in mouse embryos (Figures 2,3) in which even the smallest marker barely penetrated between the cells of the ventricular zone at E17-E19. This difference in permeability of the ventricular zone in the two studies could be due to the difference in species or to the stages of development examined. 
Results presented here demonstrate that at early stages of fetal mouse brain development the CSF-brain interchange of inert lipid insoluble molecules is restricted for ones as small as $286 \mathrm{Da}$ $\mathrm{BED}$ (Figure 2). The $3 \mathrm{kDa}$ probe was not detected in the brain extracellular spaces until P0 (Figure 3). The size restriction for free diffusion appeared to progressively relax and after P20, the CSF-brain barrier no longer hindered diffusion of protein-sized molecules such as BDA70 $\mathrm{kDa}$ (Figure 3). However, both BED and BDA $3 \mathrm{kDa}$ were detected intracellular in the brain ventricular zone in some cells lining the lateral ventricles (Figure 2). This indicates that the primary mechanism involved in the potential penetration of lipid insoluble molecules, including larger compounds such as proteins (Cavanagh and Warren, 1985), into the early developing brain is restricted to specific cellular uptake and does not occur by non-specific intercellular diffusion.

In the present study the distance to which each sized probe diffused at each stage of brain development was estimated. However, in such an approach, penetration of a probe in vivo does not occur in an entirely free, unrestricted diffusion pattern. Values calculated in the present study were mostly less than the calculated theoretical rate using Fick's second law (a mathematical representation of free diffusion) even after allowing longer times for larger molecules to diffuse into the brain. The difference is most likely to be due to (i) CSF flow, (ii) the ventricular surface barrier, (iii) the cellular architecture of the brain into which the probes diffuse, and (iv) bulk flow of the interstitial fluid of the brain. Nevertheless, the results obtained from such calculations indicated that by adulthood the passive diffusion of molecules as large as $70 \mathrm{kDa}$ was unrestricted while earlier in development the size restriction was progressively relaxed as the brain matured (Figure 4).

There is little information available about the rate of CSF flow in immature brains (Saunders, 1992) but the flow of brain interstitial fluid was studied previously by Cserr and her colleagues (e.g., Cserr et al., 1981; Szentistványi et al., 1984; Cserr, 1988). Their findings have been followed up recently using 2-photon microscopy (Iliff and Nedergaard, 2013; Thrane et al., 2013). The brain does not appear to possess a true lymphatic system like the rest of the body, although drainage from brain interstitial fluid into the cervical lymphatics has been demonstrated by studies over more than the past 100 years (see Abbott, 2004). Iliff and Nedergaard (2013) have coined the term, the glymphatic system to describe this drainage of the bulk flow of solutes. Fluid first flows via the para-arterial space-or Virchow-Robin space-and diffuses into the interstitial fluid. The interstitial fluid and solutes then diffuse into the brain parenchyma facilitated by astrocytes that possess aquaporin-4 (AQP-4) which assist in water transport. AQP-4 is expressed on the endfeet of astrocytes that line the para-arterial and para-venous spaces. Interstitial fluid and solutes are then drained via the para-venous spaces, which surround major drainage veins (Iliff and Nedergaard, 2013; Thrane et al., 2013; Xie et al., 2013).

In this study BDA probes of all three sizes were visible in the brain within many blood vessels but on closer inspection under the microscope it became apparent that these molecules were confined to the para-vascular (Virchow-Robin) spaces. This is illustrated in Figure 9 where an example of large vessels in the
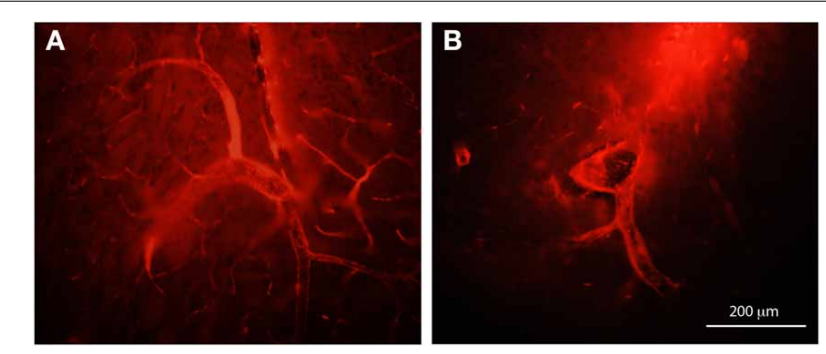

FIGURE 9 | Paravascular distribution of biotin dextrans, following intraventricular injection. Coronal sections of adult brains following intraventricular injection of BDA70K (A) or BDA10K (B). Note that most of the tracer is present in the paravascular or Virchow-Robin space and not in the lumen of blood vessels. Scale bar $200 \mu \mathrm{m}$.

adult brain after an intraventricular injection of BDA70 kDa or BDA $10 \mathrm{kDa}$ is shown.

The importance of our findings lies primarily in the observation of the impermeability of the CSF-brain interface early in development and its gradual opening up to progressively larger molecules in the postnatal period in the mouse. These permeability changes and those describer earlier in fetal sheep (Fossan et al., 1985) correlate with the presence of strap junctions in the ventricular zone of the developing brain and their subsequent disappearance at later stages of development (Møllgård et al., 1987).

\section{CSF AND PLASMA PROTEIN CONCENTRATION}

Part of the hypothesis of this study was that changes in the permeability of the CSF-brain barrier could be related to changes in protein concentration in the CSF (see Introduction). Results showed that the CSF protein concentration in the mouse embryo was highest at E15 followed by E17 and E19. There was a decline in protein concentration from P0 to P20. This drop in CSF protein concentration between $\mathrm{P} 0$ and P10 seems to coincide with the appearance of unhindered diffusion of BDA3 kDa and BDA $10 \mathrm{kDa}$ into the brain. The unrestricted diffusion of BDA70 kDa coincides with the drop in protein concentration between P10 and P20. By adulthood all three probes were visible in the brain extracellular space lining the ventricular system and the CSF protein concentration was at a minimum (Figure 5B).

Previous studies have shown that the premature infiltration of protein into the brain during development is closely linked with altered neurogenesis (Stolp et al., 2011b). Neurogenesis begins in the mouse ventricular zone at approximately E13.5 and astrogliogenesis begins to occur in the brain between the approximate ages of E16 and E18 (Götz and Huttner, 2005; Stolp et al., 2011a,b). This is around the age when the CSF protein concentration began to drop, the permeability properties of the barrier were transforming, and when progressive remodeling of the barrier with respect to junctional proteins was beginning to occur (see below).

\section{MOLECULAR MAKE-UP OF STRAP JUNCTIONS}

Strap junctions between cells lining the ventricular zone have only been described in embryonic brains (Møllgård et al., 1987). Their molecular structure is not known but freeze fracture images show 
cell-cell junctional interactions that are thought to exclude intercellular passage of molecules between CSF and the brain (Fossan et al., 1985; Møllgård et al., 1987). Permeability studies described in the present study identified changes in the size of molecules able to diffuse across this interface. This is the first study in which an attempt was made to describe possible molecular make up of strap junctional complexes.

Because the molecular structure of strap junctions is not known it seems reasonable to suggest that genes that are expressed exclusively in the embryonic ventricular zone, or at least at a higher level than in the adult, will be involved in their formation. By the same token it would seem unlikely that genes expressed only or at a higher level in the adult would be involved in strap junction structure. However, the genes that are expressed at both ages, but not differentially so, could not be ruled out as contributing to strap junction structure. In fact only three such genes were identified (Actn4, Ctnna1, and Ctnnd2, Table 5).

A striking finding was that very few of the genes known to be involved in intercellular tight junction formation (see Bauer et al., 2011; Van Itallie and Anderson, 2014) appear to be expressed in the ventricular zone of the lateral wall of the lateral ventricle in mouse embryos. Claudin- 5 has been associated with cerebral endothelial tight junctions (Nitta et al., 2003). In this study its gene was only detected in the embryo, albeit at a rather low level (FPKM-1.00, the lowest level of detection using this method). Our immunohistochemical results showed prominent staining for claudin-5 in endothelial cells of blood vessels in the subventricular zone at this age; but there was also some distinctive immunostaining of the apical-most part of the ventricular zone cells (Figures 6, 7). Claudin-5 has also been demonstrated in the ventricular zone of zebrafish embryos (Zhang et al., 2010). These authors and others (e.g., Chang and Sive, 2012a) have interpreted this as indicating that the ventricular zone/neuroepithelial cells are joined by tight junctions. The transmission electron micrographs in Zhang et al. (2010) are too low resolution for identification of the nature of the intercellular junctions, but loss of claudin-5 resulted in disruption of these intercellular junctions and allowed passage of lanthanum. As shown by Møllgård et al. (1987) it requires freeze fracture to identify these junctions as strap junctions. Otherwise in our data set transcripts for traditionally important tight junction protein genes such as occludin, MAGUK-like proteins, including ZOs, tricellulin (MarvelD2), JAMs, ESAM, were not detectable. This is in spite of their presence, as detected by immunohistochemistry, as early as E12 mouse (Møllgård et al., unpublished observations) and immunostaining for ZO-1 in zebrafish embryonic neuroepithelium (Zhang et al., 2010). The expression of Cldn5 (Table 4) and its presence demonstrated by immunohistochemistry (see Figure 7A) together with lack of expression of many other tight junction genes suggests strongly that claudin- 5 may be a component of strap junctions in the embryonic ventricular zone. In addition, representatives of all three of the key gene families involved in cell adhesion in epithelia (Adams et al., 1996) were present and expressed either exclusively or at a higher level in the embryo (Table 4). These were seven actin or actin-related genes, two cadherins, one protocadherin and four catenins. It has been thought for a long time (Adams et al., 1996) that the actin cytoskeleton stabilizes epithelial intercellular junctions by attachment to cadherins (Hirano et al., 1987; Matsuzaki et al., 1990) via cytoplasmic interactions with catenins (Nagafuchi and Takeichi, 1988; Ozawa and Kemler, 1992). However, more recently this concept has been challenged and it appears that the linkage between the cadherin-catenin complex and actin filaments may be more dynamic than previously thought (Drees et al., 2005; Yamada et al., 2005; Weis and Nelson, 2006). Zhang et al. (2010) illustrate quite widespread immunostaining for actin in $30 \mathrm{~h}$ post fertilization zebrafish embryos but do not mention which antibodies were used.

In epithelia of metazoans specialized cell-cell adhesion proteins comprise the classical cadherin $/ \beta$-catenin $/ \alpha$-catenin complex and it is the cadherins that provide the link between adjacent cells and catenins that link the cadherins to the underlying actin cytoskeleton (Miller et al., 2013). Our current RNA-Seq data shows that all of the key components of intercellular adhesion junctions are present in the ventricular zone of mouse embryo lateral ventricles although some of the specific genes are different from those usually identified in adherens junctions of mammalian epithelia. The expression of these adherens junctional genes in the ventricular zone of the embryonic mouse brain is consistent with previous ultrastructural studies of the ventricular zone in the developing sheep and human brain showing the presence of well-formed adherens junctions between the cells in this region (Saunders and Møllgård, 1984; Møllgård and Saunders, 1986).

The ultrastructural information available shows that the ventricular zone junctions have a particular configuration of a single strand in freeze fracture, which has an orientation perpendicular to the CSF surface of the cells rather than a circumferential "belt-like" configuration typical of tight and adherens junctions; although in thin-section electron microscopy these junctions have been described as having tight junction and adhesion-like elements (Møllgård et al., 1987). The typical intercellular junctions in the adult ependyma are prominent gap junctions, whereas only few and small gap junctions associated with the strap junctional elements are present in the embryonic ventricular zone (see Figure 9A in Møllgård et al., 1987). In marked contrast to this, strap junctions are only present in the ventricular zone of the early developing brain (Møllgård et al., 1987). It is noteworthy that in the current study it was only in the adult that gap junction genes were identified (Gja1, Gjb1, Gjc3, see Table 4). Also in adult ependyma only one actin-related gene was expressed at a higher level (Actr1b); in addition, one claudin (Cldn11), two Jams (Jam2 and Jam3) and one plakophilin (Pkp4) were expressed at a higher level. Two catenin genes were present in adult ependyma but expressed at a much lower level than in the embryo, whereas another was expressed at the same level at the two ages. Thus, it is quite clear that the gene profile of junction-related genes is quite different in embryonic ventricular zone and in adult ependyma.

Actins, particularly $\beta$-actin are also important for a variety of other cellular functions some of which may be related to their presence in the ventricular zone of embryonic brain. $\beta$ actin has been suggested to comprise between a half to two thirds of mammalian brain actin (Choo and Bray, 1978; Otey et al., 1987) although in a $\beta$-actin knock out actin levels were 
maintained by upregulation of $\gamma$ - and $\alpha$-smooth muscle actin; however there was about $70 \%$ perinatal lethality (Cheever et al., 2012). $\beta$-actin is involved in a range of cellular functions during development including migration (Ayala et al., 2007) and growth cone guidance (Dent and Gertler, 2003). These functions may be important for the differentiation and migration of ventricular zone cells, which contribute to the formation of the layered neocortex (Molnár and Clowry, 2012). It does seem therefore that the main families of proteins involved in strap junction formation are likely to be actins, cadherins, catenins and claudin-5. Some of these proteins were also investigated by immunohistochemistry to establish their cellular distribution (see below).

\section{LIMITATIONS OF THE STUDY Permeability}

A number of factors may have affected the outcomes of the permeability experiments. During development the volume of the lateral ventricles changes and the distance between the ventricles and from the cerebral surface to the interior of the ventricles increases (Liddelow et al., 2011) as does the flow of CSF (Saunders, 1992). Thus, the likelihood of a successfully injected probe reaching the contralateral ventricle can be variable. In order to overcome these limitations, the volume of dextran that was injected was increased with age and the time allowed for the dextran to diffuse into the contralateral ventricle was also increased. These limitations appear to be more significant in the older ages and this was reflected in the reduced number of successful injections in P20 and adult animals.

Great care was taken to inject the same volume into each ventricle at each age, but it was difficult to determine how much of this diffused into the contralateral ventricle. This limited the ability to properly measure the diffusion distances and then accurately compare them between animals. Intraperitoneal injections of these probes would at first appear to be the solution to this problem; however, with age, the amount of probe that needed to be introduced would have to be increased dramatically to offset the loss through renal excretion. Obstruction of the renal system has been used to restrict such losses thus retaining higher concentrations of marker in blood, but may represent an unphysiological state and was deemed unnecessarily stressful for the animals (Habgood et al., 1993). The most consistent approach to such experiments is an in vivo ventricular perfusion (as was done in fetal sheep, Fossan et al., 1985). This is impracticable in mice due to their very small size, especially at fetal stages.

\section{Animal ages, numbers and injection route}

The small size and vulnerability of exposed fetuses meant that numbers of fetal animals with successful injections ranged between one and three experimental animals per age, mostly two separate litters. For each probe all postnatal experiments were conducted on pups from a minimum of two different litters.

\section{Morphology}

For the permeability studies brains were embedded in agar and cut into $100 \mu \mathrm{m}$ sections using the vibratome (Leica). This method allowed for clearer observation of the diffusion of molecules into the brain. Some brains were embedded in paraffin and cut into $5 \mu \mathrm{m}$ thick coronal sections. This method better indicated the cellular uptake of the probes.

\section{Cellular distribution of junctional proteins}

There is no information available about the molecular composition of strap junctions limiting the ability to use immunohistochemistry to detect cellular changes at this interface. Immunohistochemistry of candidate proteins was based on the RNA-Seq results. It is possible that some other junctional proteins, that were either below the detection levels or were simply not identified in the transcriptomic analysis, could also be involved. We are preparing a more comprehensive immunohistochemical study of strap junctions using all available antibodies to junctional proteins (Møllgård et al., unpublished observations).

\section{Sampling of the lateral wall of the lateral ventricles and RNA-Seq analysis}

The lateral wall of both lateral ventricles was carefully dissected out under a dissecting microscope. However, it is inevitable that some tissue from the sub-ventricular layer was also obtained. This probably explains the finding of a high level of cldn11 in adult mice. Tight junctions of myelin sheaths in developing subependymal oligodendrocytes were strongly positive for claudin-11/OSP (oligodendrocyte-specific protein, Figure 8B) after P10 and the developing claudin-11 positive arachnoid barrier layer present in E16 (see, Figure 8A) was not within the dissected sample. The dissected samples from E17/18 lateral telencephalic wall would have included claudin-5 positive blood vessels in the subventricular zone (Figure 7A), but also in the adult subependymal layer. Claudin-5, occludin and ZO-1 are known to be present in the blood vessel tight junctions from their earliest development. In this study $\mathrm{Occl}$ and Tjp1 (ZO-1) expression were not detected presumably because they were below the detection level. Small gap junctions also connect adjacent neuroepithelial cells in the ventricular zone but no gap junctional proteins were detected, i.e., were also below the detection level.

We avoided contamination by the choroid plexus and checked RNA-Seq datasets for choroid plexus specific genes such as transthyretin (Dickson et al., 1985; Thomas et al., 1988). Samples used in this study were transthyretin negative. Because of the unknown degree of possible contamination of the sample by underlying sub-ventricular zone cells, the datasets were only mined for known junctional proteins. This, in principle, limited our analysis to intercellular complexes mostly occurring in the ventricular zone/ependymal layer. It is possible that there are as yet unrecognized junctional genes in our data set that contribute to the structure of strap junctions.

\section{CONCLUSION}

We have studied the permeability properties and junctionalprotein characteristics of the inner CSF-brain interface during mouse brain development. Permeability of this barrier gradually changed from highly restrictive (to molecules even as small as $286 \mathrm{Da}$ size) in fetal ages to unrestricted diffusion (to molecules at least as large as $70 \mathrm{KDa}$ ) in the adult. Molecular characterization, using RNA Sequencing of the ventricular zone (fetus) and ependyma (adult), identified several genes of known junctional 
proteins that are expressed at this inner CSF-brain barrier in a developmentally regulated manner. It was also found that the agedependent changes in permeability characteristics correlate with changes in the cellular distribution of junctional proteins: cadherins, catenins and claudin-5. We suggest that these junctional proteins are involved in the formation of strap junctions and in regulation of the changing diffusional properties of this barrier. Further work will be required to establish the scope of the control mechanisms in the CSF-brain interface during development and their significance for normal brain development.

\section{REFERENCES}

Abbott, N. J. (2004). Evidence for bulk flow of brain interstitial fluid: significance for physiology and pathology. Neurochem. Int. 45, 545-552. doi: 10.1016/j.neuint.2003.11.006

Adams, C. L., Nelson, W. J., and Smith, S. J. (1996). Quantitative analysis of cadherin-catenin-actin reorganization during development of cell-cell adhesion. J. Cell Biol. 135, 1899-1911.

Adinolfi, M. (1985). The development of the human blood-CSF-brain barrier. Dev. Med. Child Neurol. 27, 532-537.

Anders, S., Pyl, P. T., and Huber, W. (2015). HTSeq-a Python framework to work with high-throughput sequencing data. Bioinformatics 31, 166-169. doi: 10.1093/bioinformatics/btu638

Ayala, R., Shu, T., and Tsai, L.-H. (2007). Trekking across the brain: the journey of neuronal migration. Cell 128, 29-43. doi: 10.1016/j.cell.2006.12.021

Bauer, H. C., Traweger, A., Zweimueller-Mayer, J., Lehner, C., Tempfer, H., Krizbai, I., et al. (2011). New aspects of the molecular constituents of tissue barriers. J. Neural. Transm. 118, 7-21. doi: 10.1007/s00702-010-0484-6

Bradford, M. M. (1976). A rapid and sensitive method for the quantitation of microgram quantities of protein utilizing the principle of protein-dye binding. Anal. Biochem. 72, 248-254.

Brightman, M. W., and Reese, T. S. (1969). Junctions between intimately apposed cell membranes in the vertebrate brain. J. Cell Biol. 40, 648-677.

Cavanagh, M. E., and Warren, A. (1985). The distribution of native albumin and foreign albumin injected into lateral ventricles of prenatal and neonatal rat forebrains. Anat. Embryol. 172, 345-351.

Chang, J. T., and Sive, H. (2012a). An assay for permeability of the zebrafish embryonic neuroepithelium. J. Vis. Exp. e4242. doi: 10.3791/4242

Chang, J. T., and Sive, H. (2012b). Manual drainage of the zebrafish embryonic brain ventricles. J. Vis. Exp. e4243. doi: 10.3791/4243

Cheever, T. R., Li, B., and Ervasti, J. M. (2012). Restricted morphological and behavioral abnormalities following ablation of $\beta$-actin in the brain. PLoS ONE 7:e32970. doi: 10.1371/journal.pone.0032970

Choo, Q. L., and Bray, D. (1978). Two forms of neuronal actin. J. Neurochem. 31, 217-224.

Cserr, H. F. (1988). Role of secretion and bulk flow of brain interstitial fluid in brain volume regulation. Ann. N.Y. Acad. Sci. 529, 9-20.

Cserr, H. F., Cooper, D. N., Suri, P. K., and Patlak, C. S. (1981). Efflux of radiolabeled polyethylene glycols and albumin from rat brain. Am. J. Physiol. 240, F319-F328.

Dent, E. W., and Gertler, F. B. (2003). Cytoskeletal dynamics and transport in growth cone motility and axon guidance. Neuron 40, 209-227. doi: 10.1016/S0896-6273(03)00633-0

Desmond, M. E., and Jacobson, A. G. (1977). Embryonic brain enlargement requires cerebrospinal fluid pressure. Dev. Biol. 57, 188-198.

Dickson, P. W., Aldred, A. R., Marley, P. D., Tu, G. F., Howlett, G. J., and Schreiber, G. (1985). High prealbumin and transferrin mRNA levels in the choroid plexus of rat brain. Biochem. Biophys. Res. Commun. 127, 890-895.

Drees, F., Pokutta, S., Yamada, S., Nelson, W. J., and Weis, W. I. (2005). Alphacatenin is a molecular switch that binds E-cadherin-beta-catenin and regulates actin-filament assembly. Cell 123, 903-915. doi: 10.1016/j.cell.2005.09.021

Duckett, S. (1968). The germinal layer of the growing human brain during early fetal life. Anat. Rec. 161, 231-245. doi: 10.1002/ar.1091610208

Dziegielewska, K. M., Evans, C. A., Lai, P. C., Lorscheider, F. L., Malinowska, D. H., Møllgård, K., et al. (1981). Proteins in cerebrospinal fluid and plasma of fetal rats during development. Dev. Biol. 83, 193-200.
Dziegielewska, K. M., Evans, C. A., Malinowska, D. H., Møllgård, K., Reynolds, J. M., Reynolds, M. L., et al. (1979). Studies of the development of brain barrier systems to lipid insoluble molecules in fetal sheep. J. Physiol. (Lond). 292, 207-231.

Dziegielewska, K. M., Evans, C. A., Malinowska, D. H., Møllgård, K., Reynolds, M. L., and Saunders, N. R. (1980). Blood-cerebrospinal fluid transfer of plasma proteins during fetal development in the sheep. J. Physiol. (Lond). 300, 457-465.

Dziegielewska, K. M., Habgood, M., Jones, S. E., Reader, M., and Saunders, N. R. (1989). Proteins in cerebrospinal fluid and plasma of postnatal Monodelphis domestica (grey short-tailed opossum). Comp. Biochem. Physiol. B 92, 569-576.

Dziegielewska, K. M., Hinds, L. A., Sarantis, M. E., Saunders, N. R., and TyndaleBiscoe, C. H. (1986). Proteins in cerebrospinal fluid and plasma of the pouch young tammar wallaby (Macropus eugenii) during development. Comp. Biochem. Physiol. B 83, 561-567.

Dziegielewska, K. M., and Saunders, N. R. (1988). "The development of the bloodbrain barrier: proteins in fetal and neonatal CSF, their nature and origins," in Handbook of Human Growth and Developmental Biology, Vol 1. Neural, Sensory, Motor and Integrative Development, eds E. Meisami and P. S. Timiras (Boca Raton: CRC Press), 169-191.

Dziegielewska, K. M., and Saunders, N. R. (2002). The ins and outs of brain-barrier mechanisms. Trends Neurosci. 25, 69-71. doi: 10.1016/S0166-2236(02)02068-4

Ek, C. J., Dziegielewska, K. M., Habgood, M. D., and Saunders, N. R. (2012). Barriers in the developing brain and neurotoxicology. Neurotoxicology 33, 586-604. doi: 10.1016/j.neuro.2011.12.009

Engelhardt, B., and Liebner, S. (2014). Novel insights into the development and maintenance of the blood-brain barrier. Cell Tissue Res. 355, 687-699. doi: 10.1007/s00441-014-1811-2

Fossan, G., Cavanagh, M. E., Evans, C. A., Malinowska, D. H., Møllgård, K., Reynolds, M. L., et al. (1985). CSF-brain permeability in the immature sheep fetus: a CSF-brain barrier. Brain Res. 350, 113-124.

Gentleman, R. C., Carey, V. J., Bates, D. M., Bolstad, B., Dettling, M., Dudoit, S., et al. (2004). Bioconductor: open software development for computational biology and bioinformatics. Genome Biol. 5:R80. doi: 10.1186/gb-2004-510-r80

Götz, M., and Huttner, W. B. (2005). The cell biology of neurogenesis. Nat. Rev. Mol. Cell Biol. 6, 777-788. doi: 10.1038/nrm1739

Habgood, M. D., Knott, G. W., Dziegielewska, K. M., and Saunders, N. R. (1993). The nature of the decrease in blood-cerebrospinal fluid barrier exchange during postnatal brain development in the rat. J. Physiol. (Lond). 468, 73-83.

Hirano, S., Nose, A., Hatta, K., Kawakami, A., and Takeichi, M. (1987). Calciumdependent cell-cell adhesion molecules (cadherins): subclass specificities and possible involvement of actin bundles. J. Cell Biol. 105, 2501-2510.

Hubbard, T. J. P., Aken, B. L., Ayling, S., Ballester, B., Beal, K., Bragin, E., et al. (2009). Ensembl 2009. Nucleic Acids Res. 37, D690-D697. doi: $10.1093 / \mathrm{nar} / \mathrm{gkn} 828$

Iliff, J. J., and Nedergaard, M. (2013). Is there a cerebral lymphatic system? Stroke 44, S93-S95. doi: 10.1161/STROKEAHA.112.678698

Iliff, J. J., Wang, M., Liao, Y., Plogg, B. A., Peng, W., Gundersen, G. A., et al. (2012). A paravascular pathway facilitates CSF flow through the brain parenchyma and the clearance of interstitial solutes, including amyloid $\beta$. Sci. Transl. Med. 4, 147ral11. doi: 10.1126/scitranslmed.3003748

Johansson, P. A., Dziegielewska, K. M., Liddelow, S. A., and Saunders, N. R. (2008). The blood-CSF barrier explained: when development is not immaturity. Bioessays 30, 237-248. doi: 10.1002/bies.20718

Langmead, B., Trapnell, C., Pop, M., and Salzberg, S. L. (2009). Ultrafast and memory-efficient alignment of short DNA sequences to the human genome. Genome Biol. 10:R25. doi: 10.1186/gb-2009-10-3-r25

Levitt, P., Cooper, M. L., and Rakic, P. (1981). Coexistence of neuronal and glial precursor cells in the cerebral ventricular zone of the fetal monkey: an ultrastructural immunoperoxidase analysis. J. Neurosci. 1, 27-39.

Liddelow, S. A., Dziegielewska, K. M., Ek, C. J., Habgood, M. D., Bauer, H., Bauer, H.-C., et al. (2013). Mechanisms that determine the internal environment of the developing brain: a transcriptomic, functional and ultrastructural approach. PLoS ONE 8:e65629. doi: 10.1371/journal.pone.0065629

Liddelow, S. A., Dziegielewska, K. M., Møllgård, K., Whish, S. C., Noor, N. M., Wheaton, B. J., et al. (2014). Cellular Specificity of the Blood-CSF Barrier for Albumin Transfer across the Choroid Plexus Epithelium. PLoS ONE 9:e106592. doi: 10.1371/journal.pone.0106592 
Liddelow, S. A., Dziegielewska, K. M., Vandeberg, J. L., Noor, N. M., Potter, A. M., and Saunders, N. R. (2011). Modification of protein transfer across blood/cerebrospinal fluid barrier in response to altered plasma protein composition during development. Eur. J. Neurosci. 33, 391-400. doi: 10.1111/j.14609568.2010.07509.x

Liddelow, S. A., Temple, S., Møllgård, K., Gehwolf, R., Wagner, A., Bauer, H., et al. (2012). Molecular characterisation of transport mechanisms at the developing mouse blood-CSF interface: a transcriptome approach. PLoS ONE 7:e33554. doi: 10.1371/journal.pone.0033554

Matsuzaki, F., Mège, R. M., Jaffe, S. H., Friedlander, D. R., Gallin, W. J., Goldberg, J. I., et al. (1990). cDNAs of cell adhesion molecules of different specificity induce changes in cell shape and border formation in cultured S180 cells. J. Cell Biol. 110, 1239-1252.

Miller, P. W., Clarke, D. N., Weis, W. I., Lowe, C. J., and Nelson, W. J. (2013). The evolutionary origin of epithelial cell-cell adhesion mechanisms. Curr. Top. Membr. 72, 267-311. doi: 10.1016/B978-0-12-417027-8.00008-8

Mishra, P. K., and Teale, J. M. (2012). Transcriptome analysis of the ependymal barrier during murine neurocysticercosis. J. Neuroinflammation 9, 141. doi: 10.1186/1742-2094-9-141

Møllgård, K., Balslev, Y., Lauritzen, B., and Saunders, N. R. (1987). Cell junctions and membrane specializations in the ventricular zone (germinal matrix) of the developing sheep brain: a CSF-brain barrier. J. Neurocytol. 16, 433-444.

Møllgård, K., and Saunders, N. R. (1986). The development of the human bloodbrain and blood-CSF barriers. Neuropathol. Appl. Neurobiol. 12, 337-358.

Molnár, Z., and Clowry, G. (2012). Cerebral cortical development in rodents and primates. Prog. Brain Res. 195, 45-70. doi: 10.1016/B978-0-444-53860-4. 00003-9

Nagafuchi, A., and Takeichi, M. (1988). Cell binding function of E-cadherin is regulated by the cytoplasmic domain. EMBO J. 7, 3679-3684.

Nitta, T., Hata, M., Gotoh, S., Seo, Y., Sasaki, H., Hashimoto, N., et al. (2003). Sizeselective loosening of the blood-brain barrier in claudin-5-deficient mice. J. Cell Biol. 161, 653-660. doi: 10.1083/jcb.200302070

Otey, C. A., Kalnoski, M. H., and Bulinski, J. C. (1987). Identification and quantification of actin isoforms in vertebrate cells and tissues. J. Cell. Biochem. 34, 113-124. doi: 10.1002/jcb.240340205

Ozawa, M., and Kemler, R. (1992). Molecular organization of the uvomorulincatenin complex. J. Cell Biol. 116, 989-996.

Robinson, M. D., McCarthy, D. J., and Smyth, D. K. (2010). edgeR: a bioconductor package for differential expression analysis of digital gene expression data. Bioinformatics 26, 139-140. doi: 10.1093/bioinformatics/btp616

Saunders, N. R. (1992). "Ontogenetic development of brain barrier mechanisms," in Physiology and Pharmacology of the Blood-Brain Barrier, Vol. 103, Handbook of Experimental Pharmacology, ed M. W. Bradbury (Berlin; Heidelberg: Springer Berlin Heidelberg), 327-369.

Saunders, N. R., Habgood, M. D., and Dziegielewska, K. M. (1999). Barrier mechanisms in the brain, II. Immature brain. Clin. Exp. Pharmacol. Physiol. 26, 85-91.

Saunders, N. R., Knott, G. W., and Dziegielewska, K. M. (2000). Barriers in the immature brain. Cell. Mol. Neurobiol. 20, 29-40. doi: 10.1023/A:1006991809927

Saunders, N. R., Liddelow, S. A., and Dziegielewska, K. M. (2012). Barrier mechanisms in the developing brain. Front. Pharmacol. 3:46. doi: 10.3389/fphar.2012.00046

Saunders, N. R., and Møllgård, K. (1984). Development of the blood-brain barrier. J. Dev. Physiol. 6, 45-57.

Stolp, H. B. (2013). Neuropoietic cytokines in normal brain development and neurodevelopmental disorders. Mol. Cell. Neurosci. 53, 63-68. doi: 10.1016/j.mcn.2012.08.009

Stolp, H. B., Johansson, P. A., Habgood, M. D., Dziegielewska, K. M., Saunders, N. R., and Ek, C. J. (2011a). Effects of neonatal systemic inflammation on blood-brain barrier permeability and behaviour in juvenile and adult rats. Cardiovasc. Psychiatry Neurol. 2011:469046. doi: 10.1155/2011/469046

Stolp, H. B., Liddelow, S. A., Sá-Pereira, I., Dziegielewska, K. M., and Saunders, N. R. (2013). Immune responses at brain barriers and implications for brain development and neurological function in later life. Front. Integr. Neurosci. 7:61. doi: 10.3389/fnint.2013.00061

Stolp, H. B., Turnquist, C., Dziegielewska, K. M., Saunders, N. R., Anthony, D. C., and Molnár, Z. (2011b). Reduced ventricular proliferation in the foetal cortex following maternal inflammation in the mouse. Brain 134, 3236-3248. doi: 10.1093/brain/awr237

Szentistványi, I., Patlak, C. S., Ellis, R. A., and Cserr, H. F. (1984). Drainage of interstitial fluid from different regions of rat brain. Am. J. Physiol. 246, F835-F844.

Tennyson, V., and Pappas, G. (1962). An electron microscope study of ependymal cells of the fetal, early postnatal and adult rabbit. Z. Zellforsch. Mikrosk. Anat. 56, 595-618.

Thomas, T., Power, B., Hudson, P., Schreiber, G., and Dziadek, M. (1988). The expression of transthyretin mRNA in the developing rat brain. Dev. Biol. 128, 415-427.

Thrane, A. S., Takano, T., Rangroo Thrane, V., Wang, F., Peng, W., Ottersen, O. P., et al. (2013). In vivo NADH fluorescence imaging indicates effect of aquaporin-4 deletion on oxygen microdistribution in cortical spreading depression. J. Cereb. Blood Flow Metab. 33, 996-999. doi: 10.1038/jcbfm.2013.63

Van Itallie, C. M., and Anderson, J. M. (2014). Architecture of tight junctions and principles of molecular composition. Semin. Cell Dev. Biol. 36, 157-165. doi: 10.1016/j.semcdb.2014.08.011

Weis, W. I., and Nelson, W. J. (2006). Re-solving the cadherin-cateninactin conundrum. J. Biol. Chem. 281, 35593-35597. doi: 10.1074/jbc.R6000 27200

Xie, L., Kang, H., Xu, Q., Chen, M. J., Liao, Y., Thiyagarajan, M., et al. (2013). Sleep drives metabolite clearance from the adult brain. Science 342, 373-377. doi: $10.1126 /$ science. 1241224

Yamada, S., Pokutta, S., Drees, F., Weis, W. I., and Nelson, W. J. (2005). Deconstructing the cadherin-catenin-actin complex. Cell 123, 889-901. doi: 10.1016/j.cell.2005.09.020

Zhang, J., Piontek, J., Wolburg, H., Piehl, C., Liss, M., Otten, C., et al. (2010). Establishment of a neuroepithelial barrier by Claudin5a is essential for zebrafish brain ventricular lumen expansion. Proc. Natl. Acad. Sci. U.S.A. 107, 1425-1430. doi: 10.1073/pnas.0911996107

Conflict of Interest Statement: The authors declare that the research was conducted in the absence of any commercial or financial relationships that could be construed as a potential conflict of interest.

Received: 30 October 2014; accepted: 12 January 2015; published online: 12 February 2015.

Citation: Whish S, Dziegielewska KM, Møllgård K, Noor NM, Liddelow SA, Habgood MD, Richardson SJ and Saunders NR (2015) The inner CSF-brain barrier: developmentally controlled access to the brain via intercellular junctions. Front. Neurosci. 9:16. doi: 10.3389/fnins.2015.00016

This article was submitted to Neurogenomics, a section of the journal Frontiers in Neuroscience.

Copyright (c) 2015 Whish, Dziegielewska, Møllgård, Noor, Liddelow, Habgood, Richardson and Saunders. This is an open-access article distributed under the terms of the Creative Commons Attribution License (CC BY). The use, distribution or reproduction in other forums is permitted, provided the original author(s) or licensor are credited and that the original publication in this journal is cited, in accordance with accepted academic practice. No use, distribution or reproduction is permitted which does not comply with these terms. 


\section{Transport of thyroid hormones via the choroid plexus into the brain: the roles of transthyretin and thyroid hormone transmembrane transporters}

\section{Samantha J. Richardson ${ }^{1 *}$, Roshen C. Wijayagunaratne ${ }^{1}$, Damian G. D'Souza ${ }^{1}$, Veerle M. Darras ${ }^{2}$ and Stijn L. J. Van Herck ${ }^{2}$}

'School of Medical Sciences, RMIT University, Bundoora, VIC, Australia

${ }^{2}$ Laboratory of Comparative Endocrinology, Biology Department, KU Leuven, Leuven, Belgium

\section{Edited by:}

Helen B. Stolp, King's College

London, UK

Reviewed by:

Robert W. Williams, University of Tennessee Health Science Center. USA

Joao C. Sousa, University of Minho, Portugal

\section{*Correspondence}

Samantha J. Richardson, School of Medical Sciences, RMIT University, Plenty Road, PO Box 71, Bundoora VIC 3083, Australia

e-mail: samantha.richardson@ rmit.edu.au
Thyroid hormones are key players in regulating brain development. Thus, transfer of appropriate quantities of thyroid hormones from the blood into the brain at specific stages of development is critical. The choroid plexus forms the blood-cerebrospinal fluid barrier. In reptiles, birds and mammals, the main protein synthesized and secreted by the choroid plexus is a thyroid hormone distributor protein: transthyretin. This transthyretin is secreted into the cerebrospinal fluid and moves thyroid hormones from the blood into the cerebrospinal fluid. Maximal transthyretin synthesis in the choroid plexus occurs just prior to the period of rapid brain growth, suggesting that choroid plexus-derived transthyretin moves thyroid hormones from blood into cerebrospinal fluid just prior to when thyroid hormones are required for rapid brain growth. The structure of transthyretin has been highly conserved, implying strong selection pressure and an important function. In mammals, transthyretin binds T4 (precursor form of thyroid hormone) with higher affinity than T3 (active form of thyroid hormone). In all other vertebrates, transthyretin binds T3 with higher affinity than T4. As mammals are the exception, we should not base our thinking about the role of transthyretin in the choroid plexus solely on mammalian data. Thyroid hormone transmembrane transporters are involved in moving thyroid hormones into and out of cells and have been identified in many tissues, including the choroid plexus. Thyroid hormones enter the choroid plexus via thyroid hormone transmembrane transporters and leave the choroid plexus to enter the cerebrospinal fluid via either thyroid hormone transmembrane transporters or via choroid plexus-derived transthyretin secreted into the cerebrospinal fluid. The quantitative contribution of each route during development remains to be elucidated. This is part of a review series on ontogeny and phylogeny of brain barrier mechanisms.

Keywords: blood-cerebrospinal fluid barrier, brain, choroid plexus, development, evolution, thyroid hormones, transthyretin, thyroid hormone transporters

\section{THYROID HORMONES}

Thyroid hormones (THs) are key players in regulating development of the brain. Insufficient THs during prenatal development in humans can lead to cretinism and mental retardation, whereas insufficient THs in adult life can result in fatigue, lethargy, mental impairment, weight gain, cold intolerance and in severe cases, clinical depression. THs act mainly by regulating transcription of specific genes. THs are synthesized in the thyroid gland and are secreted into the blood. Most of the TH secreted from the thyroid gland is the precursor form (T4). About $80 \%$ of the active form (T3) is generated by deiodination of T4 to T3 in target tissues. In the blood, $>99 \%$ of $\mathrm{TH}$ is bound to specific plasma proteins known as TH distributor proteins. Because THs are lipophilic they partition between the lipid phase and the aqueous phase with a ratio of 20,000:1 (Dickson et al., 1987). The binding of $\mathrm{TH}$ to $\mathrm{TH}$ distributor proteins prevents avid partitioning of $\mathrm{THs}$ into the lipid membranes of cells and ensures that there is a sufficiently large pool of TH circulating in the blood (Mendel et al., 1987). In humans, the main TH distributor proteins are albumin, transthyretin (TTR) and thyroxine-binding globulin (TBG), all of which are synthesized by the liver. In addition, a small fraction is distributed by lipoproteins including ApoB100 (Benvenga et al., 1989). The free hormone hypothesis states that it is the free hormone in blood (not the protein-bound hormone) that is important for biological activity (Mendel, 1989). Thus, THs must dissociate from the distributor proteins before they can exert their effects.

In human blood $99.97 \%$ of $\mathrm{T} 4$ and $99.70 \%$ of $\mathrm{T} 3$ is bound to the TH distributor proteins (Mendel, 1989). Of these, TBG has the highest affinity for T4 and T3 $\left(1.0 \times 10^{10} \mathrm{M}^{-1}\right.$ and $4.6 \times$ $10^{8} \mathrm{M}^{-1}$, respectively), TTR has intermediate affinity $\left(7.0 \times 10^{7}\right.$ $\mathrm{M}^{-1}$ and $1.4 \times 10^{7} \mathrm{M}^{-1}$, respectively) and albumin has the 
lowest affinity $\left(7.0 \times 10^{5} \mathrm{M}^{-1}\right.$ and $1.0 \times 10^{5} \mathrm{M}^{-1}$, respectively). Together, these three $\mathrm{TH}$ distributor proteins form a buffering network for free T4 in blood, which could help to protect against hypothyroidism (abnormally low levels of free $\mathrm{TH}$ in blood) or hyperthyroidism (abnormally high levels of free $\mathrm{TH}$ in blood) (Schreiber and Richardson, 1997). The older literature and some modern text books state that the role of $\mathrm{TH}$ distributor proteins in blood is to overcome low TH solubility. However, the concentration of free T4 in human blood is about $25 \mathrm{pM}$ and the maximum solubility of $\mathrm{T} 4$ in physiological saline is $2.3 \mu \mathrm{M}$, i.e., about 100,000 times the concentration of free T4 (Schreiber and Richardson, 1997). Therefore, TH distributor proteins must play a role other than to aid the solubility of THs in the blood and CSF.

The concentrations of each of the $\mathrm{TH}$ distributor proteins in adult human blood varies greatly: TBG at $0.015 \mathrm{~g} / \mathrm{l}$, TTR at $0.25 \mathrm{~g} / \mathrm{l}$ and albumin at $42 \mathrm{~g} / \mathrm{l}$. Traditionally it has been believed that in blood, TBG distributes about $75 \%$ of thyroid hormones, TTR distributes $15 \%$ and albumin transports about $10 \%$. Because TBG binds about $75 \%$, it is sometimes referred to as the "most important" thyroid hormone distributor. However, this is too simplistic, as the biological importance is related to the delivery of THs to cells, which is dependent on the dissociation rates of T3 and T4 from the distributor proteins and capillary transit times. To determine which of the three $\mathrm{TH}$ distributor proteins contributes most effectively to hormone delivery to tissues, the dissociation rates and the capillary transit times have to be considered. In brief, the dissociation rates for T4 and T3 from TBG are 0.018 and $0.16 \mathrm{~s}^{-1}$, respectively; from TTR are 0.094 and $0.69 \mathrm{~s}^{-1}$, respectively and from albumin are 1.3 and $2.2 \mathrm{~s}^{-1}$, respectively (Mendel and Weisiger, 1990). Thus, given the capillary transit times for various tissues (Mendel et al., 1989), TTR is responsible for much of the immediate delivery of THs to tissues (Robbins, 2000).

THs have multiple mechanisms of action which can be grouped into "genomic" and "non-genomic" mechanisms of action. Non-genomic mechanisms include membrane-initiated mechanisms via binding to specific integrins (Davis et al., 2005, 2011; Lin et al., 2012); cytosolic kinase-initiated interactions which have non-genomic effects on glucose metabolism (Moeller et al., 2006, 2011) and rapid activation of kinases in signaling cascades in mitochondria resulting in altered fatty acid metabolism (Sayre and Lechleiter, 2012). The genomic pathway, which is generally better known and studied, involves thyroid hormone receptors (TRs). Free (unbound) THs enter target cells via the $\mathrm{TH}$ transmembrane transporters (Hennemann et al., 2001), which are responsible for influx and efflux of THs from cells. For genomic actions of THs within the cell nucleus, T4 can be deiodinated to T3 (considered the "active" form of TH). The family of deiodinases can either activate (e.g., T4 to T3) or inactivate (e.g., T3 to T2; T4 to rT3) THs within the cell (Darras et al., 2015). T3 exerts genomic effects by binding to TRs which are nuclear transcription factors. T3 can bind TRs either in the cytoplasm and translocate into the nucleus, or bind TRs in the nucleus. T3 binding to TR hetero-dimer complexes on thyroid response elements in the promotor of specific genes causes dissociation of co-repressor proteins and association of co-activator proteins. The resulting complex then initiates transcription of genes that are positively regulated by THs. Some genes are negatively regulated by THs, whereby binding of T3 induces the opposite changes and turns off transcription. Thus, it is crucial that the appropriate concentration of THs is present in the critical regions of the brain at each stage of development, to ensure normal brain maturation. This requires that sufficient (but not excess) THs move from the blood into the brain at critical stages of development. There are several pathways for THs to move from the blood into the CSF, but this review will focus on the route via the choroid plexus i.e., the blood-CSF barrier.

\section{THE CHOROID PLEXUS}

The choroid plexus is a villous structure located in the lateral, third and fourth ventricles of the brain and is the site of the bloodCSF barrier. The structure of the choroid plexus is similar in all vertebrates studied to date (Ek et al., 2005). The blood supply to the choroid plexus comes from two posterior choroidal arteries, which branch from the internal carotid arteries. The rate of blood flow to the choroid plexus has been estimated to be about 3.5 faster than that to the rest of the brain (Zheng, 2005). The choroid plexus epithelial cells are joined by tight junctions, giving the tissue its barrier properties (Ek et al., 2005). The choroid plexus produces about $70 \%$ of the CSF, and thus has a major influence on its composition (Cserr, 1971). In humans the total CSF is replaced about four times per day (Brown et al., 2005). In comparison to the blood, which mixes, the CSF has a pipeline type of flow which does not mix. Consequently, the composition of the CSF can vary depending on the site from which it is sampled (Cserr, 1971). The choroid plexus barrier ensures that the composition of the CSF is very different to that of the blood. For example, in adult mammals, the CSF has only about $0.5 \%$ total protein concentration compared to that in the blood (Brown et al., 2005) and the relative ratios of proteins in the blood compared to the CSF differ markedly. However, the total protein concentration in the CSF changes during development, peaking early in development and then gradually decreasing to adult levels (Saunders, 1992). For a comprehensive summary of the development of the choroid plexus (see Ek et al., 2005).

\section{TTR SYNTHESIS BY THE CHOROID PLEXUS}

The first description of TTR synthesis in the brain was in 1985 (Soprano et al., 1985). Soon after, Schreiber's group identified TTR mRNA as being located only in the choroid plexus of the brain (Dickson et al., 1985) and then specifically in the choroid plexus epithelial cells (Stauder et al., 1986). The first experiments suggesting that TTR synthesis by the choroid plexus was involved in the movement of $\mathrm{TH}$ from the blood into the CSF were published in $1987 .{ }^{125} \mathrm{I}-\mathrm{T} 4$ was injected into the blood of rats and was detected in various brain structures: initially it accumulated in the choroid plexus to $\sim 250 \%$ the level in blood. Thereafter, ${ }^{125} \mathrm{I}-\mathrm{T} 4$ accumulated in the CSF, after which it began to appear in the cortex and striatum of the brain. However, when the analogous experiment was repeated using ${ }^{125} \mathrm{I}-\mathrm{T} 3$, there was no specific accumulation of ${ }^{125} \mathrm{I}-\mathrm{T} 3$ in the choroid plexus. This was interpreted as TTR synthesis by the choroid plexus being involved in the movement of T4 but not T3, from the blood across the choroid plexus into the CSF (Dickson et al., 1987). TTR 
synthesized by the choroid plexus was shown to be secreted into the CSF and not into the blood (Schreiber et al., 1990; Duan et al., 1991) i.e., unidirectional secretion of TTR by the choroid plexus $100 \%$ into the CSF. Then Köhrle's group demonstrated that the TTR-T4 complex from the blood is not transported as such into the CSF; and the movement of T4 from the blood into the CSF is dependent on the concentration of free T4 in the serum and on T4 binding to choroid plexus-derived TTR i.e., if the TH binding capacity of TTR was disrupted, then TH did not cross the choroid plexus (Chanoine et al., 1992). Schreiber's group showed that if protein synthesis was blocked in a choroid plexus epithelial monolayer cell culture system, then T4 did not accumulate in the apical chamber (Southwell et al., 1993). Furthermore, the concentration of free T4 is higher in CSF than in the blood, thus, the unidirectional secretion of TTR (or TTR-T4) was moving T4 against the free T4 gradient (Southwell et al., 1993). These were the key experiments suggesting that the synthesis and secretion of TTR by the choroid plexus was involved in movement of T4 from the blood into the CSF.

The choroid plexus is the tissue with the highest concentration of TTR mRNA per gram tissue weight. The adult rat choroid plexus has an 11 times higher concentration of TTR mRNA compared to the liver (4.4 vs. $0.39 \mu \mathrm{g}$ TTR mRNA per gram wet weight) (Schreiber et al., 1990). In chickens, the adult choroid plexus has a 22 times higher concentration of TTR mRNA compared to the liver ( 7.2 vs. $0.33 \mu \mathrm{g}$ TTR mRNA per gram wet) (Duan et al., 1991). Furthermore, at the protein level, TTR is the major protein synthesized and secreted by the choroid plexus of eutherians, marsupials, monotremes, birds, and reptiles (Harms et al., 1991). The common ancestor of those animals whose choroid plexus specializes in TTR synthesis and secretion are the stem-reptiles, whose brains showed the first traces of neocortex (Kent, 1987). As THs are lipophilic, the increase in lipid volume of the brain could have been a selection pressure in turning on TTR synthesis in the choroid plexus and secreting it into the CSF, to act as a TH-binding protein in the CSF, thereby reducing the partitioning of THs into the lipid membranes and allowing a greater distribution of $\mathrm{TH}$ throughout the CSF (Schreiber and Richardson, 1997). Thus, the onset of TTR synthesis in the choroid plexus is suggested to have occurred at the stage of the stem-reptiles, about 300 million years ago (Schreiber and Richardson, 1997). TTR is synthesized in the liver of amphibians and fish during specific stages of development (see Richardson, 2007), but until now synthesis of TTR in the choroid plexus of amphibians and fish has not been described [although TTR mRNA has been detected in whole brain of sea bream (Santos and Power, 1999)]. The main protein synthesized and secreted by amphibian choroid plexus is a lipocalin (Achen et al., 1992), which could be the functional precursor of choroid plexusderived TTR, as lipocalins are known to bind small hydrophobic molecules. That the choroid plexus specializes in the synthesis and secretion of TTR in such a great range of vertebrates is also suggestive of an important function. Furthermore, the amino acid sequence of TTRs across all vertebrates is so highly conserved, that it forms a single motif (Hennebry et al., 2006). Such high conservation of primary structure can be explained by extremely strong selection pressures during the past 500 million years.

\section{ALTRICIAL vS. PRECOCIAL BRAIN DEVELOPMENT AND TTR SYNTHESIS BY THE CHOROID PLEXUS}

The brain develops at different rates in different vertebrate species. At the time of birth, the brain of precocial animals (e.g., chickens, sheep) is significantly further developed than the brains of altricial animals (e.g., rats, mice). Therefore, it is of interest to compare the ontogeny of TTR synthesis in the choroid plexus of altricial animals compared with precocial animals.

In rats (23 days gestation), TTR mRNA was first detected in the choroid plexus at E12.5 in the 4th ventricle, at E13.5 in the lateral ventricles, and at E17.5 in the third ventricle. The proportion of TTR mRNA in total RNA increased 40-fold from E12.5 until birth (Fung et al., 1988). Because the choroid plexus develops faster than many other parts of the brain (Sturrock, 1979), part of this 40-fold increase in TTR mRNA could have been due to the increase in size of the choroid plexus with respect to the size of the brain. The peak of TTR mRNA as a proportion of total brain mRNA (about 140\% the level in adults) occurred 2 days before birth, just prior to the period of fastest brain growth. By 8 days after birth, TTR mRNA levels had decreased to adult levels (Fung et al., 1988).

In sheep (155 days gestation), TTR mRNA was detected in choroid plexus from very early embryos (Tu et al., 1990). In sheep, the maximum increase in size of the choroid plexus (E70) occurred before that of the rest of the brain (E105). At E40 the proportion of TTR mRNA in total mRNA was 34\% of that in adult choroid plexus. By E90, this had increased to $70 \%$ of the adult value, and stayed constant at this level during the remainder of gestation (Tu et al., 1990).

In sheep (precocial), the maximal increase in brain weight occurred 50 days before birth (Tu et al., 1990), whereas in rats (altricial), the maximal increase in brain weight was about 9 days after birth (Fung et al., 1988). The ratio of choroid plexus weight to total brain weight in sheep decreased to a stable value about 20 days before birth (Tu et al., 1990), whereas that for rats occurred about 15 days after birth (Fung et al., 1988). For both species, the choroid plexus had its maximal growth rate prior to that of the rest of the brain.

The critical period of brain development which is dependent on THs is earlier for precocial animals than for altricial animals (Fisher and Polk, 1989). The maximum TTR mRNA level in the choroid plexus of sheep (precocial) occurred during the first half of gestation, whereas in rats (altricial), it occurred only 2 days prior to birth. If TTR is involved in moving THs across the choroid plexus into the brain, and if this TTR then has a role in $\mathrm{TH}$ distribution within the CSF, the timing of the maximal synthesis of TTR by the choroid plexus is highly appropriate.

In summary, in the 1990s it was proposed that T4 bound to a TH distributor protein in blood, is in equilibrium with free T4 which is lipophilic, so partitions into the choroid plexus. Within the choroid plexus, the newly synthesized TTR by the epithelial cells binds to the T4 that entered the choroid plexus from the blood. Choroid plexus-derived TTR is $100 \%$ secreted into the CSF (not into the blood), so the TTR-T4 complex would be secreted into the CSF, followed by its distribution via the CSF to the brain tissue. Thus, the TTR synthesized by the choroid plexus is involved in moving T4 from the blood into the CSF, against 
the free T4 gradient (Chanoine et al., 1992; Southwell et al., 1993; Schreiber and Richardson, 1997). We now know some of these concepts to be outdated (see below).

\section{HUMANS LACKING TTR HAVE NOT BEEN REPORTED}

Cases of humans lacking either of the other two TH distributor proteins: albumin (Minchiotti et al., 2013) or thyroxinebinding globulin (Refetoff, 1989) have been described. Such people are apparently healthy and without overt clinical symptoms. However, no humans lacking TTR have been reported. In the 1990s the hypothesis was that because TTR is the only TH distributor protein synthesized in the brain, is secreted unidirectionally across the apical surface of the choroid plexus epithelial cells into the CSF, its role in the movement of TH from the blood into the CSF, its intermediate affinity for T4 (between those of albumin and thyroxine-binding globulin), and its role is being the most effective at distributing T4 to cells (see above), that it was thought to be crucial for survival, most probably due to the requirement of adequate amounts of TH for normal CNS development (Harms et al., 1991).

There are cases of humans with mutations in TTR resulting in higher affinity for THs, including Gly6Ser $(\sim 12 \%$ of the population), Thr119Met, Ala109Thr, and Ala109Val (Refetoff et al., 1996 and references therein). Of these four variants, only Ala109Thr resulted in affected individuals consistently having elevated serum T4 levels (Moses et al., 1982) [although Ala109Val individuals had elevated rT3 levels (Refetoff et al., 1996)]. More than 100 point mutations in human TTR result in TTR amyloidosis (familial amyloidotic polyneuropathy) (Benson, 2009). Whilst the variant TTRs can have differing affinities for T4 (Rosen et al., 1993) the patients are generally euthyroid (no publications indicating otherwise; and personal communication from Merrill Benson). This is possibly because in the blood, only about 1 in 200 TTRs have a TH bound. Therefore, if some of this TTR is deposited as amyloid then it might not affect overall TH distribution. The point being made here is that whilst there are diseases associated with TTR, there are no humans without TTR, whereas there are healthy humans without either albumin or thyroxine-binding globulin.

However, the function of TTR synthesized by the choroid plexus has been debated. Some believe it to be involved with the movement of T4 from the blood into the CSF (based on the functional, developmental and evolutionary data presented above), whilst others dispute this (read below). The controversy arose when TTR null mice were created and were essentially without an overt phenotype.

\section{TTR NULL MICE}

The production of TTR null mice (Episkopou et al., 1993) that were viable and fertile threw the above hypothesis on the role of choroid plexus-derived TTR into debate. TTR null mice had reduced levels of total T4, total T3, retinol, and retinol-binding protein in their blood, but did not show any overt phenotype. Consequently, some concluded that TTR was not important for THs to reach the brain e.g., (Palha et al., 1994, 1997, 2000). One study concluded that there were no differences in the distribution of the THs to tissues of TTR null mice compared with wildtype mice, except in the choroid plexus, where the T4 level was
$14 \%$ in the TTR null mice compared to wild-type mice (Palha et al., 2000). A semi-quantitative morphological study suggested that wildtype and TTR null mice that had been injected intravenously with ${ }^{125} \mathrm{I}-\mathrm{T} 4$ or ${ }^{125} \mathrm{I}$-T3 did not show any difference in TH distribution throughout their brains (Palha et al., 2002). This bolstered the interpretation that TTR is not an important TH distributor and that TTR null mice should be considered euthyroid (Palha et al., 1994, 2000; Palha, 2002). However, this did not appear to correspond to the earlier data showing that choroid plexus-derived TTR was involved with movement of T4 from the blood into the brain (Dickson et al., 1987; Schreiber et al., 1990; Chanoine et al., 1992; Southwell et al., 1993).

Soon thereafter, the regulation of neural stem cell cycle in the subventricular zone of adult rodent brain was shown to be dependent on TR $\alpha / \mathrm{TH}$ by influencing both cell proliferation and apoptosis (Lemkine et al., 2005). Consequently, it was hypothesized that if TTR was involved in the delivery of THs to the neural stem cell niche, then this population of cells in TTR null mice would be affected. Indeed, TTR null mice were shown to have reduced apoptosis of neural stem cells in the subventricular zone compared to wildtype mice. Furthermore, the level of apoptosis was similar to that of hypothyroid wildtype mice, implying a significant reduction of TH delivery to neural stem cells in this niche (Richardson et al., 2007). It was suggested that because the dissociation rate of $\mathrm{T} 4$ from albumin differs so significantly to that of T4 from TTR, and because the concentration of albumin in the CSF is significantly lower than that in the blood, that in the absence of TTR, albumin would be unlikely to distribute significant amounts of TH beyond the ependymal layer of the brain, resulting in the reduction of $\mathrm{TH}$ reaching the subventricular zone dur to a shallower TH gradient (Richardson et al., 2007). This study highlighted the importance of TTR in delivering THs to at least one of the two populations of neural stem cells in adult mammalian brain.

Until then, all studies involving TTR null mice had been focussed on adult mice. However, it is well known that THs affect growth and development, particularly of the CNS (Morreale de Escobar et al., 1987). In severe cases, TH deficiency in human babies during gestation results in stunted growth and mental retardation. Therefore, several aspects of growth known to be influenced by $\mathrm{TH}$ were examined during post-natal development in TTR null mice. The absence of TTR resulted in delays in several TH-regulated events including bone growth, CNS maturation, intestine and muscle development and in weaning (Monk et al., 2013). Furthermore, there was no specific accumulation of ${ }^{125} \mathrm{I}-\mathrm{T} 4$ in the choroid plexus of adult TTR null mice, following intravenous injection. This was in stark contrast to the accumulation of ${ }^{125} \mathrm{I}-\mathrm{T} 4$ in the choroid plexus of adult wildtype mice, following intravenous injection (Monk, 2006). This delay in THregulated events in the CNS of TTR null mice indicated that TTR has a role in TH delivery in the CNS during development.

In summary, there was strong evolutionary and developmental evidence that choroid plexus-derived TTR was important for moving THs into the brain, but this was not backed-up by analyses of TTR null mice. Therefore, there had to be another mechanism for THs to enter the brain and presumably the choroid plexus. 


\section{TH TRANSMEMBRANE TRANSPORTERS}

The first publication on TH transport that indicated an energydependent process for TH uptake into cells was in 1954 (Christensen et al., 1954) but was "lost" in the literature for decades. In the 1970s Rao demonstrated that TH entry into cells was temperature-dependent, saturable, high-affinity, and lowcapacity, ligand specific, could transport against a gradient and required metabolic energy (Rao, 1981). Yet some researchers missed these publications and believed that because THs could avidly partition into cell membranes, they could diffuse across the membrane as a mode of entry into cells. For a history of hypotheses about TH entry into cells, see (Hennemann et al., 2001). Currently, it is known that THs enter cells via TH transmembrane transporters, which participate in the influx and efflux of THs into and out of cells. Several classes of TH transmembrane transporter proteins that belong to the family of solute carrier (Slc) transporters have been identified e.g. organic anion transporting polypeptides (OATPs), monocarboxylate transporters (MCTs) and L-type amino acid transporters (LATs), and actively participate in entry and exit of THs into and out of cells (Visser et al., 2011).

Several TH transmembrane transporters have been localized to the choroid plexus (Mayerl et al., 2012) and their distribution in the choroid plexus differs between species (Wirth et al., 2014). Most TH transmembrane transporters identified to date are promiscuous with respect to the range of metabolites they transport. Only MCT8 has been identified as being specific to transport of THs and their derivatives (Kinne et al., 2010). Intriguingly, there exists dramatic species-specificity in TH transmembrane transporters deficiency phenotypes. For example, human males with MCT8 mutations present with mental retardation (IQ <40), speech deficits and severe neurological abnormalities (known as the Allan-Herndon-Dudley syndrome), whereas MCT8 null mice are without an overt neurological phenotype (Kersseboom and Visser, 2011). The discovery of the suite of TH transmembrane transporters, particularly in the choroid plexus, was important in understanding how TTR null mice were without an overt phenotype. The difference in phenotype between MCT8 variant human males and MCT8 null mice, could point toward elucidating why TTR null mice are viable, yet no human lacking TTR has been reported. Possibly, this could be due to different combinations of TH transmembrane transporters in the choroid plexus and at the blood-brain barrier, thereby partially compensating for the lack of choroid plexus-derived TTR in mice (but equivalent mechanisms lacking in humans). For example, Lat2 is synthesized in the choroid plexus of mice but not in the choroid plexus of humans (Wirth et al., 2009) or chickens (Darras et al., 2015). This may be an explanation for the mild phenotype of TTR null mice, yet the apparent absence of humans lacking TTR. For a more detailed discussion on the role of TH transmembrane transporters, deiodinases and the establishment of TH gradients across membranes, see a comprehensive review by Schweizer and Kohrle (2013).

The majority of current information on TH transmembrane transporters has been derived from only human and rodent data. A recent, comprehensive review on expression of TH transmembrane transporters during development in zebrafish, Xenopus tropicalis, chicken, and mouse (Darras et al., 2015) highlights how much is still unknown. Whilst the developing choroid plexus has only been analyzed for $\mathrm{TH}$ transmembrane transporters in a few species, interesting differences are emerging. For example, zebrafish choroid plexus does not synthesize MCT8, which contrasts with MCT8 synthesis in the choroid plexus of chickens and rodents. Furthermore, Lat2 is synthesized in the choroid plexus of mice but not humans (Wirth et al., 2009) and appears to be totally absent in chickens (Darras et al., 2015).

From an evolutionary perspective, we need to elucidate the developmental expression profiles for each of the $\mathrm{TH}$ transmembrane transporters in the choroid plexus from a variety of vertebrates representing each of the vertebrate classes. In particular, a comparison of expression of TH transmembrane transporters in the choroid plexus of animals who synthesize TTR in their choroid plexus (reptiles, birds, and mammals) with those who do not synthesize TTR in their choroid plexus (fish and amphibians) should be made. Furthermore, comparisons of TH transmembrane transporter expression profiles in the choroid plexus between precocial and altricial species would be intriguing. A comparison of the expression profiles of $\mathrm{TH}$ transmembrane transporters in mammalian choroid plexus vs. those in choroid plexus from non- mammals should be made, as mammalian TTRs are the exception in having higher affinity for T4 than T3. TTRs from all other vertebrates have higher affinity for T3 than for T4 (see below). Such studies could shed light on the relative roles of each of the $\mathrm{TH}$ transmembrane transporters and choroid plexus-derived TTR in the overall movement of THs from the blood into the CSF during development.

\section{MAMMALIAN TTRs ARE THE EXCEPTION}

In all studied species of fish, amphibians, reptiles and birds, TTR has higher affinity for T3 than T4, whereas in mammals TTR has higher affinity for T4 than for T3 (Richardson, 2007). Thus, mammals are the exception in this regard. Is it possible that whilst TTR made by the choroid plexus of mammals moves T4 into the brain, TTR from other animals moves T3 into the brain? What could have been the selection pressure for the change to TTR from distributing T3 to distributing T4? One possibility could be a greater level of control, requiring an additional level of regulation (i.e., deiodination of $\mathrm{T} 4$ to $\mathrm{T} 3$ ) at the target site. For example, different regions of the brain generate different proportions of T3 by local deiodination (van Doorn et al., 1985). Two features that distinguish mammalian brains from non-mammalian brains that are regulated (at least in part) by thyroid hormones are the corpus callosum which is heavily myelinated, and the highly developed cortex. The point here is that mammals may be the exception rather than the rule, when it comes to modes of TH entry into the CNS, so we should not just consider mammalian data. Furthermore, we have seen examples between eutherian species, of significant differences in TH transmembrane transporters (e.g., Lat2).

The literature is often skewed toward data on mammals, especially humans (due to our self-centerd interests in medicine/disease) and mice (due to the ease of working with them: inbred strains, short generation times, knock-in/out genetic manipulations etc), so we should be careful in analysing data from humans and rodents or eutherian mammals and 
drawing generalized conclusions, which may not be applicable to all mammals, let alone to all vertebrates. Furthermore, data from knock-out mice can sometimes raise more questions than they answer.

\section{CONFUSION ARISING FROM MOUSE KO MODELS OF TH MOVEMENT INTO THE BRAIN}

TTR null mice have 36\% T4 levels in their brain compared to wildtype mice (Palha et al., 1997). This implies that TTR is responsible for the other $64 \%$ of $\mathrm{T} 4$ moving into the brain. However, Mct8/Oatp1c1 double knock-out mice are cited as having only $10 \% \mathrm{TH}$ concentration in their brains compared to wildtype mice (Muller and Heuer, 2014). However, careful analysis of the original paper reveals that only the forebrain (and not the entire brain) was analyzed for TH content (Mayerl et al., 2014). Furthermore, if $\mathrm{TH}$ transmembrane transporters are responsible for moving significant amounts of $\mathrm{TH}$ into and out of the choroid plexus, then why do TTR null mice have only 14\% T4 in their choroid plexus compared with wildtype mice? How do these data from TH transmembrane transporter and TTR null mice correlate with the data from Chanoine et al. (1992)? The quantitative contributions of the various mechanisms for TH moving across the blood-CSF barrier (the choroid plexus) and across the blood-brain barrier need to be investigated in greater detail.

\section{CURRENT HYPOTHESIS FOR TH MOVEMENT FROM BLOOD VIA THE CHOROID PLEXUS TO THE CSF}

Our current hypothesis for the movement of $\mathrm{TH}$ from the blood via the choroid plexus is depicted in Figure 1. In this Figure, T4 (major form of TH secreted by the thyroid gland) is depicted.

1. T4 dissociates from $\mathrm{TH}$ distributor proteins in the blood.

2. T4 enters the choroid plexus via TH transporters.

3. TTR is synthesized by the choroid plexus epithelial cells.

4. Some T4 will bind TTR synthesized in the choroid plexus epithelial cells.

5. T4 moves out of the choroid plexus into the CSF via either secretion of the T4-TTR complex; or via TH transmembrane transporters.

It is worth noting that there is a higher concentration free T4 in CSF than in blood, so net movement of TH from the blood to the CSF is against this gradient. Therefore, it would not be surprising if several mechanisms for moving $\mathrm{TH}$ from the blood via the choroid plexus (and the blood-brain barrier) had evolved.

\section{CONCLUSION}

From the above controversy regarding the importance of TTR synthesis by the choroid plexus, several points support the

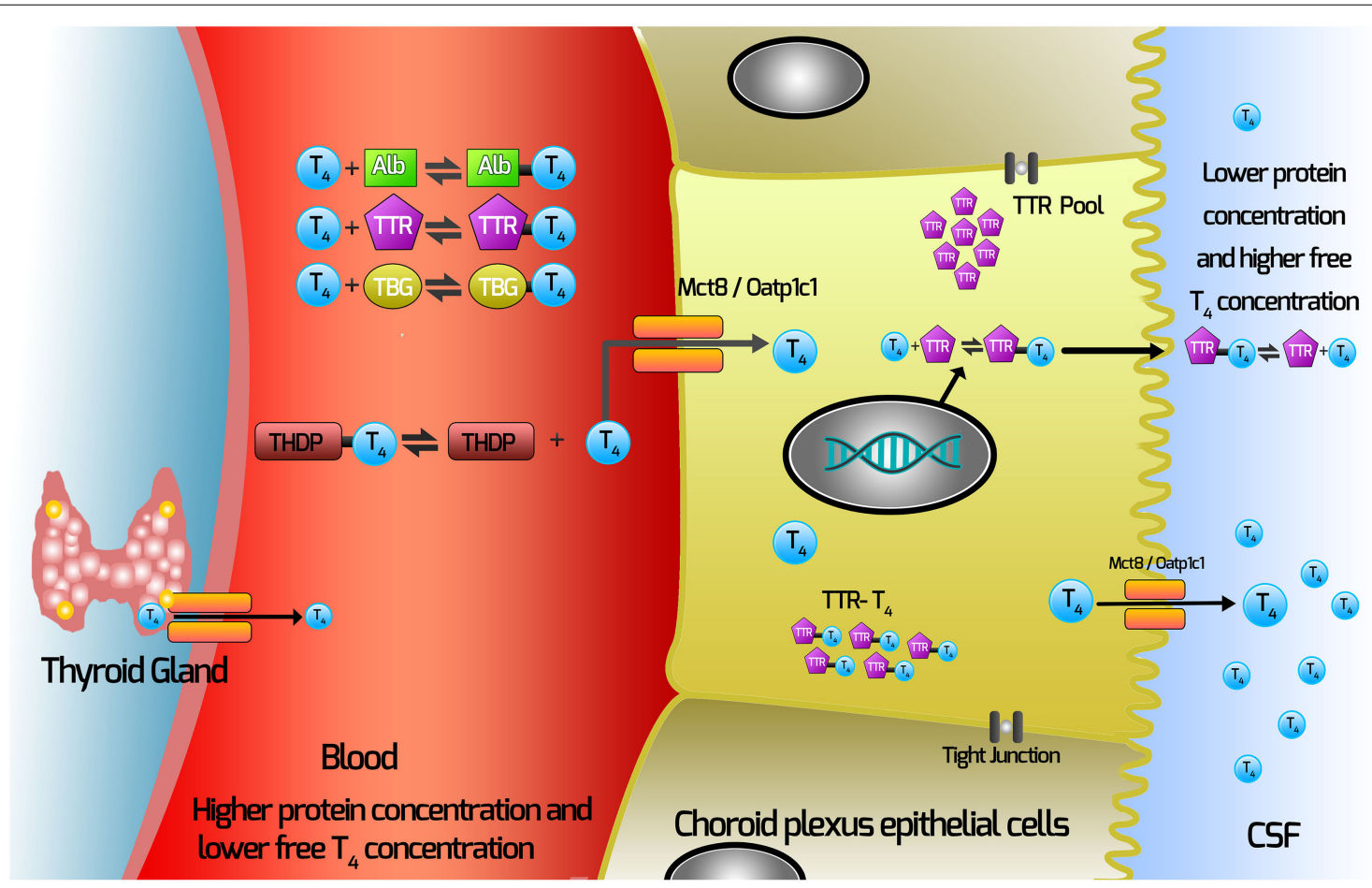

FIGURE 1 | Current hypothesis of mechanisms involved with TH movement from the blood across the choroid plexus into the CSF. THs are synthesized in the thyroid gland and secreted into the blood, where $>99 \%$ are bound by the TH distributor proteins (THDPs) which include albumin (Alb), transthyretin (TTR), thyroxine-binding globulin (TBG) and to a lesser extent by specific lipoproteins, depending on the species and the stage of development. The main form of $\mathrm{TH}$ released from the thyroid gland is T4, so T4 is used as the example in this schematic. T4 dissociates from TH distributor proteins in the blood and enters the choroid plexus via $\mathrm{TH}$ transmembrane transporters (which may include Mct8 and Oatp1c1, depending on the species). TTR is synthesized by the choroid plexus epithelial cells and is secreted into the CSF. Some T4 binds TTR synthesized in the choroid plexus epithelial cells. T4 moves out of the choroid plexus into the CSF via either secretion of the T4-TTR complex or via TH transmembrane transporters. 
hypothesis that choroid plexus-derived TTR plays a role in the movement of THs from the blood to the brain and in distributing THs in the blood and CSF. These points include evolutionary, developmental, molecular and functional data. Therefore, it would follow that TTR synthesized by the choroid plexus would appear to have an important regulatory role in determining the delivery of thyroid hormones into the CSF.

In conclusion, there is evidence for two mechanisms for THs to move out of the choroid plexus into the CSF: (i) secretion of $\mathrm{TH}$ bound to choroid plexus-derived TTR, and (ii) efflux of THs via $\mathrm{TH}$ transmembrane transporters. The relative quantitative contribution of each of these mechanisms during each stage of CNS development is currently unknown and should be investigated. Furthermore, perhaps the role played by choroid plexus-derived TTR is more significant in non-mammalian vertebrates than in mammals. It would be interesting to analyze the developmental profiles of $\mathrm{TH}$ transmembrane transporters in the choroid plexus (and in other blood-brain barrier structures) in non-mammalian vertebrates during development. The delivery of the requisite concentrations of THs to specific areas of the brain at each stage of CNS development is so important, that it would be understandable for evolution to have positively selected for more than one strategy for THs to enter the CNS.

\section{REFERENCES}

Achen, M. G., Harms, P. J., Thomas, T., Richardson, S. J., Wettenhall, R. E., and Schreiber, G. (1992). Protein synthesis at the blood-brain barrier. The major protein secreted by amphibian choroid plexus is a lipocalin. J. Biol. Chem. 267, 23170-23174.

Benson, M. D. (2009). “Genetics: clinical implications of TTR amyloidosis," in Recent Advances in Transthyretin Evolution, Structure and Biological Functions, eds S. J. Richardson and V. Cody (Berlin: Springer), 173-189. doi: 10.1007/9783-642-00646-3_11

Benvenga, S., Cahnmann, H., Gregg, R., and Robbins, J. (1989). Binding of thyroxine to human plasma low density lipoprotein through specific interaction with apolipoprotein B (apoB-100). Biochimie 71, 263-268. doi: 10.1016/03009084(89)90063-1

Brown, P. D., Speake, T., Davies, S. L., and Millar, I. D. (2005). "Ion transporters and channels involved in csf formation," in The Blood-Cerebrospinal Fluid Barrier, ed W. A. C. A. Zheng (Boca Raton, FL: Taylor \& Francis Group), 119-145.

Chanoine, J. P., Alex, S., Fang, S. L., Stone, S., Leonard, J. L., Korhle, J., et al. (1992). Role of transthyretin in the transport of thyroxine from the blood to the choroid plexus, the cerebrospinal fluid, and the brain. Endocrinology 130, 933-938.

Christensen, H. N., Hess, B., and Riggs, T. R. (1954). Concentration of taurine, beta-alanine, and triiodothyronine by ascites carcinoma cells. Cancer Res. 14, 124-127.

Cserr, H. F. (1971). Physiology of the choroid plexus. Physiol. Rev. 51, 273-311.

Darras, V. M., Houbrechts, A. M., and Van Herck, S. L. (2015). Intracellular thyroid hormone metabolism as a local regulator of nuclear thyroid hormone receptor-mediated impact on vertebrate development. Biochim. Biophys. Acta. 1849, 130-141. doi: 10.1016/j.bbagrm.2014.05.004

Davis, P. J., Davis, F. B., and Cody, V. (2005). Membrane receptors mediating thyroid hormone action. Trends Endocrinol. Metab. 16, 429-435. doi: 10.1016/j.tem.2005.09.007

Davis, P. J., Davis, F. B., Mousa, S. A., Luidens, M. K., and Lin, H. Y. (2011). Membrane receptor for thyroid hormone: physiologic and pharmacologic implications. Annu. Rev. Pharmacol. Toxicol. 51, 99-115. doi: 10.1146/annurevpharmtox-010510-100512

Dickson, P. W., Aldred, A. R., Marley, P. D., TU, G. F., Howlett, G. J., and Schreiber, G. (1985). High prealbumin and transferrin messenger RNA levels in the choroid plexus of rat brain. Biochem. Biophys. Res. Commun. 127, 890-895. doi: 10.1016/S0006-291X(85)80027-9
Dickson, P. W., Aldred, A. R., Menting, J. G., Marley, P. D., Sawyer, W. H., and Schreiber, G. (1987). Thyroxine transport in choroid plexus. J. Biol. Chem. 262, 13907-13915.

Duan, W., Achen, M. G., Richardson, S. J., Lawrence, M. C., Wettenhall, R. E., Jaworowski, A., et al. (1991). Isolation, characterization, cDNA cloning and gene expression of an avian transthyretin. Implications for the evolution of structure and function of transthyretin in vertebrates. Eur. J. Biochem. 200, 679-687. doi: 10.1111/j.1432-1033.1991.tb16232.x

Ek, C. J., Dziegielewska, K. M., and Saunders, N. R. (2005). "Devlopment of the blood-cerebrospinal fluid barrier," in The Blood-Cerebrospinal Fluid Barrier, ed W. A. C. A. Zheng (Boca Raton, FL: Chapman \& Hall/CR, Taylor \& Francis Group), 3-23105-3-23120.

Episkopou, V., Maeda, S., Nishiguchi, S., Shimada, K., Gaitanaris, G. A., Gottesman, M. E., et al. (1993). Disruption of the transthyretin gene results in mice with depressed levels of plasma retinol and thyroid-hormone. Proc. Natl. Acad. Sci. U.S.A. 90, 2375-2379. doi: 10.1073/pnas.90.6.2375

Fisher, D. A., and Polk, D. H. (1989). Development of the thyroid. Baillieres Clin. Endocrinol. Metab. 3, 627-657. doi: 10.1016/S0950-351X(89)80046-1

Fung, W. P., Thomas, T., Dickson, P. W., Aldred, A. R., Milland, J., Dziadek, M., et al. (1988). Structure and expression of the rat transthyretin (prealbumin) gene. J. Biol. Chem. 263, 480-488.

Harms, P. J., Tu, G. F., Richardson, S. J., Aldred, A. R., Jaworowski, A., and Schreiber, G. (1991). Transthyretin (prealbumin) gene expression in choroid plexus is strongly conserved during evolution of vertebrates. Comp. Biochem. Physiol. B 99, 239-249. doi: 10.1016/0305-0491(91)90035-C

Hennebry, S. C., Wright, H. M., Likic, V. A., and Richardson, S. J. (2006). Structural and functional evolution of transthyretin and transthyretin-like proteins. Proteins 64, 1024-1045. doi: 10.1002/prot.21033

Hennemann, G., Docter, R., Friesema, E. C., de Jong, M., Krenning, E. P., and Visser, T. J. (2001). Plasma membrane transport of thyroid hormones and its role in thyroid hormone metabolism and bioavailability. Endocr. Rev. 22, 451-476. doi: 10.1210/edrv.22.4.0435

Kent, G. C. (1987). Comparative Anatomy of the Vertebrates. St. Louis, MO: Time Mirror/Mosby College.

Kersseboom, S., and Visser, T. J. (2011). Tissue-specific effects of mutations in the thyroid hormone transporter MCT8. Arq. Bras. Endocrinol. Metabol. 55, 1-5. doi: 10.1590/S0004-27302011000100001

Kinne, A., Kleinau, G., Hoefig, C. S., Gruters, A., Kohrle, J., Krause, G., et al. (2010). Essential molecular determinants for thyroid hormone transport and first structural implications for monocarboxylate transporter 8. J. Biol. Chem. 285, 28054-28063. doi: 10.1074/jbc.M110.129577

Lemkine, G. F., Raj, A., Alfama, G., Turque, N., Hassani, Z., Alegria-Prevot, O., et al. (2005). Adult neural stem cell cycling in vivo requires thyroid hormone and its alpha receptor. FASEB J. 19, 863-865. doi: 10.1096/fj.04-2916fje

Lin, H. Y., Tang, H. Y., Davis, F. B., Mousa, S. A., Incerpi, S., Luidens, M. K., et al. (2012). Nongenomic regulation by thyroid hormone of plasma membrane ion and small molecule pumps. Discov. Med. 14, 199-206.

Mayerl, S., Muller, J., Bauer, R., Richert, S., Kassmann, C. M., Darras, V. M., et al. (2014). Transporters MCT8 and OATP1C1 maintain murine brain thyroid hormone homeostasis. J. Clin. Invest. 124, 1987-1999. doi: 10.1172/JCI70324

Mayerl, S., Visser, T. J., Darras, V. M., Horn, S., and Heuer, H. (2012). Impact of Oatplcl deficiency on thyroid hormone metabolism and action in the mouse brain. Endocrinology 153, 1528-1537. doi: 10.1210/en.2011-1633

Mendel, C. M. (1989). The free hormone hypothesis: a physiologically based mathematical model. Endocr. Rev. 10, 232-274. doi: 10.1210/edrv-10-3-232

Mendel, C. M., Cavalieri, R. R., Gavin, L. A., Pettersson, T., and Inoue, M. (1989). Thyroxine transport and distribution in nagase analbuminemic rats. J. Clin. Invest. 83, 143-148. doi: 10.1172/JCI113851

Mendel, C. M., and Weisiger, R. A. (1990). Thyroxine uptake by perfused rat liver. No evidence for facilitation by five different thyroxine-binding proteins. J. Clin. Invest. 86, 1840-1847. doi: 10.1172/JCI114914

Mendel, C. M., Weisiger, R. A., Jones, A. L., and Cavalieri, R. R. (1987). Thyroid hormone binding proteins in plasma facilitate uniform distribution of thyroxine within tissues - a perfused rat liver study. Endocrinology 120, 1742-1749. doi: 10.1210/endo-120-5-1742

Minchiotti, L., Galliano, M., Caridi, G., Kragh-Hansen, U., and Peters, T. Jr. (2013). Congenital analbuminaemia: molecular defects and biochemical and clinical aspects. Biochim. Biophys. Acta 1830, 5494-5502. doi: 10.1016/j.bbagen.2013.04.019 
Moeller, L. C., Cao, X., Dumitrescu, A. M., Seo, H., and Refetoff, S. (2006). Thyroid hormone mediated changes in gene expression can be initiated by cytosolic action of the thyroid hormone receptor beta through the phosphatidylinositol 3-kinase pathway. Nucl. Recept. Signal. 4:e020. doi: 10.1621/nrs.04020

Moeller, L. C., Haselhorst, N. E., Dumitrescu, A. M., Cao, X., Seo, H., Refetoff, S., et al. (2011). Stanniocalcin 1 induction by thyroid hormone depends on thyroid hormone receptor beta and phosphatidylinositol 3-kinase activation. Exp. Clin. Endocrinol. Diabetes 119, 81-85. doi: 10.1055/s-0030-1262860

Monk, J. A. (2006). Thyroid Hormone Homeostasis in Developing and Adult Transthyretin Null Mice. Ph.D., The University of Melbourne.

Monk, J. A., Sims, N. A., Dziegielewska, K. M., Weiss, R. E., Ramsay, R. G., and Richardson, S. J. (2013). Delayed development of specific thyroid hormoneregulated events in transthyretin null mice. Am. J. Physiol. Endocrinol. Metab. 304, E23-E31. doi: 10.1152/ajpendo.00216.2012

Morreale de Escobar, G., Obregon, M. J., and Escobar del Rey, F. (1987). Fetal and maternal thyroid hormones. Horm. Res. 26, 12-27. doi: 10.1159/000180681

Moses, A. C., Lawlor, J., Haddow, J., and Jackson, I. M. (1982). Familial euthyroid hyperthyroxinemia resulting from increased thyroxine binding to thyroxine-binding prealbumin. N. Engl. J. Med. 306, 966-969. doi: 10.1056/NEJM198204223061605

Muller, J., and Heuer, H. (2014). Expression pattern of thyroid hormone transporters in the postnatal mouse brain. Front. Endocrinol. (Lausanne) 5:92. doi: 10.3389/fendo.2014.00092

Palha, J. A. (2002). Transthyretin as a thyroid hormone carrier: function revisited. Clin. Chem. Lab. Med. 40, 1292-1300. doi: 10.1515/CCLM.2002.223

Palha, J. A., Episkopou, V., Maeda, S., Shimada, K., Gottesman, M. E., and Saraiva, M. J. M. (1994). Thyroid-hormone metabolism in a transthyretin-null mouse strain. J. Biol. Chem. 269, 33135-33139.

Palha, J. A., Fernandes, R., de Escobar, G. M., Episkopou, V., Gottesman, M., and Saraiva, M. J. (2000). Transthyretin regulates thyroid hormone levels in the choroid plexus, but not in the brain parenchyma: Study in a transthyretinnull mouse model. Endocrinology 141, 3267-3272. doi: 10.1210/endo.141. 9.7659

Palha, J. A., Hays, M. T., Deescobar, G. M., Episkopou, V., Gottesman, M. E., and Saraiva, M. J. M. (1997). Transthyretin is not essential for thyroxine to reach the brain and other tissues in transthyretin-null mice. Am. J. Physiol. Endocrinol. Metab. 35, E485-E493.

Palha, J. A., Nissanov, J., Fernandes, R., Sousa, J. C., Bertrand, L., Dratman, M. B., et al. (2002). Thyroid hormone distribution in the mouse brain: the role of transthyretin. Neuroscience 113, 837-847. doi: 10.1016/S0306-4522(02)00228-2

Rao, G. S. (1981). MOde of entry of steroid and thyroid hormones into cells. Mol. Cell. Endocrinol. 21, 97-108. doi: 10.1016/0303-7207(81)90047-2

Refetoff, S. (1989). Inherited thyroxine-binding globulin abnormalities in man. Endocr. Rev. 10, 275-293. doi: 10.1210/edrv-10-3-275

Refetoff, S., Marinov, V. S., Tunca, H., Byrne, M. M., Sunthornthepvarakul, T., and Weiss, R. E. (1996). A new family with hyperthyroxinemia caused by transthyretin Val109 misdiagnosed as thyrotoxicosis and resistance to thyroid hormone-a clinical research center study. J. Clin. Endocrinol. Metab. 81, 3335-3340.

Richardson, S. J. (2007). Cell and molecular biology of transthyretin and thyroid hormones. Int. Rev. Cytol. 258, 137-193. doi: 10.1016/S0074-7696(07)58003-4

Richardson, S. J., Lemkine, G. F., Alfama, G., Hassani, Z., and Demeneix, B. A. (2007). Cell division and apoptosis in the adult neural stem cell niche are differentially affected in transthyretin null mice. Neurosci. Lett. 421, 234-238. doi: 10.1016/j.neulet.2007.05.040

Robbins, J. (2000). "Thyroid hormone transport proteins and the physiology of hormone binding," in Werner and Ingbar's the Thyroid - A Fundamental and Clinical Text, 8th Edn., eds L. E. Braverman and R. D. Utiger (Philadelphia, PA: Lippincott Williams \& Wilkins), 105-120.

Rosen, H. N., Moses, A. C., Murrell, J. R., Liepnieks, J. J., and Benson, M. D. (1993). Thyroxine interactions with transthyretin: a comparison of 10 different naturally occurring human transthyretin variants. J. Clin. Endocrinol. Metab. 77, 370-374.

Santos, C. R. A., and Power, D. M. (1999). Identification of transthyretin in fish (Sparus aurata): cDNA cloning and characterisation. Endocrinology 140, 2430-2433. doi: 10.1210/endo.140.5.6898
Saunders, N. R. (1992). "Ontogenetic development of brain barrier mechanisms," in Handbook of Experimental Pharmacology, Vol. 103, Physiology and Pharmacology of the Blood-Brain Barrier, ed M. W. B. Bradbury (Berlin, Heidelberg: Springer-Verlag), 327-369. doi: 10.1007/978-3-642-76894-1_14

Sayre, N. L., and Lechleiter, J. D. (2012). Fatty acid metabolism and thyroid hormones. Curr. Trends Endocinol. 6, 65-76.

Schreiber, G., Aldred, A. R., Jaworowski, A., Nilsson, C., Achen, M. G., and Segal, M. B. (1990). Thyroxine transport from blood to brain via transthyretin synthesis in choroid plexus. Am. J. Physiol. 258, R338-R345.

Schreiber, G., and Richardson, S. J. (1997). The evolution of gene expression, structure and function of transthyretin. Comp. Biochem. Physiol B Biochem. Mol. Biol. 116, 137-160. doi: 10.1016/S0305-0491(96)00212-X

Schweizer, U., and Kohrle, J. (2013). Function of thyroid hormone transporters in the central nervous system. Biochim. Biophys. Acta 1830, 3965-3973. doi: 10.1016/j.bbagen.2012.07.015

Soprano, D. R., Herbert, J., Soprano, K. J., Schon, E. A., and Goodman, D. S. (1985). Demonstration of transthyretin mRNA in the brain and other extrahepatic tissues in the rat. J. Biol. Chem. 260, 11793-11798.

Southwell, B. R., Duan, W., Alcorn, D., Brack, C., Richardson, S. J., Kohrle, J., et al. (1993). Thyroxine transport to the brain: role of protein synthesis by the choroid plexus. Endocrinology 133, 2116-2126.

Stauder, A. J., Dickson, P. W., Aldred, A. R., Schreiber, G., Mendelsohn, F. A., and Hudson, P. (1986). Synthesis of transthyretin (pre-albumin) mRNA in choroid plexus epithelial cells, localized by in situ hybridization in rat brain. J. Histochem. Cytochem. 34, 949-952. doi: 10.1177/34.7.3458812

Sturrock, R. R. (1979). A morphological study of the development of the mouse choroid plexus. J. Anat. 129, 777-793.

Tu, G. F., Cole, T., Southwell, B. R., and Schreiber, G. (1990). Expression of the genes for transthyretin, cystatin C and beta A4 amyloid precursor protein in sheep choroid plexus during development. Brain Res. Dev. Brain Res. 55, 203-208. doi: 10.1016/0165-3806(90)90201-9

van Doorn, J., Roelfsema, F., and van der Heide, D. (1985). Concentrations of thyroxine and 3,5,3'-triiodothyronine at 34 different sites in euthyroid rats as determined by an isotopic equilibrium technique. Endocrinology 117, 1201-1208. doi: 10.1210/endo-117-3-1201

Visser, W. E., Friesema, E. C., and Visser, T. J. (2011). Minireview: thyroid hormone transporters: the knowns and the unknowns. Mol. Endocrinol. 25, 1-14. doi: 10.1210/me.2010-0095

Wirth, E. K., Roth, S., Blechschmidt, C., Holter, S. M., Becker, L., Racz, I., et al. (2009). Neuronal 3,3,5-triiodothyronine (T3) uptake and behavioral phenotype of mice deficient in Mct8, the neuronal T3 transporter mutated in Allan-Herndon-Dudley syndrome. J. Neurosci. 29, 9439-9449. doi: 10.1523/JNEUROSCI.6055-08.2009

Wirth, E. K., Schweizer, U., and Kohrle, J. (2014). Transport of thyroid hormone in brain. Front. Endocrinol. (Lausanne) 5:98. doi: 10.3389/fendo.2014.00098

Zheng, W. (2005). The Blood-Cereprospinal Fluid Barrier. Boca Raton, FL: Taylor \& Franscis Group.

Conflict of Interest Statement: The authors declare that the research was conducted in the absence of any commercial or financial relationships that could be construed as a potential conflict of interest.

Received: 24 September 2014; accepted: 16 February 2015; published online: 03 March 2015.

Citation: Richardson SJ, Wijayagunaratne RC, D'Souza DG, Darras VM and Van Herck SLJ (2015) Transport of thyroid hormones via the choroid plexus into the brain: the roles of transthyretin and thyroid hormone transmembrane transporters. Front. Neurosci. 9:66. doi: 10.3389/fnins.2015.00066

This article was submitted to Neurogenomics, a section of the journal Frontiers in Neuroscience.

Copyright (c) 2015 Richardson, Wijayagunaratne, D'Souza, Darras and Van Herck. This is an open-access article distributed under the terms of the Creative Commons Attribution License (CC BY). The use, distribution or reproduction in other forums is permitted, provided the original author(s) or licensor are credited and that the original publication in this journal is cited, in accordance with accepted academic practice. No use, distribution or reproduction is permitted which does not comply with these terms. 


\title{
Efflux transporters in blood-brain interfaces of the developing brain
}

\author{
Nathalie Strazielle ${ }^{1,2}$ and Jean-François Ghersi-Egea ${ }^{2,3 *}$ \\ ${ }^{1}$ Brain-i, Lyon, France \\ 2 Oncoflam Team, Lyon Neuroscience Research Center, INSERM, U1028, CNRS, UMR5292, Université Lyon 1, Lyon, France \\ ${ }^{3}$ BIP Platform, Lyon Neuroscience Research Center, INSERM, U1028, CNRS, UMR5292, Université Lyon 1, Lyon, France
}

\section{Edited by:}

Helen B. Stolp, King's College

London, UK

Reviewed by:

Gert Fricker, University of

Heidelberg, Germany

David S. Miller, National Institute of

Environmental Health Sciences,

USA

\section{*Correspondence:}

Jean-François Ghersi-Egea,

Blood-Brain Interface Group,

Oncoflam Team, INSERM U 1028,

CNRS UMR5292, Lyon

Neuroscience Research Center,

Oncoflam Team, Faculté de

Médecine RTH Laennec, Rue

Guillaume Paradin, 69372 Lyon,

France

e-mail: jean-francois.ghersi-egea@ inserm.fr
The cerebral microvessel endothelium forming the blood-brain barrier (BBB) and the epithelium of the choroid plexuses forming the blood-CSF barrier (BCSFB) operate as gatekeepers for the central nervous system. Exposure of the vulnerable developing brain to chemical insults can have dramatic consequences for brain maturation and lead to life-long neurological diseases. The ability of blood-brain interfaces to efficiently protect the immature brain is therefore an important pathophysiological issue. This is also key to our understanding of drug entry into the brain of neonatal and pediatric patients. Non-specific paracellular diffusion through barriers is restricted early during development, but other neuroprotective properties of these interfaces differ between the developing and adult brains. This review focuses on the developmental expression and function of various classes of efflux transporters. These include the multispecific transporters of the ATP-binding cassette transporter families ABCB, ABCC, ABCG, the organic anion and cation transporters of the solute carrier families SLC21/SLCO and SLC22, and the peptide transporters of the SLC15 family. These transporters play a key role in preventing brain entry of blood-borne molecules such as drugs, environmental toxicants, and endogenous metabolites, or else in increasing the clearance of potentially harmful organic ions from the brain. The limited data available for laboratory animals and human highlight transporter-specific developmental patterns of expression and function, which differ between blood-brain interfaces. The BCSFB achieves an adult phenotype earlier than BBB. Efflux transporters at the BBB appear to be regulated by various factors subsequently secreted by neural progenitors and astrocytes during development. Their expression is also modulated by oxidative stress, inflammation, and exposure to xenobiotic inducers. A better understanding of these regulatory pathways during development, in particular the signaling pathways triggered by oxidative stress and xenobiotics, may open new opportunities to therapeutic manipulation in view to improve or restore neuroprotective functions of the blood-brain interfaces in the context of perinatal injuries.

Keywords: blood-brain barrier, choroid plexus, development, ABC transporter, SLC transporter, perinatal injury, hypoxia, xenobiotic

\section{INTRODUCTION}

Blood-brain interfaces comprise the cerebral microvessel endothelium forming the blood-brain barrier (BBB) and the epithelium of the choroid plexuses forming the blood-CSF barrier (BCSFB). These interfaces operate as gatekeepers for the central nervous system, hence providing the cerebral homeostasis necessary for normal neuronal growth and activity.

During brain development, these CNS barriers display stage specific properties. For instance, in rat, the activity of gamma-glutamyl-transferase, an enzymatic marker of brain

Abbreviations: BBB, blood-brain barrier; BCSFB, blood-CSF barrier; ABC, ATPBinding Cassette; OATP, organic anion transport polypeptide; HNF-4 $\alpha$, hepatic nuclear factor $4 \alpha$; Nrf2, nuclear factor erythroid 2-related factor 2; AhR, aryl hydrocarbon receptor; CAR, constitutive androstane receptor; PXR, pregnane X receptor. microvessels, continuously increases from birth to adulthood (Betz and Goldstein, 1981; Gazzin et al., 2008). Conversely, the metabolic barrier to DOPA mediated by DOPA-decarboxylase is most effective in young animals (Betz and Goldstein, 1981). Blood to brain influx of pyruvate, lactate and $\beta$-hydroxybutyrate, as well as large neutral amino acids is greater in suckling rats compared to adult animals, while active efflux of amino acids across the BBB may not occur before 21 days of age (review in Betz and Goldstein, 1981). In the choroid plexus epithelium, the composition in tight junction proteins changes during brain development. Notably, expression of the pore-forming claudin-2 is upregulated and that of the tightening claudin- 3 is downregulated, probably reflecting perinatal changes in inorganic anion transport and related CSF secretion rate at the BCSFB (Kratzer et al., 2012). These various examples illustrate changes in barrier functions that occur 
to face specific needs of the brain at different developmental stages.

The vulnerability of the developing brain to chemical insults, especially during the late prenatal/early postnatal period (herein referred to as perinatal period) can have dramatic consequences for brain maturation, leading to life-long neurological diseases (Kaindl et al., 2009; Landrigan and Goldman, 2011; Miodovnik, 2011). The ability of blood-brain interfaces to efficiently protect the immature brain is therefore a major pathophysiological issue. It is also key to our understanding of therapeutic drug entry into the brain of neonate and pediatric patients. Little is known about the mechanisms protecting the developing brain from harmful compounds and regulating the cerebral entry of pharmacologically active compounds. The cerebral interstitial fluid concentration of xenobiotics is set by their ability to cross the brain endothelium and the choroidal epithelium, as well as a number of other parameters. These include protein binding, local cerebral capillary density and cerebral blood flow, intraparenchymal diffusion rate, movement by fluid flow along perivascular spaces and within ventricular and cisternal spaces, and CSF turnover (reviewed in Ghersi-Egea et al., 2009; Westerhout et al., 2011). These parameters change during development. Plasma protein concentration is lower during development than in adult, so are cerebral blood flow and capillary density (Caley and Maxwell, 1970; Nehlig et al., 1989). Intracerebral fluid dynamic differs between adult and developing brain. CSF turnover increases at birth, and the total CSF (cisternal, subarachnoid, and ventricular) to brain volume ratio is largely higher in perinatal brain (unpublished data). Taken together, these observations indicate that the impact of blood-CSF exchanges on the overall cerebral bioavailability of drugs or toxic compounds is likely to be more important in young than in adult individuals. At the level of the BBBs proper, the neuroprotective functions result from intercellular tight junctions sealing the barrier cell layers, as well as detoxification enzymes and efflux transporters located in the barrier cells (Strazielle and Ghersi-Egea, 2013). It is now clearly established that non-specific paracellular diffusion through the cerebral endothelium and the choroidal epithelium is efficiently restricted by the tight junctions early during development (Liddelow et al., 2013).

This review focuses on efflux transporters and analyses the data relative to their developmental pattern of localization, expression and function. These multispecific transport systems play a key role in preventing the entry into the brain and CSF of numerous blood-borne xenobiotics, including drugs, or in increasing the clearance of potentially harmful organic ions from the brain. They include ATP-Binding Cassette (ABC) transporters of the $\mathrm{ABCB}, \mathrm{ABCC}$, and $\mathrm{ABCG}$ families, solute carriers for organic anions and cations of the SLC21/SLCO and SLC22 families, and peptide transporters of the SLC15 family (Figure 1). These efflux transporters also recognize biologically active endogenous compounds, including steroid hormones and eicosanoids (leukotrienes, prostaglandins), thus protecting the

\footnotetext{
${ }^{1}$ Capitalized characters are used when referring to human transporters or to transporters listed in a general context. Minor characters are used when specifically referring to rodent transporters.
}

brain from a potentially deleterious overproduction of these compounds.

\section{ATP-BINDING CASSETTE TRANSPORTERS}

Multispecific efflux transporters that belong to the ABC transporter superfamily and are found in blood-brain interfaces include ABCB1 (P-glycoprotein), ABCG2 (breast cancer resistance protein, Bcrp), and several ABCCs (multidrug-related resistance proteins, Mrp) (Schinkel et al., 1996; Nishino et al., 1999; Wijnholds et al., 2000; Cooray et al., 2002; Strazielle and GhersiEgea, 2013). Located at the blood-facing membranes they prevent brain penetration of their substrates by an energy-dependent, unidirectional, outwardly directed transport mechanism. They are responsible for the efflux of many structurally unrelated lipophilic and amphiphilic environmental toxic compounds or drugs, including anti-inflammatory, immunosuppressive, antiinfectious, and antineoplastic drugs, some antiepileptic, antidepressant and psychotropic agents, and drug conjugates in the case of ABCCs (Schinkel and Jonker, 2003; Leslie et al., 2005; Staud and Pavek, 2005; Strazielle and Ghersi-Egea, 2005). These $\mathrm{ABC}$ transporters are differentially localized between the two main blood-brain interfaces (Figure 1). ABCB1 and ABCG2 are highly expressed at the $\mathrm{BBB}$, while $\mathrm{ABCC} 1$ is a landmark of the BCSFB (Daood et al., 2008; Gazzin et al., 2008). Abcc2 has been described in the rodent BBB (e.g., Wang et al., 2014), and Abcc4 expression and function have been described in both interfaces in mice (Leggas et al., 2004). Species differences are found, namely for ABCG2 which is likely to play a more important protective function in human than in rodent (Shawahna et al., 2011), and $\mathrm{ABCB} 1$, which is encoded by a single gene in human and 2 genes in rat and mouse. Species difference may also exist for ABCC2 whose expression at the BBB in normal human brain is still an open question (Kubota et al., 2006; Dauchy et al., 2008).

Several studies suggest that ABC transporters are already functional at the blood-brain interfaces in neonates, but that their expression is developmentally regulated in a protein- and barrierspecific manner.

\section{Developmental pattern of ABCB localization, expression and activity at blood-brain interfaces}

In mouse and rat, the Abcb1-dependent phenotype of multidrug resistance is associated with 2 genes Abcbla and Abcblb, both homologs to the human ABCB1 transporter gene. Abcbla and $A b c b 1 b$ are expressed in a tissue-specific manner, and also differ to some extent in their transport properties. Abcbla is closer to the human transporter in terms of amino acid primary sequence and gene regulatory region, and is the predominant gene expressed at the $\mathrm{BBB}$. Its expression is already detectable in endothelial cells in the neural tube of 10.5-day-old mouse embryos (Qin and Sato, 1995). Immunohistochemical analysis performed with different antibodies on human tissues showed that Abcb1 protein consistently localizes at the membrane of brain microvessels by mid-gestation. Before this stage, the signal is heterogeneous and displays regional differences (Schumacher and Mollgard, 1997; Virgintino et al., 2008). Accordingly, Gazzin and coll. reported that this transporter is present in rat brain 


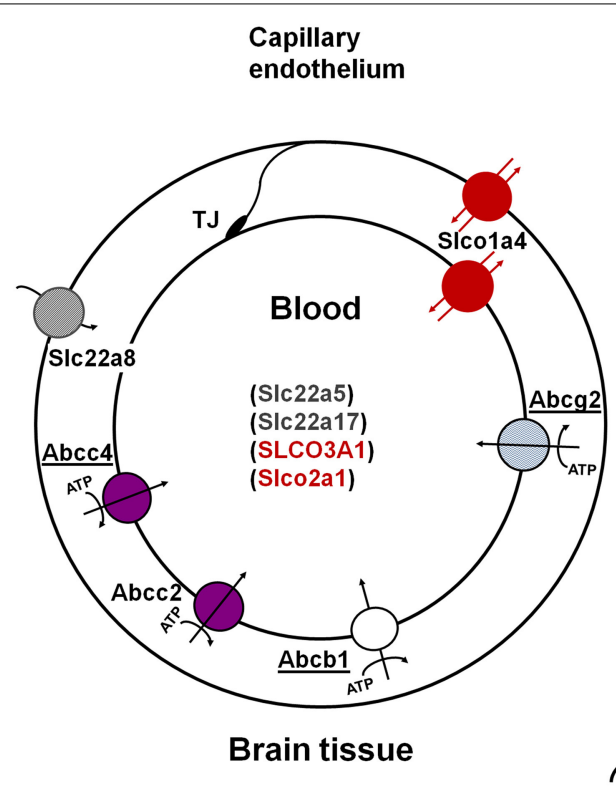

FIGURE 1|Schematic representation of the main multispecific transporters involved in neuroprotective efflux at the blood-brain (left) and blood-CSF (right) barriers. Underlined names represent transporters for which evidence has also been reported in human. The
Choroidal epithelium

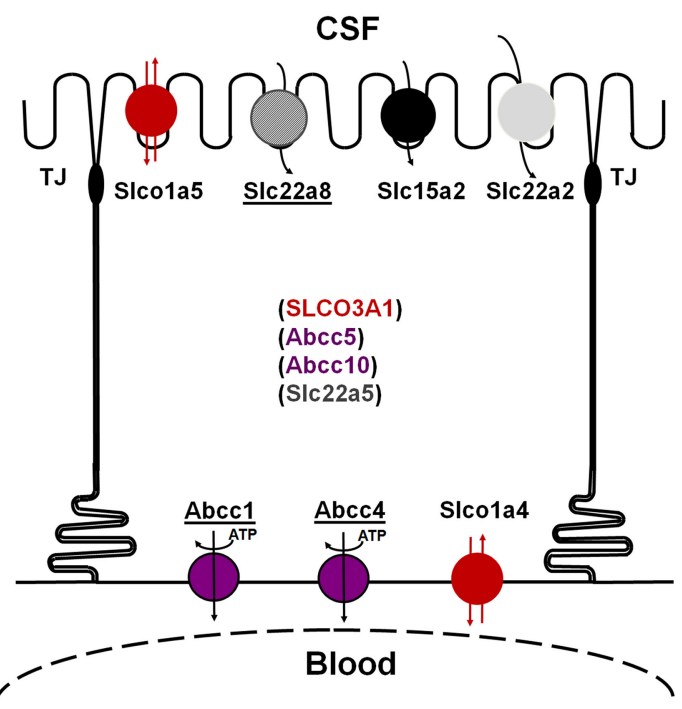

ATP-dependency is shown only for primary ATP-dependent transporters. Transporters in parenthesis are carriers for which evidence of their involvement in CNS-blood efflux processes is still limited. See text for references. TJ, tight junction. microvessels at birth, a developmental stage which corresponds to preterm development in human (Gazzin et al., 2008). As would be required for its neuroprotective efflux function, Abcb1 immunoreactivity was found at the luminal membrane of the endothelial wall. Because the cerebral capillary density increases greatly during development, the amount of Abcb1 protein at the $\mathrm{BBB}$ cannot be compared between stages using total brain homogenates. A standard curve-based quantitative Western blot analysis performed on isolated microvessels showed that the amount of Abcb1 in the BBB in 9- and 17-day-old rats was only one-fifth of that found in microvessels isolated from adult animals (Gazzin et al., 2008, 2011). This is in agreement with the more intense immunohistochemical signal observed in brain microvessels from adult as compared to perinatal or postnatal developmental stages in human, rat or mouse (Matsuoka et al., 1999; Tsai et al., 2002; Daood et al., 2008; Gazzin et al., 2011). It is also consistent with the 3-to-4-fold increase in Abcbla mRNA levels measured in endothelial cells from adult mice or rats compared to levels in endothelial cells of developing animals (2- to 8-day-old mouse, 14-day-old rat) (Daneman et al., 2010a; Harati et al., 2012a). The low expression and protein level of Abcb1 observed at the BBB in different species at late prenatal and early postnatal developmental stages may therefore result in a weaker protection of the brain against circulating compounds that are handled by this transporter. Accordingly, a 80-90\% lower brain accumulation of cyclosporin A and digoxin, assessed as brainblood/plasma ratio $2 \mathrm{~h}$ following administration, was observed in adult mice compared with 1-day-old mice, a difference which was abolished in Abcbla deficient animals (Goralski et al., 2006). A number of other functional studies have been conducted using digoxine as Abcb1 substrate. A greater accumulation of this molecule was observed in the brain of 15.5-day-old embryonic mice compared to the brain of 18.5-day-old embryonic mice (Petropoulos et al., 2010). In rat, the unbound brain-to-plasma concentration ratio of the molecule measured at steady-state was not different between 3-week- and 12-week-old animals (Harati et al., 2012a). A positron emission tomography study conducted in rhesus monkeys showed a significantly higher clearance value for verapamil uptake in 9-month-old animals than in 2- and 4year-old animals (Takashima et al., 2011). With the reservation that substrate specificities for Abcb1 are not absolute, in particular for digoxin which can be recognized by other transporters such as Slco1a4/oatp2, overall, these partial data suggest that ABCB1 functional activity parallels gene expression levels, both increasing from prenatal to pre-adult stages in various animal species, including non-human primates.

The amount of $\mathrm{ABCB} 1$ protein in rat and human choroid plexus is low by comparison to the levels measured in brain microvessels (Gazzin et al., 2008), and its cellular and subcellular localization within the choroidal tissue is subject to conflictual results. Its implication in the regulation of exchanges across the BCSFB is likely limited. In contrast to the BBB, Abcbla mRNAs are not detected by RT-PCR in the BCSFB. The gene expressed in rat choroid plexus is Abcblb. The level of expression, very low in adult rat tissue, is $4-8$ times higher during the perinatal period of development, with a peak on postnatal day 2 (Kratzer et al., 2013). The actual functional activity of the transporter and the significance of this perinatal upregulation of choroidal Abcblb expression are not known.

\section{Developmental pattern of ABCG2 localization, expression and activity at blood-brain interfaces}

There are few data available on the developmental expression of ABCG2, but there is a general agreement that the transporter 
is associated with the brain vasculature and localizes at the luminal membrane of brain endothelium early during development, specifically by week 22 of gestation in human (Daood et al., 2008), embryonic day 12.5 in mouse and in rat (Tachikawa et al., 2005; Orford et al., 2009; Ek et al., 2010). No apparent difference in signal intensity was observed throughout development in these studies. Only a moderate (2-fold or less) increase in Abcg2 mRNA levels was observed in endothelial cells isolated from adult vs. postnatal mouse brain (Daneman et al., 2010a) or between cerebral capillary preparations of comparable purity isolated from 14-day-old rats vs. adult animals (Harati et al., 2012a). In the latter study no difference in Abcg2 protein level was observed by Western blot between the two stages. Functional studies on Abcg2 mediated transport during development have been performed only in rat on postnatal day 21 . At that stage, blood-to-brain prazosin transport was not different from that measured in adult rats (Harati et al., 2012a). Overall, ABCG2 seems to reach high levels of expression at the BBB early during development. It may compensate for the lack of Abcb1-dependent protection at a period of high brain vulnerability.

Studies on the expression and function of Abcg2 in choroid plexus generated apparently conflicting results, possibly linked to species differences, or due to difficulties in achieving specific immunolabeling in this tissue. The level of protein assessed by Western blot is clearly much lower in choroidal tissue than in brain capillaries, as illustrated in the work performed by Reichel and colleagues in rat (Reichel et al., 2011). They loaded 10 times more proteins from choroidal tissue than from microvessels to be able to detect Abcg2 in the former sample, while the capillary fraction yielded an intense signal. In line with that report, an immunohistochemical study conducted on human tissue failed to detect ABCG2 in the choroid plexus at any developmental stage (extending from 22 weeks of gestation to adult), while the transporter was clearly detected in the microvasculature (Daood et al., 2008). Using transgenic mice expressing GFP under the control of Abcg2 promoter/enhancer sequences, Orford and colleagues showed that the transporter is associated with choroidal vessels from early on during development but not with the choroidal epithelium (Orford et al., 2009). By contrast, the protein was detected at the apical membrane of the choroidal epithelium in adult mouse, a signal that was not seen in Abcg2 knock-out animals (Tachikawa et al., 2005; Zhuang et al., 2006). No developmental data was provided in these studies. Other studies failed to detect the protein in choroid plexus epithelium of the adult rat (Roberts et al., 2008; Ek et al., 2010). One of the latter studies reported Abcg2 immunoreactivity at the basolateral membrane of the choroidal epithelium only at early developmental stages (embryonic day 15 and postnatal day 1) (Ek et al., 2010). This pattern of Abcg2 protein expression in rat parallels the temporal profile of choroidal Abcg 2 mRNA levels which are also higher in pre- and perinatal stages than in adult (Ek et al., 2010; Kratzer et al., 2013). It has to be noted that mRNA levels are low in choroid plexus in both rat and mouse (Tachikawa et al., 2005; Kratzer et al., 2013). Overall, if Abcg2 has any significant transport function at the BCSFB, it should be especially relevant to the developing period.

\section{Developmental localization, expression and activity of efflux transporters of the ABCC family at blood-brain interfaces}

An immunohistochemical signal for ABCC1 was consistently observed in human choroid plexus epithelium early in gestation, from week 22 onwards (Daood et al., 2008). In rat, Abccl protein was detected in choroid plexus epithelial cells as early as embryonic day 15, with a basolateral localization consistent with its efflux transporter activity and a neuroprotective function (Gazzin et al., 2008; Ek et al., 2010). Choroidal mRNA levels were 2to 3-fold lower in 15-day-old embryos and 7-day-old rat pups than in adult (Ek et al., 2010; Kratzer et al., 2013), but at birth, protein levels measured by quantitative Western blot are similar to those in adult (Gazzin et al., 2008), suggesting that Abcc1mediated efflux at the BCSFB is active early during development. Protein and mRNA levels of Abccl are low in both the rodent and human microvasculature in comparison to choroidal levels, and the actual localization and efflux activity of the protein at the BBB proper remains controversial (reviewed in Gazzin et al., 2008). One study investigated $\mathrm{ABCC} 1$ protein in the human $\mathrm{BBB}$ during development and failed to detect the transporter at any developmental stage (Daood et al., 2008). Only rat mRNA expression data are available concerning the developmental pattern of other Abcc transporters. In the choroid plexus, the temporal expression profile for Abcc4, for which functional evidence of transport has been provided in vivo in adult mice (Leggas et al., 2004), follows a developmental pattern similar to that of Abcc1, with a limited increase in mRNA level in adult tissue compared to tissue from embryonic day 19. As in choroid plexus, mRNA levels in brain endothelial cells are moderately (3.3 times) lower in young mice (postnatal day 2-8) than in adult animals (Daneman et al., 2010a). The concurrent expression of this transporter in the BCSFB and the BBB from early stages on, and its polarized localization at blood-facing membranes in both barriers suggest that it plays an important role in the clearance of endogenous substrates in young and adult individuals.

Abcc5, Abcc9, and Abcc10 genes investigated in rat choroid plexus are expressed at a similar or higher level in developing animals compared to adults (Kratzer et al., 2013). Functionally, an Abcc-dependent basolateral efflux of intracellularly formed drug conjugates has been demonstrated in freshly isolated choroid plexuses or in choroid plexus epithelial cells, both obtained from 2-day-old rats (Strazielle and Ghersi-Egea, 1999; GhersiEgea et al., 2006). These ex vivo/in vitro studies indicated that one or several Abcc proteins are efficiently participating to a neuroprotective enzymatic BCSFB during development.

\section{SOLUTE CARRIER PROTEINS}

The SLC superfamily comprises virtually all transporters that do not belong to the ABC family. Multispecific SLC transporters responsible for the neuroprotective efflux of exogenous and endogenous toxic molecules from brain or CSF to blood belong mainly to three subfamilies. The SLC22 subfamily includes organic anion and cation efflux transporters also referred to as OAT, OCT, and OCTN proteins. SLCO transporters, previously known as SLC21 form another subfamily of organic anion transport polypeptides (OATPs). Of note, several SLCO rodent genes have no homologs in human, and vice-versa. SLCO and SLC22 
transporters accept as substrates a broad range of cationic and anionic compounds, including environmental pollutants, and various drugs such as antibiotics and nucleosidic antiviral drugs, non-steroidal anti-inflammatory agents, and some antiepileptic drugs. They also transport steroid or drug conjugates, and lipid mediators (Strazielle et al., 2003; Hagenbuch and Stieger, 2013; Koepsell, 2013). Finally, members of the SLC15 family, known as peptide/proton cotransporters, transport in addition to endogenous di- and tripeptides, some peptidomimetic drugs, e.g., $\beta$-lactam antibiotics, antiviral nucleoside prodrugs or angiotensin converting enzymes (Daniel and Rubio-Aliaga, 2003).

Some of these solute carriers are bidirectional. Others function as unidirectional inwardly-directed multispecific transport systems and exchange their substrates with specific intracellular divalent organic anions by a coupled secondary energydependent mechanism. SLC transporters that are involved in brain efflux localize at the abluminal (BBB) or apical (BCSFB) membrane of barrier cells, where they remove drugs and toxic compounds from brain extracellular and CSF spaces. The most documented SLC efflux transporters at blood-brain interfaces are the following. SLC22A8/OAT3 is present at both brain endothelial cells and choroidal epithelial cells in rodents, and is also detected in human choroid plexus (Nagata et al., 2002; Alebouyeh et al., 2003; Kikuchi et al., 2003; Mori et al., 2003; Ose et al., 2009). Slco1a5/Oatp3, Slc15a2/Pept2, and possibly Slc22a2/Oct2 are restricted to choroid plexus (Kusuhara et al., 2003; Ohtsuki et al., 2003). Other organic anion transporters are found at blood-brain interfaces, but their possible involvement in brain efflux rather than influx processes remaining to be established (Figure 1). Slco1a4/oatp2 is found at both luminal and abluminal membranes of brain endothelial cells, and only at the basolateral membrane of the choroidal epithelium in rat (Gao et al., 1999). In human, two SLCO3A1 variants v1 and v2, of similar and broad substrate specificity, are respectively located on the basolateral and apical membranes of the choroidal epithelium, suggesting that SLCO3A1 may function both as an influx and an efflux transporter (Huber et al., 2007). SLCO1A2 and SLCO2B1, also expressed at the human $\mathrm{BBB}$, are likely to mediate brain influx rather than brain efflux, on the basis of their localization at the luminal membrane of the endothelium (Gao et al., 2000; Bronger et al., 2005).

\section{Developmental localization, expression and activity of SLC22 and SLCO efflux transporters at blood-brain interfaces}

Data on the developmental expression of efflux transporters that belong to the SLC22 and SLCO gene families are scarce and obtained only from rat and mouse studies. Transcriptomic analysis of endothelial cells isolated from adult vs. postnatal mouse brains suggests that developmental profiles are heterogeneous among genes (Daneman et al., 2010a). While mRNA levels for Slc22a5 (octn2) and Slcola4 were 2-to 5-fold lower in endothelial cells from developing animals compared to adults, Slc22a8 expression was similar at both stages. Slc22a17 and Slco2b1, whose precise functions are currently unknown at brain barriers, did not display developmental changes either. Of note in this study, the mRNA levels of Slco2al, the prostaglandin transporter with an unknown directionality of transport, were much higher at the BBB from developing vs. adult mice. This suggests a developmentally regulated mechanism for blood-brain exchange of prostaglandins, possibly related to the function of these lipid mediators in brain maturation. In rat, a lower expression was reported for Slcola4 between developing and adult animal, while there was no change for Slc22a8 (Harati et al., 2012a). This was assessed by quantitative PCR and Western blot on isolated capillaries and confirms the mouse transcriptomic observations.

The analysis of transcriptomic profiles established in rat choroid plexus for 14 Slco and Slc22 members showed that most genes, including Slc22a5, Slco1a4, and Slcola5 are expressed at similar or higher levels during the perinatal period than at the adult stage. One exception is Slc22a8, for which expression levels are respectively 4 -fold and 2 -fold lower in 19-day-old embryos and 2-day-old rats than in adults. At an earlier stage, in 15day-old embryos, gene expression levels relative to adult were more heterogeneous (Kratzer et al., 2013). A functional analysis showed that ex vivo uptake of bromosulfophthalein, a typical Slco substrate, was 3-fold higher in choroid plexuses isolated from newborn rat compared to adult rat (Angeletti et al., 1998). Western blot and immunohistochemical localization of Slco were also performed in this study, but are difficult to interpret as, at that time, Slcola5 was mistakenly identified as Slcolal in this tissue. As further evidence of organic anion transport maturity at the BCSFB, choroidal epithelial cells isolated from newborn rats efficiently mediate the transport of Slc22 and Slco substrates such as 2,4-dichlorophenoxyacetic acid, benzylpenicillin, zidovudine or taurocholate in an unidirectional CSF-to-blood direction (Strazielle et al., 2003).

\section{Developmental localization and expression of SLC15 efflux transporters at the choroid plexus}

Among members of the SLC15 family, Slc15a2 is well expressed in the brain, with the highest level in the choroid plexus (Shen et al., 2004). Both mRNA levels and immunohistochemical signals for Slc15a2 were similar in rat choroid plexuses at perinatal and adult stages (Shen et al., 2004; Kratzer et al., 2013). The apical localization of this choroidal transporter suggests its efficacy in exporting neuropeptides, peptide fragments, and peptide-like drugs out of CSF from early on during brain development.

The overall developmental profiles of all ABC and SLC efflux transporters reviewed above are summarized in Table 1. It shows that regarding the expression of efflux transporters, an overall adult-like phenotype is reached earlier at the BCSFB than at the BBB.

\section{REGULATION OF EFFLUX TRANSPORTER ASSOCIATED BARRIER PROPERTIES DURING DEVELOPMENT}

Signaling pathways involved in the early specification of brain barrier cells have been identified in the last decade, pointing out a few molecular cues that specifically regulate the developmental expression of efflux transporters. In addition, these transport systems are modulated in disease and by various xenobiotics via mechanisms that may bear developmental specificities. 
Table 1 | Comparative analysis of the developmental expression pattern of efflux transporters between the blood-brain- and blood-CSF barriers.

\begin{tabular}{|c|c|c|c|c|c|}
\hline \multicolumn{3}{|c|}{ Blood-brain barrier } & \multicolumn{3}{|c|}{ Blood-CSF barrier } \\
\hline ABCG2/Abcg2 & $=$ & mRNA, (Prot) & Abcc1 & $=$ & mRNA, Prot \\
\hline Abcc4 & $\nearrow$ & mRNA & Abcc4 & $\leq$ & mRNA \\
\hline \multirow[t]{2}{*}{ Slc22a8 } & $=$ & mRNA, Prot & Slc22a8 & $\nearrow$ & mRNA \\
\hline & & & Pept2/SIc15 & $=$ & mRNA (Prot) \\
\hline Other multispecific transporters & \multicolumn{2}{|c|}{ Developmental pattern } & Other multispecific transporters & \multicolumn{2}{|c|}{ Developmental pattern } \\
\hline Slc22a5 & $\nearrow$ & mRNA & Abcb1b & $\searrow$ & mRNA \\
\hline \multirow{2}{*}{ Slco2a1 $\rightarrow$} & & & Slc22a5 & $=$ & mRNA \\
\hline & & & Slco1a4 & $=$ & mRNA \\
\hline
\end{tabular}

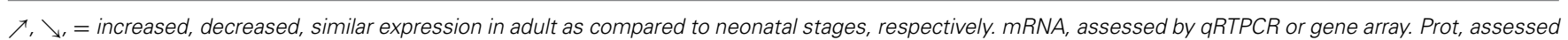
by quantitative western blot. (Prot), assessed by immunohistochemical signal intensity. Fc, assessed by functional studies, using specific substrates. For references, see text.

\section{Pathways involved in the developmental regulation of efflux transporters at blood-brain interfaces}

The unique phenotype of brain microvessel endothelial cells compared to other endothelia in the body has long ago raised the hypothesis that surrounding neural cells were responsible for inducing BBB characteristics. A series of grafting experiments generated controversial results regarding this theory (reviewed in Bauer and Bauer, 2000). Arguments in favor of a role for adjacent cells, notably astrocytes emerged from many in vitro studies. It was thought for example, that the BBB hallmark transporter Abcb1 was under astrocytic regulation on the basis that its expression and functionality, which are partially lost in brain microvessel endothelial cells in culture were reinduced in endothelial cells co-cultured with glial cells. However, because of the temporal pattern of neural cell differentiation and the late apparition of mature astrocytes which in rat and mouse occurs only around birth, these cells obviously cannot impart the inducing signals that trigger BBB formation in neovessels. Astrocytes nonetheless are essential later for further maturation of the $\mathrm{BBB}$ (see infra), or for maintenance of its properties as suggested by the link between some astrocyte diseases and BBB dysfunction (Keller, 2013).

A large number of studies have recently been directed toward identifying the molecules, pathways, and cells responsible for CNS specific vascular differentiation. The Wnt/ $\beta$-catenin canonical pathway has emerged as a prominent signaling mechanism, promoting angiogenesis and concurrently inducing hallmark features of the $\mathrm{BBB}$, such as the expression of the glucose transporter Slc2a1 (reviewed in Liebner et al., 2011). Wnt factors are secreted as early as embryonic day 9 by neural progenitors. The various studies dedicated to this topic did not examine the direct effects of Wnt signaling on the expression of ABC and SLC efflux transporters at the BBB. DNA binding sites for $\mathrm{T}$ cell factor/lymphoid enhancer-binding factor TCF/LEF, the transcriptional activating factor downstream of the Wnt/ $\beta$-catenin pathway, are present in the human ABCB1 gene promoter (Yamada et al., 2000). Functional evidence linking $\beta$-catenin accumulation with intestinal expression of the human ABCB1 gene or the mouse Abcbla gene has been reported in intestinal cells (Scotto, 2003). Regarding the BBB, activation of $\beta$-catenin signaling by GSK-3 inhibition in the human brain endothelial cell line hMEC/D3 as well as in rat brain endothelial cells in primary culture similarly increased $\mathrm{ABCB} 1$ expression and function (Lim et al., 2008). The expression of ABCC4 and ABCG2 genes was also modulated in the hMEC/D3 cells in response to $\beta$-catenin signaling activation or inhibition, suggesting that the early embryonic expression of Abcg2 described in rat and mouse could depend on the same regulatory pathway. Although direct evidence in the embryonic brain is lacking, these data support the idea that the range of genes associated with the $\mathrm{BBB}$ phenotype and induced by the canonical Wnt pathway in newly formed cerebral vessels include efflux transporters. Other regulatory mechanisms are expected to add on this triggering mechanism to explain the different profiles observed among efflux transporters, such as between Abcb1 and Abcg2 for example (see supra).

Retinoic acid, a potent morphogen directing the development of the nervous system, has been proposed as another signaling cue contributing to the induction of the specific $\mathrm{BBB}$ phenotype at an early stage of angiogenesis. In human fetal brain, radial glial cells express the final enzyme involved in retinoic acid biosynthesis, while endothelial cells express one of the receptors for 
this signaling factor (Mizee et al., 2013). Treatment of mice from day 10.5 to day 16.5 of gestation with a retinoic acid receptor antagonist led to the downregulation of several BBB distinct properties in embryos, in particular to a decrease in the expression level of Abcg2 gene. One limitation of this result was that RNA levels were measured on the whole brain including a priori the choroid plexus as well as the subventricular zone, both regions of the brain that express the Abcg2 gene at substantial levels during this period. It is possible that the decrease observed in treated embryos on day 16.5 may not reflect changes at the BBB per se, but rather in either one or both of these regions (Orford et al., 2009). Immunostaining of Abcg2 in these animals should clarify this point.

In addition to neural progenitors and radial glial cells, pericytes are present in the early phase of cerebral angiogenesis, and are recruited by the nascent vessels invading the neuroectoderm. A major role of pericytes in influencing the endothelial phenotype has been clearly demonstrated in vivo for a number of features, including the loss of transcytotic vesicles, or the induction of the glucose transporter Slc2al (Armulik et al., 2010; Daneman et al., 2010b). By contrast, transcript levels for Abcbla and Abcg2 in endothelial cells of 18-day-old mouse embryos did not differ between pericyte deficient Pdgfrb-/- animals and littermate controls (Daneman et al., 2010b), suggesting that initial induction of these efflux transporters is not under pericyte control.

Because similar deletion of astrocytes in vivo is not possible, current evidence for the influence of these cells on the differentiated status of BBB endothelial cells has been gathered using culture systems. One bias in these in vitro studies arises from the fact that endothelial cells are isolated from adult brain, thus with an already mature phenotype, and they downregulate some of their BBB-specific properties. For that reason, astrocytic effects observed in vitro may reflect not only maturation processes occurring when these cells appear, but also repair mechanisms triggered under pathological conditions. With regard to efflux transporters, the focus has been mainly on ABCB1. Its protein level and function are increased in endothelial cells cocultured with astrocytes, compared to endothelial cells alone (see for example Fenart et al., 1998; Gaillard et al., 2000). This reinduction occurs in the absence of any direct contact between the two cell populations, inferring the role of astrocytic soluble factors. Some of these astrocytederived molecules that act on endothelial receptors to promote BBB-specific properties have been identified (Abbott et al., 2006; Alvarez et al., 2011, for a review). They include sonic hedgehog ligands, angiopoietin 1 which is a ligand for the endothelial Tie 2 tyrosine kinase receptor, or TGF- $\beta 1$.Their role on the expression and function of ABC and SLC efflux transporters still need to be evaluated in vivo, using animals deficient for these pathways or pharmacological inhibition approaches. Evidence for the role of these signaling molecules in regulating the expression and activity of efflux transporters has been provided in other cell types. For example, activation of the sonic hedgehog pathway associates with elevated therapeutic resistance in cancer cells, in part due to increased protein level and activity of both ABCB1 and ABCG2 (Sims-Mourtada et al., 2007). Consistently, the promoter region of the ABCG2 gene contains a binding site for the downstream transcription factor Gli and is a direct target of sonic hedgehog signaling (Singh et al., 2011). Tie2 activation also promotes a chemoresistance phenotype in gliomas by inducing ABCB1 expression level and activity (Martin et al., 2009), and TGF- $\beta 1$-mediated increase in Abcb1 expression and activity has been demonstrated in guinea pig brain endothelial cells in culture (Baello et al., 2014).

If significant progress has been achieved in the understanding of how the BBB gains its distinct phenotype, the signaling pathways responsible for the early specification of the choroidal epithelium vs. ependyma remain obscure, in particular concerning the regulation of expression of Slc and Abcc genes. The hepatic nuclear factor $4 \alpha(\mathrm{HNF}-4 \alpha)$ is a key regulator of differentiation in the liver or the intestine, where it stimulates in particular the transcription of drug metabolizing enzymes and efflux transporters. HNF- $4 \alpha$ is expressed in choroid plexus epithelial cells in rat and human at the adult stage, and can bind to regulatory sites in the ABCC1 gene promoter (Niehof and Borlak, 2009). Further work is needed to confirm its potential role in controlling the early choroidal-specific transcription of Abccl.

\section{Modulation of efflux transporters in response to injury and environmental insults during development}

The pathogenesis of perinatal injuries most frequently involves hypoxia/ischemia, maternal-fetal infection or inflammation (Aden et al., 2010). These insults are associated with the secretion of proinflammatory cytokines, as well as oxidative/electrophilic stress for which the neonatal brain shows a selectively high susceptibility (Ferriero, 2001). Neuroprotective genes including anti-oxidant genes and efflux transporter genes of the $\mathrm{ABC}$ and SLC families are known to be regulated by these stress signals as part of the coordinated defense mechanisms implemented by the cells in response to oxidative stress. The regulatory pathways involve transcription factors that generally act as sensors in the cytoplasm and translocate to the nucleus to increase the transcription of target genes. Oxidative species and the lack of oxygen can be sensed by the nuclear factor erythroid 2-related factor 2 (Nrf2) and the hypoxia inducible factor 1alpha HIF-1 $\alpha$, respectively. There is mounting evidence that these stress sensing factors are expressed and functional in cells of the blood-braininterfaces. Nrf2-dependent signaling increases Abcb1, Abcg2, and Abcc 2 expression levels in rat brain microvessels, and accordingly decreases brain uptake of verapamil, a substrate of Abcb1 (Wang et al., 2014). The efficacy of Nrf2 signaling in improving neuroprotection at the microvasculature during brain development has not been explored. The functionality of the Nrf2-dependent pathway at the BCSFB was assessed in vitro, in choroidal epithelial cells, through Nrf2 translocation in the nucleus upon isothiocyanate treatment and transcriptional upregulation of downstream genes (e.g., heme oxygenase, Xiang et al., 2012). It is notable that in rat choroid plexus, Nrf2, HIF- $1 \alpha$, and HIF- $1 \beta$ display a strikingly steady expression from embryonic day 15 to the adult stage (Kratzer et al., 2013). We can therefore speculate that the choroid plexus will react to oxidative/electrophilic and hypoxic stresses at least, by increasing neuroprotective genes at the BCSFB early during development. Yet, this needs further investigation, since hypoxia/ischemia in 9-day-old mice did not change the level of expression of Abccl in the choroid plexus, 
although other target genes for Nrf2, such as heme oxygenase or glutathione-S-transferase a1 were strongly upregulated (D'Angelo et al., 2013). This suggests that Nrf2 signaling is functional in the choroid plexus of young animals, but that not all target genes are equally sensitive at that stage, or share the same kinetic of induction.

Regarding the consequences of inflammatory challenges on efflux transporters, Abcb1 regulation has been investigated in details in freshly isolated rat capillaries (reviewed in Hartz and Bauer, 2010). Its expression and functional activity in adult is differently modulated by short term and long term exposure to pro-inflammatory stimulus. The extent and direction of change in ABCB1 expression is also disease-specific (reviewed in Stolp et al., 2013). During development, inflammation-associated changes in BBB efflux transporters can be stage-dependent. For instance, Abcb1 and Abcg2 efflux function at the BBB increase in response to endothelin-1 in adult, but not in juvenile (21-day-old) rats. This difference may be related to the lower cytokinic response of the $\mathrm{BBB}$ to endothelin-1 treatment in juvenile compared to adult animals (Harati et al., 2012b). More work is needed to delineate the role of inflammation in the regulation of efflux transporters at the BBB during development, and the actual window of susceptibility. The choroid plexus is very sensitive to inflammatory challenges, rapidly responding through increased secretion of inflammatory mediators including chemoattractants and proinflammatory cytokines. Exposure of the choroidal epithelium in vitro to activated immune cells or cytokines leads to a decrease in CSF-to-blood efflux of prostaglandin $\mathrm{E}_{2}$, a transport process which is likely mediated by SLC and ABC efflux transporters (Khuth et al., 2005; Batisson et al., 2006). Little is known so far on the regulation of efflux transporters in vivo at the BCSFB in the context of perinatal inflammation. Changes in such transport mechanisms that set the CSF levels of different biologically active endogenous organic anions including prostanoids may have dramatic consequences for brain development and maturation, and deserves further exploration.

In different peripheral organs such as the liver, exposure to environmental toxicants or food components can activate additional transcription factors belonging to the nuclear factor family, which in turn trigger the induction of protective genes including efflux transporter genes. Among these xenosensors, the aryl hydrocarbon receptor (AhR), the constitutive androstane receptor (CAR), or the pregnane $\mathrm{X}$ receptor (PXR) have efflux transporters among their target genes (see Scotto, 2003 for a review of the mechanisms involved). Current knowledge about these nuclear receptors indicates that they are expressed in cells of the blood-brain interfaces in animals and human. AhR is expressed in human brain microvessels, while additional expression of PXR and CAR genes has been reported for the rodent BBB (Dauchy et al., 2008; Hartz and Bauer, 2010). Upon ligand activation, these nuclear factors were shown to induce the expression and/or function of Abcb1, Abcc2, or Abcg2 (reviewed in Hartz and Bauer, 2010). It is still unknown whether nuclear receptors are expressed and modulate efflux transporters at the BBB during development.

In choroid plexus, gene expression can be detected for a wide range of these transcription factors in prenatal, postnatal, and adult stages of rat development, and their role in the coordinated induction of detoxifying enzymes and transporters at the BCSFB has been suggested (Kratzer et al., 2013).

\section{CONCLUSION}

The current limited data available in the literature reveal transporter-dependent developmental profiles of expression in blood-brain interfaces both in laboratory animals and in human. The developmental pattern of a given transporter can also differ between the BBB and the BCSFB (Table 1). The BCSFB achieves an "adult-like" phenotype earlier than the BBB. For instance, in the latter, Abcbl displays a major increase in expression postnatally. On the basis of recent studies on early BBB differentiation, we can speculate that neural progenitors and radial glial cells have a major role in triggering the endothelial expression of certain efflux transporters via Wnt and retinoic acid signaling. This would provide some degree of protection against deleterious compounds to the developing brain. Once vascular networks have formed, newly generated astrocytes induce further differentiation of the BBB, through the sonic hedgehog pathway. The concept of barrier immaturity during brain development may therefore hold for selected efflux transporters at the BBB. This novel view of a restricted barrier capacity of the developing brain endothelium is independent from the efficacy of its tight junctions sealing the paracellular pathway, which is established early during prenatal development. It needs to be taken into account when neonatal and pediatric treatments are discussed. The developmental regulation of efflux transporters at the choroid plexus remains obscure. Further work is needed on the signaling cues responsible for specifying the choroidal phenotype of the neuroepithelium and in particular for governing the expression of efflux transporter genes.

A number of choroidal ABC transporters, as well as choroidal and endothelial SLCO transporters display a higher expression or activity in developing animals than in adults. Additional studies, tackling especially the functionality and substrate specificity of these transporters, are necessary to better appreciate the significance of their stage-specific expression.

In the context of developmental diseases, aberrations in one of the signaling pathways that induce efflux transporterdependent protective functions in blood-brain interfaces may lead to substantial changes in fetal/neonatal brain exposure to undesired substrates, and subsequently alter normal brain development.

Choroid plexus studies suggest that from early on during development, brain efflux may be enhanced following exposure to xenobiotic inducers including drugs and environmental toxins. The modulation of efflux transporter expression and function by oxidative stress and inflammation often associated with perinatal injuries deserves further investigation. A better understanding of the regulatory pathways, in particular the relative contribution of the different transcription factors sensing oxidative stress or xenobiotics during development, is clinically relevant. It may open new opportunities for therapeutic manipulation designed to improve or restore blood-brain interface neuroprotective functions in the context of perinatal injuries. 


\section{ACKNOWLEDGMENTS}

This work was supported by ANR-10-IBHU-0003 Cesame grant and by the European Union Seventh Framework Program under grant agreement No. HEALTH-F2-2009-241778 Neurobid

\section{REFERENCES}

Abbott, N. J., Ronnback, L., and Hansson, E. (2006). Astrocyte-endothelial interactions at the blood-brain barrier. Nat. Rev. Neurosci. 7, 41-53. doi: $10.1038 / \mathrm{nrn} 1824$

Aden, U., Favrais, G., Plaisant, F., Winerdal, M., Felderhoff-Mueser, U., Lampa, J., et al. (2010). Systemic inflammation sensitizes the neonatal brain to excitotoxicity through a pro-/anti-inflammatory imbalance: key role of TNFalpha pathway and protection by etanercept. Brain. Behav. Immun. 24, 747-758. doi: 10.1016/j.bbi.2009.10.010

Alebouyeh, M., Takeda, M., Onozato, M. L., Tojo, A., Noshiro, R., Hasannejad, H., et al. (2003). Expression of human organic anion transporters in the choroid plexus and their interactions with neurotransmitter metabolites. J. Pharmacol. Sci. 93, 430-436. doi: 10.1254/jphs.93.430

Alvarez, J. I., Dodelet-Devillers, A., Kebir, H., Ifergan, I., Fabre, P. J., Terouz, S., et al. (2011). The Hedgehog pathway promotes blood-brain barrier integrity and CNS immune quiescence. Science 334, 1727-1731. doi: 10.1126/science.1206936

Angeletti, R. H., Bergwerk, A. J., Novikoff, P. M., and Wolkoff, A. W. (1998). Dichotomous development of the organic anion transport protein in liver and choroid plexus. Am. J. Physiol. 275, C882-C887.

Armulik, A., Genove, G., Mae, M., Nisancioglu, M. H., Wallgard, E., Niaudet, C., et al. (2010). Pericytes regulate the blood-brain barrier. Nature 468, 557-561. doi: 10.1038/nature09522

Baello, S., Iqbal, M., Bloise, E., Javam, M., Gibb, W., and Matthews, S. G. (2014). TGF-betal regulation of multidrug resistance P-glycoprotein in the developing male blood-brain barrier. Endocrinology 155, 475-484. doi: 10.1210/en.20131472

Batisson, M., Strazielle, N., Hejmadi, M., Thomas, D., Ghersi-Egea, J. F., Etienne, J., et al. (2006). Toxic shock syndrome toxin-1 challenges the neuroprotective functions of the choroidal epithelium and induces neurotoxicity. J. Infect. Dis. 194, 341-349. doi: 10.1086/505428

Bauer, H. C., and Bauer, H. (2000). Neural induction of the blood-brain barrier: still an enigma. Cell. Mol. Neurobiol. 20, 13-28. doi: 10.1023/A:1006939825857

Betz, A. L., and Goldstein, G. W. (1981). Developmental changes in metabolism and transport properties of capillaries isolated from rat brain. J. Physiol. 312, 365-376. doi: 10.1113/jphysiol.1981.sp013633

Bronger, H., Konig, J., Kopplow, K., Steiner, H. H., Ahmadi, R., Herold-Mende, C., et al. (2005). ABCC drug efflux pumps and organic anion uptake transporters in human gliomas and the blood-tumor barrier. Cancer Res. 65, 11419-11428. doi: 10.1158/0008-5472.CAN-05-1271

Caley, D. W., and Maxwell, D. S. (1970). Development of the blood vessels and extracellular spaces during postnatal maturation of rat cerebral cortex. J. Comp. Neurol. 138, 31-47. doi: 10.1002/cne.901380104

Cooray, H. C., Blackmore, C. G., Maskell, L., and Barrand, M. A. (2002). Localisation of breast cancer resistance protein in microvessel endothelium of human brain. Neuroreport 13, 2059-2063. doi: 10.1097/00001756-20021115000014

Daneman, R., Zhou, L., Agalliu, D., Cahoy, J. D., Kaushal, A., and Barres, B. A. (2010a). The mouse blood-brain barrier transcriptome: a new resource for understanding the development and function of brain endothelial cells. PLoS ONE 5:e13741. doi: 10.1371/journal.pone.0013741

Daneman, R., Zhou, L., Kebede, A. A., and Barres, B. A. (2010b). Pericytes are required for blood-brain barrier integrity during embryogenesis. Nature 468, 562-566. doi: 10.1038/nature09513

D'Angelo, B., Ek, C. J., Sandberg, M., and Mallard, C. (2013). Expression of the Nrf2-system at the blood-CSF barrier is modulated by neonatal inflammation and hypoxia-ischemia. J. Inherit. Metab. Dis. 36, 479-490. doi: 10.1007/s10545012-9551-5

Daniel, H., and Rubio-Aliaga, I. (2003). An update on renal peptide transporters. Am. J. Physiol. Renal Physiol. 284, F885-F892. doi: 10.1152/ajprenal.00123.2002

Daood, M., Tsai, C., Ahdab-Barmada, M., and Watchko, J. F. (2008). ABC transporter (P-gp/ABCB1, MRP1/ABCC1, BCRP/ABCG2) expression in the developing human CNS. Neuropediatrics 39, 211-218. doi: 10.1055/s-0028-1103272
Dauchy, S., Dutheil, F., Weaver, R. J., Chassoux, F., Daumas-Duport, C., Couraud, P. O., et al. (2008). ABC transporters, cytochromes P450 and their main transcription factors: expression at the human blood-brain barrier. J. Neurochem. 107, 1518-1528. doi: 10.1111/j.1471-4159.2008.05720.x

Ek, C. J., Wong, A., Liddelow, S. A., Johansson, P. A., Dziegielewska, K. M., and Saunders, N. R. (2010). Efflux mechanisms at the developing brain barriers: ABC-transporters in the fetal and postnatal rat. Toxicol. Lett. 197, 51-59. doi: 10.1016/j.toxlet.2010.04.025

Fenart, L., Buee-Scherrer, V., Descamps, L., Duhem, C., Poullain, M. G., Cecchelli, R., et al. (1998). Inhibition of P-glycoprotein: rapid assessment of its implication in blood-brain barrier integrity and drug transport to the brain by an in vitro model of the blood-brain barrier. Pharm. Res. 15, 993-1000. doi: 10.1023/A:1011913723928

Ferriero, D. M. (2001). Oxidant mechanisms in neonatal hypoxia-ischemia. Dev. Neurosci. 23, 198-202. doi: 10.1159/000046143

Gaillard, P. J., Van Der Sandt, I. C., Voorwinden, L. H., Vu, D., Nielsen, J. L., De Boer, A. G., et al. (2000). Astrocytes increase the functional expression of Pglycoprotein in an in vitro model of the blood-brain barrier. Pharm. Res. 17, 1198-1205. doi: 10.1023/A:1026406528530

Gao, B., Hagenbuch, B., Kullak-Ublick, G. A., Benke, D., Aguzzi, A., and Meier, P. J. (2000). Organic anion-transporting polypeptides mediate transport of opioid peptides across blood-brain barrier. J. Pharmacol. Exp. Ther. 294, 73-79.

Gao, B., Stieger, B., Noe, B., Fritschy, J. M., and Meier, P. J. (1999). Localization of the organic anion transporting polypeptide 2 (Oatp2) in capillary endothelium and choroid plexus epithelium of rat brain. J. Histochem. Cytochem. 47, 1255-1264. doi: 10.1177/002215549904701005

Gazzin, S., Berengeno, A. L., Strazielle, N., Fazzari, F., Raseni, A., Ostrow, J. D., et al. (2011). Modulation of Mrpl (ABCc1) and Pgp (ABCb1) by bilirubin at the blood-CSF and blood-brain barriers in the Gunn rat. PLoS ONE 6:e16165. doi: 10.1371/journal.pone.0016165

Gazzin, S., Strazielle, N., Schmitt, C., Fevre-Montange, M., Ostrow, J. D., Tiribelli, C., et al. (2008). Differential expression of the multidrug resistance-related proteins $\mathrm{ABCb} 1$ and $\mathrm{ABCc} 1$ between blood-brain interfaces. J. Comp. Neurol. 510, 497-507. doi: 10.1002/cne.21808

Ghersi-Egea, J. F., Monkkonen, K. S., Schmitt, C., Honnorat, J., Fevre-Montange, M., and Strazielle, N. (2009). Blood-brain interfaces and cerebral drug bioavailability. Rev. Neurol. (Paris) 165, 1029-1038. doi: 10.1016/j.neurol.2009.09.011

Ghersi-Egea, J. F., Strazielle, N., Murat, A., Jouvet, A., Buenerd, A., and Belin, M. F. (2006). Brain protection at the blood-cerebrospinal fluid interface involves a glutathione-dependent metabolic barrier mechanism. J. Cereb. Blood Flow Metab. 26, 1165-1175. doi: 10.1038/sj.jcbfm.9600267

Goralski, K. B., Acott, P. D., Fraser, A. D., Worth, D., and Sinal, C. J. (2006). Brain cyclosporin A levels are determined by ontogenic regulation of mdrla expression. Drug. Metab. Dispos. 34, 288-295. doi: 10.1124/dmd.105. 007427

Hagenbuch, B., and Stieger, B. (2013). The SLCO (former SLC21) superfamily of transporters. Mol. Aspects Med. 34, 396-412. doi: 10.1016/j.mam.2012.10.009

Harati, R., Benech, H., Villegier, A. S., and Mabondzo, A. (2012a). Pglycoprotein, breast cancer resistance protein, Organic Anion Transporter 3, and Transporting Peptide la4 during blood-brain barrier maturation: involvement of Wnt/beta-catenin and endothelin-1 signaling. Mol. Pharm. 10, 1566-1580. doi: $10.1021 / \mathrm{mp} 300334 \mathrm{r}$

Harati, R., Villegier, A. S., Banks, W. A., and Mabondzo, A. (2012b). Susceptibility of juvenile and adult blood-brain barrier to endothelin-1: regulation of $\mathrm{P}$ glycoprotein and breast cancer resistance protein expression and transport activity. J. Neuroinflammation 9, 273. doi: 10.1186/1742-2094-9-273

Hartz, A. M., and Bauer, B. (2010). Regulation of ABC transporters at the bloodbrain barrier: new targets for CNS therapy. Mol. Interv. 10, 293-304. doi: 10.1124/mi.10.5.6

Huber, R. D., Gao, B., Sidler Pfandler, M. A., Zhang-Fu, W., Leuthold, S., Hagenbuch, B., et al. (2007). Characterization of two splice variants of human organic anion transporting polypeptide 3A1 isolated from human brain. Am. J. Physiol. Cell Physiol. 292, C795-C806. doi: 10.1152/ajpcell.00597.2005

Kaindl, A. M., Favrais, G., and Gressens, P. (2009). Molecular mechanisms involved in injury to the preterm brain. J. Child Neurol. 24, 1112-1118. doi: $10.1177 / 0883073809337920$

Keller, A. (2013). Breaking and building the wall: the biology of the bloodbrain barrier in health and disease. Swiss Med. Wkly 143:w13892. doi: 10.4414/smw.2013.13892 
Khuth, S. T., Strazielle, N., Giraudon, P., Belin, M. F., and Ghersi-Egea, J. F. (2005). Impairment of blood-cerebrospinal fluid barrier properties by retrovirus-activated $\mathrm{T}$ lymphocytes: reduction in cerebrospinal fluid-to-blood efflux of prostaglandin E2. J. Neurochem. 94, 1580-1593. doi: 10.1111/j.14714159.2005.03309.x

Kikuchi, R., Kusuhara, H., Sugiyama, D., and Sugiyama, Y. (2003). Contribution of organic anion transporter 3 (Slc22a8) to the elimination of p-aminohippuric acid and benzylpenicillin across the blood-brain barrier. J. Pharmacol. Exp. Ther. 306, 51-58. doi: 10.1124/jpet.103.049197

Koepsell, H. (2013). The SLC22 family with transporters of organic cations, anions and zwitterions. Mol. Aspects Med. 34, 413-435. doi: 10.1016/j.mam.2012.10.010

Kratzer, I., Liddelow, S. A., Saunders, N. R., Dziegielewska, K. M., Strazielle, N., and Ghersi-Egea, J. F. (2013). Developmental changes in the transcriptome of the rat choroid plexus in relation to neuroprotection. Fluids Barriers CNS 10:25. doi: 10.1186/2045-8118-10-25

Kratzer, I., Vasiljevic, A., Rey, C., Fevre-Montange, M., Saunders, N., Strazielle, N., et al. (2012). Complexity and developmental changes in the expression pattern of claudins at the blood-CSF barrier. Histochem. Cell. Biol. 138, 861-879. doi: 10.1007/s00418-012-1001-9

Kubota, H., Ishihara, H., Langmann, T., Schmitz, G., Stieger, B., Wieser, H. G., et al. (2006). Distribution and functional activity of P-glycoprotein and multidrug resistance-associated proteins in human brain microvascular endothelial cells in hippocampal sclerosis. Epilepsy Res. 68, 213-228. doi: 10.1016/j.eplepsyres.2005.11.011

Kusuhara, H., He, Z., Nagata, Y., Nozaki, Y., Ito, T., Masuda, H., et al. (2003). Expression and functional involvement of organic anion transporting polypeptide subtype 3 (Slc21a7) in rat choroid plexus. Pharm. Res. 20, 720-727. doi: 10.1023/A:1023473216759

Landrigan, P. J., and Goldman, L. R. (2011). Children's vulnerability to toxic chemicals: a challenge and opportunity to strengthen health and environmental policy. Health Aff. (Millwood) 30, 842-850. doi: 10.1377/hlthaff.2011.0151

Leggas, M., Adachi, M., Scheffer, G. L., Sun, D., Wielinga, P., Du, G., et al. (2004). Mrp4 confers resistance to topotecan and protects the brain from chemotherapy. Mol. Cell Biol. 24, 7612-7621. doi: 10.1128/MCB.24.17.7612-7621.2004

Leslie, E. M., Deeley, R. G., and Cole, S. P. (2005). Multidrug resistance proteins: role of P-glycoprotein, MRP1, MRP2, and BCRP (ABCG2) in tissue defense. Toxicol. Appl. Pharmacol. 204, 216-237. doi: 10.1016/j.taap.2004.10.012

Liddelow, S. A., Dziegielewska, K. M., Ek, C. J., Habgood, M. D., Bauer, H., Bauer, H. C., et al. (2013). Mechanisms that determine the internal environment of the developing brain: a transcriptomic, functional and ultrastructural approach. PLoS ONE 8:e65629. doi: 10.1371/journal.pone.0065629

Liebner, S., Czupalla, C. J., and Wolburg, H. (2011). Current concepts of blood-brain barrier development. Int. J. Dev. Biol. 55, 467-476. doi: 10.1387/ijdb.103224sl

Lim, J. C., Kania, K. D., Wijesuriya, H., Chawla, S., Sethi, J. K., Pulaski, L., et al. (2008). Activation of beta-catenin signalling by GSK-3 inhibition increases p-glycoprotein expression in brain endothelial cells. J. Neurochem. 106, 1855-1865. doi: 10.1111/j.1471-4159.2008.05537.x

Martin, V., Xu, J., Pabbisetty, S. K., Alonso, M. M., Liu, D., Lee, O. H., et al. (2009). Tie2-mediated multidrug resistance in malignant gliomas is associated with upregulation of $\mathrm{ABC}$ transporters. Oncogene 28, 2358-2363. doi: 10.1038/onc.2009.103

Matsuoka, Y., Okazaki, M., Kitamura, Y., and Taniguchi, T. (1999). Developmental expression of P-glycoprotein (multidrug resistance gene product) in the rat brain. J. Neurobiol. 39, 383-392.

Miodovnik, A. (2011). Environmental neurotoxicants and developing brain. $M t$. Sinai J. Med. 78, 58-77. doi: 10.1002/msj.20237

Mizee, M. R., Wooldrik, D., Lakeman, K. A., Van Het Hof, B., Drexhage, J. A., Geerts, D., et al. (2013). Retinoic acid induces blood-brain barrier development. J. Neurosci. 33, 1660-1671. doi: 10.1523/JNEUROSCI.1338-12.2013

Mori, S., Takanaga, H., Ohtsuki, S., Deguchi, T., Kang, Y. S., Hosoya, K., et al. (2003). Rat organic anion transporter 3 (rOAT3) is responsible for brainto-blood efflux of homovanillic acid at the abluminal membrane of brain capillary endothelial cells. J. Cereb. Blood Flow Metab. 23, 432-440. doi 10.1097/00004647-200304000-00007

Nagata, Y., Kusuhara, H., Endou, H., and Sugiyama, Y. (2002). Expression and functional characterization of rat organic anion transporter 3 (rOat3) in the choroid plexus. Mol. Pharmacol. 61, 982-988. doi: 10.1124/mol.61.5.982
Nehlig, A., Pereira De Vasconcelos, A., and Boyet, S. (1989). Postnatal changes in local cerebral blood flow measured by the quantitative autoradiographic [14C]iodoantipyrine technique in freely moving rats. J. Cereb. Blood Flow. Metab. 9, 579-588. doi: 10.1038/jcbfm.1989.83

Niehof, M., and Borlak, J. (2009). Expression of HNF4alpha in the human and rat choroid plexus: implications for drug transport across the blood-cerebrospinalfluid (CSF) barrier. BMC Mol. Biol. 10:68. doi: 10.1186/1471-2199-10-68

Nishino, J., Suzuki, H., Sugiyama, D., Kitazawa, T., Ito, K., Hanano, M., et al. (1999). Transepithelial transport of organic anions across the choroid plexus: possible involvement of organic anion transporter and multidrug resistance-associated protein. J. Pharmacol. Exp. Ther. 290, 289-294.

Ohtsuki, S., Takizawa, T., Takanaga, H., Terasaki, N., Kitazawa, T., Sasaki, M., et al. (2003). In vitro study of the functional expression of organic anion transporting polypeptide 3 at rat choroid plexus epithelial cells and its involvement in the cerebrospinal fluid-to-blood transport of estrone-3-sulfate. Mol. Pharmacol. 63 532-537. doi: 10.1124/mol.63.3.532

Orford, M., Mean, R., Lapathitis, G., Genethliou, N., Panayiotou, E., Panayi, H., et al. (2009). Generation of an ABCG2(GFPn-puro) transgenic line-a tool to study ABCG2 expression in mice. Biochem. Biophys. Res. Commun. 384, 199-203. doi: 10.1016/j.bbrc.2009.04.089

Ose, A., Ito, M., Kusuhara, H., Yamatsugu, K., Kanai, M., Shibasaki, M., et al. (2009). Limited brain distribution of [3R,4R,5S]-4-acetamido-5-amino-3-(1ethylpropoxy)-1-cyclohexene-1-carboxylate phosphate (Ro 64-0802), a pharmacologically active form of oseltamivir, by active efflux across the blood-brain barrier mediated by organic anion transporter 3 (Oat3/Slc22a8) and multidrug resistance-associated protein 4 (Mrp4/Abcc4). Drug Metab. Dispos. 37, 315-321. doi: $10.1124 / \mathrm{dmd} .108 .024018$

Petropoulos, S., Gibb, W., and Matthews, S. G. (2010). Developmental expression of multidrug resistance phosphoglycoprotein (P-gp) in the mouse fetal brain and glucocorticoid regulation. Brain Res. 1357, 9-18. doi: 10.1016/j.brainres.2010.08.016

Qin, Y., and Sato, T. N. (1995). Mouse multidrug resistance 1a/3 gene is the earliest known endothelial cell differentiation marker during blood-brain barrier development. Dev. Dyn. 202, 172-180. doi: 10.1002/aja.1002020209

Reichel, V., Burghard, S., John, I., and Huber, O. (2011). P-glycoprotein and breast cancer resistance protein expression and function at the blood-brain barrier and blood-cerebrospinal fluid barrier (choroid plexus) in streptozotocin-induced diabetes in rats. Brain Res. 1370, 238-245. doi: 10.1016/j.brainres.2010.11.012

Roberts, L. M., Black, D. S., Raman, C., Woodford, K., Zhou, M., Haggerty, J. E., et al. (2008). Subcellular localization of transporters along the rat bloodbrain barrier and blood-cerebral-spinal fluid barrier by in vivo biotinylation. Neuroscience 155, 423-438. doi: 10.1016/j.neuroscience.2008.06.015

Schinkel, A. H., and Jonker, J. W. (2003). Mammalian drug efflux transporters of the ATP binding cassette (ABC) family: an overview. Adv. Drug Deliv. Rev. 55, 3-29. doi: 10.1016/S0169-409X(02)00169-2

Schinkel, A. H., Wagenaar, E., Mol, C. A., and Van Deemter, L. (1996). Pglycoprotein in the blood-brain barrier of mice influences the brain penetration and pharmacological activity of many drugs. J. Clin. Invest. 97, 2517-2524. doi: 10.1172/JCI118699

Schumacher, U., and Mollgard, K. (1997). The multidrug-resistance P-glycoprotein (Pgp, MDR1) is an early marker of blood-brain barrier development in the microvessels of the developing human brain. Histochem. Cell Biol. 108, 179-182. doi: $10.1007 / \mathrm{s} 004180050159$

Scotto, K. W. (2003). Transcriptional regulation of ABC drug transporters. Oncogene 22, 7496-7511. doi: 10.1038/sj.onc. 1206950

Shawahna, R., Uchida, Y., Decleves, X., Ohtsuki, S., Yousif, S., Dauchy, S., et al. (2011). Transcriptomic and quantitative proteomic analysis of transporters and drug metabolizing enzymes in freshly isolated human brain microvessels. Mol. Pharm. 8, 1332-1341. doi: 10.1021/mp200129p

Shen, H., Smith, D. E., Keep, R. F., and Brosius, F. C. III. (2004). Immunolocalization of the proton-coupled oligopeptide transporter PEPT2 in developing rat brain. Mol. Pharm. 1, 248-256. doi: 10.1021/mp049944b

Sims-Mourtada, J., Izzo, J. G., Ajani, J., and Chao, K. S. (2007). Sonic Hedgehog promotes multiple drug resistance by regulation of drug transport. Oncogene 26, 5674-5679. doi: 10.1038/sj.onc. 1210356

Singh, R. R., Kunkalla, K., Qu, C., Schlette, E., Neelapu, S. S., Samaniego, F., et al. (2011). ABCG2 is a direct transcriptional target of hedgehog signaling and involved in stroma-induced drug tolerance in diffuse large B-cell lymphoma. Oncogene 30, 4874-4886. doi: 10.1038/onc.2011.195 
Staud, F., and Pavek, P. (2005). Breast cancer resistance protein (BCRP/ABCG2). Int. J. Biochem. Cell Biol. 37, 720-725. doi: 10.1016/j.biocel.2004.11.004

Stolp, H. B., Liddelow, S. A., Sa-Pereira, I., Dziegielewska, K. M., and Saunders, N. R. (2013). Immune responses at brain barriers and implications for brain development and neurological function in later life. Front. Integr. Neurosci. 7:61. doi: 10.3389/fnint.2013.00061

Strazielle, N., Belin, M. F., and Ghersi-Egea, J. F. (2003). Choroid plexus controls brain availability of anti-HIV nucleoside analogs via pharmacologically inhibitable organic anion transporters. AIDS 17, 1473-1485. doi: 10.1097/00002030-200307040-00008

Strazielle, N., and Ghersi-Egea, J. F. (1999). Demonstration of a coupled metabolism-efflux process at the choroid plexus as a mechanism of brain protection toward xenobiotics. J. Neurosci. 19, 6275-6289.

Strazielle, N., and Ghersi-Egea, J. F. (2005). Factors affecting delivery of antiviral drugs to the brain. Rev. Med. Virol. 15, 105-133. doi: 10.1002/rmv.454

Strazielle, N., and Ghersi-Egea, J. F. (2013). Physiology of blood-brain interfaces in relation to brain disposition of small compounds and macromolecules. $\mathrm{Mol}$. Pharm. 10, 1473-1491. doi: 10.1021/mp300518e

Tachikawa, M., Watanabe, M., Hori, S., Fukaya, M., Ohtsuki, S., Asashima, T., et al. (2005). Distinct spatio-temporal expression of ABCA and ABCG transporters in the developing and adult mouse brain. J. Neurochem. 95, 294-304. doi: 10.1111/j.1471-4159.2005.03369.x

Takashima, T., Yokoyama, C., Mizuma, H., Yamanaka, H., Wada, Y., Onoe, K., et al. (2011). Developmental changes in P-glycoprotein function in the bloodbrain barrier of nonhuman primates: PET study with R-11C-verapamil and 11C-oseltamivir. J. Nucl. Med. 52, 950-957. doi: 10.2967/jnumed.110.083949

Tsai, C. E., Daood, M. J., Lane, R. H., Hansen, T. W., Gruetzmacher, E. M., and Watchko, J. F. (2002). P-glycoprotein expression in mouse brain increases with maturation. Biol. Neonate 81, 58-64. doi: 10.1159/000047185

Virgintino, D., Errede, M., Girolamo, F., Capobianco, C., Robertson, D., Vimercati, A., et al. (2008). Fetal blood-brain barrier P-glycoprotein contributes to brain protection during human development. J. Neuropathol. Exp. Neurol. 67, 50-61. doi: 10.1097/nen.0b013e31815f65d9

Wang, X., Campos, C. R., Peart, J. C., Smith, L. K., Boni, J. L., Cannon, R. E., et al. (2014). Nrf2 upregulates ATP binding cassette transporter expression and activity at the blood-brain and blood-spinal cord barriers. J. Neurosci. 34, 8585-8593. doi: 10.1523/JNEUROSCI.2935-13.2014
Westerhout, J., Danhof, M., and De Lange, E. C. (2011). Preclinical prediction of human brain target site concentrations: considerations in extrapolating to the clinical setting. J. Pharm. Sci. 100, 3577-3593. doi: 10.1002/jps.22604

Wijnholds, J., Delange, E. C., Scheffer, G. L., Van Den Berg, D. J., Mol, C. A., Van Der Valk, M., et al. (2000). Multidrug resistance protein 1 protects the choroid plexus epithelium and contributes to the blood-cerebrospinal fluid barrier. J. Clin. Invest. 105, 279-285. doi: 10.1172/JCI8267

Xiang, J., Alesi, G. N., Zhou, N., and Keep, R. F. (2012). Protective effects of isothiocyanates on blood-CSF barrier disruption induced by oxidative stress. Am. J. Physiol. Regul. Integr. Comp. Physiol. 303, R1-R7. doi: 10.1152/ajpregu.00518.2011

Yamada, T., Takaoka, A. S., Naishiro, Y., Hayashi, R., Maruyama, K., Maesawa, C., et al. (2000). Transactivation of the multidrug resistance 1 gene by T-cell factor 4/beta-catenin complex in early colorectal carcinogenesis. Cancer Res. 60, 4761-4766.

Zhuang, Y., Fraga, C. H., Hubbard, K. E., Hagedorn, N., Panetta, J. C., Waters, C. M., et al. (2006). Topotecan central nervous system penetration is altered by a tyrosine kinase inhibitor. Cancer Res. 66, 11305-11313. doi: 10.1158/00085472.CAN-06-0929

Conflict of Interest Statement: The authors declare that the research was conducted in the absence of any commercial or financial relationships that could be construed as a potential conflict of interest.

Received: 03 December 2014; accepted: 13 January 2015; published online: 05 February 2015.

Citation: Strazielle $N$ and Ghersi-Egea J-F (2015) Efflux transporters in bloodbrain interfaces of the developing brain. Front. Neurosci. 9:21. doi: 10.3389/fnins. 2015.00021

This article was submitted to Neurogenomics, a section of the journal Frontiers in Neuroscience.

Copyright (C) 2015 Strazielle and Ghersi-Egea. This is an open-access article distributed under the terms of the Creative Commons Attribution License (CC BY). The use, distribution or reproduction in other forums is permitted, provided the original author(s) or licensor are credited and that the original publication in this journal is cited, in accordance with accepted academic practice. No use, distribution or reproduction is permitted which does not comply with these terms. 


\title{
Influx mechanisms in the embryonic and adult rat choroid plexus: a transcriptome study
}

\author{
Norman R. Saunders ${ }^{1 *}$, Katarzyna M. Dziegielewska ${ }^{1}$, Kjeld Møllgård ${ }^{2}$, Mark D. Habgood ${ }^{1}$, \\ Matthew J. Wakefield ${ }^{3}$, Helen Lindsay ${ }^{4}$, Nathalie Stratzielle ${ }^{5}$, Jean-Francois Ghersi-Egea ${ }^{5}$ \\ and Shane A. Liddelow ${ }^{1,6}$

\begin{abstract}
${ }^{1}$ Department of Pharmacology and Therapeutics, University of Melbourne, Parkville, VIC, Australia, ${ }^{2}$ Department of Cellular and Molecular Medicine, University of Copenhagen, Copenhagen, Denmark, ${ }^{3}$ Walter and Eliza Hall Institute of Medical Research, Parkville, VIC, Australia, ${ }^{4}$ Institute of Molecular Life Sciences, University of Zurich, Zurich, Switzerland, ${ }^{5}$ Lyon Neuroscience Research Center, INSERM U1028, Centre National de la Recherche Scientifique UMR5292, Université Lyon 1 ,
\end{abstract} \\ Lyon, France, ${ }^{6}$ Department of Neurobiology, Stanford University, Stanford, CA, USA
}

\section{OPEN ACCESS}

Edited by:

Joana A. Palha

University of Minho, Portugal

Reviewed by:

Fernanda Marques,

University of Minho, Portugal Hanspeter Herzel,

Humboldt University, Germany

*Correspondence:

Norman R. Saunders, Department of Pharmacology and Therapeutics, University of Melbourne,

Triradiate Building, Grattan Street,

Parkville, VIC 3010, Australia

n.saunders@unimelb.edu.au

Specialty section:

This article was submitted to

Neurogenomics,

a section of the journal

Frontiers in Neuroscience

Received: 24 December 2014 Accepted: 24 March 2015

Published: 28 April 2015

Citation:

Saunders NR, Dziegielewska KM, Møllgård K, Habgood MD, Wakefield $M J$, Lindsay $H$, Stratzielle $N$,

Ghersi-Egea J-F and Liddelow SA (2015) Influx mechanisms in the embryonic and adult rat choroid plexus: a transcriptome study. Front. Neurosci. 9:123. doi: 10.3389/fnins.2015.00123
The transcriptome of embryonic and adult rat lateral ventricular choroid plexus, using a combination of RNA-Sequencing and microarray data, was analyzed by functional groups of influx transporters, particularly solute carrier (SLC) transporters. RNA-Seq was performed at embryonic day (E) 15 and adult with additional data obtained at intermediate ages from microarray analysis. The largest represented functional group in the embryo was amino acid transporters (twelve) with expression levels 2-98 times greater than in the adult. In contrast, in the adult only six amino acid transporters were up-regulated compared to the embryo and at more modest enrichment levels (<5-fold enrichment above E15). In E15 plexus five glucose transporters, in particular Glut-1, and only one monocarboxylate transporter were enriched compared to the adult, whereas only two glucose transporters but six monocarboxylate transporters in the adult plexus were expressed at higher levels than in embryos. These results are compared with earlier published physiological studies of amino acid and monocarboxylate transport in developing rodents. This comparison shows correlation of high expression of some transporters in the developing brain with higher amino acid transport activity reported previously. Data for divalent metal transporters are also considered. Immunohistochemistry of several transporters (e.g., S/c16a10, a thyroid hormone transporter) gene products was carried out to confirm translational activity and to define cellular distribution of the proteins. Overall the results show that there is substantial expression of numerous influx transporters in the embryonic choroid plexus, many at higher levels than in the adult. This, together with immunohistochemical evidence and data from published physiological transport studies suggests that the choroid plexus in embryonic brain plays a major role in supplying the developing brain with essential nutrients.

Keywords: choroid plexus, brain development, amino acid transfer, protein transfer, brain barriers, cerebrospinal fluid

Abbreviations: E, embryonic day; CSF, cerebrospinal fluid. 


\section{Introduction}

Influx transporters, active across interfaces between the blood and the brain, are essential components of the mechanisms that contribute to the composition and stability of the internal environment of the brain, critical for normal brain function. The main interfaces between these two compartments are the blood-brain barrier across cerebral blood vessels and the bloodcerebrospinal (CSF) barrier across the choroid plexuses within the cerebral ventricles (Saunders et al., 2012). The transport mechanisms in these interfaces have been well studied in the adult brain (Davson and Segal, 1996; Brown et al., 2004; Damkier et al., 2007, 2013; Abbott et al., 2010; Abbott and Friedman, 2012) but relatively little is known about them during gestation, although they are clearly important for normal brain development and maturation. In addition, much of the research focus has been on the blood-brain barrier itself rather than on the transport properties of the choroid plexuses. Nevertheless, in spite of this neglect, it seems that in the early developing brain the choroid plexuses are probably a much more important portal of entry into the brain than are the cerebral blood vessels (Johansson et al., 2008). This is because the choroid plexuses develop to being a substantial and functional tissue at a stage when the brain is still poorly vascularised (Saunders et al., 2012, 2013). By far the largest group of influx transporters is the solute carrier gene series (SLCs), which currently comprises 52 families of 395 transporter genes (Hediger, 2013 and http://www.bioparadigms. org/slc/intro.htm). These are particularly important for ion exchange and amino acid and glucose delivery to the brain. Until recently, very little was known about their expression and function in the choroid plexuses of the developing brain. This paper is the third in a series of combined RNA-Sequencing and microarray analysis of transcriptome expression in developing and adult choroid plexus, with a focus on the plexuses in the lateral ventricles (Kratzer et al., 2013; Liddelow et al., 2013). Here we give a comprehensive analysis of the expression of solute carriers and other influx transporters in developing and adult choroid plexus, with an emphasis on nutrient transporters such as for glucose, amino acids and monocarboxylates and compare these data with available published physiological data on their transport activity in vivo. We also include results of immunohistochemical staining of selected SLCs, which confirm that their genes are translating to protein both in the embryo and in the adult. The ion transporters thought to be important for CSF secretion and its homeostasis, as well as brain interstitial fluid have been dealt with in a previous paper (Liddelow et al., 2013) together with the ABC/SLC transporters specifically involved in neuroprotective CSF-to-blood efflux mechanisms (Kratzer et al., 2013). Comparative data are also available from a microarray study of mouse choroid plexus (Liddelow et al., 2012) and an RNA-Seq study of non human primate choroid plexus (Ek et al., 2015).

\section{Materials and Methods}

The animal tissue obtained for this study came from two earlier published studies and most methods were as previously described (Kratzer et al., 2013; Liddelow et al., 2013).

\section{Ethics Statement}

Animal experiments in Melbourne were conducted in accordance with Australian code of practice for the care and use of animals for scientific purposes 7th Edition, published by the National Health and Medical Research Council. All animal research protocols were reviewed and approved by the University of Melbourne Faculty of Medicine, Dentistry and Health Sciences Animal Ethics Committee and registered under ID. Number 1011703. For experiments conducted in Lyon animal care and procedures were in accordance with the guidelines approved by the French ethical committee (decree 87-848), by the European Community (directive 86-609-EEC).

\section{Animal Husbandry}

In Melbourne, timed-pregnant (embryonic day 15, E15) and non-pregnant adult (6 week, 200-300g weight range) SpragueDawley rats were used. These ages were chosen as they have been previously shown to be appropriate for studies of the developing lateral ventricular choroid plexus in rodents (Johansson et al., 2006; Liddelow et al., 2012). Animals were supplied by the Biological Research Facility at the University of Melbourne (Victoria, Australia). For next generation RNA-Sequencing, lateral ventricular choroid plexuses from E15 $(n=30)$ and female adult $(n=30)$ rats were used. For immunohistochemistry E15 $(n=3)$ and female adult $(n=3)$ lateral ventricular choroid plexuses were dissected out and processed as described below. For microarray experiments Sprague-Dawley rats (adult males, pregnant time-dated females, or females with their litters) were obtained from Janvier (Le Genest Saint Isle, France). All animals were kept under similar conditions in standard cages, with free access to food and tap water under a controlled environment (12 h day/light cycles).

\section{Collection of Lateral Ventricular Choroid Plexus}

The procedure for collection of choroid plexus tissues has previously been described (Liddelow et al., 2012; Kratzer et al., 2013). Briefly, animals were killed by an overdose of inhaled isoflurane (Veterinary Companies of Australia) and brains dissected out under ice-cold RNase-free phosphate buffered saline (PBS, $\mathrm{pH}$ 7.3). Both left and right lateral ventricular choroid plexuses were carefully dissected out and placed in fresh ice-cold RNase-free PBS. All steps were performed under RNasefree conditions. The collected tissues were snap-frozen in liquid nitrogen and kept at $-80^{\circ} \mathrm{C}$ until used.

For Illumina RNA-Sequencing, lateral ventricular choroid plexuses from $\mathrm{E} 15(n=30)$ and adult $(n=30)$ rats were used (three pooled samples of ten plexuses at each age). Total RNA was extracted using the RNeasy Mini Kit, Qiashredder columns and gDNA removal columns (Qiagen, Valencia, CA, USA) according to standard supplier protocols. All RNA samples were quantified using a NanoDrop ND-1000 UVVIS spectrophotometer (Thermo Scientific, Wilmington, DE, USA) and quality checked with the Agilent 2100 Bioanalyzer (Agilent Technologies, Palo Alto, CA, USA). Plexuses were pooled ( $n=10$ animals) and centrifuged at $1000 \mathrm{rpm}$ for $30 \mathrm{~s}$, excess PBS removed, snap frozen in liquid nitrogen and stored at $-80^{\circ} \mathrm{C}$. For Affymetrix microarrays, choroid plexuses were pooled from 3 to 5 animals. Total RNA was isolated from two 
pools of choroid plexuses sampled from E19, P2, or adult rats using the RNeasy ${ }^{\circledR}$ Micro Kit (Qiagen, Valencia, CA, USA), and DNase-treated according to the manufacturer's protocol.

The choroid plexus consists of epithelium as well as blood vessels, neural innervation and mesenchymal stroma. However, the epithelium is the predominant cell type, suggested to represent up to $90 \%$ of the plexus tissue (Keep and Jones, 1990; Liddelow et al., 2012). In this study lateral ventricular choroid plexus was taken in toto.

\section{Illumina Next Generation RNA Sequencing}

RNA Sequencing was performed at the Australian Genome Research Facility (Melbourne, VIC, Australia). A cDNA library was prepared from $10 \mu \mathrm{g}$ of total RNA using the mRNASeq Sample Preparation Kit (Illumina, San Diego, CA, USA) according to standard manufacturer protocol. Quality of the library was verified using a DNA 1000 chip using the Agilent 2100 Bioanalyzer (Agilent). The library was subjected to $100 \mathrm{bp}$ single end read cycles of sequencing on an Illumina HiSeq 2000 sequencer as per manufacturer protocol. Cluster generation was performed on a c-Bot (Illumina) with a single read cluster generation kit.

\section{Data Analysis}

Short reads were trimmed to remove ambiguous bases from the start and segments with low quality scores from the end, as indicated by the ascii character " $\mathrm{B}$ " in Illumina 1.5 phred score encoding. Trimmed reads were mapped with Bowtie version 0.12.7 (Langmead et al., 2009) to the Ensembl (Hubbard et al., 2009) rat genome, release 61. Reads that did not map uniquely were discarded. The number of reads mapped to nuclear genes was determined with HTSeq (Anders, 2010) version $0.4 .7 \mathrm{p} 4$, using the default "union" counting option. Differential expression between the adult and embryonic samples was detected using an exact test in the Bioconductor (Gentleman et al., 2004) edgeR package, version 2.4.0 (Robinson et al., 2010), with common dispersion used to estimate variance between samples. Genes considered significantly differentially expressed were those with a $p$-value of less than 0.05 after BenjaminiHochberg false discovery rate correction. All fold changes during development were calculated from these normalized data. However, where appropriate we have also included raw counts for each transcript. As noted in the legends to relevant tables (Tables 4-6) ratios for individual genes calculated from raw counts may not correspond to the fold changes (as shown in the tables) calculated from normalized data. We have included both the normalized fold changes and raw cell counts to enable the reader to see both relative expression changes throughout development, as well as the absolute abundance of each transcript at an individual age. A combination of gene ontology annotation and manual curation was used to select genes encoding proteins with integral transporter function. Gene ontology descriptions for rat were downloaded from Biomart (Hubbard et al., 2009), and genes with "transport" mentioned in their gene ontology description were selected. Genes of interest were then extracted from this list. For initial analysis genes with $>100$ sequence reads and age-related fold changes $(\mathrm{FC})>2.0\left(\log _{2} \mathrm{FC}>1.0\right)$ were collated and are summarized in Tables 4-6, and Supplementary
Tables S1-S3; this level of gene expression was taken as likely to be functionally significant. For identification of the presence of a particular gene a lower cut-off of 10 sequence reads was used; these are also included in Tables 4-6. Illumina RNA sequencing data have been deposited with the Gene Expression Omnibus (http://www.ncbi.nlm.nih.gov/geo/) under accession code GSE44072.

\section{Microarray}

As previously described (Kratzer et al., 2013) microarray analysis was performed using a high-density oligonucleotide array (GeneChip Rat Genome 2302.0 array, Affymetrix, Santa Clara, CA, USA). Total RNA (100 ng) was amplified and biotin-labeled using GeneChip ${ }^{\circledR} \quad 3^{\prime}$ IVT Express target labeling and control reagents according to Affymetrix protocol (http://www.affymetrix.com). Before amplification, all samples were spiked with synthetic mRNAs at different concentrations, which were used as positive controls to ascertain the quality of the process. Biotinylated antisense cRNA for microarray hybridization was prepared. After final purification using magnetic beads, cRNAs were quantified using a NanoDrop and quality checked with Agilent 2100 Bioanalyzer. Hybridization was performed according to the Affymetrix protocol. Briefly, $10 \mu \mathrm{g}$ of labeled cRNA was fragmented and denaturated in hybridization buffer, then hybridized on the chip for $16 \mathrm{~h}$ at $45^{\circ} \mathrm{C}$ with constant mixing by rotation at $60 \mathrm{rpm}$ in a Genechip hybridization oven 640 (Affymetrix). After hybridization, arrays were washed and stained with streptavidin-phycoerythrin (GeneChip ${ }^{\circledR}$ Hybridization Wash and Stain Kit) in a fluidic station 450 (Affymetrix) according to the manufacturer's instruction. The arrays were read with a confocal laser (Genechip scanner 3000, Affymetrix). CEL files summarizing the probe cell intensity data were generated using the Affymetrix GeneChip Command Console (AGCC) software 3.0. Data were normalized with Affymetrix Expression Console software using MAS5 statistical algorithm. Data have been deposited into the Gene Expression Omnibus repository (http://www.ncbi.nlm.nih.gov/ geo) under accession number GSE44056.

\section{Immunohistochemistry}

Sagittal sections through E15 and adult rat brain including lateral choroid plexus were selected from the collection of rat tissue at the Faculty of Health and Medical Sciences, University of Copenhagen and used for immunohistochemical detection of SLC16a10, Slc38a5 (SNAT5), Slc4a1, Slc1la1 (NRAMP), Slc39a4 (ZIP4), and Slc1a3 (EAAT1). Sections were deparaffinised in xylene, rehydrated through graded alcohols followed by treatments in $0.5 \%$ hydrogen peroxide in methanol for $15 \mathrm{~min}$ and rinsing in TRIS buffered saline (TBS) as described previously (Liddelow et al., 2012). Following removal of nonspecific binding by incubation for $30 \mathrm{~min}$ with blocking buffer (ChemMate antibody diluent S2022, DakoCytomation, Glostrup, Denmark) at room temperature sections were incubated in primary antibodies as listed in Table $\mathbf{1}$.

After overnight incubation sections were washed in TBS and incubated for $30 \mathrm{~min}$ in EnVisionTM+ System/HRP (DAKO), K5007, for rabbit antibodies and RPN1025 from GE Healthcare for donkey anti-goat antibodies and then Vector: Vectastain 
TABLE 1 | List of primary antibodies used for immunohistochemistry.

\begin{tabular}{llcclc}
\hline Primary antibody & Host IgG & Dilution & Retrieval & Producer & Code number \\
\hline Slc16a10 (MCT10) & Rabbit & $1: 20$ & M6 & Abcam & Ab121519 \\
Slc38a5 (SNAT5) & Goat & $1: 150$ & - & Santa Cruz & Sc-50682 \\
Slc4a1 (AE1) & Rabbit & $1: 100$ & - & Proteintech & $18566-1-A P$ \\
Slc11a1 (NRAMP) & Rabbit & $1: 70$ & - & Santa Cruz & Sc-20113 \\
Slc1a3 (EAAT1) & Rabbit & $1: 600$ & - & Abcam & Ab416 \\
Slc5a5 (NIS) & Rabbit & $1: 200$ & - & Proteintech & $24324-1-A P$ \\
SLC 39a4 (ZIP4) & Rabbit & $1: 200$ & - & Proteintech & $20625-1-A P$ \\
\hline
\end{tabular}

RTU elite ABC reagent PK 7100. This was followed by 6 min incubation with DAB-chromogen solution (DAKO) and counterstaining with Mayer's haematoxylin, dehydrated and mounted with DPX. Control sections contained no primary antibodies and were always blank.

\section{Photography and Image Preparation}

Digitized images were obtained using an Olympus DP70 camera housing (Olympus, Tokyo, Japan) attached to an Olympus BX50 light microscope (Olympus). A10 $\times$ eyepiece and $40 \times$ objective lens were used. Raw image files were process in Adobe Photoshop CS5 ${ }^{\circledR}$ (Adobe ${ }^{\circledR}$ Systems, San Jose, CA, USA). The brightness and curve functions were used to obtain images with background close to white. There was no other manipulation of images.

\section{Results}

\section{Solute Carriers (SLCs) Identified by RNA-Sequencing and Microarray}

Examination of the whole transcriptome data set from RNASequencing showed 48 families of the 52 known SLC families (Hediger, 2013) and 64\% of transcripts in these families were identifiable at functionally significant levels of expression (>100 transcript reads, Figure 1, Supplementary Table S1). The distribution of genes at the two ages that showed increased, decreased or unchanged expression is illustrated in Figure 2. For each SLC family the distribution of gene expression in embryonic and adult choroid plexus is shown in Figure 3.

The $S l c$ genes that were expressed at a higher level ( $>2$ fold) in the embryonic choroid plexus than in the adult are listed in Table 2; microarray data from E19 and P2 are also shown. The Slc genes that were expressed at a higher level ( $>2$ fold) in the adult plexus are shown in Table 3; data comparing adult with E19 and P2, from microarray, are also shown. The main focus of this report is the $S l c$ transporter genes involved in nutrient transport into CSF, particularly amino acids, monocarboxylates and glucose.

In the present study the numerous members of the SLC families of transport proteins that were present in the lateral ventricular choroid plexus at E15 and in the adult had strikingly different patterns of gene expression at these two ages: of 250 total genes identified by RNA sequencing (Supplementary Table S2), 81 were expressed at higher levels at E15 (Supplementary Table S2 and Table 2). Developing-to-adult fold change differences between E19 and P2 were modest, apart from Slc7a11, Slc7a1, and Slc6a15, suggesting that birth is not a key milestone for developmental changes in the choroidal Slc transcriptome. A number of genes such as Slc4a1, Slc2a3, Slc11a1 had strikingly higher E15/Adult fold change than E19-P2/Adult fold changes. This suggests very specific choroidal function for brain development at the early stages of gestation. 107 genes were up-regulated in the adult (Supplementary Table S3 and Table 3). For these genes the array analysis shows that the E19/Adult and P2/Adult fold changes were modest and lower than E15/Adult fold changes for most of these genes, indicating that the choroid plexus starts to acquire its "adult" phenotype early in development, i.e., at late prenatal stages in the rat. Finally, 62 genes showed no difference in expression level between the ages (Supplementary Table S3), indicating the early maturity and life-long duration of different choroidal functions, in addition to early development-specific functions assumed from the E15 specific genes identified. Many fewer Slc transporter genes have been identified at the blood-brain barrier (Daneman et al., 2010supplementary material) of about 56 transcripts, which is around a quarter of that identified in this choroid plexus study. This finding supports the previously suggested proposition that in the developing brain the choroid plexus is the main route of entry for amino acid and ion transport and other molecules essential for brain development (Johansson et al., 2008).

\section{Glucose and Monocarboxylate Transporters}

Table 4 lists the glucose and monocarboxylate transporters differentially regulated in the embryo and in the adult with a fold change (FC) of two or more. Slc5a10 (sodium/glucose co-transporter) was expressed only in the embryonic plexus, but at a low copy number. Gene array shows that it was still expressed at E19, but disappeared after birth. The number of glucose transporters expressed at a higher level in the embryo was strikingly more than those higher in the adult (five compared to two, note that Klf15 is a regulator of GLUT4, not a transporter itself, Gray et al., 2002). The converse was the case for monocarboxylate transporters; there was only one in the embryo, but 6 in the adult.

Slc2a3 (GLUT3) was only detected in the embryo using RNASeq, but in microarray data was expressed at similar levels in the adult (ratio of $0.7-0.8$ ). However, the transcript numbers in RNA-Seq were very low, so this result is of dubious functional significance. Three other glucose transporters (Slc2a4, Slc2a5, Slc45a1) had transcript numbers $<100$ at both ages and were not detected in microarray, so these genes are unlikely to be contributing to glucose transport via the choroid plexuses. More relevant is GLUT1 (Slc2a1) which is well expressed in embryonic and E19 fetal choroid plexus relative to P2 or adult. This transporter is crucial for glucose delivery to the brain. Its expression in the developing brain choroid plexus argues for a specific role of the plexus in nutrient transport at these ages. Amongst monocarboxylate transporters the same single one (Slc10a16) was increased in the developing choroid plexus in both RNA-Seq and microarray results. The most highly expressed of all the monocarboxylate transporters (in terms of transcript numbers) was Slc16a2, at a slightly higher (2.1 fold) level in 


\section{SLC Transporters Present in Rat Lateral Ventricular Choroid Plexus}

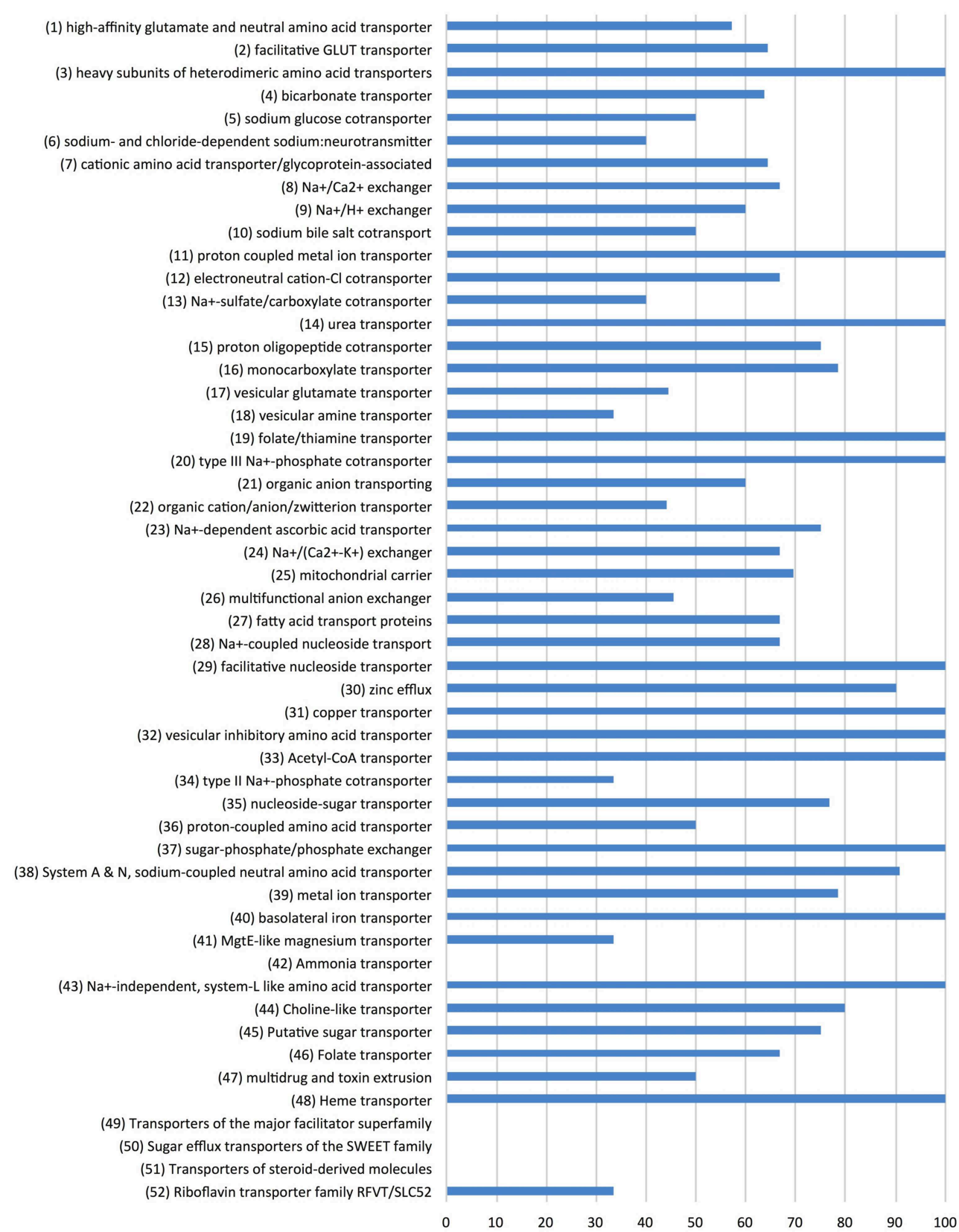

FIGURE 1 | SIc transporter genes identified in rat lateral ventricular choroid plexus. Expression of SLC family class transcripts in the rat lateral ventricular choroid plexus regardless of age, as determined by RNA sequencing. The 52 known functional groups of SLC transporters (Hediger, 2013) are shown on the left. Data are expressed as percentage total of each family of transporters (transcript number > 10)-for example, $100 \%$ of proton-coupled metal ion transporters (SLC11 family) are present in the rat lateral ventricular choroid plexus, whereas no steroid-derived molecule transporters (SLC51 family) were detected. the adult than in the embryo; this transporter was not detected in microarray, possibly because the probe was not a good match. Slc16a8 was expressed at a high level in adult plexus, but expression was $<100$ transcript counts in the embryo in the RNA-Seq analysis and was also not detected in microarray. Three other monocarboxylate transporters were expressed at high level in adult plexus in both microarray and RNA-Seq (Tables 3, 4).

\section{Amino Acid Transporters}

There were twice as many genes coding for amino acid transporters in the embryonic choroid plexus expressed at a 

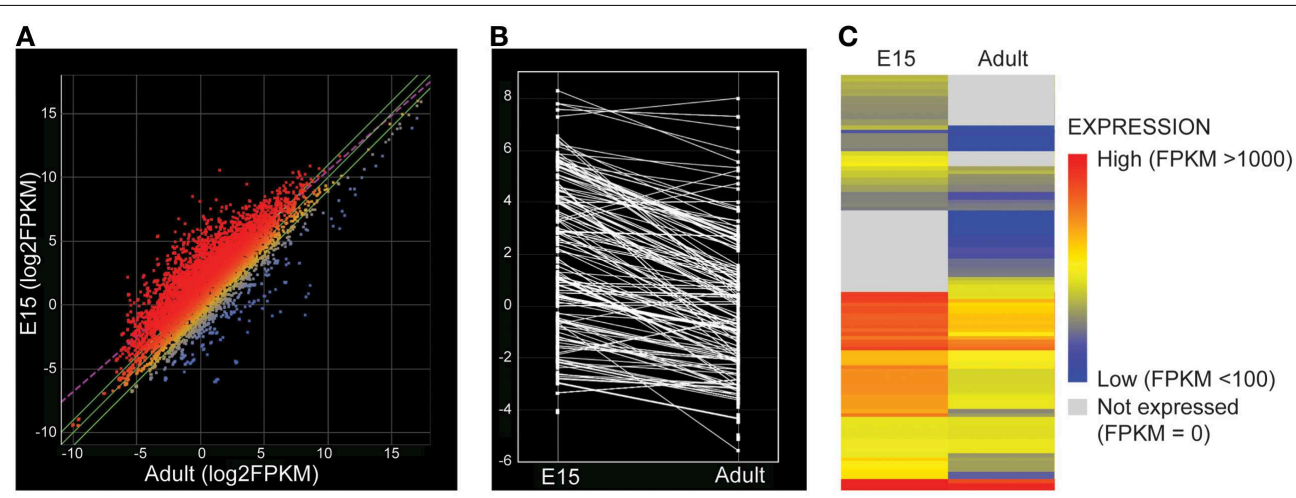

FIGURE 2 | (A) Scatter plot comparing log2(FPKM), i.e., fragment count per kilobase of exonic length per million reads mapped, for all transcripts detected by RNA sequencing of the rat lateral ventricular choroid plexus, averaged across replicates from embryonic day 15 (E15) and adult. Red points show transcripts with higher expression at E15 (greater than 2-fold) while blue points are those with higher expression in the adult. FPKM was estimated using a standard ArrayStar pipeline (http://www.dnastar.com/t-help-arraystar.aspx). There is a greater number of transcripts enriched in the embryo. (B) Parallel coordinate plot of change in average expression (log2(FPKM)) of SLC transporters in E15 and Adult lateral ventricular choroid plexus. Lines connect the same transcript present at both ages. (C) Heat map of average expression $(\log 2(\mathrm{FPKM}))$ for SLC transporters. Red depicts high expression values, blue low expression. Gray depicts transcripts that were not detected at one age. The expression level of SLC transporters was much higher at E15-as shown by more red expression data. higher level than in the adult (12 compared to 6, Table 5). The level of expression of these transporters in the embryo was also generally higher; thus in the embryo nine of the transporters were expressed at $>5$ fold compared to adult, whereas in the adult only one transporter gene was expressed $>5$ fold compared to the embryo. The microarray results were generally consistent with those from RNA-Seq (Tables 2, 4, 5). For both methods the expression levels of Slc7a11, Slc7a1, Slc6a6, and Slc6a15 were notably high in the embryonic plexus. For two genes (Slc3a1, Slc7a10) the expression levels were higher in the adult with RNA-Seq, but lower with microarray (Tables 3, 5).

\section{Divalent Metal Transporters}

It has been known for many years that zinc is important for normal brain development and for function in the adult (Sandstead, 1985; Marger et al., 2014; Tyszka-Czochara et al., 2014). In the present study there were 4 zinc transporter genes that were expressed at a higher level in embryonic choroid plexus than in the adult in the fold range of 2.5-44 (Table 6). In the adult there were more up-regulated zinc transporter genes (6), but their expression level was generally much lower (2.7-12 fold). Both of the major zinc transporter families (Slc30, ZnT, and Slc39, ZIP) are represented in our dataset. The members of the two families function in opposite directions, thus maintaining cellular zinc homeostasis. ZnT proteins of Slc30 genes efflux zinc whereas ZIP proteins function to increase zinc uptake when cellular zinc is depleted (Marger et al., 2014; Tepaamorndech et al., 2014). Ten Slc30 genes were identified in the present study (two higher in the embryo, Slc30a2, $44 \mathrm{FC}$; Slc30a10, $35 \mathrm{FC}$ and four in the adult, Slc30a3-6, with only modest 2.7-4.5 FC). Ten Slc39 genes were identified; Slc39a8 was $37 \mathrm{FC}$ higher in embryo. Three other members, Slc39a1, Slc39a12, and Slc39a13 (FC 12, 12 and 3.9 respectively), were higher in adult (Table 6).
The transferrin gene, $T f$, was expressed in terms of transcript numbers at a high level in both the embryo and adult (Table 6), but much higher in the latter (183 fold), with a continuous pre-and post natal increase from embryo to adult stages. The transferrin receptor was expressed at a higher level in embryonic choroid plexus (3.1 fold) with intermediate expression levels at the perinatal stage of development. As indicated in Table 6 several other iron carriers were expressed at a higher level in the embryo than in the adult: sideroflexin1, Sfxn1 (14 fold), ferritin light chain 1-like (LOC100360087), 3.6 fold), and this difference in expression was maintained perinatally.

Slc11a1 was expressed at much higher levels in the embryonic choroid plexus than in the adult (8.9 fold), and at still higher level at birth, while Slc11a2 (DMT1) was found expressed at a similar level at all ages examined by both RNA-Seq and microarray approaches (Table 6). Several members of these SLC families of divalent metal transporters have been implicated in iron and manganese transport. Their expression in developing choroid plexus correlates with the higher uptake of manganese by postnatal choroid plexus cells in vitro as shown by Schmitt et al. (2011), and is discussed further below.

\section{Immunohistochemistry}

Immunohistochemical investigations of the distribution of some efflux transporter proteins selected from the gene expression data included five enriched in the fetus: SLC 16a10 (TAT1), SLC $38 \mathrm{a} 5$ (SNAT5), SLC 4a1 (AE1), SLC 11a1 (NRAMP), SLC 1a3 (EAAT1), one enriched in the adult: SLC $5 \mathrm{a} 5$ (NIS) and one expressed at a low level in both fetal and adult choroid plexus: SLC 39a4 (ZIP4).

The seven tested antibodies resulted in 3 different patterns of immunoreactivity in E15 plexus epithelial cells ("differential immunoreactivity, regional distribution-type," 


\section{SLC Transporters Present in Rat Lateral Ventricular Choroid Plexus}

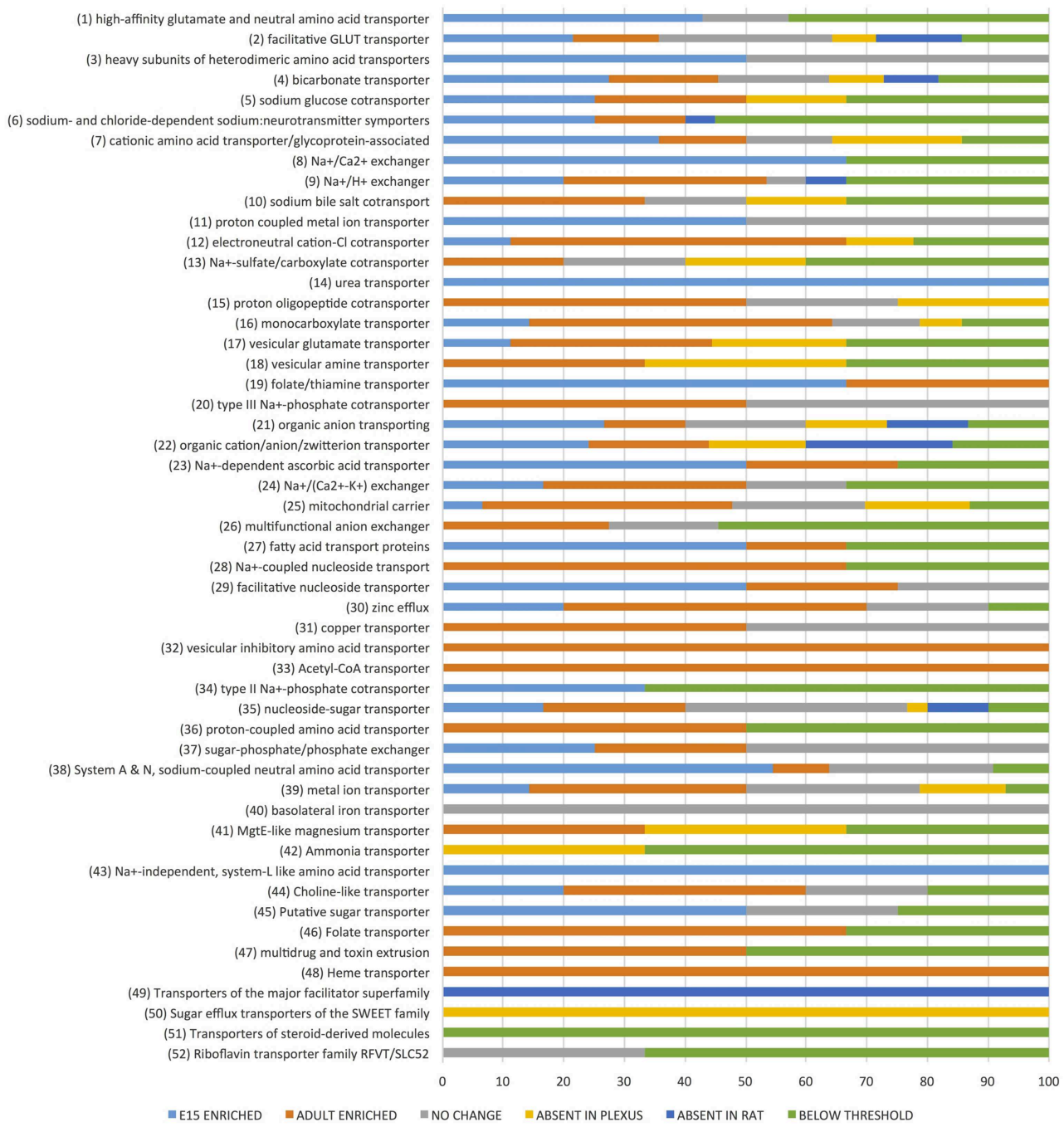

FIGURE 3 | SIc transporter genes identified in rat lateral ventricular choroid plexus. Expression of SLC family class transcripts in the embryonic and adult lateral ventricular choroid plexus as determined by RNA sequencing. Data are expressed as percentage total of each family of transporters. The 52 known functional groups of SLC transporters (Hediger, 2013) are shown on the left. The horizontal bars show the percentage of total number of members of each group identified "Enriched" refers to transcripts with greater than 2-fold expression in one age over the other, while "no change" refers to transcripts with similar expression at both ages. A transcript was considered absent in the choroid plexus if no single copy of transcript was detected (while those with "below threshold" had raw count values below 10). A transcript was considered absent in the rat if no annotation was found in the online ensemble database (http://www.ensembl.org/Rattus_norvegicus). "strong immunoreactivity, uniform distribution-type," "weak immunoreactivity, uniform distribution-type") and in 2 different patterns of immunoreactivity in adult plexus epithelial cells ("weak or lack of immunoreactivity," "strong immunoreactivity"). Representative micrographs from the 5 different patterns of immunoreactivity are shown in Figure 4.

\section{SLC 16a10}

An example of "differential immunoreactivity, regional distribution-type" is shown in E15 and of "weak or lack of immunoreactivity" is presented in adult plexus. The embryonic plexus epithelial cell layer in the "plexus-fold" next to the ganglionic eminence but opposite to the developing hippocampus showed a stronger immunostaining than the "plexus-fold" facing the hippocampus (Figure 4A). At high magnification the prominent reactivity in the epithelial cell layer facing the ganglionic eminence was shown to be due to a combination of a marked fine granular reactivity in apical cytoplasm and along both apical and basolateral cell membranes, which were particularly strongly immunostained (arrowheads in Figure 4A1). In contrast the epithelial cell layer facing the hippocampus showed a more uniform immunoreactivity both in cytoplasm and along cell membranes. Adult choroid plexus epithelial cells exhibited 
TABLE 2 | Expression and function of solute carrier (SIc) transcripts that were enriched in lateral ventricular choroid plexus of rat E15 and E19 embryos and P2 neonates compared to adult.

\begin{tabular}{|c|c|c|c|c|c|c|}
\hline Gene ID & Protein & Transport function & Ref. & E15/A & E19/A (E19/P2) & P2/A \\
\hline Slc38a5 & SN2, JM24, & $\mathrm{Na}^{+}$-dependent AAs & 2 & $E$ & $P(0.6)$ & $P$ \\
\hline Slc39a10 & ZIP10 & $\mathrm{Zn}^{2+}$ & & $E$ & - & - \\
\hline S/c35f1 & FLJ13018 & Nucleoside-sugar & & 60 & - & - \\
\hline Slc7a11 & $\mathrm{xCT}$ & Cysteine, glutamate & 2,5 & 44 & 24 & 5.4 \\
\hline S/c30a2 & ZnT2 & $\mathrm{Zn}^{2+}$ & 9 & $E$ & $\mathrm{P}(1.7)$ & $P$ \\
\hline Slc14a2_v1 & UTR, UT2 & Urea transport & & 39 & - & - \\
\hline S/c39a8 & ZIP8 & $\mathrm{Zn}^{2+}, \mathrm{Cd}^{2+}$ & 9 & 37 & $P(1.3)$ & $P$ \\
\hline Slc6a4 & SERT & $\mathrm{Na}$-dependent $5 \mathrm{HT}$ transport & & 35 & - & - \\
\hline S/c8a3 & NCX3 & $\mathrm{Na}^{+} / \mathrm{Ca} 2+$ exchanger & & 15 & - & - \\
\hline Slc38a1 & NAT2 & Glutamine ( $\mathrm{Na}^{+}$-dependent) & 3 & 15 & $P(1.0)$ & $P$ \\
\hline Slc6a6 & TAUT & Taurine, $\beta$-alanine & 2,5 & 14 & 6.9 & 6.1 \\
\hline Sfxn1 & Sideroflexin 1 & $\mathrm{Fe}^{2+}$ & & 14 & 8.1 & 7.2 \\
\hline Slc6a15 & BOAT2 & Neutral AAs & 2,5 & 14 & 9.8 & 4.3 \\
\hline Slc22a6 $6^{*}$ & OAT1, NKT & Organic anions ( $\mathrm{Na}^{+}$-dependent), kynurenic acid, $\mathrm{Hg}^{2+}$ & & 14 & - & - \\
\hline Slc23a1 & SVCT1 & Na dependent ascorbic acid & & 13 & - & - \\
\hline Slc39a11 & ZIP11 & $\mathrm{Zn}^{2+}$ transporter & & 12 & - & - \\
\hline S/c2a3 & GLUT3 & Glucose transporter & & 11 & 0.8 & 0.7 \\
\hline S/c5a7 & $\mathrm{CHT}$ & Choline, $\mathrm{Na}^{+}$/glucose co-transport & & 11 & - & - \\
\hline Slc19a3 & THTR2 & Thiamine & & 6.0 & - & - \\
\hline Slco2a1 & PGT, OATP2A1 & Prostaglandin D2, E1, E2 and F2A, organic anions & & 5.7 & $\mathrm{P}(1.7)$ & $\mathrm{P}$ \\
\hline Slc38a11 & - & $\mathrm{Na}^{+}$-dependent $\mathrm{AA} /$ proton antiporter (putative) & & 5.3 & - & - \\
\hline S/c5a8 & SMCT1 & Monocarboxylate transporter & & 5.2 & - & - \\
\hline Slc16a14 & MCT14 & Monocarboxylate transporter & & 5.2 & - & - \\
\hline Slc38a4 & ATA3 & Acidic and neutral AAs & 2. 3,5 & 5.2 & - & - \\
\hline S/c35d2 & HFRC & Nucleotide sugars & & 5.1 & - & - \\
\hline Slc1a4 & SATT & Glutamate, neutral AAsalanine, serine, cysteine, threonine & 3,4 & 5.1 & - & - \\
\hline Slc1a3 & GLU-T & Glutamate, neutral AAs & 3,4 & 4.5 & 3.3 & 2.8 \\
\hline Slc6a13 & GAT3 & GABA transporter & 8 & 4.4 & $P(0.4)$ & $\mathrm{P}$ \\
\hline S/c9a5 & NHE5 & $\mathrm{Na}^{+} / \mathrm{H}^{+}$exchanger & 6,7 & 4.4 & $P(0.9)$ & $\mathrm{P}$ \\
\hline S/c37a2 & SPX2 & Glucose-6-phosphate antiporter & & 4.2 & - & - \\
\hline Slc4a4 & NBC1 & Electrogenic $\mathrm{NaHCO}_{3}$ & 6,7 & 3.9 & - & - \\
\hline LOC100360087 & Ferritin Light Chain, Flt1-like & $\mathrm{Fe}^{3+}$ storage & & 3.6 & 2.4 & 1.7 \\
\hline Slc22a2 & OCT2 & Organic cations, anions, zwitterions & & 3.4 & - & - \\
\hline Slc29a2 & ENT2 & Nucleosides & & 3.2 & 1.2 & 1.1 \\
\hline Trfr & TFR & Transferrin receptor & & 3.1 & 0.5 & 0.6 \\
\hline
\end{tabular}




\begin{tabular}{|c|c|c|c|c|c|c|}
\hline S/c27a3 & FATP3 & Long-chain fatty acids & & 2.9 & 2.3 & 2.9 \\
\hline Slc24a6 & - & $\mathrm{Na}^{+} / \mathrm{Li}^{2+} / \mathrm{Ca}^{2+}$ exchanger & & 2.8 & 1.2 & 1.2 \\
\hline Slc7a2 & CAT2 & Low affinity cationic AAs & & 2.7 & $\mathrm{P}$ & - \\
\hline Slc38a7 & SNAT7 & Putative $\mathrm{Na}^{+}$-coupled neutral AAs & & 2.7 & - & - \\
\hline Slc22a15 & FLIPT1 & Organic cations, anions, zwitterions & & 2.6 & 2.1 & 1.8 \\
\hline S/c35e1 & FLJ14251 & Nucleotide sugars & & 2.5 & 1.2 & 0.9 \\
\hline S/c22a9 & OAT7 & Organic anions & & 2.5 & - & - \\
\hline Slc14a1 & UT-B1-B2 & Urea & & 2.4 & - & - \\
\hline S/c29a3 & ENT3 & Nucleosides & & 2.1 & 0.8 & 0.7 \\
\hline Slc1a5 & ASCT2 & Neutral AAs & 3,4 & 2.1 & 0.8 & 0.7 \\
\hline
\end{tabular}

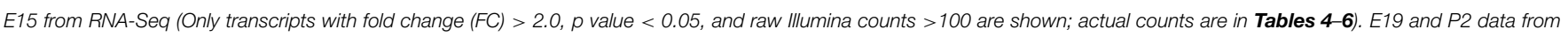

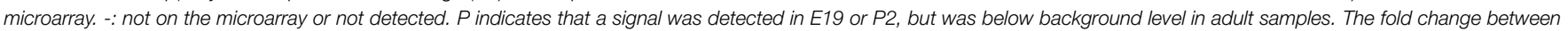

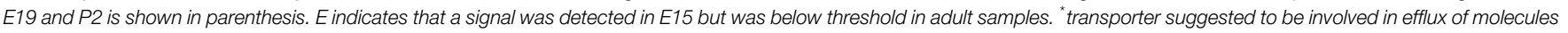

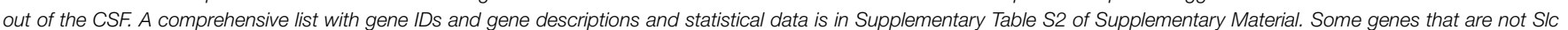

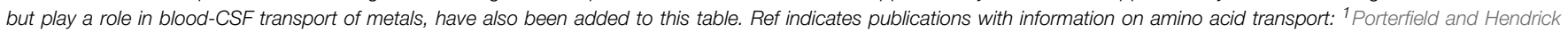

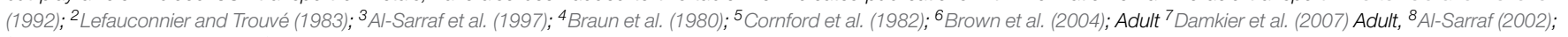
${ }^{9}$ Chowanadisai et al. (2005); ${ }^{10}$ Moos and Morgan (1998); ${ }^{11}$ Morgan and Moos (2002).

virtually no immunostaining of either membranes or cytoplasm (Figure 4B).

\section{SLC1a3}

This protein showed an example of "strong immunoreactivity," uniform distribution type at E15 and of "weak or lack of immunoreactivity" in adult plexus. Both embryonic plexus epithelial cell layers of the "plexus-fold"-the leaflet next to the ganglionic eminence and the other next to the developing hippocampus - showed a strong uniform cytoplasmic immunostaining (Figure 4C). At high magnification the prominent reactivity was shown to be due to a combination of a marked fine granular reactivity in apical cytoplasm and along both apical and basolateral cell membranes, which were particularly strongly immunostained (arrowheads in Figure 4C1) comparable to those stained for SLC16a10 in the plexus fold next to the ganglionic eminence shown in Figure 4A1. Adult choroid plexus epithelial cells showed virtually no cytoplasmic immunostaining and a lack of apical and basolateral cell membrane immunoreactivity (Figure 4D).

\section{SLC5a5}

Shown as an example of "weak immunoreactivity," uniform distribution at E15 and of "strong immunoreactivity" in adult plexus. Embryonic plexus epithelial cells showed no cytoplasmic reactivity and no immunostaining of the apical cell membrane in marked contrast to the adjacent strongly stained CSFhippocampal interface (Figure 4E). A weak basolateral cell membrane staining of plexus epithelial cells could be identified at high magnification (arrowheads in Figure 4E1). Adult choroid plexus exhibited a uniform fine and coarse granular cytoplasmic staining of most epithelial cells (open arrows in Figure 4F) and a distinct apical membrane staining (arrowheads in Figure 4F).

\section{SLC39a4 (ZIP4), SLC38a5 (SNAT5), SLC4a1 (AE1), SLC11a1 (NRAMP)}

Following immunostaining for SLC39a4 plexus epithelial cells were characterized by "weak immunoreactivity," uniformly distributed in both E15 and adult plexus, whereas staining for SLC38a5, SLC4a1, and SLC11a1 resulted in immunoreactivity of the uniform distribution-type, however stronger along the basal and basolateral cell membrane in E15, and very little cytoplasmic staining and virtually no basolateral or apical membrane staining in adult plexus apart from SLC38a5 which showed a patchy immunostaining of the apical cell membrane (data not shown).

\section{Discussion}

All but four of the 52 known families of SCL transporters were represented in the dataset from RNA sequencing of lateral ventricular choroid plexuses in the rat embryo (E15) and in the adult (Supplementary Table S1). Of the 389 individual SCLs, $64 \%$ had transcript numbers above the threshold taken to indicate likely functional significance ( $>100$ sequence reads). 81 of these were expressed 2 fold higher or more in the embryonic plexus compared to the adult, 107 were expressed higher in the adult and 62 were expressed at similar levels at the two ages. Some genes were identifiable with transcript counts of 10-100, but thought less likely to be of functional significance; they have nonetheless been included in Tables 4-6. The results for 
TABLE 3 | Expression and function of solute carrier (SIc) transcripts that were enriched in lateral ventricular choroid plexus of adult rats compared to E15 RNA-Seq) and E19 and P2 (microarray).

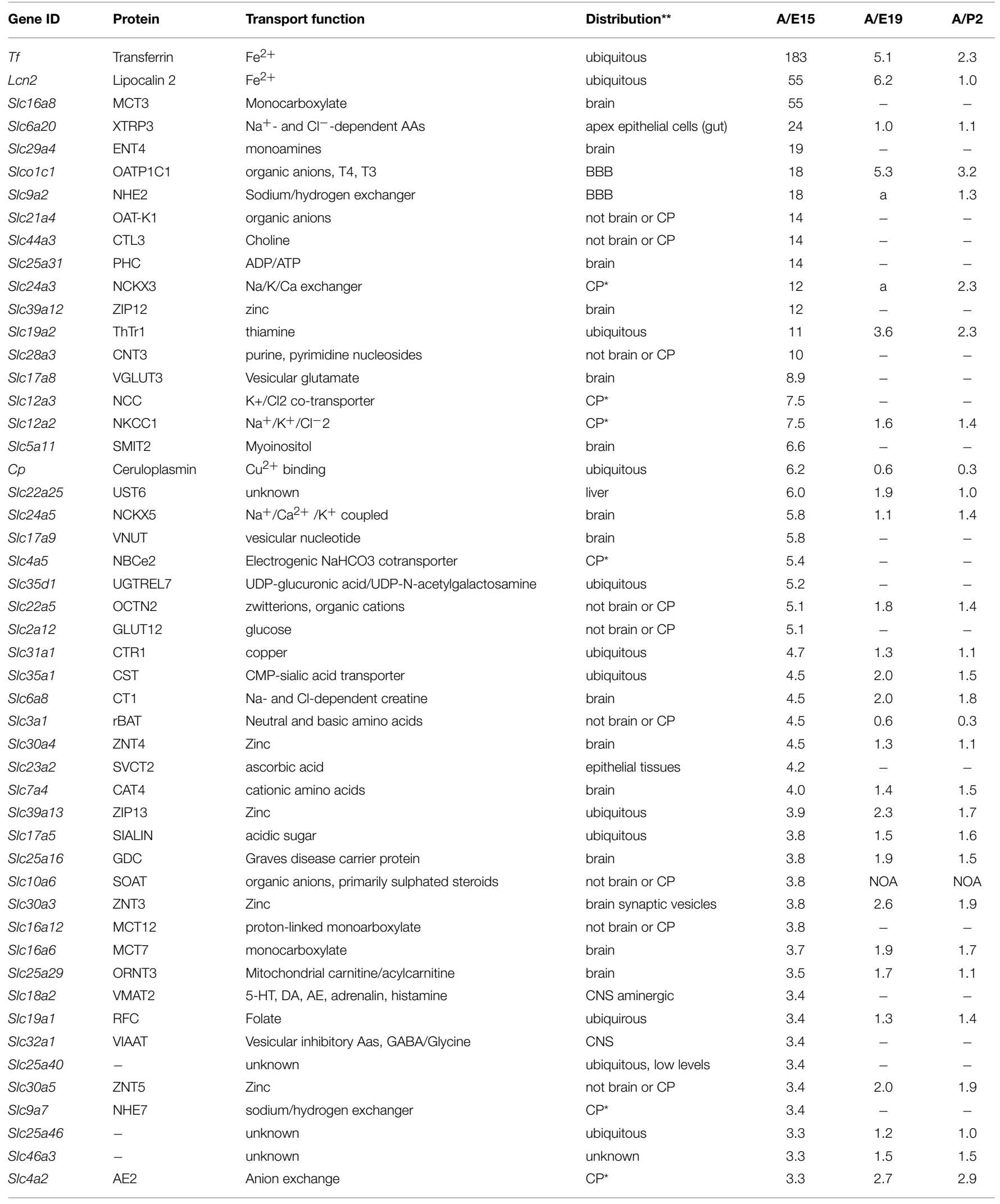


TABLE 3 | Continued

\begin{tabular}{|c|c|c|c|c|c|c|}
\hline Gene ID & Protein & Transport function & Distribution $^{\star *}$ & A/E15 & A/E19 & A/P2 \\
\hline Slc5a6 & SMVT & Sodium-dependent multivitamin, biotin & brain & 3.2 & 1.7 & 1.5 \\
\hline Slc25a17 & PMP34 & peroxisomal membrane protein & brain & 3.1 & 1.6 & 1.5 \\
\hline Slc15a3 & PHT2 & di- and tri-peptides & "faintly" in brain & 3.1 & - & - \\
\hline Slc12a4 & $\mathrm{KCC} 1$ & $\mathrm{~K}^{+} / \mathrm{Cl}^{-}$ & $\mathrm{CP}^{\star}$ & 3.1 & 2.2 & 2.0 \\
\hline Slc10a4 & P4 & Sodium/bile acid cotransporter & brain (cholinergic neurons) & 3.0 & - & - \\
\hline S/c25a12 & AGC1 & L-glutamate, aspartate & brain & 3.0 & 1.9 & 1.8 \\
\hline Slc25a3 & $\mathrm{PHC}$ & Phosphate, mitochondrial & brain & 2.9 & 1.3 & 1.2 \\
\hline Slc26a3 & DRA/CLD & Chloride anion exchanger & not brain or $\mathrm{CP}$ & 2.9 & - & - \\
\hline Slc25a4 & ANT1 & ADP/ATP & brain & 2.9 & 1.1 & 1.1 \\
\hline Slc15a2 & РEPT2 & Oligopeptides, antibiotics & choroid plexus apical surface & 2.8 & 0.7 & 0.8 \\
\hline S/c25a5 & ANT2 & ADP/ATP & brain & 2.8 & 1.2 & 1.2 \\
\hline S/c38а3 & SNAT3 & Sodium-coupled neutral amino acids & not brain or CP & 2.7 & 1.2 & 1.2 \\
\hline Slc25a14 & UCP5 & Brain mitochondrial carrier protein & highest in brain and testis & 2.7 & 1.6 & 1.4 \\
\hline S/c30a6 & ZNT6 & Zinc & brain & 2.7 & 1.3 & 1.1 \\
\hline S/c22a18 & - & Probably organic anions & not brain or $\mathrm{CP}$ & 2.6 & 1.4 & 1.6 \\
\hline Slc37a1 & SPX1 & Glycerol-3-phosphate & ubiquitous & 2.6 & 2.1 & 1.4 \\
\hline S/c25a35 & - & unknown & unknown & 2.6 & - & - \\
\hline Slc33a1 & ACATN1 & Acetyl-coenzyme A & brain & 2.6 & 1.4 & 1.3 \\
\hline Slc36a1 & PAT1 & Neutral amino acids, glycine, proline, GABA & brain & 2.4 & - & - \\
\hline Slc16a1 & MCT1 & Monocarboxylates & ubiquitous & 2.4 & 3.1 & 1.4 \\
\hline Slc20a1 & PIT1 & Sodium-dependent phosphate & ubiquitous & 2.4 & 1.7 & 2.0 \\
\hline Slc36a4 & PAT4 & Proton-coupled AAs, tryptophan, proline & ubiquitous & 2.3 & - & - \\
\hline Slc48a1 & HRG1 & Heme transporter & brain & 2.3 & 1.9 & 1.3 \\
\hline S/c25a32 & MFT & Mitochondrial folate & unknown & 2.3 & 1.1 & 1.0 \\
\hline Slc41a1 & MgtE & $\mathrm{Mg}^{2+}$, other metal cations & not brain or CP & 2.3 & a & 1.7 \\
\hline Slc16a13 & MCT13 & Monocarboxylate & not brain or CP & 2.3 & 1.9 & 1.7 \\
\hline Slc26a4 & PENDRIN & Anions & epithelial cells & 2.2 & - & - \\
\hline Slc16a2 & MCT8 & Monocarboxylate transporter 8, thyroxine & brain & 2.1 & - & - \\
\hline Slc25a20 & CACT & Mitochondrial carnitine/acylcarnitine & brain & 2.1 & 1.1 & 0.9 \\
\hline S/c35b3 & PAPST2 & Adenosine 30-phospho 50-phosphosulphate & ubiquitous & 2.1 & 1.7 & 1.5 \\
\hline Slc7a10 & ASC1 & Asc-type amino acids, D-serine & brain & 2.1 & 0.6 & 0.8 \\
\hline Slc31a2 & CTR2 & $\mathrm{Cu}^{2+}$ & ubiquitous & - & 1.7 & 1.0 \\
\hline
\end{tabular}

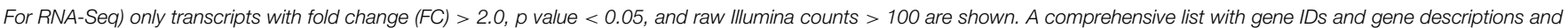

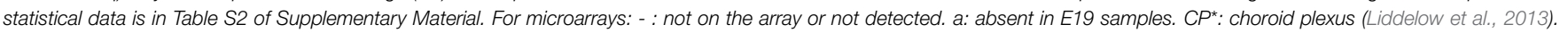

${ }^{* *}$ Previously described tissue distribution from reviews in Hediger (2013). Note that in studies involving brain extraction it is often not stated if choroid plexuses were included or excluded.

microarray were generally consistent with those for RNA-Seq in their developmental profile.

It is apparent from the above analysis that many genes identified are from families involved in transport of ions important for homeostatic mechanisms in the CSF and interstitial fluid of the brain (Tables 2, 3, and Supplementary Tables S2, S3). The monovalent inorganic ion transporters known to be functionally active in adult choroid plexus and involved in CSF composition and secretion have been described in detail and their expression levels correlated with functional data in the paper by Liddelow et al. (2013). Divalent metal ions such as $\mathrm{Ca}^{2+}$, $\mathrm{Zn}^{2+}$, and $\mathrm{Mg}^{2+}$ may be involved in specific cellular functions in brain such a synaptic transmission of nerve impulses and are considered briefly below.

The immunohistochemical data presented above indicate that at least some of the most highly expressed Slc genes are able to produce their protein products. A more difficult problem is to establish the functional effectiveness of individual Slcs for example for glucose, particular amino acids or specific monocarboxylates. This is because of the overlap in substrates between many SLCs (see Tables 2, 3). Because of this and of upregulation of unaffected genes, even selective knockouts of single genes may not reveal the contribution of that particular gene to the transport of specific substrates (see Keep and Smith, 2011). However, it is possible to suggest that at least some of the genes are indeed functionally more active in embryonic choroid plexus by examining results of transport studies for glucose, amino acids, monocarboxylates, and divalent cations in developing brain.

\section{Glucose Transport}

The best known and ubiquitously distributed glucose transporter Slc2a1 (GLUT1) was expressed at a 3-fold higher level in the embryonic choroid plexus at transcript levels (Table 4) 
TABLE 4 | Glucose and Monocarboxylate transporters in developing rat choroid plexus.

\begin{tabular}{|c|c|c|c|c|}
\hline \multirow[t]{2}{*}{ Gene name } & \multirow[t]{2}{*}{ Gene description } & \multirow[t]{2}{*}{ FC } & \multicolumn{2}{|c|}{ Raw transcript counts } \\
\hline & & & EMBRYO (E15) & ADULT \\
\hline \multicolumn{5}{|c|}{ GLUCOSE TRANSPORTERS } \\
\hline Embryo & & E/A & & \\
\hline Slc5a10 & Slc family 5 , sodium/glucose cotransporter 10 & $E$ & 62 & 0 \\
\hline S/c2a3 & Slc family 2, facilitated glucose transporter 3, GLUT3 & $E$ & 150 & 8 \\
\hline Slc2a4 & Slc family 2, facilitated glucose transporter 4, GLUT4 & $E$ & 90 & 6 \\
\hline S/c35d2 & UDP-N-acetylglucosamine/UDP-glucose/GDP-mannose & 5.1 & 224 & 25 \\
\hline Slc45a1 & Proton-associated sugar transporter A, glucose & $E$ & 33 & 5 \\
\hline Slc2a1 & Slc family 2 , facilitated glucose transporter 1 , GLUT1 & 3.0 & 368 & 111 \\
\hline Adult & & A/E & & \\
\hline Slc2a12 & Slc family 2 , facilitated glucose transporter 12 , GLUT12 & 5.1 & 3569 & 10437 \\
\hline Slc2a5 & Slc family 2, facilitated glucose transporter 5, GLUT5 & 2.3 & 20 & 27 \\
\hline Klf15 & Krueppel-like factor 15, regulator of GLUT4 & 2.2 & 292 & 373 \\
\hline \multicolumn{5}{|c|}{ MONOCARBOXYLATE TRANSPORTERS } \\
\hline Embryo & & E/A & & \\
\hline Slc16a10 & Monocarboxylate transporter 10, MCT10, TAT1, T3, T4 & $E$ & 3089 & 51 \\
\hline Adult & & A/E & & \\
\hline Slc16a8 & Monocarboxylate transporter 3, MCT3 & 55 & 49 & 1524 \\
\hline Slc16a6 & Monocarboxylate transporter 7, MCT6, MCT7 & 3.7 & 1524 & 3239 \\
\hline Slc16a1 & Monocarboxylate transporter 1, MCT1 & 2.4 & 577 & 1235 \\
\hline Slc16a7 & Monocarboxylate transporter 2, MCT2 & 2.3 & 44 & 58 \\
\hline Slc16a13 & Monocarboxylate transporter 13, MCT13-human & 2.3 & 1354 & 1756 \\
\hline Slc16a2 & Monocarboxylate transporter 8, MCT8-human, thyroid hormone & 2.1 & 7599 & 9323 \\
\hline
\end{tabular}

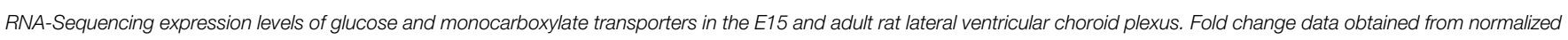

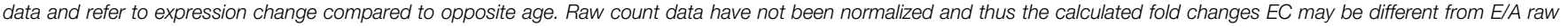
count ratios. E is embryo only. $A$ is adult only.

suggesting that it would have significant functional activity at both ages, although higher in the embryo. It has been identified in developing choroid plexus in a number of previous studies (Vannucci, 1994; Baud et al., 2003); the level was highest during gestation followed by a $50 \%$ decline by P1 and a further decline during the first postnatal week (Bauer, 1998). Bauer (1998) suggests this reflects a decline in cerebral glycogen content (Kohle and Vannucci, 1977). Glycogen is particularly prominent in choroid plexus epithelial cells in the late embryonic and early neonatal period, after which it disappears (Schachenmayr, 1967 and see Netsky and Shuangshoti, 1975). Slc2a3 (GLUT3) in the present study was expressed only at E15 in RNA-Seq compared to adult and in microarray was below adult at E19 and P2. The protein product of this gene was not identified in a previous study of postnatal rat choroid plexus (Vannucci, 1994). Slc2a4 (GLUT4) was also expressed only in the embryo, although at a low transcript count (90). This appears to be consistent with the finding of Vannucci et al. (2000) in mouse choroid plexus where it was detected as early as at E14. In addition, in the present study Slc5a10 (SGLT5), a sodium-glucose transporter was expressed exclusively in the embryonic choroid plexus. Previously it has only been identified in kidney cortex (Wright, 2013). However, the raw transcript count for this gene was low, thus its functional significance is unclear.

\section{Monocarboxylate Transport}

It has been long accepted that although glucose is a fundamentally important energy substrate in the brain, during development the brain also uses lactate and other monocarboxylates to a substantial degree. In the postnatal period in rodents it has been suggested that a switch occurs from a combination of glucose, lactate, and ketone body metabolism in the immature brain to a reliance on glucose in the adult (Cremer, 1982; Nehlig, 1997). Several monocarboxylate transporters have been found in the developing brain of rodents. Slc16a1 (MCT1) is expressed and its protein product present in cerebrovascular endothelial cells of rodents at higher levels in early postnatal ages compared to later (Leino et al., 1999; Vannucci and Simpson, 2003). In contrast the level of Slc16a7 (MCT2) expression and protein product, present predominantly in neuronal cells, do not appear to be developmentally regulated (Vannucci and Simpson, 2003). Slc16a1 (MCT1), but not Slc16a7 (MCT2), has been 
TABLE 5 | Expression of amino acid transporters in embryonic (E15) and adult rat choroid plexus.

\begin{tabular}{|c|c|c|c|c|}
\hline \multirow[t]{2}{*}{ Gene name } & \multirow[t]{2}{*}{ Gene description } & \multirow[t]{2}{*}{ FC } & \multicolumn{2}{|c|}{ Raw transcript counts } \\
\hline & & & EMBRYO (E15) & ADULT \\
\hline \multicolumn{5}{|c|}{ AMINO ACID TRANSPORTERS } \\
\hline Embryo & & E/A & & \\
\hline S/c38a5 & Sodium-coupled neutral amino acid transporter 5, glycine & $\mathrm{E}$ & 1021 & 6 \\
\hline S/c43a3 & Putative transporter (by similarity) & $E$ & 914 & 8 \\
\hline Slc7a11 & Solute carrier family 7 (cationic amino acid transporter, y+ system), member 11 & 44 & 13298 & 178 \\
\hline Slc43a1 & Large neutral amino acids transporter small subunit 3 & $E$ & 204 & 5 \\
\hline Slc7a1 & High affinity cationic amino acid transporter 1 & 16 & 7273 & 264 \\
\hline Slc38a1 & Sodium-coupled neutral amino acid transporter 1, glutamine & 15 & 1188 & 47 \\
\hline Slc6a6 & Sodium- and chloride-dependent taurine transporter & 14 & 813 & 33 \\
\hline Slc6a15 & Orphan sodium- and chloride-dependent neurotransmitter transporter NTT73 & 14 & 5446 & 227 \\
\hline S/c7a3 & Cationic amino acid transporter 3, I-lysine, I-ornithine, I-arginine & 9.1 & 42.0 & 2.7 \\
\hline Slc38a4 & Sodium-coupled neutral amino acid transporter 4 & 5.2 & 102 & 11 \\
\hline S/c1a4 & Neutral amino acid transporter A, I-serine, I-alanine, I-cystine, I-proline, & 5.1 & 436 & 49 \\
\hline S/c1a3 & Excitatory amino acid transporter 1, I-glutamate transport, malate-aspartate & 4.5 & 4372 & 577 \\
\hline Slc7a2 & Low affinity cationic amino acid transporter 2, I-arginine, I-lysine, I-ornithine & 2.7 & 179 & 38 \\
\hline S/c38a7 & Putative sodium-coupled neutral amino acid transporter 7 & 2.7 & 337 & 73 \\
\hline S/c43a2 & Large neutral amino acids transporter small subunit 4 & 2.4 & 651 & 156 \\
\hline Slc1a5 & Neutral amino acid transporter B(0) I-serine, & 2.1 & 1119 & 308 \\
\hline Adult & & A/E & & \\
\hline Slc6a20 & Sodium- and chloride-dependent transporter XTRP3, proline IMINO transporter & 24 & 44 & 594 \\
\hline Slc6a11 & Sodium- and chloride-dependent GABA transporter 3 & 5.0 & 10 & 28 \\
\hline S/c3a1 & Neutral and basic amino acid transport protein rBAT & 4.5 & 746 & 1936 \\
\hline Slc7a4 & Cationic amino acid transporter 4 & 4.0 & 706 & 1618 \\
\hline S/с38а3 & Sodium-coupled neutral amino acid transporter 3. I-asparagine, I-glutamine, I-histidine & 2.7 & 4507 & 6922 \\
\hline Slc36a1 & Proton-coupled neutral amino acid transporter 1, I-glycine, I-proline & 2.4 & 97 & 136 \\
\hline Slc36a4 & Proton-coupled amino acid transporter 4 & 2.3 & 718 & 969 \\
\hline Slc7a10 & Asc-type neutral amino acid transporter 1 , d-serine, I-serine & 2.1 & 783 & 935 \\
\hline
\end{tabular}

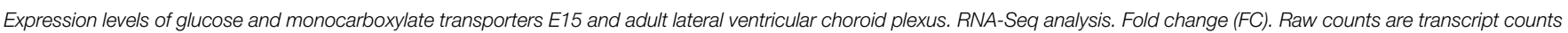

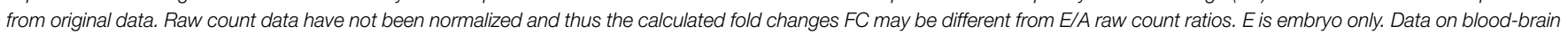

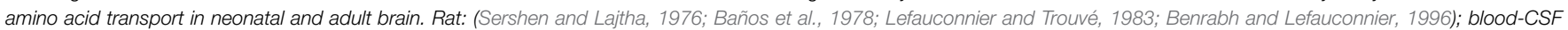

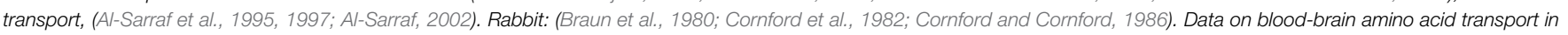
adult brain. (Davson and Segal, 1996; Segal, 2001).

identified in adult mouse choroid plexus (Koehler-Stec et al. (1998).

The expression of monocarboxylate transporters appears to have been less studied in the embryonic brain barrier interfaces. Apart from a high expression of Slc16a10 (MCT10) in embryonic mouse choroid plexus (Liddelow et al., 2012) nothing seems to be known about monocarboxylate transporters in the embryonic choroid plexus. This high expression in embryonic mouse choroid plexus is confirmed in the present study for embryonic rat choroid plexus. This is a particularly noteworthy finding because MCT10 is a transporter for tri-iodothyronine, T3 and thyroxine, T4 (Porterfield and Hendrick, 1992), which are essential for normal brain development. Inadequate delivery of T4 to the developing brain, usually due to iodine deficiency, results in cretinism (Rivas and Naranjo, 2007; Skeaff, 2011). Given the prominence of the choroid plexuses in the embryonic brain compared to vascularisation (Johansson et al., 2008), it may be that MCT10 in the choroid plexuses along with TTR, a thyroid hormone carrier highly expressed throughout development, is the major mechanism by which thyroxine is delivered to the brain in early stages of its development. This is also in line with the low developmental expression of Slco1c1, the main thyroid hormone transporter expressed at the blood-brain interfaces in the adult (Kratzer et al., 2013).

Published data demonstrating at the protein level, that some of the glucose transporters (Vannucci, 1994; Bauer, 1998; Baud et al., 2003) and monocarboxylate transporters (Leino et al., 1999; Vannucci and Simpson, 2003) are present in the choroid plexus barrier interface between blood and CSF in the developing brain indicate that these transporters are probably functional. In addition, it has been shown that brain uptake of both glucose (Braun et al., 1980; Cornford and Cornford, 1986) 
TABLE 6 | Expression of zinc and other metal transporters in embryonic (E15) and adult rat choroid plexus.

\begin{tabular}{|c|c|c|c|c|}
\hline Gene name & Gene description & FC & \multicolumn{2}{|c|}{ Raw transcript counts } \\
\hline \multicolumn{5}{|l|}{ EMBRYO } \\
\hline ZINC & & E/A & & \\
\hline Slc30a2 & Zinc transporter 2, ZnT2 & $\mathrm{E}$ & 608 & 8 \\
\hline S/c39a8 & Zinc transporter ZIP8 & 37 & 1493 & 24 \\
\hline Slc39a10 & Zinc transporter, 10 (Predicted), ZIP10 & 2.5 & 5547 & 1304 \\
\hline \multicolumn{5}{|l|}{ OTHER METALS } \\
\hline Sfxn1 & Sideroflexin-1, iron transport & 14 & 1103 & 45 \\
\hline Slc11a1 & Natural resistance-associated macrophage protein $1, \mathrm{Mn}+, \mathrm{Cd}+$ transporter & $\mathrm{E}$ & 130 & 8 \\
\hline \multicolumn{5}{|l|}{ ADULT } \\
\hline ZINC & & A/E & & \\
\hline Slc39a1 & ZIP1, zinc transport & 12 & 2143 & 17839 \\
\hline S/c39a12 & Zinc transporter ZIP12 & 12 & 489 & 3318 \\
\hline Slc30a4 & Zinc transporter 4, ZnT4 & 4.5 & 2025 & 5248 \\
\hline Slc39a13 & Zinc transporter ZIP13 & 3.9 & 758 & 1681 \\
\hline S/c30a3 & Zinc transporter 3, ZnT3 & 3.8 & 54 & 117 \\
\hline Slc30a5 & Zinc transporter 5, Co, ZnT5 & 3.4 & 1397 & 2719 \\
\hline S/c30a6 & Zinc transporter 6, Co, ZnT6 & 2.7 & 491 & 752 \\
\hline
\end{tabular}

\section{OTHER METALS}

\begin{tabular}{|c|c|c|c|c|}
\hline Tf & Transferrin, iron transport & 183 & 2027 & 212407 \\
\hline Lcn2 & Lipocalin 2, Signal recognition particle receptor subunit beta, iron transport & 55 & 12 & 365 \\
\hline$C p$ & Ceruloplasmin copper transport & 6.2 & 188 & 675 \\
\hline Slc31a1 & High affinity copper uptake protein 1 & 4.6 & 6673 & 17839 \\
\hline Slc41a1 & MgtE-like magnesium transporter, member 1 & 2.3 & 401 & 530 \\
\hline \multicolumn{5}{|c|}{ NO CHANGE WITH AGE } \\
\hline Slc30a6 & ZNT6, zinc transport & - & 491 & 752 \\
\hline Slc30a7 & ZNT7, zinc transport & - & 424 & 400 \\
\hline Slc30a9 & ZNT9, zinc transport & - & 1512 & 1526 \\
\hline Slc39a3 & ZIP3 zinc transport & - & 322 & 315 \\
\hline S/c39a4 & ZIP4, zinc transport & - & 58 & 38 \\
\hline Slc11a2 & DMT1, divalent ion transport, including iron & - & 1929 & 1518 \\
\hline \multicolumn{5}{|c|}{ OTHER METALS } \\
\hline Slc40a1 & iron-regulated transporter & - & 889 & 455 \\
\hline Slc31a2 & CTR2, copper transport & - & 63 & 144 \\
\hline S/c41a2 & magnesium transporter & - & 20 & 63 \\
\hline
\end{tabular}

Expression levels of $\mathrm{Zn}$ and other metal transporters comparing $\mathrm{E} 15$ embryo and adult lateral ventricular choroid plexus obtained from RNA-Seq analysis. Fold change (FC), Embryo only (E). Raw counts are transcript counts from original data. Raw count data have not been normalized and thus the calculated fold changes FC may be different from E/A raw count ratios. 

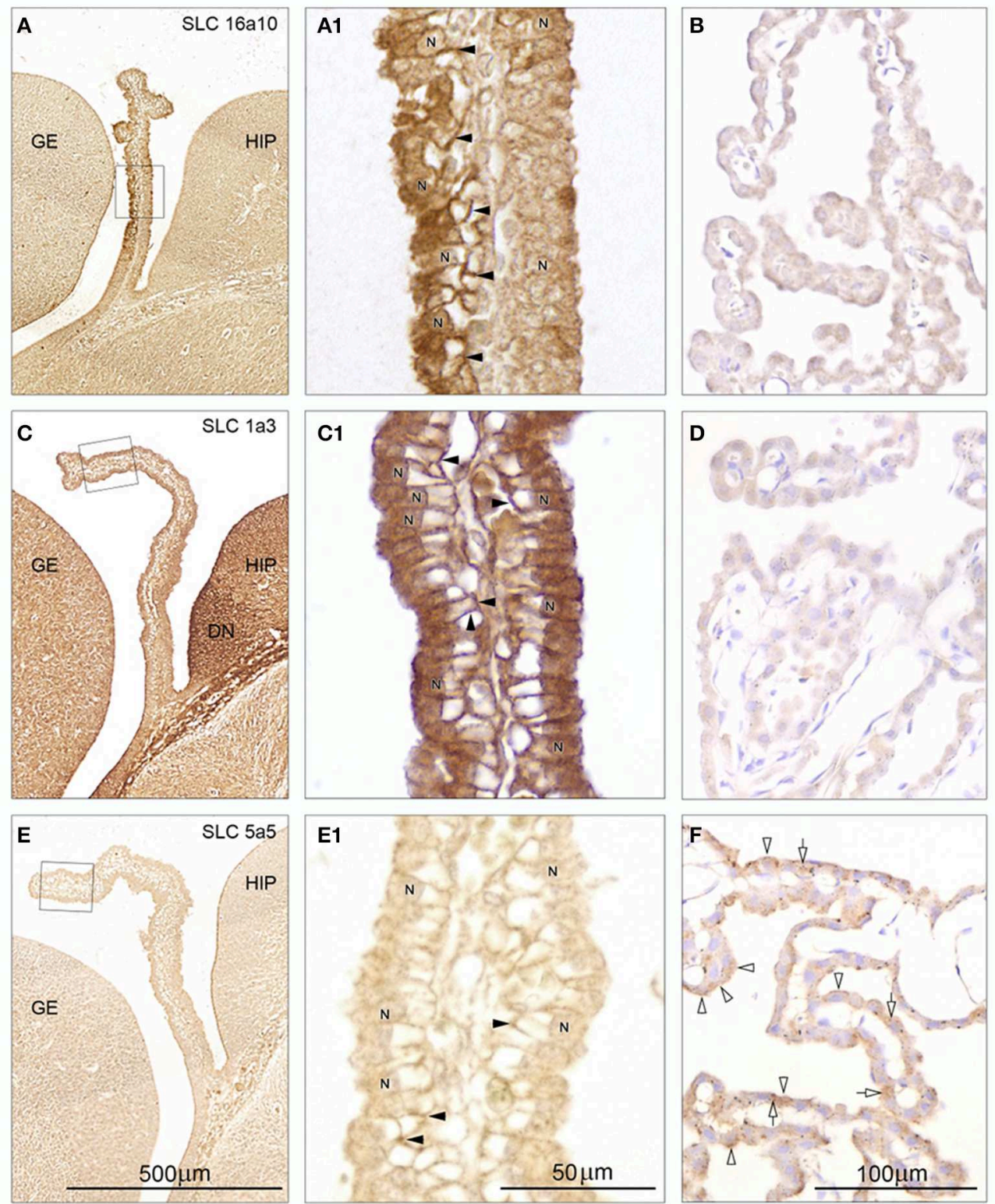

FIGURE 4 | Immunohistochemistry of influx transporters in sagittal and coronal sections from $\mathrm{E} 15$ and adult rat choroid plexus: SLC 16a10 (A,A1,B), SLC 1a3 (C,C1,D), and SLC 5a5 (E,E1,F). The rectangles in $(\mathbf{A}, \mathbf{C}, \mathbf{E})$ from $\mathrm{E} 15$ are shown at higher magnification in $\mathbf{A 1}, \mathbf{C 1}, \mathbf{E 1}$. For comparison adult choroid plexus stained for the same three transporters is shown in $\mathbf{B , D}, \mathbf{F}$. Note the differences in distribution of immunoreactivity between E15 stained for the three different transporters and between E15 and adult choroid plexus stained for a given transporter. (A) The rostral-most leaflet of the lateral ventricular choroid plexus facing the ganglionic eminence (GE) demonstrates a very strong reactivity in contrast to the caudal-most leaflet facing the developing hippocampus (HIP). (A1) At higher magnification the Immunostaining fills the entire cytoplasm apical to the nuclei $(\mathrm{N})$ and down along the basolateral membrane (arrowheads) just sparing the empty glycogen space in the epithelial cells of the rostral leaflet. The caudal leaflet is also positively stained and shows a more uniform cytoplasmic reactivity although condensed at the perinuclear membrane. (B) Adult choroid plexus shows virtually no immunostaining for SLC 16a10. (C) Both leaflets of the choroid plexus demonstrate a uniform cytoplasmic immunoreactivity for SLC 1a3 although not as strong as that of the adjacent dentate neuroepithelium (DN). (C1) At higher magnification the immunostaining of the entire plexus shows a pattern similar to that described for the rostral leaflet stained for SLC $16 a 10$ (compare with A1). (D) Adult choroid plexus shows virtually no immunostaining for SLC 1 a3. (E) The E15 choroid plexus shows very little immunoreactivity following staining for SLC $5 \mathrm{a} 5$ which on the other hand outlines the CSF surface of the hippocampus. (E1) At higher magnification a weak Immunostaining is localized to some basolateral cell membranes (arrowheads) of the choroid plexus epithelial cells. (F) Adult choroid plexus epithelial cells exhibit a uniform very fine granular cytoplasmic reactivity following immunostaining for SLC 5a5. Many cells also exhibit a coarse granular staining (open arrows) and apical membrane reactivity (open arrowheads). (A,C,E) Same magnification, scale bar $500 \mu \mathrm{m}$. (A1,C1,E1) Same magnification, scale bar $50 \mu \mathrm{m}$. (B,D,F) Same magnification, scale bar $100 \mu \mathrm{m}$. 
and lactate (Cornford et al., 1982; Cornford and Cornford, 1986) is greater in newborn rabbits than in adults, which again supports the likelihood that transporters for these metabolically important nutrients are indeed functionally more active in the developing brain. It is not clear from the transport studies, which specific glucose transporters would have been involved, but the transcript numbers perhaps give some indication of the relative functional importance of the different transporters. The most highly expressed glucose transporter at both ages was Slc2a12, although 5 fold more in the adult.

The finding in the present study of a high expression of glucose transporters in the embryonic brain, but not of those transporting lactate and other monocarboxylates, suggests that the embryonic brain is like the adult brain in being exclusively dependent on glucose metabolism as a source of energy, as suggested previously by Vannucci and Vannucci (2000) and unlike the postnatal brain in which monocarboxylate transport should be functionally significant. However, of all the monocarboxylate transporters detected on the arrays, three genes, Slc16a3, Slc16a4 (not detected by RNA-Seq) and Slc16a10, were expressed at a higher level at E19 than at P2 and adult, and the others were expressed at a higher level in adult. It is possible that a transient up-regulation occurs later in post-natal development for some of these transporters. This was shown for Slc16a1 (MCT1) in the postnatal period at least in rodents, and may relate to the high fat content in human and rodent maternal milk, which generates substantial ketone body concentrations in blood, as a consequence of liver metabolism (Vannucci and Vannucci, 2000; Vannucci and Simpson, 2003).

\section{Amino Acid Transport}

There is a limited amount of information concerning the effectiveness of amino acid transport in vivo, that could support expression data. Experiments mainly carried out in the 1970s80 s were mostly performed in neonatal rats (e.g., Baños et al., 1978; Lefauconnier and Trouvé, 1983, see Table 6) but some were in fetal and postnatal rabbits (Braun et al., 1980; Cornford et al., 1982; Cornford and Cornford, 1986). Very few of these studies estimated amino acid entry from blood into CSF. Most studied brain tissue; an exception is the papers of Al-Sarraf and colleagues (Al-Sarraf et al., 1995, 1997; Al-Sarraf, 2002). However, given the paucity of blood vessels in very immature brain it is likely that much of the amino acid detected in brain tissue of, for example neonatal rodent, arrived there via the choroid plexuses and CSF. Another interpretational difficulty is that these experiments were done before data on individual transporters were available. Thus, at best we can only compare individual or classes of amino acids shown to be transported in vivo in the developing brain with the various families of SLC transporters known to transport individual or classes of amino acids (Table 5). However, the finding (described in the papers cited above) that transport of many amino acids in developing brain is greater than in the adult strongly supports the proposition that the higher expression of many amino acid transporters in the developing brain is indeed reflected in greater transport activity.

\section{Metal Transport}

Both of the major zinc transporter families (Slc30, ZnT and Slc39, ZIP) are represented in our two datasets. The members of the two families function in opposite directions, thus maintaining cellular zinc homeostasis. ZnT proteins of Slc30 gene efflux zinc whereas ZIP proteins function to increase zinc uptake when cellular zinc is depleted (Marger et al., 2014; Tepaamorndech et al., 2014). In the context of transfer of zinc across the epithelial cells of the choroid plexus it may be that they operate in tandem. Support for this comes from the observation that SCL39A6 (LIV1) has been immunolocalised to the apical membrane of the neonatal choroid plexus epithelial cells (Chowanadisai et al., 2005).

None of the six Slc30 genes identified in this study (two high in the embryo, Slc30a2, Slc30a10, and four in the adult, Slc30a3$6,2.7-4.5$ FC) have previously been reported in choroid plexus. Of the three Slc39a genes identified (Slc39a8, 37 FC higher in embryo; Slc39a12 and Slc39a13, FC 12 and 3.9 higher in adult, respectively) none appear to have been identified previously in choroid plexus. The microarray data show that Slc30 and Slc39 genes identified in E15, but not in adult, are still expressed at the perinatal stages. Other genes of these families whose expression is lower at E15 display a rapid developmental increase in expression, to reach adult level shortly after birth. Altogether this suggests that choroidal zinc transport is substantial throughout development.

Zinc is an essential nutrient for many cellular biochemical processes (Tyszka-Czochara et al., 2014) and is essential for normal brain development and function (Sandstead, 1985). The requirement for zinc is increased during embryonic development and deficiency results in birth defects (McKenzie et al., 1975; Sandstead et al., 1975), including multiple defects in neuronal development, by mechanisms that are unclear (Chowanadisai et al., 2013a; Grabrucker et al., 2014). Slc39a12 (ZIP12) has been found to be highly expressed in brain of human and mouse adults and in development appears to be involved in early stages of neuronal differentiation and neurite outgrowth, through its role as a zinc transporter (Chowanadisai et al., 2013a,b). Zinc has specific functions in synaptic transmission. 5-15\% of the brain's zinc content is concentrated in synaptic vesicles of glutamatergic neurons. This is of importance for neuronal function in the developing and adult brain, but the identification of several genes for glutamate receptors and other transmitters, as reported earlier (Liddelow et al., 2013) suggests that the zinc transporters in choroid plexus may have a function in relation to the innervation of the choroid plexus. A more general consideration of the importance for neural function in the brain is outside the scope of this paper. Finally, zinc transporters, like other metal transporters, may transport this metal for use by the epithelial cells themselves, as discussed below.

Iron uptake into the brain occurs across both the blood-brain and blood-CSF barriers (Moos and Morgan, 2000). Transferrin, which binds iron, makes an important contribution at both interfaces, but there are some differences in the mechanisms involved. At the blood-brain barrier, transferrin receptors on the cerebral endothelial cells bind transferrin-carrying iron, which is taken up into the cells. This mechanism and subsequent 
steps in iron delivery to the brain have been described in detail (e.g., Leitner and Connor, 2012; Singh et al., 2014) and will not be considered further here. However, the uptake of both iron and transferrin is greater in the developing brain, the more so for iron itself (Morgan and Moos, 2002). The involvement of the choroid plexuses in iron transport into the brain in addition to transferrin uptake via transferrin receptors also involves the synthesis and secretion of transferrin into the CSF (Leitner and Connor, 2012). In the adult brain it has been suggested that the choroid plexuses, compared to the cerebral vasculature may play a much larger role in iron uptake than previously thought (Rouault et al., 2009). As mentioned above in relation to other transport properties, the early development of the choroid plexuses compared to brain vasculature (Johansson et al., 2008) indicates that the plexuses are likely to be even more important for iron transport in the developing brain. The likely importance of the transferrin gene, $T f$, for iron transport in both the embryonic and adult choroid plexus is emphasized by the high transcript numbers (Table 6), although much higher in the adult (183 fold). Tf expression however increases rapidly, and is only 5- and 2.3 times lower at E19 and P2 respectively than in adult. Several other iron transporters were expressed at a higher level in the embryo than in the adult: sideroflexin-1, $S f x n 1$ and ferritin light chain 1-like (LOC100360087), but in terms of transcripts both were expressed at lower levels than $T f$ (Table 6). Slc11a1 and hephaestin were only expressed in the embryonic plexus but at low levels, so their functional contribution is doubtful. The transferrin receptor was expressed at a higher level in embryonic choroid plexus (Table 6) but this was reversed at birth. Several studies have described developmentally regulated immunohistochemical staining for several of the iron transporters in the choroid plexus (e.g., Siddappa et al., 2003; Wang et al., 2008). Moos and Morgan (1998) have provided direct evidence for the transfer of ${ }^{59} \mathrm{Fe}$ from blood into brain and CSF. The importance for iron in brain function lies in its involvement in cellular respiration and cell proliferation during development. The brain also requires iron for myelination and neurotransmission (Leitner and Connor, 2012).

Manganese $(\mathrm{Mn})$ is an essential biological element and a necessary cofactor in a number of important enzymatic reactions (Gunter et al., 2013) and is an essential micronutrient for normal brain development (Santamaria and Sulsky, 2010). Manganese transport has been demonstrated in choroid plexus freshly isolated from $\mathrm{P} 2$ and adult rats; uptake into plexus cells was found to be greater in P2 choroid plexuses. Unidirectional blood-toCSF transport of manganese was demonstrated using P2-derived in vitro reconstituted epithelium (Schmitt et al., 2011). As these authors point out it is not possible to link this transport to specific transporters but suggest the divalent metal transporters SLC11a2, SLC11a3, SLC39a8, and SCL39a14 as potential candidates. In the RNA-Seq and array screens in the present study Slc11a1 (8.9 fold) and Slc39a8 (37 fold) were expressed at higher levels in the embryonic and perinatal choroid plexus, which correlated with the higher uptake shown by Schmitt et al. (2011). In addition, transport of manganese by transferrin has been demonstrated (Gunter et al., 2013). Copper is an essential nutrient for normal growth and development of the fetus and neonate (Uriu-Adams et al., 2010). It is a co-factor for several enzymes and also a structural component with involvement in many physiological pathways in the brain; in particular it is a component of cytochrome $c$ oxidase, a member of the super-family of heme-copper-containing oxidases (Scheiber et al., 2014). In addition to divalent ion transporters thought to transport copper that were identified in the present study (e.g., Slc11a2) at least two more specific copper transporters were expressed in the choroid plexus. Ceruloplasmin was expressed at modest transcript numbers some 6.2 fold higher in the adult plexus (Table 6). The high affinity copper transporter, Slc31al, was amongst the highest expressed transporter genes at both ages identified in this study and was 4.6 fold higher in the adult (Table 6). These data are in accordance with in situ hybridization showing the expression of Slc31a1 in choroid plexus and its rapid, choroid plexus-restricted increase from E14 to E18.5 in mice (Kuo et al., 2001). These authors suggest that the choroid plexus is a major pathway of copper supply to the developing brain.

Thus, genes for all of the main metal transporters have been identified in the choroid plexus, several of them expressed at higher levels in the embryo than in the adult, presumably reflecting the importance of their involvement in brain development. Metals are also needed for normal choroidal epithelial functions. Iron-containing cytochrome levels are high in choroid plexus as a result of high mitochondrial activity. Copper, zinc, and manganese are cofactors of several enzymatic systems, in particular superoxide dismutases (SOD) involved in regulating oxidative stress. Both $\mathrm{Mn}-\mathrm{SOD}$ and $\mathrm{Cu}-\mathrm{Zn}-\mathrm{SOD}$ are well expressed in choroid plexus throughout development (Yon et al., 2008, 2011). Thus, a primary function of metal transporters in the choroid plexus may be to supply metals required for proper functioning of the blood-CSF barrier. Of note, copper deficiency leads to a specific increase in Slc31a1 expression at the choroid plexus, a finding interpreted as a compensatory mechanism to spare the choroid plexus itself from copper deficiency (Gybina and Prohaska, 2006).

\section{Immunohistochemistry}

The protein products and their cellular distribution were demonstrated in E15 and adult choroid plexus for a selection of the Slcs identified in the gene screens, using immunohistochemistry. Three patterns of immunostaining were identified in E15 plexus epithelial cells: (i) differential immunoreactivity with regional distribution (e.g., SLC16a10), (ii) strong immunoreactivity with uniform distribution (SLC1a3), and (iii) weak immunoreactivity with uniform distribution (SLC5a5). In the adult plexus two different patterns of immunoreactivity in epithelial cells were noted: (i) strong immunoreactivity (SLC5a5) and (ii) weak or lack of immunoreactivity (SLC1a3, SLC16a10, SLC39a4). In a previous study (Liddelow et al., 2013) we illustrated immunostaining for SLC4a1 that was strong and uniformly distributed in E15 rat choroid plexus. Immunostaining for AQP1 has also been shown to have a uniform cellular distribution which was strong in E15 rat choroid plexus with characteristic localization in the apical 
TABLE 7 | Comparison of expression level (transcript numbers) and immunostaining for protein gene product.

\begin{tabular}{|c|c|c|c|c|c|}
\hline Gene ID & Protein ID & E15 transcript raw counts & E15 immunostaining & Adult transcript raw counts & Adult immunostaining \\
\hline Slc16a10 & MCT10 & 3089 & Strong, regional & 51 & Weak, uniform \\
\hline Slc38a5 & SNAT5 & 1021 & Moderate, uniform & 6 & Minimal, few patches \\
\hline Slc4a1 & $\mathrm{AE} 1^{*}$ & 7109 & Strong, uniform & 70 & Minimal-absent \\
\hline Slc11a1 & NRAMP & 130 & Moderate, uniform & 8 & Absent \\
\hline S/c1a3 & EAAT1 & 4372 & Strong, uniform & 577 & Weak, uniform \\
\hline Slc5a5 & NIS & 163 & Weak, uniform & 4916 & Strong, uniform \\
\hline Slc39a4 & ZIP4 & 58 & Weak, uniform & 38 & Apical, membrane \\
\hline Aqp1 & $A Q P 1 * *$ & 20112 & Strong, uniform & 32149 & Very strong, uniform \\
\hline
\end{tabular}

Uniform indicates all cells were positive. Regional indicates staining confined to only some (contiguous) cells.

* from Liddelow et al. (2013), "* from Johansson et al. (2005).

membrane of all epithelial cells with weaker staining in the basolateral membrane; in the adult plexus the immunostaining was even stronger (Johansson et al., 2005). There was good correspondence between gene expression level and strength of protein immunostaining (Table 7).

\section{Limitations of Study}

There are several limitations in this study. One concerns the use of whole choroid plexus tissue. However, as discussed previously (Liddelow et al., 2013) the epithelium is the predominant cell type, representing up to $90 \%$ of the plexus tissue (Keep and Jones, 1990; Liddelow et al., 2012). The absence of transcript for Cldn5 (a brain barrier cerebral endothelial-specific claudin family member) in the current RNA-Seq dataset suggests that any possible contamination is below detectable limits (Liddelow et al., 2013). The immunohistochemical evidence in that paper and in the current study for a range of protein products of the genes identified in the choroid plexus samples showed that these proteins were largely confined to the epithelial cells of the plexus. The analysis of large RNA sequencing databases is still quite new and relies on the accuracy of the annotations for each species studied. The rat genome is well annotated and the number of unknown genes is very low, however, there is still the possibility that some transcripts were missed. The papers based on microarray studies of adult mouse choroid plexus (Marques et al., 2011 and Janssen et al., 2013) on adult human and mouse adult choroid plexus provide important additional data on gene expression in the choroid plexuses of other species. These together with that of Liddelow et al. (2012) a mouse microarray study and that of Ek et al. (2015) an RNA-Seq study in primate choroid plexus provide comprehensive datasets for interspecies comparison reviews; however, this is outside the scope of this research paper.

We did not attempt to analyze all functional groups represented in the transcriptomic dataset. For example Slc16a10 showed strikingly high expression (Table 4) exclusively in the embryo; it has been included primarily because it is a monocarboxylate transporter (MCT10) although its little investigated putative function as a thyroid hormone transporter may well prove to be more functionally important. This high expression in the embryonic choroid plexus is in striking contrast to the expression of the best-known thyroxine transporter, Slc16a2, which barely changed expression during development.

Another limitation is that, as pointed out above, expression data alone do not necessarily equate to functional activity. The immunohistochemical evidence in this paper, in Liddelow et al. (2013) and in earlier reports summarized in the Discussion shows that at least some of the mRNAs are being translated to protein. The summary of in vivo physiological transport studies for glucose, amino acids, monocarboxylates and metals in developing brain show a good correlation with the level of expression of relevant transporter genes, although because of overlap and redundancy it is generally not possible to pinpoint the specific contribution of any one gene.

\section{Conclusions}

The results from this study and two earlier ones on gene expression in embryonic and adult rat choroid plexus (Kratzer et al., 2013; Liddelow et al., 2013) as well as a study of embryonic and adult mouse choroid plexus (Liddelow et al., 2012) show that a very large number of transporter genes are expressed in the embryonic lateral ventricular choroid plexuses as early as E15 which is only a day after these plexuses appear in the rat brain (Johansson et al., 2005). In the present study $64 \%$ of all the 389 known SLC transporters, with representatives of all but 4 of the 52 SLC families, were identified. Many of these were expressed at higher or similar levels in the embryonic compared to the adult plexus. This suggests that the lateral ventricular choroid plexuses make an important contribution to supply of nutrients and micronutrients to the brain early in development. This conclusion is supported by the results of immunohistochemistry for a number of the protein products of genes expressed in the embryonic and adult choroid plexus (Figure 4), as well as data from published transport studies for glucose, monocarboxylates, amino acids and divalent metals summarized in the Discussion.

\section{Author Contributions}

All of the listed authors contributed to the conception, design, research, drafting and final approval of the work. They each agree to be accountable for all aspects of the work. 


\section{Acknowledgments}

KD, MH and NRS are supported by the National Health and Medical Research Council (Australia) - Grants No.567205 and 1049287. SL, KD, NRS, NS and J-FG-E were supported by the European Union Seventh Framework Program (FP7) Neurobid consortium - Agreement No. HEALTH-F2-2009241778, J-FG-E is supported by ANR-10-IBHU-0003 Cesame grant, SL was supported as a Neurological Fellow by the

\section{References}

Abbott, N. J., and Friedman, A. (2012). Overview and introduction: the blood-brain barrier in health and disease. Epilepsia 53(Suppl. 6), 1-6. doi: 10.1111/j.1528-1167.2012.03696.x

Abbott, N. J., Patabendige, A. A., Dolman, D. E., Yusof, S. R., and Begley, D. J. (2010). Structure and function of the blood-brain barrier. Neurobiol. Dis. 37, 13-25. doi: 10.1016/j.nbd.2009.07.030

Al-Sarraf, H. (2002). Transport of 14C-gamma-aminobutyric acid into brain, cerebrospinal fluid and choroid plexus in neonatal and adult rats. Brain Res. Dev. Brain Res. 139, 121-129. doi: 10.1016/S0165-3806(02)00537-0

Al-Sarraf, H., Preston, J. E., and Segal, M. B. (1995). The entry of acidic amino acids into brain and CSF during development, using in situ perfusion in the rat. Dev. Brain Res. 90, 151-158. doi: 10.1016/0165-3806(96)83495-X

Al-Sarraf, H., Preston, J. E., and Segal, M. B. (1997). Changes in the kinetics of the acidic amino acid brain and CSF uptake during development in the rat. Dev. Brain Res. 102, 127-134. doi: 10.1016/S0165-3806(97)00089-8

Anders, S. (2010). HTSeq 0.4.7p4. Available online at: http://www-huber.embl.de/ users/anders/HTSeq/.

Baños, G., Daniel, P. M., and Pratt, O. E. (1978). The effect of age upon the entry of some amino acids into the brain, and their incorporation into cerebral protein. Dev. Med. Child Neurol. 20, 335-346.

Baud, O., Fayol, L., Gressens, P., Pellerin, L., Magistretti, P., Evrard, P., et al. (2003). Perinatal and early postnatal changes in the expression of monocarboxylate transporters MCT1 and MCT2 in the rat forebrain. J. Comp. Neurol. 465, 445-454. doi: 10.1002/cne.10853

Bauer, H. (1998). "Glucose transporters in mammalian brain development," in Introduction to the Blood-Brain Barrier, ed W. M. Pardridge (Cambridge: Cambridge University press), P175-P187.

Benrabh, H., and Lefauconnier, J. M. (1996). Blood-endothelial cell and bloodbrain transport of L-proline, alpha-aminoisobutyric acid, and L-alanine. Neurochem. Res. 21, 1227-1235. doi: 10.1007/BF02532400

Braun, L. D., Cornford, E. M., and Oldendorf, W. H. (1980). Newborn rabbit blood-brain barrier is selectively permeable and differs substantially from the adult. J. Neurochem. 34, 147-152. doi: 10.1111/j.1471-4159.1980. tb04633.x

Brown, P. D., Davies, S. L., Speake, T., and Millar, I. D. (2004). Molecular mechanisms of cerebrospinal fluid production. Neuroscience 129, 957-970. doi: 10.1016/j.neuroscience.2004.07.003

Chowanadisai, W., Graham, D. M., Keen, C. L., Rucker, R. B., and Messerli, M. A. (2013a). Neurulation and neurite extension require the zinc transporter ZIP12 (slc39a12). Proc. Natl. Acad. Sci. U.S.A. 2110, 9903-9908. doi: $10.1073 /$ pnas. 1222142110

Chowanadisai, W., Graham, D. M., Keen, C. L., Rucker, R. B., and Messerli, M. A. (2013b). A zinc transporter gene required for development of the nervous system. Commun. Integr. Biol. 6:e26207. doi: 10.4161/cib.26207

Chowanadisai, W., Kelleher, S. L., and Lönnerdal, B. (2005). Zinc deficiency is associated with increased brain zinc import and LIV-1 expression and decrease ZnT-1 expression in neonatal rats. J. Nutr. 135, 1002-1007.

Cornford, E. M., Braun, L. D., and Oldendorf, W. H. (1982). Developmental modulations of blood-brain barrier permeability as an indicator of changing nutritional requirements in the brain. Pediatr. Res. 16, 324-328. doi: 10.1203/00006450-198204000-00017
American-Australian Association and as an NHMRC CJ Martin Fellow.

\section{Supplementary Material}

The Supplementary Material for this article can be found online at: http://journal.frontiersin.org/article/10.3389/fnins. 2015.00123/abstract

Cornford, E. M., and Cornford, M. E. (1986). Nutrient transport and the bloodbrain barrier in developing animals. Fed. Proc. 45, 2065-2072.

Cremer, J. E. (1982). Substrate utilization and brain development. J. Cereb. Blood Flow Metab. 2, 394-407. doi: 10.1038/jcbfm.1982.45

Damkier, H. H., Brown, P. D., and Praetorius, J. (2013). Cerebrospinal fluid secretion by the choroid plexus. Physiol. Rev. 93, 1847-1892. doi: 10.1152/physrev.00004.2013

Damkier, H. H., Nielsen, S., and Praetorius, J. (2007). Molecular expression of SLC4-derived Na+-dependent anion transporters in selected human tissues. Am. J. Physiol. Regul. Integr. Comp. Physiol. 293, R2136-R2146. doi: 10.1152/ajpregu.00356.2007

Daneman, R., Zhou, L., Agalliu, D., Cahoy, J. D., Kaushal, A., and Barres, B. A. (2010). The mouse blood-brain barrier transcriptome: a new resource for understanding the development and function of brain endothelial cells. PLoS ONE. 5:e13741. doi: 10.1371/journal.pone.0013741

Davson, H., and Segal, M. B. (1996). Physiology of the CSF and Blood-Brain Barriers. Boca Raton, FL: CRC Press.

Ek, C. J., Nathanielsz, P., Li, C., and Mallard, C. (2015). Transciptomal changes and functional annotation of the developing non human primate choroid plexus. Front. Neurosci. 9:82. doi: 10.3389/fnins.2015.00082

Gentleman, R. C., Carey, V. J., Bates, D. M., Bolstad, B., Dettling, M., Dudoit, S., et al. (2004). Bioconductor: open software development for computational biology and bioinformatics. Genome Biol. 5, R80. doi: 10.1186/gb-2004-5-10r80

Grabrucker, S., Jannetti, L., Eckert, M., Gaub, S., Chhabra, R., Pfaender, S., et al. (2014). Zinc deficiency dysregulates the synaptic ProSAP/Shank scaffold and might contribute to autism spectrum disorders. Brain 137(Pt 1), 137-152. doi: 10.1093/brain/awt303

Gray, S., Feinberg, M. W., Hull, S., Kuo, C. T., Watanabe, M., Sen-Banerjee, S., et al. (2002). The Krüppel-like factor KLF15 regulates the insulin-sensitive glucose transporter GLUT4. J. Biol. Chem. 277, 34322-34328. doi: 10.1074/jbc.M2013 04200

Gunter, T. E., Gerstner, B., Gunter, K. K., Malecki, J., Gelein, R., Valentine, W. M., et al. (2013). Manganese transport via the transferrin mechanism. Neurotoxicology 34, 118-127. doi: 10.1016/j.neuro.2012.10.018

Gybina, A. A., and Prohaska, J. R. (2006). Variable response of selected cuproproteins in rat choroid plexus and cerebellum following perinatal copper deficiency. Genes Nutr. 1, 51-59. doi: 10.1007/BF02829936

Hediger, M. A. (2013). The ABCs of membrane transporters in health and disease (SLC series). Mol. Aspects Med. 34, 95-752. doi: 10.1016/j.mam.2012.12.009

Hubbard, T. J., Aken, B. L., Ayling, S., Ballester, B., Beal, K., Bragin, E., et al. (2009). Ensembl 2009. Nucleic Acids Res. 37, D690-D697. doi: 10.1093/nar/gkn828

Janssen, S. F., van der Spek, S. J., Ten Brink, J. B., Essing, A. H., Gorgels, T. G., van der Spek, P. J., et al. (2013). Gene expression and functional annotation of the human and mouse choroid plexus epithelium. PLOS ONE 8:e83345. doi: 10.1371/journal.pone.0083345

Johansson, P. A., Dziegielewska, K. M., Ek, C. J., Habgood, M. D., Liddelow, S. A., Potter, A. M., et al. (2006). Blood-CSF barrier function in the rat embryo. Eur. J. Neurosci. 24, 65-76.

Johansson, P. A., Dziegielewska, K. M., Ek, C. J., Habgood, M. D., Møllgård, K., Potter, A., et al. (2005). Aquaporin-1 in the choroid plexuses of developing mammalian brain. Cell Tissue Res. 322, 353-364. doi: 10.1007/s00441-005$1120-\mathrm{x}$ 
Johansson, P. A., Dziegielewska, K. M., Liddelow, S. A., and Saunders, N. R. (2008). The blood-CSF barrier explained: when development is not immaturity. Bioessays 30, 237-248. doi: 10.1002/bies.20718

Keep, R. F., and Jones, H. C. (1990). A morphometric study on the development of the lateral ventricular choroid plexus, choroid plexus capillaries and ventricular ependyma in the rat. Dev. Brain Res. 56, 47-53. doi: 10.1016/01653806(90)90163-S

Keep, R. F., and Smith, D. E. (2011). Choroid plexus transport: gene deletion studies. Fluids Barriers CNS 8:26. doi: 10.1186/2045-8118-8-26

Koehler-Stec, E. M., Simpson, I. A., Vannucci, S. J., Landschulz, K. T., and Landschulz, W. H. (1998). Monocarboxylate transporter expression in mouse brain. Am. J. Physiol. 275(3 Pt 1), E516-E524.

Kohle, S. J., and Vannucci, R. C. (1977). Glycogen metabolism in fetal and postnatal rat brain: influence of birth. J. Neurochem. 28, 441-443. doi: 10.1111/j.14714159.1977.tb07768.x

Kratzer, I., Liddelow, S. A., Saunders, N. R., Strazielle, N., and Ghersi-Egea, J. F. (2013). Developmental changes in the transcriptome of the rat choroid plexus in relation to neuroprotection. Fluids Barriers CNS. 10:25. doi: 10.1186/2045-81 18-10-25

Kuo, Y. M., Zhou, B., Cosco, D., and Gitschier, J. (2001). The copper transporter CTR1 provides an essential function in mammalian embryonic development. Proc. Natl. Acad. Sci. U.S.A. 98, 6836-6841. doi: 10.1073/pnas.111057298

Langmead, B., Trapnell, C., Pop, M., and Salzberg, S. L. (2009). Ultrafast and memory efficient alignment of short DNA sequences to the human genome. Genome Biol. 10:R25. doi: 10.1186/gb-2009-10-3-r25

Lefauconnier, J.-M., and Trouvé, R. (1983). Developmental changes in the pattern of amino acid transport at the blood-brain barrier in rats. Brain Res. 283, 175-182. doi: 10.1016/0165-3806(83)90095-0

Leino, R. L., Gerhart, D. Z., and Drewes, L. R. (1999). Monocarboxylate transporter (MCT1) abundance in brains of suckling and adult rats: a quantitative electron microscopic immunogold study. Brain Res. Dev. Brain Res. 113, 47-54. doi: 10.1016/S0165-3806(98)00188-6

Leitner, D. F., and Connor, J. R. (2012). Functional roles of transferrin in the brain. Biochim. Biophys. Acta 1820, 393-402. doi: 10.1016/j.bbagen.2011.10.016

Liddelow, S. A., Dziegielewska, K. M., Ek, C. J., Habgood, M. D., Bauer, H., Bauer, H.-C., et al. (2013). Mechanisms that determine the internal environment of the developing brain: a transcriptomic, functional and ultrastructural approach. PLoS ONE 8:e65629. doi: 10.1371/journal.pone.0065629

Liddelow, S. A., Temple, S., Møllgård, K., Gehwold, R., Wagner, A., Bauer, H., et al. (2012). Molecular characterisation of transport mechanisms at the developing mouse blood-CSF interface: a transcriptome approach. PloS ONE 7:e33554. doi: 10.1371/journal.pone.0033554

Marger, L., Schubert, C. R., and Bertrand, D. (2014). Zinc: an underappreciated modulatory actor of brain function. Biochem. Pharmacol. 91, 426-435. doi: 10.1016/j.bcp.2014.08.002

Marques, F., Sousa, J. C., Coppola, G., Gao, F., Puga, R., Brentani, H., et al. (2011). Transcriptome signature of the adult mouse choroid plexus. Fluids Barriers CNS 8:10. doi: 10.1186/2045-8118-8-10

McKenzie, J. M., Fosmire, G. J., and Sandstead, H. H. (1975). Zinc deficiency during the latter third of pregnancy: effects on fetal rat brain, liver, and placenta. J. Nutr. 105, 1466-1475.

Moos, T., and Morgan, E. H. (1998). Evidence for low molecular weight, nontransferrin-bound iron in rat brain and cerebrospinal fluid. J. Neurosci. Res. 54, 486-494.

Moos, T., and Morgan, E. H. (2000). Transferrin and transferrin receptor function in brain barrier systems. Cell. Mol. Neurobiol. 20, 77-95. doi: 10.1023/A:1006948027674

Morgan, E. H., and Moos, T. (2002). Mechanism and developmental changes in iron transport across the blood-brain barrier. Dev. Neurosci. 24, 106-113. doi: $10.1159 / 000065699$

Nehlig, A. (1997). Cerebral energy metabolism, glucose transport and blood flow: changes with maturation and adaptation to hypoglycaemia. Diabetes Metab. 23, 18-29.

Netsky, M. G., and Shuangshoti, S. (1975). The Choroid Plexus in Health and Disease. Bristol: Wright.

Porterfield, S. P., and Hendrick, C. E. (1992). Tissue iodothyronine levels in fetuses of control and hypothyroid rats at 13 and 16 days gestation. Endocrinology 131, 195-200. doi: 10.1210/en.131.1.195
Rivas, M., and Naranjo, J. R. (2007). Thyroid hormones, learning and memory. Genes Brain Behav. 6 Suppl. 1, 40-44. doi: 10.1111/j.1601-183X.2007.00321.x

Robinson, M. D., McCarthy, D. J., and Smyth, D. K. (2010). edgeR: a Bioconductor package for differential expression analysis of digital gene expression data. Bioinformatics 26, 139-140. doi: 10.1093/bioinformatics/btp616

Rouault, T. A., Zhang, D-L., and Jeong, S. Y. (2009). Brain iron homeostasis, the choroid plexus, and localization of iron transport proteins. Metab. Brain Dis. 24, 673-684. doi: 10.1007/s11011-009-9169-y

Sandstead, H. H. (1985). WO Atwater memorial lecture. Zinc: essentiality for brain development and function. Nutr. Rev. 43, 129-137.

Sandstead, H. H., Fosmire, G. J., McKenzie, J. M., and Halas, E. S. (1975). Zinc deficiency and brain development in the rat. Fed. Proc. 34, 86-88. doi: 10.1007/978-1-4684-2631-1_19

Santamaria, A. B., and Sulsky, S. (2010). Risk assessment of an essential element: manganese. J. Toxicol. Environ. Health A 73, 128-55. doi: $10.1080 / 15287390903337118$

Saunders, N. R., Daneman, R., Dziegielewska, K. M., and Liddelow, S. A. (2013). Transporters of the blood-brain and blood-CSF interfaces in development and in the adult. Mol. Aspects Med. 34, 742-752. doi: 10.1016/j.mam.2012.11.006

Saunders, N. R., Liddelow, S. A., and Dziegielewska, K. M. (2012). Barrier mechanisms in the developing brain. Front. Pharmacol. 3:46. doi: 10.3389/fphar.2012.00046

Schachenmayr, W. Z. (1967). On the development of ependyma and plexus choriodeus of the rat. Zellforsch. Mikrosk. Anat. 77, 25-63.

Scheiber, I. F., Mercer, J. F., and Dringen, R. (2014). Metabolism and functions of copper in brain. Prog. Neurobiol. 116, 33-57. doi: 10.1016/j.pneurobio.2014.01.002

Schmitt, C., Strazielle, N., Richaud, P., Bouron, A., and Ghersi-Egea, J.-F. (2011). Active transport at the blood-CSF barrier contributes to manganese influx into the brain. J. Neurochem. 117, 747-756 doi: 10.1111/j.1471-4159.2011.07246.x

Segal, M. B. (2001). Transport of nutrients across the choroid plexus. Microsc. Res. Tech. 52, 38-48. doi: 10.1002/1097-0029(20010101)52:1\&1t;38::AIDJEMT6\&gt;3.0.CO;2-J

Sershen, H., and Lajtha, A. (1976). Capillary transport of amino acids in the developing brain. Exp. Neurol. 53, 465-474. doi: 10.1016/0014-4886(76) 90086-8

Siddappa, A. J., Rao, R. B., Wobken, J. D., Casperson, K., Leibold, E. A., Connor, J. R., et al. (2003). Iron deficiency alters iron regulatory protein and iron transport protein expression in the perinatal rat brain. Pediatr. Res. 53, 800-807. doi: 10.1203/01.PDR.0000058922.67035.D5

Singh, N., Haldar, S., Tripathi, A. K., Horback, K., Wong, J., Sharma, D., et al. (2014). Brain iron homeostasis: from molecular mechanisms to clinical significance and therapeutic opportunities. Antioxid. Redox Signal. 20, 1324-1363. doi: 10.1089/ars.2012.4931

Skeaff, S. A. (2011). Iodine deficiency in pregnancy: the effect on neurodevelopment in the child. Nutrients 3, 265-273. doi: 10.3390/nu3020265

Tepaamorndech, S., Kirschke, C. P., and Huang, L. (2014). Linking cellular zinc status to body weight and fat mass: mapping quantitative trait loci in $\mathrm{ZnT7}$ knockout mice. Mamm Genome. 25, 335-353. doi: 10.1007/s00335-014-9512-4

Tyszka-Czochara, M., Grzywacz, A., Gdula-Argasiñska, J., Librowski, T., Wiliñski, B., and Opoka, W. (2014). The role of zinc in the pathogenesis and treatment of central nervous system (CNS) diseases. Implications of zinc homeostasis for proper CNS function. Acta Pol. Pharm. 71, 369-377.

Uriu-Adams, J. Y., Scherr, R. E., Lanoue, L., and Keen, C. L. (2010). Influence of copper on early development: prenatal and postnatal considerations. Biofactors 36, 136-152. doi: 10.1002/biof.85

Vannucci, R. C., and Vannucci, S. J. (2000). Glucose metabolism in the developing brain. Semin. Perinatol. 24, 107-115. doi: 10.1053/sp.2000.6361

Vannucci, S. J. (1994). Developmental expression of GLUT1 and GLUT3 glucose transporters in rat brain. J. Neurochem. 62, 240-246.

Vannucci, S. J., Rutherford, T., Wilkie, M. B., Simpson, I. A., and Lauder, J. M. (2000). Prenatal expression of the GLUT4 glucose transporter in the mouse. Dev. Neurosci. 22, 274-282. doi: 10.1159/000017451

Vannucci, S. J., and Simpson, I. A. (2003). Developmental switch in brain nutrient transporter expression in the rat. Am. J. Physiol. Endocrinol. Metab. 285, E1127-E1134. doi: 10.1152/ajpendo.00187.2003

Wang, X., Miller, D. S., and Zheng, W. (2008). Intracellular localization and subsequent redistribution of metal transporters in a rat choroid plexus 
model following exposure to manganese or iron. Toxicol. Appl. Pharmacol. 230:167-174. doi: 10.1016/j.taap.2008.02.024

Wright, E. M. (2013). Glucose transport families SLC5 and SLC50. Mol. Aspects Med. 34, 183-196. doi: 10.1016/j.mam.2012.11.002

Yon, J. M., Baek, I. J., Lee, B. J., Yun, Y. W., and Nam, S. Y. (2011). Dynamic expression of manganese superoxide dismutase during mouse embryonic organogenesis. Int. J. Dev. Biol. 55, 327-334. doi: 10.1387/ijdb.103270jy

Yon, J. M., Baek, I. J., Lee, S. R., Kim, M. R., Lee, B. J., Yun, Y. W., et al. (2008). Immunohistochemical identification and quantitative analysis of cytoplasmic $\mathrm{Cu} / \mathrm{Zn}$ superoxide dismutase in mouse organogenesis. J. Vet. Sci. 9, 233-240. doi: $10.4142 /$ jvs.2008.9.3.233
Conflict of Interest Statement: The authors declare that the research was conducted in the absence of any commercial or financial relationships that could be construed as a potential conflict of interest.

Copyright $\odot 2015$ Saunders, Dziegielewska, Møllgård, Habgood, Wakefield, Lindsay, Stratzielle, Ghersi-Egea and Liddelow. This is an open-access article distributed under the terms of the Creative Commons Attribution License (CC BY). The use, distribution or reproduction in other forums is permitted, provided the original author(s) or licensor are credited and that the original publication in this journal is cited, in accordance with accepted academic practice. No use, distribution or reproduction is permitted which does not comply with these terms. 


\section{Barrier mechanisms in the Drosophila blood-brain barrier}

\section{Samantha J. Hindle* and Roland J. Bainton*}

Department of Anesthesia and Perioperative Care, University of California, San Francisco, San Francisco, CA, USA

\section{Edited by:}

Norman Ruthven Saunders,

University of Melbourne, Australia

\section{Reviewed by:}

Åsa M. E. Winther, Karolinska

Institutet, Sweden

Christian Klämbt, University of

Münster, Germany

Brigitte Dauwalder, University of

Houston, USA

*Correspondence:

Samantha J. Hindle and Roland J. Bainton, Department of Anesthesia and Perioperative Care, University of California, San Francisco, Genentech Hall, Room S312C 600 16th Street.

San Francisco, CA 94158, USA

e-mail:hindles@

anesthesia.ucsf.edu;

baintonr@anesthesia.ucsf.edu
The invertebrate blood-brain barrier (BBB) field is growing at a rapid pace and, in recent years, studies have shown a physiologic and molecular complexity that has begun to rival its vertebrate counterpart. Novel mechanisms of paracellular barrier maintenance through G-protein coupled receptor signaling were the first demonstrations of the complex adaptive mechanisms of barrier physiology. Building upon this work, the integrity of the invertebrate $\mathrm{BBB}$ has recently been shown to require coordinated function of all layers of the compound barrier structure, analogous to signaling between the layers of the vertebrate neurovascular unit. These findings strengthen the notion that many BBB mechanisms are conserved between vertebrates and invertebrates, and suggest that novel findings in invertebrate model organisms will have a significant impact on the understanding of vertebrate BBB functions. In this vein, important roles in coordinating localized and systemic signaling to dictate organism development and growth are beginning to show how the BBB can govern whole animal physiologies. This includes novel functions of BBB gap junctions in orchestrating synchronized neuroblast proliferation, and of BBB secreted antagonists of insulin receptor signaling. These advancements and others are pushing the field forward in exciting new directions. In this review, we provide a synopsis of invertebrate BBB anatomy and physiology, with a focus on insights from the past 5 years, and highlight important areas for future study.

\section{Keywords: invertebrates, blood-brain barrier, chemoprotection, drug delivery, conserved physiology}

\section{INTRODUCTION}

In order for the nervous system to perform efficiently in coordinating sophisticated movements, behaviors and cognitive functions, it requires chemical isolation from the fluctuating, and potentially damaging, components in the blood. The blood-brain barrier (BBB) has evolved to provide this role, becoming more sophisticated with increasing complexity and demand of the nervous system (Abbott, 2005). Therefore, there are alternative cellular strategies for making BBB structures. In insects, crustacea, cephalopod molluscs and cartilaginous fish the BBB is formed by glial cells, whereas in higher order vertebrates the BBB is formed primarily by the brain vascular endothelium. The formation and functions of the vertebrate $\mathrm{BBB}$ are covered extensively by various reviews (e.g., Abbott, 2005; Abbott et al., 2010; Obermeier et al., 2013) and within this BBB series; the aim of this review is to describe the comparative compound structure and associated physiologies of the invertebrate BBB. In recent years, the invertebrate $\mathrm{BBB}$ field has flourished with interesting insights into the mechanisms of BBB maintenance, as well as discovering novel metabolic and signaling roles. In addition, both established and emerging invertebrate models are proving useful as drug screening tools to advance therapies for neurodegenerative diseases. These findings, and others, are providing many exciting avenues for future BBB investigations.

Abbreviations: $\mathrm{BBB}$, blood-brain barrier; SPG, subperineurial glia; PG, perineurial glia; CNS, central nervous system; ABC, ATP-binding cassette; SLC, solute carrier; GPCR, G-protein coupled receptor; ARSs, actin-rich structures; IPCs, insulin producing cells; dILPs, Drosophila insulin-like peptides

\section{ANATOMICAL OVERVIEW OF THE INVERTEBRATE BBB}

Unlike in vertebrates, the blood (hemolymph) in insects does not circulate in a closed capillary network; instead, the hemolymph is freely flowing in the body cavity. As a result, protection of the insect nervous system from the high and often fluctuating concentrations of hemolymph potassium requires that the BBB entirely surrounds the nervous system. Therefore, at the gross anatomical level, the invertebrate BBB is quite different from that of the vascular endothelium in vertebrates. However, like its vertebrate counterpart, the invertebrate BBB is a compound structure (Figure 1), analogous to the vertebrate neurovascular unit (Stork et al., 2008; Mayer et al., 2009; DeSalvo et al., 2011, 2014). The invertebrate compound BBB is primarily composed of two surface glia cell types [the apical perineurial glia (PG) and the basal subperineurial glia (SPG)], and the exterior extracellular matrix layer known as the neural lamella (Figures 1, 2). The absence of almost all glial cells in the Drosophila glial cells missing $(\mathrm{gcm})$ mutant renders the nervous system permeable to a broad range of fluorescent dextran molecules ranging from 10 to $500 \mathrm{kDa}$ (Stork et al., 2008), confirming the importance of glial cells for the invertebrate BBB.

The protective functions of the invertebrate BBB are apparent from embryonic stage 17 throughout development and into the adult stage (Bainton et al., 2005; Schwabe et al., 2005; Stork et al., 2008; Mayer et al., 2009). The PG, however, are not thought to contribute toward these BBB properties early in development as PG cells do not proliferate and completely surround the CNS until late larval stages (Stork et al., 2008). The roles of the BBB 
during early development are therefore attributed to the SPG, which entirely encapsulate the embryonic nervous system and maintain a tight barrier throughout development and into the adult stage (Stork et al., 2008). In order to maintain this tight diffusion barrier, the SPG cells do not proliferate as the nervous system grows; instead the SPG increase in size and become polyploid (Unhavaithaya and Orr-Weaver, 2012). The polyploid

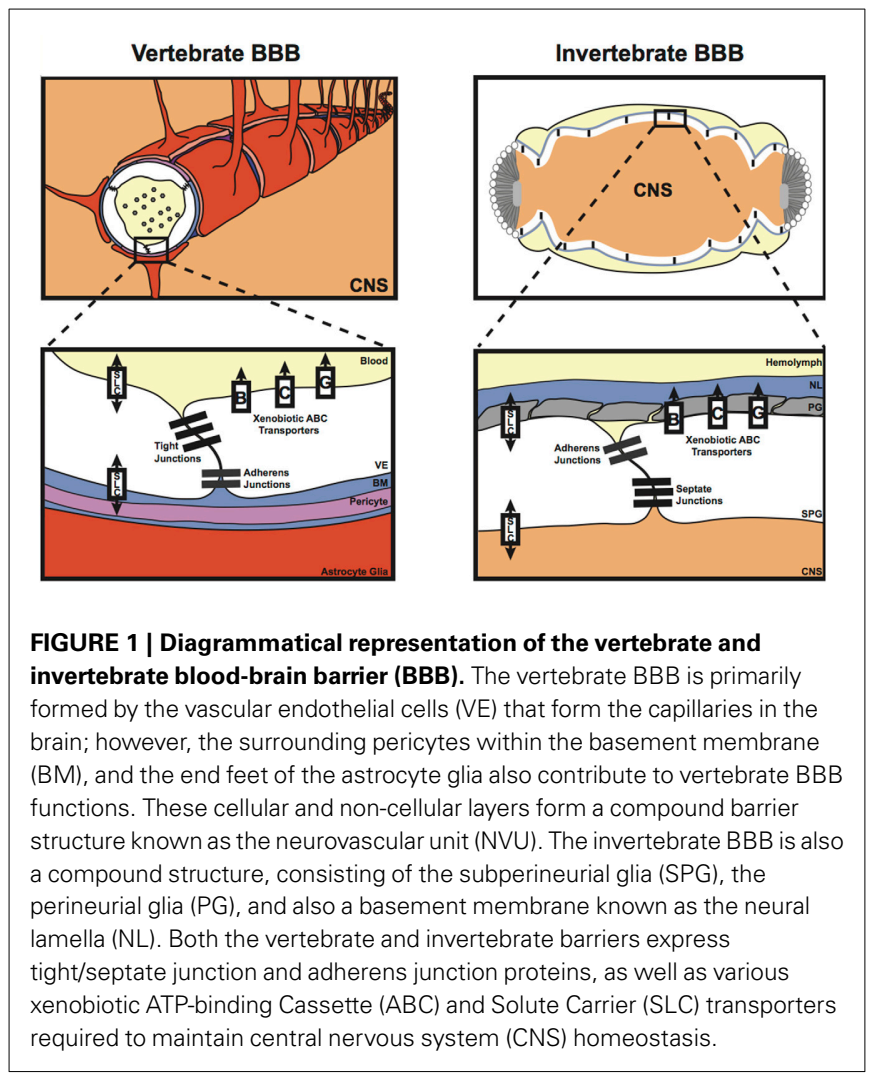

nature of the SPG is essential to maintain the integrity of the septate junctions that are present between the SPG cells, as the nervous system grows. Inhibition of polyploidy in the SPG causes the septate junctions to rupture and diffusion barrier integrity to be lost (Unhavaithaya and Orr-Weaver, 2012). The formation of the invertebrate BBB has been covered extensively elsewhere (Stork et al., 2008; Edwards and Meinertzhagen, 2010; DeSalvo et al., 2011).

The majority of research on the invertebrate BBB has focused on the main BBB layer, the SPG. However, recent investigations have started to uncover additional roles for cellular and noncellular layers external to the SPG cells. When the septate junction protein Neurexin IV was mutated in Drosophila, the brain penetration of $500 \mathrm{kDa}$ dextran was delayed compared to in the glial cells missing mutant, which lacks almost all glial cells (Stork et al., 2008). This suggests that barriers other than the SPG septate junctions are present that can reduce the brain access of certain high molecular weight molecules. It is therefore possible that the PG and neural lamella layers act as non-specific, large molecular-weight filters. Moreover, DeSalvo et al. (2011, 2014) and Meyer et al. (2014) have recently suggested that the PG may have signaling and metabolic roles in BBB function, and in maintaining the neural lamella. Collagen IV, which forms a major part of the neural lamella, is secreted by hemocytes in the embryo and the fat body during post-embryonic development (Mirre et al., 1988; Pastor-Pareja and Xu, 2011); but Meyer et al. (2014) showed that, in addition to the fat body, the Drosophila $\mathrm{BBB}$ can also contribute to the integrity of the neural lamella. Mutations that affect the deposition of Collagen IV into the neural lamella, result in deformation of the central nervous system (CNS) and deficits in nervous system function (Olofsson and Page, 2005; Meyer et al., 2014). Knocking out matrix metalloproteinases specifically in the SPG or PG cells resulted in larval lethality or an extended ventral nerve cord phenotype, respectively (Meyer et al., 2014). This suggests that
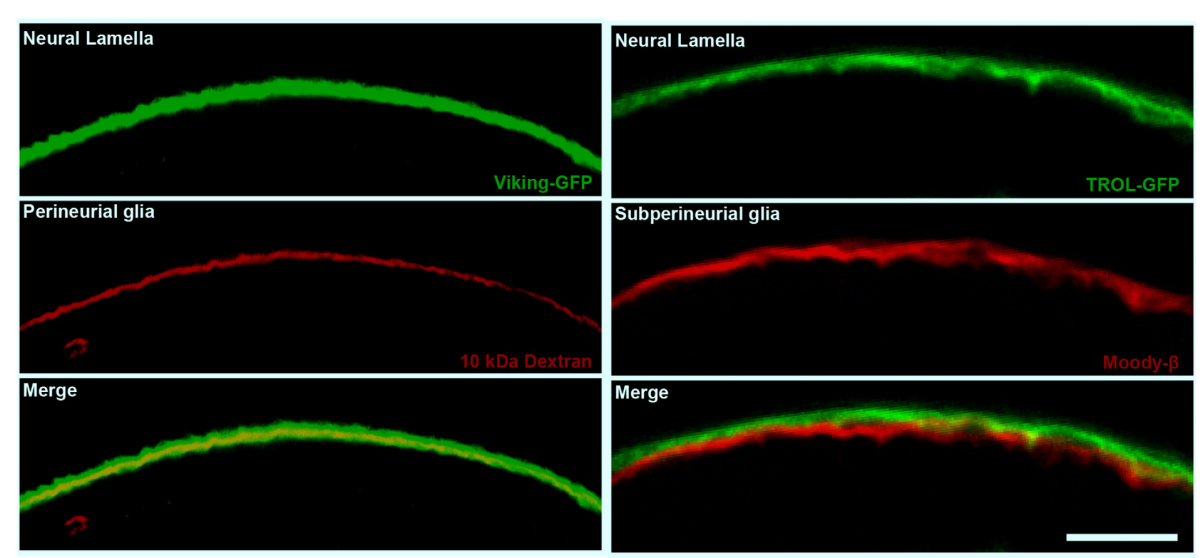

FIGURE 2 | The cellular and non-cellular layers of the Drosophila compound BBB. Confocal micrographs of cross sections of the adult Drosophila optic lobe showing the localization of the neural lamella (green, left and right panels), the perineurial glia (PG) layer (red, left panels) and the subperineurial glia (SPG) layer (red, right panels). The left panels show the positioning of the neural lamella (identified by the Collagen IV marker Viking-GFP) with respect to the PG (marked by $10 \mathrm{kDa}$ Texas Red Dextran staining). The right panels show the closely apposed neural lamella (marked by the extracellular matrix proteoglycan TROL-GFP) and SPG layer (stained by Moody- $\beta$ antibody). Scale bar, $20 \mu \mathrm{m}$. 
the $\mathrm{BBB}$ glia are required to maintain the structural integrity of the lamella, which is necessary for correct CNS shape. It therefore appears that the glial cells of the $\mathrm{BBB}$ may coordinate with the fat body to maintain and restructure the neural lamella to respond to changing developmental and possibly metabolic demands.

In addition to coordination between the $\mathrm{BBB}$ and fat body for maintenance of $\mathrm{BBB}$ functions, evidence suggests that neurons also have a role to play. Rbp9, which is homologous to the RNA-binding proteins elav (invertebrates) and hu (vertebrates), has been shown to function in maintaining BBB integrity; loss of Rbp9 function leads to down-regulation of septate junction proteins (primarily Neurexin IV and Coracle), and a resultant porous BBB (Kim et al., 2010). Interestingly, Rbp9 is expressed in neurons, but not in glia, and is therefore not expressed in the BBB itself (Kim-Ha et al., 1999). As the Rbp9 mutants initially form an intact $\mathrm{BBB}$ and only show a porous BBB phenotype upon adult eclosion (Kim et al., 2010), it appears that neuronal Rbp9 plays an important role in BBB maintenance in invertebrates, analogous to the involvement of neuronal signaling in vertebrate BBB integrity (Stewart and Wiley, 1981; Stenman et al., 2008; Daneman et al., 2009). In conclusion, it appears that, like the vertebrate neurovascular unit, there is an important involvement of all layers of the invertebrate compound $\mathrm{BBB}$ in the formation and maintenance of the BBB.

\section{MOLECULAR OVERVIEW OF THE INVERTEBRATE BBB}

As was mentioned above, the vertebrate vascular endothelium of the brain is specialized to provide an extremely tight barrier to transcellular and paracellular molecular transit. This is due to the following vascular endothelium properties: tight junction complexes between the vascular endothelium cells; enrichment of efflux $\mathrm{ABC}$ transporters, which are localized to the apical (luminal) face of the vascular endothelium; low rates of trancytosis due to reduced vesicular trafficking (Muir and Peters, 1962; Reese and Karnovsky, 1967; Brightman and Reese, 1969; Daneman et al., 2010; for reviews see Zlokovic, 2008; Abbott et al., 2010; Obermeier et al., 2013). Many of these molecular properties are also shared by the invertebrate BBB. As alluded to above, the SPG cells of the invertebrate BBB form a tight barrier to paracellular transit due to the presence of pleated septate junctions. These complexes are equivalent to the septate junctions that perform electrical and chemical insulation properties at the axo-glial paranodal junctions (Bhat et al., 2001; Bhat, 2003). SPG cells also express efflux ABC transporters, in particular the $\mathrm{ABC} \mathrm{B} 1$ xenobiotic efflux transporter Mdr1/P-glycoprotein homolog (Mdr65) is both highly enriched and shows apical localization in the SPG cells (Mayer et al., 2009; DeSalvo et al., 2014). In addition to the chemoprotective properties, the $\mathrm{BBB}$ must also regulate the brain access of endogenous molecules, such as glucose and ions. For this, the $\mathrm{BBB}$ expresses various solute carrier proteins (SLCs). Recent evidence from Drosophila has also suggested interesting roles for the gap junctions that are expressed in both the vertebrate and invertebrate BBB. These findings are discussed in more detail below.

\section{CHEMOPROTECTIVE ROLES OF THE INVERTEBRATE BBB Septate junction formation and maintenance}

The primary barrier of the invertebrate $\mathrm{BBB}$ is provided by the septate junctions, the functional equivalent of the vertebrate tight junctions and paranodal septate junctions. The formation and maintenance of a tight diffusion barrier protects the invertebrate nervous system from the high concentrations of potassium in the hemolymph; without this barrier, the embryos are unable to fire action potentials and cannot coordinate movements to hatch from the embryonic cuticle. Septate junction mutations therefore cause a porous BBB and embryonic lethality (e.g., Baumgartner et al., 1996; Schwabe et al., 2005; Stork et al., 2008). The 5 main components of the septate junctions are the membrane components Neurexin IV (Caspr in vertebrates), Neuroglian (vertebrate Neurofascin 155), ATP $\alpha$ and Nervana2 $\left(\mathrm{Na}^{+} / \mathrm{K}^{+}\right.$ATPase $\alpha$ and $\beta$ subunits), and the intracellular component Coracle (protein 4.1 in vertebrates); however additional proteins are also thought to contribute toward the integrity and maintainance of the septate junctions, including Gliotactin (vertebrate Neuroligin-like), Contactin (vertebrate Contactin), Discs large (Dlg; vertebrate PSD-95-like), Fasciclin III, Moody, Sinuous, and Megatrachea (the latter 2 are vertebrate claudin-like proteins) (Fehon et al., 1994; Auld et al., 1995; Baumgartner et al., 1996; Behr et al., 2003; Genova and Fehon, 2003; Paul et al., 2003; Schulte et al., 2003; Faivre-Sarrailh et al., 2004; Bainton et al., 2005; Schwabe et al., 2005; Stork et al., 2008; Oshima and Fehon, 2011). The septate junction proteins interact with each other to form stable septae contacts between SPG cells that surround the CNS (Figure 3). Disruption of any septate junction component leads to a porous $\mathrm{BBB}$ phenotype, suggesting that they function interdependently to maintain $\mathrm{BBB}$ function. To further address the relationship between septate junction components, Oshima and Fehon (2011) investigated the dynamics of septate junction-associated proteins. Using fluorescence recovery after photobleaching (FRAP), they elegantly showed that the 5 main septate junction components form a very stable core unit, whereas other septate junctionassociated proteins (e.g., Dlg) are more mobile. In addition, they discovered that mutating different septate junction-associated proteins had different mechanisms for causing a defective BBB. Loss of some septate junction-associated proteins (e.g., Dlg) caused delayed formation of the stable septate junction core; however, the dynamics of this stable core, once formed, was not affected by $D l g$ mutation. Furthermore, the loss of Gliotactin or endocytic proteins, such as clathrin heavy chain or the Drosophila dynamin homolog shibire, led to a mislocalisation of the core septate junction proteins, but had no effect on septate junction core formation (Oshima and Fehon, 2011).

Interestingly, a particular splice form of Neurexin IV (containing exon 3) was shown to be the septate junction-forming version of Neurexin IV and has been shown to be preferentially expressed in glia over neurons (Rodrigues et al., 2012). The mechanism of cell-specific splicing has recently been elucidated in Drosophila using elegantly designed genetic reagents that revealed the transcript pattern of the Neurexin IV isoform(s) present in neurons vs. glia (Rodrigues et al., 2012). The authors found that the cellspecific splicing of Neurexin IV involves the RNA-binding protein HOW (Held out wings). HOW is not expressed in neurons, but 


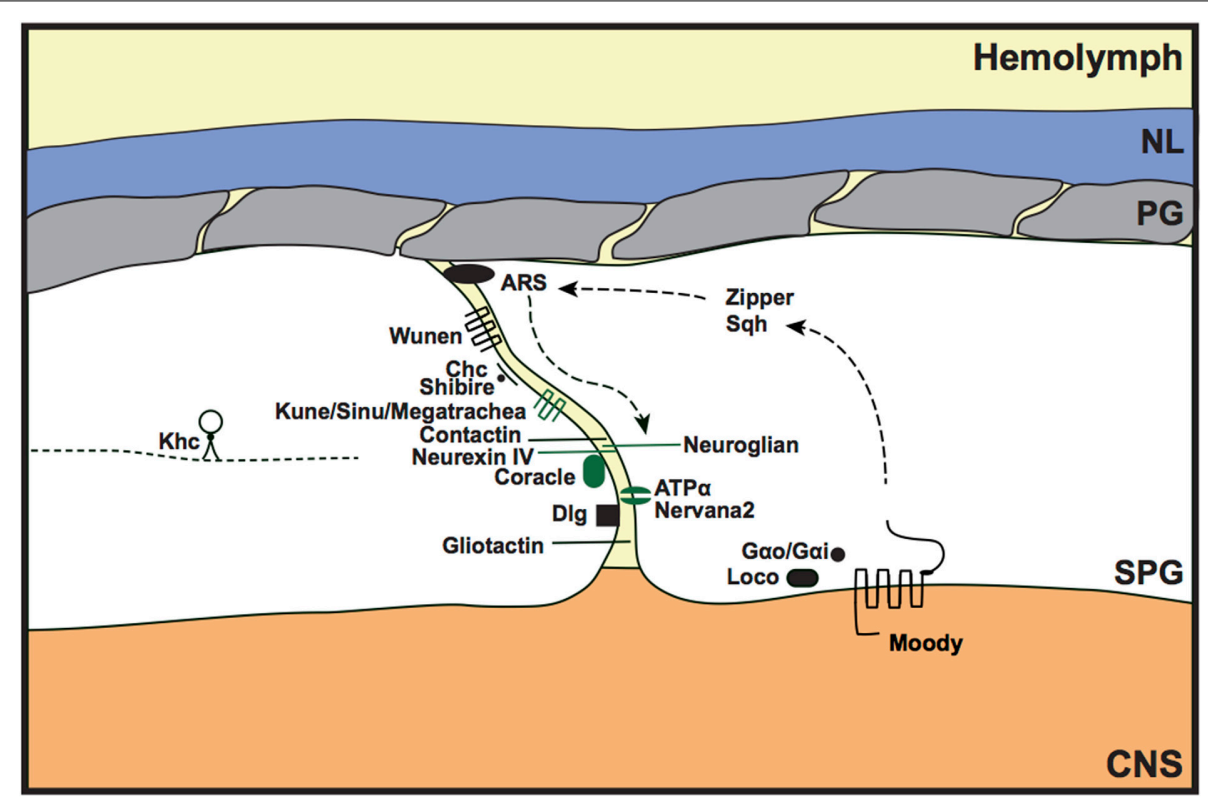

FIGURE 3 | Septate junction formation and maintenance in the invertebrate BBB. Components of the core septate junction (green) and the associated proteins that maintain BBB integrity (black) were identified from loss of function mutants that led to a porous BBB phenotype. Discs large (D/g) mutants showed delayed assembly of the core septate junction components, whereas mutants of Gliotactin, and the endocytic proteins Clathrin-heavy chain (Chc) and Shibire, showed a mislocalization of the septate junction core. The Moody GPCR signaling pathway (Moody, $\mathrm{G}_{\alpha o} / \mathrm{G}_{\alpha i}$, loco) was shown to genetically interact with actin and non-muscle myosin components [actin-rich structures (ARS), Zipper, Spaghetti squash (Sqh)] to maintain septate junction integrity. Kinesin heavy chain (Khc) may regulate septate junction integrity by transporting Neurexin IV as a cargo protein. Loss of septate junction integrity by disrupting the function of wunen, the lipid phosphate phosphatase, suggests a role for bioactive lipids in paracellular barrier function. NL, neural lamella; PG, perineurial glia; SPG, subperineurial glia; CNS, central nervous system; kune (kune-kune). ectopic neuronal expression of the constitutively nuclear form of HOW leads to a shift in Neurexin IV isoform expression to that of a glial pattern, leading to larval lethality. This showed that the expression and nuclear localization of HOW in glia predisposes glia to expression of the Neurexin IV (exon 3) form, promoting the formation of septate junctions. Rodrigues et al. (2012) showed the additional involvement of the kinase Cdk12 and the splicesomal component Prp40 in HOW function.

Recently, another claudin-like protein (Kune-kune) and a leukocyte antigen 6 family protein (Coiled) were identified in screens for disrupted BBB integrity (Nelson et al., 2010; Hijazi et al., 2011; Syed et al., 2011). The protein Kune-kune colocalizes with the septate junction component Coracle. Its localization is dependent on the presence of various septate junction components (e.g., Coracle, ATP $\alpha$, Sinuous and Megatrachea) and, reciprocally, septate junction component localization is dependent on the presence of Kune-kune (Nelson et al., 2010). The authors discovered that Kune-kune regulates both septate junction localization and levels, whereas the other claudin-like proteins Sinuous and Megatrachea regulate septate junction component levels and localization, respectively. Analysis of septate junction levels and localization in single, double and triple mutants of these three claudin-like proteins showed that Kune-kune seems to have a more important role in coordinating septate junction components than Sinuous and Megatrachea (Nelson et al., 2010). The Ly6 family protein Coiled is required for septate junction formation in the embryo; Coiled mutations cause disrupted localization of septate junction proteins, including Neurexin IV, Coracle and Discs large, therefore abrogating BBB integrity (Hijazi et al., 2011; Syed et al., 2011).

The G protein-coupled receptors (GPCRs) encoded by the moody gene (Moody- $\alpha$ and Moody- $\beta$ ) are required to regulate BBB permeability (Bainton et al., 2005; Schwabe et al., 2005). In addition, the trimeric $G$ proteins $G \alpha i$ and $G \alpha o$, and the regulator of $G$ protein signaling (RGS) loco are thought to signal in the same Moody pathway to regulate septate junction formation and maintenance (Schwabe et al., 2005). Mutations in any of these four components leads to a porous $\mathrm{BBB}$ and locomotor dysfunction; however, the moody null has a weaker BBB permeability to $10 \mathrm{kDa}$ dextran compared to the $G \alpha i, G \alpha o$, and loco mutants, suggesting that these latter components of the GPCR signaling pathway may have additional upstream or downstream signaling partners involved in septate junction integrity. Interestingly, because loco is a negative regulator of GPCR signaling, this means that both loss and gain of GPCR signaling disrupts BBB integrity. This suggests that discreet, and localized control over this signaling pathway is required for correct septate junction formation and maintenance. That being said, the effect on septate junction integrity appears to be a secondary effect of disrupted GPCR signaling on the actin cytoskeleton and cell size/shape, which leads to reduced undulations of the SPG cells and a shortening of the septate junction length (Schwabe et al., 2005). As the length of the septate junction dictates the tightness of the BBB, this creates a more permeable septate junction barrier in moody mutants. 
Additional evidence that $\mathrm{BBB}$ integrity requires the coordination of Moody signaling and actin dynamics was recently provided by Hatan et al. (2011). They discovered that highly dynamic actin-rich structures (ARSs) are localized in close proximity to, but not overlapping with, the septate junctions; the ARSs colocalized with the fusion proteins Neuroglian-GFP and Scribble-GFP, but not with Neurexin-IV. The regulation of ARS morphology and size was shown to require Moody signaling and also activation of non-muscle myosin components Zipper and Spaghetti squash, which are thought to be downstream of Moody signaling (Hatan et al., 2011). Disruption of ARSs by knocking down the actin nucleation components Arp2 and Arp3 led to dissociation of ARSs and Neuroglian, discontinuous Neuroglian labeling at the septate junction, and breakdown of BBB integrity. These findings suggest an interesting possibility that ARSs act as dynamic docking sites for septate junction components to allow their rapid incorporation into the septate junctions during development to maintain a tight $\mathrm{BBB}$ as the brain grows (Figure 3). Although the ARSs were only detectable during development, and not during adult stages (Hatan et al., 2011), it would be interesting to determine whether similar structures have a transitory role in the adult, perhaps during circumstances of BBB challenge (chemical, physical or pathological).

Another regulator of BBB integrity, the Kinesin heavy chain, was recently identified from a 5000 candidate-gene screen for locomotion defects (Schmidt et al., 2012). Kinesin heavy chain is classically known for its role in axonal transport. However, Schmidt et al. (2012) discovered an important role within SPG cells. Knocking down Kinesin heavy chain specifically in SPG cells led to disrupted axonal excitability resulting in spasticity in adult flies. They found that one of the cargoes of a Kinesin heavy chain-dependent Rab protein is Neurexin-IV, suggesting that Kinesin heavy chain may contribute toward the control of neuronal excitability by regulating Neurexin-IV incorporation into the septate junctions of the SPG cells. The vertebrate homologs of Drosophila Kinesin heavy chain are members of the KIF5 family. Both KIF5A and KIF5C are mainly expressed in neurons, but KIF5B shows some expression in glia (Kanai et al., 2000); however, no specific roles have been found for KIF5B in glia. Interestingly, the kinesin KIF13B has been shown to transport Discs large 1 (Dlg1) to sites of membrane remodeling during myelin formation in the Schwann glial cells that insulate the peripheral nervous system (Bolis et al., 2009). Therefore, homologous roles may exist for Kinesin heavy chain proteins in regulating tight junction integrity in the vertebrate BBB.

Wunen is a lipid phosphate phosphatase (LPP), an integral membrane enzyme that, in vertebrates, is required in the regulation of bioactive lipids (e.g., sphingosine-1-phosphate). In Drosophila, Wunen is known for its role in germ cell survival and migration (Zhang et al., 1997; Starz-Gaiano et al., 2001); however, it has recently been shown to also localize to septate junctions and to function in septate junction maintenance both in trachea and the BBB (Ile et al., 2012; Figure 3). Wunen mutant nerve cords show increased permeability to $10 \mathrm{kDa}$ dextran, comparable to that of a Neurexin-IV mutant (Ile et al., 2012). A role for bioactive lipids in the maintenance of $\mathrm{BBB}$ integrity has previously been show in mammalian brain microvascular endothelial cells grown in culture (Lee et al., 2006). Treatment with sphingosine1-phosphate caused relocalization of tight junction/cell-cell contact proteins (including ZO-1 and claudin-5) and increased the trans-epithelial electrical resistance. Also, an in vivo role for the mammalian LPP3 in the formation of vascular endothelial cell interactions was shown during development (Escalante-Alcalde et al., 2003). This shows that the role for bioactive lipids in $\mathrm{BBB}$ formation and maintenance is evolutionarily conserved, and supports the notion that the invertebrate septate junctions are functionally and mechanistically equivalent to the vertebrate tight junctions.

\section{Xenobiotic efflux transporters}

In the vertebrate vascular endothelium, the tight junctions provide chemoprotection to the brain by preventing the entry of molecules via the paracellular route. However, another mechanism is required to prevent transcellular access to the brain. This is provided by the xenobiotic efflux transporters (Figure 4). These transporters belong to the ATP-binding cassette (ABC) B, C, and $\mathrm{G}$ classes, and are localized to the apical membrane of the vascular endothelium. The xenobiotic efflux transporters are wellcharacterized for their role in removing lipophilic xenobiotics from the plasma membrane (Leslie et al., 2005), preventing access into the brain. Hence, these transporters are a major hindrance to the successful delivery of therapeutics into the brain (Abbott and Romero, 1996; Abbott, 2013). The main xenobiotic efflux transporters at the $\mathrm{BBB}$ are $\mathrm{P}$-glycoprotein (P-gp/ABCB1/Mdr1) and Breast Cancer Resistance Protein (BCRP/ABCG2). In invertebrates a transcellular transport-barrier has been identified in the SPG cells, which is provided by the homolog of P-glycoprotein (Mdr65; Mayer et al., 2009). Drosophila Mdr65 shares 42\% sequence identity with P-glycoprotein. In the Drosophila brain, Mdr65 expression is restricted to the SPG layer of the BBB and, like P-glycoprotein, localizes to the apical membrane. Mayer et al. (2009) identified Mdr65 in a screen for increased BBB permeability to the P-glycoprotein substrate Rhodamine B. Mdr65 is also required at the $\mathrm{BBB}$ to protect the brain from various $\mathrm{P}$ glycoprotein xenobiotic substrates, and sensitivity of the Mdr65 mutants to these xenobiotics can be rescued by expression of human P-glycoprotein specifically in the SPG cells, confirming that Mdr65 provides an evolutionarily conserved chemoprotective transport barrier at the invertebrate BBB. Interestingly, recent work in the migratory locust (Locusta migratoria) and the desert locust (Schistocerca gregaria) has shown that P-glycoprotein-like xenobiotic transporters also provide a transcellular barrier in the locust brain (Nielsen et al., 2011; Andersson et al., 2013). These findings advance the possibilities of using insect models to study the roles of xenobiotic efflux transporters at the BBB (see below).

In addition to Mdr65, other potential homologs of mammalian chemoprotective $\mathrm{ABC}$ transporters are expressed at the invertebrate BBB (DeSalvo et al., 2014). These candidates were identified based on their expression or enrichment in the adult Drosophila BBB; these include putative homologs for $A B C C-/ M R P-1,4,5$ and 6 (CG5789, SUR, CG11897, and $M R P)$ and $A B C G 2 / B C R P$ (W, CG31689 and CG3164). Currently, there are no published reports showing whether these gene 


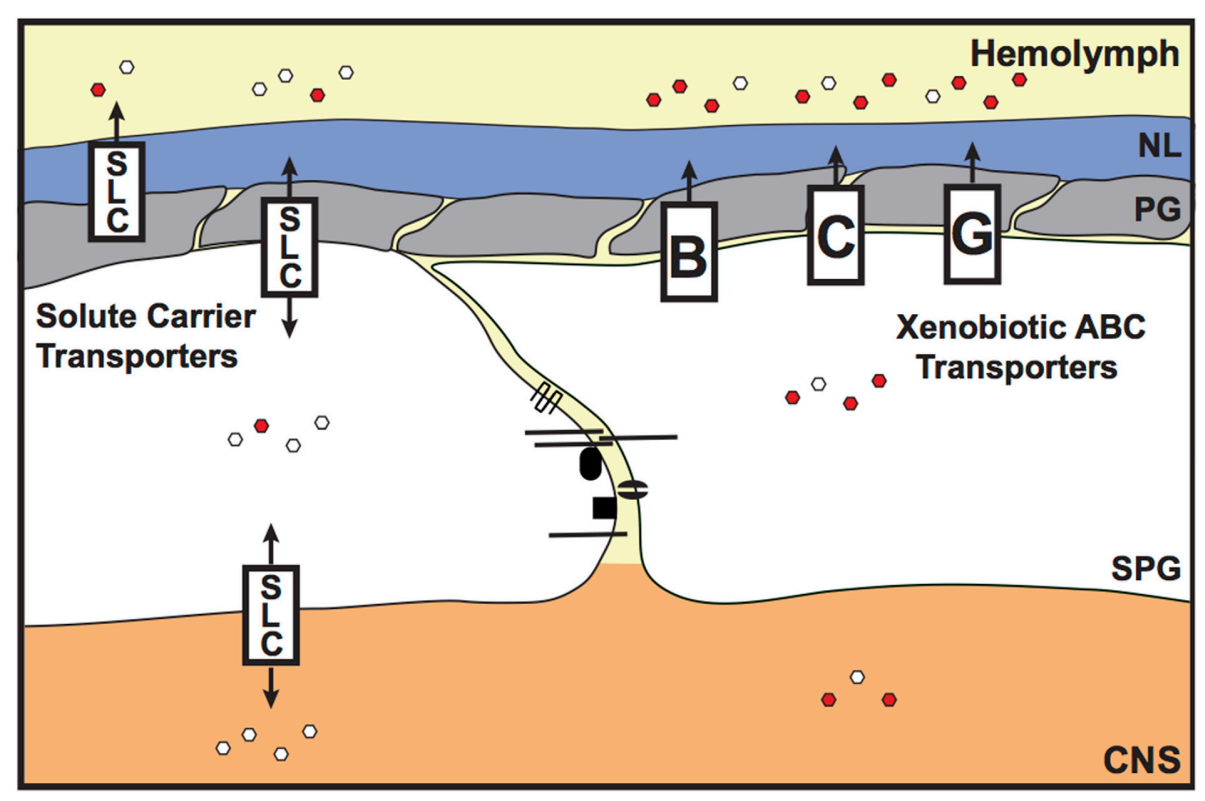

FIGURE 4 | Chemoprotective and endogenous molecule transporters of the invertebrate BBB. The transcellular chemoprotective barrier is primarily provided by the ATP-binding Cassette $(A B C)$ transporters. These xenobiotic efflux transporters are localized to the apical surface of the subperineurial glia (SPG) and remove lipophilic molecules from the SPG membrane, preventing access to the brain. Members of the Solute Carrier (SLC) class of

transporters also have some chemoprotective roles, but are mainly known as transporters of endogenous molecules. The SLCs control the entry of hydrophilic/charged molecules (e.g., glucose, amino acids and ions) into the brain, to support optimal brain function. SLCs are present both on the apical and basal surfaces of the SPG cells. Some SLC members, e.g., Oatp58Dc, are also expressed in the perineurial glia (PG). Red and white hexagons represent xenobiotics and endogenous molecules, respectively. NL, neural lamella; CNS, central nervous system. products can perform chemoprotective functions at the invertebrate $\mathrm{BBB}$.

The vertebrate organic anion transporters (Oats and Oatps) are primarily known for their roles in controlling the brain access of endogenous anions (Kusuhara and Sugiyama, 2005); however, they also have chemoprotective functions (Anderson and Thwaites, 2010). Potential homologs of vertebrate Oats and Oatps are also expressed at the Drosophila BBB (CG6126, CG3168, CG6231, ORCT2, CG4630, ORCT, Oatp58Dc, and Oatp74D; DeSalvo et al., 2014), providing candidates for determining whether Oat/Oatps can also contribute toward chemoprotection of the brain in invertebrates. The Drosophila genome encodes eight Oatp transporters, two of which have enriched transcript number in the Drosophila brain (Oatp58Dc and Oatp74D; http:// flyatlas.org/). Seabrooke and O'Donnell (2013) have recently shown that Oatp58Dc is expressed in the PG, SPG and postmitotic neurons, and is required specifically in PG cells for maintaining chemical protection of the brain from fluorescein. In the absence of Oatp58Dc, levels of hemolymph-injected fluorescein are higher in the brains of Oatp58Dc Drosophila brains. This shows that, at least for Oatp58Dc, invertebrate organic anion transporters can also provide chemoprotective functions to the BBB.

\section{ENDOGENOUS MOLECULE REGULATION}

Transcellular access to the brain is not only controlled by ATPdependent xenobiotic efflux transporters; secondary/facilitated transporters and channel proteins are also expressed at the BBB in both vertebrates and invertebrates (Figure 4). The largest group of the facilitated transporters is the solute carrier (SLC) transporters. In vertebrates, the SLCs are grouped into 48 classes (SLC 1-48) based on sequence identity (Hediger et al., 2004). It was recently documented that Drosophila glia express SLCs from eight of these vertebrate SLC classes (SLC1, SLC5, SLC6, SLC7, SLC24, and SLC29), but it was not determined whether these SLCs were expressed or enriched in the BBB (Featherstone, 2011). Microarray analysis of the adult Drosophila BBB glia has recently identified which of these SLCs are expressed or enriched at the BBB, and also identified additional BBB SLC class candidates (DeSalvo et al., 2014). Of note, there are 18 SLC homologs that are both highly expressed and enriched at the Drosophila BBB (members from the SLCO, 2, 7, 16, 19, 22, and 39 classes) and would therefore be good candidates for further study.

A role for the SLC12 transporter Ncc69 (a cation chloride cotransporter) and its serine/threonine kinase regulator Fray has been shown in controlling extracellular volume in peripheral nerves (Leiserson et al., 2011). Mutants in either Ncc69 or Fray lead to the accumulation of fluid between axons and glia, resulting in peripheral neuropathy. Both Ncc69 and Fray were also shown to function in the SPG cells of the blood-nerve barrier.

Eight potential SLC21 (Oatp) transporters have been identified in Drosophila (Seabrooke and O'Donnell, 2013). Of these eight, four are expressed in the Drosophila head or brain (Oatp30B, Oatp33Ea, Oatp58Dc, and Oatp74D; http://flyatlas.org/; Torrie et al., 2004; Seabrooke and O'Donnell, 2013). According to the data in DeSalvo et al. (2014), Oatp30B is primarily expressed in 
neurons, however, Meyer et al. (2014) suggested a glial role for Oatp30B in neural lamella integrity and CNS shape. Oatp33Ea shows very low expression in all cell types of the adult brain; as its expression was previously shown in the fly head, Oatp33Ea may therefore localize to the fat bodies of the head rather than the brain. As mentioned above, the organic anion transporter Oatp58Dc is expressed in the PG, SPG and post-mitotic neurons of the Drosophila CNS; however, its function was shown to be specifically required in the PG layer of the BBB (Seabrooke and O'Donnell, 2013). This suggests a novel role for the PG in regulating organic anion access to the brain. Oatp74D was also shown by DeSalvo et al. (2014) to be highly enriched in the BBB (21-fold and 64-fold enrichment compared to whole brain and neuron levels, respectively). It would be interesting to determine the function of this transporter, whether it is required in the SPG or PG, and whether there is any overlap or coordination in the BBB functions of Oatp58Dc and Oatp74D.

Although the BBB provides a paracellular barrier to the movement of inorganic ions between the hemolymph and the brain, transcellular transit does occur (Treherne, 1966). This involves both energy-dependent mechanisms $\left(\mathrm{Na}^{+} / \mathrm{K}^{+}\right.$ATPases $)$and also diffusion down the electrochemical gradient, which may be partially set up by V-type $\mathrm{H}^{+}$-ATPases (Kocmarek and O'Donnell, 2011).

Another mode of transit across the BBB is provided by receptor-mediated transcytosis. In general, vesicular transcytosis is reduced in the vascular endothelial cells of the vertebrate $\mathrm{BBB}$; however, small and large molecular weight molecules can transit the BBB by a specific, receptor-mediated route (see Xiao and Gan, 2013 for review). In Drosophila, the mechanism of receptor-mediated transcytosis of lipoprotein via the low-density lipoprotein receptor (LRP) has recently been investigated (Brankatschk and Eaton, 2010; Brankatschk et al., 2014). Lipophorin is the main lipoprotein present in Drosophila. The protein component of lipophorin (apolipophorin) can be cleaved into ApoLI and ApoLII, and this cleavage was shown to affect the localization of the lipoprotein once it had transited across the BBB. The full length ApoL localized to neurons, whereas ApoLII was found in the optic anlage. The authors further investigated the function of lipophorin in the brain and found that knocking down the production of lipophorin in its synthesis organ (the fat body) blocked neuroblast proliferation in the first/second instar larvae. They suggest that this may be due to the reduced $\mathrm{BBB}$ transit of GPI-linked proteins, which they show requires the presence of lipophorin. Brankatschk and Eaton (2010) also showed that lipophorin-associated, sterol-linked fluorescent proteins only have limited access to the brain and primarily accumulate at the BBB. From this, one might conclude that sterol-linked proteins don't play a role in neuroblast proliferation as they scarcely enter the brain; however, recent work in the Brand lab discovered a role for synchronized BBB calcium signaling in coordinated neuroblast proliferation (Speder and Brand, 2014; see below). Therefore, sterol-linked proteins may not need to enter the brain to induce neuroblast proliferation. Instead their accumulation in the BBB may trigger a signaling cascade that induces the underlying neuroblasts to proliferate. Brankatschk et al. (2014) have also shown that BBB calcium release is required for the $\mathrm{BBB}$ transit and normal brain localization of Lipid Transfer Particle. This was shown to be important for the insulin-dependent regulation of systemic growth (see below).

\section{METABOLIC AND SIGNALING ROLES OF THE INVERTEBRATE BBB}

The previous sections have primarily dealt with the anatomy and function of the invertebrate BBB in chemoprotection of the brain, regulation of endogenous molecule access to the brain, and the maintenance of these functions. However, the BBB acts as more than just a selective filter. Exciting new findings have revealed that the Drosophila BBB can also sense and respond to systemic metabolic signals (Chell and Brand, 2010; Speder and Brand, 2014). Thus, the BBB appears to be a dynamic and communicative layer between the brain and the body (Figure 5). The insulin signaling pathway has long been known to sense nutritional status and dictate organism growth, reproductive output and lifespan, and this is evolutionarily conserved (Partridge et al., 2011; Wigby et al., 2011; Mirth and Shingleton, 2012). This systemic signaling is coordinated by the secretion of Drosophila insulin-like peptides (dILPs), which are secreted by insulin producing cells (IPCs) in the brain. The secretion of dILPs is triggered by a factor produced by the fat body (the Drosophila liver and adipose tissue equivalent) in response to dietary amino acids [through the Slimfast and target of rapamycin (TOR) signaling pathways; Geminard et al., 2009], and fat and sugar (through the Drosophila cytokine Unpaired2; Rajan and Perrimon, 2012; reviewed in Nassel et al., 2013). In addition to the IPCs, the BBB glia have recently been shown to be a source of dILPs required for the reactivation of proliferation in the underlying neuroblasts (Chell and Brand, 2010; Speder and Brand, 2014; Figure 5). The BBB glia expression of dILP2 and dILP6 is nutrient-dependent, suggesting that a humoral signal from the fat body (possibly Unpaired2; Rajan and Perrimon, 2012) is sensed by the surface glia leading to dILP production and secretion to reactivate neuroblast proliferation in the larval brain (Chell and Brand, 2010). It would be interesting to determine whether this humoral signal is the same signal that depends upon lipophorin particles to induce neuroblast proliferation, as shown by Brankatschk and Eaton (2010).

Additional evidence for the role of $\mathrm{BBB}$ glia in coordinating neuroblast proliferation was provided by Speder and Brand (2014) who discovered that gap junctional proteins expressed in the SPG cells were required for synchronized calcium oscillations within the SPG, and for neuroblast proliferation. The synchronized calcium oscillations were nutrient-dependent, and were therefore suggested to be a bridge in the gap between the nutrition-dependent fat body-derived signal and the synchronous nutrient-dependent exit of neuroblasts from quiescence. Although this work suggests a direct role for the BBB glia in neuroblast proliferation, Sousa-Nunes et al. (2011) suggest that neuroblast proliferation requires IILP6 expression by the underlying cortex glia rather than the SPG cells. Therefore, the role of the BBB may be to coordinate a complex signaling network, bringing together systemic nutritional signals and relaying this information to the underlying cortex glia to regulate neuroblast proliferation (Figure 5). 


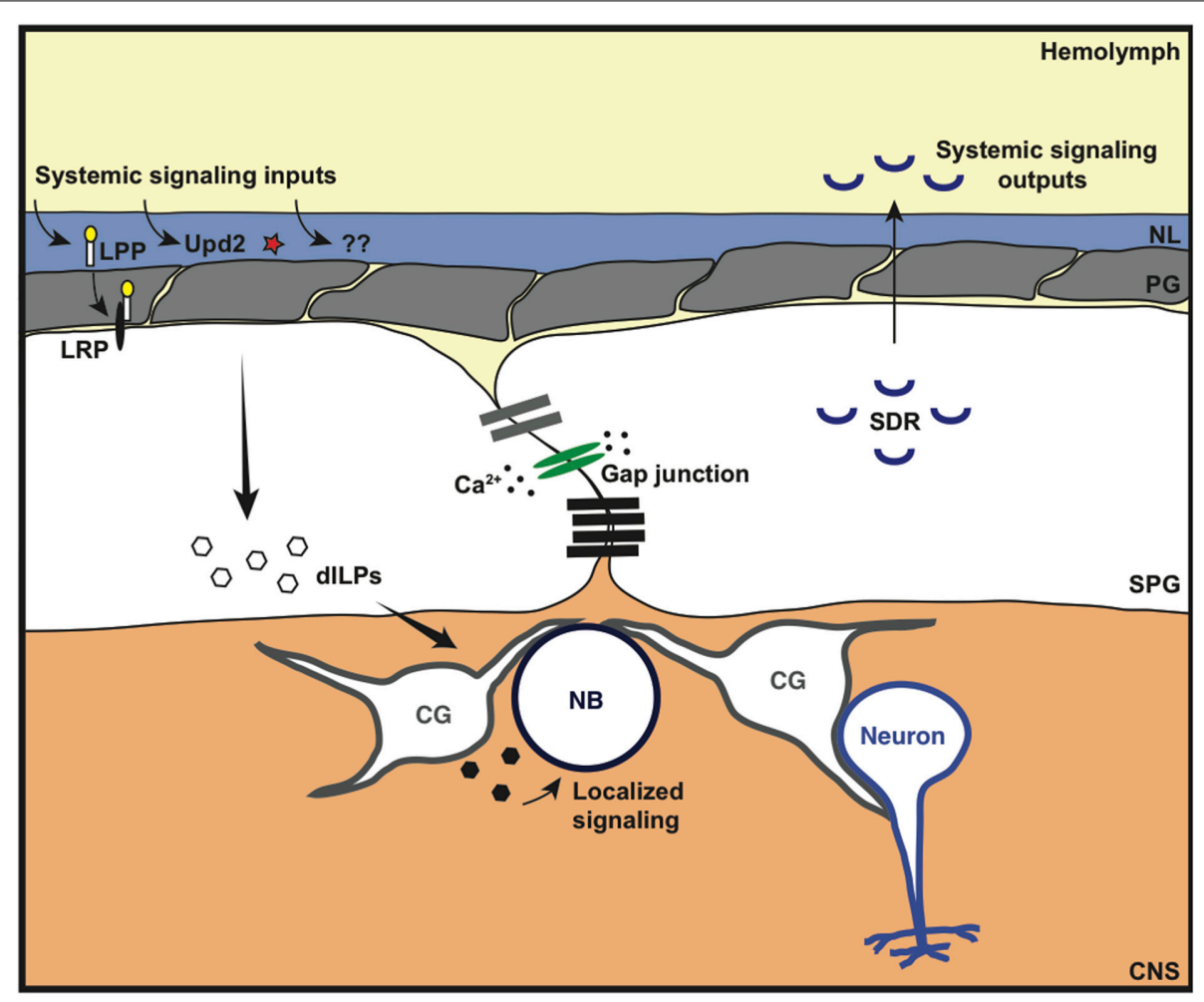

FIGURE 5 | Metabolic and signaling roles of the invertebrate BBB. The Drosophila BBB can sense and respond to various systemic signaling inputs. This includes the lipoprotein-like receptor (LRP) ligand lipophorin (LPP), the cytokine-like molecule Unpaired-2 (Upd2) and other unidentified signaling molecules (??) that are released in response to metabolic/nutritional triggers. This leads to localized secretion of Drosophila insulin-like peptide (dILP) by the subperineurial glia (SPG) and/or cortex glia (CG) to coordinate neuroblast
(NB) proliferation. The coordinated response of the SPG is thought to occur through synchronous calcium $\left(\mathrm{Ca}^{2+}\right)$ oscillations via gap junction proteins. In addition to localized growth regulation, the Drosophila BBB can also regulate systemic growth (whole organism growth). The SPG cells both express and constitutively secrete a negative regulator of insulin signaling: the secreted decoy of insulin receptor (SDR); SDR mutants lead to excessive growth of the whole animal.
In extension of this work, Brankatschk et al. (2014) have shown that the BBB can also regulate growth systemically (whole organism growth) in addition to localized growth control (neuroblast proliferation). They found that the BBB controls Lipid Transfer Particle brain access and localization to Dilp2-recruiting neurons in the brain; this resulted in systemic secretion of Dilp2 from insulin producing cells in the brain, impacting on whole organism growth. This BBB function is nutrition-dependent and requires calcium signaling in the BBB. In fact, genetic manipulations that forced an increase in BBB calcium release resulted in normal systemic insulin signaling despite an inappropriate nutritional state. Together, these fascinating findings show that the BBB can have a master role in linking nutritional input to both local and systemic growth outputs.

A further role for the Drosophila BBB in growth control was shown by Okamoto et al. (2013). The authors discovered a negative regulator of insulin signaling, the secreted decoy of insulin receptor (SDR), in an RNAi screen for secreted regulators of body growth; SDR mutants had a larger body weight. SDR is expressed in the BBB glia of the CNS, the midgut muscles and imaginal discs of the larva; however, its function in body growth was shown to be dependent on its BBB glia expression (Okamoto et al., 2013). Although SDR expression and secretion was shown to be constitutive, and not dependent on nutritional status, it proved to be crucial for controlling body weight during adverse nutritional conditions; SDR mutants were lethal when grown in adverse nutritional conditions whereas wild type larvae survived. These results suggest a further role for the BBB in controlling body growth during development. The body size of the adult fly is predetermined during the larval and pupal stages (Mirth and Shingleton, 2012); however, DeSalvo et al. (2014) showed that $S D R$ is highly expressed and enriched in the BBB glia of the adult brain. Therefore, this begs a number of questions. What is the role of SDR in the adult? Is its secretion regulated or constitutive in the adult? Does its expression level change with age? Does SDR have a role in regulating aging? These are exciting questions that can easily be addressed in the Drosophila model organism.

This additional role of the BBB in coordinating both localized and systemic insulin signaling to regulate localized and organismwide growth is likely to be one in a number of ways that the BBB can communicate with various organs of the body.

\section{THE INVERTEBRATE BBB AS A TOOL FOR ADVANCING THERAPEUTIC INTERVENTIONS}

One of the major hindrances in neuropharmacology is the resistance of the $\mathrm{BBB}$ for drug delivery to the brain. This is primarily 
due to the efficient functioning of P-glycoprotein. Furthermore, the pre-clinical to clinical translation of CNS drug penetration following the use of P-glycoprotein inhibitors has proved unsuccessful due to incomplete P-glycoprotein inhibition (see Kalvass et al., 2013 for a detailed review). Therefore, being able to develop drugs that are not P-glycoprotein substrates would be a beneficial strategy. Drug screening largely depends upon in vitro tests using brain microvascular endothelial cell monolayers. However, studies have shown that brain microvascular endothelial cells grown in culture do not maintain their full BBB properties; of particular note, they show a down regulation of P-glycoprotein (Calabria and Shusta, 2008), suggesting that this methodology is not ideal. Early phase drug screening using rodents would be too costly; therefore, there is a need for a cost-effective in vivo approach to further the development of CNS drugs. With this in mind, DeSalvo et al. (2011) developed an approach to perform live screening of chemical fluor penetration into the Drosophila brain. This approach involved visualizing a change in the fluorescence intensity in the fly eye, due to a change in permeability of the blood-eye barrier. DeSalvo et al. (2011) showed this approach could be used to screen for genetic modifiers of BBB integrity and identified various interesting candidate genes that were expressed in different layers of the compound BBB, supporting the notion that all layers of the compound barrier are important for full BBB integrity. In addition to genetic modifiers, this live imaging approach can be used for large-scale drug screening to identify drugs that will penetrate the $\mathrm{BBB}$ and are therefore not likely P-glycoprotein (Mdr65) substrates.

Interestingly, other invertebrate BBB models have been developed to allow in vivo drug screening. Using the grasshopper system, Nielsen et al. (2011) showed that co-administration of a P-glycoprotein inhibitor with a test P-glycoprotein substrate rendered the $\mathrm{BBB}$ more permeable to the P-glycoprotein substrate. Furthermore, Andersson et al. (2013) were able to use this methodology to distinguish P-glycoprotein substrates from non-substrates using liquid chromatography-mass spectrometry to measure the drug content in the brain, either in the absence or presence of a P-glycoprotein inhibitor. This confirmed that P-glycoprotein substrates could be identified using this model system. These invertebrate BBB models provide a valuable bridge between endothelial cell culture models and vertebrate P-glycoprotein knock-out models for drug screening.

In addition to using invertebrate BBB models to assess $\mathrm{BBB}$ permeability of test drugs, these model systems can also be used to test new technologies for increasing drug delivery to the brain. The cost of performing these trials in invertebrates would be comparatively small and would therefore allow for a much broader scaled testing strategy than can be applied to vertebrate systems. The use of Drosophila to address the problem of brain drug delivery was shown by Sarantseva et al. (2011), where they tested the ability of dendrimers to cross the BBB. Dendrimers are nanopolymers that can encapsulate various cargo and therefore have been utilized in many biological (and non-biological) fields (see Kesharwani et al., 2014 for a review). The advantage of using these nanopolymers as a CNS drug delivery strategy is the ability to customize the dendrimer to very specific requirements. For example, the functional group on the polymer surface can be modified to allow tissue-specific targeting, and they are also able to "carry" various types of cargo, including nucleic acids, proteins and drugs across cell membranes (Kesharwani et al., 2014). The use of these biological vectors in drug delivery have been shown for epithelial barriers, such as the skin and intestine (e.g., Kaminskas et al., 2012). Moreover, the ability of the lysine-based dendrimer D5 to carry peptides across the Drosophila BBB has been shown (Sarantseva et al., 2011), suggesting that this nanotechnology could be a useful strategy for improving CNS drug delivery in vertebrates.

\section{FUTURE QUESTIONS AND EMERGING AREAS OF BBB RESEARCH}

We have highlighted that the invertebrate $\mathrm{BBB}$ is a compound structure, like the vertebrate neurovascular unit, and each of its cellular and non-cellular layers appears to have a coordinated role in maintaining BBB integrity and for signaling purposes. However, there is still much to learn about the nature of the communication between these layers, how this is regulated, and how the individual layers respond to physical, chemical or pathological perturbations. In particular, understanding the compensations that occur within these BBB layers during a disease state will likely be required to advance therapeutic design to combat these disorders. Expression changes of chemoprotective transporters, such as P-glycoprotein and Breast Cancer Resistance Protein, have already been shown to occur in disease states including Alzheimer's disease and epilepsy (Vogelgesang et al., 2002; Loscher and Potschka, 2005; Miller, 2010). These expression changes are thought to explain the multidrug resistance phenotypes seen in some neurological disorders. Therefore, investigations that show ways to counter these compensations would be valuable for treating these diseases. Furthermore, being able to parse the contribution of the PG and SPG toward CNS protection would be a valuable endeavor. This could involve a genetic approach, like used by DeSalvo et al. (2014), to produce separate PG and SPG transcriptomes in both the wild type and diseased states.

In addition to parsing the contributions of the SPG and PG toward BBB function, an important future question for the field is to determine whether there are regional specializations across the invertebrate compound BBB. We have seen evidence of coordinated BBB functions via gap junction signaling within the SPG cells, which impacted upon the synchrony of neuroblast proliferation (Speder and Brand, 2014); equally possible is the presence of regional $\mathrm{BBB}$ responses to localized signals, in terms of developmental and pathological signals, as well as localized immune cell transmigration. In extension of this, it is important to understand how the different layers of the compound BBB network can respond to localized signals and perturbations. For example, it would be interesting to determine whether there are different subsets of underlying cortex glia that can sense and respond to chemical perturbations of the BBB and other subsets that can respond to the metabolic status of the CNS, to provide both broad and localized "feedback circuitry" to relay information about CNS homeostasis to the BBB. Due to the powerful genetic strengths of the Drosophila system and the accessible architecture of the invertebrate $\mathrm{BBB}$, this is an area where invertebrate model organisms can help to pave the way forward. 
An exciting, emerging area of $\mathrm{BBB}$ research that deserves further attention is whether there are sex-specific traits of the BBB. It is already known that there are sex-specific differences in the CNS, a concept that is evolutionarily conserved (Cachero et al., 2010). However, very little is known about sex-specific components of the BBB and its impact on physiology. This is another research area where Drosophila can advance the field, as it is possible to specifically change the sex of the BBB using targeted genetics. This allows the separation of sex-specific BBB effects from sex-specific effects of the whole organism. This approach is possible because sex determination is cell autonomous in flies; therefore, one can express a master regulator of sex determination (e.g., $\mathrm{TraF}$ ) in a specific cell type and alter the sex identity of that cell type. This has been shown for the Drosophila liver and adipose tissue equivalent (the fat body), showing that male courtship behavior can be altered by changing the sex identity of the fat body cells (Lazareva et al., 2007). This technique was recently applied to the BBB, showing a behavioral impact of sex-specificity in the BBB (Hoxha et al., 2013). BBB-specific, ectopic expression of a gene that feminizes cells ( $\mathrm{Tr} a \mathrm{~F}$ ) led to reduced courtship behavior in males. Hoxha et al. (2013) showed that this was not solely a developmental effect, as BBB-specific expression of TraF only during the adult stages of the fly still reduced courtship in males. The authors also showed that this reduction in courtship behavior required the GPCR Moody, which Bainton et al. (2005) previously showed to have separable roles in maintaining BBB integrity (a structural role) and behavioral drug responses (a signaling role). Interestingly, there are sex-specific enrichments of Moody isoforms in the BBB (Hoxha et al., 2013). Of the four Moody isoforms, there is an enrichment of one of the Moody- $\alpha$ isoforms in male heads and an enrichment of both Moody- $\beta$ isoforms in female heads. Therefore, Hoxha et al. (2013) have already identified that sex-specific transcripts do exist in the BBB and that sex identity of the BBB can impact upon behavior. It would be interesting to determine whether sex-specific differences in the BBB can also impact upon pharmacokinetics, as this could have major implications for treatments of CNS disorders.

\section{REFERENCES}

Abbott, N. J. (2005). Dynamics of CNS barriers: evolution, differentiation, and modulation. Cell. Mol. Neurobiol. 25, 5-23. doi: 10.1007/s10571-004-1374-y

Abbott, N. J. (2013). Blood-brain barrier structure and function and the challenges for CNS drug delivery. J. Inherit. Metab. Dis. 36, 437-449. doi: 10.1007/s10545013-9608-0

Abbott, N. J., Patabendige, A. A., Dolman, D. E., Yusof, S. R., and Begley, D. J. (2010). Structure and function of the blood-brain barrier. Neurobiol. Dis. 37, 13-25. doi: 10.1016/j.nbd.2009.07.030

Abbott, N. J., and Romero, I. A. (1996). Transporting therapeutics across the bloodbrain barrier. Mol. Med. Today 2, 106-113. doi: 10.1016/1357-4310(96)88720-X Anderson, C. M., and Thwaites, D. T. (2010). Hijacking solute carriers for protoncoupled drug transport. Physiology (Bethesda) 25, 364-377. doi: 10.1152/physiol.00027.2010

Andersson, O., Hansen, S. H., Hellman, K., Olsen, L. R., Andersson, G., Badolo, L., et al. (2013). The grasshopper: a novel model for assessing vertebrate brain uptake. J. Pharmacol. Exp. Ther. 346, 211-218. doi: 10.1124/jpet.113.205476

Auld, V. J., Fetter, R. D., Broadie, K., and Goodman, C. S. (1995). Gliotactin, a novel transmembrane protein on peripheral glia, is required to form the bloodnerve barrier in Drosophila. Cell 81, 757-767. doi: 10.1016/0092-8674(95)9 0537-5

Bainton, R. J., Tsai, L. T., Schwabe, T., DeSalvo, M., Gaul, U., and Heberlein, U. (2005). moody encodes two GPCRs that regulate cocaine behaviors and blood-brain barrier permeability in Drosophila. Cell 123, 145-156. doi: 10.1016/j.cell.2005.07.029

Baumgartner, S., Littleton, J. T., Broadie, K., Bhat, M. A., Harbecke, R., Lengyel, J. A., et al. (1996). A Drosophila neurexin is required for septate junction and blood-nerve barrier formation and function. Cell 87, 1059-1068. doi: 10.1016/S0092-8674(00)81800-0

Behr, M., Riedel, D., and Schuh, R. (2003). The claudin-like megatrachea is essential in septate junctions for the epithelial barrier function in Drosophila. Dev. Cell 5, 611-620. doi: 10.1016/S1534-5807(03)00275-2

Bhat, M. A. (2003). Molecular organization of axo-glial junctions. Curr. Opin. Neurobiol. 13, 552-559. doi: 10.1016/j.conb.2003.09.004

Bhat, M. A., Rios, J. C., Lu, Y., Garcia-Fresco, G. P., Ching, W., St Martin, M., et al. (2001). Axon-glia interactions and the domain organization of myelinated axons requires neurexin IV/Caspr/Paranodin. Neuron 30, 369-383. doi: 10.1016/S0896-6273(01)00294-X

Bolis, A., Coviello, S., Visigalli, I., Taveggia, C., Bachi, A., Chishti, A. H., et al. (2009). Dlg1, Sec8, and Mtmr2 regulate membrane homeostasis in Schwann cell myelination. J. Neurosci. 29, 8858-8870. doi: 10.1523/JNEUROSCI.142309.2009

Brankatschk, M., Dunst, S., Nemetschke, L., and Eaton, S. (2014). Delivery of circulating lipoproteins to specific neurons in the Drosophila brain regulates systemic insulin signaling. Elife 3:e02862. doi: 10.7554/eLife.02862

Brankatschk, M., and Eaton, S. (2010). Lipoprotein particles cross the blood-brain barrier in Drosophila. J. Neurosci. 30, 10441-10447. doi: 10.1523/JNEUROSCI.5943-09.2010

Brightman, M. W., and Reese, T. S. (1969). Junctions between intimately apposed cell membranes in the vertebrate brain. J. Cell Biol. 40, 648-677. doi: $10.1083 /$ jcb. 40.3 .648

Cachero, S., Ostrovsky, A. D., Yu, J. Y., Dickson, B. J., and Jefferis, G. S. (2010). Sexual dimorphism in the fly brain. Curr. Biol. 20, 1589-1601. doi: 10.1016/j.cub.2010.07.045

Calabria, A. R., and Shusta, E. V. (2008). A genomic comparison of in vivo and in vitro brain microvascular endothelial cells. J. Cereb. Blood Flow Metab. 28, 135-148. doi: 10.1038/sj.jcbfm.9600518

Chell, J. M., and Brand, A. H. (2010). Nutrition-responsive glia control exit of neural stem cells from quiescence. Cell 143, 1161-1173. doi: 10.1016/j.cell.2010.12.007

Daneman, R., Agalliu, D., Zhou, L., Kuhnert, F., Kuo, C. J., and Barres, B. A. (2009). Wnt/beta-catenin signaling is required for CNS, but not non-CNS, angiogenesis. Proc. Natl. Acad. Sci. U.S.A. 106, 641-646. doi: 10.1073/pnas.08051 65106

Daneman, R., Zhou, L., Agalliu, D., Cahoy, J. D., Kaushal, A., and Barres, B. A. (2010). The mouse blood-brain barrier transcriptome: a new resource for understanding the development and function of brain endothelial cells. PLoS ONE 5:e13741. doi: 10.1371/journal.pone.0013741

DeSalvo, M. K., Hindle, S. J., Rusan, Z. M., Orng, S., Eddison, M., Halliwell, K., et al. (2014). The Drosophila surface glia transcriptome: evolutionary conserved blood-brain barrier processes. Front. Neurosci. 8:346. doi: 10.3389/fnins.2014.00346

DeSalvo, M. K., Mayer, N., Mayer, F., and Bainton, R. J. (2011). Physiologic and anatomic characterization of the brain surface glia barrier of Drosophila. Glia 59, 1322-1340. doi: 10.1002/glia.21147

Edwards, T. N., and Meinertzhagen, I. A. (2010). The functional organisation of glia in the adult brain of Drosophila and other insects. Prog. Neurobiol. 90, 471-497. doi: 10.1016/j.pneurobio.2010.01.001

Escalante-Alcalde, D., Hernandez, L., Le Stunff, H., Maeda, R., Lee, H. S., Gang, C. Jr., et al. (2003). The lipid phosphatase LPP3 regulates extraembryonic vasculogenesis and axis patterning. Development 130, 4623-4637. doi: 10.1242/dev.00635

Faivre-Sarrailh, C., Banerjee, S., Li, J., Hortsch, M., Laval, M., and Bhat, M. A. (2004). Drosophila contactin, a homolog of vertebrate contactin, is required for septate junction organization and paracellular barrier function. Development 131, 4931-4942. doi: 10.1242/dev.01372

Featherstone, D. E. (2011). Glial solute carrier transporters in Drosophila and mice. Glia 59, 1351-1363. doi: 10.1002/glia.21085

Fehon, R. G., Dawson, I. A., and Artavanis-Tsakonas, S. (1994). A Drosophila homologue of membrane-skeleton protein 4.1 is associated with septate junctions and is encoded by the coracle gene. Development 120, 545-557. 
Geminard, C., Rulifson, E. J., and Leopold, P. (2009). Remote control of insulin secretion by fat cells in Drosophila. Cell Metab. 10, 199-207. doi: 10.1016/j.cmet.2009.08.002

Genova, J. L., and Fehon, R. G. (2003). Neuroglian, Gliotactin, and the Na+/K+ ATPase are essential for septate junction function in Drosophila. J. Cell Biol. 161, 979-989. doi: 10.1083/jcb.200212054

Hatan, M., Shinder, V., Israeli, D., Schnorrer, F., and Volk, T. (2011). The Drosophila blood brain barrier is maintained by GPCR-dependent dynamic actin structures. J. Cell Biol. 192, 307-319. doi: 10.1083/jcb.201007095

Hediger, M. A., Romero, M. F., Peng, J. B., Rolfs, A., Takanaga, H., and Bruford, E. A. (2004). The ABCs of solute carriers: physiological, pathological and therapeutic implications of human membrane transport proteinsIntroduction. Pflugers Arch. 447, 465-468. doi: 10.1007/s00424-003-1192-y

Hijazi, A., Haenlin, M., Waltzer, L., and Roch, F. (2011). The Ly6 protein coiled is required for septate junction and blood brain barrier organisation in Drosophila. PLoS ONE 6:e17763. doi: 10.1371/journal.pone.0017763

Hoxha, V., Lama, C., Chang, P. L., Saurabh, S., Patel, N., Olate, N., et al. (2013). Sex-specific signaling in the blood-brain barrier is required for male courtship in Drosophila. PLoS Genet. 9:e1003217. doi: 10.1371/journal.pgen.1003217

Ile, K. E., Tripathy, R., Goldfinger, V., and Renault, A. D. (2012). Wunen, a Drosophila lipid phosphate phosphatase, is required for septate junction-mediated barrier function. Development 139, 2535-2546. doi: 10.1242/dev.077289

Kalvass, J. C., Polli, J. W., Bourdet, D. L., Feng, B., Huang, S. M., Liu, X., et al. (2013). Why clinical modulation of efflux transport at the human blood-brain barrier is unlikely: the ITC evidence-based position. Clin. Pharmacol. Ther. 94, 80-94. doi: 10.1038/clpt.2013.34

Kaminskas, L. M., Boyd, B. J., and Porter, C. J. H. (2012). Dendrimer pharmacokinetics: the effect of size, structure and surface characteristics on ADME properties (vol 6, pg 1063, 2011). Nanomedicine 7, 167-168. doi: 10.2217/nnm.11.67

Kanai, Y., Okada, Y., Tanaka, Y., Harada, A., Terada, S., and Hirokawa, N. (2000). KIF5C, a novel neuronal kinesin enriched in motor neurons. J. Neurosci. 20, 6374-6384.

Kesharwani, P., Jain, K., and Jain, N. K. (2014). Dendrimer as nanocarrier for drug delivery. Prog. Polymer Sci. 39, 268-307. doi: 10.1016/j.progpolymsci.20 13.07.005

Kim, J., Kim, Y. J., and Kim-Ha, J. (2010). Blood-brain barrier defects associated with Rbp9 mutation. Mol Cells 29, 93-98. doi: 10.1007/s10059-010-0040-0

Kim-Ha, J., Kim, J., and Kim, Y. J. (1999). Requirement of RBP9, a Drosophila Hu homolog, for regulation of cystocyte differentiation and oocyte determination during oogenesis. Mol. Cell. Biol. 19, 2505-2514.

Kocmarek, A. L., and O’Donnell, M. J. (2011). Potassium fluxes across the blood brain barrier of the cockroach, Periplaneta americana. J. Insect Physiol. 57, 127-135. doi: 10.1016/j.jinsphys.2010.09.011

Kusuhara, H., and Sugiyama, Y. (2005). Active efflux across the blood-brain barrier: role of the solute carrier family. NeuroRx 2, 73-85. doi: 10.1602/neurorx.2.1.73

Lazareva, A. A., Roman, G., Mattox, W., Hardin, P. E., and Dauwalder, B. (2007). A role for the adult fat body in Drosophila male courtship behavior. PLoS Genet. 3:e16. doi: 10.1371/journal.pgen.0030016

Lee, J. F., Zeng, Q., Ozaki, H., Wang, L., Hand, A. R., Hla, T., et al. (2006). Dual roles of tight junction-associated protein, zonula occludens-1, in sphingosine 1phosphate-mediated endothelial chemotaxis and barrier integrity. J. Biol. Chem. 281, 29190-29200. doi: 10.1074/jbc.M604310200

Leiserson, W. M., Forbush, B., and Keshishian, H. (2011). Drosophila glia use a conserved cotransporter mechanism to regulate extracellular volume. Glia 59, 320-332. doi: 10.1002/glia.21103

Leslie, E. M., Deeley, R. G., and Cole, S. P. (2005). Multidrug resistance proteins: role of P-glycoprotein, MRP1, MRP2, and BCRP (ABCG2) in tissue defense. Toxicol. Appl. Pharmacol. 204, 216-237. doi: 10.1016/j.taap.2004.10.012

Loscher, W., and Potschka, H. (2005). Drug resistance in brain diseases and the role of drug efflux transporters. Nat. Rev. Neurosci. 6, 591-602. doi: 10.1038/ nrn 1728

Mayer, F., Mayer, N., Chinn, L., Pinsonneault, R. L., Kroetz, D., and Bainton, R. J. (2009). Evolutionary conservation of vertebrate blood-brain barrier chemoprotective mechanisms in Drosophila. J. Neurosci. 29, 3538-3550. doi: 10.1523/JNEUROSCI.5564-08.2009

Meyer, S., Schmidt, I., and Klambt, C. (2014). Glia ECM interactions are required to shape the Drosophila nervous system. Mech. Dev. 133C, 105-116. doi: 10.1016/j.mod.2014.05.003
Miller, D. S. (2010). Regulation of P-glycoprotein and other ABC drug transporters at the blood-brain barrier. Trends Pharmacol. Sci. 31, 246-254. doi: 10.1016/j.tips.2010.03.003

Mirre, C., Cecchini, J. P., Le Parco, Y., and Knibiehler, B. (1988). De novo expression of a type IV collagen gene in Drosophila embryos is restricted to mesodermal derivatives and occurs at germ band shortening. Development 102, 369-376.

Mirth, C. K., and Shingleton, A. W. (2012). Integrating body and organ size in Drosophila: recent advances and outstanding problems. Front. Endocrinol. (Lausanne) 3:49. doi: 10.3389/fendo.2012.00049

Muir, A. R., and Peters, A. (1962). Quintuple-layered membrane junctions at terminal bars between endothelial cells. J. Cell Biol. 12, 443-448. doi: 10.1083/jcb.12.2.443

Nassel, D. R., Kubrak, O. I., Liu, Y., Luo, J., and Lushchak, O. V. (2013). Factors that regulate insulin producing cells and their output in Drosophila. Front. Physiol. 4:252. doi: 10.3389/fphys.2013.00252

Nelson, K. S., Furuse, M., and Beitel, G. J. (2010). The Drosophila Claudin Kunekune is required for septate junction organization and tracheal tube size control. Genetics 185, 831-839. doi: 10.1534/genetics.110.114959

Nielsen, P. A., Andersson, O., Hansen, S. H., Simonsen, K. B., and Andersson, G. (2011). Models for predicting blood-brain barrier permeation. Drug Discov. Today 16, 472-475. doi: 10.1016/j.drudis.2011.04.004

Obermeier, B., Daneman, R., and Ransohoff, R. M. (2013). Development, maintenance and disruption of the blood-brain barrier. Nat. Med. 19, 1584-1596. doi: 10.1038/nm.3407

Okamoto, N., Nakamori, R., Murai, T., Yamauchi, Y., Masuda, A., and Nishimura, T. (2013). A secreted decoy of InR antagonizes insulin/IGF signaling to restrict body growth in Drosophila. Genes Dev. 27, 87-97. doi: 10.1101/gad.2044 79.112

Olofsson, B., and Page, D. T. (2005). Condensation of the central nervous system in embryonic Drosophila is inhibited by blocking hemocyte migration or neural activity. Dev. Biol. 279, 233-243. doi: 10.1016/j.ydbio.2004.12.020

Oshima, K., and Fehon, R. G. (2011). Analysis of protein dynamics within the septate junction reveals a highly stable core protein complex that does not include the basolateral polarity protein Discs large. J. Cell Sci. 124, 2861-2871. doi: $10.1242 /$ jcs. 087700

Partridge, L., Alic, N., Bjedov, I., and Piper, M. D. (2011). Ageing in Drosophila: the role of the insulin/Igf and TOR signalling network. Exp. Gerontol. 46, 376-381. doi: 10.1016/j.exger.2010.09.003

Pastor-Pareja, J. C., and Xu, T. (2011). Shaping cells and organs in Drosophila by opposing roles of fat body-secreted Collagen IV and perlecan. Dev. Cell 21, 245-256. doi: 10.1016/j.devcel.2011.06.026

Paul, S. M., Ternet, M., Salvaterra, P. M., and Beitel, G. J. (2003). The Na+/K+ ATPase is required for septate junction function and epithelial tube-size control in the Drosophila tracheal system. Development 130, 4963-4974. doi: 10.1242/dev.00691

Rajan, A., and Perrimon, N. (2012). Drosophila cytokine unpaired 2 regulates physiological homeostasis by remotely controlling insulin secretion. Cell 151, 123-137. doi: 10.1016/j.cell.2012.08.019

Reese, T. S., and Karnovsky, M. J. (1967). Fine structural localization of a blood-brain barrier to exogenous peroxidase. J. Cell Biol. 34, 207-217. doi: 10.1083/jcb.34.1.207

Rodrigues, F., Thuma, L., and Klambt, C. (2012). The regulation of glial-specific splicing of Neurexin IV requires HOW and Cdk12 activity. Development 139, 1765-1776. doi: 10.1242/dev.074070

Sarantseva, S. V., Bolshakova, O. I., Timoshenko, S. I., Kolobov, A. A., and Schwarzman, A. L. (2011). Dendrimer D5 is a vector for peptide transport to brain cells. Bull. Exp. Biol. Med. 150, 429-431. doi: 10.1007/s10517-011-1160-z

Schmidt, I., Thomas, S., Kain, P., Risse, B., Naffin, E., and Klambt, C. (2012). Kinesin heavy chain function in Drosophila glial cells controls neuronal activity. J. Neurosci. 32, 7466-7476. doi: 10.1523/JNEUROSCI.0349-12.2012

Schulte, J., Tepass, U., and Auld, V. J. (2003). Gliotactin, a novel marker of tricellular junctions, is necessary for septate junction development in Drosophila. J. Cell Biol. 161, 991-1000. doi: 10.1083/jcb.200303192

Schwabe, T., Bainton, R. J., Fetter, R. D., Heberlein, U., and Gaul, U. (2005). GPCR signaling is required for blood-brain barrier formation in drosophila. Cell 123, 133-144. doi: 10.1016/j.cell.2005.08.037

Seabrooke, S., and O'Donnell, M. J. (2013). Oatp58Dc contributes to blood-brain barrier function by excluding organic anions from the Drosophila brain. Am. J. Physiol. Cell Physiol. 305, C558-C567. doi: 10.1152/ajpcell.00408.2012 
Sousa-Nunes, R., Yee, L. L., and Gould, A. P. (2011). Fat cells reactivate quiescent neuroblasts via TOR and glial insulin relays in Drosophila. Nature 471, 508-512. doi: $10.1038 /$ nature09867

Speder, P., and Brand, A. H. (2014). Gap junction proteins in the blood-brain barrier control nutrient-dependent reactivation of Drosophila neural stem cells. Dev. Cell 30, 309-321. doi: 10.1016/j.devcel.2014.05.021

Starz-Gaiano, M., Cho, N. K., Forbes, A., and Lehmann, R. (2001). Spatially restricted activity of a Drosophila lipid phosphatase guides migrating germ cells. Development 128, 983-991.

Stenman, J. M., Rajagopal, J., Carroll, T. J., Ishibashi, M., McMahon, J., and McMahon, A. P. (2008). Canonical wnt signaling regulates organ-specific assembly and differentiation of CNS vasculature. Science 322, 1247-1250. doi: $10.1126 /$ science. 1164594

Stewart, P. A., and Wiley, M. J. (1981). Developing nervous tissue induces formation of blood-brain barrier characteristics in invading endothelial cells: a study using quail-chick transplantation chimeras. Dev. Biol. 84, 183-192. doi: 10.1016/0012-1606(81)90382-1

Stork, T., Engelen, D., Krudewig, A., Silies, M., Bainton, R. J., and Klambt, C. (2008). Organization and function of the blood-brain barrier in Drosophila. J. Neurosci. 28, 587-597. doi: 10.1523/JNEUROSCI.4367-07.2008

Syed, M. H., Krudewig, A., Engelen, D., Stork, T., and Klambt, C. (2011). The CD59 family member Leaky/Coiled is required for the establishment of the blood-brain barrier in Drosophila. J Neurosci 31, 7876-7885. doi: 10.1523/JNEUROSCI.0766-11.2011

Torrie, L. S., Radford, J. C., Southall, T. D., Kean, L., Dinsmore, A. J., Davies, S. A., et al. (2004). Resolution of the insect ouabain paradox. Proc. Natl. Acad. Sci. U.S.A. 101, 13689-13693. doi: 10.1073/pnas.0403087101

Treherne, J. E. (1966). The effect of ouabain on the efflux of sodium ions in the nerve cords of two insect species (Periplaneta americana and Carausius morosus). J. Exp. Biol. 44, 355-362.

Unhavaithaya, Y., and Orr-Weaver, T. L. (2012). Polyploidization of glia in neural development links tissue growth to blood-brain barrier integrity. Genes Dev. 26, 31-36. doi: 10.1101/gad.177436.111
Vogelgesang, S., Cascorbi, I., Schroeder, E., Pahnke, J., Kroemer, H. K., Siegmund, W., et al. (2002). Deposition of Alzheimer's beta-amyloid is inversely correlated with P-glycoprotein expression in the brains of elderly non-demented humans. Pharmacogenetics 12, 535-541. doi: 10.1097/00008571-20021000 0-00005

Wigby, S., Slack, C., Gronke, S., Martinez, P., Calboli, F. C., Chapman, T., et al. (2011). Insulin signalling regulates remating in female Drosophila. Proc. Biol. Sci. 278, 424-431. doi: 10.1098/rspb.2010.1390

Xiao, G., and Gan, L. S. (2013). Receptor-mediated endocytosis and brain delivery of therapeutic biologics. Int. J. Cell Biol. 2013:703545. doi: 10.1155/2013/7 03545

Zhang, N., Zhang, J., Purcell, K. J., Cheng, Y., and Howard, K. (1997). The Drosophila protein Wunen repels migrating germ cells. Nature 385, 64-67. doi: $10.1038 / 385064 \mathrm{a} 0$

Zlokovic, B. V. (2008). The blood-brain barrier in health and chronic neurodegenerative disorders. Neuron 57, 178-201. doi: 10.1016/j.neuron.2008.01.003

Conflict of Interest Statement: The authors declare that the research was conducted in the absence of any commercial or financial relationships that could be construed as a potential conflict of interest.

Received: 02 October 2014; accepted: 24 November 2014; published online: 16 December 2014.

Citation: Hindle SJ and Bainton RJ (2014) Barrier mechanisms in the Drosophila blood-brain barrier. Front. Neurosci. 8:414. doi: 10.3389/fnins.2014.00414

This article was submitted to Neurogenomics, a section of the journal Frontiers in Neuroscience.

Copyright (C) 2014 Hindle and Bainton. This is an open-access article distributed under the terms of the Creative Commons Attribution License (CC BY). The use, distribution or reproduction in other forums is permitted, provided the original author(s) or licensor are credited and that the original publication in this journal is cited, in accordance with accepted academic practice. No use, distribution or reproduction is permitted which does not comply with these terms. 


\title{
The Drosophila blood-brain barrier: development and function of a glial endothelium
}

\author{
Stefanie Limmer, Astrid Weiler, Anne Volkenhoff, Felix Babatz and Christian Klämbt* \\ Institut für Neuro- und Verhaltensbiologie, Universität Münster, Münster, Germany
}

\section{Edited by:}

Norman Ruthven Saunders,

University of Melbourne, Australia

Reviewed by:

Alfredo Ghezzi, The University of

Texas at Austin, USA

Brigitte Dauwalder, University of

Houston, USA

Marko Brankatschk, Max Planck

Institute of Molecular Cell Biology

and Genetics, Germany

${ }^{*}$ Correspondence:

Christian Klämbt, Institut für Neuround Verhaltensbiologie, Universität

Münster, Badestr. 9,

48140 Münster, Germany

e-mail:klaembt@uni-muenster.de
The efficacy of neuronal function requires a well-balanced extracellular ion homeostasis and a steady supply with nutrients and metabolites. Therefore, all organisms equipped with a complex nervous system developed a so-called blood-brain barrier, protecting it from an uncontrolled entry of solutes, metabolites or pathogens. In higher vertebrates, this diffusion barrier is established by polarized endothelial cells that form extensive tight junctions, whereas in lower vertebrates and invertebrates the blood-brain barrier is exclusively formed by glial cells. Here, we review the development and function of the glial blood-brain barrier of Drosophila melanogaster. In the Drosophila nervous system, at least seven morphologically distinct glial cell classes can be distinguished. Two of these glial classes form the blood-brain barrier. Perineurial glial cells participate in nutrient uptake and establish a first diffusion barrier. The subperineurial glial (SPG) cells form septate junctions, which block paracellular diffusion and thus seal the nervous system from the hemolymph. We summarize the molecular basis of septate junction formation and address the different transport systems expressed by the blood-brain barrier forming glial cells.

\section{Keywords: Drosophila, glia, blood-brain barrier, septate junction formation, transmembrane transporter, astrocyte- neuron lactate shuttle hypothesis}

\section{INTRODUCTION}

In all animals, an efficient separation of metabolic and ionic balance between nervous system and circulation is necessary. This in consequence led to the evolution of the so-called blood-brain barrier (Abbott et al., 2006). Vertebrates are characterized by a highly vascularized nervous system, while the insect nervous system floats in the hemolymph, which circulates through the body by the action of a primitive heart (Figures 1A-C). In the mammalian nervous system, the blood-brain barrier is established by an interplay of polarized endothelial cells and pericytes that leads to the formation of endothelial tight junctions (Armulik et al., 2010, 2011; Daneman et al., 2010b). These tight junctions prevent uncontrolled paracellular leakage of solutes into the brain. In more primitive vertebrates such as in elasmobranch fish (sharks, skates, and rays), but also in some bony fish (sturgeon), the blood-brain barrier is formed by perivascular astrocytes. These glial cells form interdigitating lamellae but do not establish tight junctions (Bundgaard and Abbott, 2008). A morphologically similar blood-brain barrier is found in insects. Here, only the outer surface of the nervous system, which is formed exclusively by glial cells, contacts the hemolymph. Although this glial barrier appears to be related to the evolutionary ancestral form of the blood-brain barrier, Drosophila has only recently emerged as a genetic model to study blood-brain barrier biology (Carlson et al., 2000; Abbott et al., 2006). Here, we summarize what is currently known on the

Abbreviations: ABC, ATP binding cassette; ATP, Adenosine triphosphate; FACS, fluorescence-activated cell sorting; GCM, Glial Cells Missing; GFP, green flourescent protein; GPCR, G protein-coupled receptor; IPC, Insulin-Producing Cell; RNA, Ribonucleic acid; SPG, subperineurial glia; TCA cycle, tricarboxylic acid cycle; UDP, Uridine diphosphate. organization and the physiological properties of the Drosophila blood-brain barrier.

Why is Drosophila a good system to study blood-brain barrier properties? One of the advantages is that the nervous system is small and almost all cells are known. In addition, geneticists established rich resource and tool kits, which allow the easy manipulation of individual cells at any time of development (Dietzl et al., 2007; Venken et al., 2011; Jenett et al., 2012; Li et al., 2014). The basis of neuronal transmission is identical in flies and man and, moreover, even complex behavioral aspects appear to be controlled by similar mechanisms (Davis, 2011; Anholt and Mackay, 2012). Thus, based on the overwhelming wealth of data documenting the evolutionary conservation of central biological processes, one can expect that work on the Drosophila blood-brain barrier, which correctly should be termed hemolymph-brain barrier, might provide further insights into the general biology of this essential boundary.

\section{THE NERVOUS SYSTEM OF DROSOPHILA}

Drosophila is a holometabolic insect. Following 1 day of embryogenesis, three larval stages spread over the next 4 days. During the subsequent pupal stage, which covers another 5 days, metamorphosis takes place and the imago, the adult fly, emerges. Accordingly, the nervous system of Drosophila develops in two phases.

The larval nervous system originates from stem cells called neuroblasts that delaminate in five waves shortly after gastrulation into the interior of the embryo (Campos-Ortega and Hartenstein, 1997). Analysis of the nervous system is simplified by the fact that the thoracic and abdominal segments are mostly alike and 


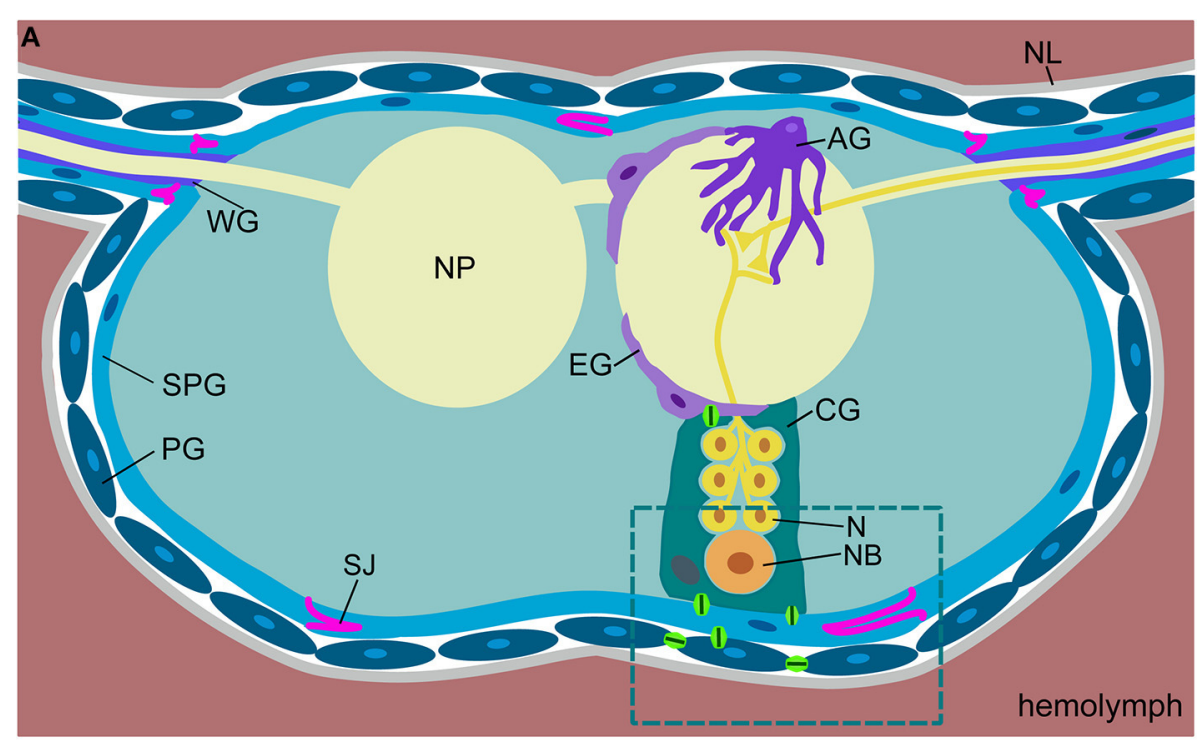

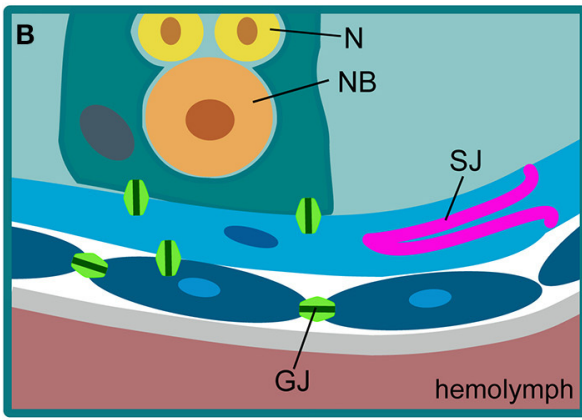

FIGURE 1 | Comparison of Drosophila and mammalian blood-brain barriers. (A) Schematic view of a cross-section of a Drosophila ventral nerve cord. The nervous system is covered by a sheath of extracellular matrix, called neural lamella (NL). The outermost glial layer consists of perineurial glial cells (PG). The subperineurial glia (SPG) forms pleated septate junctions (SJ) and blocks paracellular transport. Neurons (N) project into the neuropil (NP). Neuronal cell bodies and neuroblasts (NB) are surrounded by cortex glia (CG). The neuropil is covered by ensheathing glia (EG). Astrocytes (AG) invade the neuropil. In the peripheral nerves, wrapping glia (WG) ensheath axons.

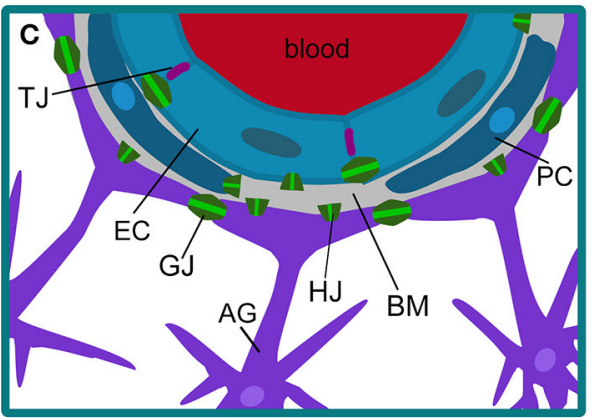

(B) In Drosophila, the blood-brain barrier is built by perineurial and subperineurial glia. The latter form septate junctions (SJ) to prevent paracellular diffusion. The different glial cells are connected via gap junctions (GJ). (C) The mammalian blood-brain barrier is built by endothelial cells (EC) that form tight junctions (TJ) to prevent paracellular diffusion. The endothelium is in close contact with pericytes (PC). Both are surrounded by the basal membrane (BM). Gap junctions (GJ) can be found between the endothelial cells and between the astrocytes (AG). Gap junction hemichannels $(\mathrm{HJ})$ can be found in all the cell types. thus, a large part of the nervous system called ventral nerve cord represents an array of repeated and almost identical neuromeric units. Currently, the identity and the lineage of all neuroblasts are known and by the end of embryogenesis about 650 neurons and 65 glial cells are found in each neuromer of the ventral nerve cord (Broadus et al., 1995; Landgraf et al., 1997; Schmid et al., 1999; Wheeler et al., 2006; Beckervordersandforth et al., 2008; Rickert et al., 2011). The brain lobes of the Drosophila larvae originate from somewhat less well-defined head neuroblasts (Urbach and Technau, 2003). The neuronal circuits established by the many neurons in the larval brain are currently being deciphered by analyzing serial TEM sections of a larval brain (Cardona et al., 2010). Thus, it can be anticipated that within the next years the complete anatomical building plan of the larval nervous system is known.

The adult nervous system, including the elaborate compound eyes, develops during early pupal stages. Neuroblast proliferation is reactivated at the end of the larval stage to generate a large number of neurons, particularly in the two brain lobes. When fully developed, the fly central nervous system harbors about 30,000 neurons (Lovick et al., 2013). Upon GFP labeling of neurons or glial cells and subsequent FACS sorting we counted about 25,000 neuronal and 10,000 glial cells per adult brain (Limmer et al., unpublished).

\section{DROSOPHILA GLIAL CELLS}

As in primitive vertebrates, the Drosophila blood-brain barrier is formed by glial cells (Stork et al., 2008). The fly nervous system harbors seven morphologically and molecularly distinct glial subtypes, namely midline glia, perineurial glia, subperineurial glia (SPG), cortex glia, ensheathing glia, astrocytes, and wrapping glia (Figures 1A,B) (Ito et al., 1995; Pereanu et al., 2005; Silies et al., 2007; Awasaki et al., 2008; Stork et al., 2008, 2012, 2014; Hartenstein, 2011). The entire nervous system is covered 
by a layer of perineurial glial cells. These cells participate in blood-brain barrier function although their exact contribution is currently unknown. The subjacent glial cell layer is represented by the SPG cells. These flat and interdigitating cells form elaborate septate junctions, which prevent paracellular diffusion (Bainton et al., 2005; Stork et al., 2008; Mayer et al., 2009). The cortex glial cells engulf neuronal stem cells and their progeny and most likely exert some nutritional functions. The ensheathing glial cells form a sheath around the neuropil area, which lacks cell bodies and harbors only axons and dendrites. Astrocytes invade the neuropil to modulate synaptic transmission as it is known from their vertebrate homologs (Awasaki et al., 2008; Stork et al., 2014). The wrapping glia is mostly found in the peripheral nervous system where these cells engulf individual axons (Pereanu et al., 2005; Awasaki et al., 2008; Franzdóttir et al., 2009).

All glial subtypes are generated in the Drosophila embryo and emerge from stem cells programmed by the expression of the gene glial cells missing $(\mathrm{gcm})$ (Hosoya et al., 1995; Jones et al., 1995; Vincent et al., 1996). The presence of the $\mathrm{Gcm}$ transcription factor specifies glial identity except for the midline glia, which requires the activity of the master regulator gene single minded (Crews et al., 1988). Gcm subsequently activates a cascade of transcription factors. The transcription factor Pointed promotes glial differentiation and the Zn-finger protein Tramtrack inhibits neuronal differentiation in glial cells (Klaes et al., 1994; Giesen et al., 1997). Together with other transcriptional regulators such as Prospero, Distal-less and Deadringer, these factors most likely are involved in specifying the glial subtype identity (Shandala et al., 2003; Thomas and van Meyel, 2007; Schmidt et al., 2011). The relevant transcriptional regulators that specify blood-brain barrier identity are currently unknown.

\section{THE DROSOPHILA BLOOD-BRAIN BARRIER}

The establishment of the Drosophila blood-brain barrier occurs at the end of embryogenesis and requires the SPG cells. Only 16 of these cells are formed in every neuromere and four additional SPG cells are generated along every segmental nerve (Beckervordersandforth et al., 2008; von Hilchen et al., 2013). During larval stages, when the animal grows in size by a factor of 100 , and likewise during metamorphosis, no additional SPG cells are formed but the blood-brain barrier remains intact (Awasaki et al., 2008; Stork et al., 2008; Unhavaithaya and Orr-Weaver, 2012). Thus, the few SPG cells generated during embryonic stages must grow enormously in size during development, and at the same time they have to maintain their elaborate junctional contacts that prevent paracellular diffusion (see below).

Once the SPG cells are born at about mid-embryogenesis, they form numerous filopodia-like processes and eventually spread to touch their neighbors at the end of embryogenesis (Schwabe et al., 2005). The SPG cells now establish a contiguous, very flat, endothelial-like sheet that covers the entire nervous system and the cell contact zones interdigitate extensively. Interestingly, in cuttlefish as well as in sturgeon, the glial blood-brain barrier also involves highly overlapping glial lamellae (Lane and Abbott, 1992; Bundgaard and Abbott, 2008). In Drosophila, glial cells of the blood-brain barrier in addition form extensive septate junctions that further restrict the paracellular diffusion between different glial cells (Carlson et al., 2000; Schwabe et al., 2005; Stork et al., 2008). The first experimental confirmation of the physiological relevance of septate junctions for blood-brain barrier integrity was provided by the genetic analysis of the septate junction component NeurexinIV. In the absence of this protein, which is homologous to the Caspr protein found in septate-like junctions in vertebrate paranodes, the blood-brain barrier is permissive to even large molecules like dextran and in consequence, the high potassium content of the hemolymph can spread into the nervous system where it blocks any neuronal activity (Baumgartner et al., 1996).

The SPG cells are very large: a single SPG can cover the size of one half of the eye imaginal disc thus covering an area equivalent to about 10,000 epithelial cells (Silies et al., 2007). In order to achieve this enormous cell growth, the SPG cells undergo polyploidization (Unhavaithaya and Orr-Weaver, 2012). All SPG cells form a polarized endothelium that in most areas does not even reach a thickness of $1 \mu \mathrm{m}$. The thin nature of these barrierforming cells has hindered detailed electron microscopic studies for a long time. Only the ability to label individual cells with GFP using subperineurial specific Gal4 driver strains such as gliotactinGal4 or moodyGal4 has allowed the description of the intricate morphology of these cells (Schwabe et al., 2005; Silies et al., 2007; Stork et al., 2008; Hatan et al., 2011; Unhavaithaya and OrrWeaver, 2012). Currently, only few molecular markers for the blood-brain barrier are available. The multidrug resistance protein Mdr65 was shown to reside in the apical domain of the SPG cell, whereas the GPCR Moody is found at the basal portion of the cell (DeSalvo et al., 2011).

\section{FORMATION OF SEPTATE JUNCTIONS}

The most characteristic feature of the SPG is the formation of extensive septate junctions. This type of cell-cell junction has been particularly well studied in ectodermal cells, such as tracheal cells (Tepass and Hartenstein, 1994). Septate junctions are a complex crystalline array of comb-like structures built by a bewildering number of different proteins that connect individual cells, as revealed by freeze-fracture studies (Figure 2) (Lane and Swales, 1979; Lane, 1991). The cell-cell distance in these junctions is about $20 \mathrm{~nm}$ and thus a bit larger than in tight junctions that seal the brain endothelial cells in mammals (Farquhar and Palade, 1963, 1965). Many membrane-associated proteins are known to be involved in septate junction formation (Table 1). The core group of septate junction proteins contains the cation pump ATPalpha, the claudin family members Megatrachea and Sinous, the Ig-domain protein Neuroglian, the potassium pump subunit Nervana2, the Caspr homolog NeurexinIV and the two cytoplasmic proteins Coracle and Varicose (Oshima and Fehon, 2011, see also references in Table 1). These proteins recruit a large number of additional membrane proteins that together build the septate junctions (Table 1). Loss of most of these proteins results in the disruption of septate junctions.

Septate junction structure becomes even more elaborate at tricellular junctions. Here, septate junction strands of three cells meet and appear to be linked to a central core in the extracellular space between three neighboring cells (Figure 2). The tricellular junctions are among the first junctional complexes 

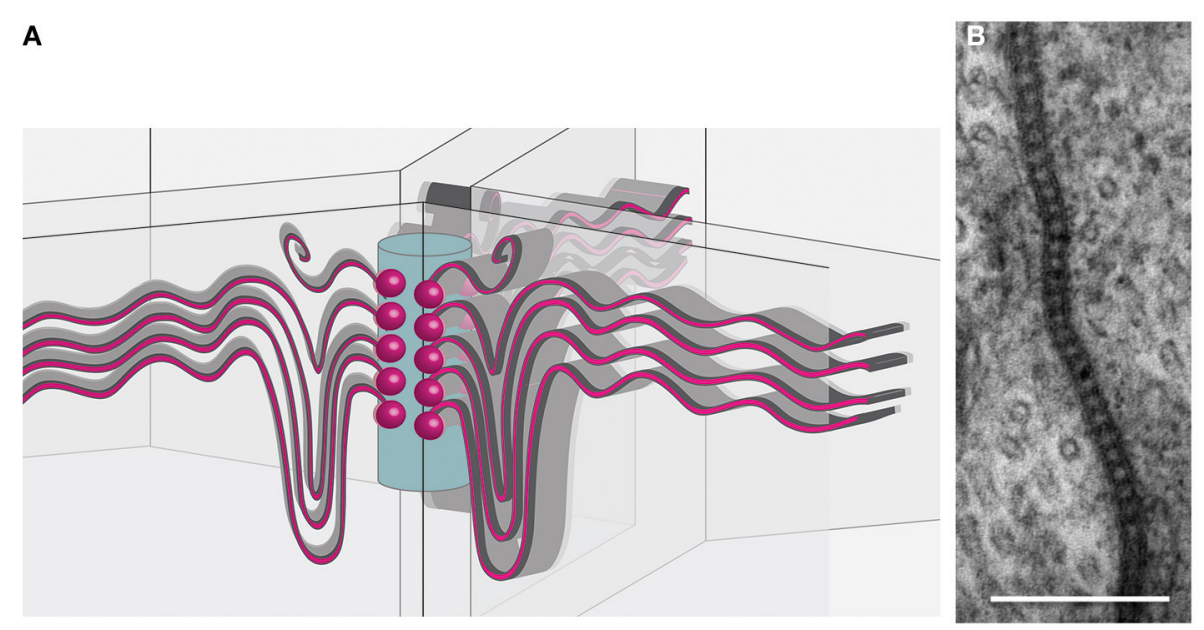

FIGURE 2 | Organization of tricellular junctions. (A) Schematic view of septate junctions at tricellular contacts according to the tricellular plug model (Graf et al., 1982; Noirot-Timothée et al., 1982; Schulte et al. 2003). Septate junctions (red sinuous lines) span the membranes of two adjacent cells. At a central core (blue cylinder), emanating from transmembrane proteins (red balls), the septate junctions extend. (B) Transmission electron microscopic image of pleated septate junctions between two SPG cells. to differentiate and are characterized by several specific proteins such as Gliotactin or Macroglobin (Auld et al., 1995; Genova and Fehon, 2003; Schulte et al., 2003; Padash-Barmchi et al., 2010; Bätz et al., 2014; Furuse et al., 2014; Oda et al., 2014). Possibly septate junction formation initiates from these positions to match the stretch growth of the SPG cells during larval development (Figure 2). The extent of septate junction formation is in part controlled by the $\mathrm{G}$ protein-coupled receptor Moody (Bainton et al., 2005; Schwabe et al., 2005). The Moody protein is continuously required for septate junction formation and a temporal lack of moody function, evoked by conditional RNA interference, results in a transient opening of the blood-brain barrier (Bainton et al., 2005). It is important to note that Moody, like possibly other developmentally required proteins, may affect both, development and the physiology of the blood-brain barrier. Indeed some effects of Moody on barrier function, such as increased cocaine sensitivity, can be seen under experimental conditions (Bainton et al., 2005). However, in the normal life of a fly no serious defects are observed when moody function is lacking. It was noted by Schwabe et al. (2005) that moody mutations are lethal, but it was also mentioned that few homozygous females and hemizygous males survive to adulthood. Interestingly, homozygous moody flies can be easily kept as a living stock, suggesting that bloodbrain barrier physiology can be efficiently regulated by additional pathways.

How Moody signaling regulates septate junction dynamics remains unclear. moody null mutants show normal septate junction morphology but a somewhat reduced junctional length (Schwabe et al., 2005). In addition, Moody was shown to affect the formation of actin-rich structures along the lateral borders of the SPG cells, which also contributes to the effects on blood-brain barrier integrity (Hatan et al., 2011).

In conclusion, SPG cells are intimately interconnected by septate junctions. They set up a tight seal around the nervous system, such that metabolite import into the brain, and the exit of waste products and xenobiotics out of the nervous system can be tightly controlled.

\section{TRANSPORT OF METABOLITES ACROSS THE BLOOD-BRAIN BARRIER}

A classical function of the blood-brain barrier is to control transport of ions and metabolites. Once the septate junctions are formed between individual SPG cells, paracellular diffusion is blocked. This, in consequence, calls for efficient and highly active transport mechanisms that shuttle all required metabolites into the brain. The relevant transporters are mostly defined by bioinformatic criteria and their expression has been globally addressed by tissue specific transcriptomics and is summarized in FlyAtlas (Chintapalli et al., 2013).

Metabolite transport across the SPG cell layer not only requires an efficient uptake mechanism into the SPG cells, but also an efficient secretion of metabolites into the nervous system. This aspect may not be confined to the blood-brain barrier, since glial cells in general and in particular SPG cells establish extensive gap junctions, which is similarly observed in endothelial cells (Figure 1) (Lane and Swales, 1979; Lane, 1991; Holcroft et al., 2013; Gaete et al., 2014). Thus, once metabolites have entered the blood-brain barrier forming cells, they might be easily distributed throughout the whole nervous system via these intercellular connections. Gap junctions are a hallmark of invertebrate glia and most glial cells express several Drosophila innexins, which constitute the functional connexin homologs of invertebrates (Holcroft et al., 2013). The direct coupling of glial cells by gap junctions is also reflected by glial $\mathrm{Ca}^{2+}$ waves, which can be seen in the blood-brain barrier, the cortex and the astrocyte glial population (Melom and Littleton, 2013; Spéder and Brand, 2014; Stork et al., 2014).

In addition to the transport of metabolites into the brain, waste products and xenobiotics need to be shuttled out of the brain. In vertebrates, chemoprotection is mediated by ATP-binding 
Table 1 | Septate junction proteins.

\begin{tabular}{|c|c|c|c|c|}
\hline Gene & Vertebrate homolog & Structural domains & Function & References \\
\hline boudin & & Ly-6, GPI & Secreted, non-autonomous & Hijazi et al., 2009 \\
\hline crimpled & & Ly-6, GPI & & Nilton et al., 2010 \\
\hline crooked & & Ly-6, GPI & & Nilton et al., 2010 \\
\hline Fasciclin III & & $\lg$ & Cell-adhesion & Narasimha et al., 2008 \\
\hline Lachesin & & $\lg , \mathrm{GPI}$ & Cell-adhesion & Llimargas et al., 2004 \\
\hline Neuroglian & Neurofascin 155 & $\lg$, Fnlll & Cell-adhesion & Genova and Fehon, 2003 \\
\hline Neurexin IV & Caspr/Paranodin & $\begin{array}{l}\text { Laminin } \mathrm{G} \text { and } \mathrm{EGF} \\
\text { domains }\end{array}$ & Cell-adhesion & Baumgartner et al., 1996 \\
\hline coracle & Protein 4.1 & FERM & Linker protein & Fehon et al., 1994 \\
\hline kune-kune & Claudin & PDZ-domain & Cell-adhesion & Nelson et al., 2010 \\
\hline scribble & hscrib1 & PDZ-Domain, LRR & Linker protein & $\begin{array}{l}\text { Bilder and Perrimon, } \\
2000\end{array}$ \\
\hline Gliotactin & Neuroligin3 & $\begin{array}{l}\text { Noncatalytic } \\
\text { cholinesterase like } \\
\text { molecule }\end{array}$ & Cell-adhesion & Auld et al., 1995 \\
\hline $\begin{array}{l}\text { Macroglobulin } \\
\text { complement-related }\end{array}$ & $\alpha 2 \mathrm{M}$ & $\begin{array}{l}\text { Thioester protein (TEP) } \\
\text { family }\end{array}$ & & $\begin{array}{l}\text { Bätz et al., 2014; Hall } \\
\text { et al., } 2014\end{array}$ \\
\hline Melanotransferrin & MTF & Iron binding, GPI & Iron-binding, endocytosis & Tiklová et al., 2010 \\
\hline varicose & Pals2 & MAGUK & Linker protein & Wu et al., 2007 \\
\hline wunen & & $\begin{array}{l}\text { Lipid phosphat } \\
\text { phosphatase }\end{array}$ & & Ile et al., 2012 \\
\hline yurt & & & & Laprise et al., 2009 \\
\hline
\end{tabular}

The known Drosophila septate junction proteins are listed. Structural domains and the predicted molecular functions are indicated. Ly-6, lymphocyte antigen-6; GPI, glycosyl phosphatidylinositol; Ig, immunoglobuline; FnIII, fibronectin type III; EGF, epidermal growth factor; MAGUK, membrane-associated guanylate kinases; SH3, $S R C$ homology 3; $L R R$, leucine-rich repeat. 
cassette $(\mathrm{ABC})$ transporters such as the Multidrug-resistance protein 1 (Mdr1). Quite similar, $m d r 65$ performs related functions in the Drosophila nervous system (Mayer et al., 2009). In line with this, Mdr65 levels are elevated upon exposure to insecticides (Dermauw and Van Leeuwen, 2014). Mdr65 belongs to a large class of transporters with 56 members in Drosophila of which several are expressed in the nervous system (Dermauw and Van Leeuwen, 2014). Their function, however, is largely unknown and will not be further discussed here.

Neural cells need to take up specific lipids from the hemolymph, which feeds into specific anabolic pathways (Palm et al., 2012) or is directly used as energy source during Boxidation (Palanker et al., 2009). In the following we will focus on metabolite import across the blood-brain barrier and review the main classes of the relevant transport systems. Due to space constrains, however, we will neglect lipid metabolism. We anticipate that the wealth of tools available in Drosophila will promote a deeper understanding of the physiological roles of this important barrier.

\section{WATER HOMEOSTASIS}

Mechanisms for controlling influx and efflux of water are essential for osmotic control of fluids in the nervous system. Water can enter the nervous system through aquaporins, small membranespanning proteins that form channels to facilitate water flow along the osmotic gradient. 14 aquaporins are known in humans and in particular aquaporin-4 (AQP4) has been linked to bloodbrain barrier function. AQP4 localizes to astrocyte endfeet at the CSF-CNS and blood-CNS barriers and is crucially required for the regulation of water homeostasis and the definition of the extracellular space in the CNS (Nagelhus and Ottersen, 2013; Papadopoulos and Verkman, 2013).

The Drosophila genome harbors eight aquaporin-encoding genes (FlyBase, CV: water transmembrane transporter activity, Table 2). The DRIP protein has the highest sequence similarity to vertebrate AQP4 but shows no prominent CNS expression (Chintapalli et al., 2013). big brain (bib) was initially identified as a neurogenic gene and encodes a protein related to aquaporins (Rao et al., 1990). Although Bib has water transport properties, this function is still controversial (Tatsumi et al., 2009). The Drosophila aquaporin most prominently expressed in the CNS is CG7777 (Chintapalli et al., 2013), but currently no functional analysis has been performed.

\section{ION HOMEOSTASIS}

The hemolymph is an ion rich fluid (Table 3). In particular the high potassium concentration, which is characteristic for the invertebrate hemolymph, would be problematic for normal neuronal function. In the mammalian blood, potassium levels are only around $1-5 \mathrm{mM}$ and thus almost a factor of 10 lower compared to the Drosophila hemolymph. The blood-brain barrier prevents any uncontrolled influx of ions into the nervous system. In consequence, the potassium concentration in the Drosophila brain fluid is about $5 \mathrm{mM}$ or less (Armstrong et al., 2012).

The Drosophila genome harbors a large number of proteins linked to potassium influx or export (Chintapalli et al., 2013). However, only in few cases, their relevance for regulated ion transport across the blood-brain barrier has been demonstrated. One example is the Fray kinase and its target Ncc69, the homolog of the human SLC12 Na $/ \mathrm{K}^{+} / \mathrm{Cl}^{-}$cotransporter (Leiserson et al., 2000, 2011). Ncc69 is expressed in SPG cells and Ncc69 mutant larvae develop a peripheral neuropathy with fluid accumulations between glia and axons (Leiserson et al., 2011). The effect of Ncc69 on CNS physiology is not reported. Several other ion transport proteins of the SLC12 family are also expressed in the Drosophila brain (Sun et al., 2010) but their possible glial functions are unexplored.

In a genetic screen for temperature-sensitive conditional seizure mutants the glial-specific $\mathrm{Na}^{+} / \mathrm{Ca}^{2+}, \mathrm{K}^{+}$exchanger Zydeco was identified. Zydeco is mostly expressed by the cortex glia and not by the blood-brain barrier glia (Guan et al., 2005; Melom and Littleton, 2013). In addition to channels, active ion pumps such as the $\mathrm{Na}^{+} / \mathrm{K}^{+}$ATPase are involved in regulating ion homeostasis in the brain. The two Drosophila nervana genes ( $n r v 1$ and $n r v 2$ ) encode beta subunits of the $\mathrm{Na}^{+} / \mathrm{K}^{+}$ATPase. Nrv2 and the ATPase alpha are expressed in the SPG, where they are localized to septate junctions. Hypomorphic mutants for the ATPase alpha subunit show a bang and ouabain sensitivity (Schubiger et al., 1994). Septate junction formation, however, does not depend on ATPase function (Genova and Fehon, 2003; Paul et al., 2007).

\section{UPTAKE OF AMINO ACIDS INTO THE NERVOUS SYSTEM}

Amino acids are not only required for protein synthesis, but particular amino acids or their direct derivatives are also used as neurotransmitters. Cells are able to generate most amino acids from intermediates of the citric acid cycle. However, some amino acids cannot be produced and thus their uptake and transport is essential. In Drosophila, 10 proteinogenic L-amino acids are essential: tryptophan, phenylalanine, leucine, histidine, valine, isoleucine, lysine, methionine, arginine, and threonine (Boudko, 2012). Therefore, specific transport systems for these amino acids must be expressed by the SPG cells.

In vertebrates, nine distinct amino acid transport systems have been reported to be present at the brain capillary endothelium (Smith, 2000): the X-system is a high affinity, sodium-independent transport system for anionic amino acids, the L-system transports large neutral amino acids, the A-and ASC-systems transport small neutral amino acids, the $\mathrm{Y}^{+}$-system is a sodium-independent cationic amino acid transport system, the $\mathrm{B}^{\mathrm{o}+}$-system represents a high affinity transport system and transports both neutral and basic amino acids and the $B$-system is a low capacity, sodium-dependent transporter for taurine and $B$ alanine. The $\mathrm{N}$-system mediates the sodium-dependent transport of L-glutamine, L-histidine and L-asparagine and the T-system transports thyroid hormones.

Two well-known X-system transporters are EAAT1 and EAAT2, which in mammals are expressed by astrocytes (Rothstein et al., 1994). In Drosophila, both proteins have been described, but are expressed in glial cells other than the blood-brain barrier cells (Soustelle et al., 2002; Freeman et al., 2003; Stacey et al., 2010). In total, 51 Drosophila genes are annotated to have an "organic acid transmembrane transporter activity" or as "amino acid transporter" (Table 4). For a few of these proteins 
Table 2 | Drosophila Aquaporins.

\begin{tabular}{|c|c|c|c|c|c|c|}
\hline \multirow[t]{2}{*}{ Gene } & \multirow[t]{2}{*}{ CG number } & \multirow[t]{2}{*}{ Human homolog } & \multicolumn{3}{|c|}{ CNS expression } & \multirow[t]{2}{*}{ References } \\
\hline & & & FlyAtlas & BDGP & Literature & \\
\hline big brain & CG4722 & AQP4 & Yes & Yes & Yes & Rao et al., 1990 \\
\hline aquaporin & CG12251 & AQP12 & No & No & - & \\
\hline CG7777 & CG7777 & AQP1 & Yes & No & Yes & Kaufmann et al., 2005 \\
\hline CG4019 & CG4019 & MIWC, AQP4 & Low & No & No & Kaufmann et al., 2005 \\
\hline CG5398 & CG5398 & AQP2 & No & - & No & Kaufmann et al., 2005 \\
\hline
\end{tabular}

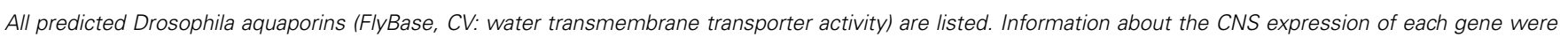
obtained from FlyAtlas, the Berkely Drosophila Genome Project (BDGP), and the literature shown. AQP, aquaporin; MIWC, mercurial-insensitive water channel.

Table 3 | lon concentration in the hemolymph.

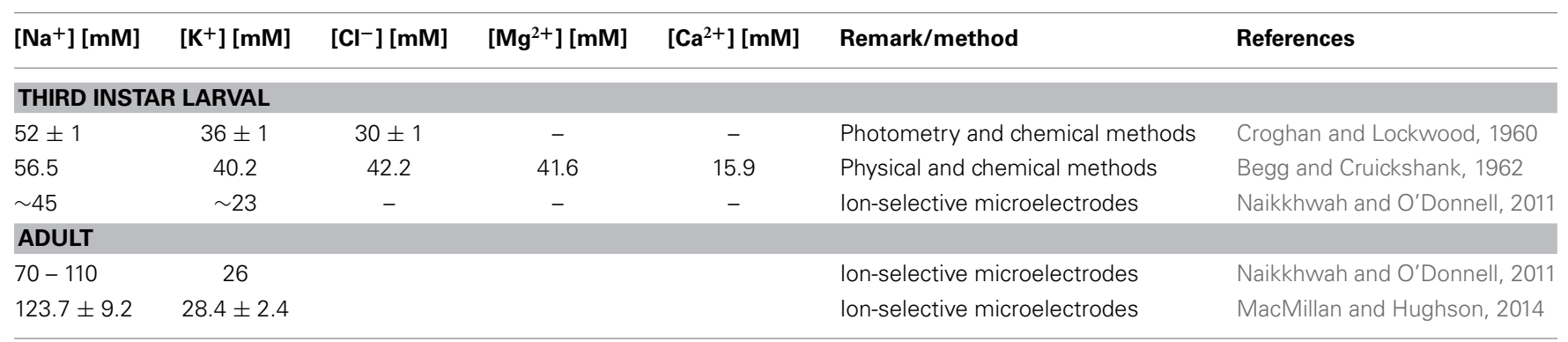

Drosophila third instar larval and adult hemolymph concentrations of selected ions are listed. The method of concentration determination is indicated.

expression in CNS glial cells has been noted (Soustelle et al., 2002; Besson et al., 2005; Augustin et al., 2007; Grosjean et al., 2008; Featherstone, 2011). In some cases, expression in the bloodbrain barrier was found (e.g., CG15088, Thimgan et al., 2006) but functional analysis is lacking so far.

\section{ENERGY SUPPLY OF THE BRAIN}

The energy expenditure for normal neuronal function is enormous. The human brain requires about $20 \%$ of the total resting oxygen consumption of the entire body although it comprises only about $2 \%$ of the body mass. Likewise in flies, just the photoreceptor cells in the retina consume about $10 \%$ of the total ATP production (Laughlin et al., 1998). Therefore, an efficient transport of energy-rich nutrients such as sugars needs to be established at the blood-brain barrier.

\section{THE ASTROCYTE-NEURON LACTATE SHUTTLE HYPOTHESIS}

The intense metabolic interactions between glial cells and neurons led to the astrocyte-neuron lactate shuttle (ANLS) hypothesis (Pellerin and Magistretti, 1994, 2012; Allaman et al., 2011). In the mammalian brain, the main energy source is glucose, which is shuttled into the nervous system via the Glutl transporter. Glut1 is asymmetrically expressed in endothelial cells and is also found in astrocytes surrounding the endothelium (Leybaert, 2005). Glucose that is taken up by astrocytes is then metabolized through glycolysis to lactate or pyruvate. These small $\mathrm{C}_{3}$ metabolites are then released into the extracellular space to be utilized by neurons, which is supported by experimental and as well as theoretical considerations (Rouach et al., 2008; Jolivet et al., 2009; Harris et al., 2012). Enhanced neuronal activity might be linked to an increase in Glut1 expression, which would account for increased energy supply (Leybaert, 2005). This energetic coupling may be of more general relevance, since also the survival of myelinated axons depends on metabolic support by the corresponding glial cells (Fünfschilling et al., 2012; Lee et al., 2012). In invertebrates, a similar compartmentalization of energy metabolism is likely to be established as well. In the honeybee retina, glucose is exclusively taken up by glial cells and alanine, which is generated from pyruvate through transamination, seems to be shuttled to neurons to fuel the TCA cycle (Tsacopoulos et al., 1994).

\section{TREHALOSE TRANSPORTERS IN THE DROSOPHILA BLOOD-BRAIN BARRIER}

Several different sugars are present in the Drosophila hemolymph (Table 5). The main carbohydrate found in the insect hemolymph is trehalose, a non-reducing disaccharide with two D-glucose units linked by an $\alpha, \alpha-1,1$-glycosidic bond. Smaller amounts of glucose and fructose are also found, but their respective concentrations appear to vary depending on the metabolic state of the animal (Blatt and Roces, 2001). Upon feeding, carbohydrates are taken up by the intestinal epithelium and glucose is secreted into the hemolymph. The glucose is then shuttled to the fat body, where trehalose is synthesized from glucose-6-phosphate and UDP-glucose by the enzyme Trehalose phosphate synthase, 
Table 4 | Drosophila amino acid transporters.

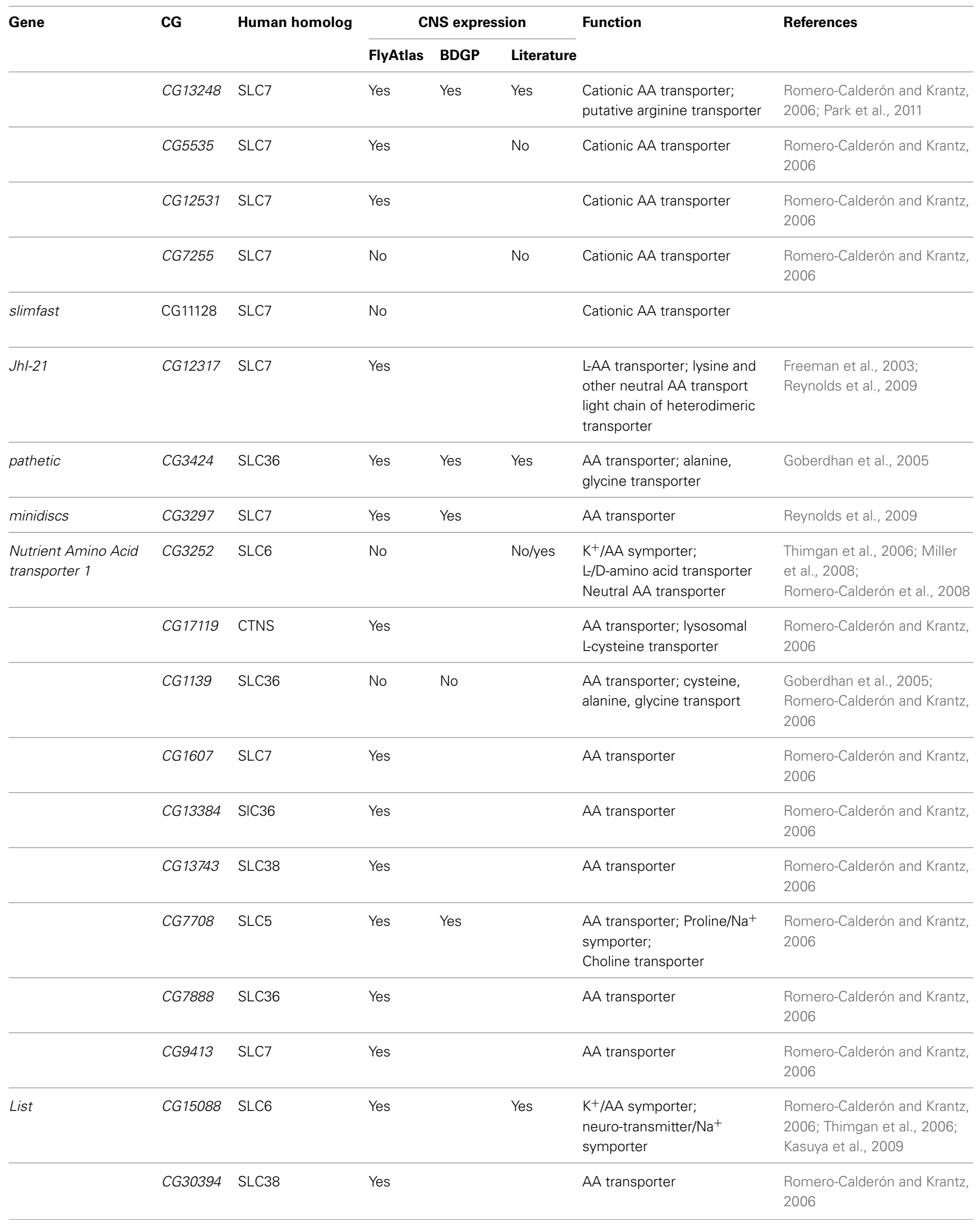


Table 4 | Continued

\begin{tabular}{|c|c|c|c|c|c|c|c|}
\hline \multirow[t]{2}{*}{ Gene } & \multirow[t]{2}{*}{ CG } & \multirow[t]{2}{*}{ Human homolog } & \multicolumn{3}{|c|}{ CNS expression } & \multirow[t]{2}{*}{ Function } & \multirow[t]{2}{*}{ References } \\
\hline & & & FlyAtlas & BDGP & Literature & & \\
\hline \multirow[t]{8}{*}{$\operatorname{tadr}$} & CG9264 & SLC7 & Yes & & & AA transporter & \\
\hline & CG4991 & SLC36 & Yes & & & AA transporter & $\begin{array}{l}\text { Romero-Calderón and Krantz, } \\
2006\end{array}$ \\
\hline & CG1628 & SLC25 & Yes & & & $\begin{array}{l}\text { AA transporter; L-ornithine } \\
\text { transporter }\end{array}$ & $\begin{array}{l}\text { Romero-Calderón and Krantz, } \\
2006\end{array}$ \\
\hline & CG8785 & SLC36 & No & & & AA transporter & $\begin{array}{l}\text { Romero-Calderón and Krantz, } \\
2006\end{array}$ \\
\hline & CG12943 & SLC36 & No & & & AA transporter & $\begin{array}{l}\text { Romero-Calderón and Krantz, } \\
2006\end{array}$ \\
\hline & CG13646 & no & No & & & AA transporter & $\begin{array}{l}\text { Romero-Calderón and Krantz, } \\
2006\end{array}$ \\
\hline & CG32079 & SLC36 & No & & No & AA transporter & $\begin{array}{l}\text { Romero-Calderón and Krantz, } \\
2006\end{array}$ \\
\hline & CG32081 & SLC36 & No & & & AA transporter & \\
\hline hoepel1 & CG12787 & OCA2 & Yes & & & L-tyrosine transporter & \\
\hline hoepel2 & CG15624 & OCA2 & Yes & & & L-tyrosine transporter & \\
\hline \multirow[t]{2}{*}{ kazachoc } & CG5594 & SLC12 & Yes & Yes & Yes & $\begin{array}{l}\mathrm{K}^{+} / \mathrm{Cl}^{-} \text {symporter; } \\
\mathrm{AA} \text { transporter }\end{array}$ & $\begin{array}{l}\text { Hekmat-Scafe et al., 2006; } \\
\text { Sun et al., } 2010 \\
\text { Filippov et al., } 2003\end{array}$ \\
\hline & CG31547 & SLC12 & Yes & & Yes & $\begin{array}{l}\text { AA transporter; } \mathrm{Na}^{+} / \mathrm{K}^{+} / \mathrm{Cl}^{-} \\
\text {symporter }\end{array}$ & $\begin{array}{l}\text { Filippov et al., 2003; } \\
\text { Romero-Calderón and Krantz, } \\
\text { 2006; Sun et al., } 2010\end{array}$ \\
\hline \multirow[t]{3}{*}{ Ncc69 } & CG4357 & SLC12 & Yes & & No/yes & $\begin{array}{l}\mathrm{Na}^{+} / \mathrm{K}^{+} / \mathrm{Cl}^{-} \text {cotransporter; } \\
\text { AA transporter }\end{array}$ & $\begin{array}{l}\text { Filippov et al., 2003; } \\
\text { Romero-Calderón and Krantz, } \\
\text { 2006; Sun et al., 2010; } \\
\text { Leiserson et al., } 2011\end{array}$ \\
\hline & CG12773 & SLC12 & Yes & & No/yes & $\begin{array}{l}\text { AA transporter; } \mathrm{Na}^{+} / \mathrm{K}^{+} / \mathrm{Cl}^{-} \\
\text {symporter }\end{array}$ & $\begin{array}{l}\text { Filippov et al., 2003; } \\
\text { Romero-Calderón and Krantz, } \\
\text { 2006; Sun et al., } 2010\end{array}$ \\
\hline & CG1698 & SLC6 & No & & No & $\mathrm{K}^{+} / \mathrm{Cl}^{-}$symporter & $\begin{array}{l}\text { Romero-Calderón and Krantz, } \\
\text { 2006; Thimgan et al., } 2006\end{array}$ \\
\hline karmoisin & CG12286 & SLC16 & Yes & & & $\begin{array}{l}\text { Monocarboxylic acid } \\
\text { transporter }\end{array}$ & \\
\hline Silnoon & CG8271 & SLC16 & Yes & & & $\begin{array}{l}\text { Secondary active } \\
\text { monocarboxylate transporter; } \\
\text { Butyrate, lactate transport }\end{array}$ & Jang et al., 2008 \\
\hline outsiders & CG8062 & SLC16 & No & & & $\begin{array}{l}\text { Monocarboxylic acid } \\
\text { transporter }\end{array}$ & \\
\hline $\begin{array}{l}\text { Dietary and metabolic } \\
\text { glutamate transporter }\end{array}$ & CG5304 & SLC17 & Yes & & No & $\begin{array}{l}\text { High affinity inorganic } \\
\text { phosphate/Na+ symporter; } \\
\text { Glutamate transporter; } \\
\mathrm{Na}^{+} \text {-independent glutamate } \\
\text { transporter }\end{array}$ & $\begin{array}{l}\text { Laridon et al., 2008; Shim } \\
\text { et al., } 2011\end{array}$ \\
\hline
\end{tabular}




\section{Table 4 | Continued}

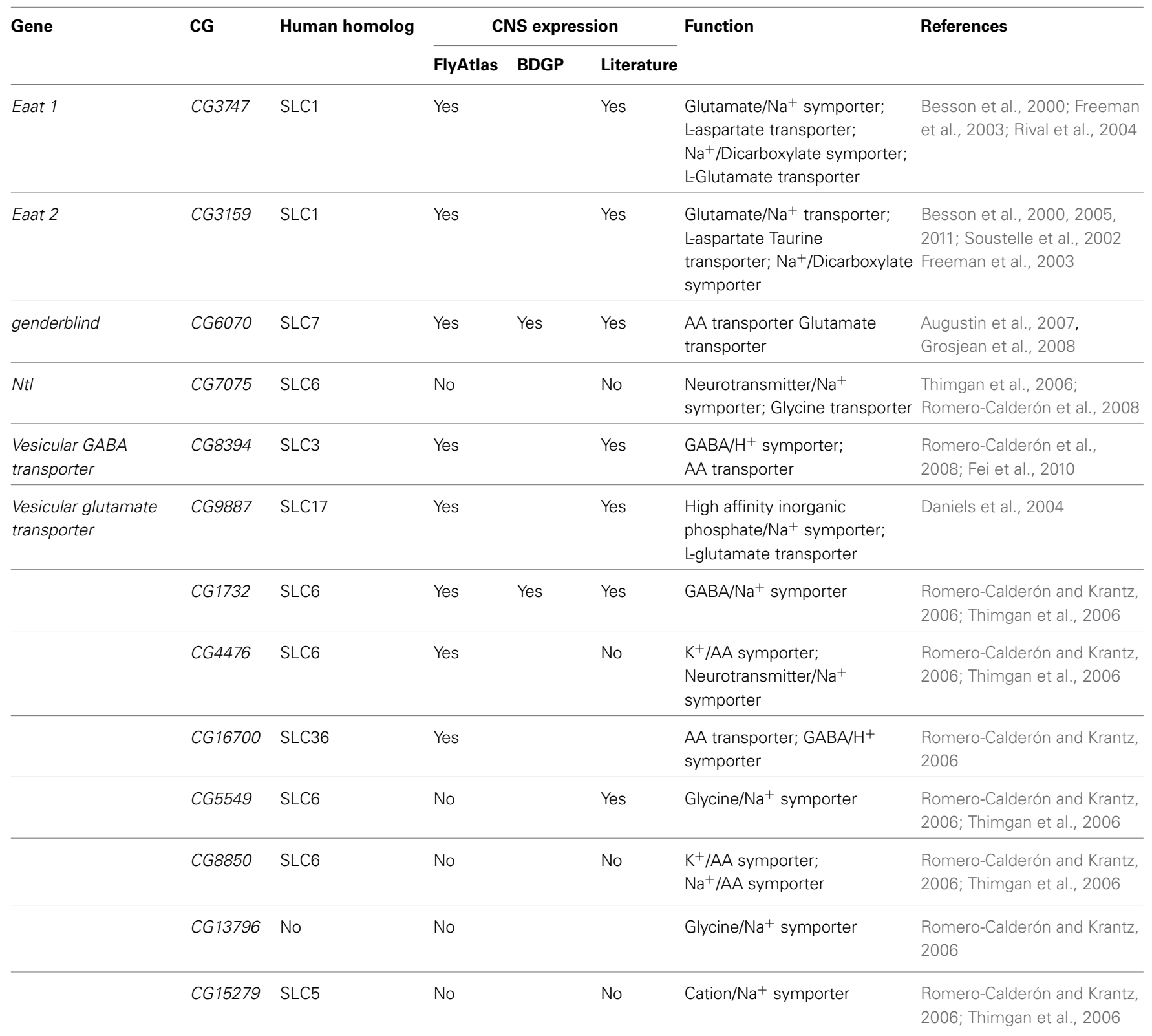

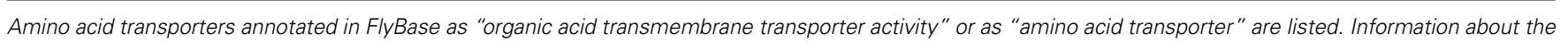

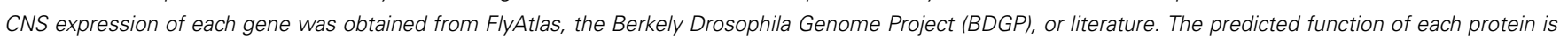
indicated. SLC, solute carrier; AA, amino acid; OCA2, oculocutaneous albinism II; GABA, $\gamma$-Aminobutyric acid.

which is encoded by an essential gene in Drosophila (Tps1, CG4104, Chen and Haddad, 2004). Among others, trehalose is used for the maintenance of energy metabolism during fasting and non-feeding periods (Friedman, 1978; Arrese and Soulages, 2010; Chen et al., 2010).

These observations suggest that specific sugar transporters exist, which import either trehalose or glucose into the brain. Two dedicated trehalose transporters, Tret1-1 and Tret1-2, have been described in Drosophila melanogaster (Kikawada et al., 2007). A comparison with other insect species shows that only one trehalose transporter gene is conserved in insects whereas the second trehalose transporter gene found in Drosophila melanogaster (Tret1-2) arose from a recent gene duplication event. Tret1-1 but not Tret1-2 is able to transport trehalose when expressed in Xenopus oocytes suggesting that the two trehalose transporters exert non-redundant functions (Kanamori et al., 2010). Trehalose transporters belong to the solute carrier 2 (SLC2) facilitated glucose transporter family with strongest homology to the human SLC2A8 protein. According to microarray data, Tret11 is strongly expressed in the brain. Tret1-2 is expressed only at very low levels. Its function is currently unknown (Kanamori et al., 2010). In conclusion, the Trehalose transporter is in a prime 
Table 5 | Sugar concentration in the insect hemolymph.

\begin{tabular}{|c|c|c|c|c|c|}
\hline Organism & Trehalose & Glucose & Fructose & Remark & References \\
\hline Several insects & $2-50 \mathrm{mg} / \mathrm{ml}$ & $\begin{array}{l}\text { Generally low amounts, } \\
\text { but Apis mellifica } \\
6-32 \mathrm{mg} / \mathrm{ml} \text {, Phormia } \\
\text { regina } 7-12.5 \mathrm{mg} / \mathrm{ml}\end{array}$ & $\begin{array}{l}\text { Generally low amounts, } \\
\text { but Apis mellifica } \\
2-16 \mathrm{mg} / \mathrm{ml}\end{array}$ & $\begin{array}{l}\text { Review on hemolymph } \\
\text { composition in insects }\end{array}$ & Jeuniaux, 1971 \\
\hline Apis mellifica & $40 \mathrm{mg} / \mathrm{ml}$ & $10 \mathrm{mg} / \mathrm{ml}$ & $10 \mathrm{mg} / \mathrm{ml}$ & $\begin{array}{l}\text { HPLC, trehalose } \\
\text { concentration changes } \\
\text { depending on metabolic } \\
\text { rate }\end{array}$ & $\begin{array}{l}\text { Blatt and Roces, } \\
2001\end{array}$ \\
\hline Drosophila larvae & $6 \mathrm{mg} / \mathrm{ml}$ & $1 \mathrm{mg} / \mathrm{ml}$ & Not tested & Commercial kit & $\begin{array}{l}\text { Pasco and } \\
\text { Léopold, } 2012\end{array}$ \\
\hline Drosophila adults & $17.2 \mathrm{mg} / \mathrm{ml}$ & $1.8 \mathrm{mg} / \mathrm{ml}$ & Not tested & Commercial kit & $\begin{array}{l}\text { Broughton et al., } \\
2008\end{array}$ \\
\hline
\end{tabular}

Trehalose, glucose, and fructose concentrations in the hemolymph of different organisms and developmental stages are listed. Methods for concentration measurements are indicated.

position to control the import of high-energy carbohydrates at the blood-brain barrier.

\section{CARBOHYDRATE TRANSPORTERS IN THE DROSOPHILA BLOOD-BRAIN BARRIER}

In total, 78 genes of Drosophila harbor a sugar transporter motif (Interpro domain search; IPR005829, Table 6). Most of the encoded proteins, however, probably will not transport sugar across the plasma membrane but, as the SLC35 member Meigo, organize intracellular trafficking of different sugars to ensure glycosylation (Sekine et al., 2013). As an alternative to trehalose, other sugars such as glucose or fructose could be taken up by the nervous system to meet the high neuronal energy demand. In vertebrates, the SLC2 family includes Glut 2 and Glut 5 transporters, which have been characterized as glucose and fructose transporters (Douard and Ferraris, 2008; Kellett et al., 2008; Mueckler and Thorens, 2013). No clear homolog of Glut 2 and Glut 5 can be identified in Drosophila, so it remains uncertain whether fructose can be imported into the nervous system. Interestingly, however, a fructose receptor ( $\mathrm{Gr} 43 \mathrm{a})$ has been shown to be expressed on several CNS neurons (Miyamoto et al., 2012). Thus, a possible explanation for the apparent lack of Glut2 or Glut 5 homologs is that fructose might be either generated in the brain or that the fructose sensing CNS neurons form dendrites that leave the CNS to detect fructose in the hemolymph.

Unlike in the Drosophila hemolymph, glucose is the main energy supply in the mammalian blood. Glucose is transported by Glut1 and Glut3, which are also members of the SLC2 family. For both of these facilitated glucose transporters, glucose, galactose, and mannose transport activities have been found (Uldry and Thorens, 2004). Glut1 functions in the mammalian endothelial blood-brain barrier and in astrocytes, whereas Glut3 is expressed by neurons (Leino et al., 1997). In Drosophila, Glut1 is specifically expressed in the embryonic nervous system and microarray data indicate continued expression in brain tissue (FlyBase). Future work needs to discriminate whether Glut1 is expressed in neurons or glial cells or both. In contrast, Glut3 is only expressed in imaginal discs of late larval stages and in adult testis (FlyBase). Thus, Glut1 might be responsible for glucose uptake into the nervous system. In support of this notion, loss of glut1 function is lethal (Saito et al., 2002) and expression of glut1 is able to improve locomotor behavior and survival of flies when mitochondrial activity is reduced in glial cells (Besson et al., 2010).

In addition, members of the SLC5A family (SLC5A1 and SLC5A2) have been shown to mediate sodium-dependent glucose uptake (Featherstone, 2011). One Drosophila SLC5A family member is CG9657, which is also expressed in glial cells (Freeman et al., 2003).

\section{BLOOD-BRAIN BARRIER AND HORMONAL FUNCTION}

In addition to the control of metabolism, the blood-brain barrier must also permit the entry and exit of hormones into or out of the nervous system. This is especially true for the Drosophila neuroendocrine system, which consists of neurosecretory cells (NSCs) in the brain. The Drosophila genome encodes eight insulin-like peptides (Dilps), which are the functional homologs 
Table 6 | Drosophila sugar transporters.

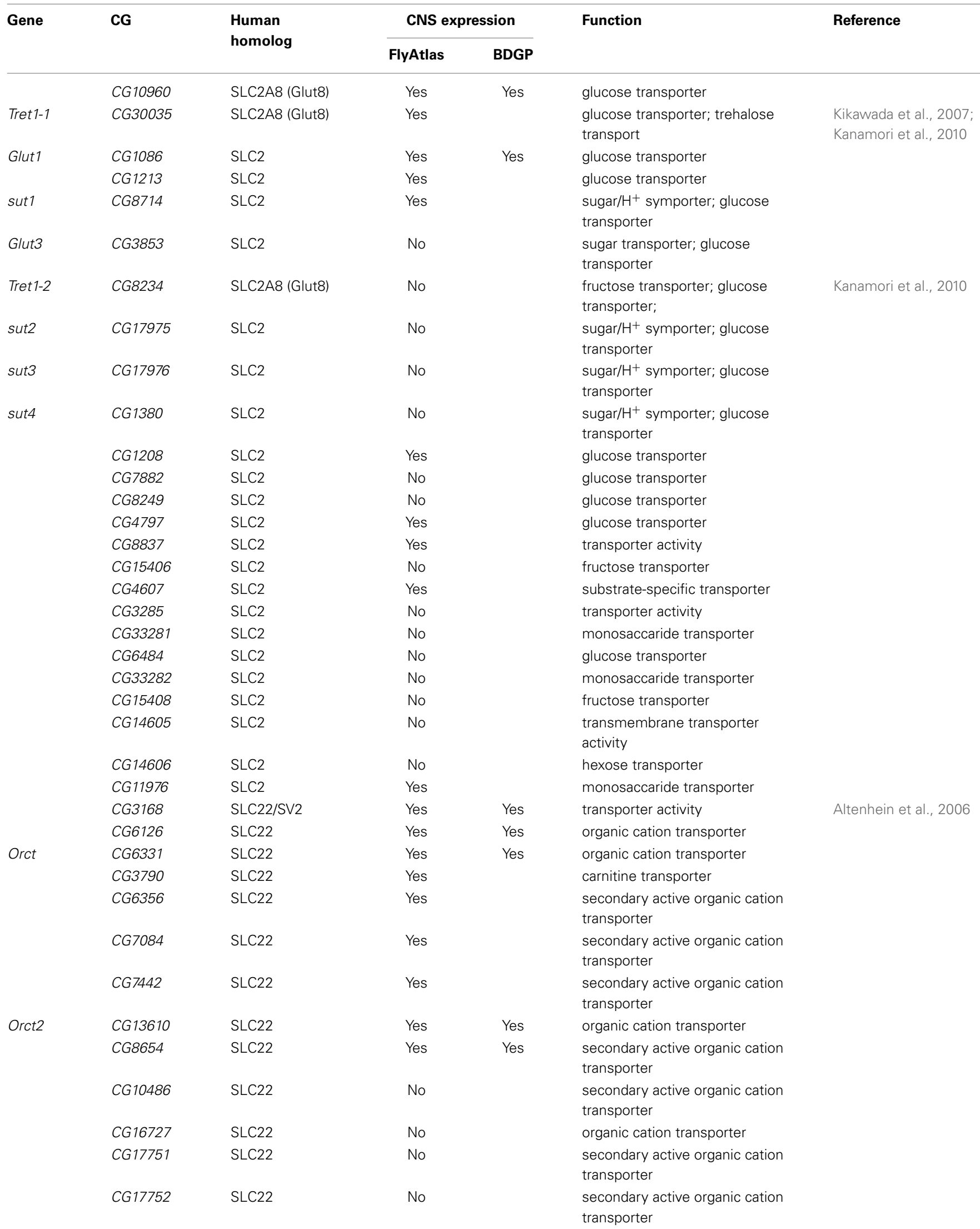


Table 6 | Continued

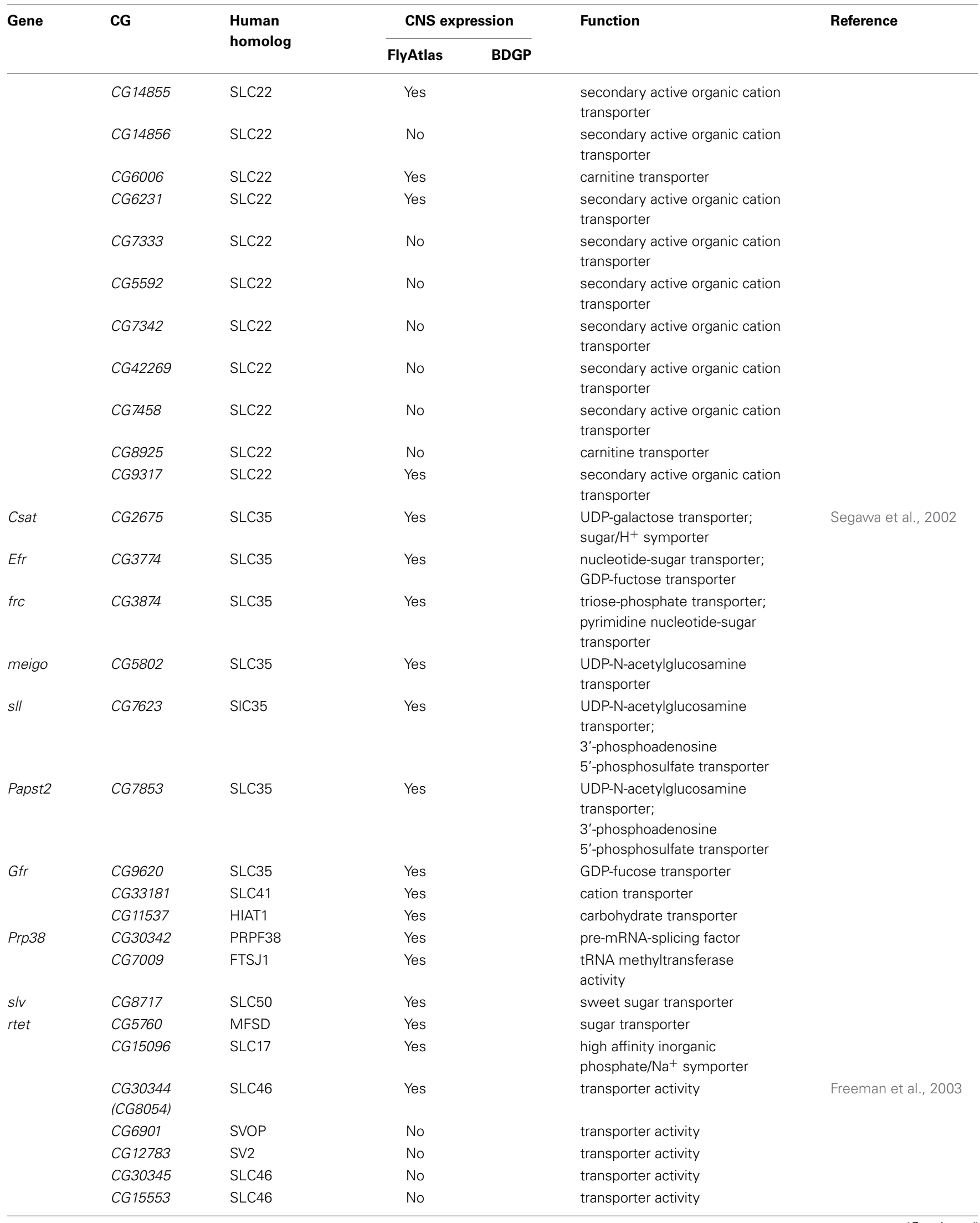


Table 6 | Continued

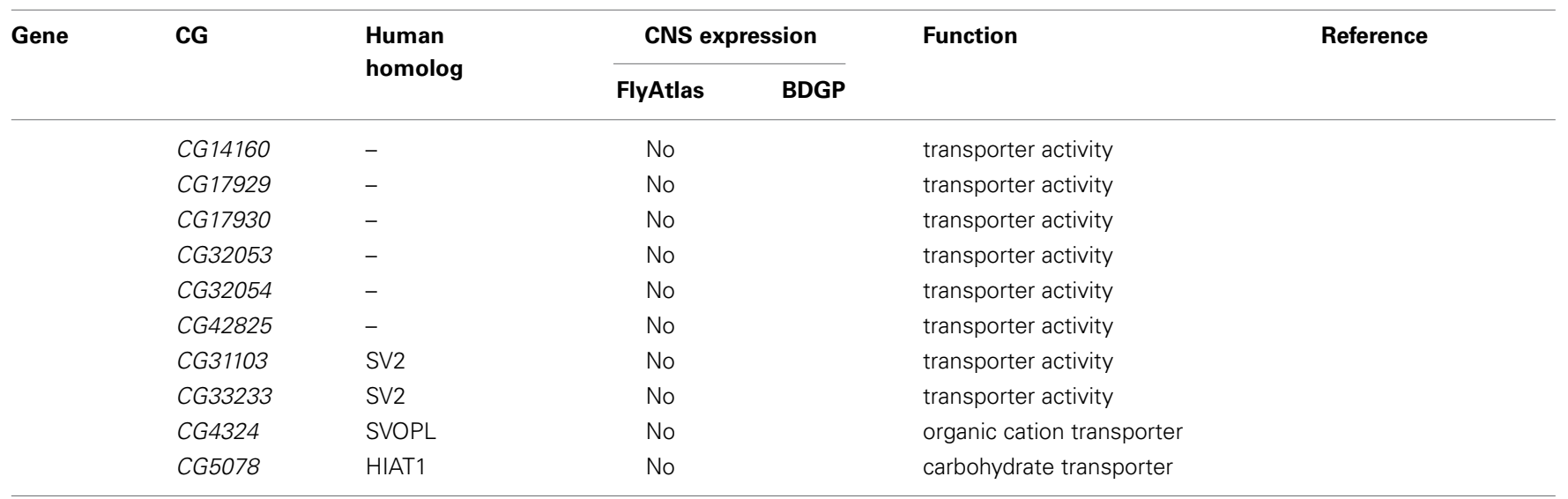

Carbohydrate transporters harboring a sugar transporter motif (Interpro domain search, IPR005829) are listed. Information about the CNS expression of each gene was obtained as indicated. The predicted function of each protein is noted. SLC, solute carrier; HIAT, hippocampus abundant transcript 1 ; PRPF, pre-mRNA processing factor; FTSJ1, FtsJ RNA methyltransferase homolog 1; MFSD10, major facilitator superfamily domain containing 10; SVOP, SV2 related protein homolog; SV2, synaptic vesicle glycoprotein 2; SVOPL, SVOP-like.

of vertebrate insulin and insulin-like growth factors (IGFs) that affect a wide range of processes (Erion and Sehgal, 2013; Shim et al., 2013). Dilp2, 3 and 5 are expressed by 14 insulin-producing cells (IPCs) and released into the hemolymph to regulate growth, metabolism, reproduction, and life span (Nässel et al., 2013). A key trigger of Dilp release from IPCs is food intake. The associated increase in hemolymph sugar and amino acid levels is sensed by the fat body and causes release of the leptin-like peptide Unpaired 2 (Upd2), which acts on IPCs via GABAergic neurons (Rajan and Perrimon, 2012). However, how this fat body-derived signal passes the blood-brain barrier or how it is sensed by the surface glia and then further transmitted into the nervous system is not known.

An additional fat body derived signal coordinates the second wave of neurogenesis at the end of larval stages, which must be matched to the nutritional status of the animal. This still elusive signal triggers expression of Dilp6 in the SPG, which in turn activates the proliferation of larval neuroblasts (Chell and Brand, 2010; Sousa-Nunes et al., 2011). Interestingly, both, the expression and the secretion of Dilp6 from the SPG cells depend on the presence of gap junctions in these cells (Spéder and Brand, 2014).

Moreover, the blood-brain barrier influences the physiology of the animal. It is known that some hemolymph proteins modulate the mating behavior of Drosophila (Lazareva et al., 2007). Interestingly, the sex of the blood-brain barrier matters and malespecific factors of the blood-brain barrier are required for normal male courtship behavior (Hoxha et al., 2013). These aspects of blood-brain barrier function are currently not extensively studied but it appears likely, that in the near future more surprising findings will be made.

\section{CONCLUSIONS}

Nervous system function strongly depends on a well-balanced ion and metabolite milieu. To ensure this homeostasis in the brain, the blood-brain barrier fulfills a variety of functions. It seals the brain from circulation and, in consequence, active transport systems are required for all solutes that have to be shuttled into or out of the brain. Therefore, a number of specific transporters must be expressed in the barrier forming cells. In Drosophila, as in primitive vertebrates, the blood-brain barrier is formed by glia. To date, our knowledge about essential transporters expressed in the glia is very limited. However, genomic information and transcriptomic data as already available for the mouse (Daneman et al., 2010a) will soon enable us to identify many relevant genes in Drosophila and the wealth of genetic tools will ease their analysis. One of the future challenges will be to decipher how the metabolic supply through the blood-brain barrier is matched to neuronal activity. In light of the apparently well-conserved blood-brain barrier, functional studies using Drosophila are expected to deepen our understanding of how the blood-brain barrier keeps our nervous system functional.

\section{ACKNOWLEDGMENTS}

We are thankful to S. Rumpf and N. Saunders for comments on the manuscript. Work in the lab of Christian Klämbt is supported by the Deutsche Forschungsgemeinschaft (DFG, SFB 1009). Stefanie Limmer is supported by a postdoctoral fellowship of the DFG.

\section{REFERENCES}

Abbott, N. J., Rönnbäck, L., and Hansson, E. (2006). Astrocyte-endothelial interactions at the blood-brain barrier. Nat. Rev. Neurosci. 7, 41-53. doi: $10.1038 / \mathrm{nrn} 1824$

Allaman, I., Bélanger, M., and Magistretti, P. J. (2011). Astrocyte-neuron metabolic relationships: for better and for worse. Trends Neurosci. 34, 76-87. doi: 10.1016/j.tins.2010.12.001

Altenhein, B., Becker, A., Busold, C., Beckmann, B., Hoheisel, J. D., and Technau, G. M. (2006). Expression profiling of glial genes during Drosophila embryogenesis. Dev. Biol. 296, 545-560. doi: 10.1016/j.ydbio.2006.04.460

Anholt, R. R. H., and Mackay, T. F. C. (2012). Genetics of aggression. Annu. Rev. Genet. 46, 145-164. doi: 10.1146/annurev-genet-110711-155514

Armstrong, G. A. B., Rodríguez, E. C., and Meldrum Robertson, R. (2012). Cold hardening modulates $\mathrm{K}+$ homeostasis in the brain of Drosophila 
melanogaster during chill coma. J. Insect Physiol. 58, 1511-1516. doi: 10.1016/j.jinsphys.2012.09.006

Armulik, A., Genové, G., and Betsholtz, C. (2011). Pericytes: developmental, physiological, and pathological perspectives, problems, and promises. Dev. Cell 21, 193-215. doi: 10.1016/j.devcel.2011.07.001

Armulik, A., Genové, G., Mäe, M., Nisancioglu, M. H., Wallgard, E., Niaudet, C., et al. (2010). Pericytes regulate the blood-brain barrier. Nature 468, 557-561. doi: $10.1038 /$ nature09522

Arrese, E. L., and Soulages, J. L. (2010). Insect fat body: energy, metabolism, and regulation. Annu. Rev. Entomol. 55, 207-225. doi: 10.1146/annurev-ento112408-085356

Augustin, H., Grosjean, Y., Chen, K., Sheng, Q., and Featherstone, D. E. (2007). Nonvesicular release of glutamate by glial xCT transporters suppresses glutamate receptor clustering in vivo. J. Neurosci. 27, 111-123. doi: 10.1523/JNEUROSCI.4770-06.2007

Auld, V. J., Fetter, R. D., Broadie, K., and Goodman, C. S. (1995). Gliotactin, a novel transmembrane protein on peripheral glia, is required to form the blood-nerve barrier in Drosophila. Cell 81, 757-767. doi: 10.1016/0092-8674(95)90537-5

Awasaki, T., Lai, S.-L., Ito, K., and Lee, T. (2008). Organization and postembryonic development of glial cells in the adult central brain of Drosophila. J. Neurosci. 28, 13742-13753. doi: 10.1523/JNEUROSCI.4844-08.2008

Bainton, R. J., Tsai, L. T.-Y., Schwabe, T., DeSalvo, M., Gaul, U., and Heberlein, U. (2005). moody encodes two GPCRs that regulate cocaine behaviors and blood-brain barrier permeability in Drosophila. Cell 123, 145-156. doi: 10.1016/j.cell.2005.07.029

Bätz, T., Förster, D., and Luschnig, S. (2014). The transmembrane protein Macroglobulin complement-related is essential for septate junction formation and epithelial barrier function in Drosophila. Development 141, 899-908. doi: 10.1242/dev. 102160

Baumgartner, S., Littleton, J. T., Broadie, K., Bhat, M. A., Harbecke, R., Lengyel, J. A., et al. (1996). A Drosophila neurexin is required for septate junction and blood-nerve barrier formation and function. Cell 87, 1059-1068. doi: 10.1016/S0092-8674(00)81800-0

Beckervordersandforth, R. M., Rickert, C., Altenhein, B., and Technau, G. M. (2008). Subtypes of glial cells in the Drosophila embryonic ventral nerve cord as related to lineage and gene expression. Mech. Dev. 125, 542-557. doi: 10.1016/j.mod.2007.12.004

Begg, M., and Cruickshank, W. J. (1962). A partial analysis of Drosophila larval hæmolymph. Proc. $R$ Soc. Edinb. B Biol. 68, 215-236. doi: 10.1017/S0080455X00001053

Besson, M.-T., Dupont, P., Fridell, Y.-W. C., and Liévens, J.-C. (2010). Increased energy metabolism rescues glia-induced pathology in a Drosophila model of Huntington's disease. Hum. Mol. Genet. 19, 3372-3382. doi: $10.1093 / \mathrm{hmg} / \mathrm{ddq} 249$

Besson, M.-T., Ré, D. B., Moulin, M., and Birman, S. (2005). High affinity transport of taurine by the Drosophila aspartate transporter dEAAT2. J. Biol. Chem. 280, 6621-6626. doi: 10.1074/jbc.M412440200

Besson, M. T., Sinakevitch, I., Melon, C., Iché-Torres, M., and Birman, S. (2011). Involvement of the Drosophila taurine/aspartate transporter dEAAT2 in selective olfactory and gustatory perceptions. J. Comp. Neurol. 519, 2734-2757. doi: $10.1002 /$ cne. 22649

Besson, M. T., Soustelle, L., and Birman, S. (2000). Selective high-affinity transport of aspartate by a Drosophila homologue of the excitatory amino-acid transporters. Curr. Biol. 10, 207-210. doi: 10.1016/S0960-9822(00)00339-0

Bilder, D., and Perrimon, N. (2000). Localization of apical epithelial determinants by the basolateral PDZ protein Scribble. Nature 403, 676-680. doi: $10.1038 / 35001108$

Blatt, J., and Roces, F. (2001). Haemolymph sugar levels in foraging honeybees (Apis mellifera carnica): dependence on metabolic rate and in vivo measurement of maximal rates of trehalose synthesis. J. Exp. Biol. 204, 2709-2716.

Boudko, D. Y. (2012). Molecular basis of essential amino acid transport from studies of insect nutrient amino acid transporters of the SLC6 family (NAT-SLC6). J. Insect Physiol. 58, 433-449. doi: 10.1016/j.jinsphys.2011.12.018

Broadus, J., Skeath, J. B., Spana, E. P., Bossing, T., Technau, G., and Doe, C. Q. (1995). New neuroblast markers and the origin of the aCC/pCC neurons in the Drosophila central nervous system. Mech. Dev. 53, 393-402. doi: 10.1016/09254773(95)00454-8

Broughton, S., Alic, N., Slack, C., Bass, T., Ikeya, T., Vinti, G., et al. (2008). Reduction of DILP2 in Drosophila triages a metabolic phenotype from lifespan revealing redundancy and compensation among DILPs. PLoS ONE 3:e3721. doi: 10.1371/journal.pone.0003721

Bundgaard, M., and Abbott, N. J. (2008). All vertebrates started out with a glial blood-brain barrier 4-500 million years ago. Glia 56, 699-708. doi: 10.1002/glia.20642

Campos-Ortega, J., and Hartenstein, V. (1997). The Embryonic Development of Drosophila Melanogaster. 2nd Edn. Berlin: Springer-Verlag. doi: 10.1007/9783-662-22489-2

Cardona, A., Saalfeld, S., Preibisch, S., Schmid, B., Cheng, A., Pulokas, J., et al. (2010). An integrated micro- and macroarchitectural analysis of the Drosophila brain by computer-assisted serial section electron microscopy. PLoS Biol 8:e1000502. doi: 10.1371/journal.pbio. 1000502

Carlson, S. D., Juang, J. L., Hilgers, S. L., and Garment, M. B. (2000) Blood barriers of the insect. Annu. Rev. Entomol. 45, 151-174. doi: 10.1146/annurev.ento.45.1.151

Chell, J. M., and Brand, A. H. (2010). Nutrition-responsive glia control exit of neural stem cells from quiescence. Cell 143, 1161-1173. doi: 10.1016/j.cell.2010.12.007

Chen, J., Tang, B., Chen, H., Yao, Q., Huang, X., Chen, J., et al. (2010). Different functions of the insect soluble and membrane-bound trehalase genes in chitin biosynthesis revealed by RNA interference. PLoS ONE 5:e10133. doi: 10.1371/journal.pone.0010133

Chen, Q., and Haddad, G. G. (2004). Role of trehalose phosphate synthase and trehalose during hypoxia: from flies to mammals. J. Exp. Biol. 207, 3125-3129. doi: $10.1242 /$ jeb.01133

Chintapalli, V. R., Wang, J., Herzyk, P., Davies, S. A., and Dow, J. A. (2013). Datamining the FlyAtlas online resource to identify core functional motifs across transporting epithelia. BMC Genomics 14:518. doi: 10.1186/1471-2164-14-518

von Hilchen, C. M., Bustos, Á. E., Giangrande, A., Technau, G. M., and Altenhein, B. (2013). Predetermined embryonic glial cells form the distinct glial sheaths of the Drosophila peripheral nervous system. Development 140, 3657-3668. doi: 10.1242/dev.093245

Crews, S. T., Thomas, J. B., and Goodman, C. S. (1988). The Drosophila single-minded gene encodes a nuclear protein with sequence similarity to the per gene product. Cell 52, 143-151. doi: 10.1016/0092-8674(88) 90538-7

Croghan, P. C., and Lockwood, A. P. M. (1960). The composition of the hemolymph of the larva of Drosophila melanogaster. J. Exp. Biol. 37, 339-343.

Daneman, R., Zhou, L., Agalliu, D., Cahoy, J. D., Kaushal, A., and Barres, B. A. (2010a). The mouse blood-brain barrier transcriptome: a new resource for understanding the development and function of brain endothelial cells. PLoS ONE 5:e13741. doi: 10.1371/journal.pone.0013741

Daneman, R., Zhou, L., Kebede, A. A., and Barres, B. A. (2010b). Pericytes are required for blood-brain barrier integrity during embryogenesis. Nature 468, 562-566. doi: 10.1038/nature09513

Daniels, R. W., Collins, C. A., Gelfand, M. V., Dant, J., Brooks, E. S., Krantz, D. E., et al. (2004). Increased expression of the Drosophila vesicular glutamate transporter leads to excess glutamate release and a compensatory decrease in quantal content. J. Neurosci. 24, 10466-10474. doi: 10.1523/JNEUROSCI.3001-04.2004

Davis, R. L. (2011). Traces of Drosophila memory. Neuron 70, 8-19. doi: 10.1016/j.neuron.2011.03.012

Dermauw, W., and Van Leeuwen, T. (2014). The ABC gene family in arthropods: comparative genomics and role in insecticide transport and resistance. Insect Biochem. Mol. Biol. 45, 89-110. doi: 10.1016/j.ibmb.2013.11.001

DeSalvo, M. K., Mayer, N., Mayer, F., and Bainton, R. J. (2011). Physiologic and anatomic characterization of the brain surface glia barrier of Drosophila. Glia 59, 1322-1340. doi: 10.1002/glia.21147

Dietzl, G., Chen, D., Schnorrer, F., Su, K.-C., Barinova, Y., Fellner, M., et al. (2007). A genome-wide transgenic RNAi library for conditional gene inactivation in Drosophila. Nature 448, 151-156. doi: 10.1038/nature05954

Douard, V., and Ferraris, R. P. (2008). Regulation of the fructose transporter GLUT5 in health and disease. Am. J. Physiol. Endocrinol. Metab. 295, E227-E237. doi: 10.1152/ajpendo.90245.2008

Erion, R., and Sehgal, A. (2013). Regulation of insect behavior via the insulinsignaling pathway. Front. Physiol. 4:353. doi: 10.3389/fphys.2013.00353

Faivre-Sarrailh, C., Banerjee, S., Li, J., Hortsch, M., Laval, M., and Bhat, M. A. (2004). Drosophila contactin, a homolog of vertebrate contactin, is required for septate junction organization and paracellular barrier function. Development 131, 4931-4942. doi: 10.1242/dev.01372 
Farquhar, M. G., and Palade, G. E. (1963). Junctional complexes in various epithelia. J. Cell Biol. 17, 375-412. doi: 10.1083/jcb.17.2.375

Farquhar, M. G., and Palade, G. E. (1965). Cell junctions in amphibian skin. J. Cell Biol. 26, 263-291. doi: 10.1083/jcb.26.1.263

Featherstone, D. E. (2011). Glial solute carrier transporters in Drosophila and mice. Glia 59, 1351-1363. doi: 10.1002/glia.21085

Fehon, R. G., Dawson, I. A., and Artavanis-Tsakonas, S. (1994). A Drosophila homologue of membrane-skeleton protein 4.1 is associated with septate junctions and is encoded by the coracle gene. Development 120, 545-557.

Fei, H., Chow, D. M., Chen, A., Romero-Calderón, R., Ong, W. S., Ackerson, L. C., et al. (2010). Mutation of the Drosophila vesicular GABA transporter disrupts visual figure detection. J. Exp. Biol. 213, 1717-1730. doi: 10.1242/jeb.036053

Filippov, V., Aimanova, K., and Gill, S. S. (2003). Expression of an Aedes aegypti cation-chloride cotransporter and its Drosophila homologues. Insect Mol. Biol. 12, 319-331. doi: 10.1046/j.1365-2583.2003.00415.x

Franzdóttir, S. R., Engelen, D., Yuva-Aydemir, Y., Schmidt, I., Aho, A., and Klämbt, C. (2009). Switch in FGF signalling initiates glial differentiation in the Drosophila eye. Nature 460, 758-761. doi: 10.1038/nature08167

Freeman, M. R., Delrow, J., Kim, J., Johnson, E., and Doe, C. Q. (2003). Unwrapping glial biology: $\mathrm{Gcm}$ target genes regulating glial development, diversification, and function. Neuron 38, 567-580. doi: 10.1016/S08966273(03)00289-7

Friedman, S. (1978). Trehalose regulation, one aspect of metabolic homeostasis. Ann. Rev. Entomol 23, 389-407. doi: 10.1146/annurev.en.23.010178.002133

Fünfschilling, U., Supplie, L. M., Mahad, D., Boretius, S., Saab, A. S., Edgar, J., et al. (2012). Glycolytic oligodendrocytes maintain myelin and long-term axonal integrity. Nature 485, 517-521. doi: 10.1038/nature11007

Furuse, M., Izumi, Y., Oda, Y., Higashi, T., and Iwamoto, N. (2014). Molecular organization of tricellular tight junctions. Tissue Barriers 2, e28960. doi: $10.4161 /$ tisb. 28960

Gaete, P. S., Lillo, M. A., and Figueroa, X. F. (2014). Functional role of connexins and pannexins in the interaction between vascular and nervous system. J. Cell. Physiol. 229, 1336-1345. doi: 10.1002/jcp.24563

Genova, J. L., and Fehon, R. G. (2003). Neuroglian, Gliotactin, and the $\mathrm{Na}^{+} / \mathrm{K}^{+}$ ATPase are essential for septate junction function in Drosophila. J. Cell Biol. 161, 979-989. doi: 10.1083/jcb.200212054

Giesen, K., Hummel, T., Stollewerk, A., Harrison, S., Travers, A., and Klämbt, C. (1997). Glial development in the Drosophila CNS requires concomitant activation of glial and repression of neuronal differentiation genes. Development 124, 2307-2316.

Goberdhan, D. C. I., Meredith, D., Boyd, C. A. R., and Wilson, C. (2005). PATrelated amino acid transporters regulate growth via a novel mechanism that does not require bulk transport of amino acids. Development 132, 2365-2375. doi: $10.1242 /$ dev.01821

Graf, F., Noirot-Timothée, C., and Noirot, C. (1982). The specialization of septate junctions in regions of tricellular junctions. I. Smooth septate junctions (=continuous junctions). J. Ultrastruct. Res. 78, 136-151. doi: 10.1016/S00225320(82)80019-1

Grosjean, Y., Grillet, M., Augustin, H., Ferveur, J.-F., and Featherstone, D. E. (2008). A glial amino-acid transporter controls synapse strength and courtship in Drosophila. Nat. Neurosci. 11, 54-61. doi: 10.1038/nn2019

Guan, Z., Saraswati, S., Adolfsen, B., and Littleton, J. T. (2005). Genome-wide transcriptional changes associated with enhanced activity in the Drosophila nervous system. Neuron 48, 91-107. doi: 10.1016/j.neuron.2005.08.036

Hall, S., Bone, C., Oshima, K., Zhang, L., McGraw, M., Lucas, B., et al. (2014). Macroglobulin complement-related encodes a protein required for septate junction organization and paracellular barrier function in Drosophila. Development 141, 889-898. doi: 10.1242/dev.102152

Harris, J. J., Jolivet, R., and Attwell, D. (2012). Synaptic energy use and supply. Neuron 75, 762-777. doi: 10.1016/j.neuron.2012.08.019

Hartenstein, V. (2011). Morphological diversity and development of glia in Drosophila. Glia 59, 1237-1252. doi: 10.1002/glia.21162

Hatan, M., Shinder, V., Israeli, D., Schnorrer, F., and Volk, T. (2011). The Drosophila blood brain barrier is maintained by GPCR-dependent dynamic actin structures. J. Cell Biol. 192, 307-319. doi: 10.1083/jcb.201007095

Hekmat-Scafe, D. S., Lundy, M. Y., Ranga, R., and Tanouye, M. A. (2006). Mutations in the $\mathrm{K}+/ \mathrm{Cl}$ - cotransporter gene kazachoc (kcc) increase seizure susceptibility in Drosophila. J. Neurosci. 26, 8943-8954. doi: 10.1523/JNEUROSCI.4998-05.2006
Hijazi, A., Masson, W., Augé, B., Waltzer, L., Haenlin, M., and Roch, F. (2009). boudin is required for septate junction organisation in Drosophila and codes for a diffusible protein of the Ly6 superfamily. Development 136, 2199-2209. doi: 10.1242/dev.033845

Holcroft, C. E., Jackson, W. D., Lin, W.-H., Bassiri, K., Baines, R. A., and Phelan, P. (2013). Innexins Ogre and Inx2 are required in glial cells for normal postembryonic development of the Drosophila central nervous system. J. Cell Sci. 126, 3823-3834. doi: 10.1242/jcs.117994

Hosoya, T., Takizawa, K., Nitta, K., and Hotta, Y. (1995). glial cells missing: a binary switch between neuronal and glial determination in Drosophila. Cell 82, 1025-1036. doi: 10.1016/0092-8674(95)90281-3

Hoxha, V., Lama, C., Chang, P. L., Saurabh, S., Patel, N., Olate, N., et al. (2013). Sex-specific signaling in the blood-brain barrier is required for male courtship in Drosophila. PLoS Genet. 9:e1003217. doi: 10.1371/journal.pgen. 1003217

Ile, K. E., Tripathy, R., Goldfinger, V., and Renault, A. D. (2012). Wunen, a Drosophila lipid phosphate phosphatase, is required for septate junction-mediated barrier function. Development 139, 2535-2546. doi: 10.1242/dev.077289

Ito, K., Urban, J., and Technau, G. M. (1995). Distribution, classification, and development ofDrosophila glial cells in the late embryonic and early larval ventral nerve cord. Roux's Arch. Dev. Biol. 204, 284-307. doi: 10.1007/ BF02179499

Jang, C., Lee, G., and Chung, J. (2008). LKB1 induces apical trafficking of Silnoon, a monocarboxylate transporter, in Drosophila melanogaster. J. Cell Biol. 183, 11-17. doi: $10.1083 /$ jcb.200807052

Jaspers, M. H. J., Nolde, K., Behr, M., Joo, S.-H., Plessmann, U., Nikolov, M., et al. (2012). The claudin Megatrachea protein complex. J. Biol. Chem. 287, 36756-36765. doi: 10.1074/jbc.M112.399410

Jenett, A., Rubin, G. M., Ngo, T.-T. B., Shepherd, D., Murphy, C., Dionne, H., et al. (2012). A GAL4-driver line resource for Drosophila neurobiology. Cell Rep. 2, 991-1001. doi: 10.1016/j.celrep.2012.09.011

Jeuniaux, C. (1971). "Hemolymph-Arthropoda," in Chemical Zoology, eds M. Florkin and B. T. Scheer (New York; London: Academic Press), 64-118.

Jolivet, R., Magistretti, P. J., and Weber, B. (2009). Deciphering neuron-glia compartmentalization in cortical energy metabolism. Front. Neuroenergetics 1:4. doi: 10.3389/neuro.14.004.2009

Jones, B. W., Fetter, R. D., Tear, G., and Goodman, C. S. (1995). glial cells missing: a genetic switch that controls glial versus neuronal fate. Cell 82, 1013-1023. doi: 10.1016/0092-8674(95)90280-5

Kanamori, Y., Saito, A., Hagiwara-Komoda, Y., Tanaka, D., Mitsumasu, K., Kikuta, S., et al. (2010). The trehalose transporter 1 gene sequence is conserved in insects and encodes proteins with different kinetic properties involved in trehalose import into peripheral tissues. Insect Biochem. Mol. Biol. 40, 30-37. doi: 10.1016/j.ibmb.2009.12.006

Kasuya, J., Kaas, G. A., and Kitamoto, T. (2009). A putative amino acid transporter of the solute carrier 6 family is upregulated by lithium and is required for resistance to lithium toxicity in Drosophila. Neuroscience 163, 825-837. doi: 10.1016/j.neuroscience.2009.07.027

Kaufmann, N., Mathai, J. C., Hill, W. G., Dow, J. A. T., Zeidel, M. L., and Brodsky, J. L. (2005). Developmental expression and biophysical characterization of a Drosophila melanogaster aquaporin. Am. J. Physiol. Cell Physiol. 289, C397-C407. doi: 10.1152/ajpcell.00612.2004

Kellett, G. L., Brot-Laroche, E., Mace, O. J., and Leturque, A. (2008). Sugar absorption in the intestine: the role of GLUT2. Annu. Rev. Nutr. 28, 35-54. doi: 10.1146/annurev.nutr.28.061807.155518

Kikawada, T., Saito, A., Kanamori, Y., Nakahara, Y., Iwata, K.-I., Tanaka, D., et al. (2007). Trehalose transporter 1, a facilitated and high-capacity trehalose transporter, allows exogenous trehalose uptake into cells. Proc. Natl. Acad. Sci. U.S.A. 104, 11585-11590. doi: 10.1073/pnas.0702538104

Klaes, A., Menne, T., Stollewerk, A., Scholz, H., and Klämbt, C. (1994). The Ets transcription factors encoded by the Drosophila gene pointed direct glial cell differentiation in the embryonic CNS. Cell 78, 149-160. doi: 10.1016/00928674(94)90581-9

Landgraf, M., Bossing, T., Technau, G. M., and Bate, M. (1997). The origin, location, and projections of the embryonic abdominal motorneurons of Drosophila. J. Neurosci. 17, 9642-9655.

Lane, N. J. (1991). Morphology of glial blood-brain barriers. Ann. N.Y. Acad. Sci. 633, 348-362. doi: 10.1111/j.1749-6632.1991.tb15626.x 
Lane, N. J., and Abbott, N. J. (1992). Freeze-fracture evidence for a novel restricting junction at the blood-brain barrier of the cuttlefish Sepia officinalis. J. Neurocytol. 21, 295-303. doi: 10.1007/BF01224762

Lane, N. J., and Swales, L. S. (1979). Intercellular junctions and the development of the blood-brain barrier in Manduca sexta. Brain Res. 168, 227-245. doi: 10.1016/0006-8993(79)90166-5

Laprise, P., Lau, K. M., Harris, K. P., Silva-Gagliardi, N. F., Paul, S. M., Beronja, S., et al. (2009). Yurt, Coracle, Neurexin IV and the Na+,K+-ATPase form a novel group of epithelial polarity proteins. Nature 459, 1141-1145. doi: $10.1038 /$ nature08067

Laridon, B., Callaerts, P., and Norga, K. (2008). Embryonic expression patterns of Drosophila ACS family genes related to the human sialin gene. Gene Expr. Patterns 8, 275-283. doi: 10.1016/j.gep.2007.12.003

Laughlin, S. B., de Ruyter van Steveninck, R. R., and Anderson, J. C. (1998). The metabolic cost of neural information. Nat. Neurosci. 1, 36-41. doi: 10.1038/236

Lazareva, A. A., Roman, G., Mattox, W., Hardin, P. E., and Dauwalder, B. (2007). A role for the adult fat body in Drosophila male courtship behavior. PLoS Genet. 3:e16. doi: 10.1371/journal.pgen.0030016

Lee, G., and Park, J. H. (2004). Hemolymph sugar homeostasis and starvationinduced hyperactivity affected by genetic manipulations of the adipokinetic hormone-encoding gene in Drosophila melanogaster. Genetics 167, 311-323. doi: 10.1534/genetics.167.1.311

Lee, Y., Morrison, B. M., Li, Y., Lengacher, S., Farah, M. H., Hoffman, P. N., et al. (2012). Oligodendroglia metabolically support axons and contribute to neurodegeneration. Nature 487, 443-448. doi: 10.1038/nature11314

Leino, R. L., Gerhart, D. Z., van Bueren, A. M., McCall, A. L., and Drewes, L. R. (1997). Ultrastructural localization of GLUT 1 and GLUT 3 glucose transporters in rat brain. J. Neurosci. Res. 49, 617-626.

Leiserson, W. M., Forbush, B., and Keshishian, H. (2011). Drosophila glia use a conserved cotransporter mechanism to regulate extracellular volume. Glia 59, 320-332. doi: 10.1002/glia.21103

Leiserson, W. M., Harkins, E. W., and Keshishian, H. (2000). Fray, a Drosophila serine/threonine kinase homologous to mammalian PASK, is required for axonal ensheathment. Neuron 28, 793-806. doi: 10.1016/S0896-6273(00) 00154-9

Leybaert, L. (2005). Neurobarrier coupling in the brain: a partner of neurovascular and neurometabolic coupling? J. Cereb. Blood Flow Metab. 25, 2-16. doi: 10.1038/sj.jcbfm.9600001

Li, H.-H., Kroll, J. R., Lennox, S. M., Ogundeyi, O., Jeter, J., Depasquale, G., et al. (2014). A GAL4 driver resource for developmental and behavioral studies on the larval CNS of Drosophila. Cell Rep. 8, 897-908. doi: 10.1016/j.celrep.2014.06.065

Llimargas, M., Strigini, M., Katidou, M., Karagogeos, D., and Casanova, J. (2004). Lachesin is a component of a septate junction-based mechanism that controls tube size and epithelial integrity in the Drosophila tracheal system. Development 131, 181-190. doi: 10.1242/dev.00917

Lovick, J. K., Ngo, K. T., Omoto, J. J., Wong, D. C., Nguyen, J. D., and Hartenstein, V. (2013). Postembryonic lineages of the Drosophila brain: I. Development of the lineage-associated fiber tracts. Dev. Biol. 384, 228-257. doi: 10.1016/j.ydbio.2013.07.008

MacMillan, H. A., and Hughson, B. N. (2014). A high-throughput method of hemolymph extraction from adult Drosophila without anesthesia. J. Insect Physiol. 63, 27-31. doi: 10.1016/j.jinsphys.2014.02.005

Mayer, F., Mayer, N., Chinn, L., Pinsonneault, R. L., Kroetz, D., and Bainton, R. J. (2009). Evolutionary conservation of vertebrate blood-brain barrier chemoprotective mechanisms in Drosophila. J. Neurosci. 29, 3538-3550. doi: 10.1523/JNEUROSCI.5564-08.2009

Melom, J. E., and Littleton, J. T. (2013). Mutation of a NCKX eliminates glial microdomain calcium oscillations and enhances seizure susceptibility. J. Neurosci. 33, 1169-1178. doi: 10.1523/JNEUROSCI.3920-12.2013

Miller, M. M., Popova, L. B., Meleshkevitch, E. A., Tran, P. V., and Boudko, D. Y. (2008). The invertebrate $\mathrm{B}(0)$ system transporter, D. melanogaster NAT1, has unique d-amino acid affinity and mediates gut and brain functions. Insect Biochem. Mol. Biol. 38, 923-931. doi: 10.1016/j.ibmb.2008.07.005

Miyamoto, T., Slone, J., Song, X., and Amrein, H. (2012). A fructose receptor functions as a nutrient sensor in the Drosophila brain. Cell 151, 1113-1125. doi: 10.1016/j.cell.2012.10.024

Moussian, B., Tång, E., Tonning, A., Helms, S., Schwarz, H., Nüsslein-Volhard, C., et al. (2006). Drosophila Knickkopf and Retroactive are needed for epithelial tube growth and cuticle differentiation through their specific requirement for chitin filament organization. Development 133, 163-171. doi: 10.1242/dev.02177

Mueckler, M., and Thorens, B. (2013). The SLC2 (GLUT) family of membrane transporters. Mol. Aspects Med. 34, 121-138. doi: 10.1016/j.mam.2012. 07.001

Nagelhus, E. A., and Ottersen, O. P. (2013). Physiological roles of aquaporin-4 in brain. Physiol. Rev. 93, 1543-1562. doi: 10.1152/physrev.00011.2013

Naikkhwah, W., and O'Donnell, M. J. (2011). Salt stress alters fluid and ion transport by Malpighian tubules of Drosophila melanogaster: evidence for phenotypic plasticity. J. Exp. Biol. 214, 3443-3454. doi: 10.1242/jeb.057828

Narasimha, M., Uv, A., Krejci, A., Brown, N. H., and Bray, S. J. (2008). Grainy head promotes expression of septate junction proteins and influences epithelial morphogenesis. J. Cell Sci. 121, 747-752. doi: 10.1242/jcs.019422

Nässel, D. R., Kubrak, O. I., Liu, Y., Luo, J., and Lushchak, O. V. (2013). Factors that regulate insulin producing cells and their output in Drosophila. Front. Physiol. 4:252. doi: 10.3389/fphys.2013.00252

Nelson, K. S., Furuse, M., and Beitel, G. J. (2010). The Drosophila Claudin Kunekune is required for septate junction organization and tracheal tube size control. Genetics 185, 831-839. doi: 10.1534/genetics.110.114959

Nilton, A., Oshima, K., Zare, F., Byri, S., Nannmark, U., Nyberg, K. G., et al. (2010). Crooked, coiled and crimpled are three Ly6-like proteins required for proper localization of septate junction components. Development 137, 2427-2437. doi: 10.1242/dev.052605

Noirot-Timothée, C., Graf, F., and Noirot, C. (1982). The specialization of septate junctions in regions of tricellular junctions. II. Pleated septate junctions. J. Ultrastruct. Res. 78, 152-165. doi: 10.1016/S0022-5320(82)80020-8

Oda, Y., Otani, T., Ikenouchi, J., and Furuse, M. (2014). Tricellulin regulates junctional tension of epithelial cells at tricellular contacts via Cdc42. J. Cell Sci. doi: 10.1242/jcs.150607. [Epub ahead of print].

Oshima, K., and Fehon, R. G. (2011). Analysis of protein dynamics within the septate junction reveals a highly stable core protein complex that does not include the basolateral polarity protein Discs large. J. Cell Sci. 124, 2861-2871. doi: $10.1242 /$ jcs. 087700

Padash-Barmchi, M., Browne, K., Sturgeon, K., Jusiak, B., and Auld, V. J. (2010). Control of Gliotactin localization and levels by tyrosine phosphorylation and endocytosis is necessary for survival of polarized epithelia. J. Cell Sci. 123, 4052-4062. doi: 10.1242/jcs.066605

Palanker, L., Tennessen, J. M., Lam, G., and Thummel, C. S. (2009). Drosophila HNF4 regulates lipid mobilization and $\beta$-oxidation. Cell Metab. 9, 228-239. doi: 10.1016/j.cmet.2009.01.009

Palm, W., Sampaio, J. L., Brankatschk, M., Carvalho, M., Mahmoud, A. Shevchenko, A., et al. (2012). Lipoproteins in Drosophila melanogasterassembly, function, and influence on tissue lipid composition. PLoS Genet. 8:e1002828. doi: 10.1371/journal.pgen.1002828

Papadopoulos, M. C., and Verkman, A. S. (2013). Aquaporin water channels in the nervous system. Nat. Rev. Neurosci. 14, 265-277. doi: 10.1038/ nrn3468

Park, D., HadŽiæ, T., Yin, P., Rusch, J., Abruzzi, K., Rosbash, M., et al. (2011). Molecular organization of Drosophila neuroendocrine cells by dimmed. Curr. Biol. 21, 1515-1524. doi: 10.1016/j.cub.2011.08.015

Pasco, M. Y., and Léopold, P. (2012). High sugar-induced insulin resistance in Drosophila relies on the lipocalin Neural Lazarillo. PLoS ONE 7:e36583. doi: 10.1371/journal.pone.0036583

Paul, S. M., Palladino, M. J., and Beitel, G. J. (2007). A pump-independent function of the Na,K-ATPase is required for epithelial junction function and tracheal tube-size control. Development 134, 147-155. doi: 10.1242/dev.02710

Pellerin, L., and Magistretti, P. J. (1994). Glutamate uptake into astrocytes stimulates aerobic glycolysis: a mechanism coupling neuronal activity to glucose utilization. Proc. Natl. Acad. Sci. U.S.A. 91, 10625-10629. doi: 10.1073/pnas.91.22.10625

Pellerin, L., and Magistretti, P. J. (2012). Sweet sixteen for ANLS. J. Cereb. Blood Flow Metab. 32, 1152-1166. doi: 10.1038/jcbfm.2011.149

Pereanu, W., Shy, D., and Hartenstein, V. (2005). Morphogenesis and proliferation of the larval brain glia in Drosophila. Dev. Biol. 283, 191-203. doi: 10.1016/j.ydbio.2005.04.024

Rajan, A., and Perrimon, N. (2012). Drosophila cytokine unpaired 2 regulates physiological homeostasis by remotely controlling insulin secretion. Cell 151, 123-137. doi: 10.1016/j.cell.2012.08.019 
Rao, Y., Jan, L. Y., and Jan, Y. N. (1990). Similarity of the product of the Drosophila neurogenic gene big brain to transmembrane channel proteins. Nature 345 , 163-167. doi: 10.1038/345163a0

Reynolds, B., Roversi, P., Laynes, R., Kazi, S., Boyd, C. A. R., and Goberdhan, D. C. I. (2009). Drosophila expresses a CD98 transporter with an evolutionarily conserved structure and amino acid-transport properties. Biochem. J. 420, 363-372. doi: 10.1042/BJ20082198

Rickert, C., Kunz, T., Harris, K.-L., Whitington, P. M., and Technau, G. M. (2011). Morphological characterization of the entire interneuron population reveals principles of neuromere organization in the ventral nerve cord of Drosophila. J. Neurosci. 31, 15870-15883. doi: 10.1523/JNEUROSCI.400911.2011

Rival, T., Soustelle, L., Strambi, C., Besson, M.-T., Iché, M., and Birman, S. (2004). Decreasing glutamate buffering capacity triggers oxidative stress and neuropil degeneration in the Drosophila brain. Curr. Biol. 14, 599-605. doi: 10.1016/j.cub.2004.03.039

Romero-Calderón, R., and Krantz, D. E. (2006). Transport of polyamines in Drosophila S2 cells: kinetics, pharmacology and dependence on the plasma membrane proton gradient. Biochem. J. 393, 583. doi: 10.1042/BJ20050981

Romero-Calderón, R., Uhlenbrock, G., Borycz, J., Simon, A. F., Grygoruk, A., Yee, S. K., et al. (2008). A glial variant of the vesicular monoamine transporter is required to store histamine in the drosophila visual system. PLoS Genet. 4:e1000245. doi: 10.1371/journal.pgen.1000245

Rothstein, J. D., Martin, L., Levey, A. I., Dykes-Hoberg, M., Jin, L., Wu, D., et al. (1994). Localization of neuronal and glial glutamate transporters. Neuron 13, 713-725. doi: 10.1016/0896-6273(94)90038-8

Rouach, N., Koulakoff, A., Abudara, V., Willecke, K., and Giaume, C. (2008). Astroglial metabolic networks sustain hippocampal synaptic transmission. Science 322, 1551-1555. doi: 10.1126/science. 1164022

Saito, M., Awasaki, T., and Hama, C. (2002). Genetic analyses of essential genes in cytological region 61D1-2 to 61F1-2 of Drosophila melanogaster. Mol. Genet. Genomics 268, 446-454. doi: 10.1007/s00438-002-0770-6

Schmid, A., Chiba, A., and Doe, C. Q. (1999). Clonal analysis of Drosophila embryonic neuroblasts: neural cell types, axon projections and muscle targets. Development 126, 4653-4689.

Schmidt, I., Franzdóttir, S. R., Edenfeld, G., Rodrigues, F., Zierau, A., and Klämbt, C. (2011). Transcriptional regulation of peripheral glial cell differentiation in the embryonic nervous system of Drosophila. Glia 59, 1264-1272. doi: 10.1002/glia.21123

Schubiger, M., Feng, Y., Fambrough, D. M., and Palka, J. (1994). A mutation of the Drosophila sodium pump alpha subunit gene results in bang-sensitive paralysis. Neuron 12, 373-381. doi: 10.1016/0896-6273(94)90278-X

Schulte, J., Tepass, U., and Auld, V. J. (2003). Gliotactin, a novel marker of tricellular junctions, is necessary for septate junction development in Drosophila. J. Cell Biol. 161, 991-1000. doi: 10.1083/jcb.200303192

Schwabe, T., Bainton, R. J., Fetter, R. D., Heberlein, U., and Gaul, U. (2005). GPCR signaling is required for blood-brain barrier formation in drosophila. Cell 123, 133-144. doi: 10.1016/j.cell.2005.08.037

Segawa, H., Kawakita, M., and Ishida, N. (2002). Human and Drosophila UDP-galactose transporters transport UDP-N-acetylgalactosamine in addition to UDP-galactose. Eur. J. Biochem. 269, 128-138. doi: 10.1046/j.00142956.2001.02632.x

Sekine, S. U., Haraguchi, S., Chao, K., Kato, T., Luo, L., Miura, M., et al. (2013). Meigo governs dendrite targeting specificity by modulating Ephrin level and N-glycosylation. Nat. Neurosci. 16, 683-691. doi: 10.1038/nn.3389

Shandala, T., Takizawa, K., and Saint, R. (2003). The dead ringer/retained transcriptional regulatory gene is required for positioning of the longitudinal glia in the Drosophila embryonic CNS. Development 130, 1505-1513. doi: $10.1242 /$ dev.00377

Shim, J., Gururaja-Rao, S., and Banerjee, U. (2013). Nutritional regulation of stem and progenitor cells in Drosophila. Development 140, 4647-4656. doi: 10.1242/dev.079087

Shim, M. S., Kim, J. Y., Lee, K. H., Jung, H. K., Carlson, B. A., Xu, X. M., et al. (2011). 1(2)01810 is a novel type of glutamate transporter that is responsible for megamitochondrial formation. Biochem. J. 439, 277-286. doi: 10.1042/BJ20110582

Silies, M., Yuva, Y., Engelen, D., Aho, A., Stork, T., and Klämbt, C. (2007). Glial cell migration in the eye disc. J. Neurosci. 27, 13130-13139. doi: 10.1523/JNEUROSCI.3583-07.2007
Smith, Q. R. (2000). Transport of glutamate and other amino acids at the bloodbrain barrier. J. Nutr. 130, 1016S-1022S.

Sousa-Nunes, R., Yee, L. L., and Gould, A. P. (2011). Fat cells reactivate quiescent neuroblasts via TOR and glial insulin relays in Drosophila. Nature 471, 508-512. doi: 10.1038/nature09867

Soustelle, L., Besson, M.-T., Rival, T., and Birman, S. (2002). Terminal glial differentiation involves regulated expression of the excitatory amino acid transporters in the Drosophila embryonic CNS. Dev. Biol. 248, 294-306. doi: $10.1006 /$ dbio. 2002.0742

Spéder, P., and Brand, A. H. (2014). Gap junction proteins in the blood-brain barrier control nutrient-dependent reactivation of drosophila neural stem cells. Dev. Cell. 30, 309-321. doi: 10.1016/j.devcel.2014.05.021

Stacey, S. M., Muraro, N. I., Peco, E., Labbé, A., Thomas, G. B., Baines, R. A., et al. (2010). Drosophila glial glutamate transporter Eaatl is regulated by fringemediated notch signaling and is essential for larval locomotion. J. Neurosci. 30, 14446-14457. doi: 10.1523/JNEUROSCI.1021-10.2010

Stork, T., Bernardos, R., and Freeman, M. R. (2012). Analysis of glial cell development and function in Drosophila. Cold Spring Harb. Protoc. 2012, 1-17. doi: 10.1101/pdb.top067587

Stork, T., Engelen, D., Krudewig, A., Silies, M., Bainton, R. J., and Klämbt, C. (2008). Organization and function of the blood-brain barrier in Drosophila. J. Neurosci. 28, 587-597. doi: 10.1523/JNEUROSCI.4367-07.2008

Stork, T., Sheehan, A., Tasdemir-Yilmaz, O. E., and Freeman, M. R. (2014). Neuron-glia interactions through the heartless FGF receptor signaling pathway mediate morphogenesis of Drosophila astrocytes. Neuron $83,388-403$. doi: 10.1016/j.neuron.2014.06.026

Sun, Q., Tian, E., Turner, R. J., and Ten Hagen, K. G. (2010). Developmental and functional studies of the SLC12 gene family members from Drosophila melanogaster. Am. J. Physiol. Cell Physiol. 298, C26-C37. doi: 10.1152/ajpcell.00376.2009

Syed, M. H., Krudewig, A., Engelen, D., Stork, T., and Klämbt, C. (2011). The CD59 family member leaky/coiled is required for the establishment of the blood-brain barrier in Drosophila. J. Neurosci. 31, 7876-7885. doi: 10.1523/JNEUROSCI.0766-11.2011

Tatsumi, K., Tsuji, S., Miwa, H., Morisaku, T., Nuriya, M., Orihara, M., et al. (2009). Drosophila big brain does not act as a water channel, but mediates cell adhesion. FEBS Lett. 583, 2077-2082. doi: 10.1016/j.febslet.2009. 05.035

Tepass, U., and Hartenstein, V. (1994). The development of cellular junctions in the Drosophila embryo. Dev. Biol. 161, 563-596. doi: 10.1006/dbio.1994.1054

Thimgan, M. S., Berg, J. S., and Stuart, A. E. (2006). Comparative sequence analysis and tissue localization of members of the SLC6 family of transporters in adult Drosophila melanogaster. J. Exp. Biol. 209, 3383-3404. doi: 10.1242/jeb. 02328

Thomas, G. B., and van Meyel, D. J. (2007). The glycosyltransferase Fringe promotes delta-notch signaling between neurons and glia, and is required for subtype-specific glial gene expression. Development 134, 591-600. doi: 10.1242/dev.02754

Tiklová, K., Senti, K.-A., Wang, S., Gräslund, A., and Samakovlis, C. (2010). Epithelial septate junction assembly relies on melanotransferrin iron binding and endocytosis in Drosophila. Nat. Cell Biol. 12, 1071-1077. doi: $10.1038 /$ ncb2111

Tsacopoulos, M., Veuthey, A. L., Saravelos, S. G., Perrottet, P., and Tsoupras, G. (1994). Glial cells transform glucose to alanine, which fuels the neurons in the honeybee retina. J. Neurosci. 14, 1339-1351.

Uldry, M., and Thorens, B. (2004). The SLC2 family of facilitated hexose and polyol transporters. Pflugers Arch. 447, 480-489. doi: 10.1007/s00424-003-1085-0

Unhavaithaya, Y., and Orr-Weaver, T. L. (2012). Polyploidization of glia in neural development links tissue growth to blood-brain barrier integrity. Genes Dev. 26 , 31-36. doi: 10.1101/gad.177436.111

Urbach, R., and Technau, G. M. (2003). Molecular markers for identified neuroblasts in the developing brain of Drosophila. Development 130, 3621-3637. doi: $10.1242 /$ dev.00533

Venken, K. J. T., Simpson, J. H., and Bellen, H. J. (2011). Genetic manipulation of genes and cells in the nervous system of the fruit fly. Neuron 72, 202-230. doi: 10.1016/j.neuron.2011.09.021

Vincent, S., Vonesch, J. L., and Giangrande, A. (1996). Glide directs glial fate commitment and cell fate switch between neurones and glia. Development 122 $131-139$. 
Wheeler, S. R., Kearney, J. B., Guardiola, A. R., and Crews, S. T. (2006). Singlecell mapping of neural and glial gene expression in the developing Drosophila CNS midline cells. Dev. Biol. 294, 509-524. doi: 10.1016/j.ydbio.2006. 03.016

Woods, D. F., Hough, C., Peel, D., Callaini, G., and Bryant, P. J. (1996). Dlg protein is required for junction structure, cell polarity, and proliferation control in Drosophila epithelia. J. Cell Biol. 134, 1469-1482. doi: 10.1083/jcb.134.6.1469

Wu, V. M., and Beitel, G. J. (2004). A junctional problem of apical proportions: epithelial tube-size control by septate junctions in the Drosophila tracheal system. Curr. Opin. Cell Biol. 16, 493-499. doi: 10.1016/j.ceb.2004.07.008

Wu, V. M., Yu, M. H., Paik, R., Banerjee, S., Liang, Z., Paul, S. M., et al. (2007). Drosophila Varicose, a member of a new subgroup of basolateral MAGUKs, is required for septate junctions and tracheal morphogenesis. Development 134, 999-1009. doi: 10.1242/dev.02785

Wyatt, G. R., and Kalf, G. F. (1957). The chemistry of insect hemolymph II. Trehalose and other carbohydrates. J. Gen. Physiol. 40, 833-847. doi: 10.1085/jgp.40.6.833
Conflict of Interest Statement: The authors declare that the research was conducted in the absence of any commercial or financial relationships that could be construed as a potential conflict of interest.

Received: 11 September 2014; paper pending published: 14 October 2014; accepted: 23 October 2014; published online: 14 November 2014

Citation: Limmer S, Weiler A, Volkenhoff A, Babatz F and Klämbt C (2014) The Drosophila blood-brain barrier: development and function of a glial endothelium. Front. Neurosci. 8:365. doi: 10.3389/fnins.2014.00365

This article was submitted to Neurogenomics, a section of the journal Frontiers in Neuroscience.

Copyright (c) 2014 Limmer, Weiler, Volkenhoff, Babatz and Klämbt. This is an openaccess article distributed under the terms of the Creative Commons Attribution License (CC BY). The use, distribution or reproduction in other forums is permitted, provided the original author(s) or licensor are credited and that the original publication in this journal is cited, in accordance with accepted academic practice. No use, distribution or reproduction is permitted which does not comply with these terms. 


\title{
The Drosophila surface glia transcriptome: evolutionary conserved blood-brain barrier processes
}

\section{Michael K. DeSalvo' , Samantha J. Hindle ${ }^{1}$, Zeid M. Rusan ${ }^{1}$, Souvinh Orng ${ }^{1}$, Mark Eddison ${ }^{2}$, Kyle Halliwill ${ }^{3}$ and Roland J. Bainton ${ }^{1 *}$}

\author{
Department of Anesthesia and Perioperative Care, University of California San Francisco, San Francisco, CA, USA \\ 2 Janelia Farm Research Campus, The Howard Hughes Medical Institute, Ashburn, VA, USA \\ ${ }^{3}$ Pharmaceutical Sciences and Pharmacogenomics, University of California San Francisco, San Francisco, CA, USA
}

Edited by:

Shane Antony Liddelow, Stanford University, USA

Reviewed by:

Alfredo Ghezzi, The University of

Texas at Austin, USA

Christian Klämbt, University of

Münster, Germany

\section{*Correspondence:}

Roland J. Bainton, Department of Anesthesia and Perioperative Care University of California San

Francisco, Genentech Hall, Room S312C, 600 16th Street, San

Francisco, CA 94158, USA

e-mail:baintonr@

anesthesia.ucsf.edu
Central nervous system (CNS) function is dependent on the stringent regulation of metabolites, drugs, cells, and pathogens exposed to the CNS space. Cellular blood-brain barrier (BBB) structures are highly specific checkpoints governing entry and exit of all small molecules to and from the brain interstitial space, but the precise mechanisms that regulate the BBB are not well understood. In addition, the BBB has long been a challenging obstacle to the pharmacologic treatment of CNS diseases; thus model systems that can parse the functions of the BBB are highly desirable. In this study, we sought to define the transcriptome of the adult Drosophila melanogaster BBB by isolating the BBB surface glia with fluorescence activated cell sorting (FACS) and profiling their gene expression with microarrays. By comparing the transcriptome of these surface glia to that of all brain glia, brain neurons, and whole brains, we present a catalog of transcripts that are selectively enriched at the Drosophila BBB. We found that the fly surface glia show high expression of many ATP-binding cassette $(A B C)$ and solute carrier (SLC) transporters, cell adhesion molecules, metabolic enzymes, signaling molecules, and components of xenobiotic metabolism pathways. Using gene sequence-based alignments, we compare the Drosophila and Murine BBB transcriptomes and discover many shared chemoprotective and small molecule control pathways, thus affirming the relevance of invertebrate models for studying evolutionary conserved BBB properties. The Drosophila BBB transcriptome is valuable to vertebrate and insect biologists alike as a resource for studying proteins underlying diffusion barrier development and maintenance, glial biology, and regulation of drug transport at tissue barriers.

Keywords: blood-brain barrier, transcriptome, surface glia, Affymetrix array

\section{INTRODUCTION}

Endothelial cells constituting the capillaries of the vertebrate central nervous system (CNS) have special properties that enable a potent blood-brain barrier (BBB). The BBB preserves CNS homeostasis by preventing the entry of harmful molecules and facilitating the passage of essential molecules such as metabolites. While the brain vascular endothelial cells are the anatomic $\mathrm{BBB}$, all members of the neurovascular unit (NVU—endothelial cells, pericytes, astrocytes, extracellular matrix, and neurons) are thought to contribute to the development and maintenance of BBB processes (Janzer and Raff, 1987; Sobue et al., 1999; Armulik et al., 2010; Daneman et al., 2010b). That being said, the barrier functions of the BBB are largely provided by vascular endothelial machinery: intercellular protein complexes, active efflux transporters, and carrier-mediated transporters (Zlokovic, 2008). In particular, the formation of tight junction (TJ) complexes between endothelial cells renders paracellular fluid flux impossible (Hirase et al., 1997; Saitou et al., 2000; Nitta et al., 2003). Also, endothelial cell expression of ABCB1 (i.e., MDR1/Pglycoprotein), an ATP-binding cassette (ABC) transporter found at the luminal surface, is responsible for the efflux of unwanted substrates back into the blood (Cordon-Cardo et al., 1989; Loscher and Potschka, 2005), and expression of SLC2A1 (i.e., GLUT1), a solute carrier (SLC) transporter found at both surfaces, shuttles glucose between the blood and the brain (Boado and Pardridge, 1990; Pardridge et al., 1990). Characterizing the entire repertoire of genes underlying BBB physiology is of paramount importance given that (1) little is known about the regulatory mechanisms that grant the $\mathrm{BBB}$ its properties, (2) the etiologies of numerous CNS diseases that include BBB dysfunctions (Daneman, 2012), and (3) CNS disease treatments that depend on efficient delivery of therapeutics across the BBB (Pardridge, 2005).

Genomic approaches to characterizing cellular BBB structures have yielded important resources for understanding the BBB. Enerson and Drewes (Enerson and Drewes, 2006) isolated blood microvessels, which contained endothelial cells, pericytes, extracellular matrix, and remnant astrocytic end feet, from rat brains and used serial analysis of gene expression to produce the first profile of a vertebrate BBB transcriptome. Daneman et al. 
(2010a) used fluorescence activated cell sorting (FACS) to purify brain vascular endothelial cells from mice and used Affymetrix GeneChips to survey the transcriptome of the endothelial BBB component. Both of these studies identified known BBB transcripts, but more importantly, they also identified novel transcripts enriched at the $\mathrm{BBB}$. Indeed, the $\mathrm{BBB}$ genes involved in CNS disease progression are not known in most cases (Daneman, 2012). In addition, strategies for delivering pharmaceuticals to the CNS have often centered on disrupting previously identified BBB drug efflux transporters such as ABCB1, but these direct strategies have been met with limited success (de Vries et al., 2007; Wu et al., 2011; Lin et al., 2013). Thus, in-depth exploration of the genes that regulate the integrated chemical protection physiologies present at the BBB is needed for overcoming BBB-related challenges.

A major limitation to studying the hundreds of $\mathrm{BBB}$ genes in vertebrates is that obtaining mutants is both time consuming and costly. For this reason, we have focused on using the Drosophila $\mathrm{BBB}$ as a model for the study of BBB function. Previous studies have shown that the insect $\mathrm{BBB}$ is analogous to the vertebrate BBB (Stork et al., 2008; Mayer et al., 2009; DeSalvo et al., 2011). The Drosophila CNS is similarly protected by a BBB with one main noteworthy difference: insects have an open circulatory system where molecules are dissolved in a fluid called hemolymph that bathes organs, instead of being distributed in a vascular system. For this reason, the insect BBB encapsulates the CNS (Stork et al., 2008). Resembling the multiple cell type architecture of the vertebrate NVU, the insect $\mathrm{BBB}$ is composed of two glial subtypes collectively known as surface glia-the apical perineurial glia (PG) and basal subperineurial glia (SPG) (Stork et al., 2008) (Figure 1). In addition, endothelial cells of the vertebrate NVU are surrounded by an extracellular matrix in which pericytes are embedded, and in the insect glial BBB, a similar matrix termed the neural lamella is apical to the PG (Stork et al., 2008). Moreover, cellular junctions precluding small molecule diffusion between cells are paramount to BBB function. The insect equivalent of the vertebrate TJ is the septate junction (SJ), consisting of similar molecular components, present between the SPG to prevent paracellular molecule diffusion (Wu and Beitel, 2004). Finally, the ABC drug efflux transporter Mdr65 is expressed in the SPG and is involved in chemical protection of the CNS, analogous to the function of its vertebrate homolog ABCB1 (Mayer et al., 2009). Fundamental CNS homeostasis requirements imposed across animal phyla suggest that numerous conserved BBB physiologies will likely be discovered in Drosophila.

We present here a transcriptome of the Drosophila BBB surface glia. We assess the relative enrichment of genes expressed at the BBB by comparing the surface glia transcriptome to that of all brain glia, brain neurons, and whole brains. By way of example we show that many genes found in the highly purified transcriptome are both highly expressed and enriched in the surface glia. We use gene set enrichment analyses to validate our surface glia transcriptome and show that the Drosophila surface glia possess many cellular processes and molecular functions also resident at the vertebrate BBB. Furthermore, we demonstrate that our data can be used to find many novel genes expressed in the surface glia. Lastly, we use BLAST to identify genes expressed in both invertebrate and vertebrate BBBs, pointing to likely evolutionary conserved mechanisms of BBB function that can now be tested using the Drosophila model system. Together with the vast and readily available molecular genetic tools for Drosophila, our data provide a wealthy resource for rapidly screening through a large group of conserved BBB proteins for phenotypes of interest.

\section{MATERIALS AND METHODS FLY GENETICS}

The following GAL4/UAS-GFP reporter lines were used to facilitate FACS of different CNS cell types: (1) 9-137-GAL4 (a P-element insertion line found in a screen of a large P-GAL4 collection (Ulrike Heberlein, Janelia Farm Research Campus, VA) that drives expression in the surface glia), crossed to pJFRC2, a previously published UAS-mCD8-GFP line (Pfeiffer et al., 2010)

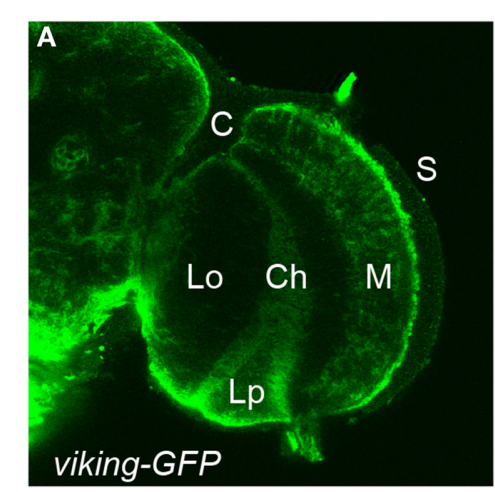

B

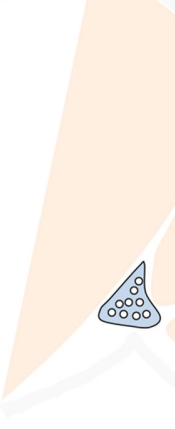

C

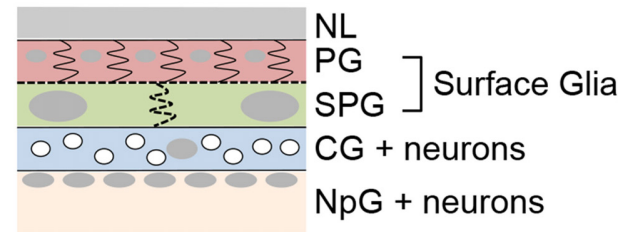

D

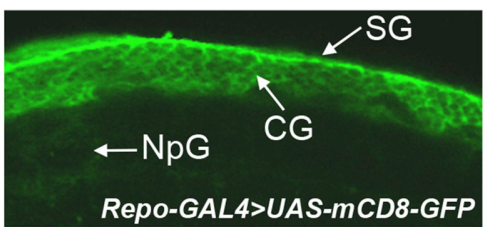

FIGURE 1 | Types of glia in the adult brain of Drosophila. (A) Different regions of the optic lobe of an adult brain as visualized by a GFP-fusion protein of the pan-glial gene, viking. Glia are located at the brain surface (S) and the cortex (C), in addition to the medulla (M), lobula (Lo), and lobular plate (Lp) neuropils. The chiasm $(\mathrm{Ch})$ is a glial region dividing the different neuropils. (B) A schematic of the optic lobe illustrating the different glial subtypes. Perineurial glia (PG) are seen in red, subperineurial glia (SPG) in green, and cortex glia (CG) in blue. The white circles within each cortex glia are neuronal cell bodies. Nuclei of neuropil glia (NpG) are in gray at the surface of the medulla neuropil. Nuclei of chiasm glia are in purple. (C) A cross section of the brain surface moving from the neural lamella (NL) to the neuropil. (D) Different glial subtypes at the brain surface as visualized by UAS-mCD8-GFP under control of the pan-glial driver repo-GAL4. 
available from the Bloomington Stock Center; (2) repo-GFP, a recombinant line of repo-GAL4 and UAS-mCD8-GFP that drives expression in all glia (Marc Freeman, UMass, MA); and (3) elav-GFP, a recombinant line of elav-GAL4 and UAS-mCD8GFP that drives expression in all neurons. Whole brain controls were of wildtype Canton-S. For anatomic characterization of 9-137-GAL4, we crossed this line to nuclear-localized GFP (UASStingerGFP) (Barolo et al., 2000) in addition to pJFRC2. FlyTrap lines are available from http://flytrap.med.yale.edu/ using genotype IDs stated in Figure 5.

\section{WHOLE BRAIN IMAGING}

Whole brain confocal images were acquired using previously reported methods (Mayer et al., 2009; DeSalvo et al., 2011; Pinsonneault et al., 2011). Briefly, flies were injected with $12.5 \mathrm{mg} / \mathrm{ml} 70 \mathrm{kDa}$ Dextran Texas Red ${ }^{\circledR}$ (Invitrogen, D1864) and left to recover overnight. Dextran labeling of the brains allows for demarcation of the surface glia barrier. Fly heads were fixed in situ for 15 min with $3.7 \%$ paraformaldehyde prior to brain dissection. Fixed brains were then incubated for $1 \mathrm{~h}$ at room temperature in a blocking buffer (PBS containing 5\% goat serum and $4 \%$ Tween $\left.{ }^{\circledR} 20\right)$, and then probed with rabbit anti-GFP antibody (Abcam ab6556, 1:1000 dilution) overnight at $4^{\circ} \mathrm{C}$. Brains were washed three times for $30 \mathrm{~min}$ in $1 \times$ PBS and incubated with FITC-conjugated goat anti-rabbit antibody (Jackson Immuno Research Laboratories, 1:100 dilution) for $45 \mathrm{~min}$ at room temperature. Brains were washed three times for $45 \mathrm{~min}$ in PBS and mounted in Dakocytomation Fluorescent Mounting Medium. Brains were visualized on a Zeiss LSM510 confocal microscope at $40 \times$ magnification.

\section{TISSUE PROCESSING AND FACS}

Brains were dissected under Schneider's media (SM) containing 1\% BSA (filter sterilized) and transferred directly to a tube containing ice-cold $500 \mu \mathrm{L}$ SM/BSA. Each tube contained 10-15 brains. Brains were washed with $1 \mathrm{~mL}$ SM/BSA and re-suspended in $220 \mathrm{uL} \mathrm{SM/BSA.} \mathrm{Collagenase} \mathrm{A} \mathrm{(Roche} \mathrm{No.} \mathrm{10103586001)}$ and DNase I were added to final concentrations of $2 \mathrm{mg} / \mathrm{mL}$ and 20 units, respectively. Brains were dissociated at $37^{\circ} \mathrm{C}$ in a Thermomixer according to the following conditions: $1000 \mathrm{rpm}$ for $20 \mathrm{~min}$ (elav/neurons), $500 \mathrm{rpm}$ for $20 \mathrm{~min}$ (repo/all glia), and $500 \mathrm{rpm}$ for $5 \mathrm{~min}$ (9-137/surface glia). EDTA ( $\mathrm{pH} 7$ ) was added to a final concentration of $5 \mathrm{mM}$ to inactivate the collagenase. Dissociated tissue was filtered through $100 \mu \mathrm{m}$ filter $(70 \mu \mathrm{m}$ for neurons) and immediately sorted using a $100 \mu \mathrm{m}$ nozzle on a BD FACSAria at the Laboratory for Cell Analysis at UCSF. Except whilst in the Thermomixer and the FACS machine, brains/cells were kept on ice.

FACS sorting was performed according to the following gating procedure: (1) an initial SSC-A/FSC-A gate to minimize debris; (2) a FSC-W/FSC-A gate to minimize doublets and large cellular aggregates; and (3) a PE-A/FITC-A gate to choose only GFPpositive cells. GFP-positive cells from multiple tubes were sorted into a single tube containing a small volume of sheath fluid. These cells were sorted again to increase purity, and during this sort, the initial scatter gate was modified to capture neurons and glia according to their unique scatter properties (see Figure 3). At this step, cells were sorted directly into ice-cold RNA lysis buffer (Ambion RNAqueous Micro Kit). When possible, re-sorted cells were analyzed again to determine their final purity levels. Of the five replicates for elav, an average of 9705 cells were sorted at an average purity of $95 \%$; repo, 8849 cells at $96 \%$ purity; and 9-137, 6093 cells at $93 \%$ purity.

\section{MICROARRAY DATA ACOUISITION AND ANALYSIS}

Each replicate for the microarray analysis represented sorted cells originating from different growth bottles and processed on different days. Five total replicates were run for each of the following genotypes: wildtype whole brain, and sorted GFP-positive cells from repo (all glia), elav (neurons), and 9-137 (surface glia). RNA was isolated using Ambion RNAqueous Micro columns and amplified using NuGEN's Ovation FFPE WTA System. Amplified RNA was processed and hybridized to Affymetrix Drosophila Genome 2.0 GeneChips at the Gladstone Institutes Genomics Core facility. All microarray data analysis was performed using $\mathrm{R} /$ Bioconductor packages (Gentleman et al., 2004). CEL files were read and raw data normalized using RMA in the affy package (Irizarry et al., 2003; Gautier et al., 2004). Prior to statistical analysis, probes were filtered if the Present sum was less than 4 for all genotypes; in other words, a probe needed to be expressed in at least one genotype to be included. This metric was shown to significantly reduce false positives (McClintick and Edenberg, 2006). 7090 probes were filtered according to this criterion. The remaining probes were used for statistical analyses in the limma package (Smyth, 2004). Pairwise comparisons were performed for all possible combinations, and fold changes and standard errors were estimated by fitting a linear model for each gene. Empirical Bayes smoothing to the standard errors was applied and differentially expressed genes were chosen according to an FDR-adjusted $P<0.05$. Lists of differentially expressed genes were trimmed to ensure that genes were indeed expressed in the enriched genotype (expression $>100$ AND Present sum $\geq 4$ ). Gene set enrichment analyses of the differentially expressed genes were performed using default settings in DAVID Bioinformatics (Huang et al., 2009a,b). Significantly enriched gene sets were identified using a Benjamini-adjusted $P<0.05$.

\section{BLAST ANALYSIS}

Using Ensembl Biomart (Kinsella et al., 2011), RefSeq protein IDs were retrieved for 144 known mouse BBB proteins according to Daneman (2012) and Zlokovic (2008). We chose to ignore proteins listed by Daneman (2012) that were up-regulated in the mouse BBB during disease. A fasta file containing all protein sequences was generated using Batch Entrez (http://www.ncbi. nlm.nih.gov/sites/batchentrez), and these sequences were compared to a BLAST-able database containing all Drosophila proteins using the BLAST+ command line (blastp with a E-value cutoff of $10^{-5}$ ). The blast2table perl script was used to parse the output file showing only the top HSP for each BLAST hit. We then linked the RefSeq protein ID for each fly BLAST hit to its corresponding Affymetrix probe IDs, which allowed us to annotate each BLAST hit with its expression and enrichment values in the surface glia transcriptome. Positive expression in the surface glia was assessed by having a Present sum $\geq 4$ AND an expression 
level $\geq 100$. Surface glia enrichment was assessed by having a positive enrichment relative to neurons OR whole brain.

\section{RESULTS}

\section{ISOLATION OF SURFACE GLIA RNA}

To purify the BBB surface glia from adult Drosophila brains, we used a GAL4/UAS genetic approach (Brand and Perrimon, 1993) to fluorescently label the surface glia. To do this, we first identified the 9-137 enhancer trap line, which drives GAL4 expression specifically in the surface glia. When crossed to UAS-GFP reporter lines, the 9-137-GAL4 results in specific GFP labeling of the PG and SPG (Figure 2), allowing the specific isolation of surface glia to a purity of $>90 \%$ using FACS (Figure 3 ). We used a similar protocol to isolate neurons and a more inclusive population of glia. All brain glial subtypes (Figure 1), including surface glia, were isolated from flies expressing UAS-mCD8-GFP under the control of the pan-glial driver repo-GAL4 (Xiong et al., 1994); neurons were specifically isolated using the pan-neuronal driver elav-GAL4 (Campos et al., 1987; Robinow and White, 1988). Total RNA from five replicate samples for each of surface glia, all glia, neurons, and whole brains were amplified and hybridized to Affymetrix Drosophila Genome 2.0 GeneChips for downstream transcriptomic analysis. All microarray data is deposited in Gene Expression Omnibus (GSE45344), and the master matrix of filtered normalized data used as input for statistical analyses is found in Table S1. While we chose to focus on the surface gliaenriched transcriptome in this study, the comprehensive data set generated here is valuable for other avenues of research. For example, the data may provide broad insight into glial biology when analyzed for gene enrichment of additional glial subtypes such as the adult cortex and neuropil glia (Figure 1).

\section{THE SURFACE GLIA TRANSCRIPTOME}

To gain insight into the genes required for specialized BBB functions in Drosophila, we first looked at transcript abundance in the surface glia. The microarray data were normalized using RMA in the R/Bioconductor package affy (Irizarry et al., 2003; Gautier et al., 2004). Table 1 reports the 50 most abundant transcripts in the surface glia together with their ratiometric enrichments relative to whole brain, neurons, and all glia. We acknowledge that Affymetrix expression signals are not perfect reflections of gene expression levels since signal values are the result of several factors both biological and technical. However, this list does appear to reveal specialized gene expression in the surface glia. For example, extracellular matrix collagens ( $v \mathrm{~kg}$ and $\mathrm{Cg} 25 \mathrm{C}$ ) are both highly expressed and highly enriched, and a SLC5 sodiumiodide symporter (CG5687) is the most enriched gene among the top 50 most abundant surface glia transcripts. Therefore, the data in Table 1 suggest that Affymetrix signal abundance for the surface glia samples is somewhat indicative of surface glia functional requirements.

To further investigate the specialized functions of the BBB, we determined the differentially expressed genes in surface glia by performing pairwise comparisons to whole brain, neurons, and all glia using limma software (Smyth, 2004). Table S2 contains differentially expressed genes for all pairwise comparisons performed in limma. Table 2 reports the top 50 enriched surface glia genes for each comparison. Relative to whole brain, there are 1010 genes up-regulated (i.e., enriched) and 3899 genes down-regulated in the surface glia. Relative to neurons, there are 1183 genes up-regulated and 2979 genes downregulated in surface glia. Relative to all glia, there are 543 genes up-regulated and 568 genes down-regulated in surface glia. Expression fold changes are greatest in the surface glianeuron comparison $(\max =729$, mean $=15.6$, median $=3.7)$, followed by the surface glia-brain comparison $(\max =247$, mean $=6.0$, median $=2.9$ ) and the surface glia-all glia comparison $(\max =35$, mean $=4.5$, median $=3.5)$. This enrichment trend can in part be explained by the amount of surface glia RNA in each comparison sample. Neuronal samples contain
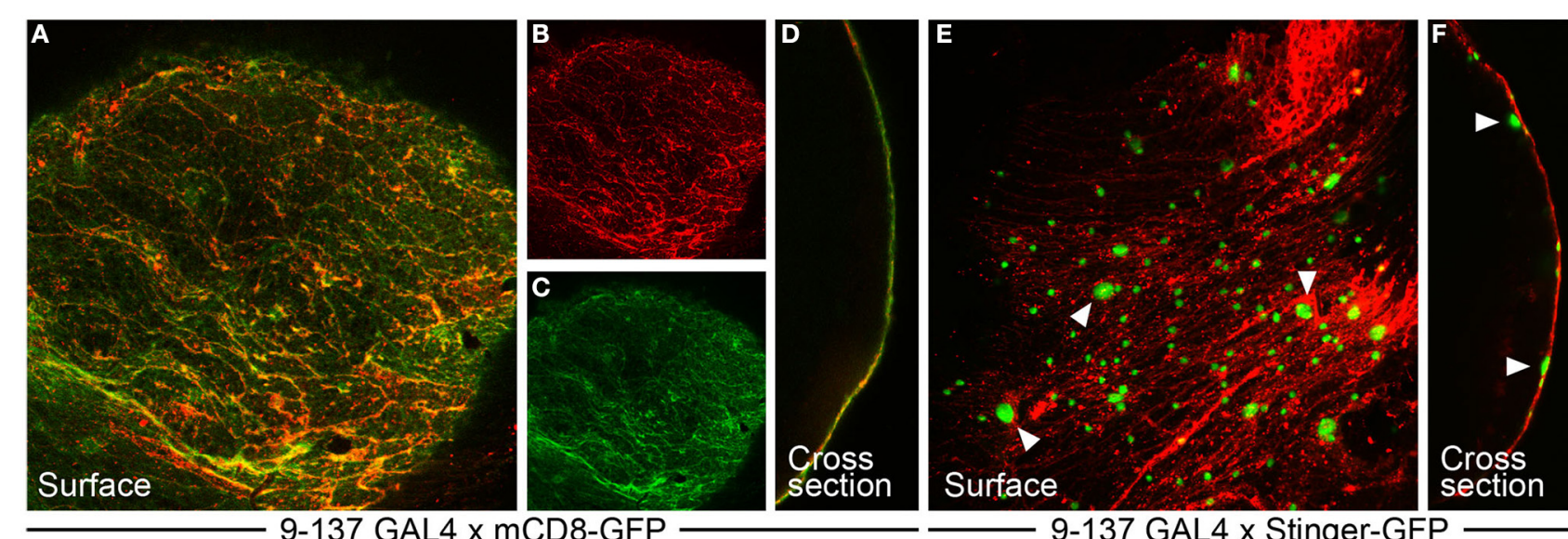

9-137 GAL4 x Stinger-GFP

FIGURE 2 | The 9-137 P-GAL4 line specifically drives expression in the surface glia layer. (A-D) 9-137-GAL4 crossed to mCD8-GFP (membrane-bound) shows hallmarks of surface glia expression. (A-C) GFP expression at the surface colocalizes with dextran, which marks the boundaries of the PG cells in a characteristic flagstone pattern. (D) The cross-section image shows complete overlap between dextran and GFP. (E,F) 9-137-GAL4 crossed to Stinger-GFP (nuclear-localized) demarcates small PG and large SPG nuclei. Arrowheads mark representative large SPG nuclei. (F) The cross-section image shows PG nuclei embedded in the dextran layer with SPG nuclei positioned below the dextran/PG layer. 


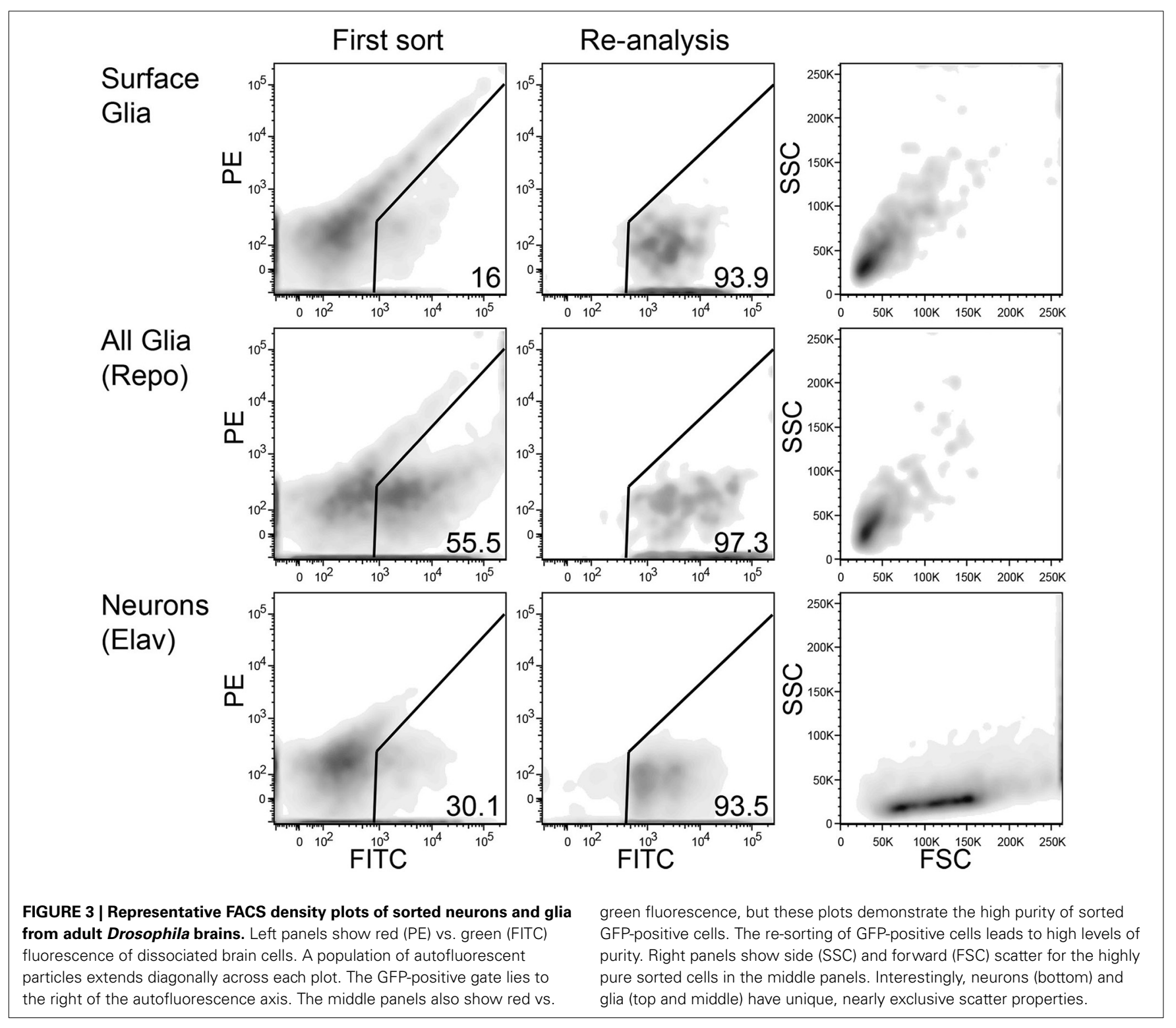

no surface glia, brain samples contain a small proportion of surface glia, and all glia samples contain a substantial proportion of surface glia mixed with other glial subtypes. Thus, as expected, the number of differentially expressed genes and their fold changes is maximal in the surface glia-neuron comparison reflecting sample cell-type homogeneity and likely functional specialization.

\section{VALIDATING THE SURFACE GLIA TRANSCRIPTOME AS A BBB GENE PROFILE}

To infer specialized molecular pathways present in the surface glia from their transcriptome, we performed gene set enrichment analyses using DAVID Bioinformatics (Huang et al., 2009a,b). Table 3 lists selected enriched Gene Ontology (GO) categories, KEGG pathways, Interpro domains, and PIR superfamilies among genes enriched in surface glia relative to brain, neurons, and all glia (for all results see Table S3). The DAVID Bioinformatics results support the view of the surface glia being the primary component of the Drosophila BBB.

Consistent with the surface glia being a chemical protection interface, we see numerous enriched categories associated with drug metabolism, cell adhesion, and transport (Table 3). Selected genes in these categories are listed in Table 4 and reveal striking signatures of chemical protection physiology. For example, there are numerous cytochrome P450 (CYP), glutathione S-transferase (GST), and UDP-glucuronosyltransferase (UGT) enzymes enriched in the surface glia. These enrichment results account for all phases of drug metabolism. Phase I reactions include oxidation reactions by CYPs; phase II reactions include conjugation reactions, such as glucoronidation by UGTs and glutathionylation by GSTs; and phase III reactions involve excretion of drug metabolites by transporters (Sheweita, 2000; Homolya et al., 2003). These excretory transporters are often $\mathrm{ABC}$ transporters. Notable $\mathrm{ABC}$ transporters involved in drug 
Table 1 | The 50 highest expressed genes in the surface glia (SG) transcriptome.

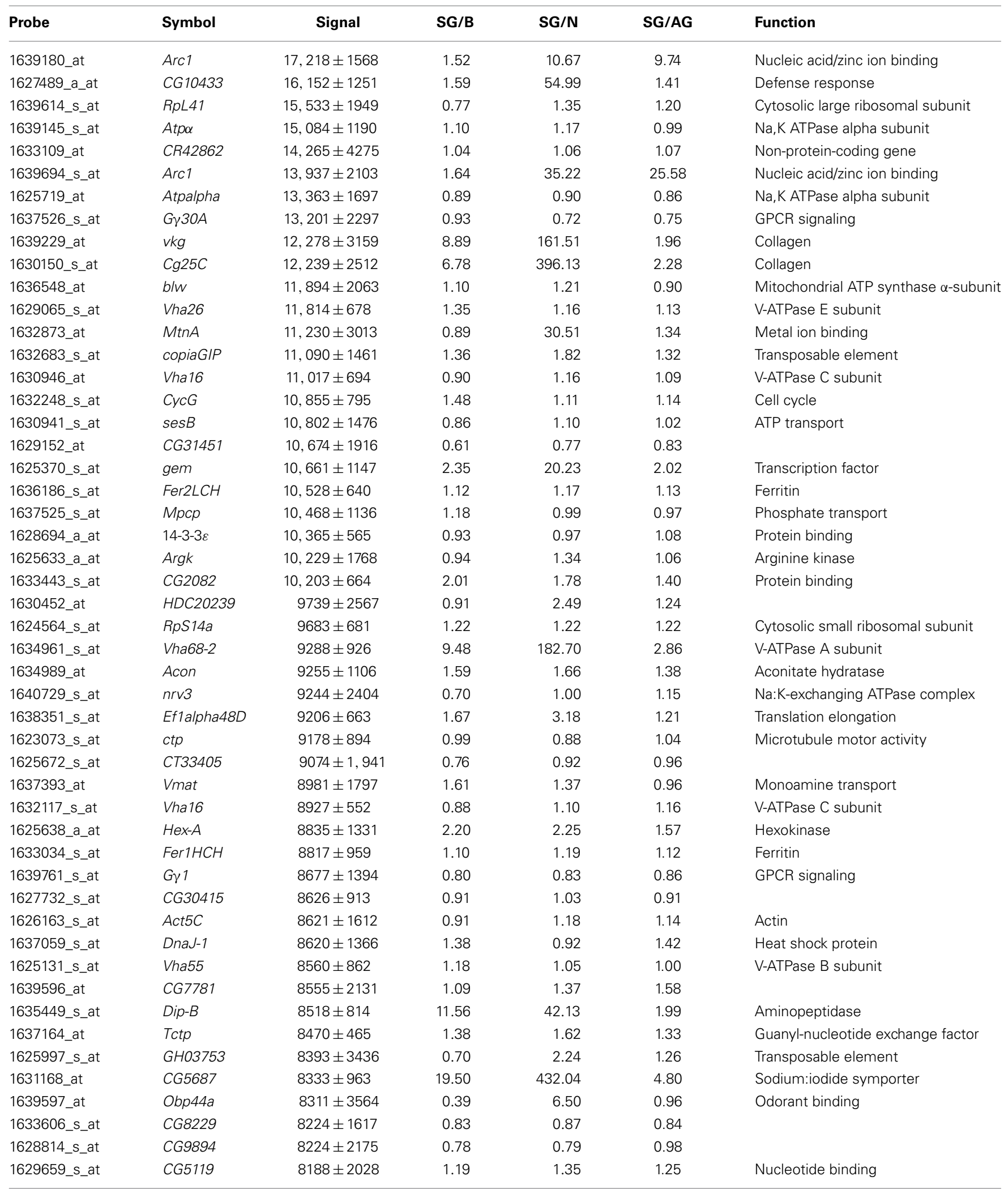

Signals (with standard deviations) represent the average of five replicate normalized expression values. Enrichments in SG relative to brain (B), neurons (N), and all glia (AG) are simple ratios and do not reflect statistically significant differences. 
Table 2 | The 50 most enriched surface glia genes according to three separate comparisons.

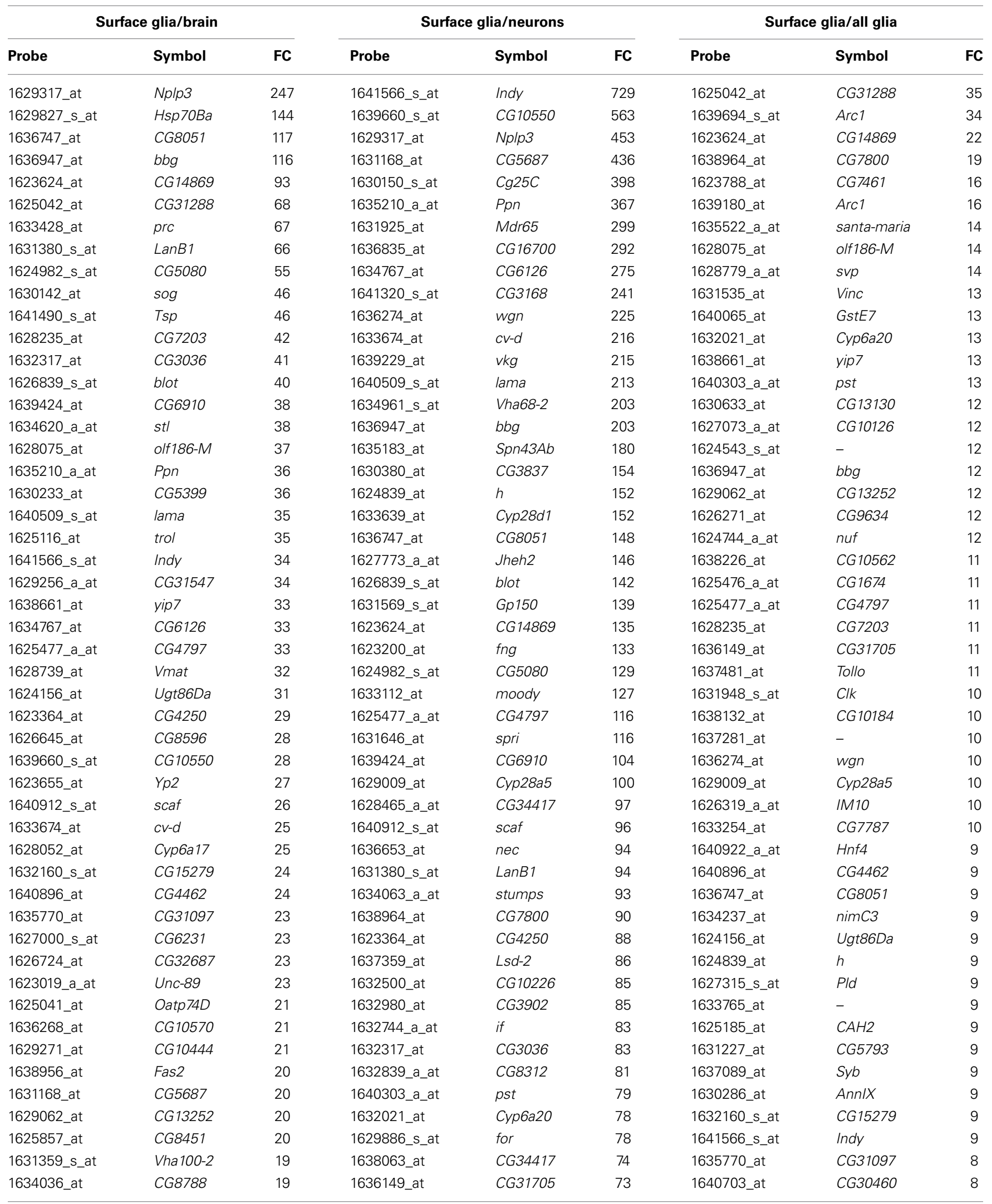

Fold changes (FC) are limma-estimated, and all genes are statistically significant according to an FDR-adjusted $P<0.05$. 
Table 3 | Selected results from gene set enrichment analyses performed using DAVID Bioinformatics on differentially expressed genes enriched in surface glia (SG) relative to brain (B), neurons (N), and all glia (AG).

\begin{tabular}{|c|c|c|c|c|c|c|c|}
\hline Enriched term & Category & \multicolumn{2}{|c|}{ SG/B } & \multicolumn{2}{|c|}{ SG/N } & \multicolumn{2}{|c|}{ SG/AG } \\
\hline dme00040:Pentose and glucuronate interconversions & KEGG pathway & & & 12 & 4.6E-02 & & \\
\hline dme00980:Metabolism of xenobiotics by cytochrome P450 & KEGG pathway & & & 20 & 1.6E-03 & & \\
\hline GO:0004364 glutathione transferase activity & GO MF & & & 11 & $3.2 \mathrm{E}-02$ & & \\
\hline GO:0007155 cell adhesion & GO BP & 22 & $2.8 \mathrm{E}-02$ & 30 & 8.5E-04 & 21 & 4.5E-03 \\
\hline \multicolumn{8}{|l|}{ TRANSPORTERS } \\
\hline GO:0015293 symporter activity & GO MF & 16 & 2.4E-02 & 19 & 8.4E-03 & 14 & 1.4E-02 \\
\hline GO:0015849 organic acid transport & GO BP & & & 12 & 4.7E-02 & & \\
\hline IPR001140:ABC transporter, transmembrane region & Interpro domain & 8 & $5.0 \mathrm{E}-02$ & & & & \\
\hline GO:0005605 basal lamina & $\mathrm{GO} C \mathrm{C}$ & 5 & 1.4E-03 & 5 & 3.3E-03 & & \\
\hline GO:0031012 extracellular matrix & GO CC & 18 & $5.5 \mathrm{E}-07$ & 19 & 2.4E-06 & 11 & 4.1E-03 \\
\hline \multicolumn{8}{|l|}{ METABOLISM } \\
\hline dme00071:Fatty acid metabolism & KEGG pathway & 11 & 1.2E-02 & 14 & 2.3E-03 & 9 & $2.2 \mathrm{E}-02$ \\
\hline GO:0016052 carbohydrate catabolic process & GO BP & & & 23 & 4.1E-06 & & \\
\hline \multicolumn{8}{|l|}{ MISCELLANEOUS } \\
\hline GO:0001666 response to hypoxia & GO BP & 7 & 3.0E-02 & & & & \\
\hline GO:0005811 lipid particle & GO CC & 39 & $6.2 \mathrm{E}-06$ & 73 & $2.4 \mathrm{E}-21$ & 23 & $1.9 \mathrm{E}-02$ \\
\hline GO:0006952 defense response & GO BP & 22 & $2.8 \mathrm{E}-02$ & 26 & 2.3E-02 & & \\
\hline GO:0022626 cytosolic ribosome & GO CC & & & 31 & 1.2E-09 & & \\
\hline GO:0045185 maintenance of protein location & GO BP & 12 & $1.5 \mathrm{E}-03$ & & & & \\
\hline
\end{tabular}

GO MF, Gene ontology molecular function; BP, biological process; and CC, cellular component. P-values are Benjamini-adjusted.

metabolism and efflux include members of the $\mathrm{B}$ and $\mathrm{C}$ class, such as ABCB1 and MRP1-4 (ABCC1-4). Our results show three $B$ class $A B C$ transporters to be highly enriched in the surface glia (Mdr65, Mdr49, and CG10226). These three transporters are all highly homologous to vertebrate $\mathrm{ABCB} 1$, and we have previously shown that Mdr65 has a conserved function in drug efflux at the apical surface of the Drosophila BBB (Mayer et al., 2009). Other enriched transporters in the surface glia include numerous SLC transporters, reflecting the function of the surface glia as a metabolic barrier similar to the vertebrate vascular endothelial BBB component.

In compliance with the BBB functioning as a diffusion barrier, numerous genes constituting SJs are enriched in the surface glia, including the components Fasciclin 2 (Fas2), lethal (2) giant larvae (l(2)gl), Neuroglian ( $\mathrm{Nrg})$, and nervana 2 (nrv2) (Table 4). Also enriched is Moody, a GPCR involved in a signaling pathway that regulates SJ formation (Bainton et al., 2005). Interestingly, the innexin gap junction genes ogre (inx1) and in $x 2$ are enriched in the surface glia and Drosophila inx1 and inx2 have recently been linked to coordination of neural stem cell proliferation in response to the metabolic status of the animal (Spéder and
Brand, 2014). Thus, the surface glia transcriptome may provide insight into higher order BBB processes. Overall, gene set enrichment analyses of the surface glia transcriptome confirms that the surface glia possess characteristics of BBB physiology.

Although the expression and large enrichment of genes listed in Table 4, especially moody and Mdr65, indicate that our cell isolation techniques are of high purity, we wanted to further validate our approach. Therefore, we searched the literature for genes known to be expressed and functional in the surface glia. We selected 29 genes represented by 36 probe IDs (listed in Table 5 ). We primarily focused on genes where mutations and/or RNAimediated knockdown lead to BBB leakiness indicating that the expressed genes function to maintain BBB integrity. If our surface glia transcriptome is valid, we would expect these 29 genes to be present in our data. To decide whether a gene is expressed, we look at two values: (1) the average normalized expression level of five replicate samples and (2) the sum of five replicate sample Present calls for the gene's probe(s). While there are no standards for calling a gene expressed based on Affymetrix data, we set a threshold of a Present sum $\geq 4$ and/or an expression level $>100$. For example, moody and Mdr65, two genes enriched 
Table 4 | Differentially expressed genes enriched in surface glia (SG) relative to brain (B), neurons (N), and all glia (AG) involved in characteristic BBB structures (the basal lamina) and physiologies (drug metabolism, cellular junctions, and transport).

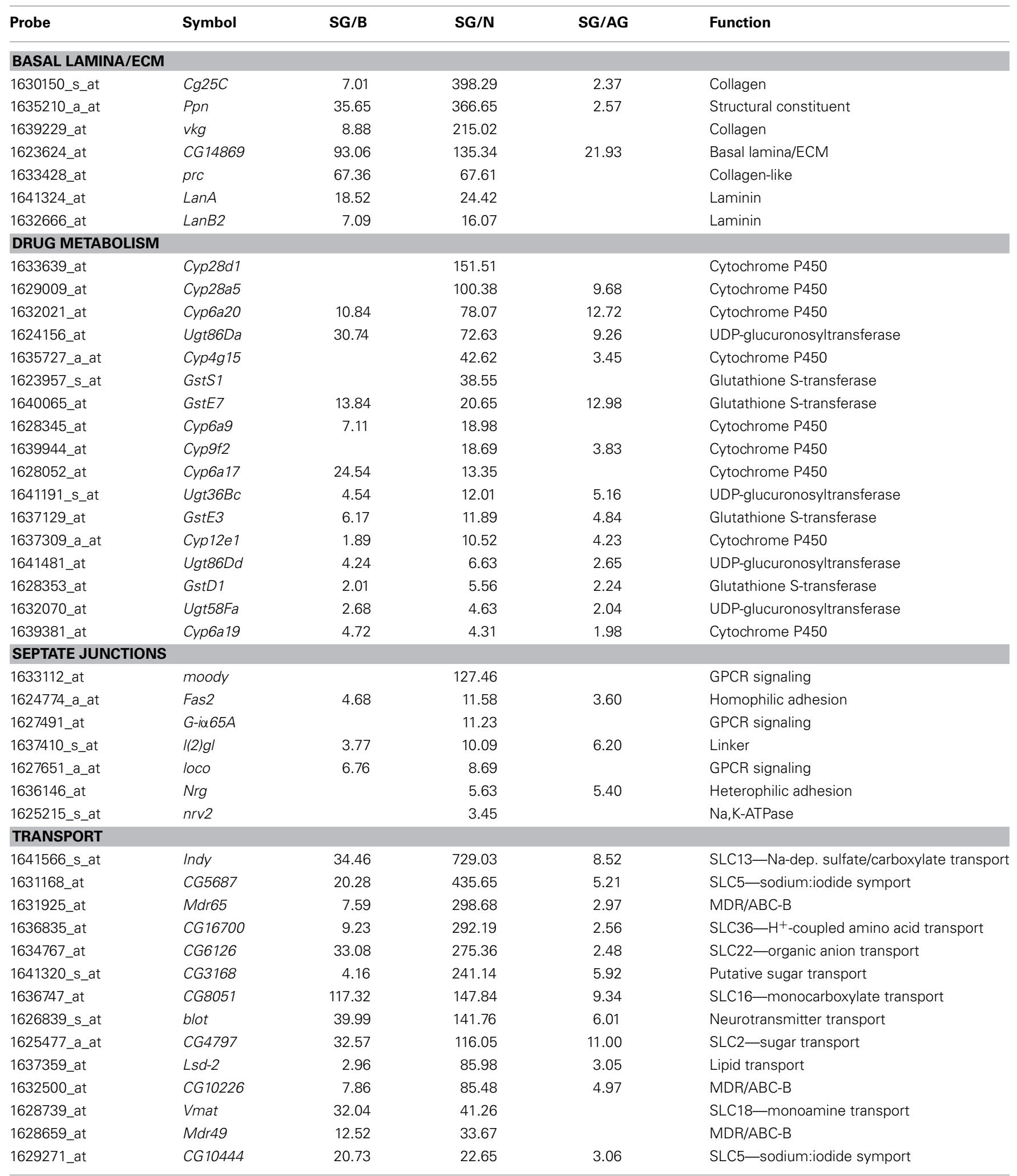

Enrichment values are limma-estimated fold changes, and all genes are significant according to an FDR-adjusted $P<0.05$. 
Table 5 | Gene expressed in surface glia at any stage of development according to the literature.

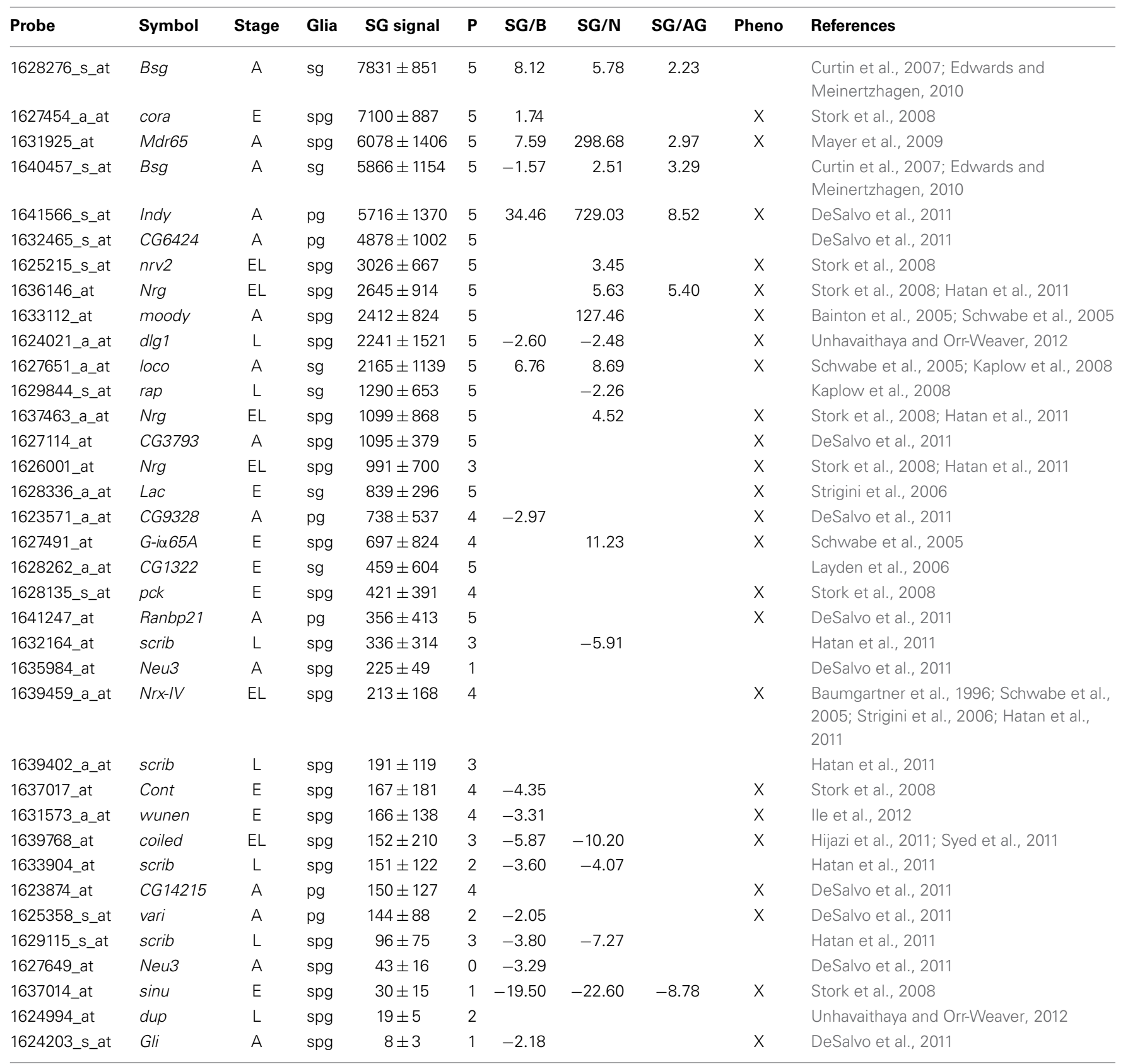

We found 29 genes for which 36 probes are listed here-genes are often represented by multiple probes (e.g., Nrg). For "Stage," E, embryo; L, larvae; and A, adult. The "Glia" column specifies which glia layer the gene is expressed in-sg, both surface glia layers; spg, subperineurial glia; and pg, perineurial glia. Signals (with standard deviations) represent the average of five replicate normalized expression values. $P=$ the $P$ sum, or the number of replicates (max $=5$ ) for which the probe was called present. Enrichment values are limma-estimated fold changes significant at an FDR-adjusted $P<0.05$. An $X$ in the "Pheno" column denotes that disrupting expression or function of the gene produces a BBB phenotype-usually leakage of large MW dextrans into the brain.

in the surface glia, have average normalized expression levels of 2412 and 6078 units, respectively. Both genes also have Present call sums of 5, meaning they were called Present in all 5 replicates of sorted surface glia samples. According to this metric, most of the genes in Table 5 can be classified as being expressed in our samples of surface glia. However, our microarray methods did not recognize expression of the following known surface glia genes
(Present sum < 4 AND expression < 100): sinuous (sinu), double parked (dup), and Gliotactin (Gli). In addition, it is not clear via Affymetrix GeneChips data whether the following genes are expressed in surface glia (Present sum $<4$ OR expression $<100$ ): Neu3, scrib, vari, and coiled (cold). However, preliminary data from deep sequencing of the surface glia transcriptome indicate that the above undetected genes are expressed in the BBB glia, 
and that dup, Gli, Neu3, scrib, and vari are also enriched in the BBB glia (data not shown). This suggests that although we can be reasonably confident in the genes present in our surface glia microarray, we need to be cautious in our interpretation of the absent calls, as these could be false negatives.

Next, we tested whether the 29 surface glia genes, as a group, have greater expression levels and Present sums compared to all other genes on the microarray. With respect to gene expression level, a two-sample Kolmogorov-Smirnov test revealed a significant difference in the distribution of expression values for known surface glia genes and all other genes on the microarray $(D=$ $0.3442, p=0.0004)$. This difference can be seen graphically in Figure 4: a density plot (Figure 4A) and a cumulative frequency plot (Figure 4B) of $\log 2$ surface glia expression for both groups

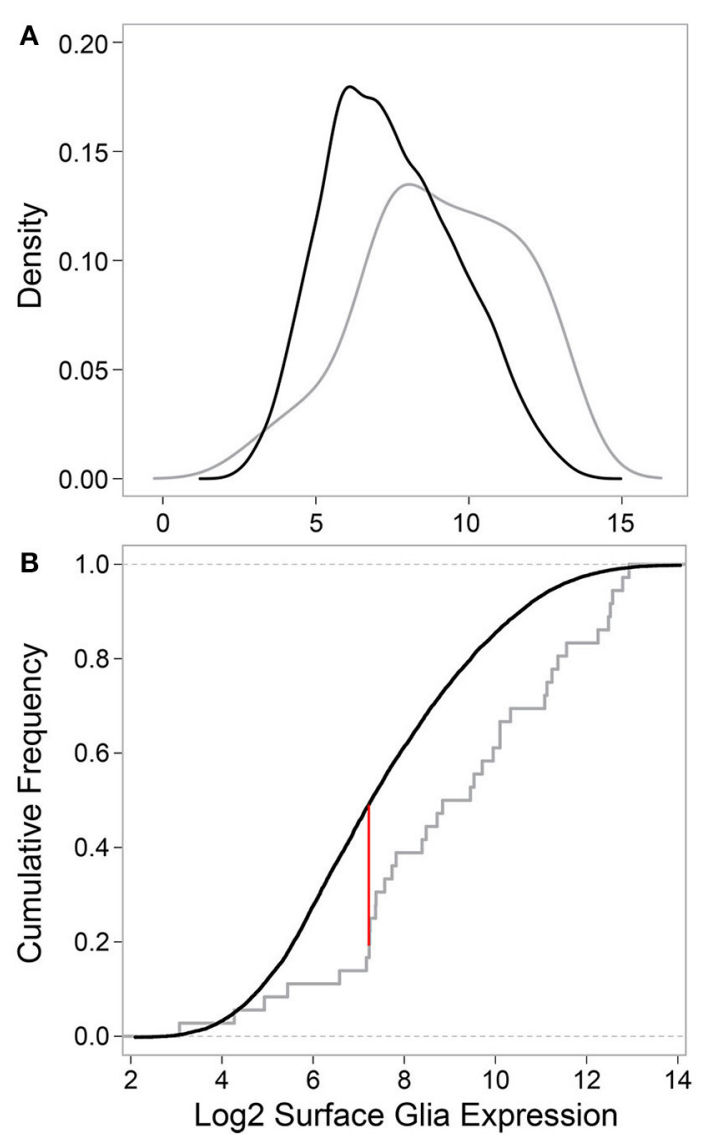

FIGURE 4 | Comparing data for known surface glia genes to all other probes on the microarray validates the surface glia transcriptome.

(A) Kernal density estimates based on surface glia log2 gene expression for known surface glia genes (gray) and all other probes on the microarray (black). The distribution of data for known surface glia genes is significantly greater than that for all other probes on the microarray (Two-sample Kolmogorov-Smirnov test, $D=0.3435, P=0.0004$ ). (B) A cumulative frequency plot of the same data also illustrates the significant difference in distributions between known surface glia genes and all other probes on the microarray. The red line corresponds to the D-statistic, the point of greatest separation between the curves. At this point, $81.6 \%$ of known surface glia genes have higher expression levels, which is in stark contrast to $51.7 \%$ for all other probes on the microarray. of genes showing that, by and large, known surface glia genes have greater expression values than all other genes on the microarray. With respect to Present sum data, we used a hypergeometric test to determine whether the distribution of Present sums in known surface glia genes was significantly different (and greater) than that for all other genes on the microarray. According to our stringent standard of requiring a Present sum $\geq 4$ to call a probe expressed, we find that 24 of 36 (67\%) probes representing known surface glia genes have a Present sum $\geq 4$. Comparing this to 4488 of 11645 (39\%) of the remaining probes on the array, the hypergeometric test indeed finds a significant difference between these distributions $(p=0.0002)$. Even if we loosen our criteria and allow a Present sum $\geq 3$, there is still a significant difference ( 29 of 36 [81\%] for known surface glia genes and 6063 of 11645 [52\%] for all other probes- $\left.p=9.5 \times 10^{-5}\right)$. Overall, we find that our microarray data shows positive expression for known surface glia genes leading us to conclude that the surface glia transcriptome is highly quantitative and accurate.

Central to validating our cell isolation and transcriptomic techniques is showing that genes identified as enriched in the surface glia are indeed expressed in these cell layers. To demonstrate surface glia-localized expression, we searched the FlyTrap GFP Protein Trap database (http://flytrap.med.yale.edu/) (Morin et al., 2001; Kelso et al., 2004; Buszczak et al., 2007; QuinonesCoello et al., 2007) for protein and enhancer traps available for surface glia-enriched genes. As a control, we also included one gene (Vha55) that, based on our transcriptome, was roughly equally expressed in neurons, glia, and surface glia. We also searched the Bloomington Stock Collection for transgenic lines containing GFP-fusion proteins for any surface glia-enriched genes, and found one for hairy $(h)$. Lastly, we targeted the expression of VMAT using polyclonal antibodies specific to the gliaspecific isoform of VMAT (DVMAT-B) previously found to store histamine in the Drosophila visual system (Romero-Calderon et al., 2008). Microarray data for all genes in Figure 5 are listed in Table S4. The images in Figure 5 indeed demonstrate that surface glia-enriched genes identified in the microarray data are expressed in these cell layers. However, surface glia enrichment does not equate to specific expression in the surface glia. Many surface glia-enriched genes are also expressed in other glial subtypes. For example, the first gene shown in Figure $\mathbf{5}$ is viking $(v k g)$, which encodes a collagen IV protein, and is enriched 9-fold relative to whole brain and 215 -fold relative to neurons. The GFP protein trap for $v \mathrm{~kg}$ shows pan-glial expression (see also Figure 1 for another image of the $v k g$ protein trap), which is consistent with a previous study (Freeman et al., 2003). Interestingly, the $v k g$ protein trap also allows for visualization of the neural lamella, and its enrichment in surface glia suggests deposition of the neural lamella by the surface glia.

Surface glia-localized expression can be assessed using both brain surface and cross-section confocal images (Figure 5). Colocalization with injected $70 \mathrm{kDa}$ dextran helps to differentiate the PG from the SPG since dextran demarcates the PG layer (DeSalvo et al., 2011). Expression in the surface glia can be identified by any of the following characteristics: (1) surface images show a "flagstone" pattern of cell junctions characteristic of the PG, e.g., Vha55, I'm not dead yet (Indy), and l(2)08717; (2) 

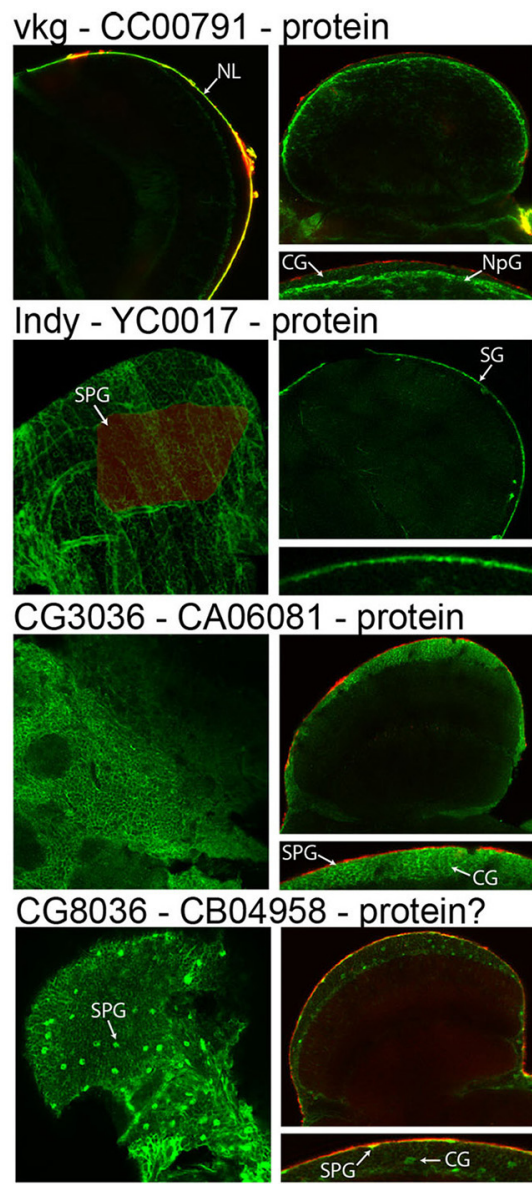

for - CB02956 - enhancer

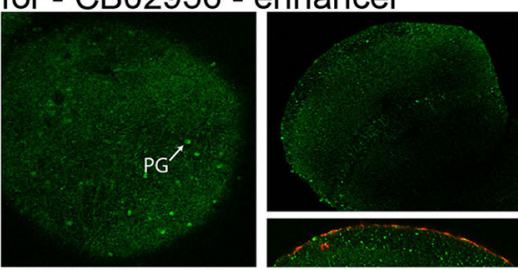

VMAT-B - antibody

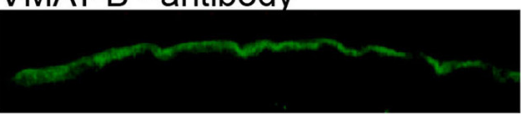

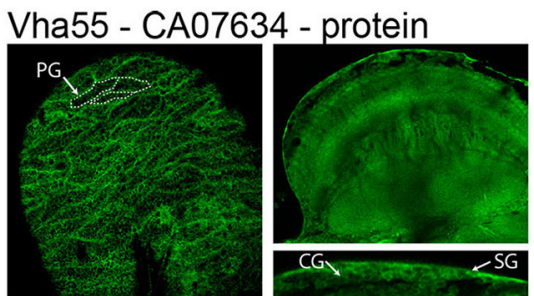

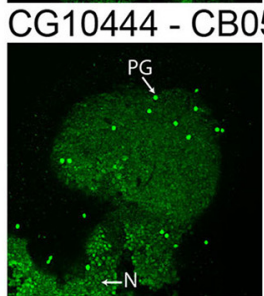

h - protein

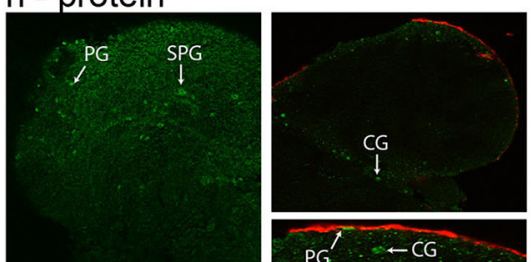

Tsp42Ed - YC0084 - protein

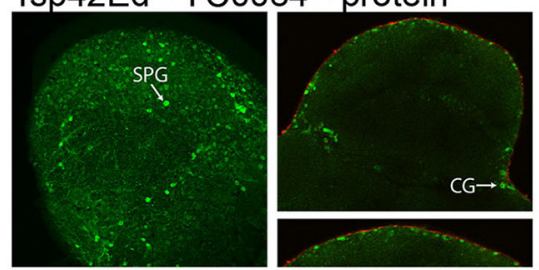

Ilk - ZCL3111 - protein

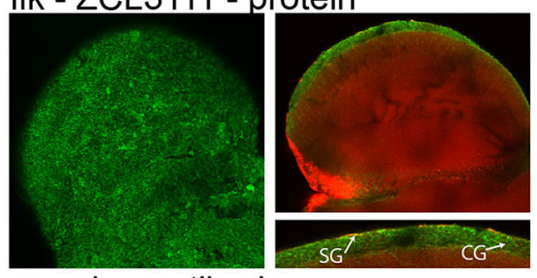

moody - antibody

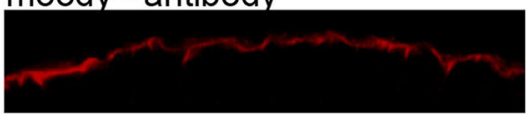

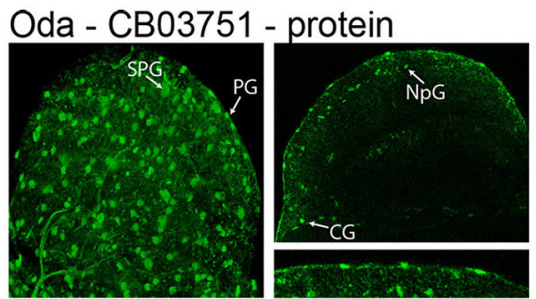

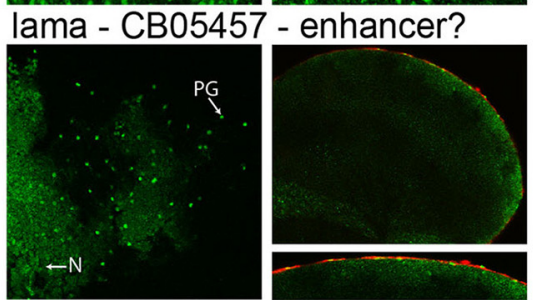

inx2 - P01999 - enhancer

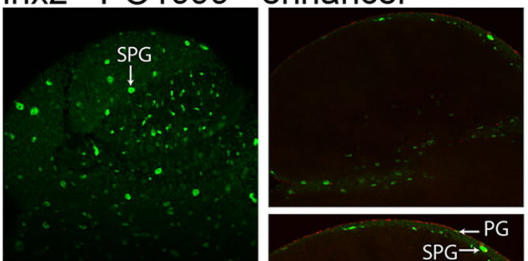

(2)08717 - CA06962 - protein

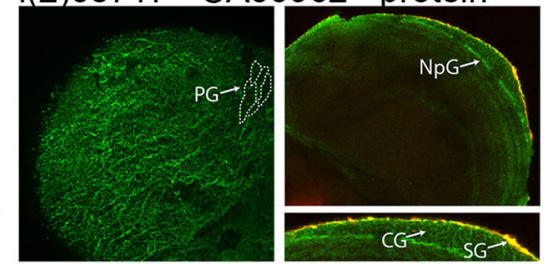

apt - CC01186 - protein
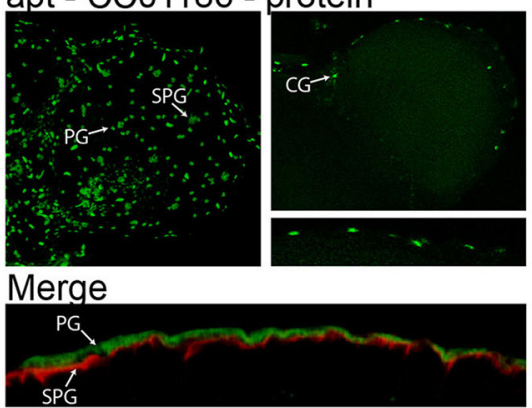

FIGURE 5 | Expression and localization of surface glia-enriched genes. Gene symbols are followed by the FlyTrap database ID number and whether the GFP exon results in a GFP-fusion protein (Protein), likely results in a GFP-fusion protein (Protein?), is controlled by nearby enhancers (Enhancer), or is likely controlled by nearby enhancers (Enhancer?). With the exception of $v k g$, each FlyTrap line has a surface image followed by a cross section image and a zoomed-in image of the surface glia layer in cross section. For $v k g$, instead of a surface image, we present a cross-section showing the GFP-positive neural lamella. Localization to the surface glia layer can be assessed using surface images that show membrane GFP at the boundaries of perineurial glia (PG) [see dotted lines in Vha55 and /(2)08717] or subperineurial glia (SPG) (see shaded cell in Indy). In cross section images of membrane GFP, localization to the surface glia can be determined because injection with $70 \mathrm{kDa}$ rhodamine dextran demarcates the PG layer (no dextran was used for Vha55, Oda, apt, and VMAT-B). For nuclear GFP, surface images reveal small PG nuclei and large SPG nuclei. In cross section, these nuclei are within the dextran layer (PG-localized) or slightly below (SPG-localized). Many surface glia-enriched genes are also expressed in other glial subtypes, such as cortex glia (CG) and neuropil glia (NpG), and in rare cases we found that surface glia-enriched genes were also expressed in neurons (N). VMAT-B expression is visualized with polyclonal antibodies (green) and localized by co-staining with antibodies to the SPG-specific Moody protein (red). surface images show the SJ boundaries characteristic of the SPG, e.g., Indy; (3) surface and cross-section images show numerous, small PG nuclei, e.g., Ornithine decarboxylase antizyme (Oda), CG10444, innexin 2 (inx2), lamina ancestor (lama), foraging (for), and apontic (apt); and (4) surface and cross-section images show sparse, large SPG nuclei, e.g., Oda, CG8036, $h$, inx2, and apt. Furthermore, many of the images (e.g., Vha55, Indy, CG3036, and CG8036) show a pattern of GFP expression similar to that seen 
when staining adult brains with antibodies to the Moody protein (Schwabe et al., 2005; Mayer et al., 2009). This pattern resembles a mosaic of small circular cell junctions, which is due to either (1) contact between the basal surface of the SPG and the underlying cortex glia, or (2) expression in both the SPG and cortex glia. Indeed, many of the surface glia-enriched genes are also expressed in the cortex glia. Membrane-bound GFP expression in cortex glia is seen in CG3036, CG8036, l(2)08717, and Integrin-linked kinase (Ilk). Nuclear-GFP expression in cortex glia is seen in $\mathrm{Oda}, h$, Tetraspanin 42Ed (Tsp42Ed), and apt.

Regarding expression of the glia-specific DVMAT-B, we co-stained adult brains with Moody and DVMAT-B antibodies. Moody is specifically expressed in the SPG (Bainton et al., 2005; Mayer et al., 2009), and the results show DVMAT-B expression apical and non-overlapping with Moody, thus pinpointing DVMAT-B to the PG layer (Figure 5). Of the 16 surface gliaenriched genes shown in Figure 5, we found that Vmat and Indy were the only surface glia-specific genes in the adult brain. Furthermore, we also found that one of the protein traps (Ilk GFP) caused the BBB to be leaky, indicated by the large accumulation of $70 \mathrm{kDa}$ dextran in the brains. Ilk is expressed in surface and cortex glia. In this case, GFP fusion likely disrupts protein function leading to $\mathrm{BBB}$ leakiness. In addition to showing that surface glia-enriched genes are indeed expressed in these cell types, we also found that our control gene Vha55 had a more global expression pattern in the brain. This is consistent with our transcriptome, further confirming the validity of our cell isolation and transcriptomic methods. Thus, these data provide a suitable starting point for an investigator interested in elucidating gene function in surface glia-localized processes.
While little is known about the fly surface glia, there is a wealth of knowledge on neuronal physiology where many proteins and processes are highly conserved among metazoans (Venter et al., 1988; Anderson and Greenberg, 2001). Thus, we can further validate our cell isolation and transcriptomic methods by analyzing neuronal genes and pathways. As anticipated, overrepresented gene set enrichment categories related to neuronal physiology are seen for genes enriched in whole brains, neurons, and all glia samples relative to those of the surface glia (Table 6). Neurons are 3-fold more numerous than glia in the fly brain (Pfrieger and Barres, 1995), thus, it was to be expected that the genes enriched in our whole brain and neuron samples largely function in synaptic transmission, axonogenesis, vesicle-mediated transport, regulation of neurotransmitter levels, and neurotransmitter receptor activity. Genes enriched in all glia relative to the surface glia include those involved in axon guidance, neuron development, and synapse formation. These functions are consistent with the activities of the abundant cortex and neuropil glia, which are more intimately involved in neuronal development and function (Edwards and Meinertzhagen, 2010).

\section{ADULT BBB TRANSCRIPTOME POINTS TO GENES REQUIRED THROUGHOUT DEVELOPMENT FOR BBB FUNCTION}

Having shown that our adult BBB transcriptome is a reliable resource, we were now well positioned to ask how the adult BBB profile compares to that of the embryo. We used data from the Berkeley Drosophila Genome Project (BDGP) in situ database (http://insitu.fruitfly.org/cgi-bin/ex/insitu.pl) to obtain gene expression patterns in the embryonic CNS. The BDGP in situ database classifies in situ gene expression patterns during embryonic development according to a controlled vocabulary

Table 6 | Selected results from gene set enrichment analyses performed using DAVID Bioinformatics on differentially expressed genes enriched in brain (B), neurons (N), and all glia (AG) relative to surface glia (SG).

\begin{tabular}{|c|c|c|c|c|c|c|c|}
\hline Enriched term & Category & \multicolumn{2}{|c|}{ SG/B } & \multicolumn{2}{|c|}{ SG/N } & \multicolumn{2}{|c|}{ SG/AG } \\
\hline GO:0007268 synaptic transmission & GO BP & 90 & 6.7E-10 & 61 & 3.8E-06 & & \\
\hline GO:0007409 axonogenesis & GO BP & 80 & 1.3E-05 & 76 & $9.1 \mathrm{E}-12$ & 17 & $1.4 \mathrm{E}-02$ \\
\hline GO:0007411 axon guidance & GO BP & 58 & 8.4E-05 & 57 & 2.2E-10 & 13 & $2.8 \mathrm{E}-02$ \\
\hline GO:0007611 learning or memory & GO BP & 32 & 2.4E-02 & 26 & 8.0E-03 & & \\
\hline GO:0008038 neuron recognition & GO BP & & & 23 & 1.1E-04 & & \\
\hline GO:0008355 olfactory learning & GO BP & & & 18 & 2.2E-02 & & \\
\hline GO:0016192 vesicle-mediated transport & GO BP & 136 & $1.8 \mathrm{E}-05$ & 85 & 4.2E-02 & & \\
\hline GO:0016319 mushroom body development & GO BP & & & 20 & $5.2 \mathrm{E}-03$ & & \\
\hline GO:0019933 cAMP-mediated signaling & GO BP & 11 & 3.3E-02 & 11 & 1.6E-03 & & \\
\hline GO:0045202 synapse & $\mathrm{GO} C \mathrm{C}$ & 65 & 6.4E-08 & 53 & 3.7E-09 & & \\
\hline GO:0048512 circadian behavior & GO BP & 22 & $1.5 \mathrm{E}-03$ & 17 & 4.3E-03 & & \\
\hline GO:0048666 neuron development & GO BP & 131 & 2.9E-07 & 114 & 1.0E-12 & 26 & $2.9 \mathrm{E}-03$ \\
\hline
\end{tabular}

GO BP, Gene ontology biological process; MF, molecular function; and CC, cellular component. P-values are Benjamini-adjusted. 
that corresponds to specific anatomic structures (Tomancak et al., 2002, 2007). The BDGP vocabulary includes gene expression in the lateral cord surface glia and central brain surface glia for embryos in stages 13-16. We asked whether surface glia-enriched genes in the adult brain are more likely to have embryonic surface glia in situ staining compared to non-surface glia-enriched genes. Indeed, we found that genes enriched in adult surface glia relative to whole brain are more likely to contain embryonic surface glia in situ staining (Chi-squared test with Yates' continuity correction: $X^{2}=4.76, d f=1, p$-value $=0.0291$, odds ratio $=2.12$ ). We also obtained a significant result for genes enriched in surface glia relative to neurons $\left(X^{2}=7.32, d f=\right.$ $1, p$-value $=0.0068$, odds ratio $=2.26)$. The results point to 18 genes that are expressed in surface glia during all stages of development and adulthood (in situ images can be found on the BDGP website). These genes include: (1) six known glial

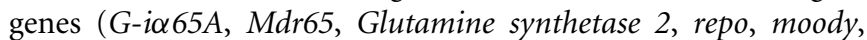
and $n r v 2$ ) with previously published in situ images (Xiong et al., 1994; Auld et al., 1995; Freeman et al., 2003; Schwabe et al., 2005); (2) six annotated genes that to our knowledge were not known to be expressed in surface glia throughout development (babos, Minichromosome maintenance 5, Hsp27, Major Facilitator Superfamily Transporter 3, pericardin, and mutagen-sensitive 209); and (3) six non-annotated genes (CG10702, CG11164, CG3168, CG4829, CG5080, and CG6126). These findings already highlight a good starting point for furthering our knowledge of embryonic $\mathrm{BBB}$ formation, maintenance and function. Together with profiling of the embryonic $\mathrm{BBB}$, our adult $\mathrm{BBB}$ transcriptome would provide a valuable resource to gain further insight into BBB dynamics during development.

\section{NEURONAL PHYSIOLOGY GENES IDENTIFIED IN THE MICROARRAY DATA}

Interestingly, we observed some SJ components [e.g., sinuous (sinu), scribbled (scrib), varicose (vari), Contactin (Cont), and discs large $1(d \lg 1)$ ] were enriched in brain and neurons relative to surface glia (Table S2). Assuming these genes are mainly involved in SJ formation, this observation suggests the existence of SJs amongst cells of the adult brain besides the surface glia. Although neuropil glia in the adult CNS ensheath axons (Edwards and Meinertzhagen, 2010), very little has been published on SJs among cells other than the surface glia. It seems likely that other cells form SJs given that this has been found in the peripheral nervous system (Banerjee and Bhat, 2008). Furthermore, enrichment of SJ genes in neurons points to neuron-localized expression of these genes. In the embryonic and larval peripheral nervous system, axons are ensheathed by inner and outer glial membranes involving the expression of SJ proteins Neurexin IV (Nrx-IV), Cont, and Nrg (Banerjee et al., 2006). All three of these proteins are expressed in the glial cells, while Nrg is also expressed by the neurons. Similarly, Nrx-IV is expressed by neurons in the larval CNS where it mediates glial wrapping but is independent of SJ formation (Stork et al., 2008). Given these results, it seems likely that neurons of the adult CNS express SJ components thereby mediating axon insulation by ensheathing glia. This is consistent with axo-glial SJs at the nodes of Ranvier of myelinated axons in the vertebrate nervous system (Bhat et al., 2001; Bhat, 2003).

\section{BBB GENES CONSERVED BETWEEN DROSOPHILA AND VERTEBRATES}

Using BLAST, we compared the fly and mouse BBB transcriptomes to assess what BBB genes are conserved across evolution. We took a focused approach by targeting known molecular components of the vertebrate BBB covered in reviews by Zlokovic (2008) and Daneman (2012). These proteins are central to the BBB's role as a diffusion barrier (TJs), chemoprotective interface (drug transporters), and conduit for metabolite passage between the blood and brain (SLC transporters). In short, we retrieved sequences for 144 known proteins expressed at the mouse BBB and searched for BLAST homologs $\left(E<10^{-5}\right)$ in the fly genome. Fly BLAST hits were then annotated with our surface glia transcriptome data to determine which fly homologs are expressed and/or enriched at the BBB.

\section{TIGHT AND ADHERENS JUNCTION PROTEINS}

Of the $16 \mathrm{TJ}$ components targeted in our comparative analysis, nine have fly homologs expressed in the surface glia, six of which have homologs enriched in the surface glia (Table 7). According to our methods, the fly genome does not contain true homologs for seven TJ proteins: claudin 3, claudin 5, claudin 12 , occludin, immunoglobulin superfamily member 5 , peripheral myelin protein 22, and lipolysis stimulated lipoprotein receptor. The importance of some of these proteins to TJ formation is well documented (Hirase et al., 1997; Saitou et al., 2000; Nitta et al., 2003), and the absence of true fly homologs perhaps highlights the differing composition of cell-cell junctions between flies and vertebrates (Wu and Beitel, 2004). We note that claudinlike proteins have been characterized in Drosophila (i.e., Sinu, Megatrachea, and Kune-kune), and they are involved in SJ formation (Behr et al., 2003; Wu et al., 2004; Nelson et al., 2010); however, their level of homology with vertebrate claudins is less than the significance level chosen in our BLAST analysis.

The calcium/calmodulin-dependent serine protein kinase (MAGUK family) protein (CASK-same symbol in mouse and fly) is the only highly conserved TJ protein $(E=0)$ co-expressed at both the fly and mouse BBBs. Other strong BLAST hits $(E<$ $10^{-40}$ ) exist for: membrane associated guanylate kinase, WW and PDZ domain containing 1 (MAGI1); multiple PDZ domain protein (MPDZ); and TJ proteins 1 and 2 (TJP1 and TJP2), which are nearly equally homologous to fly PYD. Interestingly, CASK, MAGI1, TJP1, and TJP2 are all membrane-associated proteins containing guanylate kinase and PDZ domains. MPDZ also contains PDZ domains. These proteins help link transmembrane proteins to the cytoskeleton and bind signaling complexes together (PDZ domain) (Ranganathan and Ross, 1997), and function in signaling themselves (guanylate kinase domain). Our results thus point to strong evolutionary conservation of such proteins at the BBB.

With the exception of PYD, most of the fly homologs of mouse TJ proteins, while expressed in the surface glia, are not specifically enriched. Most of the enriched fly homologs are weakly homologous $\left(E>10^{-30}\right)$; for example, PPN, FAS2, and CG7981 are highly enriched in the surface glia but are weakly homologous $\left(E>10^{-10}\right)$ to mouse JAM-A. However, with respect to adherens junction proteins, there are various highly conserved proteins $(E=0)$ co-expressed at the fly and mouse BBBs, which 
Table 7 | Fly genes homologous to known junctional components expressed at the mouse BBB.

\begin{tabular}{|c|c|c|c|c|c|c|c|c|c|c|c|c|}
\hline Mouse prot & No. exp. & Best hit & $\mathbf{E}$ & SG exp. & SG/B & SG/N & No. enr. & Fly prot & $\mathbf{E}$ & SG exp. & SG/B & SG/N \\
\hline \multicolumn{13}{|l|}{ Adherens junctions } \\
\hline CDH5 (VE-cadherin) & 4 & CADN & 2.E-44 & 1863 & & -2.4 & 0 & & & & & \\
\hline CTNNA1 ( $\alpha$-catenin) & 3 & $\alpha$-CAT & 0 & 924 & & -3.7 & 1 & VINC & 4.E-13 & 2475 & 11.8 & 29.9 \\
\hline JUP ( $\gamma$-catenin) & 1 & ARM & 0 & 3511 & & -1.9 & 0 & & & & & \\
\hline PECAM1 & 1 & CG42330 & 1.E-07 & 518 & -4.9 & -1.9 & 0 & & & & & \\
\hline \multirow{4}{*}{ CASK } & & & & & & & & Unc-89 & 1.E-29 & 952 & 22.5 & 20.6 \\
\hline & & & & & & & & $\mathrm{PHK} \gamma$ & 3.E-50 & 1931 & -2.8 & 2.3 \\
\hline & & & & & & & & Lk6 & 3.E-35 & 1352 & -2.3 & 2.2 \\
\hline & & & & & & & & Par-1 & 1.E-37 & 1182 & -2.2 & 1.7 \\
\hline CGNL1 (JACOP) & 1 & ZIP & $6 . \mathrm{E}-21$ & 2240 & & & 0 & & & & & \\
\hline F11R (JAM-A) & & & & & & & & NRG & 4.E-07 & 2645 & & 5.6 \\
\hline \multirow[t]{2}{*}{ MAGI1 } & 15 & Magi & 4.E-92 & 297 & & & 2 & CG33967 & 3.E-17 & 997 & 2.3 & 6.3 \\
\hline & & & & & & & & GRIP & 2.E-09 & 325 & 10.0 & 4.5 \\
\hline MARVELD2 (TRIC) & 1 & Su(Tpl) & 4.E-11 & 1811 & & & 0 & & & & & \\
\hline MPDZ (MUPP1) & 11 & Patj & 2.E-68 & 810 & & & 1 & PYD & 2.E-07 & 3927 & 5.6 & -6.9 \\
\hline TJP1 (ZO1) & 3 & PYD & 6.E-94 & 3927 & 5.6 & -6.9 & 1 & PYD & 6.E-94 & 3927 & 5.6 & -6.9 \\
\hline TJP2 (ZO2) & 4 & PYD & 2.E-92 & 3927 & 5.6 & -6.9 & 1 & PYD & 2.E-92 & 3927 & 5.6 & -6.9 \\
\hline
\end{tabular}

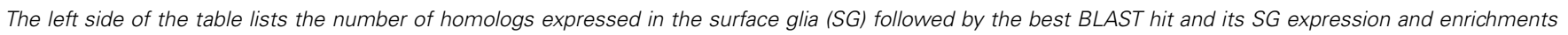
relative to brain (B) and neurons (N). The right side lists all homologs enriched in the surface glia. No significant BLAST hits for Cldn5, Cldn12, Cldn3, Ocln, Igsf5, Pmp22, and Lsr.

include $\alpha$-Catenin, and $\beta$ - and $\gamma$-Catenin (equally homologous to fly Armadillo). A high BLAST hit $\left(E=2 \times 10^{-44}\right)$ also exists for VE-cadherin. These results indicate that adherens junction proteins are conserved throughout evolution and function at the BBB in both flies and mice.

Overall, strong conservation between TJ proteins is absent, but we do see conservation between junctional adaptor proteins and adherens junction proteins. These trends were found previously (Knust and Bossinger, 2002) and are not surprising given the ultrastructural differences between the TJ and SJ. In vertebrates, the TJ is apical to the adherens junction, but in Drosophila the $\mathrm{SJ}$ is basal to the adherens junction. Some of the fly homologs to TJ proteins are localized at a comparable site termed the marginal zone or subapical region (Knust and Bossinger, 2002; Wu and Beitel, 2004), similarly, some of the mouse homologs to SJ proteins are localized at a comparable site termed the basal region (Wu and Beitel, 2004). In conclusion, our results point to the Drosophila BBB and SJ complex being a relevant model for the role of adaptor proteins and adherens junction proteins in regulating the diffusion barrier at the $\mathrm{BBB}$.

\section{TRANSPORTERS}

Besides junctional proteins, the best-studied components of the $\mathrm{BBB}$ are the diverse array of ABC and SLC transporters. BLAST hits expressed and enriched at the fly $\mathrm{BBB}$ to our targeted set of extensively studied, functionally important mouse BBB transporters (Table 8) reveal striking sequence conservation. For example, mouse BBB transporters that have highly conserved homologs $\left(E<10^{-90}\right)$ expressed at the fly BBB include: GLUT1, CAT1, LAT1, EAAT1, ABCB1A, BCRP, MRP1, and MRP5. Furthermore, the same homologs for LAT1, ABCB1A, BCRP, MRP1, and MRP5 are not only expressed but also enriched at the fly $\mathrm{BBB}$, and they are among a group of highly conserved homologs enriched at the fly BBB (e.g., there are three fly proteins enriched at the $\mathrm{BBB}$ with high homology to $\mathrm{ABCB} 1 \mathrm{~A}$ ). These results point to strong selective pressure for the conservation of BBB-localized transport of glucose, amino acids, and the numerous $\mathrm{ABC}$ transporter substrates, thus indicating that across species it is these proteins that are essential for the function of a selective barrier.

Interestingly, we also see that the best BLAST hit is often not the most BBB-enriched homolog. For example, fly GLUT1 is the most homologous fly protein to mouse GLUT1 $\left(E=10^{-124}\right)$. Fly GLUT1 is expressed at the BBB (surface glia expression $=711$ ), but it is enriched in neurons. However, six other less homologous BLAST hits are highly enriched in surface glia. CG3168 and CG4797 are both annotated as putative sugar transporters with weak homology to GLUT1 $\left(E=6 \times 10^{-9}\right.$ and $2 \times 10^{-15}$, respectively), but they are enriched 214 - and 116-fold in surface glia relative to neurons, respectively. We obtained similar results for MCT1 and LRP1, thus indicating that neurons and surface glia in the adult fly brain might use different transporters to transport 
Table 8 | Fly genes homologous to known transporters expressed at the mouse BBB (for notes on table format, see Table 7).

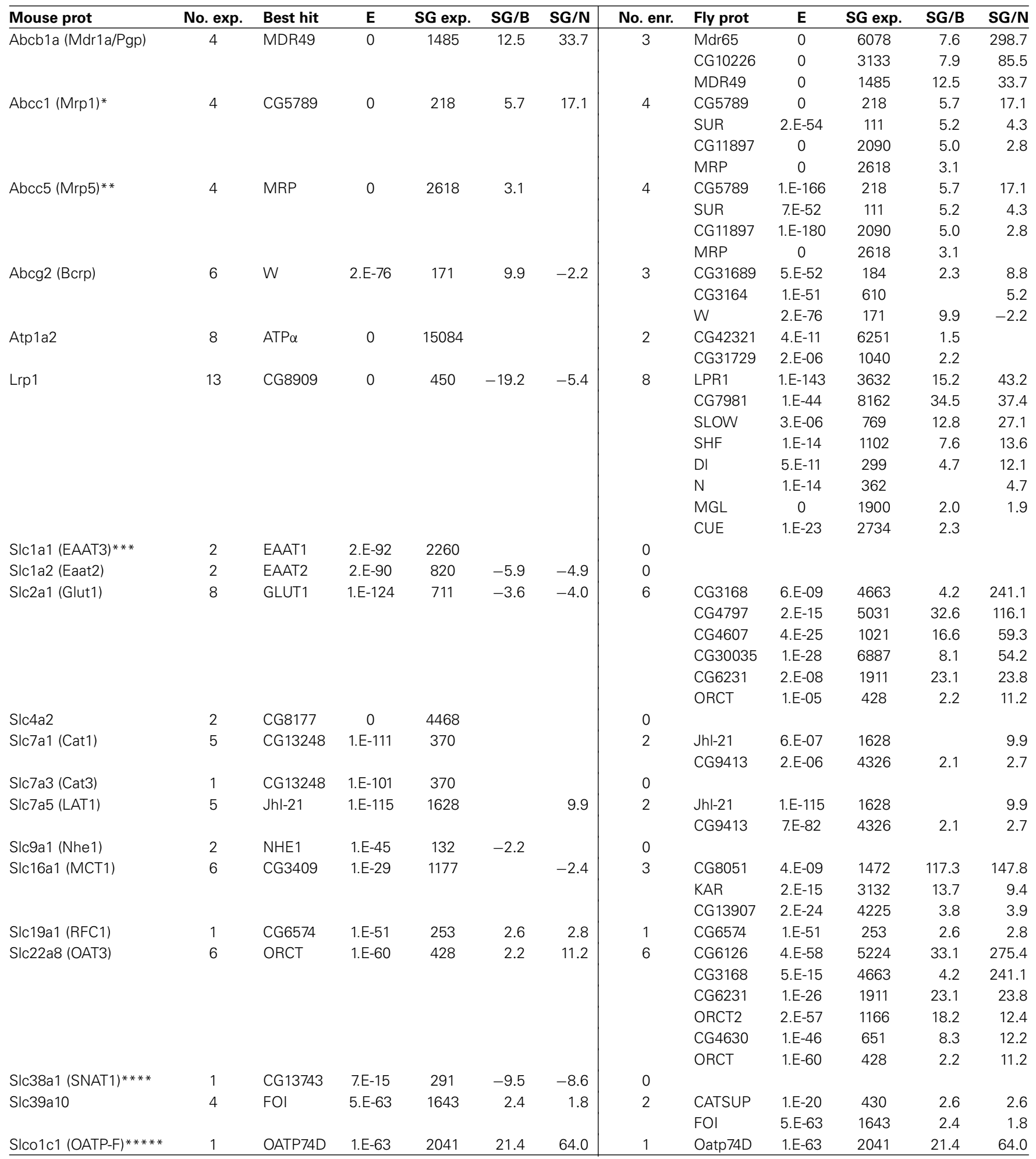

Significant BLAST hits that are not expressed in surface glia: Slc19a2, Slc19a3, Slc30a1, and Tfrc.

*Nearly identical results for Abcc4 (Mrp4);

**Nearly identical results for Abcc6 (Mrp6);

$* *$ Nearly identical results for Slc1a3 (Eaat1);

****Nearly identical results for Slc38a3 (SNAT3) and SIc38a5 (SNAT5);

****Nearly identical results for Slco1a4 (Oatp2) and Slco2b1 (OATP-B). 
sugar, monocarboxylates, and lipoproteins, and may allow differential regulation over substrate entry into the brain vs. neuronal uptake.

Overall, we see that the fly and mouse BBBs contain highly homologous transporters, which highlights the importance of these transporters in chemical protection and the transport of metabolites at the BBB regardless of the cell type that expresses them. Our previous investigations on the efflux transporter Mdr65 (Mayer et al., 2009), a fly homolog of ABCB1A, taken together with the elucidation of many more highly homologous BBB transporters in the present study, points to the Drosophila surface glia $\mathrm{BBB}$ as a relevant model for studying evolutionary conserved $\mathrm{BBB}$-localized transport properties despite the fact that it is of glial rather than endothelial origin.

\section{OTHER NOTABLE GENES CO-EXPRESSED AT THE BBB}

Noteworthy results among eight additional mouse BBB genes and their fly homologs (Table 9) include the co-enrichment of carbonic anhydrases at the mouse and fly BBBs, which indicates a conserved $\mathrm{BBB}$ physiology focused on brain carbon dioxide and bicarbonate homeostasis. We also see very high homology between mouse and fly gamma-glutamyltranspeptidase (GGT1) protein sequences. In vertebrates, GGT1 is expressed at the luminal surface of the BBB where it functions in both amino acid transport and the regulation of glutathione levels (and thus the detoxification processes involving GSTs) (Courtay et al., 1992; Hawkins et al., 2006). An alternative explanation might be that the surface glia express GGT1 similar to astroctyes, where they function to facilitate glutathione synthesis in neurons (Valdovinos-Flores and Gonsebatt, 2012). Another striking result in Table 9 is the conservation of insulin signaling at the mouse and fly BBBs. The Drosophila insulin receptor (InR) is the best BLAST hit to both mouse INSR and IGF1R ( $E=0$ for both). Our data suggest that $\operatorname{InR}$ is expressed in surface glia at low levels; however, another BLAST hit to mouse INSR, CG3837 $\left(E=3 \times 10^{-75}\right)$, is highly enriched in the surface glia. CG3837 was recently identified as a secreted decoy of the insulin receptor (SDR) (Okamoto et al., 2013). SDR acts as an antagonist of insulin signaling and its secretion into the hemolymph by the surface glia of larvae controls body growth; SDR mutants have an abnormally rapid growth rate resulting in larger adult body size. FlyAtlas (Chintapalli et al., 2007) data indicate that $I n R$ is nearly equally expressed in all tissues, whereas CG3837/SDR expression is more restricted, with the highest expression in the CNS. Our microarray data and the data in (Okamoto et al., 2013)

Table 9 | Fly genes homologous to known genes expressed at the mouse BBB (for notes on table format, see Table 7).

\begin{tabular}{|c|c|c|c|c|c|c|c|c|c|c|c|c|}
\hline Mouse prot & No. exp. & Best hit & $\mathbf{E}$ & SG exp. & SG/B & SG/N & No. enr. & Fly prot & $\mathbf{E}$ & SG exp. & SG/B & SG/N \\
\hline $\mathrm{Bsg}$ & 4 & $\mathrm{ROBO}$ & 4.E-10 & 901 & -6.1 & & 1 & CG31605 & 2.E-09 & 7831 & 8.1 & 5.8 \\
\hline \multirow[t]{3}{*}{ Car4 } & 4 & $\mathrm{CAH} 2$ & 2.E-26 & 860 & 10.0 & & 3 & $\mathrm{CAH} 1$ & 8.E-19 & 799 & -2.4 & 3.6 \\
\hline & & & & & & & & CG11284 & 7.E-18 & 1756 & 5.6 & 2.5 \\
\hline & & & & & & & & $\mathrm{CAH} 2$ & 2.E-26 & 860 & 10.0 & \\
\hline Ggt1 & 2 & CG4829 & 1.E-103 & 1132 & -3.6 & 2.6 & 1 & CG4829 & 1.E-103 & 1132 & -3.6 & 2.6 \\
\hline \multirow[t]{8}{*}{$\operatorname{lgf1r}$} & 37 & INR & 0 & 135 & & & 8 & CG3837 & 3.E-78 & 2097 & 5.7 & 153.9 \\
\hline & & & & & & & & $\mathrm{HTL}$ & 2.E-53 & 422 & 3.0 & 19.9 \\
\hline & & & & & & & & CG10702 & 2.E-56 & 635 & 3.6 & 16.5 \\
\hline & & & & & & & & FPS85D & 2.E-46 & 1257 & 14.1 & 9.7 \\
\hline & & & & & & & & PVR & 4.E-36 & 944 & 5.5 & 6.1 \\
\hline & & & & & & & & $\mathrm{PHK} \gamma$ & 1.E-11 & 1931 & -2.8 & 2.3 \\
\hline & & & & & & & & Par-1 & 1.E-09 & 1182 & -2.2 & 1.7 \\
\hline & & & & & & & & HOP & 7.E-40 & 2470 & 2.0 & -3.3 \\
\hline \multirow[t]{9}{*}{ Insr (IR) } & 41 & INR & 0 & 135 & & & 9 & CG3837 & 3.E-75 & 2097 & 5.7 & 153.9 \\
\hline & & & & & & & & FOR & 3.E-09 & 697 & 10.9 & 77.9 \\
\hline & & & & & & & & HTL & 1.E-53 & 422 & 3.0 & 19.9 \\
\hline & & & & & & & & CG10702 & 7.E-72 & 635 & 3.6 & 16.5 \\
\hline & & & & & & & & Fps85D & 7.E-48 & 1257 & 14.1 & 9.7 \\
\hline & & & & & & & & PVR & 2.E-35 & 944 & 5.5 & 6.1 \\
\hline & & & & & & & & $\mathrm{PHK} \gamma$ & 9.E-13 & 1931 & -2.8 & 2.3 \\
\hline & & & & & & & & Par-1 & 1.E-10 & 1182 & -2.2 & 1.7 \\
\hline & & & & & & & & HOP & 9.E-37 & 2470 & 2.0 & -3.3 \\
\hline \multirow[t]{6}{*}{$\mathrm{Kdr}(\mathrm{Flk}-1)$} & 32 & HTL & 4.E-68 & 422 & 3.0 & 19.9 & 7 & for & 2.E-11 & 697 & 10.9 & 77.9 \\
\hline & & & & & & & & UNC-89 & 2.E-10 & 952 & 22.5 & 20.6 \\
\hline & & & & & & & & $\mathrm{HTL}$ & 4.E-68 & 422 & 3.0 & 19.9 \\
\hline & & & & & & & & FPS85D & 3.E-31 & 1257 & 14.1 & 9.7 \\
\hline & & & & & & & & PVR & 1.E-51 & 944 & 5.5 & 6.1 \\
\hline & & & & & & & & SGG & 5.E-10 & 6594 & 1.7 & -1.6 \\
\hline Lef1 & 2 & PAN & 2.E-51 & 6202 & & -1.8 & 0 & & & & & \\
\hline Ptch1 & 1 & PTR & 2.E-12 & 1241 & 10.1 & 9.4 & 1 & PTR & 2.E-12 & 1241 & 10.1 & 9.4 \\
\hline
\end{tabular}


suggest that this high CNS expression of CG3837/SDR is specifically located in the surface glia (6-fold enriched relative to brain and 154-fold enriched relative to neurons). As Drosophila adult body size is predetermined in the larval stages, the maintained expression of CG3837/SDR in the adult surface glia suggests the possibility of novel roles for the adult $\mathrm{BBB}$ in insulin regulated physiologies independent of body size control.

Lastly, we note that the surface glia also express homologs of the vertebrate BBB proteins LEF1 and PTCH1. LEF1 is a Wntresponsive transcription factor (Liebner et al., 2008; Stenman et al., 2008; Daneman et al., 2009), and PTCH1 is a mediator of sonic hedgehog signaling (Alvarez et al., 2011). This final set of genes again illustrates that the Drosophila surface glia can be used to model evolutionary conserved BBB-localized signaling and regulatory proteins. Overall, our surface glia transcriptome will continue to provide an evolutionary comparative framework as more essential BBB proteins are identified in vertebrates.

\section{DISCUSSION}

Here we have described techniques for the isolation and transcriptome characterization of the adult brain Drosophila surface glia. We have shown that our techniques are of high quality yielding a quantitative transcriptomic portrait of the surface glia constituting the BBB. While organ-specific transcriptomes exist for Drosophila (Wang et al., 2004; Chintapalli et al., 2007), cell-typespecific data sets have been slower to emerge (Salmand et al., 2011; Berger et al., 2012; Bryantsev and Cripps, 2012; Siddiqui et al., 2012). Ultimately, cell-type-specific transcriptomes will enable us to discern how different cells interact to produce the emergent physiologic properties of a tissue or organ. In our case, we have sampled the two outermost cell layers of the Drosophila CNS, and our results confirm that this surface glia layer indeed possesses the hallmarks of a potent chemical protection interface equivalent to the vertebrate brain vascular endothelial BBB component.

That being said, the Drosophila model for vertebrate BBB physiology can and should be refined. First, the surface glia transcriptome contains two glial subtypes, the PG and SPG, thus having separate transcriptomes for these cell layers will increase the resolution at which to assign the conserved gene expression patterns discussed in this report. Second, the cortex glia that lie directly underneath the SPG are well positioned to influence BBB properties similar to astrocyte end feet positioning in the vertebrate CNS. Obtaining a transcriptome for these glia will likely add to our understanding of how conserved BBB properties are manifested in the Drosophila equivalent of the vertebrate NVU. We have shown that such refinements are feasible by our methodologies, and most importantly, that they are necessary to further research into the mechanisms of development, maintenance, and regulation of conserved $\mathrm{BBB}$ properties.

Failure to efficiently circumvent the BBB for the treatment of neurological diseases highlights the complex homeostatic mechanisms that exist at the BBB. Without a model system for which many of the interacting biological processes can be assessed in vivo, there will be little progress into understanding $\mathrm{BBB}$ development, maintenance, and regulation. The surface glia BBB of Drosophila is exactly the model system that is needed. Pharmacokinetics can be measured in vivo, hypotheses can be tested with forward and reverse genetics, different cell populations can be manipulated simultaneously, and small molecule modifiers of BBB homeostasis can be found with highthroughput screens. Previously, it was known that the Drosophila $\mathrm{BBB}$ possesses a few characteristics of the vertebrate BBB; for example, cellular junctions similar to vertebrate TJs (Juang and Carlson, 1994; Schwabe et al., 2005; Stork et al., 2008), a single drug efflux transporter similar to vertebrate ABCB1A (Mayer et al., 2009), and lipoprotein transport (Brankatschk and Eaton, 2010). Now, our characterization of the surface glia transcriptome indicates that numerous processes/structures are evolutionarily conserved between flies and vertebrates. These include: drug efflux (i.e., many B and C class ABC transporters), adherens junctions, insulin signaling, and the basal lamina. The results alone for SLC transporters are staggering. The fly and mouse BBBs co-express highly homologous SLC transporters involved in the transport of amino acids, bicarbonate, organic anions, monocarboxylates, folates, glucose, and zinc. Preliminary deep sequencing of the surface glia transcriptome suggests that about $50 \%$ more SLC transporters than revealed by GeneChips are conserved in fly and vertebrate BBBs (data not shown). With this foundation of conserved $\mathrm{BBB}$ gene expression patterns, we can begin to perform in vivo, translatable experiments in the Drosophila BBB model system at a scale unattainable to vertebrate researchers.

\section{INTERESTING GENES FOR FUTURE STUDY}

In addition to various surface glia enriched genes, our investigation revealed two surface glia-specific genes, Vmat and Indy. Using immunostaining, we showed that DVMAT-B specifically localized to the PG layer of the BBB. DVMAT-B has been shown to localize to the fenestrated glia of the Drosophila visual system, where it is thought to function in histamine storage (RomeroCalderon et al., 2008). The fenestrated glia are thought to be the visual system equivalent of PG cells (DeSalvo et al., 2011). Relatively little is known about the contribution of the PG cells to BBB functions; however, the PG-localized expression of DVMAT$B$ may suggest a role for PG cells in the storage of monoamines, and may function to isolate peripheral and CNS effects of monoamines. Indy, the other surface glia-specific gene we identified, encodes a sodium-independent dicarboxylate cotransporter (homologous to mammalian $\mathrm{NaDC} 1, \mathrm{NaDC} 3$, and $\mathrm{NaCT}$ ) and, like its mammalian counterparts, has been shown to transport intermediates of the Krebs cycle (Rogina et al., 2000; Inoue et al., 2002; Knauf et al., 2002, 2006). Intermediates of the Krebs cycle also have signaling roles, and can act through various G-protein coupled receptors with potential roles in regulating blood pressure and as a hypoxia sensor (Sadagopan et al., 2007; Sapieha et al., 2008). NaDC3 has also been postulated as a glutathione transporter, with implications in oxidative stress regulation (Lash, 2005; Li et al., 2012). NaDC3 and NaCT have been shown to be expressed in neurons and astrocytic glia (Lamp et al., 2011). We have previously shown that Drosophila Indy is expressed in both the SPG and the PG layers (DeSalvo et al., 2011), though the polarity of its expression is currently not known. Due to its high similarity to the mammalian SLC13 transporters, its surface glia expression may suggest a role for Indy as a metabolic regulator or 
sensor of oxidative stress in the BBB. Furthermore, the transcriptional repressor hairy has been suggested as a metabolic switch protein in Drosophila, with its upregulation causing hypoxia resistance (Zhou et al., 2008). We found hairy to be significantly enriched in the adult surface glia, further suggesting an important metabolic role for the BBB.

We also discovered a leaky BBB phenotype caused by a protein trap in the surface glia-enriched gene Integrin linked kinase (Ilk). Mammalian integrin linked kinase is involved in transducing signals from the extracellular matrix, through integrins, to initiate downstream intracellular signaling cascades (reviewed in Wu and Dedhar, 2001). Integrin signaling is required to maintain BBB integrity in mammalian endothelial cells; disrupting this critical link between the ECM and intracellular targets led to disruption of TJs and resulted in BBB leakiness (Osada et al., 2011). Our results, therefore, suggest there might be a conserved role in the Drosophila BBB for integrin/Ilk signaling from the ECM/basal lamina to regulate $\mathrm{BBB}$ integrity. As the role for integrin/Ilk signaling in maintaining BBB integrity has also been linked to collagen composition in the ECM (Gould et al., 2005; Vahedi et al., 2007), it might be of interest to investigate the role of the PG cells, which we have shown to express the collagen IV gene $V k g$, in neural lamella (basal lamina) composition and SPG septate junction integrity, and the response of $\mathrm{PG}$ cells during conditions that perturb BBB function.

Overall, our surface glia transcriptome has identified a number of interesting, conserved genes present in the adult Drosophila BBB. We are now well poised to interrogate the interactions between the BBB, underlying neurons and glia, and hemolymph-facing neural lamella to understand the possible feedback mechanisms that occur between the CNS and the whole organism.

\section{ACKNOWLEDGMENTS}

We thank Rich Daneman and all members of his lab at UCSF for fruitful lab meeting discussions regarding all techniques and analyses described here. Charlie Kim and Sharon Bergquist are thanked for their help in developing the brain dissociation protocols, and Sarah Elmes is thanked for her help developing FACS isolation methods. This research was supported by NIH GRANTs R01GM081863 and R21NS082856, and NIEHS R21 ES021412 (P.I. Roland J. Bainton). Also the UCSF Department of Anesthesia and Perioperative Care and the Sandler foundation.

\section{SUPPLEMENTARY MATERIAL}

The Supplementary Material for this article can be found online at: http://www.frontiersin.org/journal/10.3389/fnins.2014. 00346/abstract

\section{REFERENCES}

Alvarez, J. I., Dodelet-Devillers, A., Kebir, H., Ifergan, I., Fabre, P. J., Terouz, S., et al. (2011). The Hedgehog pathway promotes blood-brain barrier integrity and CNS immune quiescence. Science 334, 1727-1731. doi: 10.1126/science. 1206936

Anderson, P. A., and Greenberg, R. M. (2001). Phylogeny of ion channels: clues to structure and function. Comp. Biochem. Physiol. B Biochem. Mol. Biol. 129, 17-28. doi: 10.1016/S1096-4959(01)00376-1
Armulik, A., Genove, G., Mae, M., Nisancioglu, M. H., Wallgard, E., Niaudet, C., et al. (2010). Pericytes regulate the blood-brain barrier. Nature 468, 557-561. doi: 10.1038/nature09522

Auld, V. J., Fetter, R. D., Broadie, K., and Goodman, C. S. (1995). Gliotactin, a novel transmembrane protein on peripheral glia, is required to form the bloodnerve barrier in Drosophila. Cell 81, 757-767. doi: 10.1016/0092-8674(95) 90537-5

Bainton, R. J., Tsai, L. T., Schwabe, T., DeSalvo, M., Gaul, U., and Heberlein, U. (2005). moody encodes two GPCRs that regulate cocaine behaviors and blood-brain barrier permeability in Drosophila. Cell 123, 145-156. doi: 10.1016/j.cell.2005.07.029

Banerjee, S., and Bhat, M. A. (2008). Glial ensheathment of peripheral axons in Drosophila. J. Neurosci. Res. 86, 1189-1198. doi: 10.1002/jnr.21574

Banerjee, S., Pillai, A. M., Paik, R., Li, J., and Bhat, M. A. (2006). Axonal ensheathment and septate junction formation in the peripheral nervous system of Drosophila. J. Neurosci. 26, 3319-3329. doi: 10.1523/JNEUROSCI.538305.2006

Barolo, S., Carver, L. A., and Posakony, J. W. (2000). GFP and betagalactosidase transformation vectors for promoter/enhancer analysis in Drosophila. Biotechniques 29, 726, 728, 730, 732.

Baumgartner, S., Littleton, J. T., Broadie, K., Bhat, M. A., Harbecke, R., Lengyel, J. A., et al. (1996). A Drosophila neurexin is required for septate junction and blood-nerve barrier formation and function. Cell 87, 1059-1068. doi: 10.1016/S0092-8674(00)81800-0

Behr, M., Riedel, D., and Schuh, R. (2003). The claudin-like megatrachea is essential in septate junctions for the epithelial barrier function in Drosophila. Dev. Cell 5, 611-620. doi: 10.1016/S1534-5807(03)00275-2

Berger, C., Harzer, H., Burkard, T. R., Steinmann, J., van der Horst, S., Laurenson, A. S., et al. (2012). FACS purification and transcriptome analysis of drosophila neural stem cells reveals a role for Klumpfuss in self-renewal. Cell Rep. 2, 407-418. doi: 10.1016/j.celrep.2012.07.008

Bhat, M. A. (2003). Molecular organization of axo-glial junctions. Curr. Opin. Neurobiol. 13, 552-559. doi: 10.1016/j.conb.2003.09.004

Bhat, M. A., Rios, J. C., Lu, Y., Garcia-Fresco, G. P., Ching, W., St. Martin, M., et al. (2001). Axon-glia interactions and the domain organization of myelinated axons requires neurexin IV/Caspr/Paranodin. Neuron 30, 369-383. doi: 10.1016/S0896-6273(01)00294-X

Boado, R. J., and Pardridge, W. M. (1990). The brain-type glucose transporter mRNA is specifically expressed at the blood-brain barrier. Biochem. Biophys. Res. Commun. 166, 174-179. doi: 10.1016/0006-291X(90) 91927-K

Brand, A. H., and Perrimon, N. (1993). Targeted gene expression as a means of altering cell fates and generating dominant phenotypes. Development 118 , 401-415

Brankatschk, M., and Eaton, S. (2010). Lipoprotein particles cross the blood-brain barrier in Drosophila. J. Neurosci. 30, 10441-10447. doi: 10.1523/JNEUROSCI.5943-09.2010

Bryantsev, A. L., and Cripps, R. M. (2012). Purification of cardiac cells from Drosophila embryos. Methods 56, 44-49. doi: 10.1016/j.ymeth.2011. 11.004

Buszczak, M., Paterno, S., Lighthouse, D., Bachman, J., Planck, J., Owen, S., et al. (2007). The carnegie protein trap library: a versatile tool for Drosophila developmental studies. Genetics 175, 1505-1531. doi: 10.1534/genetics.106. 065961

Campos, A. R., Rosen, D. R., Robinow, S. N., and White, K. (1987). Molecular analysis of the locus elav in Drosophila melanogaster: a gene whose embryonic expression is neural specific. EMBO J. 6, 425-431.

Chintapalli, V. R., Wang, J., and Dow, J. A. (2007). Using FlyAtlas to identify better Drosophila melanogaster models of human disease. Nat. Genet. 39, 715-720. doi: $10.1038 / \mathrm{ng} 2049$

Cordon-Cardo, C., O’Brien, J. P., Casals, D., Rittman-Grauer, L., Biedler, J. L., Melamed, M. R., et al. (1989). Multidrug-resistance gene (P-glycoprotein) is expressed by endothelial cells at blood-brain barrier sites. Proc. Natl. Acad. Sci. U.S.A. 86, 695-698. doi: 10.1073/pnas.86.2.695

Courtay, C., Oster, T., Michelet, F., Visvikis, A., Diederich, M., Wellman, M., et al. (1992). Gamma-glutamyltransferase: nucleotide sequence of the human pancreatic cDNA. Evidence for a ubiquitous gamma-glutamyltransferase polypeptide in human tissues. Biochem. Pharmacol. 43, 2527-2533. doi: 10.1016/0006-2952(92)90140-E 
Curtin, K. D., Wyman, R. J., and Meinertzhagen, I. A. (2007). Basigin/EMMPRIN/CD147 mediates neuron-glia interactions in the optic lamina of Drosophila. Glia 55, 1542-1553. doi: 10.1002/glia.20568

Daneman, R. (2012). The blood-brain barrier in health and disease. Ann. Neurol. 72, 648-672. doi: 10.1002/ana.23648

Daneman, R., Agalliu, D., Zhou, L., Kuhnert, F., Kuo, C. J., and Barres, B. A. (2009). Wnt/beta-catenin signaling is required for CNS, but not non-CNS, angiogenesis. Proc. Natl. Acad. Sci. U.S.A. 106, 641-646. doi: 10.1073/pnas.0805 165106

Daneman, R., Zhou, L., Agalliu, D., Cahoy, J. D., Kaushal, A., and Barres, B. A. (2010a). The mouse blood-brain barrier transcriptome: a new resource for understanding the development and function of brain endothelial cells. PLoS ONE 5:e13741. doi: 10.1371/journal.pone.0013741

Daneman, R., Zhou, L., Kebede, A. A., and Barres, B. A. (2010b). Pericytes are required for blood-brain barrier integrity during embryogenesis. Nature 468, 562-566. doi: 10.1038/nature09513

DeSalvo, M., Mayer, N., Mayer, F., and Bainton, R. J. (2011). Physiologic and anatomic characterization of the brain surface glia barrier of Drosophila. Glia 59, 1322-1340. doi: 10.1002/glia.21147

de Vries, N. A., Zhao, J., Kroon, E., Buckle, T., Beijnen, J. H., and van Tellingen, O. (2007). P-glycoprotein and breast cancer resistance protein: two dominant transporters working together in limiting the brain penetration of topotecan. Clin. Cancer Res. 13, 6440-6449. doi: 10.1158/1078-0432.CCR07-1335

Edwards, T. N., and Meinertzhagen, I. A. (2010). The functional organisation of glia in the adult brain of Drosophila and other insects. Prog Neurobiol 90, 471-497. doi: 10.1016/j.pneurobio.2010.01.001

Enerson, B. E., and Drewes, L. R. (2006). The rat blood-brain barrier transcriptome. J. Cereb. Blood Flow Metab. 26, 959-973. doi: 10.1038/sj.jcbfm. 9600249

Freeman, M. R., Delrow, J., Kim, J., Johnson, E., and Doe, C. Q. (2003). Unwrapping glial biology: $\mathrm{Gcm}$ target genes regulating glial development, diversification, and function. Neuron 38, 567-580. doi: 10.1016/S08966273(03)00289-7

Pfrieger, F. W., and Barres, B. A. (1995). What the fly's glia tell the fly's brain. Cell 83, 671-674. doi: 10.1016/0092-8674(95)90178-7

Gautier, L., Cope, L., Bolstad, B. M., and Irizarry, R. A. (2004). affy-analysis of Affymetrix GeneChip data at the probe level. Bioinformatics 20, 307-315. doi: 10.1093/bioinformatics/btg405

Gentleman, R. C., Carey, V. J., Bates, D. M., Bolstad, B., Dettling, M., Dudoit, S., et al. (2004). Bioconductor: open software development for computational biology and bioinformatics. Genome Biol. 5, R80. doi: 10.1186/gb-2004-5-10-r80

Gould, D. B., Phalan, F. C., Breedveld, G. J., van Mil, S. E., Smith, R. S., Schimenti, J. C., et al. (2005). Mutations in Col4al cause perinatal cerebral hemorrhage and porencephaly. Science 308, 1167-1171. doi: 10.1126/science. 1109418

Hatan, M., Shinder, V., Israeli, D., Schnorrer, F., and Volk, T. (2011). The Drosophila blood brain barrier is maintained by GPCR-dependent dynamic actin structures. J. Cell Biol. 192, 307-319. doi: 10.1083/jcb.201007095

Hawkins, R. A., O’Kane, R. L., Simpson, I. A., and Vina, J. R. (2006). Structure of the blood-brain barrier and its role in the transport of amino acids. J. Nutr. 136(1 Suppl):218S-226S.

Hijazi, A., Haenlin, M., Waltzer, L., and Roch, F. (2011). The Ly6 protein coiled is required for septate junction and blood brain barrier organisation in Drosophila. PLoS ONE 6:e17763. doi: 10.1371/journal.pone.0017763

Hirase, T., Staddon, J. M., Saitou, M., Ando-Akatsuka, Y., Itoh, M., Furuse, M., et al. (1997). Occludin as a possible determinant of tight junction permeability in endothelial cells. J Cell Sci 110(Pt 14):1603-1613.

Homolya, L., Varadi, A., and Sarkadi, B. (2003). Multidrug resistance-associated proteins: Export pumps for conjugates with glutathione, glucuronate or sulfate. Biofactors 17, 103-114. doi: 10.1002/biof.5520170111

Huang, D. W., Sherman, B. T., and Lempicki, R. A. (2009a). Bioinformatics enrichment tools: paths toward the comprehensive functional analysis of large gene lists. Nucleic Acids Res. 37, 1-13. doi: 10.1093/nar/gkn923

Huang, D. W., Sherman, B. T., and Lempicki, R. A. (2009b). Systematic and integrative analysis of large gene lists using DAVID bioinformatics resources. Nat. Protoc. 4, 44-57. doi: 10.1038/nprot.2008.211

Ile, K. E., Tripathy, R., Goldfinger, V., and Renault, A. D. (2012). Wunen, a Drosophila lipid phosphate phosphatase, is required for septate junction-mediated barrier function. Development 139, 2535-2546. doi: 10.1242/dev.077289

Inoue, K., Fei, Y. J., Huang, W., Zhuang, L., Chen, Z., and Ganapathy, V. (2002). Functional identity of Drosophila melanogaster Indy as a cation-indeendent, electroneutral transporter for tricarboxylic acid-cycle intermediates. Biochem. J. 367(Pt 2):313-319. doi: 10.1042/BJ20021132

Irizarry, R. A., Bolstad, B. M., Collin, F., Cope, L. M., Hobbs, B., and Speed, T. P. (2003). Summaries of affymetrix genechip probe level data. Nucleic Acids Res. 31:e15. doi: 10.1093/nar/gng015

Janzer, R. C., and Raff, M. C. (1987). Astrocytes induce blood-brain barrier properties in endothelial cells. Nature 325, 253-257. doi: 10.1038/ $325253 \mathrm{a} 0$

Juang, J. L., and Carlson, S. D. (1994). Analog of vertebrate anionic sites in blood-brain interface of larval Drosophila. Cell Tissue Res. 277, 87-95. doi: 10.1007/BF00303084

Kaplow, M. E., Korayem, A. H., and Venkatesh, T. R. (2008). Regulation of glia number in Drosophila by Rap/Fzr, an activator of the anaphasepromoting complex, and Loco, an RGS protein. Genetics 178, 2003-2016. doi: 10.1534/genetics.107.086397

Kelso, R. J., Buszczak, M., Quinones, A. T., Castiblanco, C., Mazzalupo, S., and Cooley, L. (2004). Flytrap, a database documenting a GFP protein-trap insertion screen in Drosophila melanogaster. Nucleic Acids Res. 32(Database issue):D418-D420. doi: 10.1093/nar/gkh014

Kinsella, R. J., Kahari, A., Haider, S., Zamora, J., Proctor, G., Spudich, G., et al. (2011). Ensembl BioMarts: a hub for data retrieval across taxonomic space. Database (Oxford) 2011:bar030. doi: 10.1093/database/bar030

Knauf, F., Mohebbi, N., Teichert, C., Herold, D., Rogina, B., Helfand, S., et al. (2006). The life-extending gene Indy encodes an exchanger for krebs-cycle intermediates. Biochem. J. 397, 25-29. doi: 10.1042/BJ20060409

Knauf, F., Rogina, B., Jiang, Z., Aronson, P. S., and Helfand, S. L. (2002). Functional characterization and immunolocalization of the transporter encoded by the life-extending gene Indy. Proc. Natl. Acad. Sci. U.S.A. 99, 14315-14319. doi: $10.1073 /$ pnas. 222531899

Knust, E., and Bossinger, O. (2002). Composition and formation of intercellular junctions in epithelial cells. Science 298, 1955-1959. doi: 10.1126/science. 1072161

Lamp, J., Keyser, B., Koeller, D. M., Ullrich, K., Braulke, T., and Muhlhausen, C. (2011). Glutaric aciduria type 1 metabolites impair the succinate transport from astrocytic to neuronal cells. J. Biol. Chem. 286, 17777-17784. doi: 10.1074/jbc.M111.232744

Lash, L. H. (2005). Role of glutathione transport processes in kidney function. Toxicol. Appl. Pharmacol. 204, 329-342. doi: 10.1016/j.taap.2004. 10.004

Layden, M. J., Odden, J. P., Schmid, A., Garces, A., Thor, S., and Doe, C. Q. (2006). Zfh1, a somatic motor neuron transcription factor, regulates axon exit from the CNS. Dev. Biol. 291, 253-263. doi: 10.1016/j.ydbio.2005. 12.009

Li, B., Lee, M. S., Lee, R. S., Donaldson, P. J., and Lim, J. C. (2012). Characterization of glutathione uptake, synthesis, and efflux pathways in the epithelium and endothelium of the rat cornea. Cornea 31, 1304-1312. doi: 10.1097/ICO.0b013e31823f76bd

Liebner, S., Corada, M., Bangsow, T., Babbage, J., Taddei, A., Czupalla, C. J., et al. (2008). Wnt/beta-catenin signaling controls development of the blood-brain barrier. J. Cell Biol. 183, 409-417. doi: 10.1083/jcb.200806024

Lin, F., Marchetti, S., Pluim, D., Iusuf, D., Mazzanti, R., Schellens, J. H., et al. (2013). Abcc4 together with Abcb1 and Abcg2 form a robust cooperative drug efflux system that restricts the brain entry of camptothecin analogs. Clin. Cancer Res. 19, 2084-2095. doi: 10.1158/1078-0432.CCR12-3105

Loscher, W., and Potschka, H. (2005). Blood-brain barrier active efflux transporters: ATP-binding cassette gene family. NeuroRx 2, 86-98. doi: 10.1602/neurorx.2.1.86

Mayer, F., Mayer, N., Chinn, L., Pinsonneault, R. L., Kroetz, D., and Bainton, R. J. (2009). Evolutionary conservation of vertebrate blood-brain barrier chemoprotective mechanisms in Drosophila. J. Neurosci. 29, 3538-3550. doi: 10.1523/JNEUROSCI.5564-08.2009

McClintick, J. N., and Edenberg, H. J. (2006). Effects of filtering by Present call on analysis of microarray experiments. BMC Bioinformatics 7:49. doi: $10.1186 / 1471-2105-7-49$ 
Morin, X., Daneman, R., Zavortink, M., and Chia, W. (2001). A protein trap strategy to detect GFP-tagged proteins expressed from their endogenous loci in Drosophila. Proc. Natl. Acad. Sci. U.S.A. 98, 15050-15055. doi: $10.1073 /$ pnas. 261408198

Nelson, K. S., Furuse, M., and Beitel, G. J. (2010). The Drosophila Claudin Kunekune is required for septate junction organization and tracheal tube size control. Genetics 185, 831-839. doi: 10.1534/genetics.110.114959

Nitta, T., Hata, M., Gotoh, S., Seo, Y., Sasaki, H., Hashimoto, N., et al. (2003). Sizeselective loosening of the blood-brain barrier in claudin-5-deficient mice. J. Cell Biol. 161, 653-660. doi: 10.1083/jcb.200302070

Okamoto, N., Nakamori, R., Murai, T., Yamauchi, Y., Masuda, A., and Nishimura, T. (2013). A secreted decoy of InR antagonizes insulin/IGF signaling to restrict body growth in Drosophila. Genes Dev. 27, 87-97. doi: 10.1101/gad.204479.112

Osada, T., Gu, Y.-T., Kanazawa, M., Tsubota, Y., Hawkins, B. T., Spatz, M., et al. (2011). Inter-endothelial claudin-5 expression depends upon cerebral endothelial cell matrix adhesion by b1-integrins. J. Cereb. Blood Flow Metab. 31, 1972-1985. doi: 10.1038/jcbfm.2011.99

Pardridge, W. M. (2005). The blood-brain barrier: bottleneck in brain drug development. NeuroRx 2, 3-14. doi: 10.1602/neurorx.2.1.3

Pardridge, W. M., Boado, R. J., and Farrell, C. R. (1990). Brain-type glucose transporter (GLUT-1) is selectively localized to the blood-brain barrier. Studies with quantitative western blotting and in situ hybridization. J. Biol. Chem. 265, 18035-18040.

Pfeiffer, B. D., Ngo, T. T., Hibbard, K. L., Murphy, C., Jenett, A., Truman, J. W., et al. (2010). Refinement of tools for targeted gene expression in Drosophila. Genetics 186, 735-755. doi: 10.1534/genetics.110.119917

Pinsonneault, R. L., Mayer, N., Mayer, F., Tegegn, N., and Bainton, R. J. (2011). Novel Models for studying the blood-brain and blood-eye barriers in Drosophila. Methods Mol. Biol. 686, 357-369. doi: 10.1007/978-1-60761-9383_17

Quinones-Coello, A. T., Petrella, L. N., Ayers, K., Melillo, A., Mazzalupo, S., Hudson, A. M., et al. (2007). Exploring strategies for protein trapping in Drosophila. Genetics 175, 1089-1104. doi: 10.1534/genetics.106.065995

Ranganathan, R., and Ross, E. M. (1997). PDZ domain proteins: scaffolds for signaling complexes. Curr. Biol. 7, R770-R773. doi: 10.1016/S09609822(06)00401-5

Robinow, S., and White, K. (1988). The locus elav of Drosophila melanogaster is expressed in neurons at all developmental stages. Dev. Biol. 126, 294-303. doi: 10.1016/0012-1606(88)90139-X

Rogina, B., Reenan, R. A., Nilsen, S. P., and Helfand, S. L. (2000). Extended lifespan conferred by cotransporter gene mutations in Drosophila. Science 290 2137-2140. doi: 10.1126/science.290.5499.2137

Romero-Calderon, R., Uhlenbrock, G., Borycz, J., Simon, A. F., Grygoruk, A., Yee, S. K., et al. (2008). A glial variant of the vesicular monoamine transporter is required to store histamine in the Drosophila visual system. PLoS Genet. 4:e1000245. doi: 10.1371/journal.pgen.1000245

Sadagopan, N., Li, W., Roberds, S. L., Major, T., Preston, G. M., Yu, Y., et al. (2007). Circulating succinate is elevated in rodent models of hypertension and metabolic disease. Am. J. Hypertens. 20, 1209-1215. doi: 10.1016/j.amjhyper.2007.05.010

Saitou, M., Furuse, M., Sasaki, H., Schulzke, J. D., Fromm, M., Takano, H., et al. (2000). Complex phenotype of mice lacking occludin, a component of tight junction strands. Mol. Biol. Cell 11, 4131-4142. doi: 10.1091/mbc.11.12.4131

Salmand, P. A., Iche-Torres, M., and Perrin, L. (2011). Tissue-specific cell sorting from Drosophila embryos: application to gene expression analysis. Fly (Austin) 5, 261-265. doi: 10.4161/fly.5.3.16509

Sapieha, P., Sirinyan, M., Hamel, D., Zaniolo, K., Joyal, J. S., Cho, J. H., et al. (2008). The succinate receptor GPR91 in neurons has a major rle in retinal angiogenesis. Nat. Med. 14, 1067-1076. doi: 10.1038/nm.1873

Schwabe, T., Bainton, R. J., Fetter, R. D., Heberlein, U., and Gaul, U. (2005). GPCR signaling is required for blood-brain barrier formation in drosophila. Cell 123 , 133-144. doi: 10.1016/j.cell.2005.08.037

Sheweita, S. A. (2000). Drug-metabolizing enzymes: mechanisms and functions. Curr. Drug Metab. 1, 107-132. doi: 10.2174/1389200003339117

Siddiqui, N. U., Li, X., Luo, H., Karaiskakis, A., Hou, H., Kislinger, T., et al. (2012). Genome-wide analysis of the maternal-to-zygotic transition in Drosophila primordial germ cells. Genome Biol. 13, R11-2012-13-2-r11. doi: 10.1186/gb2012-13-2-r11
Smyth, G. K. (2004). Linear models and empirical Bayes methods for assessing differential expression in microarray experiments. Stat. Appl. Genet. Mol. Biol. 3, 1-25. doi: 10.2202/1544-6115.1027

Sobue, K., Yamamoto, N., Yoneda, K., Hodgson, M. E., Yamashiro, K., Tsuruoka N., et al. (1999). Induction of blood-brain barrier properties in immortalized bovine brain endothelial cells by astrocytic factors. Neurosci. Res. 35, 155-164. doi: 10.1016/S0168-0102(99)00079-6

Spéder, P., and Brand, A. H. (2014). Gap junction proteins in the blood-brain barrier control nutrient-dependent reactivation of Drosophila neural stem cells. Dev Cell 30, 309-321. doi: 10.1016/j.devcel.2014.05.021

Stenman, J. M., Rajagopal, J., Carroll, T. J., Ishibashi, M., McMahon, J., and McMahon, A. P. (2008). Canonical Wnt signaling regulates organ-specific assembly and differentiation of CNS vasculature. Science 322, 1247-1250. doi: 10.1126/science. 1164594

Stork, T., Engelen, D., Krudewig, A., Silies, M., Bainton, R. J., and Klambt, C. (2008). Organization and function of the blood-brain barrier in Drosophila. J. Neurosci. 28, 587-597. doi: 10.1523/JNEUROSCI.4367-07.2008

Strigini, M., Cantera, R., Morin, X., Bastiani, M. J., Bate, M., and Karagogeos, D. (2006). The IgLON protein Lachesin is required for the blood-brain barrier in Drosophila. Mol. Cell. Neurosci. 32, 91-101. doi: 10.1016/j.mcn.2006.03.001

Syed, M. H., Krudewig, A., Engelen, D., Stork, T., and Klambt, C. (2011). The CD59 family member Leaky/Coiled is required for the establishment of the blood-brain barrier in Drosophila. J. Neurosci. 31, 7876-7885. doi: 10.1523/JNEUROSCI.0766-11.2011

Tomancak, P., Beaton, A., Weiszmann, R., Kwan, E., Shu, S., Lewis, S. E., et al. (2002). Systematic determination of patterns of gene expression during Drosophila embryogenesis. Genome Biol. 3:RESEARCH0088. doi: 10.1186/gb2002-3-12-research0088

Tomancak, P., Berman, B. P., Beaton, A., Weiszmann, R., Kwan, E., Hartenstein, V., et al. (2007). Global analysis of patterns of gene expression during Drosophila embryogenesis. Genome Biol. 8, R145. doi: 10.1186/gb-2007-8-7-r145

Unhavaithaya, Y., and Orr-Weaver, T. L. (2012). Polyploidization of glia in neural development links tissue growth to blood-brain barrier integrity. Genes Dev. 26, 31-36. doi: 10.1101/gad.177436.111

Vahedi, K., Kubis, N., Boukobza, M., Arnoult, M., Massin, P., Tournier-Lasserve, E. et al. (2007). COL4A1 mutation in a patient with sporadic, recurrent intracerebral hemorrhage. Stroke 38, 1461-1464. doi: 10.1161/STROKEAHA.106.475194

Valdovinos-Flores, C., and Gonsebatt, M. E. (2012). The role of amino acid transporters in GSH synthesis in the blood-brain barrier and central nervous system. Neurochem. Int. 61, 405-414. doi: 10.1016/j.neuint.2012.05.019

Venter, J. C., di Porzio, U., Robinson, D. A., Shreeve, S. M., Lai, J., Kerlavage, A. R., et al. (1988). Evolution of neurotransmitter receptor systems. Prog. Neurobiol. 30, 105-169. doi: 10.1016/0301-0082(88)90004-4

Wang, J., Kean, L., Yang, J., Allan, A. K., Davies, S. A., Herzyk, P., et al. (2004). Function-informed transcriptome analysis of Drosophila renal tubule. Genome Biol. 5:R69. doi: 10.1186/gb-2004-5-9-r69

Wu, C., and Dedhar, S. (2001). Integrin-linked kinase (ILK) and its interactors: a new paradigm for the coupling of extracellular matrix to actin cytoskeleton and signaling complexes. J. Cell Biol. 155, 505-510. doi: 10.1083/jcb.200108077

Wu, V. M., and Beitel, G. J. (2004). A junctional problem of apical proportions: epithelial tube-size control by septate junctions in the Drosophila tracheal system. Curr. Opin. Cell Biol. 16, 493-499. doi: 10.1016/j.ceb.2004.07.008

Wu, V. M., Schulte, J., Hirschi, A., Tepass, U., and Beitel, G. J. (2004). Sinuous is a Drosophila claudin required for septate junction organization and epithelial tube size control. J. Cell Biol. 164, 313-323. doi: 10.1083/jcb.200309134

Wu, W., Dnyanmote, A. V., and Nigam, S. K. (2011). Remote communication through solute carriers and ATP binding cassette drug transporter pathways: an update on the remote sensing and signaling hypothesis. Mol. Pharmacol. 79, 795-805. doi: 10.1124/mol.110.070607

Xiong, W. C., Okano, H., Patel, N. H., Blendy, J. A., and Montell, C. (1994) repo encodes a glial-specific homeo domain protein required in the Drosophila nervous system. Genes Dev. 8, 981-994. doi: 10.1101/gad.8.8.981

Zhou, D., Xue, J., Lai, J. C., Schork, N. J., White, K. P., and Haddad, G. G. (2008). Mechanisms underlying hypoxia tolerance in Drosophila melanogaster: hairy as a metabolic switch. PLoS Genet. 4:e1000221. doi: 10.1371/journal.pgen. 1000221

Zlokovic, B. V. (2008). The blood-brain barrier in health and chronic neurodegenerative disorders. Neuron 57, 178-201. doi: 10.1016/j.neuron.2008.01.003 
Conflict of Interest Statement: The authors declare that the research was conducted in the absence of any commercial or financial relationships that could be construed as a potential conflict of interest.

Received: 19 August 2014; accepted: 10 October 2014; published online: 07 November 2014.

Citation: DeSalvo MK, Hindle SJ, Rusan ZM, Orng S, Eddison M, Halliwill K and Bainton RJ (2014) The Drosophila surface glia transcriptome: evolutionary conserved blood-brain barrier processes. Front. Neurosci. 8:346. doi: 10.3389/fnins.2014.00346
This article was submitted to Neurogenomics, a section of the journal Frontiers in Neuroscience.

Copyright (c) 2014 DeSalvo, Hindle, Rusan, Orng, Eddison, Halliwill and Bainton. This is an open-access article distributed under the terms of the Creative Commons Attribution License (CC BY). The use, distribution or reproduction in other forums is permitted, provided the original author(s) or licensor are credited and that the original publication in this journal is cited, in accordance with accepted academic practice. No use, distribution or reproduction is permitted which does not comply with these terms. 


\title{
Functional and genetic analysis of choroid plexus development in zebrafish
}

\author{
Hannah E. Henson ${ }^{1,2+\neq}$, Chaithanyarani Parupalli ${ }^{1 \neq}$, Bensheng Ju ${ }^{1}$ and Michael R. Taylor ${ }^{1,3 *}$ \\ Chemical Biology and Therapeutics, St. Jude Children's Research Hospital, Memphis, TN, USA \\ 2 Integrated Program in Biomedical Sciences, College of Graduate Health Sciences, University of Tennessee Health Science Center, Memphis, TN, USA \\ ${ }^{3}$ Pharmaceutical Sciences Division, School of Pharmacy, University of Wisconsin-Madison, Madison, WI, USA
}

\section{Edited by:}

Norman Ruthven Saunders,

University of Melbourne, Australia

\section{Reviewed by:}

Kyo Yamasu, Saitama

University, Japan

Brian Eliceiri, University of

California, San Diego, USA

\section{*Correspondence:}

Michael R. Taylor, Pharmaceutical

Sciences Division, School of

Pharmacy, University of

Wisconsin-Madison, 777 Highland

Avenue, Madison, WI

53705-2222, USA

e-mail: michael.taylor@wisc.edu

${ }^{\dagger}$ Present address:

Hannah E. Henson, Department of

Biology, University of Kentucky,

Lexington, USA

₹ These authors have contributed equally to this work.
The choroid plexus, an epithelial-based structure localized in the brain ventricle, is the major component of the blood-cerebrospinal fluid barrier. The choroid plexus produces the cerebrospinal fluid and regulates the components of the cerebrospinal fluid. Abnormal choroid plexus function is associated with neurodegenerative diseases, tumor formation in the choroid plexus epithelium, and hydrocephaly. In this study, we used zebrafish (Danio rerio) as a model system to understand the genetic components of choroid plexus development. We generated an enhancer trap line, Et(cp:EGFP)si2, that expresses enhanced green fluorescent protein (EGFP) in the choroid plexus epithelium. Using immunohistochemistry and fluorescent tracers, we demonstrated that the zebrafish choroid plexus possesses brain barrier properties such as tight junctions and transporter activity. Thus, we have established zebrafish as a functionally relevant model to study choroid plexus development. Using an unbiased approach, we performed a forward genetic dissection of the choroid plexus to identify genes essential for its formation and function. Using Et(cp:EGFP)sj2, we isolated 10 recessive mutant lines with choroid plexus abnormalities, which were grouped into five classes based on GFP intensity, epithelial localization, and overall choroid plexus morphology. We also mapped the mutation for two mutant lines to chromosomes 4 and 21, respectively. The mutants generated in this study can be used to elucidate specific genes and signaling pathways essential for choroid plexus development, function, and/or maintenance and will provide important insights into how these genetic mutations contribute to disease.

Keywords: choroid plexus, zebrafish, forward genetic screen, Claudin 5, transporters, fluorescein, genetic mapping

\section{INTRODUCTION}

The choroid plexus consists of polarized epithelial cells projecting into the brain ventricle to create a blood-cerebrospinal fluid barrier between fenestrated capillaries and the cerebrospinal fluid (Wolburg and Paulus, 2010). These epithelial cells are modified ependymal cells derived from embryonic neuroepithelial cells that form intercellular tight junctions and express multiple families of transporters that create both a physical and chemical barrier. The choroid plexus also plays an important role in the production and secretion of the cerebrospinal fluid and the regulation of central nervous system homeostasis. Although the choroid plexus of the lateral brain ventricles was documented by Herophilus, an ancient Greek physician (Dohrmann, 1970; Dziegielewska et al., 2001), there has been little research aimed toward identifying the genetic pathways essential for choroid

Abbreviations: Cldn5, Claudin 5; CNS, central nervous system; CP, choroid plexus; CPe, choroid plexus epithelium; CSF, cerebrospinal fluid; dpf, days postfertilization; dCP, diencephalic choroid plexus; EGFP, enhanced green fluorescent protein; Et(cp:EGFP) $)^{s i 2}$, Enhancer trap(choroid plexus:enhanced green fluorescent protein) $)^{s i 2}$; hpf, hours post-fertilization; mCP, myelencephalic choroid plexus; MDR1, Multidrug Resistance Transporter 1; MRP1, Multidrug ResistanceAssociated Protein 1; OAT3, Organic Anion Transporter 3; PTU, N-Phenylthiourea. plexus formation and function. Previous studies have classified choroid plexus development by observing modifications in cell morphology, measuring glycogen content as it matures, and examining gene expression (Dohrmann, 1970; Strazielle and Ghersi-Egea, 2000; Dziegielewska et al., 2001; Kratzer et al., 2012, 2013; Liddelow et al., 2014). Signaling pathways such as Sonic hedgehog (Huang et al., 2009; Nielsen and Dymecki, 2010), BMP (Currle et al., 2005), and Notch (Irvin et al., 2004) have been found to contribute to choroid plexus formation. However, how these pathways interact with one another to generate the choroid plexus and the downstream components involved in its development, function, and maintenance are unknown. Understanding normal development and function in the choroid plexus is crucial to unraveling how these mechanisms become altered in diseases. The choroid plexus has been associated with various disease conditions, including choroid plexus tumors (Wolburg and Paulus, 2010), hydrocephalus (Wodarczyk et al., 2009), and neurodegenerative diseases such as Alzheimer's disease (AlviraBotero and Carro, 2010) and multiple sclerosis (Vercellino et al., 2008). Identifying altered genes and signaling pathways in the choroid plexus that cause disease initiation or progression can help determine potential therapeutic targets for treatment. 
Models used to study the choroid plexus have included (1) mice, because of their similarity to the human choroid plexus in gene expression patterns (Janssen et al., 2013); (2) marsupials, because post-natal lateral ventricle development occurs outside of the womb (Liddelow et al., 2010); and (3) in vitro cell culture systems to study choroid plexus transport (Monnot and Zheng, 2013). However, these models do not provide real-time, in vivo developmental information. We propose, as have others (Bill et al., 2008; Garcia-Lecea et al., 2008), that zebrafish are a suitable vertebrate model to analyze and genetically dissect choroid plexus development and function in vivo, as they offer several advantages such as having central nervous system structures similar to those in mammals, rapid ex utero development, transparency, large numbers of offspring, and genetic tractability (Goldsmith and Jobin, 2012).

Previous studies have examined the role of Shh and Notch signaling in choroid plexus development using zebrafish (Bill et al., 2008; Garcia-Lecea et al., 2008). However, these studies did not examine functional choroid plexus barrier properties such as tight junctions and transporters. Here, we generated an enhancer trap line, $E t(c p: E G F P)^{s j 2}$, that expresses enhanced green fluorescent protein (EGFP) in epithelial cells of the diencephalic choroid plexus and myelencephalic choroid plexus. We used this enhancer trap line to visualize choroid plexus development in vivo and demonstrated that several components of the zebrafish choroid plexus are comparable to human. We also performed a smallscale ENU-mutagenesis screen and identified 10 recessive mutant lines. These mutants were classified into five categories on the basis of EGFP expression and choroid plexus morphology. We also genetically mapped the mutations from two mutant lines using bulked-segregant analysis. The cloning and characterization of these mutants will provide important insights into the genetic pathways that regulate formation and function of the choroid plexus in health and disease.

\section{MATERIALS AND METHODS FISH LINES AND MAINTENANCE}

Zebrafish were maintained at $28.5^{\circ}$ on a $14 \mathrm{~h}$ light/dark cycle. Embryos used for imaging or for screening $\mathrm{F}_{3}$ larvae were collected in egg water $(0.03 \%$ Instant Ocean) containing $0.02 \%$ methylene blue and treated at $24 \mathrm{~h}$ post-fertilization (hpf) with 0.003\% 1-phenyl-2-thiourea (PTU) (Sigma) to prevent pigment formation. Zebrafish were maintained in accordance with established protocols and all experiments were approved by the St. Jude Children's Research Hospital Institutional Animal Care and Use Committee.

\section{GENERATION OF THE Et(cp:EGFP)sj2 LINE}

To generate the $E t(c p: E G F P)^{s j 2}$ line, EGFP was released from the pEGFP-N1 vector (Clontech) by BamHI and NotI digestion and inserted into the pTRE-Tight vector (Clontech). The TRE-tight:EGFP fragment was released by XhoI and ClaI digestion and inserted into the pT2AL200R150G vector (Urasaki et al., 2006). Approximately $30 \mathrm{pg}$ of the resulting T2 (TREtight:EGFP) plasmid DNA was co-injected with $30 \mathrm{pg}$ of in vitro transcribed Tol 2 transposase mRNA into single-cell embryos of the $A B$ strain. The embryos were raised to adults and screened for germline transmission by examining their offspring for EGFP expression.

\section{IMMUNOHISTOCHEMISTRY (IHC)}

Larvae were anesthetized in $0.02 \%$ tricaine and fixed in $4 \%$ paraformaldehyde (PFA) (Electron Microscopy Sciences) at $4^{\circ}$ overnight and washed the next day in $1 \times$ phosphate buffered serum (PBS) (Calbiochem). Samples were sunk in 30\% sucrose/PBS at $4^{\circ}$ overnight. Larval 1-month-old brain tissues, and adult brains were embedded in Optimal Cutting Temperature (O.C.T.) Compound (Tissue-Tek), frozen on dry ice, and stored at $-80^{\circ}$. Tissues were sectioned using a Leica CM 1950 cryostat. Sections were washed in PBS for $5 \mathrm{~min}$ followed by three $5 \mathrm{~min}$ washes in PBST [PBS/0.03\% Triton X-100 (Sigma)]. Sections were incubated in blocking buffer [PBST with 5\% goat serum (Gibco) and 1\% BSA (Sigma)] for $3 \mathrm{~h}$ at room temperature. Primary antibodies were incubated at $4^{\circ}$ overnight followed by secondary antibody incubation for $2 \mathrm{~h}$ at room temperature. Sections were washed in PBST four times for 15 min after primary and secondary antibody incubations. Primary antibodies included rabbit anti-GFP (1:500; Invitrogen) and mouse antiZpr1 (1:100; ZIRC). Secondary antibodies included Alexa Fluor goat anti-rabbit 488 (1:200; Invitrogen) and Alexa Fluor goat antimouse 555 (1:200; Invitrogen). Antibody dilutions were prepared in blocking buffer. Sections were counterstained with $1 \mu \mathrm{g} / \mathrm{mL}$ DAPI (Roche) for $1 \mathrm{~min}$, rinsed briefly in PBS, and mounted with Fluoromount (Electron Microscopy Sciences). Images were taken on a Nikon AZ100 microscope and analyzed using NIS-Elements AR 3.2 software.

\section{WHOLE-MOUNT IHC}

Embryos were incubated in egg water with $0.003 \%$ PTU to prevent pigment formation. At 4 and 6 days post-fertilization (dpf), larvae were anesthetized in $0.02 \%$ tricaine and fixed in $4 \%$ PFA overnight. The next day, samples were washed in $1 \times$ PBS followed by $1 \times$ PBST and treated with $20 \mu \mathrm{g} / \mathrm{mL}$ Proteinase $\mathrm{K}$ (New England Biolabs) for $15 \mathrm{~min}$. The reaction was stopped by adding 10\% lamb serum (Gibco) followed by additional washes in PBST. Samples were blocked with $10 \%$ lamb serum for $1-4 \mathrm{~h}$ and incubated in primary antibody at $4^{\circ}$ overnight. Samples were washed the next day in PBST and incubated in secondary antibody at $4^{\circ}$ overnight. Antibodies used included rabbit antiGFP (1:100; Invitrogen), mouse anti-Zpr1 (1:50; ZIRC), mouse anti-Cldn5 (1:100; Invitrogen), Alexa Fluor goat anti-rabbit 488 (1:200; Invitrogen), and Alexa Fluor goat anti-mouse 555 (1:200; Invitrogen). Additional washes in PBST were done the following day and stored in $1 \times$ PBS. Samples were embedded in $0.8 \%$ lowmelting point agarose (Invitrogen) made in $1 \times$ PBS and imaged on Nikon C1Si laser scanning confocal microscope. Z-stacks were compiled to create maximum intensity projection images using Nikon NIS-Elements 3.1 imaging software.

\section{FLUORESCENT TRACER INJECTIONS}

To observe transporter activity in the choroid plexus, $E t(c p: E G F P)^{s j 2}$ larvae at $4 \mathrm{dpf}$ were anesthetized in $0.02 \%$ tricaine and injected intravenously into the common cardinal vein with approximately $1-2 \mathrm{~nL}$ of $100 \mu \mathrm{M}$ rhodamine 123 
(Sigma-Aldrich) using a micromanipulator and a pneumatic picopump (World Precision Instruments). The total blood volume of zebrafish embryos at $2 \mathrm{dpf}$ has been estimated to be $60 \mathrm{~nL}$ (Craig et al., 2012), so the blood volume is not significantly altered upon injection. Immediately after the injections, larvae were embedded in 1.2\% low-melting-point agarose (Invitrogen) made in egg water. Larvae were imaged on a Nikon C1Si confocal microscope and analyzed using Nikon EZC1 3.91 software.

To study choroid plexus function, Casper larvae (lacking melanocytes and iridophores) (White et al., 2008) were anesthetized in $0.02 \%$ tricaine and intravenously injected into the common cardinal vein with approximately $0.3-2 \mathrm{~nL}$ of $10 \mathrm{mg} / \mathrm{mL}$ rhodamine- and fluorescein-labeled dextrans at 2, 3, and $4 \mathrm{dpf}$. Fluorescent tracers included a $3-\mathrm{kDa}$ fluorescein dextran, a $10-\mathrm{kDa}$ rhodamine dextran, and a $40-\mathrm{kDa}$ anionic fluorescein dextran (Invitrogen). Immediately after the injections, larvae were laterally embedded in $1.2 \%$ low-melting-point agarose made in egg water. To observe tracer permeability into the brain ventricle, time-lapse images were collected every $2 \mathrm{~min}$ for $1 \mathrm{~h}$ with a Nikon AZ100 microscope equipped with a shutter (Sutter Instruments) and analyzed with Nikon NIS-Elements 3.2 software. $\operatorname{Et}(c p: E G F P)^{s j 2}$ larvae were also injected intravenously with the $10-\mathrm{kDa}$ rhodamine dextran at 2 and $4 \mathrm{dpf}$ and imaged on a Nikon C1Si confocal microscope with Nikon EZC1 3.91 software.

To visualize brain ventricle morphology in wild-type and mutant Et (cp:EGFP) $)^{s j 2}$, PTU-treated larvae at $4 \mathrm{dpf}$ were anesthetized and intraventricularly injected with approximately $2 \mathrm{~nL}$ of $40-\mathrm{kDa}$ fluorescein-labeled dextran as described previously (Gutzman and Sive, 2009). Larvae were imaged immediately after the injection using a Nikon SMZ1500 epifluorescence stereomicroscope and analyzed with Nikon NIS-Elements 3.1 software. Ventricular injections of rhodamine 123 and fluorescein were performed as described previously (Gutzman and Sive, 2009) in Casper larvae at $4 \mathrm{dpf}$ and imaged on a Nikon C1Si confocal microscope with Nikon EZC1 3.91 software.

\section{FLUORESCEIN TREATMENT}

Casper and $E t(c p: E G F P)^{s j 2}$ larvae were incubated for $4 \mathrm{~h}$ in the dark with $50 \mu \mathrm{M}$ fluorescein (Fluka). Larvae were briefly rinsed 12 times in egg water, anesthetized in $0.02 \%$ tricaine, and embedded in $1.2 \%$ low-melting-point agarose. After $30 \mathrm{~min}$, larvae were imaged using a Nikon SMZ1500 epifluorescence stereomicroscope and analyzed with Nikon NIS-Elements 3.1 software.

\section{STATISTICAL ANALYSIS OF TRACER PERMEABILITY}

The ratio of the fluorescence intensity in the ventricle to that in the heart was calculated at the 30-min timepoint when the leakiest tracer reached saturation. The ratio was then normalized against the tracer having the greatest mean intensity in the ventricle at $30 \mathrm{~min}$. A One-Way ANOVA test was performed in Microsoft Excel to determine whether there was a significant difference in fluorescent intensity between the tracers and developmental timepoints. A Tukey's post-hoc test was performed to determine which tracers were statistically significant to one another at each developmental timepoint. A significance value of $\alpha=0.05$ was used, and $P$-values were calculated using the GraphPad software $P$-value calculator. Error bars were based on the mean of 7 observations using standard error.

\section{N-ETHYL-N-NITROSOUREA (ENU) MUTAGENESIS AND FORWARD GENETIC SCREENING}

Twenty Et(cp:EGFP) sj2 males were treated three times with $3 \mathrm{mM}$ ENU (Sigma) for $1 \mathrm{~h}$ each at weekly intervals as described previously (Driever et al., 1996). After 1 month, ENU-treated males were mated to $E t(c p: E G F P)^{s j 2}$ females to produce the $\mathrm{F}_{1}$ generation. $F_{1}$ pairwise crosses were performed to produce $F_{2}$ families. $\mathrm{F}_{2}$ families that did not contain at least 4 male/female pairs were sacrificed. Pairwise crosses for each $\mathrm{F}_{2}$ family were done at least six times if possible to identify F3 offspring with homozygous recessive mutations. $\mathrm{F}_{2}$ pairs deemed heterozygous for a recessive mutation were maintained in miniboxes for use in genetic mapping experiments. To screen for choroid plexus mutants, $\mathrm{F}_{3}$ larvae at $4 \mathrm{dpf}$ were anesthetized and visualized using a Nikon SMZ1500 epifluorescence stereomicroscope to identify abnormal GFP expression or patterning in the choroid plexus. A mutant line was confirmed if approximately $25 \%$ of the total offspring displayed a choroid plexus phenotype.

\section{GENOMIC DNA ISOLATION}

Adult zebrafish were anesthetized in $0.02 \%$ tricaine (SigmaAldrich) and tail clipped. As the fish recovered in system water, the tail clip was immediately immersed into $50 \mu \mathrm{L}$ digest buffer (0.2 M NaCl, $10 \mathrm{mM}$ Tris 8.0, $5 \mathrm{mM}$ EDTA, $0.1 \%$ SDS, $0.1 \mathrm{mg} / \mathrm{ml}$ Proteinase K). Samples were incubated overnight at $50^{\circ}$. After brief centrifugation, $450 \mu \mathrm{L}$ of $100 \%$ ethanol (EtOH) added. Samples were centrifuged at $21,130 \mathrm{~g}$ for $10 \mathrm{~min}$, the supernatant was removed, and $500 \mu \mathrm{L}$ of $70 \% \mathrm{EtOH}$ added to wash the pellet. Samples were centrifuged at $21,130 \mathrm{~g}$ or $15,000 \mathrm{rpm}$ for $5 \mathrm{~min}$. After removing the supernatant, the samples were stored at room temperature for about $30 \mathrm{~min}$ to evaporate excess EtOH. The DNA pellets were resuspended with $50 \mu \mathrm{L}$ of TE buffer $(10 \mathrm{mM}$ Tris and $1 \mathrm{mM}$ EDTA, $\mathrm{pH}$ 8.0).

For zebrafish larvae, wild-type and mutants were anesthetized, separated into $1.5 \mathrm{~mL}$ eppendorf tubes, and immersed in $1 \mathrm{~mL}$ $100 \%$ methanol (MeOH) (Sigma-Aldrich). Samples were stored at $-20^{\circ}$ overnight. The following day, individual wild-type and mutant larvae were transferred into $0.5 \mathrm{~mL}$ tubes and the excess $\mathrm{MeOH}$ removed. Once all the $\mathrm{MeOH}$ evaporated, $10 \mu \mathrm{L}$ of digest buffer (same as above) was added to each tube. Samples were incubated overnight at $50^{\circ}$. DNA was isolated as described above for adults except the volume of $100 \% \mathrm{EtOH}$ and $70 \% \mathrm{EtOH}$ was $180 \mu \mathrm{L}$ and the volume of TE buffer was $25 \mu \mathrm{L}$.

\section{GENETIC MAPPING}

After identifying a mutant line, the $E t(c p: E G F P)^{s j 2} \mathrm{~F}_{2}$ heterozygous parents were each outcrossed to the polymorphic TL strain. From this outcross, we identified AB/TL hybrids heterozygous for the recessive mutation by screening their offspring. The wildtype and mutant offspring from these heterozygous hybrids were collected and their DNA was extracted (see above). A genome scan using 192 polymorphic Z-markers was performed with DNA pooled from 30 wild-type and 30 mutant larvae as previously described (Muto et al., 2005). Wild-type and mutant 
DNA was amplified using the Accuprime Taq DNA Polymerase System (Invitrogen). The resulting PCR products were analyzed for genetic linkage by agarose gel electrophoresis. For fine genetic mapping, individual wild-type and mutant DNA was amplified using Platinum PCR Supermix (Invitrogen). Z-markers used for genetic mapping were acquired from the zebrafish genome database at www.ensembl.org. Additional markers were designed by examining the Ensembl genome browser for polymorphic regions in the genome.

\section{LIVE CONFOCAL IMAGING AND TIME-LAPSE MOVIES}

Confocal imaging was performed on a Nikon C1Si confocal microscope and analyzed using Nikon EZC1 3.91 software. Scans of DIC and GFP images were acquired using a $20 \times$ objective at $4 \mu \mathrm{m}$ intervals to create a $\mathrm{z}$-stack maximum intensity projection image. To observe choroid plexus development, larvae were collected at $\sim 30 \mathrm{hpf}$ and prepared for imaging as described above. Z-stack images were acquired every $30 \mathrm{~min}$ over a $65 \mathrm{~h}$ period. Using NIS-Elements AR 4.0 software, images were smoothed and Z-stacks were compiled to create an enhanced depth of focus (EDF) image. To compare choroid plexus development, wild-type and mutant larvae were imaged beginning at $54 \mathrm{hpf}$ with images acquired every $30 \mathrm{~min}$ over a $48 \mathrm{~h}$ period. Supplemental movies run for the $48 \mathrm{~h}$ period except for Supplemental movie 6 which runs for $42 \mathrm{~h}$.

\section{RESULTS \\ GENERATION AND CHARACTERIZATION OF THE ZEBRAFISH CHOROID PLEXUS ENHANCER TRAP LINE Et(cp:EGFP)sj2}

In the process of producing a tetracycline-inducible transgenic line, we fortuitously generated the zebrafish choroid plexus line by an enhancer trap effect where EGFP became "trapped" within the genome and is now regulated by an enhancer. To generate this line, we co-injected a TRE-EGFP construct with Tol2 transposase mRNA into single-cell zebrafish embryos. The TRE-EGFP construct was integrated into an enhancer region that regulates gene expression in the choroid plexus. Using Southern blot analysis, we identified a single TRE-EGFP insertion on chromosome 13 (data not shown). By sequencing genes within this region, we identified the TRE-GFP transgene between two genes TATA box binding protein ( $t b p)$ and asparaginase-like1 (asrgl1), both of which according to zfin.org are not spatially restricted in their mRNA expression patterns. After performing in situ hybridization on asrgll, we did not observe expression within the choroid plexus (data not shown). Because we were unable to identify choroid plexus specific genes along this region of Chromosome 13, we concluded that the GFP expression in the choroid plexus is the result of an enhancer trap affect where an enhancer localized upstream or downstream of GFP is regulating GFP expression. The insertion was transmitted to subsequent generations, thereby creating a stable line termed $\operatorname{Et}(c p: E G F P)^{s j 2}$.

GFP expression was specifically localized to the diencephalic choroid plexus in the third ventricle and the myelencephalic choroid plexus in the fourth ventricle at $3 \mathrm{dpf}$ corresponding to the mammalian choroid plexus in third and fourth ventricles, respectively (Figure 1A). We identified that this integration resulted in EGFP expression in the diencephalic choroid plexus and myelencephalic choroid plexus based on two previously published papers also identifying GFP expression in the diencephalic choroid plexus and myelencephalic choroid plexus of zebrafish (Bill et al., 2008; Garcia-Lecea et al., 2008). Unlike mammals, the zebrafish choroid plexus is dorsal to the brain allowing for easy visualization of choroid plexus development. As mentioned in the two previous zebrafish choroid plexus studies, the myelencephalic choroid plexus in teleosts exists as a layer of GFP positive cells on the dorsal surface of the fourth ventricle and ventral to the outer skin epithelium, while the diencephalic choroid plexus is at the most dorsal portion of the third ventricle (Bill et al., 2008; GarciaLecea et al., 2008). This allows choroid plexus development to be easily visualized in the zebrafish because it is not buried inside the brain as in other vertebrates. Similarly as described in the dogfish shark, the myelencephalic choroid plexus lies over the brain ventricle rather than obstructed between the medulla and cerebellum (Villalobos et al., 2002). Other studies on zebrafish brain development describe how the development of the telencephalon folds outward with the proliferating cells on the outside rather than inward as seen in other vertebrates (Schmidt et al., 2013), suggesting the zebrafish has more of an "inside out" morphology. This type of development in zebrafish, termed the eversion model, begins with brain ventricular formation followed by the movement of surrounding neural tissue migrating into the ventricle space and results in an overlying ventricle surrounding the brain tissue (Folgueira et al., 2012). Because brain ventricular formation occurs prior to choroid plexus development, the eversion process may be responsible for this "inside out" morphology.

To gain further insight into the location of the choroid plexus in relation to other central nervous system structures, we used whole-mount immunohistochemistry (IHC) to stain Et(cp:EGFP $)^{s j 2}$ larvae with a Zpr-1 antibody labeling cone photoreceptors of the eye and the pineal gland. In other teleosts and mammals, the pineal gland directly interacts with the choroid plexus of the third ventricle (Omura et al., 1985; Skinner and Malpaux, 1999). Although the diencephalic choroid plexus is distinct from the pineal gland, we found a close association between the two structures, where the diencephalic choroid plexus was found anterior to the pineal gland (Figure 1B). Transverse sections showed the diencephalic choroid plexus positioned beneath the pineal gland (Figure 1C). Additional transverse sections through the myelencephalic choroid plexus showed a cluster of GFP-positive epithelial cells directly beneath the skin surface (Figure 1D). GFP expression in the diencephalic choroid plexus and myelencephalic choroid plexus was retained throughout development and at later stages, as shown in a 1-month-old $E t(c p: E G F P)^{s j 2}$ (Figures 1E,F).

To further demonstrate that this is indeed the choroid plexus, we cut sections from adult brains and showed expression of GFP in a structure historically referred to as the saccus dorsalis (Figure 2). This structure is homologous to the mammalian choroid plexus of the third ventricle. The saccus dorsalis in rainbow trout (Salmo gairdneri), has been described as a folded monolayer of epithelium that develops out of the diencephalic roof plate (Jansen et al., 1976). Because the saccus dorsalis has also been described to partially cover the pineal stalk (Tsuneki, 1986), similar to what we show in Figure 1B, we conclude that this is the 


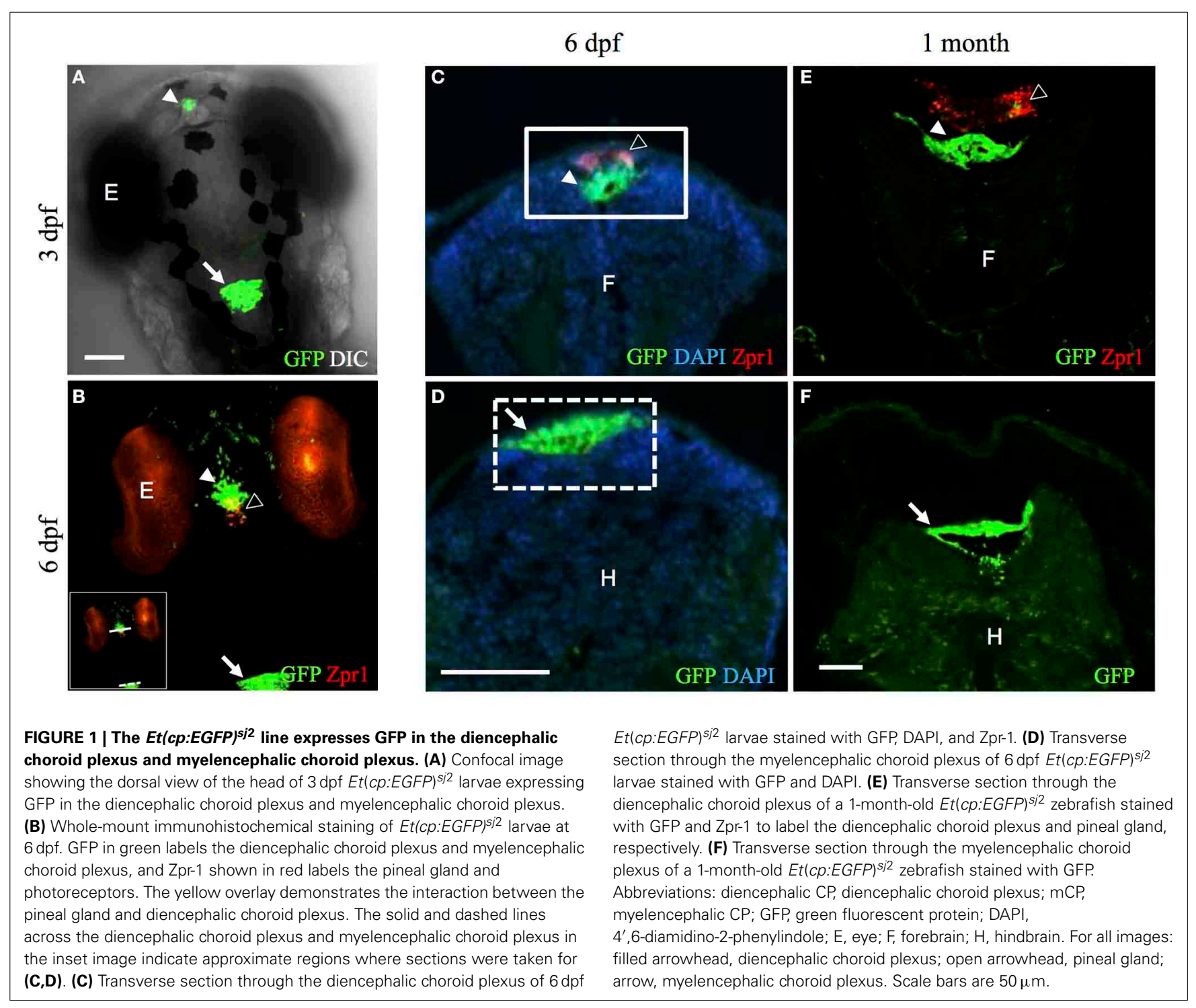

choroid plexus of the third ventricle in zebrafish. We also detected GFP expression in the swimbladder (data not shown). The swimbladder consists of epithelial cells and has been compared to the mammalian lung (Winata et al., 2009). Using time-lapse confocal microscopy, GFP expression was detected in a subset of diencephalic choroid plexus cells as early as $30 \mathrm{hpf}$ (see Supplemental Movie 1). We found that the myelencephalic choroid plexus epithelial cells migrated from the outer rhombomeres into the midbrain-hindbrain boundary as described previously (GarciaLecea et al., 2008) and also anteriorly from the developing spinal cord. These cells increase in GFP intensity as they coalesce to form a compact structure by approximately $72 \mathrm{hpf}$. There were no additional changes in choroid plexus morphology by 96 hpf.

\section{ZEBRAFISH CHOROID PLEXUS EXPRESSES THE TIGHT JUNCTION PROTEIN CLAUDIN 5 AND POSSESSES TRANSPORTER ACTIVITY}

The mammalian choroid plexus expresses many tight-junction proteins such as claudins (Lippoldt et al., 2000; Kratzer et al.,

2012), occludin (Lagaraine et al., 2011), and junction adhesion molecules (Lagaraine et al., 2011). These tight junctions create a physical barrier between the blood and cerebrospinal fluid. To demonstrate that the zebrafish choroid plexus express tight junction protein similar to mammals, we studied Claudin 5 (Cldn5) expression because of the availability of an antibody that cross-reacts with zebrafish Cldn5 (Xie et al., 2010; Zhang et al., 2010). As predicted, whole-mount IHC analysis revealed Cldn5 expression at the cell membrane of zebrafish choroid plexus epithelium (Figures $\mathbf{3 A - C}$ ). Cldn5 expression was also observed in the eyes, blood vessels, and neighboring ependymal cells (Figures 3B,C).

In mice, multidrug resistance transporters, such as MRP1, are expressed on the basolateral side of choroid plexus epithelium and regulate drug accumulation in the cerebrospinal fluid (Wijnholds et al., 2000). In addition to drug transport, MRP1 and MDR1, efflux fluorescent dyes such as rhodamine 123 (Saengkhae et al., 2003). To the best of our knowledge, no previous studies have demonstrated transporter function in zebrafish choroid 

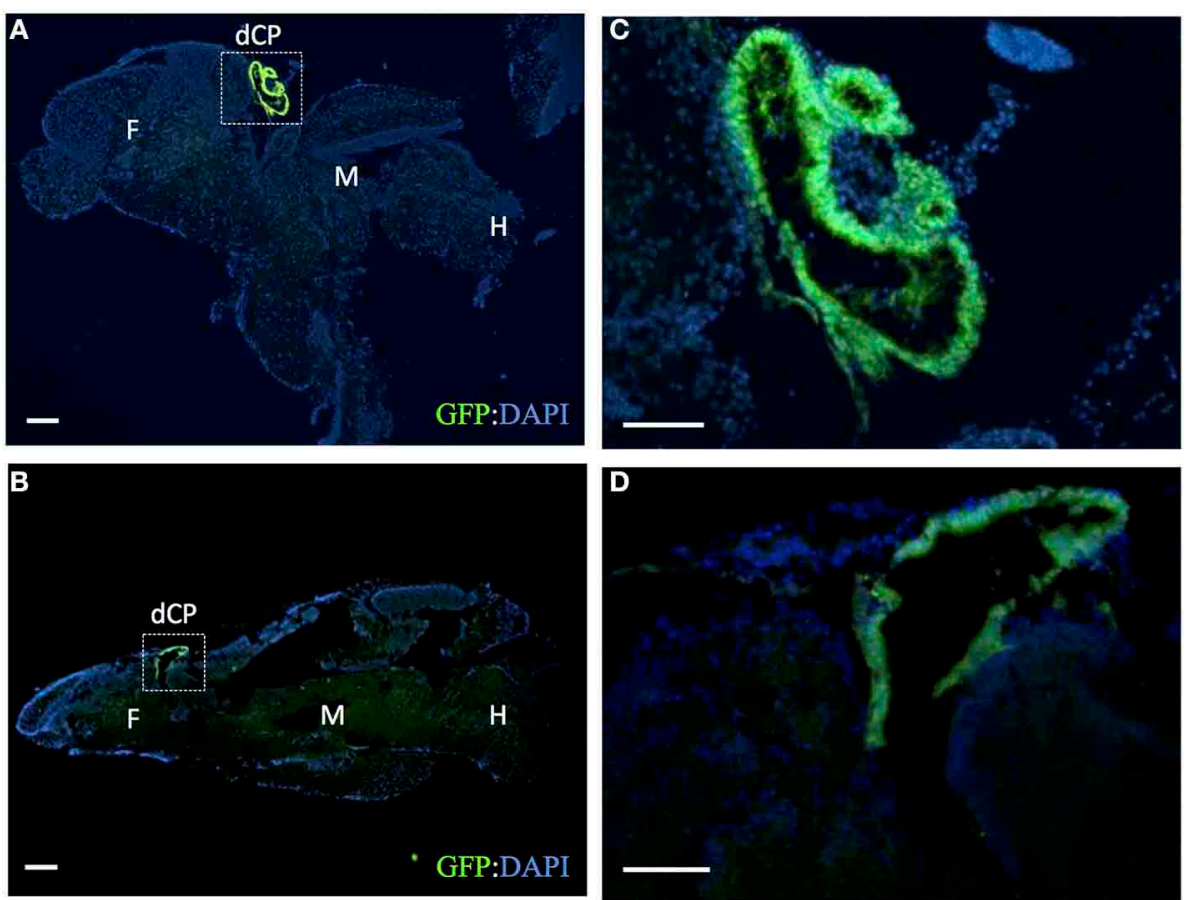

FIGURE 2 | The adult Et(cp:EGFP)sj2 expresses GFP in the saccus dorsalis. Sagital sections through adult brains were labeled with rabbit anti-GFP antibody (green) and counterstained with DAPI (blue). $(\mathbf{A}, \mathbf{B})$ shows GFP expression in the adult CP of the third ventricle taken through two different sagittal planes of the adult brain. Dashed-line boxes refer to magnified images shown in $\mathbf{( C , D )}$. dCP. diencephalic CP; F: forebrain; $\mathrm{M}$ : midbrain; $\mathrm{H}$ : hindbrain. Scale bars are $100 \mu \mathrm{m}$. plexus. To determine whether transporters contribute to the blood-cerebrospinal fluid barrier at the zebrafish choroid plexus, we intravenously injected rhodamine 123 into the $E t(c p: E G F P)^{s j 2}$ line at $4 \mathrm{dpf}$ and observed its accumulation within the choroid plexus epithelium (Figures 3D-F). After injecting rhodamine 123 into the brain ventricle/cerebrospinal fluid, we also observed accumulation in the choroid plexus (Figures 4A-D), indicating that independent of what interface the rhodamine 123 resides (whether blood or cerebrospinal fluid), the dye is transported by the choroid plexus.

Another transporter that is highly expressed in the choroid plexus is organic anion transporter 3 (OAT3) (Keep and Smith, 2011). Fluorescein, a known substrate for OAT3, is taken up by the transporter on the apical side of the choroid plexus epithelium in a $\mathrm{Na}^{+}$-dependent manner (Sykes et al., 2004). Because fluorescein is a low-molecular-weight green fluorescent dye, we soaked Casper zebrafish in $50 \mu \mathrm{M}$ fluorescein to observe accumulation in the choroid plexus. The dye was ingested in the gut, entered the circulation, and concentrated in the diencephalic choroid plexus and myelencephalic choroid plexus (Figures 3G,H). Fluorescein injected into the brain ventricle had a similar pattern of accumulation in the myelencephalic choroid plexus and diencephalic choroid plexus (Figures 4E-H).

\section{ZEBRAFISH CHOROID PLEXUS POSSESSES SIZE-SELECTIVE BARRIER PROPERTIES}

To examine the function of the zebrafish choroid plexus in regulating passage from the bloodstream into the cerebrospinal fluid, we intravenously injected fluorescently labeled dextrans of various molecular weights into the transparent Casper line at 2, 3 , and $4 \mathrm{dpf}$. Time-lapse imaging at $2 \mathrm{dpf}$, before the choroid plexus has fully developed, showed that $3-\mathrm{kDa}$ fluorescein and $10-\mathrm{kDa}$ rhodamine dextrans leaked from the bloodstream into the brain ventricle, whereas little to no $40-\mathrm{kDa}$ fluorescein dextran escaped from the bloodstream into the ventricle (Figure 5A). By $3 \mathrm{dpf}$, there was still a significant difference with a $P$-value of $<0.0001$ between the 3 - and $10-\mathrm{kDa}$ dextrans and the $40-$ $\mathrm{kDa}$ dextran; however, the overall permeability was lower at $3 \mathrm{dpf}$ compared to $2 \mathrm{dpf}$ (Figure 5B). By $4 \mathrm{dpf}$, when the choroid plexus was fully formed, there were no significant differences between the individual tracers (Figure 5B). Dorsal views of an intravenous injection of the $10-\mathrm{kDa}$ dextran into $E t(c p: E G F P)^{s j 2}$ larvae at $2 \mathrm{dpf}$ showed leakage into the brain ventricle as the choroid plexus continued to develop. Once formed by $4 \mathrm{dpf}$, dextran did not accumulate within the brain ventricle (Figure 6A). In addition, the 3 - and $10-\mathrm{kDa}$ dextrans were significantly more permeable at $2 \mathrm{dpf}(P<0.0001)$ compared to 3 and $4 \mathrm{dpf}$, but there was no significant difference in permeability for the $40-\mathrm{kDa}$ dextran (Figure 6B).

\section{IDENTIFICATION OF CHOROID PLEXUS MUTANTS}

After demonstrating that the zebrafish choroid plexus develops and functions as the blood-cerebrospinal fluid barrier beginning at $3 \mathrm{dpf}$, we initiated a small-scale forward genetic screen to identify mutants with choroid plexus abnormalities. This screen focused primarily on myelencephalic choroid plexus defects. 

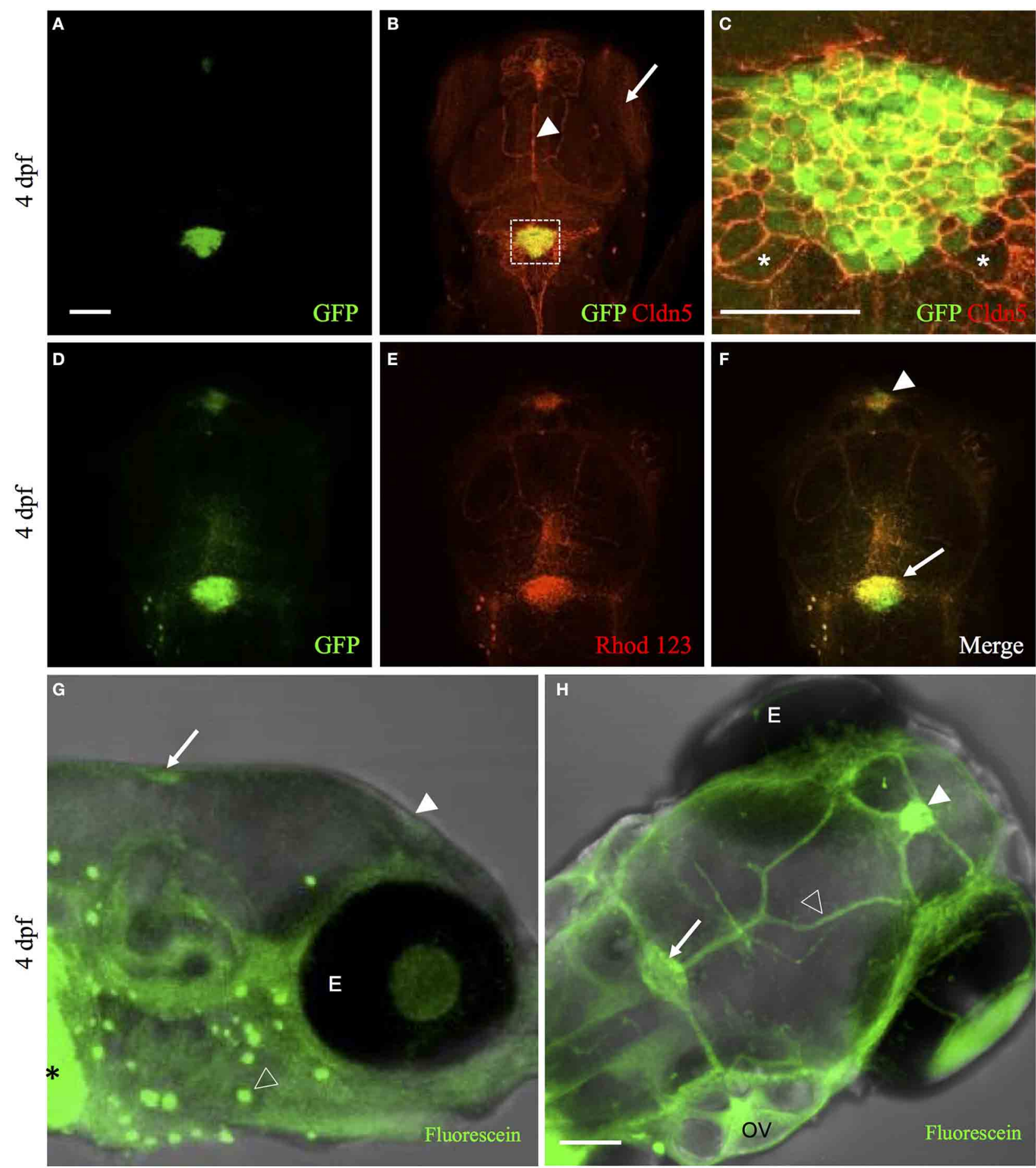

\section{FIGURE 3 | The zebrafish CP possesses tight junction and transporter} properties. (A) Whole-mount immunohistochemical staining of

Et(cp:EGFP)sj2 larvae at $4 \mathrm{dpf}$ with GFP labeling the diencephalic choroid plexus and myelencephalic choroid plexus. (B) Whole-mount immunohistochemical staining with Claudin 5 antibody (red) showed expression in the eye (arrow), surface vessels (arrowhead), and CP. (C) Overlay of GFP and Claudin 5 staining revealed Claudin 5 expression surrounding the perimeter of $\mathrm{CPe}$ and ependymal cells. Examples of ependymal cells are shown with an asterisk $(*)$. Images in $(\mathbf{A}, \mathbf{B})$ taken at $20 \times$ magnification and (C) at $40 \times$ magnification. (D) Live imaging of Et(cp:EGFP)sj2 larvae at $4 \mathrm{dpf}$ showed GFP expression in the CP. (E,F)
Accumulation of rhodamine 123 in the diencephalic choroid plexus (arrowhead) and myelencephalic choroid plexus (arrow). (G) Side view of live Casper zebrafish treated at $4 \mathrm{dpf}$ with $50 \mu \mathrm{M}$ fluorescein for $4 \mathrm{~h}$ showed accumulation of fluorescein (represented in green) in the diencephalic choroid plexus (filled arrowhead) and myelencephalic choroid plexus (arrow) along with the gut (asterisk), and lateral line (open arrowhead). (H) Similar image as in (G) except for dorsal view showing accumulation in the diencephalic choroid plexus (filled arrowhead), myelencephalic choroid plexus (arrow), and overlying vasculature (open arrowhead). Abbreviations: dCP, diencephalic $\mathrm{CP}$; $\mathrm{mCP}$, myelencephalic $\mathrm{CP}$; $\mathrm{E}$, eye; OV; Otic vesicle. Scale bar in $A$ and $H$ is $50 \mu \mathrm{m}$. Scale bar in $C$ is $20 \mu \mathrm{m}$. 

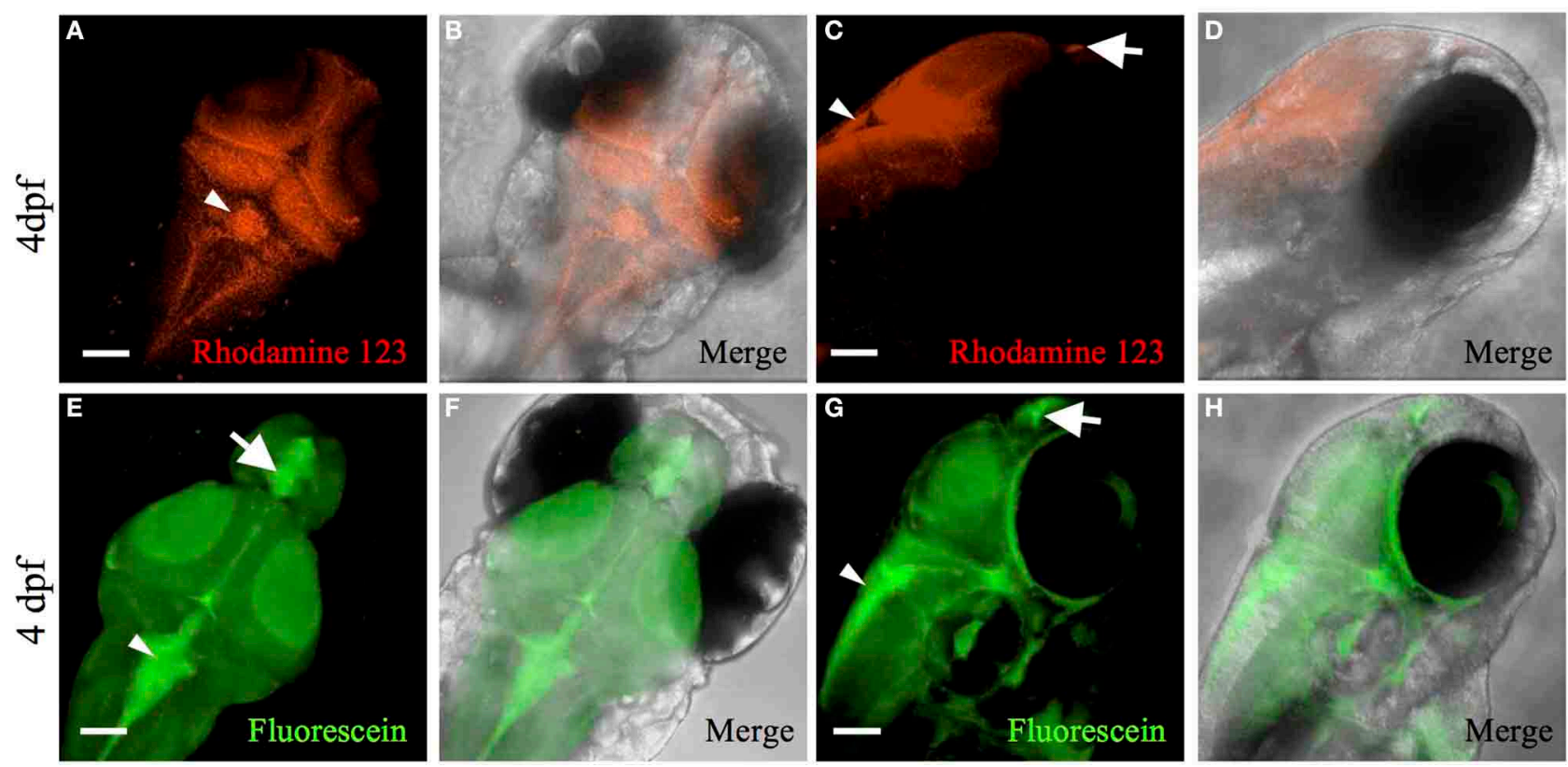

FIGURE 4 | Rhodamine 123 and fluorescein ventricle injection demonstrates transporter activity in the CP. Dorsal fluorescent (A) and merged (bright-field and Rhodamine 123) (B) confocal image of $4 \mathrm{dpf}$ Casper larvae with Rhodamine 123 (red) injection into the brain ventricle. The rhodamine 123 concentrates within the myelencephalic choroid plexus (arrowhead). Rhodamine 123 also fluoresces green and co-localizes with red emission (not shown). Lateral fluorescent (C) and merged (bright-field and Rhodamine 123) (D) confocal image of $4 \mathrm{dpf}$ Casper larvae with Rhodamine 123 (red) injection into the brain ventricle. The rhodamine 123 concentrates within the myelencephalic choroid plexus (arrowhead) and diencephalic choroid plexus (arrow). Dorsal fluorescent (E) and merged (bright-field and fluorescein) (F) confocal image of $4 \mathrm{dpf}$ Casper larvae with fluorescein (green) injected into the brain ventricle. Fluorescein concentrates within the myelencephalic choroid plexus (arrowhead) and diencephalic choroid plexus (arrow). Lateral fluorescent (G) and merged(bright-field and fluorescein) (H) confocal image of $4 \mathrm{dpf}$ Casper larvae with fluorescein (green) injected into the brain ventricle. Fluorescein concentrates within the myelencephalic choroid plexus (arrowhead) and diencephalic choroid plexus (arrow).

Abbreviations: $\mathrm{mCP}$, myelencephalic choroid plexus; $\mathrm{dCP}$, diencephalic choroid plexus. Scale bar is $50 \mu \mathrm{m}$.
Table 1 shows the numbers of fish generated from this screen. Briefly, $20 \mathrm{~F}_{0}$ Et $(c p: E G F P)^{s j 2}$ males were mutagenized with ENU. One month after treatment, ENU efficiency was tested by mating the males to Casper and screening for nacre or roy phenotypes. Approximately 1 in $250(0.4 \%)$ larvae showed the nacre or roy phenotype. The mutagenized males were mated with $E t(c p: E G F P)^{s j 2}$ females to generate $409 \mathrm{~F}_{1}$ fish. Adult $\mathrm{F}_{1}$ pairs were mated together or with non-mutagenized $E t(c p: E G F P)^{s j 2}$ fish to produce $224 \mathrm{~F}_{2}$ families. Of the $\mathrm{F}_{2}$ families, 73 contained sufficient numbers to screen 4-6 pairs for mutants in the $\mathrm{F}_{3}$ generation. We set up $1330 \mathrm{~F}_{2} \times \mathrm{F}_{2}$ crosses (including re-crosses), with 421 crosses producing $\mathrm{F}_{3}$ embryos. Larvae were screened at $4 \mathrm{dpf}$ to identify choroid plexus defects based on GFP expression patterns. In total, we generated approximately16,339 $\mathrm{F}_{3}$ embryos and screened approximately 12,196.

We identified 24 mutant lines with 10 recessive mutant lines having choroid plexus deformities. Time-lapse imaging showing choroid plexus development for wild-type and some mutants are demonstrated in Supplemental Movies 2-7. In addition to choroid plexus deformities, each of the10 recessive lines had other defects such as heart edema, small eyes, and small head. While several of the mutants exhibited brain cell death, many did not develop cell death until after the choroid plexus started to form. A description of the onset of brain cell death for each line is listed in Table 2. We also found spontaneous mutants that had normal morphology overall, but severely abnormal or no choroid plexus (data not shown). In addition, we found many mutants (some recessive and some spontaneous) with severe brain cell death and normal choroid plexus development (data not shown). The choroid plexus mutant lines were named based on the original $F_{2}$ family and pair number within that family. No mutants were adult viable and most did not survive past $4 \mathrm{dpf}$, except for $c p 26.6$ and $c p 105.2$, which lived until 5 and $6 \mathrm{dpf}$, respectively. $c p 27.5$ and $c p 44.10$ mutants displayed similar phenotypes; however, complementation analysis showed that they were not in the same complementation group. On the basis of GFP intensity, epithelial localization, and overall choroid plexus morphology, we identified five classes: (I) mutants with reduced GFP expression, dispersed epithelial cells, and irregularly shaped choroid plexus; (II) mutants with normal GFP expression, small epithelial aggregates, and irregularly shaped choroid plexus; (III) mutants with normal GFP expression, small epithelial aggregates, and expanded choroid plexus; (IV) mutants with variable GFP expression, small epithelial aggregates, and expanded choroid plexus; and $(\mathrm{V})$ mutants with normal GFP expression, compact epithelial cells, and enlarged choroid plexus. While complementation analysis was not performed between all of these mutants, we are fairly certain that these mutations are in different genes. However, we acknowledge that it is possible that different types of mutations in the same gene from different mutants could result in additional 
A
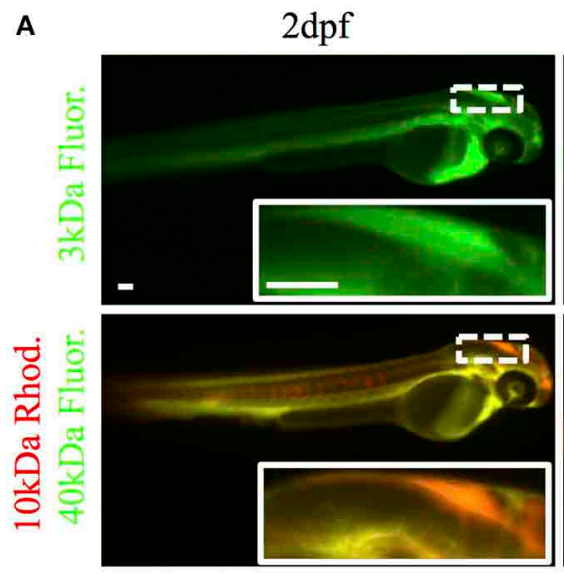
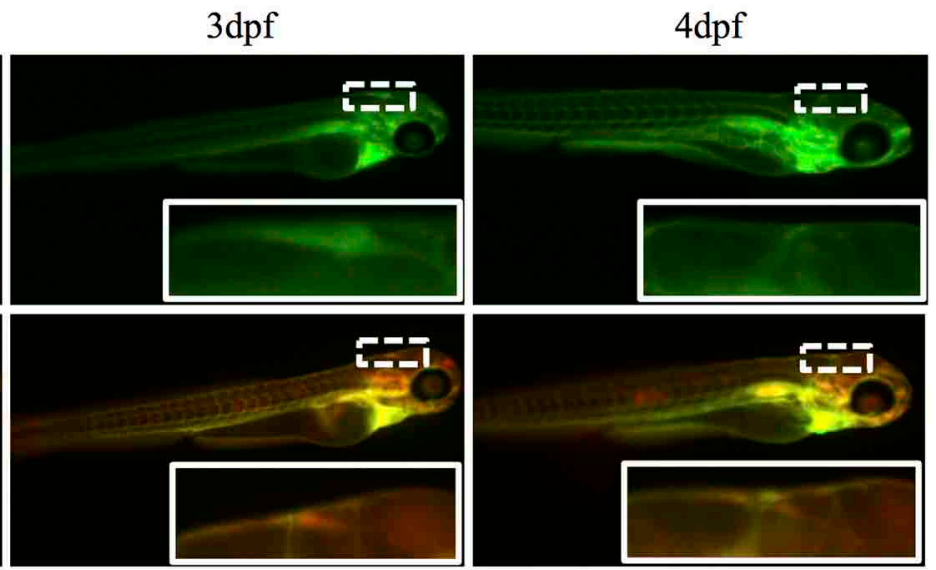

B
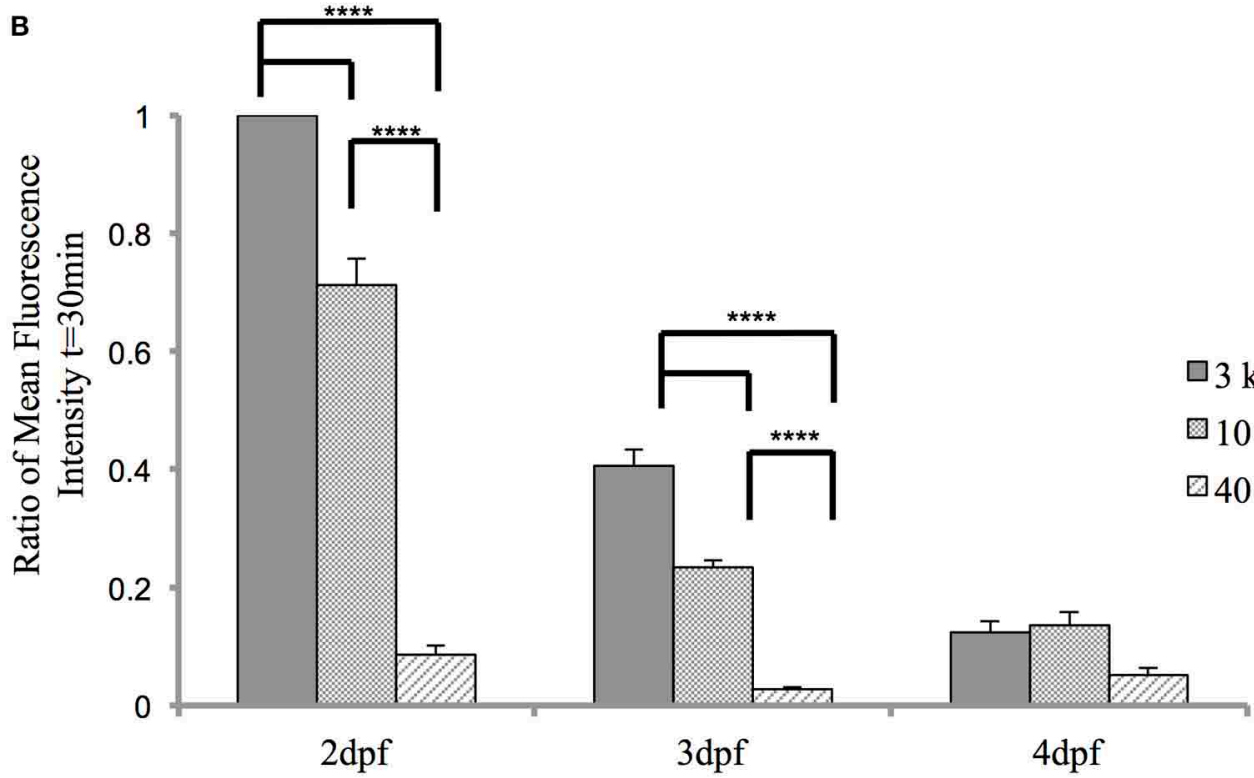

FIGURE 5 | The zebrafish CP is functional and possesses size-selective properties. Casper zebrafish were intravenously injected with fluorescent tracers at 2, 3, and $4 \mathrm{dpf}$ to identify tracer leakage from blood into the cerebrospinal fluid. (A) The top panel shows severe leakage of the $3-\mathrm{kDa}$ fluorescein dextran into the brain ventricle (inset) at $2 \mathrm{dpf}$, minor leakage at $3 \mathrm{dpf}$, and little, if any, dextran penetration at $4 \mathrm{dpf}$. The bottom panel shows permeability of the 10-kDa rhodamine dextran at $2 \mathrm{dpf}$ (inset), which decreases by $4 \mathrm{dpf}$. The $40-\mathrm{kDa}$ fluorescein dextran was the least permeable at each developmental timepoint. Images were taken at $1 \mathrm{~h}$ post-injection.
(B) A ratio of fluorescence intensity in the brain ventricle relative to the heart was measured at $30 \mathrm{~min}$ post-injection as a readout for tracer leakage into the brain ventricle. A One-Way ANOVA shows that the 3-kDa fluorescein dextran and the 10-kDa rhodamine dextran are significantly more permeable (i.e., greater mean fluorescence intensity) than the $40-k D a$ fluorescein dextran at 2 and $3 \mathrm{dpf}$. By $4 \mathrm{dpf}$, there was no significant difference in fluorescence intensity. Measurements are expressed as mean $\pm S E$ for $n=7 ;{ }^{* * * *} p<0.0001$. Abbreviations: Rhod, rhodamine dextran; Flour: fluorescein dextran. Scale bar is $50 \mu \mathrm{m}$. phenotypes. A description of these mutants and choroid plexus morphology are presented in Table 2 and Figure 7, respectively. Wild-type $E t(c p: E G F P)^{s j 2}$ larvae at 3 and $4 \mathrm{dpf}$ are shown in Figures $\mathbf{7 A - \mathbf { D } ^ { \prime }}$ as a reference to compare with mutant classes.

To further classify these mutants, we analyzed brain ventricle morphology and transporter activity in the choroid plexus. To examine brain ventricle morphology, we injected a $40-\mathrm{kDa}$ fluorescein dextran into the ventricle of $E t(c p: E G F P)^{s j 2}$ mutants that were generated by outcrossing the mutant lines to the wild-type
TL strain, were GFP negative, and selected based upon phenotypic traits. Descriptions of brain ventricles and their morphology are presented in Table 2 and Figure 7, respectively. None of the mutants except $c p 26.6$ and $c p 105.2$ had normal ventricle morphology, While some mutants such as cp79.6 and $c p 79.8$ had a third, fourth, and lateral ventricles, the shape or size of the ventricle differed from wild-type. None of the mutants except cp26.6 and cp105.2 had normal ventricle morphology. Severe cases having no defined ventricular boundaries were observed in mutants cp9.6, cp27.5, cp44.10, and cp151.2. 

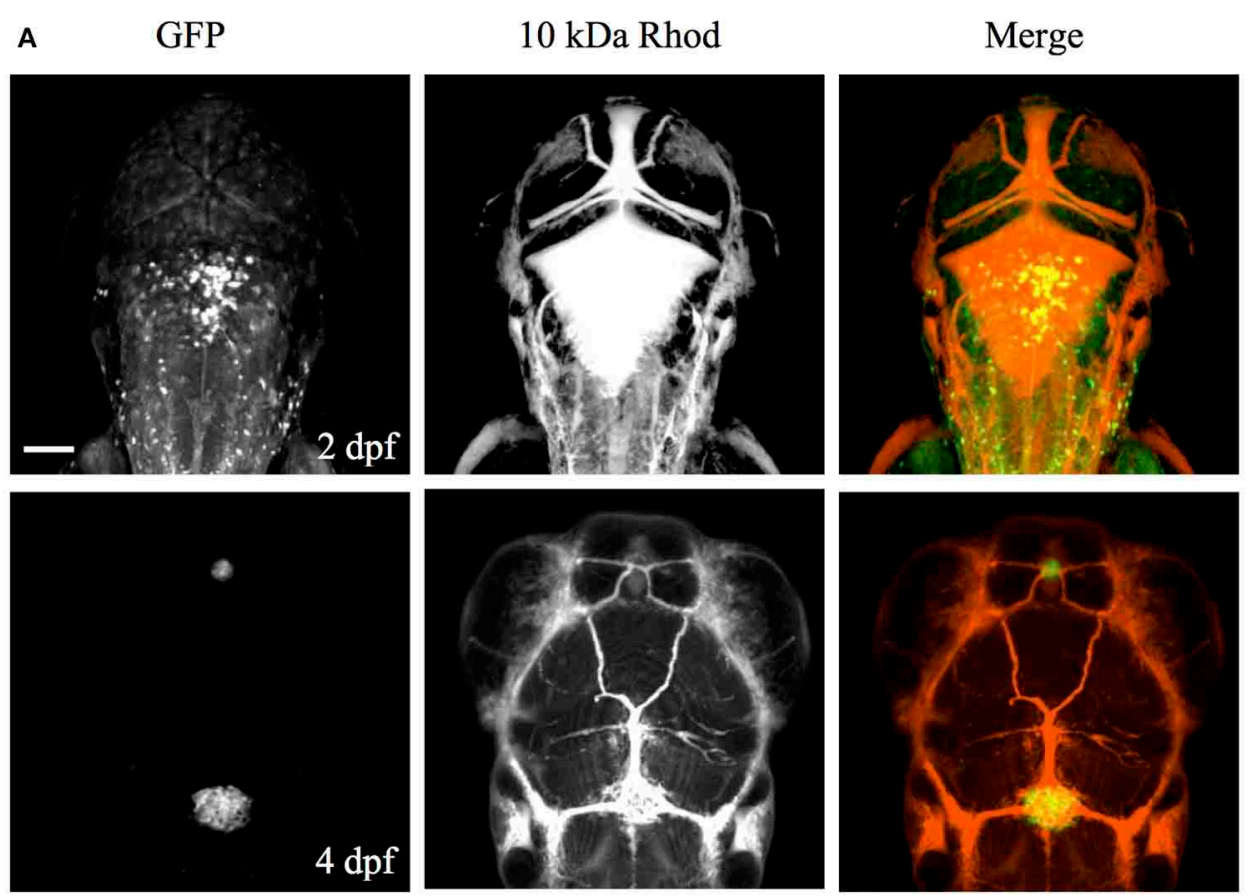

B

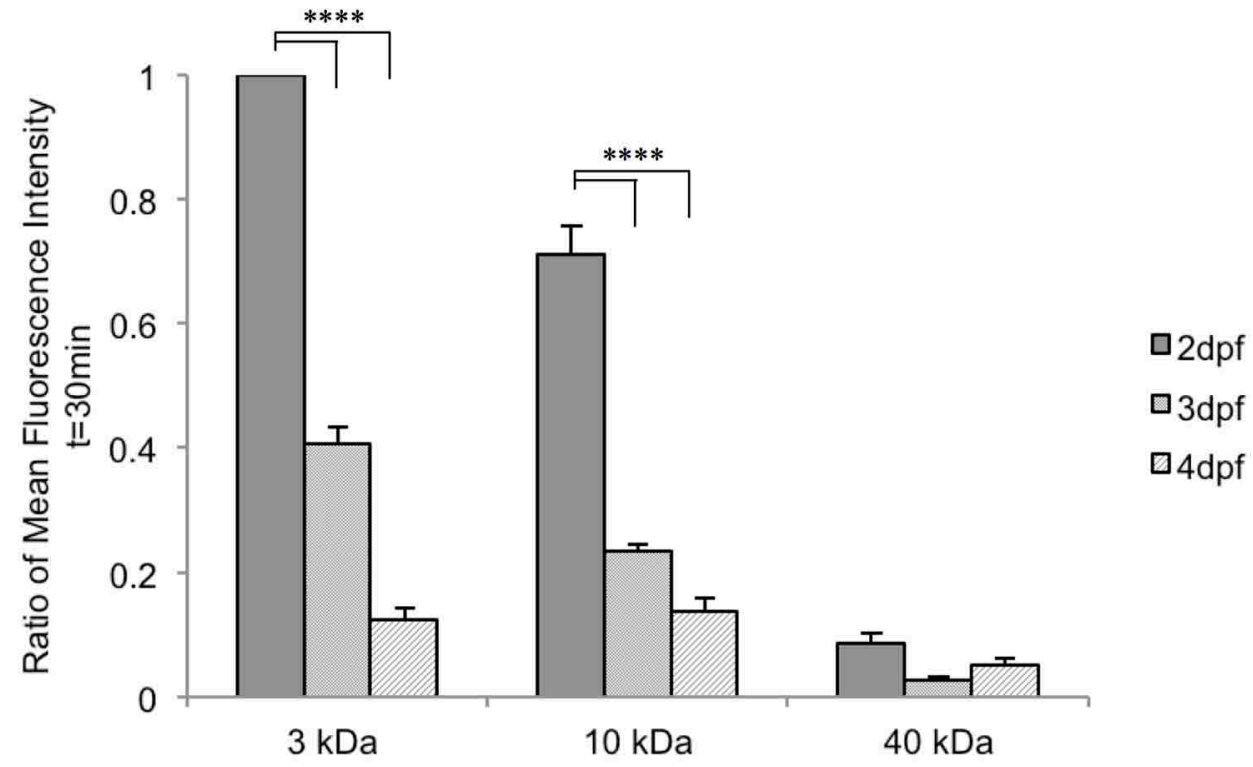

FIGURE 6 | The zebrafish CP becomes size-selective as it develops. (A) Et(cp:EGFP)sj2 larvae were intravenously injected with a $10 \mathrm{kDa}$ rhodamine-dextran and the CP was imaged dorsally by confocal microscopy. At $2 \mathrm{dpf}$, the CP is not fully developed as observed by GFP expression and the $10 \mathrm{kDa}$ rhodamine-dextran inundates the brain ventricle. By $4 \mathrm{dpf}$, the $\mathrm{CP}$ is fully formed and the $10 \mathrm{kDa}$ rhodamine-dextran is retained within the vasculature and does not enter the brain ventricle. (B) A One-Way ANOVA analysis revealed that the $3 \mathrm{kDa}$ fluorescein dextran and $10 \mathrm{kDa}$ rhodamine dextran have a significantly higher fluorescent intensity in the brain ventricle (i.e., more permeable) at $2 \mathrm{dpf}$ compared to 3 and $4 \mathrm{dpf}$. There is no significant difference between developmental time points for the $40 \mathrm{kDa}$ fluorescein dextran. Measurements are means $\pm S E$ for $n=7 ;{ }^{* * * *} p<0.0001$. Scale bar is $50 \mu \mathrm{m}$.

\section{FLUORESCEIN ASSAY TO DETERMINE CHOROID PLEXUS TRANSPORT}

Fluorescein transport was used as a secondary assay to measure transporter activity in the choroid plexus and overall permeability (refer to Figures $\mathbf{3 G}, \mathbf{H}$ ). To determine if the choroid plexus could transport fluorescein in the mutants, larvae were treated at $3 \mathrm{dpf}$ with $50 \mu \mathrm{M}$ fluorescein as described above (Figure 8). In Class I mutants, $c p 26.3$ had fluorescein accumulation toward the anterior part of the brain and no fluorescein accumulated within the choroid plexus (Figure 8E). Fluorescein did not accumulate in the choroid plexus epithelium in the $c p 140.2$ mutant, but was 
Table 1 | Number of zebrafish generated from the forward genetic screen.

\begin{tabular}{lc}
\hline Generation & Fish (n) \\
\hline$G_{0}$ males mutagenized & 20 \\
$G_{0}$ males survived & 9 \\
$F_{1}$ & 409 \\
$F_{2}$ families started & 224 \\
$F_{2}$ families screened & 73 \\
$F_{2} \times F_{2}$ crosses & 1330 \\
$F_{2}$ families that spawned & 421 \\
$F_{3}$ embryos screened & 12,196 \\
Mutants lines identified & 24 \\
Mutant lines maintained & 10 \\
\hline
\end{tabular}

localized throughout the brain ventricle. These mutants also had a higher overall fluorescein uptake than wildtype. (Figure $\mathbf{8 M}$ ).

In Class II mutants, $c p 9.6$ mutants also exhibited a higher overall uptake of fluorescein than wild-type and concentrated within the brain ventricle, gut, and heart. There was no distinct accumulation within the choroid plexus (Figure 8F). For $c p 79.6$, the overall uptake of fluorescein was comparable to wild-type. We observed very faint punctate accumulate of fluorescein within the general area of the choroid plexus indicating possible transport (Figure 8N).

Class III mutants (cp79.8 and $c p 151.2$ ) had increased uptake of fluorescein compared to wild-type and both had accumulation within the brain ventricle, gut, and heart. No fluorescein accumulated within the choroid plexus epithelium (Figures 8G-O). A similar phenotype was observed in Class IV mutants ( $c p 27.5$ and $c p 44.10$ ) in that they also had increased uptake of fluorescein concentrated within the brain ventricle, gut, and heart, but no localization within choroid plexus epithelium (Figures 8H-P). We were not able conclude whether Class V mutants (cp26.6 and cp 105.2) demonstrated transport activity using this assay because of the inability to confidently identify GFP negative mutants at 3 and $4 \mathrm{dpf}$. Once we identified mutants by late $4-5 \mathrm{dpf}$, the fluorescein was rapidly cleared by the gut and was not distributed throughout the embryo (data not shown).

\section{GENETIC MAPPING OF $c p 9.6$ AND $c p 27.5$}

To identify the mutated gene for lines $c p 9.6$ and $c p 27.5$, which displayed hydrocephaly phenotypes, we performed genetic mapping using 192 polymorphic markers distributed evenly across the genome. By bulked segregant analysis, we found linkage to marker z3275 that mapped cp9.6 to chromosome 4. With z3275, we tested 100 mutants and identified 4 recombinants in 200 meiotic events. The critical interval along the chromosome was defined by recombination analysis. We identified polymorphic markers on either side of z3275, z23802, and z15751; however, the lack of additional polymorphic markers within this region made it difficult to define a reasonable mapping distance.

For $c p 27.5$, we identified markers z2363 and z15891 by bulked segregant analysis to map the mutation to chromosome 21 . Within this critical interval, we identified the marker z9233 and found no recombinants out of 48 meioses. We then tested additional polymorphic markers and counted the number of recombinants on either side of z9233 to refine the critical interval. By testing polymorphic markers, BX530031.5 and BX530023.5, that we selected from the zebrafish genome published in Ensembl $\mathrm{Zv} 9$, we narrowed the region to $\sim 0.4 \mathrm{Mb}$. In total, we analyzed 450 meioses. Future analysis will include testing candidate genes within this interval to identify the mutated gene.

\section{DISCUSSION}

The goal of our study was to establish zebrafish as a functionally relevant model to study choroid plexus development and as a screening tool to genetically dissect genes involved in choroid plexus formation, function, and maintenance. Although the choroid plexus has been studied in various in vivo and in vitro models (Dohrmann, 1970; Strazielle and Preston, 2003), the developmental and genetic aspects of this structure remain poorly understood largely due to the lack of innovative strategies and functionally relevant systems (Saunders et al., 2008). Also, current models do not easily allow for choroid plexus development to be visualized in real time. To overcome this limitation, we created an enhancer trap zebrafish line, Et $(c p: E G F P)^{s j 2}$, which expresses EGFP within the diencephalic choroid plexus and myelencephalic choroid plexus. Using this line, choroid plexus development was easily observed in a live vertebrate by monitoring EGFP expression.

Previous studies have also generated enhancer trap lines in zebrafish expressing GFP in the choroid plexus (Bill et al., 2008; Garcia-Lecea et al., 2008). However, there are variations within these lines, including the subsets of cells expressing GFP. For example, in the $E t^{M n 16}$ line (Bill et al., 2008), only a subset of cells of the myelencephalic choroid plexus express GFP. In addition, the SqET33-E20 (Gateways) line has additional GFP expression in the rhombomeres (Garcia-Lecea et al., 2008). In our study, there was specific GFP expression in the diencephalic choroid plexus, myelencephalic choroid plexus, and swimbladder. In regards to GFP expression in the swimbladder, studies have shown that Sonic hedgehog (Shh) is expressed in the epithelial cells lining the swimbladder (Winata et al., 2009). Interestingly, the choroid plexus of other vertebrates has also been compared to the mammalian lung in a study by Nielsen and Dymecki (2010) demonstrating that Shh (also expressed in choroid plexus epithelia) is required for the synchronized outgrowth of the choroid plexus and fenestrated vasculature, similar to the need for Shh in coordinating the outgrowth of the lung (Nielsen and Dymecki, 2010). Since the zebrafish swimbladder has been compared to the mammalian lung (Winata et al., 2009), common underlying developmental pathways, such as Shh, required in both choroid plexus and swimbladder development, maybe be regulating GFP expression in these tissues. The variations in other enhancer trap lines may be due to transgenic DNA constructs integrating into different regions of the genome. Our PCR and Southern blot analysis revealed a single integration on chromosome 13 . In contrast, the Gateways line has a single insertion on chromosome 24 (Garcia-Lecea et al., 2008), which likely accounts for the differences in expression patterns between lines. Similar to the previously published zebrafish choroid plexus papers (Bill et al., 2008; Garcia-Lecea et al., 2008), we were unable to identify the 
Table 2 | Characterization of Et(cp:EGFP) mutants generated from the forward genetic screen.

\begin{tabular}{|c|c|c|c|c|c|c|c|}
\hline Class & Allele & $\begin{array}{l}\text { Map } \\
\text { position }\end{array}$ & DPF & Other phenotypes(4 dpf) & $\begin{array}{l}\text { Fluorescein } \\
\text { transport }\end{array}$ & $\begin{array}{l}\text { Onset of brain } \\
\text { cell death (up } \\
\text { to } 4 \mathrm{dpf} \text { ) }\end{array}$ & $\begin{array}{l}\text { Initial } \\
\text { circulation loss } \\
\text { (up to } 4 \mathrm{dpf} \text { ) }\end{array}$ \\
\hline \multirow[t]{2}{*}{$\begin{array}{l}\text { I: reduced GFP expression, } \\
\text { dispersed epithelia, and } \\
\text { irregularly shaped CP }\end{array}$} & $c p 26.3^{\mathrm{a}}$ & ND & $2-3$ & $\begin{array}{l}\text { No swimbladder; brain and } \\
\text { tail cell death; brain ventricle } \\
\text { hemorrhaging; faint } \\
\text { heartbeat; no circulation; no } \\
\text { midbrain-hindbrain } \\
\text { boundaries }\end{array}$ & $\begin{array}{l}\text { Accumulates within } \\
\text { third ventricle }\end{array}$ & $3 \mathrm{dpf}$ & $3 \mathrm{dpf}$ \\
\hline & cp140.2 & ND & 2 & $\begin{array}{l}\text { Small head; small eyes; no } \\
\text { swimbladder; heart edema; } \\
\text { brain cell death; faint } \\
\text { heartbeat and circulation; } \\
\text { slightly larger third ventricle } \\
\text { and wider lateral ventricles } \\
\text { ventricle }\end{array}$ & $\begin{array}{l}\text { Increased uptake; } \\
\text { accumulates within } \\
\text { brain ventricle }\end{array}$ & $4 \mathrm{dpf}$ & $4 \mathrm{dpf}$ \\
\hline \multirow[t]{2}{*}{$\begin{array}{l}\text { II: normal GFP expression, } \\
\text { smaller epithelial aggregates, } \\
\text { and irregularly shaped CP }\end{array}$} & cp9.6 & LG 4 & 2 & $\begin{array}{l}\text { Small head; small eyes; no } \\
\text { swimbladder; heart edema; } \\
\text { occasional hydrocephalus; } \\
\text { brain cell death; no } \\
\text { circulation; no distinct third } \\
\text { ventricle or lateral ventricles } \\
\text { and severely reduced fourth } \\
\text { ventricle }\end{array}$ & $\begin{array}{l}\text { Increased uptake; } \\
\text { accumulates within } \\
\text { brain ventricle }\end{array}$ & $2 \mathrm{dpf}$ & $4 \mathrm{dpf}$ \\
\hline & cp79.6 & ND & 2 & $\begin{array}{l}\text { Small head; small eyes; no } \\
\text { swimbladder; heart edema; } \\
\text { many still in chorion; brain cell } \\
\text { death; hemorrhaging around } \\
\text { heart; slow heartbeat; little to } \\
\text { no circulation; wider lateral } \\
\text { ventricles }\end{array}$ & $\begin{array}{l}\text { Dispersed punctate } \\
\text { staining on the } \\
\text { dorsal ventricle } \\
\text { surface }\end{array}$ & $4 \mathrm{dpf}$ & $3 \mathrm{dpf}$ \\
\hline \multirow[t]{2}{*}{$\begin{array}{l}\text { III: normal GFP expression, } \\
\text { smaller epithelial aggregates, } \\
\text { and expanded CP }\end{array}$} & cp79.8 & ND & 2 & $\begin{array}{l}\text { Small head; small eyes; no } \\
\text { swimbladder; brain cell } \\
\text { death; hemorrhaging around } \\
\text { heart; decreased heartbeat } \\
\text { and circulation; wider lateral } \\
\text { ventricles }\end{array}$ & $\begin{array}{l}\text { Increased uptake; } \\
\text { accumulates within } \\
\text { brain ventricle }\end{array}$ & $4 \mathrm{dpf}$ & $3 \mathrm{dpf}$ \\
\hline & cp151.2 & ND & $2-3$ & $\begin{array}{l}\text { Small head; small eyes; no } \\
\text { swimbladder; brain cell } \\
\text { death; minor swelling around } \\
\text { head; occasional } \\
\text { hemorrhaging around heart; } \\
\text { smaller third ventricle and no } \\
\text { defined lateral ventricles }\end{array}$ & $\begin{array}{l}\text { Increased uptake; } \\
\text { accumulates within } \\
\text { brain ventricle }\end{array}$ & $4 \mathrm{dpf}$ & $4 \mathrm{dpf}$ \\
\hline $\begin{array}{l}\text { IV: variable GFP expression, } \\
\text { smaller epithelial aggregates, } \\
\text { and expanded CP }\end{array}$ & $c p 27.5$ & LG 21 & 2 & $\begin{array}{l}\text { Small head; small eyes; no } \\
\text { swimbladder; brain cell } \\
\text { death; heart edema; small } \\
\text { arched body; curved tail; } \\
\text { hydrocephalus; occasional } \\
\text { hemorrhaging in brain } \\
\text { ventricle; heartbeat, but little } \\
\text { to no circulation; no defined } \\
\text { ventricle boundaries }\end{array}$ & $\begin{array}{l}\text { Increased uptake; } \\
\text { accumulates within } \\
\text { brain ventricle }\end{array}$ & $\begin{array}{l}2 \mathrm{dpf} \text { (localized } \\
\text { to forebrain) }\end{array}$ & $3 \mathrm{dpf}$ \\
\hline
\end{tabular}


Table 2 | Continued

\begin{tabular}{|c|c|c|c|c|c|c|c|}
\hline Class & Allele & $\begin{array}{l}\text { Map } \\
\text { position }\end{array}$ & DPF & Other phenotypes(4 dpf) & $\begin{array}{l}\text { Fluorescein } \\
\text { transport }\end{array}$ & $\begin{array}{l}\text { Onset of brain } \\
\text { cell death (up } \\
\text { to } 4 \mathrm{dpf} \text { ) }\end{array}$ & $\begin{array}{l}\text { Initial } \\
\text { circulation loss } \\
\text { (up to } 4 \mathrm{dpf} \text { ) }\end{array}$ \\
\hline & cp44.10 & ND & 2 & $\begin{array}{l}\text { Small head; small eyes; no } \\
\text { swimbladder; brain cell } \\
\text { death; heart edema; small } \\
\text { arched body; curved tail; } \\
\text { hydrocephalus; occasional } \\
\text { hemorrhaging around heart; } \\
\text { heartbeat, but little to no } \\
\text { circulation; no third ventricle } \\
\text { or lateral ventricles and } \\
\text { irregularly shaped fourth } \\
\text { ventricle }\end{array}$ & $\begin{array}{l}\text { Increased uptake; } \\
\text { accumulates within } \\
\text { brain ventricle }\end{array}$ & $\begin{array}{l}2 \mathrm{dpf} \text { (localized } \\
\text { to forebrain) }\end{array}$ & $4 \mathrm{dpf}$ \\
\hline \multirow[t]{2}{*}{$\begin{array}{l}\text { V: normal GFP expression, } \\
\text { compact epithelia, and } \\
\text { enlarged CP }\end{array}$} & $\operatorname{cp} 26.6^{\mathrm{b}}$ & ND & 4 & $\begin{array}{l}\text { Rounded head, protruding } \\
\text { lower jaw; no swimbladder; } \\
\text { edema around heart, eyes, } \\
\text { and gut; faint heartbeat and } \\
\text { circulation; normal brain } \\
\text { ventricle }\end{array}$ & N/A & N/A & $4 \mathrm{dpf}$ \\
\hline & $\operatorname{cp} 105.2^{\mathrm{b}}$ & ND & $4-5$ & $\begin{array}{l}\text { Rounded head, protruding } \\
\text { lower jaw; no swimbladder; } \\
\text { severe edema around heart, } \\
\text { eyes, and gut; faint heartbeat } \\
\text { and circulation; slightly } \\
\text { smaller fourth ventricle }\end{array}$ & N/A & N/A & $6 \mathrm{dpf}$ \\
\hline
\end{tabular}

${ }^{a}$ Observations recorded at $3 \mathrm{dpf} ;{ }^{b}$ observations recorded at $5 \mathrm{dpf} ; \mathrm{ND}$, not determined; N/A, not applicable.

enhancer regulating GFP expression in the choroid plexus. This is often true with enhancer trap effects where is difficult to identify the enhancer if it acts at distances upstream or downstream of the reporter gene.

We have demonstrated blood-cerebrospinal fluid barrier properties in zebrafish by identifying tight junction proteins and transporter activity. We have shown Cldn5 expression within the choroid plexus and demonstrated that the zebrafish choroid plexus possess a chemical barrier that transports and concentrates rhodamine 123 and fluorescein in the choroid plexus epithelium. Rhodamine 123 directly interacts with MRP1 and MDR1 in an ATP-dependent manner (Shapiro and Ling, 1998; Daoud et al., 2000). Because previous studies have shown the specificity of rhodamine 123 to multidrug resistance proteins (MDRs), similar drug transporters are likely expressed in the zebrafish choroid plexus. Studies in mice have identified known transporters of rhodamine 123 such as MRP1 to be localized at the basolateral side of choroid plexus epithelium and have been shown to keep drugs such as etoposide out of the cerebrospinal fluid (Wijnholds et al., 2000; Keep and Smith, 2011). Our findings also confirm that transporters are present in the zebrafish choroid plexus by showing that: when coming from the bloodstream, rhodamine 123 accumulates within the choroid plexus and is prevented from entering the cerebrospinal fluid. When injected directly into the cerebrospinal fluid, the choroid plexus removed rhodamine 123 from the fluid as shown by dye accumulation within choroid plexus epithelium. Unfortunately, due to the lack of antibodies that cross-react with zebrafish proteins, we were unable to demonstrate the expression of specific transporters in the zebrafish choroid plexus. We tested an antibody against MDR1 (C219), but found expression only within brain endothelial cells of the blood-brain barrier (Umans and Taylor, 2012) and not the choroid plexus (data not shown). We also tested several commercially available antibodies against OAT3, but none crossreacted with zebrafish. Additionally, while we did not observe Glut1 expression within the zebrafish choroid plexus, a recent review by Keep and Smith indicated that the BBB, not the blood-cerebrospinal fluid barrier, may be the major source of transporting glucose into the brain and that the choroid plexus may play a minor role in glucose transport (Keep and Smith, 2011).

We demonstrated barrier function in the zebrafish choroid plexus by injecting fluorescent tracers of different molecular weights and observing permeability across the choroid plexus epithelium into the cerebrospinal fluid. Once the choroid plexus fully formed by $4 \mathrm{dpf}$, the smallest tracer tested, a $3-\mathrm{kDa}$ fluorescein dextran, was no longer permeable across the bloodcerebrospinal fluid barrier as it was in earlier developmental stages. This result indicated a tightening of the barrier as it develops. Although we did not distinguish between transcellular and paracellular transport, there appears to be a correlation between choroid plexus formation and decreased permeability, suggesting that a physical barrier is preventing molecule entry rather than being cleared by a cerebrospinal fluid drainage pathway. For 

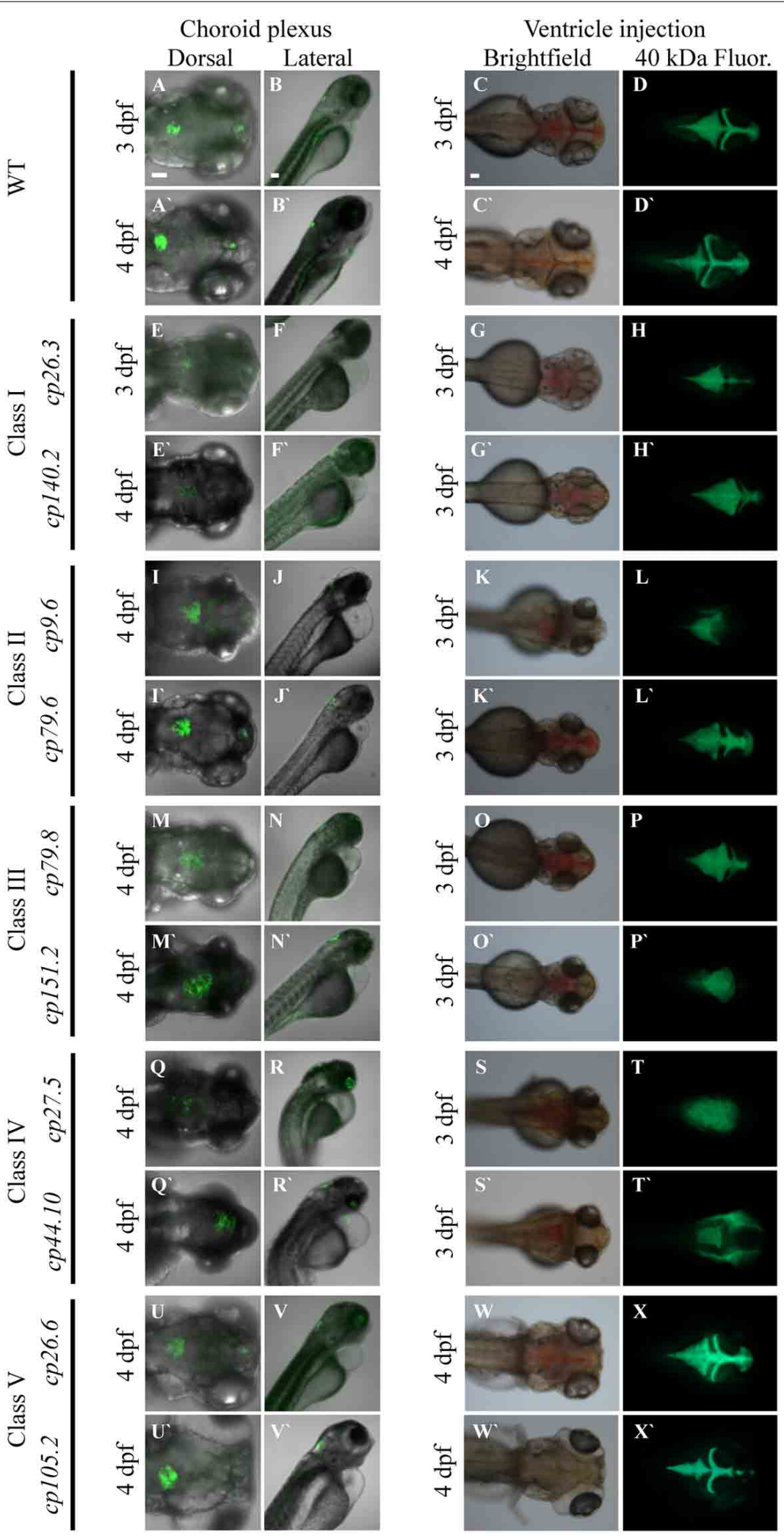

FIGURE 7 | Forward genetic screen identifies five classes of CP mutants. (A-B') Wild-type Et(cp:EGFP)sj2 larvae at $3 \mathrm{dpf}$ and $4 \mathrm{dpf}$. (C-C') Bright field image of 3 and $4 \mathrm{dpf}$ wild-type after ventricle injection of the $40-\mathrm{kDa}$ fluorescein dextran. (D-D') Fluorescent image of 3 and 4 dpf wild-type after ventricle injection of the 40-kDa fluorescein dextran. Class I: (E,F) cp26.3 at

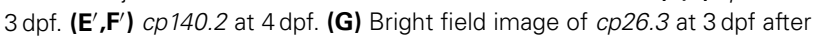
ventricle injection of the $40-\mathrm{kDa}$ fluorescein dextran. (G') Bright field

(Continued) 


\section{FIGURE 7 | Continued}

image of cp140.2 at $3 \mathrm{dpf}$ after ventricle injection of the 40-kDa fluorescein dextran. (H) Fluorescent image of $c p 26.3$ at $3 \mathrm{dpf}$ after ventricle injection of the $40-\mathrm{kDa}$ fluorescein dextran. (H') Fluorescent image of cp140.2 at $3 \mathrm{dpf}$ after ventricle injection of the 40-kDa fluorescein dextran. Class II: (I,J) cp9.6 at $4 \mathrm{dpf}$. $\left(\mathbf{I}^{\prime}, \mathbf{J}^{\prime}\right)$ cp79.6 at $4 \mathrm{dpf}$. (K) Bright field image of cp9.6 at $3 \mathrm{dpf}$ after ventricle injection of the $40-\mathrm{kDa}$ fluorescein dextran. (K') Bright field image of cp79.6 at $3 \mathrm{dpf}$ after ventricle injection of the 40-kDa fluorescein dextran. (L) Fluorescent image of cp9.6 at $3 \mathrm{dpf}$ after ventricle injection of the $40-\mathrm{kDa}$ fluorescein dextran. (L') Fluorescent image of cp79.6 at $3 \mathrm{dpf}$ after ventricle injection of the 40-kDa fluorescein dextran. Class III: (M,N) cp79.8 at $4 \mathrm{dpf}$. $\left(\mathbf{M}^{\prime}, \mathbf{N}^{\prime}\right)$ cp151.2 at $4 \mathrm{dpf}$. (0) Bright field image of $c p 79.8$ at $3 \mathrm{dpf}$ after ventricle injection of the $40-\mathrm{kDa}$ fluorescein dextran. $\left(\mathbf{O}^{\prime}\right)$ Bright field image of cp151.2 at $3 \mathrm{dpf}$ after ventricle injection of the 40-kDa fluorescein dextran. (P) Fluorescent image of $c p 79.8$ at $3 \mathrm{dpf}$ after ventricle injection of the 40-kDa fluorescein dextran. ( $\left.\mathbf{P}^{\prime}\right)$ Fluorescent image of cp151.2 at $3 \mathrm{dpf}$ after ventricle injection of the $40-k D a$ fluorescein dextran. Class IV: $(\mathbf{Q}, \mathbf{R}) \operatorname{cp} 27.5$ at $4 \mathrm{dpf}$. $\left(\mathbf{Q}^{\prime}, \mathbf{R}^{\prime}\right)$ cp44. 10 at $4 \mathrm{dpf}$. (S) Bright field image of $c p 27.5$ at $3 \mathrm{dpf}$ after ventricle injection of the $40-k D a$ fluorescein dextran. $\left(\mathbf{S}^{\prime}\right)$ Bright field image of cp44.10 at $3 \mathrm{dpf}$ after ventricle injection of the $40-\mathrm{kDa}$ fluorescein dextran. (T) Fluorescent image of $c p 27.5$ at $3 \mathrm{dpf}$ after ventricle injection of the $40-\mathrm{kDa}$ fluorescein dextran. ( $\left.\mathbf{T}^{\prime}\right)$ Fluorescent image of $c p 44.10$ at $3 \mathrm{dpf}$ after ventricle injection of the 40-kDa fluorescein dextran. Class V: (U,V) cp26.6 at $4 \mathrm{dpf}$. $\left(\mathbf{U}^{\prime}, \mathbf{V}^{\prime}\right)$ cp105.2 at $4 \mathrm{dpf}$. (W) Bright field image of cp26.6 at $4 \mathrm{dpf}$ after ventricle injection of the $40-\mathrm{kDa}$ fluorescein dextran. ( $\left.\mathbf{W}^{\prime}\right)$ Bright field image of cp105.2 at $4 \mathrm{dpf}$ after ventricle injection of the 40-kDa fluorescein dextran. (X) Fluorescent image of $c p 26.6$ at $4 \mathrm{dpf}$ after ventricle injection of the 40-kDa fluorescein dextran. ( $\left.\mathbf{X}^{\prime}\right)$ Fluorescent image of cp105.2 at $4 \mathrm{dpf}$ after ventricle injection of the $40-k D a$ fluorescein dextran. The first, third, and fourth panels for each class are dorsal views while the second panel is a lateral view. The first and second panels for each class were acquired by confocal microscopy. The third and fourth panels were acquired by fluorescent stereoscopy. The first panel for each class is at $20 \times$ magnification, the second panel is at $10 \times$ magnification, and the third and fourth panels are at $16 \times$ magnification. $40 \mathrm{kDa}$ Fluor, $40 \mathrm{kDa}$ Fluorescein Dextran. Scale bars in A, B, and C are $50 \mu \mathrm{m}$. example, this is demonstrated when we injected the $40-\mathrm{kDa}$ tracer into the bloodstream on day 4 and never observed tracer accumulation within the ventricle throughout the course of the timelapse. Rather than suggesting that the tracer is being cleared from the ventricle by cerebrospinal fluid flow, we observed that the tracer never entered the ventricle in the first place. This indicated to us that a physical barrier is preventing the dextran from crossing the barrier. Had we observed accumulation of the dextran within the cerebrospinal fluid/brain ventricle after injection, and then observed a decrease in the amount of tracer in the ventricle over time, then we would suggest that cerebrospinal fluid flow is the contributing factor and not a physical blockage of the dextran by the barrier as we observed. In immature brain barriers, it has been reported that the accumulation of molecules within the cerebrospinal fluid is not due to increased permeability of the barrier but reduced cerebrospinal fluid flow (Saunders et al., 2008). In contrast, more mature brain barriers have increased cerebrospinal fluid flow that limits the accumulation of molecules within the ventricular space (Saunders et al., 2008). Our study showed that after the formation of the choroid plexus there was no tracer penetration into the ventricle. Therefore, a decrease in tracer accumulation in the brain ventricle does not appear to be due to increased cerebrospinal fluid clearance, but due to physical barrier properties of the choroid plexus. The decrease in barrier permeability as the choroid plexus develops establishes the zebrafish choroid plexus as a functioning organ with size-selective barrier properties.

Forward genetic screens have been performed in various multicellular organisms such as plants (Meinke and Sussex, 1979), flies (Nusslein-Volhard and Wieschaus, 1980), worms (Sin et al., 2014), zebrafish (Driever et al., 1996), and mice (Nolan et al., 2000). These screens have been very successful in discovering genes involved in embryonic patterning, organ development, and behavior (Stemple et al., 1996; Horvitz, 1999; Muto et al., 2005). To characterize the mutants in our study, we examined not only GFP expression in the choroid plexus but also ventricle morphology and transporter activity. Previous studies have grouped zebrafish brain ventricle mutants into four categories: midline separation defects, reduced ventricle size, lateral ventricles and fourth ventricle abnormalities, and absence of lumen inflation
(Lowery et al., 2009). Because none of our choroid plexus mutants exhibited a phenotype at $24 \mathrm{hpf}$, we did not expect to see defects in midline separation as described in Lowery et al. (2009). Choroid plexus mutants such as $c p 79.6$ and $c p 140.2$ have a brain ventricle that closely resembles normal larvae at $2 \mathrm{dpf}$ (Lowery et al., 2007), indicating these mutants may have a developmental delay and not necessarily a brain ventricle defect. Also, cp140.2 mutants at $3 \mathrm{dpf}$ are similar to wild-type larvae at $2 \mathrm{dpf}$ in that the fluorescein accumulated within the brain ventricle rather than concentrating within choroid plexus epithelium as it does normally on day 3 . Many mutants exhibited an increased overall uptake of fluorescein, which may indicate defects in the skin epithelial barrier as well and increased permeability to absorb small molecules from their surroundings.

While mutants exhibit little to no heart beat and circulation when screened on day 4 , as described in Table 2, the majority of mutants do have a strong heart beat and rapid circulation indistinguishable from wild-type prior to this timepoint. The initial onset of circulation loss describes when we first observed mutants with decreased or no circulation. Not all of the mutants had loss of circulation at the same time, but the onset describes when we observed the first mutant from a spawn having no circulation. Because most began to have decreased heartbeat and circulation at $4 \mathrm{dpf}$ after the choroid plexus has already developed, we conclude that other factors are regulating choroid plexus development. However, we cannot rule out that circulation is required for choroid plexus maintenance. We also do not attribute the lack of blood flow to abnormal choroid plexus development, because we identified spontaneous mutants with a normal choroid plexus but no circulation (data not shown). After injecting single-cell embryos with a cardiac troponin morpholino to block heartbeat (Sehnert et al., 2002), we identified no choroid plexus defects resembling abnormalities of the five mutant classes (data not shown). Therefore, we conclude that if loss of circulation alone was the factor governing abnormal choroid plexus development in these mutants, we should see a choroid plexus that resembled that of troponin morphants. Because many mutants continued to have a heartbeat and circulation at $3 \mathrm{dpf}$, which is essential for brain ventricle expansion (Lowery and Sive, 2005), we also did not expect ventricle expansion defects in these mutants. While 

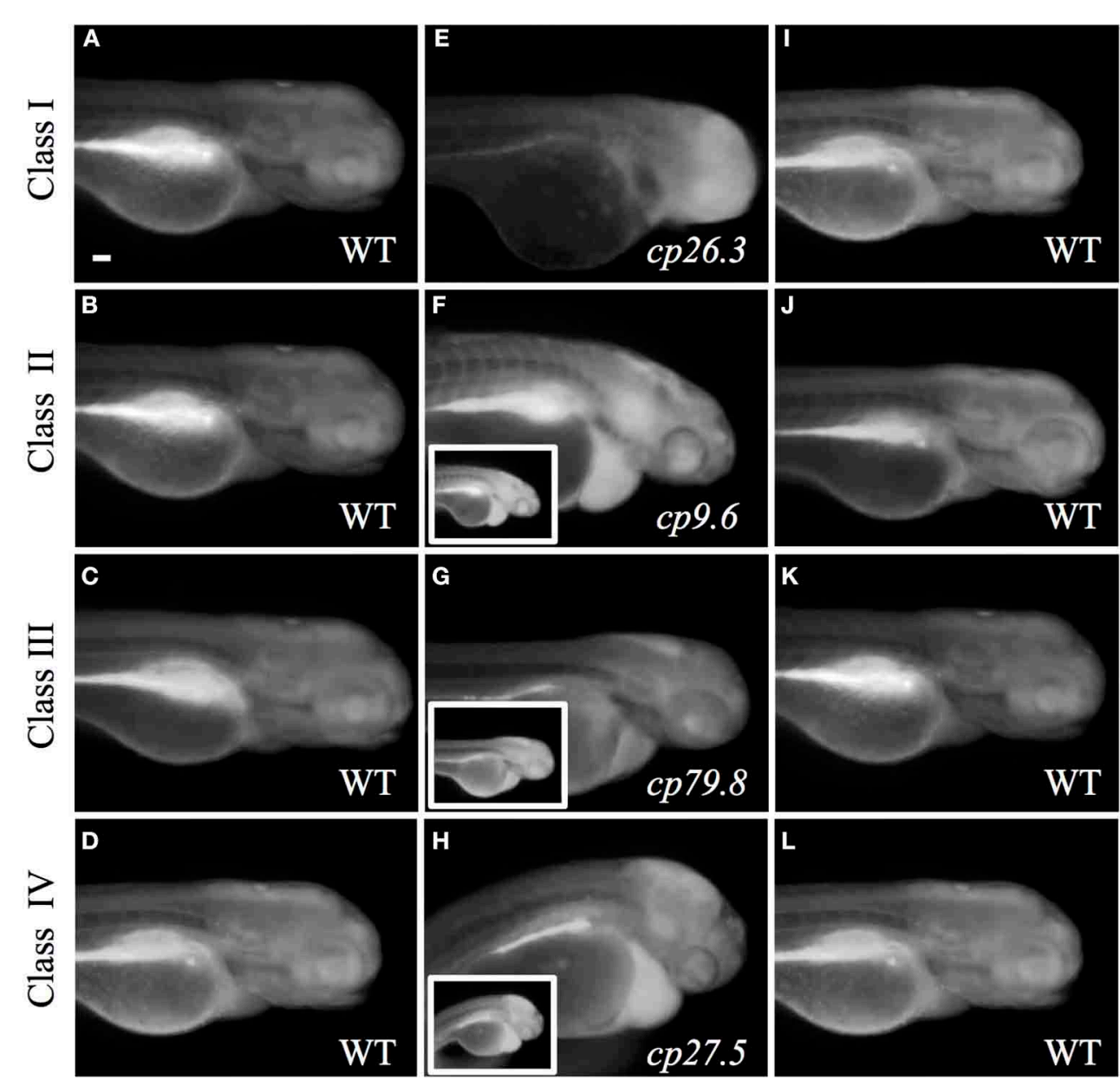
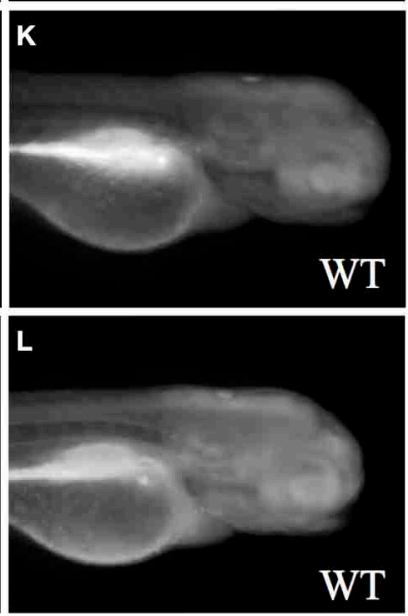
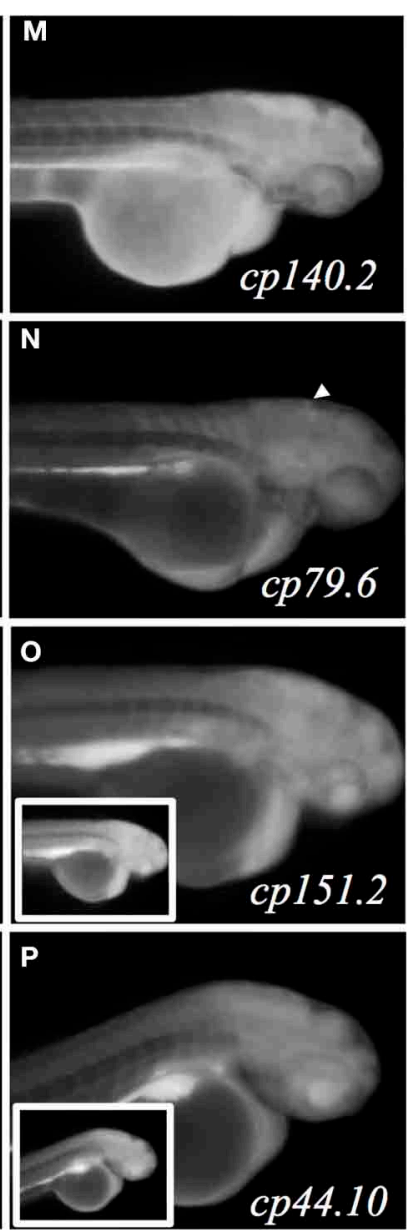

FIGURE 8 | Fluorescein assay determines CP transport activity in mutants. (A-D) and (I-L) Wild-type larvae treated at $3 \mathrm{dpf}$ with $50 \mu \mathrm{M}$ fluorescein. (E) cp26.3 mutant at $3 \mathrm{dpf}$. (F) cp9.6 mutant at $3 \mathrm{dpf}$. Inset image is acquired at the same exposure to the corresponding wild-type in (B). (G) cp79.8 mutant at $3 \mathrm{dpf}$. Inset image is acquired at the same exposure to the corresponding wild-type in (C). (H) $c p 27.5$ mutant at $3 \mathrm{dpf}$. Inset image is acquired at the same exposure to the corresponding wild-type in (D). (M) cp140.2 mutant at $3 \mathrm{dpf}$. (N) cp79.6 mutant at $3 \mathrm{dpf}$.
Punctate accumulation of fluorescein in the brain ventricle is shown with an arrowhead. (O) cp151.2 mutant at $3 \mathrm{dpf}$. Inset image is acquired at the same exposure to the corresponding wild-type in (K). (P) cp44.10 mutant at $3 \mathrm{dpf}$. Inset image is acquired at the same exposure to the corresponding wild-type in (L). The larger images for $(\mathbf{F}, \mathbf{G}, \mathbf{H}, \mathbf{O}, \mathbf{P})$ were taken at a lower exposure compared to the corresponding wild-type in order to visualize where the fluorescein was concentrating throughout the larvae. Scale bar is $50 \mu \mathrm{m}$. ventricle morphology varied between mutants, all had expanded ventricles.

The mutants identified in our screen will provide the tools necessary to investigate several genes associated with choroid plexus development and barrier function. After isolating the defective genes from these mutants, we can define the correlation between the genes involved and the phenotypic abnormalities we observed. Although we identified mutants with brain cell death, we do not contribute cell death alone as a factor responsible for abnormal choroid plexus development and believe that the processes can be unrelated. This is supported by the identification of larvae with brain cell death developed a normal choroid plexus. Furthermore, for the mutants we characterized further, we did not observe the onset of cell death until after the choroid plexus had already started to develop for many of the lines, or if cell death was present, it was not near the choroid plexus itself. We also found larvae that had normal morphology overall, but abnormal or no choroid plexus indicating again that cell death is not the sole mechanism responsible for abnormal choroid plexus development and that specific pathways exist in regulation choroid plexus formation and function.

We expect some of the genes we identify will be specifically related to a choroid plexus defect rather than housekeeping genes causing an overall severely abnormal animal. Recent discoveries have shed light on the importance of the choroid plexus and its contribution to disease. For example, transcriptome analysis during early development revealed that the choroid plexus expresses numerous genes essential for choroid plexus function, including those for tight junctions, transporters, and metabolic function (Liddelow et al., 2012), protecting the brain from potentially harmful insults (Kratzer et al., 2013). Previous 
studies have also identified genetic components associated with choroid plexus dysfunction and disease. Genes involved in these pathologies include Polycystin-1 deficiency, which contributes to hydrocephaly (Wodarczyk et al., 2009). Because some types of hydrocephalus can be congenital, we plan to look at the mutant lines displaying hydrocephalus-like phenotypes with expanded ventricles (e.g., cp27.5 and cp9.6) and determine whether we can find genetic linkage to this phenotype. In addition, mutations in Twist-related protein-1 and constitutively active Notch 3 promote proliferation and invasion in choroid plexus tumors (Wolburg and Paulus, 2010). Furthermore, disruption of the blood-cerebrospinal fluid barrier in multiple sclerosis can cause inflammation and leakage of $\mathrm{T}$ lymphocytes into the cerebrospinal fluid (Vercellino et al., 2008; Wolburg and Paulus, 2010). In patients with Alzheimer's disease, the choroid plexus has decreased function in amyloid beta plaque clearance (AlviraBotero and Carro, 2010; Wolburg and Paulus, 2010). Since the mutations in the choroid plexus lines we identified are embryonic lethal, they may not be good representations to model later life diseases such as multiple sclerosis or Alzheimer's disease. However, we can investigate whether the choroid plexus is compromised in these mutants in regards to specific aspects of these diseases, such as an increased influx of immune cells into the cerebrospinal fluid characterized in multiple sclerosis, or reduced cerebrospinal fluid flow found in Alzheimer's disease patients. Identifying the genetic mutations that produce similar pathologies of these diseases may provide insight into the mechanisms that occur in the choroid plexus at later stages in life for these conditions.

The choroid plexus mutants identified in our study will be used to identify genes essential for maintaining epithelial barrier integrity as well as genes essential for initial barrier formation. Reverse genetic studies have shown that transcription factors such as Otx2 aid in choroid plexus development and maintenance (Johansson et al., 2013). Also, augurin, a protein encoded by the esophageal cancer related gene-4 (Ecrg4) and thought to act as a tumor suppressor, has a higher expression in the choroid plexus than other tissues. Interestingly, morpholino knockdown of Ecrg4 in zebrafish results in increased central nervous system cell proliferation and hydrocephaly (Gonzalez et al., 2011). Additional studies in zebrafish showed that the clusterin gene, which encodes a secreted glycoprotein that aids in aggregation and contributes to Alzheimer's disease by colocalizing with plaques (Thambisetty et al., 2010), is similar to its human counterpart and is expressed in the diencephalic choroid plexus and myelencephalic choroid plexus (Jiao et al., 2011). Demonstrating the similarity in gene expression in zebrafish and human choroid plexus is important when considering the use of zebrafish as a disease model for choroid plexus-related conditions. Our study provides evidence that the barrier properties and function of zebrafish choroid plexus are similar to those of mammalian choroid plexus. Ultimately, the genes discovered from our choroid plexus mutants will provide a better understanding of the genetic and molecular mechanisms involved in choroid plexus formation and function and new insights into how these normal processes become altered in disease.

\section{AUTHOR CONTRIBUTIONS}

Hannah E. Henson wrote the manuscript and Michael R. Taylor edited the manuscript. Hannah E. Henson performed the characterization study including Claudin 5 staining, transporter experiments, and fluorescent tracer experiments. Hannah E. Henson also performed the forward genetic screen, fluorescein assay, and genetic mapping. Chaithanyarani Parupalli performed the forward genetic screen. Chaithanyarani Parupalli and Hannah E. Henson performed the time-lapse imaging on wild-type and choroid plexus mutants. Bensheng Ju and Michael R. Taylor generated the $E t(c p: E G F P)^{s j 2}$ line.

\section{ACKNOWLEDGMENTS}

We thank Jennifer Peters and Victoria Frohlich from the St. Jude Children's Research Hospital Cell and Tissue Imaging Center for their assistance with the confocal images. Images were acquired at the Cell \& Tissue Imaging Center which is supported by St Jude Children's Research Hospital and NCI P30 CA021765-34. We thank Vani Shanker from St. Jude Children's Research Hospital Scientific Editing for editing the manuscript. We also thank members of the Taylor lab for assistance and support. This work was funded by The Hartwell Foundation, St. Jude Children's Research Hospital, and ALSAC.

\section{SUPPLEMENTARY MATERIAL}

The Supplementary Material for this article can be found online at: http://www.frontiersin.org/journal/10.3389/fnins.2014. 00364/abstract

\section{REFERENCES}

Alvira-Botero, X., and Carro, E. M. (2010). Clearance of amyloid-beta peptide across the choroid plexus in Alzheimer's disease. Curr. Aging Sci. 3, 219-229. doi: 10.2174/1874609811003030219

Bill, B. R., Balciunas, D., McCarra, J. A., Young, E. D., Xiong, T., Spahn, A. M., et al. (2008). Development and Notch signaling requirements of the zebrafish choroid plexus. PLoS ONE 3:e3114. doi: 10.1371/journal.pone.0003114

Craig, M. P., Gilday, S. D., Dabiri, D., and Hove, J. R. (2012). An optimized method for delivering flow tracer particles to intravital fluid environments in the developing zebrafish. Zebrafish 9, 108-119. doi: 10.1089/zeb.2012.0740

Currle, D. S., Cheng, X., Hsu, C. M., and Monuki, E. S. (2005). Direct and indirect roles of CNS dorsal midline cells in choroid plexus epithelia formation. Development 132, 3549-3559. doi: 10.1242/dev.01915

Daoud, R., Kast, C., Gros, P., and Georges, E. (2000). Rhodamine 123 binds to multiple sites in the multidrug resistance protein (MRP1). Biochemistry 39, 15344-15352. doi: 10.1021/bi0020574

Dohrmann, G. J. (1970). The choroid plexus: a historical review. Brain Res. 18, 197-218. doi: 10.1016/0006-8993(70)90324-0

Driever, W., Solnica-Krezel, L., Schier, A. F., Neuhauss, S. C., Malicki, J., Stemple, D. L., et al. (1996). A genetic screen for mutations affecting embryogenesis in zebrafish. Development 123, 37-46.

Dziegielewska, K. M., Ek, J., Habgood, M. D., and Saunders, N. R. (2001). Development of the choroid plexus. Microsc. Res. Tech. 52, 5-20. doi: 10.1002/1097-0029(20010101)52:1<5::AID-JEMT3>3.0.CO;2-J

Folgueira, M., Bayley, P., Navratilova, P., Becker, T. S., Wilson, S. W., and Clarke, J. D. (2012). Morphogenesis underlying the development of the everted teleost telencephalon. Neural Dev. 7, 32. doi: 10.1186/1749-8104-7-32

Garcia-Lecea, M., Kondrychyn, I., Fong, S. H., Ye, Z. R., and Korzh, V. (2008). In vivo analysis of choroid plexus morphogenesis in zebrafish. PLoS ONE 3:e3090. doi: 10.1371/journal.pone.0003090

Goldsmith, J. R., and Jobin, C. (2012). Think small: zebrafish as a model system of human pathology. J. Biomed. Biotechnol. 2012:817341. doi: $10.1155 / 2012 / 817341$ 
Gonzalez, A. M., Podvin, S., Lin, S. Y., Miller, M. C., Botfield, H., Leadbeater, W. E., et al. (2011). Ecrg4 expression and its product augurin in the choroid plexus: impact on fetal brain development, cerebrospinal fluid homeostasis and neuroprogenitor cell response to CNS injury. Fluids Barriers CNS 8:6. doi: 10.1186/2045-8118-8-6

Gutzman, J. H., and Sive, H. (2009). Zebrafish brain ventricle injection. J. Vis. Exp. 26:e1218. doi: 10.3791/1218

Horvitz, H. R. (1999). Genetic control of programmed cell death in the nematode Caenorhabditis elegans. Cancer Res. 59, 1701s-1706s.

Huang, X., Ketova, T., Fleming, J. T., Wang, H., Dey, S. K., Litingtung, Y., et al. (2009). Sonic hedgehog signaling regulates a novel epithelial progenitor domain of the hindbrain choroid plexus. Development 136, 2535-2543. doi: 10.1242/dev.033795

Irvin, D. K., Nakano, I., Paucar, A., and Kornblum, H. I. (2004). Patterns of Jagged1, Jagged2, Delta-like 1 and Delta-like 3 expression during late embryonic and postnatal brain development suggest multiple functional roles in progenitors and differentiated cells. J. Neurosci. Res. 75, 330-343. doi: 10.1002/jnr. 10843

Jansen, W. F., De Weger, R. A., Woutersen, R. A., Van Loveren, H., and Van De Kamer, J. C. (1976). The saccus dorsalis of the rainbow trout, Salmo gairdneri Richardson: histological, cytochemical, electron microscopical and autoradiographical observations. Cell Tissue Res. 167, 467-491. doi: 10.1007/BF002 15179

Janssen, S. F., Van Der Spek, S. J., Ten Brink, J. B., Essing, A. H., Gorgels, T. G., Van Der Spek, P. J., et al. (2013). Gene expression and functional annotation of the human and mouse choroid plexus epithelium. PLOS ONE 8:e83345. doi: 10.1371/journal.pone.0083345

Jiao, S., Dai, W., Lu, L., Liu, Y., Zhou, J., Li, Y., et al. (2011). The conserved clusterin gene is expressed in the developing choroid plexus under the regulation of notch but not IGF signaling in zebrafish. Endocrinology 152, 1860-1871. doi: 10.1210/en.2010-1183

Johansson, P. A., Irmler, M., Acampora, D., Beckers, J., Simeone, A., and Gotz, M. (2013). The transcription factor Otx2 regulates choroid plexus development and function. Development 140, 1055-1066. doi: 10.1242/dev.090860

Keep, R. F., and Smith, D. E. (2011). Choroid plexus transport: gene deletion studies. Fluids Barriers CNS 8:26. doi: 10.1186/2045-8118-8-26

Kratzer, I., Liddelow, S. A., Saunders, N. R., Dziegielewska, K. M., Strazielle, N., and Ghersi-Egea, J. F. (2013). Developmental changes in the transcriptome of the rat choroid plexus in relation to neuroprotection. Fluids Barriers CNS 10:25. doi: 10.1186/2045-8118-10-25

Kratzer, I., Vasiljevic, A., Rey, C., Fevre-Montange, M., Saunders, N., Strazielle, N., et al. (2012). Complexity and developmental changes in the expression pattern of claudins at the blood-CSF barrier. Histochem. Cell Biol. 138, 861-879. doi: 10.1007/s00418-012-1001-9

Lagaraine, C., Skipor, J., Szczepkowska, A., Dufourny, L., and Thiery, J. C. (2011). Tight junction proteins vary in the choroid plexus of ewes according to photoperiod. Brain Res. 1393, 44-51. doi: 10.1016/j.brainres.2011.04.009

Liddelow, S. A., Dziegielewska, K. M., Mollgard, K., Whish, S. C., Noor, N. M., Wheaton, B. J., et al. (2014). Cellular Specificity of the Blood-CSF barrier for albumin transfer across the choroid plexus epithelium. PLoS ONE 9:e106592. doi: 10.1371/journal.pone.0106592

Liddelow, S. A., Dziegielewska, K. M., Vandeberg, J. L., and Saunders, N. R. (2010). Development of the lateral ventricular choroid plexus in a marsupial, monodelphis domestica. Cerebrospinal Fluid Res. 7:16. doi: 10.1186/1743-84 54-7-16

Liddelow, S. A., Temple, S., Mollgard, K., Gehwolf, R., Wagner, A., Bauer, H., et al. (2012). Molecular characterisation of transport mechanisms at the developing mouse blood-CSF interface: a transcriptome approach. PLOS ONE 7:e33554. doi: 10.1371/annotation/2a9099a5-688b-4def-95a7-6ac13b10d096

Lippoldt, A., Liebner, S., Andbjer, B., Kalbacher, H., Wolburg, H., Haller, H., et al. (2000). Organization of choroid plexus epithelial and endothelial cell tight junctions and regulation of claudin-1, -2 and -5 expression by protein kinase C. Neuroreport 11, 1427-1431. doi: 10.1097/00001756-200005150-00015

Lowery, L. A., De Rienzo, G., Gutzman, J. H., and Sive, H. (2009). Characterization and classification of zebrafish brain morphology mutants. Anat. Rec. (Hoboken) 292, 94-106. doi: 10.1002/ar.20768

Lowery, L. A., Rubin, J., and Sive, H. (2007). Whitesnake/sfpq is required for cell survival and neuronal development in the zebrafish. Dev. Dyn. 236, 1347-1357. doi: $10.1002 /$ dvdy.21132
Lowery, L. A., and Sive, H. (2005). Initial formation of zebrafish brain ventricles occurs independently of circulation and requires the nagie oko and snakehead/atplala.1 gene products. Development 132, 2057-2067. doi: 10.1242/dev.01791

Meinke, D. W., and Sussex, I. M. (1979). Embryo-lethal mutants of Arabidopsis thaliana. A model system for genetic analysis of plant embryo development. Dev. Biol. 72, 50-61. doi: 10.1016/0012-1606(79)90097-6

Monnot, A. D., and Zheng, W. (2013). Culture of choroid plexus epithelial cells and in vitro model of blood-CSF barrier. Methods Mol. Biol. 945, 13-29. doi: 10.1007/978-1-62703-125-7_2

Muto, A., Orger, M. B., Wehman, A. M., Smear, M. C., Kay, J. N., Page-Mccaw, P. S., et al. (2005). Forward genetic analysis of visual behavior in zebrafish. PLoS Genet. 1:e66. doi: 10.1371/journal.pgen.0010066

Nielsen, C. M., and Dymecki, S. M. (2010). Sonic hedgehog is required for vascular outgrowth in the hindbrain choroid plexus. Dev. Biol. 340, 430-437. doi: 10.1016/j.ydbio.2010.01.032

Nolan, P. M., Peters, J., Strivens, M., Rogers, D., Hagan, J., Spurr, N., et al. (2000). A systematic, genome-wide, phenotype-driven mutagenesis programme for gene function studies in the mouse. Nat. Genet. 25, 440-443. doi: 10.1038/ 78140

Nusslein-Volhard, C., and Wieschaus, E. (1980). Mutations affecting segment number and polarity in Drosophila. Nature 287, 795-801. doi: 10.1038/287 $795 \mathrm{a} 0$

Omura, Y., Korf, H. W., and Oksche, A. (1985). Vascular permeability (problem of the blood-brain barrier) in the pineal organ of the rainbow trout, Salmo gairdneri. Cell Tissue Res. 239, 599-610. doi: 10.1007/BF00219238

Saengkhae, C., Loetchutinat, C., and Garnier-Suillerot, A. (2003). Kinetic analysis of rhodamines efflux mediated by the multidrug resistance protein (MRP1). Biophys. J. 85, 2006-2014. doi: 10.1016/S0006-3495(03)74628-1

Saunders, N. R., Ek, C. J., Habgood, M. D., and Dziegielewska, K. M. (2008). Barriers in the brain: a renaissance? Trends Neurosci. 31, 279-286. doi: 10.1016/j.tins.2008.03.003

Schmidt, R., Strahle, U., and Scholpp, S. (2013). Neurogenesis in zebrafish - from embryo to adult. Neural Dev. 8:3. doi: 10.1186/1749-8104-8-3

Sehnert, A. J., Huq, A., Weinstein, B. M., Walker, C., Fishman, M., and Stainier, D. Y. (2002). Cardiac troponin T is essential in sarcomere assembly and cardiac contractility. Nat. Genet. 31, 106-110. doi: 10.1038/ng875

Shapiro, A. B., and Ling, V. (1998). Stoichiometry of coupling of rhodamine 123 transport to ATP hydrolysis by P-glycoprotein. Eur. J. Biochem. 254, 189-193. doi: 10.1046/j.1432-1327.1998.2540189.x

Sin, O., Michels, H., and Nollen, E. A. (2014). Genetic screens in Caenorhabditis elegans models for neurodegenerative diseases. Biochim. Biophys. Acta 1842, 1951-1959. doi: 10.1016/j.bbadis.2014.01.015

Skinner, D. C., and Malpaux, B. (1999). High melatonin concentrations in third ventricular cerebrospinal fluid are not due to Galen vein blood recirculating through the choroid plexus. Endocrinology 140, 4399-4405.

Stemple, D. L., Solnica-Krezel, L., Zwartkruis, F., Neuhauss, S. C., Schier, A. F., Malicki, J., et al. (1996). Mutations affecting development of the notochord in zebrafish. Development 123, 117-128.

Strazielle, N., and Ghersi-Egea, J. F. (2000). Choroid plexus in the central nervous system: biology and physiopathology. J. Neuropathol. Exp. Neurol. 59, 561-574.

Strazielle, N., and Preston, J. E. (2003). Transport across the choroid plexuses in vivo and in vitro. Methods Mol. Med. 89, 291-304. doi: 10.1385/1-59259-419-0:291

Sykes, D., Sweet, D. H., Lowes, S., Nigam, S. K., Pritchard, J. B., and Miller, D. S. (2004). Organic anion transport in choroid plexus from wild-type and organic anion transporter 3 (Slc22a8)-null mice. Am. J. Physiol. Renal Physiol. 286, F972-F978. doi: 10.1152/ajprenal.00356.2003

Thambisetty, M., Simmons, A., Velayudhan, L., Hye, A., Campbell, J., Zhang, Y., et al. (2010). Association of plasma clusterin concentration with severity, pathology, and progression in Alzheimer disease. Arch. Gen. Psychiatry 67, 739-748. doi: 10.1001/archgenpsychiatry.2010.78

Tsuneki, K. (1986). A survey of occurrence of about seventeen circumventricular organs in brains of various vertebrates with special reference to lower groups. J. Hirnforsch. 27, 441-470.

Umans, R. A., and Taylor, M. R. (2012). Zebrafish as a model to study drug transporters at the blood-brain barrier. Clin. Pharmacol. Ther. 92, 567-570. doi: $10.1038 /$ clpt.2012.168

Urasaki, A., Morvan, G., and Kawakami, K. (2006). Functional dissection of the Tol 2 transposable element identified the minimal cis-sequence and a 
highly repetitive sequence in the subterminal region essential for transposition. Genetics 174, 639-649. doi: 10.1534/genetics.106.060244

Vercellino, M., Votta, B., Condello, C., Piacentino, C., Romagnolo, A., Merola, A., et al. (2008). Involvement of the choroid plexus in multiple sclerosis autoimmune inflammation: a neuropathological study. J. Neuroimmunol. 199, 133-141. doi: 10.1016/j.jneuroim.2008.04.035

Villalobos, A. R., Miller, D. S., and Renfro, J. L. (2002). Transepithelial organic anion transport by shark choroid plexus. Am. J. Physiol. Regul. Integr. Comp. Physiol. 282, R1308-R1316. doi: 10.1152/ajpregu.00677.2001

White, R. M., Sessa, A., Burke, C., Bowman, T., Leblanc, J., Ceol, C., et al. (2008). Transparent adult zebrafish as a tool for in vivo transplantation analysis. Cell Stem Cell 2, 183-189. doi: 10.1016/j.stem.2007.11.002

Wijnholds, J., Delange, E. C., Scheffer, G. L., Van Den Berg, D. J., Mol, C. A., Van Der Valk, M., et al. (2000). Multidrug resistance protein 1 protects the choroid plexus epithelium and contributes to the blood-cerebrospinal fluid barrier. J. Clin. Invest. 105, 279-285. doi: 10.1172/JCI8267

Winata, C. L., Korzh, S., Kondrychyn, I., Zheng, W., Korzh, V., and Gong, Z. (2009). Development of zebrafish swimbladder: the requirement of Hedgehog signaling in specification and organization of the three tissue layers. Dev. Biol. 331, 222-236. doi: 10.1016/j.ydbio.2009.04.035

Wodarczyk, C., Rowe, I., Chiaravalli, M., Pema, M., Qian, F., and Boletta, A. (2009). A novel mouse model reveals that polycystin-1 deficiency in ependyma and choroid plexus results in dysfunctional cilia and hydrocephalus. PLOS ONE 4:e7137. doi: 10.1371/journal.pone.0007137

Wolburg, H., and Paulus, W. (2010). Choroid plexus: biology and pathology. Acta Neuropathol. 119, 75-88. doi: 10.1007/s00401-009-0627-8
Xie, J., Farage, E., Sugimoto, M., and Anand-Apte, B. (2010). A novel transgenic zebrafish model for blood-brain and blood-retinal barrier development. BMC Dev. Biol. 10:76. doi: 10.1186/1471-213 $\mathrm{X}-10-76$

Zhang, J., Piontek, J., Wolburg, H., Piehl, C., Liss, M., Otten, C., et al. (2010). Establishment of a neuroepithelial barrier by Claudin $5 \mathrm{a}$ is essential for zebrafish brain ventricular lumen expansion. Proc. Natl. Acad. Sci. U.S.A. 107, 1425-1430. doi: $10.1073 /$ pnas.0911996107

Conflict of Interest Statement: The authors declare that the research was conducted in the absence of any commercial or financial relationships that could be construed as a potential conflict of interest.

Received: 14 August 2014; accepted: 22 October 2014; published online: 10 November 2014.

Citation: Henson HE, Parupalli $C, J u$ B and Taylor MR (2014) Functional and genetic analysis of choroid plexus development in zebrafish. Front. Neurosci. 8:364. doi: 10.3389/fnins.2014.00364

This article was submitted to Neurogenomics, a section of the journal Frontiers in Neuroscience.

Copyright (c) 2014 Henson, Parupalli, Ju and Taylor. This is an open-access article distributed under the terms of the Creative Commons Attribution License (CC BY). The use, distribution or reproduction in other forums is permitted, provided the original author(s) or licensor are credited and that the original publication in this journal is cited, in accordance with accepted academic practice. No use, distribution or reproduction is permitted which does not comply with these terms. 


\title{
Dissecting gene expression at the blood-brain barrier
}

\author{
Melanie A. Huntley ${ }^{1+}$, Nga Bien-Ly ${ }^{2+}$, Richard Daneman ${ }^{3 *}$ and Ryan J. Watts ${ }^{2 *}$ \\ ${ }^{1}$ Department of Bioinformatics and Computational Biology, Genentech Inc., South San Francisco, CA, USA \\ ${ }^{2}$ Department of Neuroscience, Genentech Inc., South San Francisco, CA, USA \\ ${ }^{3}$ Department of Pharmacology, University of California, San Diego, La Jolla, CA, USA
}

\section{Edited by:}

Norman Ruthven Saunders,

University of Melbourne, Australia

Reviewed by:

Joel Pachter, University of

Connecticut Health Center, USA

Stefan Liebner, Goethe University

Clinic, Germany

\section{*Correspondence:}

Richard Daneman, Department of Pharmacology, University of

California San Diego, 9500 Gilman

Drive 0636, La Jolla, 92093 CA, USA

e-mail: rdaneman@ucsd.edu;

Ryan J. Watts, Department of

Neuroscience, Genentech Inc.,

1 DNA Way, South San Francisco,

94080 CA, USA

e-mail: watts.ryan@gene.com

${ }^{\dagger}$ These authors have contributed

equally to this work.
The availability of genome-wide expression data for the blood-brain barrier is an invaluable resource that has recently enabled the discovery of several genes and pathways involved in the development and maintenance of the blood-brain barrier, particularly in rodent models. The broad distribution of published data sets represents a viable starting point for the molecular dissection of the blood-brain barrier and will further direct the discovery of novel mechanisms of blood-brain barrier formation and function. Technical advances in purifying brain endothelial cells, the key cell that forms the critical barrier, have allowed for greater specificity in gene expression comparisons with other central nervous system cell types, and more systematic characterizations of the molecular composition of the blood-brain barrier. Nevertheless, our understanding of how the blood-brain barrier changes during aging and disease is underrepresented. Blood-brain barrier data sets from a wider range of experimental paradigms and species, including invertebrates and primates, would be invaluable for investigating the function and evolution of the blood-brain barrier. Newer technologies in gene expression profiling, such as RNA-sequencing, now allow for finer resolution of transcriptomic changes, including isoform specificity and RNA-editing. As our field continues to utilize more advanced expression profiling in its ongoing efforts to elucidate the blood-brain barrier, including in disease and drug delivery, we will continue to see rapid advances in our understanding of the molecular mediators of barrier biology. We predict that the recently published data sets, combined with forthcoming genomic and proteomic blood-brain barrier data sets, will continue to fuel the molecular genetic revolution of blood-brain barrier biology.

\section{HISTORICAL PERSPECTIVE ON DATASETS}

In 1967 using exogenous peroxidase, Reese and Karnovsky demonstrated the subcellular localization of the blood-brain barrier (BBB) to be the endothelium forming the walls of vessels in the brain (Reese and Karnovsky, 1967). Two structural characteristics distinguished these endothelial cells from those in the heart and skeletal muscle: the presence of tight junctions, and the paucity of micropinocytotic vesicles. Early studies used microscopy and immunohistochemistry to visualize the presence and localization of a handful of known proteins within brain endothelial cells (BECs) (Risau et al., 1986), while in situ hybridization filled in the gaps for questions about expression patterns when antibodies were not available for specific targets. Nearly fifty years later, the underlying genes and developmental cascades that result in the unique traits of BECs are just beginning to be understood. Current critical advancements in our ability to both purify and culture BECs now allow for the study of their

Abbreviations: ABC, ATP binding cassette; BBB, blood-brain barrier; BEC, brain endothelial cell; FACS, fluorescence activated cell sorting; HBMEC, human brain microvessel endothelial cell; hCMEC/D3, human cerebral microvascular endothelial cell line; HUVEC, human umbilical vein endothelial cell; LCM, laser capture microdissection; miRNA, microRNA. molecular mechanisms at an unprecedented level of breadth and depth.

Since the seminal discovery that brain endothelial cells limit the diffusion of blood-borne molecules, the following decades of work have made it clear that the BBB is not just a physical wall but a complex regulated physiology that allows the BECs to determine the movement of ions, molecules and cells between the blood and the neural tissue. At the heart of this physiology are molecular and structural specializations of BECs that differentiate them from endothelial cells in non-neural tissue. BECs are held together by tight junctions, which form a tight paracellular barrier that polarizes the cells into distinct luminal and abluminal membrane compartments. This tight paracellular junction coupled with the extremely low levels of transcytosis creates a physical barrier to hydrophilic molecules and allows cellular transport properties to determine movement of molecules between the blood and the brain. To regulate this movement, BECs express a series of different transporters that generally fall into two categories: efflux transporters and nutrient transporters (for an in depth review of BBB transporters see Saunders et al., 2013). Briefly, efflux transporters, such as ABCB1 (also known as p-glycoprotein, or Pgp), face the luminal surface and use energy generated from the hydrolysis of ATP to pump a wide 
array of lipophilic substrates up their concentrations gradients into the blood, thus limiting the passive diffusion of hydrophobic molecules into the brain. Nutrient transporters, such as Glut-1 (glucose transporter SLC2A1), Lat-1 (amino acid transporter light chain, SLC7A5) and Mct-1 (monocarboxylate transporter, SLC16A1), facilitate the movement of specific substrates down their concentration gradients, allowing specific molecules into or out of the brain. Thus, the BBB is not solely a physical barrier but it is a series of complex regulated physiological specializations of the BECs that control the microenvironment of the brain. Furthermore, work has demonstrated that many of these specializations of the BECs are not intrinsic, but regulated by interactions with neural cells including CNS pericytes and astrocytes, and immune cells including microglia and macrophages (Hudson et al., 2005; Armulik et al., 2011). Identifying the molecular signature of BECs and how they differ from ECs in other tissues, as well as key cell-cell signaling interactions in the neurovascular unit, are questions at the forefront in understanding the physiology of the BBB.

\section{THE UTILITY OF DATASETS AS RESOURCES}

High quality gene expression data has significantly impacted the direction of investigation by allowing for a better molecular understanding of BBB development, function, and dysfunction. Well-powered gene expression studies can validate hypothesis driven queries or open previously unexplored avenues of research in unbiased directions. Models of acute BBB dysfunction, such as stroke or ischemia, aid in elucidating mechanisms of impairment for development of therapeutics or preventative treatments. An understanding of BBB-specific transporter, receptor, and ion channel expression levels could benefit drug development efforts by aiding in the design of selective compounds for those that could evade efflux pumps (Pottiez et al., 2009) or utilize receptors to enhance therapeutic uptake in brain (Yu and Watts, 2013).

The variety of questions and issues that can be addressed related to BBB development and disease have vastly improved, thanks in part to genetic engineering approaches in rodents and other species, improved cellular isolation techniques, and the availability of bioinformatics software and experts that facilitate the mining of data sets for relevant genes worthy of further investigation. The combination of "big data" sets and moleculargenetic tools is beginning to elucidate biological mechanisms in many arenas, and our understanding of BBB biology is benefiting from this revolution.

\subsection{KEY PARAMETERS FOR BBB GENOMIC STUDIES}

The cellular complexity of the BBB presents a significant obstacle in the gathering of meaningful gene expression data. Brain endothelial cells are surrounded by the mural cells-pericytes and smooth muscle cells-and are further encapsulated by a basement membrane (see Figure 1C). The ensheathment of the vessels by astrocytic endfeet, likely receiving signals from neurons and microglia, serve to further regulate brain vascular function (Abbott, 2005). Therefore, these various cell types are intimately connected and unite in maintaining the integrity of the BBB. Thus a global analysis of BBB gene expression may necessitate the co-purification and co-analysis of these important non-BEC cell types along with BECs (Shusta, 2005). Early efforts to harvest brain vasculature for genomic analysis captured a majority of the cell types associated with BECs of the neurovascular unit. These earlier protocols relied on mechanical brain dissociation to isolate microvascular components, a technique known as vascular enrichment (Triguero et al., 1990; Boado and Pardridge, 1991), which uses a series of filtrations through nylon membranes or with glass beads followed with gradient centrifugation steps (Betz et al., 1979; Yousif et al., 2007). This contrasted with laser capture microdissection (LCM) methods for single capillary fragment isolation, which allows for highly localized sampling from specific human and mouse brain sections and could be restricted to diseased regions (Ball et al., 2002; Mojsilovic-Petrovic et al., 2004). Again, both methods do not specifically yield pure populations of BECs specifically, however, they do allow for a sampling of cells associated with the neurovascular unit and generally enrich for BECs for inferences to be drawn about CNS microvasculature.

Thus, several important considerations must be taken into account when designing genomic experiments to analyze the BBB. The most important parameter is to determine the desired cells or tissues to be analyzed and the method of purification. Because the key properties of the BBB are specializations of BECs, many studies have used methods to purify ECs from brain tissue, whereas others have used crude approaches to purify vessels (endothelial cells and pericytes) or entire fragments of the neurovascular unit (see Figure 1). In addition, the vasculature of the brain contains many different segments of the vascular tree including arteries, arterioles, capillaries, post-capillary venules, venules, and veins (Dorr et al., 2007), all of which contain barrier properties but each segment may have different transport, metabolic, signaling and cell adhesion properties. Gradient centrifugation has been used to specifically isolate microvessels (capillaries and post-capillary venules), whereas purification based on markers of ECs will contain BECs from all segments of the vascular tree. Also taken into consideration should be the species from which the samples are isolated, whether human, rodent or other model organism, and whether to examine acutely purified or cultured cells. The second parameter is the method of analysis. For transcriptomic generation of EST libraries, subtractive hybridization, microarrays and RNA sequencing are all common methods to examine RNA. Different methods of RNA extraction, including whole RNA, mRNA, small RNA, and tagged-ribosome isolation can highlight specific RNAs of interest. In addition, proteomics, metabolomics, lipidomics, epigenomics, and other "omics" analysis can give an even broader overview of the molecular composition of the BBB. The third parameter is the effective comparisons used for differential gene expression analysis. Identifying all the transcripts in a given cell can be challenging to decipher, however, generating a comparison of two cells that vary only for a given function can greatly narrow down the search for important genes. In the case of the $\mathrm{BBB}$, several important comparisons have been made, including comparing BECs with whole brain extracts to identify EC specific genes, as well as comparing BECs with peripheral ECs to identify BBB-specific specializations (see Table $\mathbf{1}$ for a summary of recent BBB expression profiling studies). The fourth important parameter to determine is the physiological setting in which to analyze the BBB. Much work has been done to identify 


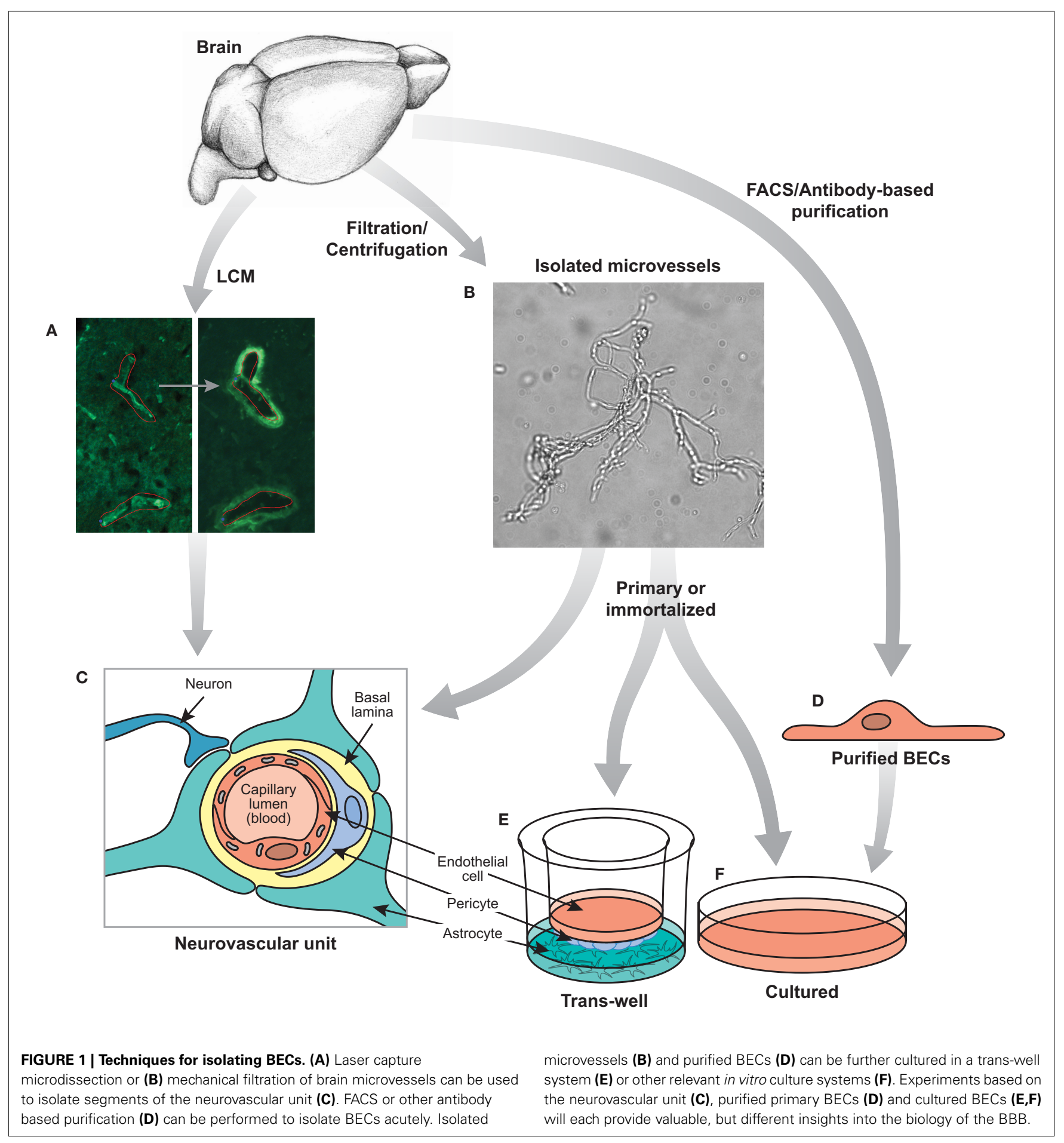

the gene expression signature of the $\mathrm{BBB}$ in a healthy adult animal, but studies have also examined the BBB during development, in different neurological disease settings, in mice with different mutations that affect BBB formation and function, or with the addition of different pharmacological agents. Each of these parameters will greatly influence the data set obtained and therefore careful consideration must be taken to ensure the best method of BEC purification and analysis.

\subsection{METHODS OF ANALYSIS}

Earlier transcriptomic approaches that yielded important initial insights on brain microvascular specific expression data included suppression subtractive hybridization and serial analysis of gene expression (SAGE) (Li et al., 2001; Shusta et al., 2002; Enerson and Drewes, 2006; Calabria and Shusta, 2008). For a detailed review on these techniques and early results, see Shusta (2005), Pottiez et al. (2009). A distinguishing aspect of the SAGE study of 
Table 1 | Recent genomewide microarray expression data sets for the BBB.

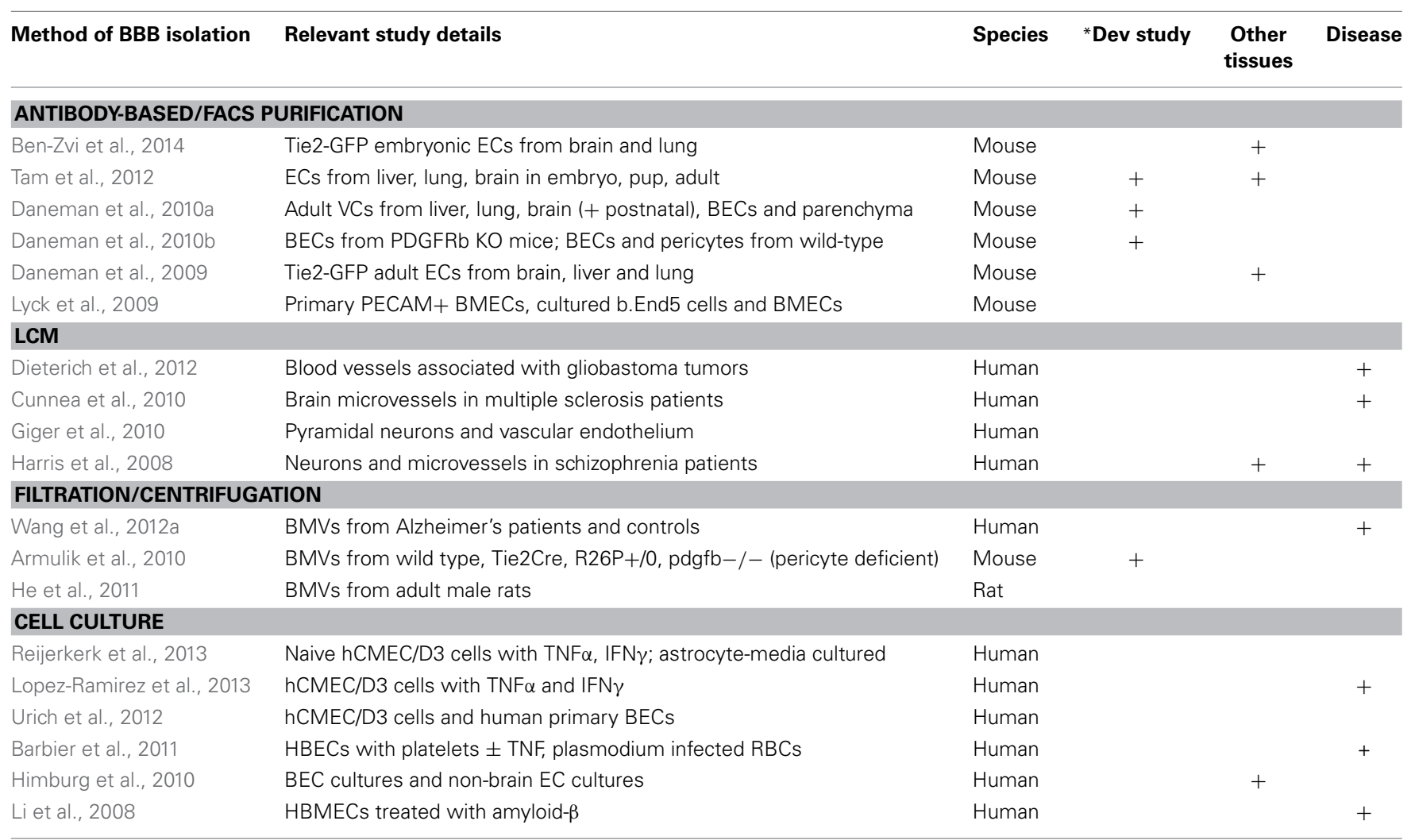

*Developmental stages. BEC, brain endothelial cell; BMEC, brain microvascular endothelial cell; BMV, brain microvessel; HBEC, human brain endothelial cell; $H B M E C$, human brain microvessel endothelial cells; $E C$, endothelial cell; $R B C$, red blood cell; VC, vascular cell.

rat brain microvessels was the comparison to rat neocortex and hippocampus tag catalogs and the resulting identification of 864 BBB enriched genes.

Today, expression microarrays offer a mature and commonly used platform to differentially compare gene expression between two samples. One of the earliest $\mathrm{BBB}$ gene expression studies using microarray established a protocol to profile human brain endothelial cells co-cultured with astrocytes under dynamic conditions with controlled intraluminal flow (Marroni et al., 2003). The authors observed that exposure to increased flow up-regulated cytoskeletal genes while also inhibiting cell cycling genes.

In the era of microarray data sets on the BBB (see Table 1), the number of genes that are queried on commercially available chips has increased several orders of magnitude, moving the field into truly high-throughput experiments and data. In addition, relatively small amounts of cells or tissue are now required for genome-wide transcriptomic approaches, thus facilitating a wide range of studies. Advancements and maturity of computational tools for this type of data analysis are now available allowing investigators to dive into these data sets and identify meaningful connections (Irizarry et al., 2003; Gentleman et al., 2004; Smyth, 2004; Falcon and Gentleman, 2007; Kauffmann et al., 2009). Online repositories, such as Gene Expression Omnibus and the European Nucleotide Archive, now allow researchers to deposit genome wide expression data sets to be queried and re-used by other groups. Indeed, many of the studies listed in Table 1 have made use of previously published expression data sets from the $\mathrm{BBB}$ as an integral part of their analyses. The importance of continuing to expand the public availability of such data sets as an ongoing resource for the community cannot be overstated.

A discussion of newer techniques, such as next generation RNA-sequencing, will follow at the conclusion of this review.

\section{EXPERIMENTAL PARADIGMS ANALYZED}

Figure 1 summarizes the most common methods for isolation and purification used in BBB genome-wide expression studies. As many recent studies have shown, in vitro models of the BBB do not fully recapitulate the complexity of an intact organism. The use of newer techniques such as fluorescence activated cell sorting (FACS), immunopanning, magnetic bead immunoprecipitation, as well as advances to LCM may aid in acutely harvesting specifically BECs, or an enriched population of BECs, for downstream transcriptomic and proteomic studies. Navone et al. (2013) document recent protocols for the isolation of human and mouse brain microvascular endothelial cells. However, no technique or protocol is perfect as there are subtle considerations to be aware of which may factor largely into downstream data analysis. Even careful acute isolation and genomic profiling of BECs may be susceptible to general expression changes induced by mechanical shearing of brain parenchyma and/or microvessel tissue, enzymatic digestion, temperature fluctuations, etc. Careful pair-wise 
comparisons with the proper controls undergoing similar handling are critical to minimize bias in the data.

\subsection{ACUTE ISOLATION AND PURIFICATION OF BRAIN MICROVASCULAR CELLS}

The use of antibody-based techniques to isolate rodent brain endothelial cells has been reported in several recent studies. Magnetic beads coupled to murine or rat antibodies against CD31/PECAM allow for immunoprecipitation of endothelial cells from homogenates of brain (Lyck et al., 2009) and possibly other vascular organs for direct comparisons. Likewise, antibodies against CD31 can be immobilized onto culture plates for immunopanning enrichment of brain homogenates for acute isolation of BECs (Zhou et al., 2014a) or the use of other cell surface receptors such as anti-PDGFR $\beta$ for pericytes (Daneman et al., 2010b). This approach also allows for a negative selection step against microglia or pericytes to remove these non-BEC subtypes from the overall homogenate. A third BEC-specific antibody or antigen-selection technique utilizes fluorescence-activated cell sorting which would use either a fluorophore-coupled anti-BEC cellular marker (Daneman et al., 2010b; Tam et al., 2012) or transgenic mice that harbor BEC-specific promoters to direct expression of a fluorescent protein, such as Tie2-GFP mice (Daneman et al., 2009, 2010a; Ben-Zvi et al., 2014).

Moreover, LCM has experienced many improvements including the addition of immunostaining protocols and brighter, more specific dyes for identification of brain endothelia (Macdonald et al., 2008). Recent immuno-LCM studies have described differences in gene expression of brain capillaries vs. venules, and highlighted important roles for their heterogeneity (Macdonald et al., 2010). Increased antibody availability for other neurovascular unit cell types have also enabled cross comparison of gene expression as well as validation of cell type specificity of the data sets generated by mass spectrometry (Murugesan et al., 2011). Advances in equipment such as microscopes with automated stages, higher quality cameras, and sophisticated software have enabled more consistent tissue sampling and higher throughput.

\subsection{IN VITRO BBB MODELS}

Immortalized cell lines derived from rodent or human brain endothelial cells have been frequently used as a model system for gene expression studies despite their questionable physiological relevance. Cultured cells, especially those that can be passaged indefinitely, represent a key model system that allows for a high level of cellular homogeneity, consistency, and experimental reproducibility. Key components of the neurovascular unit can be reconstituted in vitro by growing BECs on filter inserts that sit above pericytes and/or astrocytes, allowing signaling mechanisms between these cell types to remain largely intact. Conversely, in vitro models may still lack the complex interactions between these cell types and the endogenous extracellular matrix, as well as surrounding neurons and microglia that exist in vivo. Complicated disease processes that are also in play in vivo are likely difficult to fully model in a transwell culture system and suffer from numerous drawbacks (Hawkins and Egleton, 2008; Pottiez et al., 2009). In other words, the neurovascular unit is essentially an organ system with numerous cellular interactions that make it virtually impossible to replicate outside of the organism. However, these culture paradigms also do enable investigators to determine what individual cell types can do in the absence of other cells, or with defined cellular interactions. For instance, culturing BECs alone can give an idea of which $\mathrm{BBB}$ properties are independent of signaling interactions within the neurovascular niche. Furthermore, by adding feeder layers of individual cells, such as astrocytes or pericytes, or signaling molecules or pharmacological agents, in vitro models can aid in determining how these factors directly influence BEC gene expression.

Primary BEC culture models obtained from bovine or rodent acutely isolated brain microvessels present another system for directed studies on BBB maintenance and function. Early efforts relied on isolation and enrichment of brain capillaries from rats followed by enzymatic digestion of the basement membrane to allow migration of endothelial cells for growth on an artificial substrate (Bowman et al., 1981). Bovine models have allowed for several benefits including greater amounts of starting material obtained from one animal, less variability between cultures from the same starting material, and importantly, retention of endothelial characteristics after serial passaging (Goetz et al., 1985).

The establishment of human cerebral microvessel cultures also yielded early, important insights into BBB function (Vinters et al., 1987). These culture systems were improved upon as tighter barriers were introduced with co-culture models containing astrocytes and by introducing intraluminal flow (Marroni et al., 2003). Human brain microvessel endothelial cells (HBMEC) have also been treated with amyloid- $\beta$ to determine their gene expression changes using a cDNA array and identified a putative protective role for STC1 (Li et al., 2008).

\subsection{COMPARISONS OF ACUTE VS. CULTURED BBB MODELS}

Whether in vitro BBB models were derived from immortalized cell lines or acutely isolated from mouse or human brains, extensive efforts to characterize their gene expression signature have repeatedly revealed their limited approximation of in vivo complexity. Comparisons of $\mathrm{BBB}$ gene expression profiles from acutely isolated mouse brain endothelial cells vs. a period of brief culturing, or compared against a commonly used mouse brain endothelioma line (bEND5) revealed evidence of dedifferentiation of BEC qualities (i.e., reduced levels of tight junction proteins and amino acid transporters) induced by primary culture or immortalization (Lyck et al., 2009). A similar study which used suppression subtractive hybridization revealed that some transport-related brain endothelial genes were subsequently downregulated after BECs spent several days in culture compared to freshly isolated rat brain microvessels (Calabria and Shusta, 2008). The same study compared cultures containing puromycin (which decreases numbers of contaminating pericytes) for BEC expression and also the effect of hydrocortisone on cultures. More recently, transcriptome data from immortalized human BEC line hCMEC/D3 and primary human BEC (Urich et al., 2012) were compared with published data on acutely isolated mouse BECs (Daneman et al., 2010a) to highlight differences in levels of endothelial marker expression. 
The authors found that although the human cells demonstrated expression of tight junction proteins, transporters and receptors, their levels were dramatically reduced compared to acutely isolated mouse BECs (Urich et al., 2012).

Nevertheless, using in vitro BBB models may yield some important initial insights into BEC response to external stimuli or stress. hCMEC/D3 cells have been recently profiled for their transcriptional response to $\mathrm{TNF} \alpha$ and IFN $\gamma$ and gene expression signatures identified span from factors involved in antigen presentation, cellular adhesion, cytokine-induced signaling pathways, to reduced transporter expression (Lopez-Ramirez et al., 2013). These data were compared to similar historical data sets that included TNF $\alpha$-treated human umbilical vein (HUVEC) and primary human cerebral endothelial cell cells (Franzen et al., 2003).

\subsection{MODELS OF BBB DEVELOPMENT}

$\mathrm{Wnt} / \beta$-catenin signaling has been implicated in driving CNS specific angiogenesis and regulating BBB formation and maintenance (Liebner et al., 2008; Stenman et al., 2008; Daneman et al., 2009; Wang et al., 2012b; Zhou et al., 2014b). Using microarray expression profiling comparing BECs with ECs from non-neural tissue, Daneman and colleagues identified that genes downstream of $\mathrm{Wnt} / \beta$-catenin signaling were greatly enriched in endothelial cells of the brain but not liver or lung (Daneman et al., 2009, 2010a). Performing sophisticated genetic and functional experiments, the authors demonstrate that $\mathrm{Wnt} / \beta$-catenin signaling is required to drive CNS-specific angiogenesis but not angiogenesis in other tissues. To identify if this CNS-specific angiogeneic program also induced BBB-specific gene expression in the endothelial cells, the authors utilized gene expression profiling of purified mouse brain endothelial cells cultured with and without Wnt ligands to find that Wnt induced the expression of BBB-specific nutrient transporters in the endothelial cells, and further showed that loss of Wnt signaling in vivo led to loss of Glut-1 expression in endothelial cells. More recently, Zhou et al. (2014b) demonstrated nearly identical canonical Wnt signaling mechanisms of development and maintenance between both the blood-brain and blood-retinal barriers.

The characterization of molecular cues and signaling pathways that guide both physiological and pathophysiological CNS angiogenesis may also stand to benefit greatly from expanded efforts at expression profiling of BECs within various parts of the neurovascular unit. Common guidance molecules that regulate both nerve and vascular remodeling such as Sema/Plex/Nrp, Slit/Robo, Netrin/Unc5, VEGF, and its tyrosine kinase receptors Flt-1 and Flk-1, have been extensively studied, however, the role of the canonical axon guidance molecules in CNS angiogenesis is less studied (Tam and Watts, 2010). Transcriptomics may also enable future studies on understanding the role of TGFb or EphB receptors and ligands in determining arteriovenous identity within the brain vasculature (Adams and Alitalo, 2007; Engelhardt and Liebner, 2014).

In addition to the involvement of Wnt signaling in $\mathrm{BBB}$ angiogenesis and barriergenesis, the role of growth factors and kinases beyond VEGF and Notch have also been further characterized. Mice deficient in PDGF-B lack microvascular pericytes and succumb to lethal microaneurisms during development due to incomplete brain vascularization (Lindahl et al., 1997). More recent studies on the importance of pericytes in barriergenesis and maintenance reported that significantly reduced pericyte coverage in $\operatorname{PDGFR} \beta$ null fetal mice translated into increased permeability (Daneman et al., 2010b). Transcriptome signatures of BECs isolated from null mice were compared to wild type mice, which revealed increases in Angpt2, a Tie2 ligand involved in vascular permeability of peripheral endothelial cells but no downregulation of $\mathrm{BBB}$-enriched genes. Thus pericytes may regulate the $\mathrm{BBB}$ by inhibiting the expression of genes that normally make endothelial cells leaky. In addition, the expression profile of pericytes acutely isolated from wild type mice was also generated, further providing a valuable data set for BBB neurovascular unit transcript comparisons (Daneman et al., 2010b).

For an in depth review on recent studies of developmental barriergenesis and repair, see Obermeier et al. (2013), Siegenthaler et al. (2013), Engelhardt and Liebner (2014).

\subsection{EVOLUTIONARY STUDIES OF THE BBB}

Two distinct cellular mechanisms of BECs appear to be evolutionarily conserved across all vertebrates and some invertebrates, including the fruit fly model organism, Drosophila melanogaster. These conserved traits include the tight junctions that prevent paracellular diffusion, and ATP binding cassette (ABC) transporters that efflux molecules back into the vascular luminal space. The Drosophila genome encodes proteins which are nearly identical in sequence to those that comprise vertebrate tight junctions (Wu and Beitel, 2004; Banerjee et al., 2006), and whose disruption leads to defects in humoral-CNS barrier function (Schwabe et al., 2005; Stork et al., 2008). Further, Mayer et al. (2009) established that the fly protein Mdr65 functions as an $\mathrm{ABC}$ transporter in the Drosophila hemolymph-brain barrier.

Validation of gene expression data in other species with a simplistic BBB may expedite the search for candidate genes that warrant further study in rodents or human tissue. For example, we recently profiled the rodent $\mathrm{BBB}$ at three developmental stages, used zebrafish to confirm the necessity of candidate genes in regulating angiogenesis, and followed with subsequent analysis of genetic interactions of two death receptors, DR6 and TROY, by returning to mice to confirm a role for these genes in rodent $\mathrm{BBB}$ development (Tam et al., 2012). As such, conserved pathways can be discovered in one species and further characterized in more tractable models. In fact, fruit fly, grasshopper, and zebrafish are gaining popularity as models for high throughput screening of BBB drug permeability studies (Nielsen et al., 2011; Geldenhuys et al., 2012) It is this underlying premise of evolutionary conservation which provides confidence that understanding the BBB in rodent and other model organisms will be relevant to the human BBB.

Primate studies, on the other hand, can illuminate more recent evolutionary innovations relevant to the human $\mathrm{BBB}$. Gene expression differences between neurons and BECs from human postmortem tissue isolated by LCM were compared with published data sets on chimpanzees and rhesus macaques for analysis of rates of transcriptome evolution in neuronal vs. endothelial cell types (Giger et al., 2010). The authors found that transcriptomes 
of neurons and endothelial cells evolve at different rates within brain tissue. This finding adds complexity to the challenge of translating $\mathrm{BBB}$ results from other species, even closely related species, into human models.

\subsection{HUMAN BBB DATA SETS FOCUSED ON AGING, DISEASE, AND DYSFUNCTION}

During normal aging, the blood brain barrier experiences functional changes, which can result in impairment and dysfunction of the barrier. Marques et al. (2013) provide a review of these changes in both the $\mathrm{BBB}$ and blood-CSF barriers. In the $\mathrm{BBB}$, these changes include loosening of tight junctions, increases in pinocytotic vesicles, and decreases in mitochondrial density within ECs. The authors highlight the similarity between normal aging, and CNS disease related changes of the BBB. Since human BBB data from patients typically comes from those with obviously progressed symptoms, it is a challenge to determine if the changes in BBB function are causal or consequential to disease progression. Improvements in animal models of CNS diseases with BBB impairment may help answer such questions.

Nevertheless, several human studies in diverse disease paradigms have sought to understand vascular expression differences between healthy control and disease tissue. Microvascular endothelial cells from postmortem schizophrenia brains were isolated by LCM and their functional profile was compared to neurons and control tissue in a study by Harris et al. (2008). They found that the cerebral microvasculature of schizophrenic patients displayed a hypo-inflammatory status and this observation was reproducible on a separate microarray platform. This gene set could be useful in comparison studies with similar rodent models of cognitive dysfunction or with human BBB gene sets from patients with other cognitive or behavioral impairments. Likewise, the use of LCM to isolate vessels from glioblastoma multiforme brain tissue followed by microarray analysis and $\mathrm{qPCR}$ validation identified uniquely upregulated genes specific to tumor tissue and absent in nonmalignant human brain (Pen et al., 2007). Interestingly, a similar study from another group did not identify the same upregulated genes and describe a different expression signature (Dieterich et al., 2012). Another recent study also used LCM to query genes expressed at the BBB from multiple sclerosis lesions isolated from postmortem tissue (Cunnea et al., 2010).

Brain endothelial cell transcriptomes from human neurodegenerative diseases have been distinctly underrepresented. However, there is no shortage of studies reporting genome-wide data sets from Alzheimers disease brain or sub-regions such as hippocampus (Blalock et al., 2004; Dunckley et al., 2006; Webster et al., 2009; Blalock et al., 2011). To the best of our knowledge, the only published data set generated thus far representing the $\mathrm{BBB}$ in human neurodegeneration is from a study where brain microvessels from control and Alzheimers disease tissue were transcriptionally profiled to identify abnormal gene expression or differentially expressed genes (Wang et al., 2012a). A comparison of this data set with brain microvasculature gene expression data derived from mouse models of Alzheimer's disease may serve as a useful tool for model validation.

\subsection{RODENT BBB DATA SETS IN MODELS OF NEURODEGENERATION AND DISEASE}

Transgenic mouse models of Alzheimers disease have been instrumental to the study of amyloid- $\beta$ accumulation and its role in neuronal impairment and cognitive dysfunction. Transcriptome analysis of whole brain, specific subregions, various disease time points, and in multiple Alzheimer's disease transgenic models or after experimental treatments has been performed (Dickey et al., 2003; Reddy et al., 2004; Lazarov et al., 2005; Gatta et al., 2014). Genomic studies specifically using mouse brain vasculature from Alzheimer's disease models, on the other hand, remain sparse. The most recent study used a proteomics approach from homozygous Tg-SwDI mice which harbor three familial, autosomal dominant mutations on APP, and demonstrate sustained increases in vascular amyloid- $\beta$ accumulation over time (Searcy et al., 2014). The authors compared brain microvessel fractions from 3- and 9-month old wild type and transgenic mice for aging-related effects on the mouse vascular proteome as well as amyloid- $\beta$-induced effects. They found distinct sets of proteins changed in young and old wild type and transgenic mice, indicating a differential effect of age on the cerebrovasculature.

An experimental mouse model of multiple sclerosis, experimental autoimmune encephalomyelitis, is characterized primarily by dysfunction of the BBB. Functional studies have produced insights into the role of particular genes, such as the chemokine CXCL12, and the permeability of the BBB in experimental autoimmune encephalomyelitis (McCandless et al., 2006, 2009). However, to our knowledge, there does not yet exist a genome wide expression data set derived from the BBB in this important model of multiple sclerosis.

Metabolic diseases, with obesity a leading factor, may also have a multitude of effects on the BBB and as a result, CNS function. A recent study on diet-induced obesity using a mouse model profiled the proteomic changes in brain vasculature after 2 months of high fat diet chow administered starting at 2 months of age (Ouyang et al., 2014). The samples were compared directly with mice maintained on normal chow and the authors found 47 downregulated and 2 upregulated proteins, indicating that in general, diet-induced obesity suppressed metabolic activity in brain microvessels (Ouyang et al., 2014).

Rodent models exist for other neurological diseases associated with BBB dysfunction, including stroke, epilepsy, ALS, and neuromyelitis optica. However, to date, there is a void of genome wide expression data on brain endothelial cells from these important models. Future data sets derived from these models will be invaluable in bridging the knowledge gap between our understanding of healthy BBB maintenance and BBB dysfunction in disease.

Another genomic data set that is publicly available and could be used as a valuable cross-reference tool describes the transcriptional changes in four rat brain tissues/regions including striatum, and parietal cortex, choroid plexus, and the meninges and its associated vasculature (Bowyer et al., 2013). This study also included a time course analysis to profile the effects of amphetamines or environment-induced hypothermia on choroid plexus and the meninges. 


\section{SUMMARY OF INSIGHTS FROM BBB GENOMIC STUDIES 4.1. OVERVIEW}

Genomic studies on the BBB have provided a number of large data sets identifying genes that are expressed by BECs, enriched in BECs compared to peripheral ECs, and also genes that change levels during development or disease context. The challenge now is to sort through these vast data sets and untangle which of these large numbers of genes are functionally important for the formation and function of the $\mathrm{BBB}$, and which can be targeted to either modulate the $\mathrm{BBB}$ during neurological disease or to use as carriers for drug delivery across the BBB. Two positive outcomes have arisen from these data sets: (1) many of these analyses that use completely different methodologies have identified the same BBB genes, and (2) virtually all of the data sets have verified the expression and BBB-enrichment of commonly studied genes important for BBB function including tight junctions (claudin 5, occludin, Zo-1, Zo-2), efflux transport (P-glycoprotein, and ABCG2, also known as Bcrp), nutrient transport (Glut-1, Mct-1, Lat-1) and others (basigin, carbonic anhydrase 4 ). The strong reproducibility and ability to verify positive hits suggests that these data sets will indeed provide an extremely strong blueprint for identification of novel genes important for BBB function.

\subsection{SIGNALING REGULATORS}

Several important discoveries have already been generated from these data sets. For instance, the identification that a cluster of Wnt/ $\beta$-catenin signaling components and downstream targets (Fzd6, Lef1, Axin2, Dixdc1, Apcdd1, Troy) are greatly enriched in BECs compared to peripheral ECs led to the hypothesis that Wnt may be an important regulator of the BBB. As discussed above, several groups have verified and identified that $\mathrm{Wnt} / \beta$-catenin signaling is required for driving CNS-specific angiogenesis, BBB induction and maintenance (Liebner et al., 2008; Stenman et al., 2008; Daneman et al., 2009; Wang et al., 2012b). Beyond the morphogens Wnt and Shh, and the canonical VEGF and Notch signaling pathways, a further understanding of other growth factors and kinases involved in angiogenesis have also been possible from expression data. Moreover, gene cluster/pathway analysis can provide an important method to sift through large data sets to find groups of genes that are functionally important. Other clusters associated with BBB-enrichment are genes involved in retinoid signaling, amino acid metabolism and glycolysis, suggesting that these may be important for unique function of BECs.

\subsection{MOLECULAR COMPONENTS OF THE BARRIER}

Many groups have used these data sets to identify individual genes that are important for BBB function. Lipolysis stimulated receptor (Lsr) was identified as enriched in BECs compared to peripheral ECs (Daneman et al., 2010a), has recently been shown to recruit tricellulin/Marveld2 to tricellular tight junctions for the formation of epithelial barriers (Masuda et al., 2011), and could potentially have a similar function at the BBB. Like Lsr, Marveld2 was identified as enriched at the $\mathrm{BBB}$ along with other tight junction components such as Jam4, Mpp7 and Cgnl1 (Daneman et al., 2010a), suggesting that these may also be important for formation of BBB tight junctions. Interestingly, the genomic data sets have not added much clarity as to which claudins may be important for BBB function. While it is well established that claudin 5 is most critical in transmembrane adhesion for BEC tight junctions (Nitta et al., 2003), various studies have also implicated claudin 1, claudin 3, claudin 12 and other claudins as expressed by BECs (Liebner et al., 2000; Nitta et al., 2003; Wolburg et al., 2003; Krause et al., 2008; Pfeiffer et al., 2011). Several different genomic data sets have essentially come to different conclusions as to which claudins are expressed by BECs. Array studies on purified mouse BECs have suggested that in addition to claudin 5 , claudin 12 is the other claudin expressed by BECs (Daneman et al., 2010a; Tam et al., 2012), while cultured cells have identified claudin 3 as a component of BEC tight junctions (Liebner et al., 2008), and proteomics on human passaged endothelial cells have identified a wider array of claudins present at these junctions (Ohtsuki et al., 2013). These differences may result from species differences, differences in acutely isolated vs. cultured cells, or different sensitivities of analyses, and further work needs to be done to determine which are the important molecules.

\subsection{TRANSPORTERS AND DRUG DELIVERY TARGETS}

The genomic data sets have also identified a huge number of molecular transporters highly expressed and enriched in BECs compared to peripheral ECs. In addition to widely studied BBB transporters (Glut-1, Lat-1, Mct-1), a number of other transporters have been identified and include molecules that transport neurotransmitters (Slc6a6, Slc6a17), thiamine (Slc19a3), nucleotides (Slc25a33), carnitine (Slc25a20), zinc (Slc20a1, Slc39a10) and riboflavin (Slc52a2/Gpr172b) (Lyck et al., 2009; Daneman et al., 2010a). Determining the expression and function of this wide array of transporters may be important to understand the nutrient requirements of the CNS. A recent study by Ben-Zvi et al. (2014) selectively profiled E15.5 embryos for transcripts enriched at the BBB vs. lung endothelium and identified Mfsd2a, a lipid transporter required for docosahexaenoic acid transport (Nguyen et al., 2014), further confirming its high expression levels. Analyzing the expression of transporters and signaling components may also identify novel molecules to target for drug delivery to the CNS. The most commonly utilized target for BBB-specific receptor-mediated drug delivery to the CNS is transferrin receptor, which has been identified as highly expressed and enriched in BECs by a number of data sets (Li et al., 2001; Enerson and Drewes, 2006), suggesting that other targets may also be identified. Interestingly, a peptide targeting Lrp1 has been shown to target molecules across the BBB, however, several genomic studies identified Lrp8, and not Lrp1, as highly expressed and enriched in BECs (Daneman et al., 2010a; Tam et al., 2012). For a detailed review on the expression of transporters at the blood-brain and blood-CSF interfaces in the developing and adult brain, see Saunders et al. (2013).

\section{EMERGING FRONTIERS 5.1. PROTEOMICS}

One major criticism of transcriptomic approaches is that transcript abundance does not necessarily correlate with protein levels and actual functional activity. To that end, proteomic approaches 
may nicely complement gene expression studies. For a detailed review of proteomic studies which describe how various cell culturing conditions can significantly affect protein profiles and also how protein expression is explored using in vivo models of ischemia, stroke, inflammation, and disease, see Pottiez et al. (2009). Additionally, global genomic approaches are often followed with validation by qPCR assays and new developments in microfluidic devices have enabled higher throughput of expression validation studies from the same profiled samples, as well as the inclusion of a multitude of other samples, with or without experimental treatments, or from disease models and patients.

However, a major consideration of current proteomics BBB data is again the purity of the cell population being queried. Many BBB proteomics data are largely obtained from brain microvessel preparations consisting of additional pericytic or astrocytic connections that are in direct contact with BECs which are known to contain concentrated levels of cell-type specific receptors or channel proteins (see Table 2). Detection of known proteins such as those expressed on astrocytic endfeet (Chun et al., 2011), can be easily separated, however, lesser known hits may not be readily segregated by cell type or fall within a general category. A recent effort to profile one specific branch of CNS vasculature, the arteries comprising the Circle of Willis, generated an extensive data set of 6630 proteins, of which a third are specific to the cerebral arterial landscape (Badhwar et al., 2014). Interestingly, because isolation of the sampled tissue was via manual dissection and performed under light microscopy, whole units of arterial mediators such as neuronal regulators of vasoactivity, presumably from nerve terminals, as well as regulators of smooth muscle relaxation and contractility were identified. The cerebral artery-specific proteome was compared to the mouse microvessel proteome which revealed that intracortical microvessels had more BBB-unique protein numbers and that Circle of Willis arteries were less developed and specialized compared to microvessels (Badhwar et al., 2014). Additional studies have also compared human brain microvessel proteomic signatures with mouse (Uchida et al., 2011).

Investigators have tried to circumvent these issues by coupling gene expression with proteomics in analyses of human brain vasculature isolated from resected glioma or epileptic lesions (Dauchy et al., 2008; Shawahna et al., 2011). Furthermore, a proteomics report of young and aged primate brain microvasculature provides a highly quantitative snapshot of membrane proteins expressed at the BBB at two stages of development (Ito et al., 2011). The data were compared to mouse BBB proteomic findings, which revealed significant differences. These comparisons may provide a cautionary note when translating findings between rodents and primates, likely impacting nervous system drug development studies focused on using mouse BBB models to understand transporter and receptor expression levels (Kamiie et al., 2008; Ito et al., 2011).

Overall, proteomic studies have been incredibly useful in identifying novel molecules. In particular, proteomic analysis of lipid rafts isolated from cultured cells has identified leukocyte adhesion molecules, ninjurin, Alcam, and Mcam, that are critical for trafficking of specific subsets of immune cells into the CNS during neuroinflammatory disease (Wosik et al., 2007; Cayrol et al.,

Table 2 | Recent proteomics data sets for the BBB.

\begin{tabular}{|c|c|c|c|c|c|}
\hline Method of BBB isolation & Relevant study details & Species & $\begin{array}{l}{ }^{*} \text { Dev } \\
\text { study }\end{array}$ & $\begin{array}{l}\text { Other } \\
\text { tissues }\end{array}$ & Disease \\
\hline \multicolumn{6}{|l|}{ LCM } \\
\hline Lu et al., 2008 & Microvessels from CD31+ immunostained brain & Mouse & & & \\
\hline Haqqani et al., 2005 & In vivo ischemia & Rat & & & + \\
\hline \multicolumn{6}{|c|}{ FILTRATION/CENTRIFUGATION } \\
\hline Badhwar et al., 2014 & Surgical dissection of circle of Willis brain artery & Mouse & & & \\
\hline Ouyang et al., 2014 & Mouse model of diet-induced obesity and control & Mouse & & & + \\
\hline Searcy et al., 2014 & Effect of aging on BMVs from wild type and Tg-SwDI mice & Mouse & + & & + \\
\hline Agarwal et al., 2012 & BMVs from wild type and P-gp/Bcrp knockout & Mouse & & & \\
\hline Chun et al., 2011 & Membrane fraction of BMVs & Mouse & & & \\
\hline Ito et al., 2011 & BMVs from neonate, child, and adult & Cyno monkey & + & & \\
\hline Shawahna et al., 2011 & BMVs from epilepsy patients and glioma patients & Human & & & + \\
\hline Uchida et al., 2011 & Human and mouse BMVs & Human and mouse & & & \\
\hline Kamiie et al., 2008 & Membrane fraction of microvessels from brain, liver and kidney & Mouse & & + & \\
\hline \multicolumn{6}{|l|}{ CELL CULTURE } \\
\hline Ohtsuki et al., 2013 & hCMEC/D3 compared to isolated human BMVs and HUVECs & Human & & + & \\
\hline Pottiez et al., 2010 & BCECs co-cultured with glial cells & Cow & & & \\
\hline Pottiez et al., 2009 & BMECs cultured with and without astrocytes & Cow & & & \\
\hline Lu et al., 2007 & MECs from brain and heart & Rat & & + & \\
\hline Haqqani et al., 2007 & Immortalized BECs and in vitro ischemia & Rat & & & + \\
\hline
\end{tabular}

*Developmental stages. BCEC, brain capillary endothelial cell; BEC, brain endothelial cell; BMEC, brain microvessel endothelial cell; BMV, brain microvessel; hCMEC/D3, human cerebral microvascular endothelial cell line; HUVEC, human umbilical vein endothelial cell. 
2008; Ifergan et al., 2011). This suggests that the cultured cells may provide a model for "activated" endothelial cells and thus a good model to study their interaction with immune cells that might occur during neurological disease. In addition to these established functions of the BBB, these data sets may identify novel and unknown genes that could point to novel functions of the BBB. Although a daunting task, examining the function and regulation of each of these genes and pathways should provide incredible insight into how our brain interacts with the humoral space.

\subsection{MicroRNA}

MicroRNA (miRNA) arrays have also been used to identify and understand their role in regulating gene expression levels at the BBB in disease. Using hCMEC/D3 cells treated with TNF $\alpha$ and IFN $\gamma$, Reijerkerk et al. (2013) set out to profile the microRNAs that modulate BBB function under inflammatory conditions. With a panel of miRNAs identified to have differential expression induced by inflammatory mediators that also displayed opposing changes after barrier induction in the cell line, the authors further examined their expression in brain capillaries isolated via filtration from resected multiple sclerosis patient lesions. These samples showed decreased expression of miR-125a-5p (Reijerkerk et al., 2013). In another study using a miRNA array of 318 candidates, mouse brain endothelioma line bEND3 cells were profiled for altered miRNA expression in response to lupus serum or C5a (Eadon et al., 2014). Studies similar to these, for example evaluating freshly isolated BECs for miRNA expression at different developmental times or in diverse disease models, will further expand our knowledge of how gene expression is regulated at the $\mathrm{BBB}$.

\subsection{RNA-SEQUENCING}

RNA sequencing is a high-throughput, quantitative transcriptomic approach that is rapidly gaining popularity (Lister et al., 2008; Mortazavi et al., 2008; Nagalakshmi et al., 2008; Wilhelm et al., 2008), especially as more genome annotations are completed for more and more species. RNA-seq data also allows for the relatively unbiased discovery of alternative splicing within transcripts. Where the bulk of our transcriptome based knowledge has been driven by methods that illuminate overall gene expression (including microarray and SAGE), we have until recently, been lacking much of the important resolution afforded by identification of alternative splicing events. Many of the genes encoding proteins that form tight junctions and $\mathrm{ABC}$ transporters, for example, have distinct isoforms resulting from alternative splicing events. Future additional RNA-seq experiments with $\mathrm{BBB}$ data will provide a foundation for our future understanding of the relevance of such splicing behavior with respect to barrier development, maintenance, and dysfunction.

The high-throughput sequencing of transcriptomes afforded by RNA-seq technologies also facilitates the study of RNA editing. The process of RNA editing, which is most prevalent in the brain, is a post-transcriptional modification that can influence splicing patterns, coding sequence, and a host of other gene regulatory functions. Indeed, some of the best-studied RNA-editing events that result in altered protein isoforms occur within neuronal glutamate receptors. Future RNA-seq experiments will be needed to explore the role of RNA-editing within brain endothelial cells specifically.

Currently, BBB RNA-seq data is limited to a single dataset from mouse (at the time this review was submitted), which is already providing an extensive view of the expression landscape of not only BECs, but also neurons, astrocytes, oligodendrocytes, and microglia (Zhang et al., 2014). The authors of this study primarily used FACs and immunopanning methods to purify specific cell types, thus minimizing cross contamination and enabling greater quantitative comparisons. In addition, this valuable dataset has been made available to the public via a searchable website which includes differential splicing data (Zhang et al., 2014).

To supplement the paucity of RNA-seq data derived from the $\mathrm{BBB}$, a recent developmental study using RNA sequencing to understand brain-CSF barrier formation assembled a comprehensive list of transcripts differentially enriched between E15 embryo and P42 adult rat lateral ventricle choroid plexus (Liddelow et al., 2013). This served as a quantitative approach to update a prior microarray study (Liddelow et al., 2012). The authors performed immunohistochemistry to demonstrate embryonic and choroid plexus epithelium-specific expression of genes they found enriched via RNA-seq (Liddelow et al., 2013).

The RNA-seq approach has also been used to profile expression signatures of single cells. In a study where cultured cortical neuron transcriptomes of single cells were compared to individual layer 5 pyramidal neurons in situ, the authors found that gene expression levels correlated better amongst single cultured neurons compared to the single neurons sampled in situ (Qiu et al., 2012). This in vivo heterogeneity may also apply to cells of the BBB and thus future gene expression studies sampling single BECs or capillary fragments from distinct disease regions or developmental stages of individual vascular branches may reveal further complexity in expression of specific transporters or receptors.

\subsection{FINAL REMARKS}

Advancements in techniques for the isolation and purification of BECs and in the technologies to study the resulting transcriptomes and proteomes make for an exciting and promising future for the BBB research community. Transcriptome wide data for human diseases associated with BBB dysfunction, and corresponding animal models are under-represented in the current selection of BBB data sets. Future studies may also identify sexual dimorphisms to the $\mathrm{BBB}$, how the $\mathrm{BBB}$ changes in response to neuronal activity, during normal aging, as well as following intake of different drugs. Furthermore, understanding heterogeneity of the BBB between different segments of the vascular tree as well as between different regions of the brain may be important in our understanding of how the BBB interacts with neural cells to regulate brain function and behavior. Filling these gaps with data that are as close to the in vivo setting as possible, and utilizing techniques such as FACS or LCM, will be of great benefit. Finally, the continued commitment to share newly generated data sets in publicly accessible repositories will undoubtedly propel forward our understanding of BBB biology at an unprecedented pace. 


\section{ACKNOWLEDGMENTS}

We sincerely thank Allison Bruce for her graphics, Sabya Biswas for LCM imaging, and all the investigators who have shared their data sets publicly for the benefit of the research community.

\section{REFERENCES}

Abbott, N. J. (2005). Dynamics of CNS barriers: evolution, differentiation, and modulation. Cell Mol. Neurobiol. 25, 5-23. doi: 10.1007/s10571-004-1374-y

Adams, R. H., and Alitalo, K. (2007). Molecular regulation of angiogenesis and lymphangiogenesis. Nat. Rev. Mol. Cell Biol. 8, 464-478. doi: 10.1038/nrm2183

Agarwal, S., Uchida, Y., Mittapalli, R. K., Sane, R., Terasaki, T., and Elmquist, W. F. (2012). Quantitative proteomics of transporter expression in brain capillary endothelial cells isolated from Pglycoprotein (P-gp), breast cancer resistance protein (Bcrp), and P-gp/Bcrp knockout mice. Drug Metab. Dispos. 40, 1164-1169. doi: 10.1124/dmd.112.044719

Armulik, A., Genove, G., and Betsholtz, C. (2011). Pericytes: developmental, physiological, and pathological perspectives, problems, and promises. Dev. Cell. 21, 193-215. doi: 10.1016/j.devcel.2011.07.001

Armulik, A., Genove, G., Mae, M., Nisancioglu, M. H., Wallgard, E., Niaudet, C., et al. (2010). Pericytes regulate the blood-brain barrier. Nature 468, 557-561. doi: $10.1038 /$ nature09522

Badhwar, A., Stanimirovic, D. B., Hamel, E., and Haqqani, A. S. (2014). The proteome of mouse cerebral arteries. J. Cereb. Blood Flow. Metab. 34, 1033-1046. doi: $10.1038 /$ jcbfm.2014.52

Ball, H. J., McParland, B., Driussi, C., and Hunt, N. H. (2002). Isolating vessels from the mouse brain for gene expression analysis using laser capture microdissection. Brain Res. Brain Res. Protoc. 9, 206-213. doi: 10.1016/S1385299X(02)00147-2

Banerjee, S., Sousa, A. D., and Bhat, M. A. (2006). Organization and function of septate junctions, an evolutionary perspective. Cell Biochem. Biophys. 46, 65-77. doi: 10.1385/CBB:46:1:65

Barbier, M., Faille, D., Loriod, B., Textoris, J., Camus, C., Puthier, D., et al. (2011). Platelets alter gene expression profile in human brain endothelial cells in an in vitro model of cerebral malaria. PLoS One 6:e19651. doi: 10.1371/journal.pone.0019651

Ben-Zvi, A., Lacoste, B., Kur, E., Andreone, B. J., Mayshar, Y., Yan, H., et al. (2014). Mfsd2a is critical for the formation and function of the blood-brain barrier. Nature 509, 507-511. doi: 10.1038/nature13324

Betz, A. L., Csejtey, J., and Goldstein, G. W. (1979). Hexose transport and phosphorylation by capillaries isolated from rat brain. Am. J. Physiol. 236, c96-c102.

Blalock, E. M., Buechel, H. M., Popovic, J., Geddes, J. W., and Landfield, P. W. (2011). Microarray analyses of laser-captured hippocampus reveal distinct gray and white matter signatures associated with incipient Alzheimer's disease. J. Chem. Neuroanat. 42, 118-126. doi: 10.1016/j.jchemneu.2011. 06.007

Blalock, E. M., Geddes, J. W., Chen, K. C., Porter, N. M., Markesbery, W. R., and Landfield, P. W. (2004). Incipient Alzheimer's disease: microarray correlation analyses reveal major transcriptional and tumor suppressor responses. Proc. Natl. Acad. Sci. U.S.A. 101, 2173-2178. doi: 10.1073/pnas.0308512100

Boado, R. J., and Pardridge, M. M. (1991). A one-step procedure for isolation of poly $(A)+$ mRNA from isolated brain capillaries and endothelial cells in culture. J. Neurochem. 57, 2136-2139. doi: 10.1111/j.1471-4159.1991.tb06433.x

Bowman, P. D., Betz, A. L., Ar, D., Wolinsky, J. S., Penney, J. B., Shivers, R. R., et al. (1981). Primary culture of capillary endothelium from rat brain. In Vitro 17, 353-362. doi: 10.1007/BF02618147

Bowyer, J. F., Patterson, T. A., Saini, U. T., Hanig, J. P., Thomas, M., Camacho, L., et al. (2013). Comparison of the global gene expression of choroid plexus and meninges and associated vasculature under control conditions and after pronounced hyperthermia or amphetamine toxicity. BMC Genom. 14, 147-2164. doi: 10.1186/1471-2164-14-147

Calabria, A. R., and Shusta, E. V. (2008). A genomic comparison of in vivo and in vitro brain microvascular endothelial cells. J. Cereb. Blood Flow Metab. 28, 135-148. doi: 10.1038/sj.jcbfm.9600518

Cayrol, R., Wosik, K., Berard, J. L., Dodelet-Devillers, A., Ifergan, I., Kebir, H., et al. (2008). Activated leukocyte cell adhesion molecule promotes leukocyte trafficking into the central nervous system. Nat. Immunol. 9, 137-145. doi: $10.1038 /$ ni1551
Chun, H. B., Scott, M., Niessen, S., Hoover, H., Baird, A., Yates, J., et al. (2011). The proteome of mouse brain microvessel membranes and basal lamina. J. Cereb. Blood Flow Metab. 31, 2267-2281. doi: 10.1038/jcbfm.2011.104

Cunnea, P., McMahon, J., O’Connell, E., Mashayekhi, K., Fitzgerald, U., and McQuaid, S. (2010). Gene expression analysis of the microvascular compartment in multiple sclerosis using laser microdissected blood vessels. Acta Neuropathol. 119, 601-615. doi: 10.1007/s00401-009-0618-9

Daneman, R., Agalliu, D., Zhou, L., Kuhnert, F., Kuo, C. J., and Barres, B. A. (2009). Wnt/beta-catenin signaling is required for CNS, but not non-CNS, angiogenesis. Proc. Natl. Acad. Sci. U.S. A. 106, 641-646. doi: 10.1073/pnas.0805165106

Daneman, R., Zhou, L., Agalliu, D., Cahoy, J. D., Kaushal, A., and Barres, B. A. (2010a). The mouse blood-brain barrier transcriptome: a new resource for understanding the development and function of brain endothelial cells. PLoS ONE 5:e13741. doi: 10.1371/journal.pone.0013741

Daneman, R., Zhou, L., Kebede, A. A., and Barres, B. A. (2010b). Pericytes are required for blood-brain barrier integrity during embryogenesis. Nature 468, 562-566. doi: 10.1038/nature09513

Dauchy, S., Dutheil, F., Weaver, R. J., Chassoux, F., Daumas-Duport, C., Couraud, P. O., et al. (2008). ABC transporters, cytochromes P450 and their main transcription factors: expression at the human blood-brain barrier. J. Neurochem. 107, 1518-1528. doi: 10.1111/j.1471-4159.2008.05720.x

Dickey, C. A., Loring, J. F., Montgomery, J., Gordon, M. N., Eastman, P. S., and Morgan, D. (2003). Selectively reduced expression of synaptic plasticity-related genes in amyloid precursor protein + presenilin-1 transgenic mice. J. Neurosci. 23, 5219-5226.

Dieterich, L. C., Mellberg, S., Langenkamp, E., Zhang, L., Zieba, A., Salomaki, H., et al. (2012). Transcriptional profiling of human glioblastoma vessels indicates a key role of VEGF-A and TGFbeta2 in vascular abnormalization. J. Pathol. 228, 378-390. doi: 10.1002/path.4072

Dorr, A., Sled, J. G., and Kabani, N. (2007). Three-dimensional cerebral vasculature of the CBA mouse brain: a magnetic resonance imaging and micro computed tomography study. Neuroimage 35, 1409-1423. doi: 10.1016/j.neuroimage.2006.12.040

Dunckley, T., Beach, T. G., Ramsey, K. E., Grover, A., Mastroeni, D. Walker, D. G., et al. (2006). Gene expression correlates of neurofibrillary tangles in Alzheimer's disease. Neurobiol. Aging. 27, 1359-1371. doi: 10.1016/j.neurobiolaging.2005.08.013

Eadon, M. T., Jacob, A., Cunningham, P. N., Quigg, R. J., Garcia, J. G., and Alexander, J. J. (2014). Transcriptional profiling reveals that C5a alters miRNA in brain endothelial cells. Immunology. 143, 363-373. doi: 10.1111/imm.12314

Enerson, B. E., and Drewes, L. R. (2006). The rat blood-brain barrier transcriptome. J. Cereb. Blood Flow Metab. 26, 959-973. doi: 10.1038/sj.jcbfm.9600249

Engelhardt, B., and Liebner, S. (2014). Novel insights into the development and maintenance of the blood-brain barrier. Cell Tissue Res. 355, 687-699. doi: 10.1007/s00441-014-1811-2

Falcon, S., and Gentleman, R. (2007). Using GOstats to test gene lists for GO term association. Bioinformatics 23, 257-258. doi: 10.1093/bioinformatics/btl567

Franzen, B., Duvefelt, K., Jonsson, C., Engelhardt, B., Ottervald, J., Wickman, M., et al. (2003). Gene and protein expression profiling of human cerebral endothelial cells activated with tumor necrosis factor-alpha. Brain Res. Mol. Brain Res. 115, 130-146. doi: 10.1016/S0169-328X(03)00185-2

Gatta, V., D'Aurora, M., Granzotto, A., Stuppia, L., and Sensi, S. L. (2014). Early and sustained altered expression of aging-related genes in young $3 \times \mathrm{Tg}-\mathrm{AD}$ mice. Cell Death Dis. 5:e1054. doi: 10.1038/cddis.2014.11

Geldenhuys, W. J., Allen, D. D., and Bloomquist, J. R. (2012). Novel models for assessing blood-brain barrier drug permeation. Expert Opin. Drug Metab Toxicol. 8, 647-653. doi: 10.1517/17425255.2012.677433

Gentleman, R. C., Carey, V. J., Bates, D. M., Bolstad, B., Dettling, M., Dudoit, S., et al. (2004). Bioconductor: open software development for computational biology and bioinformatics. Genome Biol. 5:R80. doi: 10.1186/gb-2004-5-10-r80

Giger, T., Khaitovich, P., Somel, M., Lorenc, A., Lizano, E., Harris, L. W., et al. (2010). Evolution of neuronal and endothelial transcriptomes in primates. Genome Biol. Evol. 2, 284-292. doi: 10.1093/gbe/evq018

Goetz, I. E., Warren, J., Estrada, C., Roberts, E., and Krause, D. N. (1985). Longterm serial cultivation of arterial and capillary endothelium from adult bovine brain. In Vitro Cell Dev. Biol. 21, 172-180. doi: 10.1007/BF02621355

Harris, L. W., Wayland, M., Lan, M., Ryan, M., Giger, T., Lockstone, H., et al. (2008). The cerebral microvasculature in schizophrenia: a laser capture microdissection study. PLoS ONE 3:e3964. doi: 10.1371/journal.pone.0003964 
Haqqani, A. S., Kelly, J., Baumann, E., Haseloff, R. F., Blasig, I. E., and Stanimirovic, D. B. (2007). Protein markers of ischemic insult in brain endothelial cells identified using 2D gel electrophoresis and ICAT-based quantitative proteomics. $J$. Proteome Res. 6, 226-239. doi: 10.1021/pr0603811

Haqqani, A. S., Nesic, M., Preston, E., Baumann, E., Kelly, J., and Stanimirovic, D. (2005). Characterization of vascular protein expression patterns in cerebral ischemia/reperfusion using laser capture microdissection and ICAT-nanoLCMS/MS. FASEB J. 19, 1809-1821. doi: 10.1096/fj.05-3793com

Hawkins, B. T., and Egleton, R. D. (2008). Pathophysiology of the blood-brain barrier: animal models and methods. Curr. Top Dev. Biol. 80, 277-309. doi: 10.1016/S0070-2153(07)80007-X

He, Z., Cui, L., Patterson, T. A., and Paule, M. G. (2011). Defining the phosphodiesterase superfamily members in rat brain microvessels. ACS Chem. Neurosci. 2, 600-607. doi: 10.1021/cn2000487

Himburg, H. A., Muramoto, G. G., Daher, P., Meadows, S. K., Russell, J. L., Doan, P., et al. (2010). Pleiotrophin regulates the expansion and regeneration of hematopoietic stem cells. Nat. Med. 16, 475-482. doi: 10.1038/nm.2119

Hudson, L. C., Bragg, D. C., Tompkins, M. B., and Meeker, R. B. (2005). Astrocytes and microglia differentially regulate trafficking of lymphocyte subsets across brain endothelial cells. Brain Res. 1058, 148-160. doi: 10.1016/j.brainres.2005.07.071

Ifergan, I., Kebir, H., Terouz, S., Alvarez, J. I., Lecuyer, M. A., Gendron, S., et al. (2011). Role of Ninjurin-1 in the migration of myeloid cells to central nervous system inflammatory lesions. Ann. Neurol. 70, 751-763. doi: 10.1002/ana. 22519

Irizarry, R. A., Hobbs, B., Collin, F., Beazer-Barclay, Y. D., Antonellis, K. J., Scherf, U., et al. (2003). Exploration, normalization, and summaries of high density oligonucleotide array probe level data. Biostatistics 4, 249-264. doi: 10.1093/biostatistics/4.2.249

Ito, K., Uchida, Y., Ohtsuki, S., Aizawa, S., Kawakami, H., Katsukura, Y., et al. (2011). Quantitative membrane protein expression at the blood-brain barrier of adult and younger cynomolgus monkeys. J. Pharm. Sci. 100, 3939-3950. doi: 10.1002/jps.22487

Kamiie, J., Ohtsuki, S., Iwase, R., Ohmine, K., Katsukura, Y., Yanai, K., et al. (2008). Quantitative atlas of membrane transporter proteins: development and application of a highly sensitive simultaneous LC/MS/MS method combined with novel in-silico peptide selection criteria. Pharm. Res. 25, 1469-1483. doi: 10.1007/s11095-008-9532-4

Kauffmann, A., Gentleman, R., and Huber, W. (2009). arrayQualityMetrics-a bioconductor package for quality assessment of microarray data. Bioinformatics 25 , 415-416. doi: 10.1093/bioinformatics/btn647

Krause, G., Winkler, L., Mueller, S. L., Haseloff, R. F., Piontek, J., and Blasig, I. E. (2008). Structure and function of claudins. Biochim. Biophys. Acta. 1778, 631-645. doi: 10.1016/j.bbamem.2007.10.018

Lazarov, O., Robinson, J., Tang, Y. P., Hairston, I. S., Korade-Mirnics, Z., Lee, V. M., et al. (2005). Environmental enrichment reduces Abeta levels and amyloid deposition in transgenic mice. Cell 120, 701-713. doi: 10.1016/j.cell.2005. 01.015

Li, J. Y., Boado, R. J., and Pardridge, W. M. (2001). Blood-brain barrier genomics. J. Cereb. Blood Flow Metab. 21, 61-68. doi: 10.1097/00004647-200101000-00008

Li, K., Dong, D., Yao, L., Dai, D., Gu, X., and Guo, L. (2008). Identification of STC1 as an beta-amyloid activated gene in human brain microvascular endothelial cells using cDNA microarray. Biochem. Biophys. Res. Commun. 376, 399-403. doi: 10.1016/j.bbrc.2008.08.158

Liddelow, S. A., Dziegielewska, K. M., Ek, C. J., Habgood, M. D., Bauer, H., Bauer, H. C., et al. (2013). Mechanisms that determine the internal environment of the developing brain: a transcriptomic, functional and ultrastructural approach. PLoS ONE 8:e65629. doi: 10.1371/journal.pone.0065629

Liddelow, S. A., Temple, S., Mollgard, K., Gehwolf, R., Wagner, A., Bauer, H., et al. (2012). Molecular characterisation of transport mechanisms at the developing mouse blood-CSF interface: a transcriptome approach. PLoS ONE 7:e33554. doi: 10.1371/journal.pone.0033554

Liebner, S., Corada, M., Bangsow, T., Babbage, J., Taddei, A., Czupalla, C. J., et al. (2008). Wnt/beta-catenin signaling controls development of the blood-brain barrier. J. Cell Biol. 183, 409-417. doi: 10.1083/jcb.200806024

Liebner, S., Fischmann, A., Rascher, G., Duffner, F., Grote, E. H., Kalbacher, H., et al. (2000). Claudin-1 and claudin-5 expression and tight junction morphology are altered in blood vessels of human glioblastoma multiforme. Acta Neuropathol. 100, 323-331. doi: 10.1007/s004010000180
Lindahl, P., Johansson, B. R., Leveen, P., and Betsholtz, C. (1997). Pericyte loss and microaneurysm formation in PDGF-B-deficient mice. Science 277, 242-245. doi: 10.1126/science.277.5323.242

Lister, R., O’Malley, R. C., Tonti-Filippini, J., Gregory, B. D., Berry, C. C., Millar, A. H., et al. (2008). Highly integrated single-base resolution maps of the epigenome in Arabidopsis. Cell 133, 523-536. doi: 10.1016/j.cell.2008.03.029

Lopez-Ramirez, M. A., Male, D. K., Wang, C., Sharrack, B., Wu, D., and Romero, I. A. (2013). Cytokine-induced changes in the gene expression profile of a human cerebral microvascular endothelial cell-line, hCMEC/D3. Fluids Barriers CNS 10, 27-8118. doi: 10.1186/2045-8118-10-27

Lyck, R., Ruderisch, N., Moll, A. G., Steiner, O., Cohen, C. D., Engelhardt, B., et al. (2009). Culture-induced changes in blood-brain barrier transcriptome: implications for amino-acid transporters in vivo. J. Cereb. Blood Flow Metab. 29, 1491-1502. doi: 10.1038/jcbfm.2009.72

Lu, L., Yang, P.-Y., Rui, Y.-Ch., Kang, H., Zhang, J., Zhang, J.-P., et al. (2007). Comparative proteome analysis of rat brain and coronary microvascular endothelial cells. Physiol. Res. 56, 159-168.

Lu, Q., Murugesan, N., Macdonald, J. A., Wu, S. L., Pachter, J. S., and Hancock, W. S. (2008). Analysis of mouse brain microvascular endothelium using immuno-laser capture microdissection coupled to a hybrid linear ion trap with Fourier transform-mass spectrometry proteomics platform. Electrophoresis 29, 2689-2695. doi: 10.1002/elps.200700936

Macdonald, J. A., Murugesan, N., and Pachter, J. S. (2008). Validation of immunolaser capture microdissection coupled with quantitative RT-PCR to probe blood-brain barrier gene expression in situ. J. Neurosci. Methods. 174, 219-226. doi: 10.1016/j.jneumeth.2008.07.009

Macdonald, J. A., Murugesan, N., and Pachter, J. S. (2010). Endothelial cell heterogeneity of blood-brain barrier gene expression along the cerebral microvasculature. J. Neurosci. Res. 88, 1457-1474. doi: 10.1002/jnr.22316

Marques, F., Sousa, J. C., Sousa, N., and Palha, J. A. (2013). Blood-brain-barriers in aging and in Alzheimer's disease. Mol. Neurodegener. 8, 38-1326. doi: 10.1186/1750-1326-8-38

Marroni, M., Kight, K. M., Hossain, M., Cucullo, L., Desai, S. Y., and Janigro, D. (2003). Dynamic in vitro model of the blood-brain barrier. Gene profiling using cDNA microarray analysis. Methods Mol. Med. 89, 419-434. doi: 10.1385/1-59259-419-0:419

Masuda, S., Oda, Y., Sasaki, H., Ikenouchi, J., Higashi, T., Akashi, M., et al. (2011). LSR defines cell corners for tricellular tight junction formation in epithelial cells. J. Cell Sci. 124, 548-555. doi: 10.1242/jcs.072058

Mayer, F., Mayer, N., Chinn, L., Pinsonneault, R. L., Kroetz, D., and Bainton, R. J. (2009). Evolutionary conservation of vertebrate blood-brain barrier chemoprotective mechanisms in Drosophila. J. Neurosci. 29, 3538-3550. doi 10.1523/JNEUROSCI.5564-08.2009

McCandless, E. E., Budde, M., Lees, J. R., Dorsey, D., Lyng, E., and Klein, R. S. (2009). IL-1R signaling within the central nervous system regulates CXCL12 expression at the blood-brain barrier and disease severity during experimental autoimmune encephalomyelitis. J. Immunol. 183, 613-620. doi: 10.4049/jimmunol.0802258

McCandless, E. E., Wang, Q., Woerner, B. M., Harper, J. M., and Klein, R. S. (2006). CXCL12 limits inflammation by localizing mononuclear infiltrates to the perivascular space during experimental autoimmune encephalomyelitis. $J$. Immunol. 177, 8053-8064. doi: 10.4049/jimmunol.177.11.8053

Mojsilovic-Petrovic, J., Nesic, M., Pen, A., Zhang, W., and Stanimirovic, D. (2004). Development of rapid staining protocols for laser-capture microdissection of brain vessels from human and rat coupled to gene expression analyses. $J$. Neurosci. Methods. 133, 39-48. doi: 10.1016/j.jneumeth.2003.09.026

Mortazavi, A., Williams, B. A., McCue, K., Schaeffer, L., and Wold, B. (2008). Mapping and quantifying mammalian transcriptomes by RNA-Seq. Nat. Methods 5, 621-628. doi: 10.1038/nmeth.1226

Murugesan, N., Macdonald, J. A., Lu, Q., Wu, S. L., Hancock, W. S., and Pachter, J. S. (2011). Analysis of mouse brain microvascular endothelium using laser capture microdissection coupled with proteomics. Methods Mol. Biol. 686, 297-311. doi 10.1007/978-1-60761-938-3-14

Nagalakshmi, U., Wang, Z., Waern, K., Shou, C., Raha, D., Gerstein, M., et al. (2008). The transcriptional landscape of the yeast genome defined by RNA sequencing. Science 320, 1344-1349. doi: 10.1126/science.1158441

Navone, S. E., Marfia, G., Invernici, G., Cristini, S., Nava, S., Balbi, S., et al. (2013). Isolation and expansion of human and mouse brain microvascular endothelial cells. Nat. Protoc. 8, 1680-1693. doi: 10.1038/nprot.2013.107 
Nguyen, L. N., Ma, D., Shui, G., Wong, P., Cazenave-Gassiot, A., Zhang, X., et al. (2014). Mfsd2a is a transporter for the essential omega-3 fatty acid docosahexaenoic acid. Nature 509, 503-506. doi: 10.1038/nature13241

Nielsen, P. A., Andersson, O., Hansen, S. H., Simonsen, K. B., and Andersson, G. (2011). Models for predicting blood-brain barrier permeation. Drug Discov. Today. 16, 472-475. doi: 10.1016/j.drudis.2011.04.004

Nitta, T., Hata, M., Gotoh, S., Seo, Y., Sasaki, H., Hashimoto, N., et al. (2003). Sizeselective loosening of the blood-brain barrier in claudin-5-deficient mice. J. Cell Biol. 161, 653-660.

Obermeier, B., Daneman, R., and Ransohoff, R. M. (2013). Development, maintenance and disruption of the blood-brain barrier. Nat. Med. 19, 1584-1596. doi: 10.1038/nm.3407

Ohtsuki, S., Ikeda, C., Uchida, Y., Sakamoto, Y., Miller, F., Glacial, F., et al. (2013). Quantitative targeted absolute proteomic analysis of transporters, receptors and junction proteins for validation of human cerebral microvascular endothelial cell line hCMEC/D3 as a human blood-brain barrier model. Mol. Pharm. 10, 289-296. doi: 10.1021/mp3004308

Ouyang, S., Hsuchou, H., Kastin, A. J., Wang, Y., Yu, C., and Pan, W. (2014). Diet-induced obesity suppresses expression of many proteins at the blood-brain barrier. J. Cereb. Blood Flow Metab. 34, 43-51. doi: 10.1038/jcbfm.2013.166

Pen, A., Moreno, M. J., Martin, J., and Stanimirovic, D. B. (2007). Molecular markers of extracellular matrix remodeling in glioblastoma vessels: microarray study of laser-captured glioblastoma vessels. Glia 55, 559-572. doi: 10.1002/glia. 20481

Pfeiffer, F., Schafer, J., Lyck, R., Makrides, V., Brunner, S., Schaeren-Wiemers, N., et al. (2011). Claudin-1 induced sealing of blood-brain barrier tight junctions ameliorates chronic experimental autoimmune encephalomyelitis. Acta Neuropathol. 122, 601-614. doi: 10.1007/s00401-011-0883-2

Pottiez, G., Deracinois, B., Duban-Deweer, S., Cecchelli, R., Fenart, L., Karamanos, Y., et al. (2010). A large-scale electrophoresis- and chromatography-based determination of gene expression profiles in bovine brain capillary endothelial cells after the re-induction of blood-brain barrier properties. Proteome Sci. 8:57. doi: 10.1186/1477-5956-8-57

Pottiez, G., Flahaut, C., Cecchelli, R., and Karamanos, Y. (2009). Understanding the blood-brain barrier using gene and protein expression profiling technologies. Brain Res. Rev. 62, 83-98. doi: 10.1016/j.brainresrev.2009.09.004

Qiu, S., Luo, S., Evgrafov, O., Li, R., Schroth, G. P., Levitt, P., et al. (2012). Singleneuron RNA-Seq: technical feasibility and reproducibility. Front. Genet. 3:124. doi: 10.3389/fgene.2012.00124

Reddy, P. H., McWeeney, S., Park, B. S., Manczak, M., Gutala, R. V., Partovi, D., et al. (2004). Gene expression profiles of transcripts in amyloid precursor protein transgenic mice: up-regulation of mitochondrial metabolism and apoptotic genes is an early cellular change in Alzheimer's disease. Hum. Mol. Genet. 13, 1225-1240. doi: 10.1093/hmg/ddh140

Reese, T. S., and Karnovsky, M. J. (1967). Fine structural localization of a blood-brain barrier to exogenous peroxidase. J. Cell. Biol. 34, 207-217. doi: 10.1083/jcb.34.1.207

Reijerkerk, A., Lopez-Ramirez, M. A., van Het Hof, B., Drexhage, J. A., Kamphuis, W. W., Kooij, G., et al. (2013). MicroRNAs regulate human brain endothelial cell-barrier function in inflammation: implications for multiple sclerosis. J. Neurosci. 33, 6857-6863. doi: 10.1523/JNEUROSCI.3965-12.2013

Risau, W., Hallmann, R., and Albrecht, U. (1986). Differentiation-dependent expression of proteins in brain endothelium during development of the blood-brain barrier. Dev. Biol. 117, 537-545. doi: 10.1016/0012-1606(86) 90321-0

Saunders, N. R., Daneman, R., Dziegielewska, K. M., and Liddelow, S. A. (2013). Transporters of the blood-brain and blood-CSF interfaces in development and in the adult. Mol. Aspects Med. 34, 742-752. doi: 10.1016/j.mam.2012.11.006

Schwabe, T., Bainton, R. J., Fetter, R. D., Heberlein, U., and Gaul, U. (2005). GPCR signaling is required for blood-brain barrier formation in drosophila. Cell 123, 133-144. doi: 10.1016/j.cell.2005.08.037

Searcy, J. L., Le Bihan, T., Salvadores, N., McCulloch, J., and Horsburgh, K. (2014). Impact of age on the cerebrovascular proteomes of wild-type and Tg-SwDI mice. PLoS ONE 9:e89970. doi: 10.1371/journal.pone.0089970

Shawahna, R., Uchida, Y., Decleves, X., Ohtsuki, S., Yousif, S., Dauchy, S., et al. (2011). Transcriptomic and quantitative proteomic analysis of transporters and drug metabolizing enzymes in freshly isolated human brain microvessels. Mol. Pharm. 8, 1332-1341. doi: 10.1021/mp 200129 p
Shusta, E. V. (2005). Blood-brain barrier genomics, proteomics, and new transporter discovery. NeuroRx 2, 151-161. doi: 10.1602/neurorx.2.1.151

Shusta, E. V., Boado, R. J., Mathern, G. W., and Pardridge, W. M. (2002). Vascular genomics of the human brain. J. Cereb. Blood Flow Metab. 22, 245-252. doi: 10.1097/00004647-200203000-00001

Siegenthaler, J. A., Sohet, F., and Daneman, R. (2013). 'Sealing off the CNS': cellular and molecular regulation of blood-brain barriergenesis. Curr. Opin. Neurobiol. 23, 1057-1064. doi: 10.1016/j.conb.2013.06.006

Smyth, G. K. (2004). Linear models and empirical bayes methods for assessing differential expression in microarray experiments. Stat. Appl. Genet. Mol. Biol. 3:3. doi: 10.2202/1544-6115.1027

Stenman, J. M., Rajagopal, J., Carroll, T. J., Ishibashi, M., McMahon, J., and McMahon, A. P. (2008). Canonical Wnt signaling regulates organ-specific assembly and differentiation of CNS vasculature. Science 322, 1247-1250. doi: 10.1126/science.1164594

Stork, T., Engelen, D., Krudewig, A., Silies, M., Bainton, R. J., and Klambt, C. (2008). Organization and function of the blood-brain barrier in Drosophila. J. Neurosci. 28, 587-597. doi: 10.1523/JNEUROSCI.4367-07.2008

Tam, S. J., Richmond, D. L., Kaminker, J. S., Modrusan, Z., Martin-McNulty, B., Cao, T. C., et al. (2012). Death receptors DR6 and TROY regulate brain vascular development. Dev. Cell 22, 403-417. doi: 10.1016/j.devcel.2011.11.018

Tam, S. J., and Watts, R. J. (2010). Connecting vascular and nervous system development: angiogenesis and the blood-brain barrier. Annu. Rev. Neurosci. 33, 379-408. doi: 10.1146/annurev-neuro-060909-152829

Triguero, D., Buciak, J., and Pardridge, W. M. (1990). Capillary depletion method for quantification of blood-brain barrier transport of circulating peptides and plasma proteins. J. Neurochem. 54, 1882-1888. doi: 10.1111/j.14714159.1990.tb04886.x

Uchida, Y., Ohtsuki, S., Katsukura, Y., Ikeda, C., Suzuki, T., Kamiie, J., et al. (2011). Quantitative targeted absolute proteomics of human blood-brain barrier transporters and receptors. J. Neurochem. 117, 333-345. doi: 10.1111/j.14714159.2011.07208.x

Urich, E., Lazic, S. E., Molnos, J., Wells, I., and Freskgard, P. O. (2012). Transcriptional profiling of human brain endothelial cells reveals key properties crucial for predictive in vitro blood-brain barrier models. PLOS ONE 7:e38149. doi: 10.1371/journal.pone.0038149

Vinters, H. V., Reave, S., Costello, P., Girvin, J. P., and Moore, S. A. (1987). Isolation and culture of cells derived from human cerebral microvessels. Cell Tissue Res. 249, 657-667. doi: 10.1007/BF00217338

Wang, S., Qaisar, U., Yin, X., and Grammas, P. (2012a). Gene expression profiling in Alzheimer's disease brain microvessels. J. Alzheimers Dis. 31, 193-205. doi: 10.3233/JAD-2012-120454

Wang, Y., Rattner, A., Zhou, Y., Williams, J., Smallwood, P. M., and Nathans, J. (2012b). Norrin/Frizzled4 signaling in retinal vascular development and blood brain barrier plasticity. Cell 151, 1332-1344. doi: 10.1016/j.cell.2012.10.042

Webster, J. A., Gibbs, J. R., Clarke, J., Ray, M., Zhang, W., Holmans, P., et al. (2009). Genetic control of human brain transcript expression in Alzheimer disease. Am. J. Hum. Genet. 84, 445-458. doi: 10.1016/j.ajhg.2009.03.011

Wilhelm, B. T., Marguerat, S., Watt, S., Schubert, F., Wood, V., Goodhead, I., et al. (2008). Dynamic repertoire of a eukaryotic transcriptome surveyed at singlenucleotide resolution. Nature 453, 1239-1243. doi: 10.1038/nature07002

Wolburg, H., Wolburg-Buchholz, K., Kraus, J., Rascher-Eggstein, G., Liebner, S., Hamm, S., et al. (2003). Localization of claudin-3 in tight junctions of the blood-brain barrier is selectively lost during experimental autoimmune encephalomyelitis and human glioblastoma multiforme. Acta Neuropathol. 105, 586-592. doi: 10.1007/s00401-003-0688-z

Wosik, K., Cayrol, R., Dodelet-Devillers, A., Berthelet, F., Bernard, M., Moumdjian, R., et al. (2007). Angiotensin II controls occludin function and is required for blood brain barrier maintenance: relevance to multiple sclerosis. J. Neurosci. 27, 9032-9042. doi: 10.1523/JNEUROSCI.2088-07.2007

Wu, V. M., and Beitel, G. J. (2004). A junctional problem of apical proportions: epithelial tube-size control by septate junctions in the Drosophila tracheal system. Curr. Opin. Cell Biol. 16, 493-499. doi: 10.1016/j.ceb.2004.07.008

Yousif, S., Marie-Claire, C., Roux, F., Scherrmann, J. M., and Decleves, X. (2007). Expression of drug transporters at the blood-brain barrier using an optimized isolated rat brain microvessel strategy. Brain Res. 1134, 1-11. doi: 10.1016/j.brainres.2006.11.089

Yu, Y. J., and Watts, R. J. (2013). Developing therapeutic antibodies for neurodegenerative disease. Neurotherapeutics 10, 459-472. doi: 10.1007/s13311-013-0187-4 
Zhang, Y., Chen, K., Sloan, S. A., Bennett, M. L., Scholze, A. R., O'Keeffe, S., et al. (2014). An RNA-Sequencing Transcriptome and Splicing Database of Glia, Neurons, and Vascular Cells of the Cerebral Cortex. J. Neurosci. 34, 11929-11947. doi: 10.1523/JNEUROSCI.1860-14.2014

Zhou, L., Sohet, F., and Daneman, R. (2014a). Purification and culture of central nervous system endothelial cells. Cold Spring Harb. Protoc. 2014, 44-46. doi: 10.1101/pdb.top070987

Zhou, Y., Wang, Y., Tischfield, M., Williams, J., Smallwood, P. M., Rattner, A., et al. (2014b). Canonical WNT signaling components in vascular development and barrier formation. J. Clin. Invest. 124, 3825-3846. doi: 10.1172/JCI76431

Conflict of Interest Statement: The authors declare that the research was conducted in the absence of any commercial or financial relationships that could be construed as a potential conflict of interest.
Received: 19 August 2014; paper pending published: 07 September 2014; accepted: 15 October 2014; published online: 06 November 2014.

Citation: Huntley MA, Bien-Ly N, Daneman R and Watts RJ (2014) Dissecting gene expression at the blood-brain barrier. Front. Neurosci. 8:355. doi: 10.3389/fnins. 2014.00355

This article was submitted to Neurogenomics, a section of the journal Frontiers in Neuroscience.

Copyright (c) 2014 Huntley, Bien-Ly, Daneman and Watts. This is an open-access article distributed under the terms of the Creative Commons Attribution License (CC BY). The use, distribution or reproduction in other forums is permitted, provided the original author(s) or licensor are credited and that the original publication in this journal is cited, in accordance with accepted academic practice. No use, distribution or reproduction is permitted which does not comply with these terms. 


\title{
Markers for blood-brain barrier integrity: how appropriate is Evans blue in the twenty-first century and what are the alternatives?
}

\author{
Norman R. Saunders ${ }^{1 *}$, Katarzyna M. Dziegielewska ${ }^{1}$, Kjeld Møllgård ${ }^{2}$ and \\ Mark D. Habgood ${ }^{1}$ \\ ${ }^{1}$ Laboratory of Developmental Neurobiology and Neurotrauma, Department of Pharmacology and Therapeutics, University of \\ Melbourne, Parkville, VIC, Australia, ${ }^{2}$ Department of Cellular and Molecular Medicine, University of Copenhagen, \\ Copenhagen, Denmark
}

\section{OPEN ACCESS}

Edited by:

Jessica J. Connelly,

University of Virginia, USA

Reviewed by:

Peter Brunjes,

University of Virginia, USA

William Kenkel,

Northeastern University, USA

*Correspondence:

Norman R. Saunders

n.saunders@unimelb.edu.au

Specialty section:

This article was submitted to

Neurogenomics,

a section of the journal

Frontiers in Neuroscience

Received: 21 August 2015 Accepted: 05 October 2015 Published: 29 October 2015

Citation:

Saunders NR, Dziegielewska KM, Møllgård K and Habgood MD (2015)

Markers for blood-brain barrier integrity: how appropriate is Evans blue in the twenty-first century and what are the alternatives?

Front. Neurosci. 9:385

doi: 10.3389/fnins.2015.00385
In recent years there has been a resurgence of interest in brain barriers and various roles their intrinsic mechanisms may play in neurological disorders. Such studies require suitable models and markers to demonstrate integrity and functional changes at the interfaces between blood, brain, and cerebrospinal fluid. Studies of brain barrier mechanisms and measurements of plasma volume using dyes have a long-standing history, dating back to the late nineteenth-century. Their use in blood-brain barrier studies continues in spite of their known serious limitations in in vivo applications. These were well known when first introduced, but seem to have been forgotten since. Understanding these limitations is important because Evans blue is still the most commonly used marker of brain barrier integrity and those using it seem oblivious to problems arising from its in vivo application. The introduction of HRP in the mid twentieth-century was an important advance because its reaction product can be visualized at the electron microscopical level, but it also has limitations. Advantages and disadvantages of these markers will be discussed together with a critical evaluation of alternative approaches. There is no single marker suitable for all purposes. A combination of different sized, visualizable dextrans and radiolabeled molecules currently seems to be the most appropriate approach for qualitative and quantitative assessment of barrier integrity.

Keywords: blood-brain barrier, embryo, fetus, newborn, permeability, tight junctions

\section{INTRODUCTION}

The realization that brain barriers may play a critical role in a wide range of neurological disorders prompted a renewed interest in studies of their function and integrity (Saunders et al., 2008). Such studies require suitable models and especially markers to demonstrate integrity of the interfaces between the blood, the brain and the cerebrospinal fluid, CSF. Similarly markers are also required for the study of barriers in the developing brain and in its evolution, which are the main focus of the Frontiers Topic "Ontogeny and Phylogeny of Brain Barrier Mechanisms." Dyes have a venerable history dating back to the end of the nineteenth-century, in studies of brain barrier mechanisms

Abbreviations: CNS, central nervous system; CSF, cerebrospinal fluid; HRP, horseradish peroxidase. 
in both the developing and adult brain, although many ascribe incorrectly the first use of dyes for this purpose to Ehrlich in the mid nineteenth-century or to Goldmann $(1909,1913)$, in the early twentieth century (see Saunders et al., 2014). In times when there were no alternatives it seems reasonable that dyes should have been used as markers for brain barrier integrity, similar to their use in cardiovascular studies for measurement of plasma volume (Dawson et al., 1920). Particularly in the latter field scientists were well aware of the limitations of dyes and as soon as more satisfactory alternatives became available, notably radiolabeled proteins such as albumin, dyes were rapidly abandoned. In striking contrast, the use of dyes remained widespread in blood-brain barrier field in spite of their limitations, which have been well described since the mid twentieth-century. One dye in particular, Evans blue (Figure 1) is still the most commonly used marker of brain barrier integrity (Figure 2) and its use has increased substantially in recent years (Figure 3). Limitations of Evans blue as applied to studies of brain barriers, as well as of other dyes, will be reviewed here together with their properties. Another commonly used marker for brain barrier integrity is HRP (introduced in mid twentiethcentury for electron microscopy studies by Straus (1959), Reese and Karnovsky (1967), and Brightman and Reese (1969) will also be discussed in this review.

The characteristics of an "ideal" marker for evaluating brain barrier integrity are that it should be metabolically inert, nontoxic, not bound to other molecules such as proteins in plasma or tissues, be available in a range of molecular sizes, have the ability to be visualized in the range from the naked eye to the electron

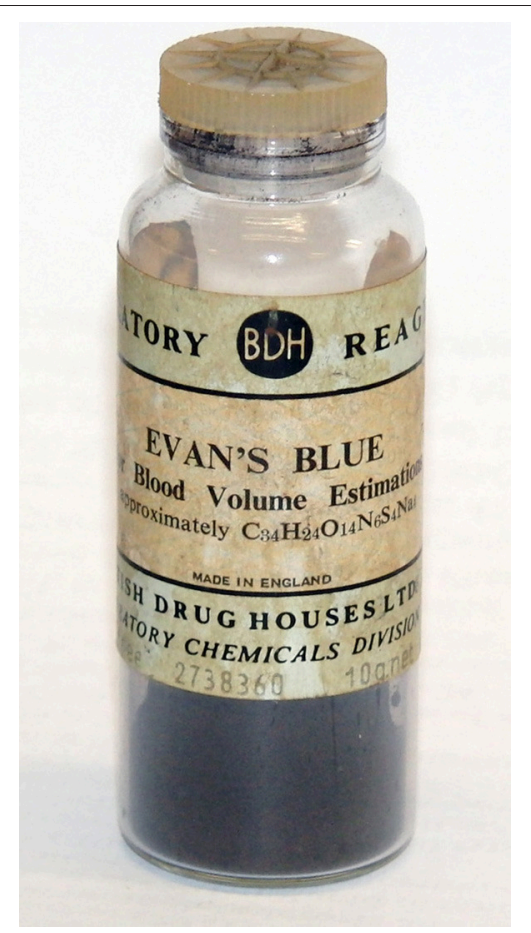

FIGURE 1 | Evans blue. As used at University College London, Department of Physiology circa 1960 for in vivo plasma volume estimation. microscopical level and be reliably quantifiable. The only merit of Evans blue dye is that is visible to the unaided eye and can thus give a gross indication of its distribution. However, it fails on all other counts. Thus, studies using only Evans blue should no longer be published, as there are much better markers available.

There is no single marker currently available that fulfills all of the specified criteria, however different molecular sized dextrans labeled with either biotin or a fluorescent tag come close. Their main limitation is probably that they are tedious to quantify; for this purpose it is better to use radiolabeled markers, which are available in a wide range of molecular sizes but have the disadvantage that currently they cannot be visualized with sufficient resolution. Thus, a combination of different dextrans and radiolabeled molecules is currently the most appropriate approach to assessing barrier integrity in the developing or pathological brain.

\section{USE OF DYES FOR ESTIMATION OF BLOOD VOLUME}

A summary of this field is included because it was the studies of the properties of dyes in circulating blood as established by cardiovascular physiologists, which provided much of the later justification for their use by blood-brain barrier biologists. The significance of this is that in the early blood-brain barrier studies it was not well appreciated that the dyes being used, such as trypan blue, bind to proteins in plasma, although there were some early hints that this might be the case (see review by Saunders et al., 2014). The earliest dye dilution estimation of plasma volume appears to be Keith et al. (1915). These authors highlighted the unsatisfactory, unreliable and potentially injurious methods previously used, which included bleeding to death (of convicts) and inhalation of, or injection of blood samples saturated with carbon monoxide. They proposed the alternative method of a non-toxic, slowly absorbable dye that remained in the circulation and suggested a colorimetric determination of its concentration in plasma by comparison with a mixture of dye and serum. On the advice of Dr. Evans in the Anatomical Department (University of California, San Francisco) Keith et al. (1915) used vital red, which Evans supplied. This resulted in several extensive published studies in dogs and humans. In later work in dogs brilliant vital red was used (Hooper et al., 1920; Smith, 1920). This dye had a number of disadvantages including difficulty in identifying when haemolysis had occurred in the blood samples. Dawson et al. (1920) investigated a series of dyes and concluded that the blue azo dye (T-1824) was slightly superior to all the vital red dyes tested. T-1824 was one of a large series of azo dyes developed in the late nineteenth early twentieth centuries by the German dye industry. T-1824 was its industrial code number. It is so named because it can be synthesized by coupling together one mole of diazotized o-toluidine and two moles of $\tau$-amino-8-naphthol-2, 4-disulfonic acid (Allen and Orahovats, 1950).

One of the co-authors of Dawson et al. (1920) was Evans. The name of the dye was much later changed from T-1824 to Evans blue in recognition of his contributions to the use of dyes in 


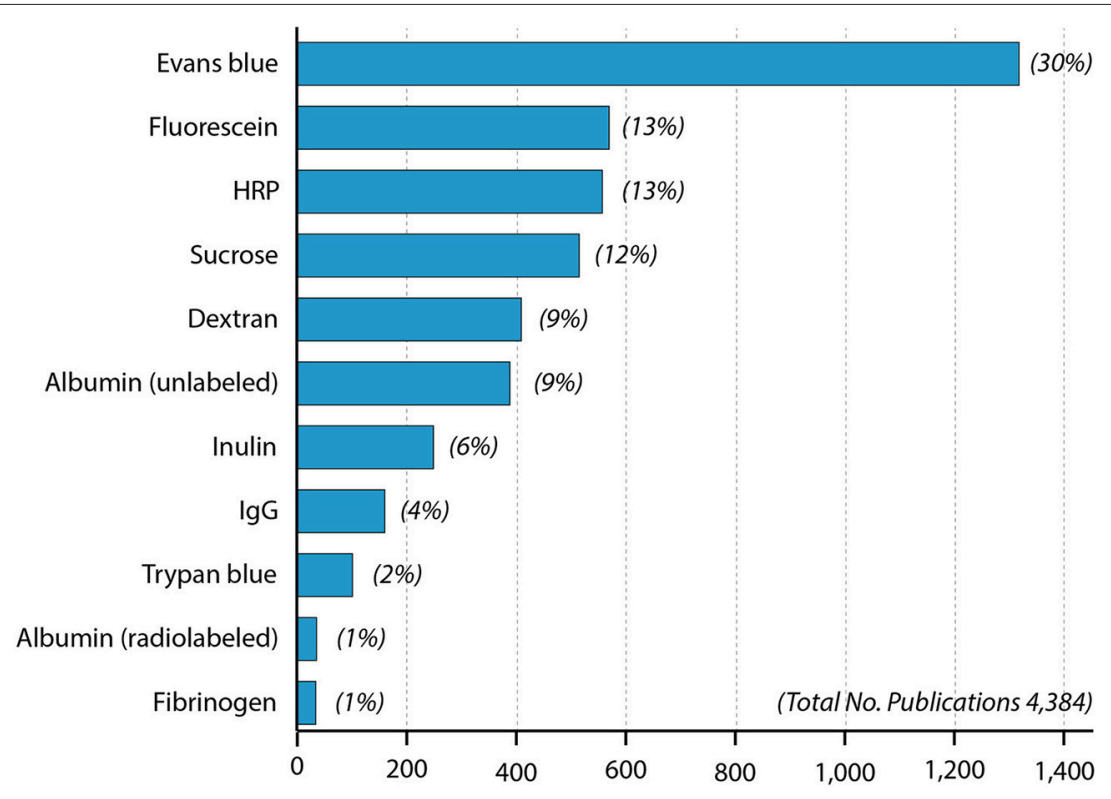

FIGURE 2 | Papers in PubMed using different blood-brain barrier markers since 1953. One curiosity is that radiolabeled albumin was used more than a decade (Ashkenazy and Crawley, 1953) before the first use of Evans blue (Rössner and Temple, 1966) but has only been infrequently used since then compared to Evans blue.

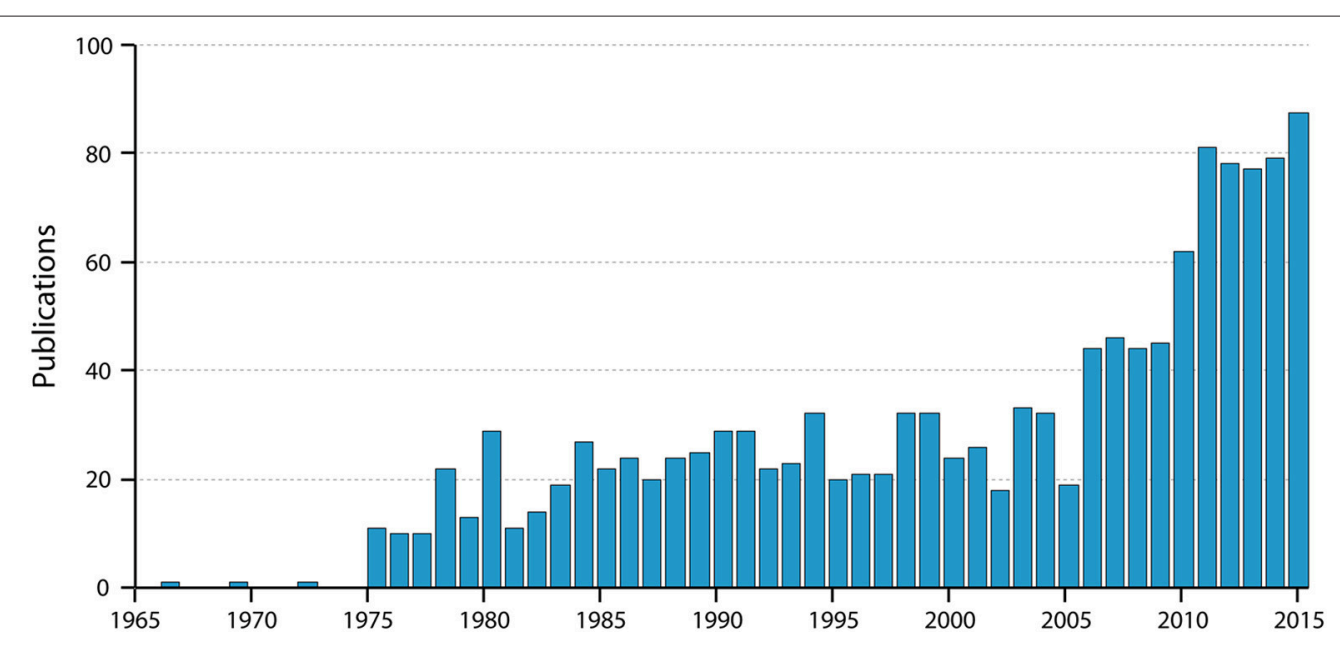

FIGURE 3 | Numbers of papers by year listed in PubMed for "blood-brain barrier Evans blue." Note the very steep increase in the past 10 years; note also that the value for 2015 is for only 9 months of the year. Thus, the use of Evans blue is still clearly increasing substantially.

animal studies; this designation has been suggested to have been introduced by the Eastman Kodak Company, which marketed the dye with this name (Cooksey, 2013). However, this name was deplored by Gregersen and Stewart (1938) and Gregersen's group persisted with the original designation of T-1824 right up to their last publication in the field (Gregersen and Rawson, 1959). In the rest of the cardiovascular field the designation T-1824 is rarely used unless in combination with Evans blue and is not used at all in the blood-brain barrier field. To avoid confusion, when discussing early literature in which only the designation T-1824 has been used, we have retained it in the form: T-1824 (Evans blue).
A key criterion for a suitable dye was that it remained in the circulation at a constant level for several minutes (optimum was considered to be $4 \mathrm{~min}$ ). Many of the dyes were found to be unsuitable because they disappeared from the blood rapidly, in some cases via the kidneys. The authors had no explanation for this difference compared to the azo dyes like T-1824 (Evans blue), which was relatively stable in the blood. It seems most likely that the excreted dyes, in contrast to Evans blue, were not bound to plasma proteins, see below. The conclusion of Dawson and his colleagues was that T-1824 (Evans blue) was marginally superior to previously used vital red dyes and others that they tested for measurement of plasma (blood) volume 
because colorimetric estimation was both easier and more accurate.

One of the first uses of T-1824 (Evans blue) in humans appears to have been by Gregersen and Stewart (1938). Prior to its introduction various red dyes or trypan blue were used. One advantage of T-1824 (Evans blue) was that much smaller concentrations could be estimated; thus some clinicians judged it to be superior to trypan blue because the large amount of that dye required for plasma volume estimations gave the patients a blue tinge, thus adding a cyanotic color to their already rather sickly appearance (Gregersen, 1938). The use of T-1824 (Evans blue) for estimation of plasma volume was extensively studied by Gregersen and colleagues over the period from 1933 to 1956. Gregersen (1938) in particular seems to have been preoccupied with the need to develop more accurate colorimetric methods than those of Keith et al. (1915) and modified by Smith (1920) and by Dawson et al. (1920). This was prompted by his initial studies undertaken at Harvard Medical School on the effects of thirst and reduced salivary flow on plasma volume in which he found these methods to give results that were of "questionable accuracy" (Gregersen, 1932). With a fortitude that would probably not be tolerated by modern grant agencies, he spent the next 5 years improving the method, with a particular focus on colorimetric methods used for measuring concentrations of vital red dyes or T-1824 in plasma. He concluded that T-1824 (Evans blue) was a substantially better dye for plasma volume measurements partly because much lower concentrations were required but also because haemolysis was much less of a problem for colorimetric measurements. Gregersen and colleagues at Columbia University published extensively on the evaluation and use of T-1824 (Evans blue) with comparisons with radioisotopic methods once these were introduced (reviewed in Gregersen and Rawson, 1959). Since that time T-1824 (Evans blue) has largely been replaced by radio-iodinated human serum albumin for indicator dilution estimations of plasma volume (Margouleff, 2013). This is in contrast to the persistence in the use of Evans blue in studies of blood-barrier barrier integrity to the present time; this is in spite of a similar availability of better methods for many years, as discussed below. One paper from the Columbia group of particular relevance for blood-brain barrier studies is that of Rawson (1943) not least because it has frequently been mis-cited. It provided a detailed analysis of the concentration dependence of binding of Evans blue to human albumin, but also provided evidence of binding to globulins. Because of the importance of this paper for blood brain barrier permeability it will be considered in some detail in a separate section on dye binding to plasma proteins, below.

\section{EVANS BLUE FOR ASSESSMENT OF BLOOD-BRAIN BARRIER INTEGRITY}

One of the earliest uses of Evans blue for blood-brain barrier experiments appears to be that of Rössner and Temple (1966); these authors cite Bauer et al. (1956) as having used Evans blue, although Bauer et al. (1956) actually used Geigy Blau, the properties of which seem to be poorly researched (Gregersen and Rawson, 1959). Rössner and Temple (1966) were primarily concerned with efforts to develop a method for measuring Evans blue in tissue for which purpose they used about 300 rats and 50 guinea pigs. They do not appear to have considered the appropriate concentration to use or in what form the dye was present, although they do appear to have been aware of at least some of the papers published by Gregersen and colleagues. They used a large amount of dye $(1 \mathrm{ml}, 2 \%$ Evans blue per $100 \mathrm{~g}$ body weight) compared to the Gregersen group (e.g., 0.005$0.01 \mathrm{ml} / 100 \mathrm{~g}$ body weight, Gregersen and Stewart, 1938). It is unclear why such a large amount of dye was used by Rössner and Temple (1966) and in most subsequent studies by others, given that an important reason for using Evans blue for blood volume studies was that a much smaller amount of dye was required (Gregersen, 1938). Possibly it followed on from the protocols used for many years from the original experiments of Goldmann (1913) in which trypan blue was used to study blood-brain barrier permeability. Goldmann (1913) injected cats intravenously with $30-50 \mathrm{ml} 1 \%$ trypan blue. In more recent times dye concentrations have usually been $2-4 \%$ but in lesser volumes. It is frequently stated in blood-brain barrier studies that Evans blue binds rapidly, tightly and exclusively to plasma albumin (Reeve, 1957; Stoelinga and van Munster, 1967; Wolman et al., 1981; Manaenko et al., 2011; Yen et al., 2013). However, this is not the case as will be considered below. The problems of using Evans blue as a marker for blood-brain barrier dysfunction can be summarized as (i) the likelihood of a substantial amount of free dye being present in an animal following the amounts injected, (ii) lack of specific binding to albumin (although this is widely believed, to the extent that many authors assume that the concentration of Evans blue in the brain is synonymous with penetration of albumin across a disrupted blood-brain barrier), (iii) injection of dye dissolved in "physiological saline" or other physiological solution, which have been suggested to affect the structure of the dye, (iv) there is evidence that Evans blue binds to tissues. (v) attempts to make quantitative assessments of damage to the blood-brain barrier in the brain are confounded by problems with the various spectroscopic methods that have been used to estimate the amount dye in brain tissue (not to mention the uncertainty about how much dye is bound to albumin and other plasma proteins, how much to brain tissue and how much may be free) (vi) in vivo potential lethal toxicity. Each of these problems is considered in some detail in the next sections of this review.

\section{(I) Binding of Evans Blue (T-1824) and other Dyes to Plasma Proteins: How Much Free Dye is there?}

Ehrlich (1885) suggested that the reason why some of the dyes, which he studied in animals, did not appear in the urine was that they might be bound to plasma albumin. This seems to have been overlooked by the blood-brain barrier field until the observations of Tschirgi (1950) on trypan blue and its exclusion from the brain. He compared the lack of staining of the brain when trypan blue was injected intravenously with the intense staining of brain that occurred when the same concentration 
$(0.2 \%)$ of the dye was injected dissolved in Tyrode solution. He suggested that the lack of staining of the brain was due to binding of the dye to plasma albumin, which he confirmed by adding bovine albumin to the Tyrode-dye solution prior to injection. The earliest suggestion that Evans blue and other related dyes may bind to proteins in the blood appears to have been made by Gregersen and Rawson (1943) and was studied in detail by Rawson (1943) and by Allen and Orahovats (1950). Rawson (1943) used the electrophoresis method of Tiselius (1937) to study the binding of T-1824 (Evans blue) and structurally related diazo dyes to proteins in human plasma and to investigate the binding capacity of human albumin for T-1824 (Evans blue), trypan blue (T-1826), Niagara sky blue 6B and Niagara sky blue. In Rawson's (1943) experiments at low concentrations $(0.004 \%$ or less) the dyes were wholly bound by albumin in either serum or plasma. But at higher concentrations this was no longer the case. Rawson (1943) estimated the binding capacity of human albumin as 8-14 moles of T-1824 (Evans blue); Rawson also found evidence for binding of the dye to globulins at higher concentrations. From their measurements of T-1824 (Evans blue) binding to albumin Allen and Orahovats (1950) showed that with an injection of $4 \mathrm{ml}$ of $0.43 \%$ of the dye in a plasma volume and albumin concentration corresponding to that in vivo there would be measureable free dye (see Table 1).

Most studies using Evans blue to assess brain barrier integrity have used $2 \%$ or $4 \%$ solutions (e.g., Petito, 1979; Wolman et al., 1981; Abraham et al., 1996; Chen et al., 1996) thus at least when first entering the bloodstream a proportion of the dye will be free. An important aspect of Rawson's (1943) study and that of Allen and Orahovats (1950), which is almost universally ignored, is that as the dye concentration was increased an increasing proportion of the dye was free (i.e., not bound to albumin).

In blood-brain barrier experiments the volume injected is usually given, but rarely the size of the animals used, so it is often not possible to calculate the likely plasma concentration of the dye. However, a few papers do give the relevant information. Kaya and Ahishali (2011) published a methodological review of

TABLE 1 | Effect of albumin (Alb) molar concentration on binding of Evans blue dye (T-1824).

\begin{tabular}{lcccc}
\hline $\begin{array}{l}\text { Albumin } \\
\text { Molar conc. }\end{array}$ & $\begin{array}{c}\text { T-1824 } \\
\text { Molar conc. }\end{array}$ & $\begin{array}{c}\text { Unbound T-1824 } \\
\text { Molar conc. }\end{array}$ & $\begin{array}{c}\text { T-1824:Alb } \\
\text { Molar ratio }\end{array}$ & $\begin{array}{c}\text { Unbound, } \\
\text { T-1824(\%) }\end{array}$ \\
\hline 1.23E-04 & 3.50E-03 & $2.13 \mathrm{E}-03$ & 28.5 & 60.9 \\
3.77E-04 & 1.76E-03 & 5.42E-06 & 4.7 & 0.31 \\
5.03E-04 & 8.80E-04 & 1.40E-06 & 1.7 & 0.16 \\
5.77E-04 & 3.52E-04 & 4.37E-07 & 0.61 & 0.12 \\
6.04E-04 & 1.76E-04 & 2.00E-07 & 0.29 & 0.11 \\
6.27E-04 & $1.76 \mathrm{E}-05$ & $1.91 \mathrm{E}-08$ & 0.03 & 0.11 \\
\hline
\end{tabular}

Data in the first three columns are from the study of Allen and Orahovats (1950) in which $4 \mathrm{ml}$ of a $0.43 \%$ solution of $T-1824$ was mixed with different volumes of a $4.4 \%$ albumin solution. At a ratio of 28.5 moles of T-1824 per mole of albumin, only around $40 \%$ of the T1824 is actually bound to albumin (i.e., each albumin molecule binds a maximum of around 10 molecules of $T$-1824). Note that at all molar ratios less than this maximum binding capacity, a small proportion of T-1824 (0.11-0.31\%) remains free in solution unbound to albumin. various blood-brain barrier integrity markers including Evans blue. In their description they indicate the use of $2 \%$ Evans blue dissolved in "physiological" saline solution administered intravenously at a dose of $4 \mathrm{ml}$ per $\mathrm{Kg}$ body weight of animal. From this information the plasma concentration can be calculated assuming that all of the injected dye mixes with plasma before any significant losses, a haematocrit of $45 \%$ and a blood volume of $8 \%$ body weight for different species. This gives a dye concentration in plasma of $1.82 \mathrm{mg} / \mathrm{ml}$ or $0.18 \%$. In rats the blood volume has been estimated as $6.44 \mathrm{ml} / 100 \mathrm{~g}$ (Lee and Blaufox, 1985) and the albumin concentration around $2250 \mathrm{mg} / 100 \mathrm{ml}$ or $22.5 \mathrm{mg} / \mathrm{ml}$ (Dziegielewska et al., 1981). Thus, in this species, the plasma dye concentration would be $2.17 \mathrm{mg} / \mathrm{ml}(0.217 \%)$ at an Evans blue: albumin molar ratio of 6.52 .

Spigelman et al. (1983) used $0.5 \mathrm{ml}$ of a $2 \%$ Evans blue simultaneously with sodium fluorescein and ${ }^{99 \mathrm{~m}} \mathrm{Tc}-\mathrm{DTPA}$ injected i.v. into 200-250 g rats; as all of these bind to plasma proteins it is likely there might have been some interference with the binding of Evans blue to albumin. However, assuming that this was not significant, the plasma concentration of Evans blue can be calculated as $1.12 \mathrm{mg} / \mathrm{ml}(0.11 \%)$ at an Evans blue: albumin molar ratio of 3.37 .

Using an in vitro test of albumin concentration on the binding of Evans blue to cellophane, Rawson (1943) estimated a maximum binding capacity of less than 14 molecules of Evans blue per molecule of albumin. The data from Allen and Orahovats (1950) investigating the effect of albumin concentration on binding of Evans blue suggests a maximum binding capacity of 10 molecules of Evans blue per molecule of albumin (see Table 1). In both the Kaya and Ahishali (2011) and Spigelman et al. (1983) studies, the plasma concentration of Evans blue would probably not have exceeded the theoretical maximum binding capacity of albumin. However, an important point to note from the Allen and Orahovats (1950) study is that even at very low Evans blue: albumin ratios $(<1.0)$ a small proportion of Evans blue always remains unbound to albumin (see Table 1).

Moos and Møllgård (1993) re-investigated the problem of free dye following intraperitoneal or subcutaneous injections of different amounts of either trypan blue or Evans blue in mice. For concentrations of Evans blue of 1 or $2 \%$ in $0.9 \%$ saline $(0.08 \mathrm{ml} / 20 \mathrm{~g}$ body weight $)$ they found spectrophotometric evidence of substantial amounts of free dye in plasma at $30 \mathrm{~min}$ and $6 \mathrm{~h}$ following injection. For example using a $2 \%$ compared to $0.5 \%$ solution of Evans blue there was about $70 \%$ more free dye at $6 \mathrm{~h}$ after injection. On examining the brains of mice injected with this higher concentration of Evans blue, the dye was detected intraneuronally at several sites in the brain. Moos and Møllgåd (1993) suggested a number of pathological mechanisms by which free dye might have entered the brain in their experiments and those of others.

Thus, it seems likely that there would be measureable amounts of free dye when concentrations of 2 or $4 \%$ were used, particularly in the first minutes after injection. That dye would be expected to rapidly enter the extracellular space of many tissues and bind to cells and matrix that have a higher affinity for Evans blue than the proteins in plasma. 


\section{(ii) Evans Blue does not Bind Exclusively to Albumin in Plasma}

It is widely claimed that Evan blue binds tightly and exclusively to plasma albumin and that its visualization and/or quantitation can be used to define increases in blood brain barrier permeability to albumin (Wolman et al., 1981; Kitagawa et al., 1991; Nagaraja et al., 2008). As evidence some authors refer to Rawson (1943) but in most cases they simply refer to an earlier paper making this claim (e.g., Kang et al., 2013 refer to Spigelman et al., 1983 who do not actually mention Evans blue binding to albumin). Consideration of the literature on use of Evans blue for plasma volume measurements shows that such a claim is not supported by the experimental evidence. There are important species differences in the specificity and tightness of the binding and of particular importance is the fact that the binding of the dye to albumin (and other proteins, including tissue proteins) is a reversible equilibrium. Most of the original studies of Evans blue as a plasma marker were carried out in humans and dogs, species where the dye binding to albumin appears to be the strongest (Alle et al., 1953; Reeve, 1957). However, even in these species there were significant discrepancies in plasma volume measurements made with Evans blue (assumed to be bound to albumin) and with $\mathrm{I}^{125}$-albumin, suggesting that not all of the injected dye became bound to protein in the blood; thus "Evans blue-albumin" was found to have a larger distribution volume than $\mathrm{I}^{125}$-albumin (Carvalho, 1989). In some species e.g., rabbits, the discrepancy was even larger (Zizza and Reeve, 1958). The most cited study of Evans blue binding to albumin is that of Rawson (1943) who examined the binding to human albumin at different dye concentrations in vitro (not in vivo as stated by some authors; e.g., Uyama et al., 1988). However, Rawson used human albumin for which the binding of Evans blue is much tighter than for many animal species including rats (Alle et al., 1953; Emmett et al., 1985); at the concentrations used the dye would have been bound to other proteins in plasma (Rawson, 1943; LeVeen and Fishman, 1947; Emmett et al., 1985). This can be deduced from studies showing that Evans blue can bind to other proteins in plasma including globulins (LeVeen and Fishman, 1947), the post-albumin fraction (Linder and Heinle, 1982) as well as $\alpha_{1}$-lipoprotein, hemopexin, and transferrin (Emmett et al., 1985). This is illustrated in Figure 4, where the authors used the elegant but little applied technique of crossed immunoeletrophoresis originally devised by Laurell (1965). The binding to plasma proteins in commonly used experimental animal species appears to be less tight than for human albumin (Alle et al., 1953; Emmett et al., 1985) and at the concentrations of the injected dye the binding capacity of albumin is likely to be exceeded, as discussed in the previous paragraph. As long ago as 1982 Linder and Heine concluded, "at the concentrations used by many investigators areas dyed by Evans blue may not be equated with the presence of diffusible protein-dye complexes." This is a good, if neglected, summary of the problem. Because there is good evidence, as outlined above that Evans blue does not bind exclusively to albumin, its detection cannot be equated with the presence of albumin, especially as albumin in many cases is much better visualized using immunohistochemistry.

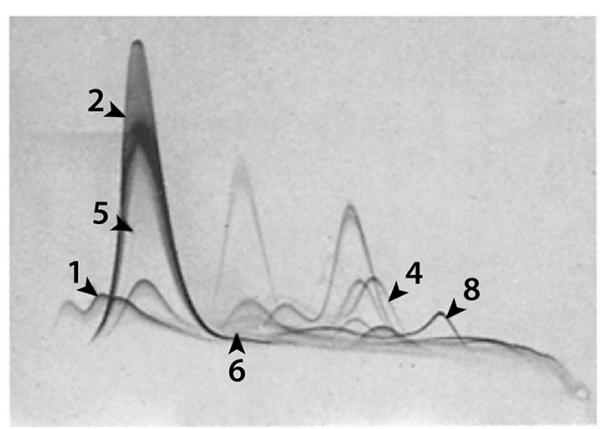

FIGURE 4 | Evans blue binding to 1, $\alpha_{1}$-lipoprotein; 2, albumin; 4, hemopexin; 5, prealbumin; 6, $\alpha_{1} X$ and 8, transferrin. Identified by anodal shifts. From Figure 3 in Emmett et al. (1985).

\section{(iii) Appropriate Carrier Solutions for Evans Blue}

According to Gregersen and Stewart (1938) Evans blue is not stable in "saline" solutions unless some protein is also present. They do not present any evidence for this, but given their extensive experience of working with the dye it seems appropriate to take note of this possible concern. Gregersen's group generally appears to have used water as the vehicle.

Some of the earlier studies, particularly those in which Evans blue was used to estimate plasma volume (e.g., Carvalho, 1989) the dye was mixed with plasma before injection. However, most if not all of the many studies of blood-brain barrier integrity published over the past 60 years appear to have used a "physiological solution" as the carrier (e.g., Ribatti et al., 1993; Kaya and Ahishali, 2011; Manaenko et al., 2011; Jiang et al., 2014) and in some there is no mention of the solution in which the Evans blue was dissolved (Kitagawa et al., 1991; Dhillon et al., 1999; Whalen et al., 1999; Stahel et al., 2000). In summary, the stability of Evans blue in many of the blood-brain barrier experiments is unclear.

\section{(iv) Tissue Binding of Albumin}

The binding of Evans blue to albumin is an equilibrium, so an additional factor to consider is the affinity of tissues with which the dye may be exchanged from that bound to albumin (LeVeen and Fishman, 1947). This has been little investigated, but it has been shown in the aorta and common carotid arteries (Linder and Heinle, 1982) and in the lungs (Dallal and Chang, 1994) that in addition to binding to albumin a significant amount of the dye is tightly bound to the tissue. The most extensive study of tissue binding of Evans blue appears to be that of Clasen et al. (1970) which will be discussed in detail in the next section because of its particular relevance to attempts to quantitate Evans blue in brain tissue, as a measure of blood-brain barrier dysfunction.

\section{(v) Quantitation of Blood-Brain Barrier Disruption using Evans Blue}

The most problematic use of Evans blue in blood-brain barrier studies is in attempts to use it to quantify the 
magnitude of a "leak" or barrier dysfunction. This is usually done by extracting Evans blue from brain tissue at various times following intravenous or intra-arterial dye injection. Different solvents have been used to extract the dye and the amount of dye in the brain extract estimated colorimetrically, spectrophotometrically, or fluoro-spectrometrically (Evans blue exhibits natural fluorescence). There are several problems in addition to the ones discussed above concerning whether Evans blue in brain is bound to plasma proteins (and which ones) or is free and the extent to which the dye may have exchanged with brain substance or has entered cells. Colorimetric and spectrophotometric measurements are heavily dependent on the standards used because Evans blue shows spectral shifts when in protein-containing solutions. This is something that exercised physiologists considered when they were developing Evans blue for measurement of blood volume (Gregersen, 1938). These problems are likely to be worse with brain extracts because of the heterogeneity of the material compared with plasma and differences between pathological and normal brain tissue if the latter is used for standard Evans blue solutions (Hellal et al., 2004). As mentioned above many authors do not mention the composition of the standard solution used for Evans blue determinations e.g., Dhillon et al. (1999), Whalen et al. (1999), and Stahel et al. (2000). Evans blue has been suggested to be unstable in "saline" or other salt solutions, unless some protein is also present, which is why earlier workers used water as the vehicle (Gregersen and Stewart, 1938).

There is the further problem that some of the Evans blue in brain samples will also be present in contaminating blood inevitably trapped in brain capillaries. Some authors have attempted to remove blood by perfusing the circulatory system prior to removing brain samples. It is unclear how successful this is and the possibility that the dye may be washed out of the brain tissue itself does not seem to have been considered.

Estimating the size of blood-brain barrier breakdown following trauma seems particularly problematic. The initial "breakdown" is probably due to rupture of blood vessels as occurs in injury to any tissue or organ in the body, rather than dysfunction due to disruption of specific brain barrier properties (Hellal et al., 2004). No one talks about, for example, bloodmuscle barrier disruption following limb trauma. There seems to be general agreement that following brain trauma there is a period of few hours when plasma proteins or other large molecules will enter the brain tissue (Habgood et al., 2007) but this probably has more to do with the time of vessel disruption and subsequent clotting of blood in the injured area of brain than actual barrier malfunction. Subsequently there is a period that may last several days when the barrier is permeable to molecules smaller than proteins (Habgood et al., 2007) as will be considered further below. The presence of clots in the vessels at the site of a brain injury makes it unlikely that a marker solution will enter the damaged region adequately. In the case of Evans blue estimates of the size of a "leak" this is likely to add to the unsatisfactory nature of attempts to quantitate the size of a barrier dysfunction using this marker.

Wang and Lai (2014) have recently published a critique of the use of Evans blue for quantitation of blood volume and blood brain barrier "leaks." They cite some of the early literature when Evans blue was being developed for blood volume measurements and claim that Evans blue is a commonly used tracer for estimation of blood volume in humans, yet the most recent paper they cite is that of Theron and Wilson (1949). As discussed above, the use of Evans blue for that purpose was replaced by better markers many decades ago. Wang and Lai (2014) assume that Evans blue and albumin can be equated, which from the extensive discussion above about its binding to other plasma proteins and tissues, is clearly not the case. They state that it is non-toxic, which as discussed in the next section is not the case either, although toxicity depends on the amount of dye and the concentrations used. They also state that Evans blue is not taken up by cells, citing Gregersen et al. (1935). However, these authors do not make such a statement. They suggest that Evans blue may be absorbed by red cells, which might mean that it is taken up but equally that it sticks to the surface of cells. More relevant is the evidence mentioned above that Evans blue sticks to tissues, some of which may have a higher affinity for the dye than albumin. Wang and Lai (2014) cite Clasen et al. (1970) in support of their assertion that "its [Evans blue] extravasation into central and peripheral organs following a more prolonged time period correlates with vascular leakage of serum albumin, and its leakage into the brain parenchyma indicates blood-brain barrier (BBB) disruption." Yet Clasen et al. (1970) were at great pains to explain and study experimentally the nature of binding of the dye to albumin and to tissues (and the equilibrium nature of this relationship) as well as citing evidence that Evans blue may be taken up by astrocytes. Clasen et al. (1970) estimate that as much as $15 \%$ of Evans blue in some tissues is free dye bound to tissue components, such as connective tissue. They state unequivocally that "..... the degree of coloration is not simply a reflection of the total albumin content of the tissue."

Thus, the published estimates of the size of blood-brain barrier impairment or "leakage" are unreliable. However, some of the concerns outlined here would apply equally to other markers, particularly in the case of traumatic damage to the blood-brain barrier.

\section{(vi) Toxicity of Evans Blue in vivo}

It seems to have been little considered that Evans blue might have toxic properties in vivo. In a brief report Gibson and Gregersen (1935) found that rats injected with $45 \mathrm{mg} / \mathrm{Kg} \mathrm{T}-1824$ (Evans blue) showed decreased rate of growth and on autopsy were found to have multiple pulmonary emboli and intracellular dye in renal epithelium and hepatic parenchyma. Malaowalla and Fong (1962) injected four monkeys with different doses of Evans blue. One receiving $25 \mathrm{mg} / \mathrm{Kg}$ survived and was apparently normal. The other three injected with 50, 100, or $200 \mathrm{mg} / \mathrm{Kg}$ died within days of injection. The only systematic study appears to be that of Hueper and Ichniowski (1944) who reported on the long term toxic effects of intravenously administration of different dose levels in dogs, cats, rabbits and rats. Aqueous solutions of $0.5 \%$ were used, which is in fact a lower concentration than generally used in blood brain barrier experiments (2\%). Rats were given $1,3,5$, or $10 \mathrm{ml} / \mathrm{Kg}$ injections of the dye. Three of ten animals in the two larger dose groups died at 1-3 months, 
with widespread pathological changes in the brain, lungs, heart and liver. Other animals were killed at 6 months; about half of those examined showed substantial degenerative lesions in the epithelium of the seminiferous tubules. This was examined in shorter-term experiments in which 25 rats were administered two injections of $1 \mathrm{ml}$ of a $1 \%$ aqueous solution of Evans blue with 3 days between injections. Fourteen rats died within 14 days, the survivors were killed and all animals examined histologically. Nearly $50 \%$ of the 25 animals showed testicular degeneration to varying degrees. Other organs were not commented upon, but it seems unlikely that the animals would have died from a sole pathology of testicular degeneration, suggesting other severe pathology in response to the Evans blue injections. Many blood-brain barrier experiments employ $4-5 \mathrm{ml} / \mathrm{Kg}$ Evans blue solutions in concentrations of $2 \%$ (e.g., Uyama et al., 1988; Dhillon et al., 1994; Whalen et al., 1999; Kaya and Ahishali, 2011; Manaenko et al., 2011) but sometimes as high as $4 \%$ (Jiang et al., 2014). The duration of the experiments is generally much less than in Hueper and Ichniowski (1944), in the range of $10 \mathrm{~min}$ to $24 \mathrm{~h}$. So it is unclear whether there would have been histologically detectable lesions within that time span. There appear to be no reports on this, apart from the observations of Moos and Møllgård (1993) from which they concluded that the Evans blue they could detect in brain a few hours after injecting $2 \%$ Evans blue into mice might be due to toxic effects on cerebral endothelial or ependymal cells; this suggests that at the most commonly used concentration of Evans blue (2\%) there may well have been toxic effects.

Thus, there is good reason to be concerned about possible toxic effects of Evans blue in short-term experiments involving the concentration of dye most commonly used (2\%).

In summary, it seems that all of the frequently asserted properties of Evans blue as an exclusive marker for plasma albumin are sufficiently suspect as to make it unsuitable for use in studies of blood-brain barrier integrity. Evans blue detected in brain is likely to be a mixture of dye bound to plasma proteins (which ones and the quantitative extent depending upon the species), dye bound to brain tissue and free dye. This point does not seem to have been specifically studied in the brain, but as mentioned above, has been examined in the lungs, following systemic injection of Evans blue dye (Dallal and Chang, 1994). There are other markers, which do not suffer from these problems. It seems baffling that Evans blue continues to be used so widely.

\section{HORSERADISH PEROXIDASE IN ASSESSMENT OF BRAIN BARRIER PERMEABILITY}

The first mention of horseradish peroxidase (HRP) exclusion from the brain after injection of a solution into the circulation appears to be that of Straus (1959) although he is rarely credited with this observation. The introduction of HRP into bloodbrain barrier studies was an important technical achievement, which led to significant advances in understanding bloodbrain barrier biology. Of particular note are the papers by
Karnovsky (1967), Reese and Karnovsky (1967), and Brightman and Reese (1969). The importance of the introduction of HRP is that the reaction product of this peroxidase can be made electron-dense, so it is possible to visualize it at the electron microscopical level. The paper of Brightman and Reese (1969) involved both intravascular and intrathecal injection experiments. This milestone paper established the ultrastructural basis for the barrier at the cerebrovascular interface between blood and brain. These detailed studies showed that the primary barrier was the intercellular tight junction between adjacent endothelial cells although an additional barrier feature was the paucity of intracellular pinocytotic vesicles in the endothelial cells. They also showed tight junctions between the epithelial cells of the choroid plexus providing a barrier to HRP at the blood-CSF interface. The experiments described in these papers (Karnovsky, 1967; Reese and Karnovsky, 1967; Brightman and Reese, 1969) were all performed in mice, with no reports of HRP toxicity.

However, when different strains of rats (and also guinea pigs) were used subsequently, some technical problems with HRP became apparent. These were well understood by the people who developed the use of HRP for permeability studies in various tissues, most notably Graham and Karnovsky (1966) and Cotran and Karnovsky $(1967,1968)$. It was demonstrated that HRP can cause degranulation of mast cells with the release of histamine and serotonin, which have been shown to affect vascular permeability (Majno et al., 1961) in some commonly used strains of rats (Cotran and Karnovsky, 1967) but not in others (Cotran et al., 1968). In early studies of barrier permeability these rat strains (e.g., Sprague-Dawley) were treated with anti-histamine and anti-serotonergic agents (Cotran and Karnovsky, 1968). However, in later studies this precaution seems to have been overlooked by some (e.g., Farrell and Shivers, 1984; Lotocki et al., 2009; Kaya and Ahishali, 2011; Cunningham et al., 2014) or only antihistamines were used (Tanno et al., 1992; Dietrich et al., 1994; Ueno et al., 2002). In other studies the problem was avoided by using Wistar rats (Ugrumov et al., 1983; Dietrich et al., 1993; Cevik et al., 2013) in which HRP has been reported not to produce mast cell degranulation (Cotran et al., 1968) although it is unclear whether Wistar rats were chosen with this problem in mind. Given these studies were aimed at evaluating blood-brain barrier permeability, in studies in which no inhibitors were used in strains of rats that are known to be sensitive to HRP, this throws the value of results from such experiments in doubt. Even in mice deleterious effects of HRP have been demonstrated, but they appear to be dose dependent and possibly also on the type of HRP used. Thus, Clementi (1970) showed that doses of $24 \mathrm{mg} / 100 \mathrm{~g}$ in Swiss albino mice affected lung capillary permeability, but not when $1 \mathrm{mg} / 100 \mathrm{~g}$ was used. Also in Wistar rats $5-10 \mathrm{mg} / 100 \mathrm{~g}$ body weight HRP (but not $1 \mathrm{mg} / 100 \mathrm{~g}$ ) resulted in profound hypotension due to histamine release which could be inhibited by pre-treatment with the antihistamine promethazine (Deimann et al., 1976). A wide range of HRP doses has been used in both rats and mice, ranging from $0.4 \mathrm{mg} / 100 \mathrm{~g}$ body weight in Sprague-Dawley rats (Cotran and Karnovsky, 1968) to $33 \mathrm{mg} / 100 \mathrm{~g}$ in adult Wistar rats (Pluta et al., 1994), $90 \mathrm{mg} / 100 \mathrm{~g}$ in postnatal Wistar rats (Ugrumov et al., 
1983 ) and $20-40 \mathrm{mg} / 100 \mathrm{~g}$ in adult white mice (Brightman and Reese, 1969).

Ototoxicity of HRP has been described in guinea pigs (Ross et al., 1977). There is also evidence of anomalous permeability results when comparing HRP with other barrier permeability markers, which, as was suggested, could be due to membrane damage (Mazariegos et al., 1984).

We suggest that an additional factor, which does not seem to have been considered, is the possibility that anesthesia might modify or mask the toxic responses to HRP, although we are not aware of any evidence for this. Some of the early experiments did not involve anesthesia because they were confined to tail vein injections (e.g., Brightman and Reese, 1969), but later experiments involved a range of volatile and injected anesthetic agents. In addition, it often seems not to be appreciated that what is visualized in electron microscopy of HRP-containing tissues is the reaction product of the peroxidase, not the protein itself. Which raises the possibility of diffusion artifacts resulting in a distribution of the reaction product that may not reflect that of the actual protein.

Overall it seems reasonable to be cautious about interpreting the results of experiments using HRP, especially when large doses were employed and there was no pre-treatment with antihistamines.

\section{MORE APPROPRIATE MARKERS OF BLOOD-BRAIN BARRIER DYSFUNCTION}

\section{Radiolabeled Markers}

The use of radiolabeled sucrose and inulin for blood-brain barrier permeability studies was pioneered by three giants in the blood-brain barrier field: Dixon Woodbury, Hugh Davson and Bill Oldendorf (e.g., Reed and Woodbury, 1963; Reed et al., 1964; Davson and Bradbury, 1965; Davson and Oldendorf, 1967; Oldendorf and Davson, 1967; Davson and Segal, 1969, 1996). An important advantage of these markers is that they allow a quantitative determination of blood-brain or blood-CSF permeability. However, experiments in which they are used need to be designed carefully if spurious results are not to be obtained. This includes (i) ensuring that steady-state plasma levels of maker are achieved, (ii) that blood contamination of brain samples by the marker is estimated and (iii) the retention of the isotopic label on the marker is secure.

Sucrose is a small water-soluble molecule that is not metabolized to a significant extent if injected parenterally. It distributes in the extracellular space of most tissues and organs of the body, but because of the limited permeability of the bloodbrain and blood-CSF barriers and the turnover of CSF, sucrose does not reach a concentration in brain that reflects the true extracellular space. This is a phenomenon Davson described as the "sink effect" (see Davson and Segal, 1996). A better estimate of brain extracellular space using labeled sucrose has been obtained by a combination of intravascular administration and perfusion through the ventricular system (Oldendorf and Davson, 1967). Because sucrose is excreted via the kidneys, a single injection causes a rapid rise in blood level, followed by a rapid decline with mixing and distribution into the extracellular fluid and then a slower but steady decline due to loss in the urine. This means that unless measures are taken to maintain a steady-state plasma level of marker results expressed as a ratio brain/plasma or CSF/plasma, then the falling plasma level will give rise to a spurious finding that these ratios increase over the period of an experiment in some cases up to $24 \mathrm{~h}$ (e.g., Ferguson and Woodbury, 1969). As described in detail by Davson in many papers and text books (e.g., Davson and Segal, 1996) an approximately steady state level in plasma can be obtained by nephrectomy and either continuous infusion or intermittent injection of sucrose during the course of the experiment. Plasma samples collected throughout the experiment can then be used to calculate a time-weighted mean plasma concentration for calculation of a ratio based on terminal brain and CSF samples. Although nephrectomy might appear to be a severe and unphysiological intervention, in short term experiments lasting only a few hours it is probably not affecting the results of such experiments. In fetal sheep, nephrectomy is unnecessary as the kidneys are not yet producing much urine but similar experiments with radiolabeled sucrose have been obtained (Evans et al., 1974). With respect to CSF samples it is essential that the samples are checked for blood contamination (see Habgood et al., 1992). For brain samples an estimate of blood contamination is important otherwise ratios will be inflated by the presence of marker in blood within the brain sample. This can be achieved by injecting a second marker a few minutes before the end of an experiment. It is necessary for this marker be present in the circulation long enough to mix properly but not to penetrate into the brain to any measurable extent. Radiolabeled markers such as ${ }^{113 \mathrm{~m}}$ Indium, which binds to transferrin and has the advantage of a very short half-life, have been used for this purpose (Evans et al., 1974) but other markers such as radiolabeled albumin or inulin would also be suitable. Some authors have attempted to deal with the problem of blood contamination of brain samples by perfusing the brain with some form of "physiological" solution prior to removing the brain. It is not clear how successful this is, particularly in experiments involving brain trauma where part of the cerebral circulation will be obstructed by post trauma blood coagulation within vessels. Care is also required to make sure the radiolabel is exclusive to the marker in use and remains attached to the marker molecule throughout the experiment. Providing these factors are taken into consideration the use of radiolabeled markers is a valuable way of obtaining a quantitative estimate of any brain barrier dysfunction. Their disadvantage is that the radiolabel cannot be visualized in tissue sections to a satisfactory level of resolution, so the morphological nature of the dysfunction cannot be ascertained. This problem has been overcome by the use of biotin labeled dextrans (see below).

\section{Endogenous and Exogenous Plasma Proteins}

Immunohistochemical detection in brain sections of proteins in plasma such as albumin (e.g., Dziegielewska et al., 1991; Liddelow et al., 2009; O'Shea et al., 2014) immunoglobulin (e.g., Garbuzova-Davis et al., 2012; O'Shea et al., 2014; Blair et al., 2015) and fibrinogen (e.g., Bridges et al., 2014) has been used 
to visualize breaches in the blood-brain barrier. Endogenous proteins have the advantage that they are already present in situ and do not need to be injected thus avoiding potentially unphysiological conditions. Their limitation is that once they have entered the extracellular space of the brain they will continue to diffuse and therefore are not a reliable index of the duration or progression of the leak across brain barriers (Habgood et al., 2007). This problem can be circumvented by injecting an exogenous protein and detecting it with an antibody that does not cross-react with the native protein or a fluorophor-labeled albumin (Dziegielewska et al., 1991; Pelz et al., 2013; Lehmann et al., 2014; Krueger et al., 2015). As will be discussed below this method and any other involving visualization of a marker at the light microscopical level, may be of insufficient resolution to determine the cellular nature of the barrier disruption, although it is common for dysfunction to be equated with disruption of tight junctions (Jin et al., 2014). Equally changes in immunostaining of tight junction proteins (often erroneously described as changes in expression e.g., Lucke-Wold et al., 2014) are often confused with changes in permeability. These conclusions can only be reached if appropriate ultrastructural studies are carried out in parallel. As will be discussed below, this is rarely the case.

\section{Sodium Fluorescein}

Sodium fluorescein $(376 \mathrm{Da})$ was the first visualizable small molecular sized marker to be introduced into the bloodbrain barrier field (Hoffman and Olszewski, 1961; Malmgren and Olsson, 1980). Unlike the more commonly used dyes such as Evans blue, sodium fluorescein binds only weakly to proteins and appears to be an effective low molecular weight marker for brain barrier studies in contrast to dyes that bind to proteins (Wolman et al., 1981). Kaya and Ahishali (2011) have suggested that spectrophotofluorimetric sodium fluorescein uptake measurements (excitation at $440 \mathrm{~nm}$ and emission at $525 \mathrm{~nm}$ ) may enable detection of more subtle alterations in blood-brain barrier permeability when compared to the use of radioactive tracers and may thus be a sensitive indicator of early stages of barrier permeability. No specific effects of sodium fluorescein on blood-brain barrier appear to have been reported. The LD50 in mice was estimated as $4738 \pm 1.23 \mathrm{mg} / \mathrm{Kg}$ body weight (Yankell and Loux, 1977) whereas the amount injected into mice for barrier permeability experiments was only a fraction of this, for example $50 \mathrm{mg} / \mathrm{Kg}$ body weight (Malmgren and Olsson, 1980). A single dose of $500 \mathrm{mg} / \mathrm{Kg}$ in pregnant mice was reported not to have any embryotoxic or teratogenic effects (Salem et al., 1979).

Thus, sodium fluorescein appears to be considerably less toxic than Evans blue or HRP and unlike Evans blue shows only weak binding to proteins in plasma, which given some justification for it being the second (with HRP) most commonly used marker for blood-brain barrier integrity (Figure 2).

\section{Dextrans}

These are complex branched polysaccharides made of many glucose molecules. They consist of chains of lengths varying from 3 to $2000 \mathrm{kDa}$. They are commercially available labeled either with a fluorophor or biotin. An even smaller biotin labeled molecule, ethylenediamine $(286 \mathrm{Da})$ is also available. This is smaller than sucrose (342 Da), which is a commonly used marker for quantitative studies of brain barrier permeability. There were early reports of dextran toxicity when injected into paws of rats due to histamine and 5-hydroxytrypatamine release that could be attenuated by the use of specific inhibitors (Rowley and Benditt, 1956). There has been quite extensive research on dextrans as potential plasma expanders in trauma cases or other circumstances of substantial blood loss. Proper discussion of this is outside the scope of this review. Suffice it to say that the use of dextrans clinically is limited by a significant incidence of anaphylactoid reactions (Lundsgaard-Hansen, 1969; Medby, 2014) and coagulopathy due to interference with some of the clotting factors (Hahn, 2013; Medby, 2014) although this property has been put to good use in prevention and treatment of deep vein thrombosis (Medby, 2014). In animal experiments it was shown that release of histamine and 5-hydroxytryptamine occurs from mast cell degranulation as also reported for HRP (see above). In rabbits administered intravenous dextran preparations over several weeks, there were toxic effects following daily doses of $20 \mathrm{mg} / \mathrm{Kg}$ body weight, but not with $10 \mathrm{mg} / \mathrm{Kg}$ (Hint and Richter, 1958). There are reports that the anaphylactoid effect of dextrans depended on the preparation and is less in smaller molecular weight preparations (Edlund et al., 1952; Walton, 1954); it is unclear to what extent the effects may have been due to contaminants. The biotin and fluorophor labeled dextrans now in experimental use are highly purified and because of the sensitivity of the techniques applied to visualize the labels, only small amounts are required. In our experience we have not seen any untoward effects in vivo (e.g., Ek et al., 2001, 2003).
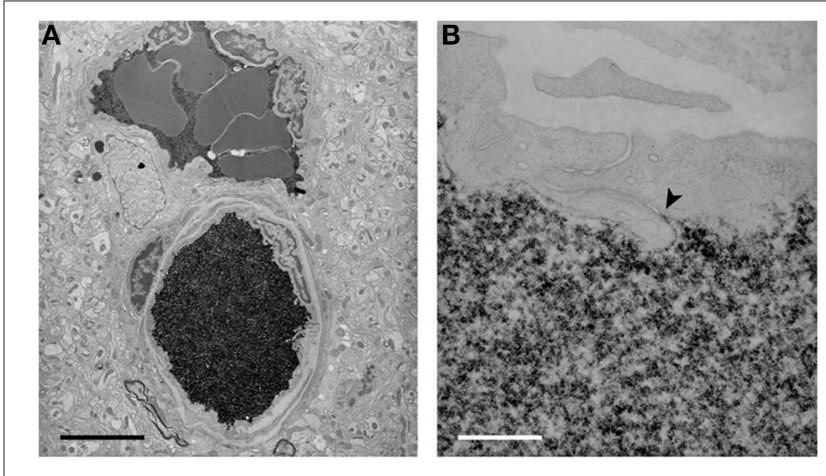

FIGURE 5 | Electron micrographs of the localization of biotin ethylenediamine (BED) in blood vessels deep inside the cortex of a 2-month-old opossum $\mathbf{1 0} \mathbf{~ m i n}$ after an intravenous injection. Similar staining is found after an intravenous injection of biotin-dextran (BDA3000). (A) Low-power micrograph showing two paired vessels with abundant reaction product within lumen. No reaction product is visible in the surrounding tissue. Pairs of arteries and veins are characteristic of the vascular pattern in marsupial brains (Wislocki and Campbell, 1937). (B) High-power micrograph of an interendothelial cleft showing that the tight junctions in the young adult restrict the passage of BED through the cleft (arrowhead). Scale bar $=4 \mu \mathrm{m}$ in (A); $300 \mathrm{~nm}$ in (B). From Ek et al. (2006). 
Biotin labeled molecules can be visualized both at the light microscopical and electron microscopical level (Ek et al., 2003, 2006; Johansson et al., 2006) as illustrated in Figure 5. The bloodCSF permeability characteristics of a range of different sized biotin labeled dextrans (and biotin ethylenediamine) have been shown in quantitative studies to be similar to the permeability of more traditional permeability markers, L-glucose, sucrose and inulin and to be consistent with diffusion across the bloodCSF interface (Ek et al., 2001, 2006). Use of fluorophor and biotin labeled dextrans led to the unexpected finding that in the developing and adult choroid plexus the route of entry from blood to CSF is an intracellular one via the plexus epithelial cells (Ek et al., 2003; Liddelow et al., 2009), as illustrated in Figure 6 rather than intercellular via the tight junctions as generally believed (Anderson, 2001; Nitta et al., 2003; Piontek et al., 2008; Abbott et al., 2010).

In summary, labeled dextrans are valuable markers of bloodbrain barrier integrity, which in the small concentrations used appear to be non-toxic. The biotin labeled form is particularly valuable as it can be visualized at both the light and electron microscopical level. Also they can be quantitated in CSF (Ek et al., 2006).
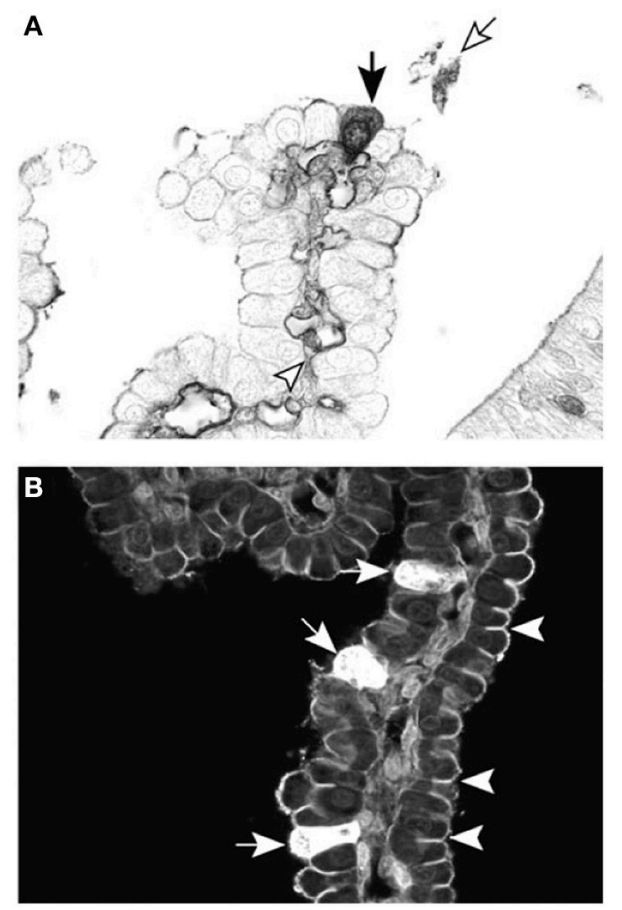

FIGURE 6 | Cellular localization of dextran probes in postnatal lateral ventricular choroid plexus of the marsupial South American opossum (Monodelphis domestica). (A) Forty-five minutes after intraperitoneal injection with BDA-3 kDa, the probe can be seen in individual epithelial cells of the choroid plexus (filled arrow), as well as in the blood vessel lumen (arrowhead) and precipitated in the CSF (unfilled arrow). (B) Ten minutes after intraventricular injection with BDA-3 kDa-Fluorescein, more epithelial cells take up the probe (filled arrows) following CSF injection compared with intraperitoneal injection (A). Penetration of the fluorescent probe between epithelial cells is stopped by the presence of tight junctions (examples highlighted by arrowheads). Scale: $50 \mu \mathrm{m}$. From Liddelow et al. (2009).

\section{BLOOD-BRAIN BARRIER DYSFUNCTION IN NEUROLOGICAL DISORDERS}

In the past $10-15$ years there has been a resurgence of interest in the possibility that the disruption or malfunction of the bloodbrain barrier may be involved in a wide range of neurological disorders: (Keep et al., 2014) including dementia (van de Haar et al., 2015) Alzheimer's disease (Provias and Jeynes, 2014), multiple sclerosis (Lengfeld et al., 2014; Kamphuis et al., 2015), stroke (Schoknecht et al., 2014; Cui et al., 2015), diabetes (Liu and Liu, 2014; Prasad et al., 2014) and trauma to the brain (Barzó et al., 1997; Whalen et al., 1998; Stahel et al., 2000; Habgood et al., 2007) and spinal cord (Oudega, 2012; Figley et al., 2014). However, it is often not clear whether barrier dysfunction is involved in the primary pathology or as a consequence of the pathology, perhaps exacerbating the effects of the disorder. There is generally a rather simple assumption that the barrier may be breached, rather than considering the nature of the breach or the possibility that the many cellular transport mechanisms (in and out of the brain) might be affected. It is perhaps the simple view of the blood-brain barrier as a mere mechanical barrier that has led to such widespread use of Evans blue, without any realization of its limitations as discussed above or the nature of the functional mechanisms that may be affected.

More recent studies are beginning to examine specific barrier mechanisms in neurological disorders, for example Cui et al. (2015) claimed to demonstrate that ATP-binding cassette, ABC1 (cholesterol efflux pump) deficiency in stroke induced in mice increased blood-brain barrier leakage of immunohistochemically detected extravascular albumin. However, what they actually showed was an association between ABCA1 deficiency and albumin leakage; as they did not do high-resolution microscopy they did not demonstrate the pathway across which the albumin leakage occurred. More importantly there are likely to have been many other transporter changes consequent upon the ischaemic injury, which could have contributed to the barrier dysfunction. The importance of this study is the demonstration of a change in a transporter, rather than a leakage of protein, which is already well known to occur in stroke (Krueger et al., 2015). Liu and Liu (2014) have summarized studies showing that a number of ABC transporters change their expression levels in blood vessels of brains of rats with induced diabetes, but there are differences in regulation that depend both on the specific transporter and on the brain region. A quite different disorder of blood-brain barrier function is heterozygous mutation in the GLUT1/SLC2A1 gene, resulting in GLUT1-deficiency syndrome (GLUT1-DS), which manifests itself in infants as motor and mental developmental delay, seizures, reduced head growth and a movement disorder with ataxia, dystonia, and spasticity (Brockmann, 2009).

The study of Krueger et al. (2015) is particularly important because it is one of the few to employ immunohistochemistry of tight junction proteins and electron microscopy of the distribution of a barrier marker (FITC-albumin). They showed that there was no change in either the tight junction proteins or the ultrastructure of the junctions post stroke in adult rats. They demonstrated that disruption of the blood-brain barrier resulted in FITC-albumin-containing transendothelial vesicle trafficking 
TABLE 2 | Characteristics of blood-brain barrier markers described in this review.

\begin{tabular}{|c|c|c|c|c|c|c|c|c|c|c|c|}
\hline \multirow[t]{2}{*}{ Marker } & \multicolumn{2}{|l|}{ Size } & \multicolumn{2}{|c|}{ Binding $^{1}$} & \multicolumn{3}{|c|}{ Visualization } & \multirow[t]{2}{*}{ Quantification ${ }^{3,4}$} & \multirow[t]{2}{*}{ Toxic $^{5}$} & \multirow{2}{*}{$\begin{array}{c}\text { Renal } \\
\text { clearance }^{6}\end{array}$} & \multirow[t]{2}{*}{ Cost } \\
\hline & $\mathrm{Da}$ & Radius nm & Protein & Tissue $^{2}$ & Macro & LM & EM & & & & \\
\hline Biotin ethylenediamine & $286^{a}$ & NR & NR & No & No & Yes & Yes & Qualitative only & NR & Yes & Med \\
\hline Radio-sucrose & $342.3^{b}$ & $0.51^{i}$ & No & No & No & No & No & Accurate & No & Yes & High \\
\hline Na fluorescein & $376^{C}$ & NR & Weak & NR & No & Yes & No & Unreliable & No & Yes & Low \\
\hline Evans blue & $960^{d}$ & NR & Yes & Yes & Yes & Yes & No & Unreliable & Yes & $\mathrm{No}^{*}$ & Low \\
\hline Trypan blue & $961^{e}$ & NR & No & Yes & Yes & Yes & No & Unreliable & Yes & $\mathrm{No}^{*}$ & Low \\
\hline Radio-inulin & $\approx 7000^{\mathrm{b}}$ & $1.3^{\mathrm{i}}$ & No & No & No & No & No & Accurate & No & Yes & High \\
\hline Horseradish peroxidase & $\approx 44,000^{f}$ & $3.0^{j}$ & $N R$ & NR & No & Yes & Yes & Unreliable & Yes & No & Low \\
\hline Albumin (unlabeled) & 69,0009 & $3.5^{i}$ & No & No & No & $\mathrm{IHC}$ & Yes & Unreliable & No & No & Low \\
\hline Radio-albumin & $69,000^{9}$ & $3.5^{\mathrm{i}}$ & No & No & No & $\mathrm{IHC}$ & No & Accurate & No & No & High \\
\hline $\lg G$ & $\approx 155,000^{9}$ & $5.3^{\mathrm{i}}$ & No & No & No & $\mathrm{IHC}$ & No & Qualitative only & No & No & Low \\
\hline Fibrinogen & 340,0009 & $11.0^{\mathrm{k}}$ & No & No & No & $\mathrm{IHC}$ & No & Qualitative only & No & No & Low \\
\hline Dextrans & 1500 to $2,000,000^{h}$ & $0.8-38.2^{1}$ & $N R$ & $N R$ & No & Yes & Yes & Qualitative in tissue & No & Only low MW & High \\
\hline
\end{tabular}

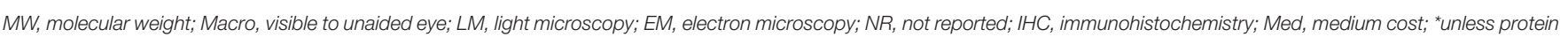
binding capacity in plasma exceeded.

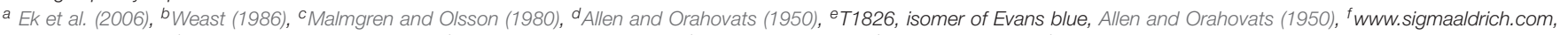
g Thompson (2005), ' hittp://www.thermofisher.com, 'Dziegielewska et al. (1979), 'ंRennke et al. (1978), 'kBoyd et al. (1969), 'Armstrong et al. (2004), Grznárová et al. (2005).

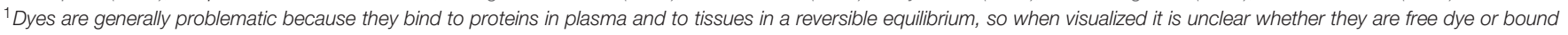
dye and how they became located at a particular site.

${ }^{2}$ Many markers may be taken up by a variety of cells, including choroid plexus epithelial cells. Also neurons and glia, but only if the blood-brain barrier is breached.

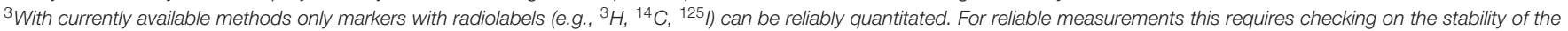

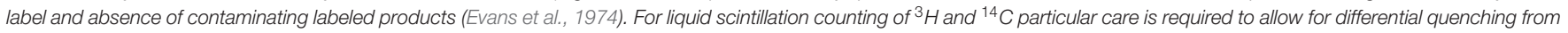

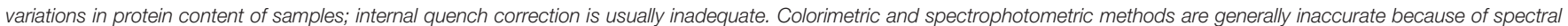
shifts produced by different composition of brain tissue and standards.

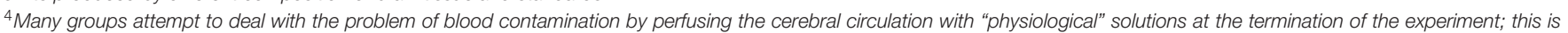

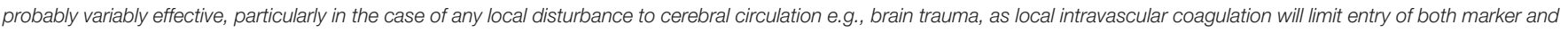
of washout fluid.

${ }^{5}$ At concentrations used in marker experiments.

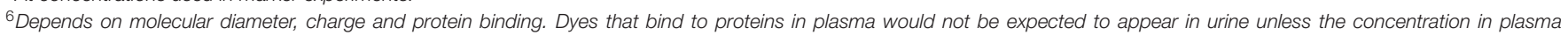

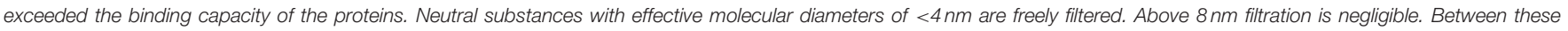
values the amount filtered depends on molecular diameter and charge (Barrett et al., 2012).

and signs of degeneration. These findings make it clear that the common assumption that a leakage of marker (often Evans blue) and/or down regulation of tight junction proteins are not synonymous with disruption of tight junctions. For this to be established requires ultrastructural studies using suitable electron dense permeability markers or ones that can be rendered electron dense by suitable processing (Ek et al., 2003, 2006).

\section{CONCLUSIONS}

The properties, advantages and disadvantages of the various markers discussed above are summarized in Table 2. For the many reasons outlined in this review, Evans blue is an unsatisfactory tool for studying blood-brain barrier dysfunction.

\section{REFERENCES}

Abbott, N. J., Patabendige, A. A. A., Dolman, D. E. M., Yusof, S. R., and Begley, D. J. (2010). Structure and function of the blood-brain barrier. Neurobiol. Dis. 37, 13-25. doi: 10.1016/j.nbd.2009.07.030

Abraham, C. S., Deli, M. A., Joo, F., Megyeri, P., and Torpier, G. (1996). Intracarotid tumor necrosis factor-alpha administration increases the
The most that can be said in its favor is that it may be useful in some circumstances as a rapid and inexpensive method for checking for the presence of a barrier leak. But for proper understanding of a barrier disruption there are now much better methods available and its seems reasonable to propose that Evans blue has had its day and is long overdue to be pensioned off from brain barrier studies as happened long ago for its use for blood volume estimates.

\section{AUTHOR CONTRIBUTIONS}

All of the listed authors contributed to the conception, design, research, drafting, and final approval of the work. They each agree to be accountable for all aspects of the work.

blood-brain barrier permeability in cerebral cortex of the newborn pig: quantitative aspects of double-labelling studies and confocal laser scanning analysis. Neurosci. Lett. 208, 85-88. doi: 10.1016/0304-3940(96) 12546-5

Alle, T. H., Ochoa, M. Jr., Roth, R. F., and Gregersen, M. I. (1953). Spectral absorption of T-1824 in plasma of various species and recovery of the dye by extraction. Am. J. Physiol. 175, 243-246. 
Allen, T. H., and Orahovats, P. D. (1950). Combination of toluidine dye isomers with plasma albumin. Am. J. Physiol. 161, 473-482.

Anderson, J. M. (2001). Molecular structure of tight junctions and their role in epithelial transport. News Physiol. Sci. 16, 126-130.

Armstrong, J. K., Wenby, R. B., Meiselman, H. J., and Fisher, T. C. (2004). The hydrodynamic radii of macromolecules and their effect on red blood cell aggregation. Biophys. J. 87, 4259-4270. doi: 10.1529/biophysj.104. 047746

Ashkenazy, M., and Crawley, J. W. (1953). The value of serial studies of cerebrovascular permeability with radioactive iodinated serum albumin and the scintillation counter particularly in the detection of neurosurgical lesions. Am. Surg. 19, 155-164.

Barrett, K. E., Barman, S. M., Boitano, S., and Brooks, H. L. (2012). Ganong's Review of Medical Physiology, 24th Edn. New York, NY; McGraw Hill Lange.

Barzó, P., Marmarou, A., Fatouros, P., Corwin, F., and Dunbar, J. G. (1997). Acute blood-brain barrier changes in experimental closed head injury as measured by MRI and Gd-DTPA. Acta Neurochir. Suppl. 70, 243-246. doi: 10.1007/978-37091-6837-0_75

Bauer, K. F., Haase, J., and Leonhardt, H. (1956). Über DosisWirkungsberiehungen bei dem durch Pentamethylenletrazol (•Cardiazol•) induzierten Zusammenbruch der Blut-Gehirn-Schranke unter Verwendung von GeigyBlau als Schrankenindikator. Arch. Psychiat. Z. Ges. Neural 195, 199.

Blair, L. J., Frauen, H. D., Zhang, B., Nordhues, B. A., Bijan, S., Lin, Y. C., et al. (2015). Tau depletion prevents progressive blood-brain barrier damage in a mouse model of tauopathy. Acta Neuropathol. Commun. 3, 8. doi: 10.1186/s40478-015-0186-2

Boyd, R. D., Hill, J. R., Humphreys, P. W., Normand, I. C., Reynold, E. O., and Strang, L. B. (1969). Permeability of lung capillaries to macromolecules in foetal and new-born lambs and sheep. J. Physiol. 201, 567-588. doi: 10.1113/jphysiol.1969.sp008773

Bridges, L. R., Andoh, J., Lawrence, A. J., Khoong, C. H., Poon, W. W., Esiri, M. M., et al. (2014). Blood-brain barrier dysfunction and cerebral small vessel disease (arteriolosclerosis) in brains of older people. J. Neuropathol. Exp. Neurol. 73, 1026-1033. doi: 10.1097/NEN.0000000000000124

Brightman, M. W., and Reese, T. S. (1969). Junctions between intimately apposed cell membranes in the vertebrate brain. J. Cell. Biol. 40, 48-77. doi: $10.1083 /$ jcb. 40.3 .648

Brockmann, K. (2009). The expanding phenotype of GLUT1-deficiency syndrome. Brain Dev. 31, 545-552. doi: 10.1016/j.braindev.2009.02.008

Carvalho, J. S. (1989). Heterologous serum albumin as tracer for plasma volume measurments evaluated in conscious nondehydrated and 48-hour water deprived rats. J. Carrdiovasc. Pharm. 13, 603-607. doi: 10.1097/00005344198904000-00013

Cevik, N. G., Orhan, N., Yilmaz, C. U., Arican, N., Ahishali, B., Kucuk, M., et al. (2013). The effects of hyperbaric air and hyperbaric oxygen on blood-brain barrier integrity in rats. Brain Res. 1531, 113-121. doi: 10.1016/j.brainres.2013.07.052

Chen, Y., Constantini, S., Trembovler, V., Weinstock, M., and Shohami, E. (1996). An experimental model of closed head injury in mice: pathophysiology, histopathology, and cognitive deficits. J. Neurotrauma 13, 557-568.

Clasen, R. A., Pandolfi, S., and Hass, G. M. (1970). Vital staining, serum albumin and the blood-brain barrier. J. Neuropathol. Exp. Neurol. 29, 266-284. doi: 10.1097/00005072-197004000-00008

Clementi, F. (1970). Effect of horseradish peroxidase on mice lung capillaries' permeability. J. Histochem. Cytochem. 18, 887-892. doi: 10.1177/18.12.887

Cooksey, C. J. (2013). Quirks of dye nomenclature. 1. Evans blue. Biotechnic. Histochem. 89, 111-113. doi: 10.3109/10520295.2013.822560

Cotran, R. S., and Karnovsky, M. J. (1967). Vascular leakage induced by horseradish peroxidase in the rat. Proc. Soc. Exp. Biol. Med. 126, 557-561. doi: 10.3181/00379727-126-32504

Cotran, R. S., and Karnovsky, M. J. (1968). Ultrastructural studies on the permeability of the mesothelium to horseradish peroxidase. J. Cell Biol. 37, 123-137. doi: $10.1083 /$ jcb.37.1.123

Cotran, R. S., Karnovsky, M. J., and Goth, A. (1968). Resistance of Wistar-Furth rats to the mast cell-damaging effect of horseradish peroxidase. J. Histochem. Cytochem. 16, 382-383. doi: 10.1177/16.5.382

Cui, X., Chopp, M., Zacharek, A., Karasinska, J. M., Cui, Y., Ning, R., et al. (2015). Deficiency of brain ATP-Binding cassette transporter A-1 exacerbates blood-brain barrier and white matter damage after stroke. Stroke 46, 827-834. doi: 10.1161/STROKEAHA.114.007145

Cunningham, T. L., Cartagena, C. M., Lu, X. C., Konopko, M., Dave, J. R., Tortella, F. C., et al. (2014). Correlations between blood-brain barrier disruption and neuroinflammation in an experimental model of penetrating ballistic-like brain injury. J. Neurotrauma 31, 505-514. doi: 10.1089/neu.2013.2965

Dallal, M. M., and Chang, S. W. (1994). Evans blue dye in the assessment of permeability-surface area product in perfused rat lungs. J. Appl. Physiol. 77, 1030-1035.

Davson, H., and Bradbury, M. (1965). The fluid exchange of the central nervous system. Symp. Soc. Exp. Biol. 19, 349-364.

Davson, H., and Oldendorf, W. H. (1967). Symposium on membrane transport. Transport in the central nervous system. Proc. R. Soc. Med. 60, 326-329.

Davson, H., and Segal, M. B. (1969). Effect of cerebrospinal fluid on volume of distribution of extracellular markers. Brain 92, 131-136. doi: 10.1093/brain/92.1.131

Davson, H., and Segal, M. B. (1996). Physiology of the CSF and Blood-Brain Barriers. Boca Raton, FL: CRC Press.

Dawson, A. B., Evans, H. M., and Whipple, G. H. (1920). Blood volume studies. III. Behaviour of large series of dyes introduced into the circulating blood. Am. J. Physiol. 51, 232-256.

Deimann, W., Taugner, R., and Fahimi, H. D. (1976). Arterial hypotension induced by horseradish peroxidase in various rat strains. J. Histochem. Cytochem. 24, 1213-1217. doi: 10.1177/24.12.1002975

Dhillon, H. S., Carman, H. M., Zhang, D., Scheff, S. W., and Prasad, M. R. (1999). Severity of experimental brain injury on lactate and free fatty acid accumulation and Evans blue extravasation in the rat cortex and hippocampus. J. Neurotrauma 16, 455-469. doi: 10.1089/neu.1999.16.455

Dhillon, H. S., Donaldson, D., Dempsey, R. J., and Prasad, M. R. (1994). Regional levels of free fatty acids and Evans blue extravasation after experimental brain injury. J. Neurotrauma 11, 405-415. doi: 10.1089/neu.1994.11.405

Dietrich, W. D., Alonso, O., and Busto, R. (1993). Moderate hyperglycemia worsens acute blood-brain barrier injury after forebrain ischemia in rats. Stroke 24, 111-116. doi: 10.1161/01.STR.24.1.111

Dietrich, W. D., Alonso, O., and Halley, M. (1994). Early microvascular and neuronal consequences of traumatic brain injury: a light and electron microscopic study in rats. J. Neurotrauma 11, 289-301. doi: 10.1089/neu.1994.11.289

Dziegielewska, K. M., Evans, C. A., Lai, P. C., Lorscheider, F. L., Malinowska, D. H., Møllgård, K., et al. (1981). Proteins in cerebrospinal fluid and plasma of fetal rats during development. Dev. Biol. 83, 193-200. doi: 10.1016/S00121606(81)80024-3

Dziegielewska, K. M., Evans, C. A., Malinowska, D. H., Møllgård, K., Reynolds, J. M., Reynolds, M. L., et al. (1979). Studies of the development of brain barrier systems to lipid insoluble molecules in fetal sheep. J. Physiol. 292, 207-231. doi: 10.1113/jphysiol.1979.sp012847

Dziegielewska, K. M., Habgood, M. D., Møllgård, K., Stagaard, M., and Saunders, N. R. (1991). Species-specific transfer of plasma albumin from blood into different cerebrospinal fluid compartments in the fetal sheep. J. Physiol. 439, 215-237. doi: 10.1113/jphysiol.1991.sp018664

Edlund, T., Lofgren, B., and Vali, L. (1952). Toxicity of dextran in rats. Nature $170,125$.

Ehrlich, P. (1885). Das Sauerstoffbedürfnis des Organismus. Eine Farbenanalytische Studie. Berlin: Hirschwald.

Ek, C. J., Dziegielewska, K. M., Stolp, H., and Saunders, N. R. (2006). Functional effectiveness of the blood-brain barrier to small water-soluble molecules in developing and adult opossum (Monodelphis domestica). J. Comp. Neurol. 496, 13-26. doi: 10.1002/cne.20885

Ek, C. J., Habgood, M. D., Dziegielewska, K. M., and Saunders, N. R. (2003). Structural characteristics and barrier properties of the choroid plexuses in developing brain of the opossum (Monodelphis domestica). J. Comp. Neurol. 460, 451-464. doi: 10.1002/cne.10661

Ek, C. J., Habgood, M. D., Dziegielewska, K. M., Potter, A., and Saunders, N. R. (2001). Permeability and route of entry for lipid-insoluble molecules across brain barriers in developing Monodelphis domestica. J. Physiol. 536, 841-853. doi: 10.1111/j.1469-7793.2001.00841.x

Emmett, M., Cerniglia, C. E., and Crowle, A. J. (1985). Differential serum protein binding of benzidine- and benzidine-congener based dyes 
and their derivatives. Arch. Toxicol. 57, 130-135. doi: 10.1007/BF003 43123

Evans, C. A., Reynolds, J. M., Reynolds, M. L., Saunders, N. R., and Segal, M. B. (1974). The development of a blood-brain barrier mechanism in foetal sheep. J. Physiol. 238, 371-386. doi: 10.1113/jphysiol.1974. sp010530

Farrell, C. L., and Shivers, R. R. (1984). Capillary junctions of the rat are not affected by osmotic opening of the blood-brain barrier. Acta Neuropathol. (Berl.) 63, 179-189. doi: 10.1007/BF00685243

Ferguson, R. K., and Woodbury, D. M. (1969). Penetration of ${ }^{14} \mathrm{C}$-inulin and ${ }^{14} \mathrm{C}$ sucrose into brain, cerebrospinal fluid, and skeletal muscle of developing rats. Exp. Brain Res. 7, 181-194. doi: 10.1007/BF00239028

Figley, S. A., Khosravi, R., Legasto, J. M., Tseng, Y. F., and Fehlings, M. G. (2014). Characterization of vascular disruption and blood-spinal cord barrier permeability following traumatic spinal cord injury. J. Neurotrauma 31, 541-552. doi: 10.1089/neu.2013.3034

Garbuzova-Davis, S., Hernandez-Ontiveros, D. G., Rodrigues, M. C., Haller, E., Frisina-Deyo, A., Mirtyl, S., et al. (2012). Impaired bloodbrain/spinal cord barrier in ALS patients. Brain Res. 1469C, 114-128. doi: 10.1016/j.brainres.2012.05.056

Gibson, J. G., and Gregersen, M. I. (1935). Toxicity of two vital dyes used in plasma volume determinations. Am. J. Physiol. 113, 50.

Goldmann, E. E. (1909). Die äussere und innere Sekretion des gesunden und kranken Organismus im Lichte der 'vitalen Färbung'. Beiträg Klinische Chirurgie 64, 192-265.

Goldmann, E. E. (1913). Vitalfärbung am Zentralnervensyatem. Beitrag zur Physio-Pathologie des plexus chorioideus und der Hirnhaute. Abh Preuss, Akad Wiss Phys-Math Kl 1, 1-60.

Graham, R. C. Jr., and Karnovsky, M. J. (1966). Glomerular permeability. Ultrastructural cytochemical studies using peroxidases as protein tracers. J. Exp. Med. 124, 1123-1134. doi: 10.1084/jem.124.6.1123

Gregersen, M. I. (1932). The physiological mechanism of thirst. Am. J. Physiol. (Proc.) 101,44

Gregersen, M. I. (1938). An analysis of colorimetric methods in relation to plasma volume determination. J. Lab. Clin. Med. 23, 423-430.

Gregersen, M. I., Gibson, J. J., and Stead, E. A. (1935). Plasma volume determination with dyes: errors in colorimetry: use of the dye T-1824. Am. J. Physiol. 113, 54.

Gregersen, M. I., and Rawson, R. A. (1943). The disappearance of T-1824 and structurally related dyes from the blood stream. Am. J. Physiol. 138, 698-707.

Gregersen, M. I., and Rawson, R. A. (1959). Blood volume. Physiol. Rev. 39, 307-342.

Gregersen, M. I., and Stewart, J. D. (1938). Simultaneous determination of the plasma volume with T-1824 and the available fluid volume with sodium thiocyanate. Am. J. Physiol. 125, 142-152.

Grznárová, G., Yu, S., Stefuca, V., and Polakovic, M. (2005). Quantitative characterization of pore structure of cellulose gels with or without bound protein ligand. J. Chromatogr. A 1092, 107-113. doi: 10.1016/j.chroma.2005.03.012

Habgood, M. D., Bye, N., Dziegielewska, K. M., Ek, C. J., Lane, M. A., Potter, A., et al. (2007). Changes in blood-brain barrier permeability to large and small molecules following traumatic brain injury in mice. Eur. J. Neurosci. 25, 231-238. doi: 10.1111/j.1460-9568.2006.05275.x

Habgood, M. D., Sedgwick, J. E., Dziegielewska, K. M., and Saunders, N. R. (1992). A developmentally regulated blood-cerebrospinal fluid transfer mechanism for albumin in immature rats. J. Physiol. 456, 181-192. doi: 10.1113/jphysiol.1992.sp019332

Hahn, R. G. (2013). Fluid therapy in uncontrolled hemorrhage-what experimental models have taught us. Acta Anaesthesiol. Scand. 57, 16-28. doi: 10.1111/j.13996576.2012.02763.x

Hellal, F., Bonnefont-Rousselot, D., Croci, N., Palmier, B., Plotkine, M., and Marchand-Verrecchia, C. (2004). Pattern of cerebral edema and hemorrhage in a mice model of diffuse brain injury. Neurosci Lett. 357, 21-24. doi: 10.1016/j.neulet.2003.12.036

Hint, H. C., and Richter, A. W. (1958). Chronic toxicity of dextran sulphate in rabbits. Brit. J. Pharmacol. 13, 109-112. doi: 10.1111/j.14765381.1958.tb00203.x
Hoffman, H. J., and Olszewski, J. (1961). Spread of sodium fluorescein in normal brain tissue. A study of the mechanism of the blood-brain barrier. Neurology 11, 1081-1085. doi: 10.1212/WNL.11.12.1081

Hooper, C. W., Smith, H. P., Belt, A. E., and Whipple, G. H. (1920). Blood volume studies, 1. Experimental control of a dye blood volume method. Am. J. Physiol. $51,205-220$

Hueper, W. C., and Ichniowski, C. T. (1944). Toxicopathological studies on the dye T-1824. Arch. Surg. 48, 17-26. doi: 10.1001/archsurg.1944.01230010020002

Jiang, Z., Li, C., Arrick, D. M., Yang, S., Baluna, A. E., and Sun, H. (2014). Role of nitric oxide synthases in early blood-brain barrier disruption following transient focal cerebral ischemia. PLoS ONE 9:e93134. doi: 10.1371/journal.pone.0093134

Jin, S., Sonobe, Y., Kawanokuchi, J., Horiuchi, H., Cheng, Y., Wang, Y., et al. (2014). Interleukin-34 restores blood-brain barrier integrity by upregulating tight junction proteins in endothelial cells. PLOS ONE 9:e115981. doi: 10.1371/journal.pone.0115981

Johansson, P. A., Dziegielewska, K. M., Ek, C. J., Habgood, M. D., Liddelow, S. A., Potter, A. M., et al. (2006). Blood-CSF barrier function in the rat embryo. Eur. J. Neurosci. 24, 65-76. doi: 10.1111/j.1460-9568.2006.04904.x

Kamphuis, W. W., Derada Troletti, C. D., Reijerkerk, A., Romero, I. A., and de Vries, H. E. (2015). The blood-brain barrier in multiple sclerosis: microRNAs as key regulators. CNS Neurol. Disord. Drug Targets. 14, 157-167. doi: 10.2174/1871527314666150116125246

Kang, E. J., Major, S., Jorks, D., Reiffurth, C., Offenhauser, N., Friedman, A., et al. (2013). Blood-brain barrier opening to large molecules does not imply blood-brain barrier opening to small ions. Neurobiol. Dis. 52, 204-218. doi: 10.1016/j.nbd.2012.12.007

Karnovsky, M. J. (1967). The ultrastructural basis of capillary permeability studied with peroxidase as a tracer. J. Cell Biol. 35, 213-236. doi: 10.1083/jcb. 35.1.213

Kaya, M., and Ahishali, B. (2011). Assessment of permeability in barrier type of endothelium in brain using tracers: Evans blue, sodium fluorescein, and horseradish peroxidase. Methods Mol. Biol. 763, 369-382. doi: 10.1007/978-161779-191-8_25

Keep, R. F., Zhou, N., Xiang, J., Andjelkovic, A. V., Hua, Y., and Xi, G. (2014). Vascular disruption and blood-brain barrier dysfunction in intracerebral hemorrhage. Fluids Barriers CNS. 11:18. doi: 10.1186/2045-8118-11-18

Keith, N. M., Rowntree, L. G., and Gerhaghty, J. T. (1915). A method for determination of plasma and blood volume. Arch. Intern. Med. (Chic.). 16, 547-576. doi: 10.1001/archinte.1915.00080040043004

Kitagawa, K., Matsumoto, M., Tagaya, M., Ueda, H., Oku, N., Kuwabara, K., et al. (1991). Temporal profile of serum albumin extravasation following cerebral ischemia in a newly established reproducible gerbil model for vasogenic brain edema: a combined immunohistochemical and dye tracer analysis. Acta Neuropathol. 82, 164-171. doi: 10.1007/BF00294441

Krueger, M., Bechmann, I., Immig, K., Reichenbach, A., Härtig, W., and Michalski, D. (2015). Blood-brain barrier breakdown involves four distinct stages of vascular damage in various models of experimental focal cerebral ischemia. J. Cereb. Blood Flow Metab. 35, 292-303. doi: 10.1038/jcbfm.2014.199

Laurell, J. C. (1965). Antigen-antibody crossed electrophoresis. Analyt. Biochem. 10, 358-361. doi: 10.1016/0003-2697(65)90278-2

Lee, H. B., and Blaufox, M. D. (1985). Blood volume ion the rat. J. Nucl. Med. 26, $72-76$.

Lehmann, J., Härtig, W., Seidel, A., Füldner, C., Hobohm, C., Grosche, J., et al. (2014). Inflammatory cell recruitment after experimental thromboembolic stroke in rats. Neuroscience 279, 139-154. doi: 10.1016/j.neuroscience.2014.08.023

Lengfeld, J., Cutforth, T., and Agalliu, D. (2014). The role of angiogenesis in the pathology of multiple sclerosis. Vasc. Cell. 6, 23. doi: 10.1186/s13221-0140023-6

LeVeen, H. H., and Fishman, W. H. (1947). Combination of Evans blue with plasma protein; its significance in capillary permeability studies, blood dye disappearance curves, and its use as a protein tag. Am. J. Physiol. 151, 26-33.

Liddelow, S. A., Dziegielewska, K. M., Ek, C. J., Johansson, P. A., Potter, A. M., and Saunders, N. R. (2009). Cellular transfer of macromolecules across the developing choroid plexus of Monodelphis domestica. Eur. J. Neurosci. 29, 253-266. doi: 10.1111/j.1460-9568.2008.06571.x 
Linder, V., and Heinle, H. (1982). Binding properties of Evans blue in rabbits as determined by disc electrophoresis. Atherosclerosis 43, 417-422. doi: 10.1016/0021-9150(82)90040-5

Liu, L., and Liu, X.-D. (2014). Alterations in function and expression of ABC transporters at blood-brain barrier under diabetes and the clinical significances. Front. Pharmacol. 5:273. doi: 10.3389/fphar.2014.00273

Lotocki, G., de Rivero Vaccari, J. P., Perez, E. R., Sanchez-Molano, J., FuronesAlonso, O., Bramlett, H. M., et al. (2009). Alterations in blood-brain barrier permeability to large and small molecules and leukocyte accumulation after traumatic brain injury: effects of post-traumatic hypothermia. J. Neurotrauma 26, 1123-1134. doi: 10.1089/neu.2008.0802

Lucke-Wold, B. P., Logsdon, A. F., Smith, K. E., Turner, R. C., Alkon, D. L., Tan, Z., et al. (2014). Bryostatin-1 restores blood brain barrier integrity following blast-induced traumatic brain injury. Mol. Neurobiol. 52, 1119-1134. doi: 10.1007/s12035-014-8902-7

Lundsgaard-Hansen, P. (1969). Treatment of shock with dextrans and gelatin. Vox Sang. 17, 161-193. doi: 10.1111/j.1423-0410.1969.tb00387.x

Majno, G., Palade, G. E., and Schoefl, G. I. (1961). Studies on inflammation. II. The site of action of histamine and serotonin along the vascular tree: a topographic study. J. Biophys. Biochem. Cytol. 11, 607-626. doi: 10.1083/jcb.11.3.607

Malaowalla, A. M., and Fong, C. (1962). Toxicity of Evans blue dye in the monkey and tracing of it in the tooth pulp. Oral Surg. Oral Med. Oral Pathol. 15, 1259-1263. doi: 10.1016/0030-4220(62)90162-7

Malmgren, L. T., and Olsson, Y. (1980). Differences between the peripheral and the central nervous system in permeability to sodium fluorescein. J. Comp. Neurol. 191, 103-107. doi: 10.1002/cne.901910106

Manaenko, A., Chen, H., Kammer, J., Zhang, J. H., and Tang, J. (2011). Comparison Evans blue injection routes: intravenous versus intraperitoneal, for measurement of blood-brain barrier in a mice hemorrhage model. J. Neurosci. Methods 195, 206-210. doi: 10.1016/j.jneumeth.2010.12.013

Margouleff, D. (2013). Blood volume determination, a nuclear medicine test in evolution. Clin. Nucl. Med. 38, 534-537. doi: 10.1097/RLU.0b013e3182 $92 \mathrm{f} 370$

Mazariegos, M. R., Tice, L. W., and Hand, A. R. (1984). Alteration of tight junction permeability in the rat parotid gland after isoproterenol stimulation. J. Cell Biol. 98, 1865-1877. doi: 10.1083/jcb.98.5.1865

Medby, C. (2014). Is there a place for crystalloids and colloids in remote damage control resuscitation? Shock 41(Suppl. 1), 47-50. doi: 10.1097/SHK.0000000000000117

Moos, T., and Møllgård, K. (1993). Cerebrovascular permeability to azo dyes and plasma proteins in rodents of different ages. Neuropathol. Appl. Neurobiol. 19, 120-127. doi: 10.1111/j.1365-2990.1993.tb00416.x

Nagaraja, T. N., Keenan, K. A., Fenstermacher, J. D., and Knight, R. A. (2008). Acute leakage patterns of fluorescent plasma flow markers after transient focal cerebral ischemia suggest large openings in blood-brain barrier. Microcirculation 15, 1-14. doi: 10.1080/10739680701409811

Nitta, T., Hata, M., Gotoh, S., Seo, Y., Sasaki, H., Hashimoto, N., et al. (2003). Sizeselective loosening of the blood-brain barrier in claudin-5-deficient mice. J. Cell Biol. 161, 653-660. doi: 10.1083/jcb.200302070

Oldendorf, W. H., and Davson, H. (1967). Brain extracellular space and the sink action of cerebrospinal fluid. Measurement of rabbit brain extracellular space using sucrose labeled with carbon 14. Arch Neurol. 17, 196-205. doi: 10.1001/archneur.1967.00470260086010

O'Shea, E., Urrutia, A., Green, A. R., and Colado, M. I. (2014). Current preclinical studies on neuroinflammation and changes in blood-brain barrier integrity by MDMA and methamphetamine. Neuropharmacology 87, 125-134. doi: 10.1016/j.neuropharm.2014.02.015

Oudega, M. (2012). Molecular and cellular mechanisms underlying the role of blood vessels in spinal cord injury and repair. Cell Tissue Res. 349, 269-288. doi: 10.1007/s00441-012-1440-6

Pelz, J., Härtig, W., Weise, C., Hobohm, C., Schneider, D., Krueger, M., et al. (2013). Endothelial barrier antigen-immunoreactivity is conversely associated with blood-brain barrier dysfunction after embolic stroke in rats. Eur. J. Histochem. 57:e38. doi: 10.4081/ejh.2013.e38

Petito, C. K. (1979). Early and late mechanisms of increased vascular permeability following experimental cerebral infarction. J. Neuropathol. Exp. Neurol. 38, 222-234. doi: 10.1097/00005072-197905000-00003
Piontek, J., Winkler, L., Wolburg, H., Müller, S. L., Zuleger, N., Piehl, C., et al. (2008). Formation of tight junction: determinants of homophilic interaction between classic claudins. FASEB J. 22, 146-158. doi: 10.1096/fj.07-8319com

Pluta, R., Lossinsky, A. S., Wiśniewski, H. M., and Mossakowski, M. J. (1994). Early blood-brain barrier changes in the rat following transient complete cerebral ischemia induced by cardiac arrest. Brain Res. 633, 41-52. doi: 10.1016/00068993(94)91520-2

Prasad, S., Sajja, R. K., Naik, P., and Cucullo, L. (2014). Diabetes mellitus and blood-brain barrier dysfunction: an overview. J. Pharmacovigil. 2, 125. doi: 10.4172/2329-6887.1000125

Provias, J., and Jeynes, B. (2014). The role of the blood-brain barrier in the pathogenesis of senile plaques in Alzheimer's disease. Int. J. Alzheimers Dis. 2014:191863. doi: 10.1155/2014/191863

Rawson, R. A. (1943). The binding of T-1824 and structurally related diazo dyes by the plasma proteins. Am. J. Physiol. 138, 708-717.

Reed, D. J., and Woodbury, D. M. (1963). Kinetics of movement of iodide, sucrose, inulin and radio-iodinated serum albumin in the central nervous system and cerebrospinal fluid of the rat. J. Physiol. 169, 816-850. doi: 10.1113/jphysiol.1963.sp007298

Reed, D. J., Woodbury, D. M., and Holtzer, R. I. (1964). Brain edema, electrolytes, and extracellular space. Effect of triethyl tin or brain and skeletal muscle. Arch. Neurol. 10, 604-616. doi: 10.1001/archneur.1964.00460180070007

Reese, T. S., and Karnovsky, M. J. (1967). Fine structural localization of a blood-brain barrier to exogenous peroxidase. J. Cell Biol. 34, 207-217. doi: 10.1083/jcb.34.1.207

Reeve, E. B. (1957). The contribution of I 131-labeled proteins to measurements of blood volume. Ann. N.Y. Acad. Sci. 70, 137-147. doi: 10.1111/j.17496632.1957.tb35384.x

Rennke, H. G., Patel, Y., and Venkatachalam, M. A. (1978). Glomerular filtration of proteins: clearance of anionic, neutral, and cationic horseradish peroxidase in the rat. Kidney Int. 13, 278-288. doi: 10.1038/ki.1978.41

Ribatti, D., Nico, B., and Bertossi, M. (1993). The development of the blood-brain barrier in the chick. Studies with Evans blue and horseradish peroxidase. Ann. Anat. 175, 85-88. doi: 10.1016/S0940-9602(11)80247-5

Ross, M. D., Nutall, A. L., and Wright, C. H. G. (1977). Horseradish peroxidase acute ototoxicity and the uptake and movement of the peroxidase in the auditory system of the guinea pig. Acta Otolaryngol. 84, 187-201. doi: $10.3109 / 00016487709123957$

Rössner, W., and Temple, K. (1966). Quantitative Bestimmung der Permeabilität der sogenannten Blut-Hirnschranke für Evans-Blau (Tl824). Med. Pharmacol. Exp. 14, 169-182.

Rowley, D. A., and Benditt, E. P. (1956). 5-Hydroxytrypatamine and histamine as mediators of the vascular injury produced by agents which damage mast cells in rats. J. Exp. Med. 103, 399-412. doi: 10.1084/jem.103.4.399

Salem, H., Loux, J. J., Smith, S., and Nichols, C. W. (1979). Evaluation of the toxicologic and teratogenic potentials of sodium fluorescein in the rat. Toxicology 12, 143-150. doi: 10.1016/0300-483X(79)90040-4

Saunders, N. R., Dreifuss, J. J., Dziegielewska, K. M., Johansson, P. A., Habgood, M. D., Møllgård, K., et al. (2014). The rights and wrongs of blood-brain barrier permeability studies: a walk through 100 years of history. Front. Neurosci. 8:404. doi: $10.3389 /$ fnins.2014.00404

Saunders, N. R., Ek, C. J., Habgood, M. D., and Dziegielewska, K. M. (2008). Barriers in the brain: a renaissance? Trends Neurosci. 31, 279-286. doi: 10.1016/j.tins.2008.03.003

Schoknecht, K., David, Y., and Heinemann, U. (2014). The blood-brain barriergatekeeper to neuronal homeostasis: clinical implications in the setting of stroke. Semin. Cell Dev. Biol. 38, 35-42. doi: 10.1016/j.semcdb.2014.10.004

Smith, H. P. (1920). Blood volume measurements. II. Repeated determination of the blood volume at short intervals by means of the dye method. Am. J. Physiol. $51,221-231$.

Spigelman, M. K., Zappulla, R. A., Malis, L. I., Holland, J. F., Goldsmith, S. J., and Goldberg, J. D. (1983). Intracarotid dehydrocholate infusion: a new method for prolonged reversible blood-brain barrier disruption. Neurosurgery 12, 606-612. doi: 10.1227/00006123-198306000-00002

Stahel, P. F., Kariya, K., Shohami, E., Barnum, S. R., Eugster, H., Trentz, O., et al. (2000). Intracerebral complement C5a receptor (CD88) expression is regulated by TNF and lymphotoxin-alpha following closed head injury 
in mice. J. Neuroimmunol. 109, 164-172. doi: 10.1016/S0165-5728(00) 00304-0

Stoelinga, G. B., and van Munster, P. J. (1967). The behaviour of Evans blue (azodye T-1824) in the body after intravenous injection. Acta Physiol. Pharmacol. Neerl. 14, 391-409.

Straus, W. (1959). Rapid cytochemical identification of phagosomes in various tissues of the rat and their differentiation from mitochondria by the peroxidase method. J. Biophys. Biochem. Cytol. 5, 193-204. doi: 10.1083/jcb.5.2.193

Tanno, H., Nockels, R. P., Pitts, L. H., and Noble, L. J. (1992). Breakdown of the blood-brain barrier after fluid percussive brain injury in the rat. Part 1: distribution and time course of protein extravasation. J. Neurotrauma 9, 21-32. doi: 10.1089/neu.1992.9.21

Theron, P. H., and Wilson, W. C. (1949). Blood changes in peritonitis. Lancet 253, 172-178. doi: 10.1016/S0140-6736(49)90466-3

Thompson, E. J. (2005). Proteins of the Cerebrospinal Fluid: Analysis and Interpretation in the Diagnosis and Treatment of Neurological Disease, $2 n d E d n$. San Diego, CA: Elsevier Academic Press.

Tiselius, A. (1937). A new apparatus for electrophoretic analysis of colloidal mixtures. Trans. Faraday Soc. 33, 524-531. doi: 10.1039/tf9373300524

Tschirgi, R. D. (1950). Protein complexes and the impermeability of the bloodbrain barrier to dyes. Am. J. Physiol. 163, 756.

Ueno, M., Tomimoto, H., Akiguchi, I., Wakita, H., and Sakamoto, H. (2002). Blood-brain barrier disruption in white matter lesions in a rat model of chronic cerebral hypoperfusion. J. Cereb. Blood Flow Metab. 22, 97-104. doi: 10.1097/00004647-200201000-00012

Ugrumov, M. V., Ivanova, I. P., and Mitskevich, M. S. (1983). Permeability of the blood-brain barrier in the median eminence during the perinatal period in rats. Cell Tissue Res. 230, 649-660. doi: 10.1007/BF00216208

Uyama, O., Okamura, N., Yanase, M., Narita, M., Kawabata, K., and Sugita, M. (1988). Quantitative evaluation of vascular permeability in the gerbil brain after transient ischemia using Evans blue fluorescence. J. Cereb. Blood Flow Metab. 8, 282-284. doi: 10.1038/jcbfm.1988.59

van de Haar, H. J., Burgmans, S., Hofman, P. A., Verhey, F. R., Jansen, J. F., and Backes, W. H. (2015). Blood-brain barrier impairment in dementia: current and future in vivo assessments. Neurosci. Biobehav. Rev. 49C, 71-81. doi: 10.1016/j.neubiorev.2014.11.022

Walton, K. W. (1954). Investigation of the toxicity of a series of dextran sulphates of varying molecular weight. Brit. J. Pharmacol. 9, 1-14. doi: 10.1111/j.14765381.1954.tb00809.x
Wang, H.-L., and Lai, T. W. (2014). Optimization of Evans blue quantitation in limited rat tissue samples. Sci. Rep. 4:6588. doi: 10.1038/srep06588

Weast, R. C. (1986). Handbook of Chemistry and Physics, 67th Edn. Boca Raton, FL: CRC press.

Whalen, M. J., Carlos, T. M., Kochanek, P. M., and Heineman, S. (1998). Bloodbrain barrier permeability, neutrophil accumulation and vascular adhesion molecule expression after controlled cortical impact in rats: a preliminary study. Acta Neurochir. Suppl. 71, 212-214. doi: 10.1007/978-3-70916475-4_61

Whalen, M. J., Carlos, T. M., Kochanek, P. M., Clark, R. S., Heineman, S., Schiding, J. K., et al. (1999). Neutrophils do not mediate blood-brain barrier permeability early after controlled cortical impact in rats. J. Neurotrauma 16, 583-594. doi: 10.1089/neu.1999.16.583

Wislocki, G. B., and Campbell, C. P. (1937). The unusual manner of vascularization of the brain of the opossum (Didelphis virginiana). Anat. Rec. 67, 177-189. doi: 10.1002/ar.1090670205

Wolman, M., Klatzo, I., Chui, E., Wilmes, F., Nishimoto, K., Fujiwara, K., et al. (1981). Evaluation of the dye-protein tracers in pathophysiology of the blood-brain barrier. Acta Neuropathol. 54, 55-61. doi: 10.1007/BF006 91332

Yankell, S. L., and Loux, J. J. (1977). Acute toxicity testing of erythrosine and sodium fluorescein in mice and rats. J. Periodontol. 48, 228-231.

Yen, L. F., Wei, V. C., Kuo, E. Y., and Lai, T. W. (2013). Distinct patterns of cerebral extravasation by Evans blue and sodium fluorescein in rats. PLoS ONE 8:e68595. doi: 10.1371/journal.pone.0068595

Zizza, F., and Reeve, E. B. (1958). Erroneous measurement of plasma volume in the rabbit by T-1824. Am. J. Physiol. 194, 522-526.

Conflict of Interest Statement: The authors declare that the research was conducted in the absence of any commercial or financial relationships that could be construed as a potential conflict of interest.

Copyright (C) 2015 Saunders, Dziegielewska, Møllgård and Habgood. This is an openaccess article distributed under the terms of the Creative Commons Attribution License (CC BY). The use, distribution or reproduction in other forums is permitted, provided the original author(s) or licensor are credited and that the original publication in this journal is cited, in accordance with accepted academic practice. No use, distribution or reproduction is permitted which does not comply with these terms. 


\section{The rights and wrongs of blood-brain barrier permeability studies: a walk through 100 years of history}

\section{Norman R. Saunders ${ }^{1}{ }^{*}$, Jean-Jacques Dreifuss ${ }^{2}$, Katarzyna M. Dziegielewska ${ }^{1}$, Pia A. Johansson ${ }^{3}$, Mark D. Habgood ${ }^{1}$, Kjeld Møllgård ${ }^{4}$ and Hans-Christian Bauer ${ }^{5,6}$}

\footnotetext{
Department of Pharmacology and Therapeutics, University of Melbourne, Parkville, VIC, Australia

2 Department of Neuroscience, University of Geneva, Geneva, Switzerland

${ }^{3}$ Institute for Stem Cell Research, Helmholtz Center Munich, Munich, Germany

${ }^{4}$ Department of Cellular and Molecular Medicine, University of Copenhagen, Copenhagen, Denmark

${ }_{5}$ Institute of Tendon and Bone Regeneration, Paracelsus Medical University, Salzburg, Austria

${ }^{6}$ Spinal Cord Injury and Tissue Regeneration Center, Paracelsus Medical University, Salzburg, Austria
}

\section{Edited by:}

Lester R. Drewes, University of Minnesota Medical School Duluth, USA

\section{Reviewed by:}

Britta Engelhardt, University of Bern Switzerland

Daniela Virgintino, Sensory Organs Bari University School of Medicine, Italy

\section{${ }^{*}$ Correspondence:}

Norman R. Saunders, Department of Pharmacology and Therapeutics, University of Melbourne, Grattan Street, Parkville, VIC 3010, Australia e-mail:n.saunders@unimelb.edu.au
Careful examination of relevant literature shows that many of the most cherished concepts of the blood-brain barrier are incorrect. These include an almost mythological belief in its immaturity that is unfortunately often equated with absence or at least leakiness in the embryo and fetus. The original concept of a blood-brain barrier is often attributed to Ehrlich; however, he did not accept that permeability of cerebral vessels was different from other organs. Goldmann is often credited with the first experiments showing dye (trypan blue) exclusion from the brain when injected systemically, but not when injected directly into it. Rarely cited are earlier experiments of Bouffard and of Franke who showed methylene blue and trypan red stained all tissues except the brain. The term "blood-brain barrier" "Blut-Hirnschranke" is often attributed to Lewandowsky, but it does not appear in his papers. The first person to use this term seems to be Stern in the early 1920s. Studies in embryos by Stern and colleagues, Weed and Wislocki showed results similar to those in adult animals. These were well-conducted experiments made a century ago, thus the persistence of a belief in barrier immaturity is puzzling. As discussed in this review, evidence for this belief, is of poor experimental quality, often misinterpreted and often not properly cited. The functional state of blood-brain barrier mechanisms in the fetus is an important biological phenomenon with implications for normal brain development. It is also important for clinicians to have proper evidence on which to advise pregnant women who may need to take medications for serious medical conditions. Beliefs in immaturity of the blood-brain barrier have held the field back for decades. Their history illustrates the importance of taking account of all the evidence and assessing its quality, rather than selecting papers that supports a preconceived notion or intuitive belief. This review attempts to right the wrongs. Based on careful translation of original papers, some published a century ago, as well as providing discussion of studies claiming to show barrier immaturity, we hope that readers will have evidence on which to base their own conclusions.

Keywords: blood-brain barrier, blood-cerebrospinal fluid barrier, embryo, fetus, newborn, permeability, tight junctions, transporters

\section{INTRODUCTION}

The very first studies of barrier properties in embryos clearly demonstrated the intactness of the interfaces between the blood, brain and cerebrospinal fluid ${ }^{1}$. Thus Wislocki (1920) injected a guinea pig embryo with trypan blue (Figure 1A), the same

\footnotetext{
Abbreviations: CNS, central nervous system; CSF, cerebrospinal fluid; HRP, horseradish peroxidase.

${ }^{1}$ Where possible we have worked from original references. Where these were not in English we have given citations of key points in the original language, together with a translation (indicated by italics). Readers will be able to decide for themselves if the translations are accurate and if our deductions from the citations are appropriate.
}

dye, which was first used by Goldmann (1909; 1913 see below). Wislocki (1920) observed that in the embryo, as in adult animals, the dye stained almost all of the tissues of the body, with the notable exception of much of the brain.

Even earlier than this Weed (1917a,b) injected an approximately isotonic solution of potassium ferrocyanide, a small molecule about the same size as sucrose, into the neural tube of E19 and older pig embryos. Weed's results showed that in an E19 pig embryo the potassium ferrocyanide (demonstrated by the Prussian blue reaction) remained entirely within the fluid filling the neural tube (Figure 1B). This suggests that even this early in development, shortly after neural tube closure, the 


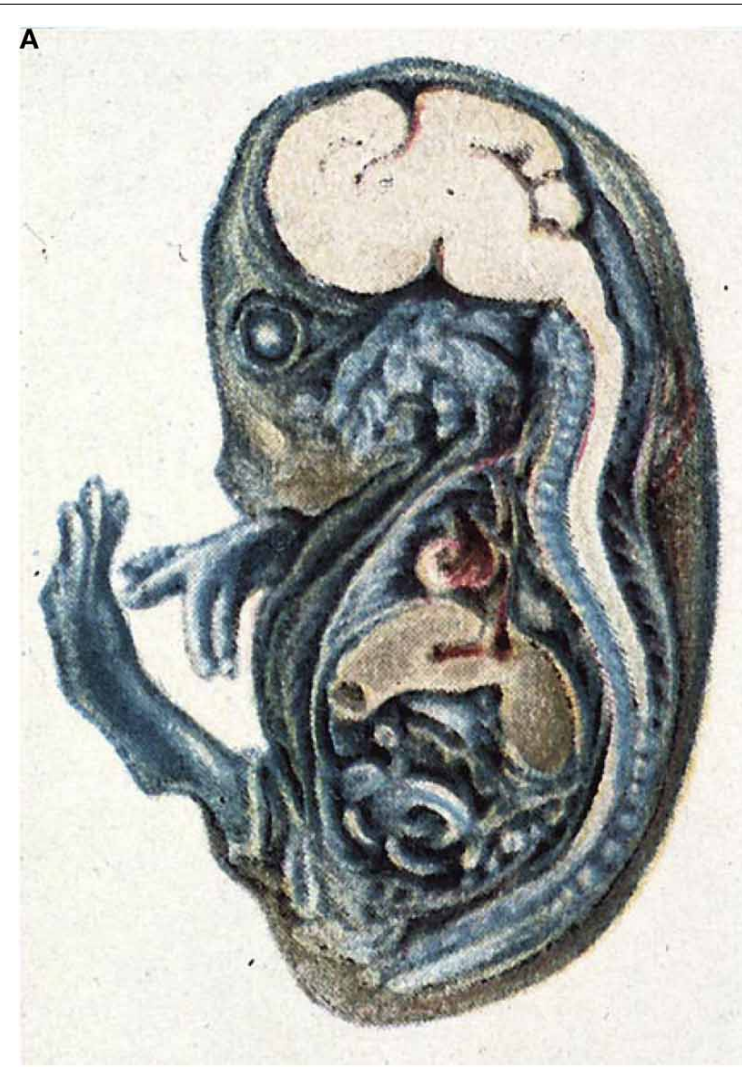

FIGURE 1 | Early demonstration of blood-brain barrier phenomenon in developing brain. (A) Mid gestation guinea pig embryo injected with trypan blue (Wislocki, 1920). Note lack of staining of brain and spinal cord as previously described in adult animals injected with trypan blue (Goldmann, 1909), methylene blue (Bouffard, 1906) and trypan red (Franke, 1905). (B) Very early pig embryo (9 mm, E19) injected with

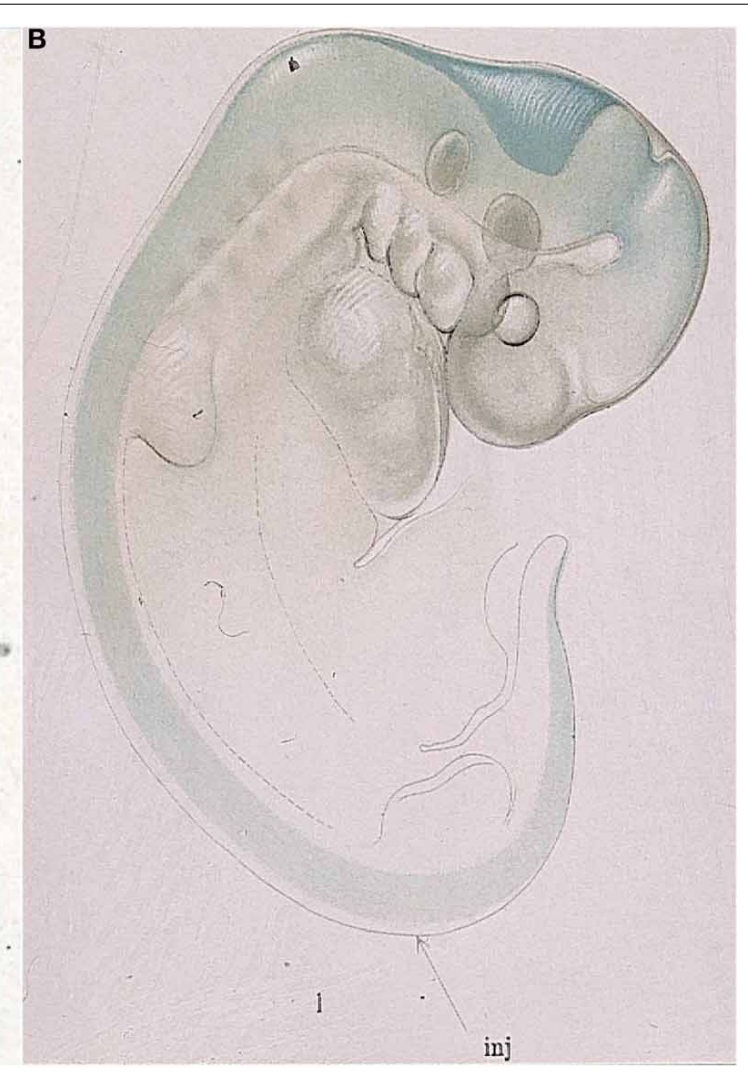

isotonic sodium ferrocyanide into spinal canal (arrow) treated with acid (Prussian blue reaction). Note that blue reaction product is confined to CSF, with a small amount of diffusion into brain stem and mid brain tissue, but no staining of somatic tissue. This indicates that the CNS is already a closed compartment separate from the rest of the embryo. From Weed (1917b). central nervous system (CNS) is separate from the rest of the embryo.

Similar results were obtained in chick embryos by Cohen and Davies (1938). The experiments of Wislocki, Weed and Cohen and Davies are the developmental equivalent of what are sometimes described as Goldmann's 1st and 2nd experiments, in which he reported iv injection (1909) and intrathecal injection (1913) of trypan blue in adult animals of various species. The evidence from the experiments of Weed, Wislocki, Chen and Davies in developing animals for the existence of a barrier between the blood and brain and blood and CSF was as convincing as Goldmann's experiments in adult animals. Yet at some point a belief, which has persisted for nearly 100 years, arose that in embryos, fetuses and even in the newborn and older, the blood-brain barrier is absent, leaky or immature (e.g., Statz and Felgenhauer, 1983; Adinolfi, 1985; Davies and Rudd, 1994; Rodier, 1994, 1995, 2004; Ahmed et al., 1996; ATSDR, 1999, 2007, 2014; Järup, 2003; Costa et al., 2004; Watson et al., 2006; Mhanna et al., 2008; Baldrick, 2010; Ehman, 2010; Srinivasan et al., 2012). These and other authors appear to equate immaturity with "leakiness." The developing brain in all respects is by definition yet to reach a mature adult state and therefore can rightly be described as immature. But this does not tell us whether its brain barriers are "leaky." The real question is their functional status, which seems to us to be more a matter of the relation between barrier functions (which are much more than the presence or absence of passive permeability) that are present at any particular age and stage of brain development. We wondered why such a dogma continues to be accepted in view of overwhelming experimental evidence to the contrary gathered over many decades, for as pointed out by Dobbing (1961): “There has never been a shred of satisfactory evidence in favor of this proposition."

The general explanation seems to be a philosophical rather than an experimentally based one. There seems to have been an underlying assumption, often not clearly enunciated, that because the embryonic and newborn brains are developing, adult barrier mechanisms would necessarily be immature or absent. This was most explicitly stated in "The brain and its environment" by the English physiologist Barcroft (1938): "There is no reason why the brain of the embryo should require an environment of very great chemical constancy. It will of course require a certain minimum of the various materials necessary for growth, but otherwise on first principles we might suppose that the good things of life may exist in and may vary in fetal blood to an extent 
much greater than in the maternal." However, this is a teleological argument, implying need/requirement or in this case, lack of need/requirement. One could as well argue that a complex organ like the brain would need (require) a specialized environment in which to develop rather than the general "soup" of the embryo as a whole. However, neither argument has any intellectual merit as they are based on opinion rather than experimental evidence. More recently, and in the face of much evidence to the contrary since Barcroft's time, Zheng (2002) has put forward a similar teleological argument: "During early development, the rapid growth of the cerebral cortex perhaps necessities a "leaky" structure of the $\mathrm{BBB}$ to accommodate the high demand of blood-borne nutrients for brain growth." However, as we discuss below, the greater entry of some amino acids into the developing brain is a reflection of much higher expression of their transporters in brain endothelial and choroid plexus epithelial cells. This higher expression, which indicates a developmental specialization, is likely to be a reflection of the importance of amino acids for developing brain rather than a manifestation of a non-specific leak.

\section{CONCEPT OF THE BLOOD-BRAIN BARRIER}

Before dealing with experimental evidence of brain barriers during development we need to consider the concept of the adult blood-brain barrier itself. This is often attributed to Ehrlich on the basis of his experiments with dyes (e.g., Gröntoft, 1954; Bakay, 1956; Dempsey and Wislocki, 1955; Lee, 1971; Davson et al., 1987; Risau and Seulberger, 1990; Risau and Wolburg, 1990; Hawkins and Davis, 2005; Nag, 2011). However, as pointed out by Bradbury (1995); Ehrlich $(1885,1906)$ himself never subscribed to the view that the lack of staining of the brain by parenterally administered dyes could be ascribed to some special property of the cerebral endothelial cells. This idea was proposed by Biedl and Kraus (1898); Roux and Borrel (1898), and Lewandowsky (1900) based on comparison of intrathecal and parenteral injections of materials with neurotoxic effects, such as bile salts, after injection by the former route. This was specifically rejected by Ehrlich: "I am unable to accept that the vascular endothelium, as such, exercises different functions in different organs, so that, for example a liver capillary is permeable for certain substances that will not pass through other capillaries" (Ehrlich, 1906). This seems a rather curious conclusion given the description of some of his earlier dye studies (Ehrlich, 1885). In his extensive MD thesis ("Habilitationsschrift") Ehrlich was primarily using dyes as a means of determining the oxygen usage in different organs by their color change (low oxygen or reduction resulting in a colorless state of the dye and on oxygenation resulted in the reverse). He used mainly Alizarin Blue-S (a compound of Alizarin blue and sodium bisulfite) and Indophenol white (which became blue when exposed to oxygen), injected usually subcutaneously into animals of various species. Both of these dyes stained the brain blue, exclusively in gray matter. Under conditions of reduced oxygen (as post mortem) the dyes became colorless. Ehrlich also used dyes treated with acids or heavy metals to give larger particle sizes-alizarin-sodium sulfite-alum and Coerulin-S. These did not stain the brain following subcutaneous injection. To explain this Ehrlich proposed, that "the brain was the most "finely porous" of the organs" he studied. Ehrlich does not say if these "pores" were in blood vessel walls or some other interface between blood and brain tissue. Later Ehrlich (1906) reported on a much larger number of dyes and concluded that acid dyes (with the exception of alizirin) did not stain the gray matter of the brain whereas many basic dyes did. He suggested that dye experiments could be explained by lack of affinity of brain tissue for the dyes rather than to a special property of brain blood vessels. So perhaps Ehrlich could best be described as the reluctant discoverer of the bloodbrain barrier phenomenon, but not as the originator of the term "blood-brain barrier."

Many authors, including Spatz (1934), Broman (1941), Davson (1967), Davson et al. (1987), Hawkins and Davis (2005), Engelhardt (2003, 2006), Ribatti et al. (2006), Saunders et al. (2012) have attributed the term "Blut-Hirnschranke" (bloodbrain barrier) to Lewandowsky's (1900) publication.

Lewandowsky (1900) used strychnine and sodium ferrocyanide, in much higher doses than the potassium ferrocyanide used by Weed (1917b), who stated that in his experiments it was not toxic. Lewandowsky demonstrated in a variety of animal species that the toxins were effective in much lower doses when injected into the brain than parenterally. He also attributed the lesser penetration of strychnine and sodium ferrocyanide into the brain following subcutaneous injection compared to subarachnoid injection to a difference in the properties of the cerebral vessels compared to other tissues: “... die Hypothese dass die Cappillarwand den Uebertritt bestimmter Stoffe, wie des Natriumferrocyanats verhindert, oder mit anderen Worten, dass seine Affinitat der Capillarzellen des Centralnervensystems nothwendig ist um bestimmte Stoffe an die Nervenzellen hindurchgelangen zu lassen.": the hypothesis that the capillary wall blocks the transfer of certain molecules like sodium ferrocyanide or in other words, that an affinity of capillary cells of the CNS is necessary in order to allow the transfer of certain molecules to nerve cells. A close reading of Lewandowsky's original paper reveals that the term "Blut-Hirnschranke" was not actually used, although as indicated by the above quotation, Lewandowsky clearly proposed that cerebral capillaries have specific restrictive properties with respect to some compounds.

The first use of the term "barrier" appears to be by Stern and Gautier (1918a,b), the latter paper is entitled "La barrière qui s'oppose au passage dans le liquide céphalo-rachidien de substances circulant dans le sang présente des différences notables suivant les espèces animales": The barrier which opposes the movement into the CSF of substances in the blood shows notable differences in different animal species (Stern and Gautier, 1918b). Stern and colleagues subsequently performed detailed studies of penetration of a wide range of molecules from blood into cerebrospinal fluid (Stern and Gautier, 1921) into brain (Stern and Gautier, 1922) and within the subarachnoid and ventricular CSF (Stern and Gautier, 1923) of adult animals. It was Stern who first described the importance of the interstitial fluid environment of the brain for its normal function, very much in the tradition of Claude Bernard's milieu interne (1865).

Malamud et al. (1928), Franceschini (1929) and Joó (1987) appear to be among only a few authors to have acknowledged that Stern was probably the first to coin the term blood-brain barrier ("barrière hémato-encéphalique," Stern and Gautier, 1921). Stern 
and Gautier were careful to point out that this term did not prejudge either the anatomical nature or functional mechanisms involved. The term "blood-brain barrier" has withstood the test of time, notwithstanding that to people outside the field it is misleading in that it implies an absolute impediment to entry into the brain and does not make it explicitly clear that exchange mechanism are involved-exactly as supposed by Stern when she proposed the term. However, Stern's important contributions to the field of blood-brain barrier biology have either been largely ignored or belittled (see below).

In addition to Lewandowsky's 1900 experiments, Biedl and Kraus (1898) injected bile and bile acids either systemically or directly into the brain. Only in the case of the latter did they see any toxic effects. They ascribed the ineffectiveness of systemically injected bile salts to "the relative impermeability of the capillary walls," although they did not use the term "barrier" to describe this effect. Roux and Borrel (1898) reported on the comparison of subcutaneous and subdural injections of tetanus toxin with the finding that much less toxin is required to provoke central manifestations of tetanus, but only in rabbits; other species were unaffected. Their explanation for the difference was "sans doute, à ce que beaucoup du poison introduit n'arrive pas aux cellules nerveuses et est detruit quelque part dans l'organisme.": without doubt little of the toxin reaches nervous tissue and is destroyed elsewhere in the organism. However, they did not comment on the mechanism that prevented toxin from reaching the brain.

Amongst the most cited papers for the concept of the blood-brain barrier are those of Goldmann (1909, 1913). He compared distribution of the dye trypan blue following parenteral or intrathecal injection. These are sometimes referred to as Goldmann's first and second experiments (Davson, 1976; Bradbury, 1979). Goldmann found that parenterally injected dye did not stain the brain (Goldmann, 1909), whereas it did when injected into the subarachnoid space, (Goldmann, 1913) as illustrated in Figure 2. Goldmann is usually given the credit for being the first to use a dye to demonstrate that some dyes administered parenterally do not enter the brain. Goldmann himself acknowledges earlier dye studies of Bouffard (1906) but incorrectly states that Bouffard used trypan blue. Bouffard makes an unreferenced mention of even earlier studies with trypan red and Congo red and describes his own studies with methylene blue. Bouffard (1906) injected this dye by various routes (subcutaneous, intravenous, intramuscular or intracardiac) into mice, guinea pigs and rabbits in single or multiple doses of increasing size. He reported unequivocally that there was no staining of the nervous tissue: "SYSTEME NERVEUX.-Aucune trace de couleur, ni dans les cellules nerveuses, ni dans les éléments
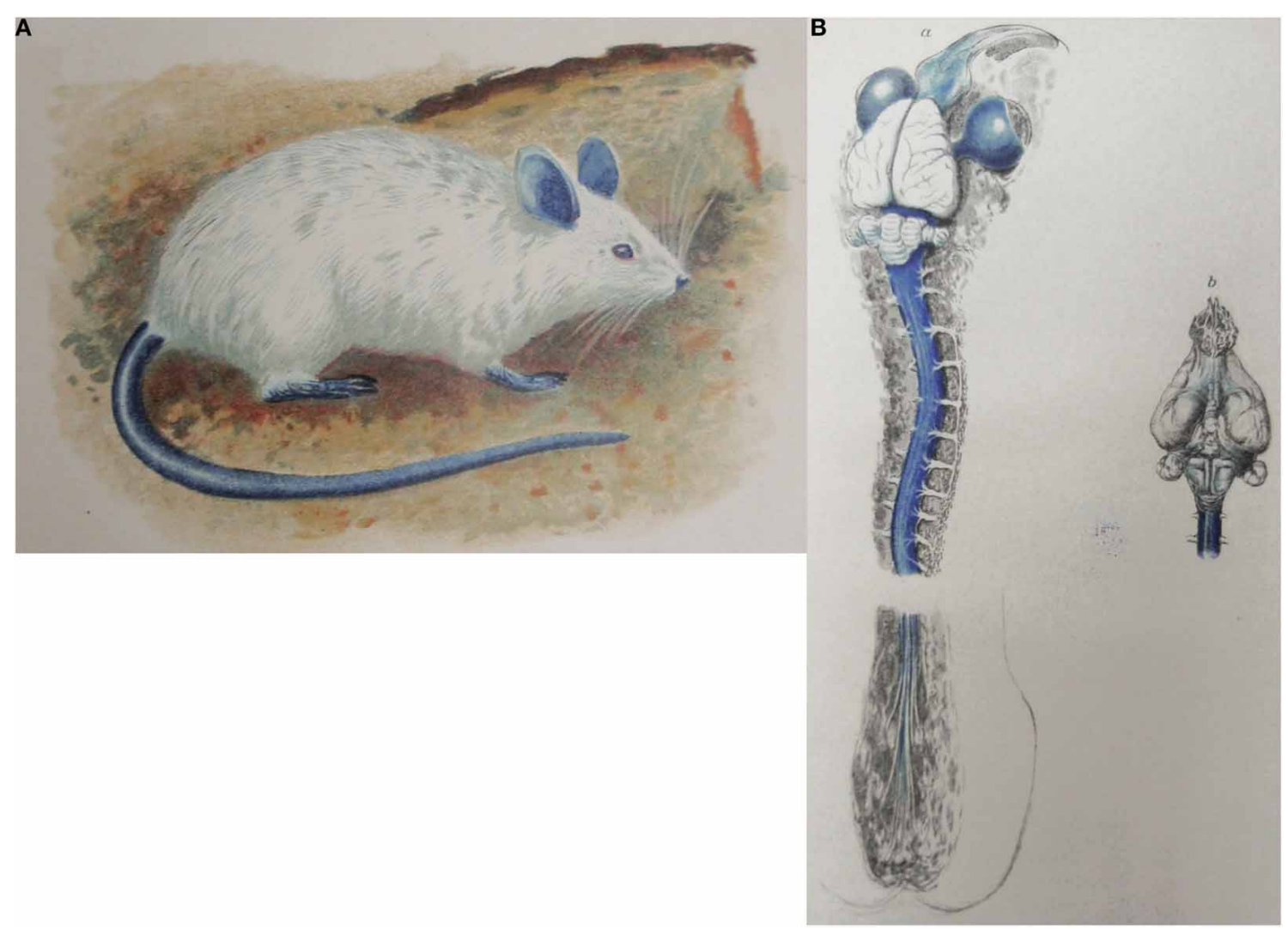

FIGURE 2 | Goldmann's trypan blue injection experiments. (A) Adult rat following systemic injection of trypan blue solution (Goldmann's "1st experiment"). From Goldmann (1909). (B) Brain and spinal cord of adult rat following lumbar subarachnoid injection of $0.5 \mathrm{ml}, 0.5 \%$ trypan blue (Goldmann's "2nd experiment"). From Goldmann (1913). 
névrogliques." Bouffard did not report any toxic effects of methylene blue and did not seem to suggest any mechanism to explain the lack of staining of the nervous system. Bouffard (1906) mentioned, but did not reference, an even earlier study by a student of Ehrlich's, Franke, who used trypan red. According to Bouffard, Franke also showed that the dye did not enter the brain although it did enter most other tissues and organs. This citation appears to have been taken from Franke's (1905) thesis, in which Franke stated "Das Trypanrot gehört zu den polytropen Farbstoffen im Sinne EHRLICHS; es wird entsprechend den Beobachtungen, die bei anderen saueren Farbstoffen gemacht worden sind, von allen Organen mit Ausnahme des Nervensystems, aufgenommen": Trypan red belongs to the polytropic dyes according to Ehrlich; it is taken up,- as observed with other acidic dyes, by all organs except the nervous system. None of these authors seems to have offered any explanation of the lack of staining of the brain by the different dyes; indeed Goldmann (1913) appears to have been surprised by this observation: "Es ist mir rätselhaft dass grosse Farbstoffmengen, welche bei der intravenösen Applikation in den Gehirngefässen zirkulieren, selbst die feinste Kapillarwand nicht zu durchdringen vermögen": I fail to understand why high concentrations of dye circulating in the blood vessels after intravenous injection are unable to penetrate even the walls of the finest capillaries (Goldmann, 1913, p. 45).

Goldmann (1913) did however, remark on the strong staining of the choroid plexus with trypan blue and suggested this represented the site at which dyes were prevented from entering the brain. Goldmann $(1909,1913)$ reported toxic effects with injected trypan blue and commented that the toxic effects that occurred following subarachnoid injections of the dye were made with amounts of trypan blue that were much less than when injected intravenously, particularly in rabbits. Because the choroid plexus stained very strongly with the dye, Goldmann suggested that it was primarily the choroid plexus that provided a protective mechanism for the brain and that this was the route by which some substances entered the brain. This subsequently led to the use of the term "Weg über den Liquor," although not by Goldmann or by von Monakow to whom it is sometimes attributed.

But Goldmann clearly thought that the choroid plexuses had a protective role in preventing entry of toxic materials into the brain: "Der Plexus vermag andererseits die Zerebrospinalflüssigkeit und damit die Nervensubstanz vor dem Eindringen von Substanzen zu schützen, die sich bei der direkten Einfuhr in den Subarachnoidealraum als ein schweres Gift für die Ganglienzellen erweisen.” (Goldmann, 1913, p. 54): On the other hand the choroid plexus is capable to protect the liquor and therefore also the nervous substance against the influx of substances which when directly transferred into the subarachnoid space are a strong toxin for the ganglionic cells. He then summarizes his view of the choroid plexus as a protective regulatory mechanism: "Wir kommen somit zu dem Schluß, dass der Plexus ein wichtiger Schutz-und Regulationsmechanismus für das zentrale Nervensystem darstellt, und dass differenten Substanzen Tür und Tor für ihren Eintritt in das zentrale Nervensystem geöffnet werden, wenn die physiologische Eintrittspforte, der Plexus chorioideus, umgangen wird und solche differente Stoffe durch die Lumbalpunktion in eine direkte Berührung mit den Nervenelementen gebracht warden.” (Goldmann, 1913, p. 55):
Thus we conclude that the plexus is an important protective-and regulatory mechanism for the CNS and that various substances will freely enter the CNS if the physiological gatekeeper, the choroid plexus, is circumvented by a lumbar puncture, those substances will come into direct contact with the nervous elements. However it should be noted that Goldmann makes no mention of any protective function of cerebral blood vessels.

\section{ROUTE OF ENTRY FROM BLOOD INTO BRAIN}

Stemming from the studies of Goldmann $(1909,1913)$ described above and supported by von Monakow (1921) there came the suggestion that the choroid plexuses, rather than the cerebral blood vessels, were the CNS protective mechanism and the primary route of entry of nutritive substances into the brain (described by the term "Weg über den Liquor" by later authors). However, this proposed nutritive function of the choroid plexus seems to have been current earlier than this (see Meek, 1907). Stern in particular initially supported this idea in her interpretation of extensive studies of entry of a wide range of molecules from blood into CSF (Stern and Gautier, 1921) and brain (Stern and Gautier, 1922) in which she emphasized the importance of the choroid plexuses in this role. Davson $(1956,1976)$ considered this an untenable hypothesis and dismissed the idea as "intellectually inadequate" as it did not take account of substantial vascular circulation of the brain parenchyma. This view was shared by Friedemann (1942): "It is utterly unlikely that in an organ of the vital importance of the brain the capillaries should lack their chief uses, namely, mediation of the exchange between blood and tissue and adaptation of blood supply to functional needs" (Friedemann, 1942, p. 126). The idea was also dismissed by several other German authors (e.g., Friedemann and Elkeles, 1931, 1934; Walter, 1934). However, this dismissal is not justified, as these authors have ignored or miss-cited Stern and Rapoport's short papers (e.g., Stern and Rapoport, 1928a,b) from which it is clear that they believed in the existence of two routes of entry from blood into brain: one directly across the blood vessels (endothelial blood-brain barrier) and the other via the choroid plexuses (Goldmann and Stern's blood-brain barrier, now referred to as the epithelial blood-cerebrospinal fluid-barrier). For example, Stern and Rapoport (1928a) reported that in animals subjected to carbon monoxide poisoning, dyes entered brain tissue directly across the cerebral blood vessels, but only sodium ferrocyanide could be detected in the CSF, which was interpreted as suggesting two different routes of entry into the brain, one (for trypan blue or similar colloids) at the vascular endothelial cells of brain capillaries, the other (for sodium ferrocyanide) via the plexuses.

In Geneva Lina Stern, who was a physiologist and biochemist, did not have anybody in her laboratory to perform a thorough post-mortem histology of the brain of the experimental animals. In Moscow she found a competent neuropathologist/histologist Yakov Rapoport, who could localize which cells in the brain had been colored by the injected trypan blue or had been revealed by the Prussian blue reaction. His contribution was therefore of great importance. Unfortunately their cooperation came to an end in the early 1930's. It seems likely that the two disagreed on the respective roles of the blood-CSF and blood-brain barriers. 
In contradistinction to her previous short papers with Rapoport, on the "two barriers model," Stern in a short paper (1934) focused her attention on the blood-CSF barrier (which she calls the barrière hémato-encéphalique). Stern admits that the use of dyes or of other yes-or-no biological markers to assess the permeability of the barrière hémato-encéphalique (bloodCSF barrier) is open to criticism. "En effet il n'est guère possible de tirer des conclusions sur le comportement du liquide en partant simplement de la pénétration ou de la non-pénétration d'une substance donnée présente [dans le plasma sanguin].": Actually the composition of the liquid cannot be directly deduced from the passage or non-passage of a substance present in blood plasma.

According to Stern (1934), the barrier has dual roles of protection and metabolic regulation of the brain. She also introduces the notions of barrier selectivity and barrier resistance. Thus in this 1934 paper, which was one of the last published in French, Stern makes a very clear statement about the importance of the blood-brain barrier in not only providing protection for the brain but also an appropriate physico-chemical environment for normal brain function: "Partant de l'idée que le liquide céphalorachidien represénte le milieu nutritif immédiat des éléments nerveux cérébro-spinaux et que la constance relative de la composition chimique et de l'état physico-chimique de ce milieu est une condition sine qua non du fonctionnment normal des centers nerveux, nous avons attributé un rôle de premier plan à l'activité de cet appareil régulateur que représente la barrière hématoencéphalique.": Starting with the idea that the CSF represents the immediate nutritive medium for the cells of the brain and spinal cord and that the relative constancy of the chemical composition and physico-chemical state of this medium is the sine qua non for normal function of the CNS we have attributed a primary role for this regulatory activity to the blood-brain barrier. The concept of the blood-brain barrier providing mechanisms that control the internal environment of the brain is a major physiological concept that was decades ahead of its time and really only began to be properly accepted and investigated with the advent of radioactive tracers in the 2 nd half of the 20th Century as outlined below.

Stern (1934) does not mention the relative importance of the cerebral vessels (blood-brain barrier) and choroid plexuses (blood-CSF barrier) in these dual functions of protection and regulation of the internal environment of the brain. This remains a technical difficulty to this day. It does seem highly unsatisfactory that later authors did not take account of Stern's direct studies on permeability of the cerebral blood vessels under both normal and pathological conditions. Another extremely important point made by Stern (1934) was her insistence on the necessity of appreciating that the barrier does not have uniform properties with respect to the wide range of molecules that she studied in earlier works: “...le fonctionnement de la barrière ne présente pas un caractère uniforme vis à vis de toutes les substances examinées...": the function of the barrier is not uniform with respect to all substances studied. This is an extremely important point, which is often still not realized to this day.

In contrast to the adult brain, in the developing brain it is now clear that the choroid plexuses are indeed the main portal of entry into the brain (Johansson et al., 2008). As will be recounted below, after returning from Geneva to Moscow in 1925 (Dreifuss and
Sigrist, 2009) Stern made important contributions to the study of brain barriers in developing animals of a number of species in a series of short papers published in the period 1927-1929. If more notice had been taken of Stern's papers, the longstanding belief in the absence of the blood-brain barrier in the fetus and newborn might have been short-lived.

\section{EARLY DYE STUDIES IN IMMATURE ANIMALS}

The important paper of Wislocki (1920), discussed above and illustrated in Figure 1A, was rarely cited in most of the literature of the 20th Century, perhaps because it did not fit with the prevailing belief in immaturity of the blood-brain barrier. Even King (1939), who published with Wislocki, does not mention the seminal 1920 paper preferring instead to cite Behnsen (1927) as evidence of barrier immaturity; the deficiencies of the latter study are discussed next. These are perhaps the most cited in support of immaturity or leakiness of the blood-brain barrier (Behnsen, 1926, 1927) particularly his 1927 paper (e.g., Spatz, 1934; King, 1939; Friedemann, 1942; Davson, 1967). In the 1926 paper Behnsen refers to sealing ("Abdichtung") of the barrier in the postnatal animals, and writes in the summary on page 1146 that the blood brain barrier is considerably more leaky (original: "erheblich durchlässiger") than in adult mice as staining with trypan blue appears in young animals more intense generally and also in different places of the CNS compared to the staining pattern of adults. However, in some slightly handicapped adult mice Behnsen also found additional areas in the brain which were stained, that he called those weak spots (orig. "schwache Stellen"), besides those where apparently no barrier exists (orig. "offene Stellen") and puts this in contrast to Goldmann's view that the central nervous is entirely sealed from dye (Goldmann, 1909, 1913). He also suggested: The fact that the blood-brain barrier in the young is more leaky may also relate to immaturity of tissues and cells and a different level of cellular maturity. (orig.: "Im Speicherverhalten der nervösen Zellen zeigen sich Unterschiede, die ... auf einen verschiedenen Reifezustand der Zellen zu beziehen sind," Behnsen, 1926. p. 1146). It may be that Behnsen's finding of dye in some parts of the brain was due to the use of an excessive amount of dye (three injections of 1\% trypan blue).

Behnsen's paper of 1927 is longer and more detailed and the results and interpretations differ in some respects to the 1926 paper. He speaks more cautiously about an increasing sealing of the barrier during postnatal development and points out that even in adult mice staining can be found in the brain due to local penetration of dye. He commented: "The sites of dye penetration in the adult animals correspond to the maximal storage in young mice. They are located 1. In the region of Retzius'ependymal wedge in the medulla oblongata; 2 . At the attachment sites of the choroid plexus; 3. In the infundibular recess" (Translation of orig.: "Die Farbstoffdurchtrittstellen der erwachsenen Tiere entsprechen den Speicherungsmaxima der jungen Mäuse...").

Behnsen (1927) himself does not appear to state unequivocally that the barrier is immature in the newborn and his experiments have a number of curious features. As was the case for many barrier studies in the early part of the 20th Century, they involved multiple injections of trypan blue into postnatal (2-3 
week old) and adult mice. He reported that areas of the brain that stain for trypan blue at postnatal ages were more extensive than in the adult, but they are located at the same sites, which Behnsen calls "Grenzflächen" i.e., interfaces/membranes between brain and bloodstream or even more generally used by Behnsen: membranes involved in barrier functions are of the same structure in young and adult animals, they only differ gradually in their function. "Die Übereinstimmung der Speicherstellen der erwachsenen Tiere mit denen der jungen macht es sehr wahrscheinlich, daß die Grenzflächen im erwachsenen Zustand, nur graduell verschieden, dieselben örtlichen Bauunterschiede aufweisen, wie sie das Speicherbild für junge Tiere offenbarte." (Behnsen, 1927, p. 570): The identical storage sites (of dye) in adult animals and in young ones makes it highly likely that the interfaces ("Grenzflächen") in the adult condition differ only gradually, but are otherwise of the same structure as revealed by the dye storage of young animals.

This is illustrated in two figures reproduced here in Figure 3. The distribution of trypan blue is illustrated in sagittal sections of mouse brain from the two ages. However, it is the same line drawing of an adult brain that is used for both ages. This gives the misleading impression that the distribution of the dye in the postnatal brain was wider than it probably was in the actual microscopical slides. This is because the neonatal brain is less well developed particularly with respect to the neocortex. It is also notable that there is no staining indicated in the postnatal neocortex as this is the region with the least well developed vasculature in the postnatal period compared to the rest of the brain; were the blood-brain barrier to dye to be immature, this is precisely where dye staining would be expected to be apparent. Bakay (1956) commented that although Behnsen's conclusions are frequently misquoted "they do not reveal a generalized increase in permeability of the barrier."

Behnsen's experiments were repeated in careful detail by Moos and Møllgård (1993). They studied the distribution of trypan blue and Evans blue, a dye that superseded trypan blue from about mid-20th century for blood-brain barrier studies. Moos and Møllgård (1993) used a range of concentrations of the dyes some of which exceeded the binding capacity of plasma albumin to which these dyes bind. Binding of dyes to plasma albumin appears to have been overlooked by the blood-brain barrier field until the observations of Tschirgi (1950), although Ehrlich himself commented that the lack of some of his small molecular weight dyes in urine, was probably accounted for by binding to plasma albumin (Ehrlich, 1885). Also, Stern and colleagues (e.g., Stern et al., 1929) made a distinction in guinea pig embryos and neonatal animals of various species between the blood-brain barrier permeability of low molecular weight sodium ferrocyanide and the lack of penetration of trypan blue, which they described as a colloid. Whether this description reflects knowledge of protein binding or aggregation of the dye in blood is not clear. However, the difference between entry of small lipid insoluble molecules (sodium ferrocyanide in early qualitative experiments and sucrose and inulin in later quantitative experiments) and the lack of entry of trypan blue turned out to be a fundamental distinction in properties of blood-brain and blood-CSF barriers, as will be described below.
Stern et al. (1924) studied the entry of sodium ferrocyanide from blood into CSF in various species at different stages of development. They reported that in the guinea pig the barrier to this molecule was already as in the adult, but in species born at a less mature stage of development (rats, mice, cats) sodium ferrocyanide could be detected in the CSF. Stern and Peyrot (1927) extended the earlier study of Stern et al. (1924) examining entry sodium ferrocyanide into the CSF. In guinea pig fetuses this marker did enter the CSF, but as reported earlier not in the newborn of this species. They confirmed the entry of sodium ferrocyanide in the newborns of rats, mice and cats and in addition rabbits.

Stern and Rapoport (1928a) studied the injection of dyes (trypan blue and Congo red) in neonates of various species. They distinguished clearly between a barrier at the level of the vascular endothelium and the choroid plexus epithelium in dye studies where cellular staining following dye injections, but unlike other authors they thought this was a post mortem phenomenon. In a following year using the same dyes Stern et al. (1929) made the important point that it was necessary to limit the amount dye injected. They stressed that in experiments in adults and developing animals using similar amounts of dye, no dye entered the CSF. But if the amounts were increased substantially (30-40 times than used normally) then staining of the CSF occurred.

Another dye study that is sometimes cited as evidence of barrier immaturity in neonates (e.g., Ribatti et al., 2006) is that of Penta (1932). Penta injected multiple doses of trypan blue or trypan red into postnatal rabbits and cats. Penta seems to have considered that the results of Behnsen and Stern were similar, although as described above Stern et al. (1929) found no entry of trypan blue in the brains of developing animals providing not too much dye was injected. Penta summarized his results as showing that the nervous system of neonates is colored more intensely than that of adults and staining occurred in the regions where the mesenchyme penetrates into the neuraxis. As in many of the adult studies using trypan blue and against the advice of Stern (Stern et al., 1929) Penta mainly used multiple large doses of trypan blue and many of the animals died from the toxic effects of the dye; therefore staining of the brain was hardly surprising. However, Penta did describe one experiment in which much less dye was used in which no staining of the brain (apart from the choroid plexus) occurred, thus confirming Stern's results, without Penta apparently realizing it.

There seems little doubt that at least part of the problem with many early dye studies, both in developing animals and adults, was that the large amounts and multiple injections used led to toxic effects. This might be indicative of a greater fragility of brain barriers in immature animals but should not be interpreted as evidence of an immature or absent blood brain barrier.

The binding of dyes to albumin in plasma was investigated systematically by Moos and Møllgård (1993). They showed that dye only entered the brains of neonatal (one day old) mice and rats if the albumin binding capacity was exceeded, except in regions of the brain known to be devoid of a blood-brain barrier. For concentrations of dye (trypan blue, 1-2\%; Evans blue, 0.5-1.0\%) that were shown not to exceed the binding capacity of albumin in plasma, neither dye penetrated into the brain by exudation 


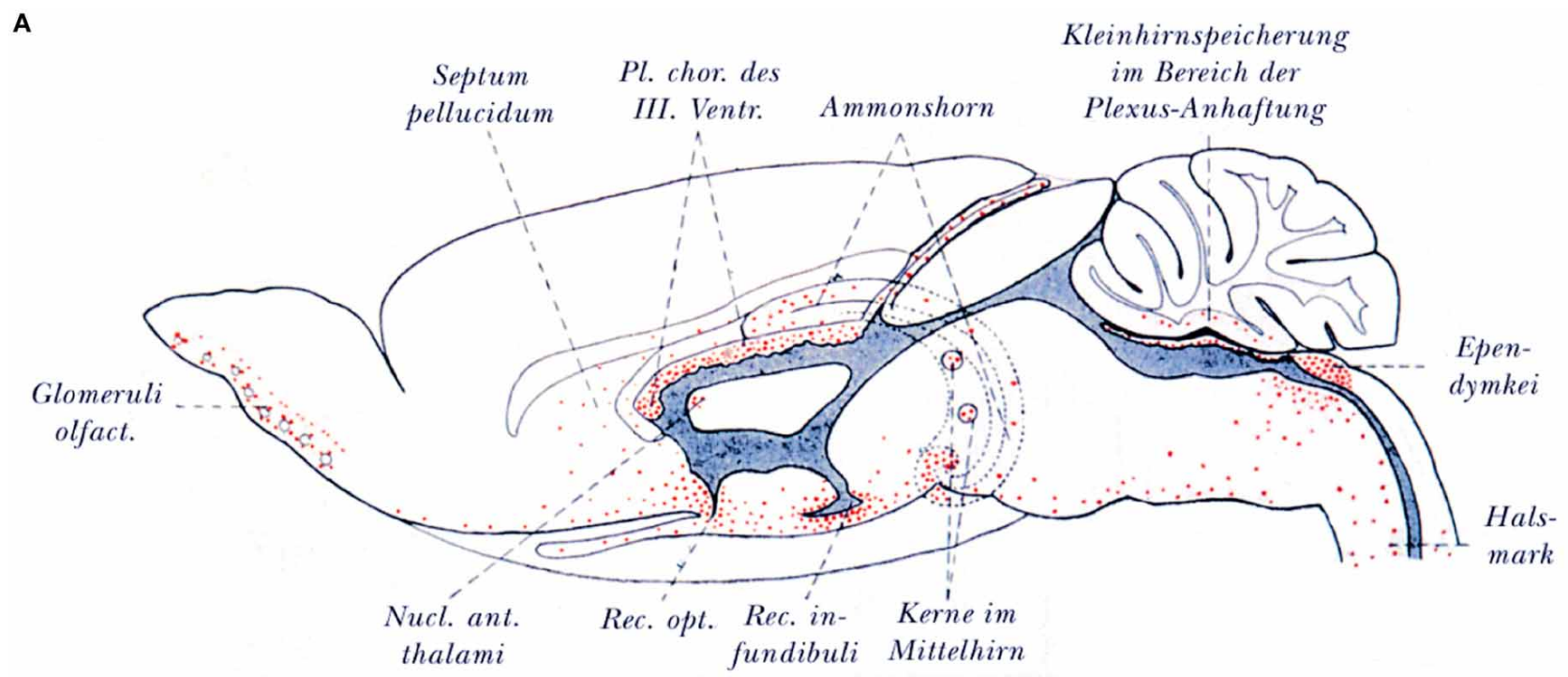

Abb. 1. Nach Serienschnitten angefertigtes Sagittalschema des Mäusehirnes; darin rot einge-
tragen die Speicherorte der jungen ( 2 bis 3 Wochen alten) Maus. (Gez. B. Schlichting.)

B

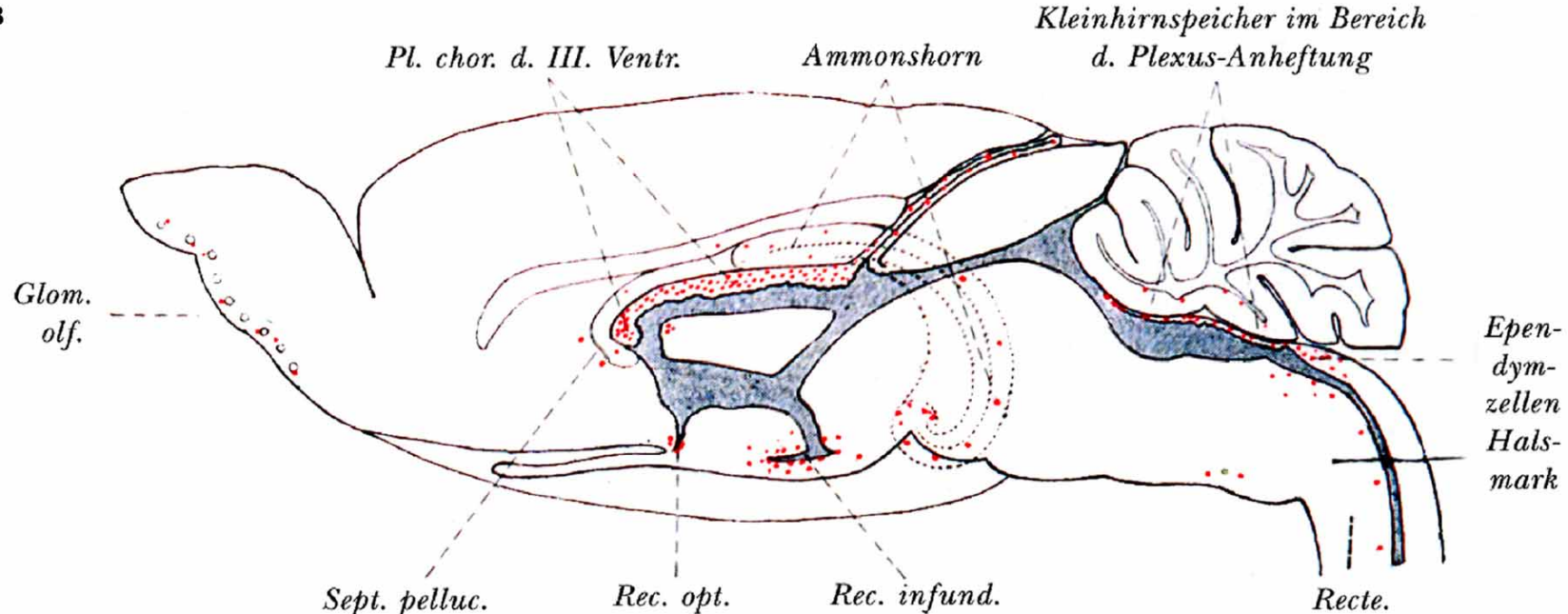

Abb. 9. Dasselbe Sagittalschema des Mäusehirnes wie Nr. I; darin rot eingetragen die Speicherorte der erwachsenen Maus. (Gez. B. Schlichting.)

FIGURE 3 | Behnsen's much cited trypan blue experiments. (A) Brain from postnatal mouse injected systemically 3 times with trypan blue at P4-P14. (B) Adult mouse brain following systemic injection of trypan blue. Note that as described by Behnsen the dye staining in the postnatal brain is in the same regions as in the adult brain, but more extensive. This is probably a visual artifact due to the use of an outline of an adult mouse brain for both ages. In postnatal mouse brain (P14) the cerebral cortex and cerebellum are less developed than illustrated here. Note also that in the postnatal brain there does not appear to be any dye staining of the neocortex-the region of the developing brain with the least mature blood vessels. From Behnsen (1927). across cerebral vessel walls in either P10 or adult mice, although there was some staining of choroid plexus epithelial and cerebral endothelial cells. At higher concentrations of the dyes (trypan blue, 2-4\%; Evans blue, $2 \%$ ) at which there was a substantial level of free dye, $60 \%$ of neonates and $20 \%$ of adults died, with higher proportions at the higher concentrations of dye. Those animals that survived showed various signs of neurotoxicity. Toxic effects of trypan blue had been reported earlier (e.g., Behnsen, 1927; Penta, 1932) but the significance of this in interpretation of barrier studies with dyes seems to have been overlooked by all but 
Stern who urged caution in the amount of dye to be injected into neonatal animals (Stern et al., 1929).

\section{LATER STUDIES OF DYES, ALBUMIN AND OTHER MARKERS IN IMMATURE ANIMALS}

The use of dyes to study blood-brain barrier permeability in immature animals began to die out in the second half of the 20th Century. This is in contrast to the adult blood-brain barrier field, which continues to place a heavy reliance on dyes, particularly Evans blue, for assessing blood-brain barrier integrity. All of the studies published after 1950 agreed in showing that dyes (usually trypan blue) did not enter the developing brain no matter what age was investigated. The most important of these studies is probably that of Gröntoft (1954) who did experiments in both human fetuses (recent abortions) and newborns. Gröntoft emphasized that the condition of the fetuses was very important in determining whether or not the dye entered the brain. In a series of carefully conducted experiments he showed clearly that when trypan blue was injected as soon as possible after separation of the placenta in human fetuses of a wide range of gestational age, the dye did not stain the brain as a whole, but only those areas (choroid plexus and circumventricular organs) that are outside the blood-brain barrier (Figure 4); this was the case even in a very immature embryo of $5 \mathrm{~cm}$ in length (approximately 10 weeks post conception). However, when the dye was injected at progressively longer intervals after placental separation of the fetuses (10-30 min depending upon the gestational age) then the whole of the brain stained. Gröntoft carried out similar well-controlled experiments in rabbit fetuses with the same findings.

Grazer and Clemente (1957) injected by various routes solutions of trypan blue into rat embryos from E10.5 to birth. They found no evidence of dye penetration into the brain. Millen and Hess (1958) injected i.p. two doses, $24 \mathrm{~h}$ apart, of either trypan blue or sodium ferrocyanide, into neonatal (P0-P8) and adult rats and the animals were examined after $30 \mathrm{~min}$. No staining of the brain for either marker was identified in the neonatal animals apart from the restricted regions (outside the blood-brain barrier) that also stained in the adult. Similar results were reported by Olsson et al. (1968) who injected fluorescently labeled bovine albumin intravascularly in rat embryos as early as E15, compared with neonates and adults.

In the second half of the 20th Century there was a switch from using dyes to using horseradish peroxidase (HRP) as a test of barrier integrity in the developing brain. HRP has a number of problems that are not always well recognized by those that use it. In the context of development the main problem is that, as

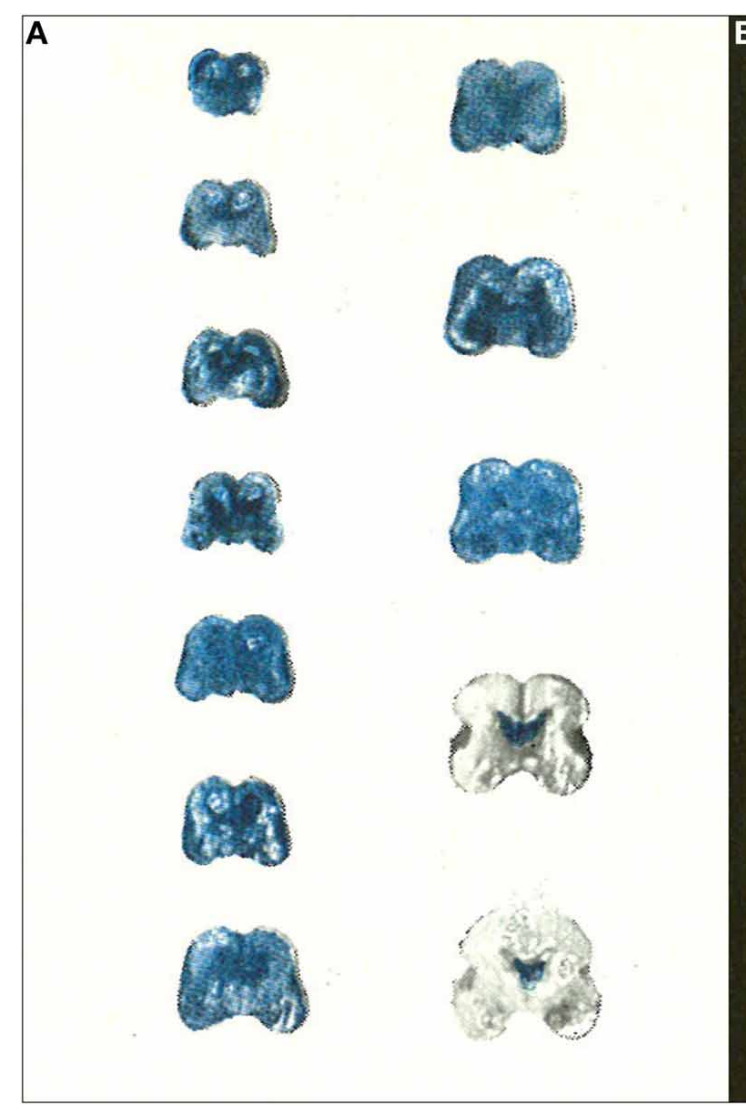

FIGURE 4 | Gröntoft's dye experiments with postmortem aborted human fetuses. (A) Human fetuses ( $14 \mathrm{~cm}-18$ weeks to $30 \mathrm{~cm}-31$ weeks gestation) injected with trypan blue solution $30 \mathrm{~min}$ after caesarian section delivery. (B) Human fetuses ( $14 \mathrm{~cm}-18$ weeks to $30 \mathrm{~cm}-31$ weeks gestation) injected with

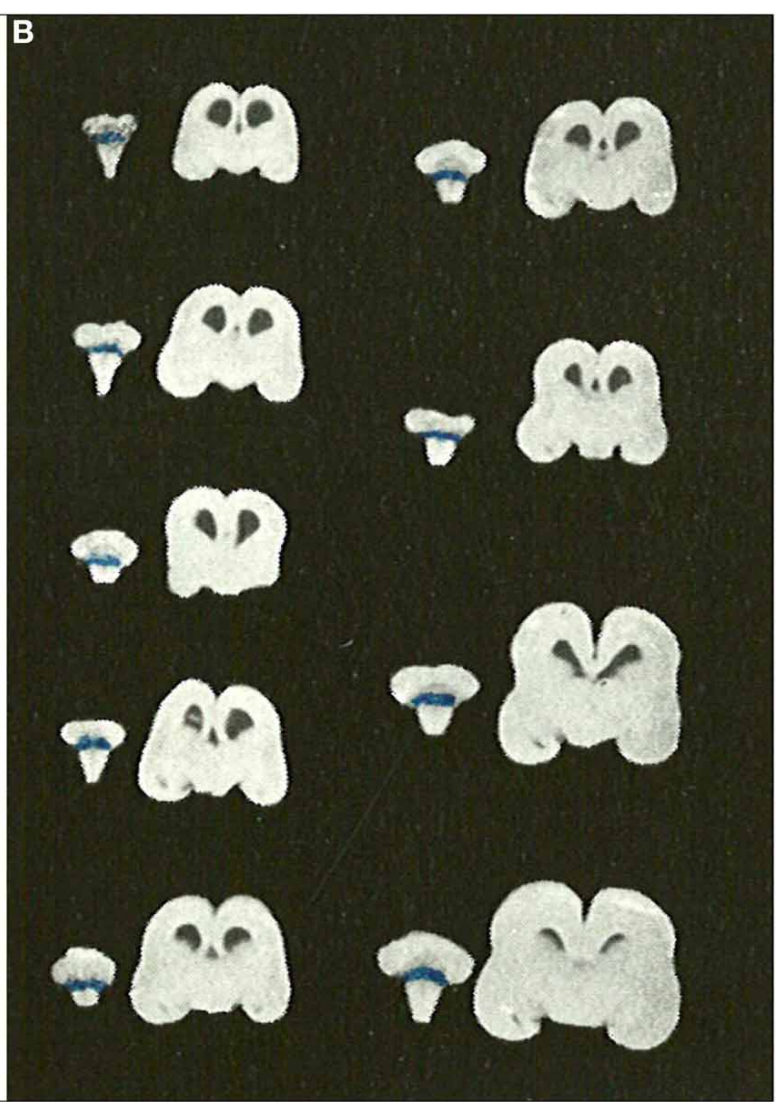

trypan blue solution 10 min after caesarian section delivery. Note that for the shorter period of anoxia none of the brains stained with trypan blue indicating intact blood-brain barrier to trypan blue even in immature human fetuses. Reproduced with permission from Gröntoft (1954), (c) Wiley. 
with the dyes in earlier experiments, too large a volume or concentration of HRP was injected, thus damaging fragile cerebral blood vessels and giving the appearance of a "leaky" blood-brain barrier (see Table 1). Several other authors reported the entry of HRP into brains of very immature embryos, for example chick embryos (Delorme et al., 1970; Delorme, 1972; Bertossi et al.,
1992). However, insufficient detail of exactly what was injected makes it difficult to evaluate the results of these studies. The finding that HRP entered the brain at different embryonic ages in the same species or at different stages of brain development in different species suggests that the findings from these studies are likely to have been artefactual. There have been a few reports of

Table 1 | Injection experiments used as test of blood-brain barrier integrity in fetal and neonatal animals.

\begin{tabular}{|c|c|c|c|c|c|c|c|}
\hline Authors & Species & $\begin{array}{c}\text { Age } \diamond \\
\text { (days) }\end{array}$ & $\begin{array}{l}\text { Body } \\
\text { weight }(g)^{a}\end{array}$ & $\begin{array}{c}\text { Injection vol } \\
\text { (\% total blood vol) }\end{array}$ & $\begin{array}{l}\text { Increase in plasma } \\
\text { protein concentration }^{c}\end{array}$ & $\begin{array}{l}\text { "Leaky" barrier } \\
\text { claimed• }\end{array}$ & $\begin{array}{l}\text { Injected } \\
\text { material }^{d}\end{array}$ \\
\hline $\begin{array}{l}\text { Grazer and } \\
\text { Clemente, } 1957\end{array}$ & Rat & E10.5 & $<0.06$ & Not stated & - & No & Trypan blue i.p./i.v. ${ }^{b}$ \\
\hline Olsson et al., 1968 & Rat & E15 & 0.14 & $<5 \%$ & - & No & $\begin{array}{l}\text { Fluorescein- } \\
\text { albumin, Umb.A. }\end{array}$ \\
\hline $\begin{array}{l}\text { Delorme et al., } \\
1970\end{array}$ & Chick & E4.5 & 0.1 & Not stated & $3 \%$ & $\begin{array}{l}\text { Reaction product in } \\
\text { neuropil ECS until } \\
\text { E10 }\end{array}$ & HRP i.v. \\
\hline $\begin{array}{l}\text { Wakai and } \\
\text { Hirokawa, 1978, } \\
1981\end{array}$ & Chick & E8 & 0.5 & $10 \%$ & $500 \%$ & Yes & HRP i.v. \\
\hline $\begin{array}{l}\text { Dziegielewska } \\
\text { et al., 1979, } 1980\end{array}$ & Sheep & E60 & 60 & $7.5 \%$ & $20 \%$ & No & $\begin{array}{l}\text { Human plasma } \\
\text { protein i.v. }\end{array}$ \\
\hline $\begin{array}{l}\text { Lossinsky et al., } \\
1986\end{array}$ & Mouse & Newborn & 1.4 & $35 \%$ & $2 \%$ & $\begin{array}{l}\text { Yes, "massive } \\
\text { leakage" }\end{array}$ & HRP i.v. \\
\hline Roncali et al., 1986 & Chick & E6 & 0.34 & $>150 \%$ & $50 \%$ & $\begin{array}{l}\text { "Unimpeded } \\
\text { extravasation" }\end{array}$ & HRP intracardiac ${ }^{e}$ \\
\hline Risau et al., 1986 & Mouse & E13 & 0.08 & $250 \%$ & $1 \%$ increase & "Fully permeable" & HRP intracardiac ${ }^{e}$ \\
\hline $\begin{array}{l}\text { Vorbrodt et al., } \\
1986\end{array}$ & Mouse & Newborn & 1.4 & $100 \%$ & $200-300 \%$ & Yes** $^{*}$ & HRP i.v. \\
\hline $\begin{array}{l}\text { Stewart and } \\
\text { Hayakawa, } 1987\end{array}$ & Mouse & E15 & 0.26 & Not stated & $100 \% *$ & Yes & HRP i.p. ${ }^{f}$ \\
\hline $\begin{array}{l}\text { Dziegielewska } \\
\text { et al., } 1988\end{array}$ & $\begin{array}{l}\text { Tammar } \\
\text { wallaby }\end{array}$ & Newborn & 0.50 & $10-20 \%$ & $25-50 \%$ & No & HRP/HSA i.v. \\
\hline $\begin{array}{l}\text { Hulsebosch and } \\
\text { Fabian, } 1989\end{array}$ & Rat & Newborn & 5.0 & $50 \% *$ & $>400 \% *$ & $\begin{array}{l}\text { IgG penetration } \\
\text { into neuraxis }\end{array}$ & $\lg G$ i.p.p \\
\hline $\begin{array}{l}\text { Dermietzel et al., } \\
1992\end{array}$ & Rat & E14.5 & 0.14 & $100-150 \%$ & $>100 \%$ & Yes & $\begin{array}{l}\text { HRP or } 40 \mathrm{kDa} \\
\text { dextran } \\
\text { intracardiac }^{\mathrm{d}}\end{array}$ \\
\hline Ribatti et al., 1993 & Chick & E6 & $0.36^{a}$ & $>150 \%$ & $50 \%$ & $\begin{array}{l}\text { "Massive } \\
\text { diffusion" }\end{array}$ & $\begin{array}{l}\text { HRP or Evans blue, } \\
\text { intracardiac }\end{array}$ \\
\hline Xu and Ling, 1994 & Rat & Newborn & 5.0 & $10 \%$ & $65 \%$ & Yes & Ferritin solution i.v. \\
\hline Knott et al., 1997 & Opossum & P3 & 0.20 & $8 \% *$ & $0.5 \% *$ & No & Human albumin i.p. \\
\hline
\end{tabular}

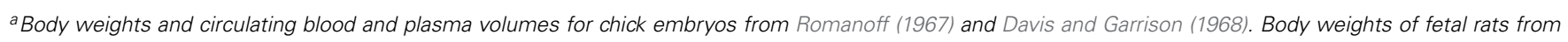
unpublished data. Body weights of opossum from Saunders et al. (1989) and unpublished. Weight of youngest animal given.

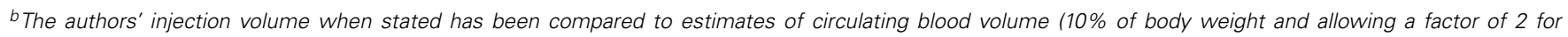

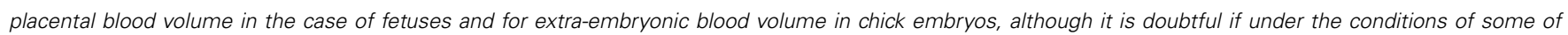
these experiments, much mixing with fetal placental blood would have occurred.

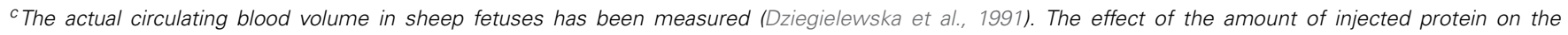

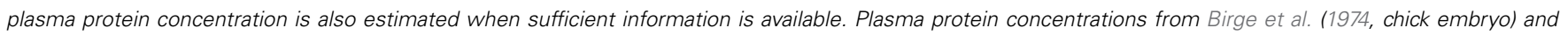
Dziegielewska et al. (1981, rat fetus).

dInformation is not usually available about the speed of injection nor about the likely distribution volume (e.g., in experiments involving intracardiac injection.

e In which the normal circulation may not have continued or i.p. injection.

${ }^{f}$ In which it is not clear what proportion of the volume and protein will have entered the circulation).

$\diamond$ Youngest age studied. E is embryonic age from day of conception.

*Depending on proportion entering circulation. "*But "not detected at E19."

-Not all authors of experiments in which marker entered brain tissue from blood vessels describe this as a "leak" or due to immaturity. 
transendothelial passage of markers such as HRP in developing brain (e.g., in tubulovesicular structures, Lossinsky et al., 1986) which may explain the entry of the marker into immature brain in some experiments, as lack of pinocytotic vesicles is a characteristic of adult cerebral endothelial cells.

\section{ENTRY OF SMALL MOLECULAR COMPOUNDS INTO THE BRAIN AND CSF OF IMMATURE ANIMALS}

Lina Stern and her colleagues published a preliminary report of studies in a range of species of developing animals (Stern et al., 1924). This was followed by a series of brief papers in the period 1927-1929 on the permeability of the "barrière hématoencéphalique" to sodium ferrocyanide and several other low molecular weight compounds in fetal guinea pigs and newborns of several species (Stern and Peyrot, 1927; Stern and Rapoport, 1928a,b; Stern et al., 1929). Following injection into fetuses via the uterine horn of pregnant guinea pigs early in gestation, sodium ferrocyanide could be demonstrated by the Prussian blue reaction in the CSF and CNS tissue. However, when sodium ferrocyanide was injected into term embryo or newborn guinea pigs its presence could not be detected in either CSF or CNS tissue. In contrast, when sodium ferrocyanide was injected into the newborns of rabbits, rats, mice, dogs, and cats it could be demonstrated in CSF and nervous system tissue and for several days into the neonatal period. Stern and Peyrot (1927) correlated the cessation of sodium ferrocyanide entry into CSF and nervous tissue with the time of eye opening and suggested that this was the time when the blood-brain barrier ("barrière hémato-encéphalique") to certain substances reached a "normal value," presumably meaning that it reached the adult condition. They concluded that in species born at less mature stages of development (which they suggested probably included human newborns) the "activity" of the barrier was insufficient to protect the CNS against many substances circulating or introduced into the blood.

These qualitative observational results with a small molecule (sodium ferrocyanide $304 \mathrm{Da}$ ) foreshadowed quantitative experiments with radiolabelled small molecules such as mannitol (182 Da), sucrose (342 Da), and inulin (5200 Da) in newborns of rats (Ferguson and Woodbury, 1969; Woodbury et al., 1974; Johanson, 1989; Habgood et al., 1993) and fetal sheep (Evans et al., 1974; Dziegielewska et al., 1979), which showed higher $\mathrm{CSF} /$ plasma and brain/plasma ratios for the these molecules in immature animals. Although initially interpreted as indicating greater permeability of the blood-brain and blood CSF barriers, it became apparent from measurements of CSF secretion in embryos (Evans et al., 1974; Fossan et al., 1985) and newborns (Bass and Lundborg, 1973; Johanson and Woodbury, 1974) and increase in volume of CSF in rat embryos (Johansson et al., 2006, 2008) that the explanation is more likely to be that the much lower turnover (sink effect) of CSF in the developing brain allowed a greater accumulation of marker in CSF and brain (Bass and Lundborg, 1973; Johansson et al., 2008). However, it took the development of the use of small molecular weight markers that could be both quantified and visualized at the electron microscopic, EM level to show that the route of penetration from blood to CSF of these small markers was via an intracellular route in a subpopulation of epithelial cells in the choroid plexuses (Ek et al.,
2001, 2003, 2006). These cells appear to be responsible for the transfer of inert dextrans and several plasma proteins from blood into CSF both in the developing and adult brain (Dziegielewska et al., 1991; Liddelow et al., 2009, 2011a). The absolute number of cells involved in this transfer actually increases with age as the choroid plexuses develop, but the CSF/plasma ratios for dextrans and proteins fall, because of the increased turnover of CSF and a larger space into which these markers are diluted (Johansson et al., 2006, 2008).

\section{KERNICTERUS IN THE NEWBORN}

One of the persistent beliefs about the blood-brain barrier in the immature organism is that the toxic effects of unconjugated bilirubin that accumulate in the blood of newborn infants suffering from erythroblastosis, generally due to Rhesus blood group incompatibility, are due to "immaturity" of the blood-brain barrier in the newborn. This is a suggestion generally attributed to Spatz (1934) e.g., Broman (1941); Davson (1967), and Lee (1971). Others stated that this was the case but without attribution (e.g., Ganong, 1993; Cotran et al., 1994). Bakay (1956) expressed the view that "the phenomenon of kernicterus appeared to offer irrefutable proof of this theory [that the blood-brain barrier in the newborn is immature]." In his later review Bakay is more circumspect and suggests that the causation of kernicterus has many components of which an immature blood-brain barrier might be one (Bakay, 1968).

Most of Spatz's lengthy paper is taken up with describing and repeating some of Behnsen's (1927) experiments with trypan blue. But he does have a short section on icterus (jaundice) in the adult, with an illustration showing that yellow pigment of bilirubin was confined to dura. He compared this (without illustration) to brain damage (kernicterus) that only occurs in the newborn with the deposition of bilirubin in the brain, characteristically in the basal ganglia and brain stem (Figure 5). He does not seem to distinguish between conjugated bilirubin, which accumulates in the circulation of adults sometimes to very high levels, and unconjugated bilirubin that accumulates in newborns because the activity of the conjugating liver enzyme, glucuronyl transferase, is very low at birth and normally increases during the first days of life. These two forms of bilirubin had been identified earlier (van den Bergh and Müller, 1916). What was not known in the 1930s was that unconjugated bilirubin binds tightly to plasma albumin and a key factor (but not the only one) in the occurrence of kernicterus is when the binding capacity of albumin for unconjugated bilirubin is exceeded; this form of bilirubin which is highly lipid soluble (in contrast to conjugated bilirubin) enters the brain without restraint.

It is noteworthy that clinicians, who have had to deal with the problem of kernicterus in the newborn for many years, rarely seem to have invoked the state of maturity of the blood-brain barrier in the neonate as a major factor in the occurrence of kernicterus (e.g., Iskander et al., 2014), although it has occasionally been mentioned (e.g., Zuelzer and Mudgett, 1950; Spiegel-Adolf et al., 1954; Ernster et al., 1957); rather they developed clinical treatments aimed at reducing the level of unconjugated bilirubin in infants who were at risk: exchange transfusion (Allen et al., 1950; Gerrard, 1952) barbiturate induction of glucuronyl 


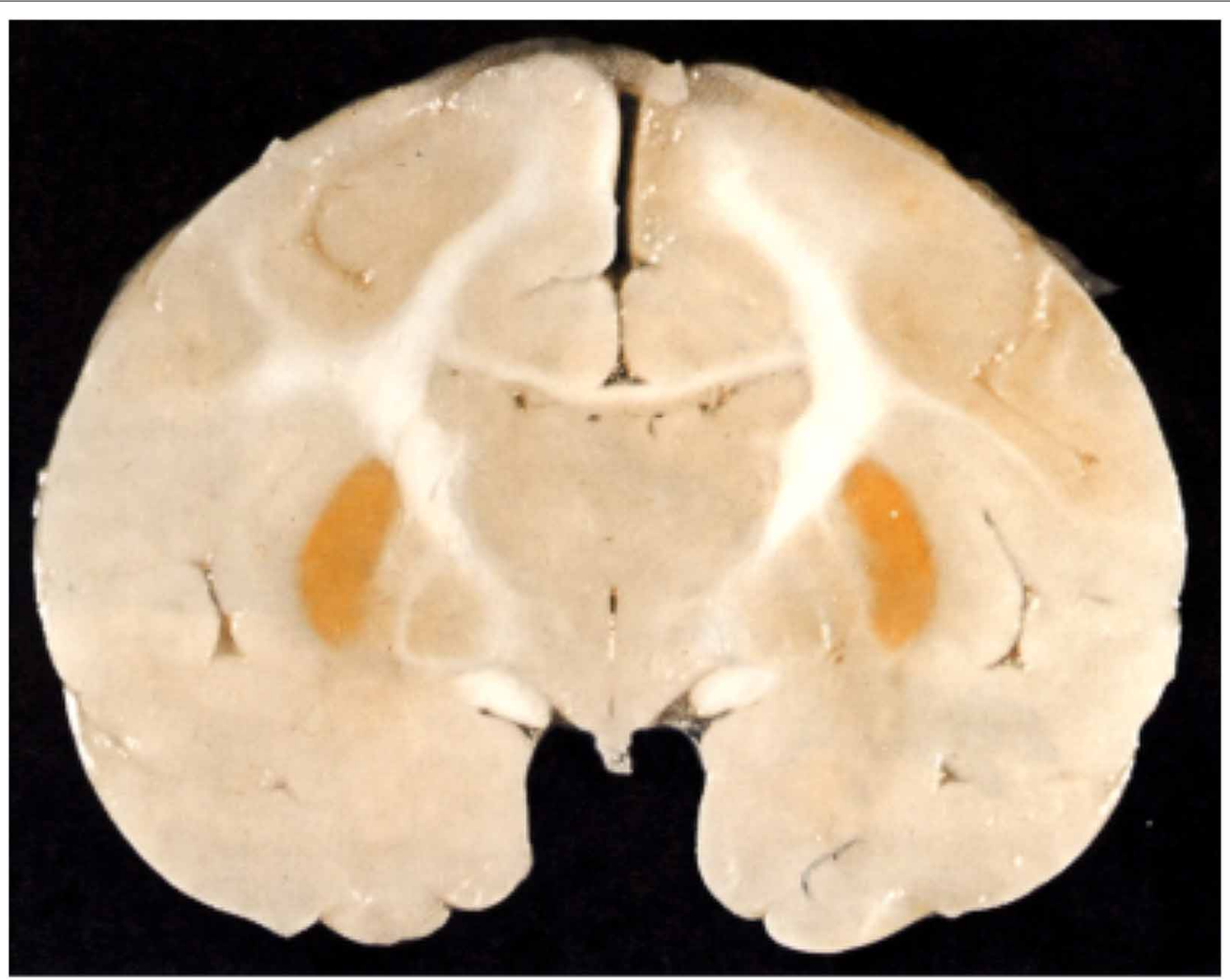

FIGURE 5 | Kernicterus in brain of neonatal monkey with jaundice. Note staining of putamen (one of the basal ganglia), which does not occur in adult brain as jaundice in adults, is generally due to high levels of conjugated bilirubin to which the blood brain barrier is impermeable. From Windle (1969).

transferase and phototherapy (Lucey, 1971) as well as prevention by immunization of $\mathrm{Rh}$-ve women against the $\mathrm{D}$ blood group antigen (Clarke, 1989). But the linking of blood-brain barrier immaturity to kernicterus has persisted amongst other groups of scientists and clinicians for decades after Spatz (1934). A good example is a physiologist, (Ganong, 1993) who stated that: "Bile pigments do not enter the brain in the adult but in the fetus and newborn infant the blood-brain barrier is not developed." Following correspondence with one author (NRS) in later editions Ganong (1999) modified this to state that "the bilirubin penetrates the brain of infants with erythroblastosis at least in part because the blood-brain barrier is more permeable in infancy"; he was not willing to take account of the effect of binding of unconjugated bilirubin to plasma albumin. Similar wording has been retained by the new authors of Ganong's Review of Medical Physiology (Barrett et al., 2012) following his unfortunate demise. Some aspects of bilirubin neural toxicity are discussed by Palmela et al. (submitted).

Some pathologists have also considered that kernicterus is due to immaturity of the blood-brain barrier (Cotran et al., 1994). A detailed consideration of kernicterus is beyond the scope of this review. But comprehensive papers are available both on the pathogenesis and treatment particularly by Wennberg and his colleagues (e.g., Wennberg, 2000; Ahlfors and Wennberg, 2004; Wennberg et al., 2006) and in Volpe (2008). However, it is becoming increasingly clear that the mechanisms involved in whether or not kernicterus occurs in newborns are much more complex than the absolute level of unconjugated bilirubin and the binding capacity of albumin in plasma. These complexities have been reviewed by Ghersi-Egea et al. (2009) and include the finding that bilirubin is a substrate for several ABC efflux transporters, which would thus be expected to contribute to limiting the entry of bilirubin into the brain.

\section{EXPERIMENTS WITH RADIOISOTOPICALLY LABELED MOLECULES IN DEVELOPING ANIMALS}

The introduction of radiolabelled markers was a major advance in experimental approaches to understanding blood-brain barrier mechanisms. The use of inert markers of permeability such as sucrose and inulin has been mentioned above. In this section we shall deal briefly with experiments involving the use of $\mathrm{P}^{32}$ and amino acids labeled with ${ }^{3} \mathrm{H}$ or ${ }^{14} \mathrm{C}$. Compared to inert markers there are potential problems with metabolically active radiolabelled molecules such as amino acids. Thus it needs to be 
shown that the radioactive tag is still attached to the molecule and that the measured radioactivity is due to transfer into the brain separate from its metabolic incorporation, for example into proteins.

\section{${ }^{32}$ PHOSPHORUS}

Bakay $(1953,1956)$ studied the uptake of ${ }^{32} \mathrm{P}$ into brain and other organs following parenteral injection into pregnant (3rd4th week), newborn, up to 7 weeks postnatal rabbits and in the adult. Animals were killed $24 \mathrm{~h}$ after injection and various organs dissected out. In some cases radioautographs of the heads or brains of fetuses were prepared. The results showed a much higher uptake of ${ }^{32} \mathrm{P}$ in the younger the animals. In fetuses and newborns the brain/blood ratio was $>1$, compared to about 0.3 in the adult, which Bakay (1953) interpreted as indicating that the vascular permeability in the fetal brain is increased. He discusses whether the increased permeability reflects a leaky, incompletely formed blood-brain barrier or increased metabolism and faster turnover in the developing nervous system. He concluded that neither explanation was satisfactory. Bakay (1953) observed that in the radioautographs of fetal brain the distribution of ${ }^{32} \mathrm{P}$ was almost uniform whereas in the brain of the mother it was concentrated in the choroid plexuses and the lining of the ventricles. Rather puzzlingly he took this as evidence that there is a bloodbrain barrier to ${ }^{32} \mathrm{P}$ in the fetus but that its permeability is greater than in the adult.

\section{AMINO ACID TRANSPORT}

In early experiments with radiolabelled amino acids there was a similar finding of a much greater entry of some (but not all) into the developing than adult brain of several species (Himwich et al., 1957; Roberts et al., 1959; Purpura and Carmichael, 1960; Lajtha and Toth, 1961; Seta et al., 1972; Baños et al., 1978) but as with ${ }^{32} \mathrm{P}$ it was not possible to distinguish between cerebral endothelial cell transport and metabolic incorporation into brain tissue in accounting for the greater uptake in developing brain. Some authors interpreted such results as indicating "lesser effectiveness" of the blood-brain barrier for amino acids in young animals (e.g., Kuttner et al., 1961; Lee, 1971). The problem of determining cerebral transport of amino acids across the blood-brain barrier without the complication of metabolic contamination of brain samples was solved by Oldendorf (1971) who developed a short pass technique in which exposure of amino acid or other metabolically active molecules to the brain from the cerebrovascular circulating blood was limited to one circulation time, thus being too quick for significant metabolic incorporation to occur. The technique was adapted for newborn and postnatal animals by Oldendorf's students (Braun et al., 1980; Cornford et al., 1982; Pardridge and Mietus, 1982) and by Lefauconnier and Trouvé (1983), Al-Sarraf (2002), and Al-Sarraf et al. (1997). These experiments showed that many amino acids and other metabolically active compounds were transported into developing brain at much higher rates than in the adult; this was interpreted as reflecting the greater metabolic demand of the developing brain rather than immaturity of the blood-brain barrier. However, some authors have continued to suggest that greater uptake may reflect barrier immaturity (e.g., Watson et al., 2006).
Thus it has been clear for sometime that transport mechanisms at the brain barrier interfaces determine the composition of the internal environment of developing brain and supply essential nutrients and other molecules important for growth and differentiation of the brain. What has been lacking until recently is information on the presence and expression of specific transporters in the brain barrier interfaces. This is now available from expression studies of isolated cerebral endothelial cells from neonatal mice (Daneman et al., 2010a) and from choroid plexuses of fetal mice and rats compared to the adult (Liddelow et al., 2012, submitted). Many of these so called Slc transporters are expressed at higher levels in the developing brain compared to the adult, thus accounting for the earlier observations of higher entry of specific amino acids into the developing brain. There are differences in the expression patterns of these Slc transporters in the cerebral endothelial cells and in the choroid plexus epithelial cells and many more are expressed at higher levels in the fetal choroid plexus (see reviews by Saunders et al., 2013 and Liddelow et al., submitted). This reflects the greater importance of the choroid plexuses for transport into the brain compared to the vasculature at very early stages of brain development. The plexuses develop much earlier than the blood supply (Johansson et al., 2008). The findings also help to explain a number of important observations on developmentally different effects of amino acids on brain function. For example it was reported many years ago that glutamate is toxic to the brain if administered in the neonatal period (Olney and Ho, 1970) which some attributed to "immaturity" of the blood-brain barrier (Viña et al., 1997). However, it can now be seen that the barrier contribution to toxicity is much more likely to be due to greater transport of glutamate by e.g., Slcla4 (see Saunders et al., 2012).

\section{THE PARACELLULAR PATHWAY AND TIGHT JUNCTIONS IN BARRIER INTERFACES IN THE DEVELOPING BRAIN}

The concept of the paracellular pathway is an important tenet of epithelial biology that was extended to cerebral endothelial interfaces. It arose from some ingenious experiments of Frömter and Diamond (1972). These authors measured transepithelial resistance in a variety of epithelia in vitro by passing a microelectrode over the external surface of the epithelium. They observed that when the electrode tip was over the region of the border between adjacent cells there was a marked drop in resistance across the epithelium. Frömter and Diamond proposed that this indicated a low resistance pathway across the epithelium for water and ion flow. They subsequently extended this concept to include the paracellular pathway as the route by which small lipid insoluble molecules such as sucrose crossed epithelia (Diamond, 1974). The physical basis for the paracellular pathway is the junctional complex between adjacent cells and in particular the tight junction component of the complex (Brightman and Reese, 1969). However, there are some important limitations to this interpretation of the sites of low resistance across epithelia. Firstly the dimensions of the tip of the microelectrodes used by Frömter and Diamond was about $5 \mu \mathrm{M}$ compared to the intercellular space at the level of tight junctions, which is measured in nanometers, if not zero where adjacent cell membranes are fused. Thus an alternative explanation for the low resistance pathway is that there is 
an intracellular pathway close to the border of the cell membrane between adjacent cells.

Diamond (1974) seems to have acknowledged this possibility as he suggested that in some epithelia the low resistance pathway might be due to "leaky" cells, although he thought this would be exceptional. Secondly, water and ions cannot yet be visualized with sufficient resolution for their route across epithelia to be defined. Until recently this was also the case for low molecular weight compounds such as sucrose and inulin; however, this problem has been solved by the use of biotin labeled small molecular sized compounds that can be visualized at both the light and electron microscopical level. At least in the case of choroid plexus epithelial and cerebral endothelial cells in the South American opossum, Monodelphis domestica, the intercellular junctions are impermeable to a compound biotin ethylenediamine that is smaller than sucrose (Ek et al., 2001, 2003, 2006). In addition it was found that biotin labeled markers transfer across a subpopulation of plexus epithelial cells and not between them (Ek et al., 2001, 2003, 2006; Liddelow et al., 2009). These studies require independent replication, preferably in several different species, but they indicate that the earliest vessels growing into the brain are structurally well enough developed for their tight junctions to be impermeable to even very small molecules. These results also suggest that the presence of a low resistance pathway across epithelial cells, rather than the paracellular route, as envisaged by Diamond (1974) as an exceptional situation may indeed exist.

Over many years the results of ultrastructural studies of tight junctions in the cerebral vasculature and choroid plexuses of the developing brain have been conflicting. Two of the earliest ultrastructural studies of the developing mammalian brain barriers were those of Donahue and Pappas, (1961, rat cerebral capillaries) and Tennyson and Pappas (1964, rabbit choroid plexus). Most of their descriptions dealt with subcellular structures, but they do comment on and illustrate what in those days were called terminal bars that were present in the earliest embryos examined (E14). These appear to correspond to structures associated with what were subsequently described as tight junctions (Brightman and Reese, 1969). Particularly considering that the 1960s were early days for electron microscopy of developing brain tissue (which in those days was regarded as very difficult to fix adequately) the micrographs of Donahue and Pappas and Tennyson and Pappas are of high quality and show clearly well formed intercellular tight junctions. Tennyson (1975) published a more comprehensive paper on choroid plexus development in human and rabbit fetuses. It includes illustrations of what she now refers to as "tight junctions" in the earliest rabbit fetuses studied (E15).

Subsequent to the early paper of Tennyson and Pappas (1964) there have been numerous transmission electron microscope studies of tight junctions in the developing brain, mainly in chick embryos (Delorme et al., 1970; Delorme, 1972; Roy et al., 1974) rodents (Stewart and Hayakawa, 1994) fetal sheep (Møllgård and Saunders, 1975, 1977; Møllgård et al., 1976, 1979) but also some in human fetuses (Møllgård and Saunders, 1975, 1986; Virgintino et al., 2004). Most, but not all, seem to agree that the typical appearance of closely apposed cell membranes of adjacent cerebral endothelial cells appears very early in brain development, although some authors (e.g., Stewart and Wiley, 1981) nonetheless conclude that the blood-brain barrier at these early stages is more permeable than in the adult. This was based on citing papers showing greater entry of both metabolically active and inert molecules into the developing brain and a high concentration of protein in CSF (cf. Sections 6, 8, and 11).

Some authors also used HRP in an attempt to delineate the age at which there was a change from blood-brain barrier permeability to one of impermeability. However, insofar as the methodological information is available it seems likely that in many experiments excessive volumes or concentrations of HRP solution or other markers were injected, thus mechanically disrupting the fragile blood vessels (e.g., Wakai and Hirokawa, 1978, 1981; Risau et al., 1986; Stewart and Hayakawa, 1987; Bertossi et al., 1988; Ribatti et al., 1993; and see Table 1). Also HRP has toxic effects and may give rise to anomalous permeability results, even in adult animals (e.g., Mazariegos et al., 1984). Nevertheless Tauc et al. (1984) were able to show that tight junctions in the choroid plexuses of rat embryos at least as early as E14 were impermeable to HRP. These observations were supported by freeze fracture studies showing a similarly early development of tight junction structure.

Møllgård et al. (1976) carried out a quantitative study of freeze fracture tight junction complexity in fetal sheep embryonic choroid plexus. This showed that there were only minor changes in junctional complexity over a large period of development in contrast to reports in the same species of large developmental changes in apparent permeability from blood to CSF for small molecular markers (Evans et al., 1974; Dziegielewska et al., 1979). It should be noted that the large change in apparent permeability was probably mainly due to an increase in CSF secretion rate (see above), but this does not diminish the significance of the finding that the freeze fracture appearance of choroid plexus tight junctions appeared very early in development.

Dermietzel et al. (1977) published a detailed freeze fracture study of the development of intercellular junctions of the chick embryo choroid plexus from E5, the day on which it begins to differentiate. A junctional network of strands was apparent by E6 and by E9 intercellular zonula occludens were more or less complete.

Attempts to correlate expression of individual tight junction genes with some supposed age when cerebral blood vessels stop being leaky, seem doomed to failure unless experiments are conducted under adequate physiological conditions. The findings that different tight junction genes are expressed at different ages and that the junctions become molecularly more complex with age seem more likely to reflect changes in the mechanical properties of tight junctions in the face of increasing hydrostatic pressure (systemic blood pressure) during embryonic and fetal development (Evans et al., 1974; Dziegielewska et al., 1979). The question of whether or not there is a stage of early vascularization of the brain when the blood vessels are leaky is a functional one that can only be answered by permeability experiments conducted under reasonably physiological conditions. In spite of several attempts (e.g., Risau et al., 1986; Mizee et al., 2013; Ben-Zvi et al., 2014, see also Bauer et al., 2014) there is no convincingly demonstrated link between the expression of one particular gene and the supposed sealing of blood vessels entering the CNS tissue, apart 
from Nitta et al. (2003) who showed increased permeability to small molecules ( $<800 \mathrm{Da})$ in claudin 5 -deficient mice; this was attributed to tight junction permeability although not confirmed at the ultrastructural level.

\section{IONIC GRADIENTS BETWEEN CSF AND PLASMA IN THE DEVELOPING BRAIN}

Some of the most convincing evidence that some critical brain barrier properties develop very early in the embryo comes from studies of ion gradients between CSF and plasma. In the adult the ionic composition of CSF is characteristically different from that of plasma and is also appreciably more stable. This reflects the importance of a homeostatically constant environment that is a prerequisite for normal brain function (Davson, 1967; Davson and Segal, 1996). The gradients are set up by ion pumps in the epithelial cells of the choroid plexuses (Damkier et al., 2013). Similar pumps in the cerebral vascular endothelial cells may also contribute via the extracellular fluid of the brain (Davson and Segal, 1996). An essential structural component of this homeostatic mechanism is the presence of tight junctions between the adjacent cells of the choroid plexus epithelium and cerebrovascular endothelium. Thus in development, if even a single ion gradient is present, it indicates the presence of both a cellular ion pump and functional integrity of the tight junctions, as without the latter a gradient simply cannot be established. In the 1970s80 s there were several studies in a variety of species showing the presence of ion gradients between CSF and plasma (Flexner, 1938; Bito and Myers, 1970; Bradbury et al., 1972; Amtorp and Sørensen, 1974; Sedlácek, 1975; Mitchell et al., 1982; Nattie et al., 1984). More recently gene expression studies have shown that key ion channel and transporter genes are expressed very early embryonic life in the choroid plexuses (Liddelow et al., 2012, 2013).

\section{CONCENTRATION AND ORIGIN OF PROTEINS IN CSF IN DEVELOPING BRAIN}

One of the pieces of evidence sometimes put forward in favor of immaturity or leakiness of the blood-brain barrier in the developing brain is that the CSF in fetal and newborn animals contains a much higher concentration of protein than in the adult (Adinolfi et al., 1976; Adinolfi and Haddad, 1977; Ramey and Birge, 1979; Adinolfi, 1985; Fishman, 1992). Strictly speaking the composition of CSF is much more determined by movements of molecules across the choroid plexuses rather than entry via the blood vessels and brain interstitial fluid, although clinicians frequently (but erroneously) interpret CSF changes as reflecting changes in permeability of the blood-brain barrier. Since the middle of the last century it has been known that the concentration of protein in human newborn CSF (e.g., Spiegel-Adolf et al., 1954; Nasralla et al., 1958) is higher than in the adult (Davson and Segal, 1996) and even more so if the infants are prematurely born (e.g., Otila, 1948; Bauer et al., 1965). Results from these and other studies are summarized in Table 2 . The mean values reported for healthy term babies were in the range $63-115 \mathrm{mg} / 100 \mathrm{ml}$. For pre-term infant the mean values in different series were generally higher (up to nearly $190 \mathrm{mg} / 100 \mathrm{ml}$ ). Little information about human fetal CSF is known but Adinolfi published important studies on
Table 2 | Total protein concentration $(\mathrm{mg} / 100 \mathrm{ml}$ ) in CSF of full term and pre-term infants estimated during the first few days of life.

\begin{tabular}{lllll}
\hline Authors & $\begin{array}{c}\text { Mean } \\
(\mathrm{mg} / 100 \mathrm{ml})\end{array}$ & S.E.M & Range $n$ & Condition \\
& & &
\end{tabular}

\begin{tabular}{|c|c|c|c|c|c|}
\hline \multicolumn{6}{|l|}{ FULL TERM } \\
\hline $\begin{array}{l}\text { Spiegel-Adolf et al., } \\
1954\end{array}$ & 103 & 9.9 & - & 14 & Normal \\
\hline Widell, 1958 & 80.9 & 6.2 & - & 11 & Healthy \\
\hline Nasralla et al., 1958 & 115 & 5.8 & 46-194 & 34 & Normal \\
\hline Naidoo, 1968 & 63 & 1.6 & $32-240$ & 135 & Healthy \\
\hline $\begin{array}{l}\text { Piliero and Lending, } \\
1959\end{array}$ & 70 & 6.0 & 25-90 & 35 & Normal \\
\hline Watson, 1964 & 77 & 5.3 & $26-180$ & 51 & $\begin{array}{l}\text { Cerebral } \\
\text { anoxia }\end{array}$ \\
\hline Heine et al., $1981^{a}$ & 73.0 & 17.6 & $53-95$ & 10 & $\begin{array}{l}\text { No cereb } \\
\text { meningitis }\end{array}$ \\
\hline Ahmed et al., 1996 & 80.8 & $30.8^{*}$ & - & 17 & $\begin{array}{l}\text { Prev. } \\
\text { healthy }\end{array}$ \\
\hline $\begin{array}{l}\text { Chadwick et al., } \\
2011\end{array}$ & 106 & - & $94-115^{* * *}$ & 54 & $\begin{array}{l}\text { No } \\
\text { meningitis }\end{array}$ \\
\hline $\begin{array}{l}\text { Srinivasan et al., } \\
2012\end{array}$ & $78^{\wedge}$ & $137^{* *}$ & $60-100$ & 130 & Normal \\
\hline \multicolumn{6}{|l|}{ PRE TERM } \\
\hline Otila, $1948^{\mathrm{b}}$ & 100 & 5.5 & $50-138$ & 19 & Healthy \\
\hline $\begin{array}{l}\text { Nasralla et al., } \\
1958^{c}\end{array}$ & 167 & 6.4 & $81-259$ & 49 & Normal \\
\hline $\begin{array}{l}\text { Gyllenswärd and } \\
\text { Malmström, 1962 }\end{array}$ & 176 & 26 & $57-292$ & 9 & Healthy \\
\hline Bauer et al., $1965^{e}$ & 187 & 28 & $30-1600$ & 70 & Various \\
\hline $\begin{array}{l}\text { Bartolozzi et al., } \\
1967^{f}\end{array}$ & 62 & 4.3 & $12-144$ & 59 & Normal \\
\hline Cole et al., 19749 & 120 & 10 & - & 9 & Normal \\
\hline Sarff et al., 1976 & 90 & - & $20-170$ & & $\begin{array}{l}\text { No } \\
\text { meningitis }\end{array}$ \\
\hline $\begin{array}{l}\text { Statz and } \\
\text { Felgenhauer, } 1983^{\text {h }}\end{array}$ & 139 & $77^{*}$ & $68-240$ & 10 & $\begin{array}{l}\text { No } \\
\text { pathology }\end{array}$ \\
\hline $\begin{array}{l}\text { Mhanna et al., } \\
2008^{i}\end{array}$ & $180^{\wedge}$ & - & $124-270$ & 10 & $\begin{array}{l}\text { Suspect } \\
\text { sepsis }\end{array}$ \\
\hline $\begin{array}{l}\text { Srinivasan et al., } \\
2012^{j}\end{array}$ & $104^{\wedge}$ & $203^{* *}$ & $79-131$ & 148 & Normal \\
\hline
\end{tabular}

${ }^{a} 0-4$ weeks. No data on birth weights or gestational age.

${ }^{b}$ Birth weights 920-2150 g, Table 19, p. 91.

${ }^{c}$ Birth weights $\leq 4.5 \mathrm{lbs}$, Table 2, p. 1404.

${ }^{d}$ Birth weights $\leq 2000 \mathrm{~g}$, Table 5, p. 60 .

${ }^{e}$ Birth weights 800-2620 g, Figure 1, p. 1018.

${ }^{f}$ Birth weights $\leq 2500 \mathrm{~g}$, Figure 2, p. 299.

g Birth weights 1080-1710 g, Table 1, p. 724.

h27-32 weeks, Table 3, p. 158.

i 27 weeks, values less at younger and older ages.

j 28-35 weeks, Tables II, p730 and III, p731.

${ }^{*}$ S.D. ${ }^{* *} 95$ th percentile. ${ }^{* *} \mathrm{Cls}$. ${ }^{*}$ Median.

CSF and plasma proteins obtained from aborted human fetuses (Adinolfi et al., 1976; Adinolfi and Haddad, 1977; Adinolfi, 1985). These studies showed that the concentration of total protein and of individual proteins such as a-fetoprotein, transferrin and IgG were much higher than reported for live-born infants; the highest 
individual total protein value reported was $730 \mathrm{mg} / 100 \mathrm{ml}$ at 24 weeks gestation (Adinolfi et al., 1976) although it needs to be born in mind that the CSF samples were obtained from aborted fetuses of uncertain physiological state.

Similar results, however, have been obtained for numerous animal species where it was possible to obtain CSF and plasma samples from a wide range of stages of fetal and neonatal development collected under good physiological conditions. The results from animal studies have been reviewed by Saunders $(1977,1992)$ and by Dziegielewska and Saunders (1988). One of the earliest studies was by Klosovskii (1963) in cat embryos in which CSF protein concentration was up to 22 times that in the adult. Klosovskii correctly interpreted this as having some nutritive function in the developing brain rather than ascribing it to barrier immaturity. The concentration of total protein and of individual proteins in fetal CSF is much higher than in the newborn and adult, but if samples were obtained early enough in development the level was lower than a peak that occurred in all species probably coinciding with the period of maximum neurogenesis. Although Adinolfi (1985) and others interpreted the high CSF concentration of protein in CSF in the developing brain as evidence of a leaky blood-brain and/or blood-CSF barrier there is now substantial evidence that it is a consequence of specific transport of proteins from blood to CSF via a subpopulation of choroid plexus epithelial cells (Dziegielewska et al., 1980, 1991; Habgood et al., 1992; Knott et al., 1997; Johansson et al., 2006, 2008; Liddelow et al., 2009, 2011a,b). In addition it should be noted that the concentration of protein in any space is a function of its amounts and volume in which they distribute. As has been shown by Johansson et al. (2008) the amount of protein in the adult CSF is actually greater in the fetus due to a combination of expanding of the ventricular system and increased CSF flow.

\section{INDUCTION OF TIGHT JUNCTIONS IN BRAIN BARRIER INTERFACES}

Another widespread misconception is that astrocytes are essential for the induction of tight junctions in cerebral blood vessels in the developing brain. In adult brain end feet of astrocytes encircle cerebral capillaries (Caley and Maxwell, 1970; Xu and Ling, 1994). They are thought to make significant contributions to blood-brain barrier functions (Abbott et al., 2006). The belief that astrocytes may be involved in tight junction formation in the developing brain seems to stem largely from in vitro experiments. In culture the presence of either astrocytes or conditioned medium from cultured astrocytes produced cells with more complex tight junctions (Tao-Cheng et al., 1987) and was essential for the preparation of cerebral endothelial monolayers with high transendothelial resistance (Dehouck et al., 1990; Rubin et al., 1991). Janzer and Raff (1987) are frequently cited as supporting the notion that astrocytes are essential for tight junction formation in vivo. However, Holash et al. (1993) showed their results were an artifact (see Saunders et al., 2012, for further discussion). It seems to have been overlooked that there are no astrocytes present in the developing brain when it is first vascularized (Caley and Maxwell, 1970; Daneman et al., 2010a). As first shown by Stewart and Wiley (1981) using chick-quail chimeras, tight junction formation in cerebral vessels is induced by some factor in the neural tissue of the developing brain. The vessels are tight to proteins and small molecules from as early as vessels first grow into the neural tissue (Bauer et al., 1993, 1995; Ek et al., 2006). It is not yet clear what the induction factor(s) is/are. However it seems that the pericytes make an important contribution to tight junction formation (Daneman et al., 2010b). The main period of differentiation of astrocytes and their association with capillaries occur in rodents in the first 3 weeks of postnatal life (Caley and Maxwell, 1970). It may be that astrocytes contribute to tight junction-induction during this period of intense vascularization, and subsequent maintenance of tight junctions, but they are not involved in earlier stages of vascularization of the brain.

\section{WHY IS IT IMPORTANT TO UNDERSTAND FUNCTIONING OF BARRIER MECHANISMS IN THE DEVELOPING BRAIN?}

It seems unsatisfactory that this belief in the "immaturity" or leakiness of the blood-brain barrier has persisted into the 21st Century in spite of much evidence to the contrary as reviewed above. There are two quite different reasons why it is important to understand the functional status of barrier mechanisms in the developing brain. The first is that they are likely to be important for different features of brain development at different stages in its development. This is suggested for example, by changes in gene expression for different efflux and influx mechanisms in both the cerebral vasculature and choroid plexuses during brain development (Virgintino et al., 2000, 2007; Daneman et al., 2010a; Liddelow et al., 2012; Kratzer et al., 2013).

The other reason is that it is clinically important. At present because of a lack of information on the extent to which drugs enter the developing brain of the embryo following administration to the mother, the general advice given by doctors to pregnant women is to avoid taking drugs. However, this is not very helpful advice in the case of patients with severe medical conditions requiring continued treatment such as epilepsy (Forsberg and Wide, 2011) depression and anxiety (El Marroun et al., 2014; Ornoy and Koren, 2014) psychosis (Galbally et al., 2014) cancer (van Hasselt et al., 2014), or severe hypertension (Brown and Garovic, 2014).

In the US it has been estimated that between 30 and $35 \%$ of women have taken psychoactive drugs during pregnancy (Goldberg and Nissim, 1994). In a study of nearly 100,000 pregnancies, Andrade et al. (2004) found that $64 \%$ of pregnant women received a drug prescription. In a four nation study in Europe (De Vigan et al., 1999) an average of 36\% of women interviewed used at least one drug during the first trimester of pregnancy. There was substantial national variation $(22.5 \%$ in Glasgow and 50 and $40 \%$ in two centers in France). Because drugs are not systematically investigated for possible deleterious effects on the developing brain the clinical management of pregnant women may be a difficult decision between what is best for the mother and limiting potential damage to the developing fetal brain. This lack of knowledge not only makes it difficult for doctors to make rational decisions about when to and when not to medicate, but commonly results in non-compliance to prescribed medication, terminating medication or self-medication, as well as alternative natural remedies being used by mothers (Wood et al., 2014). The only information available to clinicians at 
present consists of collections of reports of adverse effects or lack of such effects in compendia such as Yaffe's index (Briggs et al., 2008) or national data organizations such as the US Food and Drug Administration (FDA) or the Australian Drug Evaluation Committee in pregnancy, which classify drugs into categories $\mathrm{A} / \mathrm{B} / \mathrm{C} / \mathrm{D} / \mathrm{X}$, where $\mathrm{A}$ are drugs shown to be safe for the human fetus and $\mathrm{X}$ known to induce birth defects (see Teratology Society Public Affairs Committee, 1994). The majority of drugs fall into categories B and C, drugs for which there are not enough data in humans to know whether they are safe for the fetus. The consequences of the currently inadequate state of knowledge about the safety of medications administered during pregnancy have been highlighted by Kennedy (2011). This classification has been under sustained criticism from the Teratology Society in the US (Teratology Society Public Affairs Committee, 1994, 2007) primarily on the grounds that the lack of knowledge about drugs used in pregnancy generates difficulties in making clinical decisions and causing considerable anxiety in patients. This classification is now being abandoned in the US, in favor of a new pregnancy and lactation-labeling rule designed to improve risk versus benefit assessment of drugs used in pregnant and nursing mothers (Ramoz and Patel-Shori, 2014).

Drug use during pregnancy is likely to increase in the future due to higher average ages of mothers, increasing the likelihood of pregnancy complications as well as a higher incidence of premature births with significant medical interventions as a consequence. There is also a growing apprehension that environmental toxins may affect the developing brain. One review listed over 200 industrial compounds known to be neurotoxic in humans and experimental studies have shown over 1000 substances (Grandjean and Landrigan, 2006); this list probably only represents a very small fraction of all chemicals that are neurotoxic. It is particularly inappropriate that public bodies such as the Agency for Toxic Substances and Disease registry (ATSDR) at the Centre for Disease Control in the US should perpetuate statements about immaturity of the blood-brain barrier based on out of date information and mythology. This has been discussed in detail by Ek et al. (2012) but this discussion appears to have had no impact on current reports published by the ATSDR. A more hopeful sign is that the CDC has recently convened meetings to discuss developing a more systematic approach to safer medication use in pregnancy (Broussard et al., 2014).

Recent gene expression studies in developing animal brains (Daneman et al., 2010a; Liddelow et al., 2012, submitted; Kratzer et al., 2013) suggest that many of the protective efflux mechanism that prevent entry of drugs and toxins into the adult brain are present and in some cases more highly expressed in blood vessels and choroid plexuses of the developing brain. However, expression does not necessarily equate with functional effectiveness, so there is an urgent need for systematic drug studies in pregnant and newborn animals and people, in order to define clearly the potential hazards or lack of hazards, when drugs are administered to pregnant women.

\section{SUMMARY}

In conclusion, it seems that many of the most strongly held beliefs about the early history of the blood-brain barrier are incorrect.
The blood-brain barrier was not described first by Ehrlich (1885); indeed he did not believe that there were any differences in permeability of cerebral blood vessels compared to the rest of the body. Lewandowsky (1900) did not coin the term blood-brain barrier ("Blut-Hirnschranke"). The first person to use this term seems to have been Stern (Stern and Gautier, 1921). Goldmann (1909) was not the first person to demonstrate that some dyes (in his experiments trypan blue) do not enter the brain following parenteral injection. This appears to have been first reported by Franke (1905) who used trypan red. Behnsen (1927) although frequently cited as providing evidence for immaturity of the blood-brain barrier did not do so. Other claims of barrier immaturity or leakiness in developing animals in dye injection experiments can be explained by use of excessive volumes or concentrations of material injected into fragile animals. Early induction of tight junctions in cerebral vessels does not involve astrocytes. At least in the choroid plexus the route of transfer from blood to CSF is intracellular and not via the paracellular pathway.

The importance of discarding the notion of blood-brain barrier immaturity or leakiness in the developing brain lies in the fact that this belief has impeded research into these mechanisms in brain development and their possible relevance for normal and abnormal brain development, as well as the need for systematic studies of therapeutic agents used to treat pregnant women. Therapeutic advice to such patients should be based on evidence, not fear of the unknown.

\section{AUTHOR CONTRIBUTIONS}

All of the listed authors contributed to the conception, design, research, drafting and final approval of the work. They each agree to be accountable for all aspects of the work.

\section{REFERENCES}

Abbott, N. J., Rönnbäck, L., and Hansson, E. (2006). Astrocyte-endothelial interactions at the blood-brain barrier. Nat. Rev. Neurosci. 7, 41-53. doi: $10.1038 / \mathrm{nrn} 1824$

Adinolfi, M. (1985). The development of the human blood-brain barrier. Dev. Med. Child. Neurol. 27, 532-537. doi: 10.1111/j.1469-8749.1985.tb04581.x

Adinolfi, M., Beck, S. E., Haddad, S. A., and Seller, M. J. (1976). Permeability of the blood-cerebrospinal fluid barrier to plasma proteins during foetal and perinatal life. Nature 259, 140-141. doi: 10.1038/259140a0

Adinolfi, M., and Haddad, S. A. (1977). Levels of plasma proteins in human and rat fetal CSF and the development of the blood-CSF barrier. Neuropädiatrie 8, 345-353. doi: 10.1055/s-0028-1091530

Ahlfors, C. E., and Wennberg, R. P. (2004). Bilirubin-albumin binding and neonatal jaundice. Semin. Perinatol. 28, 334-339. doi: 10.1053/j.semperi.2004.09.002

Ahmed, A., Hickey, S. M., Ehrett, S., Trujillo, M., Brito, F., Goto, C., et al. (1996). Cerebrospinal fluid values in the term neonate. Pediatr. Infect. Dis. J. 15, 298-303. doi: 10.1097/00006454-199604000-00004

Allen, F. H. Jr., Diamond, L. K., and Vaughan, V. C. (1950). Erythroblastosis fetalis. VI. Prevention of kernicterus. Am. J. Dis. Child. 80, 779-791.

Al-Sarraf, H. (2002). Transport of 14C-gamma-aminobutyric acid into brain, cerebrospinal fluid and choroid plexus in neonatal and adult rats. Brain Res. Dev. Brain Res. 139, 121-129. doi: 10.1016/S0165-3806(02)00537-0

Al-Sarraf, H., Preston, J. E., and Segal, M. B. (1997). Changes in the kinetics of the acidic amino acid brain and CSF uptake during development in the rat. Dev. Brain Res. 102, 127-134. doi: 10.1016/S0165-3806(97)00089-8

Amtorp, O., and Sørensen, S. C. (1974). The ontogenetic development of concentration differences for protein and ions between plasma and cerebrospinal fluid in rabbits and rats. J. Physiol. 243, 387-400.

Andrade, S. E., Gurwitz, J. H., Davis, R. L., Chan, K. A., Finkelstein, J. A., Fortman, K., et al. (2004). Prescription drug use in pregnancy. Am. J. Obstet. Gynecol. 191, 398-407. doi: 10.1016/j.ajog.2004.04.025 
ATSDR. (2014). Agency for Toxic Substances and Disease Registry Home Page. Available online at: http://www.atsdr.cdc.gov (retrieved August 29 2014).

ATSDR. Tp46. (1999). Available online at: http://www.atsdr.cdc.gov/ToxProfiles/ tp46.pdf (retrieved August 29 2014).

ATSDR. Tp13. (2007). Available online at: http://www.atsdr.cdc.gov/ToxProfiles/ tp13.pdf (retrieved August 29 2014).

Bakay, L. (1953). Studies on blood-brain barrier with radioactive phosphorus. III. Embryonic development of the barrier. Arch. Neurol. Psychiatry 70, 30-39. doi: 10.1001/archneurpsyc.1953.02320310036002

Bakay, L. (1956). "Development of the blood-brain barrier," in The Blood-Brain Barrier, with Special Regard to the use of Radioactive Isotopes, ed L. Bakay (Springfield, IL: Thomas), 77-149.

Bakay, L. (1968). Changes in barrier effect in pathological states. Prog. Brain Res. 29, 315-339. doi: 10.1016/S0079-6123(08)64165-5

Baldrick, P. (2010). Juvenile animal testing in drug development - Is it useful? Regul. Toxicol. Pharmacol. 57, 291-299. doi: 10.1016/j.yrtph.2010.03.009

Baños, G., Daniel, P. M., and Pratt, O. E. (1978). The effect of age upon the entry of some amino acids into the brain, and their incorporation into cerebral protein. Dev. Med. Child. Neurol. 20, 335-346.

Barcroft, J. (1938). The Brain and its Environment 1. The Activity of the Brain in Mid-Foetal Life. New Haven, CT: Yale University Press.

Barrett, K. E., Barman, S. M., Boitano, S., and Brooks, H. L. (2012). Ganong's Review of Medical Physiology. 24th Edn. New York, NY: McGraw Hill Lange.

Bartolozzi, G., Ciampolini, M., Franchini, F., and Marianelli, L. (1967). Studio delle protein liquorate nel prematuro. I Richerche lettroforetiche. Riv. Clin. Pediatr. 80, 296-309.

Bass, N. H., and Lundborg, P. (1973). Postnatal development of bulk flow in the cerebrospinal fluid system of the albino rat: clearance of carboxyl-(14C)inulin after intrathecal infusion. Brain Res. 52, 323-332. doi: 10.1016/00068993(73)90668-9

Bauer, C. H., New, M. I., and Miller, J. (1965). Cerebrospinal fluid protein values of premature infants. J. Pediatr. 66, 1017-1022. doi: 10.1016/S0022 3476(65)80086-5

Bauer, H., Sonnleitner, U., Lametschwandtner, A., Steiner, M., Adam, H., and Bauer, H. C. (1995). Ontogenic expression of the erythroid-type glucose transporter (Glut 1) in the telencephalon of the mouse: correlation to the tightening of the blood-brain barrier. Brain Res. Dev. Brain Res. 86, 317-325. doi: 10.1016/0165-3806(95)00044-E

Bauer, H. C., Bauer, H., Lametschwandtner, A., Amberger, A., Ruiz, P., and Steiner, M. (1993). Neovascularization and the appearance of morphological characteristics of the blood-brain barrier in the embryonic mouse central nervous system. Brain Res. Dev. Brain Res. 75, 269-278. doi: 10.1016/0165-3806(93)90031-5

Bauer, H.-C., Kritzbai, I. A., Bauer, H., and Traweger, A. (2014). "You Shall Not Pass" - Tight junctions of the Blood Brain Barrier. Front. Neurosci. 8:392. doi: 10.3389/fnins.2014.00392

Behnsen, G. (1926). Farbstoffversuche mit Trypanblau an der Schranke zwischen Blut und Zentralnervensystem der wachsenden Maus. Münch. Med. Wochenschrift 73, 1143-1147.

Behnsen, G. (1927). Über die Farbstoffspeicherung im Zentralnervensystem der weissen Maus in verschiedenen Alterszuständen. Z. Zellforsch. Mikrosk. Anat. 4, 515-572. doi: 10.1007/BF00637105

Ben-Zvi, A., Lacoste, B., Kur, E., Andreone, B. J., and Mayshar, Y., Yan, H., et al (2014). Mfsd2a is critical for the formation and function of the blood-brain barrier. Nature 509, 573-590. doi: 10.1038/nature13324

Bernard, C. (1865). Introduction a l'étude de la Médecine Expérimentale. Paris: Balliere.

Bertossi, M., Mancini, L., Favia, A., Nico, B., Ribatti, D., Virgintino, D., et al. (1992). Permeability-related structures in developing and mature microvessels of the chicken optic tectum. Biol. Struct. Morphog. 4, 144-152.

Bertossi, M., Ribatti, D., Nico, B., Mancini, L., Lozupone, E., and Roncali. L. (1988). The barrier systems in the choroidal plexuses of the chick embryo studied by means of horseradish peroxidase. J. Submicrosc. Cytol. Pathol. 20, 385-395.

Biedl, A., and Kraus, R. (1898). Über eine bisher unbekannte toxische Wirkung der Gallensäuren auf das Centralnervensystem. Centralblatt. Inn. Med. 19, 1185-1200.

Birge, W. J., Rose, A. D., Haywood, J. R., and Doolin, P. F. (1974). Development of the blood-cerebrospinal fluid barrier to proteins and differentiation of cerebrospinal fluid in the chick embryo. Dev. Biol. 41, 245-254. doi: 10.1016/00121606(74)90303-0
Bito, L. Z., and Myers, R. E. (1970). The ontogenesis of haematoencephalic cation transport in the rhesus monkey. J. Physiol. (Lond.) 208, 153-170.

Bouffard, G. (1906). Injection des couleurs de benzidine aux animaux normaux. Ann. d. l'lnst. Pasteur. Paris 20, 539-548.

Bradbury, M. W. B. (1979). The Concept of a Blood-Brain Barrier. Chichester: Wiley. Bradbury, M. W. B. (1995). "Developing views of the blood-brain barrier," in New Concepts of a Blood-Brain Barrier, eds J. Greenwood, D. J. Begley, and M. B. Segal (New York, NY: Plenum), 1-9.

Bradbury, M. W. B., Crowder, J., Desai, S., Reynolds, J. M., Reynolds, M., and Saunders, N. R. (1972). Electrolytes and water in the brain and cerebrospinal fluid of the foetal sheep and guinea pig. J. Physiol. (Lond.) 227, 591-610.

Braun, L. D., Cornford, E. M., and Oldendorf, W. H. (1980). Newborn rabbit blood-brain barrier is selectively permeable and differs substantially from the adult. J. Neurochem. 34, 147-152. doi: 10.1111/j.1471-4159.1980.tb04633.x

Briggs, G. G., Freeman, R. K., and Yaffe, S. J. (2008). Drugs in Pregnancy and Lactation. Philadelphia, PA: Walters Kluwer.

Brightman, M. W., and Reese, T. S. (1969). Junctions between intimately apposed cell membranes in the vertebrate brain. J. Cell. Biol. 40, 48-77. doi: 10.1083/jcb.40.3.648

Broman, T. (1941). The possibilities of the passage of substances from the blood to the central nervous system (Is there a blood-brain-barrier and a bloodcerebrospinal fluid barrier?). Acta Psychol. Scand. 16, 1-25. doi: 10.1111/j.16000447.1941.tb07398.x

Broussard, C. S., Frey, M. T., Hernandez-Diaz, S., Greene, M. F., Chambers, C. D. Sahin, L., et al. (2014). Developing a systematic approach to safer medication use during pregnancy: summary of a centers for disease control and preventionconvened meeting. Am. J. Obstet. Gynecol. 211, 208-214. doi: 10.1016/j.ajog. 2014.05.040

Brown, C. M., and Garovic, V. D. (2014). Drug treatment of hypertension in pregnancy. Drugs 74, 283-229 doi: 10.1007/s40265-014-0187-7

Caley, D. W., and Maxwell, D. S. (1970). Development of the blood vessels and extracellular spaces during postnatal maturation of rat cerebral cortex. J. Comp. Neurol. 138, 31-47. doi: 10.1002/cne.901380104

Chadwick, S. L., Wilson, J. W., Levin, J. E., and Martin, J. M. (2011). Cerebrospinal fluid characteristics in infants who present to the Emergency Department with fever. Pediatr. Infect. Dis. J. 30, e63-e67. doi: 10.1097/INF.0b013e31820ad2ba

Clarke, C. A. (1989). Preventing rhesus babies: the Liverpool research and follow up. Arch. Dis. Child. 64, 1734-1749. doi: 10.1136/adc.64.12.1734

Cohen, H., and Davies, S. (1938). The Morphology and permeability of the roof of the fourth ventricle in some mammalian embryos. J. Anat. 72 $430-455$.

Cole, V. A., Durbin, G., Olaffson, A., Reynolds, E. O. R., Rivers, R., and Smith, J. (1974). Pathogenesis of intraventricular haemorrhage in newborn infants. Arch. Dis. Child. 49, 722-728. doi: 10.1136/adc.49.9.722

Cornford, E. M., Braun, L. D., and Oldendorf, W. H. (1982). Developmental modulations of blood-brain barrier permeability as an indicator of changing nutritional requirements in the brain. Pediatr. Res. 16, 324-328. doi: 10.1203/00006450-198204000-00017

Costa, L. G., Aschner, M., Vitalone, A., Syversen, T., and Soldin, O. P. (2004). Developmental neuropathology of environmental agents. Ann. Rev. Pharmacol. Toxicol. 44, 87-110. doi: 10.1146/annurev.pharmtox.44.101802.121424

Cotran, R. S., Kumar, V., Robbins, S. L., and Schoen, F. J. (1994). Robbins Pathologic Basis of Disease, 5th Edn. Philadelphia, PA: W. B. Saunders.

Dale, H. (1954). Paul Ehrlich. Br. Med. J. 1, 659-663. doi: 10.1136/bmj.1.4863.659

Damkier, H. H., Brown, P. D., and Praetorius, J. (2013). Cerebrospinal fluid secretion by the choroid plexus. Physiol. Rev. 93, 1847-1892. doi: 10.1152/physrev.00004.2013

Daneman, R., Zhou, L., Agalliu, D., Cahoy, J. D., Kaushal, A., and Barres, B. A. (2010a). The mouse blood-brain barrier transcriptome: a new resource for understanding the development and function of brain endothelial cells. PLoS ONE 5:e13741. doi: 10.1371/journal.pone.0013741

Daneman, R., Zhou, L., Kebede, A. A., and Barres, B. A. (2010b). Pericytes are required for blood-brain barrier integrity during embryogenesis. Nature 468, 562-566. doi: 10.1038/nature09513

Davies, P. A., and Rudd, P. T. (1994). Neonatal Meningitis. Cambridge: MacKeith Press.

Davis, J. E., and Garrison, N. E. (1968). Mean weights of chick embryos correlated with the stages of Hamburger and Hamilton. J. Morph. 124, 79-82. doi: 10.1002/jmor.1051240105 
Davson, H. (1956). Physiology of the Ocular and Cerebrospinal Fluids. London: Churchill.

Davson, H. (1967). Physiology of the Cerebrospinal Fluid. London: Churchill.

Davson, H. (1976). Review lecture: the blood-brain barrier. J. Physiol. (Lond.) 255, $1-28$.

Davson, H., Welch, K., and Segal, M. B. (1987). Physiology and Pathophysiology of the Cerebrospinal Fluid. Edinburgh: Churchill Livingstone.

Davson, H., and Segal, M. B. (1996). Physiology of the CSF and Blood-Brain Barriers. Boca Raton, FL: CRC Press.

Dehouck, M.-P., Meìresse, S., Delorme, P., Fruchart, J.-C., and Cecchelli, R. (1990). An easier, reproducible, and mass-production method to study the blood-brain barrier in vitro. J. Neurochem. 54, 1798-1801. doi: 10.1111/j.14714159.1990.tb01236.x

Delorme, P. (1972). Differenciation ultrastructurale des jonctions intercellulaires de l'endothelium des capillaires télencéphaliques chez l'embryon de poulet. Z. Zellforsch. Mikrosk. Anat. 133, 571-582. doi: 10.1007/BF003 07137

Delorme, P., Gayet, J., and Grignon, G. (1970). Ultrastructural study on transcapillary exchanges in the developing telencephalon of the chicken. Brain Res. 22, 269-283. doi: 10.1016/0006-8993(70)90471-3

Dempsey, E. W., and Wislocki, G. B. (1955). An electron microscopic study of the blood-brain barrier in the rat, employing silver nitrate as a vital stain. J. Biophys. Biochem. Cytol. 1, 245-256. doi: 10.1083/jcb.1.3.245

Dermietzel, R., Krause, D., Kremer, M., Wang, C., and Stevenson, B. (1992). Pattern of glucose transporter (Glut 1) expression in embryonic brains is related to maturation of blood-brain barrier tightness. Dev. Dyn. 193, 152-163. doi: 10.1002/aja.1001930207

Dermietzel, R., Meller, K., Tetzlaff, W., and Waelsch, M. (1977). In vivo and in vitro formation of the junctional complex in choroid epithelium. A freeze-etching study. Cell Tissue Res. 181, 427-441. doi: 10.1007/ BF00221766

De Vigan, C., De Walle, H. E. K., Cordier, S., Goujard, J., Knill-Jines, R., Aymé, S., et al. (1999). Therapeutic drug use during pregnancy: a comparison in four European countries. J. Clin. Epidemiol. 52, 977-982. doi: 10.1016/S08954356(99)00091-8

Diamond, J. M. (1974). Tight and leaky junctions of epithelia: a perspective on kisses in the dark. Fed. Proc. 33, 2220-2224.

Dobbing, J. (1961). The blood-brain barrier. Physiol. Rev. 41, 130-188.

Donahue, S., and Pappas, G. D. (1961). The fine structure of capillaries in the cerebral cortex of the rat at various stages of development. Am. J. Anat. 108, 331-347. doi: 10.1002/aja.1001080307

Dreifuss, J.-J., and Sigrist, N. T. (2009). Lina Stern, l'académicienne genevoise persécutée en URSS. Rev. Med. Suisse 5, 593-597. Available online at: http://rms.medhyg.ch/article.php?ID_ARTICLE=RMS_194_0593

Dziegielewska, K. M., Evans, C. A., Lai, P. C., Lorscheider, F. L., Malinowska, D. H., Møllgård, K., et al. (1981). Proteins in cerebrospinal fluid and plasma of fetal rats during development. Dev. Biol. 83, 193-200. doi: 10.1016/S00121606(81)80024-3

Dziegielewska, K. M., Evans, C. A. N., Malinowska, D. H., Møllgård, K., Reynolds, M. L., and Saunders, N. R. (1980). Blood-cerebrospinal fluid transfer of plasma proteins during fetal development in the sheep. J. Physiol. (Lond.) 300, 457-465.

Dziegielewska, K. M., Evans, C. A. N., Malinowska, D. H., Møllgård, K., Reynolds, J. M., Reynolds, M. L., et al. (1979). Studies of the development of brain barrier systems to lipid insoluble molecules in fetal sheep. J. Physiol. (Lond.) 292, 207-231.

Dziegielewska, K. M., Habgood, M. D., Møllgård, K., Stagaard, M., and Saunders, N. R. (1991). Species-specific transfer of plasma albumin from blood into different cerebrospinal fluid compartments in the fetal sheep. J. Physiol. (Lond.) 439, 215-237.

Dziegielewska, K. M., Hinds, L. A., Møllgård, K., Reynolds, M. L., and Saunders, N. R. (1988). Blood-brain, blood-cerebrospinal fluid and cerebrospinal fluidbrain barriers in a marsupial (Macropus eugenii) during development. J. Physiol. (Lond) 403, 367-388.

Dziegielewska, K. M., and Saunders, N. R. (1988). "The development of the bloodbrain barrier: proteins in fetal and neonatal CSF, their nature and origins," in Handbook of Human Growth and Developmental Biology. Vol 1. Neural, Sensory, Motor and Integrative Development, eds E. Meisami and P. S. Timiras (Boca Raton, FL: CRC Press), 169-191.
Ehman, K. (2010)."Development of the mammalian nervous system," in Reproductive Toxicolgy, 3rd Edn., eds R. W. Kapp and R. W. Tyl (New York, NY: Informa), 51-67.

Ehrlich, P. (1885). Das Sauerstoffbedürfnis des Organismus. Eine Farbenanalytische Studie. Berlin: Hirschwald.

Ehrlich, P. (1906). "The relations existing between chemical constitution, distribution and pharmacological action," in Collected Studies on Immunity New York: John Wiley \& Sons, translated by C Bolduana from Ch XXXIV of Gesammelte Arbeiten zur Immunitätsforschung, ed P. Ehrlich (Berlin: Hirschwald), 1904.

Ek, C. J., Dziegielewska, K. M., Habgood, M. D., and Saunders, N. R. (2012). Barriers in the developing brain and neurotoxicology. Neurotoxicology 33, 586-604. doi: 10.1016/j.neuro.2011.12.009

Ek, C. J., Habgood, M. D., Dziegielewska, K. M., and Saunders, N. R. (2003). Structural characteristics and barrier properties of the choroid plexuses in developing brain of the opossum (Monodelphis domestica). J. Comp. Neurol.460, 451-464. doi: 10.1002/cne.10661

Ek, C. J., Habgood, M. D., Dziegielewska, K. M., and Saunders, N. R. (2006). Functional effectiveness of the blood-brain barrier to small water-soluble molecules in developing and adult opossum (Monodelphis domestica). J. Comp. Neurol. 496, 13-26. doi: 10.1002/cne.20885

Ek, J., Habgood, M. D., Dziegielewska, K. M., and Saunders, N. R. (2001). Permeability of the blood-brain and blood-CSF barriers to small molecular weight lipid insoluble markers during postnatal development in the opossum, Monodelphis domestica. J. Physiol. (Lond.) 536, 841-853. doi: 10.1111/j.14697793.2001.00841.x

El Marroun, H., White, T., Verhuist, F. C., and Tiemeier, H. (2014). Maternal use of antidepressant or anxiolytic medication during pregnancy and childhood neurodevelopmental outcomes: a systematic review. Eur. Child. Adolesc. Psychiatry 23, 973-992. doi: 10.1007/s00787-014-0558-3

Engelhardt, B. (2003). Development of the blood-brain barrier. Cell Tissue Res. 314, 119-129. doi: 10.1007/s00441-003-0751-z

Engelhardt, B. (2006). "Development of the blood-brain interface," in Blood-Brain Barriers, Vol. 1, eds R. Dermietzel, D. C. Spray, and M. Nedergaard (Weinheim: Wiley-VCH), 11-39.

Ernster, L., Herlin, L., and Zetterstrom, R. (1957). Experimental studies on the pathogenesis of kernicterus. Pediatrics 20, 647-652.

Evans, C. A. N., Reynolds, J. M., Reynolds, M. L., Saunders, N. R., and Segal, M. B. (1974). The development of a blood-brain barrier mechanism in foetal sheep. $J$. Physiol. (Lond.) 238, 371-386.

Ferguson, R. K., and Woodbury, D. M. (1969). Penetration of 14C-inulin and 14Csucrose into brain, cerebrospinal fluid, and skeletal muscle of developing rats. Exp. Brain Res. 7, 181-194. doi: 10.1007/BF00239028

Fishman, R. A. (1992). Cerebrospinal Fluid in Diseases of the Nervous System, 2nd Edn. Philadelphia, FL: WB Saunders.

Flexner, L. B. (1938). Changes in the chemistry and nature of the cerebrospinal fluid during fetal life in the pig. Am. J. Physiol. 124, 131-135.

Forsberg, L., and Wide, K. (2011). Long-term consequences after exposure to antiepileptic drugs in utero.Ther. Adv. Drug Saf. 2, 227-234. doi: $10.1177 / 2042098611419003$

Fossan, G., Cavanagh, M. E., Evans, C. A. N., Malinowska, D. H., Møllgård, K., Reynolds, M. L., et al. (1985). CSF-brain permeability in the immature sheep fetus: a CSF-brain barrier. Dev. Brain Res. 18, 113-124. doi: 10.1016/01653806(85)90255-X

Franceschini, P. (1929). Sulla presenza di elementi connettivali nel sistema nervoso centrale e sopra alcune particlarità di struttura dele meningi molli die plessi coroidei. A proposito della cosi detta barriera "emato-encefalica". Sperimentale $83,419-445$.

Franke, E. (1905). Therapeutische Versuche bei Trypanosomenerkrankung. Dissertation, Giessen.

Frömter, E., and Diamond, J. (1972). Route of passive ion permeation in epithelia. Nat. New Biol. 235, 9-13. doi: 10.1038/newbio235009a0

Friedemann, U. (1942). Blood-brain barrier. Physiol. Rev. 22, 125-145.

Friedemann, U., and Elkeles, A. (1931). Über die Permeabilität der BlutHirnschranke für Bakteriengifte. I. Mitteilung. 1. Experimentelle Untersuchungen über die Diphtherie-Vergiftung. Z. Exp. Med. 74, 293-319.

Friedemann, U., and Elkeles, A. (1934). The blood-brain barrier in infectious diseases: its permeability to toxins in relation to their electrical charges. Lancet 7, 719-724. doi: 10.1016/S0140-6736(00)92558-3 
Galbally, M., Snellen, M., and Power, J. (2014). Antipsychotic drugs in pregnancy: a review of their maternal and fetal effects. Ther. Adv. Drug Saf. 5, 100-107. doi: $10.1177 / 2042098614522682$

Ganong, W. B. (1993). Review of Medical Physiology. 16th Edn. East Norwalk, CT: Appleton \& Lange.

Ganong, W. B. (1999). Review of Medical Physiology. 19th Edn. Stamford, CT: Appleton \& Lange.

Gerrard, J. (1952). Kernicterus. Brain 75, 526-570. doi: 10.1093/brain/75. 4.526

Ghersi-Egea, J.-F., Gazzin, S., and Strazielle, N. (2009). Blood-brain interfaces and bilirubin-induced neurological diseases. Curr. Pharm. Des. 15, 2893-2907. doi: 10.2174/138161209789058147

Goldberg, H. L., and Nissim, R. (1994). Psychotropic drugs in pregnancy and lactation. Int. J. Psychiatr. Med. 24, 129-147. doi: 10.2190/2BF1-0718-WE7F-A9F7

Goldmann, E. E. (1909). Die äussere und innere Sekretion des gesunden und kranken Organismus im Lichte der 'vitalen Färbung.' Beiträg Klinische Chirurgie 64, 192-265.

Goldmann, E. E. (1913). Vitalfärbung am Zentralnervensyatem. Beitrag zur PhysioPathologie des plexus chorioideus und der Hirnhäute. Abh preuss, Akad Wiss Phys-Math Kl 1, 1-60.

Grandjean, P., and Landrigan, P. J. (2006). Developmental neurotoxicity of industrial chemicals. Lancet 368, 2167-2178. doi: 10.1016/S0140-6736(06)69665-7

Grazer, F. M., and Clemente, C. D. (1957). Developing blood-brain barrier to trypan blue. Proc. Soc. Exp. Biol. Med. 94, 758-760. doi: 10.3181/00379727-9423077

Gröntoft, O. (1954). Intracranial haemorrhage and blood-brain barrier problems in the newborn. Acta Pathol. Microbiol. Scand. Suppl. 100, 1-109.

Gyllenswärd, A., and Malmström, S. (1962). The cerebrospinal fluid of newborn infants. Acta Paediatr. 135, 54-62. doi: 10.1111/j.1651-2227.1962.tb08658.x

Habgood, M. D., Knott, G. W., Dziegielewska, K. M., and Saunders, N. R. (1993). The nature of the blood-CSF barrier permeability decrease during postnatal brain development in the rat. J. Physiol. (Lond.) 468, 73-83.

Habgood, M. D., Sedgwick, J. E. C., Dziegielewska, K. M., and Saunders, N. R. (1992). A developmentally regulated blood-cerebrospinal fluid transfer mechanism for albumin in immature rats. J. Physiol. (Lond.) 456, 181-192.

Hawkins, B. T., and Davis, T. P. (2005). The Blood-brain barrier/neurovascular unit in health and disease. Pharmacol. Rev. 57, 173-185. doi: 10.1124/pr.57.2.4

Heine, W., Hobusch, D., and Drescher, U. (1981). Eiweissgehalt des Liquors und Blut-Liquor-relation der Glucose und Electrolyte im Säuglings-und Kindersalter. Helv. Paediatr. Acta 36, 217-227.

Himwich, W. A., Petersen, J. C., and Allen, M. L. (1957). Hematoencephalic exchange as a function of age. Neurology 7, 705-710. doi: 10.1212/WNL.7. 10.705

Holash, J. A., Noden, D. M., and Stewart, P. A. (1993). Re-evaluating the role of astrocytes in blood-brain barrier induction. Dev. Dyn. 197, 14-25. doi: 10.1002/aja.1001970103

Hulsebosch, C. E., and Fabian, R. H. (1989). Penetration of IgGs into the neuraxis of the neonatal rat. Neurosci. Lett. 98, 13-18. doi: 10.1016/0304-3940(89) 90365-0

Hüntelmann, A. C. (2010). Legend of science. External constructions by the extended family - the biography of Paul Ehrlich. Interdisciplines 2, 13-36. doi: 10.2390/indi-v1-i2-17

Iskander, I., Gamaleldin, R., El Houchi, S., El Shenawy, A., Seoud, I., El Gharbawi, N., et al. (2014). Serum bilirubin and bilirubin/albumin ratio as predictors of bilirubin encephalopathy. Pediatrics 134, e1330-e1339. doi: 10.1542/peds.20131764

Janzer, R. C., and Raff, M. C. (1987). Astrocytes induce blood-brain barrier properties in endothelial cells. Nature 325, 253-257. doi: 10.1038/325253a0

Järup, L. (2003). Hazards of heavy metal contamination. Br. Med. Bull. 68, 167-182. doi: $10.1093 / \mathrm{bmb} / \mathrm{ldg} 032$

Johanson, C. E. (1989). "Ontogeny and Phylogeny of the blood-brain barrier," in Implications of the Blood Brain Barrier and its Manipulation, Vol. 1. Basic aspects, ed E. A. Neuwelt (New York, NY: Plenum), 157-198.

Johanson, C. E., and Woodbury, D. M. (1974). "Changes in CSF flow and extracellular space in the developing rat," in Drugs and the Developing Brain, eds A. Vernadakis and N. Weiner (New York, NY: Plenum), 281-287.

Johansson, P. A., Dziegielewska, K. M., Ek, C. J., Habgood, M. D., Liddelow, S. A., Potter, A. M., et al. (2006). Blood-CSF barrier function in the rat embryo. Eur. J. Neurosci. 24, 65-76. doi: 10.1111/j.1460-9568.2006.04904.x
Johansson, P. A., Dziegielewska, K. M., Liddelow, S. A., and Saunders, N. R. (2008). The blood-CSF barrier explained: when development is not immaturity. Bioessays 30, 237-248. doi: 10.1002/bies.20718

Joó, F. (1987). Current aspects of the development of the blood-brain barrier. Int. J. Dev. Neurosci. 5, 369-372. doi: 10.1016/0736-5748(87)90013-X

Kennedy, D. S. (2011). A to X: the problem of categorisation of drugs in pregnancy-an Australian perspective. Med. J. Aust. 195, 572-574. doi: $10.5694 / \mathrm{mja} 11.11119$

King, L. S. (1939). The hematoencephalic barrier. Arch. Neurol. Psychiatr. 41, 51-72. doi: 10.1001/archneurpsyc.1939.02270130061003

Knott, G. W., Dziegielewska, K. M., Habgood, M. D., Li, Z. S., and Saunders, N. R. (1997). Albumin transfer across the choroid plexus of South American opossum (Monodelphis domestica). J. Physiol.(Lond.) 499, 179-194.

Klosovskii, B. N. (1963). The Development of the Brain and its Disturbance by Harmful Factors Translated by Haigh, B. Oxford: Pergamon.

Kratzer, I., Liddelow, S. A., Saunders, N. R., Dziegielewska, K. M., Strazielle, N., and Ghersi-Egea, J. F. (2013). Developmental changes in the transcriptome of the rat choroid plexus in relation to neuroprotection. Fluid Barriers CNS 10:25. doi: 10.1186/2045-8118-10-25

Kuttner, R., Sims, J. A., and Gordon, M. W. (1961). The uptake of a metabolically inert amino acid by brain and other organs. J. Neurochem. 6, 311-317. doi: 10.1111/j.1471-4159.1961.tb13481.x

Lajtha, A., and Toth, J. (1961). The brain barrier system-II. Uptake and transport of amino acids by the brain. J. Neurochem. 8, 216-225. doi: 10.1111/j.14714159.1961.tb13545.x

Lee, J. C. (1971). Evolution of the concept of the blood-brain barrier concept. Prog. Neuropathol. 1, 84-145.

Lefauconnier, J.-M., and Trouvé, R. (1983). Developmental changes in the pattern of amino acid transport at the blood-brain barrier in rats. Brain Res. 283, 175-182. doi: 10.1016/0165-3806(83)90095-0

Lewandowsky, M. (1900). Zur Lehre von der Cerebrospinalflüssgkeit. Z. Clin. Med. 40, 480-494.

Liddelow, S., Dziegielewska, K. M., Ek, C. J., Johansson, P. A., Potter, A., and Saunders, N. R. (2009). Cellular transfer of macromolecules across the developing choroid plexus of Monodelphis domestica. Eur. J. Neurosci. 29, 253-266. doi: 10.1111/j.1460-9568.2008.06571.x

Liddelow, S., Dziegielewska, K. M., Noor, N., Potter, A. M., and Saunders, N. R. (2011a). Modification of choroid plexus protein transfer from blood to cerebrospinal fluid in response to altered plasma protein composition during development. Eur. J. Neurosci. 33, 391-400. doi: 10.1111/j.1460-9568.2010.07509.x

Liddelow, S. A., Dziegielewska, K. M., Ek, C. J., Habgood, M. D., Bauer, H., Bauer, H.-C., et al. (2013). Mechanisms that determine the internal environment of the developing brain: a transcriptomic, functional and ultrastructural approach. PLoS ONE 8:e65629. doi: 10.1371/journal.pone.0065629

Liddelow, S. A., Dziegielewska, K. M., Møllgård, K., Phoenix, T. N., Temple, S., VandeBerg, J. L., et al. (2011b). Sparc/osteonectin, an endogenous mechanism for targeting albumin to the blood-CSF interface during brain development. Eur. J. Neurosci. 34, 1062-1073. doi: 10.1111/j.1460-9568.2011.07821.x

Liddelow, S. A., Temple, S., Møllgård, K., Gehwold, R., Wagner, A., Bauer, H., et al. (2012). Molecular characterisation of transport mechanisms at the developing mouse blood-CSF interface: a transcriptome approach. PLoS ONE 7:e33554. doi: 10.1371./journal.pone.0033554

Lossinsky, A. S., Vorbrodt, A. W., and Wisniewski, H. M. (1986). Characterization of endothelial cell transport in the developing mouse blood-brain barrier. Dev Neurosci. 8, 61-75. doi: 10.1159/000112242

Lucey, J. F. (1971). Changing concepts regarding exchange transfusions and neonatal jaundice. Clin. Obstet. Gynecol. 14, 586-593. doi: 10.1097/00003081197106000-00020

Malamud, W., Fuchs, D. M., and Malamud, N. (1928). Barrier between the blood and the cerebrospinal fluid. 1. Changes in permeability in mental disease. Arch. Neurol. Psychiatry 20, 780-798. doi: 10.1001/archneurpsyc.1928.02210160121010

Mazariegos, M. R., Tice, L. W., and Hand, A. R. (1984). Alteration of tight junctional permeability in the rat parotid gland after isoproterenol stimulation. J. Cell Biol. 98, 1865-1877. doi: 10.1083/jcb.98.5.1865

Meek, W. J. (1907). A study of the choroid plexus. J. Comp. Neurol. 17, 286-306.

Mhanna, M. J., Alesseh, H., Gori, A., and Aziz, H. F. (2008). Cerebrospinal fluid values in very low birth weight infants with suspected sepsis at different ages. Pediatr. Crit. Care Med. 9, 294-298. doi: 10.1097/01.PCC.0b013e31816c6e12 
Millen, J. W., and Hess, A. (1958). The blood-brain barrier: an experimental study with vital dyes. Brain 81, 248-257. doi: 10.1093/brain/81.2.248

Mitchell, W., Kim, C. S., O’Tuama, L. A., Pritchard, J. B., and Pick, J. R. (1982). Choroid plexus, brain and kidney $\mathrm{Na}+\mathrm{K}+$-ATPase: comparative activities in fetal, newborn and young adult rabbits. Neurosci. Lett. 31, 37-40. doi: 10.1016/0304-3940(82)90050-7

Mizee, M. R., Wooldrik, D., Lakeman, K. A., van het Hof, B., Drexhage, J. A., Geerts, D., et al. (2013). Retinoic acid induces blood-brain barrier development. J. Neurosci. 33, 1660-1671. doi: 10.1523/JNEUROSCI.1338-12.2013

Møllgård, K., Lauritzen, B., and Saunders, N. R. (1979). Double replica technique applied to choroid plexus from early foetal sheep: completeness and complexity of tight junctions. J. Neurocytol. 8, 139-149. doi: 10.1007/BF011 75557

Møllgård, K., Malinowska, D. H., and Saunders, N. R. (1976). Lack of correlation between tight junction morphology and permeability properties in developing choroid plexus. Nature 264, 293-294. doi: 10.1038/264293a0

Møllgård, K., and Saunders, N. R. (1975). Complex tight junctions of epithelial and of endothelial cells in early foetal brain. J. Neurocytol. 4, 453-468. doi: 10.1007/BF01261375

Møllgård, K., and Saunders, N. R. (1986).The development of the human bloodbrain and blood-CSF barriers. Neuropathol. Appl. Neurobiol. 12, 337-358. doi: 10.1111/j.1365-2990.1986.tb00146.x

Møllgård, K., and Saunders, N. R. (1977). A possible transepithelial pathway via endoplasmic reticulum in foetal sheep choroid plexus. Proc. R. Soc. Lond. B Biol. Sci. 199, 321-326. doi: 10.1098/rspb.1977.0142

Moos, T., and Møllgård, K. (1993). Cerebrovascular permeability to azo dyes and plasma proteins in rodents of different ages. Neuropathol. Appl. Neurobiol. 19, 120-127. doi: 10.1111/j.1365-2990.1993.tb00416.x

Nag, S. (2011). Morphology and properties of brain endothelial cells, Ch 1 in the blood-brain and other neural barriers: reviews and protocols. Methods Mol. Biol. 686, 3-35. doi: 10.1007/978-1-60761-938-3_1

Naidoo, B. T. (1968). The cerebrospinal fluid in the healthy newborn infant. S. Afr. Med. J. 42, 933-935.

Nasralla, M., Gawronska, E., and Hsia, D. Y.-Y. (1958). Studies on the relation between serum and spinal fluid bilirubin during early infancy. J. Clin. Invest. 37, 1403-1412. doi: 10.1172/JCI103730

Nattie, E. E., Edwards, W. H., and Marin-Padilla, M. (1984). Newborn puppy cerebral acid-base regulation in experimental asphyxia and recovery. J. Appl. Physiol. 56, 1178-1186.

Nitta, T., Hata, M., Gotoh, S., Seo, Y., Sasaki, H., Hashimoto, N., et al. (2003). Sizeselective loosening of the blood-brain barrier in claudin-5-deficient mice. J. Cell Biol. 161, 653-660. doi: 10.1083/jcb.200302070

Oldendorf, W. M. (1971). Brain uptake of radiolabeled amino acids, amines, and hexoses after arterial injection. Am. J. Physiol. 221, 1629-1639.

Olney, J. W., and Ho, O. L. (1970). Brain damage in infant mice following oral intake of glutamate, aspartate or cysteine. Nature 227, 609-611. doi: $10.1038 / 227609 \mathrm{~b} 0$

Olsson, Y., Klatzo, I., Sourander, P., and Steinwall, O. (1968). Blood-brain barrier to albumin in embryonic new born and adult rats. Acta Neuropathol. 16, 117-124. doi: 10.1007/BF00691305

Ornoy, A., and Koren, G. (2014). Selective serotonin reuptake inhibitors in human pregnancy: on the way to resolving the controversy. Semin. Fetal Neonatal Med. 19, 188-194. doi: 10.1016/j.siny.2013.11.007

Otila, E. (1948). Studies on the cerebrospinal fluid in premature infants. Acta Paediatr. 35(Suppl. 8), 9-100.

Pardridge, W. M., and Mietus, L. J. (1982). Kinetics of neutral amino acid transport through the blood-brain barrier of the newborn rabbit. J. Neurochem. 38, 955-962. doi: 10.1111/j.1471-4159.1982.tb05335.x

Penta, P. (1932). Sulla colorazione vitale del sistema nervosa negli centrale animali neonati. Riv. Neurol. 5, 62-80.

Piliero, S. J., and Lending, M. (1959). Protein studies in normal newborn infants. Paper electrophoresis of cerebrospinal fluid and serum. Am. J. Dis. Child. 97, 785-789. doi: 10.1001/archpedi.1959.02070010787004

Purpura, D. P., and Carmichael, M. W. (1960). Characteristics of blood-brain barrier to gamma-aminobutyric acid in neonatal cat. Science 131, 410-412. doi: 10.1126/science.131.3398.410

Ramey, B. A., and Birge, W. H. (1979). Development of cerebrospinal fluid and the blood-cerebrospinal fluid barrier in rabbits. Dev. Biol. 68, 292-298. doi: 10.1016/0012-1606(79)90261-6
Ramoz, L. L., and Patel-Shori, N. M. (2014). Recent changes in pregnancy and lactation labeling: retirement of risk categories. Pharmacotherapy 34, 389-395. doi: 10.1002/phar. 1385

Ribatti, D., Nico, B., and Bertossi, M. (1993). The development of the blood-brain barrier in the chick. Studies with Evans blue and horseradish peroxidase. Ann. Anat. 175, 85-88. doi: 10.1016/S0940-9602(11)80247-5

Ribatti, D., Nico, B., Crivellato, E., and Artico, M. (2006). Development of the blood-brain barrier: a historical point of view. Anat. Rec. B New Anat. 289B, 3-8. doi: 10.1002/ar.b.20087

Risau, W., Hallmann, R., and Albrecht, U. (1986). Differentiation-dependent expression of proteins in brain endothelium during development of the bloodbrain barrier. Dev. Biol. 117, 537-545. doi: 10.1016/0012-1606(86)90321-0

Risau, W., and Seulberger, H. (1990). "Angiogenesis and differentiation of bloodbrain barrier endothelium. Ch 1," in Pathophysiology of the Blood-Brain Barrier, Vol. 14, eds B. B. Johansson, C. H. Owman, and H. Widner (Amsterdam: Elsevier), 3-9.

Risau, W., and Wolburg, H. (1990). Development of the blood-brain barrier. Trends Neurosci. 13, 174-178. doi: 10.1016/0166-2236(90)90043-A

Roberts, R. B., Flexner, J. B., and Flexner, L. B. (1959). Biochemical and physiological differentiation during morphogenesis. XXIII. Further observations relating to the synthesis of amino acids and proteins by the cerebral cortex and liver of the mouse. J. Neurochem. 4, 78-90. doi: 10.1111/j.1471-4159.1959.tb13176.x

Rodier, P. M. (1994). Vulnerable periods and processes during central nervous system development. Environ. Health Perspect. 102, 121-124. doi: 10.1289/ehp.94102121

Rodier, P. M. (1995). Developing brain as a target of toxicity. Environ. Health Perspect. 103, 73-76. doi: 10.1289/ehp.95103s673

Rodier, P. M. (2004). Environmental causes of central nervous system maldevelopment. Pediatrics 113, 1076-1083. Available online at: http://pediatrics. aappublications.org/content/113/Supplement_3/1076.full.html

Romanoff, A. L. (1967). Biochemistry of the Avian Embryo. New York, NY: Wiley.

Roncali, L., Nico, B., Ribatti, D., Bertossi, M., and Mancini, L. (1986). Microscopical and ultrastructural investigations on the development of the blood-brain barrier in the chick embryo optic tectum. Acta Neuropathol. 70, 193-201. doi: 10.1007/BF00686072

Roux, E., and Borrel, A. (1898). Tétanos cérébral et immunité contre le tétanus. Ann. Institut Pasteur 12, 225-239.

Roy, S., Hirano, A., Kochen, J. A., and Zimmerman, H. M. (1974). The fine structure of cerebral blood vessels in chick embryo. Acta Neuropath. 30, 277-285. doi: 10.1007/BF00697010

Rubin, L. L., Hall, D. E., Porter, S., Barbu, K., Cannon, C., Horner, H. C., et al. (1991). A cell culture model of the blood-brain barrier. J. Cell Biol. 115, 1725-1735. doi: 10.1083/jcb.115.6.1725

Sarff, L. D., Platt, L. H., and McCracken, G. H. Jr. (1976). Cerebrospinal fluid evaluation in neonates: comparison of high-risk infants with and without meningitis. J. Pediatr. 88, 473-477. doi: 10.1016/S0022-3476(76)80271-5

Saunders, N. R. (1977). Ontogeny of the blood-brain barrier. Exp. Eye Res. 25(Suppl.), 523-550. doi: 10.1016/S0014-4835(77)80046-8

Saunders, N. R. (1992). "Ontogenetic development of brain barrier mechanisms," in Physiology and Pharmacology of the Blood Brain-barrier. Vol. 103. Handbook of Experimental Pharmacology, ed M. W. B. Bradbury (Berlin: Springer-Verlag), 327-369.

Saunders, N. R., Adam, E., Reader, M., and Møllgård, K. (1989). Monodelphis domestica (grey short-tailed opossum): an accessible model for studies of early neocortical development. Anat. Embryol. 180, 227-236. doi: 10.1007/BF003 15881

Saunders, N. R., Daneman, R., Dziegielewska, K. M., and Liddelow, S. A. (2013). Transporters of the blood-brain and blood-CSF interfaces in development and in the adult. Mol. Aspects Med. 34, 742-752. doi: 10.1016/j.mam.2012.11.006

Saunders, N. R., Liddelow, S. A., and Dziegielewska, K. M. (2012). Barrier mechanisms in the developing brain. Front. Neuropharmacol. 3:46. doi: 10.3389/fphar.2012.00046

Sedlácek, J. (1975). Some basic chemical components of the cerebrospinal fluid in developing chick embryos. Physiol. Bohemoslov. 24, 305-313.

Seta, K., Sershen, H., and Lajtha, A. (1972). Cerebral amino acid uptake in vivo in newborn mice. Brain Res. 47, 415-425. doi: 10.1016/0006-8993(72)90649-X

Spatz, H. (1934). Die Bedeutung der vitalen Färbung für die Lehre vom Stoffaustausch zwischen dem Zentralnervensystem and dem iibrigen Körper. Arch. F. Psychiatr. 101, 267-358. doi: 10.1007/BF01789978 
Spiegel-Adolf, M., Baird, H. W. 3rd., Szekely, E. G., and Wycis, H. T. (1954). Cerebrospinal fluid studies in infant children with cerebral palsy and other neurologic disorders. Pediatrics 14, 215-221.

Srinivasan, L., Shah, S. S., Padula, M. A., Abbasi, S., McGowan, K. L., and Harris, M. C. (2012). Cerebrospinal fluid reference ranges in term and preterm infants in the neonatal intensive care unit. J. Pediatr. 161, 729-734. doi: 10.1016/j.jpeds.2012.03.051

Statz, A., and Felgenhauer, K. (1983). Development of the blood-CSF barrier. Dev. Med. Child Neurol. 25, 152-161. doi: 10.1111/j.1469-8749.1983.tb13738.x

Stern, L. (1934). A propos de la méthod d'investigation du fonctionnement de la barrière hémato-encéphalique. C. R. Soc. Biol. 115, 1059-1061.

Stern, L., Battelli, F., and Peyrot, R. (1924). Fonctionnement de la barrière hématoencéphalique (H.-E.) chez les divers animaux au cours de leur développement. Schw. Med. Wschr. 5, 313.

Stern, L., and Gautier, R. (1918a). Passage simultané des substances dans le liquide céphalo-rachidien et dans les centres nerveux. R. C. R. d. Ia Soc. de Phys. et d'hist. natur. de Genève 35, 58-60.

Stern, L., and Gautier, R. (1918b). Le passage dans le liquide céphalo-rachidien de substances introduites dans la circulation et leur action sur le système nerveux central chez les différentes espèces animales. R. C. R. d. Ia Soc. de Phys. et d'hist. natur. de Genève 35, 91-94.

Stern, L., and Gautier, R. (1921). Recherches sur le liquide céphalo-rachidien. 1. Les rapports entre le liquide céphalo-rachidien et la circulation sanguine. Arch. Int. Physiol. 17, 138-192. doi: 10.3109/13813452109146211

Stern, L., and Gautier, R. (1922). Recherches sur le liquide céphalo-rachidien. II. Les rapports entre le liquide céphalo-rachidien et les éléments nerveux de l'axe cérébro-spinal. Arch. Int. Physiol. 17, 391-448. doi: $10.3109 / 13813452209146219$

Stern, L., and Gautier, R. (1923). Recherches sur le liquide céphalo-rachidien. II. Rapports entre le liquide céphalo-rachidien et des espaces ventriculaires et cellui des espaces sous-arachnodiens. Arch. Int. Physiol. 20, 403-436. doi: $10.3109 / 13813452309145975$

Stern, L., and Peyrot, R. (1927). Le fonctionnment de la barrière hématoencéphalique aux divers stades de développement chez les diverses especes animals. C. R. Soc. Biol. 96, 1124-1126.

Stern, L., and Rapoport, J. L. (1928a). Les rapports entre l'augmentation de la perméabilité de la barrière hémato-encéphalique et les altérations de son substratum morphologique. C. R. Soc. Biol. 98, 1515-1517.

Stern, L., and Rapoport, J. L. (1928b). Les échanges entre le liquide céphalorachidien et les éléments nerveux cérébro-spinaux. C. R. Soc. Biol. 98, 1518-1519.

Stern, L., Rapoport, J. L., and Lokschina, E.-S. (1929). Le fonctionnement de la barrière hémato-encéphalique chez les nouveau nés. C. R. Soc. Biol. 100, 231-223.

Stewart, P. A., and Hayakawa, E. M. (1987). Interendothelial junctional changes underlie the developmental "tightening" of the blood brain barrier. Brain Res. 429, 271-281. doi: 10.1016/0165-3806(87)90107-6

Stewart, P. A., and Hayakawa, K. (1994). Early ultrastructural changes in bloodbrain barrier vessels of the rat embryo. Brain Res. Dev. Brain Res. 78, 25-34. doi: 10.1016/0165-3806(94)90005-1

Stewart, P. A., and Wiley, M. J. (1981). Developing nervous tissue induces formation of blood-brain barrier characteristics in invading endothelial cells: a study using quail-chick transplantation chimeras. Dev. Biol. 84, 183-192. doi: 10.1016/00121606(81)90382-1

Tao-Cheng, J. H., Nagy, Z., and Brightman, M. W. (1987). Tight junctions of brain endothelium in vitro are enhanced by astroglia. J. Neurosci. 7, 3293-3299.

Tauc, M., Vignon, X., and Bouchaud, C. (1984). Evidence for the effectiveness of the blood-CSF barrier in the fetal rat choroid plexus. A freeze-fracture and peroxidase diffusion study. Tissue Cell 16, 65-74. doi: 10.1016/0040-8166(84) 90019-3

Tennyson, V. (1975). "Ultrastructural characteristics of the telencephalic and myelencephalic choroid plexus in fetus of man and rabbit, and a comparison with the adult choroid plexus in rabbit," in The Choroid Plexus in Health and Disease, eds M. G. Netsky and S. Shuangshati (Bristol: John Wright \& Sons), 36-71.

Tennyson, V. M., and Pappas, G. D. (1964). Fine structure of the developing telencephalic and myelencephalic choroid plexus in the rabbit. J. Comp. Neurol. 123, 379-412. doi: 10.1002/cne.901230307

Teratology Society Public Affairs Committee. (1994). FDA Classification of drugs for teratogenic risk. Teratology 49, 446-447. doi: 10.1002/tera.1420490603
Teratology Society Public Affairs Committee. (2007). Teratology public affairs committee position paper: pregnancy labeling for prescription drugs: ten years later. Birth Defects Res. A Clin. Mol. Teratol. 79, 627-630. doi: 10.1002/bdra.20389

Tschirgi, R. D. (1950). Protein complexes and the impermeability of the bloodbrain barrier to dyes. Am. J. Physiol. 163, 756.

van den Bergh, H. A. A., and Müller, P. (1916). Über eine direkte und indirekte Diazoreaktion auf Bilirubin. Biochem. Zeitschrift 77, 90-103.

van Hasselt, J. G., van Calsteren, K., Heyns, L., Han, S., Mhallem Gziri, M., Schellens, J. H., et al. (2014). Optimizing anti-cancer drug treatment in pregnant cancer patients: pharmacokinetic analysis of gestation-induced changes for doxorubicin, epirubicin, docetaxel and paclitaxel. Ann. Oncol. 25, 2059-2065. doi: 10.1093/annonc/mdu140

Vein, A. A. (2008). Science and fate: Lina Stern (1878-1968), a neurophysiologist and biochemist. J. Hist. Neurosci. 17, 195-206. doi: 10.1080/09647040601138478

Viña, J. R., DeJoseph, M. R., Hawkins, P. A., and Hawkins, R. A. (1997). Penetration of glutamate into brain of 7-day-old rats. Metab. Brain Dis. 12, 219-227. doi: 10.1007/BF02674614

Virgintino, D., Errede, M., Girolamo, F., Capobianco, C., Robertson, D., Vimercati, A., et al. (2007). Fetal blood-brain barrier P-glycoprotein contributes to brain protection during human development. J. Neuropathol. Exp. Neurol. 67, 50-61. doi: 10.1097/nen.0b013e31815f65d9

Virgintino, D., Errede, M., Robertson, D., Capobianco, C., Girolamo, F., Vimercati, A., et al. (2004). Immunolocalization of tight junction proteins in the adult and developing human brain. Histochem. Cell Biol. 122, 51-59. doi: 10.1007/s00418004-0665-1

Virgintino, D., Robertson, D., Benagiano, V., Errede, M., Bertossi, M., Ambrosi, G., et al. (2000). Immunogold cytochemistry of the blood-brain barrier glucose transporter GLUT1 and endogenous albumin in the developing human brain. Brain Res. Dev. Brain Res. 123, 95-101. doi: 10.1016/S0165-3806(00)00086-9

Volpe, J. J. (2008). "Bilirubin and brain injury," in Neurology of the Newborn, 5th Edn., ed J. J. Volpe (Philadelphia, PA: W. B. Saunders), 619-651.

von Monakow, C. (1921). Der Kreislauf des Liquor cerebro-spinalis. Schweiz Arch. Neurol. Psychiatry 8, 233-234.

Vorbrodt, A. W., Lossinsky, A. S., and Wisniewski, H. M. (1986). Localization of alkaline phosphatase activity in endothelia of developing and mature mouse blood-brain barrier. Dev. Neurosci. 8, 1-13. doi: 10.1159/000112236

Wakai, S., and Hirokawa, N. (1978). Development of the blood-brain barrier to horseradish peroxidase in the chick embryo. Cell Tissue Res. 195, 195-203. doi: 10.1007/BF00236719

Wakai, S., and Hirokawa, N. (1981). Development of blood-cerebrospinal fluid barrier to horseradish peroxidase in the avian choroidal epithelium. Cell Tissue Res. 214, 271-278. doi: 10.1007/BF00249211

Walter, K. (1934). Die allgemeinen Grundlagen des Stoffaustausches zwischen dem Zentralnervensystem und dem übrigen Körper. Archiv für Psychiatrie 10, 195-230. doi: 10.1007/BF01789976

Watson, D. (1964). Modern methods for determining cerebrospinal fluid protein. Clin. Chem. 10, 412-416.

Watson, R. E., Desesso, J. M., Hurtt, M. E., and Cappon, G. D. (2006). Postnatal growth and morphological Development of the brain: a species comparison. Birth Defects Res. B. Dev. Reprod. Toxicol. 77, 471-484. doi: 10.1002/bdrb. 20090

Weed, L. H. (1917a). An anatomical consideration of the cerebro-spinal fluid. Anat. Res. 12, 461-496. doi: 10.1002/ar.1090120405

Weed, L. H. (1917b). The development of the cerebrospinal fluid spaces in pig and in man. Contrib. Embryol. Carnegie Inst. 5, 3-116.

Wennberg, R. P. (2000). The blood-brain barrier and bilirubin encephalopathy. Cell. Mol. Neurobiol. 20, 97-109. doi: 10.1023/A:1006900111744

Wennberg, R. P., Ahlfors, C. E., Bhutani, V. K., Johnson, L. H., and Shapiro, S. M. (2006). Toward understanding kernicterus: a challenge to improve the management of jaundiced newborns. Pediatrics 117, 474-485. doi: 10.1542/peds.20050395

Widell, S. (1958). On the cerebrospinal fluid in normal children and in patients with acute abacterial meningoencephalitis. Acta Paediatr. 47(Suppl.), 115. doi: 10.1111/j.1651-2227.1958.tb07687.x

Wiesendanger, M. (2010). Constantin von Monakow (1853-1930) and Lina Stern (1878-1968). Schweiz Arch. Neurol. Psychiatry 161, 140-144.

Windle, W. F. (1969). Brain Damage by asphyxia at birth. Sci. Am. 221, 76-84. doi: 10.1038/scientificamerican1069-76 
Wislocki, G. B. (1920). Experimental studies on fetal absorption. I. The vitality stained fetus. Contrib. Embryol. Carnegie Inst. 11, 45-60.

Wood, K. E., Sinclair, L. L., Rysgaard, C. D., Strathman, F. G., McMillin, G. A., and Krasowski, M. D. (2014). Retrospective analysis of the diagnostic yield of newborn drug testing. BMC Pregnancy Childbirth 14:250. doi: 10.1186/14712393-14-250

Woodbury, D. M., Johanson, C., and Brønsted, M. (1974). "Maturation of the blood-brain and blood-cerebrospinal fluid barriers and transport systems," in Narcotics and the Hypothalamus, eds E. Zimmerman and R. George (New York, NY: Raven Press), 225-247.

$\mathrm{Xu}$, J., and Ling, E.-A. (1994). Studies of the ultrastructure and permeability of the blood-brain barrier in the developing corpus callosum in postnatal rat brain using electron dense tracers. J. Anat. 184, 227-237.

Zheng, W. (2002). "Blood-brain barrier and blood-CSF barrier in metal-induced neurotoxicities," in Handbook of Neurotoxicology, Vol. 1, ed E. J. Massaro (Totowa, NJ: Humana Press), 161-193.

Zuelzer, W. W., and Mudgett, R. T. (1950). KERNICTERUS: etiologic study based on an analysis of 55 cases. Pediatrics 6, 453-474.
Conflict of Interest Statement: The authors declare that the research was conducted in the absence of any commercial or financial relationships that could be construed as a potential conflict of interest.

Received: 15 September 2014; accepted: 20 November 2014; published online: 16 December 2014.

Citation: Saunders NR, Dreifuss J-J, Dziegielewska KM, Johansson PA, Habgood MD, Møllgård K and Bauer H-C (2014) The rights and wrongs of blood-brain barrier permeability studies: a walk through 100 years of history. Front. Neurosci. 8:404. doi: 10.3389/fnins.2014.00404

This article was submitted to Neurogenomics, a section of the journal Frontiers in Neuroscience.

Copyright () 2014 Saunders, Dreifuss, Dziegielewska, Johansson, Habgood, Møllgård and Bauer. This is an open-access article distributed under the terms of the Creative Commons Attribution License (CC BY). The use, distribution or reproduction in other forums is permitted, provided the original author(s) or licensor are credited and that the original publication in this journal is cited, in accordance with accepted academic practice. No use, distribution or reproduction is permitted which does not comply with these terms. 


\section{BIOGRAPHIES}

George Bernays Wislocki (1892-1956)

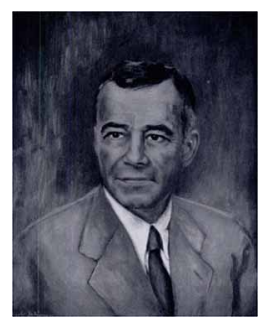

1892 Born March 5th in San Jose California

1912 Graduated from Washington University

1916 Graduated from The Johns Hopkins University School of Medicine

1916-1917 Postdoc Assistant in Anatomy under Lewis H Weed 1916-1923 Served as First Lieutenant in U.S. Army Medical Corps during WW1

Arthur Tracy Cabot Fellow at the Laboratory of Surgical Research at Harvard University

Associate in Anatomy at Johns Hopkins

1923 Associate Professor of Anatomy at Harvard University 1931-1941 Parkman Professor of Anatomy at Harvard University 1931 Married Florence Clothier

1939-1940 Member scientific advisory board, Wistar Institute of Anatomy and Biology

1941 James Stillman Professor of Comparative Anatomy at Harvard University

1941 Elected to National Academy of Sciences

1941 Honorary Master of Arts degree, Harvard University

1947 Hersey Professor of Anatomy, Harvard Medical School

1950-1951 First President of the Histochemical Society

1951 Honorary Doctor of Science, Washington University

1956 Died October 22nd in Milton, US (aged 64). Survived by his

wife Florence, 2 sons (George and Lewis) and 2 daughters (Edith and Johanna)

Scientific contributions: Wislocki is considered one of the pioneers in the use of histochemistry in anatomical studies. He greatly contributed to the description of the anatomy and function of the endocrine system as well as the comparative anatomy of the placental and brain-barriers. He was the first to publish an image of a dye experiment showing that dye (trypan blue) did not enter the brain of a guinea pig fetus (Figure 1A) except in regions known to have a barrier in the adult brain.

National Academy of Sciences (http://www.nasonline.org/ member-directory/deceased-members/20001486.html)

J. Histochem Cytochem. 1957 Jan;5(2):96. “GEORGE BERNAYS WISLOCKI, 1892-1956"

The Harvard Crimson http://www.thecrimson.com/article/1956/ 10/23/wislocki-dies-here-was-noted-scientist/

Obituary JAMA. 1956;162(18):1642-1644. doi:10.1001/jama. 1956.02970350058020

\section{Paul Ehrlich (1854-1915)}

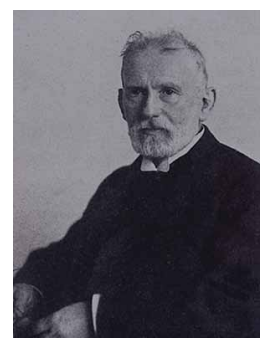

1854 Born 14th March in Strehlen in Upper Silesia (then Prussia). Educated at the Gymnasium at Breslau and subsequently at the Universities of Breslau, Strassburg, Freiburg-im-Breisgau and Leipzig

1876-1877 State examination as a medical doctor 1878 Obtained doctorate at University of Leipzig

Dissertation on theory and practice of staining animal tissues, in particular as a means of studying oxygenation of the brain

1878-1887 Physician at Berlin Medical Clinic (Charité)

1882 Ehrlich published method of staining tubercle bacillus. Gram method of staining bacteria derived from this

1882 Appointed Titular Professor

1887 Faculty of Medicine, University of Berlin, qualified as Privatdozen/Lecturer based on Habilitation thesis "Sauerstoffbedurfnis des Organismus." Later appointed Associate Professor and Senior House Physician to Charite Hospital in Berlin

1887-1890 Travels in Egypt and establishment of a private laboratory

1891-1895 Worked with Robert Koch at the Institute of Infectious Diseases in Berlin

1896 Director of the specially established Institute for Serum Research and Testing

1899 Moved to Frankfurt am Main (director of the Institute of Experimental Therapy)

1908 Nobel prize in Physiology or Medicine 1908 (together with Ilya Mechnikov) "in recognition of their work on the theory of immunity"

1914 Professor of Pharmacology at Frankfurt University

1915 Died from a Stroke (Bad Homburg, Germany)

Scientific contributions: Ehrlich made an impressive number of contributions to several research fields including hematology, immunology, chemotherapy and cancer research: the discovery of mast cells (in his Ph.D. thesis), dye experiments that were interpreted as some as early evidence for a mechanism preventing some molecules from entering the brain, although he himself did not accept this explanation (see text), major contributions to the diagnosis of blood diseases, discoveries about immunity to plant poisons and inheritance of acquired immunity via breast-milk, development of quantitative standardization methods for antiserum, the formulation of the Side-chain theory 
and the discovery of Salvarsan (first pathogen-targeted drug) for treatment of syphilis, the first successful chemotherapy.

See also Dale (1954) and Hüntelmann (2010)

The official website of the Nobel prize (http://www. nobelprize.org)

The Paul-Ehrlich-Institut (http://www.pei.de/EN/home/node. html)

\section{Max Lewandowsky (1876-1918)}

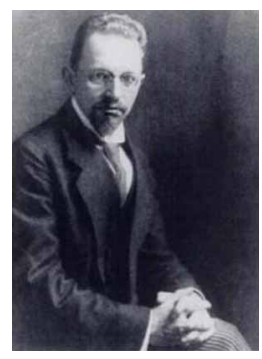

1876 Born 26 June in Berlin, Germany

1893 Awarded his Matriculation Certificate from the Friedrichs Gymnasium school in Berlin

1893 Began his studies at the University of Marburg under Hermann Cohen

1898 State examination as a medical doctor from HalleWittenberg University

1902 Habilitation at University of Berlin

1902-1904 Privat Docent in Berlin

1904 Studied Clinical Psychiatry with Nissl and Bonhofer in Heidelberg

1904-1905 Studied psychiatry with Pierre Mari at the Bicętre Clinic in Paris

1905 Physician consultant at the City Hospital in BerlinFriedrichshain

1907 Publication of Die Funktionen des zentralen Nervensystems 1908 Appointed full Professor

1910 Together with Alzheimer, founded the journal "Zeitschrift fur die gesamte Neurologie und Psychiatrie" and a series "Monographien aus dem Gesamtgebiete der Neurologie und Psychiatrie"

1910-1914 Publication of textbook "Handbuch der Neurologie" (5 volumes) of neurology Written by Lewandowsky himself (parts of each chapter) together with 40 contemporary European neurologists

1912 Publication of Praktische Neurologie für Ärzte. Later edition in 1919

1914-1917 Served at war hospitals during this period

1918 Died on 4th April in a Berlin sanatorium, probably committed suicide

Scientific contributions: His scientific inheritance contains definitive studies of neurophysiology of breathing, CSF, neuroanatomy of breathing centers, function of the sympathetic nervous system, cerebellum, discalculia and war psychosis. He was active in the Berlin neurological centers. He is frequently credited with having coined the term Blut-Hirnschranke" (blood-brain barrier) but as discussed in this review there is no evidence for this. He was also the author of the textbook Praktische Neurologie für Ärzte.

\section{Lina Stern (1878-1968)}

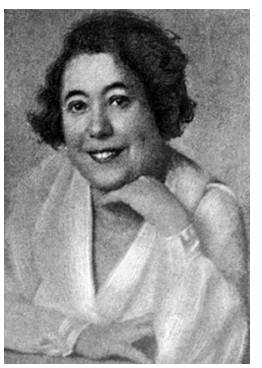

Part I. Geneva, Switzerland (1898-1925)

1878 Born August 26th in Latvia

1898-1904 Student in the Medical Faculty at the University of Geneva. Presented M.D. thesis in the field of physiology in 1904 1904-1914 Assistant, then first assistant in physiology. Research on biological oxidation, results published in German Journal of Biochemistry

1917 First studies on a barrier that slows down the penetration into the brain of substances dissolved in blood plasma

1918 First ever woman promoted to Associate Professor (in Physiological Chemistry) at the Department of Physiology

1921-1923 Coins the term barrier hemato-encephalic. This was probably the first use of the term "blood-brain barrier," although she mainly studied what was later called the blood-cerebrospinal fluid-barrier

1925 On receipt of an invitation to move her laboratory to Moscow, an attempt by the local government to promote her to full professor fails because of her leftish pro-revolutionary views and the financial crisis in Geneva

Part II. Rise, fall and rehabilitation in the USSR (1925-1953)

1925 Professor at Moscow Medical University and affiliation to several medical institutions

1925-1934 Difficult and slow adaptation to life in the Soviet Union and several failed attempts to recover her previous position in Geneva. Numerous reports on the blood-brain barrier (BBB) and on the effects of blood solutes following their parenteral or intra-ventricular injection

1929-1948 Director of the new Physiological Institute built and financed by the People's Commissariats of Education of Health, After 1939 the institute became part of the USSR Academy of Sciences. It was dismantled in 1948 (see below)

1934-1935 To mark 30 years of her scientific and educational work, Stern received an honorary doctorate of Biological Sciences and was awarded the title of "honored worker of science"

1939 First ever women elected full member of the USSR Academy of Sciences

1942 Publication in Nature and in the British Medical Journal of her article on the treatment of shock by direct action upon the 
vegetative nervous centers Another article printed in the West during WWII deals with the treatment of tetanus. During the war, Stern was a member of the Jewish Anti-Fascist Committee (JAC) organized in order to mobilize Jewish support for the USSR's war effort against Nazi Germany

1943-1945 Stalin Prize. Orders of the Red Banner and of the Red Star. Elected as full member of the USSR Academy of Medical Sciences

1947 Publication in a widely read medical journal of an unfounded attack against research on the blood-brain barrier, apparently an authorized signal from Stalin geopolitical reorientation to start persecution of Jews

1948 Closure of the Institute of Physiology and Stern "unmasked" as an "anti-Pavlovian" in 1949 she was accused of being a member of a Zionist organization, arrested and detained for 3 years and 10 months

1952 Secrete military trial of prominent members of the JAC. Except Stern, all the accused were executed on August 12, 1952. She was sentenced to five years internal exile in a small town in Central Asia

1953 Shortly after the death of Stalin, Stern was set free and allowed to return to Moscow. The same year she was reinstated in the Academy. She died on March 7, 1968

See also Vein (2008); Dreifuss and Sigrist (2009); Wiesendanger (2010)

\section{Edwin Goldmann (1862-1913)}

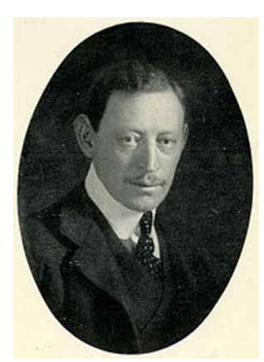

1862 Born 11th November in Burghersdorp, South Africa, Moved to Breslau (Wroclaw) in Europe with his parents, sister and brothers at age 14. Studied in Breslau, Freiburg and London 1887 Presented his dissertation "Experimental contributions to the teaching of the cystinuria and sulfur excretion in the urine." in Freiburg

1888 Received his Medical degree in London, Assistant under Prof. Weigert at the Pathological-Anatomical Institute in Frankfurt-on-Main

1888 Assistant at the University Surgical Hospital in Freiburg 1889 Practicing surgeon in Freiburg and Basel

1891 Received his Habilitation and qualified as a privat-docent 1895 Appointed Extraordinary Professor and became surgeon to the Deaconess's Hospital in Freiburg

1898-1913 Chief Physician at the hospital of the Deaconess's in Freiburg in 1898

1906 On 27th February married Lorna Lawrence Bosworth Smith (daughter of a British peer) at Bingham's Melcombe in Dorset. Associate under Paul Ehrlich at the Institute for Experimental Therapeutics, Frankfurt

1913 Ehrlich canvassed support from William Welch to establish an Institute for Vital Staining that he wished Goldmann to head. Abandoned following Goldmann's death and outbreak of 1st World War

1913 Died from cancer in Freiburg

Scientific contributions: During his time in Freiburg, in addition to his role as a physician, Goldmann worked in the field of "physiological chemistry" investigating sulfur-containing compounds in urine and later at Evangelical Sisters in the field of cancer research. He significantly contributed to the bloodbrain barrier field by his often-cited experiments showing the azo-dye trypan blue did not stain the brain when injected parenterally (Goldmann's "First experiment”), but did when injected intrathecally (Goldmann's "Second experiment").

Singer und F. T. Haneman: Goldmann, Edwin Ellen In: Jewish Encyclopedia

http://de.wikipedia.org/wiki/Edwin_Goldmann 


\title{
Understanding the rules of the road: proteomic approaches to interrogate the blood brain barrier
}

\author{
Bruce E. Torbett ${ }^{1}$, Andrew Baird ${ }^{2}$ and Brian P. Eliceiri ${ }^{2 *}$ \\ ${ }^{1}$ Molecular and Experimental Medicine, The Scripps Research Institute, La Jolla, CA, USA, ${ }^{2}$ Department of Surgery, \\ University of California, San Diego, San Diego, CA, USA
}

\section{OPEN ACCESS}

Edited by:

Shane Antony Liddelow,

Stanford University, USA

Reviewed by:

Danica Stanimirovic,

University of Ottawa, Canada

Joy Zuchero, Genentech, USA

${ }^{\star}$ Correspondence:

Brian P. Eliceiri,

Department of Surgery,

University of California, San Diego,

212 Dickinson Street, MC8236,

San Diego, CA 92103, USA beliceiri@ucsd.edu

Specialty section:

This article was submitted to Neurogenomics, a section of the

journal Frontiers in Neuroscience

Received: 20 October 2014

Accepted: 17 February 2015

Published: 04 March 2015

Citation:

Torbett BE, Baird A and Eliceiri BP (2015) Understanding the rules of the road: proteomic approaches to interrogate the blood brain barrier.

Front. Neurosci. 9:70.

doi: 10.3389/fnins.2015.00070
The blood brain barrier (BBB) is often regarded as a passive barrier that protects brain parenchyma from toxic substances, circulating leukocytes, while allowing the passage of selected molecules. Recently, a combination of molecular profiling techniques have characterized the constituents of the BBB based on in vitro models using isolated endothelial cells and ex vivo models analyzing isolated blood vessels. Characterization of gene expression profiles that are specific to the endothelium of brain blood vessels, and the identification of proteins, cells and multi-cellular structure that comprise the BBB have led to a emerging consensus that the BBB is not, in and of itself, a simple barrier of specialized endothelial cells. Instead, regulation of transcytosis, permeability, and drug translocation into the central nervous system is now viewed as a collection of neurovascular units (NVUs) that, together, give the BBB its unique biological properties. We will review recent technology advancing the understanding of the molecular basis of the BBB with a focus on proteomic approaches.

Keywords: blood brain barrier, proteomics, mass spectrometry, vascular diseases

The focus of this review is on techniques that can be applied to the analysis of in vivo models with particular attention to the complementary value of transcriptomics (Enerson and Drewes, 2006; Daneman et al., 2010) where sensitivity and standardized analytical techniques in RNA expression are powerful tools that can provide optimal coverage of gene expression in select tissues and cell types. Furthermore, we address the challenges of analyzing the protein composition of the BBB from the perspective of not only the endothelial cells, a compartment that has been the focus of in vitro models, but also the importance of considering the $\mathrm{BBB}$ as a multicellular structure with extracellular matrix (ECM) and other cell types relevant in BBB formation (Chun et al., 2011; Hoshi et al., 2013; Badhwar et al., 2014).

\section{Proteomics for Blood Brain Barrier Interrogation}

Proteomics, the global interrogation of the protein population expressed by a genome, cells, or tissue types, makes use of biochemical and physical methods to determine the identity of proteins present (Yates, 1998, 2000; Banks et al., 2000; McDonald and Yates, 2000; Pandey and Mann, 2000; Yates, 2000; Mann and Pandey, 2001; Mann et al., 2001). Proteomics

Abbreviations: BBB, Blood brain barrier; NVU, neurovascular unit; MudPIT, multi-dimensional protein identification technology; ECM, extracellular matrix; iTRAQ, Tags for Relative and Absolute Quantitation; TMT, Tandem Mass Tags; AQP4, Aquaporin 4; GFAP, glial fibrillary acidic protein; CW, circle of Willis. 
is complementary to studies of gene expression. Determination of the changes in cellular mRNA abundance provides information on gene activity and the cellular state (Enerson and Drewes, 2006; Daneman et al., 2010). For many genes, changes in mRNA abundance correspond to changes in protein abundance (Yates, 1998; Banks et al., 2000; McDonald and Yates, 2000; Pandey and Mann, 2000; Yates, 2000; Mann and Pandey, 2001; Mann et al., 2001). However, protein-based cellular analysis is essential to establish the translation of mRNAs to proteins, since mRNAs have varying stability, translational efficiency and do not address post-translational modifications. The diverse chemical properties of proteins make them difficult to separate and identify. Two-dimensional gel electrophoresis has been the standard for separation, isolation, and sequencing of individual proteins from sera, cells, and tissues for more than 25 years. However, this methodology does not provide high throughput protein identification and quantification required for the study of the cellular proteome. What revolutionized the proteomic field was the development of mass spectrometry instrumentation capable of rapidly and reproducibly fragmenting peptides and bioinformatic software to match the observed peptide fragment masses to a database of predicted masses for one of many given peptide sequences (Washburn et al., 2001; Wu et al., 2003).

Shotgun proteomics or Multi-Dimensional Protein Identification Technology (MudPIT) is the contemporary approach for identifying proteins in complex cellular samples (Washburn et al., 2001; Wu and Maccoss, 2002; Wu et al., 2003; Kislinger et al., 2005; Yates et al., 2009). The methodology relies on protease digestion of the cellular protein sample to peptides, followed by peptide separation on inline high performance chromatography, and identification of the peptides by tandem mass spectrometry (Chun et al., 2011; Hoshi et al., 2013; Badhwar et al., 2014). The uniqueness of the mass fragmentation of each peptide is used to identify the protein from which it was derived (Chun et al., 2011; Hoshi et al., 2013; Badhwar et al., 2014). The advantage of MudPIT, over classical two dimensional gel analyses, is the better resolving power, enabling a more precise protein quantification and identification of a larger number of proteins from the complex proteome of isolated tissues or cells (Washburn et al., 2001; Aebersold and Mann, 2003; Wu et al., 2003; Pottiez et al., 2009). In addition to being useful for tissues, such techniques are sensitive enough for use on desired cell populations isolated by flow cytometry or magnetic beads. Importantly, the increasing resolving power of the mass spectrometers, highly curated and annotated databases for protein identification, and bioinformatic to identify proteins from databases, provides the researcher with powerful methods to analyze complex cellular protein mixtures (Chen et al., 2006; Ruse et al., 2008; Gonzalez-Begne et al., 2009).

In contrast to transcriptomics methodology, which takes advantage of chemical similarity among RNAs allowing quantification among experimental samples, quantification of proteins in complex protein samples are hindered by the diverse chemical nature of proteins and the resolving power of mass spectrometers (Coombs, 2011). One method for relative protein quantification from different experimental samples (i.e., control vs. tests) in an experimental study relies on a label-free approach of determining peptide abundance through spectral counting in each sample, thereby allowing comparison among the experimental samples (Florens et al., 2006; Mueller et al., 2008; Neilson et al., 2011; Nahnsen et al., 2013). A more precise method for determining the relative abundance of proteins from experimental samples is to label proteins in each experimental sample with a unique stable isotope, thereby allowing the mass spectrometer to identify identical proteins with different masses $(\mathrm{McD}$ onald and Yates, 2000; Wu et al., 2004; Gouw et al., 2008; Liao et al., 2008; Uchida et al., 2013). The differentially isotope labeled samples are combined and analyzed together and the relative abundance of each protein in the mixture can be determined. Examples of these methods are Stable Isotope Labeling with Amino acids in cell Culture (SILAC), which relies on labeling of proteins in cellular samples by metabolic labeling with ${ }^{15} \mathrm{~N}$ where the differential in ${ }^{15} \mathrm{~N} /{ }^{14} \mathrm{~N}$ labeling can be used to quantify the relative abundance of identical proteins grown under different conditions (Wu et al., 2004; Lu et al., 2007; Gouw et al., 2008; Haqqani et al., 2008; Kamiie et al., 2008; Liao et al., 2008; Evans et al., 2012). This approach and other related metabolic cell labeling technologies are powerful tools where the model system, either cell culture or an animal, can be metabolically labeled to enable absolute quantification based on the relative uptake of labeled isotopes. The application of these approaches for the annotation of protein and nucleotide databases underscores the importance of multidisciplinary technologies to better understand correlations between gene expression and protein translation (Mann and Pandey, 2001; Mann et al., 2001; Ohtsuki et al., 2011; Uchida et al., 2013). Additional quantitative techniques that have been previously reviewed include labeling of a peptide mixture with $\mathrm{N}$-terminal or reactive amines using defined molecular tags, termed Isobaric Tags for Relative and Absolute Quantitation (iTRAQ) and Tandem Mass Tag (TMT) (Wu et al., 2004; Gouw et al., 2008; Christoforou and Lilley, 2012; Evans et al., 2012; Geillinger et al., 2012; Ivancic et al., 2013).

\section{Protein Prioritization Based on Functional Interactions between Capillaries, Glia, Neurons, and the Basal Lamina}

The $\mathrm{BBB}$ is generally regarded as a passive barrier that protects brain parenchyma from toxic substances. Vascular biologists studying BBB function have often ignored the neuronal components present in freshly isolated tissues and turned to endothelial cell models separated from the endogenous neuronal-astrocytic milieu. In some cases, cell models were derived from different cell types altogether to represent the BBB. However, over the course of the last several years, a general consensus has emerged that the $\mathrm{BBB}$ is not a simple barrier of specialized endothelial cells controlling transcytosis, permeability and drug translocation into the CNS (Neuwelt et al., 2008; Abbott, 2013) (Figure 1). Rather, the $\mathrm{BBB}$ is now correctly viewed as a collection of neurovascular units (NVUs) that, together, give the $\mathrm{BBB}$ its unique features (Abbott et al., 2006; Cecchelli et al., 2007; Hermann and Elali, 2012; Elali et al., 2014). NVUs are localized throughout the CNS. 
In larger microvessels (e.g., arterioles), the NVU is composed of endothelial cells, Aquaporin 4-positive $\left(\mathrm{AQP}^{+}\right)$feet vs. GFAP positive $\left(\mathrm{GFAP}^{+}\right)$astrocytes, and neuronal projections. These interactions can regulate vasodilation in arterioles (i.e., neurovascular coupling) in contrast to capillary endothelial cells that have no direct neuronal projections (Simard et al., 2003). A more precise description of the NVU requires consideration of the direct interactions of $\mathrm{AQP}^{+}{ }^{+} \mathrm{GFAP}^{-}$astrocyte end-feet upon capillary endothelial cells (Chun et al., 2011). These AQP4 ${ }^{+}$GFAP $^{-}$ astrocytes are an important class of astrocytes that are in contact with a basal lamina that envelopes the outer surface brain capillary (Muldoon et al., 1999; Simard et al., 2003). Furthermore, the isolation and analysis of intact microvessels from mouse brain identifies extracellular matrix (ECM) proteins expressed in the blood vessel microenvironment, many of which are known to

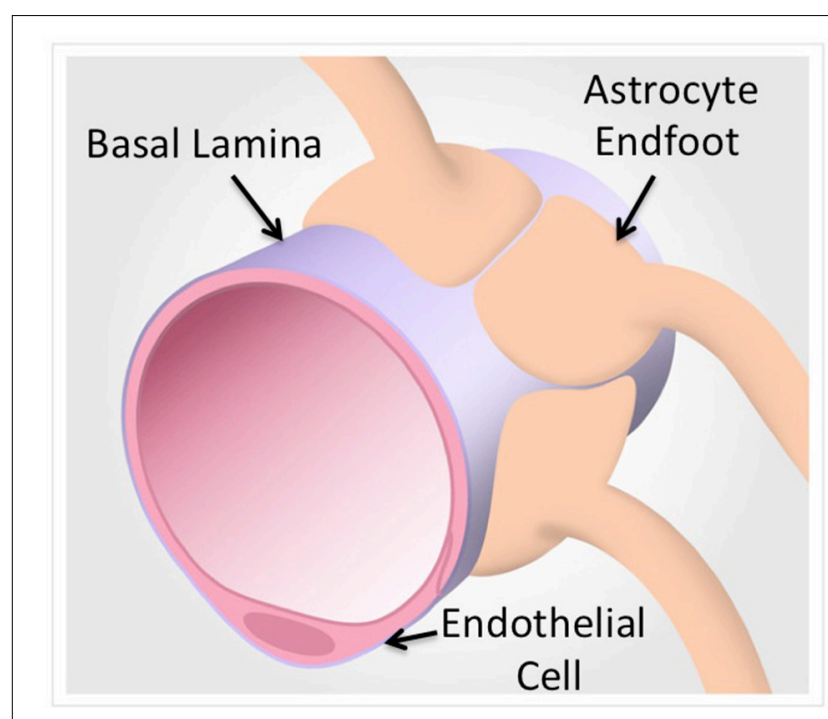

FIGURE 1 | A model of the neurovascular unit in capillaries. Proteomic technologies that focus on the protein components of brain endothelial cells and associated astrocytes from intact capillaries identify basal lamina components that are under-represented in purified cells (Chun et al., 2011). In contrast, Proteomic analyses of purified cells yields quantitative and definitive cell type specific protein expression (Reviewed in Table 1). be basal lamina proteins (Chun et al., 2011). Table 1 summarizes the approaches for isolation of blood vessels for proteomics studies where the focus is on the analysis of isolated brain blood vessels. These proteomic data sets allow for comparisons with data sets obtained from cultured brain endothelium, where the absence of ECM microenvironment and supporting cells of the NVIU limits the translation to physiological context. We propose that this model of the NVU should be front and center for prioritization of analysis of proteins identified by proteomic technologies. Specifically, physiological context can be used to categorize various proteins that may be identified as a consequence of increased/decreased BBB integrity based on the presence of various specific cell types and their response to local or distal stimuli.

\section{Proteomics Approaches Based on Isolated Blood Vessels}

Since the BBB is in fact a multicellular structure, with essential ECM contributions to the organization and maintenance of the $\mathrm{BBB}$ integrity, arguably it is the proteomic analyses of intact brain blood vessels from human, rat, and mouse where the significant growth opportunity is present for the BBB proteomics community in the coming years. As with any molecular profiling technology, attention to the technical details, and quality control of the isolation method are critical to the relevance of the data sets obtained. For example, the emergence of multiple techniques from different groups has enabled the cross-comparison between mouse capillaries and mouse arteries to assess differences in the vascular phenotypes in the brain that have distinct biological functions (Enerson and Drewes, 2006; Daneman et al., 2010; Chun et al., 2011; Ohtsuki et al., 2013; Badhwar et al., 2014). For example, the emerging importance of extracellular microvesicles in intercellular communication in $\mathrm{BBB}$ biology has been recently addressed with proteomics, advancing the understanding of the composition of an organelle that is under-represented in the BBB literature (Simpson et al., 2008; Haqqani et al., 2013). Furthermore, these studies have provided specific methods that crossvalidate the methods used for vessel isolation and proteomic analysis.

TABLE 1 | List of proteomic studies using isolated brain blood vessels.

\begin{tabular}{|c|c|c|c|c|}
\hline Species & Source & Isolation method & Analytical method & References \\
\hline Human & Microvessels & Density gradient & LC-MS/MS with in silico selection & Uchida et al., 2011 \\
\hline Mouse & Microvessels & Glass bead & LC-MS/MS MudPIT & Chun et al., 2011 \\
\hline Mouse & Microvessels & Glass bead & Tag labeling/LC-MS/MS & Uchida et al., 2013 \\
\hline Mouse & Artery & Microdissection & LC-MS/MS MudPIT & Badhwar et al., 2014 \\
\hline Rat & Vessels & Laser capture & ICAT with LC-MS/MS & Haqqani et al., 2005 \\
\hline Mouse & Vessels & Laser capture & Gel/Fourier transform MS & Murugesan et al., 2011 \\
\hline Marmoset & Microvessels & Density gradient & Tag labeling/LC-MS/MS & Hoshi et al., 2013 \\
\hline Rat & Microvessels & Density Gradient & Tag labeling/LC-MS/MS & Hoshi et al., 2013 \\
\hline
\end{tabular}


In the study by Chun et al. (2011), the focus was on the identification of membrane proteins and ECM proteins. The characterization of membrane proteins from isolated blood vessels vs. cultured endothelial studies enables comparison of proteins between intact in vivo models and more established, homogenous and quantitative assays in vitro using cultured endothelial cells. Expression of membrane transporter proteins validates the identification of ECM proteins that comprise the basal lamina, a class of proteins that is otherwise generally under-represented in RNA studies of microvessels, likely different from the ECM proteins expressed in endothelial cells cultured on plastic. Finally, the report on cell type-specific RNA expression in endothelial cells vs. associated astrocytes (Daneman et al., 2010) enables the classification of proteins based on the relative expression of the RNA that likely predicts protein expression. So while RNA expression is an imperfect assessment of protein expression profiles, the alternate proteomic techniques are complementary. The application of MudPIT to the analysis of primary mouse brain microvessels, isolated from the mouse cortex using the glass bead technique (Hartz et al., 2006; Yousif et al., 2007), established a mouse protein expression resource that can be compared with other methods. Chun et al. (2011), based their original approach on the initial work of Enerson and Drewes (2006) in the characterization of the isolated rat microvessel transcriptome, which was used as a foundation for the crosscomparison with transcriptome of isolated endothelial cells and astrocytes, and protein expression of transporters (Chun et al., 2011).

\section{Vessel Heterogeneity}

In the recent study by Badhwar et al. (2014), mouse brain arteries were dissected from the circle of Willis (CW) structure formed at the confluence of the internal carotid arteries, a portion of the vertebrobasilar artery and branching arteries. The goal of this study was to determine the protein expression profile of a CW, a vascular structure that is critical to the perfusion of deep cortical regions of the brain, which are affected by aging and neurodenegerative disorders associated with changes in vessel wall thickness and elasticity. The microsurgical isolation of the $\mathrm{CW}$ arteries in the mouse provides for a highly enriched starting material for MudPIT analysis, with a comparatively homogenous vascular phenotype. In this study both gel-based and gel-free (i.e., strong cation exchange chromatography) followed by tandem mass spectrometry were performed to obtain a comprehensive resource for mouse CW arterial vessels (Badhwar et al., 2014). Proteins identified by MudPIT were validated by Western blot analysis and exhaustively compared with the mouse microvascular proteome published by Chun et al. (2011), and were annotated with common and unique proteins in each dataset listed (Badhwar et al., 2014). The PANTHER classification identified proteins as blood-brain barrier-specific cell type proteins, tight junction and adhesion proteins, membrane transporter and channel proteins, and ECM and basal lamina proteins (Mi et al., 2013). In each case identified proteins were verified for their detection in previously reported mouse and rat transcriptome studies. Protein components related to signaling pathways, vasoactivity, arterial proteins, and neuronal proteins and were also identified.

\section{Proteomic Analyses in Disease and Animal Models}

The cross-comparison of this arterial vessel resource with microvessels provides an important database in which to analyze the effects of genetic mutants and (Agarwal et al., 2012) injury/tumor models that impact cerebrovascular biology. As more proteomic studies are completed the more comprehensive the datasets will be for the researcher. Based on the isolation of intact brain blood vessels from mice, recently characterized mouse mutant studies can be further analyzed and compared with isolation techniques such as laser capture microdissection, and analytical techniques based on gel electrophoresis, and cultured endothelial cells. Laser microdissection enables the isolation of discrete structures enriched in specific cell types that can be analyzed for gene expression with a high degree of sensitivity as well as proteomics using mass spectrometry (Haqqani et al., 2005; Murugesan et al., 2011). The power of such techniques complements the isolation of microvessels and arteries and provides opportunities to further characterize knockout mouse models, such as the P-gp/Bcrp mouse model (Agarwal et al., 2009, 2012).

Lastly, the challenges of determining differences in rodent vs. primate protein constituents of the blood brain barrier (BBB) have been recently addressed by Hoshi et al. (2013), where isolated blood vessels from rats and marmosets were subjected to MudPIT using a quantitative approach based on an in silico peptide selection. In this model, side-by-side comparison of rat vs. marmoset data sets with human proteins related to endothelial cell-related membrane transporters, receptors and tight junction proteins revealed several interesting insights. Significant 2 fold differences in several key transporters were observed between rat and human blood vessels (Hoshi et al., 2013). Interestingly few significant changes in transporters between human and marmoset blood vessels were observed, suggesting that marmosets may be a useful tool for studying select brain blood vessel proteins that are substantially different between rodents and humans (Hoshi et al., 2013). This study is an excellent example of the power of the quantitative approach to define more precisely the expression profile of cell membrane transporters that have relevance to drug development.

As the methodology and bioinformatics improve for both proteomics and transcriptomics it may soon be possible to correlate relative levels of gene expression with protein changes (Wang et al., 2014). The focused application of such technologies will enable a more complete understanding of the role of secreted factors (i.e., exosomes, growth factors) and how these components may condition the basal lamina and signal to associated cells in disease. 


\section{References}

Abbott, N. J. (2013). Blood-brain barrier structure and function and the challenges for CNS drug delivery. J. Inherit. Metab. Dis. 36, 437-449. doi: 10.1007/s10545013-9608-0

Abbott, N. J., Ronnback, L., and Hansson, E. (2006). Astrocyte-endothelial interactions at the blood-brain barrier. Nat. Rev. Neurosci. 7, 41-53. doi: $10.1038 / \mathrm{nrn} 1824$

Aebersold, R., and Mann, M. (2003). Mass spectrometry-based proteomics. Nature 422, 198-207. doi: 10.1038/nature01511

Agarwal, N., Lippmann, E. S., and Shusta, E. V. (2009). Identification and expression profiling of blood-brain barrier membrane proteins. J. Neurochem. 112, 625-635. doi: 10.1111/j.1471-4159.2009.06481.x

Agarwal, S., Uchida, Y., Mittapalli, R. K., Sane, R., Terasaki, T., and Elmquist, W. F. (2012). Quantitative proteomics of transporter expression in brain capillary endothelial cells isolated from P-glycoprotein (P-gp), breast cancer resistance protein (Bcrp), and P-gp/Bcrp knockout mice. Drug Metab. Dispos. 40, 1164-1169. doi: 10.1124/dmd.112.044719

Badhwar, A., Stanimirovic, D. B., Hamel, E., and Haqqani, A. S. (2014). The proteome of mouse cerebral arteries. J. Cereb. Blood Flow Metab. 34, 1033-1046. doi: $10.1038 / \mathrm{jcbfm} .2014 .52$

Banks, R. E., Dunn, M. J., Hochstrasser, D. F., Sanchez, J. C., Blackstock, W., Pappin, D. J., et al. (2000). Proteomics: new perspectives, new biomedical opportunities. Lancet 356, 1749-1756. doi: 10.1016/S0140-6736(00)03214-1

Cecchelli, R., Berezowski, V., Lundquist, S., Culot, M., Renftel, M., Dehouck, M.P., et al. (2007). Modelling of the blood-brain barrier in drug discovery and development. Nat. Rev. Drug Discov. 6, 650-661. doi: 10.1038/nrd2368

Chen, E. I., Hewel, J., Felding-Habermann, B., and Yates, J. R. III. (2006). Large scale protein profiling by combination of protein fractionation and multidimensional protein identification technology (MudPIT). Mol. Cell. Proteomics 5, 53-56. doi: 10.1074/mcp.T500013-MCP200

Christoforou, A. L., and Lilley, K. S. (2012). Isobaric tagging approaches in quantitative proteomics: the ups and downs. Anal. Bioanal. Chem. 404, 1029-1037. doi: $10.1007 / \mathrm{s} 00216-012-6012-9$

Chun, H. B., Scott, M., Niessen, S., Hoover, H., Baird, A., Yates, J., et al. (2011). The proteome of mouse brain microvessel membranes and basal lamina. J. Cereb. Blood Flow Metab. 31, 2267-2281. doi: 10.1038/jcbfm.2011.104

Coombs, K. M. (2011). Quantitative proteomics of complex mixtures. Expert Rev. Proteomics 8, 659-677. doi: 10.1586/epr.11.55

Daneman, R., Zhou, L., Agalliu, D., Cahoy, J. D., Kaushal, A., and Barres, B. A. (2010). The mouse blood-brain barrier transcriptome: a new resource for understanding the development and function of brain endothelial cells. PLoS ONE 5:e13741. doi: 10.1371/journal.pone.0013741

Elali, A., Theriault, P., and Rivest, S. (2014). The role of pericytes in neurovascular unit remodeling in brain disorders. Int. J. Mol. Sci. 15, 6453-6474. doi: 10.3390/ijms15046453

Enerson, B. E., and Drewes, L. R. (2006). The rat blood-brain barrier transcriptome. J. Cereb. Blood Flow Metab. 26, 959-973. doi: 10.1038/sj.jcbfm.9600249

Evans, C., Noirel, J., Ow, S. Y., Salim, M., Pereira-Medrano, A. G., Couto, N., et al. (2012). An insight into iTRAQ: where do we stand now? Anal. Bioanal. Chem. 404, 1011-1027. doi: 10.1007/s00216-012-5918-6

Florens, L., Carozza, M. J., Swanson, S. K., Fournier, M., Coleman, M. K., Workman, J. L., et al. (2006). Analyzing chromatin remodeling complexes using shotgun proteomics and normalized spectral abundance factors. Methods 40, 303-311. doi: 10.1016/j.ymeth.2006.07.028

Geillinger, K. E., Kuhlmann, K., Eisenacher, M., Meyer, H. E., Daniel, H., and Spanier, B. (2012). Dynamic changes of the Caenorhabditis elegans proteome during ontogenesis assessed by quantitative analysis with ${ }^{15} \mathrm{~N}$ metabolic labeling. J. Proteome Res. 11, 4594-4604. doi: 10.1021/pr300385v

Gonzalez-Begne, M., Lu, B., Han, X., Hagen, F. K., Hand, A. R., Melvin, J. E., et al. (2009). Proteomic analysis of human parotid gland exosomes by multidimensional protein identification technology (MudPIT). J. Proteome Res. 8, 1304-1314. doi: 10.1021/pr800658c

Gouw, J. W., Tops, B. B., Mortensen, P., Heck, A. J., and Krijgsveld, J. (2008). Optimizing identification and quantitation of ${ }^{15} \mathrm{~N}$-labeled proteins in comparative proteomics. Anal. Chem. 80, 7796-7803. doi: 10.1021/ac801249v

Haqqani, A. S., Delaney, C. E., Tremblay, T. L., Sodja, C., Sandhu, J. K., and Stanimirovic, D. B. (2013). Method for isolation and molecular characterization of extracellular microvesicles released from brain endothelial cells. Fluids Barriers CNS 10:4. doi: 10.1186/2045-8118-10-4

Haqqani, A. S., Kelly, J. F., and Stanimirovic, D. B. (2008). "Quantitative protein profiling by mass spectrometry using label-free proteomics," in Methods in Molecular Biology, eds M. Starkey and R. Elaswarapu (Totowa, NJ: Human Press, Inc.), 241-256.

Haqqani, A. S., Nesic, M., Preston, E., Baumann, E., Kelly, J., and Stanimirovic, D. (2005). Characterization of vascular protein expression patterns in cerebral ischemia/reperfusion using laser capture microdissection and ICAT-nanoLCMS/MS. FASEB J. 19, 1809-1821. doi: 10.1096/fj.05-3793com

Hartz, A. M., Bauer, B., Fricker, G., and Miller, D. S. (2006). Rapid modulation of P-glycoprotein-mediated transport at the blood-brain barrier by tumor necrosis factor-alpha and lipopolysaccharide. Mol. Pharmacol. 69, 462-470. doi: 10.1124/mol.105.017954

Hermann, D. M., and Elali, A. (2012). The abluminal endothelial membrane in neurovascular remodeling in health and disease. Sci. Signal. 5, 1-10. doi: 10.1126/scisignal.2002886

Hoshi, Y., Uchida, Y., Tachikawa, M., Inoue, T., Ohtsuki, S., and Terasaki, T. (2013). Quantitative atlas of blood-brain barrier transporters, receptors, and tight junction proteins in rats and common marmoset. J. Pharm. Sci. 102, 3343-3355. doi: 10.1002/jps.23575

Ivancic, M. M., Huttlin, E. L., Chen, X., Pleiman, J. K., Irving, A. A., Hegeman, A. D., et al. (2013). Candidate serum biomarkers for early intestinal cancer using ${ }^{15} \mathrm{~N}$ metabolic labeling and quantitative proteomics in the ApcMin/+ mouse. J. Proteome Res. 12, 4152-4166. doi: 10.1021/pr400467c

Kamiie, J., Ohtsuki, S., Iwase, R., Unine, K., Katsukura, Y., Yanai, K., et al. (2008). Quantitative atlas of membrane transporter proteins: development and application of a highly sensitive simultaneous LC/MS/MS method combined with novel in-silico peptide selection criteria. Pharm. Res. 25, 1469-1483. doi: 10.1007/s11095-008-9532-4

Kislinger, T., Gramolini, A. O., Maclennan, D. H., and Emili, A. (2005). Multidimensional protein identification technology (MudPIT): technical overview of a profiling method optimized for the comprehensive proteomic investigation of normal and diseased heart tissue. J. Am. Soc. Mass Spectrom. 16, 1207-1220. doi: 10.1016/j.jasms.2005.02.015

Liao, L., McClatchy, D. B., Park, S. K., Xu, T., Lu, B., and Yates, J. R. III. (2008). Quantitative analysis of brain nuclear phosphoproteins identifies developmentally regulated phosphorylation events. J. Proteome Res. 7, 4743-4755. doi: $10.1021 /$ pr8003198

Lu, L., Yang, P. Y., Rui, Y. C., Kang, H., Zhang, J., Zhang, J. P., et al. (2007). Comparative proteome analysis of rat brain and coronary microvascular endothelial cells. Physiol. Res. 56, 159-168.

Mann, M., Hendrickson, R. C., and Pandey, A. (2001). Analysis of proteins and proteomes by mass spectrometry. Annu. Rev. Biochem. 70, 437-473. doi: 10.1146/annurev.biochem.70.1.437

Mann, M., and Pandey, A. (2001). Use of mass spectrometry-derived data to annotate nucleotide and protein sequence databases. Trends Biochem. Sci. 26, 54-61. doi: 10.1016/S0968-0004(00)01726-6

McDonald, W. H., and Yates, J. R. III. (2000). Proteomic tools for cell biology. Traffic 1, 747-754. doi: 10.1034/j.1600-0854.2000.011001.x

Mi, H., Muruganujan, A., Casagrande, J. T., and Thomas, P. D. (2013). Large-scale gene function analysis with the PANTHER classification system. Nat. Protoc. 8 , 1551-1566. doi: 10.1038/nprot.2013.092

Mueller, L. N., Brusniak, M. Y., Mani, D. R., and Aebersold, R. (2008). An assessment of software solutions for the analysis of mass spectrometry based quantitative proteomics data. J. Proteome Res. 7, 51-61. doi: 10.1021/pr $700758 \mathrm{r}$

Muldoon, L. L., Pagel, M. A., Kroll, R. A., Roman-Goldstein, S., Jones, R. S., and Neuwelt, E. A. (1999). A physiological barrier distal to the anatomic bloodbrain barrier in a model of transvascular delivery. AJNR Am. J. Neuroradiol. 20, 217-222.

Murugesan, N., Macdonald, J. A., Lu, Q., Wu, S. L., Hancock, W. S., and Pachter, J. S. (2011). Analysis of mouse brain microvascular endothelium using laser capture microdissection coupled with proteomics. Methods Mol. Biol. 686, 297-311. doi: 10.1007/978-1-60761-938-3_14

Nahnsen, S., Bielow, C., Reinert, K., and Kohlbacher, O. (2013). Tools for label-free peptide quantification. Mol. Cell. Proteomics 12, 549-556. doi: 10.1074/mcp.R112.025163 
Neilson, K. A., Ali, N. A., Muralidharan, S., Mirzaei, M., Mariani, M., Assadourian, G., et al. (2011). Less label, more free: approaches in label-free quantitative mass spectrometry. Proteomics 11, 535-553. doi: 10.1002/pmic.2010 00553

Neuwelt, E., Abbott, N. J., Abrey, L., Banks, W. A., Blakley, B., Davis, T., et al. (2008). Strategies to advance translational research into brain barriers. Lancet Neurol. 7, 84-96. doi: 10.1016/S1474-4422(07)70326-5

Ohtsuki, S., Ikeda, C., Uchida, Y., Sakamoto, Y., Miller, F., Glacial, F., et al. (2013). Quantitative targeted absolute proteomic analysis of transporters, receptors and junction proteins for validation of human cerebral microvascular endothelial cell line hCMEC/D3 as a human blood-brain barrier model. Mol. Pharm. 10, 289-296. doi: $10.1021 / \mathrm{mp} 3004308$

Ohtsuki, S., Uchida, Y., Kubo, Y., and Terasaki, T. (2011). Quantitative targeted absolute proteomics-based adme research as a new path to drug discovery and development: methodology, advantages, strategy, and prospects. J. Pharm. Sci. 100, 3547-3559. doi: 10.1002/jps.22612

Pandey, A., and Mann, M. (2000). Proteomics to study genes and genomes. Nature 405, 837-846. doi: 10.1038/35015709

Pottiez, G., Flahaut, C., Cecchelli, R., and Karamanos, Y. (2009). Understanding the blood-brain barrier using gene and protein expression profiling technologies. Brain Res. Rev. 62, 83-98. doi: 10.1016/j.brainresrev.2009. 09.004

Ruse, C. I., McClatchy, D. B., Lu, B., Cociorva, D., Motoyama, A., Park, S. K., et al. (2008). Motif-specific sampling of phosphoproteomes. J. Proteome Res. 7, 2140-2150. doi: 10.1021/pr800147u

Simard, M., Arcuino, G., Takano, T., Liu, Q. S., and Nedergaard, M. (2003). Signaling at the gliovascular interface. J. Neurosci. 23, 9254-9262.

Simpson, R. J., Jensen, S. S., and Lim, J. W. (2008). Proteomic profiling of exosomes: current perspectives. Proteomics 8, 4083-4099. doi: 10.1002/pmic. 200800109

Uchida, Y., Ohtsuki, S., Katsukura, Y., Ikeda, C., Suzuki, T., Kamiie, J., et al. (2011). Quantitative targeted absolute proteomics of human blood-brain barrier transporters and receptors. J. Neurochem. 117, 333-345. doi: 10.1111/j.1471-4159. 2011.07208.x

Uchida, Y., Tachikawa, M., Obuchi, W., Hoshi, Y., Tomioka, Y., Ohtsuki, S., et al. (2013). A study protocol for quantitative targeted absolute proteomics (QTAP) by LC-MS/MS: application for inter-strain differences in protein expression levels of transporters, receptors, claudin-5, and marker proteins at the blood-brain barrier in ddY, FVB, and C57BL/6J mice. Fluids Barriers CNS 10, 21-21. doi: 10.1186/2045-8118-10-21

Wang, X., Liu, Q., and Zhang, B. (2014). Leveraging the complementary nature of RNA-Seq and shotgun proteomics data. Proteomics. 2676-2687. doi: 10.1002/ pmic. 201400184

Washburn, M. P., Wolters, D., and Yates, J. R. III. (2001). Large-scale analysis of the yeast proteome by multidimensional protein identification technology. Nat. Biotechnol. 19, 242-247. doi: 10.1038/85686

Wu, C. C., and Maccoss, M. J. (2002). Shotgun proteomics: tools for the analysis of complex biological systems. Curr. Opin. Mol. Ther. 4, 242-250.

Wu, C. C., Maccoss, M. J., Howell, K. E., Matthews, D. E., and Yates, J. R. III. (2004). Metabolic labeling of mammalian organisms with stable isotopes for quantitative proteomic analysis. Anal. Chem. 76, 4951-4959. doi: 10.1021/ac049208j

Wu, C. C., Maccoss, M. J., Howell, K. E., and Yates, J. R. III. (2003). A method for the comprehensive proteomic analysis of membrane proteins. Nat. Biotechnol. 21, 532-538. doi: 10.1038/nbt819

Yates, J. R. III. (1998). Mass spectrometry and the age of the proteome. J. Mass Spectrom. 33, 1-19.

Yates, J. R. III. (2000). Mass spectrometry. From genomics to proteomics. Trends Genet. 16, 5-8. doi: 10.1016/S0168-9525(99)01879-X

Yates, J. R., Ruse, C. I., and Nakorchevsky, A. (2009). Proteomics by mass spectrometry: approaches, advances, and applications. Annu. Rev. Biomed. Eng. 11, 49-79. doi: 10.1146/annurev-bioeng-061008-124934

Yousif, S., Marie-Claire, C., Roux, F., Scherrmann, J. M., and Decleves, X. (2007). Expression of drug transporters at the blood-brain barrier using an optimized isolated rat brain microvessel strategy. Brain Res. 1134, 1-11. doi: 10.1016/j.brainres.2006.11.089

Conflict of Interest Statement: The authors declare that the research was conducted in the absence of any commercial or financial relationships that could be construed as a potential conflict of interest.

Copyright (c) 2015 Torbett, Baird and Eliceiri. This is an open-access article distributed under the terms of the Creative Commons Attribution License (CC BY) The use, distribution or reproduction in other forums is permitted, provided the original author(s) or licensor are credited and that the original publication in this journal is cited, in accordance with accepted academic practice. No use, distribution or reproduction is permitted which does not comply with these terms. 


\title{
The choroid plexuses and their impact on developmental neurogenesis
}

\author{
Pia A. Johansson* \\ Institute for Stem Cell Research, Helmholtz Center Munich, German Research Center for Environmental Health, Munich, Germany
}

\section{Edited by:}

Helen B. Stolp, King's College

London, UK

\section{Reviewed by:}

Khyobeni Mozhui, University of Tennessee Health Science Center, USA

Joao C. Sousa, University of Minho, Portugal

\section{*Correspondence:}

Pia A. Johansson, Institute for Stem Cell Research, Helmholtz Center Munich, German Research Center for Environmental Health, Ingolstädterlandstr. 1, 85764 Neuherberg/Munich

Germany

e-mail:pia.johansson@

helmholtz-muenchen.de
During brain development the neural stem cells are regulated by both intrinsic and extrinsic sources. One site of origin of extrinsic regulation is the developing choroid plexuses, primely situated inside the cerebral ventricles. The choroid plexuses are very active in terms of both secretion and barrier function as soon as they appear during development and control the production and contents of cerebrospinal fluid (CSF). This suggests that regulated secretion of signaling molecules from the choroid plexuses into CSF can regulate neural stem cell behavior (as they are in direct contact with CSF) and thereby neurogenesis and brain development. Here, choroid plexus development, particularly with regards to molecular regulation and specification, is reviewed. This is followed by a review and discussion of the role of the developing choroid plexuses in brain development. In particular, recent evidence suggests a region-specific reciprocal regulation between choroid plexuses and the neural stem cells. This is accomplished by site-specific secretion of signaling molecules from the different choroid plexuses into CSF, as well as brain region specific competence of the neural stem cells to respond to the signaling molecules present in CSF. In conclusion, although in its infancy, the field of choroid plexus regulation of neurogenesis has already and will likely continue to shed new light on our understanding of the control and fine-tuning of overall brain development.

Keywords: choroid plexus, cerebrospinal fluid, neurogenesis, neural stem cells, cerebral cortex, development

\section{INTRODUCTION}

Neurogenesis, both in the adult and during development, occurs in a specialized environment established by both the neurogenic (the neural stem cells) and non-neurogenic cells within the neurogenic area, and from cells and compartments in direct contact with the niche (Johansson et al., 2010; Braun and Jessberger, 2014, Figure 1). One of these compartments is cerebrospinal fluid (CSF). During development the neural stem cells are located at the apical (ventricle facing) surface of the developing brain tissue (as exemplified in Figure $\mathbf{1}$ and Box 1). Thus, any molecule present in CSF has the potential to influence the behavior of the cells located at the ventricular surface (i.e., the neural stem cells) and thereby neurogenesis. The possible role of CSF itself in regulating neurogenesis has recently received more attention, however, there has been less focus on the tissue that produces the CSF; namely the choroid plexuses.

The most abundant proteins in CSF (albumin, transferrin, alpha-phetoprotein, fetuin) are derived from plasma and are transferred across the choroid plexus epithelium by a subset of choroid plexus epithelial cells in a highly specific and regulated manner (Johansson et al., 2006; Liddelow et al., 2009, 2011a,b). Over the last decade it has also been shown that in addition to the more classical plasma proteins, CSF contains a variety of comparatively less abundant signaling molecules (e.g., Shh, Igfl, Wnt4, Tgm2, Fgf2, see Table 1). Recent data from transcriptome analysis of the choroid plexus, in both the adult and during development (Marques et al., 2011; Johansson et al., 2013), has revealed the expression of a plethora of molecules previously not associated with the choroid plexus which can either intrinsically affect the function of the choroid plexus or be secreted into CSF. Indeed, a recent comparison of human adult CSF showed that of 1050 identified proteins in plasma 877 of these were also found in CSF whereas an astonishing 2204 proteins were found only in CSF (Guldbrandsen et al., 2014). As CSF is in direct contact with the neural stem cells in both the developing and the adult brain and as CSF is mostly regulated via the choroid plexuses, it is not farfetched to assume that the choroid plexuses could be significant regulators of neurogenesis.

So far, only two papers have described a direct link between altered choroid plexus secretion (i.e., via direct choroid plexus manipulation) and alterations in developmental neurogenesis (Huang et al., 2010; Johansson et al., 2013). Hopefully, the recent increased focus on this signaling axis will lead to further contributions to this field over the coming years. Regulation of neurogenesis via molecules secreted (or transferred) from the choroid plexus into CSF could have potentially far-reaching ramifications for the understanding and treatment of both developmental disorders and pathological situations in the adult brain where modulation of neurogenesis might be beneficial. The role of the choroid plexus-CSF signaling axis in adult neurogenesis is beyond the scope of this review but the interested reader is referred to some recent articles and reviews which highlight this interaction (Falcao et al., 2012; Silva-Vargas et al., 2013; Baruch et al., 2014). 

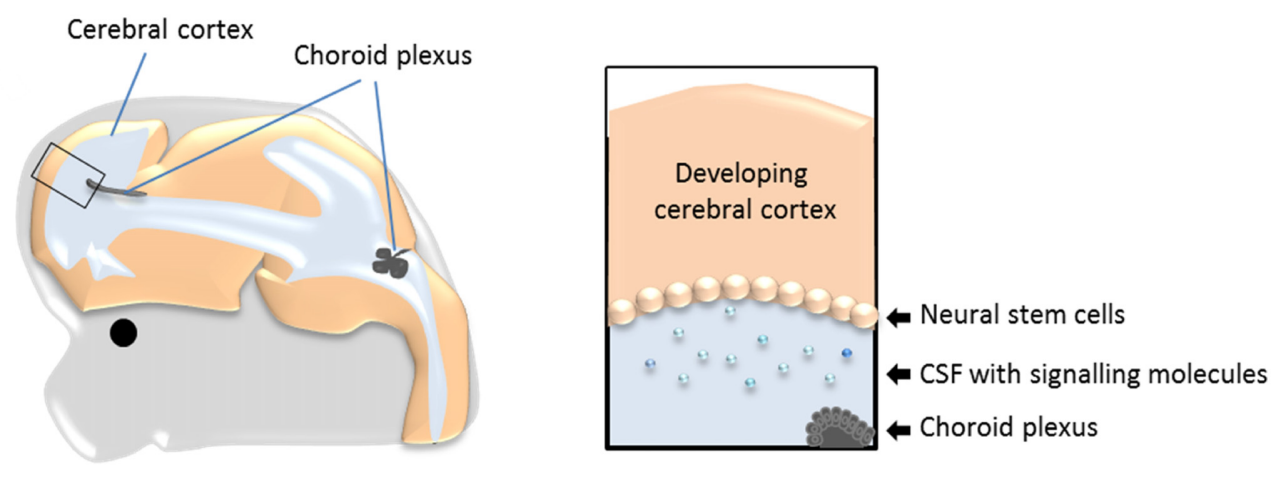

FIGURE 1 | The choroid plexuses, CSF and the neural stem cells during development. Schematic drawing showing the prime location of the choroid plexuses inside the cerebral ventricles and the bathing of the neural stem cells with CSF and molecules within it. CSF, cerebrospinal fluid.

\section{STRUCTURE AND FUNCTION OF THE CHOROID PLEXUSES}

The choroid plexuses are four modified epithelial structures suspended inside the cerebral ventricles. There are two lateral ventricular choroid plexuses, the third ventricular choroid plexus and the fourth ventricular (hindbrain) choroid plexus (Figure 2). The choroid plexuses consist of a central stroma covered by a single epithelial layer; the polarized choroid plexus epithelium (CPE). The stroma is highly vascularized, consisting of blood vessels, connective tissue and pericytes. The blood vessels are fenestrated and leaky, making them different from blood vessels in the brain parenchyma which are connected by tight junctions and form the blood-brain barrier. Thus, instead of having a barrier at the level of the blood vessels it is the cells of the CPE that are connected by continuous tight-junctional strands, restricting the entry of lipid-insoluble molecules into the CSF (the blood-CSF barrier, Figure 2, Brightman and Reese, 1969; Ek et al., 2003; Johansson et al., 2006).

In the adult, the choroid plexuses and CSF have several know functions such as: (i) protecting and regulating the internal environment of the brain via the blood-CSF barrier; (ii) secretion and modulation of CSF through the activity of the choroid plexus epithelial cells; and (iii) waste and metabolite removal, via the "CSF sink," through the continuous production and then removal of CSF into peripheral circulation. During development two out of these three functions are already performed by the choroid plexuses; protection of the brain via the blood-CSF barrier and regulation of CSF composition via specific and regulated transfer and secretion (see more below). However, due to the lack of CSF reabsorption during embryogenesis, the removal of waste and metabolites does not occur (Johansson et al., 2008a). There have also long been suggestions for additional roles for the choroid plexuses during development, such as the generation of an expansive pressure through CSF secretion (Desmond and Jacobson, 1977).

\section{CHOROID PLEXUS DEVELOPMENT}

The choroid plexuses are of dual embryonic origin, with the choroid plexus epithelium originating from the ectoderm and the central stroma from the mesoderm (Catala, 1998). The fourth ventricular choroid plexus develops first followed by the two lateral and then the third ventricular choroid plexuses (Dziegielewska et al., 2001). In the mouse the dorsal midline starts to invaginate (from dorsal to ventral) around E10, giving rise to the choroid plexus and, in the forebrain, the adjacent cortical hem (Figure 3). The choroid plexus and the hem both express Lmhx1, the hem expresses Wnt at high levels and the choroid plexus specifically expresses transthyretin (Ttr). Ttr is known as the choroid plexus marker and is expressed in the mouse lateral ventricular choroid plexus from E11 (Figure 3). However, it should be noted that Ttr identifies choroid plexus tissue rather than determining the fate of the cells, as in the Ttr knock-out mice the choroid plexuses form normally.

Other molecules and signaling pathways have been shown to be involved in the specification and formation of the choroid plexus. The inhibition of BMP signaling via Foxg1Cre mediated deletion of Bmpla receptors led to the absence of the lateral ventricular choroid plexuses (Hebert et al., 2002), however in this study the impact on brain development was not further investigated. A similar result was seen in the developing chick when the transcription factors Emxl and 2 were mis-expressed in the roof plate region, which lead to the absence of $B m p 7$ expression and a failure of the dorsal midline to invaginate (Von Frowein et al., 2006). Interestingly, what was also apparent during the latter experiments was that the transcription factor Otx2 was reciprocally inhibited by Emx1/2, suggesting a role of Otx2 in choroid plexus development. Further investigations into the role of Otx2 in choroid plexus development, this time in mice, revealed that it is indeed vital for choroid plexus specification and development (Johansson et al., 2013). Removal of Otx2 from all the choroid plexus anlage (tamoxifen mediated Cre-induction at E9) resulted in an almost complete absence of choroid plexus tissue. Both the Otx2 and Bmp1a deletion show specific roles for these molecules in choroid plexus development as the nearby cortical hem was unaffected in both mutants.

Another group of molecules involved in choroid plexus differentiation are members of the Notch signaling pathway. When the levels of Ngn2 or Hes5 were altered in the telencephalic midline (over-expressed and down-regulated respectively) the choroid plexus was reduced in size but Cajal-Retzius cells were increased in number (Imayoshi et al., 2008), suggesting a reciprocal 


\section{Box 1 | Schematic representation of neurogenesis in the developing cerebral cortex.}

Neurogenesis in the cerebral cortex goes through a differentiation cascade starting with the neural stem cells (NSCs) lining the ventricle (a.k.a radial glial cells, blue cells). The processes of the NSCs span the entire thickness of the cortical wall and their nuclei move up and down in the ventricular zone (VZ) during the cell cycle (mitosis occurs at the ventricle and S-phase at the basal end of the VZ) but they stay in contact with the ventricle via the apical process and the cilium responsive to elements present in CSF through-out the cell cycle. The NSCs divide and give rise to the next cells in the differentiation cascade, the transient amplifying cells, TAPs (yellow cells) or neurons (green cells). The TAPs are a heterogeneous population and vary in their morphology, location and abundance (see Taverna et al., 2014) but in the mouse cerebral cortex the majority of the TAPs are what is known as basal (or intermediate) progenitors which migrated away from the VZ into the subventricular zone (SVZ). The TAPs then divide again and give rise to the neurons which migrate through the intermediate zone (IZ) into the cortical plate (CP) in an inside-out manner (i.e., the newly-born neurons migrate passed the older neurons and settle in a layer above the previous-born neurons, as illustrated by different shades of green). For a recent comprehensive review see Taverna et al., 2014.

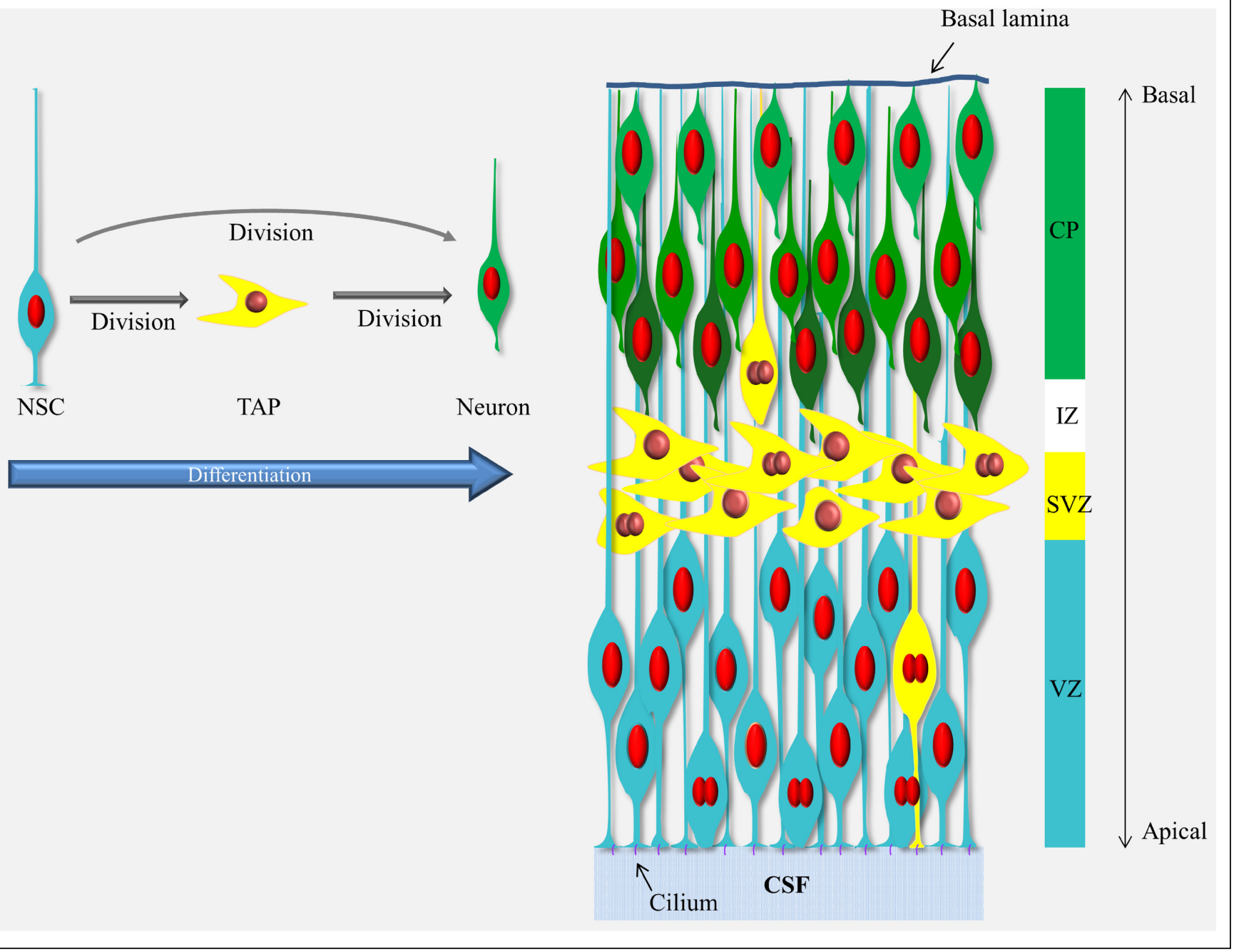

regulation of these cell types. An involvement of Notch signaling in the development of the Zebrafish (Danio rerio) choroid plexus has also been described (Bill et al., 2008). Overexpression of Notch1, on the other hand (activated Notch1 in the Gdf7lineage), massively increased the size of the mouse hindbrain choroid plexus (Hunter and Dymecki, 2007). Further evidence for the involvement of Notch in choroid plexus development includes the formation of choroid plexus tumors after E9 injection of a virus encoding constitutively active Notch3 (Dang et al., 2006). Additionally, alterations in Notch-receptor localization and expression have been found in human choroid plexus tumors when compared to normal choroid plexus tissue (Beschorner et al., 2013).

After choroid plexus specification, the choroid plexus anlage go through a characteristic thinning of the epithelium, going from pseudostratified to a proper single layer epithelium as it starts to invaginate into the ventricles (Dziegielewska et al., 2001). The majority of the choroid plexus epithelial cells are post-mitotic and the plexus grows into the ventricle through addition of cells from the proliferative zone at "the root" (Figure 3, Liddelow et al., 
Table 1 | Selected list of secreted signaling molecules expressed in the choroid plexuses.

\begin{tabular}{|c|c|c|c|}
\hline Gene symbol & Entrez & Gene title & $\begin{array}{l}\text { Functional annotations/ } \\
\text { Signaling pathways }\end{array}$ \\
\hline \multicolumn{4}{|c|}{ (A) SECRETED MOLECULES WITH CONFIRMED PRESENCE IN EMBRYONIC CSF AND ABILITY TO INFLUENCE NEUROGENESIS } \\
\hline Shh & 20423 & Sonic hedgehog & Shh-signaling \\
\hline Fgf2 & 14173 & Fibroblast growth factor 2 & Growth factor activity \\
\hline $\operatorname{Tgm} 2$ & 21817 & Transglutaminase 2, C polypeptide & Wnt-signaling \\
\hline \multicolumn{4}{|c|}{ (B) SECRETED MOLECULES WITH THE POTENTIAL OF INFLUENCING NEUROGENESIS } \\
\hline Cthrc1 & 68588 & Collagen triple helix repeat containing 1 & Wnt-signaling \\
\hline Cxcl12 & 20315 & Chemokine (C-X-C motif) ligand 12 & Chemokine/growth factor activity \\
\hline Reln & 19699 & Reelin & Nervous system development \\
\hline Dkk2 & 56811 & Dickkopf homolog 2 (Xenopus laevis) & Wnt-signaling \\
\hline Sema3c & 20348 & $\begin{array}{l}\text { Sema domain, immunoglobulin domain (lg), short basic domain, } \\
\text { secreted, (semaphorin) } 3 C\end{array}$ & Nervous system development \\
\hline Sfrp2 & 20319 & Secreted frizzled-related protein 2 & Wnt/BMP-signaling \\
\hline Sostdc1 & 66042 & Sclerostin domain containing 1 & Wnt/BMP-signaling \\
\hline Spp1 & 20750 & Secreted phosphoprotein 1 & BMP-signaling \\
\hline Sulf1 & 240725 & Sulfatase 1 & $\begin{array}{l}\text { Wnt/BMP/FGFreceptor signaling } \\
\text { pathway }\end{array}$ \\
\hline Tgfb1 & 21803 & Transforming growth factor, beta 1 & Tgf $\beta$-signaling \\
\hline Tgfb2 & 21808 & Transforming growth factor, beta 2 & Tgf $\beta$-signaling \\
\hline Tgfb3 & 21809 & Transforming growth factor, beta 3 & Tgf $\beta$-signaling \\
\hline Wfikkn2 & 278507 & $\begin{array}{l}\text { WAP, follistatin/kazal, immunoglobulin, kunitz and netrin domain } \\
\text { containing } 2\end{array}$ & Tgf $\beta$-signaling \\
\hline
\end{tabular}

Molecules in B from Johansson et al. (2013).

2010), which adds to the size of the choroid plexus throughout development. The choroid plexuses go through several stages of morphologically characterized development before reaching maturity postnatally (Dziegielewska et al., 2001).

The stroma of the choroid plexus (mesoderm derived, Catala, 1998), is induced by the choroid plexus anlage. Using chimeric transplantation experiments between chick and quail embryos, it was shown that the choroid plexus anlage (i.e., yet without stroma) from quail transplanted into the gut of a chick embryo induced the formation of a morphologically normal choroid plexus with organotypic fenestrated blood-vessels (Wilting and Christ, 1989). The blood vessels and the stroma were found to be of chick (host) origin demonstrating that the choroid plexus anlage induces the formation of the stroma and the blood vessels therein. In a different experiment they also showed that transplanting a non-choroid plexus forming neural epithelium to the normal site of choroid plexus development, in contact with the prospective stroma, did not induce choroid plexus characteristics (Wilting and Christ, 1989). However, even though neither the stroma nor the blood vessels induce choroid plexus formation, signaling from the stroma to the epithelial cells after stromal formation has been observed. This was seen recently when Shh was deleted from the choroid plexus epithelial cells, which lead to a decrease in Shh-signaling in the Shh responsive cells (choroid plexus progenitor cells and pericytes), which in turn resulted in a much smaller choroid plexus deficient in both vasculature and CPE cells (Huang et al., 2009; Nielsen and Dymecki, 2010).

Interestingly, this also leads us to one of the very few described regional differences between the choroid plexuses; Shh is not expressed in the forebrain choroid plexuses, suggesting that other molecules might be responsible for this regulation in the forebrain. Conversely, it appears that Otx2, which is expressed in and has an effect on the initial development of all the choroid plexuses, may have alternative functions in the different choroid plexuses after their specification and initial development, as deletion at E15 only affected survival in the hindbrain choroid plexus (Johansson et al., 2013). Further comparisons between the different choroid plexuses are currently under investigation.

\section{REGULATION OF CSF SECRETION AND COMPOSITION DURING DEVELOPMENT}

Three main components are required for fluid transport/secretion across an epithelium: (i) free passage of water and solutes between the cells has to be inhibited via the presence of tight-junctions 

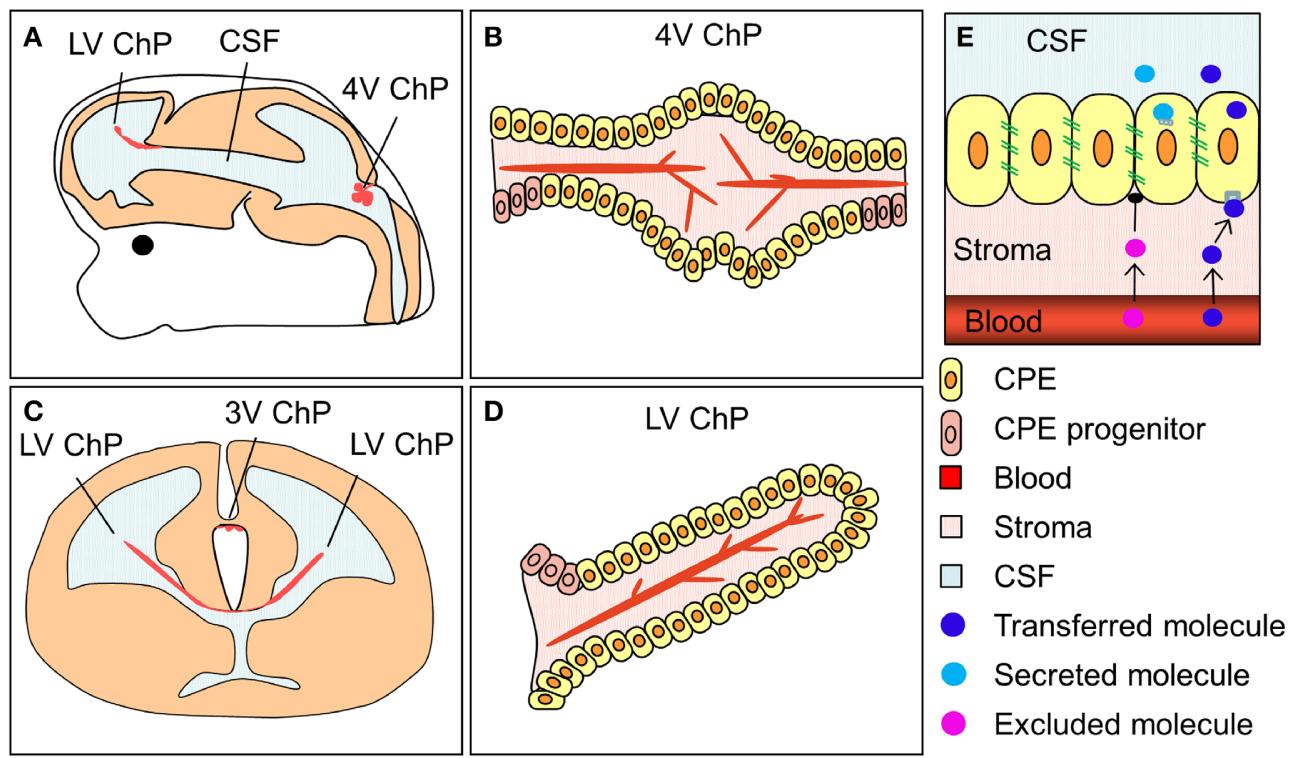

FIGURE 2 | The location and structure of the choroid plexuses and the blood-CSF barrier. (A,C) Schematic drawings of an embryonic brain in sagittal (A) and coronal (C) orientation depicting the location of the lateral, third and fourth ventricular choroid plexuses (LV ChP, 3V ChP and
4V ChP). (B,D) schematic drawing of the lateral and fourth ventricular choroid plexuses. (E) Simplified schematic depiction of the blood-CSF barrier and the transfer and secretion of molecules into CSF. ChP, choroid plexus.
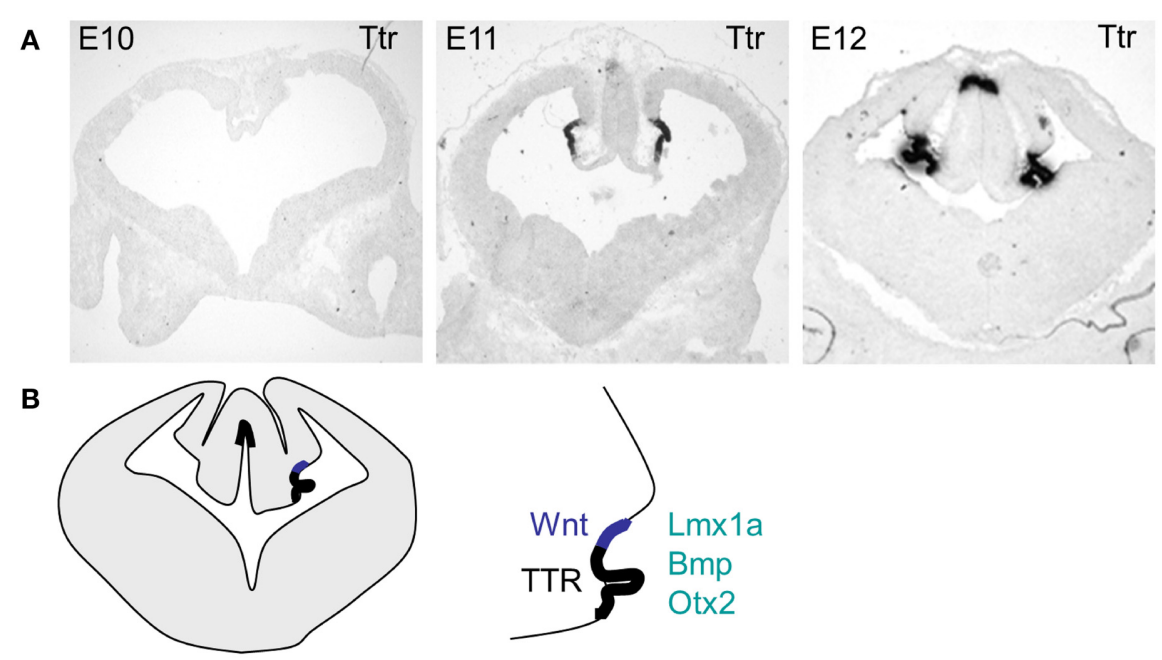

C

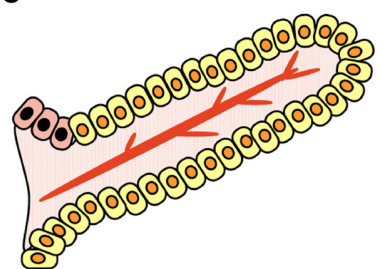

1 hour after BrdU

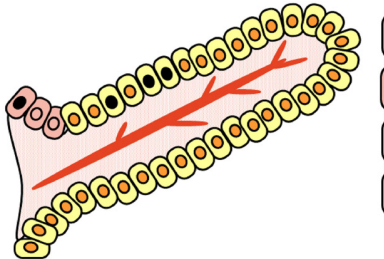

24 hours after BrdU
FIGURE 3 | The specification, differentiation and growth of the lateral ventricular choroid plexuses. (A) Photographs of transthyretin (Ttr) in situ hybridization sections, showing the choroid plexus anlage at E19, E11 and E12. (B) Schematic drawing showing the localization of the lateral ventricular choroid plexus and the cortical hem within the invaginated dorsal midline. (C) Schematic drawing (modified from Liddelow et al., 2010) showing the localization of the progenitor cells and the mode of growth of the choroid plexus. 
(i.e., a barrier), (ii) the capacity of water transport across the cells (i.e., the presence of water channels), and (iii) the presence of transporters and exchangers that can create an ionic or osmotic gradient that drives water across the epithelium. These criteria are of course all met in the adult choroid plexus via the presence of the blood-CSF barrier, the presence of the water channel AQP1 and the creation of ion gradients between blood and CSF via a multitude of ion transporters and exchangers (see e.g., Wolburg and Paulus, 2010). In development the barrier criterion is met by the presence of continuous tight junctional strands as soon as the choroid plexus invaginates into the ventricles (Møllgård and Saunders, 1975; Møllgård et al., 1976; Ek et al., 2003; Johansson et al., 2006). Aquaporin-1, the only confirmed and consistently identified water channel in the choroid plexus, is present in the choroid plexus epithelial cells as soon as they start to differentiate (Johansson et al., 2005), making water transport possible from very early stages in choroid plexus development. The gradient, however, that drives the water transport, does not appear to be regulated in the same manner as in the adult (Johansson et al., 2008a,b; Liddelow et al., 2013). For example, two of the main components in creating the ion gradients in the adult (NaK-ATPase alpha subunit and carbonic anhydrase II) are not present in the early developing choroid plexus (Johansson et al., 2008b). Instead it has been suggested that the there is a colloid-osmotic gradient, created by the high protein concentration in CSF during development, that drives the water from blood (or more accurately from interstitial fluid) into CSF (see Johansson et al., 2008a). Experimental evidence for this suggestion was recently described, where the total protein concentration in CSF was artificially increased via systemic protein injections in early development (P9 Monodelphis Domestica), resulting in a transient doubling of the size of the lateral ventricles (Liddelow et al., 2011a).

In both development and in the adult little is known about the transcriptional regulation of CSF secretion. One transcription factor that appears to have a role in regulating CSF secretion is E2F5, which is expressed in the developing choroid plexus from E12.5 (Swetloff and Ferretti, 2005). Its deletion (homozygous full knock-out mice) resulted in post-natal non-obstructive hydrocephalus (Lindeman et al., 1998), suggesting that it normally suppresses secretion. However, as E2F5 protein levels appear to decrease in the choroid plexus as they mature (and its localization is shifted from nuclear to cytoplasmic), it was suggested that E2F5 plays a role in the maturation process of CPE cells (Lindeman et al., 1998). It was also found that E2F5 is one of the target genes of choroid plexus specific micro-RNA mir449, which is expressed through-out choroid plexus development (Redshaw et al., 2009), suggesting a yet unexplored role of microRNAs in choroid plexus function, CSF secretion and potentially in the regulation of neurogenesis.

\section{THE IMPACT OF CSF PRESSURE AND COMPOSITION ON NEUROGENESIS}

The continuous CSF production (originating mostly from the choroid plexuses) creates a pressure inside the cerebral ventricles. The requirement of this pressure for normal brain development was shown by a study in chick embryos (Stage18), where the intraventricular pressure was removed via insertion of hollow tubes into the ventricular system (Desmond and Jacobson, 1977). After $24 \mathrm{~h}$ the nervous tissue had folded into the ventricles. The ventricles themselves were smaller (only $20 \%$ of their normal size) and the surrounding brain tissue was not only disorganized but also reduced in size (Desmond and Jacobson, 1977).

However, the choroid plexuses not only regulate the level of CSF production but also modify its composition. CSF composition can be regulated in two main ways; either via changes in the transfer of molecules from blood into CSF across the epithelium (receptor or carrier mediated transfer as well as exclusion of molecules via efflux pumps) or via changes in the secretion of molecules produced by the choroid plexuses themselves (Figure 2). In the developing choroid plexuses both of these mechanisms are in play. The subset of epithelial cells that transport proteins from blood into CSF are not only present in development but their proportion is larger and they appear to be more specialized as they selectively transport certain plasma proteins and not others across the barrier, a mechanism that appears to not be present in the adult (for example see Johansson et al., 2008a; Liddelow et al., 2011b, 2013). In regards to other proteins capable of modifying CSF composition in the adult (efflux pumps, amino acid transporters, solute transporters), the majority are already present in the developing (e.g., E15 rat) choroid plexus but are often expressed at different levels (both higher and lower) compared to the adult (Ek et al., 2010; Kratzer et al., 2013; Liddelow et al., 2013) again suggesting a dynamic and regulated mechanisms of CSF composition control. In addition, recent choroid plexus transcriptome analyses have shown that the developing choroid plexuses also express a large amount of signaling type of molecules that can be secreted into the ventricles and affect the behavior of the neural stem cells lining the ventricles (e.g., Johansson et al., 2013, Table 1).

That CSF can modulate brain development has been shown in in vitro experiments. Here it was shown that isolated cortical cells can be maintained on embryonic CSF alone (Miyan et al., 2006). Neuroepithelial explants from chick were shown to survive and proliferate just as well in CSF serum free media as in serum supplemented media (Gato et al., 2005). Further, CSF from different ages affects the CSF differently, as seen in rat cortical cultures where E19 cells proliferated more in CSF from E19 and E20 compared to CSF from E18 and E21 (Miyan et al., 2006). Similar results were shown with E14 rat neurospheres, which, when cultured with E17 CSF, generated more spheres than when grown in CSF from E14, P6 or adult CSF (Lehtinen et al., 2011). This is consistent with the age-dependent changes in CSF composition previously observed using both proteomics (Zappaterra et al., 2007), total protein measurements (see Johansson et al., 2008a) and individual protein measurements (Dziegielewska et al., 1981; Huang et al., 2010; Lehtinen et al., 2011). The proteome study compared embryonic mouse CSF from three different ages and reported 423 proteins in E12.5 CSF, 318 in E14.5 and 382 in E17.5 CSF (all sampled from the lateral ventricle). Only 137 proteins were common to all three ages, suggesting a highly dynamic regulation of CSF composition (Zappaterra et al., 2007). In studies investigating particular molecules, the levels of different plasma derived proteins was found to vary between different embryonic 
stages and although the individual proteins were following the same pattern (increase, peak, and decrease) the peak was reached at different ages (Dziegielewska et al., 1981). Igf2 was found in CSF from embryonic rat at all ages investigated but showed a significant increase late in embryogenesis and a decrease again perinatally (Lehtinen et al., 2011). However, in a study measuring Shh levels, the concentration of this particular molecule did not change in mouse CSF between E12.5 and E15.5 (Huang et al., 2009). Together these studies show a highly regulated control of CSF composition during development and during the period of neurogenesis.

In support of the regulation of CSF content having a physiological function, direct manipulation of CSF composition has been shown to alter the behavior of the stem cells in direct contact with the CSF. Here, both loss and gain of function experiments have been performed in vivo using either neutralizing antibodies or recombinant proteins injected into the embryonic ventricles. For example, the removal of Fgf2 from chick CSF caused a loss of proliferation and increased neural differentiation (Martin et al., 2006). Similar experiments with another type of growth factor Igf1, in embryonic mice and rats, showed an increase in proliferation after intraventricular injections of recombinant proteins and a decrease after IGF-neutralizing antibodies were injected (Mairet-Coello et al., 2009). Thus, the link between CSF composition and alteration in neural stem cell behavior has been experimentally shown but the link between choroid plexus development and brain development remains less clear.

\section{THE SPECIFIC IMPACT OF THE CHOROID PLEXUSES ON DEVELOPMENTAL NEUROGENESIS}

The choroid plexuses appear whilst neurogenesis is occurring through-out the nervous system. This gives the potential for the choroid plexuses (via their modulation of CSF) to alter the behavior of the neural stem cells along the neuroaxis (Figure 1, Box 1). As described above, the capability of the CSF to alter both proliferation and survival in different brains regions in vitro has long been known, likely due to the signaling molecules present in CSF. However, to what extent these molecules are regulated or even secreted by the choroid plexuses has not been sufficiently investigated (perhaps in part due to the technical difficulties such an approach poses).

With the advancement of region and time specific deletion techniques new strategies to isolate choroid plexuses involvement in neurogenesis are available. Along these lines, some recent work has demonstrated, via choroid plexus specific deletions, that molecules expressed in the choroid plexus have the capability to influence brain development at other sites. This was first achieved by deletion of Shh from the hindbrain choroid plexus (using the Wnt1-Cre), resulting in a more than 50\% decrease in the proliferation of the neural stem cells in the nearby cerebellum and impaired GABAergic progenitor expansion (Huang et al., 2010).

Recently, in work done by the author and colleagues, it has also been shown that when the transcription factor Otx2 was deleted from the hindbrain choroid plexus using the Gd7-Cre (only very sparse recombination in other regions) it not only significantly altered the size of the choroid plexus but also the relative expression of many secreted signaling molecules (Johansson et al., 2013). To our surprise, this deletion led to a region-specific increase in proliferation in the far distant cerebral cortex (but not in the spinal cord or in the lateral ganglionic eminence), which in turn led to site-specific alterations in the cortical neuronal layers at P7. The increase in proliferation was caused by an increase in Wnt-signaling, mediated by, at least in part, altered hindbrain choroid plexus expression levels and the ensuing increased CSF protein levels of the Wnt-signaling modulators Wnt4 and Tgm2.

This work revealed for the first time, the choroid plexus as a potential modulator of Wnt-signaling in the developing brain. It also highlighted that the hindbrain choroid plexus, being the first to appear, can alter the behavior of progenitor cells at the opposite end of the ventricular system prior to the differentiation of the other choroid plexuses. This period is also notable as the ventricular system is still an enclosed cavity, not connected with the fluid in the subarachnoid space and does not yet have any directional flow, making it more possible for molecules secreted from the fourth ventricular choroid plexus to reach the forebrain and influence the behavior of the neural stem cells (Figure 4). In support of this, in the above study we also found that the increase in proliferation and Wnt-signaling was no longer present at E16, when the influence of the hindbrain choroid plexus would be much reduced due to the now much larger (unaffected) lateral ventricular choroid plexuses in closer proximity to the cortical neural stem cells (Johansson et al., 2013).

This work also showed that not all regions in the CNS responded to the altered CSF in the same way (or at all). We suggest that select tissues are prepared for certain signaling molecules, by the expression of not only receptors but also by the expression of modulators and inhibitors of the different signaling pathways. Thus, neurogenesis can be seen as a reciprocal process between the choroid plexus and the neural stem cells mediated via the CSF (Figure 4).

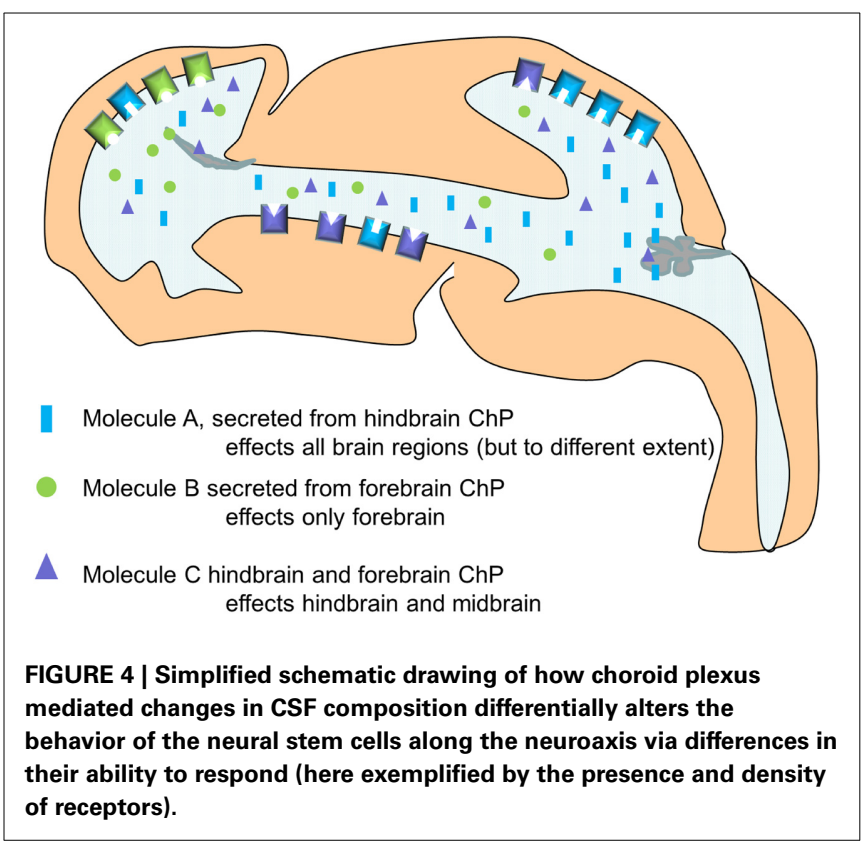




\section{CONCLUSIONS AND FUTURE DIRECTIONS}

In conclusion, there is substantial evidence that there is indeed a role played by the choroid plexus-CSF-signaling axis in the modulation of developmental neurogenesis. However, much more remains to be discovered. The specific mechanisms by which the choroid plexuses modulate brain development both in terms of the choroid plexuses as whole organs as well as specific aspects of their functions (e.g., transfer of blood-borne molecules or secretion of a particular molecule) needs to be investigated. Further detailed studies of choroid plexus impact on different brain regions and developmental time-points would increase our understanding of the whole process of brain development. Understanding the role of the choroid plexus-CSF signaling axis and its impact on brain development can also lead to novel routes and mechanisms for treatment approaches for a multitude of developmental disorders and in the long-run also for application to adult neurodegeneration and brain injuries.

\section{REFERENCES}

Baruch, K., Deczkowska, A., David, E., Castellano, J. M., Miller, O., Kertser, A., et al. (2014). Aging-induced type I interferon response at the choroid plexus negatively affects brain function. Science 346, 89-93. doi: 10.1126/science.1252945

Beschorner, R., Waidelich, J., Trautmann, K., Psaras, T., and Schittenhelm, J. (2013). Notch receptors in human choroid plexus tumors. Histol. Histopathol. 28, 1055-1063.

Bill, B. R., Balciunas, D., McCarra, J. A., Young, E. D., Xiong, T., Spahn, A. M., et al. (2008). Development and notch signaling requirements of the zebrafish choroid plexus. PLoS ONE 3:e3114. doi: 10.1371/journal.pone.0003114

Braun, S. M., and Jessberger, S. (2014). Adult neurogenesis: mechanisms and functional significance. Development 141, 1983-1986. doi: 10.1242/dev. 104596

Brightman, M. W., and Reese, T. S. (1969). Junctions between intimately apposed cell membranes in the vertebrate brain. J. Cell Biol. 40, 648-677. doi: $10.1083 /$ jcb.40.3.648

Catala, M. (1998). Embryonic and fetal development of structures associated with the cerebro-spinal fluid in man and other species. Part I: the ventricular system, meninges and choroid plexuses. Arch. Anat. Cytol. Pathol. 46, 153-169.

Dang, L., Fan, X., Chaudhry, A., Wang, M., Gaiano, N., and Eberhart, C. G. (2006). Notch3 signaling initiates choroid plexus tumor formation. Oncogene 25, 487-491. doi: 10.1038/sj.onc. 1209074

Desmond, M. E., and Jacobson, A. G. (1977). Embryonic brain enlargement requires cerebrospinal fluid pressure. Dev. Biol. 57, 188-198. doi: 10.1016/00121606(77)90364-5

Dziegielewska, K. M., Ek, J., Habgood, M. D., and Saunders, N. R. (2001). Development of the choroid plexus. Microsc. Res. Tech. 52, 5-20. doi: 10.1002/ 1097-0029(20010101)52:1<5::AID-JEMT3>3.0.CO;2-J

Dziegielewska, K. M., Evans, C. A., Lai, P. C., Lorscheider, F. L., Malinowska, D. H., Mollgard, K., et al. (1981). Proteins in cerebrospinal fluid and plasma of fetal rats during development. Dev. Biol. 83, 193-200. doi: 10.1016/S00121606(81)80024-3

Ek, C. J., Habgood, M. D., Dziegielewska, K. M., and Saunders, N. R. (2003). Structural characteristics and barrier properties of the choroid plexuses in developing brain of the opossum (Monodelphis Domestica). J. Comp. Neurol. 460, 451-464. doi: 10.1002/cne.10661

Ek, C. J., Wong, A., Liddelow, S. A., Johansson, P. A., Dziegielewska, K. M., and Saunders, N. R. (2010). Efflux mechanisms at the developing brain barriers: ABC-transporters in the fetal and postnatal rat. Toxicol. Lett. 197, 51-59. doi: 10.1016/j.toxlet.2010.04.025

Falcao, A. M., Marques, F., Novais, A., Sousa, N., Palha, J. A., and Sousa, J. C. (2012). The path from the choroid plexus to the subventricular zone: go with the flow! Front. Cell. Neurosci. 6:34. doi: 10.3389/fncel.2012.00034

Gato, A., Moro, J. A., Alonso, M. I., Bueno, D., De La Mano, A., and Martin, C. (2005). Embryonic cerebrospinal fluid regulates neuroepithelial survival, proliferation, and neurogenesis in chick embryos. Anat. Rec. A Discov. Mol. Cell. Evol. Biol. 284, 475-484. doi: 10.1002/ar.a.20185
Guldbrandsen, A., Vethe, H., Farag, Y., Oveland, E., Garberg, H., Berle, M., et al. (2014). In-depth characterization of the cerebrospinal fluid proteome displayed through the CSF Proteome Resource (CSF-PR). Mol. Cell. Proteomics. doi: 10.1074/mcp.M114.038554. [Epub ahead of print].

Hebert, J. M., Mishina, Y., and McConnell, S. K. (2002). BMP signaling is required locally to pattern the dorsal telencephalic midline. Neuron 35, 1029-1041. doi: 10.1016/S0896-6273(02)00900-5

Huang, X., Ketova, T., Fleming, J. T., Wang, H., Dey, S. K., Litingtung, Y., et al. (2009). Sonic hedgehog signaling regulates a novel epithelial progenitor domain of the hindbrain choroid plexus. Development 136, 2535-2543. doi: 10.1242/dev.033795

Huang, X., Liu, J., Ketova, T., Fleming, J. T., Grover, V. K., Cooper, M. K., et al. (2010). Transventricular delivery of Sonic hedgehog is essential to cerebellar ventricular zone development. Proc. Natl. Acad. Sci. U.S.A. 107, 8422-8427. doi: 10.1073/pnas.0911838107

Hunter, N. L., and Dymecki, S. M. (2007). Molecularly and temporally separable lineages form the hindbrain roof plate and contribute differentially to the choroid plexus. Development 134, 3449-3460. doi: 10.1242/dev.003095

Imayoshi, I., Shimogori, T., Ohtsuka, T., and Kageyama, R. (2008). Hes genes and neurogenin regulate non-neural versus neural fate specification in the dorsal telencephalic midline. Development 135, 2531-2541. doi: 10.1242/dev. 021535

Johansson, P., Dziegielewska, K., and Saunders, N. (2008b). Low levels of Na, KATPase and carbonic anhydrase II during choroid plexus development suggest limited involvement in early CSF secretion. Neurosci. Lett. 442, 77-80. doi: 10.1016/j.neulet.2008.06.068

Johansson, P. A., Cappello, S., and Gotz, M. (2010). Stem cells niches during development-lessons from the cerebral cortex. Curr. Opin. Neurobiol. 20, 400-407. doi: 10.1016/j.conb.2010.04.003

Johansson, P. A., Dziegielewska, K. M., Ek, C. J., Habgood, M. D., Liddelow, S. A., Potter, A. M., et al. (2006). Blood-CSF barrier function in the rat embryo. Eur. J. Neurosci. 24, 65-76. doi: 10.1111/j.1460-9568.2006.04904.x

Johansson, P. A., Dziegielewska, K. M., Ek, C. J., Habgood, M. D., Mollgard, K., Potter, A., et al. (2005). Aquaporin-1 in the choroid plexuses of developing mammalian brain. Cell Tissue Res. 322, 353-364. doi: 10.1007/s00441005-1120-X

Johansson, P. A., Dziegielewska, K. M., Liddelow, S. A., and Saunders, N. R. (2008a). The blood-CSF barrier explained: when development is not immaturity. Bioessays 30, 237-248. doi: 10.1002/bies.20718

Johansson, P. A., Irmler, M., Acampora, D., Beckers, J., Simeone, A., and Gotz, M. (2013). The transcription factor Otx2 regulates choroid plexus development and function. Development 140, 1055-1066. doi: 10.1242/dev.090860

Kratzer, I., Liddelow, S. A., Saunders, N. R., Dziegielewska, K. M., Strazielle, N., and Ghersi-Egea, J. F. (2013). Developmental changes in the transcriptome of the rat choroid plexus in relation to neuroprotection. Fluids Barriers CNS 10:25. doi: 10.1186/2045-8118-10-25

Lehtinen, M. K., Zappaterra, M. W., Chen, X., Yang, Y. J., Hill, A. D., Lun, M., et al. (2011). The cerebrospinal fluid provides a proliferative niche for neural progenitor cells. Neuron 69, 893-905. doi: 10.1016/j.neuron.2011.01.023

Liddelow, S. A., Dziegielewska, K. M., Ek, C. J., Habgood, M. D., Bauer, H., Bauer, H. C., et al. (2013). Mechanisms that determine the internal environment of the developing brain: a transcriptomic, functional and ultrastructural approach. PLoS ONE 8:e65629. doi: 10.1371/journal.pone.0065629

Liddelow, S. A., Dziegielewska, K. M., Ek, C. J., Johansson, P. A., Potter, A. M., and Saunders, N. R. (2009). Cellular transfer of macromolecules across the developing choroid plexus of Monodelphis domestica. Eur. J. Neurosci. 29, 253-266. doi: 10.1111/j.1460-9568.2008.06571.x

Liddelow, S. A., Dziegielewska, K. M., Møllgård, K., Phoenix, T. N., Temple, S., Vandeberg, J. L., et al. (2011b). SPARC/osteonectin, an endogenous mechanism for targeting albumin to the blood-cerebrospinal fluid interface during brain development. Eur. J. Neurosci. 34, 1062-1073. doi: 10.1111/j.14609568.2011.07821.x

Liddelow, S. A., Dziegielewska, K. M., Vandeberg, J. L., Noor, N. M., Potter, A. M., and Saunders, N. R. (2011a). Modification of protein transfer across blood/cerebrospinal fluid barrier in response to altered plasma protein composition during development. Eur. J. Neurosci. 33, 391-400. doi: 10.1111/j.14609568.2010.07509.x

Liddelow, S. A., Dziegielewska, K. M., Vandeberg, J. L., and Saunders, N. R. (2010). Development of the lateral ventricular choroid plexus in a marsupial, 
Monodelphis domestica. Cerebrospinal Fluid Res. 7:16. doi: 10.1186/17438454-7-16

Lindeman, G. J., Dagnino, L., Gaubatz, S., Xu, Y., Bronson, R. T., Warren, H. B., et al. (1998). A specific, nonproliferative role for E2F-5 in choroid plexus function revealed by gene targeting. Genes Dev. 12, 1092-1098. doi: 10.1101/gad.12.8.1092

Mairet-Coello, G., Tury, A., and Dicicco-Bloom, E. (2009). Insulin-like growth factor-1 promotes $\mathrm{G}(1) / \mathrm{S}$ cell cycle progression through bidirectional regulation of cyclins and cyclin-dependent kinase inhibitors via the phosphatidylinositol 3kinase/Akt pathway in developing rat cerebral cortex. J. Neurosci. 29, 775-788. doi: 10.1523/JNEUROSCI.1700-08.2009

Marques, F., Sousa, J. C., Coppola, G., Gao, F., Puga, R., Brentani, H., et al. (2011). Transcriptome signature of the adult mouse choroid plexus. Fluids Barriers CNS 8:10. doi: $10.1186 / 2045-8118-8-10$

Martin, C., Bueno, D., Alonso, M. I., Moro, J. A., Callejo, S., Parada, C., et al. (2006). FGF2 plays a key role in embryonic cerebrospinal fluid trophic properties over chick embryo neuroepithelial stem cells. Dev. Biol. 297, 402-416. doi: 10.1016/j.ydbio.2006.05.010

Miyan, J. A., Zendah, M., Mashayekhi, F., and Owen-Lynch, P. J. (2006). Cerebrospinal fluid supports viability and proliferation of cortical cells in vitro, mirroring in vivo development. Cerebrospinal Fluid Res. 3:2. doi: 10.1186/17438454-3-2

Møllgård, K., Milinowska, D. H., and Saunders, N. R. (1976). Lack of correlation between tight junction morphology and permeability properties in developing choroid plexus. Nature 264, 293-294. doi: 10.1038/ 264293a0

Møllgård, K., and Saunders, N. R. (1975). Complex tight junctions of epithelial and of endothelial cells in early foetal brain. J. Neurocytol. 4, 453-468. doi: 10.1007/BF01261375

Nielsen, C. M., and Dymecki, S. M. (2010). Sonic hedgehog is required for vascular outgrowth in the hindbrain choroid plexus. Dev. Biol. 340, 430-437. doi: 10.1016/j.ydbio.2010.01.032

Redshaw, N., Wheeler, G., Hajihosseini, M. K., and Dalmay, T. (2009). microRNA449 is a putative regulator of choroid plexus development and function. Brain Res. 1250, 20-26. doi: 10.1016/j.brainres.2008.11.020

Silva-Vargas, V., Crouch, E. E., and Doetsch, F. (2013). Adult neural stem cells and their niche: a dynamic duo during homeostasis, regeneration, and aging. Curr. Opin. Neurobiol. 23, 935-942. doi: 10.1016/j.conb.2013. 09.004

Swetloff, A., and Ferretti, P. (2005). Changes in E2F5 intracellular localization in mouse and human choroid plexus epithelium with development. Int. J. Dev. Biol. 49, 859-865. doi: 10.1387/ijdb.051996as

Taverna, E., Gotz, M., and Huttner, W. B. (2014). The cell biology of neurogenesis: toward an understanding of the development and evolution of the neocortex. Annu. Rev. Cell Dev. Biol. 30, 465-502. doi: 10.1146/annurev-cellbio-101011155801

Von Frowein, J., Wizenmann, A., and Gotz, M. (2006). The transcription factors Emx1 and Emx2 suppress choroid plexus development and promote neuroepithelial cell fate. Dev. Biol. 296, 239-252. doi: 10.1016/j.ydbio.2006.04.461

Wilting, J., and Christ, B. (1989). An experimental and ultrastructural study on the development of the avian choroid plexus. Cell Tissue Res. 255, 487-494. doi: 10.1007/BF00218783

Wolburg, H., and Paulus, W. (2010). Choroid plexus: biology and pathology. Acta Neuropathol. 119, 75-88. doi: 10.1007/s00401-009-0627-8

Zappaterra, M. D., Lisgo, S. N., Lindsay, S., Gygi, S. P., Walsh, C. A., and Ballif, B. A. (2007). A comparative proteomic analysis of human and rat embryonic cerebrospinal fluid. J. Proteome Res. 6, 3537-3548. doi: 10.1021/pr070247w

Conflict of Interest Statement: The author declares that the research was conducted in the absence of any commercial or financial relationships that could be construed as a potential conflict of interest.

Received: 08 August 2014; accepted: 07 October 2014; published online: 24 October 2014.

Citation: Johansson PA (2014) The choroid plexuses and their impact on developmental neurogenesis. Front. Neurosci. 8:340. doi: 10.3389/fnins.2014.00340

This article was submitted to Neurogenomics, a section of the journal Frontiers in Neuroscience.

Copyright (๑) 2014 Johansson. This is an open-access article distributed under the terms of the Creative Commons Attribution License (CC BY). The use, distribution or reproduction in other forums is permitted, provided the original author $(s)$ or licensor are credited and that the original publication in this journal is cited, in accordance with accepted academic practice. No use, distribution or reproduction is permitted which does not comply with these terms. 


\section{Barrier mechanisms in neonatal stroke}

\section{Ingrid Kratzer ${ }^{\dagger}$, Sophorn Chip ${ }^{\dagger}$ and Zinaida S. Vexler*}

Department of Neurology, University of California San Francisco, San Francisco, CA, USA

Edited by:

Helen B. Stolp, King's College

London, UK

Reviewed by:

Jun Zhang, Texas Tech University Health Sciences Center, USA

Ana A. Baburamani, King's College

London, UK

*Correspondence:

Zinaida S. Vexler, Department of Neurology, University of California San Francisco, 675 Nelson Rising

Lane, San Francisco, CA

94158-0663, USA

e-mail: zena.vexler@ucsf.edu

${ }^{\dagger}$ These authors have contributed equally to this work.
Clinical data continue to reveal that the incidence of perinatal stroke is high, similar to that in the elderly. Perinatal stroke leads to significant morbidity and severe long-term neurological and cognitive deficits, including cerebral palsy. Experimental models of cerebral ischemia in neonatal rodents have shown that the pathophysiology of perinatal brain damage is multifactorial. Cerebral vasculature undergoes substantial structural and functional changes during early postnatal brain development. Thus, the state of the vasculature could affect susceptibility of the neonatal brain to cerebral ischemia. In this review, we discuss some of the most recent findings regarding the neurovascular responses of the immature brain to focal arterial stroke in relation to neuroinflammation. We also discuss a possible role of the neonatal blood-CSF barrier in modulating inflammation and the long-term effects of early neurovascular integrity after neonatal stroke on angiogenesis and neurogenesis.

Keywords: vascular permeability, microglia, leukocyte, inflammation, CSF-brain barrier, neonatal ischemia

\section{INTRODUCTION}

The blood-brain barrier (BBB) protects the central nervous system (CNS) and prevents non-specific leakage of molecules and cells from the blood into the brain. Stroke can disintegrate the BBB in many ways by disrupting cell-cell communication between endothelial cells (ECs), pericytes, and astrocytes, cumulatively referred to as the "neurovascular unit" (NVU), and by affecting neurons and microglial cells, thereby enhancing injury. Reperfusion and re-oxygenation of previously ischemic brain regions further affect BBB function, restoring or disrupting, depending on various factors such as the extent, length of initial cerebral blood flow (CBF) disruption, genetic background, sex, age, and the presence of other confounding factors.

The timing of injury during brain development has a major impact on determining the pathophysiology of ischemic brain damage. Maturation of individual cell types, individual components of the NVU and of the extracellular matrix (ECM), and of individual brain regions is not synchronized, contributing to the existence of "windows of susceptibility" to ischemia during particular fetal and postnatal periods. Here we discuss some of the most recent findings regarding the neurovascular responses of the immature brain to focal arterial stroke in relation to neuroinflammation and long-term effects on repair.

\section{AGE AT THE TIME OF CEREBRAL ISCHEMIA AS A DETERMINANT OF THE PATHOPHYSIOLOGY OF BRAIN DAMAGE: PRETERM vs. TERM}

In preterm human babies (23-32-weeks of gestation) intracerebral hemorrhage (ICH), intraventricular hemorrhage (IVH), and periventricular white matter injury (PWMI) are the most common types of ischemia-related injuries (Volpe, 2009). Clinical aspects of perinatal stroke have been extensively discussed (Ferriero, 2004; Nelson and Lynch, 2004). Vulnerability of oligodendrocyte progenitor cells (OPCs) to ischemia and hypoxia including a concomitant loss of subplate neurons, a transient neuronal subpopulation important in corticogenesis and proper wiring of the developing brain, contribute to PWMI (McQuillen et al., 2003; Volpe, 2009). The germinal matrix with its weak, leaky vasculature, high local production of vascular endothelial growth factor (VEGF), angiopoietin-2 (Angpt2), and matrix metalloproteinases (MMPs) also make the preterm brain prone to $\mathrm{ICH}$ and IVH (Ballabh, 2010). Low pericyte coverage and ensheathement of astrocytic endfeets along blood vessels, together with immaturity of the basal membrane (BM), also make the preterm brain susceptible to ischemia-related injury (El-Khoury et al., 2006; Braun et al., 2007). At term (between birth and 28 days of life), ischemia-related white matter injury does occur (Rothstein and Levison, 2005; Van Den Broeck et al., 2008), but injury predominantly affects gray matter (Ferriero, 2004).

Several models were developed to understand the pathophysiological mechanisms of hypoxic-ischemic encephalopathy (HIE) and focal arterial stroke at term (Yager and Ashwal, 2009). A model of hypoxia-ischemia (HI) consists of a unilateral ligation of the common carotid artery (CCA) followed by a variable duration of exposure to $8 \% \mathrm{O}_{2}$ in postnatal day 7 (P7) rats (Rice et al., 1981), and P9 mice and mimics HIE, whereas transient middle cerebral artery occlusion (tMCAO) model in $\mathrm{P} 7$ rats (Derugin et al., 1998), P10 rats (Mu et al., 2003), and P9 mice (Woo et al., 2012), and a combined permanent MCAO and transient CCA occlusion in P7 rats (Renolleau et al., 1998) mimic focal arterial stroke. The $\mathrm{HI}$ is associated with increased $\mathrm{CBF}$ during systemic hypoxia whereas CBF is disrupted after MCAO. Models of HI were also developed in rabbit and sheep (Marks et al., 1999; Derrick et al., 2007).

Studies in these age-appropriate ischemic models revealed several features unique to neonatal brain injury. First, excitotoxicity-induced neuronal death is a significant injury component (Ikonomidou et al., 1989). Second, neonatal brain is 
prone to reactive oxygen species (ROS) after HI (Sheldon et al., 2004). Third, apoptosis, a major neuronal cell death mode during this period, coexists with necrosis and necroptosis, creating a "continuum" with features of both cell death types (Blomgren et al., 2007; Northington et al., 2011). Finally, inflammation associated with failure to complete apoptosis is pivotal to ischemia-induced injury (Vexler and Yenari, 2009). Figure 1 outlines identified differences in ischemic injury mechanisms between adults and neonates.

\section{BRAIN MATURATION-DEPENDENT SUSCEPTIBILITY OF THE BBB AFTER STROKE}

The BBB undergoes major disruption early after adult stroke. Depending on initial stroke severity BBB opening can be biphasic or gradual (Knowland et al., 2014). The timing, extent of leukocyte-mediated BBB disturbances and redistribution of tight junctions (TJs) can vary. Degradation of TJ proteins occludin, claudin-5, and of a TJ-associated protein, zonula occludens 1 ( $\mathrm{ZO}-1)$, is increased after stroke in part due to activation and/or de novo synthesis of MMPs (Yang et al., 2007a; Liu et al., 2012). TJ proteins can also internalize into the cytosol by caveolin-1-mediated endocytosis or redistribute to other

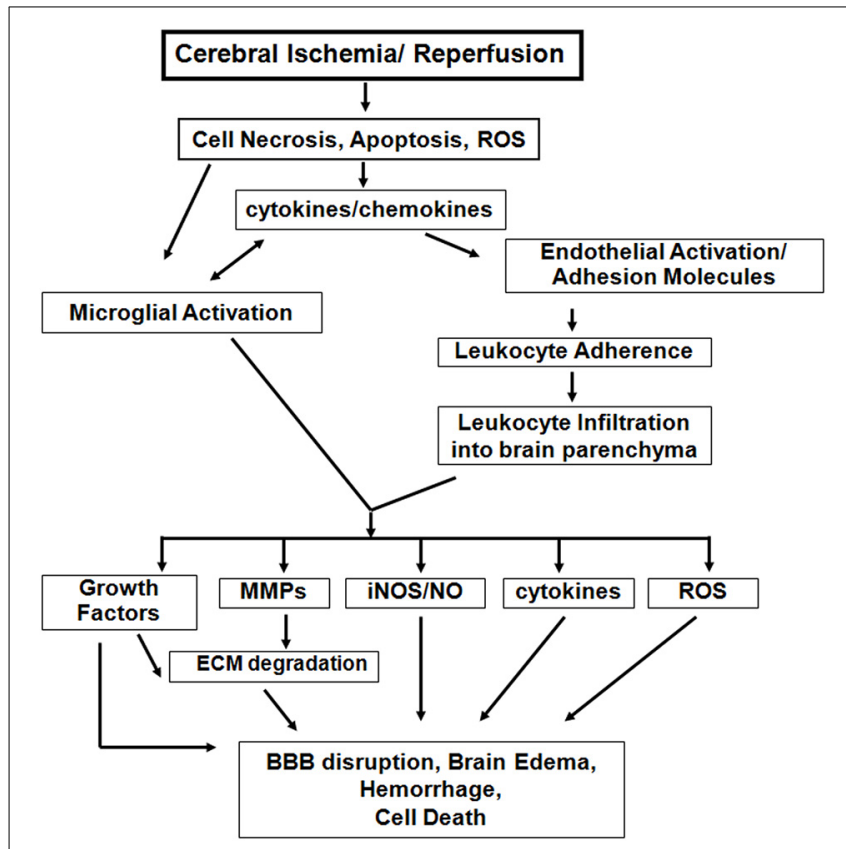

FIGURE 1 | Inflammatory mechanisms following acute cerebral

ischemia-reperfusion injury. There are both common and distinct features of the inflammatory response to cerebral ischemia between adults and neonates. However, neonatal brain is more susceptible to excitotoxic-damage and oxidative injury by ROS, resulting in necrosis and apoptosis continuum. The induction of cytokine/chemokine production, activation of microglial cells, and the systemic inflammatory response lead to neuroinflammation. Differences of adhesion molecule expression on ECs and on peripheral leukocytes exist between the injured adult and neonatal brain. Among the inflammatory mediators, activated matrix

metalloproteinases (MMPs), inducible nitric oxide synthase (iNOS), and further cytokine and ROS accumulation also contribute to the variance in magnitude and spatial distribution of BBB disruption, brain edema and injury between neonatal and adult stroke. (modified from Vexler et al., 2006). membrane domains after cerebral ischemia, coinciding with the early post-ischemic BBB opening. Adherens junction proteins support BBB properties (Petty and Lo, 2002) and their altered composition after stroke changes TJ stability, affecting BBB permeability (Dejana and Giampietro, 2012; Wacker et al., 2012).

Surprisingly little is known about BBB function after neonatal ischemic injury. Major differences in functional BBB response to acute ischemia-reperfusion between neonates and adults have been recently identified (Fernandez-Lopez et al., 2012). While $\mathrm{BBB}$ permeability to albumin or intravascular tracers of a similar size is significantly increased after acute tMCAO in adult rats, $\mathrm{BBB}$ permeability remains low in injured neonatal rats. Gene and protein expression of occludin, claudin- 5 and $\mathrm{ZO}-1$ are better preserved in injured neonatal brain than in injured adult brain, whereas gene expression of the efflux transporters ATPbinding cassette, subfamily G2 (Abcg2) and P-glycoprotein (P-gp) is reduced in both ages $24 \mathrm{~h}$ after reperfusion (Fernandez-Lopez et al., 2012). Transcript levels of several adhesion molecules and ECM components are differentially affected by injury in immature and adult brain, including E-selectin and P-selectin. Gene expression of Mmp-9 is significantly upregulated in injured adults and, while high transcript levels of collagen type IV $\alpha 1$ (Col4a1), and Col4a 2 remain unaltered in neonates, a significant increase of these two genes is evident in injured adult rats. Interestingly, transcripts of angiogenic regulators Vegfr-2, and Angpt2 are increased after stroke in adults but not in neonates (Fernandez-Lopez et al., 2012). Figure 2 summarizes these findings. In contrast, a transient leakage of much smaller tracers, sucrose and inulin, was observed in a mouse HI model, with the peak at $6 \mathrm{~h}$ and normalization by $24 \mathrm{~h}$ (D'Angelo et al., 2012), conveying that size and chemical structure of molecules affect leakage and highlighting the need for future BBB studies in injured immature brain.

The ECM proteins and their corresponding receptors on ECs and astrocytes provide both physical and biochemical glialvascular "scaffolding" while BM components laminin, collagen IV (Col-IV), fibronectin, and perlecan provide proper cell-cell interactions. Endothelial-ECM interaction via $\beta 1$ integrins regulates the expression of claudin-5 and BBB tightness whereas other ECM proteins, like galectin-3 mediate integrin-induced stabilization of focal adhesions, and activate cytokine receptors to enhance actions of growth factors (Goetz et al., 2008). Homozygous mutations in Col-IV are lethal in mid-gestation due to blockage of capillary bed development, and mutations in the COL4A1 gene cause ICH both in newborn mouse and human (Gould et al., 2005; Labelle-Dumais et al., 2011). Laminin degradation after focal stroke in adults causes detachment of astrocytic endfeet, disrupts BBB and induces ICH (Fukuda et al., 2004), while in neonates expression of this ECM protein is not reduced acutely (Fernandez-Lopez et al., 2012). The role of other ECM proteins in injured neonates is less studied but opposite effects of galectin3 in adult stroke and HI has been demonstrated (Doverhag et al., 2010; Lalancette-Hebert et al., 2012).

\section{PARENCHYMAL BRAIN CELLS AS MODULATORS OF BBB INTEGRITY AFTER NEONATAL STROKE}

Neuroinflammation is a characteristic feature of stroke progression and a major contributor to brain injury (Iadecola and Anrather, 2011). Multiple cell types (i.e. neurons, 


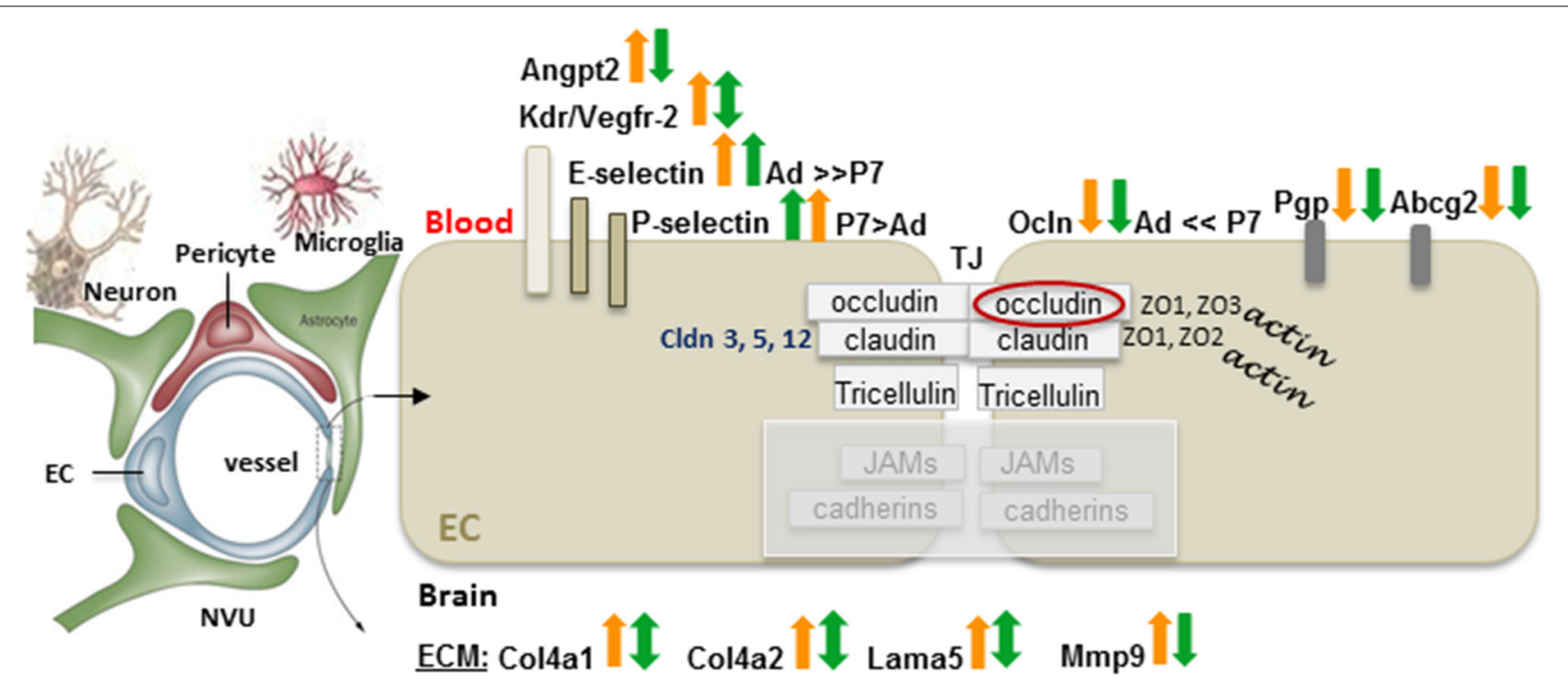

FIGURE 2 | Differences in the gene expression of the BBB components between adults and neonates $24 \mathrm{~h}$ after tMCAO. On a diagram of the NVU, arrows demarcate the gene expression changes in injured vs. matching contralateral region (ratio, ipsilateral/contralateral) in adult (orange arrows), and neonatal rats (green arrows) subjected to $3 \mathrm{~h}$ of MCAO followed by $24 \mathrm{~h}$ of reperfusion. Arrows pointing upwards demarcate increased gene expression in injured regions vs. matching contralateral regions (statistically significant $>2$-fold change). Downward arrows show reduced expression in injured regions vs. matching contralateral regions (statistically significant $>2$-fold change).
Double-sided arrows indicate no changes between injured and contralateral regions. Abbreviations: Ad: adult, P7: postnatal 7-days-old, EC, endothelial cell; angiopoetin 2, Angpt2; Kdr, Vegfr-2; Cldn, claudin; Ocln, occludin; ZO, zonula occludens; JAM, junctional adhesion molecules; TJ, tight junction; Pgp, P-glycoprotein; Abcg2, ATP-binding cassette transporter, subfamily G 2; Col4a1, collagen, type IV, alpha 1; Col4a2, collagen, type IV, alpha 2; Lama5, laminin, alpha 5; Mmp-9, matrix metalloproteinase 9. Original data are published in Fernandez-Lopez et al. (2012). The NVU contains fragments from Eichler et al. (2011). astrocytes, ECs, and microglia) increase production of inflammatory mediators post-ischemia, which can adversely affect BBB integrity and propagate injury.

ECs are sensitive to oxidative stress (Freeman and Keller, 2012). Excessive ROS accumulation generated by ischemia/reperfusion contributes to damage of TJs and other EC components, and promotes activation of cell death pathways (Rizzo and Leaver, 2010). Although less sensitive to cerebral ischemia than neurons, ECs also undergo cell death via several mechanisms (Rizzo and Leaver, 2010). Our comparison of gene expression in ECs from injured and contralateral cortex in neonatal and adult rats after tMCAO (endothelial transcriptome) revealed a markedly distinct signature of up-regulated and down-regulated transcripts in injured regions between two ages (Fernandez-Lopez et al., 2012).

Astrocytes are rather resistant to ischemic injury but impairment of water fluxes through astrocytic swelling and increased expression of aquaporin 4 (AQP4) leads to edema after adult focal ischemia (Loreto and Reggio, 2010). No edema was observed in injured P10 pups in brain regions with greatest AQP4 expression (Badaut et al., 2007), suggesting that enhanced water clearance at border regions can protect.

Microglial cells have been considered toxic after cerebral ischemia due to production of inflammatory mediators. However, phagocytotic ability and production of growth factors by microglia can be beneficial via several mechanisms, including support of neuronal and endothelial survival (Baburamani et al.,
2012; Luo and Chen, 2012). Microglial cells have several direct modulatory effects on the vasculature. During brain development they mediate vasculogenesis (Arnold and Betsholtz, 2013), and act as a physical bridge that guides vascular anastomosis, facilitating normal angiogenesis, and vascular sprouting. Microglia patrol the vasculature in the naïve brain (Davalos et al., 2012) and, upon BBB disruption, rapidly extend their processes, shielding injured sites (Nimmerjahn et al., 2005). Depletion of microglia worsens parenchymal injury in neonatal rats after tMCAO, increases levels of inflammatory mediators in acutely injured regions, and induces hemorrhagic transformation (Faustino et al., 2011; Fernandez Lopez et al., 2014) perhaps due to differing cell phenotypes in the immature brain.

Pericytes are important regulators of vessel contractility. Pericyte loss or dissociation from vessels lead to edema (Peppiatt et al., 2006) and impaired reflow (Yemisci et al., 2009). Lower than in adult pericyte coverage in the neonatal brain (Daneman et al., 2010) may differentially affect CBF regulation and BBB function after injury but this is yet to be demonstrated.

OPCs support EC survival but they are sensitive to ROS and can adversely affect $\mathrm{BBB}$ integrity via release of inflammatory mediators. Mast cells contribute to early ischemic brain swelling, BBB leakage and neutrophil infiltration (Strbian et al., 2006). Their stabilization protects and reduces hemorrhage formation (Strbian et al., 2007). Degranulation of mast cells is injurious in neonatal HI (Jin et al., 2007) and stroke (Biran et al., 2008) but direct effects on BBB have not been studied. 


\section{SYSTEMIC INFLAMMATION ALTERS BBB PERMEABILITY AFTER NEONATAL STROKE}

Parenchymal, perivascular and peripheral circulating cells independently and in concert contribute to stroke-induced production of inflammatory mediators, upregulation of integrins and adhesion molecules on ECs and leukocytes (Iadecola and Anrather, 2011).

Neutrophils rapidly, often transiently infiltrate ischemic tissue and exacerbate reperfusion injury in adults by priming the endothelium, causing "no-reflow" phenomenon, releasing ROS and proteolytic enzymes, and stimulating cytokine release (Mori et al., 1992; Amantea et al., 2009; Engelhardt and Liebner, 2014). Both loosely attached and infiltrated leukocytes can contribute to generation of pro-inflammatory molecules in the CNS (Denes et al., 2010). Neutropenia or treatments that prevent leukocyte adhesion and infiltration are neuroprotective (Kochanek and Hallenbeck, 1992; Yamasaki et al., 1995). Compared to adults, in neonates neutrophil infiltration is markedly lower in response to HI (Hudome et al., 1997; Bona et al., 1999), and tMCAO (Fernandez-Lopez et al., 2012). Neutralization of cytokineinduced neutrophil chemoattractant 1 protein (CINC-1) following tMCAO in adults holts neutrophil transmigration, reduces brain edema and protects (Yamasaki et al., 1997) whereas it promotes neutrophil infiltration following tMCAO and disrupts the BBB in neonates (Fernandez-Lopez et al., 2012).

Monocytes have been recently shown to have a dual role, elicit both inflammatory effects and maintain NVU integrity following cerebral ischemia. Monocyte depletion, chemokine (C-C motif) receptor 2 (CCR2) knockout, and bone marrow chimeric approach in murine stroke models demonstrated that CCR2 in bone marrow-derived cells alters injury and hemorrhagic transformation (Gliem et al., 2012). The stabilizing effects of monocytes are transforming growth factor beta 1 (TGF- $\beta 1$ )-dependent (Gliem et al., 2012). Compared to adult stroke, infiltration of circulating monocytes across the $\mathrm{BBB}$ is relatively low during the acute injury phase in neonates (Denker et al., 2007). It remains poorly understood whether leukocyte immaturity at the time of insult or a distinct gene expression pattern of selectins, and cytokines/chemokines account for the difference.

$\mathrm{T}$ and $\mathrm{B}$ cell infiltration may be less profound (Bona et al., 1999) or transient (Benjelloun et al., 1999) in injured neonates than in adults (Catania and Lipton, 1998; Chu et al., 2014).

\section{INDIVIDUAL INFLAMMATORY SIGNALING MECHANISMS}

Evidence is growing that the inflammatory responses after stroke are different in neonates and adults (Vexler and Yenari, 2009). Genetic deletion of various inflammatory mediators, including NADPH oxidase (Doverhag et al., 2008), or interleukin 1 (IL-1) $\beta$, IL-1 $\alpha$, or both $\alpha \beta$ (Hedtjarn et al., 2005) are not neuroprotective in the neonatal brain compared to adults. Some mediators that are upregulated and injurious during the acute injury phase, such as nitric oxide (NO), MMPs, macrophage inflammatory protein 1 alpha (MIP-1 $\alpha$ ), monocyte chemoattractant protein 1 (MCP-1), and complement, may be beneficial and mediate repair (Fernandez-Lopez et al., 2014).

MMPs degrade $\mathrm{TJ}$ and $\mathrm{BM}$ proteins, including collagen, laminin and fibronectin, thereby leading to brain edema, BBB leakage and leukocyte infiltration. MMP upregulation after stroke contributes to ECM breakdown and acute brain damage (Rosenberg et al., 1998; Asahi et al., 2000). MMP-2 and MMP-9, the two most studied MMPs in stroke, play different roles in BBB disruption (Asahi et al., 2001). MMP-3 targets several ECM components, including laminin and proteoglycans, and propagates injury by mediating BBB opening by inflammatory mediators (Cunningham et al., 2005). While activated microglia, macrophages and infiltrating leukocytes are the major sources of MMPs early after injury (Gidday et al., 2005; McColl et al., 2008), over time activated astrocytes and neurons begin producing MMPs, enhancing repair (Zhao et al., 2006). MMP-9 was shown to predict HIE in human newborns (Bednarek et al., 2012). Cerebrovascular ECs from neonates in culture contain more tissue plasminogen activator (t-PA) and gelatinases upon glutamate challenge than adult cells (Omouendze et al., 2013). MMP inhibition is protective after HI (Chen et al., 2009) but long-term MMP inhibition may holt ECM remodeling, as shown in adult stroke (Zhao et al., 2006).

The patterns of monocyte and neutrophil recruitment are cytokine- and chemokine-specific. The multifaceted roles for $\alpha$, $\beta$, and $\delta$ classes of chemokines were shown in adult stroke models (Yamasaki et al., 1995). Integrins are central for cell communication within the NVU and for leukocyte recruitment after stroke (Iadecola and Anrather, 2011) but information on the role of integrins in neonatal stroke is scant.

\section{BLOOD-CSF BARRIER (BCSFB)}

The choroid plexuses (CPs), forming the BCSFB, are involved in immune cell entry after brain injury (Shechter et al., 2013). CPs express chemokines and support transepithelial trafficking of neutrophils, monocytes and T cells (Szmydynger-Chodobska et al., 2009, 2012; Kunis et al., 2013). Engraftment of CPs in adult stroke models reduced infarct size and improved neurological function, in part via secretion of glial cell line-derived neurotrophic factor (GDNF), brain-derived neurotrophic factor (BDNF), and nerve growth factor (NGF) (Borlongan et al., 2004). $\mathrm{CPs}$ have unique functions in the developing brain (Dziegielewska et al., 2001) but their role in protection of the immature brain after stroke is unknown.

\section{NEUROVASCULAR RESPONSES AND REPAIR AFTER NEONATAL STROKE}

Long-term neural repair is less studied after neonatal stroke than after adult stroke. Cell proliferation in the subventricular zone (SVZ) after ischemia is triggered in both adults (Ohab et al., 2006), and neonates (Plane et al., 2004; Yang et al., 2007b). The dynamic changes within the SVZ neurogenic niche permit neuroblast migration into the ischemic striatum (Young et al., 2011) in the adult, where they express phenotypic region-specific mature neuronal markers (Parent et al., 2002), and into periinfarct striatum after neonatal HI (Plane et al., 2004; Yang et al., 2007b). Niche astrocytes and SVZ microglia are also involved in neuroblast migration (Young et al., 2011). The newborn SVZ contains numerous cell types, including unipotential astrocytes and OPCs as well as bipotential glial progenitors (Levison and Goldman, 1997). 
Given the dynamic nature of postnatal brain development one would expect robust repair processes in neonatal stroke but a 1-2 week delay in induction of angiogenesis was shown after tMCAO in P7 and P10 rats (Shimotake et al., 2010; Dzietko et al., 2013; Fernandez-Lopez et al., 2013) despite induction of VEGF (Mu et al., 2003). Another obstacle for endogenous repair is that proliferating cells in the postnatal SVZ differentiate into astrocytes, rather than neurons or oligodendrocytes, and astrogliosis holts the repair (Spadafora et al., 2010; Gonzalez et al., 2013). Changing the neural stem cell fate to route the differentiation into neurons and oligodendrocytes after stroke, for example, with erythropoietin, enhances the repair (Iwai et al., 2010; Gonzalez et al., 2013). Cell based therapies, including mesenchymal stem cells, improve functional outcomes after neonatal HI (Van Velthoven et al., 2010) and arterial stroke (Van Velthoven et al., 2013) by changing the microenvironment and stimulating Angpt1 and VEGF signaling, which amplify angiogenesis and "loosen" the barrier, allowing vessel remodeling and neuroblast migration.

\section{TRANSLATIONAL ASPECTS AND FUTURE DIRECTIONS}

A broad range of therapeutic agents was used in neonatal ischemic brain injury models to target the excitotoxic, oxidative, and inflammatory injury components, but, as in adult stroke, studies revealed limits in protection. To date, hypothermia is the only neuroprotective treatment for perinatal HIE with efficacy limited to moderate injury (Azzopardi et al., 2009; Edwards et al., 2010).

Importantly, recent studies have improved our understanding of the events at the $\mathrm{BBB}$ after neonatal ischemia by revealing that the developmental stage of the BBB at the time of ischemic insult is of prime importance and that careful consideration should be given to whether the $\mathrm{BBB}$ is in fact disrupted or it limits therapies from reaching an injured neonatal brain. The role of local parenchymal cells, microglia, as modulators of neurovascular integrity after neonatal stroke was also uncovered. Future studies should shed light on relationships between neurovascular integrity and interaction with neuroprogenitors, endogenous, or engrafted, including the migration and differentiation of neural progenitors during stroke-induced neurogenesis.

\section{AUTHOR CONTRIBUTIONS}

Ingrid Kratzer and Sophorn Chip wrote individual parts of the review and contributed equally. Together with Zinaida S. Vexler who provided conceptual framework and wrote parts of the review, they contributed to revisions and prepared artwork.

\section{ACKNOWLEDGMENTS}

The following grants NS080015 (Zinaida S. Vexler), NS44025 (Zinaida S. Vexler), and NS76726 (Zinaida S. Vexler) were used as principal sources of funding.

\section{REFERENCES}

Amantea, D., Nappi, G., Bernardi, G., Bagetta, G., and Corasaniti, M. T. (2009). Post-ischemic brain damage: pathophysiology and role of inflammatory mediators. FEBS J. 276, 13-26. doi: 10.1111/j.1742-4658.2008.06766.x

Arnold, T., and Betsholtz, C. (2013). The importance of microglia in the development of the vasculature in the central nervous system. Vasc. Cell 5, 4. doi: 10.1186/2045-824X-5-4

Asahi, M., Asahi, K., Jung, J. C., Del Zoppo, G. J., Fini, M. E., and Lo, E. H. (2000). Role for matrix metalloproteinase 9 after focal cerebral ischemia: effects of gene knockout and enzyme inhibition with BB-94. J. Cereb. Blood Flow Metab. 20, 1681-1689. doi: 10.1097/00004647-200012000-00007

Asahi, M., Sumii, T., Fini, M. E., Itohara, S., and Lo, E. H. (2001). Matrix metalloproteinase 2 gene knockout has no effect on acute brain injury after focal ischemia. Neuroreport 12, 3003-3007. doi: 10.1097/00001756-20010917000050

Azzopardi, D. V., Strohm, B., Edwards, A. D., Dyet, L., Halliday, H. L., Juszczak, E., et al. (2009). Moderate hypothermia to treat perinatal asphyxial encephalopathy. N. Engl. J. Med. 361, 1349-1358. doi: 10.1056/NEJMoa0900854

Baburamani, A. A., Ek, C. J., Walker, D. W., and Castillo-Melendez, M. (2012). Vulnerability of the developing brain to hypoxic-ischemic damage: contribution of the cerebral vasculature to injury and repair? Front. Physiol. 3:424. doi: 10.3389/fphys.2012.00424

Badaut, J., Ashwal, S., Tone, B., Regli, L., Tian, H. R., and Obenaus, A. (2007). Temporal and regional evolution of aquaporin-4 expression and magnetic resonance imaging in a rat pup model of neonatal stroke. Pediatr. Res. 62, 248-254. doi: 10.1203/PDR.0b013e3180db291b

Ballabh, P. (2010). Intraventricular hemorrhage in premature infants: mechanism of disease. Pediatr. Res. 67, 1-8. doi: 10.1203/PDR.0b013e3181c1b176

Bednarek, N., Svedin, P., Garnotel, R., Favrais, G., Loron, G., Schwendiman, L. et al. (2012). Increased MMP-9 and TIMP-1 in mouse neonatal brain and plasma and in human neonatal plasma after hypoxia-ischemia: a potential marker of neonatal encephalopathy. Pediatr. Res. 71, 63-70. doi: 10.1038/pr. 2011.3

Benjelloun, N., Renolleau, S., Represa, A., Ben-Ari, Y., and Charriaut-Marlangue, C. (1999). Inflammatory responses in the cerebral cortex after ischemia in the P7 neonatal Rat. Stroke 30, 1916-1923. discussion 1923-4.

Biran, V., Cochois, V., Karroubi, A., Arrang, J. M., Charriaut-Marlangue, C., and Heron, A. (2008). Stroke induces histamine accumulation and mast cell degranulation in the neonatal rat brain. Brain Pathol. 18, 1-9. doi: 10.1111/j.17503639.2007.00092.x

Blomgren, K., Leist, M., and Groc, L. (2007). Pathological apoptosis in the developing brain. Apoptosis 12, 993-1010. doi: 10.1007/s10495-007-0754-4

Bona, E., Andersson, A. L., Blomgren, K., Gilland, E., Puka-Sundvall, M., Gustafson, K., et al. (1999). Chemokine and inflammatory cell response to hypoxia-ischemia in immature rats. Pediatr. Res. 45, 500-509. doi: 10.1203/00006450-199904010-00008

Borlongan, C. V., Skinner, S. J., Geaney, M., Vasconcellos, A. V., Elliott, R. B., and Emerich, D. F. (2004). Intracerebral transplantation of porcine choroid plexus provides structural and functional neuroprotection in a rodent model of stroke. Stroke 35, 2206-2210. doi: 10.1161/01.STR.0000138954.25825.0b

Braun, A., Xu, H., Hu, F., Kocherlakota, P., Siegel, D., Chander, P., et al. (2007). Paucity of pericytes in germinal matrix vasculature of premature infants. J. Neurosci. 27, 12012-12024. doi: 10.1523/JNEUROSCI.3281-07.2007

Catania, A., and Lipton, J. M. (1998). Peptide modulation of fever and inflammation within the brain. Ann. N.Y. Acad. Sci. 856, 62-68. doi: 10.1111/j.17496632.1998.tb08313.x

Chen, W., Hartman, R., Ayer, R., Marcantonio, S., Kamper, J., Tang, J., et al. (2009). Matrix metalloproteinases inhibition provides neuroprotection against hypoxia-ischemia in the developing brain. J. Neurochem. 111, 726-736. doi: 10.1111/j.1471-4159.2009.06362.x

Chu, H. X., Kim, H. A., Lee, S., Moore, J. P., Chan, C. T., Vinh, A., et al. (2014). Immune cell infiltration in malignant middle cerebral artery infarction: comparison with transient cerebral ischemia. J. Cereb. Blood Flow Metab. 34, 450-459. doi: 10.1038/jcbfm.2013.217

Cunningham, L. A., Wetzel, M., and Rosenberg, G. A. (2005). Multiple roles for MMPs and TIMPs in cerebral ischemia. Glia 50, 329-339. doi: 10.1002/glia.20169

D’Angelo, B. D., Baburamani, A., Hagberg, H., and Mallard, C. (2012). "Role of blood-brain barrier in perinatal brain injury," in Abstract Presented at the SfN in a Nanosymposium, New Orleans, LA, USA. Abstract 831.0399. Available online at: http://am2012.sfn.org/am2012/pdf/ final_program/final_program_b6.pdf

Daneman, R., Zhou, L., Kebede, A. A., and Barres, B. A. (2010). Pericytes are required for blood-brain barrier integrity during embryogenesis. Nature 468, 562-566. doi: 10.1038/nature09513

Davalos, D., Ryu, J. K., Merlini, M., Baeten, K. M., Le Moan, N., Petersen, M. A., et al. (2012). Fibrinogen-induced perivascular microglial clustering is required for the development of axonal damage in neuroinflammation. Nat. Commun. 3, 1227. doi: $10.1038 /$ ncomms 2230 
Dejana, E., and Giampietro, C. (2012). Vascular endothelial-cadherin and vascular stability. Curr. Opin. Hematol. 19, 218-223. doi: 10.1097/MOH.0b013e3283523e1c

Denes, A., Thornton, P., Rothwell, N. J., and Allan, S. M. (2010). Inflammation and brain injury: acute cerebral ischaemia, peripheral and central inflammation. Brain Behav. Immun. 24, 708-723. doi: 10.1016/j.bbi.2009.09.010

Denker, S., Ji, S., Lee, S. Y., Dingman, A., Derugin, N., Wendland, M., et al. (2007). Macrophages are comprised of resident brain microglia not infiltrating peripheral monocytes acutely after neonatal stroke. J. Neurochem. 100, 893-904. doi: $10.1111 / j .1471-4159.2006 .04162 . x$

Derrick, M., Drobyshevsky, A., Ji, X., and Tan, S. (2007). A model of cerebral palsy from fetal hypoxia-ischemia. Stroke 38, 731-735. doi: 10.1161/01.STR.0000251445.94697.64

Derugin, N., Ferriero, D. M., and Vexler, Z. S. (1998). Neonatal reversible focal cerebral ischemia: a new model. Neurosci. Res. 32, 349-353. doi: 10.1016/S01680102(98)00096-0

Doverhag, C., Hedtjarn, M., Poirier, F., Mallard, C., Hagberg, H., Karlsson, A., et al. (2010). Galectin-3 contributes to neonatal hypoxic-ischemic brain injury. Neurobiol. Dis. 38, 36-46. doi: 10.1016/j.nbd.2009.12.024

Doverhag, C., Keller, M., Karlsson, A., Hedtjarn, M., Nilsson, U., Kapeller, E., et al. (2008). Pharmacological and genetic inhibition of NADPH oxidase does not reduce brain damage in different models of perinatal brain injury in newborn mice. Neurobiol. Dis. 31, 133-144. doi: 10.1016/j.nbd.2008.04.003

Dziegielewska, K. M., Ek, J., Habgood, M. D., and Saunders, N. R. (2001). Development of the choroid plexus. Microsc. Res. Tech. 52, 5-20. doi: 10.1002/1097-0029(20010101)52:1<5::AID-JEMT3>3.0.CO;2-J

Dzietko, M., Derugin, N., Wendland, M. F., Vexler, Z. S., and Ferriero, D. M. (2013). Delayed VEGF treatment enhances angiogenesis and recovery after neonatal focal rodent stroke. Transl. Stroke Res. 4, 189-200. doi: 10.1007/s12975-0120221-6

Edwards, A. D., Brocklehurst, P., Gunn, A. J., Halliday, H., Juszczak, E., Levene, M., et al. (2010). Neurological outcomes at 18 months of age after moderate hypothermia for perinatal hypoxic ischaemic encephalopathy: synthesis and meta-analysis of trial data. BMJ 340, c363. doi: 10.1136/bmj.c363

Eichler, A. F., Chung, E., Kodack, D. P., Loeffler, J. S., Fukumura, D., and Jain, R. K. (2011). The biology of brain metastases-translation to new therapies. Nat. Rev. Clin. Oncol. 8, 344-356. doi: 10.1038/nrclinonc.2011.58

El-Khoury, N., Braun, A., Hu, F., Pandey, M., Nedergaard, M., Lagamma, E. F., et al. (2006). Astrocyte end-feet in germinal matrix, cerebral cortex, and white matter in developing infants. Pediatr. Res. 59, 673-679. doi: 10.1203/01.pdr.0000214975.85311.9c

Engelhardt, B., and Liebner, S. (2014). Novel insights into the development and maintenance of the blood-brain barrier. Cell Tissue Res. 355, 687-699. doi: 10.1007/s00441-014-1811-2

Faustino, J., Wang, X., Jonhson, C., Klibanov, A., Derugin, N., Wendland, M., et al. (2011). Microglial cells contribute to endogenous brain defenses after acute neonatal focal stroke. J. Neurosci. 31, 12992-13001. doi: 10.1523/JNEUROSCI.2102-11.2011

Fernandez-Lopez, D., Faustino, J., Daneman, R., Zhou, L., Lee, S. Y., Derugin, N., et al. (2012). Blood-brain barrier permeability is increased after acute adult stroke but not neonatal stroke. J. Neurosci. 32, 9588-9600. doi: 10.1523/JNEUROSCI.5977-11.2012

Fernandez-Lopez, D., Faustino, J., Derugin, N., and Vexler, Z. S. (2013). Acute and chronic vascular responses to experimental focal arterial stroke in the neonate rat. Transl. Stroke Res. 4, 179-188. doi: 10.1007/s12975-012-0214-5

Fernandez Lopez, D., Faustino, J., Klibanov, A., Derugin, N., Akassoglou, K., and Vexler, Z. S., (2014). "Activated microglia contribute to neurovascular integrity and limit brain injury after acute neonatal focal stroke," in International Stroke Meeting (San Diego, CA).

Fernandez-Lopez, D., Natarajan, N., Ashwal, S., and Vexler, Z. S. (2014). Mechanisms of perinatal arterial ischemic stroke. J. Cereb. Blood Flow Metab. 34, 921-932. doi: $10.1038 / \mathrm{jcbfm} .2014 .41$

Ferriero, D. M. (2004). Neonatal brain injury. N. Engl. J. Med. 351, 1985-1995. doi: 10.1056/NEJMra041996

Freeman, L. R., and Keller, J. N. (2012). Oxidative stress and cerebral endothelial cells: regulation of the blood-brain-barrier and antioxidant based interventions. Biochim. Biophys. Acta 1822, 822-829. doi: 10.1016/j.bbadis.2011.12.009

Fukuda, S., Fini, C. A., Mabuchi, T., Koziol, J. A., Eggleston, L. L. Jr., and Del Zoppo, G. J. (2004). Focal cerebral ischemia induces active proteases that degrade microvascular matrix. Stroke 35, 998-1004. doi: 10.1161/01.STR.0000119383.76447.05

Gidday, J. M., Gasche, Y. G., Copin, J. C., Shah, A. R., Perez, R. S., Shapiro, S. D., et al. (2005). Leukocyte-derived matrix metalloproteinase- 9 mediates blood-brain barrier breakdown and is proinflammatory after transient focal cerebral ischemia. Am. J. Physiol. Heart Circ. Physiol. 289, H558-H568. doi: 10.1152/ajpheart.01275.2004

Gliem, M., Mausberg, A. K., Lee, J. I., Simiantonakis, I., Van Rooijen, N., Hartung, H. P., et al. (2012). Macrophages prevent hemorrhagic infarct transformation in murine stroke models. Ann. Neurol. 71, 743-752. doi: 10.1002/ ana.23529

Goetz, J. G., Joshi, B., Lajoie, P., Strugnell, S. S., Scudamore, T., Kojic, L. D., et al. (2008). Concerted regulation of focal adhesion dynamics by galectin3 and tyrosine-phosphorylated caveolin-1. J. Cell Biol. 180, 1261-1275. doi: $10.1083 /$ icb. 200709019

Gonzalez, F. F., Larpthaveesarp, A., McQuillen, P., Derugin, N., Wendland, M., Spadafora, R., et al. (2013). Erythropoietin increases neurogenesis and oligodendrogliosis of subventricular zone precursor cells after neonatal stroke. Stroke 44, 753-758. doi: 10.1161/STROKEAHA.111.000104

Gould, D. B., Phalan, F. C., Breedveld, G. J., Van Mil, S. E., Smith, R. S., Schimenti, J. C., et al. (2005). Mutations in Col4al cause perinatal cerebral hemorrhage and porencephaly. Science 308, 1167-1171. doi: 10.1126/science.1109418

Hedtjarn, M., Mallard, C., Iwakura, Y., and Hagberg, H. (2005). Combined deficiency of IL-1beta18, but not IL-1alphabeta, reduces susceptibility to hypoxia-ischemia in the immature brain. Dev. Neurosci. 27, 143-148. doi: $10.1159 / 000085986$

Hudome, S., Palmer, C., Roberts, R. L., Mauger, D., Housman, C., and Towfighi, J. (1997). The role of neutrophils in the production of hypoxic-ischemic brain injury in the neonatal rat. Pediatr. Res. 41, 607-616. doi: 10.1203/00006450199705000-00002

Iadecola, C., and Anrather, J. (2011). The immunology of stroke: from mechanisms to translation. Nat. Med. 17, 796-808. doi: 10.1038/nm.2399

Ikonomidou, C., Mosinger, J. L., Salles, K. S., Labruyere, J., and Olney, J. W. (1989). Sensitivity of the developing rat brain to hypobaric/ischemic damage parallels sensitivity to N-methyl-aspartate neurotoxicity. J. Neurosci. 9, 2809-2818.

Iwai, M., Stetler, R. A., Xing, J., Hu, X., Gao, Y., Zhang, W., et al. (2010). Enhanced oligodendrogenesis and recovery of neurological function by erythropoietin after neonatal hypoxic/ischemic brain injury. Stroke 41, 1032-1037. doi: 10.1161/STROKEAHA.109.570325

Jin, Y., Silverman, A. J., and Vannucci, S. J. (2007). Mast cell stabilization limits hypoxic-ischemic brain damage in the immature rat. Dev. Neurosci. 29, 373-384. doi: 10.1159/000105478

Knowland, D., Arac, A., Sekiguchi, K. J., Hsu, M., Lutz, S. E., Perrino, J., et al. (2014). Stepwise recruitment of transcellular and paracellular pathways underlies blood-brain barrier breakdown in stroke. Neuron 82, 603-617. doi: 10.1016/j.neuron.2014.03.003

Kochanek, P. M., and Hallenbeck, J. M. (1992). Polymorphonuclear leukocytes and monocytes/macrophages in the pathogenesis of cerebral ischemia and stroke. Stroke 23, 1367-1379. doi: 10.1161/01.STR.23.9.1367

Kunis, G., Baruch, K., Rosenzweig, N., Kertser, A., Miller, O., Berkutzki, T., et al. (2013). IFN-gamma-dependent activation of the brain's choroid plexus for CNS immune surveillance and repair. Brain 136, 3427-3440. doi: 10.1093/brain/awt259

Labelle-Dumais, C., Dilworth, D. J., Harrington, E. P., De Leau, M., Lyons, D., Kabaeva, Z., et al. (2011). COL4A1 mutations cause ocular dysgenesis, neuronal localization defects, and myopathy in mice and Walker-Warburg syndrome in humans. PLoS Genet. 7, e1002062. doi: 10.1371/journal.pgen. 1002062

Lalancette-Hebert, M., Swarup, V., Beaulieu, J. M., Bohacek, I., Abdelhamid, E., Weng, Y. C., et al. (2012). Galectin-3 is required for resident microglia activation and proliferation in response to ischemic injury. J. Neurosci. 32, 10383-10395. doi: 10.1523/JNEUROSCI.1498-12.2012

Levison, S. W., and Goldman, J. E. (1997). Multipotential and lineage restricted precursors coexist in the mammalian perinatal subventricular zone. J. Neurosci. Res. 48, 83-94.

Liu, J., Jin, X., Liu, K. J., and Liu, W. (2012). Matrix metalloproteinase-2-mediated occludin degradation and caveolin-1-mediated claudin-5 redistribution contribute to blood-brain barrier damage in early ischemic stroke stage. J. Neurosci. 32, 3044-3057. doi: 10.1523/JNEUROSCI.6409-11.2012 
Loreto, C., and Reggio, E. (2010). Aquaporin and vascular diseases. Curr. Neuropharmacol. 8, 105-111. doi: 10.2174/157015910791233196

Luo, X. G., and Chen, S. D. (2012). The changing phenotype of microglia from homeostasis to disease. Transl. Neurodegener. 1, 9. doi: 10.1186/2047-9158-1-9

Marks, K. A., Mallard, C. E., Roberts, I., Williams, C. E., Gluckman, P. D., and Edwards, A. D. (1999). Nitric oxide synthase inhibition and delayed cerebral injury after severe cerebral ischemia in fetal sheep. Pediatr. Res. 46, 8-13. doi: 10.1203/00006450-199907000-00002

McColl, B. W., Rothwell, N. J., and Allan, S. M. (2008). Systemic inflammation alters the kinetics of cerebrovascular tight junction disruption after experimental stroke in mice. J. Neurosci. 28, 9451-9462. doi: 10.1523/JNEUROSCI.267408.2008

McQuillen, P. S., Sheldon, R. A., Shatz, C. J., and Ferriero, D. M. (2003). Selective vulnerability of subplate neurons after early neonatal hypoxiaischemia. J. Neurosci. 23, 3308-3315.

Mori, E., Del Zoppo, G. J., Chambers, J. D., Copeland, B. R., and Arfors, K. E. (1992). Inhibition of polymorphonuclear leukocyte adherence suppresses no-reflow after focal cerebral ischemia in baboons. Stroke 23, 712-718. doi: 10.1161/01.STR.23.5.712

Mu, D., Jiang, X., Sheldon, R. A., Fox, C. K., Hamrick, S. E., Vexler, Z. S., et al. (2003). Regulation of hypoxia-inducible factor lalpha and induction of vascular endothelial growth factor in a rat neonatal stroke model. Neurobiol. Dis. 14, 524-534. doi: 10.1016/j.nbd.2003.08.020

Nelson, K. B., and Lynch, J. K. (2004). Stroke in newborn infants. Lancet Neurol. 3 , 150-158. doi: 10.1016/S1474-4422(04)00679-9

Nimmerjahn, A., Kirchhoff, F., and Helmchen, F. (2005). Resting microglial cells are highly dynamic surveillants of brain parenchyma in vivo. Science 308, 1314-1318. doi: 10.1126/science.1110647

Northington, F. J., Chavez-Valdez, R., and Martin, L. J. (2011). Neuronal cell death in neonatal hypoxia-ischemia. Ann. Neurol. 69, 743-758. doi: 10.1002/ana.22419

Ohab, J. J., Fleming, S., Blesch, A., and Carmichael, S. T. (2006). A neurovascular niche for neurogenesis after stroke. J. Neurosci. 26, 13007-13016. doi: 10.1523/JNEUROSCI.4323-06.2006

Omouendze, P. L., Henry, V. J., Porte, B., Dupre, N., Carmeliet, P., Gonzalez, B. J., et al. (2013). Hypoxia-ischemia or excitotoxin-induced tissue plasminogen activator- dependent gelatinase activation in mice neonate brain microvessels. PLoS ONE 8:e71263. doi: 10.1371/journal.pone.0071263

Parent, J. M., Vexler, Z. S., Gong, C., Derugin, N., and Ferriero, D. M. (2002). Rat forebrain neurogenesis and striatal neuron replacement after focal stroke. Ann. Neurol. 52, 802-813. doi: 10.1002/ana.10393

Peppiatt, C. M., Howarth, C., Mobbs, P., and Attwell, D. (2006). Bidirectional control of CNS capillary diameter by pericytes. Nature 443, 700-704. doi: 10.1038/nature05193

Petty, M. A., and Lo, E. H. (2002). Junctional complexes of the blood-brain barrier: permeability changes in neuroinflammation. Prog. Neurobiol. 68, 311-323. doi: 10.1016/S0301-0082(02)00128-4

Plane, J. M., Liu, R., Wang, T. W., Silverstein, F. S., and Parent, J. M. (2004). Neonatal hypoxic-ischemic injury increases forebrain subventricular zone neurogenesis in the mouse. Neurobiol. Dis. 16, 585-595. doi: 10.1016/j.nbd.2004.04.003

Renolleau, S., Aggoun-Zouaoui, D., Ben-Ari, Y., and Charriaut-Marlangue, C. (1998). A model of transient unilateral focal ischemia with reperfusion in the P7 neonatal rat: morphological changes indicative of apoptosis. Stroke 29, 1454-1460. discussion: 1461. doi: 10.1161/01.STR.29.7.1454

Rice, J. E. D., Vannucci, R. C., and Brierley, J. B. (1981). The influence of immaturity on hypoxic-ischemic brain damage in the rat. Ann. Neurol. 9, 131-141. doi: 10.1002/ana.410090206

Rizzo, M. T., and Leaver, H. A. (2010). Brain endothelial cell death: modes, signaling pathways, and relevance to neural development, homeostasis, and disease. Mol. Neurobiol. 42, 52-63. doi: 10.1007/s12035-010-8132-6

Rosenberg, G. A., Estrada, E. Y., and Dencoff, J. E. (1998). Matrix metalloproteinases and TIMPs are associated with blood-brain barrier opening after reperfusion in rat brain. Stroke 29, 2189-2195. doi: 10.1161/01.STR.29.10.2189

Rothstein, R. P., and Levison, S. W. (2005). Gray matter oligodendrocyte progenitors and neurons die caspase- 3 mediated deaths subsequent to mild perinatal hypoxic/ischemic insults. Dev. Neurosci. 27, 149-159. doi: 10.1159/000085987

Shechter, R., Miller, O., Yovel, G., Rosenzweig, N., London, A., Ruckh, J., et al. (2013). Recruitment of beneficial M2 macrophages to injured spinal cord is orchestrated by remote brain choroid plexus. Immunity 38, 555-569. doi: 10.1016/j.immuni.2013.02.012

Sheldon, R. A., Jiang, X., Francisco, C., Christen, S., Vexler, Z. S., Tauber, M. G., et al. (2004). Manipulation of antioxidant pathways in neonatal murine brain. Pediatr. Res. 56, 656-662. doi: 10.1203/01.PDR.0000139413.27864.50

Shimotake, J., Derugin, N., Wendland, M., Vexler, Z. S., and Ferriero, D. M. (2010). Vascular endothelial growth factor receptor-2 inhibition promotes cell death and limits endothelial cell proliferation in a neonatal rodent model of stroke. Stroke 41, 343-349. doi: 10.1161/STROKEAHA.109.564229

Spadafora, R., Gonzalez, F. F., Derugin, N., Wendland, M., Ferriero, D., and McQuillen, P. (2010). Altered fate of subventricular zone progenitor cells and reduced neurogenesis following neonatal stroke. Dev. Neurosci. 32, 101-113. doi: $10.1159 / 000279654$

Strbian, D., Karjalainen-Lindsberg, M. L., Kovanen, P. T., Tatlisumak, T., and Lindsberg, P. J. (2007). Mast cell stabilization reduces hemorrhage formation and mortality after administration of thrombolytics in experimental ischemic stroke. Circulation 116, 411-418. doi: 10.1161/CIRCULATIONAHA.106.655423 Strbian, D., Karjalainen-Lindsberg, M. L., Tatlisumak, T., and Lindsberg, P. J. (2006). Cerebral mast cells regulate early ischemic brain swelling and neutrophil accumulation. J. Cereb. Blood Flow Metab. 26, 605-612. doi: $10.1038 /$ s.j.jbfm. 9600228

Szmydynger-Chodobska, J., Strazielle, N., Gandy, J. R., Keefe, T. H., Zink, B. J., Ghersi-Egea, J. F., et al. (2012). Posttraumatic invasion of monocytes across the blood-cerebrospinal fluid barrier. J. Cereb. Blood Flow Metab. 32, 93-104. doi: $10.1038 / \mathrm{jcbfm} .2011 .111$

Szmydynger-Chodobska, J., Strazielle, N., Zink, B. J., Ghersi-Egea, J. F., and Chodobski, A. (2009). The role of the choroid plexus in neutrophil invasion after traumatic brain injury. J. Cereb. Blood Flow Metab. 29, 1503-1516. doi: $10.1038 / j \mathrm{jbfm} .2009 .71$

Van Den Broeck, C., Himpens, E., Vanhaesebrouck, P., Calders, P., and Oostra, A. (2008). Influence of gestational age on the type of brain injury and neuromotor outcome in high-risk neonates. Eur. J. Pediatr. 167, 1005-1009. doi: 10.1007/s00431-007-0629-2

Van Velthoven, C. T., Kavelaars, A., Van Bel, F., and Heijnen, C. J. (2010). Mesenchymal stem cell treatment after neonatal hypoxic-ischemic brain injury improves behavioral outcome and induces neuronal and oligodendrocyte regeneration. Brain Behav. Immun. 24, 387-393. doi: 10.1016/j.bbi.2009.10.017

Van Velthoven, C. T., Sheldon, R. A., Kavelaars, A., Derugin, N., Vexler, Z. S., Willemen, H. L., et al. (2013). Mesenchymal stem cell transplantation attenuates brain injury after neonatal stroke. Stroke 44, 1426-1432. doi: 10.1161/STROKEAHA.111.000326

Vexler, Z. S., and Yenari, M. A. (2009). Does inflammation after stroke affect the developing brain differently than adult brain? Dev. Neurosci. 31, 378-393. doi: $10.1159 / 000232556$

Vexler, Z. S., Tang, X. N., and Yenari, M. A. (2006). Inflammation in adult and neonatal stroke. Clin. Neurosci. Res. 6, 293-313. doi: 10.1016/j.cnr.2006.09.008

Volpe, J. J. (2009). The encephalopathy of prematurity-brain injury and impaired brain development inextricably intertwined. Semin. Pediatr. Neurol. 16, 167-178. doi: 10.1016/j.spen.2009.09.005

Wacker, B. K., Freie, A. B., Perfater, J. L., and Gidday, J. M. (2012). Junctional protein regulation by sphingosine kinase 2 contributes to blood-brain barrier protection in hypoxic preconditioning-induced cerebral ischemic tolerance. J. Cereb. Blood Flow Metab. 32, 1014-1023. doi: 10.1038/jcbfm.2012.3

Woo, M. S., Wang, X., Faustino, J., Derugin, N., Wendland, M. F., Zhou, P., et al. (2012). Genetic deletion of CD36 enhances injury after acute neonatal stroke. Ann. Neurol. 72, 961-970. doi: 10.1002/ana.23727

Yager, J. Y., and Ashwal, S. (2009). Animal models of perinatal hypoxic-ischemic brain damage. Pediatr. Neurol. 40, 156-167. doi: 10.1016/j.pediatrneurol.2008. 10.025

Yamasaki, Y., Matsuo, Y., Matsuura, N., Onodera, H., Itoyama, Y., and Kogure, K. (1995). Transient increase of cytokine-induced neutrophil chemoattractant, a member of the interleukin-8 family, in ischemic brain areas after focal ischemia in rats. Stroke 26, 318-22; discussion 322-3. doi: 10.1161/01.STR.26.2.318

Yamasaki, Y., Matsuo, Y., Zagorski, J., Matsuura, N., Onodera, H., Itoyama, Y., et al. (1997). New therapeutic possibility of blocking cytokine-induced neutrophil chemoattractant on transient ischemic brain damage in rats. Brain Res. 759, 103-111. doi: 10.1016/S0006-8993(97)00251-5

Yang, Y., Estrada, E. Y., Thompson, J. F., Liu, W., and Rosenberg, G. A. (2007a). Matrix metalloproteinase-mediated disruption of tight junction proteins in 
cerebral vessels is reversed by synthetic matrix metalloproteinase inhibitor in focal ischemia in rat. J. Cereb. Blood Flow Metab. 27, 697-709. doi: 10.1038/sj.jcbfm.9600375

Yang, Z., Covey, M. V., Bitel, C. L., Ni, L., Jonakait, G. M., and Levison, S. W. (2007b). Sustained neocortical neurogenesis after neonatal hypoxic/ischemic injury. Ann. Neurol. 61, 199-208. doi: 10.1002/ana.21068

Yemisci, M., Gursoy-Ozdemir, Y., Vural, A., Can, A., Topalkara, K., and Dalkara, T. (2009). Pericyte contraction induced by oxidative-nitrative stress impairs capillary reflow despite successful opening of an occluded cerebral artery. Nat. Med. 15, 1031-1037. doi: 10.1038/nm.2022

Young, C. C., Brooks, K. J., Buchan, A. M., and Szele, F. G. (2011). Cellular and molecular determinants of stroke-induced changes in subventricular zone cell migration. Antioxid. Redox Signal. 14, 1877-1888. doi: 10.1089/ars.2010.3435

Zhao, B. Q., Wang, S., Kim, H. Y., Storrie, H., Rosen, B. R., Mooney, D. J., et al. (2006). Role of matrix metalloproteinases in delayed cortical responses after stroke. Nat. Med. 12, 441-445. doi: 10.1038/nm1387
Conflict of Interest Statement: The authors declare that the research was conducted in the absence of any commercial or financial relationships that could be construed as a potential conflict of interest.

Received: 26 August 2014; accepted: 20 October 2014; published online: 07 November 2014.

Citation: Kratzer I, Chip S and Vexler ZS (2014) Barrier mechanisms in neonatal stroke. Front. Neurosci. 8:359. doi: 10.3389/fnins.2014.00359

This article was submitted to Neurogenomics, a section of the journal Frontiers in Neuroscience.

Copyright (c) 2014 Kratzer, Chip and Vexler. This is an open-access article distributed under the terms of the Creative Commons Attribution License (CC BY). The use, distribution or reproduction in other forums is permitted, provided the original author(s) or licensor are credited and that the original publication in this journal is cited, in accordance with accepted academic practice. No use, distribution or reproduction is permitted which does not comply with these terms. 


\section{Blood-brain barrier dysfunction in disorders of the developing brain}

\section{Raffaella Moretti ${ }^{1,2,3,4}$, Julien Pansiot ${ }^{1,2,3}$, Donatella Bettati ${ }^{1,2,3}$, Nathalie Strazielle ${ }^{5,6}$, Jean-François Ghersi-Egea ${ }^{5}$, Giuseppe Damante ${ }^{4}$, Bobbi Fleiss ${ }^{1,2,3,7}$, Luigi Titomanlio ${ }^{1,2,3,8}$ and Pierre Gressens ${ }^{1,2,3,7 *}$}

1 INSERM U1141, Robert Debre's Hospital, Paris, France

2 Université Paris Diderot, Sorbonne Paris Cité, UMRS 1141-PROTECT, Paris, France

${ }^{3}$ PremUP, Paris, France

${ }^{4}$ S. Maria della Misericordia Hospital, Università degli Studi di Udine, Udine, Italy

${ }^{5}$ Lyon Neurosciences Research Center, INSERM U1028, CNRS UMR5292 - Lyon University, Lyon, France

${ }^{6}$ Brain-i, Lyon, France

7 Department of Division of Imaging Sciences and Biomedical Engineering, Centre for the Developing Brain, St. Thomas' Hospital, London, UK

8 Pediatric Emergency Department, APHP, Robert Debré Hospital, Paris, France

\section{Edited by:}

Joana A. Palha, University of Minho, Portugal

\section{Reviewed by:}

Jun Zhang, Texas Tech University Health Sciences Center, USA

Kosman Daniel, State Univesity of New York at Buffalo, USA

Pia Johansson, Helmholtz-Zentrum München, Germany

*Correspondence:

Pierre Gressens, INSERM U-1141, Robert Debré University Hospital, 48, Bld Sérurier - 75019 Paris, France e-mail: pierre.gressens@inserm.fr
Disorders of the developing brain represent a major health problem. The neurological manifestations of brain lesions can range from severe clinical deficits to more subtle neurological signs or behavioral problems and learning disabilities, which often become evident many years after the initial damage. These long-term sequelae are due at least in part to central nervous system immaturity at the time of the insult. The blood-brain barrier (BBB) protects the brain and maintains homeostasis. BBB alterations are observed during both acute and chronic brain insults. After an insult, excitatory amino acid neurotransmitters are released, causing reactive oxygen species (ROS)-dependent changes in BBB permeability that allow immune cells to enter and stimulate an inflammatory response. The cytokines, chemokines and other molecules released as well as peripheral and local immune cells can activate an inflammatory cascade in the brain, leading to secondary neurodegeneration that can continue for months or even years and finally contribute to post-insult neuronal deficits. The role of the BBB in perinatal disorders is poorly understood. The inflammatory response, which can be either acute (e.g., perinatal stroke, traumatic brain injury) or chronic (e.g., perinatal infectious diseases) actively modulates the pathophysiological processes underlying brain injury. We present an overview of current knowledge about BBB dysfunction in the developing brain during acute and chronic insults, along with clinical and experimental data.

Keywords: WMD, TBI, BBB, stroke, hypoxia-ischemia, brain, developemental desorders

\section{INTRODUCTION}

The blood-brain barrier (BBB) is a physical barrier essential for the maintenance of a precisely regulated intracerebral microenvironment. Its characteristics limit paracellular diffusion while allowing the tightly controlled receptor-mediated endocytosis of larger molecules and the transporter-mediated intake of smaller nutrients like glucose, insulin and iron. Endothelial cells interact closely with other central nervous system (CNS) cells such as neurons, pericytes and astrocytes, through adherens junctions,

\footnotetext{
Abbreviations: $\mathrm{BBB}$, blood-brain barrier; CAV, caveolin; Cld5, claudin-5; CNS, central nervous system; GM-IVH, Intraventricular hemorrhage of the germinal matrix; HIE, hypoxic-ischemic encephalopathy; HIF, hypoxia-inducible factor; IL, interleukin; LPS, lipopolysaccharide; MMP, matrix metalloproteinase; NO, nitric oxide; NOS, nitric oxide synthase; NVU, neurovascular unit; Occl, occluding; P, postnatal day; ROS, reactive oxygen species; TBI, traumatic brain injury; TIMP, tissue inhibitor of metalloproteinases; TJ, tight junction; TNF, tumor necrosis factor; VEGF, vascular endothelial growth factor; WMD, white-matter damage; ZO, zonula occludens.
}

influx and efflux transporters, metabolic enzymes, the extracellular matrix, astrocytic endfeet etc. All these structures together constitute the Neurovascular Unit (NVU), and are believed to be essential for the regulation of BBB permeability (Ballabh et al., 2004).

For a long time, the BBB was considered "incomplete" during fetal life. However, there is ample evidence to suggest that the BBB is already well developed (Ballabh et al., 2005). For instance, the formation of tight junctions (TJs) takes place at the same time as angiogenesis (Virgintino et al., 2004), and even the first vessels to invade the avascular neuroectoderm are impermeable to albumin and immunoglobulins. Nevertheless, fetal brain vessels exhibit different, and in some respects increased, transport properties for amino acids and other metabolites, reflecting the higher demand for nutrients in the developing CNS (Ek et al., 2001, 2006; Johansson et al., 2006, 2008; Saunders et al., 2012). Understanding the changes that occur in the BBB during normal development is critical, as it is not only a site of possible 
vulnerability to injury but also a potential therapeutic route for injuries occurring during the perinatal period. Perinatal brain damage is one of the leading causes of lifelong disability, including cerebral palsy, seizure disorders, sensory impairment, and cognitive limitations (Low, 2004). This brain damage could be due to inflammatory, hypoxic-ischemic, hemorrhagic or excitotoxic mechanisms, or a combination of these. The lifetime cost of the care of one child affected by cerebral palsy is about 1 million US dollars (CDC CfDCaP, 2004), highlighting the need to develop new strategies for treatment and prevention. The only treatment with proven efficacy for perinatal brain injury is hypothermia, but this can only be applied safely to term infants with hypoxic-ischemic encephalopathy. Hypothermia in these infants reduces brain injury, doubling the chances of survival without clinical deficits (Edwards et al., 2010). However, more efforts are needed to find novel neuroprotective treatments for perinatal brain damage, especially in preterm infants. Designing neuroprotective molecules requires a detailed knowledge of the pathophysiology of brain damage, including BBB damage, in this population. This will facilitate the delivery of neuroprotective molecules, whose efficacy is strictly dependent on their capacity to cross brain barriers.

Currently, translational research is increasingly focused on the broader functional aspects of the brain response to injury, shifting from cell-oriented studies to experimental research on physiological concepts such as BBB integrity. This is due to the fact that dysfunctions of the $\mathrm{BBB}$, such as the impairment of TJ formation/function, are a contributing factor in a number of neurological diseases in adults and infants. In models of perinatal injuries in term infants, cerebrovascular endothelial cells can be damaged by hypoxic-ischemic insults, an activated excitotoxic cascade or traumatic brain injury, leading to impaired BBB function. The link between BBB dysfunction and injury due to the dysregulation of developmental processes, such as occurs to a greater extent in preterm infants, is less well studied.

The aim of this review is to describe current understanding of BBB dysfunction in the developing brain during the most common subtypes of injury, supported by clinical and experimental data.

\section{BASICS OF BBB DEVELOPMENT AND AGE-RELATED DIFFERENCES IN FUNCTION}

The specific barrier characteristics of BBB endothelial cells within the developing CNS are induced during angiogenesis by complex crosstalk with cellular and acellular elements. During development, the CNS is vascularized by the angiogenic sprouting of vascular networks from the surrounding mesoderm in a precise spatiotemporal manner that differs among species. Which cell type is responsible for $\mathrm{BBB}$ differentiation has not yet been clarified: astrocytes have long been considered as the main source of BBB-inducing signals, but barrier induction most likely takes place well before astrocyte differentiation, so there is a probable influence of neuroblast cells or pericytes (Bauer and Bauer, 2000; Armulik et al., 2010; Daneman et al., 2010a). These different structures and cells have different maturation rates across species and across developmental stages, so animal models are not always representative of the human situation in every respect.
With regard to the developmental changes in the $\mathrm{BBB}$, the presence of TJs in cerebral blood vessels and the expression of influx and efflux transporters is even higher at mid-gestation than in adulthood; for instance, TJs appear as early as 8 weeks of gestation in humans, at 13 days in mice and at postnatal day (P) 5 in Monodelphis opossums, which is when the first vessels appear in the neocortex (Ek et al., 2012). These TJ proteins are functional, as recent ultrastructural studies have demonstrated that the TJs of both endothelial cells in cerebral blood vessels and choroidal epithelial cells in embryos and neonates restrict the passage of low-molecular-weight molecules (Ek et al., 2003, 2006; Johansson et al., 2006). However, as these blood vessels do not display the properties of mature vessels with respect to pericyte coverage or junctional organization, it is probable that their BBB properties are not yet fully mature. This has been confirmed in part by a recent study showing that several hundreds of genes are differentially expressed between early postnatal and adult brain endothelial cells, indicating differences in molecular and probably physiological properties (Daneman et al., 2010b). However, the implication of these differences in the BBB across developmental stages for brain health remains unknown (Engelhardt and Liebner, 2014).

\section{PRINCIPAL INSULTS TO THE DEVELOPING BRAIN AND THEIR ANIMAL MODELS GERMINAL MATRIX HEMORRHAGE}

Intraventricular hemorrhage of the germinal matrix (GM-IVH) causes very substantial and permanent injury, and is the leading cause of hydrocephalus in children. GM-IVH occurs when a hemorrhage in the GM breaks through the ependyma and into the lateral ventricles (see Figure 1). About 12,000 premature infants develop IVH every year in the United States alone (Vohr et al., 1999; Ballabh, 2010), with the incidence in extremely premature infants being approximately 45\% (Wilson-Costello et al., 2005). Infants with a history of IVH have a higher incidence of seizures, neurodevelopmental delays, cerebral palsy and death. The incidence of IVH in very low birth weight infants $(<1500 \mathrm{~g})$ declined from $40 \%$ in the early 1980 s to $20 \%$ in the late 1980 s (Philip et al., 1989). However, because of the sharply increased survival of extremely premature infants, in the last two decades, the incidence of IVH has remained stable (Jain et al., 2009). Thus, IVH continues to be one of the major problems faced by premature infants in modern neonatal intensive care units worldwide. The etiopathogenesis of GM hemorrhage is multifactorial, with a combination of vascular and intravascular factors considered to be responsible. It is necessary to understand the reason for the vulnerability of GM microvessels to hemorrhage in order to develop therapeutic strategies.

GM-IVH has been modeled in several animal species including the dog, rabbit, sheep, rat, mouse and pig, either by directly injecting blood into the ventricles or by changing hemodynamic properties, such as blood pressure, the volume of blood circulating, serum glycerol, carbon dioxide, osmolarity or oxygenation levels (Goddard et al., 1980; Yoshioka et al., 1989; Balasubramaniam and Del Bigio, 2006; Georgiadis et al., 2008; Tosun et al., 2013). The two most-used animals models of IVH are the intracerebral injection of sterile collagenase, a hemorrhage-inducing agent 

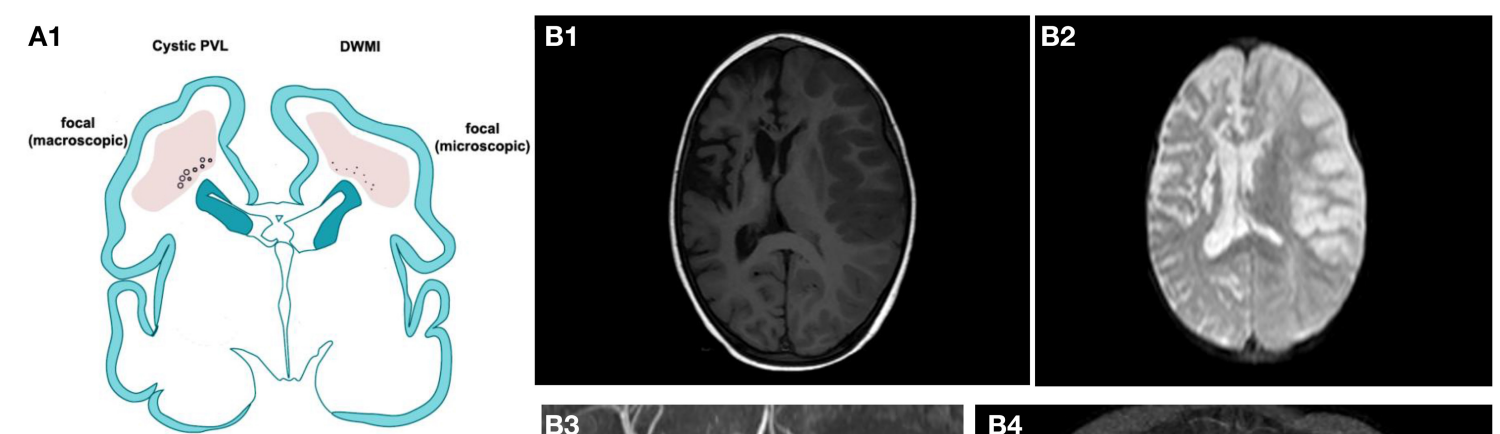

A2
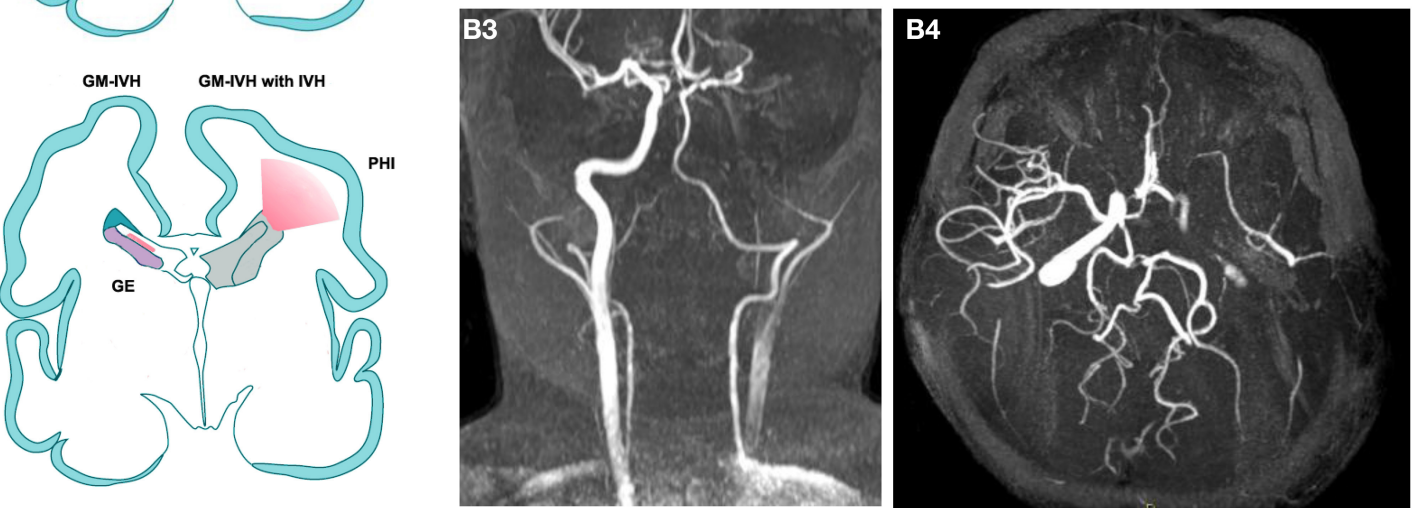

FIGURE 1 | (A) Modified by J. Volpe, Lancet Neurology 2009. (A1) Cystic Periventricular Leucomalacia (PVL) and Diffuse White Matter Injury (DWMI) and (A2) germinal matrix haemorrage-intraventricular Haemorrage (GMH-IVH) and $\mathrm{GMH}-\mathrm{IVH}$ with periventricular Haemorrage infarction (PHI). Schematic coronal sections from the brain of a 28 -week-old premature infant. Color key: Focal necrotic lesions in cystic PVL (small circles), focal necrotic lesions in DWMI (black dots), diffuse cellular changes in both cystic PVL and DWMI (pink), hemorrhage into ganglionic eminence (red) that results in IVH in the ependymal (left) or PHI (right). (B,B1) T1 weighted image illustrating an acute right ischemic stroke is a sickle cell disease children previously affected by a left ischemic stroke. (B2) Brain diffusion-weighted imaging (DWI) sequence of the same patient. (B3,B4) a 3D time-of-flight magnetic resonance angiography (MRA) suggesting dissection of the left internal carotid (B2: coronal view, B3 transverse view).
(Krafft et al., 2012; Lekic et al., 2012), in mice, and the intracerebroventricular injection of fresh homologs blood in adult Sprague-Dawley rats or in piglets at 9-22 h of life (Aquilina et al., 2007, 2012). The appropriate model is selected based on the pathophysiology of hemorrhage induction and injury progression. The blood injection model mimics a rapidly progressing hemorrhage, whereas collagenase enzymatically disrupts the basal lamina of brain capillaries, causing an active bleed that generally evolves over several hours. The effects of GM-IVH on the BBB have not been extensively assessed in any of these models.

\section{WHITE MATTER DAMAGE}

White matter damage (WMD) is a characteristic injury of preterm infants, involving destructive microcystic lesions within the white matter, the disrupted maturation of oligodendrocytes and hypomyelination (Degos et al., 2010a) (see Figure 1). Although WMD is also observed in term infants, the relative contributions of direct injury to oligodendrocytes/myelin and secondary injury due to gray matter damage are still being defined. WMD is a healthcare priority, as it is the main cause of neurological handicap in preterm infants and the principal cause of cerebral palsy (Tahraoui et al., 2001). On a pathophysiological level, based on strong clinical evidence and a wealth of studies in animal models, the hypothesis of a purely hypoxic-ischemic cause of injury has been replaced by a multifactorial hypothesis. The central role of inflammation is now appreciated, given its contribution in terms of excess cytokines, free radicals, increased excitatory amino acid release (excitotoxicity), and trophic factor imbalance (Lipton and Rosenberg, 1994; Evrard et al., 1997; Yoon et al., 1997; Back et al., 1998). Clinical spectroscopy data do support a role for hypoxiaischemia in the pathophysiology of WMD, but it is increasingly evident that inflammation-induced endothelial cell dysfunction and changes in glial metabolism may contribute substantially to these effects (Schuhmann et al., 2003; Lodygensky et al., 2014). The multifactorial hypothesis includes the concept of sensitization, whereby vulnerability to hypoxia-ischemia or excitotoxicity is enhanced by the preexistence of inflammation (Hagberg and Mallard, 2005; Favrais et al., 2007, 2011), which in the clinical setting is reported to occur in at least $40 \%$ of preterm infants.

Experimental paradigms that have addressed the pathophysiology of WMD include models in the mouse, rat and sheep using inflammation, excitotoxicity and hypoxia-ischemia (Tahraoui et al., 2001) (see Table 1). A well-characterized murine model of perinatal excitotoxic-induced WMD consists of the i.c. injection of the glutamate analog ibotenate, a potent neurotoxin that activates the N-methyl-D-aspartate receptor, in 5-day-old mouse pups (Marret et al., 1995). Ibotenate is commonly used to induce brain lesions in both the hippocampus and the white matter, in which it stimulates these receptors present on oligodendrocytes and microglia (Degos et al., 2010b), leading to microcysts similar 
Table 1 | Some of the principal animal models mentioned in this review with correlate references.

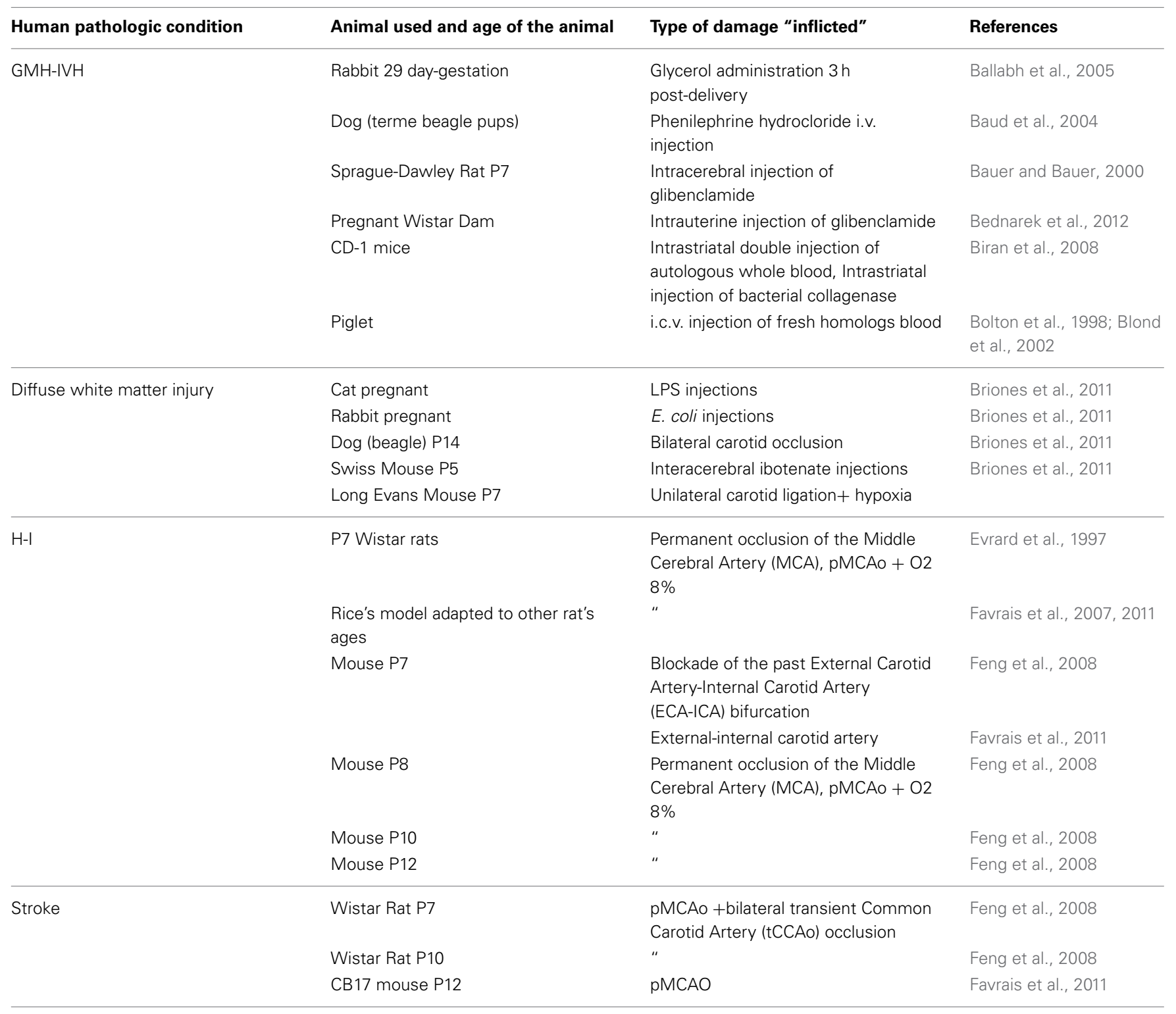

TBI

Fluid percussion injury (FPI) models

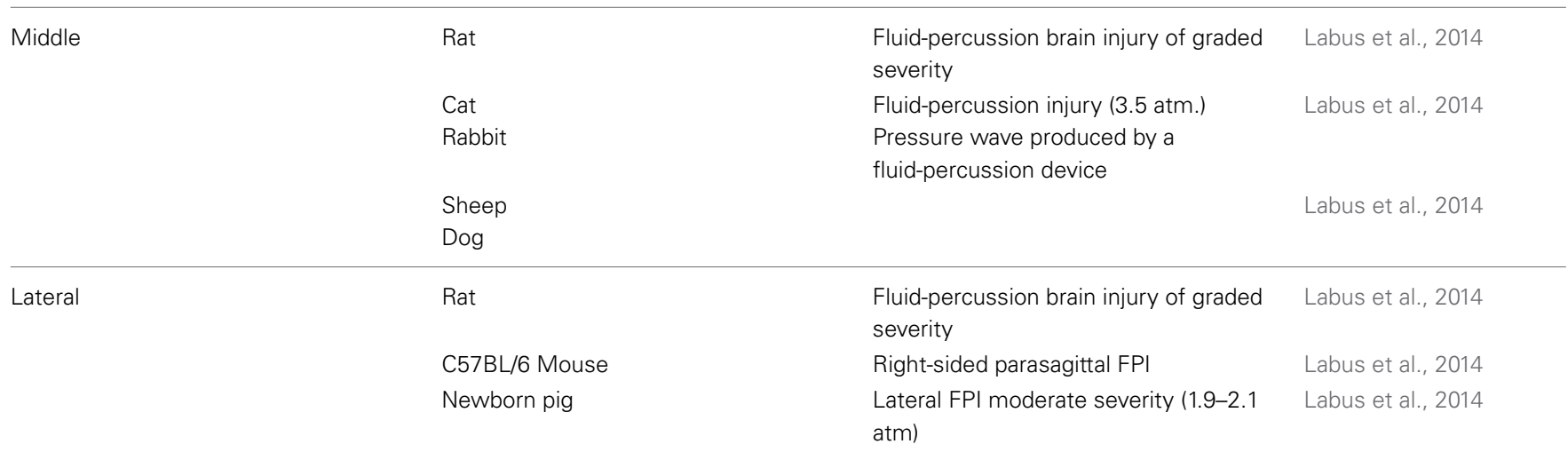


Table 1 | Continued

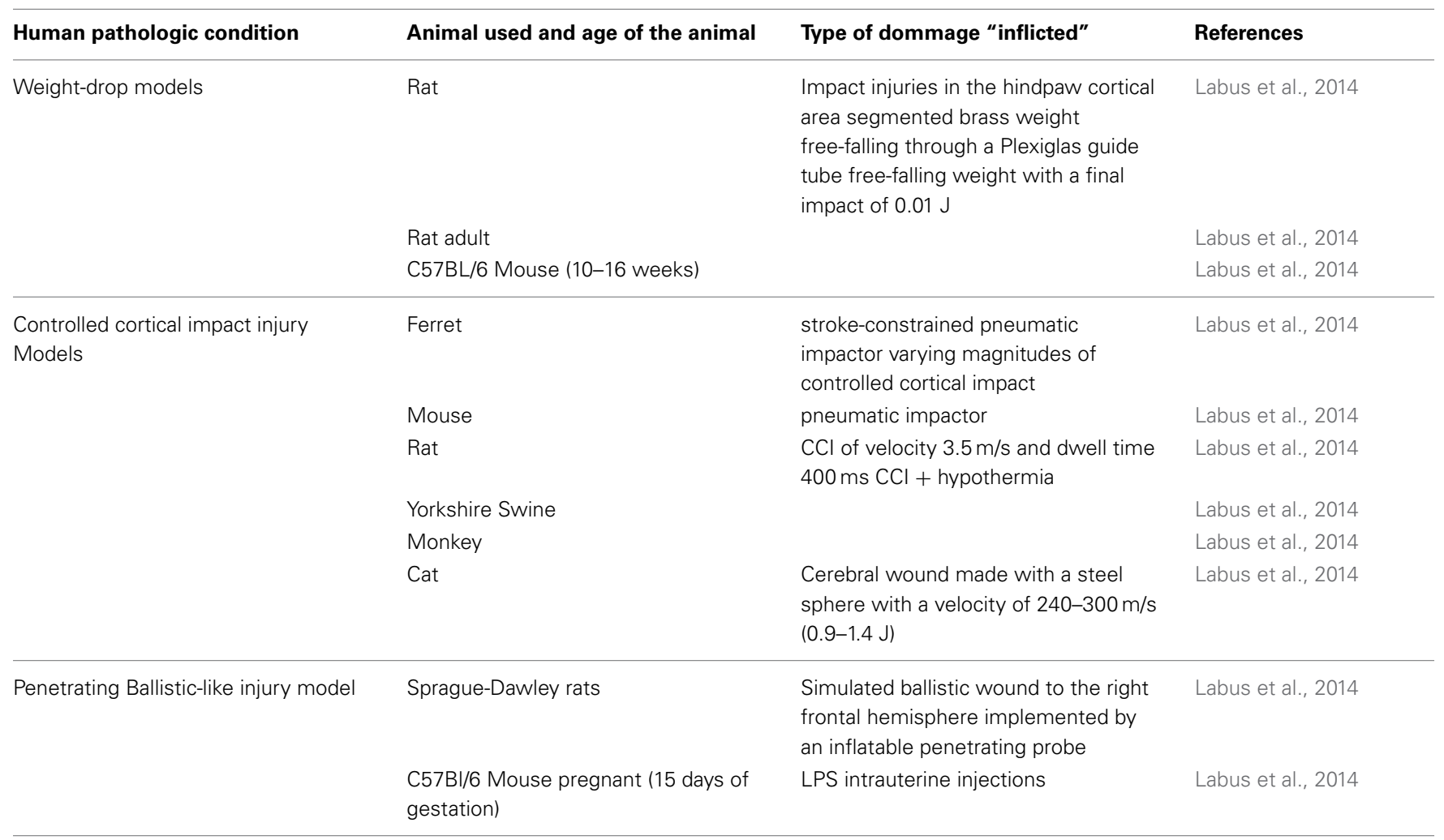

to those seen on post-mortem examination in preterm infants (Verney et al., 2012). In this model, vulnerability to WMD is increased by systemic inflammation (Dommergues et al., 2003; Favrais et al., 2007; Aden et al., 2010), and the BBB is slightly affected.

Animal models of preterm brain injury have also conclusively shown that exposure to inflammation alone, even at low levels, is sufficient to induce WMD similar to that seen clinically (Rousset et al., 2006; Bednarek et al., 2012). In addition to WMD, the exposure of fetal sheep to low-dose endotoxin leads to increased BBB permeability, as assessed by staining for plasma albumin extravasation (Yan et al., 2004). This effect has been noted in the cortical white and gray matter and the thalamus, but not the cerebellum. WMD comparable to that seen in preterm infants can also be induced in the mouse or rat brain by the Vannucci hypoxiaischemia method at P3 or P5 or by chronic exposure to hypoxia (P3-P11) (Hagberg et al., 2002). There is very little information regarding the $\mathrm{BBB}$ in these models of preterm WMD.

\section{HYPOXIC-ISCHEMIC ENCEPHALOPATHY}

Hypoxic-ischemic encephalopathy (HIE) is considered a major cause of brain injury in term neonates. At present, there are no specific serum biomarkers or other tools to easily assess neurological injury at birth, either in terms of its severity or the prediction of neurodevelopmental outcome. Because of this knowledge gap, all newborns with moderate or severe encephalopathy are treated with hypothermia in an identical fashion. Randomized controlled trials of therapeutic hypothermia have demonstrated a reduction in death or severe disability at 18 months, but death and disability continue to occur in $30-70 \%$ of infants with severe to moderate HIE despite cooling therapy (Shankaran et al., 2005; Azzopardi et al., 2009; Tagin et al., 2012). The criteria used to identify neonates with so-called HIE are (1) a $\mathrm{pH} \leq 7$ or base deficit $\geq 16 \mathrm{mEq} / \mathrm{L}$ in umbilical cord arterial plasma, and (2) a history of an acute perinatal event or non-availability of arterial blood gas or a $\mathrm{pH}$ or base deficit in the borderline range associated with a 10 -min APGAR score $\leq 5$ or assisted ventilation at birth. An understanding of the neuropathological process that leads to HIE could lead to the identification of new markers of neuronal injury that correlate with disease and neurodevelopmental outcome, and thus facilitate a more targeted therapeutic approach using adjunctive therapies (Chalak et al., 2014).

To study the effects of oxygen and nutrient deprivation on the developing brain, the species most commonly used are sheep, rabbits, piglets and, most of all, rodents. Differences in temporal phases of development between human and animal brain structures make the choice of a specific species and the determination of a precise correlation between developmental stages in the animal model and the corresponding stage in neonatal brain development very complex. Based on several experimental studies evaluating the developmental profile of the main neural cells of interest, the scientific community has established that a reasonable comparison can be drawn between $\mathrm{P} 3 / 5$ rodents and preterm infants, and P7/10 rodents and term infants (Kinney and Volpe, 2012). 
Across the wide range of species used to model HIE, the relative contribution of vessels supplying blood to the brain is variable (Purves, 1972). In rodents, brain ischemia induced by the occlusion of a single carotid artery is not always consistent, and injury is typically induced by combining this with systemic hypotension. This combination of insults in the P7 rat, known as the Rice-Vannucci model, was originally developed in 1981 and is considered the pioneering model of HIE (Rice et al., 1981). It has since been adapted to many postnatal ages and transferred to the mouse (Derugin et al., 1998; Sheldon et al., 1998; Ohshima et al., 2012; Tsuji et al., 2013). The brain's response to global hypoxia-ischemia is a multistep process. Within the first few hours, regionally specific increases of cerebral blood flow occur followed by decreases due to regional vasoconstriction or the collapse of cardiac output, with the subsequent onset of excitotoxicity, energy depletion and the generation of free radicals, leading to apoptotic and necrotic cell death and the generation of edema (Ferrari et al., 2010a). This phase is followed within a few hours/days by a second phase consisting of a neuroinflammatory response, mitochondrial permeabilization, reperfusion and loss of cerebral autoregulation, and the production of free radicals (Hamrick and Ferriero, 2003; McLean and Ferriero, 2004). Recent hypotheses describe a tertiary phase that occurs days or even years after the injury and that contributes to the persistence of inflammation and reduced efficacy of brain repair (Fleiss and Gressens, 2012).

The effects of hypoxia-ischemia on the adult BBB include the disruption of TJ proteins (in particular claudin-5 (Cld5), occludin (Occl), zonula occludens (ZO) -1 and -2 (Bolton et al., 1998; Fischer et al., 2002; Mark and Davis, 2002; Witt et al., 2003; Sandoval and Witt, 2008; Zehendner et al., 2013) and increased BBB permeability in in vitro studies (Fischer et al., 2000; Zhang et al., 2000; Mark and Davis, 2002). These events are mediated by proinflammatory cytokines such as tumor necrosis factor (TNF) $\alpha$, interleukin (IL)-1 $\beta$, IL-8, monocyte chemoattractant protein1 , intercellular adhesion molecule-1, vascular endothelial growth factor (VEGF) and nitric oxide (NO), and astrocytes seems to play a protective role (for a review, see Ballabh et al., 2004). However, differences between the mature and developing brain limit the possibility of extrapolating data from adult studies to neonates.

Concerning developmental age, there are few comparative studies between barriers in the adult and developing brain in this specific model, and the results are not always concordant; in fact, studies in sheep show that the BBB becomes less susceptible to hyperosmolar stress with age (Stonestreet et al., 2006), and in rodents, it seems to be more vulnerable to hypoxia-ischemia at early stages of development (Muramatsu et al., 1997). Studies in rodent models of neonatal hypoxia-ischemia have indicated that the integrity of brain barriers is compromised (Muramatsu et al., 1997; Ferrari et al., 2010b; Tu et al., 2011; Yang et al., 2013a), a finding confirmed by a study of cerebrospinal fluid (CSF) samples from HIE infants showing that the ratio of the albumin concentration in the CSF to that in the plasma is 5 times higher than normal, suggesting leaky brain barriers in these infants (Kumar et al., 2008). Most of these studies, however, precede more recent knowledge of $\mathrm{BBB}$ characteristics suggesting that the $\mathrm{BBB}$ is more resistant during the developmental period than after maturation
(Johansson et al., 2008; Ek et al., 2012; Saunders et al., 2012). Additionally, these early studies did not examine specific TJ disruptions or undertake a qualitative evaluation of the passage of substance and molecules, assessing only immunoglobulin (Ig) G labeling at different time points. Further investigations are needed to better understand BBB behavior in this model.

\section{STROKE}

Ischemic stroke occurs when cerebral blood flow is locally interrupted due to a clot within a vessel (Davis and Donnan, 2012) (see Figure 1). Perinatal stroke is defined as a cerebrovascular event that occurs between 28 weeks of gestation and a postnatal age of 28 days. Its incidence is similar to that in the elderly, approximately 1 in 4000 live births (Lynch et al., 2002), and it is associated with cerebral palsy [which is correlated with infarct size (Lee et al., 2005)], epilepsy, language delays and behavioral abnormalities. Classically associated with hypoxia, recent animal models have used permanent middle cerebral artery occlusion in association with transient bilateral common carotid artery occlusion to mimic an isolated acute ischemic event followed by reperfusion on resuscitation. Currently, more reproducible models are the 1998 Renolleau model in P7 Wistar rats and the 2013 Tsuji model in P9 CB-17 mice (Charriaut-Marlangue et al., 2013). After stroke in adults, BBB leakage/disruption can occur either transiently, in two distinct phases (Belayev et al., 1996; Rosenberg et al., 1998), or continuously (McColl et al., 2008; Kuntz et al., 2014). Although the basic mechanisms of neurodegeneration after stroke are shared across age groups, immaturity critically affects brain susceptibility and response to ischemiarelated insults, including modes of neuronal death, inflammation, leukocyte-mediated injury and susceptibility to reactive oxygen species (ROS) (Benjelloun et al., 1999).

\section{TRAUMATIC BRAIN INJURY}

Traumatic Brain Injury (TBI) in children can cause more severe cognitive and behavioral deficits than comparable injuries in mature brains, and is one of the most common reasons for the development of significant lifelong disability in a child (Anderson et al., 2005; Rivara et al., 2012; Stanley et al., 2012; Roozenbeek et al., 2013). This is particularly important if we consider that children under the age of 4 years more frequently undergo TBI than any other age group (Koepsell et al., 2011) and that injured infants under the age of 12 months are at high risk of requiring intensive care support (Keenan et al., 2003). A similar developmental sensitivity to TBI can be seen in a rodent model of TBI, in which TBI inflicted at P7 causes the maximum damage. In addition, during the first 3 weeks of life, rodents display high sensitivity to excitotoxicity, as seen during the developmental period in human brains (i.e., maximal brain growth, synaptogenesis, and myelination). Thus, P7 is considered the period of maximal sensitivity and the best age to evaluate effects of TBI in rodents (Bittigau et al., 1999).

TBI can be simulated in different ways in various animal models: in the 1980s, a variety of species including cats, dogs, pigs and non-human primates were used (Xiong et al., 2013), but since the 1990s the use of rodents has predominated thanks to the ease with which experiments can be carried out and managed, and the limited costs. In addition, the ability to genetically modify mice has 
confirmed their position as the most-used species in laboratory research (Longhi et al., 2001; Duhaime, 2006). Models of focal TBI are the most common, induced by the impact of an object on the head (contusion) or the sudden arrest of the head during motion (deceleration) not necessarily requiring contact with an object. The most common modes of contusion trauma induction are lateral fluid percussion injury (Thompson et al., 2005), controlled cortical impact injury (Dixon et al., 1991; Cernak, 2005; Ajao et al., 2012; Pop et al., 2013) and focal TBI through a closed skull (Chen et al., 1996). The primary injury process in TBI is mechanical damage (vascular damage and bleeding caused by shear forces), immediately followed by mast cell degranulation (Stokely and Orr, 2008), and a secondary pathological phase characterized by excitotoxicity, ischemia, mitochondrial dysfunction and apoptosis (Xiong et al., 2013). This phase leads to the activation of inflammatory processes, which are themselves neurotoxic (Hagberg et al., 2012) and mediated by immune cells resident in the brain as well as by the transmigration of systemic immune cells and BBB disruption (Ramlackhansingh et al., 2011).

An electron microscopic analysis of rat cerebral slices in an animal model of TBI (Dietrich et al., 1994) has revealed the presence of microscopic hemorrhagic contusions or petechia, especially in the white-gray matter interface underlying the somatosensory cortex and cisterna ambiens, demonstrating that primary shearstress-induced vascular damage can be found distant from the initial injury site, indicating protein leakage and the extravasation of blood cells. The choroid plexus is also activated by TBI inflicted at a distance from the ventricles, leading not to blood-cerebrospinal fluid barrier disruption but to the homing of choroidal monocytes and neutrophils and the synthesis and secretion into the CSF of inflammatory chemokines (Szmydynger-Chodobska et al., 2009, 2012).

\section{ROLE OF THE BBB IN THE PATHOPHYSIOLOGY OF DEVELOPMENTAL BRAIN INSULTS}

It would be difficult to thoroughly analyze in this review the large amount of information available concerning the possible mechanisms that influence BBB disruption in the developing brain. We will therefore focus on a few selected factors.

\section{EXCITOTOXICITY AND FREE RADICALS}

NO is a weak free radical produced by the action of nitric oxide synthase (NOS). The direct effects of this molecule on the BBB during development have not been studied so far. However, studies in adult rats demonstrate that in a model of stressinduced neurodegeneration, the production of $\mathrm{NO}$ and related oxidative-nitrosative compounds via the expression of inducible NOS (iNOS) correlates with BBB disruption, as evaluated by $\left[{ }^{14} \mathrm{C}\right]$-sucrose uptake by brain tissue. The injection of the specific iNOS inhibitor $1400 \mathrm{~W}$ prevents this increase in BBB permeability. Since the developing brain is considered to be extremely vulnerable to free radical damage due to its high lipid content (O'Brien and Sampson, 1965), relatively high oxygen consumption and capacity for ROS generation, and low concentrations of the main antioxidant enzymes (Baud et al., 2004; Vannucci and Hagberg, 2004; Miller et al., 2012), a potential role of this free radical could be suspected.
Following neonatal hypoxia-ischemia, both neuronal (n) NOS and endothelial (e) NOS are up-regulated, but while nNOS knockout mice seem to be protected following neonatal hypoxiaischemia (Ferriero et al., 1996), studies indicate that eNOS can play a neuroprotective role in the adult brain by influencing neural migration and outgrowth and acting as a downstream regulator of angiogenesis (Huang et al., 1996). Further studies are needed to clarify the different roles of specific NOS isoforms and their link with BBB disruption.

Concerning white matter insults, in the presence of an activated excitotoxic cascade, damage to cerebrovascular endothelial cells should cause alterations in BBB function that could exacerbate neuronal injury and death. Glutamic acid or glutamate the most important excitatory neurotransmitter in the CNS, also acts in the pathophysiology of neuronal damage. Excess glutamate is known to induce neuronal death through a process in which the overactivation of ionotropic and metabotropic glutamate receptors triggers several intracellular signaling pathways, leading to apoptosis, necrosis or both (Gudino-Cabrera et al., 2014). Glutamate agonists, such as ibotenate, are frequently used to reproduce excitotoxic insults in the CNS. However, there are, to our knowledge, no studies that directly evaluate the effects of excitotoxic insults on BBB permeability during development.

The pathophysiology of TBI can also be explained in part by free radical formation and the effects of these on the BBB. During TBI, the transmigration of circulating immune cells and local microglial activation leads to the production of NO, ROS and inflammatory mediators that are capable of interfering with the BBB (Perez-Asensio et al., 2005; Briones et al., 2011). For instance, the ROS-induced peroxidation of polyunsaturated fatty acids in cell membranes gives rise to active aldehydes, significantly increasing endothelial monolayer permeability in experimental studies (Chodobski et al., 2011). In addition, oxidative stress is associated with the endogenous antioxidant glutathione and the increased permeability of the BBB, although only to low-molecular-weight markers (Agarwal and Shukla, 1999), and hydrogen peroxide has been demonstrated to increase BBB permeability via the Extracellular-signal-regulated Kinase (ERK) signal transduction pathway, along with the redistribution of Occl and ZO-1 and -2 (Fischer et al., 2005).

\section{ALTERATIONS OF THE BASAL LAMINA AND ENDOTHELIAL CELLS}

One class of enzymes extensively studied for its role in the BBB is family of proteases known as the matrix metalloproteinases (MMPs). The MMPs cleave their protein substrates based on a conserved mechanism that involves the interaction of an active-site-bound water molecule with a $\mathrm{Zn}^{2+}$ ion. The MMPs participate not only in pathophysiological processes but also in many normal biological processes such as embryonic (including brain) development, organ morphogenesis, blastocyst implantation, bone remodeling and wound healing (Brew et al., 2000). Because of this dual nature of the MMPs, it would be interesting to uncover their role in the developing brain after neonatal hypoxia-ischemia and, more importantly, their long-term role in neurological function. MMPs disrupt the BBB by degrading TJ and basal lamina proteins, thereby leading to BBB leakage, leukocyte infiltration, brain edema and hemorrhage (Liu 
et al., 2012). The activity of the MMPs is regulated by a group of endogenous proteins called tissue inhibitors of metalloproteinases (TIMPs), which bind to active and alternative sites of activated MMPs.

MMPs have been implicated in cerebral ischemia, and several studies have revealed that plasma MMP-9 concentrations strongly correlate with stroke severity in patients and that MMP9 inhibition attenuates early BBB disruption and cerebral edema while promoting the expression of TJ proteins and angiogenesis (Rosenberg et al., 1998; Liu et al., 2009; Gu et al., 2012; Yang et al., 2013b). For instance, in a mouse model of focal cerebral ischemia, the deletion of the TIMP-1 gene results in increased MMP-9 protein expression and gelatinolytic activity, and is accompanied by exacerbated BBB disruption, neuronal apoptosis and ischemic injury when compared to wild-type animals (Fujimoto et al., 2008). Correspondingly, BBB leakage is reduced by TIMP- 1 overexpression, and $24 \mathrm{~h}$ infarct volumes are also reduced (Maier et al., 2006). MMP-9 has been shown to degrade TJ proteins (Cld-5, Occl, ZO-1) in animal models of focal cerebral ischemia (Yang and Rosenberg, 2011). Aberrant MMP-9 proteolytic activity degrades not only TJ proteins but also basal membrane proteins (e.g., fibronectin, laminin, collagen, and others). This degradation is associated with an increase in BBB permeability, resulting in brain infarction, edema and hypertension in both animal models (Lee et al., 2007; Rosenberg and Yang, 2007; Yang et al., 2007) and human patients (Gasche et al., 2001; Castellanos et al., 2003; Lo, 2008). In a study conducted in P7 rat pups, hypoxia-ischemia was induced by unilateral ligation of the right carotid artery followed by hypoxia, and a broadspectrum MMP inhibitor was injected intraperitoneally (i.p.). This early MMP inhibition provided both acute and long-term neuroprotection by reducing $\mathrm{TJ}$ protein degradation, preserving $\mathrm{BBB}$ integrity and reducing brain edema after neonatal hypoxicischemic injury (Chen et al., 2009). In addition, IgG accumulation in the brain is reduced in neonatal MMP-9 knockout (KO) mice compared to wild-type mice $24 \mathrm{~h}$ after hypoxia-ischemia (Svedin et al., 2007; Vexler and Yenari, 2009), suggesting that MMP-9 contributes to the opening of the BBB shortly after hypoxia-ischemia. However, there are still several points that need to be clarified. Like many biological mediators, MMPs can be used for good or bad, and this seems to be the case in the brain too. In fact, although MMPs seem to be involved in damage early after stroke, other studies demonstrate that they take on another role later in the process, contributing to repair (Stolp et al., 2005). However, it has been noted that exogenous MMPs kill neurons in culture (Stonestreet et al., 2006; Stolp et al., 2011). This could be due to temporal variations in the action of MMP-9 and/or to the divergent functions of different subtypes of MMPs. These aspects are well reviewed in Lakhan et al. (2013). Briefly, whereas MMP-2 could play a role in the initial opening of the BBB, MMP-9 seems to be more important for the secondary, delayed, opening of the $\mathrm{BBB}$ after ischemia, which is necessary for vascular angiogenesis and neuronal regeneration.

A possible explanation for the action of the MMPs is mediation by caveolin-1 (Cav-1). ROS can stimulate MMP activation through the loss of caveolin-1, a protein encoded by the cav1 gene and a critical determinant of BBB permeability ( $\mathrm{Gu}$ et al.,
2011). Recent studies have revealed that Cav-1 can prevent the degradation of TJ proteins and protect BBB integrity by inhibiting the production of reactive nitrogen species (RNS) and MMP activity. In addition, Cav-1 KO mice show higher rates of apoptotic cell death and larger infarct volumes than wild- in ischemic brains, and the production of NO induces the loss of Cav-1 in focal cerebral ischemia and reperfusion injury. The downregulation of Cav-1 is correlated with an increase in the activity of MMP-2 and -9, decreased ZO-1 expression and increased BBB permeability (Gu et al., 2012).

\section{ANGIOGENESIS}

Although hypoxia-induced angiogenesis is considered beneficial since it allows more oxygen delivery to ischemic regions, there are some associated aspects that are not necessarily positive. One of these aspects is BBB permeability. The main molecules implicated in this process of endothelial cell regression and regrowth are VEGF, placental growth factor, acidic fibroblast growth factor (FGF), TNF $\alpha$, IL-8 and erythropoietin. The VEGF family has six homologs members, of which the most widely studied is VEGF-A, and 3 tyrosine kinase receptors. Of these, VEGF receptors -1 and -2 , which bind VEGF-A, are involved in blood vessel growth, endothelial cell mitogenesis and vasodilatation (via NO-dependent pathways). VEGF is thus a key regulator of vasculogenesis, and endothelial cells that express VEGF also produce MMPs and plasminogen activators to initiate extracellular matrix degradation, the first step in angiogenesis. In response to hypoxia, hypoxia-inducible factor (HIF) - 1 is stabilized, leading to the augmented expression of the angiogenic genes mentioned above (Semenza, 2010). Three HIF isoforms are known (HIF-1 $\alpha$, HIF- $2 \alpha$, and HIF- $3 \alpha$ ), of which HIF- $2 \alpha$ is restricted to vascular endothelial cells (Skuli et al., 2009). It has been suggested that in endothelial cells, HIF- $1 \alpha$ plays a role in metabolism, proliferation and survival, while HIF- $2 \alpha$ is involved in cell migration, adhesion and vascular integrity. Following hypoxia in the neonatal brain, HIF- $1 \alpha$ starts to accumulate in neurons, an effect that has been shown to be protective and antiapoptotic (Tahraoui et al., 2001; Tagin et al., 2012). However, in a study evaluating the role of HIF- $1 \alpha$ in BBB permeability in a rat subarachnoid hemorrhage model, the elevated expression of HIF- $1 \alpha$ in brain tissues temporally coincided with brain edema formation and $\mathrm{BBB}$ disruption. In addition, BBB disruption, as determined by the extravasation of Evans Blue (a standard ink tracer used in the CNS), was downregulated by the inhibition of either HIF-1 $\alpha$ or MMP-9. In the present study, the inhibition of HIF- $1 \alpha$ reduced not only brain edema, but also the expression of aquaporin-4 (see Section on "Edema") and MMP-9, suggesting that HIF-1 $\alpha$ may be a key protein in brain edema formation and BBB disruption, possibly through aquaporin-4 and MMP-9 regulation (Tcharmtchi et al., 2000). Whether the induction of HIF- $1 \alpha$ is beneficial or harmful is thus still debatable. Some recent studies have reported that HIF- $1 \alpha$ and its target genes may contribute to cell death, tissue destruction and the formation of brain edema, primarily in the acute phase after ischemic brain damage (Thibeault et al., 2001; Thompson et al., 2005). Therefore, inhibiting this molecule immediately following damage could be effective in improving neurological outcome by protecting the brain from secondary 
injury following edema and BBB disruption (Tcharmtchi et al., 2000).

VEGF also appears to have a dual response to hypoxiaischemia depending on the time frame studied. Several studies conducted both in adults and, to a lesser extent, in the neonatal brain following hypoxia, show that the i.c. injection of VEGF results in reduced brain injury, with the reduction of brain edema and infarct volume, a decrease in apoptotic cells and BBB permeability (Kaya et al., 2005; Feng et al., 2008). In addition, the inhibition of VEGF receptor-2 decreases endothelial cell proliferation, increases cell death and worsens stroke injury in a neonatal rodent model (Shimotake et al., 2010). The most likely hypothesis is that the "early" upregulation of VEGF (between 1 and $3 \mathrm{~h}$ after MCAO) is associated with BBB permeability and contributes to ischemic injury, while conversely, the neuroprotective effects of VEGF, including neovascularization and neuronal protection, occur in the days (48 h) after hypoxia (Marti et al., 2000).

\section{EDEMA}

One of the most important complications of TBI is edema, which occurs more frequently in the pediatric than in the adult population. This is possibly due to the higher water content of the developing brain and also to the mechanism of regulation of water homeostasis. There are few in vivo studies of TBI and BBB disruption in neonates, with most focusing on juvenile rodents. The Badault group has recently evaluated the contribution of the aquaporins to the post-traumatic edema process in juvenile rats and mice (Badaut et al., 2011a,b, 2014; Fukuda et al., 2012, 2013). Pups treated with an inhibitor of aquaporin-4, siGLO siAQP4, show acute improvements in motor function (3 days after injury) and long-term improvements in spatial memory ( 60 days after injury) when compared with control animals. These improvements are associated with decreased edema formation, increased microglial activation, decreased BBB disruption, and reduced astrogliosis and neuronal death. The effectiveness of the treatment paradigm is associated with a $30 \%$ decrease in aquaporin-4 expression at the injection site. Thus, aquaporin's seems to be molecules significantly involved in post-TBI damage, and represent an interesting therapeutic target (Fukuda et al., 2013). Another study has revealed that the inhibition of a kinase involved in the development of brain edema, myosin light-chain kinase, by the administration of an inhibitor, ML-7, to P24 mice starting $4 \mathrm{~h}$ after TBI and every $24 \mathrm{~h}$ until sacrifice, significantly reduces BBB breakdown and the development of cerebral edema, and preserves neurological function (Rossi et al., 2013).

\section{INFLAMMATORY RESPONSE}

Neuroinflammation is a key pathological factor in most insults to the developing brain (Degos et al., 2010a). Neuroinflammation is induced by the invasion of pathogens or the release of damageassociated proteins by injured and dying cells. Inflammation includes the activation of microglia, astrocytes and endothelial cells, leading to the secretion of proinflammatory cytokines such as TNF $\alpha$ and IL-1 $\beta$. There is an increased expression of endothelial adhesion molecules such as vascular cell adhesion molecule 1, intercellular adhesion molecule 1 and the selectins (Simi et al., 2007). Inflammatory processes are, moreover, associated with modifications of the molecular composition or functional state of TJs (Coisne and Engelhardt, 2011). Proinflammatory cytokines also influence the expression of MMPs, which increase BBB permeability by degrading TJs and extracellular matrix components in the endothelial basement membrane (Rosenberg, 2009). These events lead to barrier leakiness, which in turn allows pathogen and immune cell invasion (Labus et al., 2014).

Resident microglia are some of the first cells to respond to inflammatory stimuli. They can migrate, together with astrocytes, to the region of injury or infection, where they clean up cellular debris and produce proinflammatory cytokines such as IL-1 $1 \beta$ and IL-6. In the case of TBI and hypoxia-ischemia, mast cells also produce large quantities of proinflammatory mediators (Biran et al., 2008; Jin et al., 2009). With regard to the BBB, these cytokines lead to increased permeability and facilitate the entry of peripheral macrophages and cytokines from the systemic circulation. In the instance of inflammation following hypoxic-ischemic injury, this has the effect of exacerbating the excitotoxic cascade by further stimulating glutamate release and free radical and NO production (McLean and Ferriero, 2004). The importance of the mast cell response in causing brain damage after TBI is supported by post-insult treatment with cromoglycate, a mast cell stabilizer that inhibits mast cell degranulation and decreases BBB opening (Strbian et al., 2006), glial activation and neuronal death (Jin et al., 2007) sufficiently to provide long-term neuroprotection.

Leukocyte migration depends on strict interactions with the vascular endothelium, mediated by 3 groups of adhesion molecules: the selectins, the immunoglobulin superfamily (such as intercellular adhesion molecule 1) and the integrins. The pattern of recruitment of monocytes and macrophages is cytokinespecific: IL-1 $\beta$ leads to neutrophil recruitment while TNF $\alpha$ attracts monocytes (Blond et al., 2002). A comparative study in P7 and adult rats subjected to transient middle cerebral artery occlusion shows that Evans Blue extravasation, a measure of paracellular diffusion, the main method of entry for bulky proteins and leukocytes into the brain, remains low during the $24 \mathrm{~h}$ period following reperfusion in neonatal rats but is profoundly increased in adult rats. The largely unaltered paracellular diffusion in neonatal rats is associated with the increased expression of several TJ proteins (Vexler and Yenari, 2009) rather than with the decrease in their expression seen in adult rats after a similar injury. In contrast to stroke in adults, in P7 hypoxic-ischemic brains, the transmigration of neutrophils is far lower (Hudome et al., 1997). This may be related to the greater resistance of the BBB to hypoxia-ischemia-induced leaks at this age but also to the immaturity of both endothelial cells, which express less P-selectin (Lorant et al., 1999), and neutrophils, which are less capable of adhering to P-selectin (Tcharmtchi et al., 2000). The lower extent of leukocyte extravasation after ischemic injury in neonatal rats may contribute to the age difference in the structural and functional changes to the BBB after ischemia.

Specific studies have been carried out to examine the effects of inflammation on the functions of the developing BBB and how this influences the development of brain injury. A rather complex and unexpected effect of inflammatory stimuli on BBB integrity 
and leukocyte transmigration has been reported during the first 3 weeks of life in rats. A comparison of the extent of IgG infiltration in different brain regions following intrastriatal injections of IL-1 or $\mathrm{TNF} \alpha$ in rats of different ages shows dramatically higher IgG accumulation in 21-day-old than in 2-h-old rat pups (Anthony et al., 1997, 1998; Schnell et al., 1999). These data demonstrate that at birth, the BBB is functional, and is in fact more resistant to inflammatory stress than in the juvenile brain.

Stolp et al. have conducted two studies of BBB behavior in neonatal, juvenile and adult rats in response to prolonged inflammatory stimuli, in particular the i.p. injection of $0.2 \mathrm{mg} / \mathrm{kg}$ lipopolysaccharide (LPS). They have demonstrated for the first time a possible link between changes in BBB permeability (evaluated by permeability to sucrose, inulin and plasma proteins and Cld-5 distribution) and behavioral alterations in animals exposed to the inflammatory stimulus early in development, i.e., on postnatal day (P) 0, P2, P4, P6, and P8. LPS treatment resulted in increased permeability only in adulthood, preceded by Cld5 alterations in a few vessels at an earlier time point. However, due to the normally low permeability of cerebral blood vessels, it is probable that even a few vessels with altered permeability could significantly contribute to the overall properties of the $\mathrm{BBB}$, and that these changes could explain the short-term changes seen in behavioral tests, in particular in the prepulse inhibition paradigm, that were distinguishable in juvenile animals but not in adult animals. Long-term changes in permeability, as shown by the sucrose permeability test, are correlated in adult rats with altered responses to the dark/light test, suggesting that the impact of inflammation could occur in several phases (short- and longterm) and that each phase could lead to different behavioral modifications (Stolp et al., 2005, 2011). A third study from the same group analyzes, in a similar model (using the opossum Monodelphis domestica), the ability of minocycline, a potent antiinflammatory molecule, to modulate the inflammation-induced changes in BBB permeability and white matter damage following acute and prolonged inflammation during development. The effects of minocycline on inflammation were evaluated in terms of IL- $1 \beta$ mRNA levels in the spleen and brain, and of white blood cell counts in animals sacrificed $1.5 \mathrm{~h}$ after the first or fifth LPS injection (single or prolonged inflammatory stimuli). No differences in IL-1 $\beta$ levels were seen between groups, although white blood cells were significantly augmented in animals given prolonged LPS stimulation compared to non-treated animals. Interestingly minocycline significantly reduced this augmentation. The same results were obtained if permeability to ${ }^{14} \mathrm{C}$-sucrose and plasma proteins were evaluated: animals that received a single injection of either LPS or LPS+minocycline showed no changes in BBB permeability to ${ }^{14} \mathrm{C}$-sucrose or plasma proteins. Thus, the inclusion of minocycline probably did not prevent barrier permeability changes after a single LPS injection but prevented barrier permeability to proteins and ${ }^{14} \mathrm{C}$-sucrose when administered during prolonged inflammation.

We have carried out further studies of the BBB in a model in which inflammation alone induces WMD, by the twice-daily i.p. injection of newborn rats or mice with IL-1 over 5 days (Favrais et al., 2011). We evaluated modifications in the expression of the most important choroid plexus TJ proteins at $\mathrm{P} 2$, the second day of injections, and 5 days after the beginning of the injections (Favrais et al., 2011; Riddle et al., 2011; Schang et al., 2014). Our results demonstrate that there is no significant non-specific disruption of BCSFB integrity, as assessed by paracellular permeability to tracers, and only a modest increase in Cld-3 expression in the choroidal tissue. In adult animal models of inflammation, the choroid plexus is known to be extremely sensitive to peripheral inflammatory stimuli, as demonstrated by the augmentation of cytokines and other inflammatory markers (Quan et al., 1999; Thibeault et al., 2001; Szmydynger-Chodobska et al., 2012), and the choroid plexus-CSF system appears to be crucial for immune cell trafficking into the CNS during neuroimmune surveillance as well as in the early stages of neuroinflammatory diseases (Reboldi et al., 2009; Schmitt et al., 2012). Thus, studies on the choroid plexus conducted in the context of moderate systemic perinatal inflammation occurring during a period approximating 28-35 weeks of gestation in humans, which alters the developmental program of the white matter, should focus more on the perturbation of specialized functions of the BCSFB, such as neuroimmune regulation, rather than on gross non-specific alterations of barrier integrity.

\section{CONCLUSION}

Inflammation, vascular reactivity and excitotoxicity are the main protagonists of perinatal diseases, and the BBB and NVU are certainly majorly implicated in these processes. As our knowledge of these processes increases, promising new targets for neuroprotection have been found. However, a lot of questions remain open, especially regarding differences between the behavior of young and adult brains. In addition, even though clinical studies are impossible for obvious ethical reasons, the use of animal models and/or in vitro studies impose great limitations in this domain, since the therapeutic options validated in these models are not always as effective when applied to humans. Funding research on the $\mathrm{BBB}$, both in the pre-clinical and clinical domains, should be encouraged in order to better understand early cerebral development and develop effective therapeutic strategies.

\section{ACKNOWLEDGMENTS}

In vivo blood-CSF barrier assessment was carried out by the BIP platform, CRNL Inserm U1028, Lyon. A special thanks to Dr. S Rasika for her priceless proofreading and critical editing of the manuscript.

\section{REFERENCES}

Aden, U., Favrais, G., Plaisant, F., Winerdal, M., Felderhoff-Mueser, U., Lampa, J., et al. (2010). Systemic inflammation sensitizes the neonatal brain to excitotoxicity through a pro-/anti-inflammatory imbalance: key role of TNFalpha pathway and protection by etanercept. Brain Behav. Immun. 24, 747-758. doi: 10.1016/j.bbi.2009.10.010

Agarwal, R., and Shukla, G. S. (1999). Potential role of cerebral glutathione in the maintenance of blood-brain barrier integrity in rat. Neurochem. Res. 24, 1507-1514. doi: 10.1023/A:1021191729865

Ajao, D. O., Pop, V., Kamper, J. E., Adami, A., Rudobeck, E., Huang, L., et al. (2012). Traumatic brain injury in young rats leads to progressive behavioral deficits coincident with altered tissue properties in adulthood. J. Neurotrauma 29, 2060-2074. doi: 10.1089/neu.2011.1883

Anderson, V., Catroppa, C., Morse, S., Haritou, F., and Rosenfeld, J. (2005). Functional plasticity or vulnerability after early brain injury? Pediatrics 116, 1374-1382. doi: 10.1542/peds.2004-1728 
Anthony, D. C., Bolton, S. J., Fearn, S., and Perry, V. H. (1997). Age-related effects of interleukin-1 beta on polymorphonuclear neutrophil-dependent increases in blood-brain barrier permeability in rats. Brain 120(Pt 3), 435-444. doi: 10.1093/brain/120.3.435

Anthony, D., Dempster, R., Fearn, S., Clements, J., Wells, G., Perry, V. H., et al. (1998). CXC chemokines generate age-related increases in neutrophil-mediated brain inflammation and blood-brain barrier breakdown. Curr. Biol. 8, 923-926. doi: 10.1016/S0960-9822(07)00373-9

Aquilina, K., Chakkarapani, E., and Thoresen, M. (2012). Early deterioration of cerebrospinal fluid dynamics in a neonatal piglet model of intraventricular hemorrhage and posthemorrhagic ventricular dilation. J. Neurosurg. Pediatr. 10, 529-537. doi: 10.3171/2012.8.PEDS11386

Aquilina, K., Hobbs, C., Cherian, S., Tucker, A., Porter, H., Whitelaw, A., et al. (2007). A neonatal piglet model of intraventricular hemorrhage and posthemorrhagic ventricular dilation. J. Neurosurg. 107, 126-136. doi: 10.3171/PED07/08/126

Armulik, A., Genove, G., Mae, M., Nisancioglu, M. H., Wallgard, E., Niaudet, C., et al. (2010). Pericytes regulate the blood-brain barrier. Nature 468, 557-561. doi: 10.1038/nature09522

Azzopardi, D. V., Strohm, B., Edwards, A. D., Dyet, L., Halliday, H. L., Juszczak, E., et al. (2009). Moderate hypothermia to treat perinatal asphyxial encephalopathy. N. Engl. J. Med. 361, 1349-1358. doi: 10.1056/NEJMoa0900854

Back, S. A., Gan, X., Li, Y., Rosenberg, P. A., and Volpe, J. J. (1998). Maturationdependent vulnerability of oligodendrocytes to oxidative stress-induced death caused by glutathione depletion. J. Neurosci. 18, 6241-6253.

Badaut, J., Ashwal, S., Adami, A., Tone, B., Recker, R., Spagnoli, D., et al. (2011b). Brain water mobility decreases after astrocytic aquaporin-4 inhibition using RNA interference. J. Cereb. Blood Flow Metab. 31, 819-831. doi: 10.1038/jcbfm. 2010

Badaut, J., Ashwal, S., and Obenaus, A. (2011a). Aquaporins in cerebrovascular disease: a target for treatment of brain edema? Cerebrovasc. Dis. 31, 521-531. doi: $10.1159 / 000324328$

Badaut, J., Fukuda, A. M., Jullienne, A., and Petry, K. G. (2014). Aquaporin and brain diseases. Biochim. Biophys. Acta 1840, 1554-1565. doi: 10.1016/j.bbagen.2013.10.032

Balasubramaniam, J., and Del Bigio, M. R. (2006). Animal models of germinal matrix hemorrhage. J. Child Neurol. 21, 365-371. doi: 10.1177/08830738060210 050201

Ballabh, P. (2010). Intraventricular hemorrhage in premature infants: mechanism of disease. Pediatr. Res. 67, 1-8. doi: 10.1203/PDR.0b013e3181c1b176

Ballabh, P., Braun, A., and Nedergaard, M. (2004). The blood-brain barrier: an overview: structure, regulation, and clinical implications. Neurobiol. Dis. 16, 1-13. doi: 10.1016/j.nbd.2003.12.016

Ballabh, P., Hu, F., Kumarasiri, M., Braun, A., and Nedergaard, M. (2005). Development of tight junction molecules in blood vessels of germinal matrix, cerebral cortex, and white matter. Pediatr. Res. 58, 791-798. doi: 10.1203/01.PDR.0000180535.14093.FB

Baud, O., Li, J., Zhang, Y., Neve, R. L., Volpe, J. J., and Rosenberg, P. A. (2004). Nitric oxide-induced cell death in developing oligodendrocytes is associated with mitochondrial dysfunction and apoptosis-inducing factor translocation. Eur. J. Neurosci. 20, 1713-1726. doi: 10.1111/j.1460-9568.2004.03616.x

Bauer, H. C., and Bauer, H. (2000). Neural induction of the blood-brain barrier: still an enigma. Cell. Mol. Neurobiol. 20, 13-28. doi: 10.1023/A:1006939 825857

Bednarek, N., Svedin, P., Garnotel, R., Favrais, G., Loron, G., Schwendiman, L., et al. (2012). Increased MMP-9 and TIMP-1 in mouse neonatal brain and plasma and in human neonatal plasma after hypoxia-ischemia: a potential marker of neonatal encephalopathy. Pediatr. Res. 71, 63-70. doi: 10.1038/pr. 2011.3

Belayev, L., Busto, R., Zhao, W., and Ginsberg, M. D. (1996). Quantitative evaluation of blood-brain barrier permeability following middle cerebral artery occlusion in rats. Brain Res. 739, 88-96. doi: 10.1016/S0006-8993(96)00815-3

Benjelloun, N., Renolleau, S., Represa, A., Ben-Ari, Y., and Charriaut-Marlangue, C. (1999). Inflammatory responses in the cerebral cortex after ischemia in the P7 neonatal Rat. Stroke 30, 1916-1923. discussion: 1923-1914.

Biran, V., Cochois, V., Karroubi, A., Arrang, J. M., Charriaut-Marlangue, C., and Heron, A. (2008). Stroke induces histamine accumulation and mast cell degranulation in the neonatal rat brain. Brain Pathol. 18, 1-9. doi: 10.1111/j.17503639.2007.00092.x
Bittigau, P., Sifringer, M., Pohl, D., Stadthaus, D., Ishimaru, M., Shimizu, H., et al. (1999). Apoptotic neurodegeneration following trauma is markedly enhanced in the immature brain. Ann. Neurol. 45, 724-735.

Blond, D., Campbell, S. J., Butchart, A. G., Perry, V. H., and Anthony, D. C. (2002). Differential induction of interleukin-1beta and tumour necrosis factor-alpha may account for specific patterns of leukocyte recruitment in the brain. Brain Res. 958, 89-99. doi: 10.1016/S0006-8993(02)03473-X

Bolton, S. J., Anthony, D. C., and Perry, V. H. (1998). Loss of the tight junction proteins occludin and zonula occludens- 1 from cerebral vascular endothelium during neutrophil-induced blood-brain barrier breakdown in vivo. Neuroscience 86, 1245-1257. doi: 10.1016/S0306-4522(98)00058-X

Brew, K., Dinakarpandian, D., and Nagase, H. (2000). Tissue inhibitors of metalloproteinases: evolution, structure and function. Biochim. Biophys. Acta 1477, 267-283. doi: 10.1016/S0167-4838(99)00279-4

Briones, T. L., Rogozinska, M., and Woods, J. (2011). Modulation of ischemiainduced NMDAR1 activation by environmental enrichment decreases oxidative damage. J. Neurotrauma 28, 2485-2492. doi: 10.1089/neu.2011.1842

Castellanos, M., Leira, R., Serena, J., Pumar, J. M., Lizasoain, I., Castillo, J., et al. (2003). Plasma metalloproteinase-9 concentration predicts hemorrhagic transformation in acute ischemic stroke. Stroke 34, 40-46. doi: 10.1161/01.STR.0000046764.57344.31

CDC CfDCaP. (2004). "Economic costs associated with mental retardation, cerbral palsy, hearing loss, and vision impairment: United States 2003," in MMWWR Morb. Mortal. Wkly. Rep. 53, (North Carolina), 57-59.

Cernak, I. (2005). Animal models of head trauma. NeuroRx 2, 410-422. doi: 10.1602/neurorx.2.3.410

Chalak, L. F., Sanchez, P. J., Adams-Huet, B., Laptook, A. R., Heyne, R. J., and Rosenfeld, C. R. (2014). Biomarkers for severity of neonatal hypoxic-ischemic encephalopathy and outcomes in newborns receiving hypothermia therapy. J. Pediatr. 164, 468-474 e461. doi: 10.1016/j.jpeds.2013.10.067

Charriaut-Marlangue, C., Bonnin, P., Leger, P. L., and Renolleau, S. (2013). Brief update on hemodynamic responses in animal models of neonatal stroke and hypoxia-ischemia. Exp. Neurol. 248, 316-320. doi: 10.1016/j.expneurol.2013.06.022

Chen, W., Hartman, R., Ayer, R., Marcantonio, S., Kamper, J., Tang, J., et al. (2009). Matrix metalloproteinases inhibition provides neuroprotection against hypoxia-ischemia in the developing brain. J. Neurochem. 111, 726-736. doi: 10.1111/j.1471-4159.2009.06362.x

Chen, Y., Constantini, S., Trembovler, V., Weinstock, M., and Shohami, E. (1996). An experimental model of closed head injury in mice: pathophysiology, histopathology, and cognitive deficits. J. Neurotrauma 13, 557-568.

Chodobski, A., Zink, B. J., and Szmydynger-Chodobska, J. (2011). Blood-brain barrier pathophysiology in traumatic brain injury. Transl. Stroke Res. 2, 492-516. doi: 10.1007/s12975-011-0125-x

Coisne, C., and Engelhardt, B. (2011). Tight junctions in brain barriers during central nervous system inflammation. Antioxid. Redox Signal. 15, 1285-1303. doi: 10.1089/ars.2011.3929

Daneman, R., Zhou, L., Agalliu, D., Cahoy, J. D., Kaushal, A., and Barres, B. A. (2010b). The mouse blood-brain barrier transcriptome: a new resource for understanding the development and function of brain endothelial cells. PLoS ONE 5:e13741. doi: 10.1371/journal.pone.0013741

Daneman, R., Zhou, L., Kebede, A. A., and Barres, B. A. (2010a). Pericytes are required for blood-brain barrier integrity during embryogenesis. Nature 468, 562-566. doi: 10.1038/nature09513

Davis, S. M., and Donnan, G. A. (2012). Clinical practice. Secondary prevention after ischemic stroke or transient ischemic attack. N. Engl. J. Med. 366, 1914-1922. doi: 10.1056/NEJMcp1107281

Degos, V., Favrais, G., Kaindl, A. M., Peineau, S., Guerrot, A. M., Verney, C., et al. (2010a). Inflammation processes in perinatal brain damage. J. Neural. Transm. 117, 1009-1017. doi: 10.1007/s00702-010-0411-x

Degos, V., Favrais, G., Kaindl, A. M., Peineau, S., Guerrot, A. M., Verney, C., et al. (2010b). Inflammation processes in perinatal brain damage. J. Neural. Transm. 117, 1009-1017. doi: 10.1007/s00702-010-0411-x

Derugin, N., Ferriero, D. M., and Vexler, Z. S. (1998). Neonatal reversible focal cerebral ischemia: a new model. Neurosci. Res. 32, 349-353. doi: 10.1016/S01680102(98)00096-0

Dietrich, W. D., Alonso, O., and Halley, M. (1994). Early microvascular and neuronal consequences of traumatic brain injury: a light and electron microscopic study in rats. J. Neurotrauma 11, 289-301. doi: 10.1089/neu.1994.11.289 
Dixon, C. E., Clifton, G. L., Lighthall, J. W., Yaghmai, A. A., and Hayes, R. L. (1991). A controlled cortical impact model of traumatic brain injury in the rat. J. Neurosci. Methods 39, 253-262. doi: 10.1016/0165-0270(91)90104-8

Dommergues, M. A., Plaisant, F., Verney, C., and Gressens, P. (2003). Early microglial activation following neonatal excitotoxic brain damage in mice: a potential target for neuroprotection. Neuroscience 121, 619-628. doi: 10.1016/S0306-4522(03)00558-X

Duhaime, A. C. (2006). Large animal models of traumatic injury to the immature brain. Dev. Neurosci. 28, 380-387. doi: 10.1159/000094164

Edwards, A. D., Brocklehurst, P., Gunn, A. J., Halliday, H., Juszczak, E., Levene, M., et al. (2010). Neurological outcomes at 18 months of age after moderate hypothermia for perinatal hypoxic ischaemic encephalopathy: synthesis and meta-analysis of trial data. BMJ 340:c363. doi: 10.1136/bmj.c363

Ek, C. J., Dziegielewska, K. M., Habgood, M. D., and Saunders, N. R. (2012). Barriers in the developing brain and Neurotoxicology. Neurotoxicology 33, 586-604. doi: 10.1016/j.neuro.2011.12.009

Ek, C. J., Dziegielewska, K. M., Stolp, H., and Saunders, N. R. (2006). Functional effectiveness of the blood-brain barrier to small water-soluble molecules in developing and adult opossum (Monodelphis domestica). J. Comp. Neurol. 496, 13-26. doi: 10.1002/cne.20885

Ek, C. J., Habgood, M. D., Dziegielewska, K. M., Potter, A., and Saunders, N. R. (2001). Permeability and route of entry for lipid-insoluble molecules across brain barriers in developing Monodelphis domestica. J. Physiol. 536, 841-853. doi: 10.1111/j.1469-7793.2001.00841.x

Ek, C. J., Habgood, M. D., Dziegielewska, K. M., and Saunders, N. R. (2003). Structural characteristics and barrier properties of the choroid plexuses in developing brain of the opossum (Monodelphis Domestica). J. Comp. Neurol. 460, 451-464. doi: 10.1002/cne.10661

Engelhardt, B., and Liebner, S. (2014). Novel insights into the development and maintenance of the blood-brain barrier. Cell Tissue Res. 355, 687-699. doi: 10.1007/s00441-014-1811-2

Evrard, P., Marret, S., and Gressens, P. (1997). Environmental and genetic determinants of neural migration and postmigratory survival. Acta Paediatr. Suppl. 422, 20-26. doi: 10.1111/j.1651-2227.1997.tb18339.x

Favrais, G., Schwendimann, L., Gressens, P., and Lelievre, V. (2007). Cyclooxygenase- 2 mediates the sensitizing effects of systemic IL-1-beta on excitotoxic brain lesions in newborn mice. Neurobiol. Dis. 25, 496-505. doi: 10.1016/j.nbd.2006.10.012

Favrais, G., van de Looij, Y., Fleiss, B., Ramanantsoa, N., Bonnin, P., StoltenburgDidinger, G., et al. (2011). Systemic inflammation disrupts the developmental program of white matter. Ann. Neurol. 70, 550-565. doi: 10.1002/ana.22489

Feng, Y., Rhodes, P. G., and Bhatt, A. J. (2008). Neuroprotective effects of vascular endothelial growth factor following hypoxic ischemic brain injury in neonatal rats. Pediatr. Res. 64, 370-374. doi: 10.1203/PDR.0b013e318180ebe6

Ferrari, D. C., Nesic, O. B., and Perez-Polo, J. R. (2010b). Oxygen resuscitation does not ameliorate neonatal hypoxia/ischemia-induced cerebral edema. J. Neurosci. Res. 88, 2056-2065. doi: 10.1002/jnr.22358

Ferrari, D. C., Nesic, O., and Perez-Polo, J. R. (2010a). Perspectives on neonatal hypoxia/ischemia-induced edema formation. Neurochem. Res. 35, 1957-1965. doi: 10.1007/s11064-010-0308-y

Ferriero, D. M., Holtzman, D. M., Black, S. M., and Sheldon, R. A. (1996). Neonatal mice lacking neuronal nitric oxide synthase are less vulnerable to hypoxic-ischemic injury. Neurobiol. Dis. 3, 64-71. doi: 10.1006/nbdi.1996.0006

Fischer, S., Wiesnet, M., Renz, D., and Schaper, W. (2005). $\mathrm{H}_{2} \mathrm{O}_{2}$ induces paracellular permeability of porcine brain-derived microvascular endothelial cells by activation of the p44/42 MAP kinase pathway. Eur. J. Cell Biol. 84, 687-697. doi: 10.1016/j.ejcb.2005.03.002

Fischer, S., Wobben, M., Kleinstuck, J., Renz, D., and Schaper, W. (2000). Effect of astroglial cells on hypoxia-induced permeability in PBMEC cells. Am. J. Physiol. Cell Physiol. 279, C935-C944.

Fischer, S., Wobben, M., Marti, H. H., Renz, D., and Schaper, W. (2002). Hypoxiainduced hyperpermeability in brain microvessel endothelial cells involves VEGF-mediated changes in the expression of zonula occludens-1. Microvasc. Res. 63, 70-80. doi: 10.1006/mvre.2001.2367

Fleiss, B., and Gressens, P. (2012). Tertiary mechanisms of brain damage: a new hope for treatment of cerebral palsy? Lancet Neurol. 11, 556-566. doi: 10.1016/S1474-4422(12)70058-3

Fujimoto, M., Takagi, Y., Aoki, T., Hayase, M., Marumo, T., Gomi, M., et al. (2008). Tissue inhibitor of metalloproteinases protect blood-brain barrier disruption in focal cerebral ischemia. J. Cereb. Blood Flow Metab. 28, 1674-1685. doi: 10.1038/jcbfm.2008.59

Fukuda, A. M., Adami, A., Pop, V., Bellone, J. A., Coats, J. S., Hartman, R. E., et al. (2013). Posttraumatic reduction of edema with aquaporin-4 RNA interference improves acute and chronic functional recovery. J. Cereb. Blood Flow Metab. 33, 1621-1632. doi: 10.1038/jcbfm.2013.118

Fukuda, A. M., Pop, V., Spagnoli, D., Ashwal, S., Obenaus, A., and Badaut, J. (2012). Delayed increase of astrocytic aquaporin 4 after juvenile traumatic brain injury: possible role in edema resolution? Neuroscience 222, 366-378. doi: 10.1016/j.neuroscience.2012.06.033

Gasche, Y., Copin, J. C., Sugawara, T., Fujimura, M., and Chan, P. H. (2001). Matrix metalloproteinase inhibition prevents oxidative stress-associated blood-brain barrier disruption after transient focal cerebral ischemia. J. Cereb. Blood Flow Metab. 21, 1393-1400. doi: 10.1097/00004647-200112000-00003

Georgiadis, P., Xu, H., Chua, C., Hu, F., Collins, L., Huynh, C., et al. (2008). Characterization of acute brain injuries and neurobehavioral profiles in a rabbit model of germinal matrix hemorrhage. Stroke 39, 3378-3388. doi: 10.1161/STROKEAHA.107.510883

Goddard, J., Lewis, R. M., Armstrong, D. L., and Zeller, R. S. (1980). Moderate, rapidly induced hypertension as a cause of intraventricular hemorrhage in the newborn beagle model. J. Pediatr. 96, 1057-1060. doi: 10.1016/S00223476(80)80641-X

Gu, Y., Dee, C. M., and Shen, J. (2011). Interaction of free radicals, matrix metalloproteinases and caveolin-1 impacts blood-brain barrier permeability. Front. Biosci. (Schol. Ed). 3, 1216-1231. doi: 10.2741/222

Gu, Y., Zheng, G., Xu, M., Li, Y., Chen, X., Zhu, W., et al. (2012). Caveolin-1 regulates nitric oxide-mediated matrix metalloproteinases activity and bloodbrain barrier permeability in focal cerebral ischemia and reperfusion injury. J. Neurochem. 120, 147-156. doi: 10.1111/j.1471-4159.2011.07542.x

Gudino-Cabrera, G., Urena-Guerrero, M. E., Rivera-Cervantes, M. C., FeriaVelasco, A. I., and Beas-Zarate, C. (2014). Excitotoxicity triggered by neonatal monosodium glutamate treatment and blood-brain barrier function. Archives Med. Res. doi: 10.1016/j.arcmed.2014.11.014. [Epub ahead of print].

Hagberg, H., Gressens, P., and Mallard, C. (2012). Inflammation during fetal and neonatal life: implications for neurologic and neuropsychiatric disease in children and adults. Ann. Neurol. 71, 444-457. doi: 10.1002/ana. 22620

Hagberg, H., Ichord, R., Palmer, C., Yager, J. Y., and Vannucci, S. J. (2002). Animal models of developmental brain injury: relevance to human disease. A summary of the panel discussion from the third hershey conference on developmental cerebral blood flow and metabolism. Dev. Neurosci. 24, 364-366. doi: $10.1159 / 000069040$

Hagberg, H., and Mallard, C. (2005). Effect of inflammation on central nervous system development and vulnerability. Curr. Opin. Neurol. 18, 117-123. doi: 10.1097/01.wco.0000162851.44897.8f

Hamrick, S. E., and Ferriero, D. M. (2003). The injury response in the term newborn brain: can we neuroprotect? Curr. Opin. Neurol. 16, 147-154. doi: 10.1097/00019052-200304000-00005

Huang, Z., Huang, P. L., Ma, J., Meng, W., Ayata, C., Fishman, M. C., et al. (1996). Enlarged infarcts in endothelial nitric oxide synthase knockout mice are attenuated by nitro-L-arginine. J. Cereb. Blood Flow Metab. 16, 981-987. doi: 10.1097/00004647-199609000-00023

Hudome, S., Palmer, C., Roberts, R. L., Mauger, D., Housman, C., and Towfighi, J. (1997). The role of neutrophils in the production of hypoxic-ischemic brain injury in the neonatal rat. Pediatr. Res. 41, 607-616. doi: 10.1203/00006450199705000-00002

Jain, N. J., Kruse, L. K., Demissie, K., and Khandelwal, M. (2009). Impact of mode of delivery on neonatal complications: trends between 1997 and 2005. J. Mat. Fetal Neonat. Med. 22, 491-500. doi: 10.1080/14767050902769982

Jin, Y., Silverman, A. J., and Vannucci, S. J. (2007). Mast cell stabilization limits hypoxic-ischemic brain damage in the immature rat. Dev. Neurosci. 29, 373-384. doi: 10.1159/000105478

Jin, Y., Silverman, A. J., and Vannucci, S. J. (2009). Mast cells are early responders after hypoxia-ischemia in immature rat brain. Stroke 40, 3107-3112. doi: 10.1161/STROKEAHA.109.549691

Johansson, P. A., Dziegielewska, K. M., Ek, C. J., Habgood, M. D., Liddelow, S. A., Potter, A. M., et al. (2006). Blood-CSF barrier function in the rat embryo. Eur. J. Neurosci. 24, 65-76. doi: 10.1111/j.1460-9568.2006. 04904.x 
Johansson, P. A., Dziegielewska, K. M., Liddelow, S. A., and Saunders, N. R. (2008). The blood-CSF barrier explained: when development is not immaturity. Bioessays 30, 237-248. doi: 10.1002/bies.20718

Kaya, D., Gursoy-Ozdemir, Y., Yemisci, M., Tuncer, N., Aktan, S., and Dalkara, T. (2005). VEGF protects brain against focal ischemia without increasing bloodbrain permeability when administered intracerebroventricularly. J. Cereb. Blood Flow Metab. 25, 1111-1118. doi: 10.1038/sj.jcbfm.9600109

Keenan, H. T., Runyan, D. K., Marshall, S. W., Nocera, M. A., Merten, D. F., and Sinal, S. H. (2003). A population-based study of inflicted traumatic brain injury in young children. JAMA 290, 621-626. doi: 10.1001/jama.290.5.621

Kinney, H. C., and Volpe, J. J. (2012). Modeling the encephalopathy of prematurity in animals: the important role of translational research. Neurol. Res. Int. 2012:295389. doi: 10.1155/2012/295389

Koepsell, T. D., Rivara, F. P., Vavilala, M. S., Wang, J., Temkin, N., Jaffe, K. M., et al. (2011). Incidence and descriptive epidemiologic features of traumatic brain injury in King County, Washington. Pediatrics 128, 946-954. doi: 10.1542/peds.2010-2259

Krafft, P. R., Rolland, W. B., Duris, K., Lekic, T., Campbell, A., Tang, J., et al. (2012). Modeling intracerebral hemorrhage in mice: injection of autologous blood or bacterial collagenase. J. Vis. Exp. e4289. doi: 10.3791/4289

Kumar, A., Mittal, R., Khanna, H. D., and Basu, S. (2008). Free radical injury and blood-brain barrier permeability in hypoxic-ischemic encephalopathy. Pediatrics 122, e722-e727. doi: 10.1542/peds.2008-0269

Kuntz, M., Mysiorek, C., Petrault, O., Petrault, M., Uzbekov, R., Bordet, R., et al. (2014). Stroke-induced brain parenchymal injury drives blood-brain barrier early leakage kinetics: a combined in vivo/in vitro study. J. Cereb. Blood Flow Metab. 34, 95-107. doi: 10.1038/jcbfm.2013.169

Labus, J., Hackel, S., Lucka, L., and Danker, K. (2014). Interleukin-1beta induces an inflammatory response and the breakdown of the endothelial cell layer in an improved human THBMEC-based in vitro blood-brain barrier model. J. Neurosci. Methods 228, 35-45. doi: 10.1016/j.jneumeth.2014.03.002

Lakhan, S. E., Kirchgessner, A., Tepper, D., and Leonard, A. (2013). Matrix metalloproteinases and blood-brain barrier disruption in acute ischemic stroke. Front. Neurol. 4:32. doi: 10.3389/fneur.2013.00032

Lee, C. Z., Xue, Z., Zhu, Y., Yang, G. Y., and Young, W. L. (2007). Matrix metalloproteinase-9 inhibition attenuates vascular endothelial growth factor-induced intracerebral hemorrhage. Stroke 38, 2563-2568. doi: 10.1161/STROKEAHA.106.481515

Lee, J., Croen, L. A., Lindan, C., Nash, K. B., Yoshida, C. K., Ferriero, D. M., et al. (2005). Predictors of outcome in perinatal arterial stroke: a population-based study. Ann. Neurol. 58, 303-308. doi: 10.1002/ana.20557

Lekic, T., Manaenko, A., Rolland, W., Krafft, P. R., Peters, R., Hartman, R. E., et al. (2012). Rodent neonatal germinal matrix hemorrhage mimics the human brain injury, neurological consequences, and post-hemorrhagic hydrocephalus. Exp. Neurol. 236, 69-78. doi: 10.1016/j.expneurol.2012.04.003

Lipton, S. A., and Rosenberg, P. A. (1994). Excitatory amino acids as a final common pathway for neurologic disorders. N. Engl. J. Med. 330, 613-622. doi: 10.1056/NEJM199403033300907

Liu, J., Jin, X., Liu, K. J., and Liu, W. (2012). Matrix metalloproteinase2-mediated claudin-5 redistribution contribute to blood brain barrier damage in early ischemic stroke stage. J. Neurosci. 9, 3044-3057. doi: 10.1523/JNEUROSCI.6409-11.2012

Liu, W., Hendren, J., Qin, X. J., Shen, J., and Liu, K. J. (2009). Normobaric hyperoxia attenuates early blood-brain barrier disruption by inhibiting MMP-9-mediated occludin degradation in focal cerebral ischemia. J. Neurochem. 108, 811-820. doi: 10.1111/j.1471-4159.2008.05821.x

Lo, E. H. (2008). A new penumbra: transitioning from injury into repair after stroke. Nat. Med. 14, 497-500. doi: 10.1038/nm1735

Lodygensky, G. A., Kunz, N., Perroud, E., Somm, E., Mlynarik, V., Huppi, P. S., et al. (2014). Definition and quantification of acute inflammatory white matter injury in the immature brain by MRI/MRS at high magnetic field. Pediatr. Res. 75, 415-423. doi: 10.1038/pr.2013.242

Longhi, L., Saatman, K. E., Raghupathi, R., Laurer, H. L., Lenzlinger, P. M., Riess, P., et al. (2001). A review and rationale for the use of genetically engineered animals in the study of traumatic brain injury. J. Cereb. Blood Flow Metab. 21, 1241-1258. doi: 10.1097/00004647-200111000-00001

Lorant, D. E., Li, W., Tabatabaei, N., Garver, M. K., and Albertine, K. H. (1999). Pselectin expression by endothelial cells is decreased in neonatal rats and human premature infants. Blood 94, 600-609.
Low, J. A. (2004). Determining the contribution of asphyxia to brain damage in the neonate. J. Obstet. Gynaecol. Res. 30, 276-286. doi: 10.1111/j.14470756.2004.00194.x

Lynch, J. K., Hirtz, D. G., DeVeber, G., and Nelson, K. B. (2002). Report of the National Institute of Neurological Disorders and Stroke workshop on perinatal and childhood stroke. Pediatrics 109, 116-123. doi: 10.1542/peds. 109.1.116

Maier, C. M., Hsieh, L., Crandall, T., Narasimhan, P., and Chan, P. H. (2006). Evaluating therapeutic targets for reperfusion-related brain hemorrhage. Ann. Neurol. 59, 929-938. doi: 10.1002/ana.20850

Mark, K. S., and Davis, T. P. (2002). Cerebral microvascular changes in permeability and tight junctions induced by hypoxia-reoxygenation. Am. J. Physiol. Heart Circ. Physiol. 282, H1485-H1494. doi: 10.1152/ajpheart.00645.2001

Marret, S., Mukendi, R., Gadisseux, J. F., Gressens, P., and Evrard, P. (1995). Effect of ibotenate on brain development: an excitotoxic mouse model of microgyria and posthypoxic-like lesions. J. Neuropathol. Exp. Neurol. 54, 358-370. doi: 10.1097/00005072-199505000-00009

Marti, H. J., Bernaudin, M., Bellail, A., Schoch, H., Euler, M., Petit, E., et al. (2000). Hypoxia-induced vascular endothelial growth factor expression precedes neovascularization after cerebral ischemia. Am. J. Pathol. 156, 965-976. doi: 10.1016/S0002-9440(10)64964-4

McColl, B. W., Rothwell, N. J., and Allan, S. M. (2008). Systemic inflammation alters the kinetics of cerebrovascular tight junction disruption after experimental stroke in mice. J. Neurosci. 28, 9451-9462. doi: 10.1523/JNEUROSCI.267408.2008

McLean, C., and Ferriero, D. (2004). Mechanisms of hypoxic-ischemic injury in the term infant. Semin. Perinatol. 28, 425-432. doi: 10.1053/j.semperi.2004. 10.005

Miller, S. L., Wallace, E. M., and Walker, D. W. (2012). Antioxidant therapies: a potential role in perinatal medicine. Neuroendocrinology 96, 13-23. doi: $10.1159 / 000336378$

Muramatsu, K., Fukuda, A., Togari, H., Wada, Y., and Nishino, H. (1997). Vulnerability to cerebral hypoxic-ischemic insult in neonatal but not in adult rats is in parallel with disruption of the blood-brain barrier. Stroke 28, 2281-2288. discussion: 2288-2289. doi: 10.1161/01.STR.28.11.2281

O'Brien, J. S., and Sampson, E. L. (1965). Lipid composition of the normal human brain: gray matter, white matter, and myelin. J. Lipid Res. 6, 537-544.

Ohshima, M., Tsuji, M., Taguchi, A., Kasahara, Y., and Ikeda, T. (2012). Cerebral blood flow during reperfusion predicts later brain damage in a mouse and a rat model of neonatal hypoxic-ischemic encephalopathy. Exp. Neurol. 233, 481-489. doi: 10.1016/j.expneurol.2011.11.025

Perez-Asensio, F. J., Hurtado, O., Burguete, M. C., Moro, M. A., Salom, J. B., Lizasoain, I., et al. (2005). Inhibition of iNOS activity by $1400 \mathrm{~W}$ decreases glutamate release and ameliorates stroke outcome after experimental ischemia. Neurobiol. Dis. 18, 375-384. doi: 10.1016/j.nbd.2004.10.018

Philip, A. G., Allan, W. C., Tito, A. M., and Wheeler, L. R. (1989). Intraventricular hemorrhage in preterm infants: declining incidence in the 1980 s. Pediatrics 84 , 797-801.

Pop, V., Sorensen, D. W., Kamper, J. E., Ajao, D. O., Murphy, M. P., Head, E., et al. (2013). Early brain injury alters the blood-brain barrier phenotype in parallel with beta-amyloid and cognitive changes in adulthood. J. Cereb. Blood Flow Metab. 33, 205-214. doi: 10.1038/jcbfm.2012.154

Purves, M. J. (1972). The physiology of the cerebral circulation. Monogr. Physiol. Soc. $1-414$.

Quan, N., Stern, E. L., Whiteside, M. B., and Herkenham, M. (1999). Induction of pro-inflammatory cytokine mRNAs in the brain after peripheral injection of subseptic doses of lipopolysaccharide in the rat. J. Neuroimmunol. 93, 72-80. doi: 10.1016/S0165-5728(98)00193-3

Ramlackhansingh, A. F., Brooks, D. J., Greenwood, R. J., Bose, S. K., Turkheimer, F. E., Kinnunen, K. M., et al. (2011). Inflammation after trauma: microglial activation and traumatic brain injury. Ann. Neurol. 70, 374-383. doi: 10.1002/ana.22455

Reboldi, A., Coisne, C., Baumjohann, D., Benvenuto, F., Bottinelli, D., Lira, S., et al. (2009). C-C chemokine receptor 6-regulated entry of TH-17 cells into the CNS through the choroid plexus is required for the initiation of EAE. Nat. Immunol. 10, 514-523. doi: 10.1038/ni.1716

Rice, J. E. 3rd, Vannucci, R. C., and Brierley, J. B. (1981). The influence of immaturity on hypoxic-ischemic brain damage in the rat. Ann. Neurol. 9, 131-141. doi: 10.1002/ana.410090206 
Riddle, A., Dean, J., Buser, J. R., Gong, X., Maire, J., Chen, K., et al. (2011). Histopathological correlates of magnetic resonance imaging-defined chronic perinatal white matter injury. Ann. Neurol. 70, 493-507. doi: 10.1002/ana.22501

Rivara, F. P., Vavilala, M. S., Durbin, D., Temkin, N., Wang, J., O’Connor, S. S., et al. (2012). Persistence of disability 24 to 36 months after pediatric traumatic brain injury: a cohort study. J. Neurotrauma 29, 2499-2504. doi: 10.1089/neu.2012.2434

Roozenbeek, B., Maas, A. I., and Menon, D. K. (2013). Changing patterns in the epidemiology of traumatic brain injury. Nat. Rev. Neurol. 9, 231-236. doi: 10.1038/nrneurol.2013.22

Rosenberg, G. A. (2009). Matrix metalloproteinases and their multiple roles in neurodegenerative diseases. Lancet Neurol. 8, 205-216. doi: 10.1016/S14744422(09)70016-X

Rosenberg, G. A., Estrada, E. Y., and Dencoff, J. E. (1998). Matrix metalloproteinases and TIMPs are associated with blood-brain barrier opening after reperfusion in rat brain. Stroke 29, 2189-2195. doi: 10.1161/01.STR.29.10.2189

Rosenberg, G. A., and Yang, Y. (2007). Vasogenic edema due to tight junction disruption by matrix metalloproteinases in cerebral ischemia. Neurosurg. Focus 22, E4. doi: 10.3171/foc.2007.22.5.5

Rossi, J. L., Todd, T., Bazan, N. G., and Belayev, L. (2013). Inhibition of Myosin light-chain kinase attenuates cerebral edema after traumatic brain injury in postnatal mice. J. Neurotrauma 30, 1672-1679. doi: 10.1089/neu.2013.2898

Rousset, C. I., Chalon, S., Cantagrel, S., Bodard, S., Andres, C., Gressens, P., et al. (2006). Maternal exposure to LPS induces hypomyelination in the internal capsule and programmed cell death in the deep gray matter in newborn rats. Pediatr. Res. 59, 428-433. doi: 10.1203/01.pdr.0000199905.08848.55

Sandoval, K. E., and Witt, K. A. (2008). Blood-brain barrier tight junction permeability and ischemic stroke. Neurobiol. Dis. 32, 200-219. doi: 10.1016/j.nbd.2008.08.005

Saunders, N. R., Liddelow, S. A., and Dziegielewska, K. M. (2012). Barrier mechanisms in the developing brain. Front. Pharmacol. 3:46. doi: 10.3389/fphar.2012.00046

Schang, A. L., Van Steenwinckel, J., Chevenne, D., Alkmark, M., Hagberg, H., Gressens, P., et al. (2014). Failure of thyroid hormone treatment to prevent inflammation-induced white matter injury in the immature brain. Brain Behav. Immun. 37, 95-102. doi: 10.1016/j.bbi.2013.11.005

Schmitt, C., Strazielle, N., and Ghersi-Egea, J. F. (2012). Brain leukocyte infiltration initiated by peripheral inflammation or experimental autoimmune encephalomyelitis occurs through pathways connected to the CSF-filled compartments of the forebrain and midbrain. J. Neuroinflammation 9, 187. doi: 10.1186/1742-2094-9-187

Schnell, L., Fearn, S., Schwab, M. E., Perry, V. H., and Anthony, D. C. (1999). Cytokine-induced acute inflammation in the brain and spinal cord. J. Neuropathol. Exp. Neurol. 58, 245-254. doi: 10.1097/00005072-19990300000004

Schuhmann, M. U., Stiller, D., Skardelly, M., Bernarding, J., Klinge, P. M., Samii, A., et al. (2003). Metabolic changes in the vicinity of brain contusions: a proton magnetic resonance spectroscopy and histology study. J. Neurotrauma 20, 725-743. doi: 10.1089/089771503767869962

Semenza, G. L. (2010). Vascular responses to hypoxia and ischemia. Arterioscler. Thromb. Vasc. Biol. 30, 648-652. doi: 10.1161/ATVBAHA.108.181644

Shankaran, S., Laptook, A. R., Ehrenkranz, R. A., Tyson, J. E., McDonald, S. A., Donovan, E. F., et al. (2005). Whole-body hypothermia for neonates with hypoxic-ischemic encephalopathy. N. Engl. J. Med. 353, 1574-1584. doi: 10.1056/NEJMcps050929

Sheldon, R. A., Sedik, C., and Ferriero, D. M. (1998). Strain-related brain injury in neonatal mice subjected to hypoxia-ischemia. Brain Res. 810, 114-122. doi: 10.1016/S0006-8993(98)00892-0

Shimotake, J., Derugin, N., Wendland, M., Vexler, Z. S., and Ferriero, D. M. (2010). Vascular endothelial growth factor receptor-2 inhibition promotes cell death and limits endothelial cell proliferation in a neonatal rodent model of stroke. Stroke 41, 343-349. doi: 10.1161/STROKEAHA.109.564229

Simi, A., Tsakiri, N., Wang, P., and Rothwell, N. J. (2007). Interleukin-1 and inflammatory neurodegeneration. Biochem. Soc. Trans. 35, 1122-1126. doi: 10.1042/BST0351122

Skuli, N., Liu, L., Runge, A., Wang, T., Yuan, L., Patel, S., et al. (2009). Endothelial deletion of hypoxia-inducible factor-2alpha (HIF-2alpha) alters vascular function and tumor angiogenesis. Blood 114, 469-477. doi: 10.1182/blood-2008-12193581
Stanley, R. M., Bonsu, B. K., Zhao, W., Ehrlich, P. F., Rogers, A. J., and Xiang, H. (2012). US estimates of hospitalized children with severe traumatic brain injury: implications for clinical trials. Pediatrics 129, e24-e30. doi: 10.1542/peds.20112074

Stokely, M. E., and Orr, E. L. (2008). Acute effects of calvarial damage on dural mast cells, pial vascular permeability, and cerebral cortical histamine levels in rats and mice. J. Neurotrauma 25, 52-61. doi: 10.1089/neu.2007.0397

Stolp, H. B., Dziegielewska, K. M., Ek, C. J., Potter, A. M., and Saunders, N. R. (2005). Long-term changes in blood-brain barrier permeability and white matter following prolonged systemic inflammation in early development in the rat. Eur. J. Neurosci. 22, 2805-2816. doi: 10.1111/j.1460-9568.2005.04483.x

Stolp, H. B., Johansson, P. A., Habgood, M. D., Dziegielewska, K. M., Saunders, N. R., and Ek, C. J. (2011). Effects of neonatal systemic inflammation on bloodbrain barrier permeability and behaviour in juvenile and adult rats. Cardiovasc. Psychiatry Neurol. 2011:469046. doi: 10.1155/2011/469046

Stonestreet, B. S., Sadowska, G. B., Leeman, J., Hanumara, R. C., Petersson, K. H., and Patlak, C. S. (2006). Effects of acute hyperosmolality on blood-brain barrier function in ovine fetuses and lambs. Am. J. Physiol. Regul. Integr. Comp. Physiol. 291, R1031-R1039. doi: 10.1152/ajpregu.00883.2005

Strbian, D., Karjalainen-Lindsberg, M. L., Tatlisumak, T., and Lindsberg, P. J. (2006). Cerebral mast cells regulate early ischemic brain swelling and neutrophil accumulation. J. Cereb. Blood Flow Metab. 26, 605-612. doi: 10.1038/sj.jcbfm.9600228

Svedin, P., Hagberg, H., Savman, K., Zhu, C., and Mallard, C. (2007). Matrix metalloproteinase-9 gene knock-out protects the immature brain after cerebral hypoxia-ischemia. J. Neurosci. 27, 1511-1518. doi: 10.1523/JNEUROSCI.439106.2007

Szmydynger-Chodobska, J., Strazielle, N., Gandy, J. R., Keefe, T. H., Zink, B. J., Ghersi-Egea, J. F., et al. (2012). Posttraumatic invasion of monocytes across the blood-cerebrospinal fluid barrier. J. Cereb. Blood Flow Metab. 32, 93-104. doi: 10.1038/jcbfm.2011.111

Szmydynger-Chodobska, J., Strazielle, N., Zink, B. J., Ghersi-Egea, J. F., and Chodobski, A. (2009). The role of the choroid plexus in neutrophil invasion after traumatic brain injury. J. Cereb. Blood Flow Metab. 29, 1503-1516. doi: 10.1038/jcbfm.2009.71

Tagin, M. A., Woolcott, C. G., Vincer, M. J., Whyte, R. K., and Stinson, D. A. (2012). Hypothermia for neonatal hypoxic ischemic encephalopathy: an updated systematic review and meta-analysis. Arch. Pediatr. Adolesc. Med. 166, 558-566. doi: 10.1001/archpediatrics.2011.1772

Tahraoui, S. L., Marret, S., Bodenant, C., Leroux, P., Dommergues, M. A., Evrard, P., et al. (2001). Central role of microglia in neonatal excitotoxic lesions of the murine periventricular white matter. Brain Pathol. 11, 56-71. doi: 10.1111/j.1750-3639.2001.tb00381.x

Tcharmtchi, M. H., Smith, C. W., and Mariscalco, M. M. (2000). Neonatal neutrophil interaction with P-selectin: contribution of P-selectin glycoprotein ligand-1 and sialic acid. J. Leukoc. Biol. 67, 73-80.

Thibeault, I., Laflamme, N., and Rivest, S. (2001). Regulation of the gene encoding the monocyte chemoattractant protein $1(\mathrm{MCP}-1)$ in the mouse and rat brain in response to circulating LPS and proinflammatory cytokines. J. Comp. Neurol. 434, 461-477. doi: 10.1002/cne.1187

Thompson, H. J., Lifshitz, J., Marklund, N., Grady, M. S., Graham, D. I., Hovda, D. A., et al. (2005). Lateral fluid percussion brain injury: a 15-year review and evaluation. J. Neurotrauma 22, 42-75. doi: 10.1089/neu.2005.22.42

Tosun, C., Koltz, M. T., Kurland, D. B., Ijaz, H., Gurakar, M., Schwartzbauer, G., et al. (2013). The protective effect of glibenclamide in a model of hemorrhagic encephalopathy of prematurity. Brain Sci. 3, 215-238. doi: 10.3390/brainsci3010215

Tsuji, M., Ohshima, M., Taguchi, A., Kasahara, Y., Ikeda, T., and Matsuyama, T. (2013). A novel reproducible model of neonatal stroke in mice: comparison with a hypoxia-ischemia model. Exp. Neurol. 247, 218-225. doi: 10.1016/j.expneurol.2013.04.015

Tu, Y. F., Tsai, Y. S., Wang, L. W., Wu, H. C., Huang, C. C., and Ho, C. J. (2011). Overweight worsens apoptosis, neuroinflammation and blood-brain barrier damage after hypoxic ischemia in neonatal brain through JNK hyperactivation. J. Neuroinflammation 8:40. doi: 10.1186/1742-2094-8-40

Vannucci, S. J., and Hagberg, H. (2004). Hypoxia-ischemia in the immature brain. J. Exp. Biol. 207, 3149-3154. doi: 10.1242/jeb.01064

Verney, C., Pogledic, I., Biran, V., Adle-Biassette, H., Fallet-Bianco, C., and Gressens, P. (2012). Microglial reaction in axonal crossroads is a hallmark 
of noncystic periventricular white matter injury in very preterm infants. J. Neuropathol. Exp. Neurol. 71, 251-264. doi: 10.1097/NEN.0b013e3182496429

Vexler, Z. S., and Yenari, M. A. (2009). Does inflammation after stroke affect the developing brain differently than adult brain? Dev. Neurosci. 31, 378-393. doi: $10.1159 / 000232556$

Virgintino, D., Errede, M., Robertson, D., Capobianco, C., Girolamo, F., Vimercati, A., et al. (2004). Immunolocalization of tight junction proteins in the adult and developing human brain. Histochem. Cell Biol. 122, 51-59. doi: 10.1007/s00418004-0665-1

Vohr, B., Allan, W. C., Scott, D. T., Katz, K. H., Schneider, K. C., Makuch, R. W., et al. (1999). Early-onset intraventricular hemorrhage in preterm neonates: incidence of neurodevelopmental handicap. Semin. Perinatol. 23, 212-217. doi: 10.1016/S0146-0005(99)80065-2

Wilson-Costello, D., Friedman, H., Minich, N., Fanaroff, A. A., and Hack, M. (2005). Improved survival rates with increased neurodevelopmental disability for extremely low birth weight infants in the 1990s. Pediatrics 115, 997-1003. doi: 10.1542/peds.2004-0221

Witt, K. A., Mark, K. S., Hom, S., and Davis, T. P. (2003). Effects of hypoxiareoxygenation on rat blood-brain barrier permeability and tight junctional protein expression. Am. J. Physiol. Heart Circ. Physiol. 285, H2820-H2831. doi: 10.1152/ajpheart.00589.2003

Xiong, Y., Mahmood, A., and Chopp, M. (2013). Animal models of traumatic brain injury. Nat. Rev. Neurosci. 14, 128-142. doi: 10.1038/nrn3407

Yan, E., Castillo-Melendez, M., Nicholls, T., Hirst, J., and Walker, D. (2004). Cerebrovascular responses in the fetal sheep brain to low-dose endotoxin. Pediatr. Res. 55, 855-863. doi: 10.1203/01.PDR.0000115681.95957.D4

Yang, D., Sun, Y. Y., Nemkul, N., Baumann, J. M., Shereen, A., Dunn, R. S., et al. (2013a). Plasminogen activator inhibitor-1 mitigates brain injury in a rat model of infection-sensitized neonatal hypoxia-ischemia. Cereb. Cortex 23, 1218-1229. doi: 10.1093/cercor/bhs115

Yang, Y., Estrada, E. Y., Thompson, J. F., Liu, W., and Rosenberg, G. A. (2007). Matrix metalloproteinase-mediated disruption of tight junction proteins in cerebral vessels is reversed by synthetic matrix metalloproteinase inhibitor in focal ischemia in rat. J. Cereb. Blood Flow Metab.27, 697-709. doi: 10.1038/sj. jcbfm.9600375

Yang, Y., and Rosenberg, G. A. (2011). MMP-mediated disruption of claudin-5 in the blood-brain barrier of rat brain after cerebral ischemia. Methods Mol. Biol. 762, 333-345. doi: 10.1007/978-1-61779-185-7_24
Yang, Y., Thompson, J. F., Taheri, S., Salayandia, V. M., McAvoy, T. A., Hill, J. W., et al. (2013b). Early inhibition of MMP activity in ischemic rat brain promotes expression of tight junction proteins and angiogenesis during recovery. J. Cereb. Blood Flow Metab. 33, 1104-1114. doi: 10.1038/jcbfm.2013.56

Yoon, B. H., Romero, R., Kim, C. J., Koo, J. N., Choe, G., Syn, H. C., et al. (1997). High expression of tumor necrosis factor-alpha and interleukin-6 in periventricular leukomalacia. Am. J. Obstet. Gynecol. 177, 406-411. doi: 10.1016/S0002-9378(97)70206-0

Yoshioka, H., Iino, S., Sato, N., Osamura, T., Hasegawa, K., Ochi, M., et al. (1989). New model of hemorrhagic hypoxic-ischemic encephalopathy in newborn mice. Pediatr. Neurol. 5, 221-225. doi: 10.1016/0887-8994(89)90079-9

Zehendner, C. M., Librizzi, L., Hedrich, J., Bauer, N. M., Angamo, E. A., de Curtis, M., et al. (2013). Moderate hypoxia followed by reoxygenation results in bloodbrain barrier breakdown via oxidative stress-dependent tight-junction protein disruption. PLoS ONE 8:e82823. doi: 10.1371/journal.pone.0082823

Zhang, W., Smith, C., Howlett, C., and Stanimirovic, D. (2000). Inflammatory activation of human brain endothelial cells by hypoxic astrocytes in vitro is mediated by IL-1beta. J. Cereb. Blood Flow Metab. 20, 967-978. doi: 10.1097/00004647-200006000-00009

Conflict of Interest Statement: The authors declare that the research was conducted in the absence of any commercial or financial relationships that could be construed as a potential conflict of interest.

Received: 17 September 2014; accepted: 27 January 2015; published online: 17 February 2015.

Citation: Moretti R, Pansiot J, Bettati D, Strazielle N, Ghersi-Egea J-F, Damante $G$, Fleiss B, Titomanlio L and Gressens $P$ (2015) Blood-brain barrier dysfunction in disorders of the developing brain. Front. Neurosci. 9:40. doi: 10.3389/fnins.2015.00040 This article was submitted to Neurogenomics, a section of the journal Frontiers in Neuroscience.

Copyright (C) 2015 Moretti, Pansiot, Bettati, Strazielle, Ghersi-Egea, Damante, Fleiss, Titomanlio and Gressens. This is an open-access article distributed under the terms of the Creative Commons Attribution License (CC BY). The use, distribution or reproduction in other forums is permitted, provided the original author(s) or licensor are credited and that the original publication in this journal is cited, in accordance with accepted academic practice. No use, distribution or reproduction is permitted which does not comply with these terms. 


\title{
Neurogenic niches in the brain: help and hindrance of the barrier systems
}

\author{
Helen B. Stolp ${ }^{1 *}$ and Zoltán Molnár ${ }^{2}$ \\ ${ }^{1}$ Division of Biomedical Engineering and Health Sciences, Department of Perinatal Imaging and Health, King's College London, London, UK \\ ${ }^{2}$ Department of Physiology, Anatomy and Genetics, University of Oxford, Oxford, UK
}

\author{
Edited by: \\ Joana A. Palha, University of Minho, \\ Portugal \\ Reviewed by: \\ Isabel Farinas, Universidad de \\ Valencia, Spain \\ Joao C. Sousa, University of Minho, \\ Portugal \\ *Correspondence: \\ Helen B. Stolp, Department of \\ Perinatal Imaging and Health, Level \\ 1, 1st Floor, South Wing, St \\ Thomas' Hospital, King's College \\ London, London, SE1 7EH, UK \\ e-mail: helen.stolp@kcl.ac.uk
}

In the developing central nervous system, most neurogenesis occurs in the ventricular and subventricular proliferative zones. In the adult telencephalon, neurogenesis contracts to the subependyma zone and the dentate gyrus (subgranular zone) of the hippocampus. These restricted niches containing progenitor cells which divide to produce neurons or glia, depending on the intrinsic and environmental cues. Neurogenic niches are characterized by a comparatively high vascular density and, in many cases, interaction with the cerebrospinal fluid (CSF). Both the vasculature and the CSF represent a source of signaling molecules, which can be relatively rapidly modulated by external factors and circulated through the central nervous system. As the brain develops, there is vascular remodeling and a compartmentalization and dynamic modification of the ventricular surface which may be responsible for the change in the proliferative properties. This review will explore the relationship between progenitor cells and the developing vascular and ventricular space. In particular the signaling systems employed to control proliferation, and the consequence of abnormal vascular or ventricular development on growth of the telencephalon. It will also discuss the potential significance of the barriers at the vascular and ventricular junctions in the influence of the proliferative niches.

\footnotetext{
Keywords: neurogenesis, neuronal progenitors, neurogenic niche, blood-brain barrier, cerebrovasculature, cerebrospinal fluid, choroid plexus
}

\section{INTRODUCTION}

It is essential that an organ and its blood supply should develop together in synchrony to allow optimal conditions for the different stages of growth, differentiation and changing functional requirements. This co-dependence is particularly evident in the brain, where the vascular network originates from the peri-neural vascular plexus in early embryonic development and undergoes multiple stages of remodeling to meet the changing needs of the complex developing environment (Bautch and James, 2009). Barrier functions of the neurovasculature start from the earliest stages of development and are coupled with other aspects of vascular and neuro development (Ruhrberg and Bautch, 2013).

Close examination of developing brain has led to the hypothesis that signals from the brain parenchyma itself regulate the sites of penetrance by the pioneering vascular branches from the perineural plexus, and there is now increasing evidence that variations in normal brain growth can alter the vasculature (Vasudevan et al., 2008) in the same way that altered vascular development affects neural patterning (Javaherian and Kriegstein, 2009). This is, at least partly, due to the presence of a variety of co-signaling systems, e.g., vascular endothelial growth factor (VEGF), Notch, Sonic Hedgehog (Shh) and their receptors, which clearly have a

Abbreviations: CSF, cerebrospinal fluid; SEZ, subependymal zone/also called the adult SVZ; SGZ, subgranular zone; Shh, sonic hedgehog; Smo, smoothened receptor; SVZ, subventricular zone; VCAM, vascular cell adhesion molecule; VEGF, vascular endothelial growth factor; VZ, ventricular zone. role in both the developing neural system as well as the developing vascular system. The involvement of signaling molecules in the neural and vascular systems, while implying close interaction between vascular and neural development, also makes independent manipulation of the systems difficult, and therefore determination of cause and effect in vascular and neural developmental processes is an intractable issue.

During development, the primary regions of proliferation are the ventricular and subventricular zones (VZ and SVZ, respectively, see Figure 1). These are substantive pools of progenitor cells which over time gradually become restricted in their differentiation capacity, and which ultimately produce the diverse neuronal populations of the brain as well as most of the glial cells. In the adult brain (see Figure 2), the proliferative zones are confined to two main areas of the brain, the subependymal zone (SEZ, also called the adult SVZ) and the subgranular zone (SGZ), and the progenitor cells have a more restricted potential fate.

The relationship between neural progenitors and the vasculature has been extensively described, for both the adult SEZ (Shen et al., 2008), and SGZ (Palmer et al., 2000), as well as for the SVZ in the developing cerebral cortex (Stubbs et al., 2009) and developing rostral migratory stream (Nie et al., 2010). In all cases, a clustering of progenitor cells in close proximity with the cerebral endothelial cells was found to occur to at a greater frequency that expected by chance. This observation has led to the hypothesis that the endothelial cells provide a support for the neural progenitors, either structural or chemical, a concept that is 


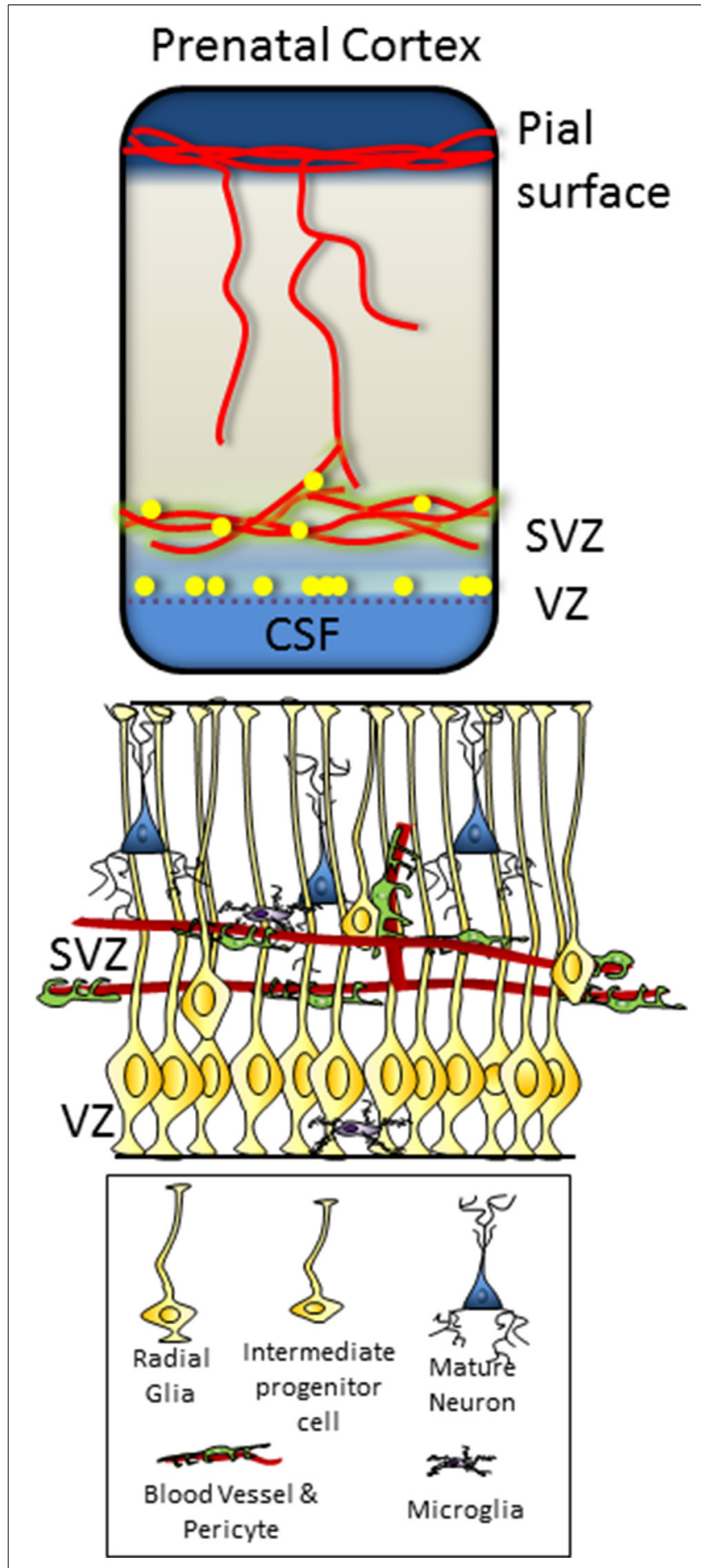

FIGURE 1 | Dorsal telencephalon in the fetus, showing pial and ventricular vascular plexi and the location of the VZ and SVZ progenitor zones. Microstructure and cellular component of the $\mathrm{VZ}$ and SVZ illustrated below, with VZ progenitors contacting the ventricular surface of the brain and SVZ progenitors in close contact with the vascular plexus.

now supported by substantial evidence and is discussed in detail below.

The structure and location of the proliferative zones suggest a second regulator of the neurogenic niche, the cerebrospinal fluid (CSF). Both the VZ in the developing brain and the SEZ in the adult have close association with the ventricular surface and the CSF. Therefore, in addition to proliferative support from the cerebrovasculature, trophic regulation/modulation is thought to come from the CSF (Dziegielewska et al., 2000).

The vasculature in the brain is specialized compared to that of the rest of the body. The presence of the blood-brain barrier means there is a tighter control of brain microenvironment as there is limited paracellular transport and reduced vesicular transport of molecules from the blood into the brain. The composition of the CSF is also tightly controlled, in this case by the choroid plexus. The presence of a structural and functional barrier at the epithelial interface of the choroid plexus with the CSF is an essential element of this control. During development, the composition of the CSF is very dynamic, and is considered to be an important for the regulation of a number of neurodevelopmental processed. The specifics of CSF composition regulation and the role of the blood-CSF barrier is beyond the scope of this work, readers are directed to recent reviews by Bueno et al. (2014) and Johansson (2014).

Very few studies have assessed whether the brain barriers actively control progenitor proliferation in neurogenic niches within the brain, but it is likely that at the least the presence of these barriers make an important contribution to the signaling environment of the neurovascular niches. In the case of the VZ, the developmental barrier between the brain and the CSF (Mollgard et al., 1987) may also contribute directly to the proliferative environment.

In this review we will explore the intertwined development of the vasculature and the progenitor pools in the developing brain, the signaling systems common to all neurogenic niches and whether the brain- barrier systems at the endothelial cell and ventricular surface contribute to the control of these specialized brain regions.

\section{REGULATION OF VASCULAR GROWTH AND BARRIER FORMATION IN THE BRAIN}

The blood vessel ingrowth from the peri-neural plexus (as shown in Figure 1) to the brain parenchyma is regulated by a number of signaling cues from the parenchyma itself. VEGF signaling is of particular importance, with James et al. (2009) showing that the stereotypical pattern of vascular ingrowth into the neural tube can be disrupted by local over expression of VEGF isotypes (causing ectopic vessel ingrowth) or the VEGF receptor Flt1 (which reduces angiogenesis).

Vasudevan et al. (2008) have shown that the formation of the vascular plexus in the cortex is guided by the neural transcription factors that regulate the proliferation in the pallium and subpallium (e.g., Pax6, Nkx2.1, Dlx1/2). They also put forward the idea that vascular plexi of different origin (e.g., ventral pallium or peri-neural plexus) are differentially controlled by the local proliferative transcription factors. This interplay between neural cells and the vasculature does not stop at proliferative stages of brain growth. The radial migration of vessels from the vascular plexus through the cortex in the human brain appears to be regulated by $\mathrm{cxcl} 12 / \mathrm{cxcr} 4 / \mathrm{cxcr} 7$ signaling between the vessels, the radial glial and the perivascular astrocytes (Virgintino et al., 2013). The cxcl12 signaling system appears to be key in regulating 


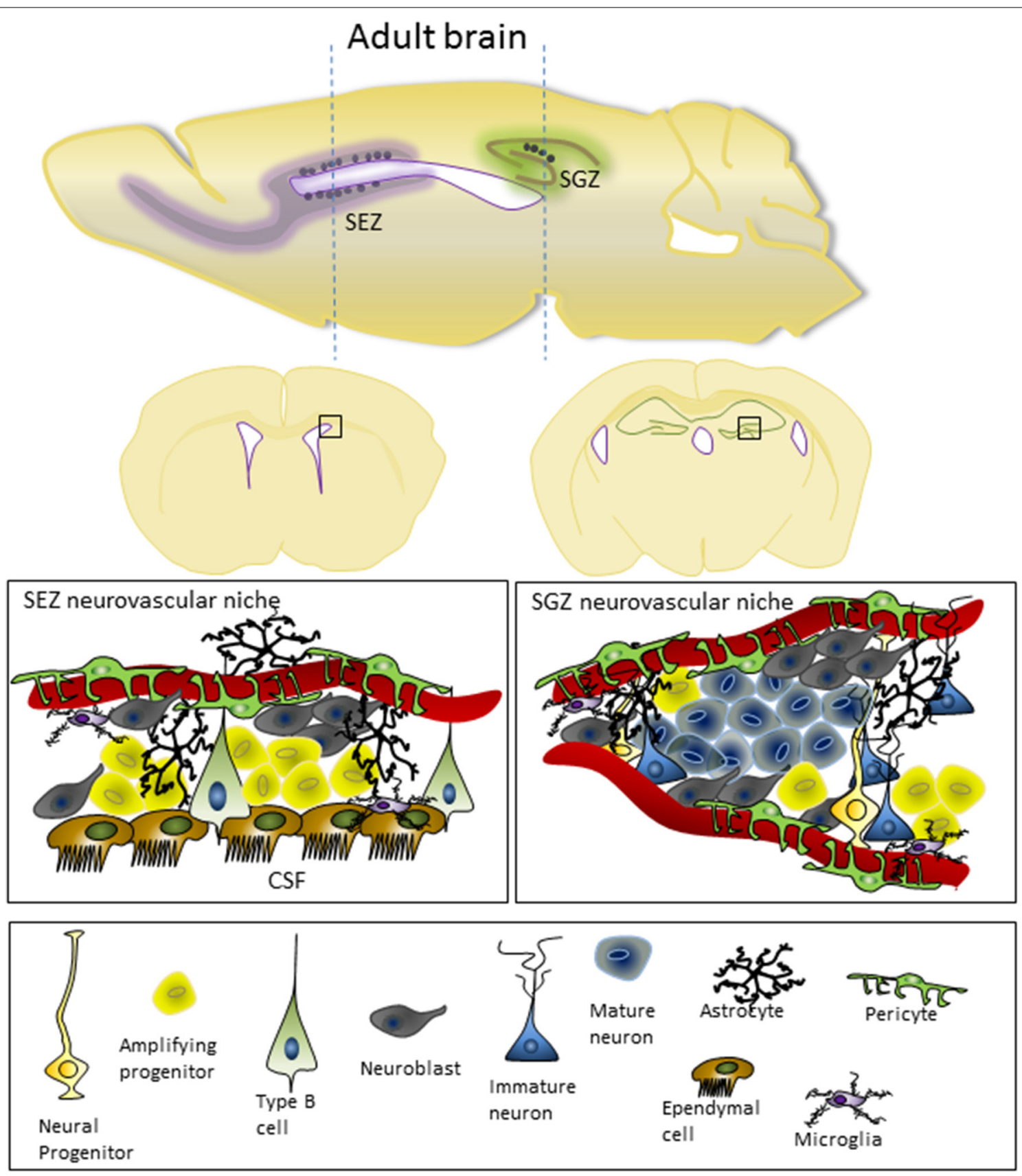

FIGURE 2 | Adult cortex in the mouse, shown from both sagittal and coronal views, indicating the location of the SEZ and SGZ neurovascular niches. The microstructure and cellular component of the SEZ and SGZ are shown underneath. In the SEZ, progenitor cells are in close contact with the
CSF and ventricular ependymal cells as well as the vasculature, while the $S G Z$ is restricted to influences from the vasculature. For details on the cell types involved and the molecular characteristics of these cells see the recent review from Fuentealba et al. (2012). many migratory processes in the developing brain, and is probably also involved in controlling progenitor differentiation and migration (discussed below).

Sonic hedgehog (Shh) is a major signaling molecule that regulates both vascular and neural development and may be important in the establishment of the neurovascular niche. Shh has a clear role in regulating neural development, and in the dorsal cortex conditional knockout of Shh or its receptor Smoothened
(Smo) results in reduced cell division, and a smaller cortex (Komada et al., 2008). There is also recent evidence that Shh regulates vascular development and blood-brain barrier formation (Alvarez et al., 2011). In vitro, Shh in astrocytes conditioned media interacts with receptors on endothelial cells facilitating formation of barrier type properties. Vascular specific knockout of Smo results in impaired blood-brain barrier formation and disrupted extracellular matrix, detectable as early as E14 (Alvarez 
et al., 2011). It is important to remember that astrocytes are not a major constituent of the brain during very early stages of neurovascular development and therefore more work is required to determine (a) if neural progenitors may be an additional source of Shh, contributing to the control of vascular development in the embryo, and (b) whether vascular Shh contributes a proliferative signal to the neural progenitors.

$\beta$-catenin and the Wnt signaling pathway also affect vessel ingrowth from the peri-neural plexus and the structure of the developing vessels. Endothelial specific modifications of $\beta$ catenin expression, Wnt7 knockout or delivery of the soluble frizzled-8 receptor to bind extracellular Wnt ligand all cause a disruption in the vascular ingrowth into the parenchyma and malformation of the vessel beds, characterized by enlarged vascular space and thickening of the vascular wall with multiple layers of endothelial cells (Stenman et al., 2008; Daneman et al., 2009). In addition, Wnt signaling has also been shown to affect the barrier properties of the developing parenchymal vessels. Knockout of Wnt7 was associated with reduced GLUT1 expression (Stenman et al., 2008; Daneman et al., 2009), although there was no change in the expression of tight junction proteins. In contrast, endothelial specific $\beta$-catenin knockout showed an increased GLUT1 expression (Daneman et al., 2009), indicating that regulation of GLUT and barrier properties by the Wnt pathway is not a clear cut mechanism, and instead is likely to respond to numerous conflicting regulatory signals. However, in general the canonical Wnt signaling has been shown to affect blood-brain barrier permeability and the presence of tight junction proteins at the endothelial cells, where $\beta$-catenin gain-of-function stabilizes barrier properties, in response to other changes in Wnt signaling (Liebner et al., 2008). It is possible that the mechanism for this variation is a change in the polarity of the endothelial cells, mediated by Wnt signaling (Artus et al., 2014), which inhibits the formation of tight junctions. The polarity of progenitor cells in the $\mathrm{VZ}$ is of clear importance for the formation of junctions and regulating neurogenesis (discussed in more detail below), which provides support for a similar mechanism in the vasculature.

Contact-dependent signaling is another local environmental regulator of growth. McCarty et al. (2002) have shown that deletion of av-integrin causes abnormal vascular ingrowth and alignment into the ganglionic eminence from as early as E10.5. These ultimately led to enlarged vascular space, thinning of the endothelial lining and hemorrhage, and are associated with disruption of the neuroepithelial cells in the surrounding tissue. These disruptions appear to be due to the lack of adhesion with the perivascular basement membrane, rather than endothelial specific signaling or pericyte localization with the vasculature (McCarty et al., 2002), and highlights the importance of all components of the brain microenvironment for the establishment of the neurovascular and neurogenic niche.

\section{VASCULAR CONTROL OF NEURAL PROLIFERATION}

In turn, vascular development can control proliferation and brain development. For example, ectopic vascular development, induced by in utero injection of VEGF into the dorsal cortex, causes a change in distribution of the Tbr2 positive SVZ progenitor cells in conjunction with the new vascular network, and a disruption of radial fibers and axonal ingrowth into the surrounding tissue (Javaherian and Kriegstein, 2009). One of the first studies confirming that cerebral endothelial cells produce neurotophic factors was performed by Leventhal et al. (1999), where co-culture of cells from the adult SEZ with endothelial cells was found to enhance cell survival in vitro. These studies confirmed that BDNF produced by the endothelial cells was in fact secreted to affect the neurogenic precursors. Further coculture studies confirmed that secretions from endothelial cells increased proliferation of precursors and ultimately facilitated the production of a larger number of neurons, of all neuronal classes (Shen et al., 2004). Early work from Tavazoie et al. (2008) suggests that elements of the blood-brain barrier may be different in the SEZ neurovascular niche. They specifically showed that progenitor cells contact blood vessels in place of some of the normal astrocytic endfeet contacts and that this appears to correlate with patches of reduced presence of tight junction proteins and increased permeability. Thus, they hypothesize that cells within the SEZ have an altered exposure to secreted proteins due to changed blood-brain barrier function.

There are certainly many studies showing both secreted protein from endothelial cells, and progenitor-endothelial cell contact is important for maintain proliferation in the SEZ. Transplant studies of neural precursor cells into the SEZ have shown that the chemokine cxcl12, which is produced by endothelial cells, is important for the localization of these cells to the vasculature. Cxcl12 appears to act through the activation of integrin that allows cell binding to the endothelial cell wall, and support migration of differentiating cells out of the SEZ (Kokovay et al., 2010).

Vascular production of VEGF is likely to be due to HIF activation in response to the low oxygen environment of the developing brain. HIF knockout in neural crest cells leads to reduced vascular density in the developing brain, and gross abnormalities in cellular migration (Tomita et al., 2003). There is also substantial hydrocephaly in these animals, possibly suggesting altered neural proliferation and/or the over production of CSF. Tamoxifeninduced knockout of HIF- $1 \alpha$ in neural stem cells in the SEZ confirms a constitutive role for this molecule in maintenance of the progenitor population as well as vascular development ( $\mathrm{Li}$ et al., 2014). As well as altering VEGF production, HIF activation can induce nitric oxide synthesis. Endogenous production of nitric oxide has been shown to negatively regulate progenitor production (Moreno-Lopez et al., 2004), and may act through one of a number of signaling pathways [reviewed by Goldman and Chen (2011)]. Neurotrophin-3 is another molecule released by endothelial cells and found in the CSF that appears to maintain progenitor quiescence/self-renewal through nitric oxide signaling (Delgado et al., 2014). Endothelial-derived pigment epithelium derived factor (PEDF) is another trophic factor that has been show to maintain stem cell renewal in the SEZ, in this case acting through notch signaling (Ramirez-Castillejo et al., 2006; AndreuAgullo et al., 2009). Local purinergic signaling has also recently be suggested to contribute to self-renewal of progenitors in the SEZ, though it is currently unclear whether these come from the endothelial cells, or other components of the neurogenic niche (see Goldman and Chen, 2011). 
Recent work has provided evidence in support of a further neurogenic niche in the adult brain, that of perivascular stem cells that do not proliferate in control conditions, but are upregulated following injury, such as ischemia (Ohira et al., 2010; Nakagomi et al., 2011). The nature of the neurogenic niche for this proliferative population is still not clear, though the recovery of the cortex after ischemia has been shown to be improved by cotransplantation of endothelial cells and neural stem cells (Nakagomi et al., 2009). However, the cells produced from the perivascular progenitors activated by ischemia appear to be more gliogenic than neurogenic (Nakagomi et al., 2011), and may, therefore, have a different relationship with the vasculature. As an extension to this, it has recently been suggested that signaling from the meninges call also regulate proliferative/differentiation decisions made in the VZ and SVZ of the developing brain [discussed by Sockanathan and Gaiano (2009)].

\section{ROLE OF CELL-TO-CELL CONTACTS IN REGULATION OF PROLIFERATION}

In addition to secreted signaling to neural precursors, the endothelial cells may also support the progenitor population in a contact-dependent manner (contact between type B and endothelial cells in the SEZ is shown in Figure 2). Ephrin and notch signaling have both been shown to be activated by contact between type B progenitors and endothelial cells (Ottone et al., 2014). Notch signaling pathways were up-regulated in cells co-cultured with endothelial cells, and Shen et al. (2004) hypothesized that the expansion of the progenitor population in connected sheets co-cultured with endothelial cells might also induce $\beta$-catenin signaling, another indication that junctions between progenitor cells may be essential for proliferation. This is certainly the case in the VZ, where the junctions between the neuroepithelial cells at the CSF-brain barrier appear to be essential for normal proliferation. $\beta$-catenin and $\mathrm{N}$-cadherin are important components of these junctions, and disruption of $\beta$-catenin in the apical endfeet of the projector cells results in reduced proliferation, increased cell death and cortical malformation (Junghans et al., 2005). Hatakeyama et al. (2014) have also clearly shown that disruption of junctions at the ventricular surface affect proliferation. Electroporation with a dominant-negative form of cadherin, which lack the extracellular domain and disrupts the adherens junctions, resulted in a retraction of apical foot process followed by the production of ectopic neurons within the VZ and a clear abolishment of immunoreactivity of the junctional protein $\mathrm{ZO}$ 1 in the adjacent area of the ventricular surface. The upshot of this process was an increased differentiation of neurons over production of progenitors within the electroporated region of the brain. Further work from these researchers suggest that in this experimental paradigm canonical $\beta$-caterinin/Wnt signaling was not affected, but that Notch signaling was down-regulated (Hatakeyama et al., 2014). In both endothelial cells and neuroepithelial cell progenitors, the production of delta-like ligand from selected cells in the population appear to regulate Notch signaling in the juxtaposed neighboring cells, and this may facilitate selective population changes like initiating vessel sprouting (e.g., Hellstrom et al., 2007) or selective neuronal differentiation. Further work is required to determine whether this sort of interaction between juxtaposed neural stem cells and endothelial cells is part of the signaling system in the neurovascular niche.

While the ventricular zone in the developing brain is characterized by specialized junctions between the neuroepithelial cells, there are no such junctions between the ependymal cells that make the border with the CSF in the adult SEZ. The absence of these junctions between the ependymal cells appears to be very important for the structure of the SEZ, as progenitor cells are intercalated between the ependymal cells (Shen et al., 2008) and the ependymal cells of the ventricular wall form a rosette like arrangement where projections from the progenitor cells protrude through the center of the rosette to make direct contact with the CSF (Mirzadeh et al., 2008). However, it has been hypothesized that while traditional junctions don't exist between ependymal cells, the maintenance of apical adhesions between cells at the ventricular surface is essential for the formation of this specialized proliferative zone (Mirzadeh et al., 2008). The finding that there is close association of the cell adhesion molecule VCAM1 with the type B1 cells at the center of the pinwheels/rosettes, and that rosette formation is disrupted when VCAM is knocked out (Kokovay et al., 2012), supports this hypothesis.

\section{CSF-NEUROGENIC NICHE}

Both the VZ in the developing brain and the SEZ in the adult brain have clear connections with the CSF (see Figures 1, 2), and it has been shown that the CSF contains many trophic factors important for regulating proliferation in these zones (Johansson et al., 2010; Falcao et al., 2012; Stolp, 2013). Johansson et al. (2013) showed that knockout of the 4 th ventricle choroid plexus by selective Otx2 deletion was sufficient to change the composition of the CSF and proliferation in the cortical VZ. These changes were likely, at least in part, to be mediated by Wnt signaling, which was found to be modulated in the CSF of transgenic animals (Johansson et al., 2013). Insulin-like growth factor (Igf) 2 is another molecule that has been shown to be produced by the choroid plexus to circulate in the CSF during early development. It is then able to interact with the Igf receptors on the ventricular surface and regulate proliferation within the $\mathrm{VZ}$ in an age dependent manner (Lehtinen et al., 2011).

The possibility that systemic signaling may directly alter the neurogenic niche has been explored by a number of authors, and it is clear that signaling through inflammatory pathways can cause short- and long-term changes in proliferation in the brain (Stolp et al., 2012; Stolp, 2013). In the developing brain, a systemic inflammatory challenge resulted in decreased proliferation in the VZ, which was associated with local changes in $\beta$-catenin immunoreactivity and the integrity of the junctions at the ventricular surface (Stolp et al., 2011). Prenatal inflammation produces a long-term reduction in proliferation, as shown in the SGZ by Graciarena et al. (2013), which is equivalent to that produced by an adult systemic inflammatory response. In this case, the SGZ was affected, rather than the SVZ, implying a tissue specific alteration, which may be more related to the vasculature or local microglia than changes in CSF signaling. Chronic inflammation, such as in models of Multiple Sclerosis, has also been found to result in reduced neurogenesis within the brain, though the mechanisms of this have not been fully explored (Pluchino 
et al., 2008; Tepavcevic et al., 2011). One potential mediator of this response is IL- $1 \beta$. The IL-1 receptor is found on the type B cell in the SEZ. IL-1 is produced by the choroid plexus cells and released into the CSF (Kokovay et al., 2012), and is upregulated by peripheral inflammation (Marques et al., 2007). Injection of IL-1 $\beta$ into the lateral ventricles increased VCAM- 1 expression in the SEZ and reduced the proliferation of type B cells (Kokovay et al., 2012). Cxcl12 is another typically inflammatory mediator that appears to play an important role in the regulation of the SEZ. As well as being produced by endothelia cells (as described above), it is highly expressed by the ependymal cells of the SEZ, and helps to maintain progenitors in their proliferative state (Kokovay et al., 2010). The contribution of cxcl12 to the neurogenic niches is a clear example of the dual influence of the CSF/ependymal and the microvasculature in neurogenesis, and the stage specific mobilization of progenitors through to differentiated neurons or glia which is essential in the brain [discussed by Kokovay et al. (2010); reviewed by Goldman and Chen (2011)].

\section{CONCLUSION}

Both the CSF and the vasculature of the brain provide regulatory niches for neurogenesis in the developing and adult brain. Variation in angiogenesis and CSF production clearly affect proliferation in the neurogenic niches as a result of cross-talk between these regions, which appears to be dependent both on secreted tropic factors and contact-dependent signaling pathways. The brain barriers, which regulate the internal environment and contribute to the contact between cells in the neurogenic niches are part of this complex system that can be altered both in systemic and central injury. The localization of neurogenic niches makes them sensitive to circulating soluble factors. Recent work from Villeda et al. (2011) highlights this point, showing that the chemokine CCL11 in the plasma and CSF during aging can regulate neurogenesis in the SGZ, which in turn affects learning and memory. The local and systemic control of the neurogenic niches therefore has significant impact on brain function throughout life. Disruption of neurovascular/CSF signaling in early development is likely to contribute to long-term disruption of neural organization and may be a contributor to neurodevelopmental disorders such as autism, schizophrenia and epilepsy. As such, it is essential that we increase our understanding of the control of neurogenic niches and how local and systemic injury/inflammation affect proliferative and differentiation cues in these regions.

\section{REFERENCES}

Alvarez, J. I., Dodelet-Devillers, A., Kebir, H., Ifergan, I., Fabre, P. J., Terouz, S., et al. (2011). The Hedgehog pathway promotes blood-brain barrier integrity and CNS immune quiescence. Science 334, 1727-1731. doi: 10.1126/science. 1206936

Andreu-Agullo, C., Morante-Redolat, J. M., Delgado, A. C., and Farinas, I. (2009). Vascular niche factor PEDF modulates Notch-dependent stemness in the adult subependymal zone. Nat. Neurosci. 12, 1514-1523. doi: 10.1038/ nn.2437

Artus, C., Glacial, F., Ganeshamoorthy, K., Ziegler, N., Godet, M., Guilbert, T., et al. (2014). The Wnt/planar cell polarity signaling pathway contributes to the integrity of tight junctions in brain endothelial cells. J. Cereb. Blood Flow Metab. 34, 433-440. doi: 10.1038/jcbfm.2013.213

Bautch, V. L., and James, J. M. (2009). Neurovascular development: the beginning of a beautiful friendship. Cell Adh. Migr. 3, 199-204. doi: 10.4161/cam. 3.2.8397
Bueno, D., Parvas, M., Hermelo, I., and Garcia-Fernandez, J. (2014). Embryonic blood-cerebrospinal fluid barrier formation and function. Front. Neurosci. 8:343. doi: 10.3389/fnins.2014.00343

Daneman, R., Agalliu, D., Zhou, L., Kuhnert, F., Kuo, C. J., and Barres, B. A. (2009). $\mathrm{Wnt} / \mathrm{beta}$-catenin signaling is required for CNS, but not non-CNS, angiogenesis. Proc. Natl. Acad. Sci. U.S.A. 106, 641-646. doi: 10.1073/pnas.0805165106

Delgado, A. C., Ferron, S. R., Vicente, D., Porlan, E., Perez-Villalba, A., Trujillo, C. M., et al. (2014). Endothelial NT-3 delivered by vasculature and CSF promotes quiescence of subependymal neural stem cells through nitric oxide induction. Neuron 83, 572-585. doi: 10.1016/j.neuron.2014.06.015

Dziegielewska, K. M., Knott, G. W., and Saunders, N. R. (2000). The nature and composition of the internal environment of the developing brain. Cell. Mol. Neurobiol. 20, 41-56. doi: 10.1023/A:1006943926765

Falcao, A. M., Marques, F., Novais, A., Sousa, N., Palha, J. A., and Sousa, J. C. (2012). The path from the choroid plexus to the subventricular zone: go with the flow! Front. Cell. Neurosci. 6:34. doi: 10.3389/fncel.2012.00034

Fuentealba, L. C., Obernier, K., and Alvarez-Buylla, A. (2012). Adult neural stem cells bridge their niche. Cell Stem Cell 10, 698-708. doi: 10.1016/j.stem.2012.05.012

Goldman, S. A., and Chen, Z. (2011). Perivascular instruction of cell genesis and fate in the adult brain. Nat. Neurosci. 14, 1382-1389. doi: 10.1038/nn.2963

Graciarena, M., Roca, V., Mathieu, P., Depino, A. M., and Pitossi, F. J. (2013). Differential vulnerability of adult neurogenesis by adult and prenatal inflammation: role of TGF-beta1. Brain Behav. Immun. 34, 17-28. doi: 10.1016/j.bbi.2013.05.007

Hatakeyama, J., Wakamatsu, Y., Nagafuchi, A., Kageyama, R., Shigemoto, R., and Shimamura, K. (2014). Cadherin-based adhesions in the apical endfoot are required for active Notch signaling to control neurogenesis in vertebrates. Development 141, 1671-1682. doi: 10.1242/dev.102988

Hellstrom, M., Phng, L. K., Hofmann, J. J., Wallgard, E., Coultas, L., Lindblom, P., et al. (2007). Dll4 signalling through Notch1 regulates formation of tip cells during angiogenesis. Nature 445, 776-780. doi: 10.1038/nature05571

James, J. M., Gewolb, C., and Bautch, V. L. (2009). Neurovascular development uses VEGF-A signaling to regulate blood vessel ingression into the neural tube. Development 136, 833-841. doi: 10.1242/dev.028845

Javaherian, A., and Kriegstein, A. (2009). A stem cell niche for intermediate progenitor cells of the embryonic cortex. Cereb. Cortex 19 Suppl. 1, i70-i77. doi: $10.1093 /$ cercor/bhp029

Johansson, P. A. (2014). The choroid plexuses and their impact on developmental neurogenesis. Front. Neurosci. 8:340. doi: 10.3389/fnins.2014.00340

Johansson, P. A., Cappello, S., and Gotz, M. (2010). Stem cells niches during development-lessons from the cerebral cortex. Curr. Opin. Neurobiol. 20, 400-407. doi: 10.1016/j.conb.2010.04.003

Johansson, P. A., Irmler, M., Acampora, D., Beckers, J., Simeone, A., and Gotz, M. (2013). The transcription factor Otx2 regulates choroid plexus development and function. Development 140, 1055-1066. doi: 10.1242/dev.090860

Junghans, D., Hack, I., Frotscher, M., Taylor, V., and Kemler, R. (2005). Betacatenin-mediated cell-adhesion is vital for embryonic forebrain development. Dev. Dyn. 233, 528-539. doi: 10.1002/dvdy.20365

Kokovay, E., Goderie, S., Wang, Y., Lotz, S., Lin, G., Sun, Y., et al. (2010). Adult SVZ lineage cells home to and leave the vascular niche via differential responses to SDF1/CXCR4 signaling. Cell Stem Cell 7, 163-173. doi: 10.1016/j.stem.2010.05.019

Kokovay, E., Wang, Y., Kusek, G., Wurster, R., Lederman, P., Lowry, N., et al. (2012). VCAM1 is essential to maintain the structure of the SVZ niche and acts as an environmental sensor to regulate SVZ lineage progression. Cell Stem Cell 11, 220-230. doi: 10.1016/j.stem.2012.06.016

Komada, M., Saitsu, H., Kinboshi, M., Miura, T., Shiota, K., and Ishibashi, M. (2008). Hedgehog signaling is involved in development of the neocortex. Development 135, 2717-2727. doi: 10.1242/dev.015891

Lehtinen, M. K., Zappaterra, M. W., Chen, X., Yang, Y. J., Hill, A. D., Lun, M., et al. (2011). The cerebrospinal fluid provides a proliferative niche for neural progenitor cells. Neuron 69, 893-905. doi: 10.1016/j.neuron.2011.01.023

Leventhal, C., Rafii, S., Rafii, D., Shahar, A., and Goldman, S. A. (1999). Endothelial trophic support of neuronal production and recruitment from the adult mammalian subependyma. Mol. Cell. Neurosci. 13, 450-464. doi: 10.1006/mcne.1999.0762

Li, L., Candelario, K. M., Thomas, K., Wang, R., Wright, K., Messier, A., et al. (2014). Hypoxia inducible factor- $1 \alpha$ (HIF- $1 \alpha)$ is required for neural stem cell 
maintenance and vascular stability in the adult mouse SVZ. J. Neurosci. 34, 16713-16719. doi: 10.1523/JNEUROSCI.4590-13.2014

Liebner, S., Corada, M., Bangsow, T., Babbage, J., Taddei, A., Czupalla, C. J., et al. (2008). Wnt/beta-catenin signaling controls development of the blood-brain barrier. J. Cell Biol. 183, 409-417. doi: 10.1083/jcb.200806024

Marques, F., Sousa, J. C., Correia-Neves, M., Oliveira, P., Sousa, N., and Palha, J. A. (2007). The choroid plexus response to peripheral inflammatory stimulus. Neuroscience 144, 424-430. doi: 10.1016/j.neuroscience.2006. 09.029

McCarty, J. H., Monahan-Earley, R. A., Brown, L. F., Keller, M., Gerhardt, H., Rubin, K., et al. (2002). Defective associations between blood vessels and brain parenchyma lead to cerebral hemorrhage in mice lacking alphav integrins. Mol. Cell. Biol. 22, 7667-7677. doi: 10.1128/MCB.22.21.7667-7677.2002

Mirzadeh, Z., Merkle, F. T., Soriano-Navarro, M., Garcia-Verdugo, J. M., and Alvarez-Buylla, A. (2008). Neural stem cells confer unique pinwheel architecture to the ventricular surface in neurogenic regions of the adult brain. Cell Stem Cell 3, 265-278. doi: 10.1016/j.stem.2008.07.004

Mollgard, K., Balslev, Y., Lauritzen, B., and Saunders, N. R. (1987). Cell junctions and membrane specializations in the ventricular zone (germinal matrix) of the developing sheep brain: a CSF-brain barrier. J. Neurocytol. 16, 433-444. doi: 10.1007/BF01668498

Moreno-Lopez, B., Romero-Grimaldi, C., Noval, J. A., Murillo-Carretero, M., Matarredona, E. R., and Estrada, C. (2004). Nitric oxide is a physiological inhibitor of neurogenesis in the adult mouse subventricular zone and olfactory bulb. J. Neurosci. 24, 85-95. doi: 10.1523/JNEUROSCI.1574-03.2004

Nakagomi, N., Nakagomi, T., Kubo, S., Nakano-Doi, A., Saino, O., Takata, M., et al. (2009). Endothelial cells support survival, proliferation, and neuronal differentiation of transplanted adult ischemia-induced neural stem/progenitor cells after cerebral infarction. Stem Cells 27, 2185-2195. doi: 10.1002/stem.161

Nakagomi, T., Molnar, Z., Nakano-Doi, A., Taguchi, A., Saino, O., Kubo, S., et al. (2011). Ischemia-induced neural stem/progenitor cells in the pia mater following cortical infarction. Stem Cells Dev. 20, 2037-2051. doi: $10.1089 / \mathrm{scd} .2011 .0279$

Nie, K., Molnar, Z., and Szele, F. G. (2010). Proliferation but not migration is associated with blood vessels during development of the rostral migratory stream. Dev. Neurosci. 32, 163-172. doi: 10.1159/000301135

Ohira, K., Furuta, T., Hioki, H., Nakamura, K. C., Kuramoto, E., Tanaka, Y., et al. (2010). Ischemia-induced neurogenesis of neocortical layer 1 progenitor cells. Nat. Neurosci. 13, 173-179. doi: 10.1038/nn.2473

Ottone, C., Krusche, B., Whitby, A., Clements, M., Quadrato, G., Pitulescu, M. E., et al. (2014). Direct cell-cell contact with the vascular niche maintains quiescent neural stem cells. Nat. Cell Biol. 16, 1045-1056. doi: 10.1038/ncb3045

Palmer, T. D., Willhoite, A. R., and Gage, F. H. (2000). Vascular niche for adult hippocampal neurogenesis. J. Comp. Neurol. 425, 479-494. doi: 10.1002/10969861(20001002)425:43.0.CO;2-3

Pluchino, S., Muzio, L., Imitola, J., Deleidi, M., Alfaro-Cervello, C., Salani, G., et al. (2008). Persistent inflammation alters the function of the endogenous brain stem cell compartment. Brain 131(Pt 10), 2564-2578. doi: 10.1093/brain/awn198

Ramirez-Castillejo, C., Sanchez-Sanchez, F., Andreu-Agullo, C., Ferron, S. R., Aroca-Aguilar, J. D., Sanchez, P., et al. (2006). Pigment epithelium-derived factor is a niche signal for neural stem cell renewal. Nat. Neurosci. 9, 331-339. doi: $10.1038 / \mathrm{nn} 1657$

Ruhrberg, C., and Bautch, V. L. (2013). Neurovascular development and links to disease. Cell. Mol. Life Sci. 70, 1675-1684. doi: 10.1007/s00018-013-1277-5

Shen, Q., Goderie, S. K., Jin, L., Karanth, N., Sun, Y., Abramova, N., et al. (2004). Endothelial cells stimulate self-renewal and expand neurogenesis of neural stem cells. Science 304, 1338-1340. doi: 10.1126/science.1095505

Shen, Q., Wang, Y., Kokovay, E., Lin, G., Chuang, S.-M., Goderie, S. K., et al. (2008). Adult SVZ Stem cells lie in a vascular niche: a quantitative analysis of niche cell-cell interactions. Cell Stem Cell 3, 289-300. doi: 10.1016/j.stem.2008.07.026 Sockanathan, S., and Gaiano, N. (2009). Preview. Meninges play a RAdical role in embryonic neural stem cell regulation. Cell Stem Cell 5, 455-456. doi: 10.1016/j.stem.2009.10.010

Stenman, J. M., Rajagopal, J., Carroll, T. J., Ishibashi, M., McMahon, J., and McMahon, A. P. (2008). Canonical Wnt signaling regulates organ-specific assembly and differentiation of CNS vasculature. Science 322, 1247-1250. doi: $10.1126 /$ science. 1164594

Stolp, H. B. (2013). Neuropoietic cytokines in normal brain development and neurodevelopmental disorders. Mol. Cell. Neurosci. 53, 63-68. doi: 10.1016/j.mcn.2012.08.009

Stolp, H. B., Turnquist, C., Dziegielewska, K. M., Saunders, N. R., Anthony, D. C., and Molnar, Z. (2011). Reduced ventricular proliferation in the foetal cortex following maternal inflammation in the mouse. Brain 134, 3236-3248. doi: 10.1093/brain/awr237

Stolp, H., Neuhaus, A., Sundramoorthi, R., and Molnar, Z. (2012). The long and the short of it: gene and environment interactions during early cortical development and consequences for long-term neurological disease. Front. Psychiatry 3:50. doi: 10.3389/fpsyt.2012.00050

Stubbs, D., DeProto, J., Nie, K., Englund, C., Mahmud, I., Hevner, R., et al. (2009). Neurovascular congruence during cerebral cortical development. Cereb. Cortex 19 Suppl. 1, i32-i41. doi: 10.1093/cercor/bhp040

Tavazoie, M., Van der Veken, L., Silva-Vargas, V., Louissaint, M., Colonna, L., Zaidi, B., et al. (2008). A specialized vascular niche for adult neural stem cells. Cell Stem Cell 3, 279-288. doi: 10.1016/j.stem.2008.07.025

Tepavcevic, V., Lazarini, F., Alfaro-Cervello, C., Kerninon, C., Yoshikawa, K., Garcia-Verdugo, J. M., et al. (2011). Inflammation-induced subventricular zone dysfunction leads to olfactory deficits in a targeted mouse model of multiple sclerosis. J. Clin. Invest. 121, 4722-4734. doi: 10.1172/JCI59145

Tomita, S., Ueno, M., Sakamoto, M., Kitahama, Y., Ueki, M., Maekawa, N., et al. (2003). Defective brain development in mice lacking the Hif-1alpha gene in neural cells. Mol. Cell. Biol. 23, 6739-6749. doi: 10.1128/MCB.23.19.67396749.2003

Vasudevan, A., Long, J. E., Crandall, J. E., Rubenstein, J. L., and Bhide, P. G. (2008). Compartment-specific transcription factors orchestrate angiogenesis gradients in the embryonic brain. Nat. Neurosci. 11, 429-439. doi: 10.1038/nn2074

Villeda, S. A., Luo, J., Mosher, K. I., Zou, B., Britschgi, M., Bieri, G., et al. (2011). The ageing systemic milieu negatively regulates neurogenesis and cognitive function. Nature 477, 90-94. doi: 10.1038/nature10357

Virgintino, D., Errede, M., Rizzi, M., Girolamo, F., Strippoli, M., Walchli, T., et al. (2013). The CXCL12/CXCR4/CXCR7 ligand-receptor system regulates neuroglio-vascular interactions and vessel growth during human brain development. J. Inherit. Metab. Dis. 36, 455-466. doi: 10.1007/s10545-012-9574-y

Conflict of Interest Statement: The authors declare that the research was conducted in the absence of any commercial or financial relationships that could be construed as a potential conflict of interest.

Received: 30 September 2014; accepted: 13 January 2015; published online: 03 February 2015.

Citation: Stolp HB and Molnár Z (2015) Neurogenic niches in the brain: help and hindrance of the barrier systems. Front. Neurosci. 9:20. doi: 10.3389/fnins.2015.00020 This article was submitted to Neurogenomics, a section of the journal Frontiers in Neuroscience.

Copyright $\odot 2015$ Stolp and Molnár. This is an open-access article distributed under the terms of the Creative Commons Attribution License (CC BY). The use, distribution or reproduction in other forums is permitted, provided the original author(s) or licensor are credited and that the original publication in this journal is cited, in accordance with accepted academic practice. No use, distribution or reproduction is permitted which does not comply with these terms. 


\title{
Hydrophilic bile acids protect human blood-brain barrier endothelial cells from disruption by unconjugated bilirubin: an in vitro study
}

\author{
Inês Palmela ${ }^{1}$, Leonor Correia ${ }^{1,2}$, Rui F. M. Silva ${ }^{1,2}$, Hiroyuki Sasaki ${ }^{3}$ Kwang S. Kim ${ }^{4}$, \\ Dora Brites $^{1,2}$ and Maria A. Brito ${ }^{1,2 *}$ \\ 1 Research Institute for Medicines (iMed.ULisboa), Faculdade de Farmácia, Universidade de Lisboa, Lisbon, Portugal, \\ ${ }^{2}$ Department of Biochemistry and Human Biology, Faculdade de Farmácia, Universidade de Lisboa, Lisbon, Portugal, \\ ${ }^{3}$ Division of Fine Morphology, Core Research Facilities, The Jikei University School of Medicine, Tokyo Japan, ${ }^{4}$ Division of \\ Infectious Diseases, Johns Hopkins University School of Medicine, Baltimore, MA, USA
}

OPEN ACCESS

Edited by:

Norman Ruthven Saunders,

University of Melbourne, Australia

Reviewed by:

Jean-François Ghersi-Egea, Institut National de la Santé et de la

Recherche Médicale, France Richard P. Wennberg,

Bilimetrix-USA, LLC, USA

${ }^{*}$ Correspondence:

Maria A. Brito,

Faculdade de Farmácia, Universidade de Lisboa, Research Institute for Medicines (iMed.ULisboa), Avenida Professor Gama Pinto, 1649-003 Lisbon, Portugal abrito@ff.ulisboa.pt

Specialty section: This article was submitted to Neurogenomics, a section of the journal Frontiers in Neuroscience

Received: 25 October 2014

Accepted: 24 February 2015

Published: 13 March 2015

Citation:

Palmela I, Correia L, Silva RFM Sasaki H, Kim KS, Brites D and Brito

MA (2015) Hydrophilic bile acids protect human blood-brain barrier endothelial cells from disruption by unconjugated bilirubin: an in vitro

study. Front. Neurosci. 9:80 doi: 10.3389/fnins.2015.00080
Ursodeoxycholic acid and its main conjugate glycoursodeoxycholic acid are bile acids with neuroprotective properties. Our previous studies demonstrated their anti-apoptotic, anti-inflammatory, and antioxidant properties in neural cells exposed to elevated levels of unconjugated bilirubin (UCB) as in severe jaundice. In a simplified model of the blood-brain barrier, formed by confluent monolayers of a cell line of human brain microvascular endothelial cells, UCB has shown to induce caspase-3 activation and cell death, as well as interleukin-6 release and a loss of blood-brain barrier integrity. Here, we tested the preventive and restorative effects of these bile acids regarding the disruption of blood-brain barrier properties by UCB in in vitro conditions mimicking severe neonatal hyperbilirubinemia and using the same experimental blood-brain barrier model. Both bile acids reduced the apoptotic cell death induced by UCB, but only glycoursodeoxycholic acid significantly counteracted caspase-3 activation. Bile acids also prevented the upregulation of interleukin- $6 \mathrm{mRNA}$, whereas only ursodeoxycholic acid abrogated cytokine release. Regarding barrier integrity, only ursodeoxycholic acid abrogated UCB-induced barrier permeability. Better protective effects were obtained by bile acid pre-treatment, but a strong efficacy was still observed by their addition after UCB treatment. Finally, both bile acids showed ability to cross confluent monolayers of human brain microvascular endothelial cells in a time-dependent manner. Collectively, data disclose a therapeutic time-window for preventive and restorative effects of ursodeoxycholic acid and glycoursodeoxycholic acid against UCB-induced blood-brain barrier disruption and damage to human brain microvascular endothelial cells.

Keywords: blood-brain barrier, glycoursodeoxycholic acid, human brain microvascular endothelial cells, interleukin-6, unconjugated bilirubin, ursodeoxycholic acid

Abbreviations: BBB, Blood-brain barrier; GUDCA, Glycoursodeoxycholic acid; HBMEC, Human brain microvascular endothelial cell; IL, Interleukin; SF, Sodium fluorescein; TUDCA, Tauroursodeoxycholic acid; UCB, Unconjugated bilirubin; UDCA, Ursodeoxycholic acid. 


\section{Introduction}

In neonatal life, increased levels and prolonged exposure to unconjugated bilirubin (UCB) may trigger bilirubin-induced neurological dysfunction (Cohen et al., 2010). Although the mechanisms underlying neurological dysfunction are still unclear, the understanding of UCB-induced neurotoxicity has increased greatly in the past years (Brites and Brito, 2012). A general impairment of membrane structure, properties, and function (Rodrigues et al., 2002b; Brito et al., 2004), with neuronal oxidative stress, the release of pro-inflammatory cytokines by glial cells and altered myelinogenesis have been demonstrated (Silva et al., 2002, 2010; Falcão et al., 2006; Fernandes et al., 2006; Brito et al., 2008; Vaz et al., 2010; Barateiro et al., 2014). The awareness of the important role of the blood-brain barrier (BBB) and particularly of brain microvascular endothelial cells (BMEC) in the course of bilirubin-induced neurological dysfunction has also grown. In fact, the influence of UCB on porcine and rat BMEC (Akin et al., 2002; Cardoso et al., 2012) and in a mouse BMEC line (Kapitulnik et al., 2012) revealed that UCB induces a loss of endothelial cell viability. Our recent studies on human BMEC (HBMEC) have shown that UCB decreases endothelial cell survival and induces the release of cytokines, such as interleukin-6 (Palmela et al., 2011), which are known to be involved in BBB disruption in pathological conditions (Kaur and Ling, 2008; Carvey et al., 2009). Furthermore, HBMEC exposure to UCB resulted in biphasic effects depending on the time of interaction, where prolonged incubation compromised the endothelial junctions and led to significant impairment of barrier integrity (Palmela et al., 2012). Interestingly, UCB-induced disruption of barrier properties of BMEC was observed even in the presence of astrocytes (Cardoso et al., 2012), an in vitro co-culture model that better resembles the in vivo condition. Importantly, these in vitro evidences have been confirmed in autopsy studies of a kernicterus premature infant presenting increased vascularization and infiltration of erythrocytes and albumin in the brain parenchyma (Brito et al., 2013). Moreover, recent studies of additional cases of kernicterus have shown that the most susceptible brain regions to UCB toxicity, as the cerebellum, hippocampus, and basal ganglia, present marked signs of $\mathrm{BBB}$ dysfunction, as reduced pericyte vascular coverage and alterations in the basement membrane (Palmela et al., submitted). Thus, these features point to an enhanced permeability of the vascular walls, at least in severely ill pre-term infants with bilirubin encephalopathy.

The bile acid ursodeoxycholic acid (UDCA), which exists in very low levels in the circulation in humans, is largely used as therapy for chronic liver diseases involving cholestasis (Poupon et al., 1994; Brites et al., 1998; Lazaridis et al., 2001). UDCA is conjugated in the liver originating tauroursodeoxycholic acid (TUDCA) and glycoursodeoxycholic acid (GUDCA), the latest accounting for approximately $80 \%$ of the bile acid conjugates produced in patients under therapy (Rudolph et al., 2002). In addition, a four-fold increase of GUDCA relatively to that of TUDCA was found in the bile of patients with complete extrahepatic biliary obstruction treated with UDCA (Rudolph et al., 2002). Several studies have suggested a potential role of UDCA in the treatment of non-liver diseases involving increased levels of apoptosis (Keene et al., 2002; Rodrigues et al., 2003) due to the anti-apoptotic properties of this bile acid (Amaral et al., 2009b). Interestingly, the anti-apoptotic properties of UDCA were recently demonstrated in osteoblasts exposed to bilirubin (Ruiz-Gaspa et al., 2014). Our own studies have shown that UDCA and GUDCA protect astrocytes from apoptosis and suppress the production of pro-inflammatory cytokines (Rodrigues et al., 2000; Silva et al., 2001b; Fernandes et al., 2007a) while also counteracting UCB-induced neuronal death and synaptic changes (Silva et al., 2012). Moreover, GUDCA abrogated UCBinduced alterations in the redox status, mitochondrial dysfunction and energy impairment in neurons (Brito et al., 2008; Vaz et al., 2010). Interestingly, the mechanism of action of UDCA and its conjugates appears to rely on the stabilization of the cell membrane structure and maintenance of its dynamic properties, derived from their ability to prevent alterations in membrane lipid polarity and fluidity, as well as in the protein order and redox status (Rodrigues et al., 2001, 2002b; Solá et al., 2002).

In regard to the beneficial effects of these bile acids on endothelial cells, little is known. Nevertheless, it was shown that TUDCA is able to protect against amyloid- $\beta$-induced apoptosis (Viana et al., 2009) and leukocyte rolling and adhesion to the endothelium induced by lipid peroxidation products (Vladykovskaya et al., 2012), as well as to promote vessel repair (Cho et al., 2015). Interestingly, UDCA was shown to inhibit endothelin-1 production (Ma et al., 2004) and to have an antiangiogenic capacity (Suh et al., 1997; Woo et al., 2010), suggesting an influence on endothelial cells in a much more complex manner. However, it remains unknown whether protective effects of UDCA and GUDCA are exerted on the endothelial cells of the human BBB, and specifically toward UCB-induced injury. Thus, we here aimed to first evaluate if such bile acids are able to protect HBMEC from UCB-induced apoptosis and ultrastructural changes. Next, we intended to investigate if UDCA and GUDCA are able to prevent the production of a mediator of endothelial permeability, interleukin-6, as well as changes in barrier integrity induced by UCB. By treating cells with UDCA and GUDCA prior to UCB exposure, or 4 and $8 \mathrm{~h}$ after the initiation of the incubation procedure, our purpose was to establish the therapeutic window of opportunity to be used in jaundiced infants at risk of bilirubin-induced neurological dysfunction requiring complementary medicines to the conventional treatments.

\section{Materials and Methods}

\section{Chemicals}

The basal medium Roswell Park Memorial Institute 1640, antibiotic-antimycotic solution, human serum albumin (fraction V, fatty acid free), bovine serum albumin, Hoechst 33258 dye, sodium fluorescein and UCB were purchased from Sigma Chemical Co. (St. Louis, MO, USA). Non-essential amino acids, sodium pyruvate, L-glutamine, fetal bovine serum and minimum essential medium vitamins were from Biochrom AG (Berlin, Germany). Nuserum IV and rat-tail collagen I were acquired from BD Biosciences (Erembodegem, Belgium). TRIzol Plus RNA Purification Kit, was from Invitrogen (Carlsbad, CA, USA). 
Caspase-3 substrate and Ac-Asp-Glu-Val-Asp- $p$-nitroanilide, were acquired from Calbiochem (San Diego, CA, USA). DuoSet ELISA kit was from R\&D systems (Minneapolis, MN, USA). Primers for real-time PCR analysis were purchased from Thermo Scientific (Soeborg, Denmark). RevertAid H Minus First Strand cDNA synthesis and Maxima SYBR Green qPCR Master Mix $(2 \times)$ were obtained from Fermentas (Burlington, ON, Canada). All other chemicals were of analytical grade and were purchased from Merck (Darmstadt, Germany).

\section{Cell Culture and Treatment}

To test whether UCB-induced injury to endothelial cells could be abrogated in the presence of UDCA and GUDCA, we used a HBMEC line as a simplified model of the human BBB. This cell line was derived from primary cultures of HBMEC transfected with SV40 large T antigen (Stins et al., 2001) and was recently proved to be the most suitable human cell line for an in vitro BBB concerning barrier tightness (Eigenmann et al., 2013). Cells were cultured in Roswell Park Memorial Institute medium supplemented with $10 \%$ fetal bovine serum, $10 \%$ NuSerum IV, $1 \%$ nonessential amino acids, $1 \%$ minimum essential medium vitamins, $1 \mathrm{mM}$ sodium pyruvate, $2 \mathrm{mM}$ L-glutamine, and $1 \%$ antibioticantimycotic solution, seeded at a density of $8 \times 10^{4} \mathrm{cell} / \mathrm{mL}$ in collagen I-coated coverslips or plates and treated after 2 days in culture, as previously described (Palmela et al., 2011). For integrity studies, based on the measurement of paracellular permeability to sodium fluorescein, cells were seeded on collagen I-coated polyester transwell inserts $(0.4 \mu \mathrm{m}$, Corning Costar Corp., USA) at a density of $8 \times 10^{4}$ cell/insert and treated after 8 days in culture (Palmela et al., 2012). Endothelial cultures were maintained at $37^{\circ} \mathrm{C}$ in a humid atmosphere enriched with $5 \% \mathrm{CO}_{2}$, and all experiments were performed at confluence.

UCB was purified (Mcdonagh and Assisi, 1972) and a $10 \mathrm{mM}$ stock solution was prepared in $0.1 \mathrm{M} \mathrm{NaOH}$ and used immediately after preparation. The $\mathrm{pH}$ value was restored to 7.4 by addition of equal amounts of $0.1 \mathrm{M} \mathrm{HCl}$, and all the procedures were performed under light protection to avoid photodegradation. Confluent monolayers of the HBMEC line were incubated with $100 \mu \mathrm{M} \mathrm{UCB}$, or with no addition (control), in the presence of $100 \mu \mathrm{M}$ human serum albumin. This experimental condition mimics the bilirubin/albumin ratio (1:1 molar ratio; $8.7 \mathrm{mg} / \mathrm{g}$ ) found in a kernicterus case described by us (Brito et al., 2012) and is within the $5.4-21.0 \mathrm{mg} / \mathrm{g}$ ratio recently associated to acute bilirubin encephalopathy in Egypt (Iskander et al., 2014). Determination of unbound bilirubin, or free bilirubin, by the widely used peroxidase method (Roca et al., 2006) showed that this experimental condition corresponds to a free bilirubin concentration of $23.6 \mathrm{nM}$, as previously reported by Palmela et al. (2012). The free bilirubin level used in the present in vitro model is within the range of values found by us in a group of moderately jaundiced neonates $(19.1 \pm 1.5 \mathrm{nM})$ (Brito, 2001) and by Ahlfors et al. (2009) (21-51 nM) in babies readmitted for jaundice. Also to mention that the apparent discrepancy between the free bilirubin value obtained in our lab and those indicated by Roca et al. (2006) may result from the fact that Roca et al. (2006) did not include cells in their system, thus not considering the fraction of bilirubin that is bound/included in cells (Brito et al., 2000; Palmela et al.,
2012), nor the non-conjugating pathways for UCB catabolism (Ahlfors et al., 2009). The incubation period used for each parameter varied between 1 and $48 \mathrm{~h}$, based on the time to obtain the maximal effect observed in prior studies (Palmela et al., 2011, 2012). The incubation medium consisted in the regular medium without fetal bovine serum and Nuserum IV, to avoid disturbance of the final concentration of albumin in the incubation medium.

Co-incubation studies were also performed with the bile acids UDCA and GUDCA, molecules with octanol/water partition coefficients of 1000 for the unconjugated form and 105 for the glycine-amidated molecule, and $\log \mathrm{P}$ values of 3.0 and 2.02 for the former and the later, respectively (Roda et al., 1990). In the coincubation studies, UDCA or GUDCA were added at a final concentration of $50 \mu \mathrm{M}$, which is found in the circulation of patients under therapy with UDCA. In particular, the concentration of $50 \mu \mathrm{M}$ GUDCA is commonly found in the serum of patients after treatment with UDCA at a dose of 13-15 mg per kilogram of body weight per day (Podda et al., 1990; Poupon et al., 1994; Brites et al., 1998). We previously showed that such concentration is not toxic to neurons (Silva et al., 2001b) and, most importantly, has beneficial properties in preventing neurodegeneration (Brito et al., 2008; Vaz et al., 2010). The bile acids were added at three different time points: $1 \mathrm{~h}$ prior to UCB addition and at 4 or $8 \mathrm{~h}$ after UCB incubation. For short periods of UCB incubation only the effects of $1 \mathrm{~h}$ pre-incubation with the bile acids were evaluated. Appropriate controls including cells treated with UDCA and GUDCA (without UCB) were also included to ascertain the absence of toxicity of these molecules.

For the integrity experiments, endothelial cells were cultured on semipermeable filters inserted in the culture plate well. With this system, there are two compartments: the apical or upper one that can be considered as the "blood-side," where UCB, bile acids and human serum albumin were added, and the basal or lower compartment, which is considered the "brain side."

\section{Assessment of Apoptosis}

Caspase-3 activity and the number of apoptotic nuclei were determined after 4 and $48 \mathrm{~h}$ of UCB exposure, respectively, since these time points represent the maximum effects of UCB alone (Palmela et al., 2011).

Activity of caspase- 3 was measured by a colorimetric method (Calbiochem, Darmstadt, Germany), as usual in our lab (Palmela et al., 2011). The results were expressed as fold change from control values. Assessment of nuclear morphology of the HBMEC line following Hoechst 33258 staining was evaluated as previously described (Palmela et al., 2011). Fluorescence was visualized using a Leica DFC 490 camera (Leica, Wetzlar, Germany) adapted to an AxioScope.A1 microscope (Zeiss, Göttingen, Germany). Values were expressed as percentage of apoptotic nuclei.

\section{Transmission Electron Microscopy}

Ultrastructural analysis was performed by transmission electron microscopy following $48 \mathrm{~h}$ exposure to UCB in HBMEC pretreated with UDCA or GUDCA. Cells were fixed with $1.2 \%$ glutaraldehyde in $0.1 \mathrm{M}$ phosphate buffer and $1 \%$ osmium tetroxide in the same buffer, dehydrated with a graded series of ethanol, and 
then embedded in epoxy resin. Ultrathin sections were stained with uranyl acetate and lead citrate and observed with a Hitachi H-7500 transmission electron microscope (Tokyo, Japan) at an acceleration voltage of $80 \mathrm{kV}$.

\section{Measurement of Interleukin-6 mRNA Expression and Protein Release}

The HBMEC line was exposed to UCB for $1 \mathrm{~h}$ for mRNA analysis, and for $4 \mathrm{~h}$ for cytokine release quantification, since the maximum effects of UCB alone in interleukin-6 expression and secretion were observed at these time points (Palmela et al., 2011).

Analysis of mRNA expression was performed by quantitative real time PCR using a SYBR Green qPCR Master Mix $(2 \times)$, as described previously (Palmela et al., 2011). This assay was performed using $\beta$-actin as an endogenous control to normalize the expression level of interleukin- 6 mRNA. The following sequences were used as primers: interleukin6 sense, 5'-GACAGCCACTCACCTCTTCA- $3^{\prime}$ and anti-sense, $5^{\prime}$-TTCACCAGGCAAGTCTCCTC-3' (Wang et al., 2006); $\beta$ actin sense, $5^{\prime}$-ACAGAGCCTCGCCTTTGCCG-3' and antisense, 5'-TGGGCCTCGTCGCCCACATA-3' (NM_001101.3). Non-specific products of PCR were not found in any case. The relative quantification was made using the Pfaffl modification of the $\Delta \Delta \mathrm{C}_{\mathrm{T}}$ equation $\left(\mathrm{C}_{\mathrm{T}}\right.$, cycle number at which the fluorescence passes the threshold level of detection), taking into account the efficiencies of individual genes. The results were normalized to $\beta$ actin and the initial amount of the template of each sample was determined as fold change from control samples (reference).

Endothelial interleukin-6 release was assessed in duplicate, using a specific DuoSet ELISA development kit, according to the manufacturer's instructions. Measurements were obtained at a wavelength of $450 \mathrm{~nm}$, with a reference filter of $620 \mathrm{~nm}$, using a microplate reader. The average control values were $135 \mathrm{pg} / \mathrm{mL}$ and the results were expressed as fold change from control.

\section{Evaluation of Barrier Integrity by Permeability Measurement}

The capacity of UDCA and GUDCA to modulate permeability was evaluated in cells treated with UCB for $48 \mathrm{~h}$, the time-point resulting in the maximum disruption of the integrity state of HBMEC monolayer by UCB (Palmela et al., 2012).

In our previous studies, we found that UCB increases the permeability to sodium fluorescein (Palmela et al., 2012), a low molecular weight tracer $(376 \mathrm{Da})$, but not to albumin-bound Evans blue, a high molecular weight tracer $(68 \mathrm{kDa})$. So, in this study HBMEC paracellular permeability assay was conducted with sodium fluorescein as previously described (Veszelka et al., 2007; Cardoso et al., 2012; Palmela et al., 2012). Briefly, cell culture inserts were transferred to 12 -well plates containing RingerHepes solution $(118 \mathrm{mM} \mathrm{NaCl}, 4.8 \mathrm{mM} \mathrm{KCl}, 2.5 \mathrm{mM} \mathrm{CaCl}$, $1.2 \mathrm{mM} \mathrm{MgSO}_{4}, 5.5 \mathrm{mM}$ D-glucose, $20 \mathrm{mM}$ Hepes, $\mathrm{pH} 7.4$ ) in the basal compartments. The sodium fluorescein solution (10 $\mathrm{mg} / \mathrm{mL}$ sodium fluorescein in Ringer-Hepes) was added to the upper chambers. The inserts were transferred to new wells at 20, 40, and $60 \mathrm{~min}$. Lower chamber solutions were collected to determine sodium fluorescein levels (Hitachi F-2000 fluorescence spectrophotometer, excitation: $440 \mathrm{~nm}$ and emission: $525 \mathrm{~nm}$ ). Flux across cell-free inserts was also measured. The endothelial permeability coefficient was calculated as previously described (Deli et al., 2005) and the average control permeability coefficient was $1.4 \times 10^{-5} \mathrm{~cm} / \mathrm{s}$.

\section{Assessment of UDCA and GUDCA Passage across the HBMEC Monolayer}

To establish whether UDCA and GUDCA are able to cross the BBB endothelium, a two-chamber culture system was used. Bile acids were added to the upper chambers and media from the lower chambers were collected after 4 and $48 \mathrm{~h}$ of incubation. The bile acid passage across the HBMEC monolayer was evaluated by measuring the concentrations of UDCA and GUDCA by an enzymatic-fluorimetric assay (Brites et al., 1998). Results were shown as average concentration $(\mu \mathrm{M}) \pm \mathrm{SEM}$.

\section{Statistical Analysis}

Results are expressed as means \pm SEM values from, at least, three separate experiments. Differences between groups were determined by one-way ANOVA with Bonferroni post-test, using Prism 5.0 (GraphPad Software, San Diego, CA). Statistical significance was considered when $P$-values were lower than 0.05 .

\section{Results}

\section{UDCA and GUDCA Protect HBMEC from UCB-Induced Apoptosis, but only GUDCA is Effective in Reducing Caspase-3 Activation}

UCB-induced apoptosis in the HBMEC line includes the presence of apoptotic features that increased with the time of exposure and reached maximal levels at $48 \mathrm{~h}$ (Palmela et al., 2011). Thus, this time was chosen to evaluate the ability of UDCA and GUDCA to protect HBMEC from UCB-induced apoptosis. The bile acids were added at three different time points, evaluating their potential when added before and after the injury. Addition of UDCA and GUDCA reduced UCB injury, regardless of the time of addition (Figure 1). This protective effect was maximal in the treatments with GUDCA, especially when added at $1 \mathrm{~h}$ prior to $\mathrm{UCB}$ addition (54\% reduction from UCB values, $P<0.001$, vs. $42 \%$ for UDCA at the same time point, $P<0.01$ ). Importantly, bile acids partially reverted UCB injury with a nearly $30 \%$ protection rate reduction compared to UCB damage (Figure 1), when added $8 \mathrm{~h}$ after UCB addition.

We next assessed the preventive bile acid effect on caspase- 3 activity at $4 \mathrm{~h}$, the time point where the maximal effect of UCB on endothelial cells was observed (Palmela et al., 2011). As seen in Figure 2, only GUDCA was able to significantly protect from UCB-induced activation of caspase- $3(P<0.05)$.

\section{Ultrastructural Changes Induced by UCB in HBMEC are Abrogated by UDCA and GUDCA}

Based on the UCB-induced effects on apoptosis after $48 \mathrm{~h}$ incubation and the rescue ability of both UDCA and GUDCA to partially restore cell functionality, we decided to further assess whether changes at HBMEC ultrastructural level were produced 


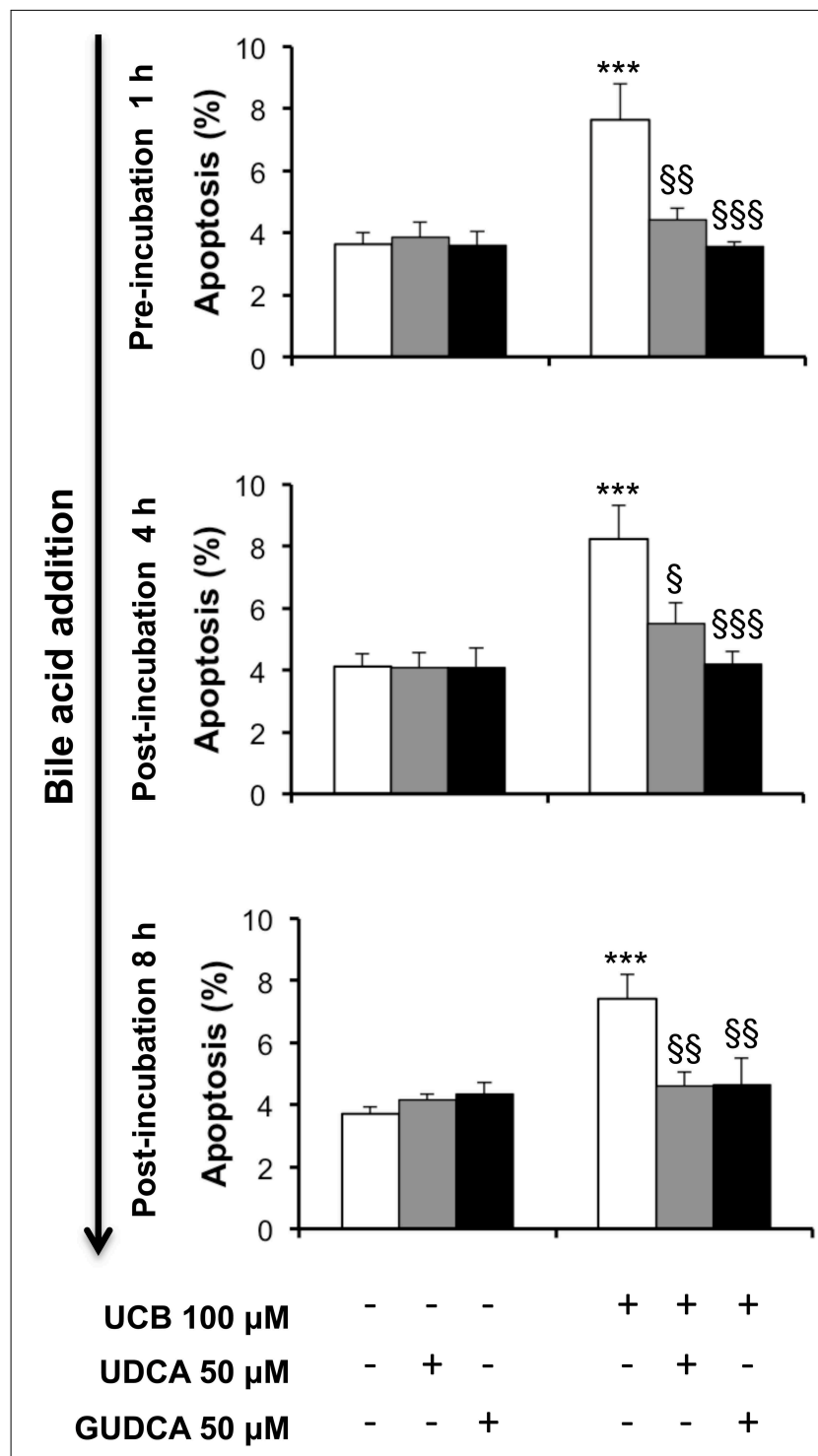

FIGURE 1 | Ursodeoxycholic acid (UDCA) and glycoursodeoxycholic acid (GUDCA) protect human brain microvascular endothelial cells (HBMEC) from unconjugated bilirubin (UCB)-induced apoptosis. A cell line of HBMEC was incubated without (control) or with $100 \mu \mathrm{M} \cup \mathrm{CB}$, in the presence of $100 \mu \mathrm{M}$ human serum albumin, for $48 \mathrm{~h}$. The bile acids $(50 \mu \mathrm{M})$ were added prior to ( $1 \mathrm{~h}$ ) or after ( 4 or $8 \mathrm{~h}$ ) UCB addition. Quantification of apoptosis is shown as percentage of apoptotic nuclei per total number of cells and results are expressed as mean \pm SEM from at least three independent experiments. ${ }^{* * *} P<0.001$ vs. control; ${ }^{\S} P<0.05$, ${ }^{\S} P P<0.01$, and $\S \S \S<0.001$ vs. UCB alone.

by UCB and prevented by bile acid treatment. The transmission electron microscopy analysis revealed a marked reduction in the amount of ribosomes in UCB-treated cells, with an evident recovery in the presence of both bile acids (Figure 3). The same occurred relatively to cellular fragments detaching from the cultured HBMEC and mitochondrial cristae disruption observed, showing the damaging effects of UCB, once again markedly reduced in the presence of each of the bile acids.

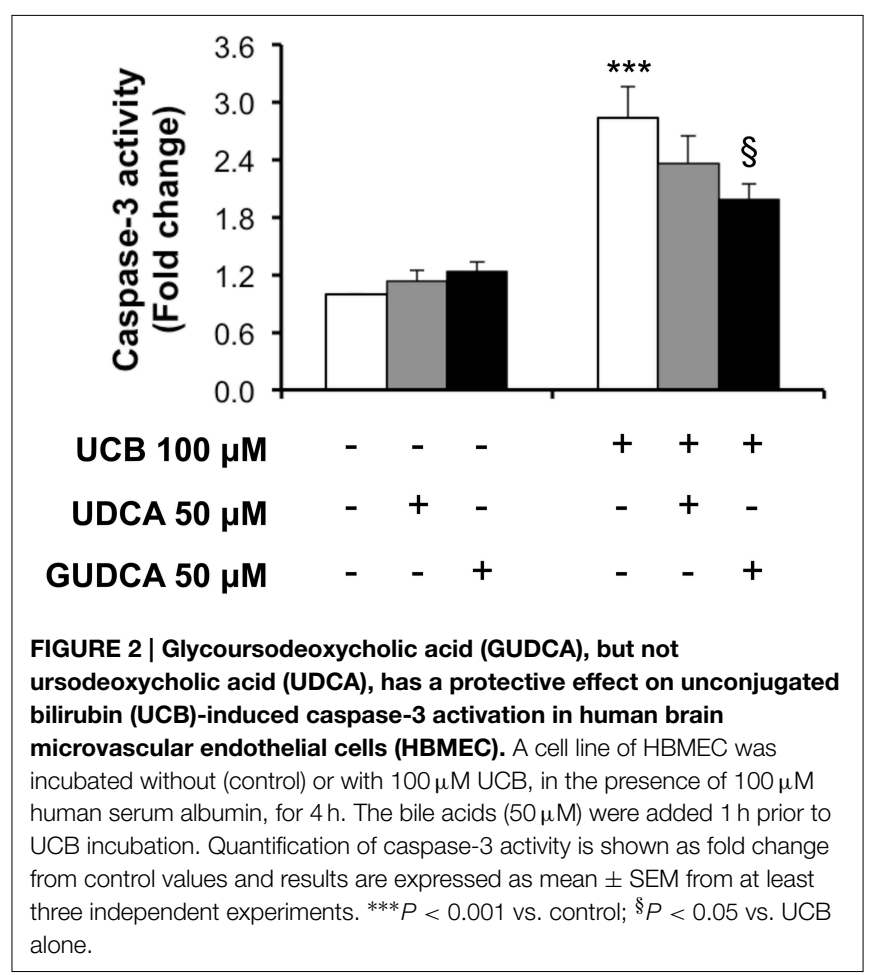

\section{UCB-Induced Increase of Interleukin-6 mRNA and Cytokine Expression in HBMEC is More Effectively Reduced by UDCA than by GUDCA}

One of the important effects of UCB on HBMEC previously observed by us was the upregulation of interleukin- 6 mRNA levels and protein secretion (Palmela et al., 2011). This previous work indicated that UCB induced the maximum cytokine secretion at $4 \mathrm{~h}$, while the highest mRNA expression was at $1 \mathrm{~h}$ following UCB exposure. These time points were then selected and the bile acids were added $1 \mathrm{~h}$ prior to UCB incubation. As seen in Figure 4, both bile acids abrogated interleukin- 6 mRNA upregulation (Figure 4A), with reductions from UCB values of $27 \%$ for GUDCA $(P<0.05)$ and $46 \%$ for UDCA $(P<0.001)$. On the other hand, only UDCA showed preventive effects on UCB-induced release of interleukin-6 (Figure 4B), decreasing UCB-induced cytokine secretion by $35 \%(P<0.001)$.

\section{UDCA and GUDCA Prevent and Rescue Disruption of HBMEC Integrity by UCB}

The ability of the tested bile acids to counteract UCB-induced upregulation of interleukin-6, led us to hypothesize that UDCA and GUDCA would protect against the consequent endothelial hyperpermeability. Thus, we measured the paracellular permeability to the low molecular weight compound, sodium fluorescein. This is a widely used indicator of the barrier properties, with several studies showing increased values in conditions associated with hyperpermeability (Hülper et al., 2013; Labus et al., 2014). In our previous study we showed that this parameter is significantly enhanced upon prolonged UCB exposure (Palmela et al., 2012), as also observed in the present study (Figure 5). Here, 


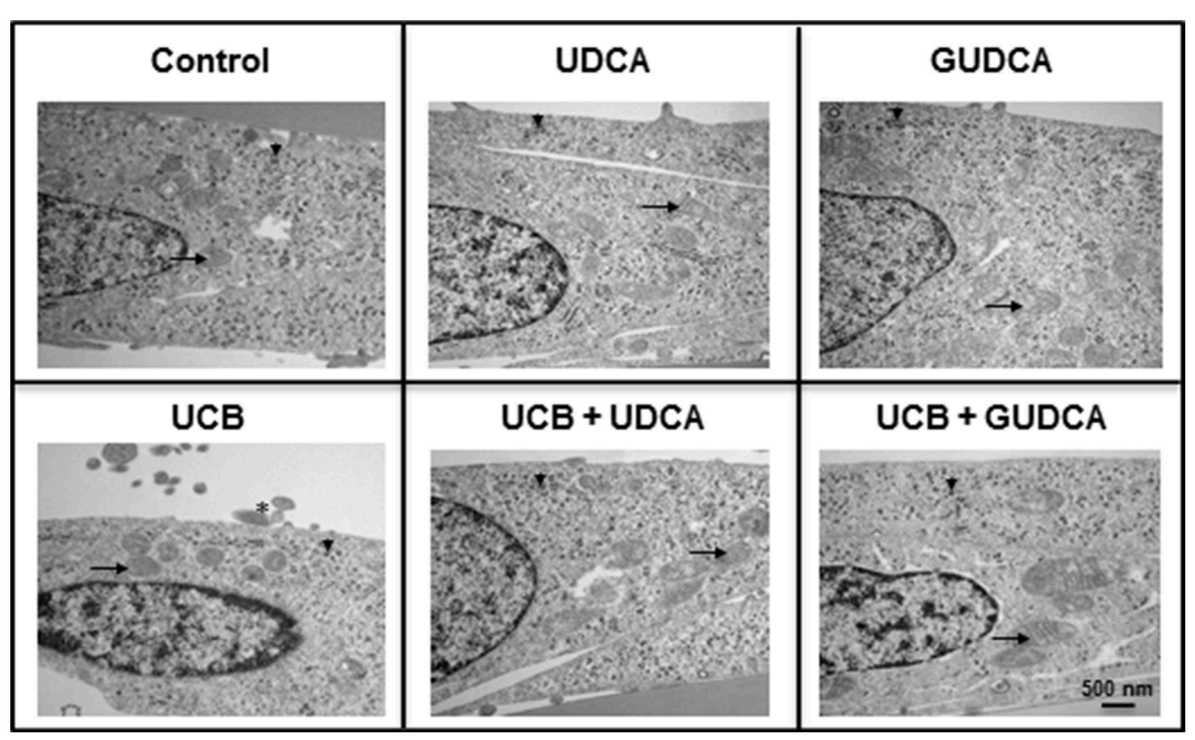

FIGURE 3 | Ursodeoxycholic acid (UDCA) and glycoursodeoxycholic acid (GUDCA) have protective effects on unconjugated bilirubin (UCB)-induced ultrastructural changes in human brain microvascular endothelial cells (HBMEC). A cell line of HBMEC was incubated without (control) or with $100 \mu \mathrm{M}$ UCB, in the presence of $100 \mu \mathrm{M}$ human serum albumin, for $48 \mathrm{~h}$. The bile acids $(50 \mu \mathrm{M})$ were added $1 \mathrm{~h}$ prior to the addition of UCB. Representative ultrastructure observations by transmission electron microscopy are shown. In UCB-treated cells note the decrease in mitochondrial cristae (arrows) and ribosomes (arrowhead) and the appearance of detaching cellular fragments (asterisk), which are abrogated by UDCA and GUDCA.
A

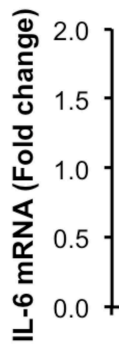

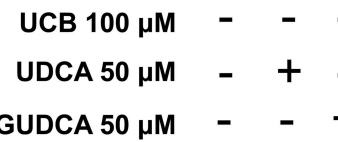

FIGURE 4 | Decrease in unconjugated bilirubin (UCB)-induced interlkeukin (IL)-6 mRNA expression and cytokine release in human brain microvascular endothelila cells (HBMEC) is higher with ursodeoxycholic acid (UDCA) than with

glycoursodeoxycholic acid (GUDCA). A cell line of HBMEC was incubated without (control) or with $100 \mu \mathrm{M} \mathrm{UCB}$, in the presence of $100 \mu \mathrm{M}$ human serum albumin, for 1 or $4 \mathrm{~h}$. The bile acids $(50 \mu \mathrm{M})$

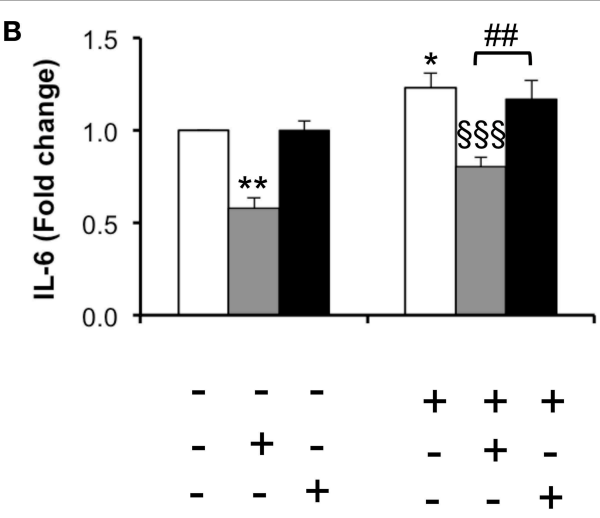

were added $1 \mathrm{~h}$ prior to the addition of UCB. Changes in IL-6 mRNA after $1 \mathrm{~h}$ (A) and protein release after $4 \mathrm{~h}$ of incubation (B) are shown. Quantification is shown as fold change from control values and results are expressed as mean \pm SEM from at least three independent experiments. ${ }^{*} P<0.05, * * P<0.01$, and $* * * P<0.001$ vs. control; $\S P<0.05$ and $\S \S \S P<0.001$ vs. UCB alone; $\# P<0.05$ and $\# \# P<0.01$ UDCA vs. GUDCA. we also observed that UDCA and GUDCA alone do not affect the HBMEC integrity, since we did not observe any changes in permeability values. However, analysis of the bile acids effect on the permeability to sodium fluorescein revealed that only UDCA prevented UCB injury, and if added before (22\% reduction from UCB values, $P<0.01)$ or at $4 \mathrm{~h}(18 \%$ protection from UCB values, $P<0.05)$. In fact, while UCB induced an increased passage of sodium fluorescein molecules from $1.42 \times 10^{-5} \mathrm{~cm} / \mathrm{s}$ in controls to $2.48 \times 10^{-5} \mathrm{~cm} / \mathrm{s}$ in UCB-treated samples, incubation with UDCA reduced such value to $1.95 \times 10^{-5} \mathrm{~cm} / \mathrm{s}$ or to $1.99 \times 10^{-5} \mathrm{~cm} / \mathrm{s}$ in cells pre-treated or treated $4 \mathrm{~h}$ after UCB addition. In contrast, the values obtained for GUDCA were $2.19 \times 10^{-5}$ and $2.24 \times 10^{-5} \mathrm{~cm} / \mathrm{s}$ (pre- and $4 \mathrm{~h}$ after UCB addition treatments, respectively), thus not different from UCB values. 


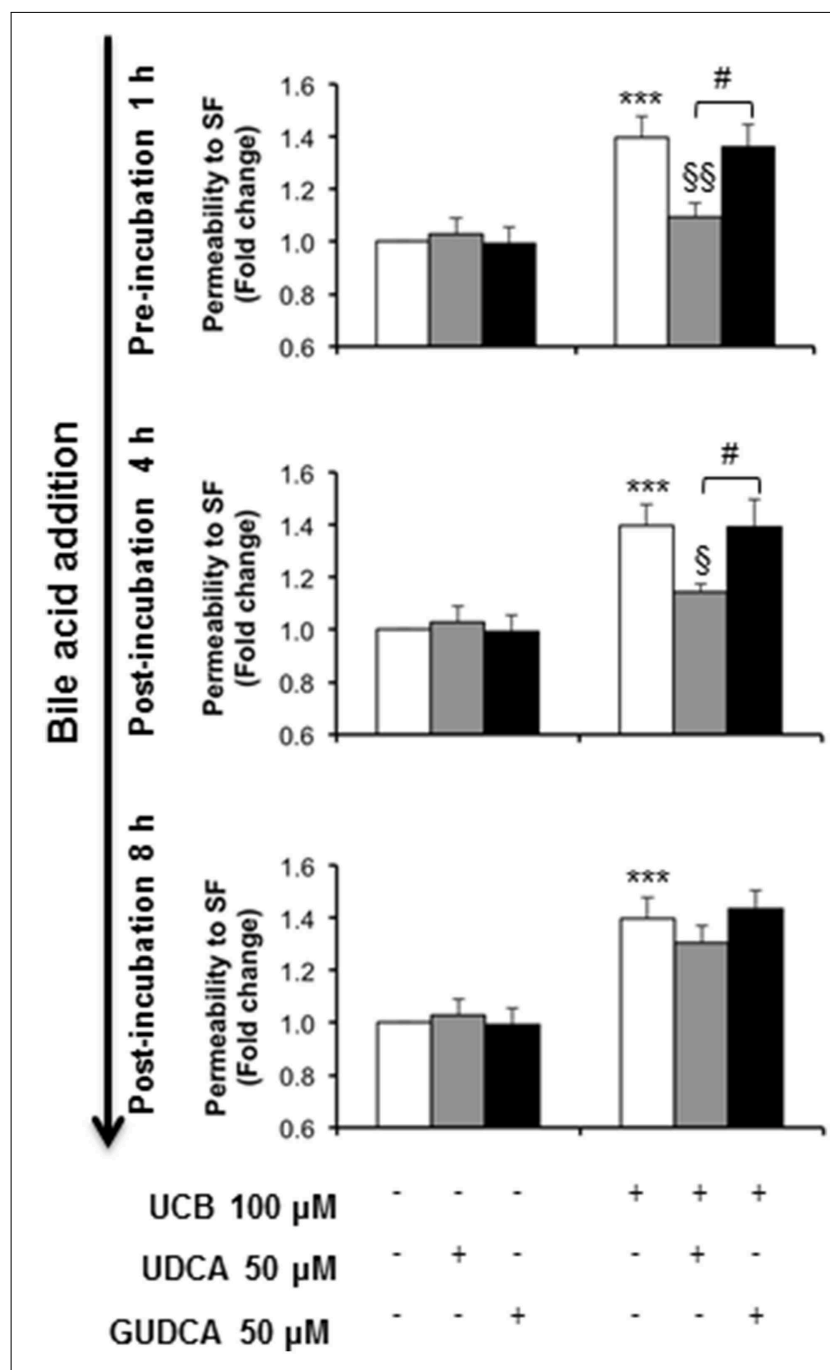

FIGURE 5 | Ursodeoxycholic acid (UDCA), but not glycoursodeoxycholic acid (GUDCA), abrogates paracellular permeability impairment to sodium fluorescein (SF) in human brain microvascular endothelial cells (HBMEC). A cell line of HBMEC was incubated without (control) or with $100 \mu \mathrm{M}$ UCB, in the presence of $100 \mu \mathrm{M}$ human serum albumin, for $48 \mathrm{~h}$. The bile acids $(50 \mu \mathrm{M})$ were added prior to $(1 \mathrm{~h})$ or after ( 4 or $8 \mathrm{~h}$ ) UCB addition. Quantification of permeability to $\mathrm{SF}$ is expressed as fold change from control values and results are shown as mean \pm SEM from at least three independent experiments. ${ }^{* * *} P<0.001$ vs. control; ${ }^{\S} P<0.05$ and $\S P<0.01$ vs. UCB alone; $\# P<0.05$ UDCA vs. GUDCA.

\section{UDCA and GUDCA Cross the HBMEC Monolayer in a Time-Dependent Manner}

The addition of $50 \mu \mathrm{M}$ of each of the studied bile acids to the upper ("blood") compartment of an insert culture system was performed to evaluate if they were able to cross the HBMEC monolayer and thus hypothetically achieve the brain parenchyma. After a short period of incubation $(4 \mathrm{~h})$ the bile acids were barely detectable in the lower chamber of the culture plate. However, when longer periods of treatment were applied $(48 \mathrm{~h})$ a significant increase in the bile acid passage through the monolayer was obtained $(18.8 \pm 4.8$ and $16.2 \pm 3.9 \mu \mathrm{M}$, for UDCA and GUDCA, respectively).

\section{Discussion}

In this study we investigated the ability of the bile acid UDCA and its glycine conjugate GUDCA to abrogate the injury caused by UCB in a simplified in vitro model of the human BBB, formed by confluent monolayers of HBMEC. The beneficial role of UDCA and its conjugates on liver-associated pathologies has been extensively addressed in the past (Lazaridis et al., 2001; Paumgartner and Beuers, 2004; Perez and Briz, 2009). Interestingly, it was also demonstrated that these bile acids act as pleiotropic agents and can be used as therapeutic molecules in other non-hepatic pathological conditions, including tumors, hemorrhagic stroke, and neurodegenerative disorders like amyotrophic lateral sclerosis (Min et al., 2012), Huntington's (Keene et al., 2002), Alzheimer's (Solá et al., 2003), and Parkinson's diseases (Duan et al., 2002). We have previously shown that UDCA and/or GUDCA have protective properties in reducing the UCBmediated induction of cell death in both neurons and astrocytes (Silva et al., 2001b; Fernandes et al., 2007b; Vaz et al., 2010). Moreover, we observed the beneficial effects of these bile acids in reducing the increased secretion of pro-inflammatory cytokines by astrocytes and microglia treated with UCB (Fernandes et al., 2007b; Silva et al., 2012) and the oxidative injury in neurons exposed to UCB (Brito et al., 2008), as reviewed by Brites (2012). Importantly, numerous studies have demonstrated that these specific therapeutic properties of UDCA and its conjugates do not apply to the more hydrophobic bile acids. In fact, deoxycholic acid (hydrophobic bile acid) was shown to increase lipid polarity and fluidity, while UDCA and TUDCA (hydrophilic bile acids) are able to reverse such effects (Solá et al., 2002). Additionally, UDCA demonstrated to protect neurons from UCB toxicity, while other hydrophobic bile acids like cholic and chenodeoxycholic acids even aggravated UCB injury (Silva et al., 2001b). The same was observed with endothelial cells where deoxycholic and taurochenodeoxycholic acids caused the cell lysis by acting as detergents (Greenwood et al., 1991) while TUDCA revealed beneficial effects in preventing cell damage by other injurious conditions (Viana et al., 2009; Vladykovskaya et al., 2012). Thus, we aimed to understand if the unconjugated species UDCA and the most predominant conjugate GUDCA derivative, with LogP values of 3.0 and 2.02, respectively (Roda et al., 1990), in humans had protective properties in our simplified in vitro model of the $\mathrm{BBB}$ in conditions mimicking severe hyperbilirubinemia.

Our previous studies have shown that the BMEC response to UCB is extremely elaborate and far more complex than formerly thought. Our in vitro findings include the elevation of endothelial cell death, the upregulation of caveolae and caveolin-1 levels, the increased production of interleukin-6, and the release of matrix metalloproteinases (Palmela et al., 2011, 2012; Cardoso et al., 2012). Consequently, these events led to junction weakness, as well as disruption of endothelial barrier integrity. To such disruption may also contribute the impairment of the cytoskeleton organization induced by UCB (Silva et al., 2001a, 2006), which is known to compromise the intercellular junctions assembly and 
lead to an increased paracellular permeability (Cardoso et al., 2010). Thus, in addition to diffusion of free bilirubin across the brain microvascular endothelium, the paracellular hyperpermeability of the microvasculature may further favor the freely movement of free bilirubin, as well as of UCB-bound to albumin, via the paracellular space. Also interesting is the increased caveolin-1 expression and the enhanced number of caveolae (Palmela et al., 2012) since caveolae are known to transport albumin, which raises the possibility of augmented entrance of albumin-bound UCB by the transcellular pathway. Validating these in vitro findings, signs of $\mathrm{BBB}$ compromise were also observed in some brain regions of human cases of neonatal kernicterus, including albumin presence in the brain parenchyma, increased vascularization and microvascular hyperpermeability (Brito et al., 2012, 2013), reduced pericyte vascular coverage and loss of basement membrane components (Palmela et al., submitted). These findings suggest that the $\mathrm{BBB}$ plays an important role in the progression of brain damage by severe hyperbilirubinemia and, thus, that this interface should be considered when studying this pathology.

Here, we intended to evaluate the effects of UDCA and GUDCA at the time-point corresponding to the most significant UCB-induced injury previously observed. Furthermore, as UCB effects on HBMEC were also concentration-dependent, we focused on the concentration with the most disruptive potential regarding the integrity of HBMEC $(100 \mu \mathrm{M}$ UCB in the presence of $100 \mu \mathrm{M}$ human serum albumin), which corresponds to the bilirubin:albumin ratio that induces acute bilirubin encephalopathy and kernicterus (Brito et al., 2012; Iskander et al., 2014) and to free bilirubin values found in jaundiced neonates (Brito, 2001; Ahlfors et al., 2009). To assess the therapeutic window of opportunity of GUDCA and UDCA we tested bile acid efficacy when used before (prevention), or at 4 and $8 \mathrm{~h}$ after UCB addition (recovery). To further understand if such bile acids can be promising candidates to rescue neural function in brain diseases we assessed their capacity to cross the HBMEC monolayer, here used as an in vitro model of BBB.

Bile acids revealed a high capacity in protecting HBMEC from UCB-induced apoptosis. Interestingly, GUDCA was the most effective against apoptotic features and caspase-3 activation, and was able to restore basal levels, even when the injury was already initiated. Although the anti-apoptotic role of UDCA has been documented in hepatic and non-hepatic cells (Amaral et al., 2009a), the findings here reported are the first in a simplified in vitro model of the human BBB. Interestingly, apoptotic cell death results in increased permeability (Erdbruegger et al., 2006), rendering conceivable that the mechanisms underlying HBMEC protection may involve the anti-apoptotic properties of the bile acids. Regardless of the mechanism(s) involved in the protection, the present study opens new avenues for treatment of neurodegenerative diseases that have increasingly been associated with endothelial demise and BBB disruption (Zlokovic, 2008).

When analysing the possible ultrastructural changes produced by UCB and their recovery by the bile acids, we noticed that the loss of ribosomes in UCB-treated samples was reverted by both UDCA and GUDCA. Alterations in ribosomes were associated with apoptosis (Nishida et al., 2002) and autophagy (Cebollero et al., 2012), phenomena already observed in HBMEC treated with UCB (Palmela et al., 2011, 2012). Indeed, Hansen et al. (2001) found elevated levels of bilirubin in ribosomes following exposure of rats to hyperbilirubinemia and hyperosmolality, recognized as a risk factor of kernicterus by increasing BBB permeability (Wennberg, 2000). Therefore, it is tentative to speculate that UCB may induce HBMEC ribophagy, a recent term do designate autophagic turnover of ribosomes (Cebollero et al., 2012), and that both bile acids are able to prevent and recover such event from occurring.

The transmission electron microscopy analysis provided further information about the toxicity of UCB to other cell organelles, particularly the mitochondria. Our observations highlighted a loss of mitochondrial cristae after UCB exposure, which appeared to be restored in HBMEC pre-incubated with each of the bile acids. In a recent study, damaged mitochondria in the presence of UCB were identified in rat BMEC (Cardoso et al., 2012). Mitochondria has been considered one of the first targets of UCB injury to the cells (Mustafa and King, 1970), showing accumulation of glycogen, a sign of impaired energetic function (Batty and Millhouse, 1976). Other studies, including several from our own group, have shown loss of mitochondrial membrane potential, release of cytochrome $c$, and impaired cytochrome $c$ oxidase activity (Rodrigues et al., 2000, 2002a; Malik et al., 2010; Barateiro et al., 2012). To note that the protective ability of the bile acids, especially UDCA, in preventing mitochondria dysfunction by UCB was shown in some of those studies (Rodrigues et al., 2000, 2002a). Moreover, the mitochondria enlargement that seems to occur in cells treated with GUDCA and UCB may represent a mechanism to protect cells from apoptotic stimuli (Chiche et al., 2010). Detachment of cellular fragments in UCB-treated samples is in line with the release of small vesicles from HBMEC already noticed by scanning electron microscopy (Palmela et al., 2012). All these features corroborate the previously demonstrated interaction of UCB with membranes (Brites and Brito, 2012) and the stabilizing effect of the bile acids at this level (Rodrigues et al., 2002b; Solá et al., 2002).

Amongst the cytokines produced by HBMEC upon UCB interaction is interleukin-6 (Palmela et al., 2011), a cytokine that has been reported to induce the disruption of the BBB (Maruo et al., 1992; De Vries et al., 1996). Only UDCA was able to reduce both interleukin- 6 mRNA expression and protein release. Compromised $\mathrm{BBB}$ by vascular leak and tight junction disassembly may lead to deregulated flux of molecules and loss of brain homeostasis. Thus, the restorative effects of the bile acids in interleukin-6 levels may contribute to sustain HBMEC integrity in the presence of UCB. While GUDCA revealed strong antiapoptotic effects, UDCA was more effective in preventing the increased interleukin- 6 secretion by HBMEC treated with UCB. Interestingly, UDCA was also more able than GUDCA in rescuing HBMEC from the increased permeability induced by UCB, an effect that was observed even if the bile acid was added $4 \mathrm{~h}$ after UCB-addition. Such dissimilarities among the bile acids species may derive from the different changes that UDCA and the conjugated species were shown to produce in the composition of membrane lipid content (Bellentani et al., 1996). 
Crossing the $\mathrm{BBB}$ is one of the main milestones for therapeutic molecules that are meant to act in the brain. Although detection of UDCA and its taurine conjugate was found in the brain parenchyma after injection in rodents (Kaemmerer et al., 2001; Rodrigues et al., 2003; Parry et al., 2010) and in the cerebrospinal fluid after oral administration in patients with amyotrophic lateral sclerosis (Parry et al., 2010), no studies were till now performed using HBMEC. Here, we show that both UDCA and GUDCA cross the HBMEC monolayer, in vitro. However, the mechanism underlying the passage of the bile acids across the $\mathrm{BBB}$ has never been reported. Among the factors that may influence $\mathrm{BBB}$ permeation are the bile acids' physico-chemical characteristics, namely their octanol/water partition coefficient (Roda et al., 1990), and the presence of several transporters at the BBB (Abbott et al., 2010) that may modulate their passage across the endothelium. Therefore, additional studies are necessary to establish the mechanism involved in these bile acids passage across the BBB. The suggested potential therapeutic role for UDCA and GUDCA also needs further evaluation in animal models of severe jaundice, such as the Gunn rat (Gunn, 1938) or the glucuronosyl-transferase knock-out mice (Nguyen et al., 2008).

Collectively, our in vitro data show that the disruption of endothelial cell function and BBB dynamic properties by exposure to UCB in conditions mimicking a severe neonatal jaundice can be prevented and partially restored by UDCA and GUDCA.

\section{References}

Abbott, N. J., Patabendige, A. A., Dolman, D. E., Yusof, S. R., and Begley, D. J. (2010). Structure and function of the blood-brain barrier. Neurobiol. Dis. 37, 13-25. doi: 10.1016/j.nbd.2009.07.030

Ahlfors, C. E., Wennberg, R. P., Ostrow, J. D., and Tiribelli, C. (2009). Unbound (free) bilirubin: improving the paradigm for evaluating neonatal jaundice. Clin. Chem. 55, 1288-1299. doi: 10.1373/clinchem.2008.121269

Akin, E., Clower, B., Tibbs, R., Tang, J., and Zhang, J. (2002). Bilirubin produces apoptosis in cultured bovine brain endothelial cells. Brain Res. 931, 168-175. doi: 10.1016/S0006-8993(02)02276-X

Amaral, J. D., Viana, R. J., Ramalho, R. M., Steer, C. J., and Rodrigues, C. M. P. (2009a). Bile acids: regulation of apoptosis by ursodeoxycholic acid. J. Lipid Res. 50, 1721-1734. doi: 10.1194/jlr.R900011-JLR200

Amaral, J. D., Viana, R. J., Ramalho, R. M., Steer, C. J., and Rodrigues, C. M. P. (2009b). Bile acids: regulation of apoptosis by ursodeoxycholic acid. J. Lipid Res. 50, 1721-1734. doi: 10.1194/jlr.R900011-JLR200

Barateiro, A., Domingues, H. S., Fernandes, A., Relvas, J. B., and Brites, D. (2014). Rat cerebellar slice cultures exposed to bilirubin evidence reactive gliosis, excitotoxicity and impaired myelinogenesis that is prevented by AMPA and TNFalpha inhibitors. Mol. Neurobiol. 49, 424-439. doi: 10.1007/s12035-013-8530-7

Barateiro, A., Vaz, A. R., Silva, S. L., Fernandes, A., and Brites, D. (2012). ER stress, mitochondrial dysfunction and calpain/JNK activation are involved in oligodendrocyte precursor cell death by unconjugated bilirubin. Neuromol. Med. 14, 285-302. doi: 10.1007/s12017-012-8187-9

Batty, H. K., and Millhouse, O. E. (1976). Ultrastructure of the Gunn rat substantia nigra I. Cytoplasmic changes. Acta Neuropathol. 35, 93-107. doi: 10.1007/BF00690556

Bellentani, S., Chao, Y. C., Ferretti, I., Panini, R., and Tiribelli, C. (1996). Chronic administration of ursodeoxycholic and tauroursodeoxycholic acid changes microsomal membrane lipid content and fatty acid compositions in rats. Biochem. Biophys. Res. Commun. 220, 479-483. doi: 10.1006/bbrc. 1996.0430
The results also show a higher efficacy when the bile acids are administered before injury, reinforcing their preventive effects. They further show that both molecules slowly permeate across the $\mathrm{BBB}$ endothelium, which points to their potential to reach the brain and elicit therapeutic properties in target cells.

\section{Author Contributions}

IP, performed most of the experimental work, analyzed data and drafted the manuscript; LC, RFMS, HS, and KSK contributed to the acquisition, analysis and interpretation of the data, as well as to the review of the manuscript. DB contributed to the analysis and interpretation of data, and critically revised the manuscript. MAB designed the work, contributed to the acquisition, analysis and interpretation of data, and critically revised the manuscript. All the authors approved the version to be published and provided agreement to be accountable for all aspects of the work.

\section{Acknowledgments}

This work was supported by FEDER (COMPETE Programme) and by National funds (FCT - Fundação para a Ciência e a Tecnologia): project PEst-OE/SAU/UI4013/2011-2013 to iMed.ULisboa, PTDC/SAU-FCF/68819/2006 (to MAB), and a $\mathrm{PhD}$ fellowship (SFRH/BD/61646/2009) to IP.
Brites, D. (2012). The evolving landscape of neurotoxicity by unconjugated bilirubin: role of glial cells and inflammation. Front. Pharmacol. 3:88. doi: 10.3389/fphar.2012.00088

Brites, D., and Brito, M. A. (2012). "Bilirubin toxicity," in Care of The Jaundiced Neonate, eds D. K. Stevenson, M. J. Maisels and J. F. Watchko (New York, NY: Mc-Graw-Hill Companies, Inc.), 115-143.

Brites, D., Rodrigues, C. M. P., Van-Zeller, H., Brito, A., and Silva, R. (1998). Relevance of serum bile acid profile in the diagnosis of intrahepatic cholestasis of pregnancy in an high incidence area: Portugal. Eur. J. Obstet. Gynecol. Reprod. Biol. 80, 31-38. doi: 10.1016/S0301-2115(98)00086-4

Brito, M. A. (2001). Interacção da Bilirrubina Com o Eritrócito. Espécies Moleculares Envolvidas, Estádios de Toxicidade e Comportamento da Célula Fetal. $\mathrm{PhD}$ Thesis, Faculty of Pharmacy, University of Lisbon.

Brito, M. A., Brites, D., and Butterfield, D. A. (2004). A link between hyperbilirubinemia, oxidative stress and injury to neocortical synaptosomes. Brain Res. 1026, 33-43. doi: 10.1016/j.brainres.2004.07.063

Brito, M. A., Lima, S., Fernandes, A., Falcão, A. S., Silva, R. F. M., Butterfield, D. A., et al. (2008). Bilirubin injury to neurons: contribution of oxidative stress and rescue by glycoursodeoxycholic acid. Neurotoxicology 29, 259-269. doi: 10.1016/j.neuro.2007.11.002

Brito, M. A., Pereira, P., Barroso, C., Aronica, E., and Brites, D. (2013). New autopsy findings in different brain regions of a preterm neonate with kernicterus: neurovascular alterations and up-regulation of efflux transporters. Pediatr. Neurol. 49, 431-438. doi: 10.1016/j.pediatrneurol.2013. 08.020

Brito, M. A., Silva, R., Tiribelli, C., and Brites, D. (2000). Assessment of bilirubin toxicity to erythrocytes. Implication in neonatal jaundice management. Eur. J. Clin. Invest. 30, 239-247. doi: 10.1046/j.1365-2362.2000. 00612.x

Brito, M. A., Zurolo, E., Pereira, P., Barroso, C., Aronica, E., and Brites, D. (2012). Cerebellar axon/myelin loss, angiogenic sprouting, and neuronal increase of vascular endothelial growth factor in a preterm infant with kernicterus. J. Child Neurol. 27, 615-624. doi: 10.1177/0883073811423975 
Cardoso, F. L., Brites, D., and Brito, M. A. (2010). Looking at the blood-brain barrier: molecular anatomy and possible investigation approaches. Brain Res. Rev. 64, 328-363. doi: 10.1016/j.brainresrev.2010.05.003

Cardoso, F. L., Kittel, A., Veszelka, S., Palmela, I., Toth, A., Brites, D., et al. (2012). Exposure to lipopolysaccharide and/or unconjugated bilirubin impair the integrity and function of brain microvascular endothelial cells. PLOS ONE 7:e35919. doi: 10.1371/journal.pone.0035919

Carvey, P. M., Hendey, B., and Monahan, A. J. (2009). The blood-brain barrier in neurodegenerative disease: a rhetorical perspective. J. Neurochem. 111, 291-314. doi: 10.1111/j.1471-4159.2009.06319.x

Cebollero, E., Reggiori, F., and Kraft, C. (2012). Reticulophagy and ribophagy: regulated degradation of protein production factories. Int. J. Cell Biol. 2012:182834. doi: 10.1155/2012/182834

Chiche, J., Rouleau, M., Gounon, P., Brahimi-Horn, M. C., Pouyssegur, J., and Mazure, N. M. (2010). Hypoxic enlarged mitochondria protect cancer cells from apoptotic stimuli. J. Cell. Physiol. 222, 648-657. doi: 10.1002/jcp.21984

Cho, J. G., Lee, J. H., Hong, S. H., Lee, H. N., Kim, C. M., Kim, S. Y., et al. (2015). Tauroursodeoxycholic acid, a bile acid, promotes blood vessel repair by recruiting vasculogenic progenitor cells. Stem Cells. 33, 792-805. doi: 10.1002/stem.1901

Cohen, R. S., Wong, R. J., and Stevenson, D. K. (2010). Understanding neonatal jaundice: a perspective on causation. Pediatr. Neonatol. 51, 143-148. doi: 10.1016/S1875-9572(10)60027-7

Deli, M. A., Abraham, C. S., Kataoka, Y., and Niwa, M. (2005). Permeability studies on in vitro blood-brain barrier models: physiology, pathology, and pharmacology. Cell. Mol. Neurobiol. 25, 59-127. doi: 10.1007/s10571-004-1377-8

De Vries, H. E., Blom-Roosemalen, M. C., Van Oosten, M., De Boer, A. G., Van Berkel, T. J., Breimer, D. D., et al. (1996). The influence of cytokines on the integrity of the blood-brain barrier in vitro. J. Neuroimmunol. 64, 37-43. doi: 10.1016/0165-5728(95)00148-4

Duan, W. M., Rodrigues, C. M. P., Zhao, L. R., Steer, C. J., and Low, W. C. (2002). Tauroursodeoxycholic acid improves the survival and function of nigral transplants in a rat model of Parkinson's disease. Cell Transplant. 11, 195-205. doi: 10.0000/096020198389960

Eigenmann, D. E., Xue, G., Kim, K. S., Moses, A. V., Hamburger, M., and Oufir, M. (2013). Comparative study of four immortalized human brain capillary endothelial cell lines, hCMEC/D3, hBMEC, TY10, and BB19, and optimization of culture conditions, for an in vitro blood-brain barrier model for drug permeability studies. Fluids Barriers CNS 10:33. doi: 10.1186/2045-81 18-10-33

Erdbruegger, U., Haubitz, M., and Woywodt, A. (2006). Circulating endothelial cells: a novel marker of endothelial damage. Clin. Chim. Acta 373, 17-26. doi: 10.1016/j.cca.2006.05.016

Falcão, A. S., Fernandes, A., Brito, M. A., Silva, R. F. M., and Brites, D. (2006). Bilirubin-induced immunostimulant effects and toxicity vary with neural cell type and maturation state. Acta Neuropathol. 112, 95-105. doi: 10.1007/s00401006-0078-4

Fernandes, A., Falcão, A. S., Silva, R. F. M., Brito, M. A., and Brites, D. (2007a). MAPKs are key players in mediating cytokine release and cell death induced by unconjugated bilirubin in cultured rat cortical astrocytes. Eur. J. Neurosci. 25, 1058-1068. doi: 10.1111/j.1460-9568.2007.05340.x

Fernandes, A., Falcão, A. S., Silva, R. F. M., Gordo, A. C., Gama, M. J., Brito, M. A., et al. (2006). Inflammatory signalling pathways involved in astroglial activation by unconjugated bilirubin. J. Neurochem. 96, 1667-1679. doi: 10.1111/j.14714159.2006.03680.x

Fernandes, A., Vaz, A. R., Falcão, A. S., Silva, R. F. M., Brito, M. A., and Brites, D. (2007b). Glycoursodeoxycholic acid and interleukin-10 modulate the reactivity of rat cortical astrocytes to unconjugated bilirubin. J. Neuropathol. Exp. Neurol. 66, 789-798. doi: 10.1097/nen.0b013e3181461c74

Greenwood, J., Adu, J., Davey, A. J., Abbott, N. J., and Bradbury, M. W. (1991). The effect of bile salts on the permeability and ultrastructure of the perfused, energydepleted, rat blood-brain barrier. J. Cereb. Blood Flow Metab. 11, 644-654. doi: $10.1038 / \mathrm{jcbfm} .1991 .116$

Gunn, C. H. (1938). Hereditary acholuric jaundice in a new mutant strain of rats. $J$ Hereditary 29, 137-139.

Hansen, T. W. R., Tommarello, S., and Allen, J. (2001). Subcellular localization of bilirubin in rat brain after in vivo i.v. administration of $[3 \mathrm{H}]$ bilirubin. Pediatr. Res. 49, 203-207. doi: 10.1203/00006450-200102000-00012
Hülper, P., Veszelka, S., Walter, F. R., Wolburg, H., Fallier-Becker, P., Piontek, J., et al. (2013). Acute effects of short-chain alkylglycerols on blood-brain barrier properties of cultured brain endothelial cells. Br. J. Pharmacol. 169, 1561-1573. doi: $10.1111 /$ bph. 12218

Iskander, I., Gamaleldin, R., El Houchi, S., El Shenawy, A., Seoud, I., El Gharbawi, N., et al. (2014). Serum bilirubin and bilirubin/albumin ratio as predictors of bilirubin encephalopathy. Pediatrics 134, e1330-e1339. doi: 10.1542/peds.20131764

Kaemmerer, W. F., Rodrigues, C. M., Steer, C. J., and Low, W. C. (2001). Creatinesupplemented diet extends purkinje cell survival in spinocerebellar ataxia type 1 transgenic mice but does not prevent the ataxic phenotype. Neuroscience 103, 713-724. doi: 10.1016/S0306-4522(01)00017-3

Kapitulnik, J., Benaim, C., and Sasson, S. (2012). Endothelial cells derived from the blood-brain barrier and islets of langerhans differ in their response to the effects of bilirubin on oxidative stress under hyperglycemic conditions. Front. Pharmacol. 3:131. doi: 10.3389/fphar.2012.00131

Kaur, C., and Ling, E. A. (2008). Blood brain barrier in hypoxic-ischemic conditions. Curr. Neurovasc. Res. 5, 71-81. doi: 10.2174/156720208783565645

Keene, C. D., Rodrigues, C. M. P., Eich, T., Chhabra, M. S., Steer, C. J., and Low, W. C. (2002). Tauroursodeoxycholic acid, a bile acid, is neuroprotective in a transgenic animal model of Huntington's disease. Proc. Natl. Acad. Sci. U.S.A. 99, 10671-10676. doi: 10.1073/pnas.162362299

Labus, J., Hackel, S., Lucka, L., and Danker, K. (2014). Interleukin-1beta induces an inflammatory response and the breakdown of the endothelial cell layer in an improved human THBMEC-based in vitro blood-brain barrier model. J. Neurosci. Methods 228, 35-45. doi: 10.1016/j.jneumeth.2014.03.002

Lazaridis, K. N., Gores, G. J., and Lindor, K. D. (2001). Ursodeoxycholic acid 'mechanisms of action and clinical use in hepatobiliary disorders'. J. Hepatol. 35, 134-146. doi: 10.1016/S0168-8278(01)00092-7

Ma, J., Iida, H., Jo, T., Takano, H., Oonuma, H., Morita, T., et al. (2004). Ursodeoxycholic acid inhibits endothelin-1 production in human vascular endothelial cells. Eur. J. Pharmacol. 505, 67-74. doi: 10.1016/j.ejphar.2004.10.042

Malik, S. G., Irwanto, K. A., Ostrow, J. D., and Tiribelli, C. (2010). Effect of bilirubin on cytochrome $c$ oxidase activity of mitochondria from mouse brain and liver. BMC Res. Notes 3:162. doi: 10.1186/1756-0500-3-162

Maruo, N., Morita, I., Shirao, M., and Murota, S. (1992). IL-6 increases endothelial permeability in vitro. Endocrinology 131, 710-714.

Mcdonagh, A. F., and Assisi, F. (1972). The ready isomerization of bilirubin IX- $\alpha$ in aqueous solution. Biochem. J. 129, 797-800.

Min, J. H., Hong, Y. H., Sung, J. J., Kim, S. M., Lee, J. B., and Lee, K. W. (2012). Oral solubilized ursodeoxycholic acid therapy in amyotrophic lateral sclerosis: a randomized cross-over trial. J. Korean Med. Sci. 27, 200-206. doi: 10.3346/jkms.2012.27.2.200

Mustafa, M. G., and King, T. E. (1970). Binding of bilirubin with lipid. A possible mechanism of its toxic reactions in mitochondria. J. Biol. Chem. 245, 1084-1089.

Nguyen, N., Bonzo, J. A., Chen, S., Chouinard, S., Kelner, M. J., Hardiman, G., et al. (2008). Disruption of the ugt1 locus in mice resembles human CriglerNajjar type I disease. J. Biol. Chem. 283, 7901-7911. doi: 10.1074/jbc.M709 244200

Nishida, J., Shiratsuchi, A., Nadano, D., Sato, T. A., and Nakanishi, Y. (2002). Structural change of ribosomes during apoptosis: degradation and externalization of ribosomal proteins in doxorubicin-treated Jurkat cells. J. Biochem. 131, 485-493. doi: 10.1093/oxfordjournals.jbchem.a003125

Palmela, I., Cardoso, F. L., Bernas, M., Correia, L., Vaz, A. R., Silva, R. F. M., et al. (2011). Elevated levels of bilirubin and long-term exposure impair human brain microvascular integrity Curr. Neurovasc. Res. 8, 153-169. doi: $10.2174 / 156720211795495358$

Palmela, I., Sasaki, H., Cardoso, F. L., Moutinho, M., Kim, K. S., Brites, D., et al. (2012). Time-dependent dual effects of high levels of unconjugated bilirubin on the human blood-brain barrier lining. Front. Cell. Neurosci. 6:22. doi: $10.3389 /$ fncel.2012.00022

Parry, G. J., Rodrigues, C. M. P., Aranha, M. M., Hilbert, S. J., Davey, C., Kelkar, P., et al. (2010). Safety, tolerability, and cerebrospinal fluid penetration of ursodeoxycholic acid in patients with amyotrophic lateral sclerosis. Clin. Neuropharmacol. 33, 17-21. doi: 10.1097/WNF.0b013e318 $1 c 47569$ 
Paumgartner, G., and Beuers, U. (2004). Mechanisms of action and therapeutic efficacy of ursodeoxycholic acid in cholestatic liver disease. Clin. Liver. Dis. 8, 67-81, vi. doi: 10.1016/S1089-3261(03)00135-1

Perez, M. J., and Briz, O. (2009). Bile-acid-induced cell injury and protection. World J. Gastroenterol. 15, 1677-1689. doi: 10.3748/wjg.15.1677

Podda, M., Ghezzi, C., Battezzati, P. M., Crosignani, A., Zuin, M., and Roda, A. (1990). Effects of ursodeoxycholic acid and taurine on serum liver enzymes and bile acids in chronic hepatitis. Gastroenterology 98, 1044-1050. doi: 10.1016/0016-5085(90)90032-V

Poupon, R. E., Poupon, R., and Balkau, B. (1994). Ursodiol for the long-term treatment of primary biliary cirrhosis. The UDCA-PBC study group. N. Engl. J. Med. 330, 1342-1347. doi: 10.1056/NEJM199405123301903

Roca, L., Calligaris, S., Wennberg, R. P., Ahlfors, C. E., Malik, S. G., Ostrow, J. D., et al. (2006). Factors affecting the binding of bilirubin to serum albumins: validation and application of the peroxidase method. Pediatr. Res. 60, 724-728. doi: 10.1203/01.pdr.0000245992.89965.94

Roda, A., Minutello, A., Angellotti, M. A., and Fini, A. (1990). Bile acid structureactivity relationship: evaluation of bile acid lipophilicity using 1-octanol/water partition coefficient and reverse phase HPLC. J. Lipid Res. 31, 1433-1443.

Rodrigues, C. M. P., Solá, S., and Brites, D. (2002a). Bilirubin induces apoptosis via the mitochondrial pathway in developing rat brain neurons. Hepatology 35 , 1186-1195. doi: 10.1053/jhep.2002.32967

Rodrigues, C. M. P., Solá, S., Brito, M. A., Brites, D., and Moura, J. J. G. (2002b). Bilirubin directly disrupts membrane lipid polarity and fluidity, protein order, and redox status in rat mitochondria. J. Hepatol. 36, 335-341. doi: 10.1016/S0168-8278(01)00279-3

Rodrigues, C. M. P., Solá, S., Brito, M. A., Brondino, C. D., Brites, D., and Moura, J. J. G. (2001). Amyloid beta-peptide disrupts mitochondrial membrane lipid and protein structure: protective role of tauroursodeoxycholate. Biochem. Biophys. Res. Commun. 281, 468-474. doi: 10.1006/bbrc.2001.4370

Rodrigues, C. M. P., Solá, S., Nan, Z., Castro, R. E., Ribeiro, P. S., Low, W. C., et al. (2003). Tauroursodeoxycholic acid reduces apoptosis and protects against neurological injury after acute hemorrhagic stroke in rats. Proc. Natl. Acad. Sci. U.S.A. 100, 6087-6092. doi: 10.1073/pnas.1031632100

Rodrigues, C. M. P., Solá, S., Silva, R., and Brites, D. (2000). Bilirubin and amyloidbeta peptide induce cytochrome $\mathrm{c}$ release through mitochondrial membrane permeabilization. Mol. Med. 6, 936-946.

Rudolph, G., Kloeters-Plachky, P., Sauer, P., and Stiehl, A. (2002). Intestinal absorption and biliary secretion of ursodeoxycholic acid and its taurine conjugate. Eur. J. Clin. Invest. 32, 575-580. doi: 10.1046/j.1365-2362.2002.01030.x

Ruiz-Gaspa, S., Dubreuil, M., Guanabens, N., Combalia, A., Peris, P., Monegal, A., et al. (2014). Ursodeoxycholic acid decreases bilirubin-induced osteoblast apoptosis. Eur. J. Clin. Invest. 44, 1206-1214. doi: 10.1111/eci.12355

Silva, R. F. M., Falcão, A. S., Fernandes, A., Gordo, A. C., Brito, M. A., and Brites, D. (2006). Dissociated primary nerve cell cultures as models for assessment of neurotoxicity. Toxicol. Lett. 163, 1-9. doi: 10.1016/j.toxlet.2005.09.033

Silva, R. F. M., Mata, L. M., Gulbenkian, S., and Brites, D. (2001a). Endocytosis in rat cultured astrocytes is inhibited by unconjugated bilirubin. Neurochem. Res. 26, 793-800. doi: 10.1023/A:1011608017870

Silva, R. F. M., Rodrigues, C. M. P., and Brites, D. (2001b). Bilirubin-induced apoptosis in cultured rat neural cells is aggravated by chenodeoxycholic acid but prevented by ursodeoxycholic acid. J. Hepatol. 34, 402-408. doi: 10.1016/S01688278(01)00015-0

Silva, R. F. M., Rodrigues, C. M. P., and Brites, D. (2002). Rat cultured neuronal and glial cells respond differently to toxicity of unconjugated bilirubin. Pediatr. Res. 51, 535-541. doi: 10.1203/00006450-200204000-00022

Silva, S. L., Vaz, A. R., Barateiro, A., Falcão, A. S., Fernandes, A., Brito, M. A., et al. (2010). Features of bilirubin-induced reactive microglia: from phagocytosis to inflammation. Neurobiol. Dis. 40, 663-675. doi: 10.1016/j.nbd.2010.08.010
Silva, S. L., Vaz, A. R., Diógenes, M. J., Van Rooijen, N., Sebastião, A. M., Fernandes, A., et al. (2012). Neuritic growth impairment and cell death by unconjugated bilirubin is mediated by NO and glutamate, modulated by microglia, and prevented by glycoursodeoxycholic acid and interleukin-10. Neuropharmacology 62, 2398-2408. doi: 10.1016/j.neuropharm.2012.02.002

Solá, S., Brito, M. A., Brites, D., Moura, J. J. G., and Rodrigues, C. M. P. (2002). Membrane structural changes support the involvement of mitochondria in the bile salt-induced apoptosis of rat hepatocytes. Clin. Sci. (Lond) 103, 475-485. doi: $10.1042 / \mathrm{CS} 20020196$

Solá, S., Castro, R. E., Laires, P. A., Steer, C. J., and Rodrigues, C. M. P. (2003). Tauroursodeoxycholic acid prevents amyloid-beta peptide-induced neuronal death via a phosphatidylinositol 3-kinase-dependent signaling pathway. Mol. Med. 9, 226-234. doi: 10.2119/2003-00042.Rodrigues

Stins, M. F., Badger, J., and Kim, K. S. (2001). Bacterial invasion and transcytosis in transfected human brain microvascular endothelial cells. Microb. Pathog. 30, 19-28. doi: 10.1006/mpat.2000.0406

Suh, H., Jung, E. J., Kim, T. H., Lee, H. Y., Park, Y. H., and Kim, K. W. (1997). Antiangiogenic activity of ursodeoxycholic acid and its derivatives. Cancer Lett. 113, 117-122. doi: 10.1016/S0304-3835(97)04604-1

Vaz, A. R., Delgado-Esteban, M., Brito, M. A., Bolaños, J. P., Brites, D., and Almeida, A. (2010). Bilirubin selectively inhibits cytochrome $c$ oxidase activity and induces apoptosis in immature cortical neurons: assessment of the protective effects of glycoursodeoxycholic acid. J. Neurochem. 112, 56-65. doi: 10.1111/j.1471-4159.2009.06429.x

Veszelka, S., Pasztoi, M., Farkas, A. E., Krizbai, I., Ngo, T. K., Niwa, M., et al. (2007). Pentosan polysulfate protects brain endothelial cells against bacterial lipopolysaccharide-induced damages. Neurochem. Int. 50, 219-228. doi: 10.1016/j.neuint.2006.08.006

Viana, R. J., Nunes, A. F., Castro, R. E., Ramalho, R. M., Meyerson, J., Fossati, S., et al. (2009). Tauroursodeoxycholic acid prevents E22Q Alzheimer's Abeta toxicity in human cerebral endothelial cells. Cell. Mol. Life Sci. 66, 1094-1104. doi: 10.1007/s00018-009-8746-x

Vladykovskaya, E., Sithu, S. D., Haberzettl, P., Wickramasinghe, N. S., Merchant, M. L., Hill, B. G., et al. (2012). Lipid peroxidation product 4-hydroxy-trans2-nonenal causes endothelial activation by inducing endoplasmic reticulum stress. J. Biol. Chem. 287, 11398-11409. doi: 10.1074/jbc.M111.320416

Wang, C. T., Lin, Y. T., Chiang, B. L., Lin, Y. H., and Hou, S. M. (2006). High molecular weight hyaluronic acid down-regulates the gene expression of osteoarthritis-associated cytokines and enzymes in fibroblast-like synoviocytes from patients with early osteoarthritis. Osteoarthr. Cartil. 14, 1237-1247. doi: 10.1016/j.joca.2006.05.009

Wennberg, R. P. (2000). The blood-brain barrier and bilirubin encephalopathy. Cell. Mol. Neurobiol. 20, 97-109. doi: 10.1023/A:1006900111744

Woo, S. J., Kim, J. H., and Yu, H. G. (2010). Ursodeoxycholic acid and tauroursodeoxycholic acid suppress choroidal neovascularization in a laser-treated rat model. J. Ocul. Pharmacol. Ther. 26, 223-229. doi: 10.1089/jop.2010.0012

Zlokovic, B. V. (2008). The blood-brain barrier in health and chronic neurodegenerative disorders. Neuron 57, 178-201. doi: 10.1016/j.neuron.2008.01.003

Conflict of Interest Statement: The authors declare that the research was conducted in the absence of any commercial or financial relationships that could be construed as a potential conflict of interest.

Copyright (c) 2015 Palmela, Correia, Silva, Sasaki, Kim, Brites and Brito. This is an open-access article distributed under the terms of the Creative Commons Attribution License (CC BY). The use, distribution or reproduction in other forums is permitted, provided the original author(s) or licensor are credited and that the original publication in this journal is cited, in accordance with accepted academic practice. No use, distribution or reproduction is permitted which does not comply with these terms. 


\section{OPEN ACCESS}

Edited by:

Norman Ruthven Saunders, University of Melbourne, Australia

Reviewed by:

Joao C. Sousa,

University of Minho, Portugal

Pia Johansson,

Helmholtz-Zentrum München,

Germany

Maria Lehtinen,

Boston Children's Hospital, USA

Richard F. Keep,

University of Michigan, USA

*Correspondence:

Patrizia Ferretti,

Stem Cells and Regenerative

Medicine Section, UCL Institute of Child Health, 30 Guilford Street,

London WC1N 1EH, UK p.ferretti@ucl.ac.uk

Specialty section:

This article was submitted to Neurogenomics, a section of the journal Frontiers in Neuroscience

Received: 06 January 2015

Accepted: 11 March 2015

Published: 31 March 2015

Citation:

Prasongchean W, Vernay B, Asgarian $Z$, Jannatul $N$ and Ferretti $P$ (2015) The neural milieu of the developing choroid plexus: neural stem cells, neurons and innervation. Front. Neurosci. 9:103. doi: 10.3389/fnins.2015.00103

\section{The neural milieu of the developing choroid plexus: neural stem cells, neurons and innervation}

\author{
Weerapong Prasongchean ${ }^{1,2}$, Bertrand Vernay ${ }^{1}$, Zeinab Asgarian ${ }^{1}$, Nahin Jannatul ${ }^{1}$ and \\ Patrizia Ferretti ${ }^{1 *}$ \\ ${ }^{1}$ Stem Cells and Regenerative Medicine Section, UCL Institute of Child Health, University College London, London, UK, \\ ${ }^{2}$ Department of Biochemistry and Microbiology, Faculty of Pharmaceutical Sciences, Chulalongkorn University, Bangkok, \\ Thailand
}

The choroid plexus produces cerebrospinal fluid and plays an important role in brain homeostasis both pre and postnatally. In vitro studies have suggested that cells from adult choroid plexus have stem/progenitor cell-like properties. Our initial aim was to investigate whether such a cell population is present in vivo during development of the choroid plexus, focusing mainly on the chick choroid plexus. Cells expressing neural markers were indeed present in the choroid plexus of chick and also those of rodent and human embryos, both within their epithelium and mesenchyme. B3-tubulin-positive cells with neuronal morphology could be detected as early as at E8 in chick choroid plexus and their morphological complexity increased with development. Whole mount immunochemistry demonstrated the presence of neurons throughout choroid plexus development and they appeared to be mainly catecholaminergic, as indicated by tyrosine-hydroxylase reactivity. The presence of cells co-labeling for BrdU and the neuroblast marker, doublecortin, in organotypic choroid plexus cultures supported the hypothesis that neurogenesis can occur from neural precursors within the developing choroid plexus. Furthermore, we found that extrinsic innervation is present in the developing choroid plexus, unlike previously suggested. Altogether, our data are consistent with the presence of neural progenitors within the choroid plexus, suggest that at least some of the choroid plexus neurons are born locally, and show for the first time that choroid plexus innervation occurs prenatally. Hence, we propose the existence of a complex neural regulatory network within the developing choroid plexus that may play a crucial role in modulating its function during development as well as throughout life.

Keywords: chick, choroid plexus, development, innervation, neurogenesis, neural stem cell

Abbreviations: Aq-1, aquaporin-1; ASPP2, apoptosis-stimulating of p53 protein 2; BrdU, 5-bromo-2'-deoxyuridine; bv blood vessel; CP, choroid plexus; CSF, cerebrospinal fluid; Dcx, doublecortin; EBSS, Earle's balanced salt solution; GAPDH, glyceraldehyde 3-phosphate dehydrogenase; GFAP, glial fibrillary acidic protein; HDBR, human developmental biology resource; ne, neuroepithelium; NF200, neurofilament $200 \mathrm{kDa}$; MB, midbrain; MBP, myelin basic protein; MTT, 3-(4,5Dimethylthiazol-2-yl)-2,5-diphenyltetrazolium bromide; Otx2, orthodenticle homeobox 2; PFA, paraformaldehyde; pH3, phosphorylated-histone 3; PI, propidium iodide; RT-PCR, reverse transcription polymerase chain reaction; RT-qPCR, quantitative reverse transcription polymerase chain reaction; Sox2, SRY (sex determining region Y)-box 2; SV2, synaptic vesicles 2; TH, tyrosine-hydroxylase; vWF, von Willebrand factor; ZO-1, zonula occludens-1. 


\section{Introduction}

The choroid plexus (CP) is important for the secretion of cerebrospinal fluid (CSF) and plays an important role in brain development, homeostasis and disease. The CP, found in lateral, third and fourth ventricles of the brain, consists of a highly vascularized stroma with loose connective tissue surrounded by a single layer of simple cuboidal epithelium. The $\mathrm{CP}$ epithelium, considered to be a specialized ependymal epithelium, is continuous with the ependyma lining the brain of lateral, III and IV ventricles. It originates from the neuroepithelium, whereas the inner CP stromal core is believed to originate from the mesenchyme (Lehtinen et al., 2013; Liddelow et al., 2013).

The CP develops early during embryogenesis and which CP is first clearly visible depends on the species. In mouse and humans, the IV ventricle $\mathrm{CP}$ anlage is the first one observed at approximately E10.5 and 41 days of gestation, respectively (Dziegielewska et al., 2001; Hunter and Dymecki, 2007). In the chick embryo, it has been indicated that a $\mathrm{CP}$ anlage is first detectable in the lateral ventricles between E6 and E8. The chick lateral ventricle $\mathrm{CP}$ rapidly grows, lengthening and branching out many times with a dip in its growth reported at around E15-E16 followed by its thinning and flattening (Smith, 1966; Stastny and Rychter, 1976).

The CP constitutes the blood-CSF barrier that is established by the tight junctions of the $\mathrm{CP}$ epithelial cells. The $\mathrm{CP}$ is a very active organ that continuously secretes CSF from early development throughout life (Johansson et al., 2008; Liddelow et al., 2012; Lehtinen et al., 2013). It has been proposed that its secretory function is controlled by its autonomic innervation (Edvinsson and Lindvall, 1978; Lindvall and Owman, 1981). CSF secretion could be inhibited by stimulation of sympathetic nerves in the neck (Lindvall et al., 1978c) and via activation of $\beta 2$ receptors (Nathanson, 1980). Sympathetic denervation in rabbits resulted in hydrocephalus and was fatal (Lindvall and Owman, 1981). It has been assumed that nerves enter the CP only after birth (Tsuker, 1947; Edvinsson et al., 1973; Lindvall et al., 1977a, 1978a,b,c; Edvinsson and Lindvall, 1978), but there is only one study where this issue was specifically addressed and that suggests that the CP becomes innervated postnatally (Lindvall and Owman, 1978). This seems peculiar, as innervation tends to occur at early stages of organ development, before a high degree of complexity is achieved. We therefore postulated that this would be the case also for the $\mathrm{CP}$ and set to test this hypothesis.

A variety of stem/progenitor cells have been hypothesized to reside within the CP. Nataf and co-workers have reported that myeloid progenitors in the $\mathrm{CP}$ are capable of differentiation toward macrophage or dendritic cell phenotypes (Nataf et al., 2006). In addition, the CP has been reported to contain neural stem-like cells able to give rise to neural cells in vitro and upon transplantation into a spinal cord injury model (Kitada et al., 2001). The transcriptome of the $\mathrm{CP}$ from adult mouse has revealed the presence of genes important for neural development (Itokazu et al., 2006; Marques et al., 2011). The neural stem cell potential observed in the CP has been suggested to reside in the CP epithelium on the basis of in vitro studies (Itokazu et al., 2006). The localization of those putative neural stem cell population(s) was not extensively studied in vivo. In another study in rat pups, the presence of nestin-expressing cells just beneath the $\mathrm{CP}$ epithelium, that decreased in number with age, was taken to indicate the presence of neural stem cells in the CP stroma (Huang et al., 2011). We hypothesized that the developing CP harbors a neurogenic niche that may account for the proposed "neurogenicity" of the $\mathrm{CP}$.

We assessed the presence of neural progenitors and time of innervation in developing $\mathrm{CPs}$, focusing on the chick $\mathrm{CP}$ in vivo and in organotypic cultures. We show for the first time that the CP mesenchymal compartment contains neural progenitorlike cells that may be the precursors of the neurons identified in this compartment, and that innervation of the $\mathrm{CP}$ is an early developmental event. Hence, we suggest a model where a neural regulatory network is present within the $\mathrm{CP}$ and may play a crucial role in modulating its function during development as well as throughout life.

\section{Materials and Methods}

Unless otherwise specified all general reagents were from Sigma and tissue culture reagents from Gibco.

\section{Chicken Embryos and Isolation of Choroid Plexus (CP)}

Fertilized Brown Leghorn chicken eggs were purchased from Henry Stewart \& Co. Ltd (Lincolnshire, UK). On arrival, eggs were stored at $15^{\circ} \mathrm{C}$ in the egg fridge (JENCONS Ltd., USA) and used within 1 week. They were maintained on cardboard egg racks in a humidified forced flow incubator at $38^{\circ} \mathrm{C}$ (MARSH automatic incubator, LYON electric company, USA) until the required developmental stages. Chick embryos were decapitated and brains removed. The meninges were carefully peeled off because its connective tissue normally attaches to the pineal gland which also connects to the 3 rd ventricle $\mathrm{CP}$. The isolated $\mathrm{CP}$ was used for whole mount immunohistochemistry, organotypic culture, or RNA extraction. Unless otherwise indicated, lateral ventricle $\mathrm{CPs}$ were used.

\section{Human and Mouse Embryos}

Mouse embryonic brains were isolated and fixed in $4 \%$ paraformaldehyde (PFA) overnight at $4^{\circ} \mathrm{C}$, cryo-protected by incubation in $30 \%$ sucrose containing $0.02 \%$ sodium azide in PBS (phosphate buffered saline) at $4^{\circ} \mathrm{C}$ for approximately $24 \mathrm{~h}$, OCT embedded, and immunohistochemistry performed. Paraffin sections of human brains at Carnegie stage 23 (Cs23; 56 days of gestation) were obtained from the Human Developmental Biology Resource (HDBR).

\section{Immunohistochemistry and Whole Mount Labeling}

Embryonic chick or mouse brains were fixed in $4 \%$ PFA overnight at $4{ }^{\circ} \mathrm{C}$, cryo-protected by incubation in $30 \%$ sucrose containing $0.02 \%$ sodium azide in PBS (phosphate buffered saline) at $4^{\circ} \mathrm{C}$ for approximately $24 \mathrm{~h}$, OCT embedded and cryosectioned (14-16 $\mu \mathrm{m}$ thick). Isolated CPs were fixed in 4\% PFA overnight 
TABLE 1 | List of antibodies used for immunohistochemistry and whole mount staining.

\begin{tabular}{|c|c|c|c|c|}
\hline & Dilution & Host & Types & Suppliers \\
\hline \multicolumn{5}{|l|}{ ANTIGEN (PRIMARY ANTIBODIES)* } \\
\hline Aquaporin 1 (Aq-1) & $1: 200$ & Mouse & $\lg G$ & Bioscience \\
\hline$\beta$ Tub3 & 1: 1000 & Mouse & $m A b \lg G$ & Promega, \\
\hline BrdU & 1: 100 & Mouse & $\lg G$ & DSHB \\
\hline Clusterin & 1: 100 & Rabbit & $\mathrm{pAb} \lg \mathrm{G}$ & Santa cruz (SC) \\
\hline Glial fibrillary acidic protein (GFAP) & $1: 1000$ & Rabbit & $\mathrm{pAb}$ & Chemicon \\
\hline Laminin & $1: 200$ & Rabbit & $\mathrm{pAb}$ & Milipore \\
\hline Myelin basic protein & 1: 100 & Mouse & $\lg G$ & Chemicon \\
\hline Nestin (human) & 1: 1000 & Rabbit & pAb IgG & Milipore \\
\hline Nestin (rat, mouse) & 1: 200 & Mouse & $\lg G$ & Developmental studies hybridoma bank (DSHB) \\
\hline Pax6 & 1: 100 & Mouse & mAb lgG1 & DSHB \\
\hline Phosphohistone3 (pH3) & 1: 100 & Rabbit & $\lg G$ & Upstate \\
\hline Sox2 & 1: 1000 & Rabbit & $\mathrm{pAb}$ & Milipore \\
\hline Synaptic vesicles 2 (SV2) & 1: 100 & Mouse & $\lg G 1$ & DSHB \\
\hline Transitin (chick) & 1: 100 & Mouse & mAb IgG1 & DSHB \\
\hline Tyrosine hydroxylase & 1: 400 & Sheep & $\mathrm{pAb}$ & Millipore \\
\hline VEGF & 1: 100 & Mouse & $\mathrm{mAb} \lg \mathrm{G} 1$ & SC \\
\hline Vimentin & 1: 100 & Mouse & $\lg G$ & DAKO \\
\hline ZO-1 & 1: 100 & Rabbit & $p A b \lg G$ & Molecular Probe \\
\hline \multicolumn{5}{|l|}{ SECONDARY ANTIBODIES } \\
\hline Anti-mouse IgG1a Alexa Fluor ${ }^{\circledR} 568$ & 1: 400 & Goat & - & Molecular probe \\
\hline
\end{tabular}

$m A b$, monoclonal antibody; pAb, polyclonal antibody. ${ }^{*}$ The same antibodies were used in the different species unless the species is indicated in brackets.

at $4^{\circ} \mathrm{C}$ and washed several times with PBS for whole mount labeling. For wax removal and epitope unmasking, human embryonic brain sections were immersed in 1:20 Declere ${ }^{\circledR}$ solution (Sigma) in PBS, heated in a microwave oven at 720 watt for $5 \mathrm{~min}$ and then at 270 watt for $20 \mathrm{~min}$, rinsed in PBS and then processed as cryosections. Brain sections or whole mount CPs were permeabilized (0.5\% Triton X-100 in PBS) and blocked in 20\% BSA in PBS for $1 \mathrm{~h}$ at room temperature. Sections were incubated with primary antibodies (Table 1) diluted in blocking solution for $3-4 \mathrm{~h}$ at room temperature or overnight at $4^{\circ} \mathrm{C}$. After several washes with PBS, sections were incubated with appropriate secondary antibodies for $1-2 \mathrm{~h}$. Citiflour ${ }^{\circledR}$ solution (Citifluor Limited) was used as a mounting medium in most of immunohistochemistry for cryosections and paraffin sections. In this case, cell nuclei were visualized with Hoechst $33258(2.5 \mu \mathrm{g} / \mathrm{ml}$ final concentration). Stained section and whole mount CPs were visualized and digitally scanned using an Axiophot 2 (Zeiss) with Hamamatsu ORCA-ER digital camera or by confocal laser scanning microscopy using an LSM 710 (Zeiss). Image analysis was performed using ImageJ software (Rasband, 1997-2009).

\section{Organotypic Culture of Chick CPs}

E12 chick embryo CPs were used to set up organotypic cultures. CPs from lateral and third ventricles were dissected and grown onto a $0.4-\mu \mathrm{M}$-Millipore ${ }^{\circledR}-\mathrm{CM}$ culture plate insert in MEM (minimal essential medium) containing Glutamax, 5\% Earle's balanced salt solution (EBSS), $36 \mathrm{mM}$ D-glucose, $1 \%$ penicillin/streptomycin and $25 \%$ horse serum.

\section{MTT Assay and PI Staining of Organotypic Chick CP Cultures}

Propidium iodide (PI) was used to identify cell death in organotypic CP cultures. Briefly, the cultures were incubated with culture medium containing $5 \mu \mathrm{g} / \mathrm{ml} \mathrm{PI}$ for $1 \mathrm{~h}$ at $37^{\circ} \mathrm{C}$ in an atmosphere of $95 \% \mathrm{O}_{2}$ and $5 \% \mathrm{CO}_{2}$. Organotypic cultures were incubated with $0.5 \mathrm{mg} / \mathrm{ml}$ MTT [3-(4,5-Dimethylthiazol2-yl)-2,5-diphenyltetrazolium bromide] solution for $1 \mathrm{~h}$ at $37^{\circ} \mathrm{C}$ to assess cell viability. The appearance of blue/purple formazan crystals is indicative of cell viability and structural integrity. 


\section{BrdU Incorporation in Organotypic Chick CP Cultures}

Organotypic CP preparations were treated with $10 \mu \mathrm{M}$ BrdU for 3 days and then processed for BrdU detection and immunohistochemistry. CPs were incubated in $1 \mathrm{~N} \mathrm{HCl}$ for $10 \mathrm{~min}$ on ice, followed by incubation in $2 \mathrm{~N} \mathrm{HCl}$ for $10 \mathrm{~min}$ at room temperature, and then for $20 \mathrm{~min}$ at $37^{\circ} \mathrm{C}$. The $\mathrm{CPs}$ were then washed three times in $0.1 \mathrm{M}$ borate buffer at room temperature, once in PBS, and immunostained as described above.

\section{Polymerase Chain Reaction (RT-qPCR)}

RNA was extracted from chick lateral ventricle $\mathrm{CP}$ and midbrain at different developmental stages (see Results) using Tri-Reagent (Life Technologies) according to the manufacturer's instructions. RNA was retro-transcribed with Moloney murine leukemia virus reverse transciptase (Promega, Madison, WI). The annealing temperature for RT-PCR was $54^{\circ} \mathrm{C}$ for Sox 2 and Otx2 (30 cycles) and $66^{\circ} \mathrm{C}$ for GAPDH ( 25 cycles). Real time quantitative polymerase chain reaction (RT-qPCR) was preformed with ABI Prism 7500 sequence detection system (Applied Biosystems) using the QuantiTect SYBR Green PCR kit (Qiagen, Hilden, Germany) according to the manufacturer's instructions. Gene expression data were normalized using GAPDH housekeeping gene as a reference using the 2- $\Delta \Delta \mathrm{Ct}$ method. The primers used for RT-PCR and RT-qPCR are listed in Table 2.

\section{Statistical Analysis}

Data are presented as mean \pm SEM. The statistical analysis was performed using GraphPad Prism version 5.00 for Windows. Statistical significance was evaluated by One-Way ANOVA. A $p$ value equal to or less than 0.05 was considered as statistically significant.

TABLE 2 | Primers used for analysis of gene expression in chick choroid plexus and midbrain by RT-PCR and RT-qPCR.

\begin{tabular}{ll}
\hline Gene & Primers $\left(\mathbf{5}^{\prime} \mathbf{-} \mathbf{3}^{\prime} \mathbf{)}\right.$ \\
\hline GAPDH & $\begin{array}{l}\text { For CCAGGTTGTCTCCTGTGACT } \\
\text { Rev CACAACACGGTTGCTGTAT }\end{array}$ \\
\hline Transitin & For CTGGAGCAGGAGAAGCAGAG \\
& Rev CTGTTGGCAGCTTGAACTC \\
\hline GFAP & For CCAGGTTGTCTCCTGTGACT \\
\hline Doublecortin (Dcx) & For CCCATTCGTTGGAGCAAG TT \\
\hline B3-tubulin & Rev CCTGTGCATAGCGGATTTT \\
\hline Sox2 & For TCTCACAAGTACGTGCCTCG \\
& Rev CCCCGCTCTGACCGAAAATG \\
\hline Otx2 & For AGGCTATGGGATGATGCAAG \\
& Rev GTAGGTAGGCGATCCGTTCA \\
\hline
\end{tabular}

\section{Results}

\section{Expression of Neural Markers in the Developing Choroid Plexus (CP)}

Expression of markers of neural stem/progenitor cells, such as Sox 2 and nestin, in the CP was first studied by fluorescent immunohistochemistry in sections from E12 chick brains (Figure 1 and Table 3). In the E12 CP, cells positive for the neural progenitor marker, Sox2, were detected in the $\mathrm{CP}$ epithelium of both lateral and 3rd ventricles (Figure 1A). The staining intensity in the $\mathrm{CP}$ was lower than in the neuroepithelium and a gradient of Sox 2 expression, from high, in the neuroepithelium, to low, in the CP epithelium, was apparent (Figure 1A, Table 3). Interestingly, Sox 2 expression in cells located at presumed $\mathrm{CP}$ branching points was stronger than in adjacent cells (see insert Figure 1A). The early CP epithelium was also positive for Otx2 and Pax6, and for several other markers that have been reported to be expressed in neural stem cells (Table 3). In contrast to the $\mathrm{CP}$ epithelium, the $\mathrm{CP}$ stroma was Sox2-negative. However, the $\mathrm{CP}$ mesenchyme contained cell expressing other neural markers, transitin, the chick nestin-like protein, and GFAP, a marker of astrocytes, radial glia and neural stem cells (Figures 1B,C). Expression of neural progenitor-associated proteins in the chick $\mathrm{CP}$ was consistent with expression of their transcripts detected by RT-PCR (Figure 2).

In order to establish whether neural progenitor-like cells were present only in the chick or also in mammals, we studied their expression in mouse $\mathrm{CP}$ at embryonic day 12.5 (E12.5) and in the Cs23 (56 days of gestation) human CP. Nestin and GFAP protein expression was found in the stromal compartment of the mouse CP paralleling that in the chick (Figures 1D,E). Proliferating nestin-positive cells were observed in the $\mathrm{CP}$ as well as in the neuroepithelium (Figure 1D). Expression of nestin and GFAP appeared to be mutually exclusive (Figure 1F), but some overlap of nestin immunoreactivity with an endothelial marker, the von Willebrand factor, was observed suggesting expression of nestin in some endothelial cells (Figure 1E). The developing human CP at Cs23 also displayed a gradient of SOX2 staining, as observed in the chick, and was also positive for vimentin and nestin (Figures 1G,H). Nestin staining in the human CP appeared to be sub-epithelial and vimentin expression was very high in the $\mathrm{CP}$ epithelium but not restricted to it (Figure 1H). This paralleled the vimentin pattern of expression observed in the chick $\mathrm{CP}$ (Table 3). In mouse CP at comparable stages of development, vimentin was detected in the $\mathrm{CP}$ stroma (not shown).

\section{Analysis of Neurogenesis in the Developing Chick Choroid Plexus (CP)}

Given the expression of several neural markers observed in the $\mathrm{CP}$, we investigated whether neurons were generated within the $\mathrm{CP}$ focusing on the chick $\mathrm{CP}$.

E12 chick lateral ventricle CPs were immunostained for the neuroblast marker, doublecourtin (Dcx), and the neuronal marker, $\beta 3$-tubulin. Extensive Dcx reactivity was detected even at a low magnification within the $\mathrm{CP}$ mesenchyme. Some scattered $\beta 3$-tubulin reactivity was observed in the E12 CP sections (Figures 3A,B). Both $D c x$ and $\beta 3$-tubulin transcripts were 

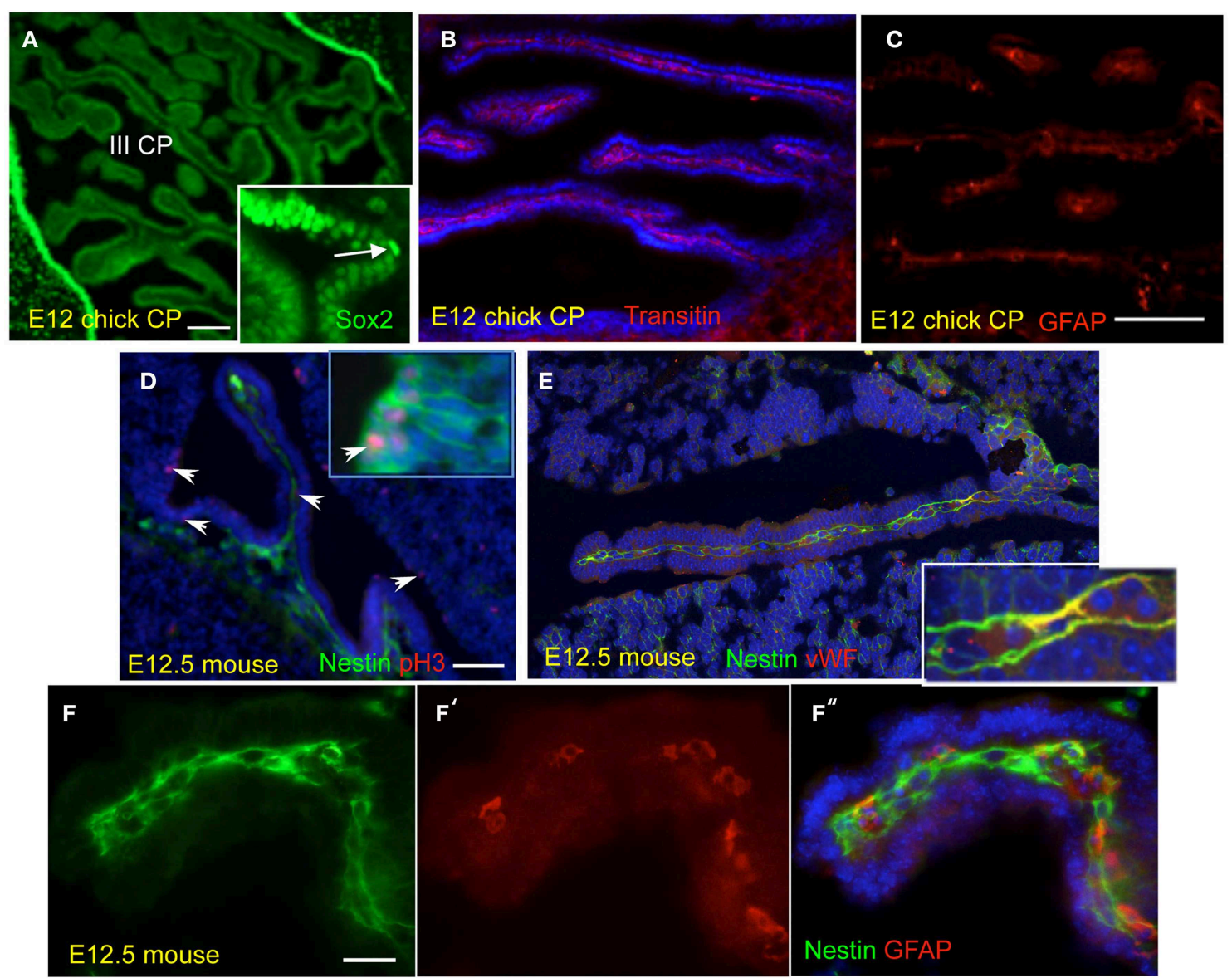

$\mathbf{F}^{\prime}$
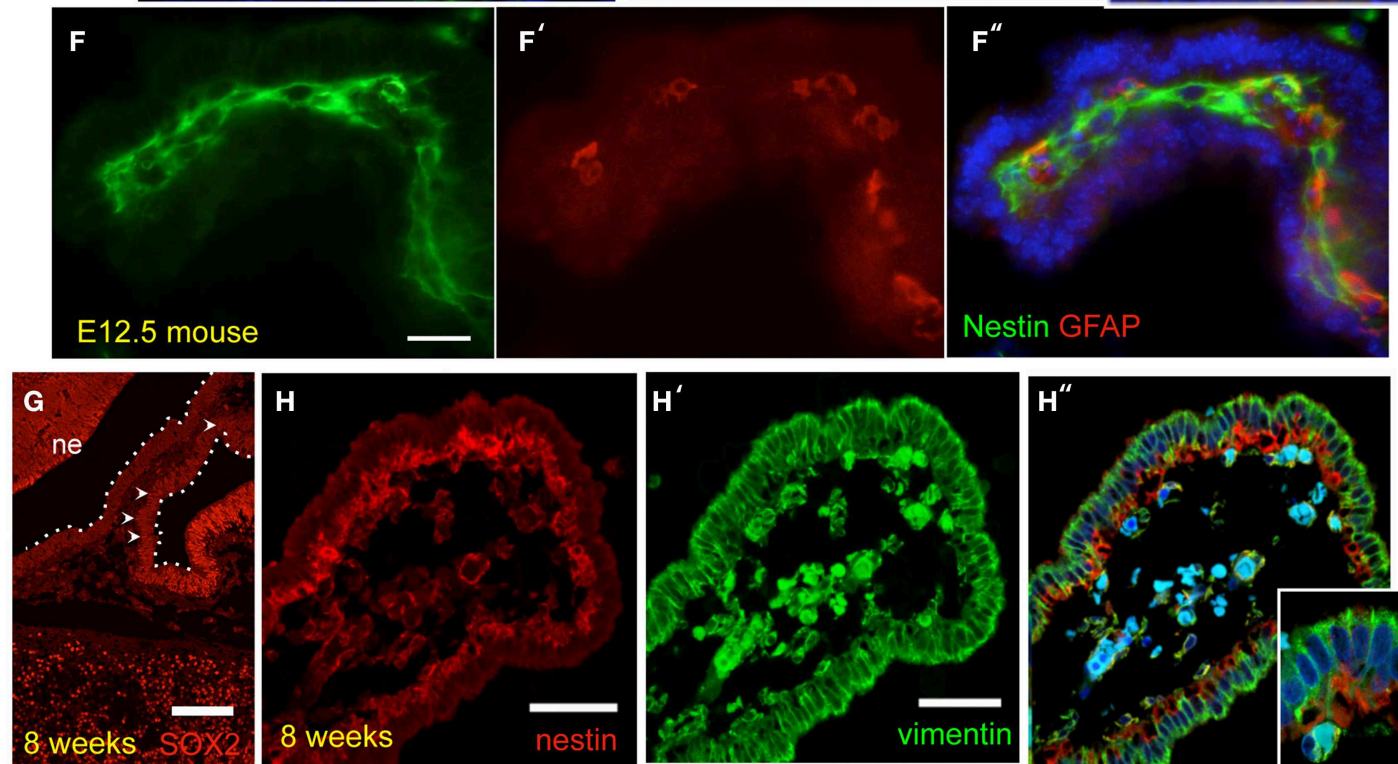

H
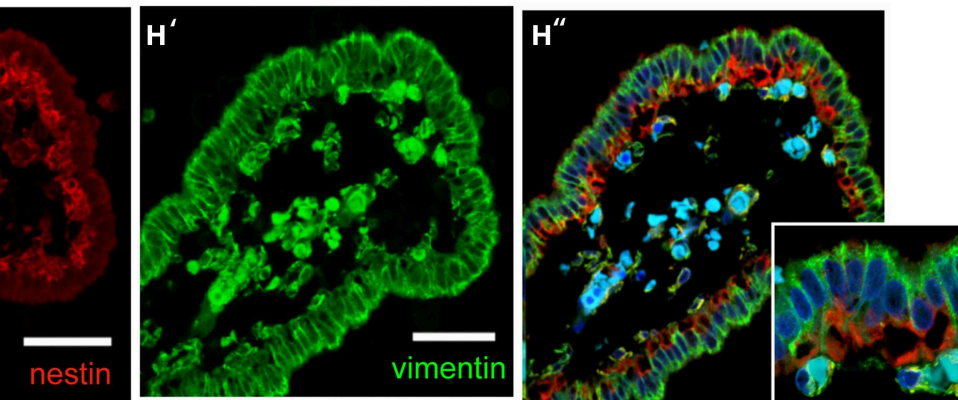

FIGURE 1 | Neural progenitor markers are expressed in the developing choroid plexus (CP), in different species. Unless otherwise indicated, micrographs show the CP of lateral ventricles. (A-C) E12 Chick CP stained for Sox2, transitin, and GFAP. III CP: third ventricle CP. (D-F) E12.5 mouse CP stained for $\mathrm{pH} 3$ (phosphorylated-histone 3), nestin, von Willebrand factor, and GFAP, either alone or in combination. Arrowheads in (D) point at proliferating cells in the CP and in the neuropepithelium (insert); only the CP is shown in (F). (G,H) Human CP at 8 weeks of gestation (CS23) stained for Sox2, nestin, and vimentin. The CP is outlined and some of the brighter SOX2-positve cells in the CP are indicated by arrowheads; note the gradient of SOX2 staining in the CP; ne, neuroepithelium. Nuclei are counterstained with Hoechst dye (blue). Scale bars are: (A) $=50 \mu \mathrm{m}$; (B,C) (same magnification) $=100 \mu \mathrm{m} ;(\mathbf{D}, \mathbf{E})($ same magnification $)=100 \mu \mathrm{m} ; \mathbf{( F )}=$ $100 \mu \mathrm{m} ;(\mathbf{G})=100 \mu \mathrm{m} ; \mathbf{( H )}=50 \mu \mathrm{m}$. detected in the CP (Figure 2). The presence of cells doublelabeled for Dcx and $\beta 3$-tubulin was also consistent with birth of neurons within the CP (Supplementary Figure 1). As it was difficult to visualize what the anti- $\beta 3$-tubulin antibody staining in $\mathrm{CP}$ sections, particularly at later stages of development when the CPs are highly branched, most of the subsequent analysis was carried out in whole mount $\mathrm{CP}$ preparations. Analysis of whole mount
E12 CPs stained for $\beta 3$-tubulin clearly showed the presence of $\beta 3$-tubulin-positive cells with extensive branching and a variety of neuronal morphologies (Figures 3 C-F, Movie 1).

We then wished to establish when these neurons first appeared in the $\mathrm{CP}$ and whether they were a transient or a stable feature of the CP. Hence we stained chick CPs for $\beta 3$-tubulin at E6, E8, E10, E18, E19, and E20 (Figures 4-6, Supplementary Figure 1). 
TABLE 3 | Summary of protein expression in the developing chick choroid plexus.

\begin{tabular}{|c|c|c|c|c|}
\hline & \multicolumn{2}{|c|}{ Stroma } & \multicolumn{2}{|c|}{ Epithelium } \\
\hline & E12 & E19/20 & E12 & $E 19 / 20$ \\
\hline \multicolumn{5}{|c|}{ NEURAL STEM/PROGENITOR CELL } \\
\hline Sox2 & - & nd & $\checkmark$ & nd \\
\hline Otx2 & - & - & $\checkmark$ & $\checkmark$ \\
\hline Vimentin* & $\checkmark($ low) & $\checkmark($ low $)$ & $\checkmark$ (high) & $\checkmark$ (high) \\
\hline Transitin & $\checkmark$ & $\sqrt{ }$ & - & - \\
\hline GFAP & $\checkmark$ & $\checkmark$ & - & - \\
\hline Cytokeratin & - & nd & $\checkmark$ & nd \\
\hline Doublecortin & $\checkmark$ & $\checkmark$ & - & - \\
\hline \multicolumn{5}{|l|}{ NEURONAL } \\
\hline B3-tubulin & $\checkmark$ & $\checkmark$ & - & - \\
\hline Neurofilament & $\checkmark$ & $\checkmark$ & - & - \\
\hline Synaptic vesicles (SV2) & $\checkmark$ & $\checkmark$ & - & - \\
\hline Tyrosine hydroxylase & $\checkmark$ & nd & - & nd \\
\hline \multicolumn{5}{|c|}{ TIGHT JUNCTION PROTEINS } \\
\hline ZO-1 & - & - & $\checkmark$ & $\checkmark$ \\
\hline ASPP2 & $\checkmark$ & $\checkmark$ & $\checkmark$ & $\checkmark$ \\
\hline Connexin-34 & - & - & $\checkmark$ & $\checkmark$ \\
\hline \multicolumn{5}{|l|}{ OTHERS } \\
\hline Laminin & $\checkmark$ & nd & - & nd \\
\hline Aquaporin-1 & - & nd & $\checkmark$ & nd \\
\hline Clusterin* & $\checkmark($ low $)$ & $\checkmark($ low $)$ & $\checkmark$ (high) & $\checkmark$ (high) \\
\hline
\end{tabular}

-, negative; nd, not done; *particularly low levels of expression in the CP stroma and high levels of expression in the $\mathrm{CP}$ epithelium are indicated.

We were unable to identify the CP at E6, but at E8 a small CP was clearly visible, and it was found to contain neurons as indicated by $\beta 3$-tubulin staining and morphological appearance (Figure 4). At this stage $\beta 3$-tubulin staining was observed also in some cells spanning the CP epithelium and contacting the CSF; some thin $\beta 3$-tubulin fibers that appeared to project into the CSF were also observed at E8 as well as at later stages when double-labeling for B3-tubulin and the tight junction marker, ZO-1, was carried out (Figure 4, Movie 2). Furthermore, nerve fibers that appeared to be entering the $\mathrm{CP}$ mesenchyme were observed at E8 as well as at E12 (Figures 4, 5).

The morphology of the CP neurons became more complex with development. At E8 neurons were mainly bipolar, at E10 some were bipolar, some tripolar and other had several branches, and from E12 the neuronal processes appeared to be longer and more convoluted (Figures 3C-F, 4, Movie 1). Staining for tyrosine-hydroxylase at E12 indicated that at least some of the neurons present in the developing CPs are catecholaminergic (Figure 3G, Supplementary Figure 2A). The morphology of some of these neurons resembles the drawing by Clark (1928) shown in Supplementary Figure 2B, though this author interpreted the bulbous structures as nerve terminals.

Nerves coursing the length of the CPs were detected at all stages studied and in all CPs, with dense clusters of neurons or fibers located at the base of the lateral ventricle CP and at branching points, where groups of neurons with highly convoluted

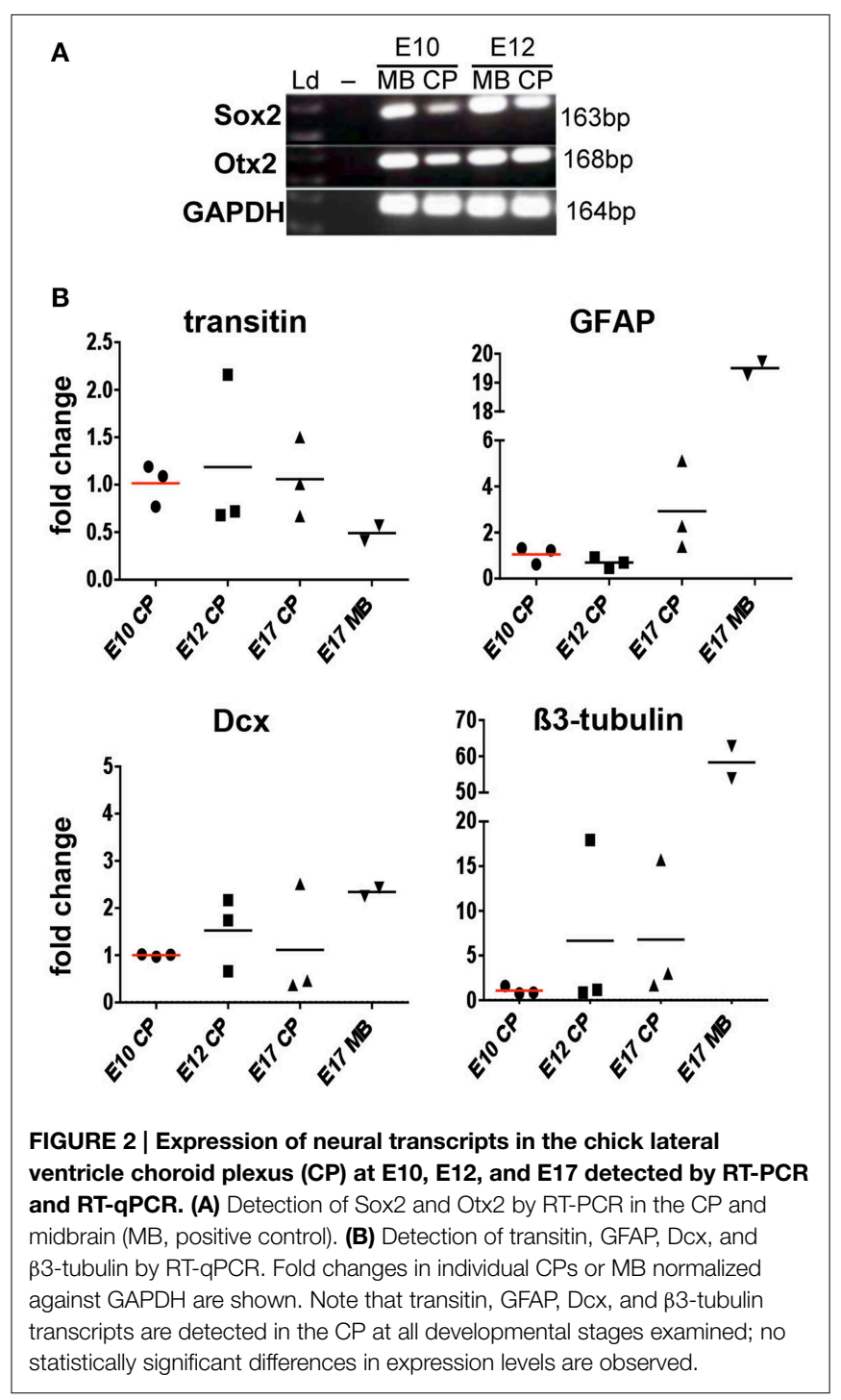

morphology could be observed (Figures 4, 5, 6A,B). The CP neural fibers did not appear to be myelinated, as staining for myelin basic protein was only detected in the nerve bundle at the base of the CP stalk (Supplementary Figure 3). Dcxpositive cells were still present at late developmental stages, consistent with detection of $D c x$ mRNA by RT-qPCR (Figure 6C). A high density of nerve terminals/fibers was observed in close proximity to blood vessels, as indicated by staining with the SV2 (synaptic vesicle protein 2) antibody that detects a glycoprotein important for synaptic vesicle function, and NF200; close correspondence between SV2 and NF200 staining could be observed, notwithstanding some background with the NF200 antibody in whole mount CPs (Figure 6D) (Xu and Bajjalieh, 2001).

Whole mount analysis of E20 pineal gland, 3rd and lateral ventricle $\mathrm{CPs}$ isolated together revealed an interesting neural network between these structures (Figure 6B). Interconnecting nerve fibers were clearly observed between the pineal gland and the 3rdV CP and between the 3rd and lateral ventricle CPs. 

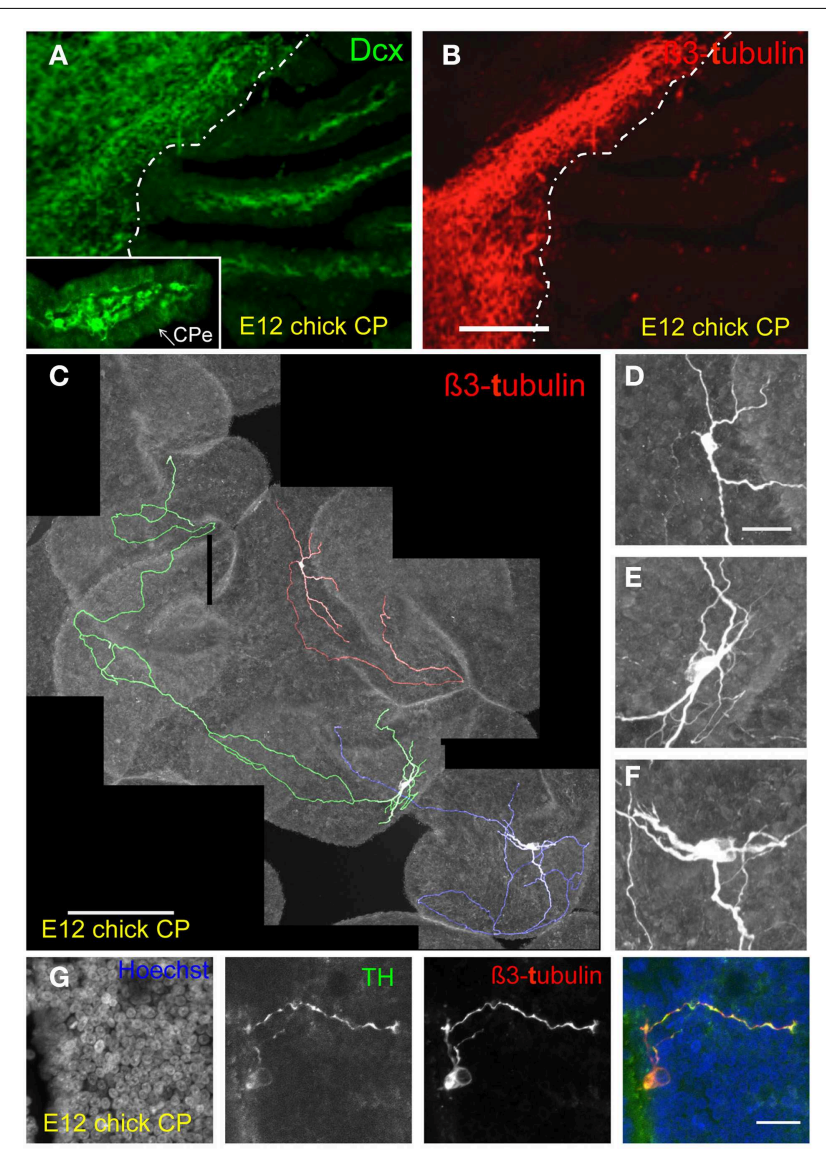

FIGURE 3 | Identification of neuroblasts and neurons in the developing chick CP. (A,B) E12 lateral CP section stained for doublecortin (Dcx) and

B3-tubulin in E12 chick CP. Note Dcx reactivity in the CP stroma (insert in A) as well as in the brain; only some punctuated staining is observed in the CP with the anti-B3-tubulin antibody whereas the brain tissue is strongly labeled. The approximate boundary between brain and CP is indicated by the dotted line. $\mathrm{CPe}, \mathrm{CP}$ epithelium. (C-F) Examples of extensive branching (individual neurons in (C) are shown in different colors for ease of visualization) and neuronal morphologies detected by B3-tubulin staining. (G) Staining of the E12 CP for tyrosine-hydroxylase (TH), B3-tubulin and Hoechst dye (nuclei) and merged image; note the presence of a positive $\mathrm{TH}$ neuron in proximity of the CP epithelium. Scale bars: (A,B) (same magnification) $=200 \mu \mathrm{m} ;(\mathbf{C})=200 \mu \mathrm{m}$; (D-F) (same magnification) $=30 \mu \mathrm{m} ;(\mathbf{G})=20 \mu \mathrm{m}$.

\section{Analysis of Choroid Plexus (CP) Organotypic Cultures}

Although expression of neural progenitor markers within the $\mathrm{CP}$ suggested that the Dcx-positive cells are born within the $\mathrm{CP}$, it could not be ruled out that post-mitotic Dcx-positive neuroblasts had migrated into the $\mathrm{CP}$ from other brain regions. In vivo experiments cannot address this issue as BrdU would label both neural precursor cells born and matured in the $\mathrm{CP}$ and neuroblasts originating from adjacent brain regions that had migrated into the $\mathrm{CP}$. Hence to address this issue, we set up $\mathrm{CP}$ organotypic cultures from E12 chick embryo that allow one to specifically study events occurring within the CP. As indicated by MTT metabolic assay, PI (Figures 7A-C) and Otx2 (not shown), the E12 organotypic $\mathrm{CP}$ cultures showed very good viability and maintenance of the

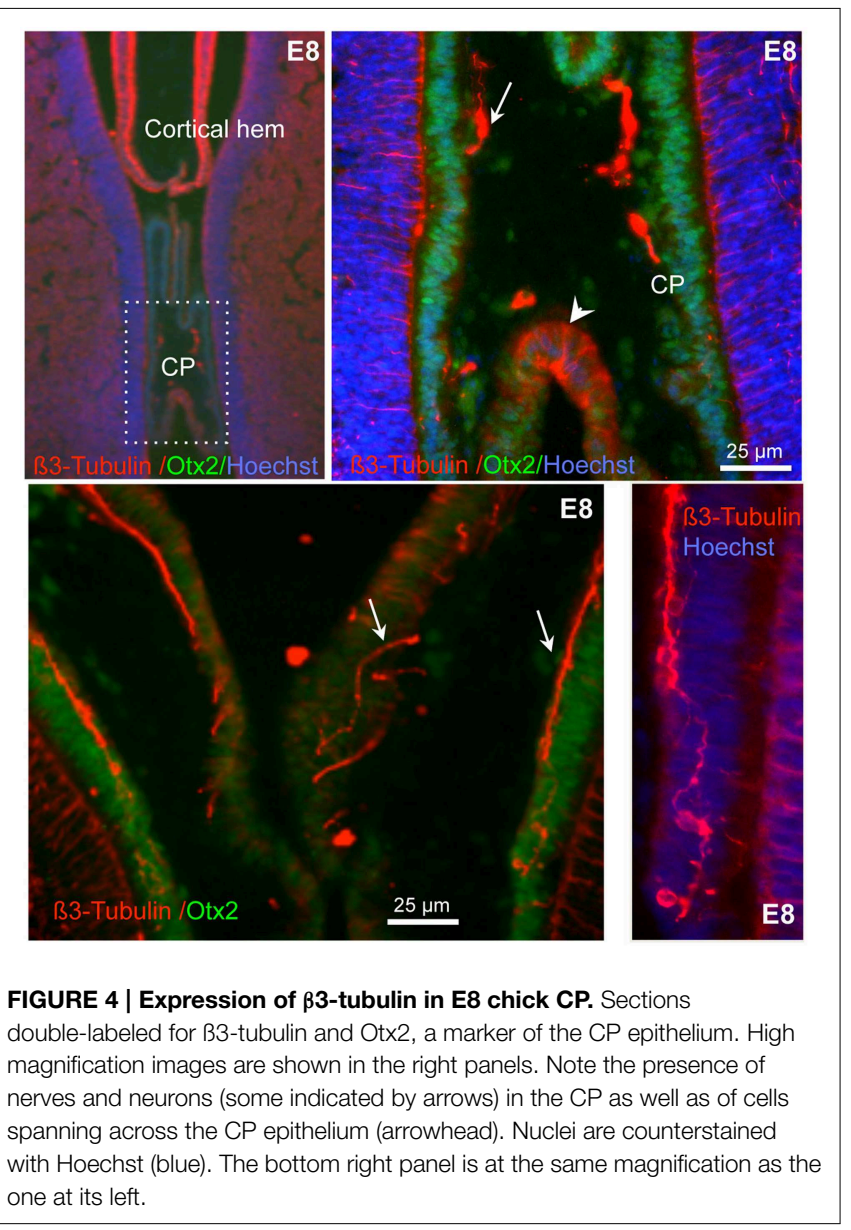

CP phenotype over 7 days in culture. After 3 days labeling with $\mathrm{BrdU}$, the CPs were stained either for BrdU and Dcx or BrdU and NF200 (Figures 7D-E). BrdU-positive cells were observed in the $\mathrm{CP}$ stroma, and some were found to be Dcx-positive; also a few BrdU and NF200 positive cells were observed. This suggests that the neuroblasts had been born within the CP.

\section{Discussion}

This study shows that neurons are present from early stages of CP development and that they appear to originate from progenitor cells within the $\mathrm{CP}$. Furthermore, it challenges the view that the $\mathrm{CPs}$ become innervated only postnatally, by clearly demonstrating innervation of the $\mathrm{CP}$ at early developmental stages.

\section{Proteins Normally Associated with Neural Progenitors are Expressed in the Immature Developing CP}

We have shown that the developing chick CPs express markers that are found in the neuroepithelium and/or radial glial cells of the developing central nervous system. Markers expressed in the neuroepithelium at early stages of development, including Sox2, vimentin, and ASPP2 (Bennett and Dilullo, 1985; Uwanogho et al., 1995; Sottocornola et al., 2010), have been found to be 


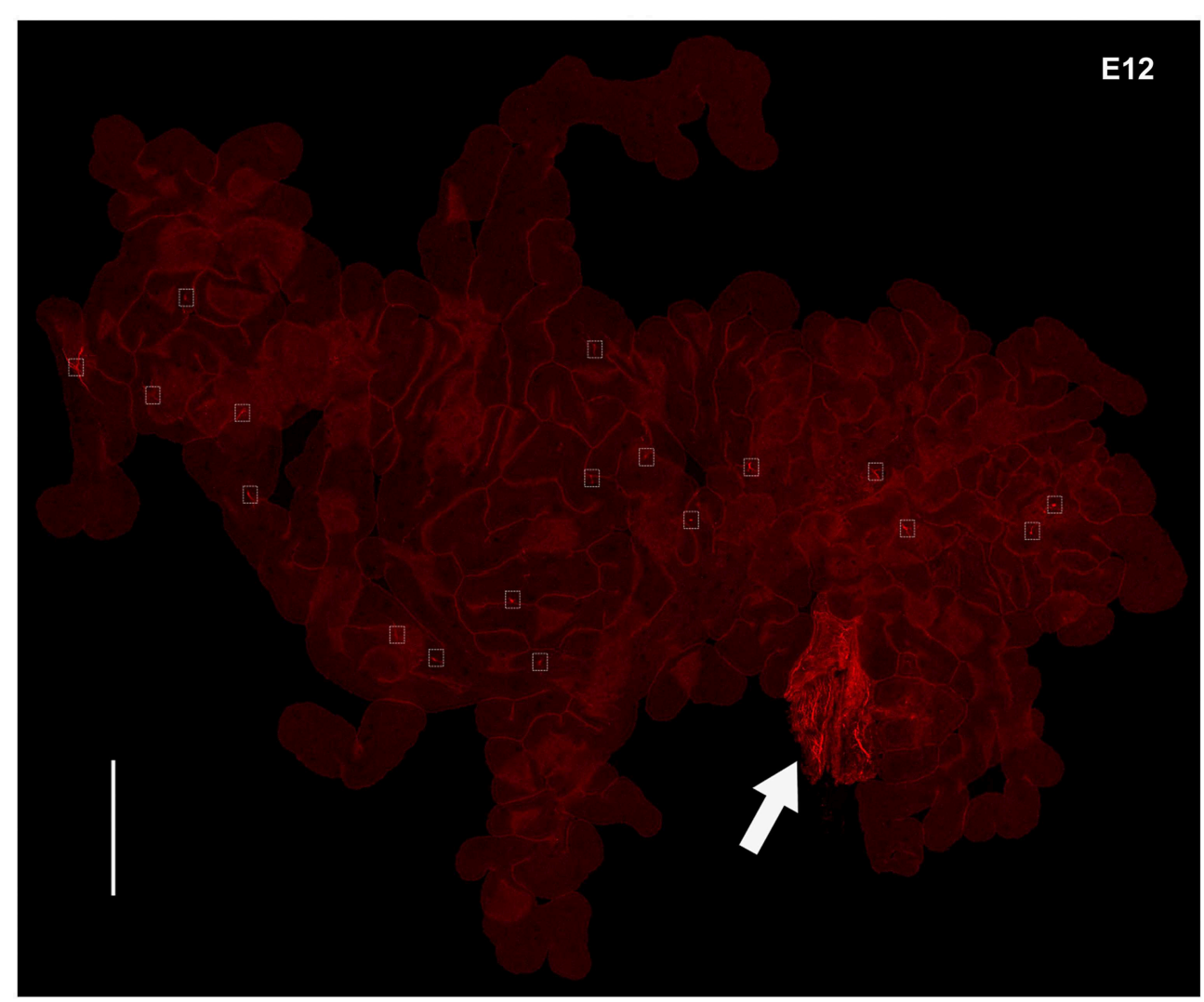

FIGURE $\mathbf{5}$ | Expression of $\boldsymbol{\beta} \mathbf{3}$-tubulin in whole mount E12 chick CP. The nerve bundle in the CP stalk is indicated by the arrow and some neurons within the CP are boxed. Scale bars $=500 \mu \mathrm{m}$.

expressed in the epithelium of chick CPs. Expression of Sox2 in the prenatal mouse $\mathrm{CP}$ epithelium as well as in a subset of CP epithelial cells in the adult mouse has been previously reported (Ferri et al., 2004). This, together with our observations in chick and human CPs, indicates that expression of Sox 2 in the $\mathrm{CP}$ is a feature conserved across species. Interestingly, whereas Sox 2 staining intensity in both chick and human embryonic CPs is overall lower than in the neuroepithelium, some strongly Sox2-positive cells, mainly localized at CP branching points, are present. Differences in Sox2 levels have also been observed in the developing mouse telencephalon, with "high Sox2" radial glial cells displaying higher neurosphere forming ability, growth rate, and self-renewal capability than "low Sox2" intermediate progenitor cells (Hutton and Pevny, 2011). To establish whether the "high-Sox2" cells within the CPs are landmarks important for CP branching morphogenesis, and/or represent a neural stem cells population from which nestin and/or GFAP-positive cells in the stroma originate is an important and challenging question that will require extensive investigation.

In contrast to Sox 2 and vimentin, the chick nestin-like protein, transitin, expressed in early radial glial cells that have neuronal differentiation potential (Kriegstein and Alvarez-Buylla, 2009), was not detected in the chick CP epithelium, but only in the mesenchyme. This was the case also for nestin in mouse and human CPs, where the nestin positive cells were located just beneath the
CP epithelium as reported in neonatal rats (Huang et al., 2011). Cells expressing GFAP, another protein expressed in neural stem cells as well as in astrocytes, were found in the mesenchymal compartment of the chick CPs. Because of antibody species of origin, we could not double stain the chick CP for transitin and GFAP. However, double-staining of mouse E12.5 CPs for nestin and GFAP indicated that at least in this species the developing CP contains two distinct populations of "neural" cells in the stroma, identified by nestin and GFAP reactivity. Consistent with population(s) of neural progenitors within the $\mathrm{CP}$ was the presence of cells expressing the neuroblast marker, Dcx. The finding that BrdU incorporation in Dcx-positive cells was observed in isolated $\mathrm{CPs}$, supports the view that the neurons present in the $\mathrm{CP}$ are born within the $\mathrm{CP}$ itself and originate from neural progenitors, rather than having migrated from the neighboring neuroepithelium. This is consistent with previous studies suggesting that cells isolated from the CP have neural potential, as they could form neurosphere-like structures and differentiated into neuronal-like cells (Itokazu et al., 2006).

The presence of subsets of highly Sox2-positive cells in the CP epithelium, that is also vimentin-positive, and of nestin-positive cells beneath it, raises the possibility, summarized in Figure 8, that the $\mathrm{CP}$ may be akin to the neurovascular niche in the subventricular region, where the neural stem cells reside close to the ependyma, in contact with blood vessels and extend their 

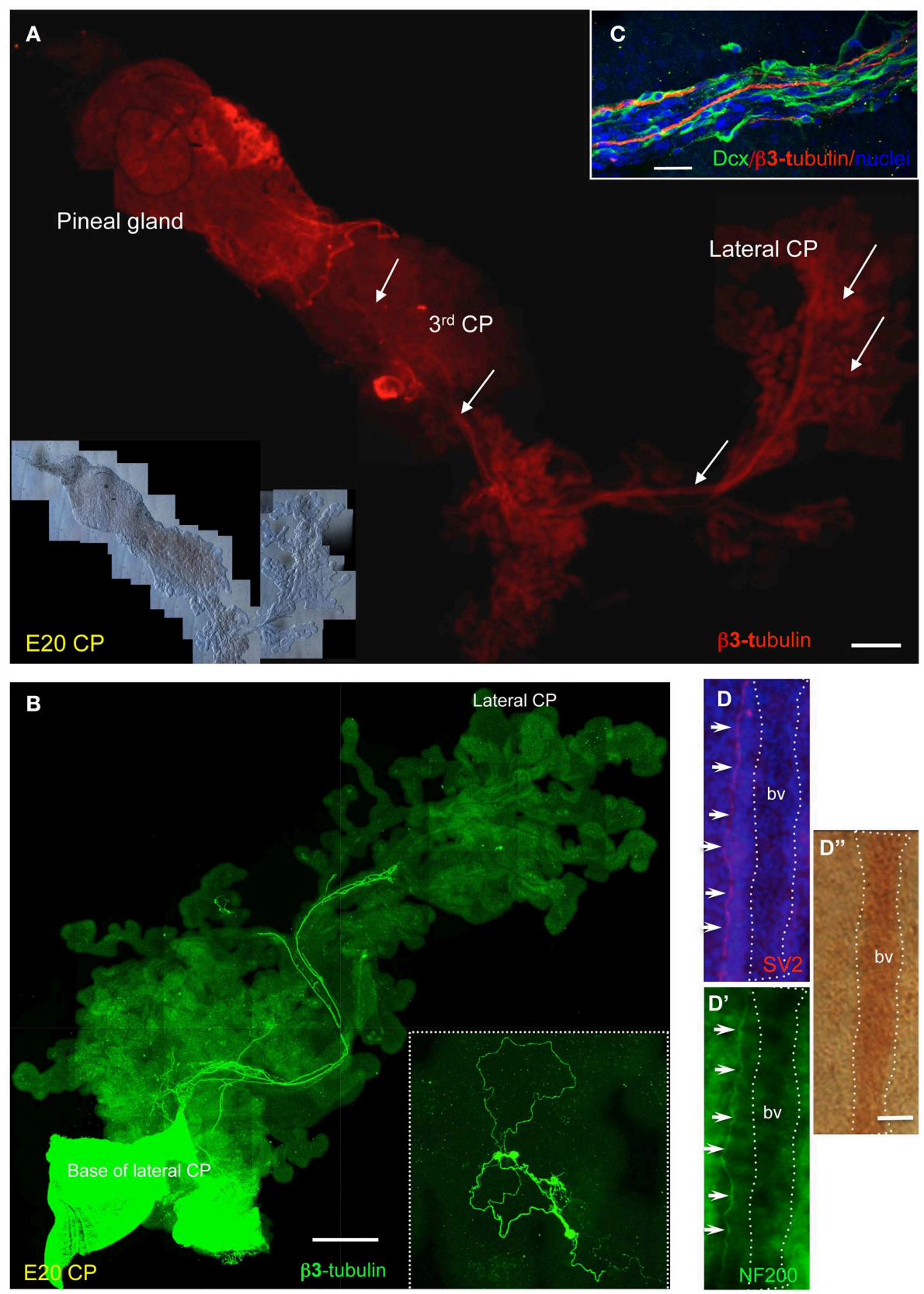

FIGURE 6 | Expression of neuronal proteins in chick CPs. (A,B) Examples of $\beta 3$-tubulin staining in whole mount E20 CPs; note the extensive network connecting lateral ventricle $\mathrm{CP}$, 3rd ventricle $\mathrm{CP}$ and pineal gland. The arrows point at some of the neurons observed at high magnification. (C) E19 CP stained for doublecortin (Dcx) and B3-tubulin; nuclei are counterstained with Hoechst dye. (D-D") Whole mount E19 chick CP double-labeled for the synaptic vesicle 2 marker, SV2, and NF200 and corresponding bright field image; nuclei are counterstained with Hoechst dye. The dotted line indicates a blood vessel (bv). Scale bars: $(\mathbf{A})=200 \mu \mathrm{m}$; (B) $=500 \mu \mathrm{m} ;(\mathbf{C}, \mathbf{D})=25 \mu \mathrm{m}$. primary cilia into the CSF of the brain ventricles (Doetsch et al., 1997).

\section{Neurogenesis Occurs within the Developing CP}

We have demonstrated for the first time that single neurons reside within the chick CP stroma from early stages of development. We have also shown that at least some of the neurons present in the developing CPs are catecholaminergic as indicated by co-expression of tyrosine-hydroxylase and $\beta 3$-tubulin. This is consistent with the presence of dopamine receptors in the epithelium and smooth muscle cells of the blood vessels of the adult rat CP (Mignini et al., 2000).

Because of the difficulties in carrying out accurate quantification on neurons in whole mounts and variability among animals, 

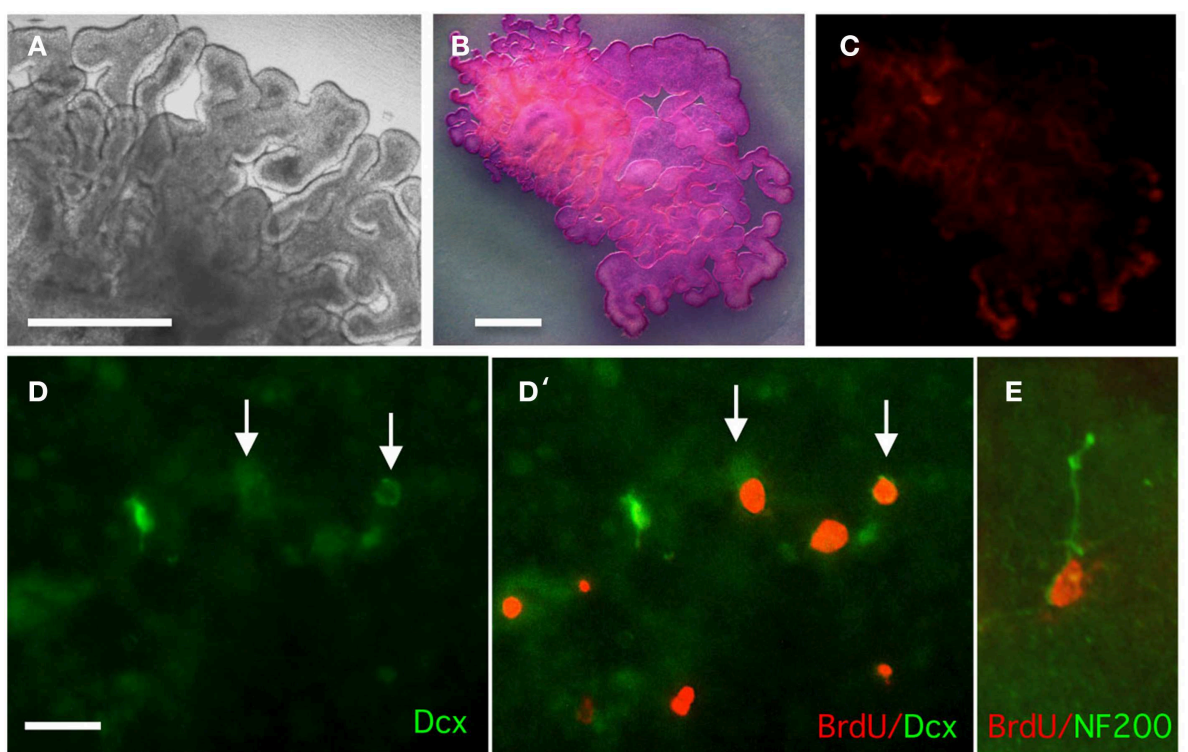

E

FIGURE 7 | Analysis of survival, proliferation, and doublecortin (Dcx) expression in organotypic cultures of E12 lateral CP. (A) Phase image of $\mathrm{CP}$ after 7 days in culture. (B) MTT staining of live CP after 7 days in culture; extensive staining is indicative cell viability. (C) Propidium iodide staining of live CP after 7 days in culture to detect dead cells; only very limited staining is observed. (D,D') CP organotypic culture treated with BrdU for 3 days and double-stained for BrdU and doublecortin (Dcx). Some double-labeled cells are observed. (E) Detection of NF200 and BrdU in an organotypic culture. Scale bars: $(\mathbf{A}-\mathbf{C})=200 \mu \mathrm{m}(\mathbf{B}$ and $\mathbf{C}$ are at the same magnification); (D,E) $=50 \mu \mathrm{m}$.

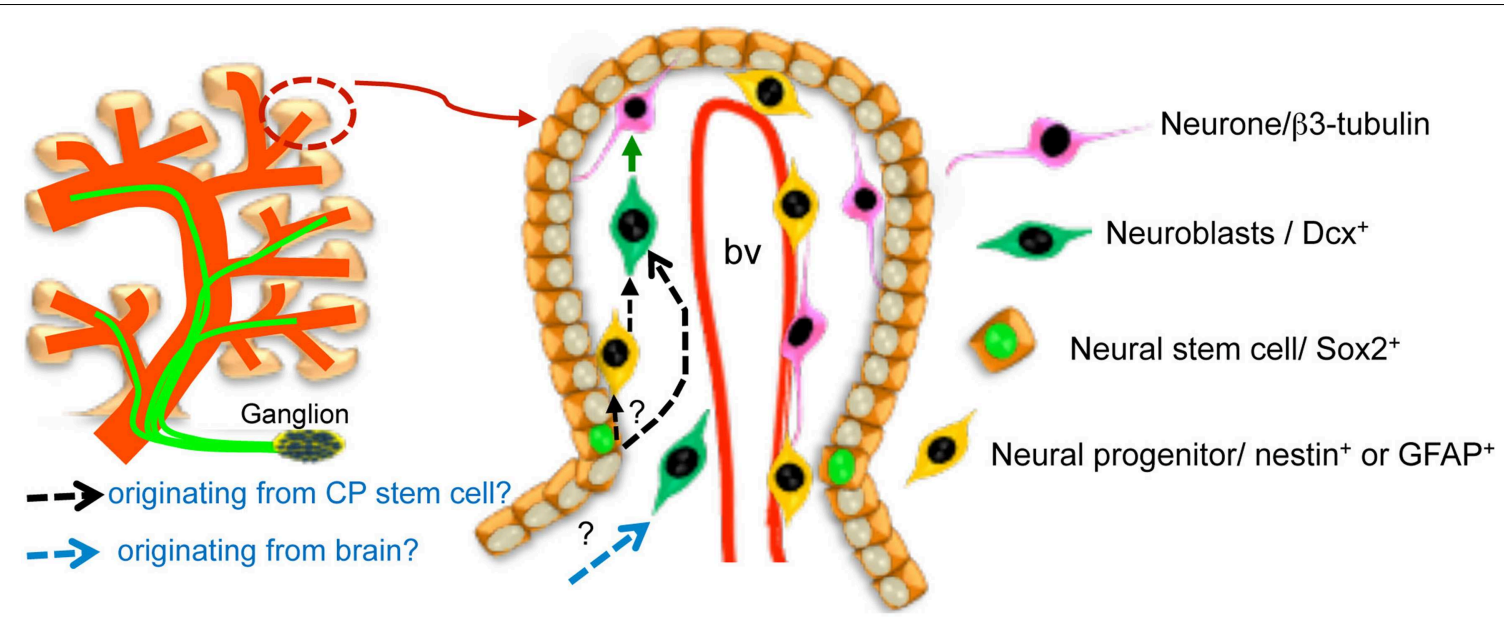

FIGURE 8 | Schematic representation of the CP neural components (innervation from ganglia outside the $\mathrm{CP}$ and neurons within the $\mathrm{CP}$ ) and of the model proposing the existence of a neurovascular niche within the developing chick CP. It is not currently known whether nestin-like-positive cells reflect a transition from high-Sox2/vimentin-positive cells to neuroblasts (black dotted arrows) nor whether some of the $\mathrm{CP}$ neurons are born from neuroblasts migrating into the $\mathrm{CP}$ from the brain rather than having been generated within it (blue dotted arrow). Please note that only the subset of highly Sox2-positive putative progenitors in the CP epithelium is indicated in the cartoon. The two sources are not mutually exclusive. bv, blood vessel. we cannot be sure when neurons stop being born in the CP. Analysis of neurons in several preparations, and the fact that Dcx staining was more widespread around E12, would suggest that most neurons are born around this stage of development. However, some neurogenesis appeared to occur also at later stages, as some Dcx-BrdU labeled cells were observed after in vivo BrdU labeling at E15 (not shown).
The morphology of the CP neurons appears to change with development and the complexity of their branching appears to increase with embryonic age. CSF secretion is crucial for brain development and its production is at least in part controlled by the nervous system (Edvinsson and Lindvall, 1978; Lindvall and Owman, 1981). Projection into the CSF of fibers from individual neurons could be detected in some of the chick whole 
mount preparations, and fibers in very close proximity to the $\mathrm{CP}$ epithelium have been detected by light and transmission electron microscopy in adult CPs (Clark, 1928; Edvinsson et al., 1975; Nakamura and Milhorat, 1978). It is conceivable that these neurons act as sensory or autonomic neurons that integrate signals between the CP/CSF and the nerves entering the CP. The presence of numerous CSF-contacting axons has been reported in the ependyma and periventricular organs (Tramu et al., 1983; Michaloudi and Papadopoulos, 1996) and a mechanism for nonsynaptic signal transmission in the brain has been proposed (Vigh et al., 2004).

\section{Innervation of the CP Occurs Pre-Natally}

The presence of nerves in adult CPs has been described in various species including mice, rats, hedgehogs, guinea-pigs, rabbits, cats, cows, and monkeys; differences in density of innervation were reported in different CPs, with the 3rd ventricle CP being the most densely innervated, and across species, with the lowest density of innervation observed in the mouse CP (Edvinsson et al., 1973; Lindvall et al., 1977a,b, 1978c; Lindvall, 1979). Notwithstanding its complexity, innervation of the CP was believed to occur postnatally (Tsuker, 1947; Lindvall and Owman, 1978). Our data clearly demonstrate that in the chick the nerves enter the CP before birth, apparently as early as at E8. The previously reported lack of evidence for prenatal innervation of the $\mathrm{CP}$ is likely due to the fact that it is very difficult to detect nerves, as well as neurons, in CP sections; apart from very early stages of development, clear visualization of $\mathrm{CP}$ innervation can be achieved only by using whole mount $\mathrm{CP}$ preparations and confocal microscopy; furthermore, nerves are easily pulled out of the CP during dissection, hence this step requires very careful handling. Expression of synaptic vesicle proteins involved in the release of neurotransmitters is indicative of neural activity within the $\mathrm{CP}$ well before birth, consistent with a role for innervation in the developing CP. Hence, innervation of the CP during development is in line with that of other secretory structures, such as the salivary glands that become innervated by parasympathetic nerves during organogenesis (Knox et al., 2010). As intestinal epithelial stem/progenitor cells in the crypts are controlled by mucosal afferent nerves (Lundgren et al., 2011), it is conceivable that the nerves might play a role in proliferation and branching of the CP. However, whether during development the nerves' main role is in controlling CSF secretion or also in $\mathrm{CP}$ growth remains to be established.

The nerves in the E20 CP did not appear to be myelinated, as indicated by lack of staining for myelin basic protein, and this is consistent with a transmission electron microscopy study of adult rat CP (Edvinsson et al., 1975; Nakamura and Milhorat, 1978).

The nature of $\mathrm{CP}$ innervation has been examined in a variety of species including adult rat, guinea-pig, hedgehog, rabbit, cat, and man; noradrenergic and cholinergic innervation, as well as substance P-positive fibers, have been described in adult CPs from different species, whereas the presence of serotoninergic fibers has so far been reported only in the rat and hedgehog CPs (Napoleone et al., 1982; Edvinsson et al., 1983; Nilsson et al., 1990; Michaloudi and Papadopoulos, 1996). However, most of these studies have not investigated comprehensively the presence of different types of fibers and their localization within the same CP. Hence, it will be important to carry out systematic comparative studies on the type of fibers present in developing and adult $\mathrm{CP}$ and innervation using whole mount preparations.

Another important finding that has emerged from staining whole mount preparations for $\beta 3$-tubulin concerns the identification of a neural network between pineal gland, 3rd ventricle $\mathrm{CP}$ and lateral ventricle $\mathrm{CP}$. This neural network may be important for development and maintenance of the CP throughout life as suggested in other organs, such as the developing teeth (Luukko et al., 1997), kidney (Tiniakos et al., 2004), pancreas (Burris and Hebrok, 2007), and heart (Hildreth et al., 2009). It is also tempting to speculate that in the developing chick, the neuron cluster between the 3rd and lateral ventricle CPs may serve as a relay station for integrating signals from the CSF and co-ordinating secretory activity throughout the ventricular system.

Together, as schematically summarized in Figure 8, the presence of innervation and of neurons adjacent to blood vessels, or projecting into the ventricles, is consistent with the hypothesis that the ventricular system contains a neural network that resembles to some extent the enteric nervous system and is important for CP development and homeostasis.

\section{Author Contributions}

WP designed and performed experiments, analyzed data and wrote the manuscript; $\mathrm{BV}$ and ZA performed experiments and critical reading of the manuscript; NJ performed experiments; PF planned research, analyzed data, and wrote the manuscript.

\section{Acknowledgments}

This work was supported by the Higher Educational Strategic Scholarship for Frontier Research Network from the Commission on Higher Education, Royal Thai Government and the Great Ormond Street Hospital Children's Charity. The human embryonic material was provided by the Human Developmental Biology Resource (http://hdbr.org) jointly funded by the Medical Research Council (grant G070089) and The Wellcome Trust (grant GR082557).

\section{Supplementary Material}

The Supplementary Material for this article can be found online at: http://www.frontiersin.org/journal/10.3389/fnins. 2015.00103/abstract

\begin{abstract}
Movie 1 | Neuron detected by B3tubulin staining (red) in an E12 whole mount chick CP. Nuclei are counterstained with Hoechst dye (blue). Scale bar = $100 \mu \mathrm{m}$.
\end{abstract}

Movie 2 | Neuron detected by B3tubulin staining (red) coursing across the CP epithelium stained by the tight junction protein, ZO-1 (green), in an E12 whole mount chick CP. Nuclei are counterstained with Hoechst dye (blue). Scale bar $=20 \mu \mathrm{m}$. 


\section{References}

Bennett, G. S., and Dilullo, C. (1985). Transient expression of a neurofilament protein by replicating neuroepithelial cells of the embryonic chick brain. Dev. Biol. 107, 107-127. doi: 10.1016/0012-1606(85)90380-X

Burris, R. E., and Hebrok, M. (2007). Pancreatic innervation in mouse development and beta-cell regeneration. Neuroscience 150, 592-602. doi: 10.1016/j.neuroscience.2007.09.079

Clark, S. L. (1928). Nerve endings inthe choroid plexus of the fourth ventricle. J. Comp. Neurol. 47, 1-21. doi: 10.1002/cne. 900470102

Doetsch, F., Garcia-Verdugo, J. M., and Varez-Buylla, A. (1997). Cellular composition and three-dimensional organization of the subventricular germinal zone in the adult mammalian brain. J. Neurosci. 17, 5046-5061.

Dziegielewska, K. M., Ek, J., Habgood, M. D., and Saunders, N. R. (2001). Development of the choroid plexus. Microsc. Res. Tech. 52, 5-20. doi: 10.1002/10970029(20010101)52:1<5::AID-JEMT3>3.0.CO;2-J

Edvinsson, L., Hakanson, R., Lindvall, M., Owman, C., and Svensson, K. G. (1975). Ultrastructural and biochemical evidence for a sympathetic neural influence on the choroid plexus. Exp. Neurol. 48, 241-251. doi: 10.1016/00144886(75)90154-5

Edvinsson, L., and Lindvall, M. (1978). Autonomic vascular innervation and vasomotor reactivity in the choroid plexus. Exp. Neurol. 62, 394-404. doi: 10.1016/0014-4886(78)90063-8

Edvinsson, L., Nielsen, K. C., and Owman, C. (1973). Cholinergic innervation of choroid plexus in rabbits and cats. Brain Res. 63, 500-503. doi: 10.1016/00068993(73)90132-7

Edvinsson, L., Rosendal-Helgesen, S., and Uddman, R. (1983). Substance P: localization, concentration and release in cerebral arteries, choroid plexus and dura mater. Cell Tissue Res. 234, 1-7. doi: 10.1007/BF00217397

Ferri, A. L., Cavallaro, M., Braida, D., Di Cristofano, A., Canta, A., Vezzani, A., et al. (2004). Sox2 deficiency causes neurodegeneration and impaired neurogenesis in the adult mouse brain. Development 131, 3805-3819. doi: $10.1242 /$ dev. 01204

Hildreth, V., Anderson, R. H., and Henderson, D. J. (2009). Autonomic innervation of the developing heart: origins and function. Clin. Anat. 22, 36-46. doi: $10.1002 /$ ca.20695

Huang, S. L., Shi, W., Jiao, Q., and He, X. J. (2011). Change of neural stem cells in the choroid plexuses of developing rat. Int. J. Neurosci. 121, 310-315. doi: 10.3109/00207454.2011.556282

Hunter, N. L., and Dymecki, S. M. (2007). Molecularly and temporally separable lineages comprise the hindbrain roof plate and contribute differentially to the choroid plexus. Development 134, 3449-3460. doi: 10.1242/dev. 003095

Hutton, S. R., and Pevny, L. H. (2011). SOX2 expression levels distinguish between neural progenitor populations of the developing dorsal telencephalon. Dev. Biol. 352, 40-47. doi: 10.1016/j.ydbio.2011.01.015

Itokazu, Y., Kitada, M., Dezawa, M., Mizoguchi, A., Matsumoto, N., Shimizu, A., et al. (2006). Choroid plexus ependymal cells host neural progenitor cells in the rat. Glia 53, 32-42. doi: 10.1002/glia.20255

Johansson, P. A., Dziegielewska, K. M., Liddelow, S. A., and Saunders, N. R. (2008). The blood-CSF barrier explained: when development is not immaturity. Bioessays 30, 237-248. doi: 10.1002/bies.20718

Kitada, M., Chakrabortty, S., Matsumoto, N., Taketomi, M., and Ide, C. (2001). Differentiation of choroid plexus ependymal cells into astrocytes after grafting into the pre-lesioned spinal cord in mice. Glia 36, 364-374. doi: 10.1002/glia.1123

Knox, S. M., Lombaert, I. M., Reed, X., Vitale-Cross, L., Gutkind, J. S., and Hoffman, M. P. (2010). Parasympathetic innervation maintains epithelial progenitor cells during salivary organogenesis. Science 329, 1645-1647. doi: $10.1126 /$ science. 1192046

Kriegstein, A., and Alvarez-Buylla, A. (2009). The glial nature of embryonic and adult neural stem cells. Annu. Rev. Neurosci. 32, 149-184. doi: 10.1146/annurev.neuro.051508.135600

Lehtinen, M. K., Bjornsson, C. S., Dymecki, S. M., Gilbertson, R. J., Holtzman, D. M., and Monuki, E. S. (2013). The choroid plexus and cerebrospinal fluid: emerging roles in development, disease, and therapy. J. Neurosci. 33, 17553-17559. doi: 10.1523/JNEUROSCI.3258-13.2013

Liddelow, S. A., Dziegielewska, K. M., Ek, C. J., Habgood, M. D., Bauer, H., Bauer, H. C., et al. (2013). Mechanisms that determine the internal environment of the developing brain: a transcriptomic, functional and ultrastructural approach. PLOS ONE 8:e65629. doi: 10.1371/journal.pone.0065629

Liddelow, S. A., Temple, S., Mollgard, K., Gehwolf, R., Wagner, A., Bauer, H., et al. (2012). Molecular characterisation of transport mechanisms at the developing mouse blood-CSF interface: a transcriptome approach. PLoS ONE 7:e33554. doi: 10.1371/journal.pone.0033554

Lindvall, M. (1979). Fluorescence histochemical study on regional differences in the sympathetic nerve supply of the choroid plexus from various laboratory animals. Cell Tissue Res. 198, 261-267. doi: 10.1007/BF00232009

Lindvall, M., Alumets, J., Edvinsson, L., Fahrenkrug, J., Hakanson, R., Hanko, J., et al. (1978a). Peptidergic (VIP) nerves in the mammalian choroid plexus. Neurosci. Lett. 9, 77-82. doi: 10.1016/0304-3940(78)90051-4

Lindvall, M., Edvinsson, L., and Owman, C. (1977a). Histochemical study on regional differences in the cholinergic nerve supply of the choroid plexus from various laboratory animals. Exp. Neurol. 55, 152-159. doi: 10.1016/00144886(77)90166-2

Lindvall, M., Edvinsson, L., and Owman, C. (1977b). Histochemical, ultrastructural and functional evidence for a neurogenic control of cerebrospinal fluid production from the choroid plexus. Acta Physiol. Scand. Suppl. 452, 77-86.

Lindvall, M., Edvinsson, L., and Owman, C. (1978b). Histochemical, ultrastructural, and functional evidence for a neurogenic control of CSF production from the choroid plexus. Adv. Neurol. 20, 111-120.

Lindvall, M., Edvinsson, L., and Owman, C. (1978c). Sympathetic nervous control of cerebrospinal fluid production from the choroid plexus. Science 201, 176-178. doi: 10.1126/science.663649

Lindvall, M., and Owman, C. (1978). Early development of noradrenalinecontaining sympathetic nerves in the choroid plexus system of the rabbit. Cell Tissue Res. 192, 195-203. doi: 10.1007/BF00220738

Lindvall, M., and Owman, C. (1981). Autonomic nerves in the mammalian choroid plexus and their influence on the formation of cerebrospinal fluid. J. Cereb. Blood Flow Metab. 1, 245-266. doi: 10.1038/jcbfm.1981.30

Lundgren, O., Jodal, M., Jansson, M., Ryberg, A. T., and Svensson, L. (2011). Intestinal epithelial stem/progenitor cells are controlled by mucosal afferent nerves. PLoS ONE 6:e16295. doi: 10.1371/journal.pone.0016295

Luukko, K., Arumae, U., Karavanov, A., Moshnyakov, M., Sainio, K., Sariola, H., et al. (1997). Neurotrophin mRNA expression in the developing tooth suggests multiple roles in innervation and organogenesis. Dev. Dyn. 210, $117-129$.

Marques, F., Sousa, J. C., Coppola, G., Gao, F., Puga, R., Brentani, H., et al. (2011). Transcriptome signature of the adult mouse choroid plexus. Fluids Barriers CNS 8:10. doi: 10.1186/2045-8118-8-10

Michaloudi, H. C., and Papadopoulos, G. C. (1996). Catecholaminergic and serotoninergic fibres innervate the ventricular system of the hedgehog CNS. J. Anat. 189(Pt 2), 273-283.

Mignini, F., Bronzetti, E., Felici, L., Ricci, A., Sabbatini, M., Tayebati, S. K., et al. (2000). Dopamine receptor immunohistochemistry in the rat choroid plexus. J. Auton. Pharmacol. 20, 325-332. doi: 10.1046/j.1365-2680.2000. 00198.x

Nakamura, S., and Milhorat, T. H. (1978). Nerve endings in the choroid plexus of the fourth ventricle of the rat: electron microscopic study. Brain Res. 153, 285-293. doi: 10.1016/0006-8993(78)90407-9

Napoleone, P., Sancesario, G., and Amenta, F. (1982). Indoleaminergic innervation of rat choroid plexus: a fluorescence histochemical study. Neurosci. Lett. 34, 143-147. doi: 10.1016/0304-3940(82)90166-5

Nataf, S., Strazielle, N., Hatterer, E., Mouchiroud, G., Belin, M. F., and Ghersi-Egea, J. F. (2006). Rat choroid plexuses contain myeloid progenitors capable of differentiation toward macrophage or dendritic cell phenotypes. Glia 54, 160-171. doi: $10.1002 /$ glia.20373

Nathanson, J. A., (1980). beta-Adrenergic-sensitive adenylate cyclase in choroid plexus: properties and cellular localization. Mol. Pharmacol. 18, 199-209.

Nilsson, C., Ekman, R., Lindvall-Axelsson, M., and Owman, C. (1990). Distribution of peptidergic nerves in the choroid plexus, focusing on coexistence of neuropeptide $\mathrm{Y}$, vasoactive intestinal polypeptide and peptide histidine isoleucine. Regul. Pept. 27, 11-26. doi: 10.1016/0167-0115(90)90201-7

Rasband, W. (1997-2009). ImageJ. Bethesda, MD: U. S. National Institutes of Health. Available online at: http://rsb.info.nih.gov/ij

Smith, D. E. (1966). Morphological changes occurring in the developing chick choroid plexus. J. Comp. Neurol. 127, 381-388. doi: 10.1002/cne.901270306 
Sottocornola, R., Royer, C., Vives, V., Tordella, L., Zhong, S., Wang, Y., et al. (2010). ASPP2 binds Par-3 and controls the polarity and proliferation of neural progenitors during CNS development. Dev. Cell 19, 126-137. doi: 10.1016/j.devcel.2010.06.003

Stastny, F., and Rychter, Z. (1976). Quantitative development of choroid plexuses in chick embryo cerebral ventricles. Acta Neurol. Scand. 53, 251-259. doi: 10.1111/j.1600-0404.1976.tb04346.x

Tiniakos, D., Anagnostou, V., Stavrakis, S., Karandrea, D., Agapitos, E., and Kittas, C. (2004). Ontogeny of intrinsic innervation in the human kidney. Anat. Embryol. (Berl) 209, 41-47. doi: 10.1007/s00429-004-0420-3

Tramu, G., Pillez, A., and Leonardelli, J. (1983). Serotonin axons of the ependyma and circumventricular organs in the forebrain of the guinea pig. An immunohistochemical study. Cell Tissue Res. 228, 297-311. doi: 10.1007/BF00204880

Tsuker, M. (1947). Innervation of the choroid plexus. Arch. Neurol. Psychiatry 58, 474-483. doi: 10.1001/archneurpsyc.1947.02300330086007

Uwanogho, D., Rex, M., Cartwright, E. J., Pearl, G., Healy, C., Scotting, P. J., et al. (1995). Embryonic expression of the chicken Sox2, Sox3 and Sox11 genes suggests an interactive role in neuronal development. Mech. Dev. 49, 23-36. doi: 10.1016/0925-4773(94)00299-3
Vigh, B., Manzano e Silva, M. J., Frank, C. L., Vincze, C., Czirok, S. J., Szabo, A., et al. (2004). The system of cerebrospinal fluid-contacting neurons. Its supposed role in the nonsynaptic signal transmission of the brain. Histol. Histopathol. 19, 607-628. Available online at: http://www.hh.um.es/pdf/Vol_19/19_2/Vigh-19-607-628-2004.pdf

$\mathrm{Xu}, \mathrm{T}$., and Bajjalieh, S. M. (2001). SV2 modulates the size of the readily releasable pool of secretory vesicles. Nat. Cell Biol. 3, 691-698. doi: 10.1038/ 35087000

Conflict of Interest Statement: The authors declare that the research was conducted in the absence of any commercial or financial relationships that could be construed as a potential conflict of interest.

Copyright $\odot 2015$ Prasongchean, Vernay, Asgarian, Jannatul and Ferretti. This is an open-access article distributed under the terms of the Creative Commons Attribution License (CC BY). The use, distribution or reproduction in other forums is permitted, provided the original author(s) or licensor are credited and that the original publication in this journal is cited, in accordance with accepted academic practice. No use, distribution or reproduction is permitted which does not comply with these terms. 


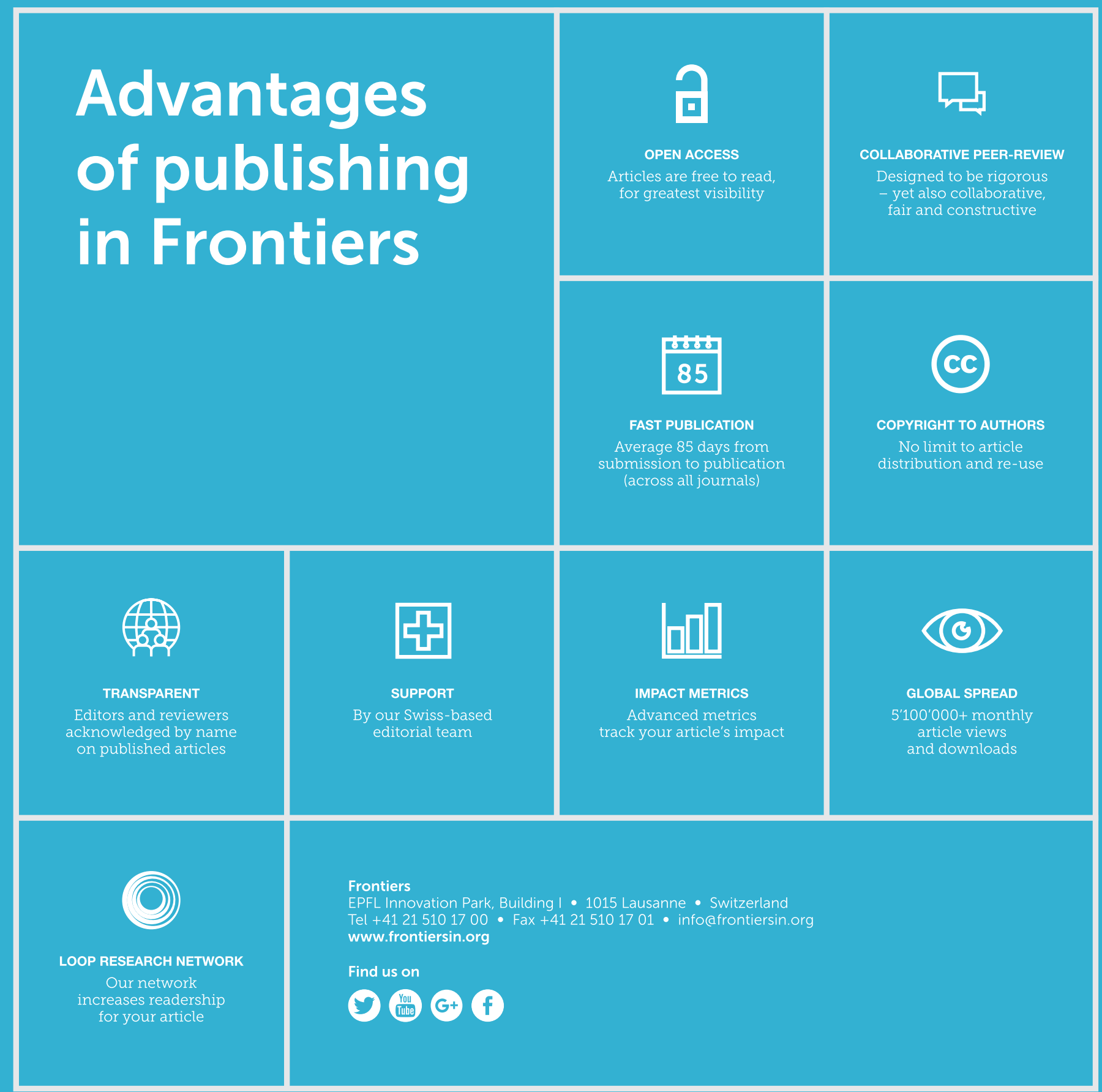

